\title{
Seismic Evaluation Procedure
}

\section{FOR EQUIPMENT IN}

U.S. DEPARTMENT OF ENERGY

\section{FACILITIES}

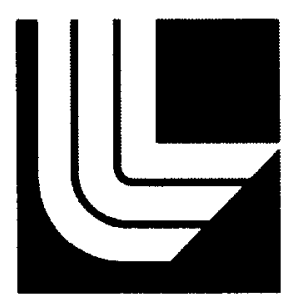

Lawrence Livermore National Laboratory

University of California

Livermore, California 94550

\section{March 1997}

\section{Prepared for \\ U. S. DEPARTMENT OF ENERGY}

Office of Defense Programs

Office of Environment, Safety and Health 


\section{DISCLAIMER}

This document was prepared as an account of work sponsored by an agency of the United States Government. Neither the United States Government nor the University of California nor any of their employees, makes any warranty, express or implied, or assumes any legal liability or responsibility for the accuracy, completeness, or usefulness of any information, apparatus, product, or process disclosed, or represents that its use would not infringe privately owned rights. Reference herein to any specific commercial product, process, or service by trade name, trademark, manufacturer, or otherwise, does not necessarily constitute or imply its endorsement, recommendation, or favoring by the United States Government or the University of California. The views and opinions of authors expressed herein do not necessarily state or reflect those of the United States Government or the University of California, and shall not be used for advertising or product endorsement purposes.

This report has been reproduced directly from the best available copy.

Available to DOE and DOE contractors from the Office of Scientific and Technical Information P.O. Box 62, Oak Ridge, TN 37831

Prices available from (615) 576-8401, FTS 626-8401

Available to the public from the National Technical Information Service

U.S. Department of Commerce 5285 Port Royal Rd., Springfield, VA 22161 


\section{Seismic Evaluation Procedure}

\section{FOR EQUIPMENT IN}

U.S. DEPARTMENT OF ENERGY

FACILITIES

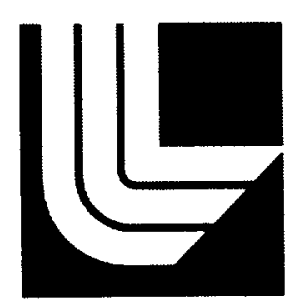

Lawrence Livermore National Laboratory

University of California

Livermore, California 94550

March 1997

Prepared for

\section{U. S. DEPARTMENT OF ENERGY}

Office of Defense Programs

Office of Environment, Safety and Health 



\section{EXECUTIVE SUMMARY}

At U.S. Department of Energy (DOE) facilities, safety analyses and facility-specific action may require the evaluation of systems and components subjected to seismic hazards. DOE has a program that provides guidance for evaluating DOE equipment and distribution systems using experience data from past seismic events and shake table tests. The program relies on the use of facility walkdowns as a means of efficiently identifying and fixing deficiencies of systems and components. Screening criteria is applied with the walkdowns such that items that pass the criteria are accepted without detailed analysis or testing. This report provides the implementation procedure for the program.

A primary objective of the DOE Seismic Evaluation Procedure is to provide comprehensive guidance for consistent seismic evaluations of equipment and distribution systems in DOE facilities. Due to the evolution of design and operating requirements, developments in engineering technology, and differing hazards and missions, DOE facilities embody a broad spectrum of design features for earthquake resistance. The earliest-vintage facilities often have the least seismic design considerations and potentially exhibit the greatest difference between their design basis and what DOE requires today for seismic design criteria for new facilities. The approach sometimes used to review the seismic capacity of equipment and distribution systems includes sophisticated evaluations or qualification testing that can be very time consuming, complex, and costly. This Procedure is designed to be a cost-effective method of enhancing the seismic safety of facilities by emphasizing the use of facility walkdowns and engineering judgment based on seismic experience data.

The DOE Seismic Evaluation Procedure is adapted from Part II of Revision 2 of the Seismic Qualification Utility Group (SQUG) Generic Implementation Procedure (GIP) (Ref. 1) used by the nuclear power industry. The SQUG GIP provided a procedure for resolving a U.S. Nuclear Regulatory Commission (NRC) unresolved safety issue through the use of experience and generic test data of equipment in industrial facilities subjected to strong motion seismic events. With a Safety Evaluation Report (Ref. 2), the NRC approved the program implemented by the SQUG GIP.

The DOE Seismic Evaluation Procedure builds on the procedures and screening criteria in the SQUG GIP by incorporating DOE-specific requirements and guidance and broadening the application of the experience-based methodology to equipment classes not contained in the SQUG GIP. The DOE Procedure has information from DOE Orders and Standards, DOE state-of-thepractice manuals for seismic upgrades of equipment, the Seismic Evaluation Procedure (SEP-6) used at the Savannah River Site (Ref. 3), and other documents from DOE sites that discuss experience-based methodologies and guidelines. The scope of equipment covered in the DOE Procedure includes classes of equipment from the SQUG GIP, such as batteries on racks, motor control centers, switchgear, valves, pumps, motor generators, tanks, cable and conduit raceway systems, and relays. In addition, the DOE Procedure includes guidelines for evaluating the seismic adequacy of piping systems, HVAC ducts, glove boxes, unreinforced masonry (URM) walls, and other classes of equipment in DOE facilities. The provisions of the DOE Procedure have been subjected to independent technical reviews as discussed in Section 1.4.2 and a letter that provides a summary of the reviews is attached at the end of the Foreword.

This report is divided into five parts in order to identify the relationships of the DOE procedure with the SQUG GIP. Part I is titled the General Approach for DOE Seismic Evaluation Procedure and contains the first four chapters. Part II is titled the Seismic Evaluation Procedures Modified from the SQUG GIP and is based on corresponding sections in the SQUG GIP. Part III, which contains Chapters 8 and 9, is titled the Seismic Evaluation Procedures Adopted Directly from the SQUG GIP and is nearly identical to corresponding sections in the SQUG GIP. Part IV is titled the Seismic Evaluation Procedures Developed Uniquely for the DOE and contains Chapter 10. Finally, Part V is titled Additional Seismic Evaluation Procedures Modified from the SQUG GIP and is based on corresponding sections in the SQUG GIP. 



\section{TABLE OF CONTENTS}

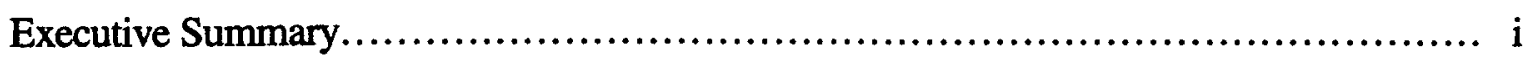

Table of Contents .................................................................... ii

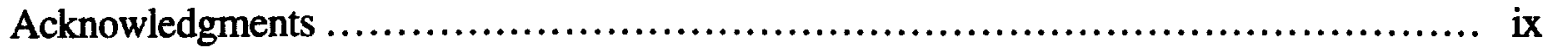

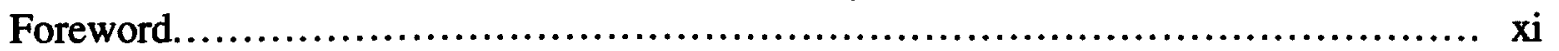

Review of DOE Seismic Evaluation Procedure.................................... xvii

\section{Part I - General Approach for DOE Seismic Evaluation Procedure}

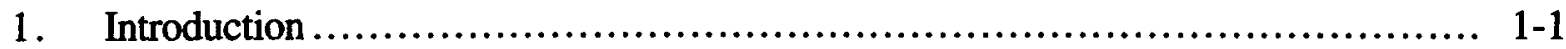

1.1 Purpose of the DOE Seismic Evaluation Procedure 1-1

1.2 DOE Orders and Standards $1-2$

1.3 Use of Seismic Experience Data in Nuclear Power Plants 1-2

1.4 Use of Seismic Experience Data in DOE Facilities $1-7$

1.5 DOE License for EPRI / SQUG Material 1-26

2. Screening Evaluation and Walkdown Procedure ............................. 2-1

2.1 Approach in the DOE Seismic Evaluation Procedure 2-1

2.2 Peer Review 2-15

$\begin{array}{ll}2.3 & \text { Preparation for the Evaluation }\end{array}$

3. Seismic Evaluation Personnel............................................... 3-1

3.1 Introduction 3-1

3.2 Seismic Capability Engineers 3-1

3.3 Other Support Personnel 3-4

3.4 Training 3-6

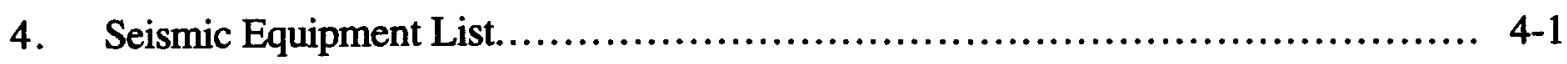

4.1 General Approach 4-1

4.2 Identification of Facility Safety Requirements 4-2

4.3 Postulated Facility Conditions 4-2

4.4 System Interaction Considerations 4-3

4.5 Seismic Vulnerability Considerations $4-4$

4.6 Operational Review 4-4 


\section{Part II - Seismic Evaluation Procedures Modified from the SQUG GIP}

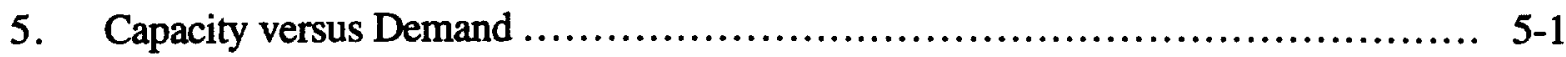

5.1 Introduction 5-1

5.2 Seismic Demand $5-1$

5.3 Equipment Capacity $5-8$

5.4 Experience-Based Capacity Compared to Seismic Demand 5-11

6. Anchorage Data and Evaluation Procedure .................................. $6-1$

6.1 Introduction 6-1

6.2 Anchorage Installation Inspection 6-2

6.3 Anchorage Capacity Determination 6-10

6.4 Anchorage Demand Determination 6-62

6.5 Comparison of Capacity to Demand 6-71

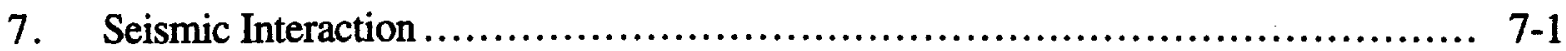

$\begin{array}{lll}7.1 & \text { Introduction } & 7-1\end{array}$

7.2 Interaction Effects

$\begin{array}{ll}7.3 & \text { DOE Guidance }\end{array}$

$\begin{array}{ll}7.4 & \text { Evaluation of Interaction Effects }\end{array}$ 


\section{Part III - Seismic Evaluation Procedures Adopted Directly from the SQUG GIP}

8. Equipment Class Evaluations Using Caveats for the Reference Spectrum and / or GERS

8.1 Electrical Equipment

8.1.1 Batteries on Racks

8.1-3

8.1.2 Motor Control Centers

8.1-8

8.1.3 Low-Voltage Switchgear

8.1-14

8.1.4 Medium-Voltage Switchgear

$8.1-20$

8.1.5 Distribution Panels

$8.1-26$

8.1.6 Transformers

8.1-31

8.1.7 Battery Chargers and Inverters

$8.1-37$

8.1.8 Instrumentation and Control Panels

$8.1-44$

8.1.9 Instruments on Racks

$8.1-47$

8.1.10 Temperature Sensors

$8.1-52$

8.2 Mechanical Equipment

8.2.1 Fluid-Operated / Air-Operated Valves

8.2-1

8.2.2 Motor-Operated / Solenoid-Operated Valves

8.2-9

8.2.3 Horizontal Pumps

$8.2-21$

8.2.4 Vertical Pumps

8.2-24

8.2.5 Chillers

8.2-27

8.2.6 Air Compressors

8.2-30

8.2.7 Motor-Generators

8.2-33

8.2.8 Engine-Generators

8.2-35

8.2.9 Air Handlers

8.2-37

8.2.10 Fans

$8.2-40$

9. Equipment Class Evaluations Using Screening Procedures....................... 9.1-1

9.1 Aboveground Tanks

9.1.1 Vertical Tanks

9.1.2 Horizontal Tanks and Heat Exchangers

9.1-2

9.2 Raceway Systems
9.2.1 Cable and Conduit Raceway Systems

$9.1-40$

9.2-1 
Part IV - Seismic Evaluation Procedures Developed Uniquely for the DOE

10. Equipment Class Evaluations Using Screening Procedures or General Guidelines.......................................................... 10.1-1

10.1 Piping Systems

10.1.1 Piping

$10.1-2$

10.1.2 Underground Piping $10.1-22$

10.2 Mechanical Equipment

10.2.1 HEPA Filters 10.2-1

10.2.2 Glove Boxes 10.2-6

10.2.3 Miscellaneous Machinery 10.2-11

10.3 Other Tanks

10.3.1 Underground Tanks

10.3-1

10.3.2 Canisters and Gas Cylinders 10.3-4

10.4 Duct Systems

10.4.1 HVAC Ducts 10.4-1

Architectural Features and Components
$10.5 .1 \quad$ Unreinforced Masonry (URM) Walls

10.5.2 Raised Floors $10.5-49$

10.5.3 Storage Racks 10.5-56 


\section{Part V - Additional Seismic Evaluation Procedures Modified from the SQUG GIP}

11. Relay Functionality Review .................................................... 11

11.1 Introduction 11-1

11.2 Seismic Capacity of Relays 11-1

11.3 Seismic Demand on Relays $11-5$

11.4 Relay Walkdown $11-7$

11.5 Outliers 11-9

12. Outlier Identification and Resolution...................................... 12-1

12.1 Introduction $12-1$

12.2 Outlier Resolution 12-1

12.3 Grouping and Pooling of Outliers 12-3

13. Documentation.............................................................. 13-1

13.1 Introduction 13-1

13.2 Screening Evaluation Work Sheets 13-1

13.3 Outlier Seismic Evaluation Sheets 13-4

13.4 Screening Evaluation and Data Sheets 13-4

13.5 Equipment Seismic Evaluation Report 13-8

14. References ....................................................................... 14 
March 1997 


\section{ACKNOWLEDGMENTS}

The following individuals participated in the development, guidance, and review of this Procedure: DOE Steering Group:

- Fred Loceff (Chairman) - Westinghouse Savannah River Company

- Larry Goen - Los Alamos National Laboratory (principal author of Sections 10.2.1 and 10.2.2)

- R. Joe Hunt - Lockheed Martin

- Leon McGovern - Rocky Flats Environmental Technology Center

- Richard Stroud and Jeffrey Wang - Mason \& Hanger Company

Technical Support and Review:

- Robert Budnitz - Future Resources Associates, Inc.

- Robert Kennedy - Structural Mechanics Consulting

- Loring Wyllie - Degenkolb Engineers

- Ed Wais - E.A. Wais and Associates

- John Reed - J. R. Benjamin and Associates

Project Team at Lawrence Livermore National Laboratory:

- Robert Murray - Project Manager (principal author of Chapter 7, Section 10.3.2, and Section 10.5.1)

- Stanley Sommer - Technical Editor of DOE Seismic Evaluation Procedure (principal author of Chapters $1,2,3,6,12,13$, and 14 as well as Sections 10.2.3, 10.5.2, and 10.5.3)

- Quazi Hossain (principal author of Chapter 4)

- Thomas Nelson (principal author of Chapter 5)

- Mark Eli

- David McCallen

Technical Support for Project Team:

- Westinghouse Savannah River Company - George Antaki (principal author of Section 10.1.1) and Gary Driesen (principal author of Section 10.4.1)

- EQE International - Farzin Beigi, John Dizon, Stephen Eder (principal author of Chapter 11), Gregory Hardy, Gayle Johnson, Kelly Merz, Michael Salmon, and Stephen Short (principal author of Sections 10.1.2 and 10.3.1)

- Science Applications International Corporation - Bhasker Tripathi 
Dawn Francis and Susan Birnbaum of Lawrence Livermore National Laboratory provided editorial assistance in the preparation of this report. Belinda Burke of EQE International provided electronic copies of Revision 2 of the SQUG GIP.

\section{DOE Support and Review:}

The following DOE organizations and individuals provided financial support and/or technical review:

- DP-45 - Jeffrey Kimball, Krishan Mutreja, Michael Davister

- EH-32 - Dan Guzy (principal author of Section 1.2)

- EH-34 - James Hill, Subir Sen

- EH-31 - Harish Chander

- DOE-SR - Brent Gutierrez 


\section{FOREWORD}

\section{Use of the DOE Seismic Evaluation Procedure}

The guidance provided in the DOE Seismic Evaluation Procedure must be used with the appropriate training and judgment as discussed throughout the Procedure. Before applying the methodology in this Procedure, the reference material for the SQUG GIP should be carefully studied. The methodology is not a "cookbook" approach because it requires an extensive use of judgment and a thorough understanding of the basis for the methodology. If differences are not marked appropriately in the sections of the DOE Seismic Evaluation Procedure which are taken directly or modified from the SQUG GIP, then the corresponding information in the SQUG GIP should be followed.

Engineers who use the DOE Seismic Evaluation Procedure are responsible for its appropriate application, their level of training, and their use of judgment. The developers of the Procedure assume no responsibility for specific applications of the methodology.

Peer review is a vitally important component of seismic evaluations of equipment and distribution systems at DOE facilities. The evaluation procedures described in the DOE Seismic Evaluation Procedure involve an extensive use of engineering judgment. This type of judgment must be independently reviewed to ensure that significant details are not overlooked or improperly evaluated.

\section{Differences Between DOE Seismic Evaluation Procedure and the SQUG (Industry) GIP}

As listed in the table following this discussion, the DOE Seismic Evaluation Procedure expands the SQUG GIP by incorporating DOE-specific requirements and guidance and by broadening the application of the experience-based methodology to equipment classes not contained in the SQUG GIP. The DOE Seismic Evaluation Procedure does not modify the technical content or numerical values of the equipment classes and anchorage procedure provided in the SQUG GIP, except where appropriately marked and referenced.

An attempt was made in the development of the DOE Seismic Evaluation Procedure to eliminate repetition in the SQUG GIP in order to make the procedure less cumbersome. This is most dramatically evidenced in Chapter 6 on anchorage and in Chapter 13 on documentation. Caveats for the Reference Spectrum and/or GERS were removed from the equipment evaluations in Chapter 8 that duplicate the requirements of Chapter 6 on anchorage, Chapter 7 on seismic interaction, or Chapter 11 on relays. The intent of these caveats is met by satisfying the procedures in Chapters 6, 7, and 11.

Throughout the DOE Seismic Evaluation Procedure, nuclear power plant and NRC-specific requirements and commitments from the SQUG GIP were removed and replaced with DOE facility information. Several of the sections in the DOE Seismic Evaluation Procedure reflect DOE guidance and standards and are considerably different than equivalent sections in the SQUG GIP. These sections have generic changes in order to integrate the experience-based methodology with DOE Orders and Standards. Portions of Chapters 1 and 3 on the DOE use of experience-based criteria, Chapter 4 on the Seismic Equipment List, Chapter 5 on comparing seismic capacity to demand, and Chapter 7 on seismic interaction were modified to reflect DOE provisions. 
The DOE Seismic Evaluation Procedure has three major adaptations for non-reactor applications:

- In the SQUG GIP, the "40-foot rule" permits the use of the Bounding Spectrum to define the capacity for equipment with fundamental frequencies greater than about 8 Hertz and mounted within $\mathbf{4 0}$ feet above effective grade. The Bounding Spectrum has a generic deamplification of 1.5 as compared to the Reference Spectrum and is a simplified way for reducing the experience-based capacity to account for in-structure amplification. Since the "40-foot rule" was developed for nuclear power plants with massive and stiff shear wall structures that are not the typical structural types at DOE facilities, the DOE Seismic Evaluation Procedure does not have the "40-foot rule" or the Bounding Spectrum. Instead, the DOE approach uses the Reference Spectrum to define equipment capacity and to compare with in-structure response spectra developed at equipment locations.

- The DOE Seismic Evaluation Procedure has equipment classes that are not in the SQUG GIP. Chapter 10 on equipment class evaluations using screening procedures and general guidelines contains the additional equipment classes. Further information on the classes of equipment in Chapter 10 is provided in Sections 2.1.3.4 and 2.1.3.4.4.

- The relay review for DOE facilities contained in Chapter 11 focuses primarily on identifying low ruggedness relays and comparing seismic capacity to demand. The detailed procedure which is required for relay functionality reviews in nuclear power plants is not included in the DOE Seismic Evaluation Procedure.

As the SQUG GIP is revised, the appropriate modifications will be made to the DOE Seismic Evaluation Procedure. Currently, Revision 3 of the SQUG GIP (Ref. 4) is being reviewed by the NRC. Modifications have been made for expansion anchor capacity reduction factors in Table 6.3-5 and for panel amplification factors in Table 11.3-1 of the DOE Seismic Evaluation Procedure.

The following table lists the sections of the DOE Seismic Evaluation Procedure that contain information from Part II of Revision 2 of the SQUG GIP. In some cases, the section in the DOE Seismic Evaluation Procedure is considerably different than the section(s) from the SQUG GIP. For the DOE Seismic Evaluation Procedure sections listed below, footnotes appear in the document that identify the corresponding SQUG GIP sections. All the tables and figures from the SQUG GIP are also identified in the DOE Seismic Evaluation Procedure. In the table below, modifications (Mod.) from the appropriate sections of the SQUG GIP are denoted in these sections of the DOE Seismic Evaluation Procedure with words in italics. 


\section{Relationship of Sections in the DOE Seismic Evaluation Procedure}

and the SQUG GIP

\begin{tabular}{|c|c|c|}
\hline $\begin{array}{c}\text { Section in } \\
\text { DOE Seismic } \\
\text { Evaluation } \\
\text { Procedure } \\
\end{array}$ & Mod. & Section(s) from the SQUG GIP \\
\hline 1.3 & & Section 1.2 \\
\hline 2.1 & & Sections $1.3,3.3,4.0,4.2,4.3,4.4$ and 4.5 \\
\hline 2.3 & & Appendices $\mathrm{E}$ and $\mathrm{F}$ \\
\hline$\overline{3.1}$ & & Section 2.0 \\
\hline$\overline{3.2}$ & & Section 2.4 \\
\hline$\overline{3.3}$ & & Sections $2.2,2.3$, and 2.5 \\
\hline$\overline{5.3}$ & & Section 4.2 \\
\hline$\overline{5.4}$ & & Section 4.2 \\
\hline 6. & & Section 4.4 and Appendix C \\
\hline 7.1 & & Section D.1 \\
\hline$\overline{7.2}$ & & Sections D.2, D.3, D.4, and D.6 \\
\hline 7.5 & & Section D.5 \\
\hline 8.1 .1 & * & Section B.15 \\
\hline 8.1 .2 & * & Section B.1 \\
\hline 8.1 .3 & * & Section B.2 \\
\hline 8.1 .4 & * & Section B.3 \\
\hline 8.1 .5 & * & Section B.14 \\
\hline 8.1 .6 & * & Section B.4 \\
\hline 8.1 .7 & * & Section B.16 \\
\hline 8.1 .8 & * & Section B.20 \\
\hline 8.1 .9 & * & Section B.18 \\
\hline 8.1 .10 & * & Section B.19 \\
\hline 8.2 .1 & * & Section B.7 \\
\hline 8.2 .2 & * & Section B.8 \\
\hline 8.2 .3 & * & Section B.5 \\
\hline 8.2 .4 & * & Section B.6 \\
\hline 8.2 .5 & * & Section B.11 \\
\hline 8.2 .6 & * & Section B.12 \\
\hline 8.2 .7 & * & Section B.13 \\
\hline 8.2 .8 & * & Section B.17 \\
\hline 8.2 .9 & * & Section B.10 \\
\hline 8.2 .10 & * & Section B.9 \\
\hline 9.1 .1 & * & Sections $7.2,7.3,7.5$, and 7.6 \\
\hline 9.1 .2 & * & Sections $7.2,7.4,7.5$, and 7.6 \\
\hline 9.2 .1 & * & Sections $8.0,8.2,8.3,8.4$, and 8.5 \\
\hline 11.2 & & Section 6.4 \\
\hline 11.3 & & Section 6.4 \\
\hline 11.4 & & Section 6.5 \\
\hline 11.5 & & Section 6.6 \\
\hline
\end{tabular}


Relationship of Sections in the DOE Seismic Evaluation Procedure and the SQUG GIP (Continued)

\begin{tabular}{|c|l|l|}
\hline $\begin{array}{c}\text { Section in } \\
\text { DOE Seismic } \\
\text { Evaluation } \\
\text { Procedure }\end{array}$ & Mod. & Section(s) from the SQUG GIP \\
\hline 12.1 & & Section 5.0 \\
\hline 12.2 & & Section 5.2 \\
\hline 12.3 & & Section 5.3 \\
\hline 12.4 & & Section 5.4 \\
\hline 13.1 & & Section 9.0 \\
\hline 13.2 & & Appendix G \\
\hline 13.3 & & Section 4.6 \\
\hline 13.4 & & Section 9.4 \\
\hline 14. & & Section 10 \\
\hline
\end{tabular}

Acronyms Used in the DOE Seismic Evaluation Procedure

AF in-cabinet Amplification Factor

DBE Design Basis Earthquake

DOE United States Department of Energy

EPRI Electric Power Research Institute

ESER Equipment Seismic Evaluation Report

$\mathrm{F}_{\mathrm{ED}} \quad$ Experience Data Factor

GERS Generic Equipment Ruggedness Spectrum

GIP Generic Implementation Procedure

IDS In-cabinet Demand Spectrum

IRS In-structure Response Spectrum

LANL Los Alamos National Laboratory

LLNL Lawrence Livermore National Laboratory

NEHRP National Earthquake Hazards Reduction Program

NRC Nuclear Regulatory Commission

OSES Outlier Seismic Evaluation Sheets

$P_{\text {all }} \quad$ Allowable pullout capacity of installed anchors 
PC Performance Category

$\mathrm{Sa}_{\mathrm{i}} \quad$ Spectral acceleration at frequency $\mathrm{f}_{\mathrm{i}}$

SAR Safety Analysis Report

SCE Seismic Capability Engineer

SDS Seismic Demand Spectrum

SEDS Screening Evaluation and Data Sheets

SEL Seismic Equipment List

SEWS Screening Evaluation Work Sheets

SF Scale Factor

SQUG Seismic Qualification Utility Group

SRS Savannah River Site

SRT Seismic Review Team

SSC Structure, Systems, and Component

SSRAP Senior Seismic Review and Advisory Panel

STD Standard

UBC Uniform Building Code

USI Unresolved Safety Issue

$\mathrm{V}_{\text {all }} \quad$ Allowable shear capacity of installed anchors

ZPA Zero Period Acceleration 


\section{March 1997}

Dr. Robert C. Murray

Geologic and Atmospheric Hazards Project, L-224

Lawrence Livermore National Laboratory

Livermore, CA 94550

\section{SUBJECT: $\quad$ Review of DOE Seismic Evaluation Procedure}

The DOE Seismic Evaluation Procedure (SEP), whose final version is dated November 11, 1996 (and including a series of revisions made through early March, 1997), has been reviewed by the team of reviewers that you constituted specially for this purpose. The team includes Robert J. Budnitz, Robert P. Kennedy, and Loring A. Wyllie Jr. The expertise of the team covers the disciplines of systems analysis and regulatory issues (Budnitz), and seismic and structural engineering (Kennedy and Wyllie). Although all three team members examined the whole report, the individual team reviewers concentrated their efforts in their fields of expertise.

Preliminary drafts of the SEP were reviewed over the past year, at a meeting in July 1996, and then through the autumn. Another draft developed recently was reviewed at a meeting that took place on November 22, 1996 at Palo Alto, CA. All three members of the review team were present, along with the principal authors, Stanley C. Sommer and Robert C. Murray of LLNL. Comments from that meeting were then incorporated into the finalversion which has since been forwarded to our team and with which we concur. This means that all of our technical concerns with previous drafts have now been resolved.

Our review comments are three in number:

1) We strongly endorse the use of the SEP procedures by DOE for seismic evaluations of existing facilities.

2) The basis for much of the SEP is the SQUG (Seismic Qualification Utility Group) seismic-review procedures developed for nuclear power plants and endorsed by the NRC, with heavy multi-year involvement by an independent review body, the Senior Seismic Review and Advisory Panel (SSRAP) and by an initiallyskeptical NRC. However, the scope of the SQUG guidance does not cover all of the safety-relevant equipment in DOE facilities, so DOE has developed several extensions to evaluate additional equipment categories. We believe that these extensions, that allow experience-based evaluation by rules in lieu of rigorous analysis or testing, are the single most cost-effective way to maximize the seismic-safety benefits achieved for any given cost expenditure. Therefore, we strongly support the extensions in the SEP to the additional equipment categories contained in the current document. 
However, it must be recognized that these extensions have not undergone the same degree of review and consensus-building as the SQUG procedures for nuclear power plants, which involved a multi-year, very costly process. We believe it important to point out that the DOE extensions have been reviewed by two of the five original SSRAP members (Kennedy and Wyllie) but the level of review has been much less than they expended on the SQUG procedures. Also, no regulatory body has been involved here that is comparable to NRC. While we are not convinced that such a costly and indepth review is necessary, it is important to note that the pedigree of the DOE extensions is not similar to that of the SQUG procedures.

3) We also support the cautious extension of these experience-based seismic evaluation methods to the design and evaluation of new equipment, if the areas of application are carefully selected. In fact, we believe that this approach can be at least as effective, and in some important areas can be an improvement over NRC's Standard Review Plan sections for many categories of equipment. Designing new facilities for improved earthquake performance can best be achieved by providing sufficient anchorage, bracing, and ductile details rather than through principal reliance on extensive and expensive dynamic analysis.

The three of us wish to thank you for the opportunity to have participated in reviewing this important project. With warmest regards,

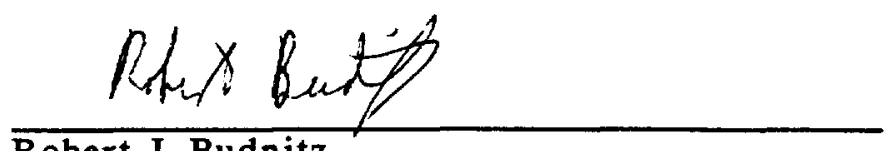

Robert J. Budnitz

Future Resources Associates Inc.
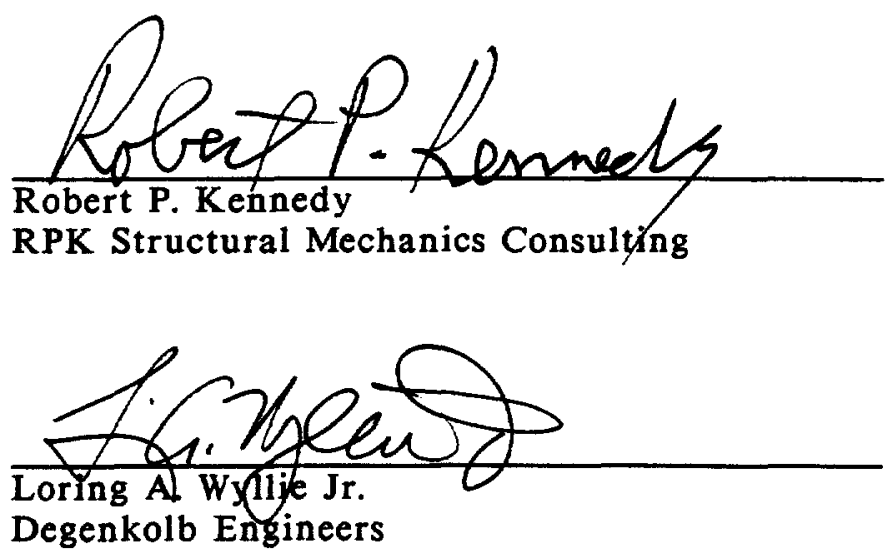


\section{Part I}

\section{General Approach for DOE Seismic Evaluation Procedure}





\section{INTRODUCTION}

\subsection{PURPOSE OF THE DOE SEISMIC EVALUATION PROCEDURE}

U.S. Department of Energy (DOE) facilities need to have adequate measures for protection of public health and safety, for on-site worker life safety, for protection of the environment, and for investment protection in the event of natural phenomena hazards, specifically earthquakes. Due to the evolutionary nature of design and operating requirements as well as developments in engineering technology, DOE facilities embody a broad spectrum of design features for earthquake resistance. These features depend on factors such as vintage of the facility design and construction and hardware supplier practices at the time of design and construction. The earliest-vintage facilities often have the least design consideration for seismic and potentially exhibit the greatest difference between their design basis and what DOE requires today for seismic design criteria for new facilities.

Seismic evaluations of essential systems and equipment at many DOE facilities will be conducted over the next several years. For many of these systems and components, few, if any, seismic requirements applied to the original design, procurement, installation, and maintenance process and therefore, the evaluation of the seismic adequacy of existing systems and components presents a difficult challenge. The purpose of this Seismic Evaluation Procedure is to summarize a technical approach and provide generic procedures and documentation requirements that can be used at DOE facilities to evaluate the seismic adequacy of mechanical and electrical equipment.

This procedure is meant to comply with DOE Policy, Orders, and Standards as discussed in Section 1.2. The scope of equipment covered in this procedure includes active mechanical and electrical equipment such as batteries on racks, motor control centers, switchgear, distribution panels, valves, pumps, HVAC equipment, engine generators, and motor generators. In addition, this generic procedure includes guidelines for evaluating the seismic adequacy of tanks, heat exchangers, cable and conduit raceway systems, piping systems, HVAC ducts, architectural features and components, and relays.

The Seismic Evaluation Procedure is intended to provide DOE facility managers, safety professionals, and engineers with a practical procedure for evaluating the seismic adequacy of equipment and distribution systems. Often the approach used to review the seismic capacity of equipment is to conduct sophisticated evaluations that can be very time consuming, complex, and costly. Much of the available funding is spent on analysis rather than on the real objective of increasing the seismic capacity of equipment and distribution systems. This procedure is designed to be an extremely cost-effective method of enhancing the seismic safety of facilities and reducing the potential for major economic loss that can result from equipment and systems damaged or destroyed by an earthquake.

The following sections provide the background for the development of the DOE Seismic Evaluation Procedure. First, DOE Orders and Standards that address natural phenomena hazards are discussed since a purpose of the DOE Seismic Evaluation Procedure is to provide a procedure that satisfies the requirements of these Orders and their supporting standards. Second, a methodology that was developed for older nuclear power plants to satisfy safety issues raised in the late $1970 \mathrm{~s}$ is discussed. This methodology or procedure is based on seismic experience data and screening evaluations. The nuclear power industry concluded that the methodology was the most viable option to resolve safety issues as compared with testing or analysis. Testing or analysis were often not viable due to problems of removal, decontamination, shipment of equipment for testing, access, and potential damage from in-situ testing. Next, the extension to DOE facilities of the procedure developed for nuclear power plants is discussed. Applications at nuclear power plants and DOE facilities have demonstrated that a seismic evaluation using the 
methodology based on experience data is the only viable option for many systems and components. Finally, the license which regulates the use of background material for the DOE Seismic Evaluation Procedure is discussed.

\subsection{DOE ORDERS AND STANDARDS}

The DOE Seismic Evaluation Procedure is intended to comply with DOE Policy, Orders, and Standards on natural hazards mitigation which allow for the seismic evaluation of systems and components by analysis, testing, or the use of earthquake experience data. These include DOE Order 420.1, "Facility Safety" (Ref. 5), and its Implementation Guide; a rule currently under development; and supporting Standards. The two supporting Standards most relevant to this procedure are DOE-STD-1020, "Natural Phenomena Hazards Design and Evaluation Criteria for Department of Energy Facilities" (Ref. 6) (see Section 2.4.1 of DOE-STD-1020) and DOE-STD1021, "Natural Phenomena Hazard Performance Categorization Guidelines for Structure, Systems, and Components" (Ref. 7). DOE Order 420.1 is a replacement order for DOE Order 5480.28, "Natural Phenomena Hazards Mitigation" (Ref. 8).

DOE Orders and Standards for natural phenomena hazards mitigation are closely linked to those for safety analysis. DOE Order 5480.23 (Ref. 9) requires that safety analyses be performed that develop and evaluate the adequacy of a DOE nuclear facility's safety basis and that the analyses be documented in a Safety Analysis Report (SAR). To assist in preparing a SAR, DOE-STD-1027 (Ref. 10) and DOE-STD-3009 (Ref. 11) provide guidance on hazard categorization and SAR implementation, respectively. Using a graded approach unique to DOE, systems and components are subjected to different seismic design and evaluation criteria that correspond to safety system and facility hazard classifications. The graded approach and wide diversity of DOE facilities' functions and designs require that the methodology developed for equipment in commercial nuclear power plants, as discussed in the next section, be modified for use at DOE facilities.

\subsection{USE OF SEISMIC EXPERIENCE DATA IN NUCLEAR POWER PLANTS}

\subsubsection{Background ${ }^{1}$}

The requirements for seismic design of nuclear power plants from 1960 to the present have evolved from the application of commercial building codes, which use a static load coefficient approach applied primarily to major building structures, to more sophisticated methods today. Current seismic design requirements for new nuclear power plants consist of detailed specifications that include dynamic analyses or testing of safety-related structures, equipment, instrumentation, controls, and their associated distribution systems, such as piping, cable trays, conduit, and ducts. In the late 1970s, the U.S. Nuclear Regulatory Commission (NRC) expressed the concern that nuclear equipment seismically qualified to standards preceding IEEE-344-1975 (Ref. 12) might not provide sufficient assurance of seismic adequacy. This concern was reinforced through field inspections of older-vintage nuclear power plants where equipment was found to lack adequate anchorage.

The NRC initiated Unresolved Safety Issue (USI) A-46, "Seismic Qualification of Equipment in Operating Plants" (Ref. 13) in December of 1980, to address the concern that a number of older operating nuclear power plants contained equipment which may not have been qualified to meet newer, more rigorous seismic design criteria. Much of the equipment in these operating plants was installed when design requirements, seismic analyses, and documentation were less formal than the rigorous practices currently being used to build and license nuclear power plants. However, it was realized that it would not be practical or cost-effective to develop the documentation for seismic

1 Based on Section 1.2 of SQUG GIP (Ref. 1) 
qualification or requalification of safety-related equipment using procedures applicable to modern plants. Therefore, the objective of USI A-46 was to develop alternative methods and acceptance criteria that could be used to verify the seismic adequacy of essential mechanical and electrical equipment in operating nuclear power plants. The NRC pursued several options for the resolution of USI A-46, including use of shake table testing, in-situ testing, deterministic and probabilistic analytical methods, and seismic experience data. Most options proved not to be viable because of the unavailability of older model components for testing, the high costs of component replacements, and complications of testing radiologically contaminated equipment. The NRC concluded that the use of experience data could provide a reasonable alternative for resolution of USI A-46.

In early 1982, the Seismic Qualification Utility Group (SQUG) was formed for the purpose of collecting seismic experience data as a cost-effective means of verifying the seismic adequacy of equipment in operating plants. One source of experience data was the numerous non-nuclear power plants and industrial facilities which had experienced major earthquakes. These facilities contained industrial grade equipment similar to that used in nuclear power plants. Another source of seismic experience data was shake table tests that had been performed since the mid 1970's to qualify safety-related equipment for licensing of nuclear plants. To use these sources of seismic experience data, SQUG and the Electric Power Research Institute (EPRI) collected and organized this information and developed guidelines and criteria for its use. The guidelines and criteria provided the generic means for applying experience data to verify the seismic adequacy of mechanical and electrical equipment required to be used in a nuclear power plant during and following a safe shutdown earthquake (SSE). According to 10CFR100 Appendix A (Ref. 14), the SSE is defined as the earthquake which is based upon the maximum earthquake potential considering both regional and local geology, seismology, and local subsurface materials. For nuclear power plants, the SSE is also referred to as the Design Basis Earthquake. The ground motion at the nuclear facility associated with the SSE is used for the design of equipment, structures, and systems necessary for: the integrity of the reactor coolant pressure boundary, the capability to shut down and maintain the reactor in a safe shutdown condition, and the capability to prevent or mitigate potential offsite exposures.

\subsubsection{Approach}

The approach developed by SQUG and EPRI for verifying the seismic adequacy of mechanical and electrical equipment is consistent with the intent of NRC Generic Letter (GL) 87-02, "Verification of Seismic Adequacy of Mechanical and Electrical Equipment in Operating Reactors, Unresolved Safety Issue (USI) A-46" (Ref. 15), NUREG-1030 (Ref. 16), and NUREG-1211 (Ref. 17). The approach is also consistent with the EPRI Seismic Margins Assessment Program described in Reference 18. NRC approval of the approach was based on research done at several DOE national laboratories and on extensive independent review by the Senior Seismic Review and Advisory Panel (SSRAP). The summary of the SSRAP review is contained in Reference 19. In 1987, NRC GL 87-02 required utilities to respond to USI A-46, and encouraged participation in generic resolution by using the SQUG approach, documented in the Generic Implementation Procedure (GIP) for Seismic Verification of Nuclear Plant Equipment (Ref. 1). NRC accepted the SQUG GIP (also referred to as the Industry GIP) with a generic safety evaluation report (Ref. 2). There were a few exceptions that have since been resolved and are being incorporated into Revision 3 of the SQUG GIP (Ref. 4). The SQUG GIP consists of four sets of criteria:

1) the experience-based capacity spectrum must bound the plant seismic demand spectrum,

2) the equipment item must be reviewed against certain inclusion rules and caveats,

3) the component anchorage must be evaluated, and

4) any potentially significant seismic systems interaction concerns that may adversely affect component safe shutdown function must be addressed. 
These SQUG criteria are in the form of screening evaluation guidelines. Items not passing the screen, called outliers, are not necessarily inadequate, but other seismic engineering methods must be used to further evaluate these items.

The screening evaluation adopted in the SQUG GIP is generally a conservative and rapid appraisal process that is used during a facility walkdown to verify acceptability or identify outliers by review of key physical attributes. A model of the screening evaluation process is shown in Figure 1.3-1. Items passing the screen are verified as acceptable and may be documented as such, or can be selected for a bounding sample analysis to validate the evaluation results. Items not passing the screen are not verified and are formally designated as outliers, which must be subject to more detailed review or upgrade before being accepted. The SQUG GIP screening evaluation process is performed primarily during in-plant walkdowns and for a limited set of equipment, or Safe Shutdown Equipment List (SSEL), required to bring a plant to hot shutdown and maintain it there for 72 hours. Prior to a screening evaluation, a systems review is conducted to assess the minimal and prioritized scope of equipment for the evaluation.

Results of the work in compiling earthquake experience data by SQUG found the following: (1) conventional power plant and industrial facility equipment are generally similar to that found in older, operating nuclear power plants and, (2) equipment, when properly anchored, will generally perform well in earthquakes at levels of shaking in excess of the SSE for many nuclear power plants. SQUG, EPRI, and SSRAP developed the caveats and inclusion rules that help to ensure functionality and structural integrity of equipment when using the experience-based methodology. Caveat and inclusion rules determine whether or not an item of equipment is sufficiently similar to data from past earthquake or testing experience. The SQUG program is considered by most, including the NRC and all of the SQUG utility members, to be a major engineering breakthrough and an overall success. Important methods utilized by SQUG include: utilization of screening criteria implemented during walkdowns that is coupled with review team engineering training, screening criteria primarily based on natural phenomena experience data that is supplemented with test and analysis, programmatic direction given by facility management and engineering, technical review and advice provided by an independent panel of industry experts, and establishing priority listing of systems and components based on systems analysis.

An important element of the SQUG GIP is its application by the use of specially trained and experienced seismic review teams who must exercise considerable judgment while performing the in-plant screening evaluations. Besides establishing strict qualification requirements for review team engineers, SQUG and EPRI provide a training course in the use of the implementation guidelines and procedures.

The EPRI / SQUG seismic evaluation methodology based on experience data has become a key element in the ongoing earthquake evaluations for commercial nuclear power plants. The experience-based evaluation methods address most plant components needed for safe shutdown in the event of a SSE. These components include 20 classes of electrical and mechanical equipment, cable trays and conduit systems, relays, anchorage, tanks and heat exchangers. For each type of component, the seismic evaluation methodology provides experience data that documents the performance of systems and components that have been subjected to earthquake motion. The data includes components in commercial and industrial facilities that were in the strong motion regions of major earthquakes. SQUG and EPRI have developed a seismic experience data base that includes the response of systems and components in about 100 (typically non-reactor) facilities located in areas of strong ground motion from 20 earthquakes. The earthquakes have Richter magnitudes in the range of 5.2 to 8.1 , have peak ground accelerations from $0.10 \mathrm{~g}$ to $0.85 \mathrm{~g}$, and have about 3 to 50 second durations. Soil conditions, building structure types, and location of equipment vary considerably within the data base. 
The facilities surveyed and documented contain a large number of mechanical and electrical equipment, and control and distribution systems that are identical or very similar to those found in nuclear power plants. Information sources consist of interviews with facility management and operating personnel, walkdown inspections of facilities, photographs and performance data records of systems and components, facility operating logs, and the facility's inspection reports. Design criteria and specifications, component data books, and design drawings are additional sources of information. There is diversity in equipment design, size, configuration, age, application, operating conditions, manufacturer, and quality of construction and maintenance. The earthquake experience data are useful for determining common sources of seismic damage or adverse effects of equipment and facilities, thresholds of seismic motion corresponding to various types of seismic performance, and standards in equipment construction and installation to ensure the ability to withstand anticipated seismic loads.

As an expansion of the earthquake experience data, EPRI and SQUG also collected data on shaketable qualification tests from utilities, manufacturers, and test laboratories. Results were compiled from about 300 shake table tests of equipment components, covering 15 generic classes of equipment. The objective was to compile the information by class, and to obtain generic insights, if any, that could be used to assist utilities in evaluating these equipment classes in their plants. These generic equipment ruggedness data represent substantially higher levels of seismic motion than the earthquake experience data, but in most cases, are applicable to a narrower range of equipment parameters. EPRI and SQUG also obtained available electro-mechanical relay chatter shake table tests and performed additional tests for other relays. The relay test experience data base provides capacities for about 150 specific models of relays.

Another important element of seismic experience data is information on the anchor bolts that are commonly used to attach systems and components to the supporting building structure. EPRI and SQUG have summarized capacity information for expansion anchor bolts, covering about 1200 ultimate capacity tension and shear tests. Capacity data have also been compiled for other anchor types including welded attachments, cast-in-place bolts and headed studs, grouted-in-place anchors, and cast-in-place J-hooks. 


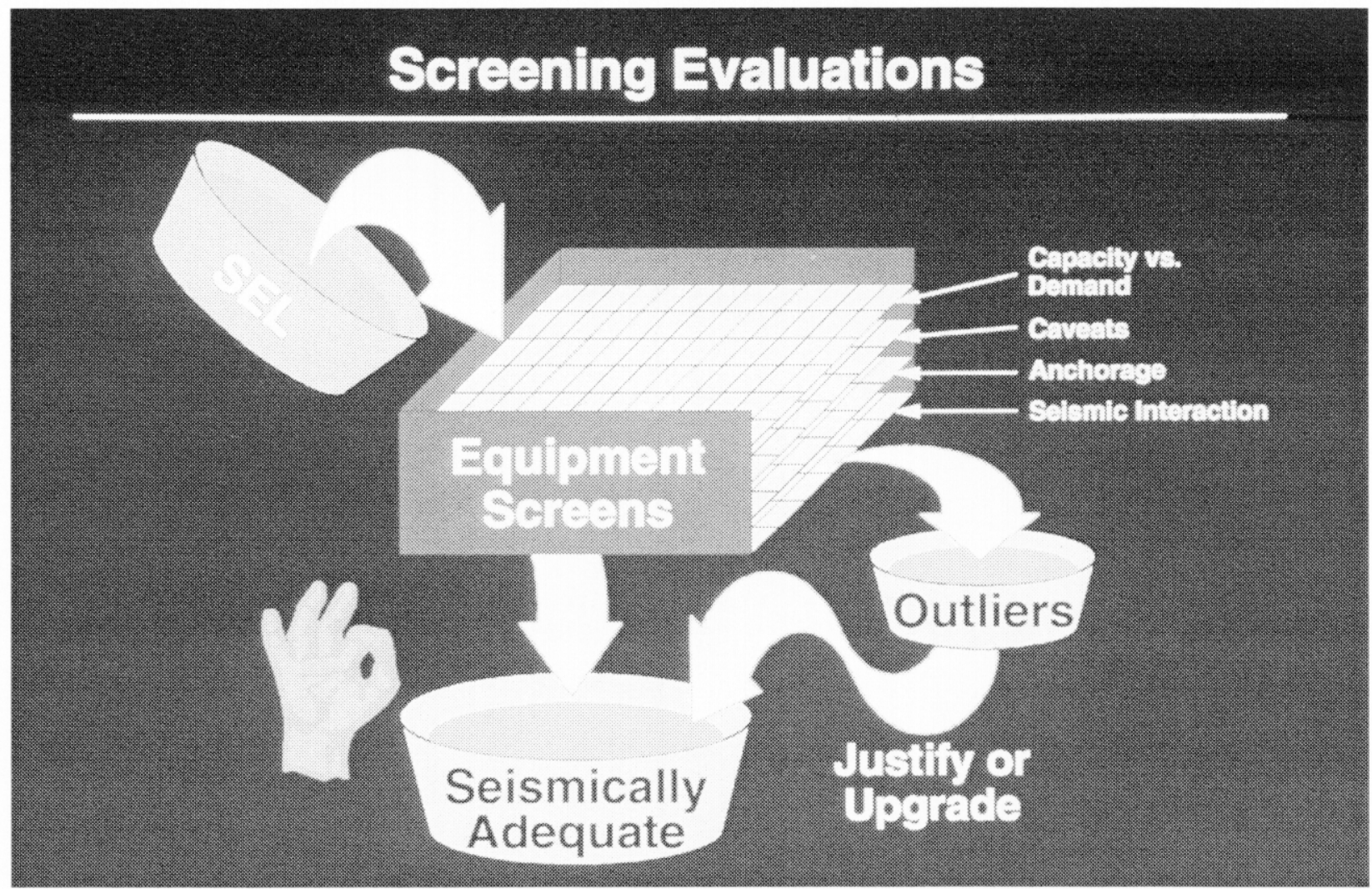

Figure 1.3-1 The DOE Seismic Evaluation Procedure contains the screening evaluation approach. The process begins with the development of the Seismic Equipment List (SEL). 


\subsection{USE OF SEISMIC EXPERIENCE DATA IN DOE FACILITIES}

\subsubsection{DOE Existing Facilities Program}

A DOE Existing Facilities Program was implemented for the development of seismic evaluation guidelines for systems and components at existing facilities. A Program Plan (Ref. 20) for the Existing Facilities Program maximizes the use of past experience in conjunction with a walkdown screening evaluation process in order to meet the policy of applicable DOE Orders and Standards. The process of evaluating existing DOE facilities for the effects of natural phenomena hazards was patterned after the SQUG program for commercial nuclear power plants, which is discussed in Section 1.3. As discussed in Section 1.5, the SQUG and EPRI reference documents, which provide the basis for the use of experience data, are being used by DOE through a special agreement between Lawrence Livermore National Laboratory (LLNL) and EPRI. The use of seismic experience data, specifically the EPRI / SQUG data, for DOE seismic evaluations was recommended in a position paper (Ref. 21) authored by personnel from many DOE facilities. In addition, a letter (Ref. 22) from Robert Kennedy, a member of SSRAP who has also been involved in the technical review of the DOE Seismic Evaluation Procedure, endorses the use of experience-based seismic evaluations for equipment in existing DOE facilities.

A Walkthrough Screening Evaluation Field Guide (Ref. 23) was developed to assist in rapidly identifying major deficiencies at existing DOE facilities. The document was developed based on walkdown experience at nuclear power plants, revised after applying it to walkdowns at selected DOE facilities, and used as an interim methodology before the DOE Seismic Evaluation Procedure was fully developed. The purpose of the Field Guide was to direct walkthrough screening evaluations of DOE facilities in the technical area involving potential hazards caused by natural phenomena. Using the Field Guide, the walkthrough screening evaluation is a facility appraisal of key physical attributes. Items that pass the screen are considered to possess no obvious deficiencies and documented evaluation may be deferred. Items not passing the screen may be of concern such that detailed review or upgrade may be appropriate for these cases depending on potential risk. The methodology in the DOE Seismic Evaluation Procedure is a more thorough extension of the concepts developed in the Field Guide.

\subsubsection{Development and Technical Review of the DOE Seismic Evaluation Procedure}

The DOE Seismic Evaluation Procedure is based on Part II of Revision 2 of the SQUG GIP. Since DOE facilities, objectives, and criteria are different from those for commercial nuclear power plants, the DOE Seismic Evaluation Procedure has been enhanced with information from the SEP6 (Ref. 3) developed for the Savannah River Site (SRS) and from several DOE guidance documents. In addition, DOE-specific requirements and guidance and equipment classes not contained in the SQUG GIP, such as piping systems and unreinforced masonry walls, have been included in the DOE Seismic Evaluation Procedure. The DOE classes of equipment are contained in Chapter 10 and their development and pedigree are discussed in Section 2.1.3.4.4. Nuclear power plant and NRC-specific requirements from the SQUG GIP have been removed and an attempt is made to reduce some of the repetition in the SQUG GIP and make the procedure less cumbersome to use. Additional information on the differences of the DOE Seismic Evaluation Procedure and the SQUG GIP is contained in the Foreword.

Since DOE facilities are not structurally equivalent to nuclear power plants, which are typically stiff, shear wall structures, the approach in the SQUG GIP for comparing seismic capacity with seismic demand has been modified for DOE usage. An assessment (Ref. 24) was done of the performance goals that are achieved when seismic experience-based screening evaluation methods are used. In contrast to the SQUG deterministic criteria, DOE facilities are required to demonstrate the ability to achieve probabilistic performance goals. As discussed in Chapter 5, experience data 
factors are used to scale in-structure response spectra that are derived from the Design Basis Earthquake (DBE) for a facility. The scaled in-structure spectra, or the Seismic Demand Spectrum (SDS), are compared with experience-based capacity spectra.

DOE facility management and operations personnel have played an important role in the development and review of the approach implemented by the DOE Seismic Evaluation Procedure. A Steering Group of selected individuals from the DOE operating contractors have ensured that appropriate priorities were established from the facility operations perspective. The Steering Group is a five-member panel, which is nominated by DOE and its consultants, and is considered a key element to the success of the overall approach presented in the DOE Seismic Evaluation Procedure. The Steering Group has the primary responsibility of reviewing the DOE Seismic Evaluation Procedure in conjunction with a check of technical content and potential impact to a site from a cost, schedule, or operations standpoint. In addition, the Steering Group played a decisive role in the selection of the technology transfer mechanisms for the facility evaluations. Members of the Steering Group and appropriate support personnel have met regularly to discuss and decide on issues affecting the procedures. Examples of issues for which the Steering Group provided a decisive role toward final outcome include implementation procedures, documentation requirements, scope of detailed system and component evaluation tools, peer review requirements, anticipated level of effort for the reviews, and system prioritization guidelines for a facility. The Steering Group also formed a technical review committee to conduct an independent and thorough technical review of the information in the DOE Seismic Evaluation Procedure. The review committee was modeled after SSRAP which was used for the technical review of the SQUG GIP. Members of the review committee were Robert Budnitz, Robert Kennedy, and Loring Wyllie. Since Robert Kennedy participated in the development of Section 10.5.1, he was not an independent technical reviewer of that section.

Two preliminary drafts of the DOE Seismic Evaluation Program were prepared in January and June of 1995 . The June 1995 Draft was technically reviewed by staff at DOE, personnel at DOE sites, and several consultants. Based on the review comments, a second Draft of the DOE Seismic Evaluation Procedure (Ref. 25) was published in September 1995 for review by the DOE, personnel from DOE sites, technical consultants, and attendees of DOE training courses on the EPRI / SQUG methodology. A Final Draft of the Procedure (Ref. 26) was published in November 1996 and it incorporated detailed review comments from the technical reviews of the September 1995 Draft of the Procedure. Following a technical review of the Final Draft, minor modifications were made to the Procedure, except for Section 10.5.1 on Unreinforced Masonry (URM) Walls. Robert Murray and Robert Kennedy extensively revised Section 10.5.1 to incorporate review comments and enhance the methodology in that section.

The technical reviews of the DOE Seismic Evaluation Procedure, which are listed in Table 1.4-1, have provided information for improving portions of the procedure and for emphasizing the appropriateness of using experience data for evaluating the seismic adequacy of equipment. The primary charter of the technical reviews was to independently determine the adequacy of the technical content of the screening evaluation guidelines, including the safety margins that result from implementation of the criteria. For sections of the DOE Seismic Evaluation Procedure that are identical or technically equivalent to corresponding sections in the SQUG GIP, the technical aspects of these sections were reviewed as part of the SSRAP and other reviews of the SQUG GIP as listed in Table 1.4-1. While the technical reviews of the DOE Seismic Evaluation Procedure were modeled after SSRAP, the technical reviews of the DOE Procedure did not involve as many reviewers as the review of the SQUG GIP and did not require formalized consensus building between the DOE and the technical reviewers. Technical reviewers of the DOE Seismic Evaluation Procedure, especially the technical consultants, have extensive experience in the evaluation of the seismic adequacy of equipment and were members of SSRAP or were involved with the development of the SQUG GIP. The emphasis of the technical review of the DOE Seismic 
Evaluation Procedure was the sections of the procedure that are different from the SQUG GIP and there was special focus on Chapter 10, which contains classes of equipment that are not in the SQUG GIP. The key technical consultants reviewing the DOE procedure included Robert Budnitz, Robert Kennedy, and Loring Wyllie as members of the technical review committee. These review efforts were supplemented by reviews by DOE staff and personnel at DOE sites, especially SRS and LLNL, and several engineers from EQE International who had extensive experience with the SQUG GIP.

In addition to the overall review of the DOE Seismic Evaluation Procedure, several sections of the procedure, as listed in Table 1.4-1, received specialized or additional review and in some cases, information about the reviews is referenced. The methodology in Reference 24, which is the basis for Chapter 5, was reviewed by John Reed and Section 10.1.1 on piping was reviewed by Ed Wais (Ref. 27). Section 10.4.1 on HVAC ducts is based on a procedure used at SRS (Ref. 28) and this procedure has been subjected to independent technical review by DOE staff, personnel at DOE sites, and technical consultants. Section 10.3.1 on underground tanks and Section 10.1.2 on underground piping are based on a DOE report that was developed at Brookhaven National Laboratory (Ref. 29) and has been reviewed by DOE staff, personnel at DOE sites, technical consultants, and the American Society of Civil Engineers, Dynamic Analysis of Nuclear Structures Committee. An independent review of Section 10.5.1 on unreinforced masonry walls was performed at the Lawrence Livermore National Laboratory.

\section{Table 1.4-1 Technical Reviews of DOE Seismic Evaluation Procedure}

\begin{tabular}{|c|c|c|c|}
\hline $\begin{array}{c}\text { Chapter or } \\
\text { Section of the } \\
\text { DOE Seismic } \\
\text { Evaluation } \\
\text { Procedure }\end{array}$ & $\begin{array}{c}\text { Technical } \\
\text { Aspects } \\
\text { Reviewed as Part } \\
\text { of SQUG GIP }\end{array}$ & $\begin{array}{l}\text { Reviewed by } \\
\text { Technical } \\
\text { Consultants for } \\
\text { the DOE }\end{array}$ & $\begin{array}{c}\text { Specialized } \\
\text { Review for the } \\
\text { DOE }\end{array}$ \\
\hline Chapter 1 & & $\overline{\mathrm{X}}$ & \\
\hline Chapter 2 & & $\bar{X}$ & \\
\hline Chapter 3 & & $\overline{\mathrm{X}}$ & \\
\hline Chapter 4 & & $\bar{X}$ & \\
\hline Chapter 5 & & $\overline{\mathrm{X}}$ & $\overline{\mathbf{X}}$ \\
\hline Chapter 6 & $\bar{X}$ & $\overline{\mathbf{X}}$ & \\
\hline Chapter 7 & $\bar{X}$ & $\bar{X}$ & \\
\hline Chapter 8 & $\bar{X}$ & $\bar{X}$ & \\
\hline Chapter 9 & $\bar{X}$ & $\bar{X}$ & \\
\hline Section 10.1.1 & & $\overline{\mathbf{X}}$ & $\bar{X}$ \\
\hline Section 10.1.2 & & $\bar{X}$ & $\bar{X}$ \\
\hline Section 10.2 & & $\bar{X}$ & \\
\hline Section 10.3.1 & & $\bar{X}$ & $\bar{X}$ \\
\hline Section 10.3 .2 & & $\bar{X}$ & \\
\hline Section 10.4 .1 & & $\bar{X}$ & $\bar{X}$ \\
\hline Section 10.5 .1 & & $\bar{X}$ & $\bar{X}$ \\
\hline Section 10.5 .2 & & $\bar{X}$ & \\
\hline Section 10.5 .3 & & $\bar{X}$ & \\
\hline Chapter 11 & $\bar{X}$ & $\bar{X}$ & \\
\hline Chapter 12 & $\bar{X}$ & $\bar{X}$ & \\
\hline Chapter 13 & $\bar{X}$ & $\bar{X}$ & \\
\hline Chapter 14 & & $\bar{X}$ & \\
\hline
\end{tabular}


Additional information for the development of the DOE Seismic Evaluation Procedure has come from trial applications of the September 1995 Draft at the SRS, Rocky Flats Environmental Technology Center (RFETC), the Los Alamos National Laboratory (LANL), the Stanford Linear Accelerator Center (SLAC) and LLNL. Feedback from these applications of the DOE procedure have been incorporated as appropriate.

The technical review of the DOE Seismic Evaluation Procedure and the endorsement of its use for the DOE is summarized in a letter (Ref. 30) from a technical review committee consisting of Robert Budnitz, Robert Kennedy, and Loring Wyllie. This letter is attached at the end of the Foreward with the following three review comments:

(1) the use of the DOE Seismic Evaluation Procedure is endorsed for the seismic evaluations of existing DOE facilities,

(2) the use of additional equipment categories beyond those in the SQUG GIP is supported for the DOE Seismic Evaluation Procedure, and

(3) the use of the DOE Seismic Evaluation Procedure for new equipment is supported with caution.

It is intended that the DOE Seismic Evaluation Procedure will be revised and updated as appropriate. As screening procedures are developed and reviewed for other classes of equipment, these procedures can be added to the DOE procedure. Section 2.1.3.4 discusses some of the other classes of equipment that can be added to future versions of the DOE procedure. As the SQUG GIP is revised and the information in the earthquake experience database and shake table testing database is enhanced, the appropriate modifications will be made to the DOE Seismic Evaluation Procedure.

\subsubsection{Applications at DOE Facilities}

The SQUG experience-based seismic evaluation approach has been used at many DOE facilities. The most extensive application has been at the SRS which has reactors that are similar to commercial nuclear power plants. The SRS reactors were built in the 1950 s when seismic qualification requirements were in their infancy. SRS became a member of SQUG in 1988, and used the SQUG GIP at its K, L and P reactors to evaluate the seismic adequacy of selected safety systems for their Design Basis Earthquake (DBE). The SRS reactor program included definition of the system scope requiring review; development of SRS facility-specific procedures; use of seismic screening evaluation walkdowns and calculations; and identification, resolution, and upgrading of outliers.

The seismic evaluation program at SRS expands the SQUG GIP in several areas including programmatic changes to enhance engineering assurance. Several technical changes were added to address unique needs at SRS such as additional steps for expansion anchor evaluation, development of capacity for lead cinch anchors, implementation of consistent guidelines for HVAC ducting (Section 10.4.1), and use of experience-based screening guidelines for piping (Section 10.1.1). SRS developed a Seismic Engineering Procedure (SEP-6) (Ref. 3) that includes sections on licensing, the SQUG GIP, and site-specific topics. Portions of the SRS-developed SEP are used in the DOE Seismic Evaluation Procedure.

The SRS seismic evaluation program was judged to be a success with roughly $60 \%$ of the items that were evaluated to be seismically adequate as-is. For the others, about $11 \%$ were resolved by additional evaluation and the remainder were resolved by upgrade. The typical upgrades consisted of anchorage enhancement and elimination of seismic interaction concerns by providing restraint or 
removal of the interaction source. The use of the experience-based evaluation approach enabled efficient identification of realistic seismic concerns at SRS. Maximum safety enhancement was achieved with a reasonable engineering effort.

The seismic experience-based approach is currently being used at SRS to evaluate non-reactor facilities. According to Reference 31, seismic qualification using experience data is a technical necessity and is the most economically attractive of the options to qualify existing equipment at SRS. At two SRS facilities, representative costs for seismic qualification using the methodology in the SRS SEP-6 demonstrate costs are 70\% lower than the costs for qualification using conventional methods such as seismic testing or detailed engineering analyses.

Similar benefits from use of experience data were realized at the High Flux Isotope Reactor (HFIR) at Oak Ridge National Laboratory. Prior to facility restart, seismic verification of essential systems and components had to be demonstrated. Experience-based screening evaluations were used as a key part of the seismic evaluation and upgrade program. Several items were determined to be acceptable in their as-installed configuration. Backfit modifications were installed to increase seismic adequacy as needed. This included providing anchorage for some components, additional restraint for items where deflection considerations governed capacity, and correction of potential seismic systems interaction hazards.

Other applications of using experience data for the evaluation of seismic design issues at DOE facilities include the Princeton Plasma Physics Laboratory, the Idaho Chemical Processing Plant (ICPP), Y-12 at Oak Ridge, and RFETC. At Princeton, active electrical and mechanical equipment, fluid pressure boundary components, and seismic interaction effects were evaluated and resolved by use of experience-based methods. The seismic adequacy of critical fire protection components was evaluated using the experience-based approach at ICPP. Using the methodology in Section 10.1.1, the seismic adequacy of piping systems have been evaluated at $Y-12$ and RFETC.

The applications at SRS, HFIR, Princeton, and ICPP have proven the viability of using the methodology developed by EPRI / SQUG based on seismic experience data. Many of the results of these evaluations have withstood strict scrutiny during technical audits, peer reviews, quality control audits, and other independent reviews. The approach is also being applied to facilities at LANL, LLNL, and SLAC. Further discussion of the use of experience data for seismic evaluations is provided in Chapter 9 of the "Seismic Safety Manual" (Ref. 32), which was prepared for the DOE. With the experience from the nuclear power industry coupled with numerous applications at DOE sites, the consistent approach in the DOE Seismic Evaluation Procedure for the application of experience data provides DOE sites with an efficient tool for performing their necessary seismic evaluations.

\subsubsection{Post-Earthquake Investigations}

An important element of the development of the DOE Seismic Evaluation Procedure has been postearthquake investigations after significant earthquakes. Each significant earthquake provides important lessons that reemphasize and provide new information about designing and retrofitting equipment for strong seismic motion. Since a major component of the EPRI / SQUG methodology is experience data, the data must be appropriately augmented and enhanced with information from recent and significant earthquakes. In many cases, recent earthquakes have provided information which emphasizes the procedures and screens already developed for the EPRI / SQUG methodology.

Post-earthquake investigations are vital to determine if any part of the methodology should be modified or developed further. With each significant earthquake, the experience database will be 
updated to reflect the results of post-earthquake investigations. Since the DOE Seismic Evaluation Procedure contains classes of equipment and distribution systems that are not included in the SQUG GIP, post-earthquake investigations sponsored by the DOE will focus on these classes of equipment. As data is gathered on these classes of equipment, rigorous procedures for determining equipment capacity can be developed based on the collected information.

Recent earthquakes have provided valuable information about the performance of equipment during seismic strong motion. Details about the performance of industrial facilities and their associated equipment during recent earthquakes are contained in many documents including References 33 and 34. Information in these references emphasizes the response of equipment similar to the types of equipment included in the DOE Seismic Evaluation Procedure. Figures 1.4-1 to 1.4-9 show examples of the performance of equipment, systems, and architectural features subjected to relatively strong seismic motion during recent earthquakes that are similar to the classes of equipment discussed in Chapters 8, 9, 10, and 11 .

As appropriate, data from recent earthquakes can be incorporated into the DOE Seismic Evaluation Procedure. In Section 12.2, a potential method for resolving outliers, or equipment that does not meet the intent of the caveats in the DOE Seismic Evaluation Procedure, involves expanding the earthquake experience database to include the equipment or specific features of the equipment. The scope of the earthquake experience data documented in References 19 and 35 represents only a portion of the total data available. Extension of the generic experience equipment classes beyond the descriptions in the DOE Seismic Evaluation Procedure is subject to DOE review and to an external peer review. The external peer review is to be of similar caliber as that required during the original development of the earthquake experience database. An extension of the database must have as rigorous a basis as the information that is currently contained in References 19 and 35 .

In addition to post-earthquake investigations, there is a significant amount of seismic data at DOE facilities in the form of shake-table test data. This DOE shake-table test data can be incorporated into the DOE Seismic Evaluation Procedure applying the same considerations for expanding the earthquake experience database as discussed above. 


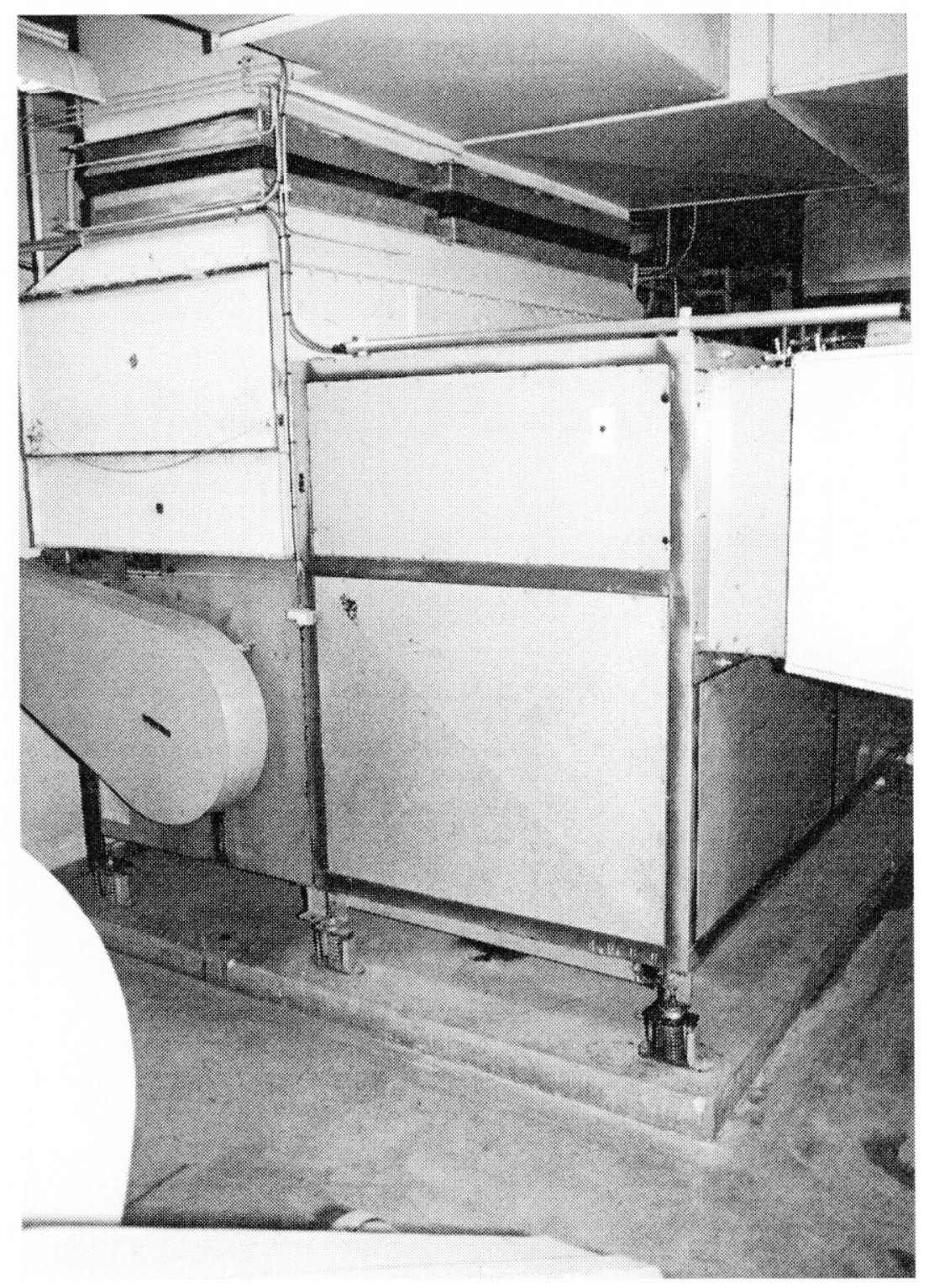

Figure 1.4-1a Shown is an example of vibration isolators without adequate seismic bumpers. This air-handler unit suffered damage at an electrical substation during the 1994 Northridge Earthquake. (Reference 33) 


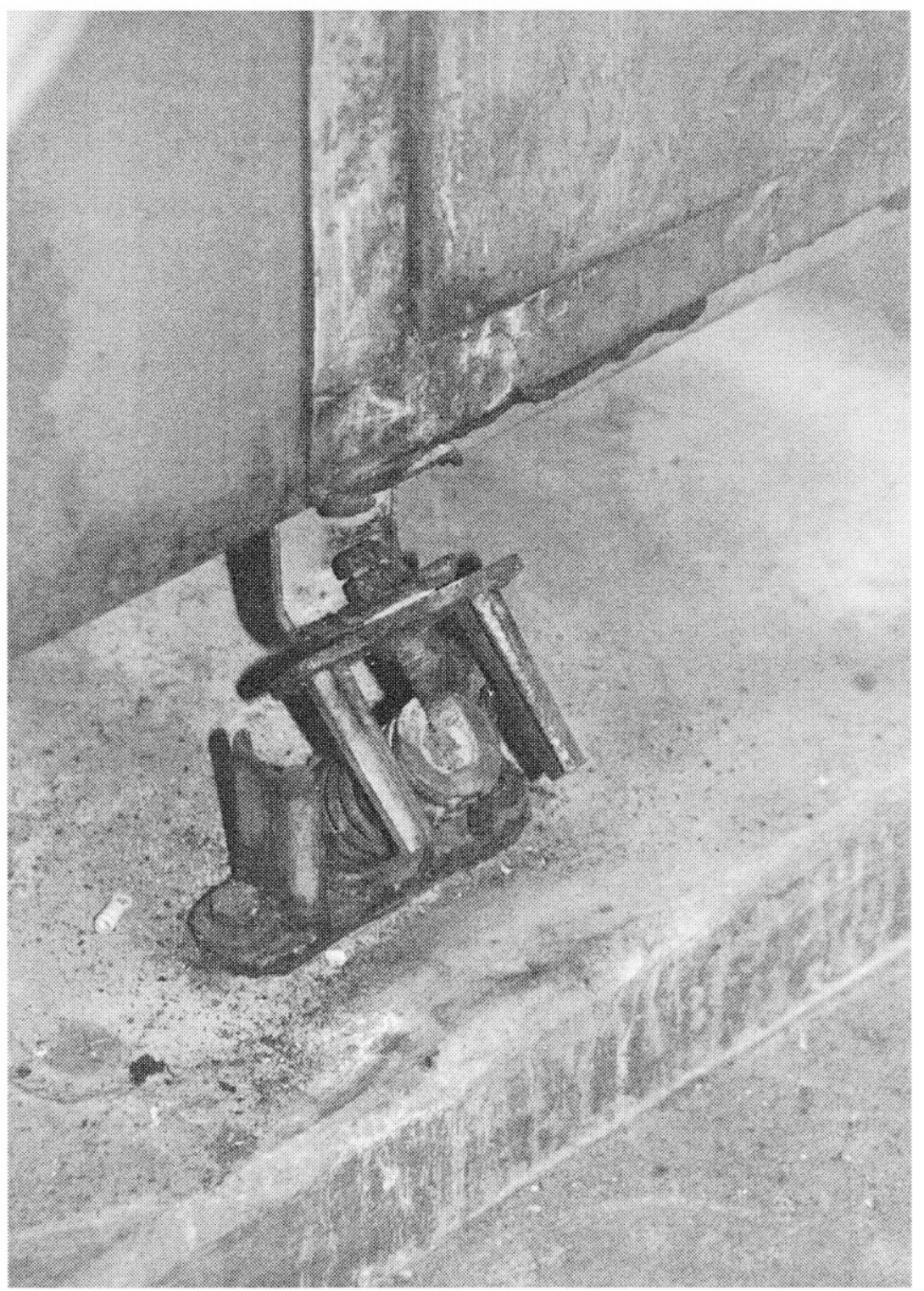

Figure 1.4-1b Shown is a close-up of vibration isolators without adequate seismic bumpers. (Reference 33) 


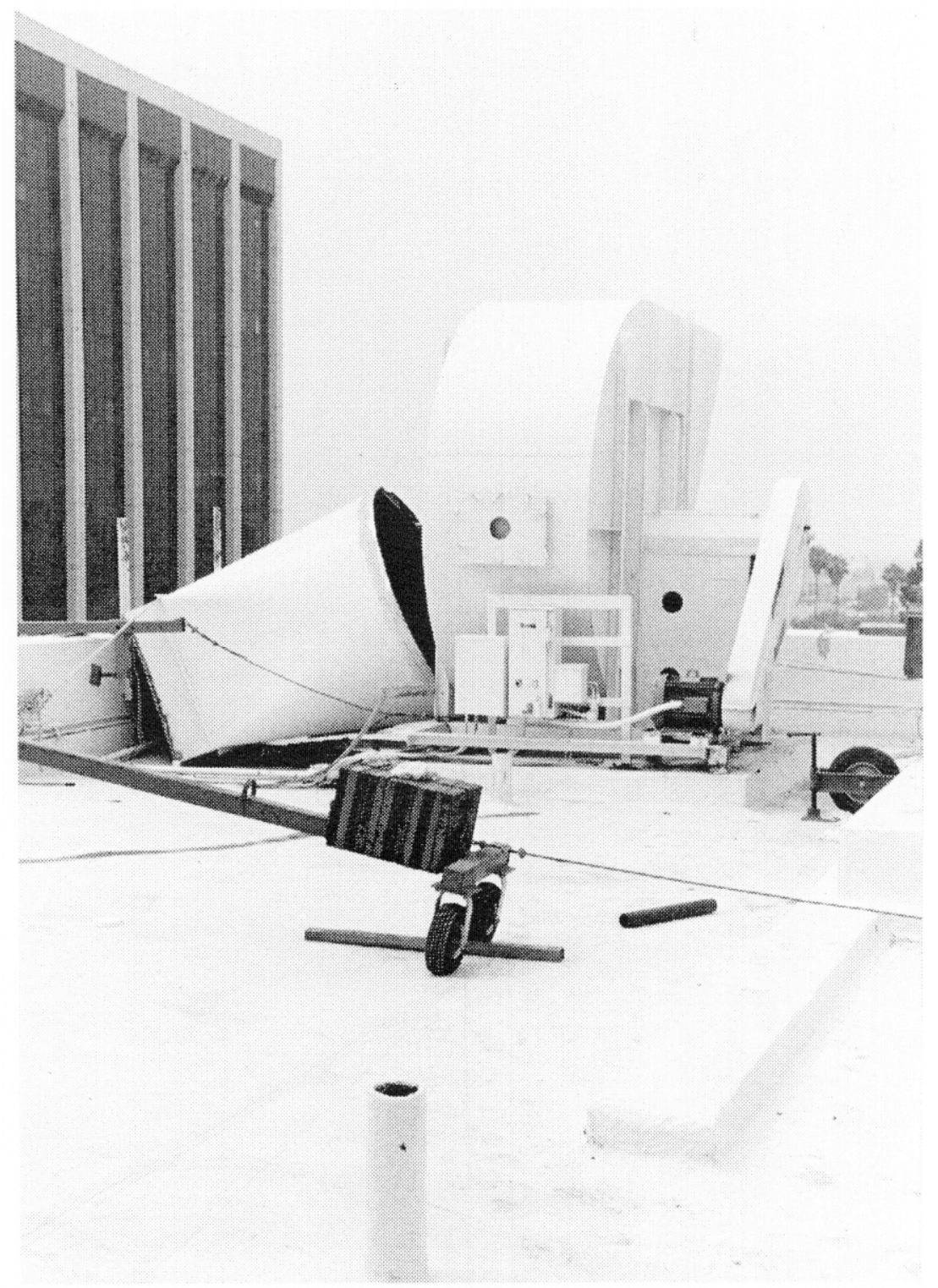

Figure 1.4-2 On the roof of a six-story hospital, a plenum pulled loose from its fan enclosure during the 1994 Northridge Earthquake. '(Reference 33) 


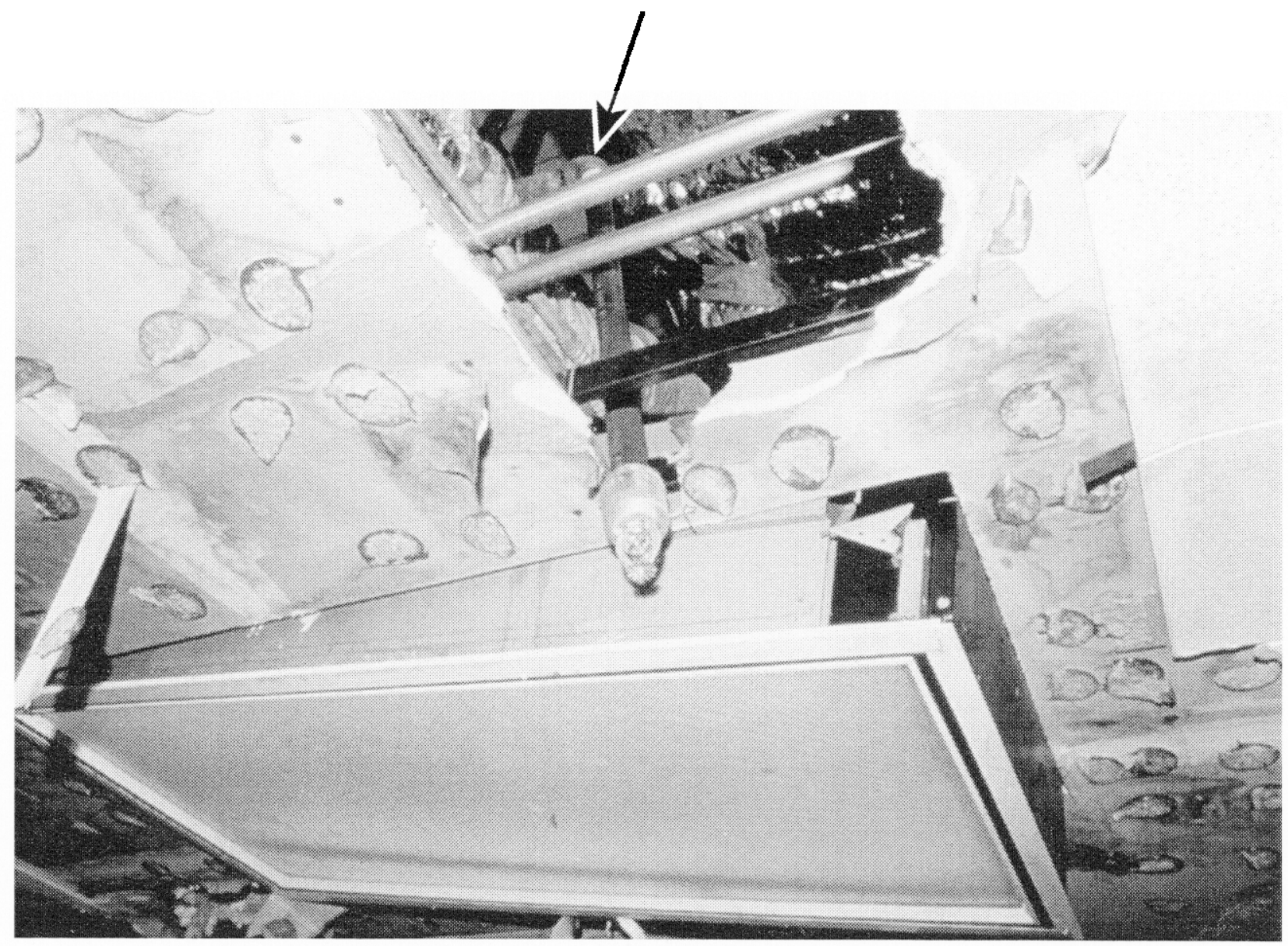

Figure 1.4-3 Water spray following an earthquake was a major seismic interaction issue during and directly after the 1994 Northridge Earthquake. As shown in this figure, fire sprinkler piping broke at threaded elbow joints of the vertical branches that suspend the sprinkler heads. Damage to the fire sprinkler piping at several facilities caused these facilities to shut down following the earthquake, even though the buildings had no structural damage. (Reference 33) 


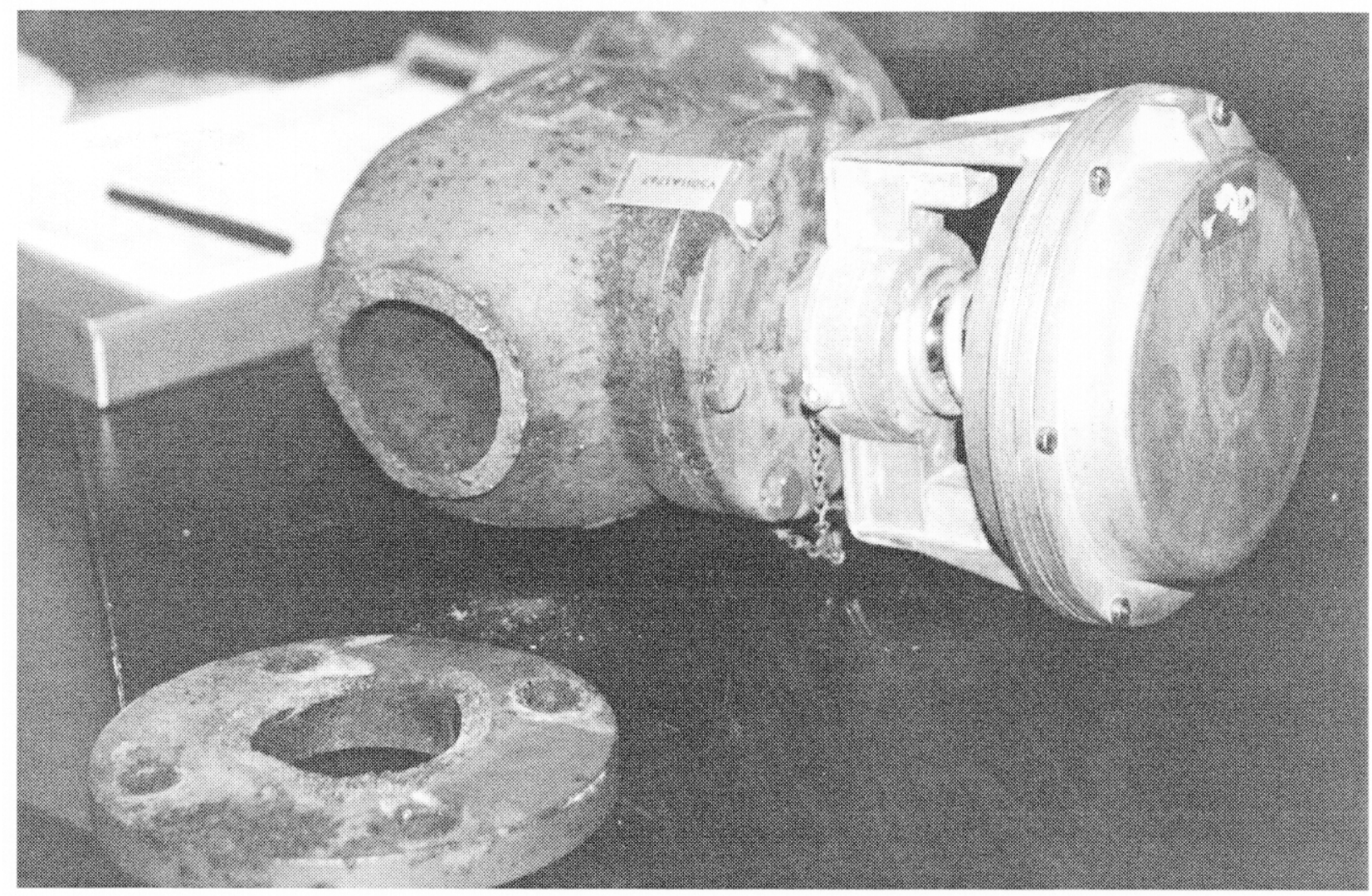

Figure 1.4-4 In a penthouse above the sixth story of a hospital, a cast-iron valve body failed near its flange due to inertial forces on a 4-inch diameter chilled water line and allowed water to leak down to the floors below. This occurred during the 1994 Northridge Earthquake. (Reference 33) 


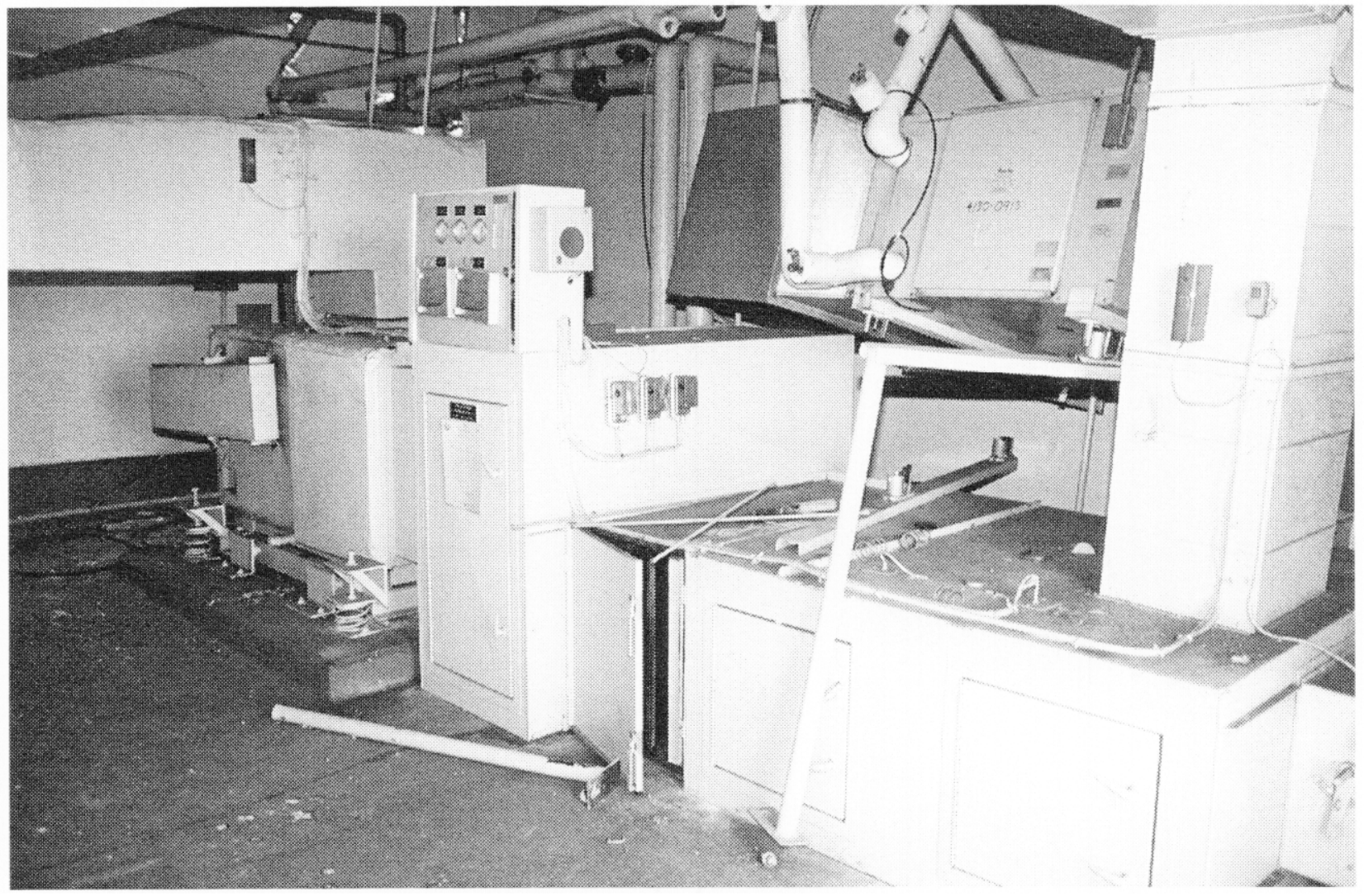

Figure 1.4-5 As a result of the pounding between the wings of a six-story building during the 1994 Northridge Earthquake, a fan came off of its support frame inside a penthouse. (Reference 33) 


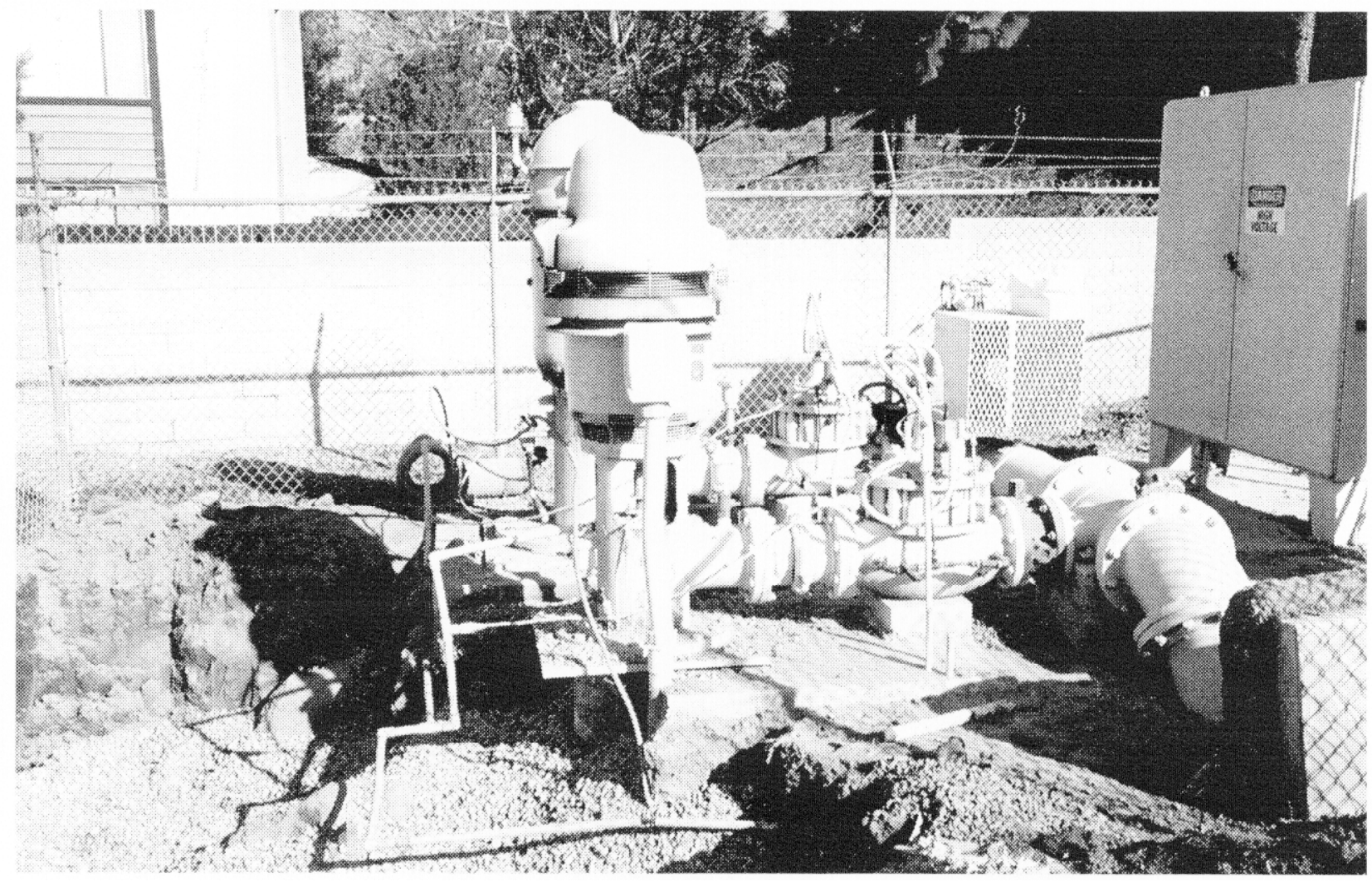

Figure 1.4-6a Ground settlement at this lift station caused underground attached piping to crack and leak after the 1994 Northridge Earthquake. (Reference 33) 


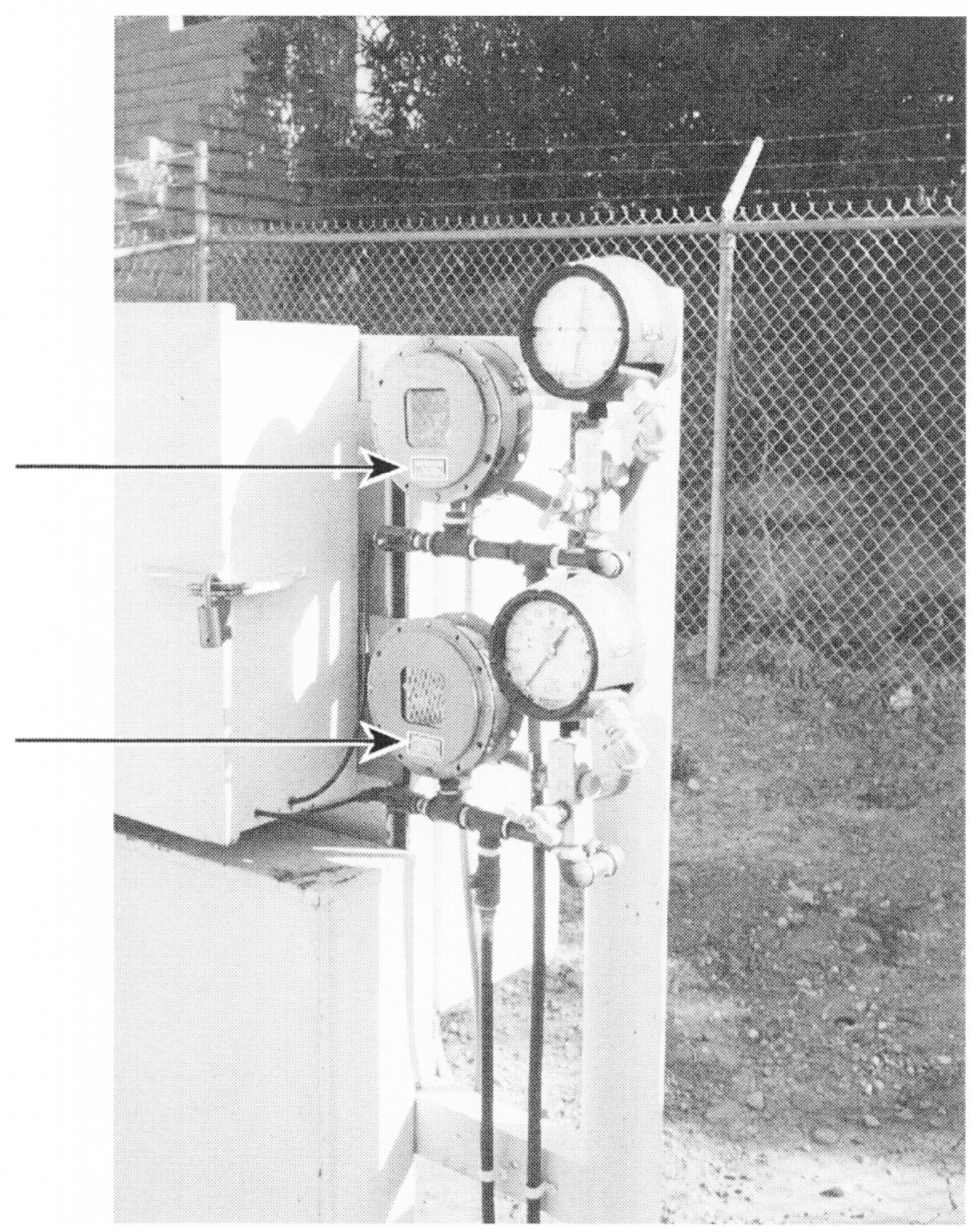

Figure 1.4-6b Mercoid Switches connected to the pressure transmitters at a lift station may have caused an inadvertent trip of relays, or change of state of the control system. (Reference 33) 


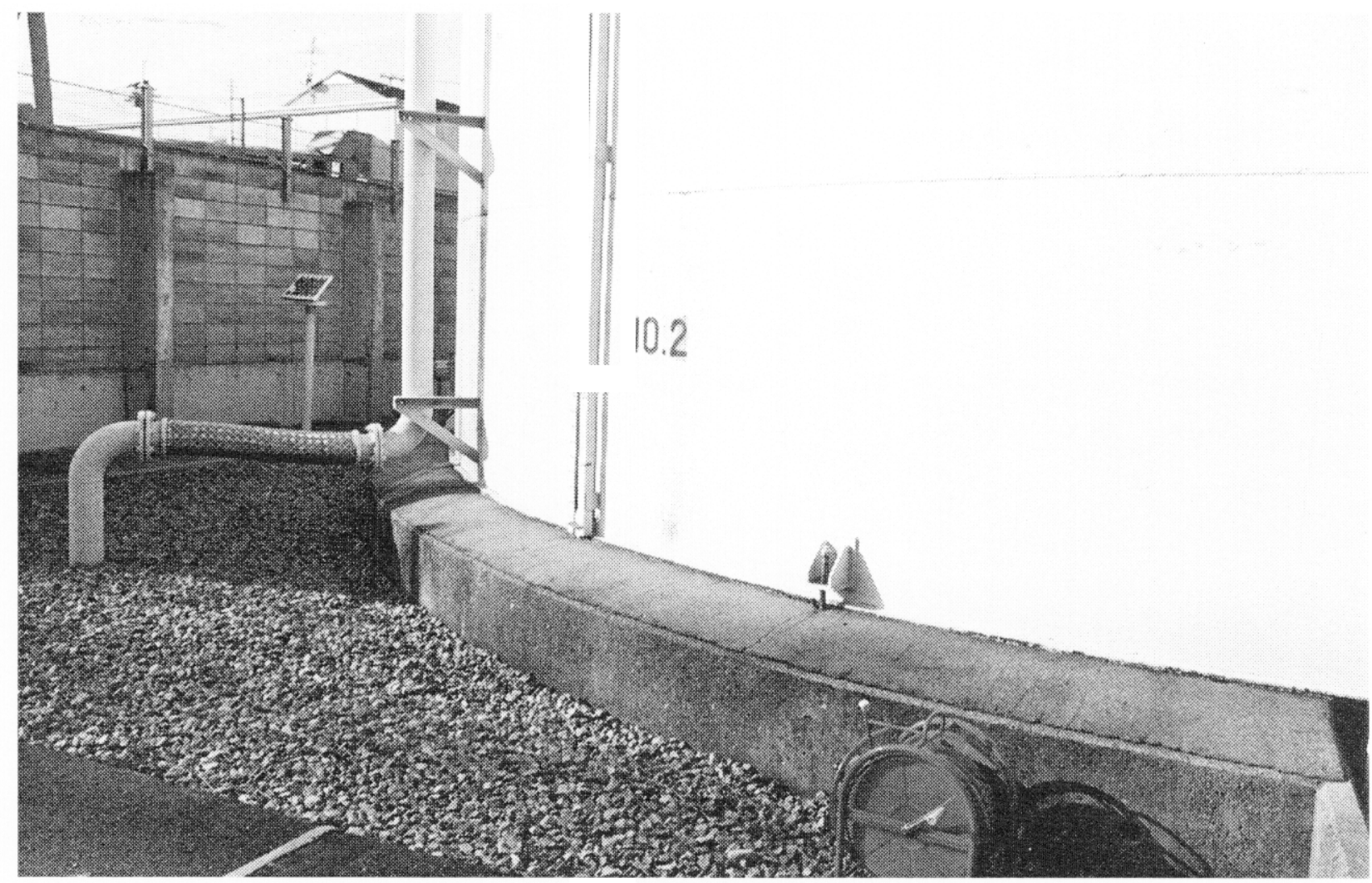

Figure 1.4-7a This vertical, flat-bottom tank experienced the 1995 Kobe Earthquake (note both the flexible connection for the attached piping and the stretched/pulled anchor bolts at the base of the tank). (Reference 34) 


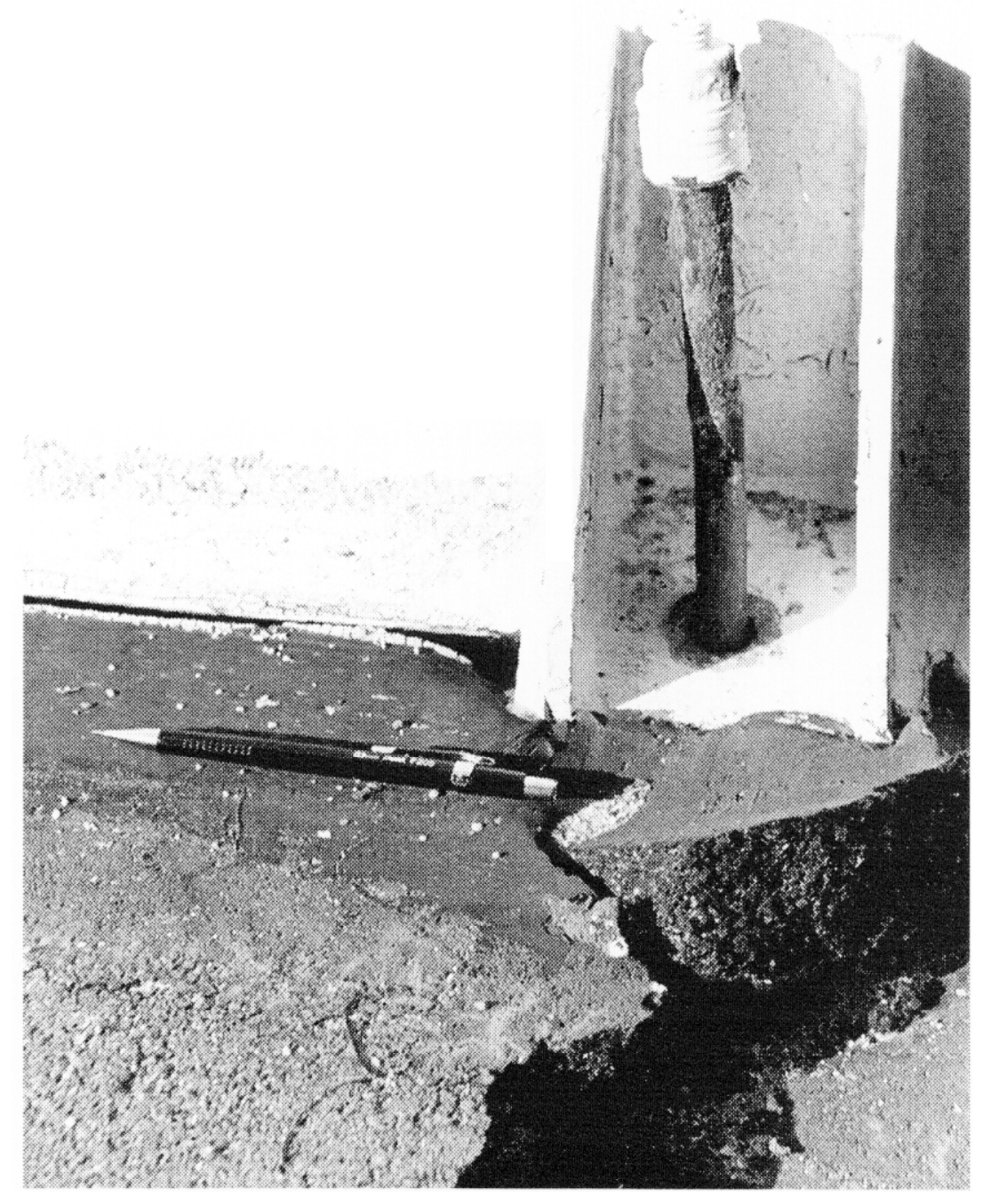

Figure 1.4-7b Close-up of one of the anchor bolts which appeared to have experienced a combination of partial pull-out as well as stretching of the bolt as the tank tried to rock. (Reference 34) 


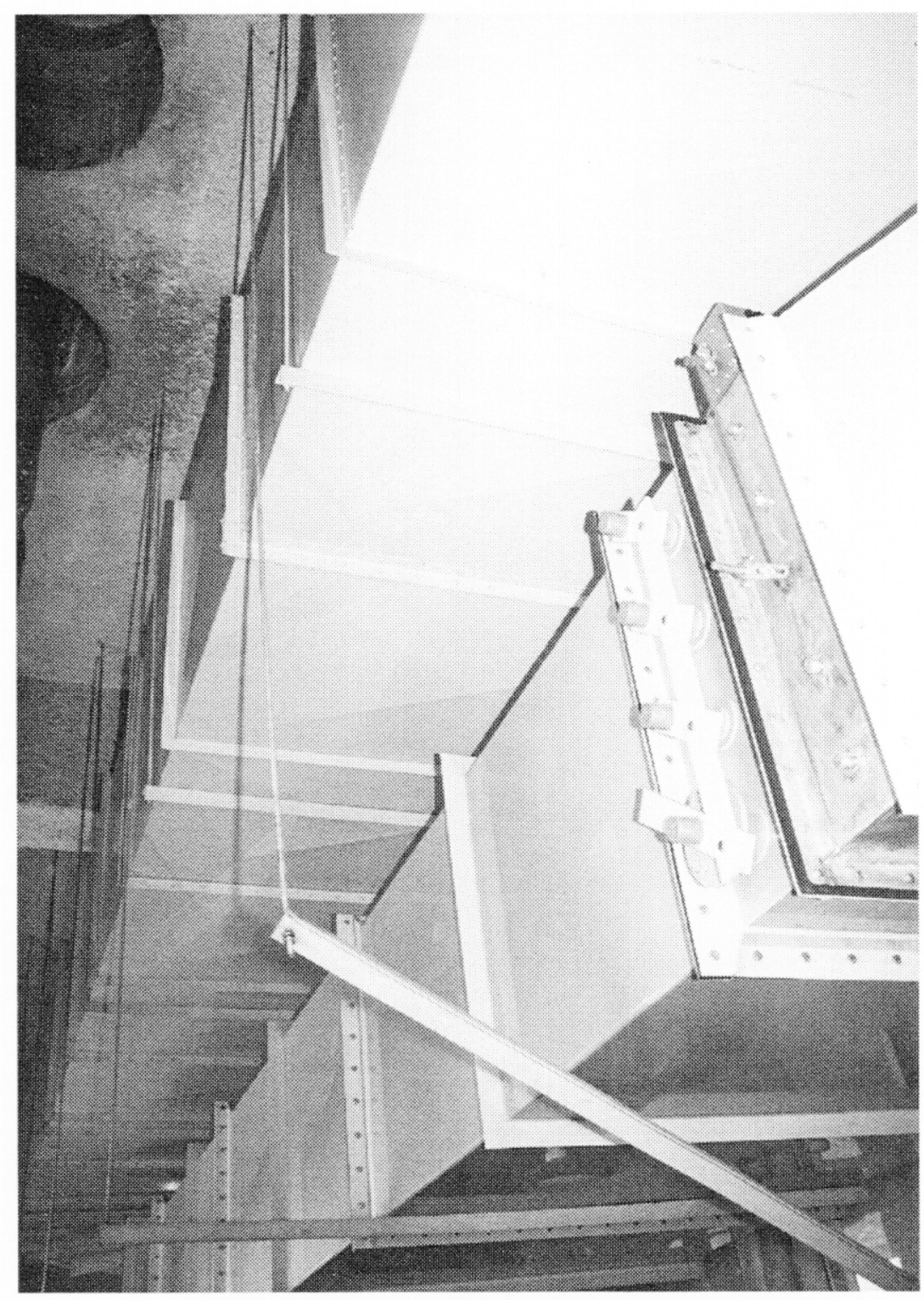

Figure 1.4-8a Shown is a ductwork trapeze that is partially collapsed. During the 1995 Kobe Earthquake, one of the expansion anchors for the threaded rod support pulled out of the reinforced concrete ceiling. (Reference 34) 


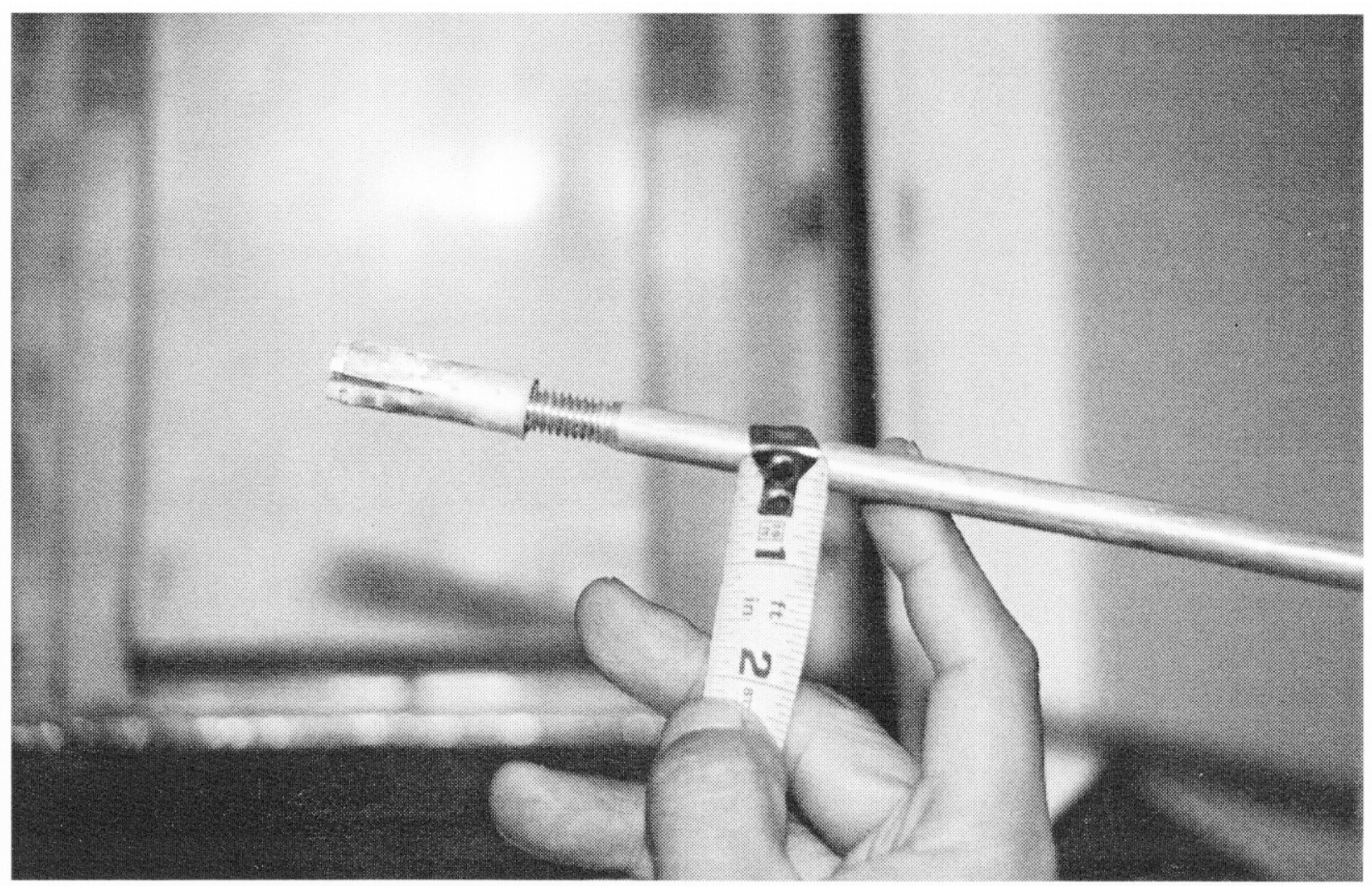

Figure 1.4-8b Shown is a close-up of the expansion anchor which pulled out of the reinforced concrete ceiling. It appears that there was inadequate expansion of the shell. (Reference 34 ) 


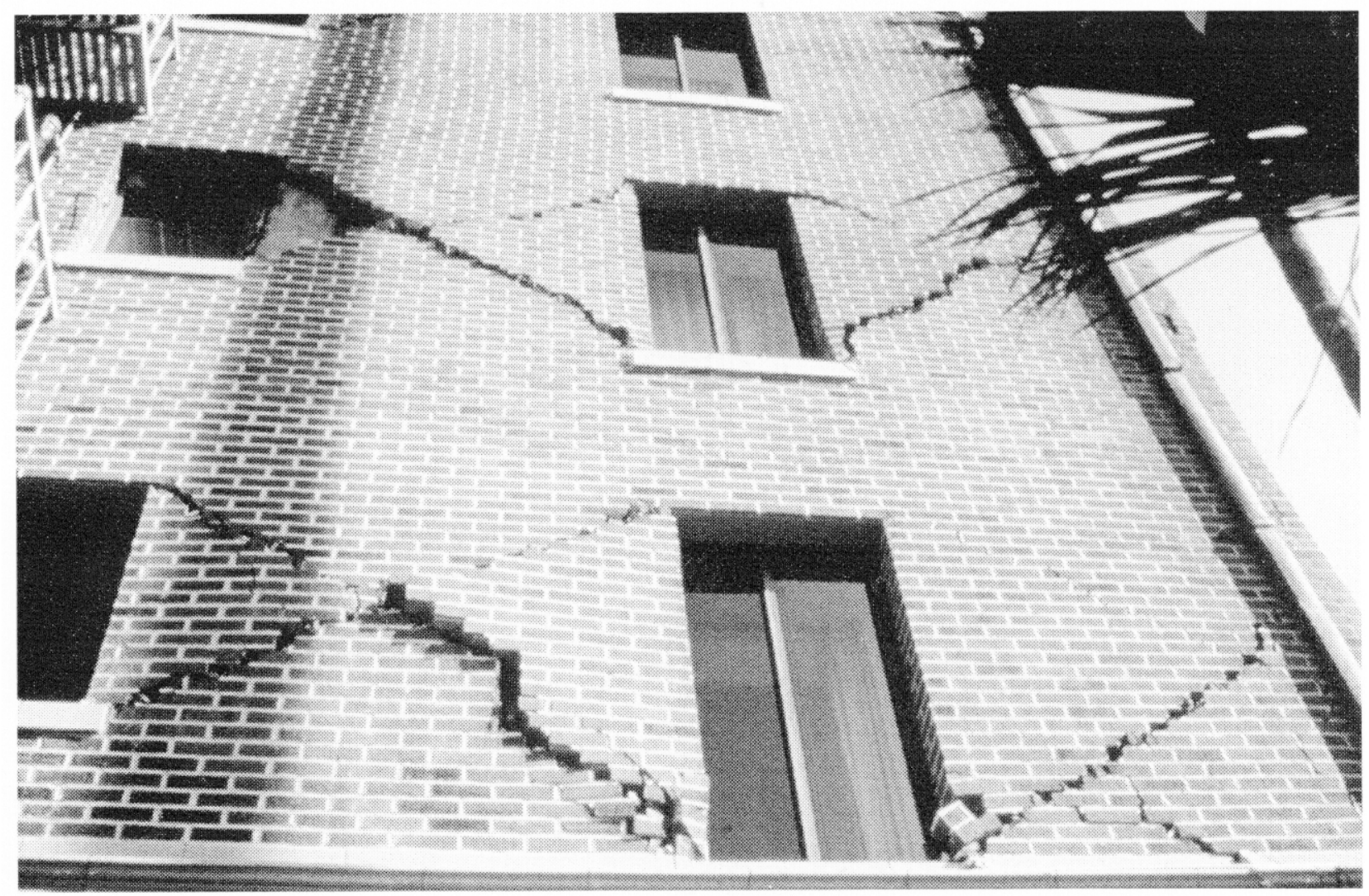

Figure 1.4-9 Large diagonal cracks in unreinforced masonry cladding (onewidth thickness) over a reinforced concrete frame in an L-shaped building experienced damage during the 1994 Northridge Earthquake. (Reference 36) 


\subsection{DOE LICENSE FOR EPRI / SQUG MATERIAL}

An important step toward development of the comprehensive natural phenomena hazard evaluation guidelines for systems and components at DOE facilities was obtaining the proprietary reference documents and procedures developed by SQUG and EPRI. This was a key element of the DOE evaluation program because it allows DOE to take advantage of all the work performed to-date for several classes of equipment at commercial nuclear reactors. The EPRI / SQUG material is arranged into six volumes and copies of the material have been distributed throughout the DOE. Within the volumes there are twelve key reference reports (Ref. 35 and 40 to 50) that cover the technical areas of 20 classes of equipment, anchorage, electrical raceways, relays, and tanks and heat exchangers. A document which develops a methodology for assessment of nuclear power plant seismic margin (Ref. 18) is also available to the DOE. In addition, the SQUG GIP is contained in the volumes of material as a basis document for the DOE Seismic Evaluation Procedure. There are several documents in the volumes that summarize the SSRAP and NRC review of the EPRI / SQUG methodology (Ref. 2, 19, and 50) and provide additional information for piping and ducting systems (Ref. 39 and 51 to 55).

The EPRI / SQUG Seismic Assessment Material is available for use when performing seismic evaluations of DOE facilities under a written licensing agreement between EPRI and LLNL. Control and use of the EPRI / SQUG Material is by a procedure (Ref. 56) that applies to all DOE staff; Management and Operations (M\&O) contractor staff; and subcontractors, who are currently under contract to DOE or a M\&O, to conduct seismic evaluations of DOE facilities. DOE, M\&O, and contractor staff may only obtain the EPRI / SQUG Material by attending a training course sponsored by DOE. All personnel who are issued a controlled set of the Material sign an acknowledgment receipt form to comply with the requirements of the procedure.

According to the procedure, all personnel having a controlled copy of the Material may use and reference the Material while performing seismic assessments of DOE facilities. In addition, the Material may be loaned within a particular DOE site by its custodian. Volumes 1 and 8 of the Material have no restrictions on its use. The SQUG GIP in Volume 2 is copyrighted by SQUG and should not be reproduced. Volumes 3 through 7 of the Material shall not be reproduced and its ownership shall not be transferred to any other personnel without following the established procedure. As discussed in Section 3.4, the Material is only issued to personnel and may only be used by personnel who attend a DOE-sponsored training course (see Section 3.4) that instructs attendees on its proper use. Attendance at the training courses and the receipt of the Material are documented by DOE. 


\section{SCREENING EVALUATION AND WALKDOWN PROCEDURE}

\section{$2.1 \quad$ APPROACH IN THE DOE SEISMIC EVALUATION PROCEDURE ${ }^{1}$}

The approach used in the DOE Seismic Evaluation Procedure for evaluating the seismic adequacy of equipment in DOE facilities is consistent with the intent of DOE Policy, Orders, and Standards. It is also consistent with the approach in the SQUG GIP (Ref. 1) and the EPRI Seismic Margins Assessment Program (Ref. 18). The four major steps used in the DOE procedure for the majority of the equipment to be evaluated are listed below, along with the chapter(s) of the procedure where these steps are covered in detail:

- Selection of Seismic Evaluation Personnel (Chapter 3)

- Determination of Seismic Equipment List (Chapter 4)

- Screening Evaluation and Walkdown

Capacity versus Demand (Chapter 5)

Anchorage (Chapter 6)

Seismic Interaction (Chapter 7)

Equipment Class Evaluations (Chapters 8, 9, and 10)

Relay Functionality (Chapter 11)

- $\quad$ Outlier Identification and Resolution (Chapter 12)

The suggested documentation for these reviews is discussed in each of the chapters and in Chapter 13. The remainder of this section summarizes the material covered in Chapters 3 through 13.

An important aspect of the methodology in the DOE Seismic Evaluation Procedure is the use of judgment that results from appropriate training, extensive experience with walkdowns, and review of the reference documents for the SQUG GIP. Guidance and discussion about the use of engineering judgment are provided in References 18, 57, and 58 that discuss the assessment of seismic margins for nuclear power plants. Since the level of expertise will differ with the seismic evaluation personnel as discussed in the following section, it is vital that the personnel identify the equipment that they do and do not have the adequate level of expertise to evaluate and that they evaluate only the equipment for which they have the appropriate experience. Engineers who use the DOE Seismic Evaluation Procedure are responsible for its appropriate application, for their level of training, and for their use of judgment. The developers of the Procedure assume no responsibility for specific applications of the methodology.

\subsubsection{Seismic Evaluation Personnel ${ }^{2}$}

Individuals from several engineering disciplines, their recommended minimum requirements or qualifications, and their responsibilities for implementing this Seismic Evaluation Procedure are described in Chapter 3. These individuals include: (1) Safety Professionals and Systems Engineers who identify the methods and the equipment needed in the Seismic Equipment List

1 Based on Section 1.3 of SQUG GIP (Ref. 1)

2 Based on Section 1.3.1 of SQUG GIP (Ref. 1) 
(SEL); (2) Operations Personnel who have a comprehensive understanding of the facility layout, the function and operation of the equipment and systems in the facility, and the facility operating procedures; (3) Seismic Capability Engineers (SCEs) who perform the Screening Evaluation and Walkdown of the equipment listed in the SEL; (4) Relay Evaluation Personnel who perform the relay functionality review; and (5) Piping Evaluation Engineers who perform the walkdown and evaluation of piping listed in the SEL.

Since the instructions and requirements contained in this procedure are guidelines and not fixed, inflexible rules, the SCEs must exercise sound engineering judgment during the Screening

Evaluation and Walkdown. Therefore, the selection and training of qualified SCEs for participation on the Seismic Review Teams (SRTs) is an important element of the DOE Seismic Evaluation Procedure. The SCEs are expected to exercise engineering judgment based upon an understanding of the guidelines given in the procedure, the basis for these guidelines given in the reference documents and presented in the DOE training course, and their own seismic engineering experience.

Chapter 3 also describes the DOE-developed training course which should be taken by individuals who perform the seismic review of a DOE facility with the DOE Seismic Evaluation Procedure. This course provides assurance that there is a minimum level of understanding and consistency in applying the guidelines contained in this procedure.

\subsubsection{Seismic Equipment List}

The Seismic Equipment List (SEL) is described in Chapter 4. This list is typically developed by Safety Professionals and Systems Engineers in consultation with Operations Personnel and other engineers. Equipment listed on the SEL is evaluated by SCEs using the screening and walkdown methodology of the Seismic Evaluation Procedure.

Screening guidelines are provided in the DOE Seismic Evaluation Procedure for evaluating the seismic adequacy of most types of equipment which could be listed in the SEL. However, if an item of equipment listed in the SEL is not covered by the screening guidelines, then it is identified as an outlier and evaluated separately as discussed in Chapter 12.

\subsubsection{Screening Evaluation and Walkdown ${ }^{3}$}

The Screening Evaluation and Walkdown of equipment listed in the SEL is described in Chapters 5 through 11. The purpose of the Screening Evaluation and Walkdown is to screen out from further consideration those items of equipment that pass certain generic, seismic adequacy criteria. The screening evaluation is based heavily on the use of seismic experience data. If the equipment does not pass the screens, other more refined or sophisticated methods for evaluating the seismic adequacy of the equipment may be used as described in Chapter 12 .

The procedure for performing the Screening Evaluation and Walkdown is depicted in Figure 2.1-1. As shown in the figure, each of the following four seismic screening guidelines should be used to evaluate the seismic adequacy of an item of equipment:

- $\quad$ Seismic Capacity Compared to Seismic Demand (Chapter 5) - The seismic capacity of the equipment, based on earthquake experience data, generic seismic testing data, or equipmentspecific seismic qualification data, should be greater than the seismic demand imposed on the equipment, system, or architectural feature.

3 Based on Section 4.0 of SQUG GIP (Ref. 1) 
- $\quad$ Anchorage (Chapter 6) - The equipment anchorage capacity, installation, and stiffness should be adequate to withstand the seismic demand at the equipment location.

- $\quad$ Seismic Interaction (Chapter 7) - The effect of possible seismic spatial interactions with nearby equipment, systems, and structures and interaction from water spray, flooding, and fire hazards should not cause the equipment to fail to perform its intended function.

- $\quad$ Equipment Class Evaluations (Chapters 8, 9, and 10) - In Chapter 8, the equipment must be similar to the equipment in the earthquake experience equipment class or the generic seismic testing equipment class and also meet the intent of the specific caveats for that class of equipment in order to use the seismic capacity defined by the earthquake experience Reference Spectrum or the generic seismic testing GERS. If equipment-specific seismic qualification data is used, then specific restrictions or caveats for that qualification data apply instead. In Chapter 9, the equipment must be similar to the equipment in the earthquake experience equipment class, meet the caveats, and satisfy the screening procedures. In Chapter 10, the equipment must be similar to the equipment classes and be evaluated using the general screening procedures or guidelines.

The evaluation of equipment against each of these four screening guidelines is to be based upon walkdown evaluations, calculations, and other supporting data. While equipment seismic evaluations can generally be performed independently from each other, there are a few areas where an interface with the Relay Functionality Review (Chapter 11) is appropriate:

- Any cabinets containing essential relays, as determined by the relay review in Chapter 11 , should be evaluated for seismic adequacy using the guidelines contained in Chapter 8 .

- Apply a capacity reduction factor to expansion anchor bolts that secure cabinets containing essential relays. This capacity reduction factor is discussed in Chapter 6.

- Seismic interaction, including even mild bumping, is not allowed on cabinets containing essential relays. This limitation is discussed in Chapters 7, 8, and 11.

- In-cabinet amplification factors for cabinets containing essential relays are to be estimated by the SCEs for use in the Relay Functionality Review.

It is suggested that items of equipment containing essential relays be identified prior to the Screening Evaluation and Walkdown so that the above evaluations may be accomplished during the Screening Evaluation and Walkdown.

\subsubsection{Seismic Capacity Compared to Seismic Demand ${ }^{4}$}

A screening guideline to be satisfied to evaluate the seismic adequacy of an item of equipment is to confirm that the seismic capacity of the equipment is greater than or equal to the seismic demand imposed on it. Chapter 5 addresses the comparison of seismic capacity to seismic demand for the equipment classes discussed in Chapter 8 . The seismic capacity of an item of equipment can be compared to a seismic demand spectrum (SDS) defined in terms of an in-structure response spectrum (IRS) with the applicable scale factors. In Chapter 9 and parts of Chapter 10, specific methods for comparing seismic capacity to seismic demand are developed for several classes of equipment. In addition, a comparison of seismic capacity to seismic demand is made in Chapter 6 for the anchorage of the equipment and in Chapter 11 for relays mounted in the equipment.

4 Based on Sections 4.2, 4.2.1, and 4.2.2 of SQUG GIP (Ref. 1) 
The seismic capacity of equipment can be represented by a "Reference Spectrum" based on earthquake experience data, or a "Generic Equipment Ruggedness Spectrum" (GERS) based on generic seismic test data. Note that these two methods of representing seismic capacity of equipment can only be used if the equipment meets the intent of the caveats for its equipment class as described in Chapter 8.

Earthquake experience data was obtained by surveying and cataloging the effects of strong ground motion earthquakes on various classes of equipment mounted in conventional facilities and other industrial facilities. The results of this effort are summarized in Reference 35 . Based on this work, a "Reference Spectrum" was developed representing the seismic capacity of equipment in the earthquake experience equipment class. A detailed description of the derivation and use of this Reference Spectrum is contained in Reference 19 and this reference should be reviewed by the SCEs before using the Reference Spectrum. The Reference Spectrum, which is shown in Chapter 5 , can be used to represent the seismic capacity of equipment in a DOE facility when this equipment is determined to have characteristics similar to the earthquake experience equipment class and meets the intent of the caveats for that class of equipment as defined in Chapter 8. Use of the Reference Spectrum for comparison with a SDS is described in Chapter 5.

A large amount of data was also collected from seismic qualification testing of equipment. This data was used to establish a generic ruggedness level for various equipment classes in the form of Generic Equipment Ruggedness Spectra (GERS). The development of the GERS and the limitations on their use (caveats) are documented in Reference 40. Copies of the non-relay GERS along with a summary of the caveats to be used with them are included in Chapter 8. SCEs should review Reference 40 to understand the basis for the GERS. GERS can be used to represent the seismic capacity of an item of equipment in a DOE facility when this equipment is determined to have characteristics that are similar to the generic testing equipment class and meets the intent of the caveats for that class of equipment as defined in Chapter 8. Use of the GERS for comparison to a SDS is described in Chapter 5.

\subsubsection{Anchorage Adequacy 5}

A screening guideline to be satisfied to evaluate the seismic adequacy of an item of equipment is to confirm that the anchorage of the equipment is adequate. Lack of anchorage or inadequate anchorage has been a significant cause of equipment failing to function properly during and following past earthquakes.

The screening approach for evaluating the seismic adequacy of equipment anchorage is based upon a combination of inspections, analyses, and engineering judgment. Inspections consist of measurements and visual evaluations of the equipment and its anchorage, supplemented by use of facility documentation and drawings. Analyses should be performed to compare the anchorage capacity to the seismic loads (demand) imposed upon the anchorage. These analyses should be done using the guidelines contained in Chapter 6 . Engineering judgment is an important element in the evaluation of equipment anchorage. Guidance for making judgments is included, where appropriate, in Chapter 6 and in the reference documents.

Section 6.4.1 contains methods for determining or estimating the natural frequency and damping of many of the classes of equipment in Chapters 8,9, and 10. Generic equipment characteristics are provided for motor control centers, low-voltage switchgear, medium-voltage switchgear, transformers, horizontal pumps, vertical pumps, air compressors, motor-generators, batteries on racks, battery chargers and inverters, engine-generators, instrument racks, equipment cabinets, and control panels.

5 Based on Section 4.4 of SQUG GIP (Ref. 1) 
There are various combinations of inspections, analyses, and engineering judgment that can be used to evaluate the adequacy of equipment anchorage. The SCEs should select the appropriate combination of elements for each anchorage installation based on the information available. For example, a simple hand calculation may be sufficient for a pump that has only a few, very rugged, anchor bolts in a symmetrical pattern. On the other hand, at times it may be advisable to use one of the anchorage computer codes to determine the loads applied to a multi-cabinet motor control center if its anchorage is not symmetrically located. Likewise a trade-off can be made between the level of inspection performed and the factor of safety used for expansion anchor bolts. These types of trade-offs and others are discussed in Chapter 6.

\subsubsection{Seismic Interaction $^{6}$}

A screening guideline to be satisfied to evaluate the seismic adequacy of an item of equipment is to confirm that there are no adverse seismic spatial interactions with nearby equipment, systems, and structures and interaction from water spray, flooding, and fire hazards that could cause the equipment to fail to perform its intended function. The interactions of concern are potential impact due to proximity, structural failure and falling, and flexibility of attached lines and cables. Guidelines for judging interaction effects when evaluating the seismic adequacy of equipment are presented in Chapter 7.

It is the intent of the seismic interaction evaluation that real (i.e., credible and significant) interaction hazards be identified and evaluated. The interaction evaluations described in Chapter 7 focus on areas of concern based on past earthquake experience. Systems and equipment that have not been specifically designed for seismic loads should not be arbitrarily assumed to fail under earthquake loads; instead, SCEs are expected to differentiate between likely and unlikely interactions, using their judgment and past earthquake experience. In addition, system interaction effects as defined in DOE-STD-1021 (Ref. 7) are also discussed in Chapter 7.

Note that special attention should be given to the seismic interaction of electrical cabinets containing relays. If the relays in the electrical cabinets are essential (i.e., the relays should not chatter during an earthquake), then any impact on the cabinet should be considered an unacceptable seismic interaction and cause for identifying that item of equipment as an outlier. Guidance for evaluating the consequences of relay chatter due to earthquake motions, including cabinet impact interactions, are presented in Chapter 11 and Reference 45.

\subsubsection{Equipment Class Evaluations ${ }^{7}$}

A screening guideline to be satisfied to evaluate the seismic adequacy of an item of equipment is to confirm that (1) the equipment characteristics are generally similar to the earthquake experience equipment class or the generic seismic testing equipment class and (2) the equipment meets the intent of the specific caveats, procedures, or guidelines for the equipment class.

The DOE Seismic Evaluation Procedure has three different types of equipment class evaluations with varying levels of rigor and technical review. Table 2.1-1 lists all the equipment classes contained in the DOE Seismic Evaluation Procedure and the type of evaluation for each equipment class.

6 Based on Section 4.5 of SQUG GIP (Ref. 1)

7 Based on Section 4.3 of SQUG GIP (Ref. 1) 
- Chapter 8 contains caveats that permit the rigorous use of the Reference Spectrum and/or GERS to define the seismic capacity of the equipment classes. The twenty classes of equipment and the procedures in Chapter 8 are from Revision 2 of the SQUG GIP. The procedures in Chapter 8 were independently reviewed by the Senior Seismic Review and Advisory Panel (SSRAP) as part of the SQUG program and were approved by the NRC with a safety evaluation report (Ref. 2).

- Chapter 9 contains equipment class evaluations based on rigorous screening procedures from Revision 2 of the SQUG GIP. The procedures in Chapter 9 were independently reviewed by SSRAP as part of the SQUG program and were approved by the NRC with a safety evaluation report (Ref. 2 ).

- Chapter 10 contains screening procedures and general guidelines for equipment classes that are not provided in the SQUG GIP and are found at DOE facilities. Sections 10.1.1, 10.4.1, and 10.5.1 contain relatively rigorous screening procedures. Sections 10.2, 10.3.2, 10.5.2, and 10.5.3, on the other hand, contain guidelines that are not rigorous, but are intended to provide cost-effective and achievable techniques for increasing the seismic capacity of equipment classes in those sections. Finally, Sections 10.3.1 and 10.1.2 are summarized versions of several chapters of a DOE document. The technical review of the Sections in Chapter 10 is discussed in Section 1.4.2.

In addition to the classes of equipment in the SQUG GIP, twenty additional classes of equipment were identified as potentially requiring seismic evaluation at DOE sites. These additional classes of equipment were identified based on the responses from questionnaires sent to DOE sites and Chapter 10 contains about half of the identified classes of equipment. As the screening procedures and guidelines for additional classes of equipment are developed and reviewed, they can be added to Chapter 10 of the DOE Seismic Evaluation Procedure. In addition, the rigor of some of the sections in Chapter 10 can be enhanced with further development and review. Other classes of equipment that exist at DOE facilities that could be added to the DOE Seismic Evaluation Procedure include:

electrical equipment - distributed control systems, computer equipment, alarm and security equipment, communication equipment, and miscellaneous electrical equipment

mechanical equipment - ventilation dampers

tanks - elevated tanks, boilers, and miscellaneous tanks

piping and raceway systems - stacks, tubing, bus ducts, and conveyors of material

architectural features - suspended ceilings, cranes, and elevators

switchyard and substation equipment - power transformers, circuit breakers, disconnect switches, current and voltage transformers, surge and lightning arresters, wave traps, capacitor banks, buswork, and miscellaneous switchyard equipment 
Table 2.1-1 Equipment Class Evaluations in the DOE Seismic Evaluation Procedure

\begin{tabular}{|c|c|c|}
\hline Section & \begin{tabular}{|l} 
Equipment \\
Class
\end{tabular} & Type of Evaluation \\
\hline \multicolumn{3}{|c|}{ ELECTRICALEQUIPMENT } \\
\hline 8.1 .1 & Batteries on Racks & Caveats \\
\hline 8.1 .2 & Motor Control Centers & Caveats \\
\hline 8.1 .3 & Low-Voltage Switchgear & Caveats \\
\hline 8.1 .4 & Medium-Voltage Switchgear & Caveats \\
\hline 8.1 .5 & \begin{tabular}{|l|} 
Distribution Panels \\
\end{tabular} & Caveats \\
\hline 8.1 .6 & Transformers & Caveats \\
\hline 8.1 .7 & Battery Chargers and Inverters & Caveats \\
\hline 8.1 .8 & Instrumentation and Control Panels & Caveats \\
\hline 8.1 .9 & Instruments on Racks & Caveats \\
\hline 8.1 .10 & Temperature Sensors & Caveats \\
\hline \multicolumn{3}{|c|}{ MECHANICAL EQUIPMENT } \\
\hline 8.2 .1 & Fluid-Operated/Air-Operated Valves & Caveats \\
\hline 8.2 .2 & Motor-Operated/Solenoid-Operated Valves & Caveats \\
\hline 8.2 .3 & Horizontal Pumps & Caveats \\
\hline 8.2 .4 & Vertical Pumps & Caveats \\
\hline 8.2 .5 & Chillers & Caveats \\
\hline 8.2 .6 & Air Compressors & Caveats \\
\hline 8.2 .7 & Motor-Generators & Caveats \\
\hline 8.2 .8 & Engine-Generators & Caveats \\
\hline 8.2 .9 & Air Handlers & Caveats \\
\hline 8.2 .10 & Fans & Caveats \\
\hline 10.2 .1 & HEPA Filters & General Guidelines \\
\hline 10.2 .2 & Glove Boxes & General Guidelines \\
\hline 10.2 .3 & Miscellaneous Machinery & General Guidelines \\
\hline \multicolumn{3}{|c|}{ TANKS } \\
\hline 9.1 .1 & Vertical Tanks & Screening Procedure \\
\hline 9.1 .2 & Horizontal Tanks and Heat Exchangers & Screening Procedure \\
\hline 10.3 .1 & Underground Tanks & General Guidelines \\
\hline 10.3 .2 & Canisters and Gas Cylinders & General Guidelines \\
\hline \multicolumn{3}{|c|}{ PIPING, RACEWAY, AND DUCT SYSTEMS } \\
\hline 9.2 .1 & Cable and Conduit Raceway Systems & Screening Procedure \\
\hline 10.1 .1 & Piping & Screening Procedure \\
\hline 10.1 .2 & Underground Piping & General Guidelines \\
\hline 10.4 .1 & HVAC Ducts & Screening Procedure \\
\hline \multicolumn{3}{|c|}{ ARCHITECTURAL FEATURES AND COMPONENTS } \\
\hline 10.5 .1 & Unreinforced Masonry (URM) Walls & Screening Procedure \\
\hline 10.5 .2 & Raised Floors & General Guidelines \\
\hline 10.5 .3 & Storage Racks & General Guidelines \\
\hline
\end{tabular}




\subsection{Rule of the Box ${ }^{8}$}

An important aspect of evaluating the seismic adequacy of equipment included within the scope of this procedure is explained by the "rule of the box". "Rule of the box" applies to "normal" components of equipment, or parts of the equipment that are included in the earthquake experience database or shake table tests database. The intent of the "rule of the box" for equipment included in either the earthquake or testing equipment database is that all of the components mounted on or in this equipment are considered to be part of that equipment and do not have to be evaluated separately. Auxiliary components that are not mounted on the item of equipment but are needed by the equipment to fulfill its intended function need to be evaluated separately. Peer review, as discussed in Section 2.2, is needed to evaluate if the earthquake experience database or shake table tests database provides the basis for a particular application of the "rule of the box".

A typical example of the "rule of the box" is a diesel generator which not only includes the engine block and generator, but also all other items of equipment mounted on the diesel generator or on its skid; such as the lubrication system, fuel supply system, cooling system, heaters, starting systems, and local instrumentation and control systems. Components needed by the diesel generator but not included in the "box" (i.e., not mounted on the diesel generator or on its skid) are to be identified and evaluated separately. Typically this would include such items as off-mounted control panels, air-start compressors and tanks, batteries, pumps for circulating coolant and lubricant, day tanks, and switchgear cabinets.

An obvious advantage to the "rule of the box" is that only the major items of equipment need be evaluated for seismic adequacy (and only documented once), i.e., if a major item of equipment is shown to be seismically adequate using the guidelines in this procedure, then all of the parts and components mounted on or in that item of equipment are also considered seismically adequate. Typically, the "rule of the box" applies for components attached to the equipment before the first anchor point of the equipment. However, the SCEs should exercise their judgment and experience to seek out suspicious details or uncommon situations (those which are "out of the ordinary", are not specifically covered in the equipment class evaluations, or are site add-ons) that may make that item of equipment vulnerable to earthquake effects. This evaluation should include any areas of concern within the "box" which could be seismically vulnerable, such as added attachments, missing anchorage, or obviously inadequate anchorage of components.

One exception to the "rule of the box" is relays (and other types of device using contacts in the control circuitry). Even though relays are mounted on or in another larger item, they should be identified and evaluated for seismic adequacy using the procedure described in Chapter 11 since they may be susceptible to chatter during seismic excitation. The relays to be evaluated are identified by first identifying the major item of equipment for the SEL which could be affected if the relays malfunctioned. Then, in Chapter 11, the particular relays used to control these major items of equipment are determined and evaluated for seismic adequacy.

\subsection{Equipment Class Evaluations Using Caveats for the Reference Spectrum and/or GERS (Chapter 8) $^{9}$}

Chapter 8 contains a summary of equipment class descriptions based on earthquake experience data and generic seismic testing data. These descriptions and the rest of Chapter 8 is from Appendix B of Revision 2 of the SQUG GIP. An item of equipment must have the same general characteristics as the equipment in the earthquake experience equipment class or the generic seismic testing equipment class to apply the methodology in Chapter 8 . The intent of this rule is to preclude items

8 Based on Section 3.3.3 of SQUG GIP (Ref. 1)

9 Based on Section 4.3 of SQUG GIP (Ref. 1) 
of equipment with unusual designs and characteristics that have not demonstrated seismic adequacy in earthquakes or tests.

"Caveats" are defined as the set of inclusion and exclusion rules that represent specific characteristics and features particularly important for seismic adequacy of a particular class of equipment. Chapter 8 contains a summary of the caveats for the earthquake experience equipment class and for the generic seismic testing equipment class. If the caveats are satisfied, then the capacity of the equipment class can be represented by the Reference Spectrum and/or the GERS. For these equipment classes, extensive use of earthquake experience and test data permits the rigorous definition of the equipment capacity and evaluation of the seismic adequacy of the equipment. The equipment capacity determined in Chapter 8 is compared to the seismic demand using the provisions of Chapter 5.

The "intent" of the caveats should be met when evaluating an item of equipment as they are not fixed, inflexible rules. Engineering judgment may be used to determine whether the specific seismic concern addressed by the caveat is met. Chapter 8 provides brief discussions of the intent of the caveats. When specific cases are identified where the intent of the caveats are considered to be met, but the specific wording of the caveat rule is not, the reason for this conclusion should be documented.

Note that the caveats in Chapter 8 are not necessarily a complete list of every seismically vulnerable detail that may exist since it is impossible to cover all such situations by meaningful caveats. Instead, the SCEs should exercise their judgment and experience to seek out suspicious details or uncommon situations (not specifically covered by the caveats) which may make equipment vulnerable to earthquake effects. For example, the SCEs should note any areas of concern within the "box" which could be seismically vulnerable such as added attachments, missing or obviously inadequate anchorage of components, heavy objects mounted on the equipment, and components that are known to be seismically sensitive.

The summaries of the equipment class descriptions and caveats in Chapter 8 are based on information contained in References 19,35, and 40. Additional information on seismic experience data is contained in Chapter 9d of Reference 32. The SCEs should use the summaries in Chapter 8 only after first thoroughly reviewing and understanding the background of the equipment classes and bases for the caveats as described in these references. These references provide more details (such as photographs of the data base equipment) and more discussion than summarized in Chapter 8. Note that in some cases, clarifying remarks have been included in Chapter 8 that are not contained in the reference documents. These clarifying remarks include such things as the reason for including a particular caveat, the intent of the caveat, and recommended allowable limits for stress analysis. The remarks are also based on experience gained during SQUG GIP reviews at operating nuclear power plants and DOE seismic evaluations at DOE facilities and they serve to help guide the SCEs in their judgment.

Certain important caveats from the reference documents are not included in Chapter 8 because they are covered in other sections of the DOE Seismic Evaluation Procedure. These caveats include:

- Equipment should be adequately anchored and base isolation should be carefully evaluated (see Chapter 6).

- Seismic interaction concerns, such as flexibility of attached lines, should not adversely affect the equipment (see Chapter 7).

- Relays for which chatter is not acceptable should be specifically evaluated. Note that although the primary responsibility for conducting the relay evaluation is the Lead Relay Reviewer, the SCEs should be alert for any seismically induced systems effects that may lead to loss of function or malfunction of the equipment being evaluated (see Chapter 11). 
In addition, caveats discussing a limiting fundamental frequency of $8 \mathrm{~Hz}$ are not included in Chapter 8 because this limiting frequency does not apply with the provisions of Chapter 5 .

Chapter 8 is organized by equipment class as listed in Table 2.1-2. For each equipment class, the class description and the caveats applicable to the Reference Spectrum are given first. A plot of the Reference Spectrum is provided in Chapter 5. Next, the class description and the caveats applicable to the GERS are given, when available. Some equipment classes have more than one GERS while other classes have none. A plot of the GERS follows the caveats for each applicable equipment class. While the GERS typically define a higher capacity, the GERS caveats are more restrictive than the reference spectrum caveats.

Table 2.1-2 Equipment Class Evaluations Using Caveats for the Reference Spectrum and/or GERS (SQUG GIP, Reference 1)

\begin{tabular}{|c|l|c|c|}
\hline Section & $\begin{array}{l}\text { Equipment } \\
\text { Class }\end{array}$ & $\begin{array}{c}\text { Reference } \\
\text { Spectrum }\end{array}$ & GERS \\
\hline 8.1 .1 & Batteries on Racks & $\mathrm{X}$ & $\mathrm{X}$ \\
\hline $\mathbf{8 . 1 . 2}$ & Motor Control Centers & $\mathrm{X}$ & $\mathrm{X}$ \\
\hline $\mathbf{8 . 1 . 3}$ & Low-Voltage Switchgear & $\mathrm{X}$ & $\mathrm{X}$ \\
\hline 8.1 .4 & Medium-Voltage Switchgear & $\mathrm{X}$ & $\mathrm{X}$ \\
\hline $\mathbf{8 . 1 . 5}$ & Distribution Panels & $\mathrm{X}$ & $\mathrm{X}$ \\
\hline $\mathbf{8 . 1 . 6}$ & Transformers & $\mathrm{X}$ & $\mathrm{X}$ \\
\hline 8.1 .7 & Battery Chargers and Inverters & $\mathrm{X}$ & $\mathrm{X}$ \\
\hline 8.1 .8 & Instrumentation and Control Panels & $\mathrm{X}$ & \\
\hline $\mathbf{8 . 1 . 9}$ & Instruments on Racks & $\mathrm{X}$ & $\mathrm{X}$ \\
\hline $\mathbf{8 . 1 . 1 0}$ & Temperature Sensors & $\mathrm{X}$ & \\
\hline 8.2 .1 & Fluid-Operated/Air-Operated Valves & $\mathrm{X}$ & $\mathrm{X}$ \\
\hline 8.2 .2 & Motor-Operated/Solenoid-Operated Valves & $\mathrm{X}$ & $\mathrm{X}$ \\
\hline $\mathbf{8 . 2 . 3}$ & Horizontal Pumps & $\mathrm{X}$ & \\
\hline 8.2 .4 & Vertical Pumps & $\mathrm{X}$ & \\
\hline 8.2 .5 & Chillers & $\mathrm{X}$ & \\
\hline $\mathbf{8 . 2 . 6}$ & Air Compressors & $\mathrm{X}$ & \\
\hline $\mathbf{8 . 2 . 7}$ & Motor-Generators & $\mathrm{X}$ & \\
\hline $\mathbf{8 . 2 . 8}$ & Engine-Generators & $\mathrm{X}$ & \\
\hline $\mathbf{8 . 2 . 9}$ & Air Handlers & $\mathrm{X}$ & \\
\hline $\mathbf{8 . 2 . 1 0}$ & Fans & $\mathrm{X}$ & \\
\hline
\end{tabular}

\subsection{Equipment Class Evaluations Using Screening Procedures (Chapter 9)}

Chapter 9 contains a summary of equipment class descriptions and parameters based on earthquake experience data, test data, and analytical derivations. The screening procedures in Chapter 9 are from Chapters 7 and 8 of Revision 2 of the SQUG GIP. An item of equipment must have the same general characteristics as the equipment in the evaluation procedures. The intent of this rule is to preclude items of equipment with unusual designs and characteristics that have not demonstrated seismic adequacy in earthquakes or tests.

The screening procedures for evaluating the seismic adequacy of the different equipment classes in Chapter 9 cover those features which experience has shown can be vulnerable to seismic loadings. These procedures are a step-by-step process through which the important equipment parameters and dimensions are determined, seismic performance concerns are evaluated, the equipment capacity is determined, and the equipment capacity is compared to the seismic demand. 
The screening procedures in Chapter 9 are based on information contained in References 42, 46, 47, and 50. The SCEs should use the information in Chapter 9 only after first thoroughly reviewing and understanding the background of the equipment classes and bases for the screening procedures as described in these references. These references provide more details and more discussion than summarized in Chapter 9. In some cases, clarifying remarks not contained in the reference documents have been included in Chapter 9. These clarifying remarks are based on experience gained during SQUG GIP reviews at operating nuclear power plants and DOE seismic evaluations at DOE facilities and they serve to help guide the SCEs apply their judgment.

The screening procedures in Chapter 9 are from Revision 2 of the SQUG GIP and Table 2.1-3 lists the equipment classes in Chapter 9.

\section{Table 2.1-3 Equipment Class Evaluations Using Screening Procedures (SQUG GIP, Reference 1)}

\begin{tabular}{|c|l|c|}
\hline Section & Equipment Class & $\begin{array}{c}\text { Source of } \\
\text { Screening } \\
\text { Procedure in } \\
\text { SQUG GIP }\end{array}$ \\
\hline 9.1 .1 & Vertical Tanks & Section 7 \\
\hline 9.1 .2 & Horizontal Tanks and Heat Exchangers & Section 7 \\
\hline 9.2 .1 & Cable and Conduit Raceway Systems & Section 8 \\
\hline
\end{tabular}

\subsection{Equipment Class Evaluations Using Screening Procedures or General Guidelines} (Chapter 10)

Chapter 10 contains a summary of equipment class descriptions and parameters based on earthquake experience data, test data, and analytical derivations. The classes of equipment contained in Chapter 10 are not from the SQUG GIP. Much of the information in Chapter 10 is from DOE references. Table 2.1-4 lists the principal references and authors for the sections in Chapter 10. An item of equipment must have the same general characteristics as the equipment in the screening procedures and general guidelines. The intent of this rule is to preclude items of equipment with unusual designs and characteristics that have not demonstrated seismic adequacy in earthquakes or tests.

The screening procedures in Sections 10.1.1, 10.4.1, and 10.5.1, for evaluating the seismic adequacy of piping, HVAC ducts, and unreinforced masonry (URM) walls respectively, cover those features which experience has shown can be vulnerable to seismic loading. These procedures are a step-by-step process through which the important equipment parameters and dimensions are determined, seismic performance concerns are evaluated, the equipment capacity is determined, and the equipment capacity is compared to the seismic demand. Sections 10.1.1 and 10.4.1 have been technically reviewed and used extensively at several DOE sites including Savannah River Site and Rocky Flats Environmental Technology Center.

The general guidelines for evaluating the seismic adequacy of the equipment classes in the other sections of Chapter 10 cover those features which experience has shown can be vulnerable to seismic loading. The sections contain practical guidelines and reference to documents that can be used to implement an equipment strengthening and upgrading program. The relatively simple seismic upgrades are designed to provide cost-effective methods of enhancing the seismic safety of the equipment classes in Chapter 10. Sections 10.3.1 and 10.1.2 summarize information from portions of a DOE document that has undergone extensive technical review. Sections 10.2.1, 
10.2.2, 10.2.3, 10.3.2, 10.5.2, and 10.5.3, on the other hand, are based on walkdown and seismic strengthening efforts at several DOE sites including Los Alamos National Laboratory and Lawrence Livermore National Laboratory.

\section{Table 2.1-4 Equipment Class Evaluations Using Screening Procedures or General Guidelines}

\begin{tabular}{|c|l|c|c|}
\hline Section & Equipment Class & $\begin{array}{c}\text { Principal } \\
\text { Reference }\end{array}$ & Principal Author \\
\hline 10.1 .1 & Piping & 59 & G. Antaki, SRS \\
\hline 10.1 .2 & Underground Piping & 29 & S. Short, EQE \\
\hline 10.2 .1 & HEPA Filters & & L. Goen, LANL \\
\hline 10.2 .2 & Glove Boxes & & L. Goen, LANL \\
\hline 10.2 .3 & Miscellaneous Machinery & 60 & S. Sommer, LLNL \\
\hline 10.3 .1 & Underground Tanks & 29 & S. Short, EQE \\
\hline 10.3 .2 & Canisters and Gas Cylinders & 60 & R. Murray, LLNL \\
\hline 10.4 .1 & HVAC Ducts & 28 & G. Driesen, SRS \\
\hline 10.5 .1 & Unreinforced Masonry (URM) Walls & & R. Murray, LLNL \\
\hline 10.5 .2 & Raised Floors & 60 & S. Sommer, LLNL \\
\hline 10.5 .3 & Storage Racks & 60 & S. Sommer, LLNL \\
\hline
\end{tabular}

\subsubsection{Outlier Identification and Resolution ${ }^{10}$}

Items listed in the SEL that do not pass the screening criteria contained in the Seismic Evaluation Procedure are considered outliers (i.e., they lay outside the cope of coverage for the screening criteria) and should be evaluated further as described in Chapter 12. An outlier may be shown to be adequate for seismic loads by performing evaluations such as the seismic qualification techniques currently being used in some DOE facilities. These additional evaluations and alternate methods should be thoroughly documented to permit independent review.

Methods of outlier resolution are typically more time consuming and expensive than the screening evaluations provided in the Seismic Evaluation Procedure. Also, outlier resolution may be somewhat open-ended because several different options or approaches are available to evaluate seismic adequacy. The most appropriate method of outlier resolution will depend upon a number of factors such as: (1) which of the screening criteria could not be met and by how much, (2) whether the discrepancy lends itself to an analytical evaluation, (3) how extensive the problem is in the facility and in other facilities, or (4) how difficult and expensive it would be to modify, test, or replace the subject items of equipment.

10 Based on Section 1.3.4 of SQUG GIP (Ref. 1) 


\subsubsection{Documentation}

The suggested types of document which should be used with the DOE Seismic Evaluation Procedure are described in Chapter 13. The five major types of documents are:

- $\quad$ Seismic Equipment List (SEL)

- $\quad$ Screening Evaluation Work Sheets (SEWS)

- $\quad$ Outlier Seismic Evaluation Sheets (OSES)

- $\quad$ Screening Evaluation and Data Sheets (SEDS)

- $\quad$ Equipment Seismic Evaluation Report (ESER)

These documents serve as tools to summarize the results of the Screening Evaluation and Walkdown and to highlight areas in need of further evaluation or upgrading. Other, informal documentation may be used by the SCEs as an aid and these may include calculations, sketches, photographs, audio tapes, and videotapes. The completed OSES, SEWS, SEDS, and ESER constitute the documentation of the Screening Evaluation and Walkdown and reflect the final judgment of the SCEs. 


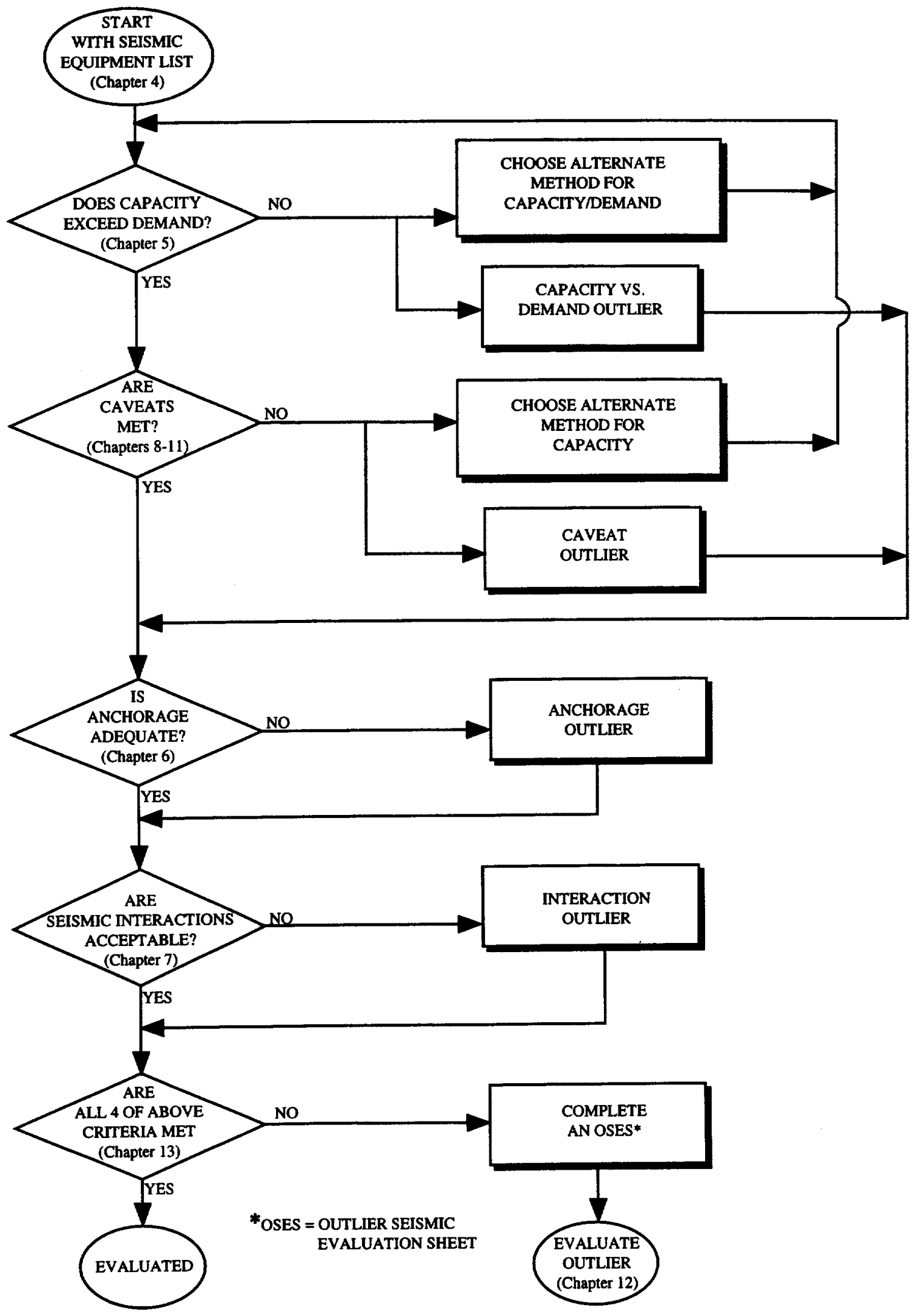

Figure 2.1-1 Overall Procedure for Performing Screening Evaluation and Walkdown (Figure 4-1 of SQUG GIP, Reference 1) 


\subsection{PEER REVIEW}

Peer review is a vitally important component of seismic evaluations of equipment and distribution systems at DOE facilities. The evaluation procedures described in the DOE Seismic Evaluation Procedure involve an extensive use of engineering judgment. This type of judgment must be independently reviewed to ensure that significant details are not overlooked or improperly evaluated. In addition, DOE Orders and Standards discuss that peer review is a necessary element of design and evaluation for natural phenomena hazards. Peer review can be provided by certified SCEs who are independent of the SRT whose evaluation is being reviewed.

Members of a peer review team should be selected and incorporated early in the evaluation process. With review occurring in parallel with evaluations, the peer review team can efficiently study the important facets of the evaluation and provide useful feedback. The peer review team should consist of engineers that have extensive experience with seismic design and evaluation as well as be knowledgeable of the methodology and procedures in the DOE Seismic Evaluation Procedure. Typically, the members of the peer review should be more qualified than the SCEs conducting the equipment evaluations and the members should have conducted many evaluations similar to the ones being reviewed. The size of the peer review team should reflect the scope of the equipment evaluations being reviewed.

The equipment evaluations and the peer review should consider the DOE requirements for quality assurance. These requirements are specified in 10CFR830.120, the DOE Nuclear Safety Management Rule, (Ref. 61) and DOE Order 5700.6C, "Quality Assurance", (Ref. 62). The Rule requires the development of quality assurance programs for DOE nuclear facilities. Information for implementing quality procedures is provided in the Rule and Order. Sections 1.4 and C.8 of DOESTD-1020 (Ref. 6) provides additional guidance on quality assurance and peer review.

\subsection{PREPARATION FOR THE EVALUATION}

\subsubsection{Systems Engineering and Facility Operations ${ }^{11}$}

Experience from facility reviews has demonstrated that preparatory work performed prior to conducting the facility screening evaluations will maximize the effectiveness of the walkdown procedure outlined in Section 2.1. Prior to the walkdown, members of the SRT including the SCEs, systems engineer(s), and facility operations representative(s) should review the facility design documents to familiarize themselves with facility design features and, in particular, those associated with equipment identified in the Seismic Equipment List (SEL). Much of the required initial information is contained in a Safety Analysis Report (SAR) or related report. In addition, piping and instrumentation diagrams (P\&IDs), electrical one-line drawings, instrument block diagrams, operating procedures, system descriptions, facility arrangement drawings, and selected topical reports and specifications should be used during the equipment identification and walkdown efforts.

Discussions with facility operations personnel are beneficial in identifying equipment within various safety systems. Systems engineers may wish to consider including equipment that does not have seismic qualification documentation, thereby upgrading its seismic qualification status. Most of the industrial-grade equipment in the earthquake experience data base has been shown to be seismically rugged even though it has not been qualified for seismic loads.

Facility arrangement drawings should be marked with the location of each item of equipment selected for review and provided to the SCEs who will be doing the seismic evaluation. In

11 Based on Sections E.1 and E.2 of SQUG GIP (Ref. 1) 
addition, the SEL, which is described in Chapter 4, should be completed in order to identify the equipment to be seismically evaluated.

\subsubsection{Pre-Walkdown Planning ${ }^{12}$}

The purpose of pre-walkdown planning is to organize the facility walkdown. Judicious planning will minimize the time spent in the field by the SRT.

The planning process should be performed with active participation from the principal walkdown participants and the facility personnel with experience in the configuration and operation of the facility under review. The following organizations or individuals will typically be involved in the walkdown and should be part of the planning effort:

- $\quad$ Facility Manager

- Safety Professionals and Systems Engineer(s)

- Facility Operations and/or Radiation Protection Personnel

- $\quad$ Seismic Capability Engineers

- Relay Evaluation Personnel

- $\quad$ Piping Evaluation Engineers

Advance planning on when to perform the walkdown is advisable. Walkdowns should not interfere with the normal operation of the facility. Security, radiation level, operations, and maintenance considerations are necessary in deciding when each area of the facility can be visited. Some areas of the facility are inaccessible during normal operation and can only be inspected during outage periods. The Screening Evaluation and Data Sheets (SEDS), discussed in Chapter 13, can be organized by facility location and thereby used as a checklist and itinerary for the walkdown. The itinerary, however, should be flexible to allow the walkdown teams time to revisit certain areas or alter their plans because of difficulties in determining seismic adequacy of particular types of equipment. It is also advisable to provide the walkdown teams with the itineraries in advance so that they can review the items of equipment assigned prior to the walkdown.

Advance planning and preparation are needed to gain access to operating facilities, particularly if contractors are used to conduct the walkdown. The SRT may be required to obtain security clearances, access badges, and radiation training. The walkdown participants may need to be accompanied by facility security and radiation protection personnel; however, such accompaniment is costly, ties up personnel, and tends to interfere with normal facility operations and maintenance. It also increases the number of individuals involved with the walkdown which tends to slow down the pace of the effort. Advance notification and scheduling can streamline the process of gaining facility access. All people concerned with the facility walkdown, including walkdown team members, facility operations personnel, health physics personnel, security personnel and facility staff, should be advised of the dates and duration of the facility walkdown well in advance of the scheduled walkdowns (e.g., two months ahead of time).

The SRT or individual team members may want to have discussions with other facility operations personnel prior to and during the walkdown to clarify the way a system or an item of equipment operates. If possible, these meetings should be planned well in advance so that people

12 Based on Section E.3 of SQUG GIP (Ref. 1) 
knowledgeable in the specific areas of concern will be available with a minimum of disruption in the normal operation of the facility.

A summary of all the available seismic design and qualification data should be prepared and provided to the SRT several weeks before their scheduled walkdown. The summary does not have to be formal, but it should be comprehensive. The SCEs performing the walkdown should become thoroughly familiar with the facility seismic design basis. The greater the understanding of the facility seismic design basis and the approaches taken for equipment qualification and anchorage, the easier it will be to exercise judgment and experience to eliminate outliers.

Construction details of the anchorage for the equipment in the SEL are essential for evaluating the seismic adequacy of the equipment. Inspection and evaluation of anchorage are difficult if not impossible without the use of construction drawings, specifications, and bills of materials.

The documents which should be available to the SRT include:

1. The Seismic Equipment List (SEL), prepared using Chapter 4.

2. List of equipment for which prior seismic qualification documentation exists.

3. Summary of the facility seismic design basis, specifically: ground response spectra for the design basis earthquake (DBE) seismic design criteria, amplified in-structure response spectra (IRS), and seismic demand spectra (SDS).

4. Standard details for equipment anchorage.

5. Facility arrangement drawings.

6. Health physics and facility security requirements.

In addition, certain facility design information should be collected to help maximize the benefit of the evaluation. The following provides a checklist of example data that, if appropriate, should be collected prior to the Screening Evaluation and Walkdown procedure:

- $\quad$ Map of site with outline of structures and structure identifiers

- Performance goals for the facility equipment which is listed on the SEL

- $\quad$ Structural drawings for buildings, including current as-built key plans where possible

- Date of construction of facility (including dates of modifications as appropriate)

- $\quad$ Available soils data

- General description of processes housed in the building

- $\quad$ Safety Analysis Reports (SARs)

- $\quad$ Emergency response procedures related to seismic

- $\quad$ Facility procedural requirements including security access 


\subsubsection{Screening Walkdown Plan ${ }^{13}$}

This section describes an approach that can be used to perform the screening evaluation of the equipment listed in the SEL during the facility walkdown. This approach is based on the experience gained in performing facility reviews. This section covers the organization and approach which can be used by the SRT, the degree of inspection to be performed, walkdown logistics, and screening walkdown completion.

\subsubsection{Organization and Approach of SRT ${ }^{14}$}

The number of individuals in each SRT should be limited to permit ready access to inspect equipment and facilitate movement. In addition to the two SCEs, a systems or operations engineer may also be involved in the walkdown as needed by the SRT to provide information on how a system or an item of equipment operates. Health physics and security personnel may also accompany the SRT as the need arises.

Each group of individuals walking down the facility should collectively have:

1. An understanding of the facility layout and location of the various system and equipment scheduled to be evaluated during that walkdown period;

2. An understanding of the scope and objectives of the walkdown including the methodology and procedures;

3. An understanding of the seismic evaluation guidelines including inspection techniques and evaluation criteria;

4. An understanding of the operational aspects of the facility and the importance of the various facility systems and equipment.

SRT decisions concerning equipment seismic adequacy should be made on the spot, if possible, and the walkdown should proceed at a pace consistent with this objective. Decisions to evaluate the seismic adequacy of equipment should be unanimous among the SCEs. Concerns which do not permit seismic evaluation during the screening walkdown should be documented and left for further review to either eliminate the equipment as a required part of the SEL or identify it as an outlier for further evaluation (as described in Chapter 12). During the walkdown, many items of equipment may have evaluation results that are unknown. The SRT should decide what information or additional action is required to resolve the issue and inform the appropriate support staff personnel so that, if possible, the issue may be resolved during the later part of the walkdown.

If several SRTs are used to conduct the screening evaluation and walkdown, then a means for coordinating the activities should be invoked to ensure that all the equipment and activities of the evaluation are covered. This coordinating function could be performed by a single individual or by a committee of individuals from the various SRTs.

\subsubsection{Degree of Inspection ${ }^{15}$}

All of the equipment on the SEL should be reviewed. Exceptions to this may occur (e.g., equipment in very high radiation areas or otherwise inaccessible locations), and each exception

13 Based on Section F.1 of SQUG GIP (Ref. 1)

14 Based on Section F.2 of SQUG GIP (Ref. 1)

15 Based on Section F.3 of SQUG GIP (Ref. 1) 
should be justified by the SRT. The level or scope of evaluation may vary depending upon the experience and judgment of the SRT.

\subsubsection{Walkdown Logistics and Cautions ${ }^{16}$}

A three-to-four hour kick-off meeting can be scheduled for the beginning of the facility walkdown. This meeting can provide a briefing on the objectives of the walkdown, the organization of the walkdown groups, the planning for the walkdown, and the breakdown of the total list of equipment for which each group was responsible. After this kick-off meeting, the SRTs can commence with the facility walkdown.

Radiation training, including whole body counts and issuance of personnel dosimetry, and facility access requirements, such as obtaining security badges, for the SRT members are done prior to this kick-off meeting. Access to contaminated and radiological areas may require DOE or sitespecific Radiological Worker II Training. DOE-sponsored radiological training may reduce delays associated with facility-specific training.

A daily morning meeting should be held in which the SRT reviews the equipment included in that day's walkdown. Anchorage drawings are also reviewed by the SRT. The walkdown can be conducted in morning and afternoon sessions. A meeting can also be held during the lunch break to discuss problem areas and the approaches used by other SRTs. At the option of the facility and the SRTs, it may be desirable to conduct the walkdown outside of normal working hours. In any case, it is not recommended that the walkdown "day" exceed 10 hours.

A short meeting can also be held at the end of each day to discuss the day's walkdown, request information as required from the appropriate support staff personnel, certify the completed documentation, review information retrieved by the support staff so that previously started evaluations could be completed, and organize the next day's activities. Any unknowns are reconciled as soon as possible after the item of equipment had been inspected.

When performing the walkdown, the SRT should have the appropriate tools to collect and record data. These tools included a clip board (e.g., for SEDS and SEWS), a tape measure capable of measuring to $1 / 16$ inch, pencils or pens, and a flashlight. The SRT may wish to use some form of carrying pack to allow hands to be free for climbing ladders, going through crawl spaces.

Other tools may be included depending on the preference of the SRT. For example, a compact camera (subject to facility policy) can be useful to record visual findings, such as each picture frame should have a designation and be fully described. A small audio cassette recorder can be used to record the subject of each picture frame and general notes about the walkdown. More elaborate visual records can be obtained by using a video recorder. However, video equipment is usually cumbersome and expensive, and has not been used extensively in past facility walkdowns. It should also be understood that the use of personal equipment is typically at the individual's own risk. If equipment is contaminated or broken, there is often no compensation by the facility.

The SRT should be aware that there is usually a need for hard hats, safety glasses, hearing protection, and sometimes safety shoes. SRT members should consider wearing light cotton clothing since temperatures inside operating DOE facilities can be relatively high. These conditions can lead to extreme personnel discomfort, especially when protective clothing is required for walkdowns in contaminated and high radiation areas.

16 Based on Section F.4 of SQUG GIP (Ref. 1) 
During the walkdown, the SRT should use caution when evaluating equipment due to the many potential dangers. Manufacturer's data should be consulted if there are any questions and/or concerns about the operation of the equipment being evaluated.

- Temporarily inactive mechanical and electrical equipment may activate while being evaluated so all manufacturer's warnings should be carefully followed.

- A common rule for evaluating equipment, especially electrical equipment, is to not break the vertical plane. Since electrical equipment may be energized, only trained personnel should provide access to this type of equipment. It is not appropriate, potentially very dangerous, and usually prohibited by facility policy to open panels on electrical equipment without approval from the appropriate facility personnel.

- Since mechanical and electrical equipment may contain vibration sensitive components, it is inappropriate to test the dynamic characteristics of the equipment by shaking it. If facility personnel indicate that the equipment does not contain vibration sensitive equipment, such as essential relays, then any field testing of the dynamic characteristics should be done within manufacturer recommendations.

- In addition, all placards with hazards control information should be reviewed, understood, and obeyed. The typical information on a hazardous material card (see Figure 2.3-1) includes level of fire hazard, level of health hazard, level of oxidation or reactivity hazard, and special information. If the information or indications on a warning label are not understood, then the appropriate facility personnel, such as hazardous material technicians or fire protection personnel, should be contacted before proceeding.

The basic rules while conducting the walkdown are to use common sense, to avoid dangerous or unpredictable situations, and to obey facility policy and safety procedures.

\subsubsection{Screening Walkdown Completion ${ }^{17}$}

At the completion of the Screening Evaluation and Walkdown, all equipment identified in the SEL and included in the walkdown should be classified as being either evaluated or an outlier. The SEDS should be completed, checked for accuracy, and certified for each item of equipment. The outlier sheets (OSES) should be completed for each item of equipment identified as an outlier. Work sheets (SEWS), if used, should also be checked so that the information noted (judgments, description, and calculations) can be reasonably followed by a reviewer. At the completion of the Screening Evaluation and Walkdown, the SRT should inform the facility management about the walkdown results in detail.

17 Based on Section F.5 of SQUG GIP (Ref. 1) 


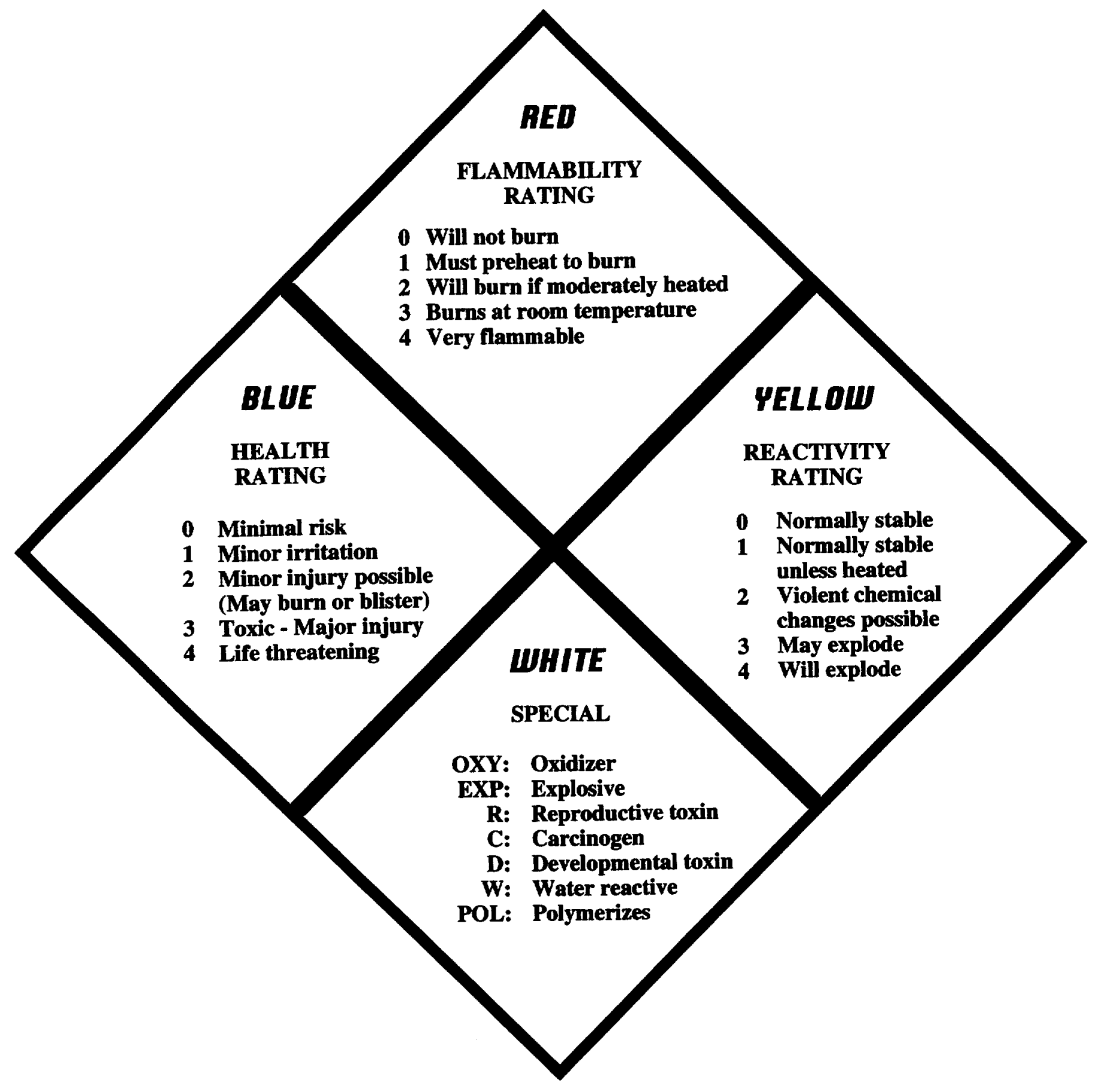

Figure 2.3-1 Hazardous Material Card 


\section{SEISMIC EVALUATION PERSONNEL}

\subsection{INTRODUCTION 1}

The purpose of this section is to define the responsibilities and recommended minimum requirements of the individuals who will implement the DOE Seismic Evaluation Procedure. The seismic evaluation personnel include individuals who develop the Seismic Equipment List (SEL), perform the facility walkdown and evaluate the seismic adequacy of equipment listed in the SEL, and perform the relay screening and evaluation. This may involve a number of safety, facility, and engineering disciplines including structural, mechanical, civil, electrical, systems, and seismic.

Most facilities may prefer to implement this procedure using a designated team of individuals; i.e., a Seismic Review Team (SRT). However, the functions and responsibilities may be assigned to existing departments or groups, without definition of a dedicated team, provided the individuals in these departments or groups have the appropriate qualifications and training and that the walkdown teams have the required collective qualifications. Similarly, the individuals who undertake the seismic review may be DOE staff; M\&O contractor staff; and subcontractors, who are currently under contract to DOE or a $M \& O$, provided the qualification and training criteria are met. This flexibility allows for the possibility that the functions may be performed by individuals of different disciplines at different times. DOE and M\&O contractor staff are responsible for evaluating the qualifications of the seismic evaluation personnel for compliance with this procedure.

\subsection{SEISMIC CAPABILITY ENGINEERS ${ }^{2}$}

\subsubsection{Responsibilities and Minimum Requirements}

The Seismic Capability Engineers (SCEs) should:

- Become familiar with the seismic experience data approach as defined in the DOE Seismic Evaluation Procedure and EPRI / SQUG reference documents.

- Become familiar with the seismic design basis of the facility being evaluated, especially the equipment on the SEL and the Design Basis Earthquake (DBE) for the facility.

- Conduct the seismic evaluations and walkdowns of equipment and systems as described in the following chapters and sections:

- Capacity versus Demand Evaluation (Chapter 5)

- Anchorage Review (Chapter 6)

- Seismic Interaction Evaluation (Chapter 7)

- Equipment Class Evaluations Using Caveats for the Reference Spectrum and/or GERS (Chapter 8)

- Equipment Class Evaluations Using Screening Procedures (Chapter 9)

1 Based on Section 2.0 of SQUG GIP (Ref. 1)

2 Based on Section 2.4 of SQUG GIP (Ref. 1) 
- Equipment Class Evaluations Using Screening Procedures or General Guidelines (Chapter 10)

- Relay Functionality Review (Chapter 11)

- Use the DOE Seismic Evaluation Procedure, along with experience and judgment, to evaluate the seismic adequacy of equipment and systems identified as necessary.

- Perform additional analyses and calculations, when necessary, to evaluate the seismic adequacy of the equipment and systems.

- Make recommendations for any additional evaluations or physical modifications to equipment or systems that may be necessary to determine the seismic adequacy of equipment identified as outliers as described in Chapter 12.

The SCEs may be assisted in fulfilling the above responsibilities by other individuals. For example, others may do background work to obtain information necessary for performing the seismic evaluations; they may also locate and assist in evaluating existing seismic qualification documentation; and they may perform backup calculations where necessary. Another example is that SCEs may ask the Systems Engineers, Safety Professionals, and the Operations Personnel for information on how an item of equipment operates in a system so they may decide whether a malfunction of certain features of the item of equipment will affect its safety performance. Regardless of what help the SCEs receive from others, they should remain fully responsible for all the seismic evaluations, engineering judgments, and documentation, including the details and backup documentation.

The recommended minimum requirements for the SCEs are:

- Bachelor of Science degree, or equivalent, in structural, mechanical, or civil engineering or related discipline,

- 5 years of experience in seismic design, testing, analysis, and/or evaluation of structures and equipment in DOE facilities and / or commercial nuclear power plants,

- $\quad$ Complete the 5-day DOE-developed training course on applying the EPRI / SQUG walkdown screening and seismic evaluation methodology for equipment at DOE facilities.

If an engineer has completed the 5-day EPRI training course on the EPRI / SQUG methodology for the commercial nuclear power industry, then the following list provides an alternative means of meeting the recommended minimum requirements for SCEs evaluating equipment at DOE facilities. The list replaces the recommended minimum requirement of attending the 5-day DOE-developed training course.

- Hold a certificate of completion for the 5-day EPRI course on the SQUG methodology presented for the commercial nuclear power industry,

- Complete the following supplemental DOE-specific training administrated under the oversight of the DOE: performance goals, capacity versus demand, and equipment classes beyond those covered in the EPRI course, as well as complete the associated case studies and quizzes. This supplemental training is conducted on a case-by-case basis depending on the qualifications of the engineer. It typically does not involve attendance of another training course. 
The Screening Evaluation and Walkdown should be conducted by one or more SRTs consisting of at least two SCEs on each team. The engineers on each team should collectively possess the following knowledge and experience:

- Experience in seismic design, seismic analysis and test qualification practices at DOE facilities. This should include active mechanical and electrical equipment, process and control equipment, and safety equipment.

- Knowledge of the performance of equipment, systems, and structures during strong motion earthquakes in industrial process and power plants. This should include active mechanical and electrical equipment, process and control equipment, and safety equipment.

- DOE facility walkdown experience.

- Knowledge of DOE orders, standards, and guidance.

It is not necessary for each SCE to possess all of the above qualifications; differing levels of expertise among the SRT engineers is appropriate. However, each SRT should collectively possess the above qualifications and each engineer on the team should have the ability to make judgments regarding the seismic adequacy of equipment.

At least one of the SCEs on each of the SRTs should be a licensed Professional Engineer to ensure that there is a measure of accountability and personal responsibility in making the judgments called for in the DOE Seismic Evaluation Procedure.

In general, the individuals who perform the seismic review walkdown may be required to wear protective clothing, wear a respirator, work in radiation areas, climb ladders, move through crawl spaces, climb over obstacles, and work in high temperatures or other difficult situations. In addition to required facility-specific training, the SRT members should be in good physical condition and have the capability and willingness to perform these tasks as necessary.

\subsubsection{Piping Evaluation Engineers}

The Piping Evaluation Engineers are responsible for conducting the walkdown and screening verification of piping that is listed in the SEL. Recommended minimum requirements for the Piping Evaluation Engineers are:

- Satisfy recommended minimum requirements for SCEs,

- 5 years of experience in seismic design and / or evaluation of piping systems and support structures is desirable with the capability to apply sound engineering judgment based on the knowledge of the behavior of piping systems in actual earthquakes and seismic tests and to recognize potential failure modes,

- Complete the 1-day DOE-developed workshop, or equivalent, on applying the walkdown screening and seismic evaluation methodology for piping at DOE facilities. 


\subsection{OTHER SUPPORT PERSONNEL}

There are several other groups of personnel who provide important assistance to the SCEs. These personnel include safety professional and systems engineers, operations personnel, and relay evaluation personnel. The combination of these personnel and the SCEs comprise a complete SRT.

\subsubsection{Safety Professionals and Systems Engineers ${ }^{3}$}

The primary responsibility of the Safety Professionals and Systems Engineers is to develop the SEL, as described in Chapter 4 . This involves identifying the various types of safety equipment that exist within the facility and determining which types will be evaluated with the Screening Evaluation and Walkdown.

If the SEL contains few outliers following the facility walkdown, further evaluation by the Safety Professionals and Systems Engineers may not be necessary. However, if as a result of the walkdown, numerous outliers are found or outliers which are difficult to resolve are identified, the Safety Professionals and Systems Engineers may be requested to further evaluate the SEL.

In addition to the primary responsibility of developing the SEL, the Safety Professionals and Systems Engineers may be asked to provide background information and guidance to the SCEs who evaluate the seismic adequacy of the equipment and the Relay Evaluation Professionals who perform the relay functionality review.

The Safety Professionals and Systems Engineers should be degreed engineers, or equivalent, and have extensive experience with the broad understanding of the systems, equipment, and procedures of the DOE facility being evaluated.

\subsubsection{Operations Personnel ${ }^{4}$}

The Operations Personnel have two types of responsibilities during implementation of this procedure. First, they are responsible for reviewing the SEL (developed in Chapter 4) to confirm that the SEL is compatible with approved normal and emergency operating procedures for the facility. Second, Operations Personnel may be asked to assist the SCEs during the Screening Evaluation and Walkdown and assist the Relay Review Personnel during the Relay Functionality Review.

To fulfill these responsibilities, the Operations Personnel should have knowledge of both steadystate and transient operations and the associated facility-specific operating procedures. They should be able to supply information on the consequences of, and operator recovery from, functional anomalies. It is their responsibility to provide information on the function and operation of individual equipment, instrumentation, and control systems.

Operations Personnel may assist the SCEs either as staff support or as members of an SRT. Though it is not required that the Operations Personnel be part of the seismic walkdown team, it is recommended. The Operations Personnel should have experience in the specific facility being seismically evaluated.

3 Based on Section 2.2 of SQUG GIP (Ref. 1)

4 Based on Section 2.3 of SQUG GIP (Ref. 1) 


\subsubsection{Relay Evaluation Personnel ${ }^{5}$}

The Relay Evaluation Personnel include those individuals who will perform the Relay Functionality Review described in Chapter 11. This evaluation includes reviewing electrical circuit drawings, documenting the review conclusions, performing the relay walkdowns, and providing associated support activities.

Electrical engineering will be the primary engineering discipline involved in the relay review; however, the evaluation may also use a number of other engineering disciplines; including structural, mechanical, civil, systems, and earthquake engineering. Information and assistance from facility personnel regarding operations and maintenance may also be required. The capabilities and responsibilities of the various Relay Evaluation Personnel are listed below.

The Lead Relay Reviewer should be a degreed, or equivalent, electrical engineer with experience who is familiar with the Relay Functionality Review procedure described Chapter 11. The relay walkdown is not expected to involve entries into radiation areas nor any special physical demands. The Lead Relay Reviewer should either perform the review or assist reviewers in interpreting electrical design drawings and in identifying essential relays in the safety systems. The Lead Relay Reviewer should have a good understanding of circuit design logic and the consequences of relay malfunction in essential circuits.

Assistant Relay Reviewers with electrical engineering, or equivalent, backgrounds can be used to document the relay review and obtain support documentation such as electrical drawings, technical specifications, operator reference manuals, and procedures

Safety Professionals, Systems Engineers and Operations Personnel who are capable of providing information on the operation of the safety systems and facility operating procedures should be used in the Relay Functionality Review. Their assistance will be needed to identify equipment for the SEL and essential control and power circuits which may be tripped as a result of an earthquake. They should also be able to provide information on the instrumentation and controls available to monitor and control the equipment affected by relays. A staff electrical and/or instrumentation and controls maintenance representative should be available to provide assistance during the Relay Functionality Review to help establish the location, mounting, types and characteristics of relays in the safety circuits.

The SCEs should perform certain appropriate evaluations in support of the Relay Functionality Review. These evaluations can be performed during the Screening Evaluation and Walkdown and include:

- Identifying potential instances of seismic spatial interaction.

- Giving special consideration to expansion anchor bolts which secure cabinets containing essential relays.

- Establishing in-cabinet amplification factors for and lowest natural frequency of cabinets containing essential relays.

5 Based on Section 2.5 of SQUG GIP (Ref. 1) 


\section{$3.4 \quad$ TRAINING}

A workshop and training course were developed by DOE to provide guidance on how to implement seismic evaluations using the DOE Seismic Evaluation Procedure and the referenced EPRI / SQUG documents.

DOE Workshop on Walkdown Field Guide and SOUG / EPRI Seismic Oualification Material (Reference 63) The workshop provides an overview of the methodology employed by the EPRI/ SQUG seismic qualification material for seismic evaluations of equipment in existing DOE facilities. By attending the workshop, participants obtain copies of the EPRI / SQUG evaluation material for use at DOE facilities. The workshop is provided for DOE staff, M\&O contractor staff, and subcontractors who were under contract to DOE or a M\&O. In addition, the workshop had training on the use of the Walkthrough Field Guide which is discussed in Section 1.4.1. The intent of the Field Guide training is to introduce techniques for efficiently identifying and upgrading significant seismic concerns at DOE facilities.

DOE Training Course on SQUG / EPRI Walkdown Screening and Seismic Evaluation Material (Reference 64) The training course provides guidance for implementing and following the procedures of the DOE Seismic Evaluation Procedure. Detailed information about the Screening Evaluation and Walkdown Procedure, capacity versus demand evaluation, anchorage review, seismic interaction evaluation, electrical and mechanical equipment review, tanks and heat exchangers review, cable tray and conduit systems review, and relay functionality review is presented in the course. As part of the course, attendees complete a study guide and pre-test, complete quizzes, and participate in a walkdown. This course is provided primarily for the SCEs, however others who may support these engineers may also take this course. Attendance at the training course is currently a mandatory step for any DOE site to obtain the EPRI / SQUG documents and to permit use of the documents for safety-basis evaluations. Videotapes of the course are available through LLNL.

The objective of the DOE training course is as follows: (1) provide additional information on the background, philosophy, and general approach developed by the DOE to conduct seismic evaluations of DOE facilities and (2) provide additional guidance in the use of the DOE Seismic Evaluation Procedure and applicable references to select the SEL and to verify their seismic adequacy. Implementation of the procedures in the DOE Seismic Evaluation Procedure require experienced, well-trained engineers applying sound engineering judgment. As a result, the training course provides for the transfer of the necessary technology to DOE sites and the training of DOE and contractor personnel to conduct seismic evaluations.

A revised version of the training course in Reference 64 provides tailored training for DOE sites. This revised version emphasizes the DOE Seismic Evaluation Procedure and the aspects of the Procedure which are of most interest to DOE sites. In addition to the material discussed in Reference 64, the revised training covers DOE-specific classes of equipment, such as piping, HVAC ducts, and architectural features and components.

DOE Workshop on Qualification of Piping Systems (Reference 65) The workshop provides guidance for implementing and following the procedures of the DOE Seismic Evaluation Procedure for piping systems. Detailed information about the codes and standards for process, instrumentation, and fire protection piping systems; loading of piping systems; qualification by analysis; testing experience data; earthquake experience data; seismic screening criteria for piping and tubing; special considerations for buried piping; and special considerations for double-wall piping are presented at the workshop. As part of the workshop, attendees participate in discussions and are given an examination. This workshop is provided primarily for SCEs who are also Piping Evaluation Engineers, however others who may support these engineers should also take this course. 


\section{SEISMIC EQUIPMENT LIST}

\subsection{GENERAL APPROACH}

The methodology and procedures for evaluating the seismic adequacy of systems and components described in the DOE Seismic Evaluation Procedure are based on the observed performance, failure, and response of various types of systems and components during and after actual earthquake motion or simulated earthquake motion on a shake table. Systems and components can be evaluated for seismic adequacy using the methods and procedures in the DOE Seismic Evaluation Procedure provided that the associated guidelines, limitations, requirements, and caveats described in the procedure are satisfied. This chapter provides guidelines and some discussion to aid in preparing a list of systems and components that can be seismically evaluated to meet the intent of DOE Orders and standards. A prescriptive method for developing the Seismic Equipment List (SEL) is not provided in this chapter because each DOE facility may utilize methods which address facility-specific issues. Even though the SEL is intended for all systems and components, it will primarily consist of systems and components which, if damaged or destroyed, could potentially harm the environment, public and/or workers.

DOE Orders and standards on natural phenomena hazards require that all systems and components be seismically evaluated, except for Performance Category (PC)-0 systems and components. All PC-1 through PC-4 systems and components could then be included in the SEL of the facility. However, the DOE Orders and standards use a "graded approach" permitting the level of rigor and thoroughness of seismic adequacy evaluation to vary in proportion to the importance and significance of the systems and components being evaluated. Consistent with this approach and recognizing the impracticality of performing seismic evaluation and upgrading of all DOE facilities simultaneously, DOE Orders and standards permit prioritization of seismic evaluation and upgrading of various systems and components on some rational basis, such as the risk reduction potential associated with the seismic evaluation and upgrading of a particular system or component. DOE Orders and standards also permit some relaxation of the requirements for older-vintage and existing facilities consistent with a backfit principle. The use of the screening methods and procedures described in the DOE Seismic Evaluation Procedure is based on similar principles.

The above-mentioned relaxation and prioritization provisions of DOE Orders and standards permit an SEL that is not all inclusive, even though all PC-1 through PC-4 systems and components could be in the SEL. Considering the availability of resources and the estimated risk-reduction potential, it is acceptable for only certain systems and components to be included in the SEL. Since a rigorous determination of the risk reduction potential for a large number of systems and components is not practical, an approximate and subjective estimation is acceptable. With appropriate guidance from facility management on resource availability and facility mission, the estimation of relative risk-reduction potential and preparation of an SEL can best be performed by a team, the SEL Team. This team should consist of safety professionals, facility system safety engineers, seismic engineers, and facility operators. For some facilities, the SEL Team may need to incorporate the specialized expertise of relay engineers, piping engineers, chemical engineers, or other professionals and facility designers.

The general approach for the development of the SEL requires the consideration of the following items: identification of facility safety requirements, postulated facility conditions, system interaction considerations, and seismic vulnerability considerations. From these considerations, it is anticipated that a preliminary SEL can be developed. To complete the SEL it is recommended that the preliminary SEL undergo an operational review for concurrence by facility operators. 


\subsection{IDENTIFICATION OF FACILITY SAFETY REQUIREMENTS}

As discussed earlier, the SEL will contain only a portion of the facility systems and components and, in many cases, the SEL will contain only safety-related systems and components which must function during or after a seismic event. To determine which systems and components belong in the SEL, the selection should be based on the results of accident analyses. These accident analyses should consider all the appropriate facility hazards as required by the applicable DOE Orders, such as DOE Order 420.1 (Ref. 5), DOE Order 5480.23 (Ref. 9) for nuclear facilities, DOE Order 5480.30 (Ref. 66) for nuclear reactors, DOE Order 5480.25 (Ref. 67) for accelerator facilities, and DOE Order 5481.1B (Ref. 68) for nonnuclear facilities.

Accident analyses and their results are typically provided in a Safety Analysis Report (SAR) for the DOE facility being evaluated and the SEL should be based on information provided in the SAR. For a nonreactor nuclear facility, DOE-STD-3009 (Ref. 11) provides guidance on the preparation of a SAR. Using the guidance in DOE-STD-3009 and the appropriate accident analyses in the SAR, systems and components can be differentiated into Safety Class or Safety Significant. The SEL can focus on those facility systems and components which are classified as Safety Class or Safety Significant. These systems and components are typically those which must function during or after a seismic event. For facilities without a SAR, hazard and/or accident analyses comparable to those required for a SAR should be performed to identify systems and components needed to perform safety functions.

Additional guidance for the development of the SEL is provided in DOE-STD-1021 (Ref. 7) and DOE-STD-1027 (Ref. 10). The results of facility hazard classification, safety classification, and performance categorization are considered in DOE-STD-1021. With these considerations, the facility systems and components can be assigned to the appropriate performance category. The SEL can focus on those facility systems and components which are classified above a specified performance category and these systems and components are typically those which must function during or after a seismic event.

\subsection{POSTULATED FACILITY CONDITIONS}

In developing the SEL, the SEL Team will need to postulate facility conditions following a seismic event. These postulated conditions will help the SEL Team to identify systems and components needed following an earthquake and serve as a basis of questions asked during the operational review.

- Offsite Utilities: Offsite utilities such as power, telephone, water, steam and gas supplies should be considered for two conditions:

1) Offsite utilities are interrupted and are not available for up to 72 hours.

2) Offsite utilities are uninterrupted.

- Seismic Induced Accidents: Postulate seismic induced accidents, such as fire and criticality, unless a hazard analysis is performed to show that such events are not credible.

- Single Active Failure: Postulate random or seismically induced failure of any single active component on the SEL. 
- Operator Actions: Consider operator actions, as necessary, provided the following conditions are met:

1) Procedures and training are in place.

2) Procedures take into account the environment which will result from the postulated earthquake.

3) Operator actions utilize seismically qualified components and instrument alarms.

4) Egress routes are confirmed viable by seismic review. An alternate egress route must be included in operator action procedures, unless a single route is structurally qualified (including opening of doors and emergency lighting). In addition, access routes for the operator to activate alarms may be required.

- Other Accidents: Do not postulate that other natural phenomena hazards (extreme winds, floods) or man-made accidents (sabotage, plane-crash) occur simultaneously with the earthquake.

\subsection{SYSTEM INTERACTION CONSIDERATIONS}

In preparing the SEL for a facility, system safety will be the primary consideration and the safety professionals and system engineers in the SEL Team will have the primary responsibility of selecting systems and components that must be seismically evaluated. This is a primary consideration for facilities that contain, store, or process nuclear or chemically hazardous materials. For such facilities, the responsibility of the system engineers of the SEL Team will be to grade the candidate systems and components according to their safety significance in relation to the consequences of their failure during or following a seismic event. Such grading may be performed on the basis of system safety studies, if any, associated with the development of SARs and with DOE-STD-1021 (Ref. 7). In addition to the data on conventional safety classification or seismic performance categorization of systems and components, additional data on the approximate number of on-site and off-site individuals that are likely to be adversely affected and the extent of potential damage to the environment will be useful in assessing the relative safety significance of the systems and components.

The SEL Team, especially the safety professionals and system engineers, should also include the following considerations in their evaluation of safety significance of the systems and components:

- Seismic Interaction Effects: The effect of one failure of a systems or component on the performance of other safety-related systems and components should be considered.

- Common-Cause Failure Effects: Since a seismic event affects all systems and components within a facility, several non-safety related systems and components may fail and result in the unacceptable performance or failure of a safety-related systems or component. The effects of such common-cause failure on non-safety related systems and components should be considered.

- Performance During a Seismic Event: Not all safety-related systems and components need to continuously function during a seismic event to meet their safety requirement, as long as they perform their safety-related function after the event. Functional failure of such systems and components during a seismic event is obviously not significant compared to those systems and components, such as some switches and relays, which must function during the event. 


\subsection{SEISMIC VULNERABILITY CONSIDERATIONS}

In developing the SEL, structural and seismic vulnerability considerations are also important. In general, the systems and components that are inherently or generically susceptible to seismic failure or malfunction should get more attention in the evaluation process than those that are inherently rugged. The determination and assessment of seismic weakness or ruggedness for the purpose of preparing the SEL will be the responsibility of the SEL Team, especially the seismic engineers. The seismic engineers will consider: (1) the structural configuration of the system or component in relation to its function, (2) its potential failure mode (ductile or brittle, large displacement, vibration sensitivity, unacceptable function even though stress or displacement is within acceptable limits, etc.), (3) generic performance during past earthquakes or during shake table test, and (4) the actual attachment and support conditions of the system or component.

A systematic walkthrough is recommended to evaluate the seismic ruggedness of the systems and components and their support and anchorage. The Walkthrough Screening Evaluation Field Guide (Ref. 23) discussed in Section 1.4 can aid this process. A brief review of seismic design documents and records is also necessary to assess the seismic vulnerability of the systems and components. Based on such walkthrough and document review, the seismic engineer of the SEL Team will subjectively evaluate the relative seismic vulnerability of the systems and components that are included in the SEL prepared by the safety professionals and system engineers. As a result of this seismic vulnerability evaluation, each system or component of the SEL, which was prepared on the basis of safety considerations, will have a qualitative seismic vulnerability rating which, when combined with the system safety significance, can provide an assessment of the relative risk associated with the seismic event.

\subsection{OPERATIONAL REVIEW}

The SEL prepared from the considerations discussed in Sections 4.2, 4.3, 4.4 and 4.5 should be reviewed by the SEL Team for operational and functional considerations. The facility operators will specially review the completeness of the list to ensure that the systems and components whose functionality and integrity are assumed essential for personnel and public safety by the operating personnel are included in the SEL. To assist the SEL Team and facility operators in reviewing the preliminary SEL, the following questions are suggested:

- What are the hazards to the public, workers, or environment upon failure of facility systems and components?

- What are the confinement systems in place to protect the public or environment from facility operations or accidents?

- What are the procedures in the event of a loss of off-site power?

- What are the facility emergency response and evacuation procedures, monitors, alarms, and routes for a major seismic event?

- Are there essential instrumentation and controls for vital components needed to provide confinement?

- What type of fire protection system does the facility have (wet systems, dry systems, any functional requirements of any pumps)?

- What type of monitoring systems and components does the facility have (continuous air monitors, high-radiation area monitors, stack monitors, and associated operational requirements)? 
- What type of alarm systems does the facility have?

- What, if any, are the operational requirements for components in the confinement systems?

- Is any operator intervention required to operate the vital components for confinement?

- What success paths are available for placing any hazardous operations into a safe state including those requiring operator action?

- Upon loss of off-site power, what is the failure state of active confinement systems (e.g., will air be needed to re-open dampers)?

- Are there any highly important and expensive experiments or unique components that if lost, would jeopardize the mission of the facility due to excessive downtime?

- Are there significant common-cause interaction effects?

- What support systems do facility systems and components depend on to fulfill their safety functions?

- What defense-in-depth features are required for the facility systems and components?

Information to help answer the above questions may be in the facility SAR or other related safety documents. After addressing these questions in the operational review and revising the preliminary SEL based on the answers to the questions, the final SEL can be developed. 


\section{.}




\section{Part II}

\section{Seismic Evaluation Procedures Modified from the SQUG GIP}





\section{CAPACITY VERSUS DEMAND}

\section{$5.1 \quad$ INTRODUCTION}

A screening guideline which should be satisfied to evaluate the seismic adequacy of an item of equipment identified in the Seismic Equipment List (SEL), as described in Chapter 4, is to confirm that the seismic capacity of the equipment is greater than or equal to the seismic demand imposed on it. This chapter addresses the determination of the seismic demand and capacity for the equipment as well as the comparison of the demand to the capacity. Note that a comparison of seismic capacity to seismic demand is also made in Chapter 6 for the equipment anchorage, in Chapter 9, Section 10.4.1, Section 10.4.3, and Section 10.5.1 for the equipment class evaluations using screening procedures, and in Chapter 11 for relays mounted in the equipment.

This chapter first presents the general description and techniques for computing the seismic demand and capacity, followed by the comparison of the demand to the capacity. In Section 5.2, the seismic demand is defined by the Seismic Demand Spectrum (SDS). The SDS is based on the Design Basis Earthquake (DBE) as defined for DOE facilities. The input motion for the equipment is determined by computing an in-structure response spectrum based on the DBE and the frequency response of the structure in which the equipment is mounted. Scaling factors are applied to the instructure response spectrum to compute the SDS. In Section 5.3, the seismic capacity is represented by the Reference Spectrum, Generic Equipment Ruggedness Spectrum (GERS), or qualification test spectrum. Note that the Reference Spectrum and GERS can be used for representing seismic capacity of equipment only if the equipment meets the intent of the caveats for its equipment class as described in Chapter 8. Finally, in Section 5.4 the SDS is compared to the appropriate capacity spectrum.

The DOE Seismic Evaluation Procedure is intended primarily for systems and components identified as Performance Category (PC)-2 or higher. As discussed in DOE Orders and standards, the performance goal description for PC-1 is to maintain occupant safety during and/or immediately following an earthquake, while PC-2 and higher categories add goals such as continued operation with minimum interruption. Within the DOE graded approach, the primary concern for PC-1 structures is to prevent major structural damage or collapse that would endanger personnel. This concern is consistent with the goal of the model building codes, such as the Uniform Building Code (UBC) (Ref. 69), for general facilities to maintain life safety during earthquakes. The provisions of the UBC or similar building code should be followed for PC-1 systems and components since continued operation is not a requirement. For PC-2 and higher systems and components, the provisions of the DOE Seismic Evaluation Procedure satisfy the qualitative description of the performance goals for those categories and can be used to evaluate their capacity to at least have continued operation with minimum interruption during and/or immediately following an earthquake.

\subsection{SEISMIC DEMAND}

\subsubsection{Design Basis Earthquake}

For DOE facilities, the Design Basis Earthquake (DBE) is a specification of the mean seismic ground motion at the facility site for the earthquake-resistant design or evaluation of the structures, systems, and components at that site. The DBE is defined by ground motion parameters determined from mean seismic hazard curves and a design response spectrum shape. These hazard curves relate hazard exceedance probabilities to response quantities, such as peak ground acceleration. The methodologies for determining the seismic environment are described in DOESTD-1022 (Ref. 70) and DOE-STD-1023 (Ref. 71). While DOE-STD-1022 provides procedures for site characterization, DOE-STD-1023 provides procedures for the development of hazard curves and spectra, such as the DBE, using parameters determined from the site characterization. 
Many DOE sites have determined their site-specific DBE and have documented information about their DBE in Safety Analysis Reports (SARs) and reports in the hazards control or plant engineering departments of the DOE site.

As discussed in DOE-STD-1020 (Ref. 6), the preferable shape for the median deterministic DBE response spectrum should be site-specific and consistent with earthquake hazard parameters such as magnitudes, distances, and soil profiles. If a site-specific response spectrum shape is unavailable, a median standardized spectral shape may be used as long as the shape is consistent with or conservative for the site conditions. A recommended standardized spectral shape is shown in Figure 5.2-1, which is the shape defined in NUREG/CR-0098 (Ref. 72). The control points for the spectral shape in Figure 5.2-1 are provided in Table 5.2-1.

Table 5.2-1 Control Points for NUREG/CR-0098 Spectral Shape

\begin{tabular}{|c|c|}
\hline $\begin{array}{c}\text { Frequency } \\
\text { (Hertz) }\end{array}$ & $\begin{array}{c}\text { Spectral Acceleration } \\
(\mathrm{g})\end{array}$ \\
\hline 0.1 & $0.395 \mathrm{~d}_{\max } / \mathrm{g}$ \\
\hline $\mathrm{v}_{\max } /\left(2 \pi \mathrm{d}_{\max }\right)$ & $\mathrm{v}_{\max }^{2} /\left(\mathrm{g} \mathrm{d}_{\max }\right)$ \\
\hline$\left(\mathrm{g} \mathrm{a}_{\max }\right) /\left(2 \pi \mathrm{v}_{\max }\right)$ & $\mathrm{a}_{\max }$ \\
\hline 8.0 & $\mathrm{a}_{\max }$ \\
\hline 33.0 & $\mathrm{a}_{\mathrm{g}}$ \\
\hline 100.0 & $\mathrm{a}_{\mathrm{g}}$ \\
\hline
\end{tabular}

where (for competent soil, $\mathrm{V}_{\mathrm{s}}<3,500 \mathrm{ft} / \mathrm{sec}$, and for $50 \%$ spectra):

PGA - peak ground acceleration

$\beta$ - percent damping

$\mathrm{g}=$ acceleration due to gravity (in $/ \mathrm{sec}^{2}$ )

$\mathrm{a}_{\mathrm{g}}=$ PGA (g)

$v_{g}=48 a_{g} \quad($ in $/ \mathrm{sec})$

$\mathrm{d}_{\mathrm{g}}=36 \mathrm{a}_{\mathrm{g}} \quad$ (in)

$\mathrm{a}_{\max }=\mathrm{a}_{\mathrm{g}}(3.21-(0.68 \ln \beta))$

$v_{\max }=v_{g}(2.31-(0.41 \ln \beta))$

$\mathrm{d}_{\max }=\mathrm{d}_{\mathrm{g}}(1.82-(0.27 \ln \beta))$

DOE-STD-1020 also discusses techniques for addressing the effective peak acceleration as compared to the predicted instrumental peak ground acceleration reported in some probabilistic seismic hazard assessments for sites at short epicentral distances. Typically, the effective peak acceleration is lower than the peak ground acceleration. While it is appropriate in seismic evaluations to remove sources of excessive conservatism, use of the effective peak acceleration for the evaluation of the functionality of active systems and components may not be conservative and should be peer reviewed on a site-specific basis. The effective peak acceleration may not be conservative because many types of active systems and components are relatively stiff and may no longer operate if the seismic demand requires inelastic response to the peak ground acceleration. 
In order to demonstrate that DOE facilities are capable of resisting a specified level of seismic demand, it must be demonstrated that there is a sufficiently low probability of damage or failure of those facilities consistent with established performance goals as defined in DOE Orders and DOESTD-1020. As discussed in Sections 1.2 and 4.1, the annual exceedance probability for a facility is determined by its performance category and the equipment in the SEL are classified into a particular performance category in accordance with DOE-STD-1021 (Ref. 7). Associated with each performance category is a different performance goal and an accompanying hazard exceedance probability which specifies the level of the DBE for each category.

DOE-STD-1020 permits some relief in the criteria for the seismic evaluation of systems and components in existing facilities. For existing facilities, the seismic evaluations may use a natural phenomena hazard exceedance probability that is twice the value specified for new facilities. This relief corresponds to a slight reduction (approximately 10-20\%) in the seismic loads for the DBE. The basis of this slight reduction is contained in Reference 73. Use of the relief for specific existing facilities should follow the provisions in DOE-STD-1020.

The DBE is established at a higher annual frequency of occurrence than the acceptable annual probability of failure of the structures, systems, or components, so scale factors and experience data factors are required to achieve the appropriate risk reduction. These scale factors are similar to safety factors or the inherent conservatism in the acceptance criteria of structural design codes. The basis for the scale factors is provided in References 24 and 73 and the scale factors are shown in Table 5.2-2.

Table 5.2-2 Scale Factors

\begin{tabular}{|c|c|}
\hline $\begin{array}{c}\text { Performance } \\
\text { Category }\end{array}$ & $\begin{array}{c}\text { Scale Factor } \\
\text { (SF) }\end{array}$ \\
\hline 2 & 0.67 \\
\hline 3 & 1.00 \\
\hline 4 & 1.25 \\
\hline
\end{tabular}

In the design of new equipment, rules are specified such that a known margin exists between the design value and the ultimate failure level. This margin has been considered in developing the provisions of DOE-STD-1020 as discussed in References 6, 24, and 73. A similar margin is required for the use of capacity obtained from experience data. Section 5.3 discusses the different types of capacity representation. The margin between the design and ultimate failure values are contained in the experience data factor, $\mathrm{F}_{\mathrm{ED}}$, defined in Reference 24 and shown in Table 5.2-3.

Table 5.2-3 Experience Data Factors

\begin{tabular}{|c|c|}
\hline $\begin{array}{c}\text { Capacity } \\
\text { Representation }\end{array}$ & $\overline{\mathrm{F}}_{\mathrm{ED}}$ \\
\hline Reference Spectrum & $1.0 \mathrm{SF}$ \\
\hline GERS & $1.4 \mathrm{SF}$ \\
\hline Relay GERS & $1.8 \mathrm{SF}$ \\
\hline Qualification Test & $1.4 \mathrm{SF}$ \\
\hline
\end{tabular}

1 The Performance Category for each item of equipment in the SEL is determined using the provisions in Chapter 4 and DOE-STD-1021 (Ref. 7).

2 Definitions for the different capacity representations are provided in Sections 2.1.3.1 and 5.3. 


\subsubsection{In-Structure Response Spectrum ${ }^{3}$}

For buildings, the DBE defines the seismic demand at the foundation of the structure. For equipment, the demand is defined in terms of the input motion applied at the appropriate attachment point(s) of the equipment. This demand or input motion is generally represented by an in-structure response spectrum (IRS). The IRS will differ significantly from the DBE spectrum because it is essentially filtered and / or amplified through the building. To use the provisions of the DOE Seismic Evaluation Procedure, the demand at the attachment point(s) of the equipment must consider the effects of structural filtering and / or amplification. Methods for determining the IRS with dynamic analyses are described in DOE-STD-1020 (Ref. 6) and ASCE 4 (Ref. 74). As discussed in ASCE 4, the IRS must account for uncertainties by spectral broadening or peak shifting. Additional guidance on computing IRS is provided in Sections 2.3 and C.4 of DOESTD-1020. In DOE-STD-1020, dynamic analyses which may use IRS are only specified for PC-3 and PC-4 systems and components. In order to use the methodology in the DOE Seismic Evaluation Procedure, IRS should be developed as well for PC-2 systems and components in the SEL. Guidance for determining in-structure spectra for PC-2 systems and components is provided in the model building codes such as the UBC (Ref. 69) and the National Earthquake Hazards Reduction Program (NEHRP) Provisions (Ref. 75).

Realistic, median-centered in-structure response spectra are defined as response spectra which are based on realistic damping levels for the structure (including the effects of embedment and wavescattering) and on structural dynamic analysis using realistic, best estimate modeling parameters and calculational methods such that no intentional conservatism enters into the process. These instructure response spectra should be based on a ground response spectrum defined by the DBE as determined in DOE-STD-1023. For existing facilities with an approved Safety Analysis Report (SAR), the in-structure response spectra included in the SAR may be used as appropriate. Examples of realistic damping values are given in DOE-STD-1020 and EPRI Report NP-6041 (Ref. 18). The effects of embedment, wave scattering, and other soil-structure interaction (SSI) effects can be accounted for by using the methods in ASCE 4 by using frequency shifting rather than peak broadening. A spectral reduction factor can be used for considering the effects of horizontal spatial variation.

DOE-STD-1020 recommends the procedures in ASCE 4 for generating in-structure response spectra. The experience data factors, $\mathrm{F}_{\mathrm{ED}}$, listed in Table 5.2-3 are appropriate when the instructure response spectra are generated in accordance with DOE-STD-1020. In some cases, instructure response spectra may be developed with varying conservatism which is different than that defined in DOE-STD-1020. Reference 24 outlines methods to account for variation in the determination of in-structure response spectra with different levels of conservatism. The Seismic Safety Margins Research Program (Ref. 57 and 58) has demonstrated the large conservatism which exists in traditionally-computed, conservative design in-structure response spectra versus realistic, median-centered in-structure response spectra. The specific assumptions made in generating in-structure response curves should be reviewed by SCEs using the guidance provided in Appendix A of Reference 19.

3 Based on Section 4.2.4 of SQUG GIP (Ref. 1) 


\subsubsection{Seismic Demand Spectrum}

To evaluate the seismic demand at the attachment point(s) of equipment, an in-structure response spectrum (IRS) is scaled by $F_{E D}$ to determine the Seismic Demand Spectrum (SDS) according to the following equation:

$\mathrm{SDS}=\mathrm{F}_{\mathrm{ED}} \times \mathrm{IRS}$

where:

SDS - Seismic Demand Spectrum or Scaled In-Structure Response Spectrum. For relays, the SDS is modified to account for in-cabinet amplification. Chapter 11 provides two methods for modifying the SDS for relays mounted in cabinets.

$F_{E D}$ - Experience Data Factor. It depends on the performance category and capacity representation of the equipment and is defined in Tables 5.2-2 and 5.2-3.

IRS - In-Structure Response Spectrum. It is determined for the appropriate attachment point(s) of the equipment and is a function of the DBE for the facility and the frequency content of the structure supporting the equipment.

Additional information on techniques for computing the seismic demand spectrum are provided in Step 1 of Section 6.4.2. In this section, an approximate technique for scaling seismic demand spectra, which are defined for different damping values, is discussed.

\subsubsection{Total Demand}

The total demand $\left(D_{\mathrm{T}}\right)$ is a combination of seismic loads $\left(D_{S I}\right)$ and concurrent non-seismic loads $\left(\mathrm{D}_{\mathrm{NS}}\right)$.

$$
\mathrm{D}_{\mathrm{TI}}=\mathrm{D}_{\mathrm{SI}}+\mathrm{D}_{\mathrm{NS}}
$$

where:
$\mathrm{D}_{\mathrm{T1}}-\quad$ Total Demand
$\mathrm{D}_{\mathrm{SI}}$ - Seismic Loads. According to DOE-STD-1020 (Ref. 6), the dynamic analyses used to compute the seismic loads for PC-3 and PC-4 systems and components must consider all three orthogonal components of earthquake ground motion (two horizontal and one vertical). In order to use the methodology in the DOE Seismic Evaluation Procedure, all three orthogonal components of earthquake ground motion should be considered for PC-2, PC-3, and PC-4 systems and components. The earthquake ground motion is described by the SDS defined in Section 5.2.3. For near-field sites, the vertical component of the DBE may exceed the horizontal components. Responses from the various directional components should be combined with acceptable combinations techniques, such as the Square-Root-Sum-of-the-Squares (SRSS) and the 100-40-40-Rule, in accordance with ASCE 4 (Ref. 74).
$\mathrm{D}_{\mathrm{NS}}$ - Non-Seismic Operational Loads 
When comparing $\mathrm{D}_{\mathrm{T}}$ to seismic capacity based on earthquake experience data as defined in Section 5.3.1 or generic seismic testing data as defined in Section 5.3.2, the effects of all three orthogonal components of the earthquake ground motion and the effects of non-seismic operational loads are typically not explicitly considered for equipment adequacy assessment as described below:

(a) According to Section 4.2.3 of the SQUG GIP (Ref. 1), the vertical component of the ground response spectrum is not explicitly considered for equipment adequacy assessment. In general, it is considered that equipment is more sensitive to horizontal motion than vertical motion. Evaluation of the effects of the vertical component is implicit in the horizontal motion assessment since the earthquake-experience facilities typically experienced relatively higher vertical motion than that explicitly considered. When using GERS, the generic seismic testing included effects of vertical motion which was consistent with that explicitly considered.

(b) Equipment in the earthquake-experience database was subjected to non-seismic operating loads concurrent with the seismic loads. In many cases, the non-seismic loads were implicitly included along with the horizontal seismic loads and in defining the caveats for the Reference Spectrum. Note that there may be facility-specific equipment that is subjected to operating loads which were not implicitly included in the experience database. For equipment subjected to both operating and seismic loads, the database may need to be reviewed to determine if the operating loads were implicitly considered. If the operating loads were not implicitly considered, then their effects should be considered concurrently with the seismic loads. 


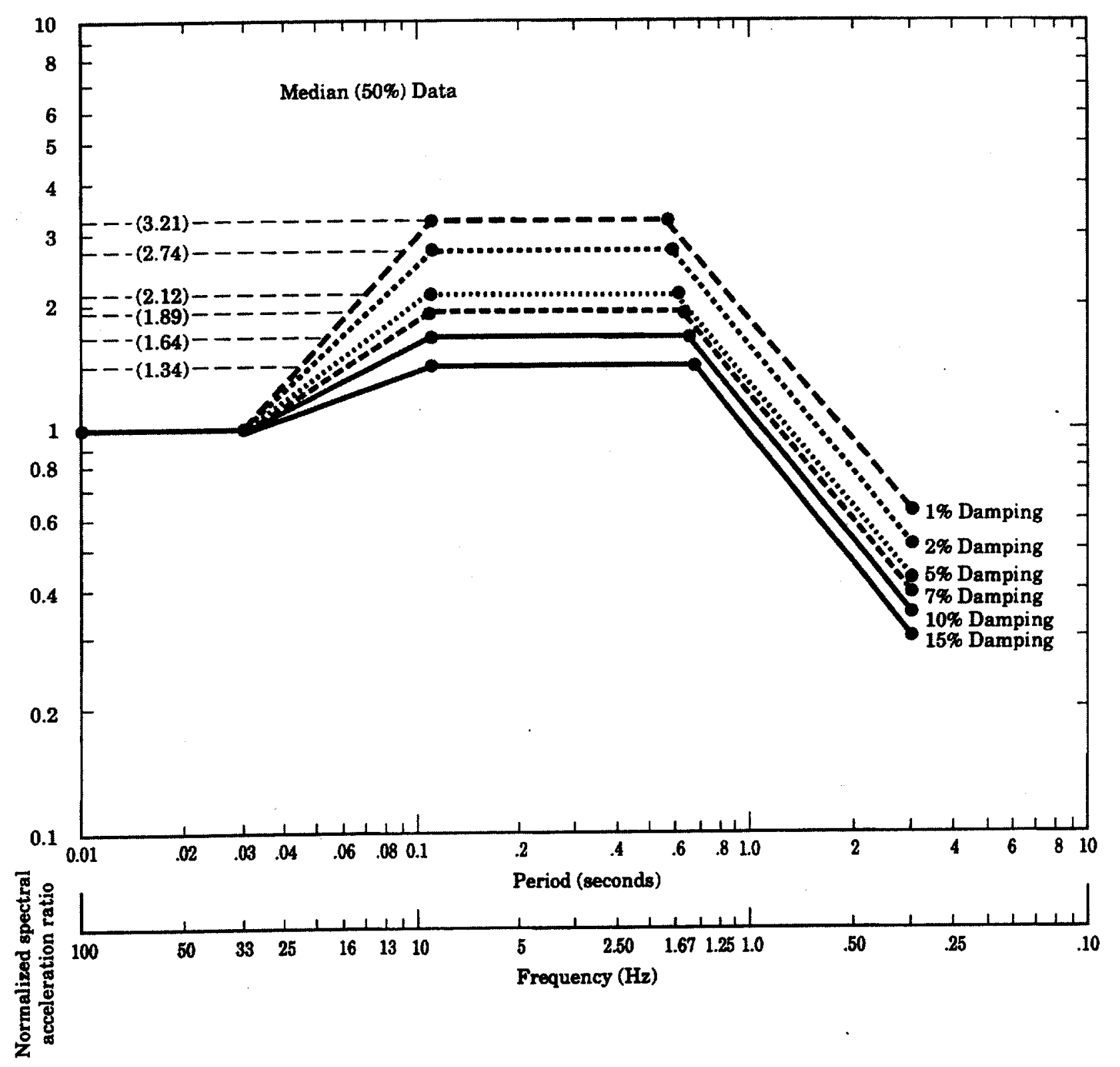

Figure 5.2-1 Median Standardized Spectral Shape Defined in NUREG/CR-0098, the Newmark and Hall 50\% Exceedance Horizontal Spectra for Soil (References 72 and 76) 


\subsection{EQUIPMENT CAPACITY}

\subsubsection{Seismic Capacity Based on Earthquake Experience Data ${ }^{4}$}

Earthquake experience data was obtained by surveying and cataloging the effects of strong ground motion earthquakes on various classes of equipment mounted in conventional power plants and other industrial facilities. The results of this effort are summarized in Reference 35. Based on this work, a Reference Spectrum was developed which represents the seismic capacity of equipment in the earthquake experience equipment class. A detailed description of the derivation and use of this Reference Spectrum is contained in Reference 19 and this reference should be reviewed by the SCEs before using the Reference Spectrum. The Reference Spectrum and the four spectra from which it is derived are shown in Figure 5.3-1. Figure 5.3-2 shows the Reference Spectrum and its defining response levels and frequencies.

The Reference Spectrum can be used to represent the seismic capacity of equipment in a DOE facility when this equipment is determined to have characteristics similar to the earthquake experience equipment class and meets the intent of the caveats for that class of equipment as defined in Chapter 8. Use of the Reference Spectrum for comparison with the Seismic Demand Spectrum (SDS) is described in Section 5.4.

\subsubsection{Seismic Capacity Based on Generic Seismic Testing Data ${ }^{5}$}

A large amount of data was collected from seismic qualification testing of nuclear power plant equipment. This data was used to establish a generic ruggedness level for various equipment classes in the form of Generic Equipment Ruggedness Spectra (GERS). The development of the GERS and the limitations on their use (caveats) are documented in Reference 40. Copies of the non-relay GERS along with a summary of the caveats to be used with them are included in Chapter 8. A copy of a relay GERS is included in Chapter 11. SCEs should review Reference 40 to understand the basis for the GERS.

GERS can be used to represent the seismic capacity of an item of equipment in a DOE facility when this equipment is determined to have characteristics which are similar to the generic testing equipment class and meets the intent of the caveats for that class of equipment as defined in Chapter 8. Use of the GERS for comparison with the Seismic Demand Spectrum (SDS) is described in Section 5.4.

\subsubsection{Equipment-Specific Seismic Qualification}

Equipment-specific seismic qualification techniques, as used in newer DOE facilities, may be used instead of the methods given in Section 5.3.1 and 5.3.2. With this technique, shake-table tests should be performed in accordance with IEEE-344-75 Standards (Ref. 12) or more current standards.

Equipment-specific seismic qualification can be useful for equipment classes discussed in Chapter 10. Some of these equipment classes do not have the Reference Spectrum or GERS to define their capacity. With seismic qualification techniques, a test spectrum can be generated for these classes of equipment and this spectrum must be scaled with the $\mathrm{F}_{\mathrm{ED}}$ for Qualification Test in Table 5.2-3.

4 Based on Section 4.2.1 of SQUG GIP (Ref. 1)

5 Based on Section 4.2.2 of SQUG GIP (Ref. 1) 


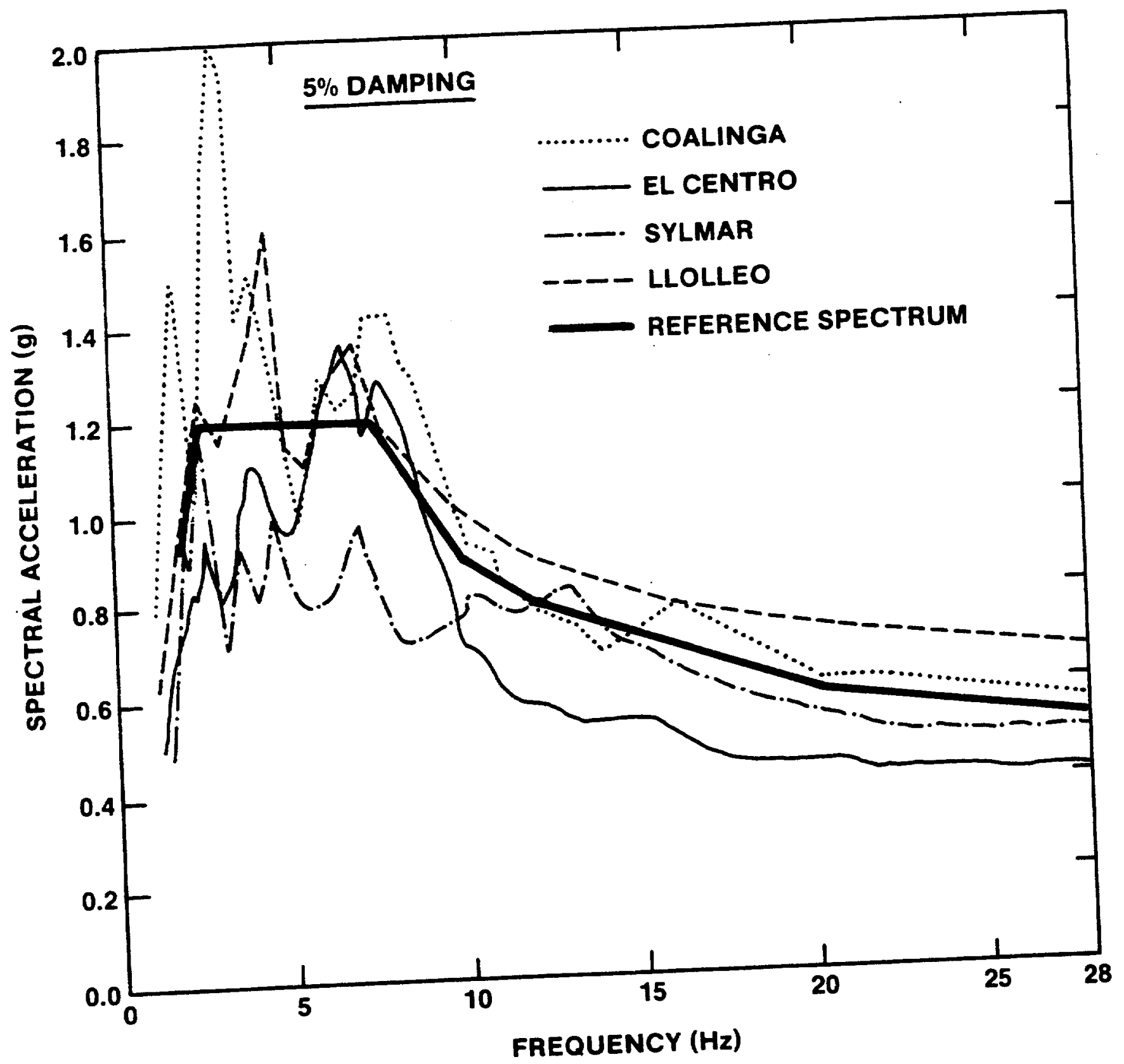

Figure 5.3-1 Horizontal Response Spectra Representing the Earthquake Experience Database (Reference 19) 


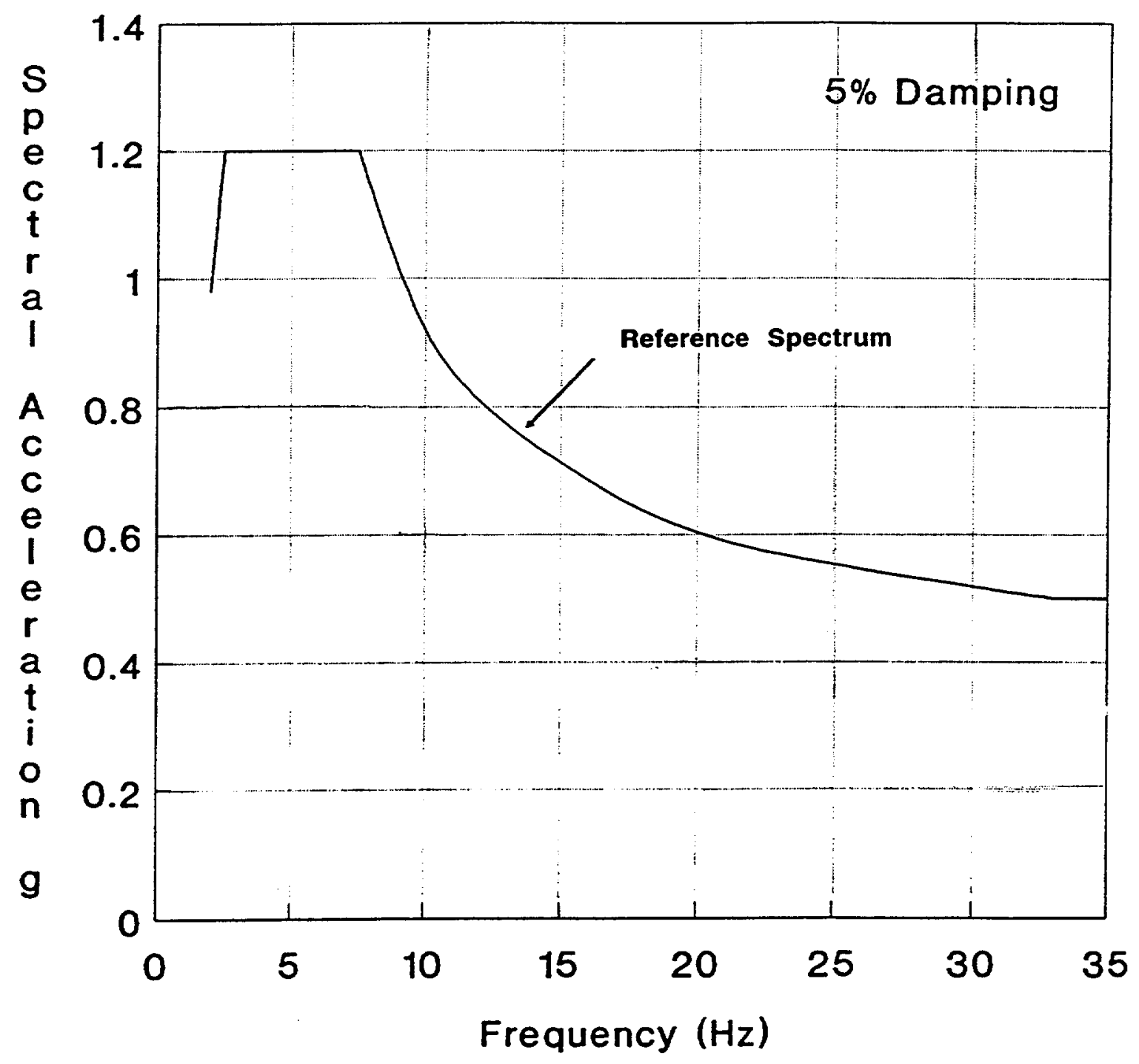

\begin{tabular}{|c|c|c|c|c|c|c|c|c|c|c|}
\hline Frequency $(\mathrm{Hz})$ & 2.0 & 2.5 & 7.5 & 8.0 & 10 & 12 & 16 & 20 & 28 & 33 \\
\hline Spectral Acceleration (g) & .98 & 1.2 & 1.2 & 1.13 & .90 & .80 & .68 & .59 & .53 & .50 \\
\hline
\end{tabular}

Figure 5.3-2 Seismic Capacity Represented by Reference Spectrum Based on Earthquake Experience Database (Figure 4-2 of SQUG GIP, Reference 1) 


\subsection{EXPERIENCE-BASED CAPACITY COMPARED TO SEISMIC DEMAND}

This section addresses the comparison of experience-based seismic capacity to seismic demand for the equipment. The seismic capacity of equipment can be represented by a Reference Spectrum based on earthquake experience data, a Generic Ruggedness Spectrum (GERS) based on generic seismic test data, or a test spectrum from equipment-specific seismic qualification as respectively described in Sections 5.3.1, 5.3.2, and 5.3.3. Note that the first two methods of representing seismic capacity of equipment can only be used if the equipment meets the intent of the caveats for its equipment class as described in Chapter 8. The seismic capacity of an item of equipment is compared to its seismic demand which is defined in terms of an in-structure response spectrum (IRS). As discussed in Section 5.2, the IRS is scaled with the applicable scale factors to determine the Seismic Demand Spectrum (SDS).

\subsubsection{Comparison of Equipment Seismic Capacity to Seismic Demand ${ }^{6}$}

An in-structure response spectrum can be used for comparison to Reference Spectrum, GERS, or test spectrum for equipment which is mounted at any elevation in the facility and/or for equipment with any natural frequency. The Reference Spectrum, GERS, or test spectrum are used to represent the capacity of the equipment. The SDS associated with the DBE for a DOE facility can be used to represent the seismic demand applied to the facility equipment. One of the following comparisons of capacity and demand, as illustrated in Figure 5.4-1, is made:

- Reference Spectrum envelops the Seismic Demand Spectrum (SDS)

Reference Spectrum (Section 5.3.1) $\quad \geq \quad$ SDS

- GERS envelops the Seismic Demand Spectrum (SDS)

GERS (Section 5.3.2) $\geq \quad$ SDS

- Test spectrum envelops the Seismic Demand Spectrum (SDS)

Seismic Qualification Tests (Section 5.3.3) $\geq \quad$ SDS

- Relay GERS envelops the In-cabinet Demand Spectrum (IDS). Section 11.3 discusses techniques for calculating the IDS using the Seismic Demand Spectrum (SDS).

$$
\text { Relay GERS (Section 11.2) } \quad \geq \text { IDS }
$$

For these comparisons, the largest horizontal component of the 5\% damped in-structure response spectra is used for the location in the facility where the item of equipment is mounted. An approximate technique for scaling in-structure response spectra by their damping ratios is provided in Section 6.4. The in-structure response spectrum used for the seismic demand should be representative of the elevation in the structure where the equipment is anchored and receives its seismic input. This elevation should be determined by the SCEs during the facility walkdown. If one of the comparisons shown above is not satisfied, then the equipment being evaluated is an outlier. Methods for resolving outliers are provided in Chapter 12.

6 Based on Section 4.2.4 of SQUG GIP (Ref. 1) 


\subsubsection{Enveloping of Seismic Demand Spectrum ${ }^{7}$}

To evaluate seismic adequacy, in general, the seismic capacity spectrum should envelop the SDS over the entire frequency range of interest (typically 1 to $33 \mathrm{~Hz}$ ). There are two special exceptions to this general rule:

- The seismic capacity spectrum needs only to envelop the SDS for frequencies at and above the conservatively estimated lowest natural frequency of the item of equipment being evaluated.

Caution should be exercised when using this exception because an equipment assembly (e.g., electrical cabinet lineup) may consist of many subassemblies, each manifesting its fundamental mode of vibration at different frequencies. The lowest natural frequency of each subassembly should be determined with high confidence using the guidance provided below in Section 5.4.3. It is noted that unless the equipment is tested with a high-level vibratory input, the fundamental frequency can be difficult to estimate, especially for complex structural equipment.

- Narrow peaks in the SDS may exceed the seismic capacity response spectrum if the average ratio of the SDS to the capacity spectrum does not exceed unity when computed over a frequency range of $10 \%$ of the peak frequency (e.g., $0.8 \mathrm{~Hz}$ range at $8 \mathrm{~Hz}$ ). Note that it is permissible to use unbroadened SDS for this comparison, however when doing so, uncertainty in the natural frequency of the building structure should be addressed by shifting the frequency of the SDS at these peaks. An acceptable method of peak shifting is described in ASCE 4 (Ref. 74). A reference or basis for establishing the degree of uncertainty in the natural frequency of the building structure should be included in the facility-specific seismic evaluation records.

If either of these exceptions are used, the Screening Evaluation Work Sheets (SEWS) should be marked to indicate the exception that has been invoked.

\subsubsection{Lowest Natural Frequency ${ }^{8}$}

When it is necessary to determine the lowest natural frequency of an item of equipment, the SCEs may, in most cases, estimate a lower bound for this frequency based on their experience, judgment, and available data. Methods for frequency estimation are provided in Reference 77. The lowest natural frequency of concern is that of the lowest natural mode of vibration that could adversely affect the safety function of the equipment. The modes of vibration which should be considered are:

- The overall structural modes of the equipment itself and

- The modes for internal structures (e.g., flexural mode for door panels) which support components needed to accomplish the safety function of the equipment.

- The modes of devices which are needed to accomplish the safety function of the equipment. A value of $5 \mathrm{Hertz}$ is recommended and higher values should be appropriately justified.

In addition, the SCEs should also be alert and note any items of concern within the "box" which could be seismically vulnerable. This would include components mounted in the "box" which have known low natural frequencies, seismic vulnerabilities, or improper mounting (e.g., loose or

7 Based on Section 4.2 of SQUG GIP (Ref. 1)

8 Based on Section 4.2 of SQUG GIP (Ref. 1) 
missing bolts). If these types of situations are found during the seismic review, their presence may constitute a third type of vibrational mode and their influence should be included in the estimate of the lowest natural frequency and the assessment of the seismic adequacy of the equipment.

\subsubsection{Guidance for Evaluating In-Line Equipment ${ }^{9}$}

The amplified response of in-line equipment which is supported by piping (e.g., valves, valve operators, and sensors) is handled differently when using the Reference Spectrum or the GERS as the seismic capacity of the equipment. When using the Reference Spectrum, it is not necessary to account for amplification of the piping system between the anchor point of the piping system (i.e., the floor or wall of the building) and the point on the piping system where the item of equipment is attached. This is because the effect of amplified response in piping systems is accounted for in the earthquake experience data base.

When using GERS as the seismic capacity of equipment, piping system amplifications should be accounted for when establishing the seismic demand on the in-line item of equipment. The amplification factor can be obtained from a dynamic piping analysis if one is available. As an alternative, the amplification factor may be estimated using judgment with peer review.

9 Based on Section 4.2.4 of SQUG GIP (Ref. 1) 


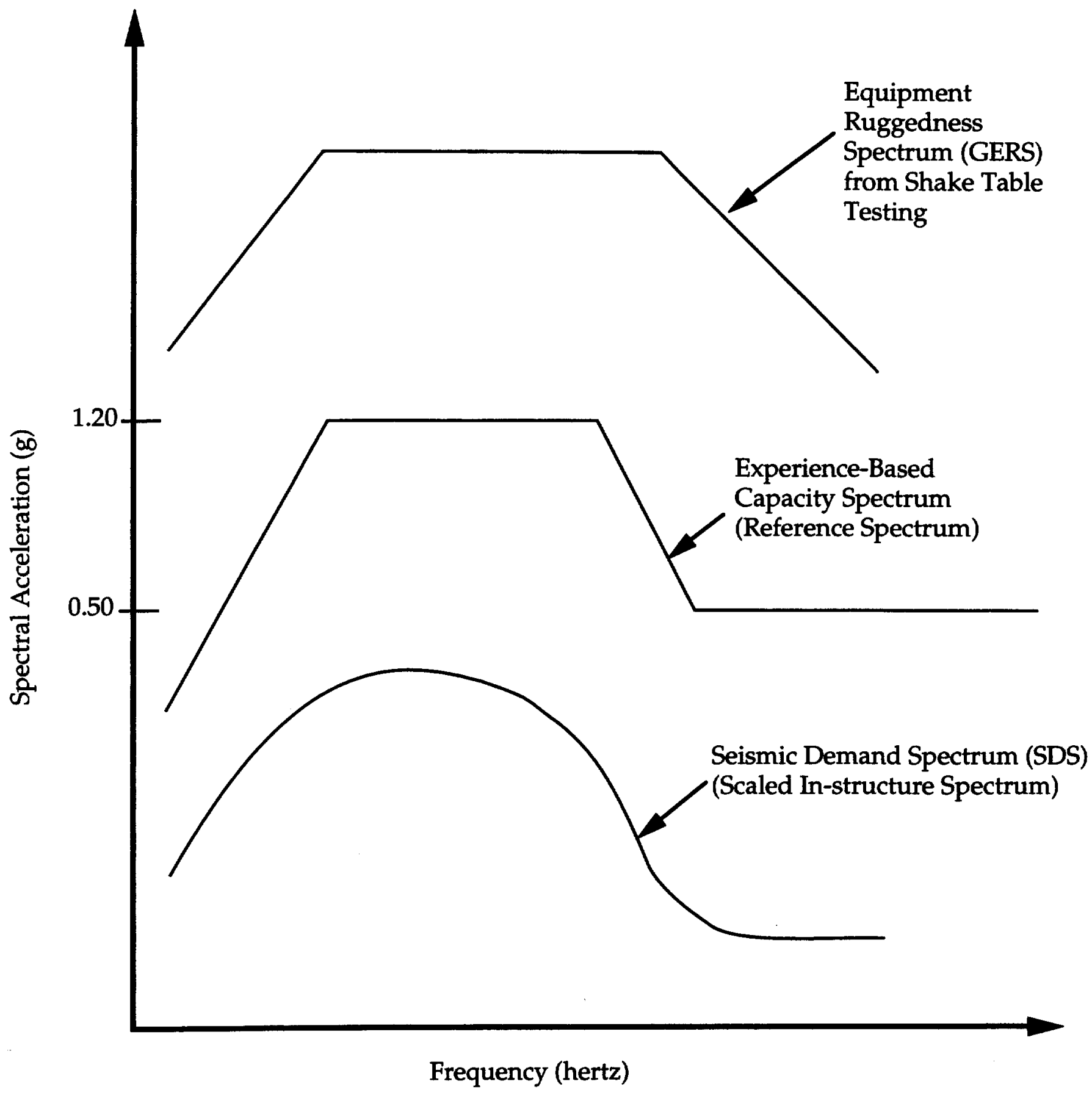

Figure 5.4-1 Comparison of Seismic Capacity Spectrum to Seismic Demand Spectrum 


\section{ANCHORAGE DATA AND EVALUATION PROCEDURE}

\subsection{INTRODUCTION}

The purpose of this chapter is to:

- Provide a general description of the anchorage evaluation procedure,

- Provide generic information on the various equipment classes for use in anchorage evaluations,

- Provide nominal allowable capacities for certain types of anchors, and

- Describe anchor-specific inspection checks and capacity reduction factors.

The four main steps for evaluating the seismic adequacy of equipment anchorage include:

1. Anchorage Installation Inspection (Section 6.2)

2. Anchorage Capacity Determination (Section 6.3)

3. Seismic Demand Determination (Section 6.4)

4. Comparison of Capacity to Demand (Section 6.5)

This chapter is organized with an evaluation of the installation adequacy and attributes of the anchorage given first. Next, the anchorage capacity is determined in Sections 6.3.1 to 6.3.9 and the steps in the capacity determination are grouped by the following anchor types:

\section{Expansion Anchors}

Cast-In-Place Bolts and Headed Studs

Cast-In-Place J-Bolts

Grouted-In-Place Bolts

The following two other anchor types are evaluated using separate procedures in Section 6.3.10:

Welds to Embedded Steel or Exposed Steel

Lead Cinch Anchors

Section 6.3 contains the main steps in the procedure for evaluating the seismic capacity of equipment anchorage. The sections contain a table of nominal allowable load capacities along with anchor-specific inspections which should be performed. In some cases a capacity reduction factor is given which may be used to lower the nominal allowable load capacities if the inspection check reveals that the installation does not meet the minimum guidelines.

Section 6.4 contains generic equipment characteristics for anchorage demand evaluations for use when equipment-specific data is not available for equipment mass, natural frequency, or damping. In addition, an approximate technique for scaling in-structure response spectra by their damping ratios is provided.

1 Based on Sections C-Introduction and 4.4 of SQUG GIP (Ref. 1) 
The material in this chapter is based on the information contained in Reference 41. The SCEs should not use the material contained in this chapter unless they have thoroughly reviewed and understood Reference 41.

Adequate anchorage is almost always essential to the survivability of an item of equipment. Lack of anchorage or inadequate anchorage has been a significant cause of equipment failing to function properly during and following past earthquakes. The screening approach for evaluating the seismic adequacy of equipment anchorage is based upon a combination of inspections, analyses, and engineering judgment. Inspections consist of measurements and visual evaluations of the equipment and its anchorage; supplemented by use of facility documentation and drawings.

Analyses should be performed to compare the anchorage capacity to the seismic loadings (demand) imposed upon the anchorage. These analyses should be done using the guidelines contained in this chapter. Engineering judgment is also an important element in the evaluation of equipment anchorage. Guidance for making judgments is included, where appropriate, in this chapter and in anchorage reference documents.

There are various combinations of inspections, analyses, and engineering judgment which can be used to evaluate the adequacy of equipment anchorage. The SCEs should select the appropriate combination of elements for each anchorage installation based on the information available. For example, a simple hand calculation may be sufficient for a pump which has only a few, very rugged, anchor bolts in a symmetrical pattern. On the other hand, at times it may be advisable to use computer codes to determine the loads applied to a multi-cabinet motor control center if its anchorage is not symmetrically located. Likewise a trade-off can be made between the level of inspection performed and the factor of safety used for expansion anchor bolts. These types of trade-offs and others are discussed in this chapter.

This chapter describes the main steps for evaluating the seismic adequacy of anchorage. In some cases, specific inspection checks and evaluations apply to only certain types of anchors. Section 13.2 describes Screening and Evaluation Work Sheets (SEWS) which can be used as checklists to evaluate that all the appropriate steps in the anchorage evaluation procedure have been completed.

It is not necessary to perform an anchorage evaluation for in-line valves which are discussed in Sections 8.2.1 and 8.2.2. Likewise temperature sensors, which are discussed in Section 8.1.10, are relatively light, normally attached to another piece of equipment, and do not need an anchorage evaluation.

\subsection{ANCHORAGE INSTALLATION INSPECTION}

\subsubsection{Installation Adequacy and Attributes ${ }^{2}$}

To evaluate the seismic adequacy of anchorage, the anchorage installation and its connection to the base of the equipment should be checked. This inspection consists of visual checks and measurements along with a review of facility documentation and drawings where necessary. All accessible anchorage should be visually inspected. All practicable means should be tried to inspect inaccessible anchorage or those obstructed from view if they are needed for strength to secure the item of equipment or if they secure equipment housing essential relays (to avoid impact or excessive cabinet motion). For example, it is not considered practicable to resort to equipment disassembly or removal to inspect inaccessible anchorage. The basis for the engineering judgment for not performing these inspections should be documented.

2 Based on Section 4.4.1 of SQUG GIP (Ref. 1) 
Several general installation checks should be made of the anchorage. For welds, a visual check of the adequacy of the welded joint should be performed. For bolt or stud installation, a visual check should be made to determine whether the bolt or nut is in place and uses a washer where necessary. Oversized washers or reinforcing plates are recommended for thin equipment bases. Lock washers are recommended where even low-level vibration exists. For expansion anchors, a tightness check should be made to detect gross installation defects (such as oversized concrete holes, total lack of preload, loose nuts, damaged subsurface concrete, and missing plug for shell types) which would leave the anchor loose in the hole. The checks to be made on expansion anchors are discussed in detail in Section 6.3.9.

A check of the following equipment anchorage attributes should be made:

- Equipment Characteristics (i.e., estimation of mass, center of gravity location, natural frequency, damping, and equipment base overturning moment center of rotation) (see Section 6.4.1)

- Type of Anchorage (see Sections 6.3.1 and 6.3.2)

- Size and Location of Anchorage (see Section 6.2.2)

- Equipment Base Stiffness and Prying Action (see Section 6.2.4)

- Equipment Base Strength and Structural Load Path (see Section 6.2.5)

- Embedment Steel and Pads (see Section 6.2.6)

- Embedment Length (see Section 6.3.3)

- Gap at Threaded Anchors (see Section 6.2.3)

- Spacing Between Anchors (see Section 6.3.4)

- Edge Distance (see Section 6.3.5)

- Concrete Strength and Condition (see Section 6.3.6)

- Concrete Crack Locations and Sizes (see Section 6.3.7)

- Essential Relays in Cabinets (see Section 6.3.8)

- Installation Adequacy (see Section 6.3.9.1)

Not all of these attributes are applicable to all types of anchors. General guidelines for performing the checks are provided in the sections provided in the list. Engineering judgment should be exercised when making these checks. For example, it is not necessary to measure the spacing between anchor bolts if it is obvious they are much farther apart than the minimum spacing guidelines. 


\subsubsection{Size and Location of Anchorage ${ }^{3}$}

The size of the anchors and the locations where they secure the item of equipment to the floor or wall are key parameters for establishing the capacity of the anchorage for that item of equipment. The nominal allowable capacities are listed according to the diameter of the anchor. Diameter is also used as a key parameter for defining the minimum embedment length, spacing between anchors, and edge distance. The number and location of the anchors which secure an item of equipment determine how the seismic loadings are distributed among all the anchors. Note that the nominal allowable capacities also apply to anchors in the tension zone of concrete; e.g., on the ceiling. Anchors in damp areas or harsh environments should be checked for corrosion deterioration if heavy surface rust is observed.

\subsubsection{Gap at Threaded Anchors ${ }^{4}$}

The size of the gap between the base of the equipment and the surface of the concrete should be less than about 1/4 inch in the vicinity of the anchors (as illustrated in Figure 6.2-1). This limitation is necessary to prevent excessive flexural stresses in the anchor bolt or stud and excessive bending moments on the concrete anchorage when shear loads are applied. Expansion anchors may have low resistance to imposed bolt bending moment which might result from gaps between base and floor. Anchorage with gaps larger than about 1/4 inch should be classified as outliers and evaluated in more detail. Guidance on resolving anchorage outliers is provided in Reference 78.

There should be no gap at the bolt or stud anchor locations for equipment containing essential relays. Gaps beneath the base of this equipment are not allowed since they have the potential for opening and closing during earthquake load reversals. This may create high frequency impact loadings on the equipment and any essential relays mounted therein could chatter.

The gap size can be checked by performing a visual inspection; a detailed measurement of the gap size is not necessary. The check for the presence of essential relays in equipment can be done in conjunction with the Relay Functionality Review described in Chapter 11.

\subsubsection{Base Stiffness and Prying Action ${ }^{5}$}

The base and anchorage load path of the equipment should be inspected to confirm that there is adequate stiffness and there is no significant prying action applied to the anchors. One special case of base flexibility is base vibration isolation systems. Guidelines for evaluating base vibration isolators are included at the end of this section.

There are two main concerns with the lack of adequate stiffness in the anchorage and load path. First, the natural frequency of the item of equipment could be lowered into the frequency range where dynamic earthquake loadings are higher. Second, the cabinet could lift up off the floor during an earthquake resulting in high frequency impact loadings on the equipment, and any essential relays mounted therein could chatter.

Prying action can result from eccentric loads within the equipment itself and between the equipment and the anchors. The concern is that these prying actions can result in a lack of adequate stiffness and strength and in additional moment loadings within the equipment or on the anchors.

3 Based on Section 4.4.1 - Check 3 of SQUG GIP (Ref. 1)

4 Based on Section 4.4.1 - Check 6 of SQUG GIP (Ref. 1)

5 Based on Section 4.4.1 - Check 12 of SQUG GIP (Ref. 1) 
Thin framing members and clip angles may lack the strength and stiffness required to transfer loads to anchor bolts. Stiff load paths with little eccentricity are preferable for anchorage. Equipment constructed of sheet metal, such as motor control centers, switchgear, and instrumentation and control cabinets, is susceptible to these effects and should be carefully inspected for lack of stiffness and prying action. Figure 6.2-2 shows examples of stiff and excessively flexible anchorage connections with prying action. In Example " $\mathrm{A}$ " of this figure, the thin sheet metal may easily bend during uplift of the cabinet.

This unacceptable condition may be corrected by welding the outside edge of the cabinet base to the embedded steel as shown in Example "C". Care should be taken during welding to avoid burning through the thin sheet metal frame of the cabinet. Example "B" shows a thin sheet metal base which can also easily bend during uplift. This unacceptable condition may be corrected by adding a thick metal plate under the nut of the anchor bolt so that the effective thickness and size of the base is similar to the bottom leg of the structural angle shown in Example "D". Note that the prying effect of the eccentric load on the anchor bolt in Example "D" should be considered. Likewise, if the weld in Example " $\mathrm{C}$ " is actually nearer the edge of the embedded plate rather than at the center as shown, then prying and/or bending will be present in the embedded plate. Thin cabinet bases should be reinforced with angle framing so that seismic loads may be transferred to anchor points. In addition, oversized washers are required when anchors are bolted directly through thin sheet metal bases.

Heavy components that are mounted on upright channel sections may rely on weak-way bending of the channel to transfer shear loads to the anchorage. Unstiffened, light-gage channels may not have sufficient strength to handle this load transfer.

The checks for adequate stiffness and lack of prying action require considerable engineering judgment and can be done by a visual inspection of the anchorage installation. SCEs should also review by visual inspection the entire anchorage load path of the equipment for adequate stiffness. If the base is flexible or if prying action could occur, then the SCEs should exercise their judgment to lower the capacity of the anchorage accordingly.

If the equipment is mounted on a base vibration isolation system, then the isolators should be evaluated for seismic adequacy using the following guidelines. Base vibration isolators are vulnerable to failure during an earthquake for several reasons. Vibration isolators consisting primarily of one or several springs have failed during earthquakes when the springs could not resist lateral loads. Isolators manufactured of cast iron can shatter when subjected to earthquakes. Rubber and elastomer products in isolators can fail when bonding adhesives or the material itself fails. Other isolators have steel sections surrounding the spring element which at first appear stout; however, detailed review can reveal that seismic loads may be carried through small fillet or tack welds and through flat bearing plates which bend along their weak axis.

For a base vibration isolator system to be acceptable for seismic loads, the isolator system should have a complete set of bumpers to prevent excessive lateral movement in all directions. The bumpers should not only prevent any excessive lateral movement and torsion, but a positive method of resisting uplift should also be provided other than the springs themselves, or the rubber or adhesives in tension. If the bumpers do not have elastomeric pads to prevent hard impact, the effect of that impact on the equipment should be evaluated. (Note: Essential relays should not be mounted in such equipment.) Isolators which were specifically designed for seismic applications (not cast iron, unbraced springs, weak elastomers, etc.) may be accepted, provided suitable check calculations determine that all possible load combinations and eccentricities within the isolator itself, including possible impact loads, can be taken by the isolator system. 


\subsubsection{Equipment Base Strength and Structural Load Path ${ }^{6}$}

The equipment base and structural load path should be checked to confirm that it has adequate strength, stiffness, and ductility to transmit the seismic loads from the center of gravity of the equipment to the anchorage. Several connections and support members may need to be checked in the evaluation to confirm that the weak link in the load path is addressed, e.g., the channel or stud embedment, the weld between the embedded steel and the cabinet base, and the connection bolts between the base of the equipment and its frame members. Friction connections, such as holddown clips, often pry off or completely slip out-of-place during seismic loading and become completely ineffective. Adequate anchorage requires positive connection.

This check should include such items as whether a washer is present under the nut or the head of the bolt, and if not present, whether one is necessary. A washer is not necessary if the base of the equipment is at least as thick as a standard washer with a hole no larger than the hole in a standard washer. Another item to check is whether the internal bolting and welds near the base of the equipment can carry the anchorage loads.

One example of inadequate strength in the equipment base was demonstrated during a shake table test of a motor control center in which all four corners of the assembly broke loose. The weld between the base channel and the shake table remained intact; however, the small 5/16-inch bolted connections between the base channel and the frame of the assembly broke. The check for adequate strength in the equipment base can be done by a visual inspection of the anchorage installation. This check should be done in conjunction with Section 6.2.4.

\subsubsection{Embedment Steel and Pads ${ }^{7}$}

If an item of equipment is welded to embedded steel or it is mounted on a grout pad or a large concrete pad, the adequacy of the embedded steel, the grout pad, or the large concrete pad should be evaluated.

Welds made to embedded steel transmit the anchor load to the embedment. The location of the weld should be such that large eccentric loads are not applied to the embedded steel. With welded anchors, the presence of weld burn-through in light-gage steel may indicate a weak connection. In addition, line welds have minimal resistance to bending moments applied about the axis of the weld. These moments may occur when there is weld only on one side of a flange. Puddle welds and plug welds used to fill bolt holes in equipment bases have relatively little capacity for applied tensile loads. Fillet welds built up across stacked shim plates may appear to be large but have very small effective throat area and thus low capacity.

If the embedment uses headed studs, the strength criteria should be used together with the generic guidelines contained in this section. Other types of cast-in-place embedments are not covered in this procedure and should be classified as outliers. The holding strength of these other types of embedments may be determined during the outlier evaluation by testing or by application of generally accepted engineering principles. Engineering judgment should be exercised to establish a conservative estimate of the concrete failure surface for outlier resolution of these other types of embedments. Manufacturer's test information or facility specific test information may be used in the outlier resolution of other types of embedments as appropriate. Factors of safety consistent with this procedure should be followed. Guidance on resolving anchorage outliers is provided in Reference 78.

6 Based on Section 4.4.1 - Check 13 of SQUG GIP (Ref. 1)

7 Based on Section 4.4.1 - Check 14 of SQUG GIP (Ref. 1) 
Equipment mounted on grout pads should be checked to confirm that the anchorage penetrate through the grout pad into the structural concrete beneath. Anchorage installed only in the grout pad have failed in past earthquakes and do not have the capacity values assigned to anchors in structural concrete. Inadequate embedment may result from use of shims or tall grout pads.

If an item of equipment is anchored to a large concrete pad, the pad should have reinforcing steel and be of sound construction (i.e., no prominent cracks). The pad/floor interface should also be evaluated to determine whether it can transmit the earthquake loads. For example, if there are sufficient reinforcement bars connecting the floor to the pad, then the connection is adequate. Also, if a chemical bonding agent was used between the pad and floor, the adhesion strength can typically develop the same strength as the concrete in tension and shear.

If there are no reinforcement bars or chemical bond between the pad and the floor, then the interface can typically resist only shear loadings (if the interface had been roughened at the time the pad was poured). It may be possible, in this case, to show that there are no tensile loads on the pad/floor interface due to either: (1) the center of gravity of the item of equipment being low, or (2) the weight of the pad itself acting as a ballast to resist the overturning moment. The adequacy check of the embedded steel, grout pad, and large concrete pad can be done with a visual inspection together with measurements and the use of drawings and other documents where necessary. 


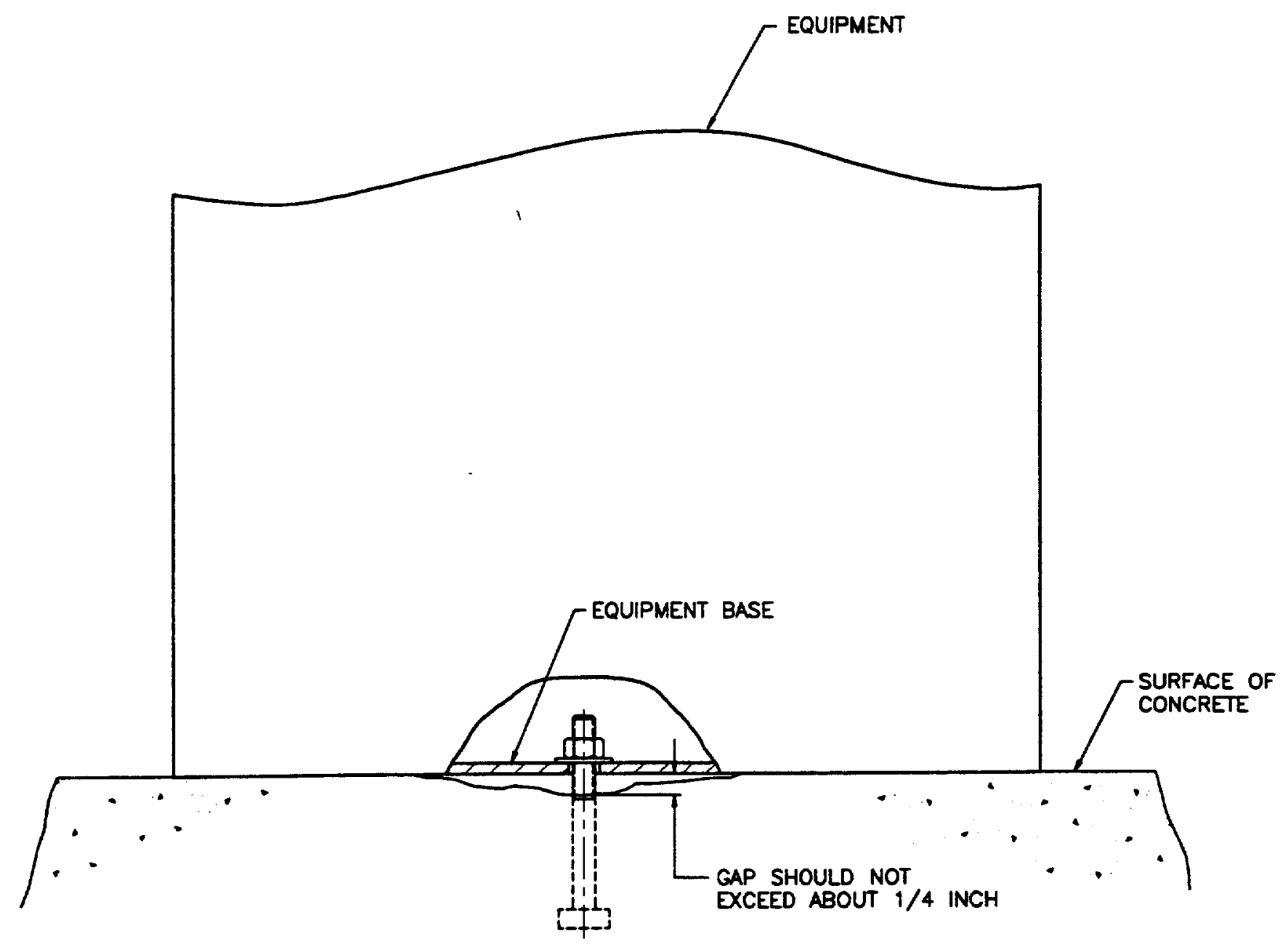

Figure 6.2-1 Equipment with Gap at Anchor Bolt (Figure 4-5 of SQUG GIP, Reference 1) 


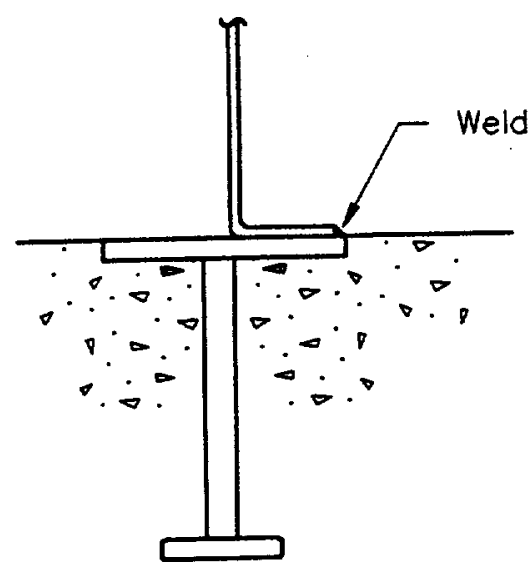

A Undesirable Flexible Welded Anchorage Uplift causes sheet metal frome to bend.

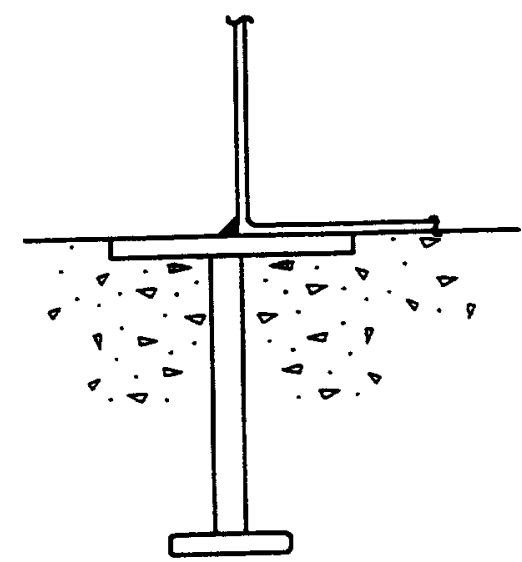

C Desirable Stiff Welded Anchorage Loods poss through sheet metal wall.

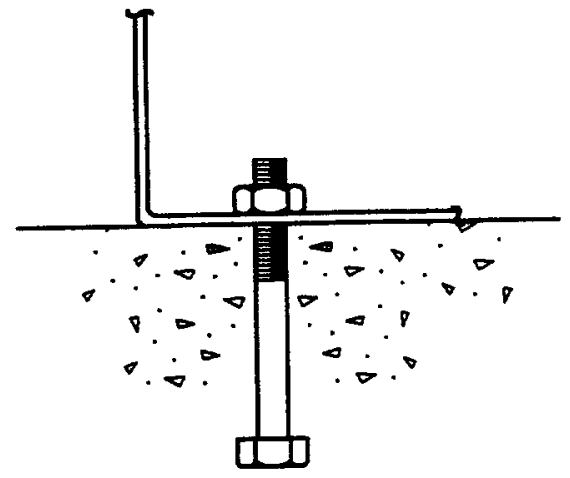

B Undesirable Flexible Bolted Anchorage Uplift causes sheet metal to bend.

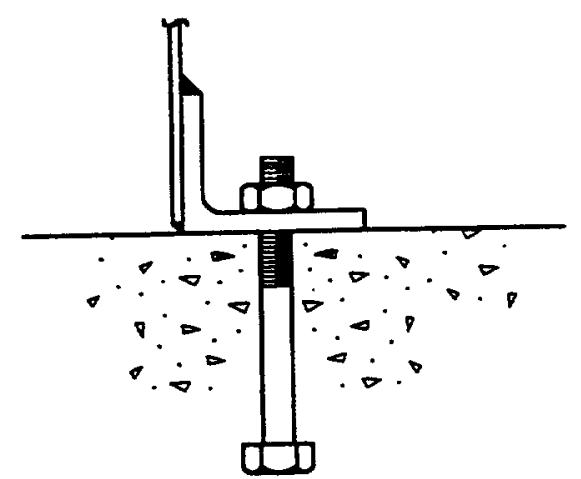

D Desirable Stiff Bolted Anchorage Structural ongle base provides adequate stiffness.

E Undesirable Flexible Base Anchorage Plate can bend from uplift loods and bose frome migr: bend from sheor looss

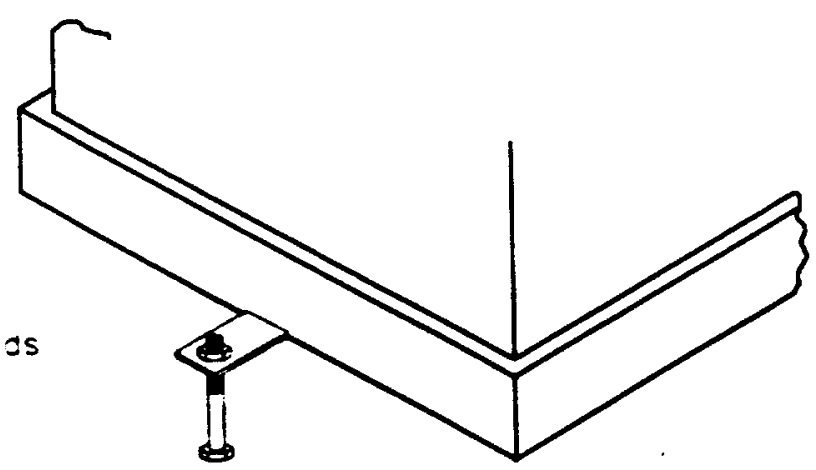

Figure 6.2-2 Examples of Stiff and Excessively Flexible Anchorage Connections (Reference 19) (Figure 4-6 of SQUG GIP, Reference 1) 


\subsection{ANCHORAGE CAPACITY DETERMINATION ${ }^{8}$}

The next step in evaluating the seismic adequacy of anchorage is to determine the allowable capacity of the anchors used to secure an item of equipment. The allowable capacity is obtained by multiplying the nominal allowable capacities by the applicable capacity reduction factors. The nominal capacities and reduction factors can be obtained from this section.

The pullout capacity allowable is based on the product of the nominal pullout capacity and the applicable capacity reduction factors:

$$
P_{\text {all }}=P_{\text {nom }} R T_{p} R_{p} R_{p} R_{p} R F_{p} R C_{p} R R_{p} R_{p}
$$

Where: $\mathrm{P}_{\text {all }}=$ Allowable Pullout capacity of installed anchor (kip)

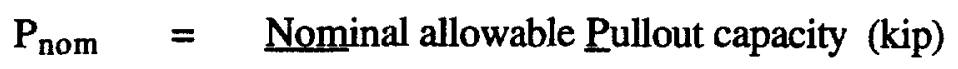

expansion anchors

cast-in-place bolts and headed studs cast-in-place J-bolts grouted-in-place bolts

Section 6.3.1.1

Section 6.3.1.2

lead cinch anchors

$\mathrm{RT}_{\mathrm{p}}=$ Reduction factor for the Type of expansion anchor

expansion anchors

Section 6.3.2

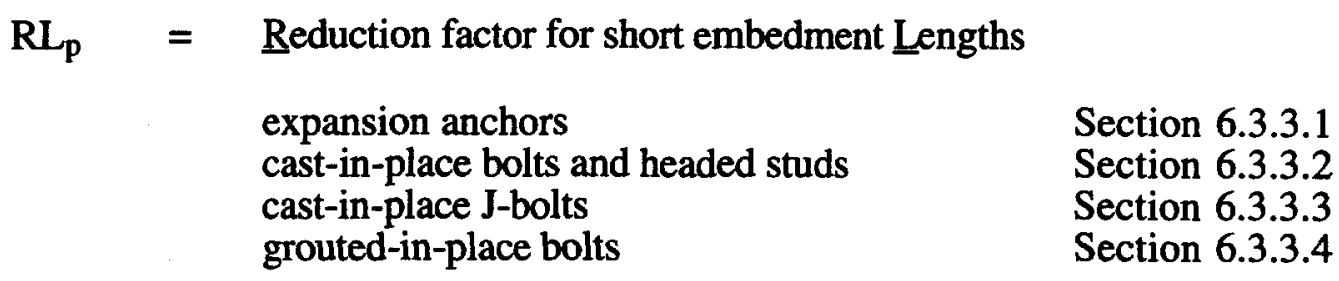

$\mathrm{RS}_{\mathrm{p}} \quad=\quad$ Reduction factor for closely $\underline{\text { Spaced anchors }}$

expansion anchors

cast-in-place bolts and headed studs cast-in-place J-bolts grouted-in-place bolts

Section 6.3.4.1

Section 6.3.4.2

Section 6.3.4.3

Section 6.3.4.4

$\mathrm{RE}_{\mathrm{p}} \quad=\quad$ Reduction factor for near Edge anchors

expansion anchors

cast-in-place bolts and headed studs

cast-in-place J-bolts

grouted-in-place bolts

Section 6.3.5.1

Section 6.3.5.2

Section 6.3.5.3

Section 6.3.5.4

8 Based on Section 4.4.2 of SQUG GIP (Ref. 1) 


$$
\begin{aligned}
& \mathrm{RF}_{\mathrm{p}}=\underline{\text { Reduction factor for low strength concrete }} \\
& \text { expansion anchors Section 6.3.6.1 } \\
& \text { cast-in-place bolts and headed studs } \quad \text { Section 6.3.6.2 } \\
& \text { cast-in-place J-bolts Section 6.3.6.3 } \\
& \text { grouted-in-place bolts } \quad \text { Section 6.3.6.4 }
\end{aligned}
$$

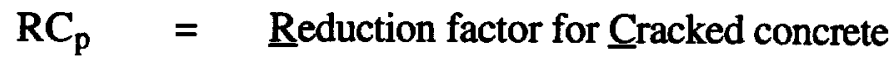

$$
\begin{aligned}
& \text { expansion anchors } \\
& \text { cast-in-place bolts and headed studs } \quad \text { Section 6.3.7.2 } \\
& \text { cast-in-place J-bolts Section 6.3.7.3 } \\
& \text { grouted-in-place bolts } \quad \text { Section 6.3.7.4 }
\end{aligned}
$$

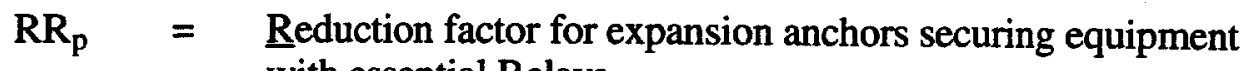

$$
\begin{aligned}
& \text { with essential Relays } \\
& \text { expansion anchors } \quad \text { Section 6.3.8 } \\
& \mathrm{RI}_{\mathrm{p}}=\text { Reduction factor for reduced Inspection procedure } \\
& \text { expansion anchors }
\end{aligned}
$$

The shear capacity allowable is based on the product of the nominal shear capacity and the applicable capacity reduction factors:

$$
V_{\text {all }}=V_{\text {nom }} R T_{s} R_{s} R S_{s} R E_{s} R F_{s} R R_{s} R_{s}
$$

Where: $\mathrm{V}_{\text {all }}=$ Allowable shear capacity of installed anchor (kip)

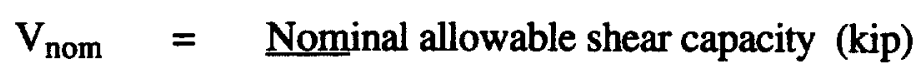

expansion anchors

cast-in-place bolts and headed studs

Section 6.3.1.1

cast-in-place J-bolts grouted-in-place bolts

lead cinch anchors

Section 6.3.1.2

Section 6.3.1.3

Section 6.3.1.4

Section 6.3.10.2

$\mathrm{RT}_{\mathrm{s}}=$ Reduction factor for the Type of expansion anchor

expansion anchors

Section 6.3.2

$R L_{S}=$ Reduction factor for short embedment Lengths

expansion anchors

cast-in-place bolts and headed studs

grouted-in-place bolts

Section 6.3.3.1

Section 6.3.3.2

Section 6.3.3.4 


$$
\begin{aligned}
& \mathrm{RS}_{\mathrm{s}}= \text { Reduction factor for closely Spaced anchors } \\
& \text { expansion anchors } \\
& \text { cast-in-place bolts and headed studs } \\
& \text { cast-in-place J-bolts } \\
& \text { grouted-in-place bolts }
\end{aligned}
$$

Section 6.3.4.1

Section 6.3.4.2

Section 6.3.4.3

Section 6.3.4.4

Section 6.3.5.1

Section 6.3.5.2

Section 6.3.5.3

Section 6.3.5.4

Section 6.3.6.1

Section 6.3.6.2

Section 6.3.6.3

Section 6.3.6.4 $\mathbf{R R}_{\mathbf{s}}=\begin{aligned} & \text { Reduction factor for expansion anchors securing equipment with } \\ & \text { essential Relays }\end{aligned}$

expansion anchors

Section 6.3.8

$\mathrm{RI}_{\mathrm{s}} \quad=\quad$ Reduction factor for reduced Inspection procedure

expansion anchors

Section 6.3.9.2

Note that the pullout and shear capacities for anchors given above are based on having adequate stiffness in the base of the equipment and on not applying significant prying action to the anchor. If Section 6.2 shows that stiffness is not adequate or that significant prying action is applied to the anchors, then the SCEs should lower the allowable capacity loads accordingly. 


\subsubsection{Type of Anchorage and Nominal Allowable Capacities 9}

It is important to identify which of these types of anchorage is used in an installation since these anchorage have different capacities and different installation parameters which should be checked during the inspection. The following four types of anchorage are covered in Sections 6.3.1 to 6.3.9:

1. Expansion Anchors - Shell and Nonshell Types

2. Cast-In-Place Bolts and Headed Studs

3. Cast-In-Place J-Bolts

4. Grouted-In-Place Bolts

Welds to embedded steel or exposed steel and lead cinch anchors are covered individually in Section 6.3.10. If any other type of anchorage is used to secure an item of equipment besides the four covered in this section and the other two covered in subsequent sections, the anchorage for that piece of equipment should be classified as an outlier and evaluated further in Chapter 12 or with the guidance in Reference 78.

In most cases, it will be necessary to use facility drawings, specifications, general notes, purchase records, manufacturer's data, or other such documents to identify the type of anchorage used for an item of equipment. Welds to embedded steel can be distinguished from bolted anchorage without using drawings; however, concrete drawings will still be needed to check the embedment details of the steel. It is not necessary to have specific documented evidence for each item of equipment installed in the facility; i.e., it is permissible to rely upon generic installation drawings or specifications so long as the SCEs have high confidence as to anchorage type and method of installation and remain alert for subtle differences in anchorage installations during the in-facility inspections. The SCEs should visually inspect the anchorage to check that the actual installation appears to be the same as that specified on the drawing or installation specification. If documents are not available to identify the type of bolted anchorage used for an installation, more detailed inspections should be done to develop a basis for the type of anchorage used and its adequacy.

For expansion anchors, it is important to identify the specific make and model of expansion anchor since there is considerable variance in seismic performance characteristics for different expansion anchor types. The makes and models of expansion anchors covered by this procedure are listed in Section 6.3.2 along with appropriate capacity reduction factors. Properly designed, deeply embedded cast-in-place headed studs and J-bolts have desirable performance since the failure mode is ductile, or steel governs. Well-designed and detailed welded connections to embedded plates or structural steel can provide a high-capacity anchorage. Special consideration should be given to grouted-in-place anchors since capacity is highly dependent on the installation practice used. If the grout shrinks any measurable amount, the anchor may have no tensile capacity.

9 Based on Section 4.4.1 - Check 2 of SQUG GIP (Ref. 1) 


\subsubsection{Expansion Anchors ${ }^{10}$}

The nominal allowable load capacities which can be used for the types of expansion anchors covered by this procedure (i.e., those listed in Section 6.3.2) are given in Table 6.3-1 below.

Table 6.3-1 Nominal Allowable Capacities for Expansion Anchors ( $f_{c}^{\prime} \geq 4000$ psi for Pullout and $f_{c}^{\prime} \geq 3500$ psi for Shear) ${ }^{1}$ (Table C.2-1 of SQUG GIP, Ref. 1)

\begin{tabular}{|c|c|c|c|c|}
\hline $\begin{array}{c}\text { Bolt/Stud } \\
\text { Diameter } \\
(\mathrm{D}, \text { in. })\end{array}$ & $\begin{array}{c}\text { Pullout } \\
\text { Capacity } \\
\left(\mathrm{P}_{\text {nom }}, \text { kip }\right)\end{array}$ & $\begin{array}{c}\text { Shear } \\
\text { Capacity } \\
\left(\mathrm{V}_{\text {nom }}, \text { kip }\right)\end{array}$ & $\begin{array}{c}\text { Minimum } \\
\text { Spacing }^{2} \\
\left(\mathrm{~S}_{\text {min }}, \mathrm{in} .\right)\end{array}$ & $\begin{array}{c}\text { Min. Edge } \\
\text { Distance }^{2} \\
\left(\mathrm{E}_{\text {min }}, \text { in. }\right)\end{array}$ \\
\hline $3 / 8$ & 1.46 & 1.42 & 3.75 & 3.75 \\
\hline $1 / 2$ & 2.29 & 2.38 & 5.00 & 5.00 \\
\hline $5 / 8$ & 3.17 & 3.79 & 6.25 & 6.25 \\
\hline $3 / 4$ & 4.69 & 5.48 & 7.50 & 7.50 \\
\hline $7 / 8$ & 6.09 & 7.70 & 8.75 & 8.75 \\
\hline 1 & 6.95 & 9.53 & 10.00 & 10.00 \\
\hline
\end{tabular}

1 The pullout and shear capacities shown here are for the expansion anchor types included in Section 6.3.2 installed in sound, uncracked concrete (i.e., no cracks passing through the anchor bolt installation) with a compressive strength $\left(f^{\prime}\right)$ of at least $4000 \mathrm{psi}$ for pullout and $3500 \mathrm{psi}$ for shear.

2 Minimum spacings and edge distances are measured from bolt center to bolt center or concrete edge. Smaller spacings and edge distances less than the minimums given here can be used with the reduction factors given in Sections 6.3.4.1 and 6.3.5.1.

10 Based on Section C.2.1 of SQUG GIP (Ref. 1) 


\subsubsection{Cast-in-Place Bolts and Headed Studs ${ }^{11}$}

The nominal allowable load capacities which can be used for cast-in-place bolts and headed studs are listed in Table 6.3-2.

Table 6.3-2 Nominal Allowable Capacities for Cast-In-Place Bolts and Headed Studs $\left(f_{c}^{\prime} \geq 3500\right.$ psi) ${ }^{1}$ (Table C.3-1 of SQUG GIP, Ref. 1)

\begin{tabular}{|c|c|c|c|c|c|}
\hline $\begin{array}{c}\text { Bolt/Stud } \\
\text { Diameter } \\
(\mathrm{D}, \text { in. })\end{array}$ & $\begin{array}{c}\text { Pullout } \\
\text { Capacity } \\
\left(\mathrm{P}_{\text {nom }} \text {, kip }\right)\end{array}$ & $\begin{array}{c}\text { Shear } \\
\text { Capacity } \\
\left(\mathrm{V}_{\text {nom }} \text {, kip }\right)\end{array}$ & $\begin{array}{c}\text { Minimum } \\
\text { Embedment }^{2} \\
\left(\mathrm{~L}_{\text {min }}, \text { in. }^{2}\right.\end{array}$ & $\begin{array}{c}\text { Minimum } \\
\text { Spacing }^{3} \\
\left(\mathrm{~S}_{\text {min }}, \text { in. }\right)\end{array}$ & $\begin{array}{c}\text { Min. Edge } \\
\text { Distance }^{3} \\
\left(\mathrm{E}_{\text {min }} \text { in. }\right)\end{array}$ \\
\hline $1 / 8$ & 3.74 & 1.87 & $3-3 / 4$ & $4-3 / 4$ & $3-3 / 8$ \\
\hline $5 / 8$ & 6.66 & 3.33 & 5 & $6-1 / 4$ & $4-3 / 8$ \\
\hline $3 / 4$ & 10.44 & 5.22 & $6-1 / 4$ & $7-7 / 8$ & $5-1 / 2$ \\
\hline $7 / 8$ & 20.44 & 10.22 & $8-3 / 4$ & 11 & $7-3 / 4$ \\
\hline 1 & 26.69 & 13.35 & 10 & $12-5 / 8$ & $8-3 / 4$ \\
\hline $1-1 / 8$ & 33.80 & 16.90 & $11-1 / 4$ & $14-1 / 4$ & $9-7 / 8$ \\
\hline $1-1 / 4$ & 41.72 & 20.86 & $12-1 / 2$ & $15-3 / 4$ & 11 \\
\hline $1-3 / 8$ & 50.40 & 25.25 & $13-3 / 4$ & $17-3 / 8$ & $12-1 / 8$ \\
\hline
\end{tabular}

1 The pullout and shear capacities shown here are for ASTM A-307 (Ref. 79) or equivalent strength bolts installed in sound, uncracked concrete (i.e., no cracks passing through the anchor bolt installation) with a compressive strength of $3500 \mathrm{psi}$ or greater. For bolt capacities in lower strength concrete see Section 6.3.6.2. For bolt capacities in cracked concrete see Section 6.3.7.2.

2 See Figure 6.3-1 for definition of embedment length (L). Smaller embedments than the minimum given here can be used with the reduction factor given in Section 6.3.3.2.

3 Minimum spacings and edge distances are measured from bolt center to bolt center or concrete edge. Spacings and edge distances less than the minimums given here can be used with the reduction factors given in Sections 6.3.4.2 and 6.3.5.2.

11 Based on Section C.3.1 of SQUG GIP (Ref. 1) 


\subsubsection{Cast-in-Place J-Bolts ${ }^{12}$}

The nominal allowable load capacities which can be used for cast-in-place J-bolts are listed in Table 6.3-3 below. The term J-bolt refers to a plain steel bar with a hook formed at the embedded end and threaded at the other end. An embedded bar can be considered as a J-bolt only if it has a hook on the embedded end meeting the minimum dimensions shown in Figure 6.3-2.

Table 6.3-3 Nominal Allowable Capacities for Cast-In-Place J-Bolts $\left(f_{c}^{\prime} \geq 3500\right.$ psi) ${ }^{1}$ (Table C.4-1 of SQUG GIP, Ref. 1)

\begin{tabular}{|c|c|c|c|c|c|c|}
\hline $\begin{array}{c}\text { Bar } \\
\text { Diameter } \\
\text { (D, in.) } \\
\end{array}$ & $\begin{array}{c}\text { Pullout } \\
\text { Capacity } \\
\left(\mathrm{P}_{\text {nom, kip) }}\right.\end{array}$ & $\begin{array}{c}\text { Shear } \\
\text { Capacity } \\
\text { ( } V_{\text {nom, kip) }}\end{array}$ & $\begin{array}{r}\text { Min } \\
\text { Embe } \\
\left(\mathrm{L}_{\mathrm{mi}}\right. \\
180^{\circ} \\
\text { Hook } \\
\end{array}$ & $\begin{array}{l}\text { num } \\
\text { nent }^{2} \\
\text { in.) } \\
90^{\circ} \\
\text { Hook } \\
\end{array}$ & $\begin{array}{l}\text { Minimum } \\
\text { Spacing }{ }^{3} \\
\left(\mathrm{~S}_{\min }, \text { in. }\right) \\
\end{array}$ & $\begin{array}{l}\text { Minimum } \\
\text { Edge } \\
\text { Distance }^{3} \\
\left(\mathrm{E}_{\mathrm{min}}, \text { in. }\right) \\
\end{array}$ \\
\hline $3 / 8$ & 3.74 & 1.87 & 16 & $20-1 / 2$ & $1-1 / 8$ & $3-3 / 8$ \\
\hline $1 / 2$ & 6.66 & 3.33 & $21-1 / 4$ & $27-1 / 4$ & $1-1 / 2$ & $4-3 / 8$ \\
\hline $5 / 8$ & 10.44 & 5.22 & $26-5 / 8$ & $34-1 / 8$ & $1-7 / 8$ & $5-1 / 2$ \\
\hline $3 / 4$ & 15.03 & 7.51 & $31-7 / 8$ & $40-7 / 8$ & $2-1 / 4$ & $6-5 / 8$ \\
\hline $7 / 8$ & 20.44 & 10.22 & $37-1 / 4$ & $47-3 / 4$ & $2-5 / 8$ & $7-3 / 4$ \\
\hline 1 & 26.69 & 13.35 & $42-1 / 2$ & 54-1/2 & 3 & $8-3 / 4$ \\
\hline $1-1 / 8$ & 33.80 & 16.90 & $47-7 / 8$ & $61-3 / 8$ & $3-3 / 8$ & $9-7 / 8$ \\
\hline $1-1 / 4$ & 41.72 & 20.86 & $53-1 / 8$ & $68-1 / 8$ & $3-3 / 4$ & 11 \\
\hline $1-3 / 8$ & 50.40 & 25.25 & $58-1 / 2$ & 75 & $4-1 / 8$ & $12-1 / 8$ \\
\hline
\end{tabular}

1 The pullout and shear capacities shown here are from J-Bolts installed in sound, uncracked concrete with a compressive strength $\left(f_{c}{ }_{c}\right)$ of at least 3500 psi.

2 Embedment length is defined in Figure 6.3-2.

3 Spacing and edge distance are measured from the center of the bolt(s).

12 Based on Section C.4.1 of SQUG GIP (Ref. 1) 


\subsubsection{Grouted-in-Place Bolts ${ }^{13}$}

The nominal allowable pullout and shear capacities which can be used for grouted-in-place bolts are listed in Table 6.3-4. Note that the values in this table are identical to those in Table 6.3-2 for cast-in-place bolts and headed studs except that the pullout capacities $\left(\mathrm{P}_{\text {nom }}\right)$ are reduced by a factor of 10. This was done since the pullout capacity of grouted-in-place bolts is significantly affected by the method of installation. Since documentation of the method used to install grouted-in-place bolts often is not available, the pullout capacities given in the table below are reduced significantly.

However, if the bolts were installed using effective installation procedures similar to those in Reference 80, then the pullout capacities of this grouted-in-place bolts may be taken to be the same as for cast-in-place bolts (i.e., use the capacities given in Table 6.3-2). Some of the installation techniques used in Reference $\mathbf{8 0}$ include such things as thorough cleansing of the concrete hole, acid etching of the concrete hole to roughen the surfaces, and use of grout which expands while it is curing.
Table 6.3-4 Nominal Allowable Capacities for Grouted-In-Place Bolts $\left(f_{c}^{\prime} \geq 3500\right.$ psi) ${ }^{1}$ (Table C.5-1 of SQUG GIP, Ref. 1 )

\begin{tabular}{|c|c|c|c|c|c|}
\hline $\begin{array}{c}\text { Bolt/Stud } \\
\text { Diameter } \\
(\mathrm{D}, \text { in. })\end{array}$ & $\begin{array}{c}\text { Pullout } \\
\text { Capacity } \\
\left(\mathrm{P}_{\text {nom }}, \mathrm{kip}\right)\end{array}$ & $\begin{array}{c}\text { Shear } \\
\text { Capacity } \\
\left(\mathrm{V}_{\text {nom }}, \mathrm{kip}\right)\end{array}$ & $\begin{array}{c}\text { Minimum } \\
\text { Embedment }^{3} \\
\left(\mathrm{~L}_{\text {min }} \text {, in. }\right)\end{array}$ & $\begin{array}{c}\text { Minimum } \\
\text { Spacing }^{4} \\
\left(\mathrm{~S}_{\text {min }}, \text { in. }\right)\end{array}$ & $\begin{array}{c}\text { Min. Edge } \\
\text { Distance } \\
\left(\mathrm{E}_{\text {min }}, \text { in. }^{4}\right.\end{array}$ \\
\hline $3 / 8$ & 0.37 & 1.87 & $3-3 / 4$ & $4-3 / 4$ & $3-3 / 8$ \\
\hline $1 / 2$ & 0.67 & 3.33 & 5 & $6-1 / 4$ & $4-3 / 8$ \\
\hline $5 / 8$ & 1.04 & 5.22 & $6-1 / 4$ & $7-7 / 8$ & $5-1 / 2$ \\
\hline $3 / 4$ & 1.50 & 7.51 & $7-1 / 2$ & $9-1 / 2$ & $6-5 / 8$ \\
\hline $7 / 8$ & 2.04 & 10.22 & $8-3 / 4$ & 11 & $7-3 / 4$ \\
\hline 1 & 2.67 & 13.35 & 10 & $12-5 / 8$ & $8-3 / 4$ \\
\hline $1-1 / 8$ & 3.38 & 16.90 & $11-1 / 4$ & $14-1 / 4$ & $9-7 / 8$ \\
\hline $1-1 / 4$ & 4.17 & 20.86 & $12-1 / 2$ & $15-3 / 4$ & 11 \\
\hline $1-3 / 8$ & 5.04 & 25.25 & $13-3 / 4$ & $17-3 / 8$ & $12-1 / 8$ \\
\hline
\end{tabular}

13 Based on Section C.5.1 of SQUG GIP (Ref. 1) 
1 The pullout and shear capacities shown here are for ASTM A-307 (Ref. 79) or equivalent strength bolts installed in sound, uncracked concrete (i.e., no cracks passing through the anchor bolt installation) with a compressive strength of 3500 psi or greater. For bolt capacities in lower strength concrete see Section 6.3.6.3. For bolt capacities in cracked concrete see Section 6.3.7.3.

2 The pullout capacities (Pnom) are based on not having used special installation practices (or not knowing whether such practices were used). However, if installation procedures similar to those in Reference 80 were used, then the pullout capacities for cast-in-place bolts (Table 6.3-2) can be used in place of the values in this table.

3 See Figure 6.3-1 for definition of embedment length (L). Smaller embedments than the minimum given here can be used with the reduction factor given in Section 6.3.3.4.

4 Minimum spacings and edge distances are measured from bolt center to bolt center or concrete edge. Spacings and edge distances less than the minimums given here can be used with the reduction factors given in Sections 6.3.4.4 and 6.3.5.4. 


\subsubsection{Type of Expansion Anchor ${ }^{14}$}

If the specific manufacturer and model of an expansion anchor is not known, then a generic capacity reduction factor as specified in Table 6.3-5 can be used. This generic factor may be used, however, only on expansion anchors made from carbon steel or better material. Concrete fasteners made from other materials or which use fastening mechanisms which are different than that of expansion anchors should be identified as outliers. This would include fasteners such as chemical anchors, plastic anchors, powder actuated fasteners, and concrete screws.

It is also important to distinguish between shell- and nonshell-type expansion anchors since different types of checks should be made to assure that they are properly installed. This section provides a description of the differences between shell and nonshell expansion anchors, how to tell them apart while they are installed, and what the capacity reduction factors are for the various makes and models. The shell type, or displacement controlled, (see Figure 6.3-3) and wedge type, or torque controlled, (see Figure 6.3-4) expansion anchors have been widely tested and have reasonably consistent capacity when properly installed in sound concrete.

Note that expansion anchors should generally not be used for securing vibratory equipment, such as pumps and air compressors. Expansion anchors used for vibrating equipment may rattle loose and have little to no tensile capacity. If such equipment is secured with expansion anchors, then there should be a large margin between the pullout loads and the pullout capacities; i.e., these expansion anchors should be loaded primarily in shear with very little pullout load. If a component which is secured with expansion anchors, has been in service for a long time and its expansion anchors remain tightly set, then this is a reasonable basis for ensuring installation adequacy. It is generally recommended that if expansion anchors need to be used for vibrating equipment, then the undercut-type of expansion anchors should be installed.

The specific manufacturers and product names of expansion anchors covered by this procedure are listed in Table 6.3-5 below. This table also lists capacity reduction factors ( $\mathrm{RT}_{\mathrm{p}}$ for pullout and $\mathrm{RT}_{\mathrm{s}}$ for shear) which should be multiplied by the nominal pullout and shear capacities $\left(\mathrm{P}_{\text {nom, }}\right.$, $\mathrm{V}_{\text {nom }}$ ) given in Table 6.3-1.

$$
\begin{aligned}
\mathrm{RT}_{\mathrm{p}}=\mathrm{RT}_{\mathrm{s}}=\quad \begin{array}{l}
\text { Pullout }(\mathrm{p}) \text { and shear }(\mathrm{s}) \text { capacity reduction } \\
\text { factors for type of expansion anchor from } \\
\text { Table 6.3-5 }
\end{array}
\end{aligned}
$$

14 Based on Sections 4.1.1 - Check 2 and C.2.2 of SQUG GIP (Ref. 1) and information from Revision 3 of SQUG GIP (Ref. 12) 
Table 6.3-5 Type of Expansion Anchors Covered by this Procedure and Associated Capacity Reduction Factors (Table C.2-2 of SQUG GIP, Ref. 1)

\begin{tabular}{|c|c|c|c|c|}
\hline Manufacturer & Product Name & Type & $\begin{array}{l}\text { Capacity } \\
\text { Reduction } \\
\text { Factors } \\
\left(\mathrm{RT}_{\mathrm{p}}\right) \\
\end{array}$ & $\begin{array}{l}\text { Capacity } \\
\text { Reduction } \\
\text { Factors } \\
\left(\mathrm{RT}_{\mathrm{s}}\right) \\
\end{array}$ \\
\hline Drillco & MaxiBolt & Nonshell & $1.0^{1}$ & $1.0^{1}$ \\
\hline Hilti & $\begin{array}{l}\text { Kwik-Bolt } \\
\text { HDI } \\
\text { Sleeve ( }(3 / 8 \text { inch) } \\
\text { Sleeve }(1 / 2 \text { to } 5 / 8 \text { inch }) \\
\end{array}$ & $\begin{array}{l}\text { Nonshell } \\
\text { Shell } \\
\text { Nonshell } \\
\text { Nonshell } \\
\end{array}$ & $\begin{array}{c}1.0 \\
1.0 \\
0.5^{2} \\
0.75^{2} \\
\end{array}$ & $\begin{array}{l}1.0^{2} \\
1.0^{2} \\
1.0^{2} \\
1.0^{2}\end{array}$ \\
\hline ITW/Ramset & $\begin{array}{l}\text { Dynaset } \\
\text { Dynabolt } \\
\text { Trubolt } \\
\end{array}$ & $\begin{array}{l}\text { Shell } \\
\text { Nonshell } \\
\text { Nonshell } \\
\end{array}$ & $\begin{array}{l}1.0 \\
0.75 \\
0.75 \\
\end{array}$ & $\begin{array}{l}1.0^{2} \\
0.75^{2} \\
0.75^{2} \\
\end{array}$ \\
\hline $\begin{array}{l}\text { ITW/Ramset/ } \\
\text { Redhead }\end{array}$ & $\begin{array}{l}\text { Multiset Drop-In } \\
\text { Self Drilling } \\
\text { Dynabolt Sleeve } \\
\text { Nondrill } \\
\text { Stud } \\
\text { TRUBOLT } \\
\end{array}$ & $\begin{array}{l}\text { Shell } \\
\text { Shell } \\
\text { Nonshell } \\
\text { Shell } \\
\text { Shell } \\
\text { Nonshell } \\
\end{array}$ & $\begin{array}{l}1.0 \\
1.0 \\
1.0 \\
1.0 \\
0.75 \\
0.75 \\
\end{array}$ & $\begin{array}{l}1.0^{2} \\
1.0^{2} \\
1.0^{2} \\
1.0^{2} \\
0.75^{2} \\
0.75^{2} \\
\end{array}$ \\
\hline Molly & $\begin{array}{l}\text { Parasleeve } \\
\text { MDI } \\
\text { Parabolt } \\
\end{array}$ & $\begin{array}{l}\text { Nonshell } \\
\text { Shell } \\
\text { Nonshell } \\
\end{array}$ & $\begin{array}{l}1.0 \\
1.0 \\
0.75 \\
\end{array}$ & $\begin{array}{l}1.0^{2} \\
1.0^{2} \\
0.75^{2} \\
\end{array}$ \\
\hline Phillips & $\begin{array}{l}\text { Self-Drilling } \\
\text { Wedge } \\
\text { Sleeve } \\
\text { Multi-Set } \\
\text { Stud } \\
\text { Non-Drilling } \\
\end{array}$ & $\begin{array}{l}\text { Shell } \\
\text { Nonshell } \\
\text { Nonshell } \\
\text { Shell } \\
\text { Shell } \\
\text { Shell } \\
\end{array}$ & $\begin{array}{l}1.0 \\
1.0 \\
1.0 \\
1.0 \\
1.0 \\
1.0\end{array}$ & $\begin{array}{l}1.0^{2} \\
1.0^{2} \\
1.0^{2} \\
1.0^{2} \\
1.0^{2} \\
1.0^{2}\end{array}$ \\
\hline Rawl & $\begin{array}{l}\text { Drop-In } \\
\text { Stud } \\
\text { Saber-Tooth } \\
\text { Bolt } \\
\end{array}$ & $\begin{array}{l}\text { Shell } \\
\text { Shell } \\
\text { Shell } \\
\text { Nonshell }\end{array}$ & $\begin{array}{l}1.0 \\
0.75 \\
0.75 \\
0.75\end{array}$ & $\begin{array}{l}1.0^{2} \\
0.75^{2} \\
0.75^{2} \\
0.75^{2} \\
\end{array}$ \\
\hline Star & $\begin{array}{l}\text { Selfdrill } \\
\text { Steel } \\
\text { Stud } \\
\end{array}$ & $\begin{array}{l}\text { Shell } \\
\text { Shell } \\
\text { Shell } \\
\end{array}$ & $\begin{array}{l}0.75 \\
0.75^{2} \\
0.75^{2} \\
\end{array}$ & $\begin{array}{c}0.75^{2} \\
1.0^{2} \\
0.75^{2} \\
\end{array}$ \\
\hline USE Diamond & $\begin{array}{l}\text { Sup-R-Drop } \\
\text { Sup-R-Stud } \\
\text { Sup-R-Sleeve } \\
\text { Sup-R-Drill } \\
\end{array}$ & $\begin{array}{l}\text { Shell } \\
\text { Shell } \\
\text { Nonshell } \\
\text { Shell }\end{array}$ & $\begin{array}{l}1.0 \\
1.0 \\
1.0 \\
0.75 \\
\end{array}$ & $\begin{array}{l}1.0^{2} \\
1.0^{2} \\
1.0^{2} \\
0.75^{2} \\
\end{array}$ \\
\hline WEJ-IT & $\begin{array}{l}\text { Drop-In } \\
\text { Sleeve } \\
\text { Wedge } \\
\text { Stud } \\
\end{array}$ & $\begin{array}{l}\text { Shell } \\
\text { Nonshell } \\
\text { Nonshell } \\
\text { Shell } \\
\end{array}$ & $\begin{array}{c}1.0 \\
1.0 \\
0.5^{2} \\
0.75^{2} \\
\end{array}$ & $\begin{array}{c}1.0^{2} \\
1.0^{2} \\
0.75^{2} \\
1.0^{2} \\
\end{array}$ \\
\hline Unknown & $\begin{array}{l}\text { Unknown }(3 / 8 \text { inch })^{2} \\
\text { Unknown }(>3 / 8 \text { inch })^{2}\end{array}$ & $\begin{array}{l}\text { Unknown } \\
\text { Unknown }\end{array}$ & $\begin{array}{l}0.5^{2} \\
0.75^{2}\end{array}$ & $\begin{array}{l}0.75^{2} \\
0.75^{2}\end{array}$ \\
\hline
\end{tabular}

1 From Table C-2 of WSRC SEP-6 (Ref. 3)

2 From Table 6.3-5 of Revision 3 of SQUG GIP (Ref. 4), which is being reviewed by the NRC 
If the specific manufacturer and product name of an expansion anchor is not known, then a generic capacity reduction factors as indicated below may be used:

$$
\begin{aligned}
& \mathrm{RT}_{\mathrm{P}}=0.5 \text { and } \mathrm{RT}_{\mathrm{S}}=0.75 \text { (for bolt diameter }=3 / 8 \text { inch) } \\
& \mathrm{RT}_{\mathrm{P}}=0.75 \text { and } \mathrm{RT}_{\mathrm{S}}=0.75 \text { (for bolt diameter } \geq 3 / 8 \text { inch) }
\end{aligned}
$$

Note, however, that this generic capacity reduction factor may only be used for expansion anchors made from carbon steel or better material. Concrete fasteners made from other materials or which use fastening mechanisms which are different than that of expansion anchors should be identified as outliers. This would include fasteners such as chemical anchors, plastic anchors, powder actuated fasteners, and concrete screws. "Unknown" anchors should be examined to ensure that they are not the WEJ-IT wedge anchor bolts, which can be distinguished from all other bolts by the two vertical slots cut along opposite sides of the bolt, parallel to the longitudinal axis of the bolt. Guidance on resolving anchorage outliers is provided in Reference 78.

In general, expansion anchors should not be used for securing vibratory equipment such as pumps and air compressors. If such equipment is secured with expansion anchors, then there should be a large margin between the pullout loads and the pullout capacities; i.e., the loads on these expansion anchors should be primarily shear.

The principal differences between shell- and nonshell-type expansion anchors are explained below.

Shell-type expansion anchors are expanded into the concrete by application of a setting force independent of the load later applied to the bolt or nut by the equipment being anchored. The key feature of this type of expansion anchor is that it relies upon its initial preset for holding it in place. Figure 6.3-3 shows the features of several types of shell-type expansion anchors.

Figure 6.3-3a shows a "Self-Drilling Type" of shell-type expansion anchor. This type of anchor is set in place by driving the shell down over the cone expander which is resting against the bottom of the hole.

Figure 6.3-3b shows a "Drop-In Type" which is set in place by driving a cone expander down through the center of the shell thereby causing the lower portion of the shell to expand into the concrete.

Figure 6.3-3c shows a "Phillips Stud Type" which is set in place by driving the stud down over the cone expander which is resting against the bottom of the hole.

Nonshell-type expansion anchors are expanded into the concrete by pulling the stud up out of the hole which causes a sleeve or a split ring to be forced into the concrete. The key feature of this type of expansion anchor is that the more the stud is loaded in tension, the greater the expansion setting force becomes. Figure 6.3-4 shows the features of two types of nonshell-type expansion anchors.

Figure 6.3-4a shows a "Sleeve Type" which is set in place by pulling the stud, with its integral cone expander on the bottom, up into the sleeve thereby forcing the lower split portion of the sleeve into the concrete. The sleeve is held in place during this setting process by butting up against the lower surface of the washer.

Figure 6.3-4b shows a "Wedge Type" which is set in place by pulling the stud, with its integral cone expander on the bottom, up though a split ring. Note that the split ring relies on friction against the concrete to stay in place during the setting operation. 
Distinguishing characteristics of shell- and nonshell-type expansion anchors in their as-installed condition are shown in Figure 6.3-5.

Figure 6.3-5a shows a nonshell-type expansion anchor in which the visible portion is characterized by a smoothly cut or mechanically finished threaded stud with a nut holding the base of the equipment in place.

Figure 6.3-5b shows the most common type of shell-type expansion anchor in which the visible portion is characterized by a head of a bolt.

Figures 6.3-5c and 6.3-5d show other types of shell-type expansion anchors in which the visible portion is characterized by a rough cut or a raised knob on the end of the threaded rod. Careful inspection is necessary to distinguish these two types of shell expansion anchors from the nonshell-type shown in Figure 6.3-5a. 


\subsubsection{Embedment Length ${ }^{15}$}

The embedment length of an anchor should be checked to confirm that it meets the minimum value so that nominal allowable anchor capacities can be used. A capacity reduction factor can be applied to the nominal allowable capacities for certain types of anchors with less embedment. Minimum embedments and reduction factors are given for each type of anchor covered in this procedure.

The minimum embedments for expansion anchors are based on the manufacturer's recommendations and cannot be reduced by applying capacity reduction factors. Expansion anchors which have deeper embedments may use the higher recommended capacities contained in the manufacturer's catalog in place of the nominal allowable capacities. The minimum embedments for cast-in-place bolts and headed studs and for grouted-in-place bolts are set to be sufficiently long so that the anchorage will fail in a ductile manner; i.e., in the bolt or stud, not in the concrete. Grouted-in-place anchor embedments are the same as those for cast-in-place anchors; a higher factor of safety is assigned to the pullout capacities of grouted-in-place anchors to account for uncertainties in the bolt installation. The minimum embedment for smooth bar J-bolts is based primarily on the bond strength between the bar and the concrete.

The embedment length of expansion anchors can be checked by confirming that the anchor is one of the makes and models covered by this procedure and performing a visual inspection of the installation. For many types of nonshell anchors, ultrasonic testing can be used to determine bolt length. Bolt embedment length may not be adequate if part of the shell is exposed or if there is a long stud protruding above the concrete surface.

It is not necessary to perform an embedment length check of an expansion anchor if the anchorage for that piece of equipment is robust, i.e., there is a large margin between the applied load and the anchorage capacity. Guidelines for evaluating whether there is sufficient margin in the anchorage are provided in Section 6.3.9.2, Reduced Inspection Alternative. The embedment length for anchor types other than expansion anchors can be determined from concrete installation drawings, ultrasonic testing, or other appropriate means.

\subsubsection{Expansion Anchors ${ }^{16}$}

If the embedment is greater than the values given in Table 6.3-6, then a pullout capacity reduction factor $\left(R L_{p}\right)$ and a shear capacity reduction factor $\left(R_{\mathrm{s}}\right)$ should be multiplied by the nominal pullout and shear capacities $\left(P_{\text {nom }}, V_{\text {nom }}\right)$ given in Table 6.3-1.

$$
\begin{aligned}
R_{p}=R_{s} & =\begin{array}{l}
\text { Pullout }(p) \text { and shear (s) capacity reduction factors for } \\
\text { expansion anchors }
\end{array} \\
& =1.0 \quad \text { for embedments greater than those listed in Table 6.3-6 } \\
& =\text { Outlier for embedments less than those listed in Table 6.3-6 }
\end{aligned}
$$

(Note: This inspection check is not needed if the Reduced Inspection Alternative is chosen, as described in Section 6.3.9.2)

15 Based on Section 4.4.1 - Check 5 of SQUG GIP (Ref. 1)

16 Based on Section C.2.4 of SQUG GIP (Ref. 1) 
The manufacturer's recommended minimum embedments listed in Table 6.3-6 are from the catalogs of each of the vendors as listed in Reference 41, page E-27. These are the most recent catalogs available when Reference 41 was published. Expansion anchors with less than the minimum embedment should be documented as outliers. Guidance for resolving anchorage outliers is provided in Reference 78.

\section{Table 6.3-6 Manufacturer's Recommended Minimum Embedment for Expansion Anchors Covered by this Procedure (Table C.2-6 of SQUG GIP, Ref. 1)}

\begin{tabular}{|c|c|c|c|c|c|c|c|}
\hline & Product Name & & $\begin{array}{r}\text { Mini } \\
\text { f }\end{array}$ & $\begin{array}{l}\mathrm{Im} E m \\
\text { Bolt/S }\end{array}$ & $\begin{array}{l}\text { edmen } \\
\text { d Diar }\end{array}$ & $\begin{array}{l}\text { L) [in } \\
\text { ter: }\end{array}$ & \\
\hline Manufacturer & ( $\mathrm{S}=$ Shell, $\mathrm{N}=$ Nonshell) & $3 / 8^{\prime \prime}$ & $1 / 2 "$ & 5/8" & $3 / 4 "$ & 7/8" & $1 "$ \\
\hline Hilti & Kwik-Bolt (N) & 1.63 & 2.25 & 2.75 & 3.25 & - & 4.50 \\
\hline & HDI (S) & $1.56^{1}$ & 2.00 & $2.56^{1}$ & 3.19 & - & -- \\
\hline & Sleeve $(\mathrm{N})$ & 1.50 & 2.00 & 2.00 & -- & - & - \\
\hline ITW/Ramset & Dynaset (S) & 1.63 & 2.00 & 2.63 & 3.25 & - & - \\
\hline & Dynabolt $(\mathbf{N})$ & 2.00 & 2.25 & 2.25 & -- & -- & - \\
\hline & Trubolt $(\mathbf{N})$ & 1.50 & 2.25 & 2.75 & 3.38 & 4.00 & 4.50 \\
\hline ITW/Ramset/ & Multiset Drop-In (S) & 1.63 & 2.00 & 2.50 & 3.19 & -- & - \\
\hline Redhead & Self Drilling (S) & 1.53 & 2.03 & 2.47 & 3.25 & -- & - \\
\hline & Dynabolt Sleeve & 1.88 & 2.00 & 2.25 & - & -- & -- \\
\hline & Nondrill (S) & 1.56 & 2.06 & 2.56 & 3.19 & -- & - \\
\hline & Stud (S) & 1.63 & 1.88 & 2.38 & 2.88 & -- & -- \\
\hline & TRUBOLT $(\mathbf{N})$ & 1.50 & 2.25 & 2.75 & 3.25 & 3.75 & 4.50 \\
\hline Molly & Parasleeve (N) & $1.50^{1}$ & $2.00^{1}$ & 2.00 & $--^{1}$ & - & -- \\
\hline & MDI (S) & $1.56^{1}$ & 2.00 & $2.50^{1}$ & $--^{1}$ & -- & -- \\
\hline & Parabolt $(\mathrm{N})$ & 1.50 & 2.25 & $2.75^{1}$ & 3.25 & 4.00 & 4.50 \\
\hline
\end{tabular}


Table 6.3-6 (Continued)

\begin{tabular}{|c|c|c|c|c|c|c|c|}
\hline & Product Name & & $\begin{array}{l}\text { Minin } \\
\text { fo }\end{array}$ & $\begin{array}{l}\mathrm{um} \text { Em } \\
\text { Bold/S }\end{array}$ & $\begin{array}{l}\text { edment } \\
\text { Id Dian }\end{array}$ & $\begin{array}{l}\mathrm{L}) \text { [in.] } \\
\text { ter: }\end{array}$ & \\
\hline & $(\mathrm{S}=\mathrm{Sh}$ & $3 / 8 "$ & $1 / 2 "$ & $5 / 8 "$ & $3 / 4 "$ & 7/8" & 1" \\
\hline Phillips & Self-Drilling (S) & 1.53 & 2.03 & 2.47 & 3.25 & 3.69 & -- \\
\hline & Wedge $(\mathrm{N})$ & 1.75 & 2.13 & 2.63 & 3.25 & 3.75 & 4.50 \\
\hline & Sleeve $(\mathbf{N})$ & 1.88 & 2.00 & 2.25 & -- & -- & -- \\
\hline & Multi-Set (S) & 1.38 & 1.75 & 2.25 & 2.50 & -- & - \\
\hline & Stud (S) & 1.63 & 1.88 & 2.38 & 2.88 & -- & -- \\
\hline & Non-Drilling (S) & 1.56 & 2.06 & 2.56 & 3.19 & - & - \\
\hline Rawl & Drop-In (S) & 1.88 & 2.38 & 3.00 & 3.50 & -- & -- \\
\hline & Stud (S) & 1.75 & 2.25 & 2.88 & 3.38 & 4.00 & 4.50 \\
\hline & Saber Tooth (S) & 1.53 & 2.03 & 2.47 & 3.25 & 3.69 & -- \\
\hline & Bolt $(\mathrm{N})$ & 2.00 & 2.50 & 2.75 & 3.00 & - & - \\
\hline Star & Selfdrill (S) & 1.53 & 2.03 & 2.47 & 3.25 & 3.69 & -- \\
\hline & Steel (S) & 1.44 & 1.94 & 2.38 & 3.00 & -- & -- \\
\hline & Stud $(\mathbf{S})$ & 1.63 & 1.75 & 2.38 & 2.88 & - & -- \\
\hline USE Diamond & Sup-R-Drop (S) & 1.56 & 2.00 & 2.53 & 3.19 & -- & -- \\
\hline & Sup-R-Stud (S) & 2.16 & 2.81 & 3.31 & 4.25 & 4.72 & 5.56 \\
\hline & Sup-R-Sleeve $(\mathrm{N})$ & $1.50^{1}$ & $2.00^{1}$ & $2.50^{1}$ & $3.00^{1}$ & -- & -- \\
\hline & Sup-R-Drill (S) & 1.53 & 2.03 & 2.47 & 3.27 & - & -- \\
\hline WEJ-IT & Drop-In (S) & 1.63 & 2.00 & 2.50 & 3.25 & -- & -- \\
\hline & Sleeve $(\mathbf{N})$ & 1.50 & 1.88 & 2.00 & 2.25 & -- & - \\
\hline & Wedge $(\mathrm{N})$ & 1.50 & 2.00 & 3.00 & 3.00 & 4.50 & 5.50 \\
\hline & Stud (S) & 1.75 & 2.13 & $3.63^{1}$ & 3.25 & -- & 4.50 \\
\hline
\end{tabular}

'From Table 6.3-6 of Revision 3 of SQUG GIP (Ref. 4), which is being reviewed by the NRC 
These minimum embedments can be evaluated by performing the following inspection checks for shell- and nonshell-type expansion anchors. Note that these checks should be performed after the tightness check (Section 6.3.9) has been performed.

Shell-Type Expansion Anchors. The embedment length of shell-type expansion anchors is predetermined by the length of the shell and how it is installed in the concrete. The appropriate shell length is assured if the expansion anchor is one of the types listed in Table 6.3-6. An appropriate installation is assured if the shell of these anchors does not protrude above the surface of the concrete.

When making this embedment check, a check should also be made to confirm that the top of the shell is not touching the bottom of the base plate of the item of equipment being anchored. This check should be performed after the tightness check (Section 6.3.9) has been done. This will assure that the expansion anchor is tight in the hole and not just tight up against the base of the equipment.

If it is necessary to remove the bolt or nut from the anchorage to make the above two checks, then it is only necessary to spot check the embedment of a few anchors. If this spot check indicates that these types of bolts may not be properly installed, then this inspection check should be expanded accordingly. When re-installing the anchor, it should be re-tightened to a "wrench tight" condition or to the recommended tightness check torque values.

Nonshell-Type Expansion Anchors. The embedment length of nonshell-type expansion anchors is predetermined by the length of the stud and the installation of the anchor. The appropriate overall length of nonshell studs is dependent upon the manufacturer, the model, and the thickness of the equipment base plate for which the anchor is designed. Table 6.3-7, below, can be used as a generic screen for assessing whether a nonshell expansion anchor has adequate embedment. A range of projections is given in Table 6.3-7 since there are differences in acceptable projections depending upon the make and model of the anchor. If a nonshell stud projects more than the lower value of this range, then anchor-specific information should be used to determine the embedment length of the anchor.

Table 6.3-7 Maximum Stud Projections Above Concrete for Nonshell-Type Expansion Anchors (Table C.2-7 of SQUG GIP, Ref. 1)

\begin{tabular}{|c|c|}
\hline $\begin{array}{c}\text { Stud } \\
\text { Diameter } \\
\text { (in.) }\end{array}$ & $\begin{array}{c}\text { Maximum Stud } \\
\text { Projections } \\
\text { Above Concrete } \\
\text { (in.) }\end{array}$ \\
\hline $3 / 8$ & $1 / 2-3 / 4$ \\
\hline $1 / 2$ & $1 / 2-3 / 4$ \\
\hline $5 / 8$ & $1 / 2-7 / 8$ \\
\hline $3 / 4$ & $7 / 8-11 / 2$ \\
\hline $7 / 8$ & $11 / 2-2$ \\
\hline 1 & $11 / 2-2$ \\
\hline
\end{tabular}


Note that careful evaluation is needed when checking the projections since larger projections than those given above may be needed if the base plate is relatively thick or if, at the time of installation in the facility, a particular bolt length may not have been available. Also, for bolts made by some manufacturers, the bolt projections may be larger than those given in the above table even for their shortest bolts. Thus, while this check need only be visual, a careful evaluation should be made to determine whether the stud projection is reasonable, given the bolt diameter, base plate thickness, and whether a grout pad is used. When projections are larger than those given in Table 6.3-7, adequate embedment should be evaluated by consulting design and construction documents and vendor catalogs. Alternately, ultrasonic inspection techniques may be used to compare the measured bolt/stud length to the manufacturer's recommended minimum embedment given in Table 6.3-6.

This embedment check should be performed on wedge- and sleeve-type, nonshell expansion anchors after the tightness check (Section 6.3.9) has been done. This is to ensure that the tightness check does not pull the expansion anchor partially out of the hole beyond the required minimum embedment.

For bolts with deeper embedments than the minimum values given in Table 6.3-6, manufacturer's catalog data may be used, if it is available, to establish the nominal allowable capacities instead of those given in Table 6.3-1. As an alternative, facility specific testing may be performed to establish the strength of the more deeply embedded expansion anchors. Guidance for resolving anchorage outliers is provided in Reference 78.

\subsubsection{Cast-in-Place Bolts and Headed Studs ${ }^{17}$}

The nominal pullout and shear capacities $\left(\mathrm{P}_{\text {nom }}, \mathrm{V}_{\text {nom }}\right)$ given in Table 6.3-2 are based on the assumption that the embedment length is sufficiently long to preclude failure in the concrete. The minimum embedments $\left(\mathrm{L}_{\mathrm{min}}\right)$ given in Table 6.3-2 are equal to 10 times the bolt diameter (D). Figure 6.3-1 shows the embedment length (L) for a cast-in-place bolt and a headed stud.

The embedment length should be evaluated by consulting existing drawings to ensure that the actual embedment length $(\mathrm{L})$ is more than the minimum $\left(\mathrm{L}_{\mathrm{min}}\right)$. If the construction drawings are not available, ultrasonic means or other appropriate methods may be used to evaluate the actual embedments.

If the embedment length $(\mathrm{L})$ is less than the minimum value $\left(\mathrm{L}_{\min }\right)$ given in Table 6.3-2, then a pullout capacity reduction factor $\left(R L_{p}\right)$ and a shear capacity reduction factor $\left(R L_{s}\right)$ should be multiplied by the nominal pullout and shear capacities $\left(P_{\text {nom }}, V_{\text {nom }}\right)$ given in Table 6.3-2.

$$
\begin{aligned}
\mathrm{RL}_{\mathrm{p}}=\mathrm{RL}_{\mathrm{s}} & =\quad \begin{array}{l}
\text { Pullout }(\mathrm{p}) \text { and shear (s) capacity reduction factors for cast-in-place } \\
\text { anchors with shallow embedment }
\end{array} \\
& =1.0 \quad \text { for } \mathrm{L} \geq 10 \mathrm{D} \\
& =\frac{(\mathrm{L}+\mathrm{D}) \mathrm{L}}{\left(\mathrm{L}_{\min }+\mathrm{D}\right) \mathrm{L}_{\min }} \quad \text { for } 4 \mathrm{D}<\mathrm{L}<10 \mathrm{D} \text { and } \mathrm{L}>3 \text { inches } \\
& =\text { Outlier } \quad \text { for } \mathrm{L}<\text { Greater of: } 4 \mathrm{D} \text { or } 3 \text { inches }
\end{aligned}
$$

17 Based on Section C.3.2 of SQUG GIP (Ref. 1) 


$\begin{array}{lll}\mathrm{L} & = & \text { Length of anchor embedment per Figure 6.3-1 } \\ \mathrm{L}_{\min } & = & \text { Minimum length of anchor embedment from Table 6.3-2 } \\ \mathrm{D} & = & \text { Diameter of anchor bolt/stud }\end{array}$

\subsubsection{Cast-in-Place J-Bolts ${ }^{18}$}

The nominal pullout capacities $\left(\mathrm{P}_{\text {nom }}\right)$ given in Table 6.3-3 are based on the assumption that the embedded length is at least as long as the minimum embedment lengths $\left(\mathrm{L}_{\text {min }}\right)$ given in Table 6.3.3.

If the embedment length ( $L)$ is less than the minimum value $\left(L_{\min }\right)$, then a pullout capacity reduction factor $\left(R L_{p}\right)$ should be multiplied by the nominal pullout capacity $\left(P_{\text {nom }}\right)$. A capacity reduction factor for shear is not needed since J-bolts develop their full shear strength even when the embedment is so small that the J-bolt becomes an outlier due to insufficient embedment for pullout (at $L=16 \mathrm{D}$ ). Guidance for resolving anchorage outliers is provided in Reference 78 .

$$
\begin{aligned}
R_{p} & =\text { Pullout capacity reduction factor for cast-in-place J-bolts } \\
& =1.0 \quad \text { for } L \geq L_{\min } \\
& =\frac{L+20 D}{62.5 D} \quad \text { for } 180^{\circ} \text { hook when } L_{\min }>L \geq 16 D \\
& =\frac{L+8 D}{62.5 D} \quad \text { for } 90^{\circ} \text { hook when } L_{\min }>L \geq 16 D \\
& =\text { Outlier } \quad \text { for } L<16 D \\
L & =\text { Length of J-Bolt embedment per Figure 6.3-2 (in.) } \\
L_{\min } & =\text { Minimum length of J-Bolt embedment from Table 6.3-3 } \\
D \quad & \text { Rod diameter (in.) }
\end{aligned}
$$

\subsubsection{Grouted-in-Place Bolts ${ }^{19}$}

For grouted-in-place bolts having embedments which are less than the minimum values given in Table 6.3-4, the capacity reduction factors given in Section 6.3.3.2 for cast-in-place bolts may be used to reduce the nominal pullout and shear capacities given in Table 6.3-4.

18 Based on Section C.4.2 of SQUG GIP (Ref. 1)

19 Based on Section C.5.2 of SQUG GIP (Ref. 1) 


\subsubsection{Spacing Between Anchors ${ }^{20}$}

The spacing from an anchor to each nearby anchor should be checked to confirm that it meets the minimum value so that nominal allowable anchor capacities can be used. A capacity reduction factor can be used when bolt-to-bolt spacing is less than the minimum specified value. Minimum spacings and reduction factors are given for each type of anchor covered in this procedure.

For expansion anchors, these spacing guidelines are based primarily on anchor capacity test results. The pullout capacity of cast-in-place anchors and headed studs is based on the shear cone theory. The minimum spacings are for distances between adjacent anchors in which the shear cones of the anchors overlap slightly, reducing the projected shear cone area for each anchor by about $13 \%$. These minimum spacings are for anchors with the minimum embedment. Greater spacings are necessary to develop the full pullout capacities of deeply embedded anchors if higher capacity values are used. About 10 bolt diameter spacing is required to gain full capacity in expansion and cast-in-place anchors.

The shear capacity of anchors is not affected as significantly as tension capacity by closely-spaced anchors. Recommended minimum spacings for shear loads are given along with the corresponding capacity reduction factors for closely-spaced anchors.

For clusters of closely-spaced anchors, a capacity reduction factor should be applied to an anchor for every other nearby anchor. For example, if there are three anchors in a line and all are closer than the minimum spacing, then the center anchor should have two reduction factors applied to its nominal capacity allowable and the outside anchors should have only one reduction factor applied.

The spacings between anchors can be checked in the field by a visual inspection and, if necessary, the spacings can be measured. Measurements should be made from anchor centerline to anchor centerline.

\subsubsection{Expansion Anchors ${ }^{21}$}

If the spacing (S) between an expansion anchor and another anchor is less than the minimum value $\left(S_{\min }\right)$ given in Table 6.3-1, then a pullout capacity reduction factor $\left(\mathrm{RS}_{\mathrm{p}}\right)$ and a shear capacity reduction factor $\left(\mathrm{RS}_{\mathrm{s}}\right)$ should be multiplied by the nominal pullout and shear capacities $\left(\mathrm{P}_{\text {nom }}\right.$, $\mathrm{V}_{\text {nom }}$ ) given in Table 6.3-1.

$$
\begin{aligned}
R_{p} & =\quad \begin{array}{l}
\text { Pullout capacity reduction factor for closely spaced } \\
\text { expansion anchors }
\end{array} \\
& =1.0 \quad \text { for } \mathrm{S} \geq 10 \mathrm{D} \\
& =\frac{\mathrm{S}}{10 \mathrm{D}} \quad \text { for } 10 \mathrm{D}>\mathrm{S} \geq 5 \mathrm{D} \\
& =0.5 \quad \text { for } 5 \mathrm{D}>\mathrm{S} \geq 2.5 \mathrm{D} \\
& =\text { Outlier } \quad \text { for } \mathrm{S}<2.5 \mathrm{D}
\end{aligned}
$$

S = Spacing between anchors measured center-to-center

20 Based on Section 4.4.1 - Check 7 of SQUG GIP (Ref. 1)

21 Based on Section C.2.5 of SQUG GIP (Ref. 1) 


$$
\begin{aligned}
\mathrm{D} & =\quad \text { Diameter of anchor bolt/stud } \\
\mathrm{RS}_{\mathrm{s}} & =\quad \begin{array}{l}
\text { Shear capacity reduction factor for closely spaced } \\
\text { expansion anchors }
\end{array} \\
& =1.0 \quad \text { for } \mathrm{S} \geq 2 \mathrm{D} \\
& =0.5 \quad \text { for } \mathrm{S}<2 \mathrm{D}
\end{aligned}
$$

A reduction factor should be applied for each nearby anchor, whether it is another expansion anchor or a different type of anchor. The spacings (S) given above are defined in terms of multiples of the anchor bolt/stud diameter (D), measured from anchor centerline to centerline.

\subsubsection{Cast-in-Place Bolts and Headed Studs 22}

If the spacing (S) between a cast-in-place anchor and another anchor is less than the minimum value $\left(S_{\min }\right)$ given in Table 6.3-2, then a pullout capacity reduction factor $\left(\mathrm{RS}_{\mathrm{p}}\right)$ and a shear capacity reduction factor $\left(\mathrm{RS}_{\mathrm{S}}\right)$ should be multiplied by the nominal pullout and shear capacities $\left(P_{\text {nom }}, V_{\text {nom }}\right)$ given in Table 6.3-2.

Note that a reduction factor should be applied for each nearby anchor, whether it is another cast-inplace anchor or a different type of anchor. For example, for 4 bolts in a line, the interior bolts would be subject to 2 reductions, while the exterior bolts would be subject to only one reduction.

Note that if there are 5 or more cast-in-place anchors in a cluster which are spaced closer together than the minimum $\left(S_{\min }\right)$ as defined in Table 6.3-2, then the pullout capacity reduction factor $\left(R_{\mathrm{p}}\right)$ cannot be used and the anchors in that cluster should instead be identified as outliers.

$$
\begin{aligned}
\mathrm{RS}_{\mathrm{p}} & =\begin{array}{l}
\text { Pullout capacity reduction factor for closely spaced cast-in- } \\
\text { place anchors }
\end{array} \\
& =1.0 \quad \text { for } \mathrm{S} \geq \mathrm{S}_{\min } \\
& =\mathrm{A}_{\mathrm{s}, \text { red }} \quad \text { for } \mathrm{S}<\mathrm{S}_{\min }
\end{aligned}
$$

22 Based on Section C.3.3 of SQUG GIP (Ref. 1) 
$\mathrm{A}_{\mathrm{s}, \mathrm{nom}}=\quad$ Nominal projected area of the nonoverlapping shear cone of a single bolt located at the minimum spacing distance $\left(S_{\text {min }}\right)$ from Table 6.3-8. The values of $A_{s, \text { nom }}$ given in Table 6.3-8 are about 13 percent less than the full, geometric shear cone projected area.

Table 6.3-8 Nonoverlapping Projected Shear Cone Areas for Bolts Meeting Minimum Spacing Requirements (Table C.3-2 of SQUG GIP, Ref. 1)

\begin{tabular}{|c|c|}
\hline $\begin{array}{c}\text { Bolt } \\
\text { Diameter }\end{array}$ & $\begin{array}{c}\text { Nonoverlapping } \\
\text { Shear Cone Area } \\
\left(\mathrm{A}_{\text {s,nom }}, \text { in. }^{2}\right)\end{array}$ \\
\hline $3 / 8$ & 41.9 \\
\hline $1 / 2$ & 74.1 \\
\hline $5 / 8$ & 116.0 \\
\hline $3 / 4$ & 167.4 \\
\hline $7 / 8$ & 227.2 \\
\hline 1 & 297.3 \\
\hline $1-1 / 8$ & 376.7 \\
\hline $1-1 / 4$ & 464.1 \\
\hline $1-3 / 8$ & 562.2 \\
\hline
\end{tabular}

$\mathrm{A}_{\mathrm{s}, \mathrm{red}}=\quad$ Reduced projected area of the nonoverlapping shear cone of a single bolt located less than the minimum spacing $\left(S_{\min }\right)$ from another bolt. The values of $A_{s, \text { red }}$ are calculated from the following equation:

$=\pi r^{2}-\frac{1}{2}\left[r^{2} \theta-r S \sin \left(\frac{\theta}{2}\right)\right]$

$r=\frac{2 L+D}{2}$

$\theta=2 \cos ^{-1}\left[\frac{S}{2 L+D}\right]$ 


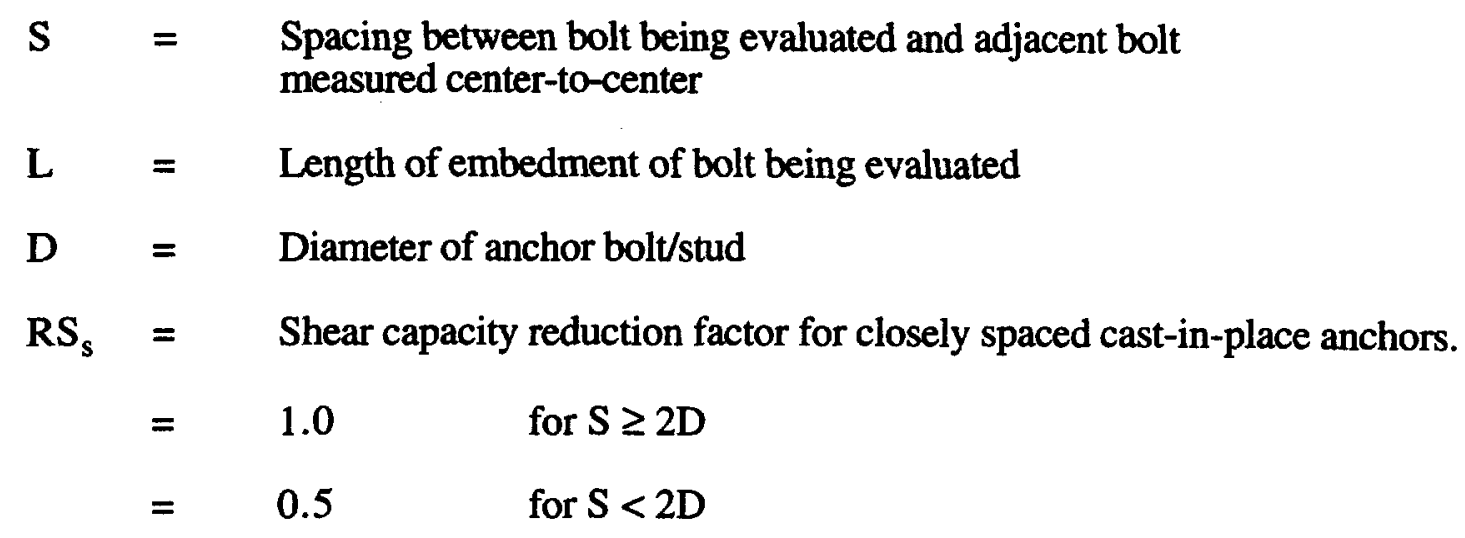

\subsubsection{Cast-in-Place J-Bolts ${ }^{23}$}

The nominal shear capacities $\left(\mathrm{V}_{\text {nom }}\right)$ for J-bolts given in Table 6.3-3 are based on a minimum spacing of 3D, where $\mathrm{D}$ is the diameter of the J-bolt.

For spacings less than $3 \mathrm{D}$, the $\mathrm{J}$-bolt is an outlier.

\subsubsection{Grouted-in-Place Bolts ${ }^{24}$}

For grouted-in-place bolts having, spacings which are less than the minimum values given in Table 6.3-4, the capacity reduction factors given in Section 6.3 .4 .2 for cast-in-place bolts may be used to reduce the nominal pullout and shear capacities given in Table 6.3-4. 


\subsubsection{Edge Distance 25}

The distance from an anchor to a free edge of concrete should be checked to confirm that it meets the minimum value so that the nominal allowable anchor capacities can be used. A capacity reduction factor can be used for an anchor which is closer to an edge than the minimum. Minimum edge distances and reduction factors are given for each type of anchor covered in this procedure.

For expansion anchors, these edge distance guidelines are based primarily on anchor capacity test results. Full pullout and shear capacity can be developed for cast-in-place anchors and headed studs which are no closer to a free edge than the radius of the projected shear cone. The minimum edge distances correspond to the shear cone just touching the free edge of concrete at the surface (no credit is taken for concrete reinforcement). These minimum edge distances apply to anchors with the minimum embedment. Greater edge distances are necessary to develop the full pullout capacities of deeply embedded anchors if higher capacities are used. About 10 bolt diameter edge distance is required to gain full capacity of expansion anchors.

When an anchor is near more than one free concrete edge, a capacity reduction factor should be applied for each nearby edge. For example, if an anchor is near a corner, then two reduction factors apply. The edge distances can be checked in the field by a visual inspection and, if necessary, the edge distances can be measured. Measurements should be made from anchor centerline to the free edge.

\subsubsection{Expansion Anchors ${ }^{26}$}

If the distance (E) from an expansion anchor to a free edge of concrete is less than the minimum value $\left(E_{\min }\right)$ given in Table 6.3-1, then a pullout capacity reduction factor $\left(\mathrm{RE}_{\mathrm{p}}\right)$ and a shear capacity reduction factor $\left(\mathrm{RE}_{\mathrm{s}}\right)$ should be multiplied by the nominal pullout and shear capacities $\left(P_{\text {nom }}, V_{\text {nom }}\right)$ given in Table 6.3-1.

$$
\begin{aligned}
\mathrm{RE}_{\mathrm{p}} & =\begin{array}{l}
\text { Pullout capacity reduction factor for near edge expansion } \\
\text { anchors }
\end{array} \\
& =1.0 \quad \text { for } \quad \mathrm{E} \geq 10 \mathrm{D} \\
& =\frac{\mathrm{E}}{10 \mathrm{D}} \quad \text { for } \quad 10 \mathrm{D}>\mathrm{E} \geq 4 \mathrm{D} \\
& =0.0 \text { (Outlier) } \quad \text { for } \quad \mathrm{E}<4 \mathrm{D} \\
\mathrm{E} & =\text { Edge distance from centerline of anchor to free edge } \\
D \quad & =\text { Diameter of anchor bolt/stud }
\end{aligned}
$$

25 Based on Section 4.4.1 - Check 8 of SQUG GIP (Ref. 1)

26 Based on Section C.2.6 of SQUG GIP (Ref. 1) 


$$
\begin{array}{rlrl}
R E_{\mathrm{s}} & =\begin{array}{l}
\text { Shear capacity reduction factor for near edge expansion } \\
\text { anchors }
\end{array} \\
& =1.0 \quad \text { for } \quad \mathrm{E} \geq 10 \mathrm{D} \\
& =\left[\frac{\mathrm{E}}{10 \mathrm{D}}\right]^{1.5} & \text { for } \quad 10 \mathrm{D}>\mathrm{E} \geq 4 \mathrm{D} \\
& =0.0 \text { (Outlier) } \quad \text { for } \mathrm{E}<4 \mathrm{D}
\end{array}
$$

A reduction factor should be applied for each nearby edge; e.g., if an anchor is near a corner, then two reduction factors apply. The edge distance (E) given in the tables above are in terms of multiples of the anchor bolt/stud diameter (D), measured from the anchor centerline to the edge.

\subsubsection{Cast-in-Place Bolts and Headed Studs ${ }^{27}$}

If the distance (E) from a cast-in-place bolt or a headed stud to a free edge of concrete is less than the minimum value $\left(\mathrm{E}_{\min }\right)$, given in Table 6.3-2, then a pullout capacity reduction factor $\left(\mathrm{RE}_{\mathrm{p}}\right)$ and a shear capacity reduction factor $\left(\mathrm{RE}_{\mathrm{s}}\right)$ should be multiplied by the nominal pullout and shear capacities $\left(\mathrm{P}_{\text {nom }}, \mathrm{V}_{\text {nom }}\right)$, given in Table 6.3-2. A reduction factor should be applied for each nearby edge; e.g., if an anchor is near a corner, then two reduction factors apply.

$$
\begin{aligned}
& \mathrm{RE}_{\mathrm{p}}=\quad \text { Pullout capacity reduction factor for near edge cast-in- } \\
& \text { place bolts and headed studs } \\
& =\quad 1.0 \text { for } E \geq E_{\min } \\
& =\quad \frac{A_{e, \text { red }}}{A_{e, \text { nom }}} \text { for } E_{\min }>E \geq 4 D \\
& =\quad 0.0 \text { (Outlier) for } E<4 D \\
& \mathrm{E}=\text { Edge distance from centerline of anchor to free edge } \\
& \mathrm{E}_{\min }=\quad \text { Minimum edge distance to develop full pullout } \\
& \text { capacity from Table 6.3-2 } \\
& \text { D }=\text { Diameter of anchor bolt/stud } \\
& A_{e, n o m}=\quad \text { Nominal projected shear cone area of a bolt which is } \\
& \text { located away from a free concrete edge at least the } \\
& \text { minimum edge distance }\left(\mathrm{E}_{\min }\right) \text { given in Table 6.3-2 } \\
& =\quad 0.96 \frac{\pi}{4}(2 L+D)^{2} \\
& \mathrm{~L}=\text { Length of embedment of bolt being evaluated }
\end{aligned}
$$

27 Based on Section C.3.4 of SQUG GIP (Ref. 1) 


$$
\begin{aligned}
A_{e, \text { red }} & =\quad \begin{array}{l}
\text { Reduced projected shear cone area of a bolt located at } \\
\text { less than the minimum edge distance from a concrete edge }
\end{array} \\
& =\quad \pi r^{2}-\frac{1}{2}\left[r^{2} \theta-2 r E \sin \left(\frac{\theta}{2}\right)\right] \\
\theta & =\quad 2 \cos ^{-1}\left[\frac{2 E}{2 L+D}\right] \\
r & =\frac{2 L+D}{2} \quad \text { Shear capacity reduction factor for near edge cast-in- } \\
R_{s} & =\quad 1.0 \quad \text { flace bolts and headed studs } \\
& =0.0131\left[\frac{E}{D}\right]^{2} \quad \text { for } 8.75 \mathrm{D}>\mathrm{E} \geq 4 \mathrm{D} \\
& =0.0(\text { Outlier }) \quad \text { for } E<4 \mathrm{D}
\end{aligned}
$$

\subsubsection{Cast-in-Place J-Bolts ${ }^{28}$}

The minimum edge distances given in Table 6.3-3 for J-bolts are the same as those for cast-inplace bolts and headed studs. Likewise the capacity reduction factors for J-bolts installed near an edge are also the same as discussed in Section 6.3.5.2 for cast-in-place bolts and headed studs.

For calculating reduction factors for near-edge J-bolts, the " $\mathrm{L}$ " dimension from Table 6.3-2 for cast-in-place bolts should be used.

\subsubsection{Grouted-in-Place Bolts ${ }^{29}$}

For grouted-in-place bolts having edge distances which are less than the minimum values given in Table 6.3-4, the capacity reduction factors given in Section 6.3.5.2 for cast-in-place bolts may be used to reduce the nominal pullout and shear capacities given in Table 6.3-4.

28 Based on Section C.4.4 of SQUG GIP (Ref. 1)

29 Based on Section C.5.2 of SQUG GIP (Ref. 1) 


\subsubsection{Concrete Strength and Condition ${ }^{30}$}

The concrete compressive strength $\left(f_{c}^{\prime}\right)$ should be obtained from design documentation or tests to confirm that it meets the minimum value so that the nominal allowable anchor capacities can be used. A capacity reduction factor can be used for concrete which has lower strength than the minimum. Minimum concrete strength and reduction factors are given for each type of anchor covered in this procedure.

In addition, the concrete in the vicinity of the anchor should be checked to be sure that it is free of gross defects which could affect the holding strength of the anchor. This check should be done in conjunction with Section 6.3.7. Surface defects such as hairline shrinkage cracks are not of concern.

Note that this procedure covers anchors installed only in poured, structural concrete. If any equipment is secured to other types of concrete or masonry structures, such as concrete block masonry walls, the anchorage for that item of equipment should be classified as an outlier and evaluated separately using guidance in Chapter 12 and Reference 78.

The compressive strength of the concrete can normally be obtained from facility construction drawings, specifications, or other documents. If this information is not available, core sample information can be used or new samples can be taken and tested.

Expansion anchors installed in masonry block walls have lower capacity than those in concrete and should be classified as outliers. Block wall adequacy (anchorage and reinforcement) should be checked as part of the outlier resolution.

\subsubsection{Expansion Anchors ${ }^{31}$}

If the concrete compressive strength $\left(f_{c}^{\prime}\right)$ is less than 4000 psi for pullout loads or 3500 psi for shear loads, then a pullout capacity reduction factor $\left(\mathrm{RF}_{\mathrm{p}}\right)$ and a shear capacity reduction factor $\left(R F_{s}\right)$ should be multiplied by the nominal pullout and shear capacities $\left(P_{\text {nom }}, V_{\text {nom }}\right)$, given in Table 6.3-1.

$$
\begin{array}{rlrl}
R_{p} & = & \begin{array}{l}
\text { Pullout capacity reduction factor for expansion anchors in } \\
\text { low strength concrete }
\end{array} \\
& =1.0 & \text { for } f_{c}^{\prime} \geq 4000 \mathrm{psi} \\
& =\frac{f_{c}^{\prime}}{4000} & \text { for } 4000 \mathrm{psi}>\mathrm{f}_{\mathrm{c}}^{\prime} \geq 2000 \mathrm{psi} \\
& = & \text { Outlier } & \text { for } \mathrm{f}_{\mathrm{c}}^{\prime}<2000 \mathrm{psi} \\
\mathrm{f}_{\mathrm{c}}^{\prime} & = & \text { Concrete compression strength (psi) }
\end{array}
$$

30 Based on Section 4.4.1 - Check 9 of SQUG GIP (Ref. 1)

31 Based on Section C.2.7 of SQUG GIP (Ref. 1) 


$$
\begin{aligned}
\mathrm{RF}_{\mathrm{s}} & =\quad \begin{array}{l}
\text { Shear capacity reduction factor for expansion anchors in } \\
\text { low strength concrete }
\end{array} \\
& =1.0 \quad \text { for } \mathrm{f}_{\mathrm{c}}^{\prime} \geq 3500 \mathrm{psi} \\
& =\frac{\mathrm{f}_{\mathrm{c}}^{\prime}}{10,000}+0.65 \text { for } 3500 \mathrm{psi}>\mathrm{f}_{\mathrm{c}}^{\prime} \geq 2000 \mathrm{psi} \\
& =\text { Outlier } \quad \text { for } \mathrm{f}_{\mathrm{c}}^{\prime}<2000 \mathrm{psi}
\end{aligned}
$$

\subsubsection{Cast-in-Place Bolts and Headed Studs ${ }^{32}$}

If the concrete compressive strength $\left(f_{c}^{\prime}\right)$ is less than $3500 \mathrm{psi}$, then a pullout capacity reduction factor $\left(R_{p}\right)$ and a shear capacity reduction factor $\left(R_{s}\right)$ should be multiplied by the nominal pullout and shear capacities $\left(\mathrm{P}_{\text {nom }}, \mathrm{V}_{\text {nom }}\right)$ given in Table 6.3-2.

$$
\begin{aligned}
R F_{p} & =R F_{s}=\quad \begin{array}{l}
\text { Pullout (p) and shear (s) capacity reduction factors } \\
\text { for cast-in-place bolts and headed studs in low } \\
\text { strength concrete }
\end{array} \\
& =1.0 \quad \text { for } \quad f_{c}^{\prime} \leq 3500 \mathrm{psi} \\
& =\sqrt{\frac{f_{c}^{\prime}}{3500}} \text { for } 3500 \mathrm{psi}>f_{c}^{\prime} \geq 2500 \mathrm{psi} \\
& =\text { Outlier for } \quad f_{c}^{\prime}<2500 \mathrm{psi} \\
f_{c}^{\prime} & =\text { Concrete compressive strength (psi) }
\end{aligned}
$$

32 Based on Section C.3.5 of SQUG GIP (Ref. 1) 


\subsubsection{Cast-in-Place J-Bolts ${ }^{33}$}

If the concrete compressive strength $\left(f_{c}^{\prime}\right)$ is less than 3500 psi, then a pullout capacity reduction factor $\left(\mathrm{RF}_{\mathrm{p}}\right)$ and a shear capacity reduction factor $\left(R F_{\mathrm{s}}\right)$ should be multiplied by the nominal pullout and shear capacities $\left(P_{n o m}, V_{\text {nom }}\right)$ given in Table 6.3-3.

$$
\begin{aligned}
\mathrm{RF}_{\mathrm{p}}=\mathrm{RF}_{\mathrm{s}} & =\begin{array}{l}
\text { Pullout }(\mathrm{p}) \text { and shear (s) capacity reduction factors } \\
\text { for J-bolts in low strength concrete }
\end{array} \\
& =1.0 \text { for } \mathrm{f}_{\mathrm{c}}^{\prime} \geq 3500 \mathrm{psi} \\
& =\sqrt{\frac{\mathrm{f}_{\mathrm{c}}^{\prime}}{3500}} \text { for } 2500 \mathrm{psi} \leq \mathrm{f}_{\mathrm{c}}^{\prime}<3500 \mathrm{psi} \\
& =\text { Outlier for } \mathrm{f}_{\mathrm{c}}^{\prime}<2500 \mathrm{psi} \\
\mathrm{f}_{\mathrm{c}}^{\prime}= & \text { Concrete compressive strength }(\mathrm{psi})
\end{aligned}
$$

\subsubsection{Grouted-in-Place Bolts ${ }^{34}$}

When grouted-in-place bolts are installed in concrete which has a compressive strength of $f_{c}^{\prime} \leq 3500$ psi, the capacity reduction factors given in Section 6.3.6.2 for cast-in-place bolts may be used to reduce the nominal pullout and shear capacities given in Table 6.3-4. 


\subsubsection{Concrete Crack Locations and Sizes ${ }^{35}$}

The concrete should be checked to confirm that it is free of significant structural cracks in the vicinity of the installed anchors so that the nominal pullout capacities can be used. A pullout capacity reduction factor can be used for concrete which has cracks which are larger than the acceptable maximum widths and are located in the vicinity of the anchor. Maximum acceptable crack sizes and capacity reduction factors are given for each type of anchor covered in this procedure.

Significant structural cracks in concrete are those which appear at the concrete surface and pass through the concrete shear cone of an anchor installation or the location of the expansion wedge. Concrete with surface (craze) cracks or shrinkage cracks which only affect the surface of the concrete should be considered uncracked.

The check for cracks in the concrete can be done by a visual inspection of the anchorage installation. It may be necessary to exercise judgment to establish whether cracks in the vicinity of an anchor actually pass through the installation. It is sufficient to estimate the width of cracks without making detailed measurements. This check should be done in conjunction with Section 6.3.6 to find other gross defects which could affect the holding strength of an anchor.

\subsubsection{Expansion Anchors ${ }^{36}$}

If there are significant structural cracks in the concrete where expansion anchors are installed, then a pullout capacity reduction factor $\left(\mathrm{RC}_{\mathrm{p}}\right)$ should be multiplied by the nominal pullout capacity $\left(\mathrm{P}_{\text {nom }}\right)$, given in Table 6.3-1. The shear capacity of expansion anchors is not significantly affected by cracks in the concrete.

$$
\begin{aligned}
\mathrm{RC}_{\mathrm{p}} & =\quad \begin{array}{l}
\text { Pullout capacity reduction factor for expansion anchors in } \\
\text { cracked concrete }
\end{array} \\
& =\quad \text { See Table } 6.3-9 \text { for values }
\end{aligned}
$$

The pullout capacity reduction factor applies only to significant structural cracks which penetrate the concrete mass and pass through the vicinity of the anchor installation. Concrete with surface (craze) cracks or shrinkage cracks which only affect the surface of the concrete should be considered uncracked. It may be necessary to exercise judgment to establish whether cracks in the vicinity of an anchor actually pass through the installation. Inspections for crack width should be visual (i.e., detailed measurement of crack widths is not necessary).

35 Based on Section 4.4.1 - Check 10 of SQUG GIP (Ref. 1)

36 Based on Section C.2.8 of SQUG GIP (Ref. 1) 
Table 6.3-9 Pullout Capacity Reduction Factors for Expansion Anchors in Cracked Concrete (Table C.2-8 of SQUG GIP, Ref. 1)

\begin{tabular}{|l|c|}
\hline \multicolumn{1}{|c|}{ Conditions } & $\begin{array}{c}\text { Reduction Factor for } \\
\text { Pullout Capacity } \\
\left(\mathrm{RC}_{\mathrm{p}}\right)\end{array}$ \\
\hline No Cracks & 1.0 \\
\hline $\begin{array}{l}\text { Crack Size }<0.01 \text { in. and the number of } \\
\text { required anchors securing the equipment } \\
\text { which are affected by these cracks is: } \\
\leq 50 \%\end{array}$ \\
$>50 \%$ & 1.0 \\
\hline 0.01 in. $\leq$ Crack Size $\leq 0.02$ in & $0.75^{*}$ \\
\hline Crack Size $>0.02$ in. & $0.75^{*}$ \\
\hline
\end{tabular}

* Capacity reduction factor applies to all required anchors securing the item of equipment, not just the anchors which are affected by the cracks.

\subsubsection{Cast-in-Place Bolts and Headed Studs ${ }^{37}$}

If there are significant structural cracks in the concrete where the cast-in-place bolts and headed studs are installed, then a pullout capacity reduction factor $\left(\mathrm{RC}_{\mathrm{p}}\right)$ should be multiplied by the nominal pullout capacity $\left(\mathrm{P}_{\text {nom }}\right)$ given in Table 6.3-2. The shear capacity of the cast-in-place bolts and headed stud anchors is not significantly affected by cracks in the concrete.

The pullout capacity reduction factor applies only to significant structural cracks which penetrate the concrete mass and pass through the vicinity of the anchor installation. Concrete with surface (craze) cracks or shrinkage cracks which only affect the surface of the concrete should be considered uncracked. It may be necessary to exercise judgment to establish whether cracks in the vicinity of an anchor actually pass through the installation. Inspections for crack width should be visual (i.e., detailed measurement of crack widths is not necessary).

$$
\begin{aligned}
\mathrm{RC}_{\mathrm{p}} & =\begin{array}{l}
\text { Pullout capacity reduction factor for cast-in-place anchors } \\
\text { in cracked concrete }
\end{array} \\
& =1.0 \quad \text { for no cracks and for } \mathrm{CS}<0.01 \mathrm{in} . \\
& =1.08-8 \mathrm{CS} \quad \text { for } 0.01 \mathrm{in} \leq \mathrm{CS} \leq 0.06 \mathrm{in} . \\
& =\text { Outlier } \quad \text { for } \mathrm{CS}>0.06 \mathrm{in} . \\
\mathrm{CS} & =\text { Crack size (approximate size based on visual observation) }
\end{aligned}
$$

37 Based on Section C.3.6 of SQUG GIP (Ref. 1) 


\subsubsection{Cast-in-Place J-Bolts ${ }^{38}$}

The areas adjacent to J-bolt installations should be inspected for significant structural cracks which penetrate the concrete mass. Concrete with surface (craze) cracks or shrinkage cracks which only affect the surface of the concrete should be considered uncracked. Inspections for crack width should be visual (i.e., detailed measurement of crack widths is not necessary). J-bolts should be classified as outliers when either of the following two crack sizes are exceeded:

- When cracks are larger than about 0.02 inch wide and traverse through the J-bolt installation, or

- When cracks are larger than about 0.05 inches wide and exist near the J-bolt installation.

\subsubsection{Grouted-in-Place Bolts ${ }^{39}$}

If there are significant structural cracks in the concrete where the grouted-in-place bolts are installed, then the pullout capacity reduction factors given in Section 6.3.7.2 for cast-in-place bolts may be used to reduce the nominal pullout capacities given in Table 6.3-4.

38 Based on Section C.4.6 of SQUG GIP (Ref. 1)

39 Based on Section C.5.3 of SQUG GIP (Ref. 1) 


\subsubsection{Essential Relays in Cabinets ${ }^{40}$}

Electrical cabinets and other equipment which are secured with expansion anchors should be checked to determine whether they house essential relays. If essential relays are present, a capacity reduction factor of 0.75 should be used for cabinets which are secured with expansion anchors. The check for the presence of essential relays in equipment can be done in conjunction with the Relay Functionality Review described in Chapter 11.

The basis for this capacity reduction factor is that expansion anchors have a tendency to loosen slightly when they are heavily loaded (i.e., they pull out of the concrete slightly). This effect does not significantly reduce the ultimate load carrying capability of expansion anchors; however, the slight gap between the base of the equipment and the surface of the concrete can open during the first part of an earthquake load cycle and then slam closed during the second part of the cycle. This creates high frequency impact loadings on the equipment, and the relays mounted therein could chatter. Use of a capacity reduction factor for the expansion anchors which secure this type of equipment lowers the maximum load which the anchor will experience; therefore this minimizes the amount of loosening and hence the potential for introducing high frequency impact loadings into the equipment.

If there are essential relays mounted in the item of equipment, then the following pullout capacity reduction factor $\left(R R_{p}\right)$ and shear capacity reduction factor $\left(R R_{s}\right)$ should be multiplied by the nominal pullout and shear capacities $\left(P_{\text {nom }}, V_{\text {nom }}\right)$ given in Table 6.3-1.

$$
\begin{aligned}
\mathbf{R R}_{\mathrm{p}}= & \begin{array}{l}
\text { Pullout capacity reduction factor for expansion anchors } \\
\text { securing equipment in which essential relays are mounted }
\end{array} \\
& =0.75 \\
\mathbf{R R}_{\mathrm{s}} & =\quad \begin{array}{l}
\text { Shear capacity reduction factor for expansion anchors } \\
\text { securing equipment in which essential relays are mounted }
\end{array} \\
& =0.75
\end{aligned}
$$

The Relay Functionality Review described in Chapter 11 identifies which cabinets and items of equipment contain essential relays.

40 Based on Sections 4.4.1 - Check 11 and C.2.9 of SQUG GIP (Ref. 1) 


\subsubsection{Tightness Check and Reduced Inspection Procedure for Expansion Anchors}

\subsubsection{Tightness Check for Expansion Anchors ${ }^{41}$}

The tightness check for expansion anchors can be accomplished by applying a torque to the anchor by hand until the anchor is "wrench tight," i.e., tightened without excessive exertion. If the anchor bolt or nut rotates less than about $1 / 4$ turn, then the anchor is considered tight. This tightness check is not intended to be a proof test of the capacity of the anchorage. This check is merely meant to provide a reasonable assurance that the expansion anchor is not loose in the hole due to gross installation defects. Loose nuts may indicate inadequate anchor set.

It is not the intent of this procedure to require disassembly of cabinets and structures or removal of electrical cabling and conduit to provide access to the expansion anchors for this tightness check. Therefore, in those cases where expansion anchors are inaccessible, either during facility operation or during shutdown, the SCEs should make a judgment as to whether the number and distribution of tightness checks which have already been made in the facility is sufficient, considering both the problem of inaccessibility and the results of the other tightness checks. One concern with not checking the tightness of inaccessible expansion anchors is that these types of anchors may not have been properly installed because access to them was limited during installation; therefore, the reason for inaccessibility should be considered when deciding not to check the tightness of expansion anchors.

For facilities which have a large number of similar expansion anchors installed, a sampling program may be used for the tightness check based on achieving 95\% confidence that no more than $5 \%$ of the expansion anchors fail the tightness test. Guidelines for conducting a sampling program are provided below.

It is not necessary to perform a tightness check of an expansion anchor if the anchorage for that piece of equipment is robust; i.e., there is a large margin between the applied load and the anchorage capacity. Guidelines for evaluating whether there is sufficient margin in the anchorage are provided below.

It is not necessary to perform a tightness check of expansion anchors which are loaded in tension due to dead weight, since the adequacy of the anchor set is effectively proof-tested by the dead weight loading. Judgment should be exercised to assess the need for tightness checks when multiple expansion anchors are used to secure a base plate loaded in tension by dead loads.

SCES should be aware that a tightness check alone for shell-type expansion anchors may not be sufficient to detect gross installation defects of expansion anchors. If the top of the shell is in contact with the equipment base, then the tightness check may simply be tightening the shell against the bottom of the equipment base as shown in Figure 6.3-6. SCEs should exercise engineering judgment and spot check for this type of installation defect by removing a few bolts from shell-type anchors and inspecting them to ensure that the shell anchor and the equipment base are not in contact. If this spot check indicates that these types of bolts may not be properly installed, then the inspection check should be expanded accordingly. Embedment length is determined from the point on the anchor to the surface of the structural concrete. Grout pads should not be included in the embedment length.

41 Based on Sections 4.4.1 - Check 4 and C.2.3 of SQUG GIP (Ref. 1) 
The tightness check can be performed by using a standard size box or open-end wrench on the bolt head or nut and applying a torque by hand until the bolt or nut is "wrench tight"; i.e., tightened without excessive exertion. For those cases where specific torque values must be used (e.g., for maintenance work orders), the "Tightness Check Torque" values given in Table 6.3-10, below, can be used for this expansion anchor tightness check. These values correspond to about $20 \%$ of the normal installation torques.

\section{Table 6.3-10 Recommended Torque Values for Expansion Anchor Tightness Check (Table C.2-3 of SQUG GIP, Ref. 1)}

\begin{tabular}{|c|c|c|}
\hline $\begin{array}{c}\text { Anchor } \\
\text { Diameter } \\
\text { (in.) }\end{array}$ & $\begin{array}{c}\text { Installation } \\
\text { Torque } \\
\text { (ft-lbs) }\end{array}$ & $\begin{array}{c}\text { Tightness Check } \\
\text { Torque } \\
\text { (ft-lbs) }\end{array}$ \\
\hline $3 / 8$ & $25-35$ & $5-7$ \\
\hline $1 / 2$ & $45-65$ & $9-13$ \\
\hline $5 / 8$ & $80-90$ & $16-18$ \\
\hline $3 / 4$ & $125-175$ & $25-35$ \\
\hline $7 / 8$ & $200-250$ & $40-50$ \\
\hline 1 & $250-300$ & $50-60$ \\
\hline $1-1 / 4^{1}$ & $400-500^{1}$ & $80-100^{1}$ \\
\hline
\end{tabular}

${ }^{1}$ Data from Table C-39 of WSRC SEP-6 (Ref. 3)

A well-installed expansion anchor should not rotate under this applied torque. A small amount of initial rotation (about 1/4 turn) is acceptable provided the nut or bolt will tighten and resist the applied torque. If a bolt turns more than about $1 / 4$ turn, but does eventually resist the torque, it should be re-torqued to the manufacturer's recommended installation torque and then considered acceptable.

A sampling program can be used to check the tightness of expansion anchors provided it achieves 95\% confidence that no more than $5 \%$ of the expansion anchors fail to meet the tightness guidelines given above. This $95 / 5$ criterion can be met using the guidelines given below for sample size, homogeneous population, allowable number of nonconforming anchors, and use of initial tightness test results. 
- $\quad$ Sample Size. The number of expansion anchors selected for tightness checking should be at least as large as given in Table 6.3-11 below for "Sample Size".

\section{Table 6.3-11 Sample Size for Expansion Anchor Tightness Check (Table C.2-4 of SQUG GIP, Ref. 1)}

\begin{tabular}{|l|c|}
\hline \multicolumn{1}{|c|}{ Condition } & $\begin{array}{c}\text { Sample } \\
\text { Size }\end{array}$ \\
\hline $\begin{array}{c}\text { Expansion Anchors Securing Equipment Which } \\
\text { Contains Essential Relays }\end{array}$ & $100 \%$ \\
\hline $\begin{array}{l}\text { Total Size of Homogeneous Anchor Population Is } \\
\text { Less Than 40 Anchors }\end{array}$ & $100 \%$ \\
\hline $\begin{array}{l}\text { Total Size of Homogeneous Anchor Population Is } \\
\text { Between 40 and 160 Anchors }\end{array}$ & 40 Anchors \\
\hline $\begin{array}{l}\text { Total Size of Homogeneous Anchor Population Is } \\
\text { More Than 160 Anchors }\end{array}$ & $20 \%$ \\
\hline
\end{tabular}

1Note: The sample sizes provided in this table are for accessible bolts.

- Homogeneous Population. The sample size is based on the total population of expansion anchors being homogeneous. Factors such as installation specifications, quality assurance procedures used in the installation specifications, quality assurance procedures used during installation, bolt manufacturer, installation contractor, etc., should be considered when judging whether or not the total population is homogeneous. If there is more than one homogeneous set of expansion anchors, then the sample size limitations given above and the allowable number of nonconforming anchors given below apply to each individual population.

- Allowable Number of Nonconforming Anchors. The criterion of $95 \%$ confidence that there are no more than $5 \%$ nonconforming anchors can be met if the number of expansion anchors which fails the tightness check does not exceed the limitations given in Table 6.312 below. If more than these number of anchors fail the tightness check, then the sample size should be increased until the failure rate does not exceed the limitations in this table.

- Use of Initial Tightness Test Results. The results of the initial torque tightness check on each expansion anchor should be used to establish the failure rate for the purposes of the sampling program. For example, if out of a total population of 400 expansion anchors 100 were tightness checked and 4 of these failed the initial check, then the sample size should be expanded. (Table 6.3-12 only allows 3 anchors to fail for 100 tests on a population of 400.) The sample size should be expanded even if all 4 of the failed anchors were able to be fully tightened up to their installation torque requirements. 
Table 6.3-12 Allowable Number of Expansion Anchors Which Need Not Pass Tightness Check (Table C.2-5 of SQUG GIP, Ref. 1)

\begin{tabular}{|c|c|c|c|c|c|c|c|c|c|c|c|c|}
\hline $\begin{array}{c}\text { Total } \\
\text { Population }\end{array}$ & \multicolumn{10}{|c|}{ Number of Anchors Which Need Not Pass Tightness Check } \\
& \multicolumn{10}{|c|}{ for Test Sample Size, (n): } \\
\hline Size N & 40 & 60 & 80 & 100 & 150 & 200 & 250 & 300 & 350 & 400 & 450 & 500 \\
\hline 100 & 1 & 2 & 3 & 5 & -- & -- & - & - & -- & -- & -- & - \\
\hline 200 & N/A & 1 & 2 & 3 & 6 & 10 & -- & - & - & -- & -- & - \\
\hline 300 & N/A & N/A & 2 & 3 & 5 & 7 & 10 & 15 & -- & -- & -- & - \\
\hline 400 & N/A & N/A & N/A & 3 & 5 & 7 & 9 & 12 & 15 & 20 & -- & - \\
\hline 500 & N/A & N/A & N/A & N/A & 5 & 7 & 9 & 12 & 14 & 17 & 20 & 25 \\
\hline 600 & N/A & N/A & N/A & N/A & 5 & 7 & 9 & 11 & 14 & 16 & 19 & 22 \\
\hline 700 & N/A & N/A & N/A & N/A & N/A & 7 & 9 & 11 & 13 & 16 & 18 & 21 \\
\hline 800 & N/A & N/A & N/A & N/A & N/A & 6 & 9 & 11 & 13 & 16 & 18 & 21 \\
\hline 900 & N/A & N/A & N/A & N/A & N/A & N/A & 8 & 11 & 13 & 15 & 18 & 20 \\
\hline 1000 & N/A & N/A & N/A & N/A & N/A & N/A & 8 & 11 & 13 & 15 & 17 & 20 \\
\hline
\end{tabular}

If certain expansion anchors are not accessible due to such things as high radiation, concrete poured over the anchorage, equipment disassembly or removal being required, etc., then other methods may be used to assess the tightness of the expansion anchors.

- $\quad$ Use the Reduced Inspection Alternative (Section 6.3.9.2) to evaluate the anchorage adequacy (the reduced inspection does not require a tightness check).

- Delay the tightness checks until radiation hazards are less.

- Use engineering judgment to assess the anchorage adequacy based on other considerations, e.g., tightness checks on similar anchors elsewhere in the facility which show that installation practices produced consistently tight installation. This method should be used as a last resort. The basis for the engineering judgment should be documented.

\subsubsection{Reduced Inspection Procedure for Expansion Anchors ${ }^{42}$}

A reduced level of inspection can be performed for expansion anchors if additional conservatism is included in the anchorage evaluation. The two inspections which can be deleted for this reduced inspection are:

- Tightness Check (Section 6.3.9.1)

- Embedment Check (Section 6.3.3)

42 Based on Section C.2.10 of SQUG GIP (Ref. 1) 
However to use this Reduced Inspection Alternative, the following conditions should be met:

Capacity Reduction Factor Applied. If the Reduced Inspection Alternative is used, then a pullout capacity reduction factor $\left(\mathrm{RI}_{\mathrm{p}}\right)$ and shear capacity reduction factor $\left(\mathrm{RI}_{\mathrm{s}}\right)$ should be multiplied by the nominal pullout and shear capacities $\left(\mathrm{P}_{\text {nom }}, \mathrm{V}_{\text {nom }}\right)$ given in Table 6.3-1.

$$
\begin{aligned}
\mathrm{RI}_{\mathrm{p}} & =\begin{array}{l}
\text { Pullout capacity reduction factor for use with Reduced } \\
\text { Inspection Alternative }
\end{array} \\
& =0.75 \\
\mathrm{RI}_{\mathrm{s}} & =\quad \begin{array}{l}
\text { Shear capacity reduction factor for use with Reduced } \\
\text { Inspection Alternative }
\end{array} \\
& =0.75
\end{aligned}
$$

- Other Effects Do Not Reduce Anchor Capacity. None of the other effects which could lower the capacity of the anchor are present. The following anchorage inspection checks, should show that the anchors have full capacity. The checks and the full capacity values are listed:

Gap Size: $\quad$ None $\quad$ (Section 6.2.3)

Spacing: $\quad S \geq 10 D \quad$ (Section 6.3.4.1)

Edge Distance: $\quad \mathrm{E} \geq 10 \mathrm{D} \quad$ (Section 6.3.5.1)

Concrete Strength:

$$
\begin{array}{ll}
\text { For Pullout: } & f_{c}^{\prime} \geq 4000 \mathrm{psi} \quad \text { (Section 6.3.6.1) } \\
\text { For Shear: } & f_{c}^{\prime} \geq 3500 \mathrm{psi} \quad \text { (Section 6.3.6.1) }
\end{array}
$$

Concrete Cracks: $\quad$ None (Section 6.3.7.1)

Essential Relays: $\quad$ None (Section 6.3.8)

- $\quad$ One Third of Anchors Not Available. The applied seismic and dead loads should be less than the allowable anchor pullout and shear capacities given above when a third of the anchors securing the item of equipment are assumed to be unavailable for carrying loads, i.e., $50 \%$ more bolts are used to secure the item of equipment than necessary to meet the allowable loads. There should be at least six anchors securing the equipment with four assumed to be carrying the load and two not. 


\subsubsection{Other Anchor Types}

\subsubsection{Welds to Embedded Steel or Exposed Steel ${ }^{43}$}

Equipment at DOE facilities are often anchored by welds to steel plates or channels which are embedded in concrete (see Figure 6.3-1). The strength of such an anchorage depends on the weld of the equipment to the steel and the shear and pullout resistance of the headed stud that anchors the steel into the concrete. The following topics are covered in this section:

- $\quad$ Allowable Loads for Typical Welds (Section 6.3.10.1.1)

- $\quad$ Summary of Equivalent Weld Sizes (Section 6.3.10.1.2)

- Weld Check (Section 6.3.10.1.3)

- $\quad$ Embedded or Exposed Steel Check (Section 6.3.10.1.4)

The specific checks described in this section should be performed in conjunction with the generic anchorage installation inspection checks described in the rest of Section 6.2.

\subsection{Allowable Loads for Typical Welds ${ }^{44}$}

The allowable loads for typical welds made with E60 electrodes are listed in Table 6.3-13. These allowable loads are based on a weld stress allowable of $30,600 \mathrm{psi}$.

Table 6.3-13 Allowable Capacities for Typical Welds (E60 Electrodes)

(Table C.6-1 of SQUG GIP, Ref. 1)

\begin{tabular}{|c|c|c|c|}
\hline \multicolumn{2}{|c|}{ Weld Sizes } & \multirow{2}{*}{$\begin{array}{c}\text { Throat Area } \\
(\mathrm{A}=.707 \mathrm{t} \mathrm{L}) \\
\text { (in. }^{2} \text { ) }\end{array}$} & \multirow{2}{*}{$\begin{array}{c}\text { Allowable } \\
\text { F }_{\mathbf{w}} \\
\text { (kips) }\end{array}$} \\
\hline $\begin{array}{c}\mathrm{t} \\
\text { (in.) } \\
\end{array}$ & $\begin{array}{c}\mathrm{L} \\
\text { (in.) }\end{array}$ & & \\
\hline $1 / 8$ & $1 / 2$ & 0.0442 & 1.35 \\
\hline $1 / 8$ & $3 / 4$ & 0.0663 & 2.03 \\
\hline $1 / 8$ & 1 & 0.0884 & 2.70 \\
\hline $3 / 16$ & $1 / 4$ & 0.0331 & 1.01 \\
\hline $3 / 16$ & $1 / 2$ & 0.0663 & 2.03 \\
\hline $3 / 16$ & $3 / 4$ & 0.0994 & 3.04 \\
\hline $3 / 16$ & 1 & 0.1326 & 4.06 \\
\hline $1 / 4$ & $1 / 4$ & 0.0442 & 1.35 \\
\hline $1 / 4$ & $1 / 2$ & 0.0884 & 2.70 \\
\hline $1 / 4$ & $3 / 4$ & 0.1326 & 4.06 \\
\hline $1 / 4$ & 1 & 0.1768 & 5.41 \\
\hline
\end{tabular}

43 Based on Section C.6 of SQUG GIP (Ref. 1)

44 Based on Section C.6.1 of SQUG GIP (Ref. 1) 
Where: $\quad t \quad=\quad$ Thickness of the weld leg

$\mathrm{L}=$ Length of the weld

A $=$ Cross-sectional area through the throat of the weld

$\begin{array}{ll} & =0.707 \mathrm{t} \mathrm{L} \\ \mathrm{F}_{\mathrm{w}} & =\quad \text { Allowable load capacity of weld }\end{array}$

\subsection{Summary of Equivalent Weld Sizes ${ }^{45}$}

A summary of equivalent weld sizes which have the same capacity as other types of fasteners is shown in Table 6.3-14.

Table 6.3-14 Summary of Equivalent Weld Sizes (Table C.6-2 of SQUG GIP, Ref. 1)

\begin{tabular}{|c|c|c|c|}
\hline \multicolumn{2}{|c|}{ Welds } & \multicolumn{2}{c|}{ Equivalent Bolt Diameter (D, in.) } \\
\hline $\begin{array}{c}\text { Typical Size } \\
\text { (L x t, in.) }\end{array}$ & $\begin{array}{c}\text { Throat Area } \\
\left(\text { in. }{ }^{2}\right)\end{array}$ & $\begin{array}{c}\text { Expansion } \\
\text { Anchor Bolts }\end{array}$ & $\begin{array}{c}\text { Cast-in-Place } \\
\text { Anchor Bolts }\end{array}$ \\
\hline $1 / 2 \times 1 / 8$ & 0.0442 & $3 / 8$ & - \\
\hline $1 \times 1 / 8$ & 0.0884 & $1 / 2$ & -- \\
\hline $1 \times 3 / 16$ & 0.1326 & $3 / 4$ & $3 / 8$ \\
\hline $1 \times 1 / 4$ & 0.1768 & $3 / 4$ & $3 / 8$ \\
\hline $2 \times 3 / 16$ & 0.2651 & $7 / 8$ & $1 / 2$ \\
\hline $2 \times 1 / 4$ & 0.3535 & 1 & $5 / 8$ \\
\hline $2 \times 3 / 8$ & 0.5305 & -- & $3 / 4$ \\
\hline
\end{tabular}

45 Based on Section C.6.2 of SQUG GIP (Ref. 1) 


\subsection{Weld Check ${ }^{46}$}

The welds used for anchoring equipment to embedded or exposed steel should be inspected in the following areas:

- $\quad$ Determine the overall length $(L)$ and thickness $(t)$ of the welds. The weld thickness should be limited to the thinnest part of either the weld itself or the connecting part.

- Check for weld burn-through on cabinets made of thin material.

- Check for weld quality, particularly in puddle welds which carry high tension loads.

- The minimum effective length of fillet welds should not be less than 4 times the nominal size of the weld, or else the size of the weld should be considered not to exceed $1 / 4$ of its effective length.

\subsection{Embedded or Exposed Steel Check ${ }^{47}$}

The embedded steel or the exposed steel to which the equipment is anchored by the weld should be evaluated to determine whether it has the capacity to carry the loads applied to it.

The allowable stresses from Part 2 of the AISC code (Ref. 81) may be used for evaluating the adequacy of exposed steel and the structural members of an embedded steel assembly. The guidelines given in Section 6.3 can be used for evaluating the cast-in-place bolts and headed studs which are a part of the embedded steel assembly.

46 Based on Section C.6.3 of SQUG GIP (Ref. 1)

47 Based on Section C.6.5 of SQUG GIP (Ref. 1) 


\subsubsection{Lead Cinch Anchors}

This section is adapted from Section 4.3 of Part III of SEP-6, Revision 1, "The Procedure for the Seismic Evaluation of SRS Systems using Experience Data" (Ref. 3), which was developed for the Savannah River Site (SRS).

Nominal allowable capacities for lead anchors are given in Table 6.3-15. These values are derived from SRS in-situ test data (Ref. 3) with a factor of safety of at least 4. The derivation of allowables for lead anchors is consistent with the anchorage methodology of the DOE Seismic Evaluation Procedure.

Table 6.3-15 Allowable Loads for Inspected Lead Anchors (Table C-1 of Ref. 3)

\begin{tabular}{|c|c|c|}
\hline $\begin{array}{c}\text { Bolt Diameter } \\
\text { (in.) }\end{array}$ & $\begin{array}{c}\text { Allowable } \\
\text { Tension (lbs.) }\end{array}$ & $\begin{array}{c}\text { Allowable Shear } \\
\text { (lbs.) }\end{array}$ \\
\hline $3 / 8$ & 600 & 400 \\
\hline $1 / 2$ & 870 & 800 \\
\hline $5 / 8$ & 970 & 1,400 \\
\hline $3 / 4$ & 1,280 & 2,000 \\
\hline 1 & 3,160 & 3,500 \\
\hline
\end{tabular}

The above allowables are to be used for all lead anchors that have been successfully inspected. Higher tension allowables may be used if the bolt can be torqued to induce the desired tension load. Figures 6.3-7 to 6.3-10 give the 95\% lower confidence bound torque tension correlation needed to evaluate the proof torque. Note that these curves cannot be extrapolated to give higher allowables. Following the additional torque check, the gap must be re-evaluated between the top of the shell and the bottom of the equipment base.

These allowables are applicable if the minimum criteria for bolt to bolt spacing (10D) and bolt to edge distance (10D) are satisfied, and installation adequacy is assured. When the edge distance and bolt-to-bolt requirements are not met, the allowables can be reduced as for expansion anchors (see Sections 6.3.4.1, 6.3.5.1, 6.3.6.1, 6.3.7.1, and 6.3.8). 


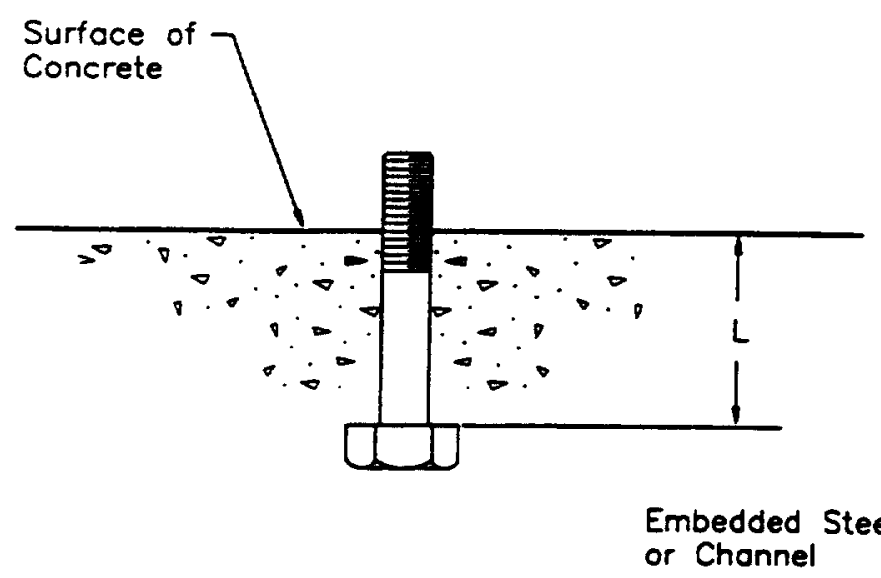

A. Cast-in-Ploce Bott

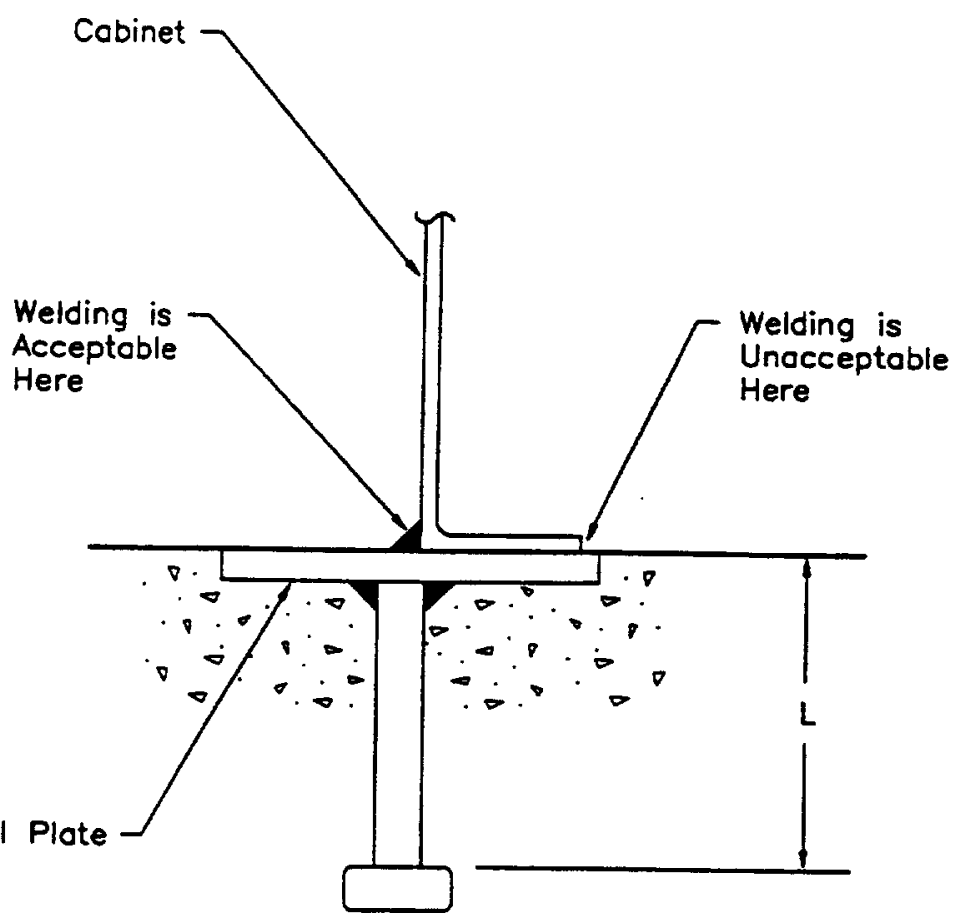

B. Heoded Stud

L = Embedment Length

Figure 6.3-1 Typical Installations of Cast-In-Place Bolt and Headed Stud (Figure C.3-1 of SQUG GIP, Reference 1) 


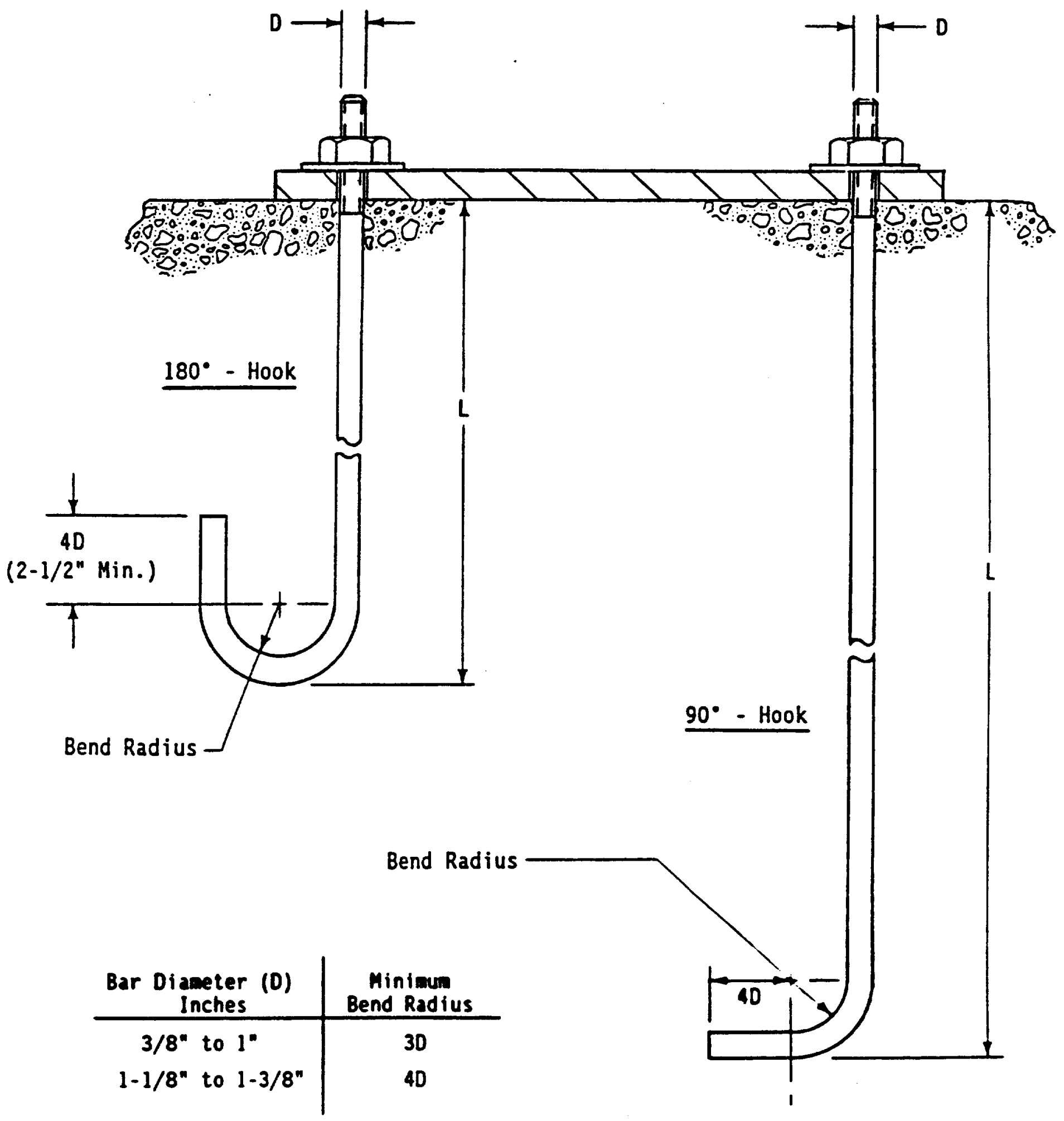

Figure 6.3-2 Typical J-Bolt Installations (Reference 41) (Figure C.4-1 of SQUG GIP, Reference 1) 


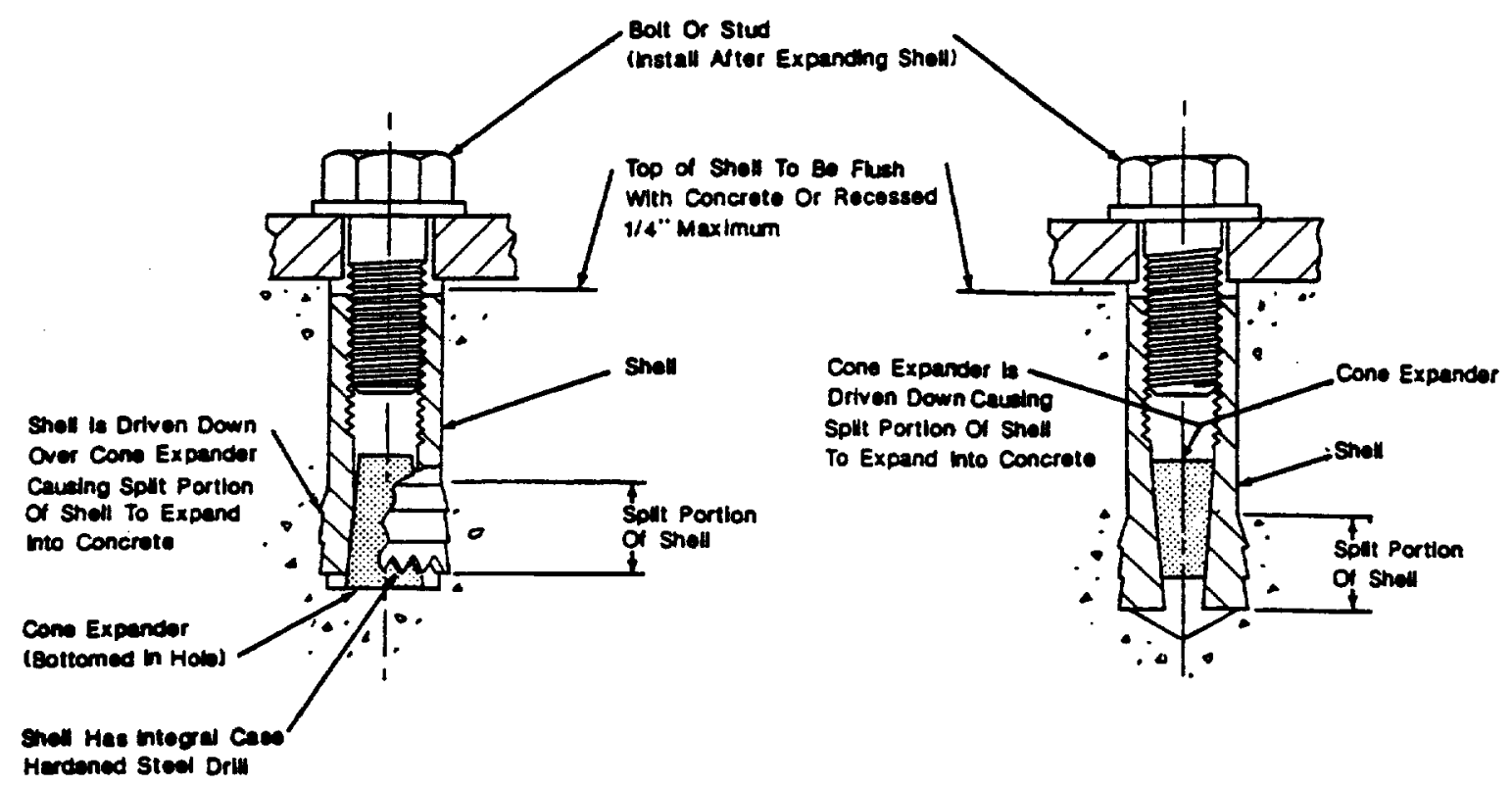

-. SELF-DRILLINO TYPE

b. DROP-IN TYPE

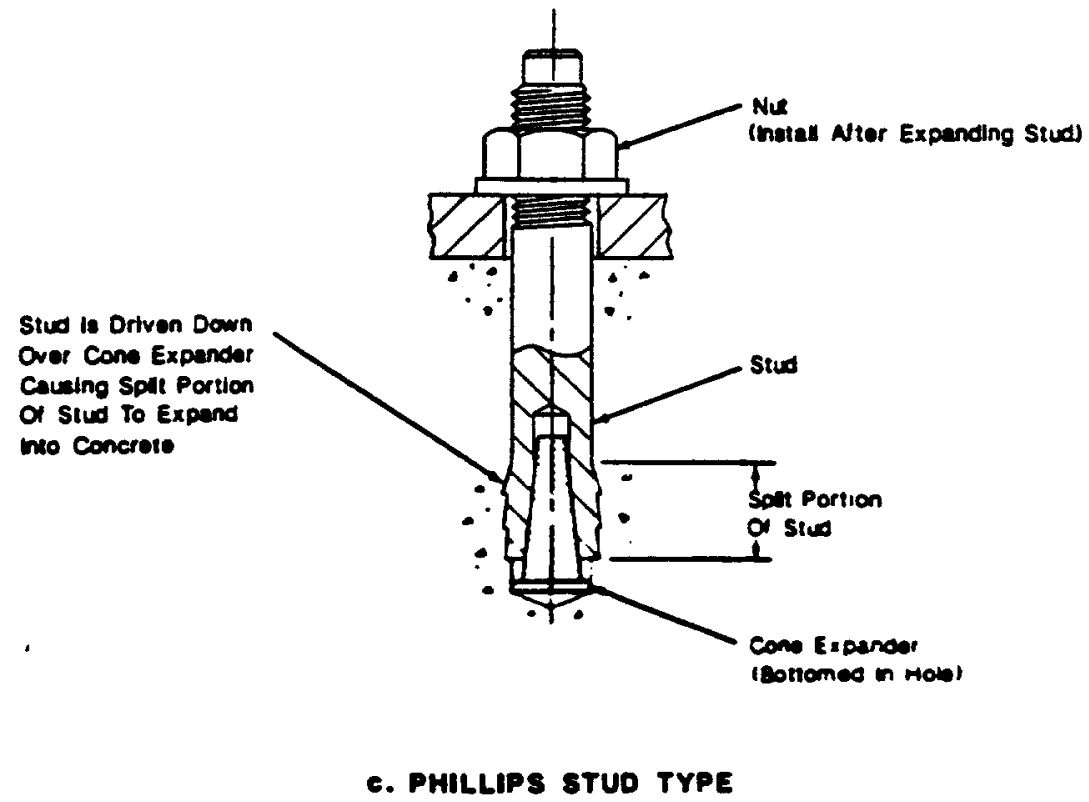

Figure 6.3-3 Features of Shell-Type Expansion Anchors (Reference 41) (Figure C.2-1 of SQUG GIP, Reference 1) 


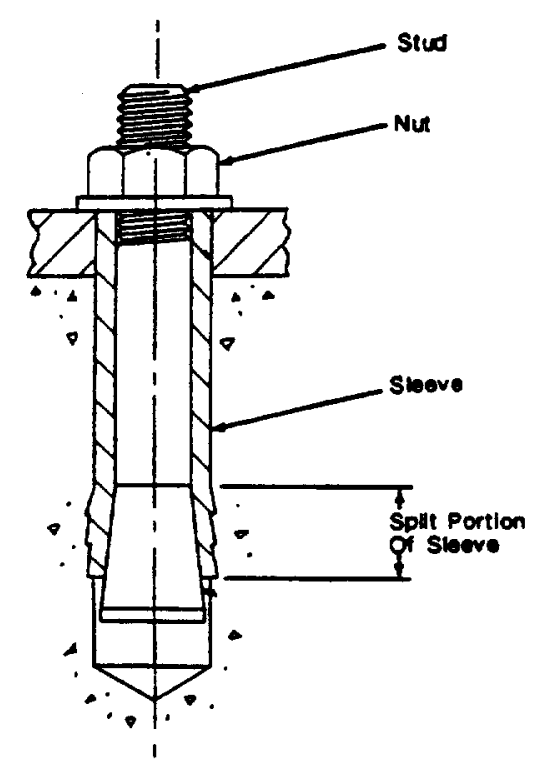

a. SLEeVE trpe

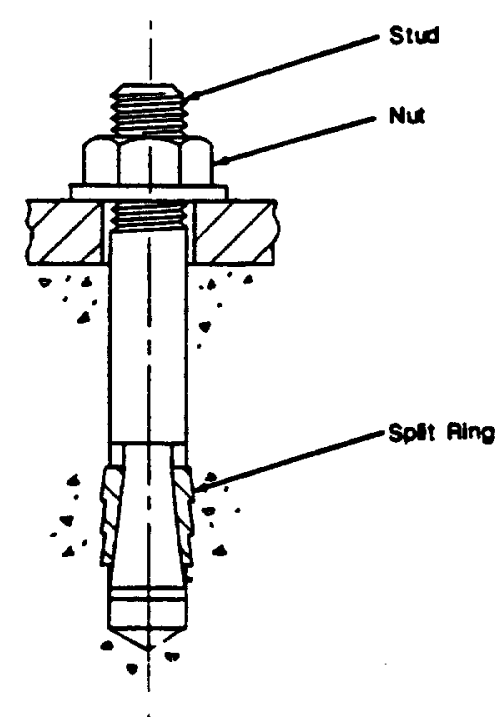

D. WEDOE TYPE

Figure 6.3-4 Features of Nonshell-Type Expansion Anchors (Reference 41) (Figure C.2-2 of SQUG GIP. Reference 1) 


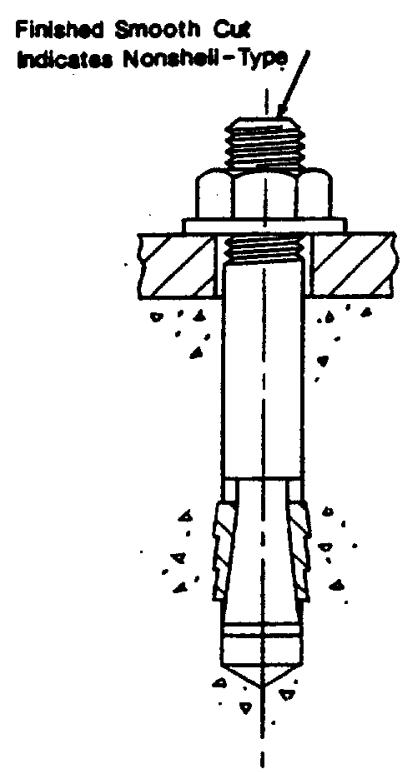

a. NONSHELL-TYPE
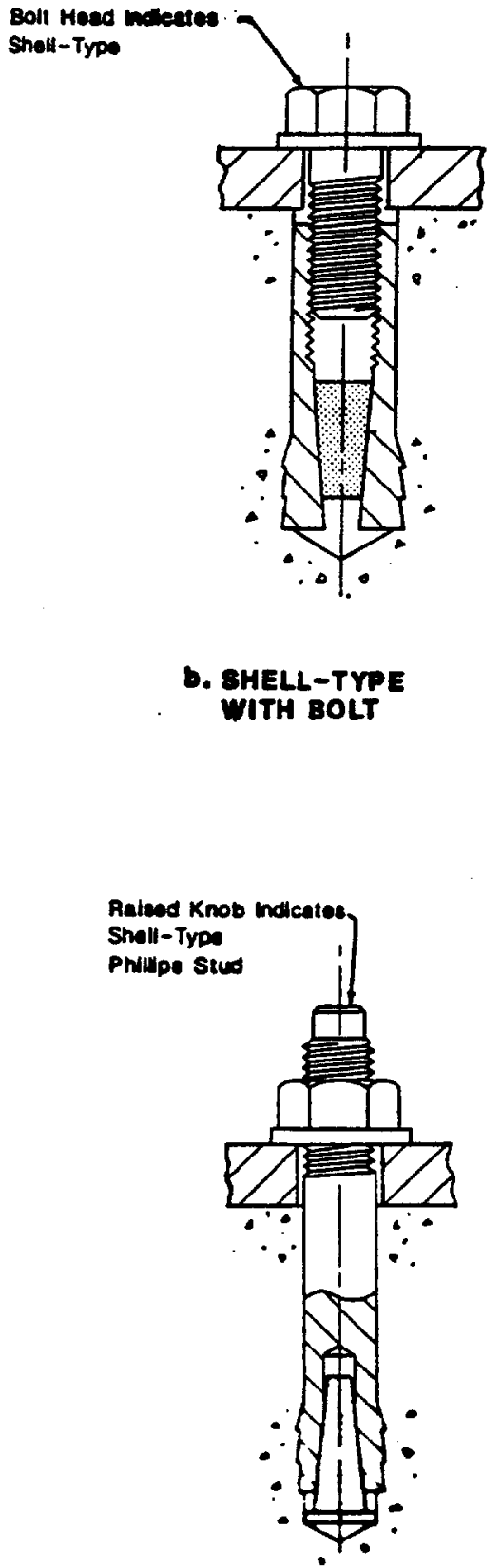

๑. SHELL-TYPE PHILLIPS STUD

Figure 6.3-5 Distinguishing Characteristics of Installed Shell- and NonshellType Expansion Anchors (Reference 41) (Figure C.2-3 of SQUG GIP, Reference 1) 


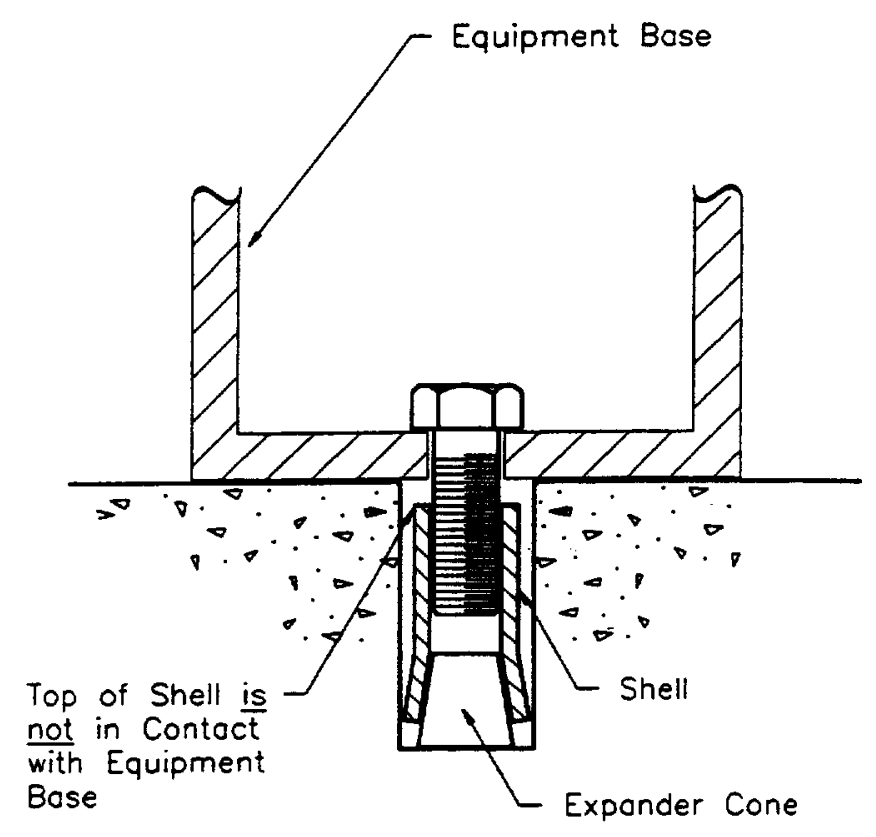

A. Property Set Shell After Tightness Check

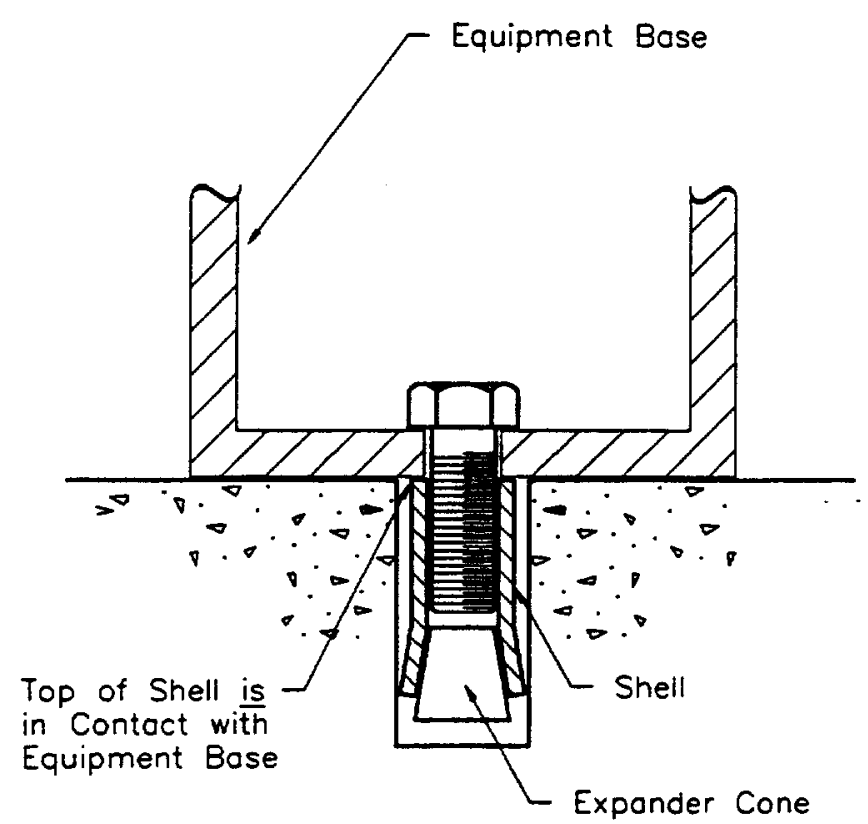

B. Improperly Set Shell After Tightness Check

Figure 6.3-6 Installation of Shell-Type Expansion Anchors (Figure 4-4 of SQUG GIP, Reference 1) 


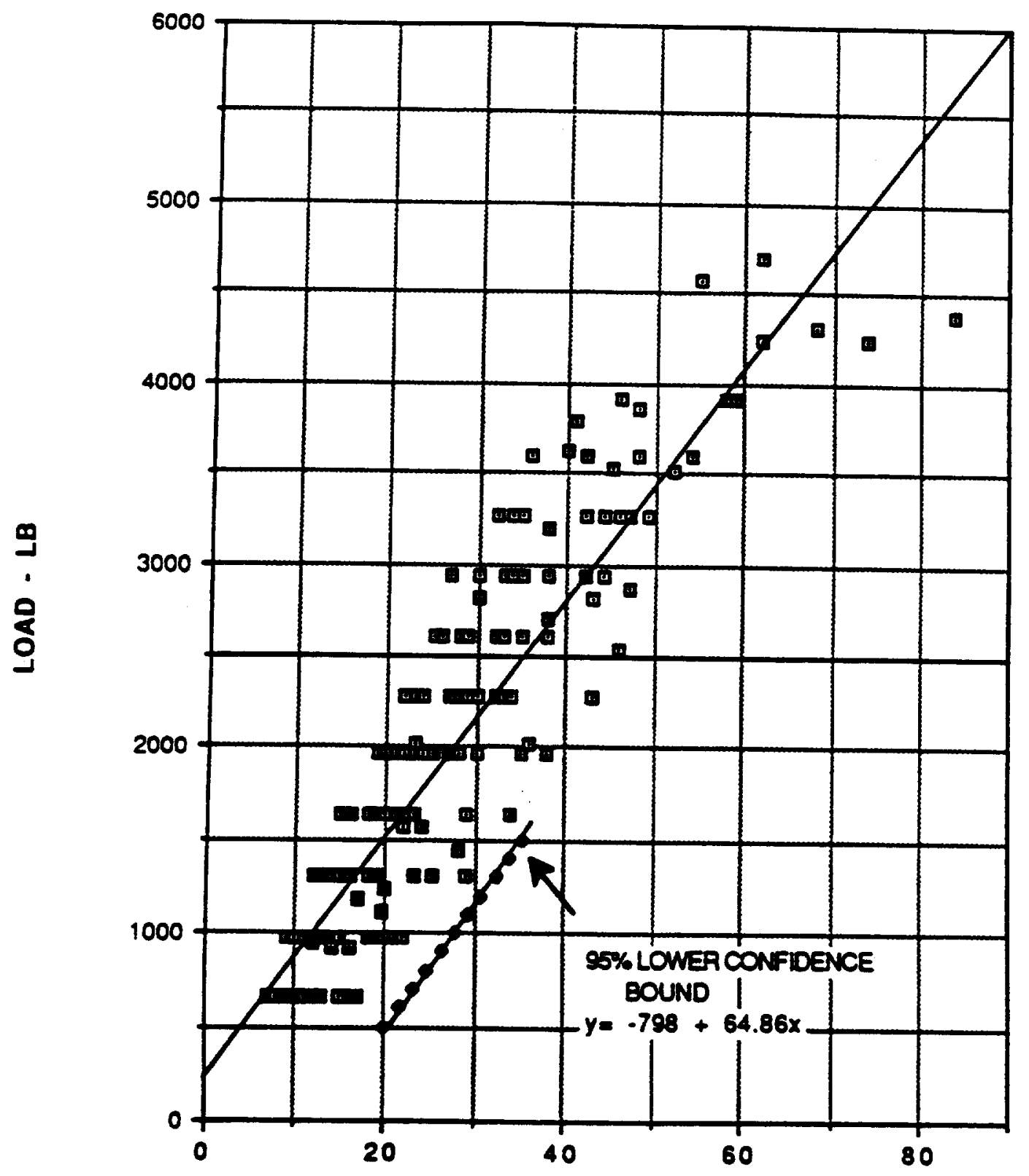

TOROUE (FT-LB)

Figure 6.3-7 Torque vs Load Tension Test for 3/8" Anchor (Figure C-1 of WSRC SEP-6, Reference 3) 


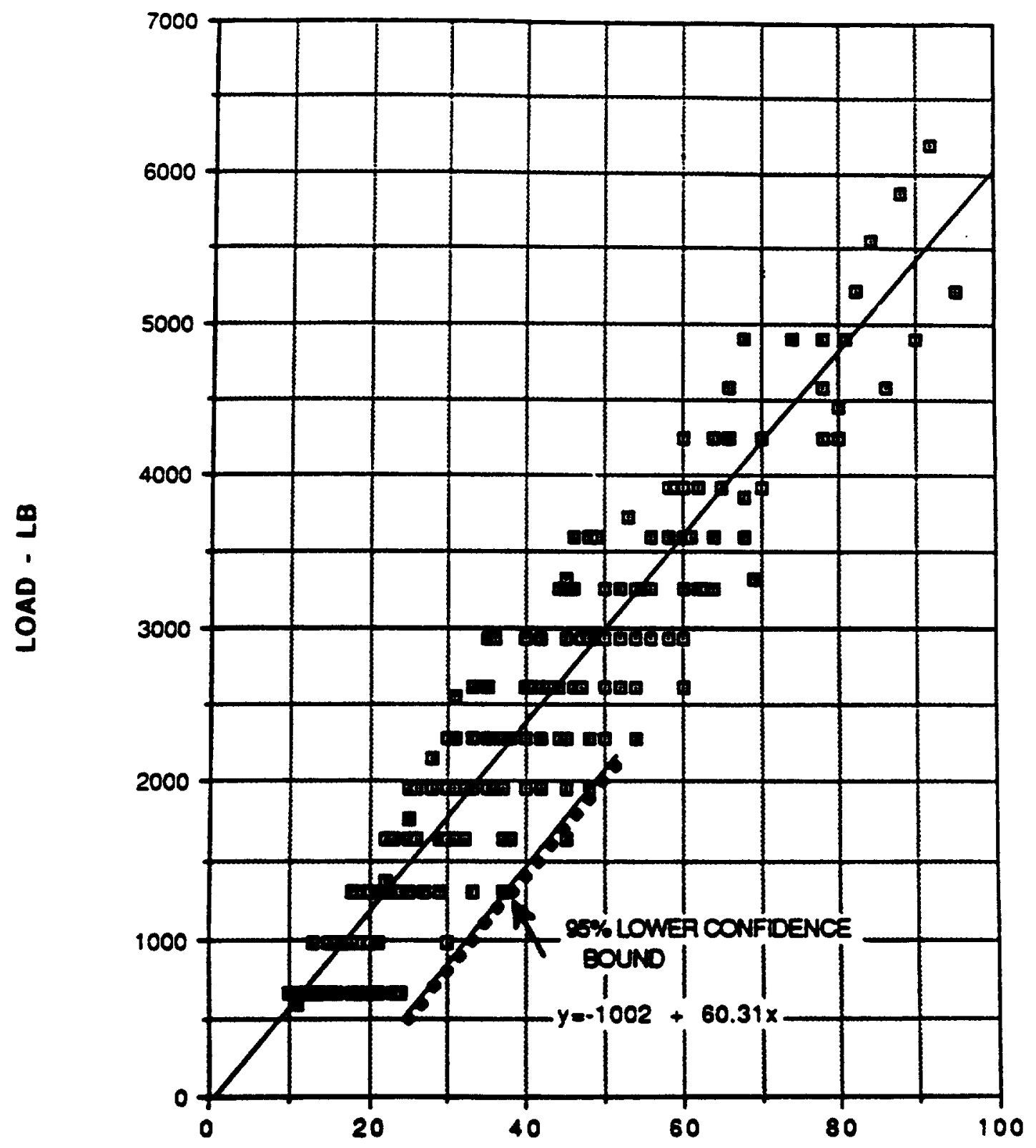

TOROUE (FT.LB)

Figure 6.3-8 Torque vs Load Tension Tests for 1/2" Anchor (Figure C-2 of WSRC SEP-6, Reference 3) 


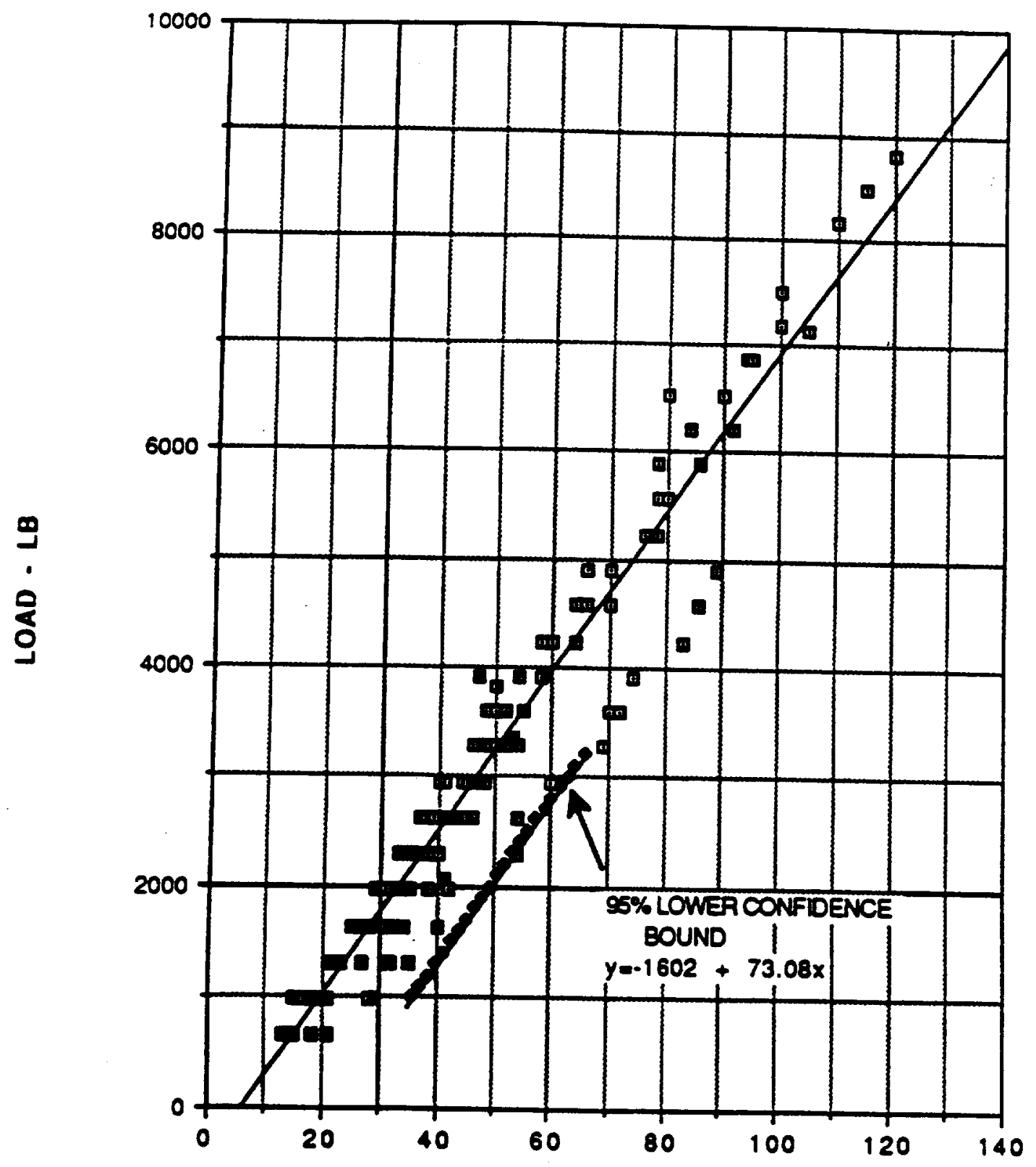

TORQUE (FT-LB)

Figure 6.3-9 Torque vs Load Tension Test for 5/8" Anchor (Figure C-3 of WSRC SEP-6, Reference 3) 


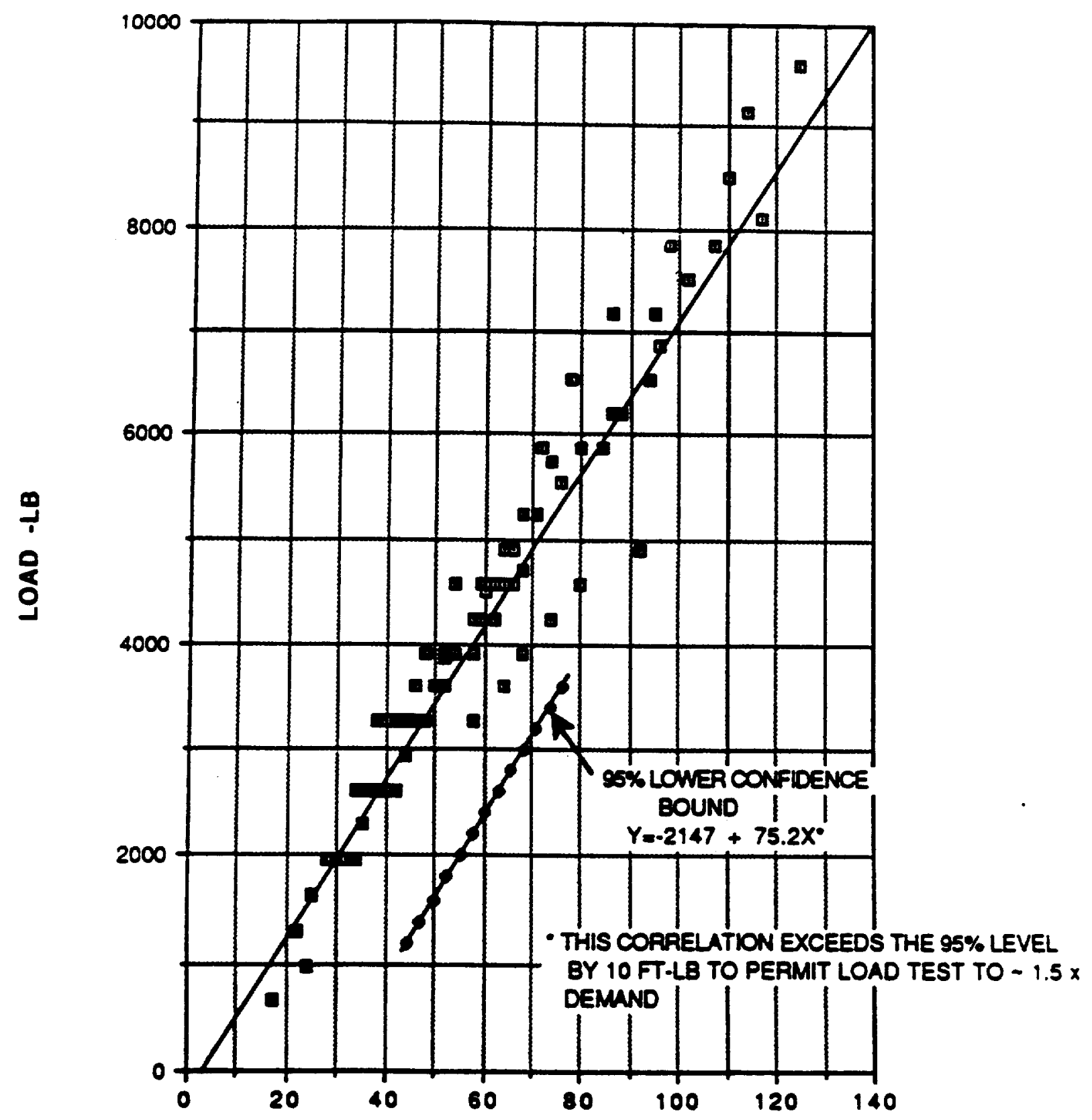

TOROUE FT-LB

Figure 6.3-10 Torque vs Load Tension Test for 3/4" Anchor (Figure C-4 of WSRC SEP-6, Reference 3) 


\subsection{ANCHORAGE DEMAND DETERMINATION}

\subsubsection{Equipment Characteristics ${ }^{48}$}

To determine the seismic demand on the anchorage of an item of equipment, the following equipment characteristics should be estimated: mass, location of the center of gravity, natural frequency, component damping, and equipment base center of rotation for overturning moment.

The mass of the equipment is a primary parameter for determining the inertial loads applied to the anchorage. Equipment weight can be obtained from drawings and/or original purchase documents, if available. However, if this information is not available, then conservative estimates of equipment weight for several equipment classes are discussed below. These estimated masses are, in general, based on the heaviest (or most dense) item identified during a survey of typical equipment in each of the equipment classes. For unusual equipment, an independent mass calculation should be performed or a conservative estimate made.

The location of the center of gravity of the equipment is used to determine the overturning moment caused by the inertial loads. It should be estimated by performing a visual inspection of the equipment. If the equipment has relatively uniform density, the center of gravity can be taken at the geometric center of the equipment. If the mass of the equipment is skewed, then appropriate adjustments should be made to the center of gravity location. If the equipment mass is centered significantly offset from the geometric centerline, then this should be noted and torsional effects included in the anchorage evaluations.

The lowest natural frequency $\left(f_{n}\right)$ of the equipment is used to determine the amplified acceleration of the equipment from the in-structure response spectrum. Only the overall structural modes of the equipment need be considered for anchorage evaluations. Since equipment-specific information is normally not available for determining the natural frequency of most types of equipment, approximate natural frequencies for certain classes of equipment are discussed below as either rigid $\left(f_{n}>\right.$ about $\left.20 \mathrm{~Hz}\right)$ or flexible $\left(f_{n}<\right.$ about $\left.20 \mathrm{~Hz}\right)$. Reference 77 also contains guidance for estimating the natural frequency of equipment.

The equipment damping should be determined for flexible equipment so that an appropriate instructure response spectrum, with the appropriate level of damping, is used to obtain spectral accelerations. The damping values for certain classes of equipment are discussed below.

The center of rotation of the equipment base is the line on the base about which the equipment would rotate due to an overturning moment. The location of the center of rotation should be estimated based on the following guidance. For very rigid equipment bases, such as heavy machinery on skid mounts, the equipment may be considered to pivot about its outer edge or far side bolt centerline. For flexible equipment bases, such as electrical cabinets with light base framing members, the center of rotation should be taken close to the equipment base centerline.

This remainder of this section contains estimates of equipment mass, natural frequency, and damping for the various classes of equipment for anchorage evaluations as summarized in Table 6.4-1. For those classes of equipment not covered in Table 6.4-1, the relative flexibility/stiffness and damping should be estimated based on engineering judgment, past experience, and comparison to the equipment provided in Table 6.4-1.

The purpose of Table 6.4-1 is to describe generic characteristics which may be used during anchorage evaluations in place of equipment-specific data. These generic characteristics typically

48 Based on Sections 4.4.1 - Check 1 and C.1 of SQUG GIP (Ref. 1) 
result in larger than actual loadings on the anchorage. However, for unusual items of equipment, e.g., motor control center weighing 800 pounds with an additional 100 pounds external weight, an independent check should be made of the reasonableness of the values contained in Table 6.4-1.

The equipment mass contained in Table 6.4-1 is based on the heaviest item found in each of the classes covered during a survey of equipment. Note that these masses are the same as those used in the screening tables given in the EPRI Anchorage Report (Ref. 41) except for the motor control centers which use 625 pounds per cabinet in the screening tables instead of the 800 pounds given in Table 6.4-1.

Equipment lowest natural frequency is given as a relative rigidity of either "rigid" or "flexible" in Table 6.4-1. Equipment with a lowest natural frequency of the overall structural mode greater than about $20 \mathrm{~Hz}$ is considered rigid. Equipment with natural frequencies below about $20 \mathrm{~Hz}$ are considered flexible. Note that "rigid" and "flexible" categories of equipment in Table 6.4-1 apply only to anchorage evaluations.

The relative rigidities given in Table 6.4-1 are for "typical" equipment in DOE facilities. These generic categories of rigid or flexible should be checked when performing the seismic evaluation, noting particularly the rigidity or flexibility of the base support system for the equipment and the rigidity of the anchorage itself. In particular, the estimate for natural frequency of equipment secured with expansion anchors should take into account the potential for slippage of these types of anchors. This would be necessary, for example, when natural frequency estimates of equipment secured with expansion anchors are based on analytical models which used fixed anchor points or when shake table test results are used in which the equipment was welded to the table.

Data for in-line equipment is not contained in Table 6.4-1.

Figure 6.4-1 provides equations for computing the lowest natural frequency of typical structural frames.

For rigid equipment, the seismic demand on the equipment can be determined by using the Zero Period Acceleration (ZPA) of the appropriate floor response spectrum. For flexible equipment, the peak of the floor response spectrum (for the damping value given in Table 6.4-1) should be used. 
Table 6.4-1 Generic Equipment Characteristics for Anchorage Evaluations (Table C.1-1 of SQUG GIP, Ref. 1)

\begin{tabular}{|c|c|c|c|}
\hline Equipment Class & \multicolumn{2}{|c|}{$\begin{array}{l}\text { Typical Maximum Weight } \\
\text { or Weight Density }\end{array}$} & $\begin{array}{l}\text { Typical Natural } \\
\text { Frequency }{ }^{(b)} \text { and } \\
\text { Damping }\end{array}$ \\
\hline $\begin{array}{l}\text { Motor Control Centers } \\
\text { (Section 8.1.2) }\end{array}$ & \multicolumn{2}{|c|}{$800 \mathrm{lb}$ per cabinet ${ }^{(\mathrm{d})}$} & $\begin{array}{c}\text { Flexible } \\
\text { 5\% Damping }\end{array}$ \\
\hline $\begin{array}{l}\text { Low-Voltage Switchgear } \\
\text { (Section 8.1.3) }\end{array}$ & \multicolumn{2}{|l|}{$35 \mathrm{lb} / \mathrm{ft}^{3}$} & $\begin{array}{c}\text { Flexible } \\
\text { 5\% Damping }\end{array}$ \\
\hline $\begin{array}{l}\text { Medium-Voltage } \\
\text { Switchgear }{ }^{(a)} \\
\quad \text { (Section 8.1.4) }\end{array}$ & \multicolumn{2}{|l|}{$31 \mathrm{lb} / \mathrm{ft}^{3}$} & $\begin{array}{c}\text { Flexible } \\
5 \% \text { Damping }\end{array}$ \\
\hline $\begin{array}{l}\text { Transformers } \\
\text { (Section 8.1.6) }\end{array}$ & $\begin{array}{c}\text { Rating(KVA) } \\
3,000 \\
2,500 \\
2,000 \\
1,000 \\
100\end{array}$ & $\begin{array}{c}\text { Weight (lb) } \\
15,000 \\
11,050 \\
9,400 \\
6,300 \\
975\end{array}$ & $\begin{array}{c}\text { Flexible } \\
\text { 5\% Damping }\end{array}$ \\
\hline $\begin{array}{l}\text { Horizontal Pumps with } \\
\text { Motors } \\
\text { (Section } 8.2 .3 \text { ) }\end{array}$ & $\begin{array}{c}\text { Power (HP) } \\
1,000 \\
600 \\
500 \\
400 \\
200 \\
100\end{array}$ & $\begin{array}{c}\text { Weight (lb) } \\
20,000 \\
16,500 \\
12,000 \\
8,600 \\
6,000 \\
3,600\end{array}$ & $\begin{array}{c}\text { Rigid } \\
\text { 5\% Damping }\end{array}$ \\
\hline $\begin{array}{l}\text { Vertical Pumps with Motors } \\
\text { (Section 8.2.4) }\end{array}$ & Power (HP) & Weight (lb) & \multirow{3}{*}{$\begin{array}{c}\text { Flexible } \\
\text { 3\% Damping } \\
\text { Rigid } \\
\text { 5\% Damping }\end{array}$} \\
\hline $\begin{array}{l}\text { a. Vertical } \\
\text { Immersion }\end{array}$ & 150 & 4,000 & \\
\hline b. Centrifugal & $\begin{array}{r}500 \\
2,000\end{array}$ & $\begin{array}{r}9,000 \\
48,000\end{array}$ & \\
\hline \multirow[t]{2}{*}{ c. Deep-Well } & 500 & $\begin{array}{r}9,000 \\
\text { (motor) }\end{array}$ & \multirow[t]{2}{*}{$\begin{array}{c}\text { Flexible } \\
\text { 3\% Damping }\end{array}$} \\
\hline & & $\begin{array}{l}14,000 \\
\text { (pump) }\end{array}$ & \\
\hline $\begin{array}{l}\text { Air Compressors } \\
\text { (Section 8.2.6) }\end{array}$ & $\begin{array}{c}\text { Power (HP) } \\
50 \\
200\end{array}$ & $\begin{array}{c}\text { Weight (lb) } \\
4,000 \\
10,000\end{array}$ & $\begin{array}{c}\text { Rigid } \\
\text { 5\% Damping } \\
{ }^{(c)}\end{array}$ \\
\hline
\end{tabular}


Table 6.4-1 (Continued)

\begin{tabular}{|c|c|c|}
\hline Equipment Class & $\begin{array}{l}\text { Typical Maximum Weight } \\
\text { or Weight Density }\end{array}$ & $\begin{array}{c}\text { Typical Natural } \\
\text { Frequency }{ }^{(b)} \text { and } \\
\text { Damping }\end{array}$ \\
\hline $\begin{array}{l}\text { Motor-Generators } \\
\text { (Section 8.2.7) }\end{array}$ & (Not Available) & $\begin{array}{c}\text { Rigid } \\
\text { 5\% Damping }\end{array}$ \\
\hline $\begin{array}{r}\text { Batteries on Racks } \\
\text { (Section 8.1.1) }\end{array}$ & $\begin{array}{l}0.11 \mathrm{lb} / \mathrm{in}^{3} \text { for batteries, } \\
\text { plus weight of racks }\end{array}$ & $\begin{array}{c}\text { Flexible } \\
\text { 5\% Damping }\end{array}$ \\
\hline $\begin{array}{l}\text { Battery Chargers and Inverters } \\
\text { (Section 8.1.7) }\end{array}$ & $45 \mathrm{lb} / \mathrm{ft}^{3}$ & $\begin{array}{c}\text { Flexible } \\
\text { 5\% Damping }\end{array}$ \\
\hline $\begin{array}{r}\text { Engine-Generators } \\
\text { (Section 8.2.8) }\end{array}$ & (Not Available) & $\begin{array}{c}\text { Rigid } \\
\text { 5\% Damping }\end{array}$ \\
\hline $\begin{array}{l}\text { Instrument Racks } \\
\text { (Section 8.1.9) }\end{array}$ & $20 \mathrm{lb} / \mathrm{ft}^{2}$ of vertical face & $\begin{array}{c}\text { Flexible } \\
\text { 3\% Damping }\end{array}$ \\
\hline $\begin{array}{l}\text { Generic Equipment Cabinets } \\
\text { (Section 8.1.5) }\end{array}$ & $\begin{array}{l}3 \text { times the weight of cabinet } \\
\text { housing }\end{array}$ & $\begin{array}{c}\text { Flexible } \\
\text { 5\% Damping }\end{array}$ \\
\hline $\begin{array}{l}\text { Walk-Through Control Panels } \\
\text { (Section 8.1.8) }\end{array}$ & $\begin{array}{l}\text { Determine and use weight } \\
\text { per foot of length }\end{array}$ & $\begin{array}{c}\text { Flexible } \\
\text { 5\% Damping }\end{array}$ \\
\hline
\end{tabular}

(a) Medium voltage switchgear are called "Metal-Clad Switchgear" in Reference 41.

(b) The lowest natural frequencies of the overall structural mode are given as either Rigid ( $>$ about $20 \mathrm{~Hz}$ ) or Flexible (< about $20 \mathrm{~Hz}$ ) and apply only to anchorage evaluations.

(c) A damping value of 5\% can be used for rigid equipment since the seismic accelerations can be taken from the ZPA which is not affected significantly by damping level.

(d) Note: When using the screening tables in the EPRI Anchorage Report (Reference 41), an average weight per MCC section of 625 pounds was used rather than the 800 pounds shown in this table.

\subsubsection{Seismic Loads ${ }^{49}$}

The next step in evaluating the seismic adequacy of anchorage is to determine the loads applied to the anchorage by the seismic demand imposed on the item of equipment. This is done using the following five steps:

1. Determine the appropriate input seismic accelerations for the item of equipment for each of the three directions of motion.

2. Determine the seismic inertial equipment loads for each of the three directions of motion using the equivalent static load method.

49 Based on Section 4.4.3 of SQUG GIP (Ref. 1) 
3. Determine the seismic inertial anchor loads by calculating the various load components for each direction of motion.

4. Calculate the combined seismic loads on each anchor from each of the three directions of seismic motion. Then combine the load components from these three directions using the Square Root Sum of the Squares (SRSS) method.

5. Calculate the total anchor loads on each anchor by adding the combined seismic loads to the equipment deadweight loads and any other loads on the equipment.

These five steps are described below:

Step 1 - Input Seismic Accelerations. The first step in determining the seismic demand loads on the anchorage is to compute the input seismic accelerations from an appropriate in-structure response spectrum, at the damping and natural frequency of the equipment, for the location in the facility where the equipment is mounted. Section 5.2.2 discusses the techniques for determining the scaled in-structure response spectrum (SDS) which is computed from the Design Basis Earthquake (DBE).

If the equipment is located in an area where there are two applicable lateral response spectra (nominally one N-S and one E-W), then one of the following alternatives can be used to define a single horizontal seismic demand acceleration for load calculation:

- Use the higher acceleration for both horizontal directions.

- Use the acceleration value (either N-S or E-W) which aligns with the direction of the "weak" anchorage for that item of equipment.

- Use the actual direction N-S and E-W accelerations for the N-S and E-W loads on each item of equipment.

The vertical component of acceleration should be the appropriate site-specific fraction of the horizontal component of acceleration. For most equipment classes, the vertical direction fundamental frequency is in the rigid range.

The following factors which should be considered in determining the input seismic accelerations are covered below: equipment damping, natural frequency of the equipment, and use of unbroadened response spectra.

Equipment Damping. A 5\% damping value can be used in anchorage evaluations for most of the equipment classes covered by this procedure. Section 6.4.1 lists the equipment classes for which $5 \%$ damping is recommended. This level of damping is adequate for these classes because the equipment either exhibits this level of damping or it is essentially rigid (natural frequency greater than about $20 \mathrm{~Hz}$ ) so that the damping level is nearly irrelevant. Section 6.4.1 also lists the classes of equipment which have lower damping (3\% damping) and which are, in general, flexible. This equipment includes electrical equipment and some types of Vertical Pumps. It should be evaluated that the equipment does not have unusual features which could lower its damping below the values given in Section 6.4.1.

In-structure response spectra for the facility may not be available at the $5 \%$ or $3 \%$ damping levels recommended in this procedure for anchorage evaluations. Therefore available response spectra may be normalized to the desired spectral damping level using one of the methods from Appendix A of Reference 19. 
For in-structure response spectra which have a shape similar to the Reference Spectrum, (without very narrow peaks) the spectral acceleration for a desired damping ratio $\beta_{D}$ can be estimated from an available response spectrum with a damping ratio of $\beta_{A}$ by using the following relationship:

$$
S a_{i D}=S a_{i A} \sqrt{\frac{\beta_{A}}{\beta_{D}}}
$$

However this spectral acceleration $\mathrm{Sa}_{\mathrm{iD}}$ is limited to:

$$
\mathrm{Sa}_{\mathrm{iD}} \geq \mathrm{ZPA}
$$

for frequencies $\left(f_{i}\right)$ in the high frequency region; i.e. frequencies greater than the frequency associated with the peak of the response spectrum.

The meaning of the symbols used above is as follows:

$$
\begin{aligned}
& \mathrm{Sa}_{\mathrm{iA}}=\quad \begin{array}{l}
\text { available spectral acceleration at frequency } \mathrm{f}_{\mathrm{i}} \text { associated with a damping } \\
\text { ratio } \beta_{A}
\end{array} \\
& \mathrm{Sa}_{\mathrm{iD}}=\begin{array}{l}
\text { desired spectral acceleration at frequency } \mathrm{f}_{\mathrm{i}} \text { associated with a damping } \\
\text { ratio } \beta_{\mathrm{D}}
\end{array} \\
& \beta_{\mathrm{A}}=\text { damping ratio of available response spectrum } \\
& \beta_{D}=\text { damping ratio of desired response spectrum } \\
& \mathrm{ZPA}=\text { Zero Period Acceleration } \\
& \mathrm{f}_{\mathrm{i}}=\text { frequency of interest }
\end{aligned}
$$

Natural Frequency. The lowest natural frequency $\left(f_{n}\right)$ of the equipment may be estimated by past experience with testing or analysis. The natural frequency of the equipment can be determined during the inspection of the anchorage installation. Note that reasonable estimates of equipment natural frequency for several equipment classes are given in Section 6.4.1 as either rigid $\left(f_{n}>\right.$ about $\left.20 \mathrm{~Hz}\right)$ or flexible $\left(f_{n}<\right.$ about $\left.20 \mathrm{~Hz}\right)$. The following classes of equipment can generally be considered as rigid (i.e., natural frequency greater than about $20 \mathrm{~Hz}$ ) if anchored stiffly:

- $\quad$ Horizontal Pump (Section 8.2.3)

- $\quad$ Air Compressors (Section 8.2.6)

- Motor-Generators (Section 8.2.7)

- $\quad$ Engine-Generators (Section 8.2.8)

Rigid equipment can use a damping value of $5 \%$ since it is not significantly amplified over the Zero Period Acceleration (ZPA). 
If the natural frequency of the equipment is estimated to be high (i.e., greater than about 20 $\mathrm{Hz}$ ), then the equipment should be considered "rigid" and the Zero Period Acceleration (ZPA) should be used for anchorage load calculations. If the natural frequency is estimated to be below about $20 \mathrm{~Hz}$, then the equipment should be considered "flexible" and the peak of the response spectrum may conservatively be used for anchorage load calculations. If the natural frequency of the equipment is known (by calculation, test, or other means), the maximum acceleration from the response spectrum for the frequency range of interest (from equipment natural frequency to $33 \mathrm{~Hz}$ ) can be used instead of the peak.

Unbroadened Response Spectra. Unbroadened in-structure response spectra can be used for comparison to seismic capacity spectra. Uncertainty in the natural frequency of the building structure should be addressed by shifting the frequency of the seismic demand response spectrum at these peaks. A reference or basis for establishing the degree of uncertainty in the natural frequency of the building structure should be included in the facility-specific seismic evaluation records. The method of peak shifting discussed in ASCE 4 (Ref. 74) may also be used.

Step 2 - Seismic Inertial Equipment Loads. The second step in determining the seismic demand loads on the anchorage is to compute the seismic inertial equipment loads for each of the three directions of motion using the equivalent static load method. In this method, the seismic analysis is performed statically by applying the inertial load at the center of gravity of the equipment. The inertial load in each direction is equal to the product of the input seismic accelerations, an equivalent static coefficient, and the mass of the equipment.

An equivalent static coefficient of 1.0 can be used for the classes of equipment covered by this procedure; the basis for this is described in Reference 41. The mass of the equipment is determined during the inspection of the anchorage installation. Note that conservative estimates of equipment mass for several equipment classes are given in Section 6.4.1.

Step 3 - Seismic Inertial Anchor Loads. The third step in determining the seismic demand loads on the anchorage is to compute the seismic inertial anchor loads for each of the three directions of motion. This is done by applying the seismic inertial equipment loads determined in the previous step to the center of gravity of the item of equipment and calculating the free-body loads on the anchors. The location of the center of gravity of the equipment is determined during the inspection of the anchorage installation. The location of the center of gravity can be taken as the geometric center of the equipment if the equipment is of uniform density. If the mass of the equipment is skewed, then appropriate adjustments should be made to the center of gravity location.

The following types of seismic inertial anchor loads should be determined. Note that these loads are applicable whether the equipment is mounted on the floor, wall, or ceiling.

- Anchor shear loads due to the lateral component of force caused by the seismic inertial equipment loads, including, if significant, the anchor shear loads due to any torsional moments (center of gravity is not in line with the centroid of the group of anchors).

- Anchor pullout loads due to the overturning moment caused by the seismic inertial equipment loads, with an appropriately estimated location of the overturning axis. Guidance on estimating the location of the overturning axis is given below.)

- Anchor pullout loads caused by the seismic inertial equipment loads due to the component of force which is in line with the axes of the anchor bolts; e.g., for floor-mounted equipment include the vertical component of the seismic load. 
The anchor loads caused by the equipment overturning moment can be based on the assumption that plane sections remain plane during loading and that the material in the equipment and the anchors behave in a linear-elastic manner. This results in a linear distribution of anchor loads for a set of anchors which are equal in stiffness and size.

The recommended location for the overturning axis is at the equipment centerline for equipment with flexible bases. For rigid base equipment, the overturning axes can be taken at the edge of the equipment. Reference 78 contains discussion on locating the overturning axes.

Step 4 - Combined Seismic Loads. The fourth step in determining the seismic demand loads on the anchorage is to compute the combined seismic anchor loads of the seismic loads on each anchor from the three directions of earthquake motion. The combined loads can be computed with a combination technique such as the Square Root Sum of the Squares (SRSS) or the 100-40-40 Rule.

Step 5 - Total Anchor Loads. The total loads on the anchorage are computed by combining the combined seismic anchor loads from the previous step to the equipment deadweight loads and any other significant loads which would be applied to the equipment, e.g.; pipe reaction loads on pumps. 


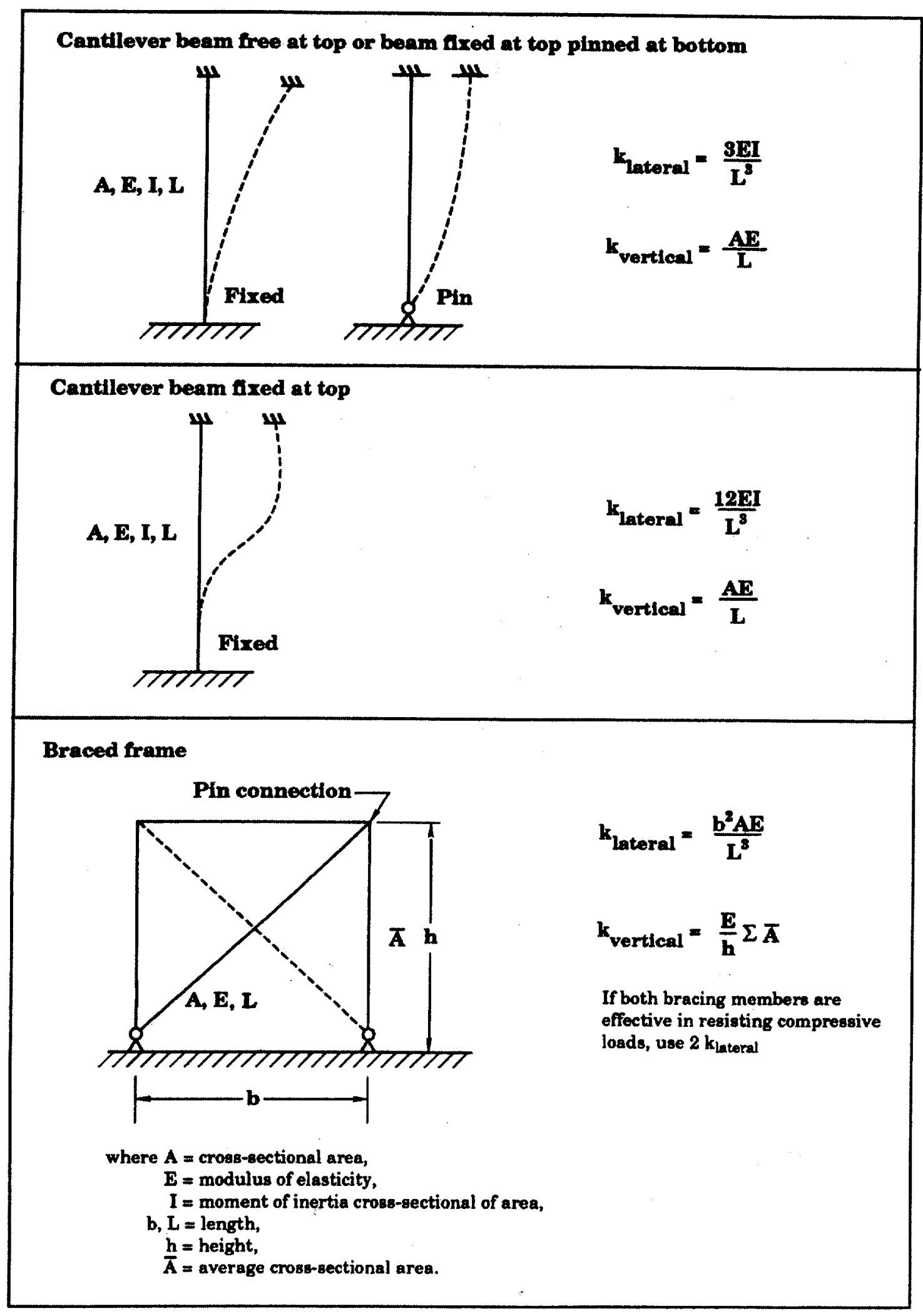

Figure 6.4-1 Stiffness Equations for Structural Frames (Reference 76) 


\subsection{COMPARISON OF CAPACITY TO DEMAND ${ }^{50}$}

The final main step in evaluating the seismic adequacy of anchorage is to compare the seismic capacity loads of the anchors (determined in Section 6.3) to the total anchor loads (determined in Section 6.4). This comparison can be done using the shear-tension interaction formulations given below for each of the anchor types covered by this procedure.

\subsubsection{Expansion Anchors ${ }^{51}$}

When expansion anchors are subjected to simultaneous shear and tension, one of the following shear-tension interaction formulations should be used. The linear formulation is conservative. The bi-linear formulation is more realistic. Figure 6.5-1 illustrates these formulations.

- $\quad$ Linear Formulation (conservative)

$$
\frac{\mathrm{V}}{\mathrm{V}_{\mathrm{all}}}+\frac{\mathrm{P}}{\mathrm{P}_{\mathrm{all}}} \leq 1.0
$$

- $\quad$ Bilinear Formulation (more realistic)

$$
\begin{array}{ll}
\frac{P}{P_{\text {all }}} \leq 1.0 & \text { for } \frac{V}{V_{\text {all }}} \leq 0.3 \\
0.7 \frac{\mathrm{P}}{\mathrm{P}_{\text {all }}}+\frac{\mathrm{V}}{\mathrm{V}_{\text {all }}} \leq 1.0 \text { for } & 0.3<\frac{\mathrm{V}}{\mathrm{V}_{\text {all }}} \leq 1.0
\end{array}
$$

Where: $\mathbf{P}=$ Applied pullout loads due to earthquake plus dead loads.

$\mathrm{V}=$ Applied shear loads due to earthquake plus dead loads.

$\mathrm{P}_{\mathrm{all}}=$ Allowable pullout capacity load for the anchor.

$\mathrm{V}_{\text {all }}=$ Allowable shear capacity load for the anchor.

\subsubsection{Cast-in-Place Bolts and Headed Studs ${ }^{52}$}

For existing cast-in-place bolts subjected to simultaneous shear and tension, the shear-tension interaction depends on the anticipated failure mode. Figure 6.5-2 presents the interaction curves for cast-in-place bolts for failure in the bolt steel or failure in the concrete. Because the anchorage criteria in this procedure and Reference 41 for cast-in-place bolts and headed studs are based on an additional factor of safety of 1.5 against failure not occurring in the concrete, it is recommended that the interaction formulation for steel failure be used.

50 Based on Section 4.4.4 of SQUG GIP (Ref. 1)

51 Based on Section C.2.11 of SQUG GIP (Ref. 1)

52 Based on Section C.3.7 of SQUG GIP (Ref. 1) 


\subsubsection{Cast-in-Place J-Bolts ${ }^{53}$}

It is left to the user to select an appropriate shear-tension interaction formulation for use with Jbolts when both tension and shear loads are significant.

\subsubsection{Grouted-in-Place Bolts ${ }^{54}$}

For grouted-in-place bolts subjected to simultaneous shear and tension, the guidelines given in Section 6.5.2 for cast-in-place bolts may be used to compare the allowable loads to the applied loads.

\subsubsection{Welds to Embedded Steel or Exposed Steel ${ }^{55}$}

When welds are subjected to simultaneous shear and tension, the allowable loads can be compared to the applied loads using the following shear-tension interaction formulation:

$$
\begin{aligned}
\left(\frac{P}{F_{W}}\right)^{2}+\left(\frac{V}{F_{W}}\right)^{2} & \leq 1 \\
\text { Where: } \quad P & =\text { Pullout (tensile) load applied to weld [kip] } \\
V & =\text { Shear load applied to weld [kip] } \\
F_{w} & =\text { Allowable load for weld from Table 6.3-13 [kip] }
\end{aligned}
$$




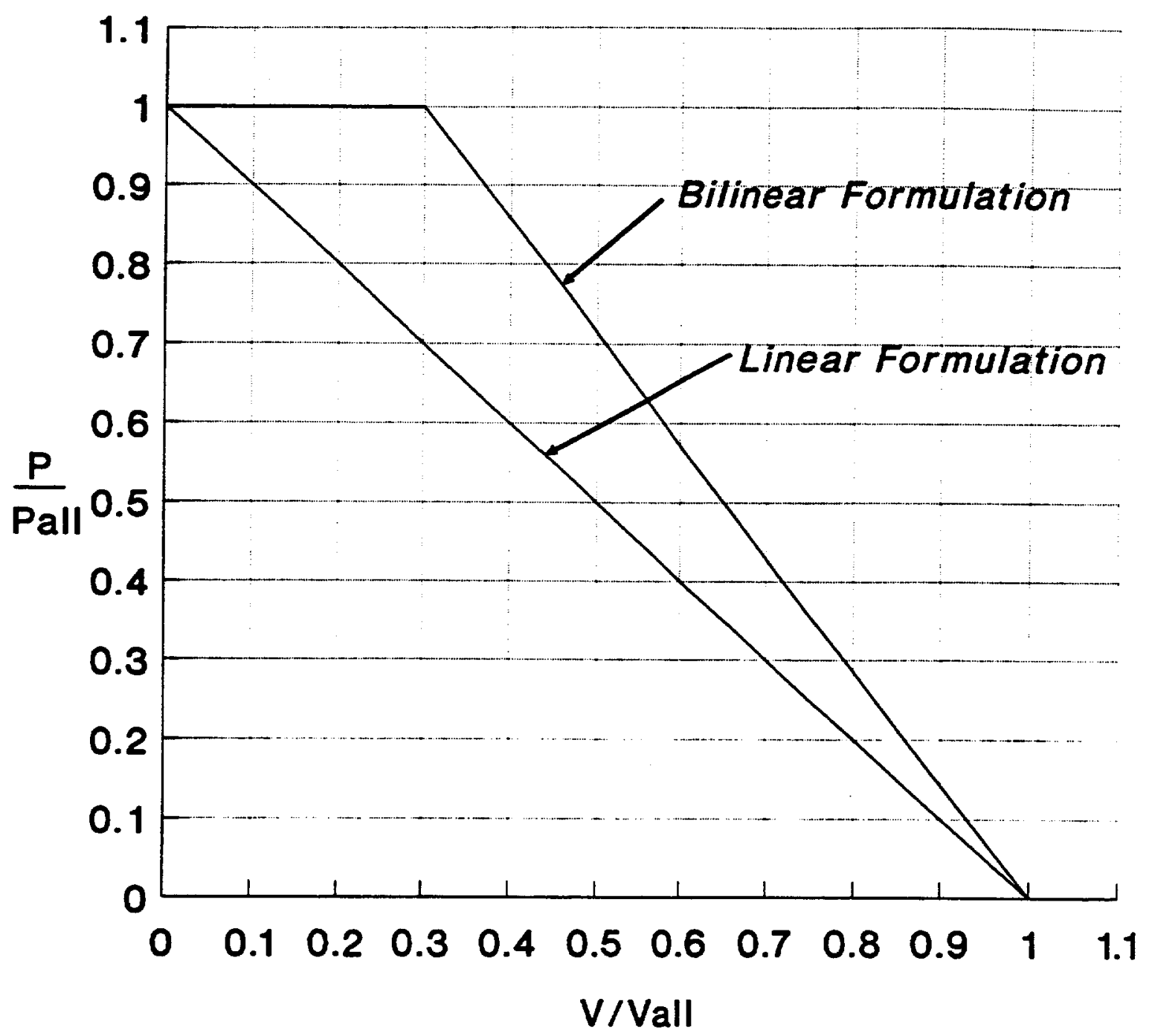

Figure 6.5-1 Shear-Tension Interaction Limitations for Expansion Anchors (Reference 41) (Figure C.2-4 of SQUG GIP, Reference 1) 


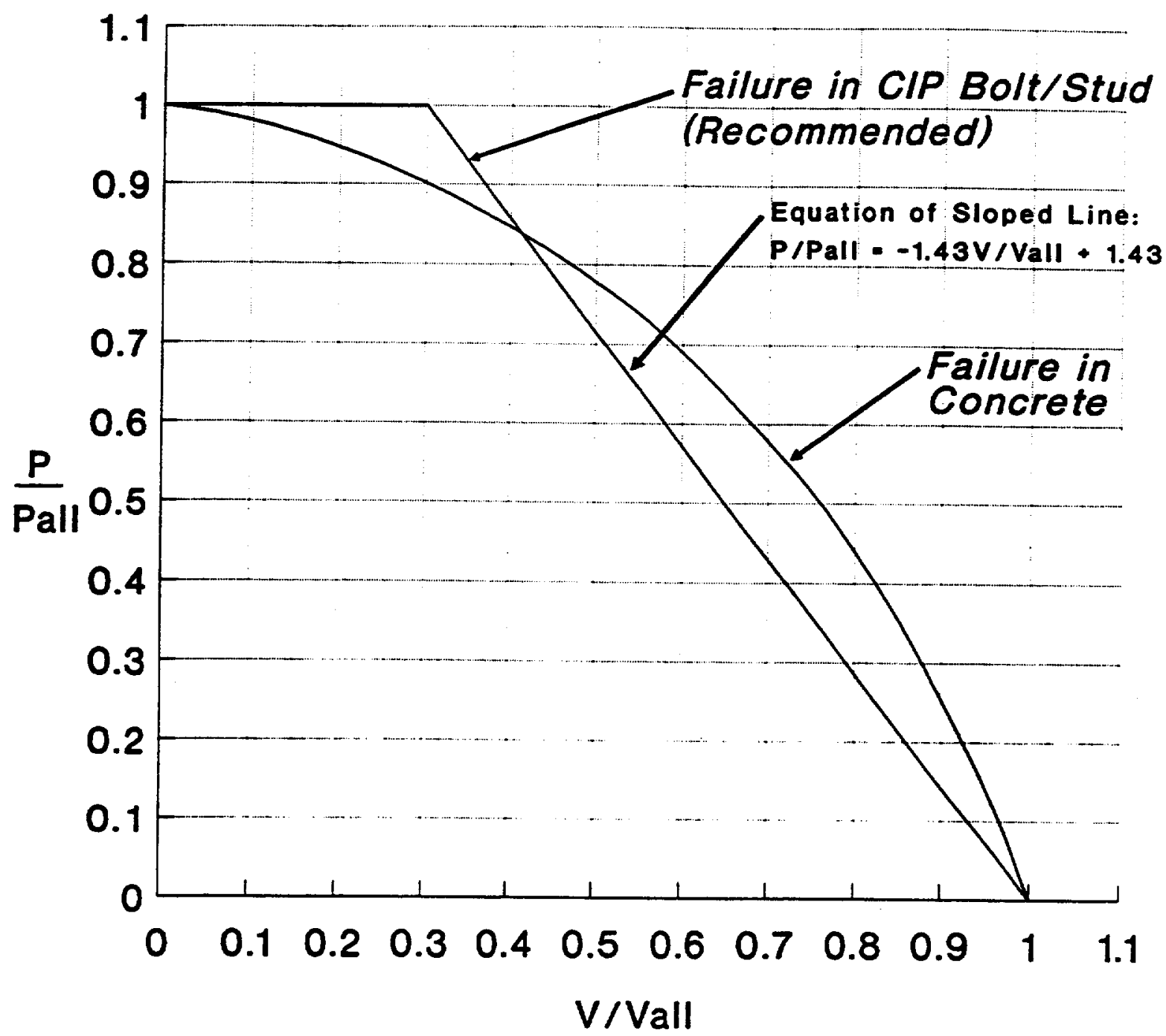

\begin{tabular}{l|ccccccc} 
V/Vall & 0.0 & 0.1 & 0.3 & 0.5 & 0.7 & 0.9 & 1.0 \\
\hline P/Pall - CIP Bolt/Stud & 1.0 & 1.0 & 1.0 & .71 & .43 & .14 & 0.0 \\
P/Pall - Concrete & 1.0 & .99 & .91 & .79 & .60 & .30 & 0.0
\end{tabular}

Figure 6.5-2 Shear-Tension Interaction Limitations for Cast-In-Place Bolts and Headed Studs (Reference 41) (Figure C.3-2 of SQUG GIP, Reference 1) 


\section{SEISMIC INTERACTION}

\subsection{INTRODUCTION ${ }^{1}$}

The purpose of this section is to describe seismic interaction and techniques for evaluating its effects on equipment in DOE facilities. Seismic interaction is the physical interaction of any structures, piping, or equipment with a nearby item of equipment caused by relative motions from an earthquake. Components with fragile appendages (such as instrumentation tubing, air lines, and glass site tubes) are most prone to damage for seismic interaction. An inspection should be performed in the area adjacent to and surrounding equipment to identify any seismic interaction condition which could adversely affect the capability of the equipment to perform its intended function.

An overview of seismic interaction is shown in Figure 7.1-1. An earthquake can cause various types of interactions such as bumping, falling, or flooding. The SCEs should identify the various types of interactions and work with other SRT members to determine the overall effect on the facility. This chapter describes the seismic interaction effects covered by the DOE Seismic Evaluation Procedure and how they can be evaluated. The seismic interaction effects which are included within the scope of this procedure are proximity; structural failure and falling; flexibility of attached lines and cables and differential displacements; and water spray, flood, and fire hazards.

Using this chapter, the SCEs should be familiar with the various types of interaction, be able to judge if an interaction is credible and its significance during a walkdown, be able to identify outliers, and be familiar with DOE Guidance related to seismic interactions.

1 Based on Section D.1 of SQUG GIP (Ref. 1) 


\section{Seismic Interaction}

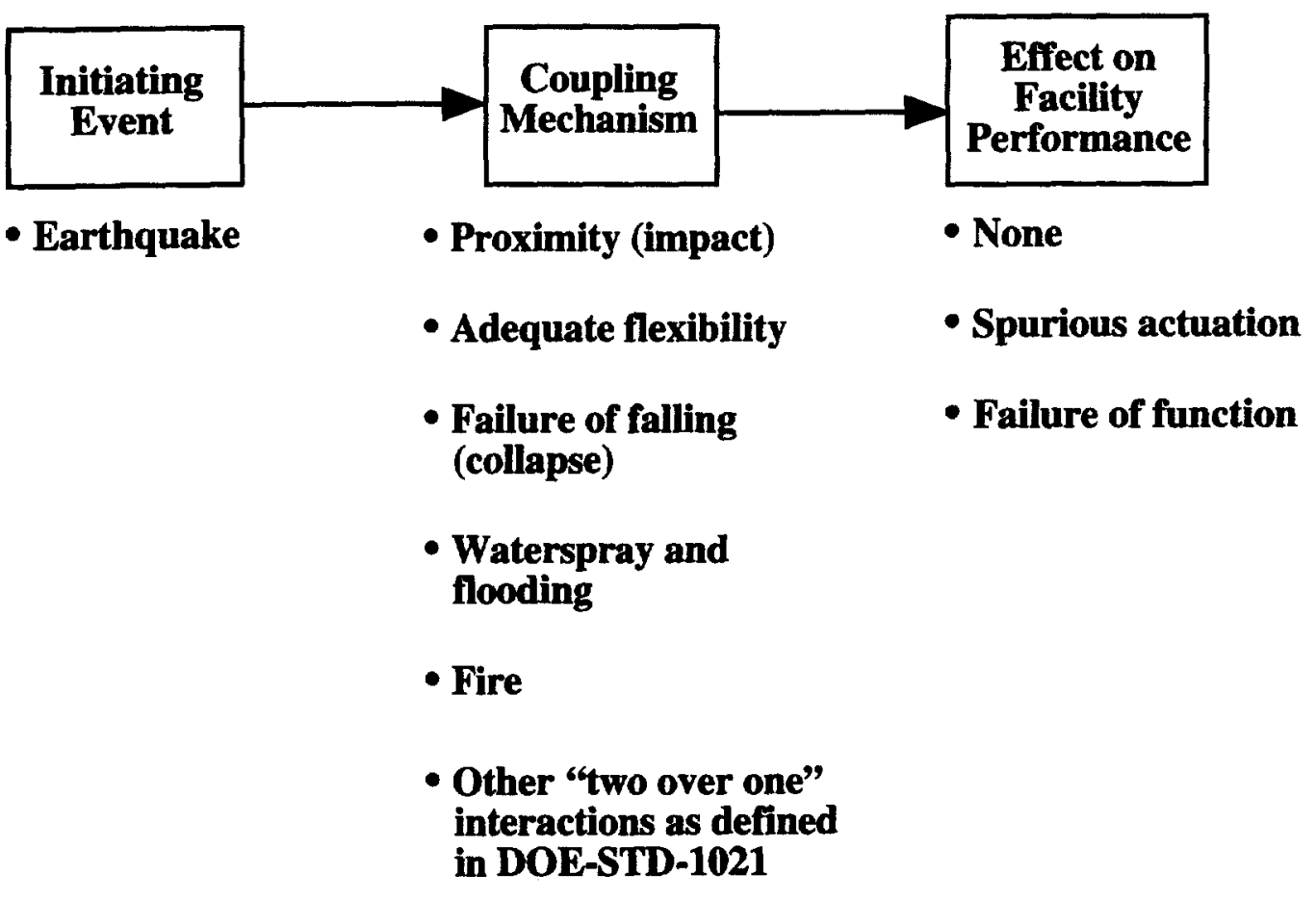

Figure 7.1-1 Overview of Seismic Interaction 


\subsection{INTERACTION EFFECTS}

An example of the effects of seismic interaction is shown in Figure 7.2-1. The hanging conduit or piping, which is free to swing during earthquake motion, is the source, while the target is the electrical cabinet. The shaded zone in the figure defines the zone of influence where the source can affect the target. For a credible interaction to occur, the source must impact or interact with the target (see Figure 7.2-2). While evaluating the effects of credible seismic interactions, the SCEs must determine if the interactions are significant or not. The screening process for interaction effects includes evaluating the target, source, credibility, and significance. If all of these screens or considerations are satisfied, then the interaction being evaluated is an outlier and should be resolved as discussed in Chapter 12.

A significant interaction will compromise the intended performance and will affect the safety function of the equipment being evaluated. Examples of a significant interaction include an airoperated valve impacting a nearby structural column (see Figure 7.2-3), rupture of water sprinkler piping above medium-voltage switchgear, or a cart impacting a motor control center which contains vibration sensitive equipment such as essential relays.

A non-significant interaction, on the other hand, will not cause appreciable damage to the equipment being evaluated. Examples of a non-significant interaction include a light weight object impacting a large diameter conduit (see Figure 7.2-4) or small diameter piping impacting the outside casing of a rugged horizontal pump.

\subsubsection{Proximity ${ }^{2}$}

Seismic interaction due to proximity is the impact of adjacent equipment or structures on equipment due to their relative motion during seismic excitation. This relative motion can be the result of the vibration and movement of the equipment itself or any adjacent equipment or structures. When sufficient anchorage, bracing, adequate clearance, or other means are provided to preclude large deflections, seismic proximity effects are not typically a concern.

Even if there is impact between adjacent equipment or structures, there may not be any significant damage to the equipment. In such cases, this seismic interaction would not be considered a reason for concern, provided the equipment can still accomplish its intended function. One exception to this is electrical cabinets containing essential relays which are required to function. Since relays are susceptible to chatter, any impact on an electrical cabinet which has such an essential relay in it should be considered an unacceptable seismic interaction and cause for identifying that electrical cabinet as an outlier.

Examples of potential seismic interaction due to proximity include the following:

- Equipment carts, dollies, chains, air bottles, welding equipment, etc., may roll into, slide, overturn, or otherwise impact equipment

- Electrical cabinets, that deflect and impact walls, structural members, another cabinet, etc., may damage devices in the cabinet or cause devices to trip or chatter

- Storage cabinets, office cabinets, files, bookcases, wall lockers, and medicine cabinets may fall or tip into equipment

2 Based on Sections D.2 and D.6 of SQUG GIP (Ref. 1) 
- The doors on electrical cabinets may swing and impact devices or cause relays to chatter.

- Inadequately anchored or braced equipment such as pumps, vessels, tanks, heat exchangers, cabinets, and switchgear may deflect or overturn and impact equipment

The judgment of the SCEs should be used to differentiate between credible and non-credible interaction hazards.

\subsubsection{Piping, Raceways, and Ductwork Deflections ${ }^{3}$}

The motion of piping, conduit, cable raceways, and other distribution lines may result in impact interactions with equipment being reviewed. Non-safety-related piping is commonly supported with rod hangers or other forms of flexible dead load support, with little or no lateral restraint. Where adequate clearance with equipment is not provided, potential impact interaction may result. The integrity of the piping is typically not a concern. (Threaded fittings, cast iron pipes and fittings, and grooved type couplings may be exceptions where large anchor movement is possible.) In general, impacts between distribution systems (piping, conduit, ducts, raceways) and equipment of comparable size are not a cause for concern; the potential for large relative motions between dissimilar size systems should be carefully evaluated to assure that a large system cannot carry away a smaller one.

Judgment should be exercised by the SCEs in estimating potential motions of distribution systems in proximity to the equipment under evaluation. For screening purposes, a clearance of 2 inches for relatively rigid cable tray and conduit raceway systems and 6 inches for relatively flexible systems would normally be adequate to prevent impacts, subject to the judgment of the SCEs.

Where potential interaction may involve systems with significant thermal movements during facility normal operating conditions, the thermal displacements should be evaluated along with those resulting from seismic deflections. Inter-equipment displacement limits may be developed from the applicable floor response spectra to assist in this effort. In-structure response spectra (IRS) are discussed in Chapter 5.

\subsubsection{Mechanical and Electrical Equipment Deflections ${ }^{4}$}

Inadequately anchored or inadequately braced mechanical and electrical equipment, such as pumps, valves, vessels, cabinets, and switchgear, may deflect or overturn during seismic loading which results in impact with nearby equipment on the SEL. Certain items, such as tanks with high height-to-diameter aspect ratios, can deflect and impact nearby equipment. Electrical cabinets in proximity to each other may pound against each other or against walls and columns. Suspended equipment components such as room heaters and air conditioning units may impact with equipment.

The SCEs should use judgment in such cases to evaluate the potential displacements and their potential effect on nearby equipment being evaluated. Cabinets with essential relays warrant special concern.

\subsubsection{Structural Failure and Falling 5}

Equipment listed on the SEL can be damaged and unable to accomplish its function due to impact caused by failure of overhead or adjacent equipment, systems, or structures. This interaction

3 Based on Section D.2.1 of SQUG GIP (Ref. 1)

4 Based on Section D.2.2 of SQUG GIP (Ref. 1)

5 Based on Sections D.3 and D.6 of SQUG GIP (Ref. 1) 
hazard is commonly referred to as a Category II over Category I concern. This seismic interaction effect can occur from nearby or overhead: (1) mechanical and electrical equipment; (2) piping, raceway, and HVAC systems; (3) architectural features; and (4) operations, maintenance, and safety equipment. The seismic interaction effects which are of concern for these types of equipment, systems, and structures are described below. It is the intent of this evaluation that realistic hazards be identified and corrected; failure of non-seismically supported equipment and systems located over equipment being evaluated should not be arbitrarily assumed.

Facility operations, safety, and maintenance equipment as well as facility architectural features are commonly overlooked in seismic design programs and present sources of seismic interaction concerns. Examples of potential seismic interaction due to failure and falling include the following:

- Partition walls and unreinforced masonry block walls

- Ceiling tiles on unrestrained T-bar grid systems

- Overhead walkway platform grating lacking tie-downs

- Suspended light fixtures and fluorescent tubes

- Storage cabinets, files, and bookcases

- Tool carts on wheels and tool chests

- Ladders and scaffolding

- Portable testing equipment

- Unrestrained gas bottles and fire extinguishers

- Unrestrained equipment on wall-mounted supports

- Unreinforced masonry walls adjacent to equipment may spall or fall and impact equipment or cause loss of support of equipment

- Emergency lighting units and batteries used for emergency lighting can fall or overturn and damage equipment by impact or spilling of acid

- Fire extinguishers may fall and impact or roll into equipment

- Intercom speakers can fall and impact equipment

- Cable trays, conduit systems, and HVAC systems, including HVAC louvers and diffusers, may fall and impact equipment

- Structures or structural elements may deform or fall and impact equipment

- Architectural features such as suspended ceilings, ceiling components such as T-bars and acoustical panels, light fixtures, fluorescent tubes, partition walls, and plate glass may deflect, overturn or break and fall and impact equipment

- Grating may slide or fall and impact equipment

The judgment of the SCEs should be used to differentiate between credible and non-credible interaction hazards. 


\subsubsection{Mechanical and Electrical Equipment ${ }^{6}$}

Equipment such as tanks, heat exchangers, and electrical cabinets that are inadequately anchored or inadequately braced have historically overturned and/or slid due to earthquake excitation (see Figure 7.2-5). In some cases this has resulted in damage to nearby equipment or systems.

\subsubsection{Piping. Raceways, and HVAC Systems ${ }^{7}$}

Falling of non-seismically designed piping, raceways, and HVAC systems have been observed in very limited numbers during earthquakes. Most commonly reported are falling of inadequately secured louvers and diffusers on lightweight HVAC ducting. Damage to piping systems is less common and usually is limited to component failures which have rarely compromised system structural integrity. Typical damage is attributed to differential motions of systems resulting from movement of unanchored equipment, attachment of systems between buildings, or extremely flexible long runs of unrestrained piping. Very long runs of raceway systems pose a potential falling hazard when the runs are resting on, but not attached to, cantilever supports.

\subsubsection{Architectural Features ${ }^{8}$}

Architectural features include such items as ceilings, light fixtures, platform grating, unreinforced masonry walls, and other structures. The seismic interaction effects for these are described below:

- Ceilings. T-bar suspended tiles, recessed fixtures, and sheet rock are used in some facility areas (such as the control room). Seismic capabilities of these ceilings may be low. The SCEs should check for details that are known to lead to failure such as open hooks, no lateral wire bracing, etc. Section 10.5.2 discusses suspended ceilings.

- Light Fixtures. Normal and emergency light fixtures are used throughout the facility. Fixture designs and anchorage details vary widely. Light fixtures may possess a wide range of seismic capabilities. Pendant-hung fluorescent fixtures and tubes pose the highest risk of failure and damage to sensitive equipment. The SCEs should check for positive anchorage, such as closed hooks and properly twisted wires. Typically this problem is not caused by lack of strength; it is usually due to poor connections. Emergency lighting units and batteries can fall and damage equipment being reviewed due to impact or spillage of acid.

- Platform Gratings. Unrestrained platform gratings and similar personnel access provisions may pose hazards to impact-sensitive equipment or components mounted on them. Some reasonable positive attachment is necessary, if the item can fall.

- Unreinforced Masonry Walls. Unreinforced, masonry block walls should be evaluated for possible failure and potential seismic interaction with equipment being reviewed unless the wall has been seismically qualified. The SCEs should review the documentation for masonry walls to determine which walls have and which walls have not been seismically qualified. Section 10.5.1 discusses the qualification of these types of walls.

- Structures. If equipment being reviewed is located in lower Performance Category structures, then potential structural vulnerabilities of the building should be identified; however, facility structures are typically seismically adequate.

6 Based on Section D.3.1 of SQUG GIP (Ref. 1)

7 Based on Section D.3.2 of SQUG GIP (Ref. 1)

8 Based on Section D.3.3 of SQUG GIP (Ref. 1) 


\subsubsection{Operations, Maintenance, and Safety Equipment ${ }^{9}$}

Facility operations and maintenance require specialized equipment, some of which may be permanently located or stored in locations near safety systems. Some operations, maintenance, and safety equipment is designed so that it may be easily relocated by facility personnel. Where equipment design or facility operating procedures do not consider anchorage for permanently located equipment, this equipment may slide, fall, overturn, or impact with equipment listed on the SEL. Typically such equipment includes:

- Cabinets and Lockers. Inadequately restrained floor and wall-mounted filing cabinets and equipment storage lockers may result in overturning or falling and impact.

- Gas Storage Bottles. Unrestrained or inadequately restrained gas bottles may result in overturning and/or rolling and this may cause impact. In addition, the gas bottles can become high velocity projectiles if the reducing valve is snapped off and the gas bottles overturn and/or roll. Section 10.3.2 discusses further considerations for gas bottles.

- Refueling Equipment. Refueling equipment such as lifting equipment and servicing and refueling tools may be stored in proximity to equipment being evaluated. Inadequately restrained equipment may pose hazards.

- Monorails. Hoists, and Cranes. Monorails and service cranes are permanently located over heavy equipment requiring movement for service. Falling of service crane components such as tool and equipment boxes may result from inadequate component anchorage. They should be restrained from falling. Judgment by the SCEs should be used to assess the potential for and consequences of such equipment falling.

- Radiation Shields. Fire Protection and Miscellaneous Equipment. Temporary and permanent radiation shielding may pose hazards. Miscellaneous maintenance tools, such as chains and dollies, test equipment, fire protection equipment, fire extinguishers, and hose reels may fall if inadequately restrained. Equipment carts may roll into equipment being evaluated.

\subsubsection{Flexibility of Attached Lines and Differential Displacements ${ }^{10}$}

Distribution lines, such as small bore piping, tubing, conduit, or cable, which are connected to equipment can potentially fail if there is insufficient flexibility to accommodate relative motion between the equipment and the adjacent equipment or structures. Straight, in-line connections in particular are prone to failure. The scope of review for flexibility of these lines extends from the item of equipment being evaluated to their supports on the building or nearby structure. In addition, the review should consider operational concerns for the lines, such as the relationship of the lines to any check valve and sources of supply for the lines.

Distribution systems that span between different structural systems need to have sufficient flexibility to accommodate differential motion of the supporting structures (see Figure 7.2-6). Piping may be vulnerable where it interfaces with a building structure foundation.

9 Based on Section D.3.4 of SQUG GIP (Ref. 1)

10 Based on Sections D.4 and D.6 of SQUG GIP (Ref. 1) 
Examples of potential seismic interaction due to flexibility of attached lines include the following:

- Piping, cable trays, conduit, and HVAC may deflect and impact equipment

- Anchor movement may cause breaks in piping, cable trays, conduit, HVAC, etc. which may fall or deflect and impact adjacent equipment

The judgment of the SCEs should be used to differentiate between credible and non-credible interaction hazards.

\subsubsection{Water Spray, Flood, and Fire Hazards}

Potential seismic-induced spray, flood, and fire interaction sources should be evaluated and a few examples include the following:

- Hazardous/flammable material stored in unanchored drums, unanchored shelves, or unlocked cabinets

- Nonductile fluid-carrying pipe (such as cast-iron or PVC pipe) (see Figure 7.2-7)

- Fire protection piping with inadequate clearance around fusible-link sprinkler heads (see Figure 7.2-8)

- Natural gas lines and their attachment to equipment or buildings

- Acetylene bottles

- Mechanical and threaded piping couplings can fail and lead to pipe deflection or falling and impact on equipment. Grooved type couplings used in fire protection piping are one example of this type of mechanical coupling

- Sheetrock may fall and impact equipment if it was previously water-damaged or if there is severe distortion of the building

- Unanchored room heaters, air conditioning units, sinks, and water fountains may fall or slide into equipment

The judgment of the SCEs should be used to differentiate between credible and non-credible interaction hazards. 


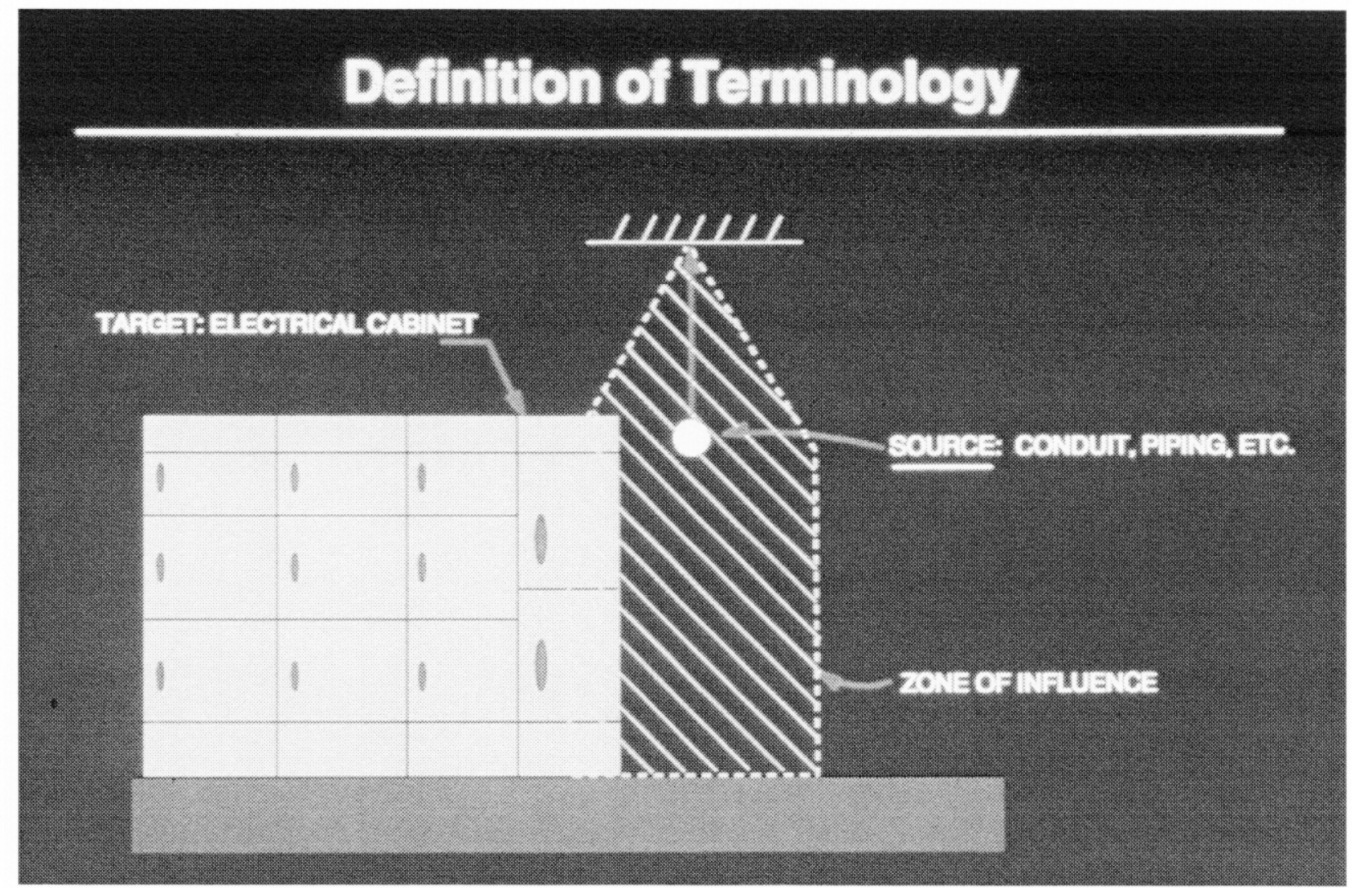

Figure 7.2-1 Example of Seismic Interaction 


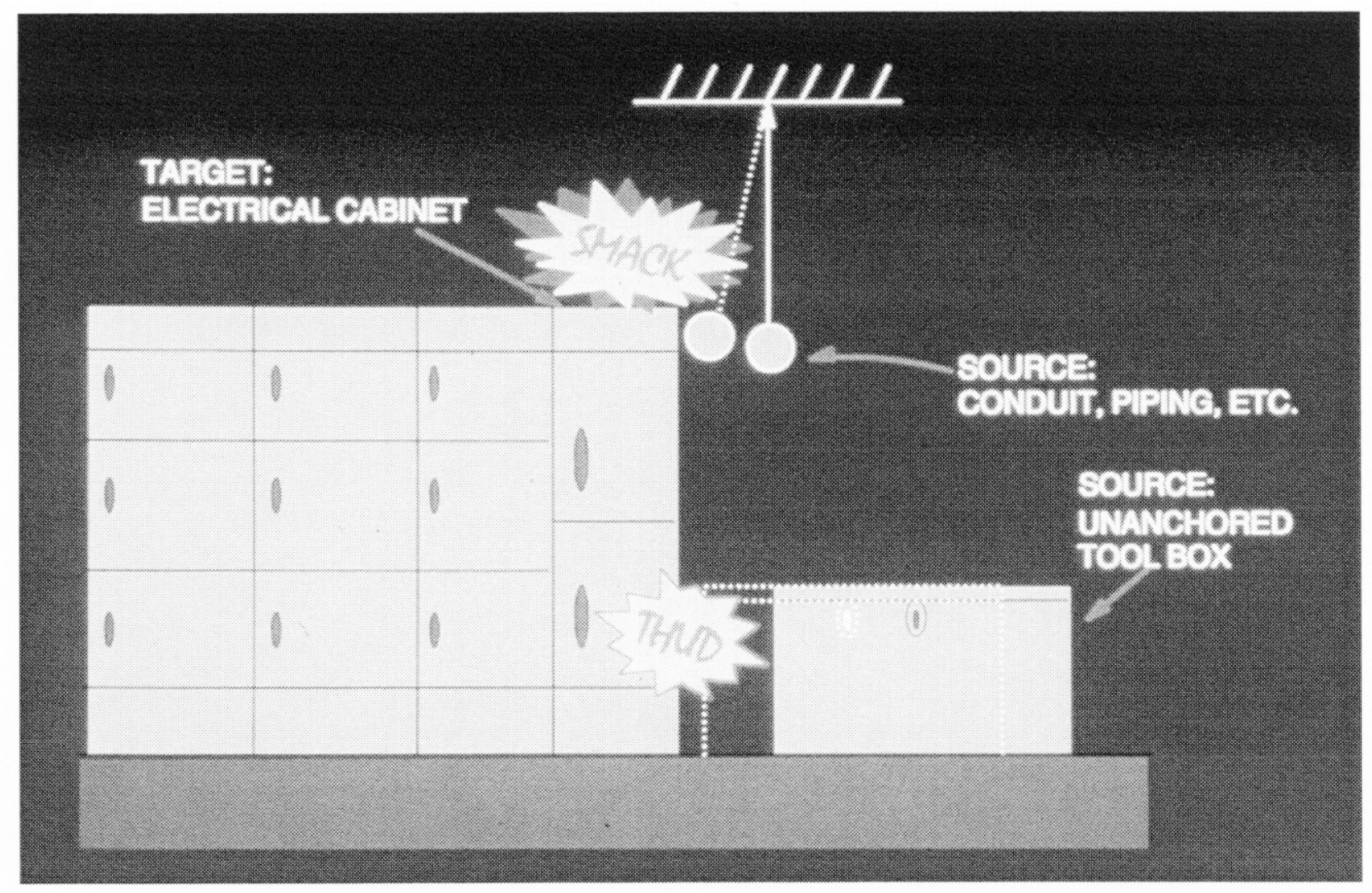

Figure 7.2-2 Example of Credible Interactions 


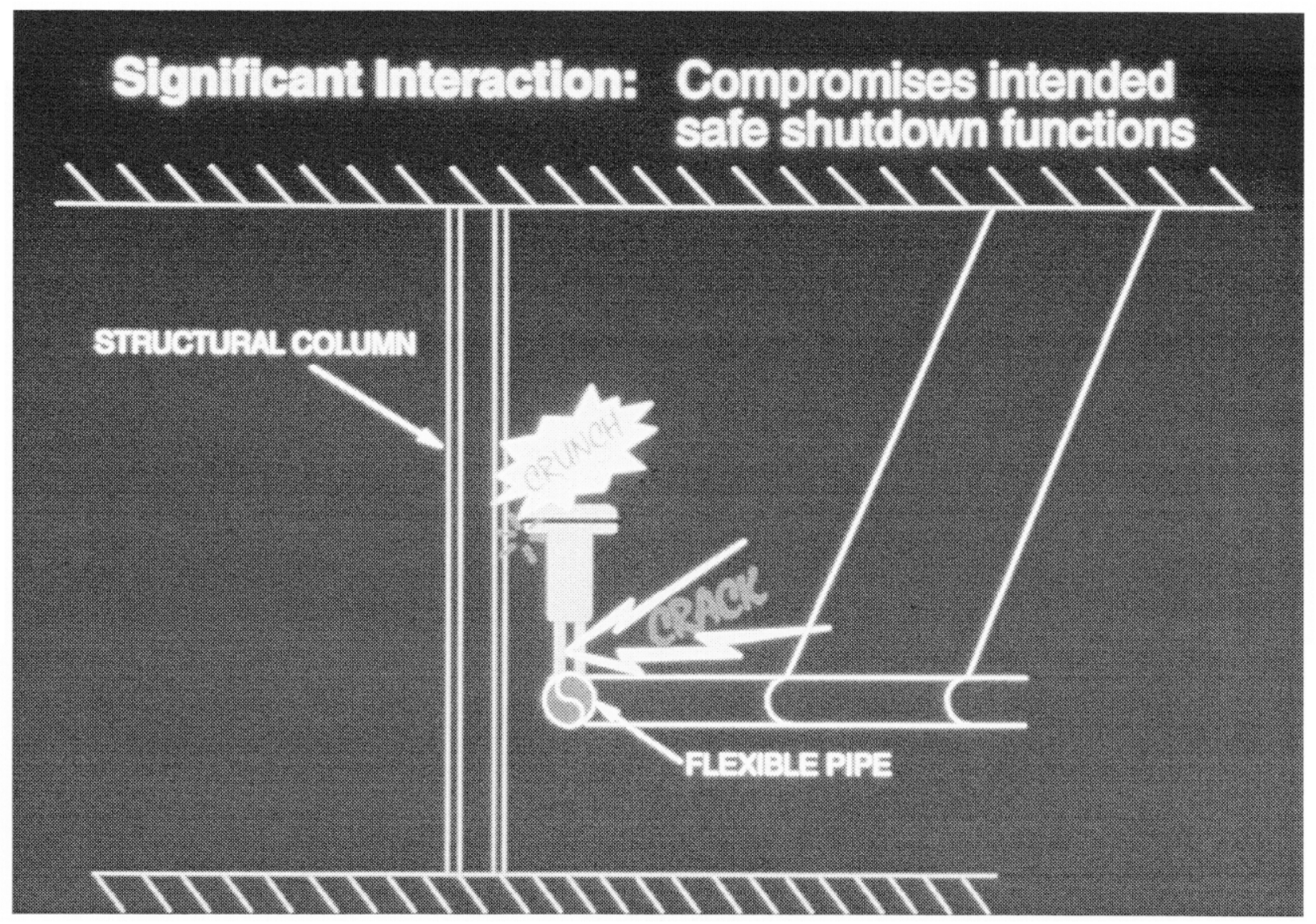

Figure 7.2-3 Example of Significant Interaction which Compromises Intended Safety Functions 


\section{Non-significant Interaction}
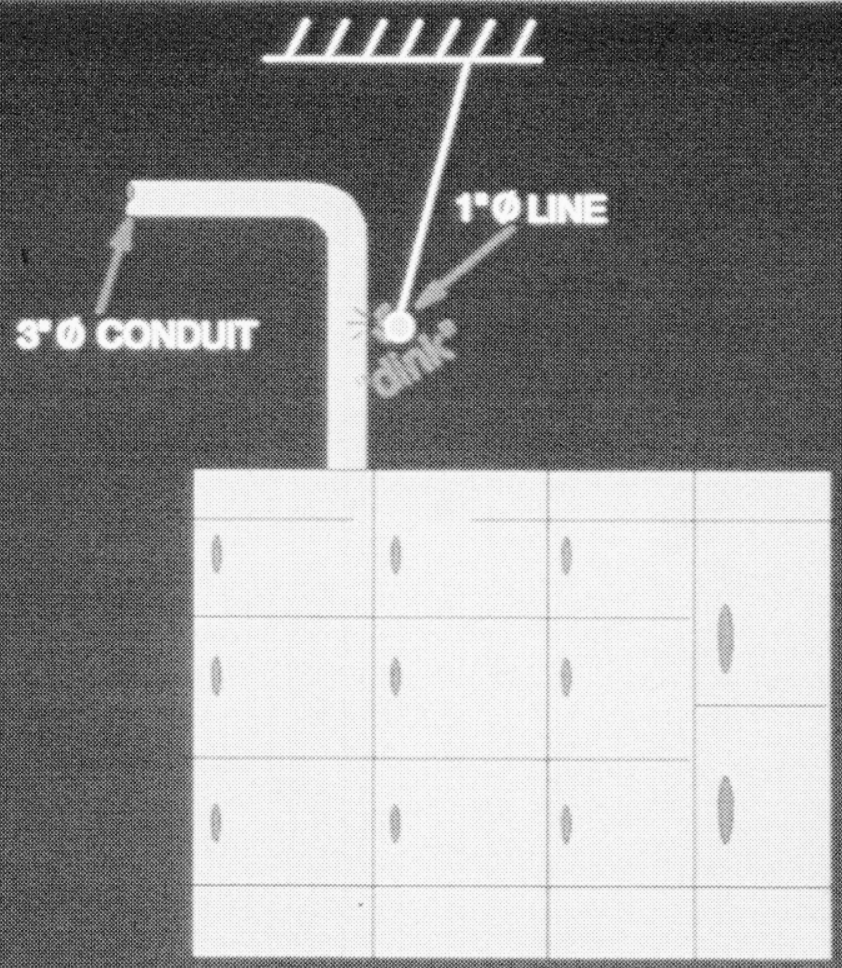

Figure 7.2-4 Example of Non-Significant Interaction 


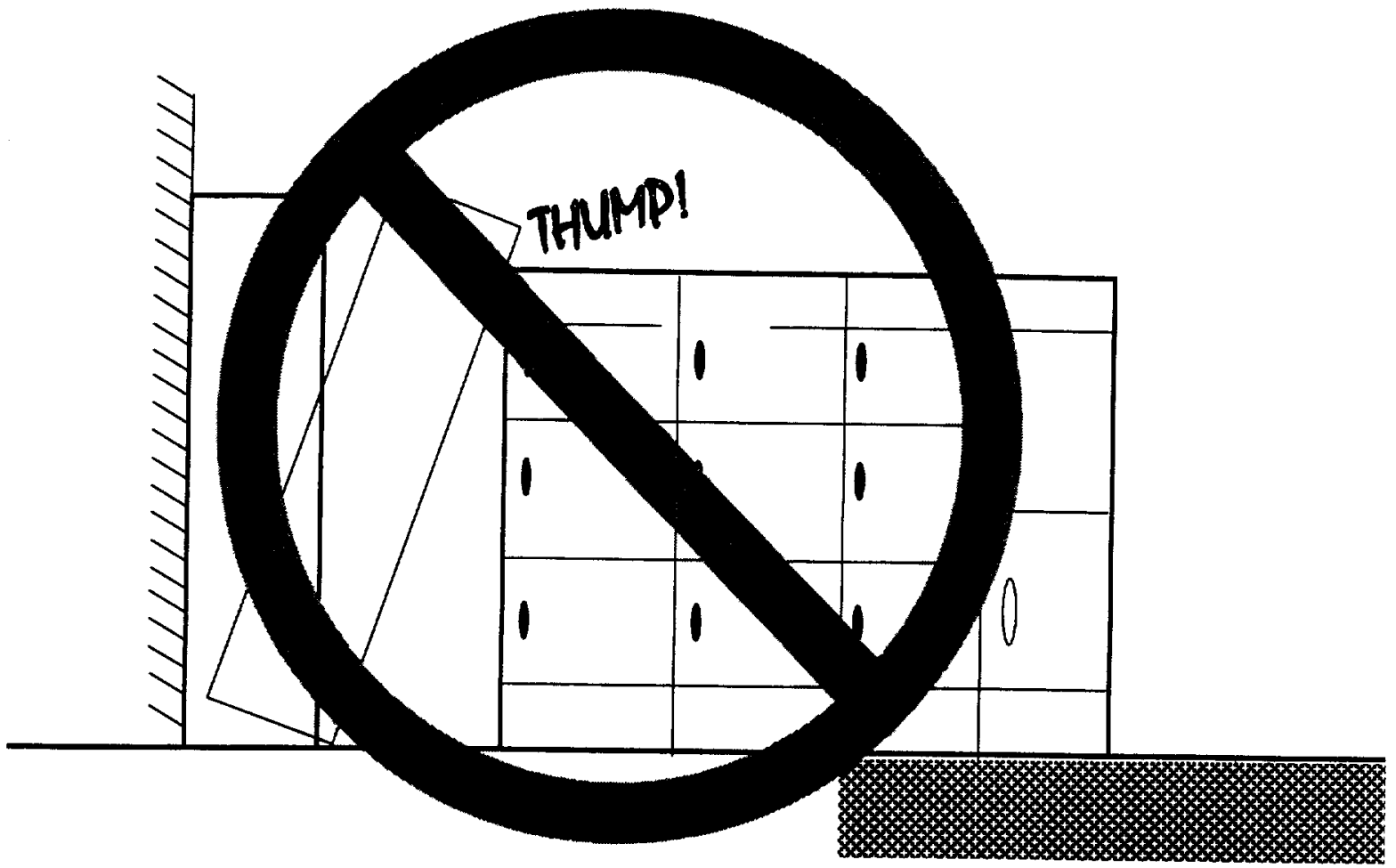

Figure 7.2-5 Failure and Falling Interaction Hazards 


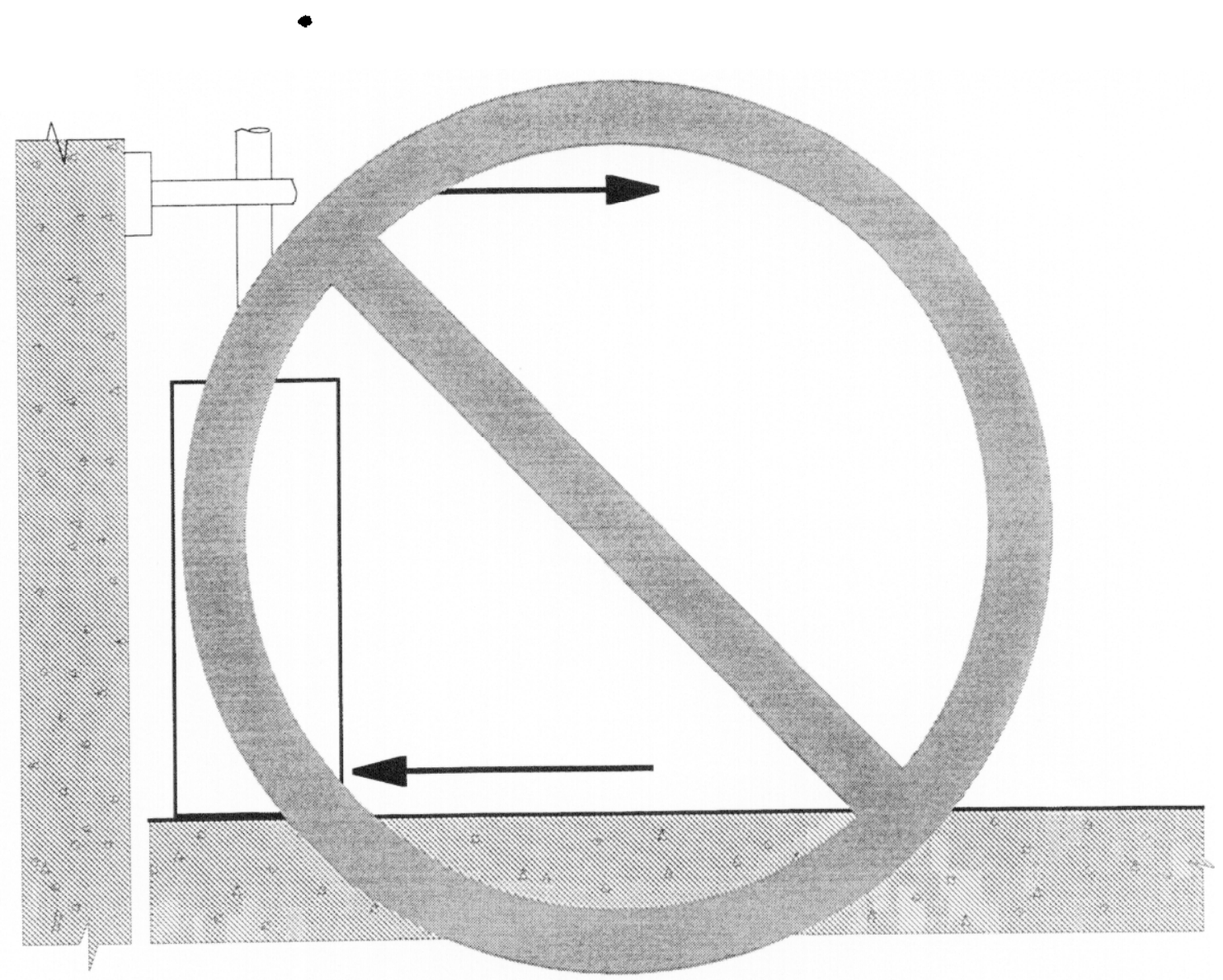

Figure 7.2-6 Differential Displacement Interaction 


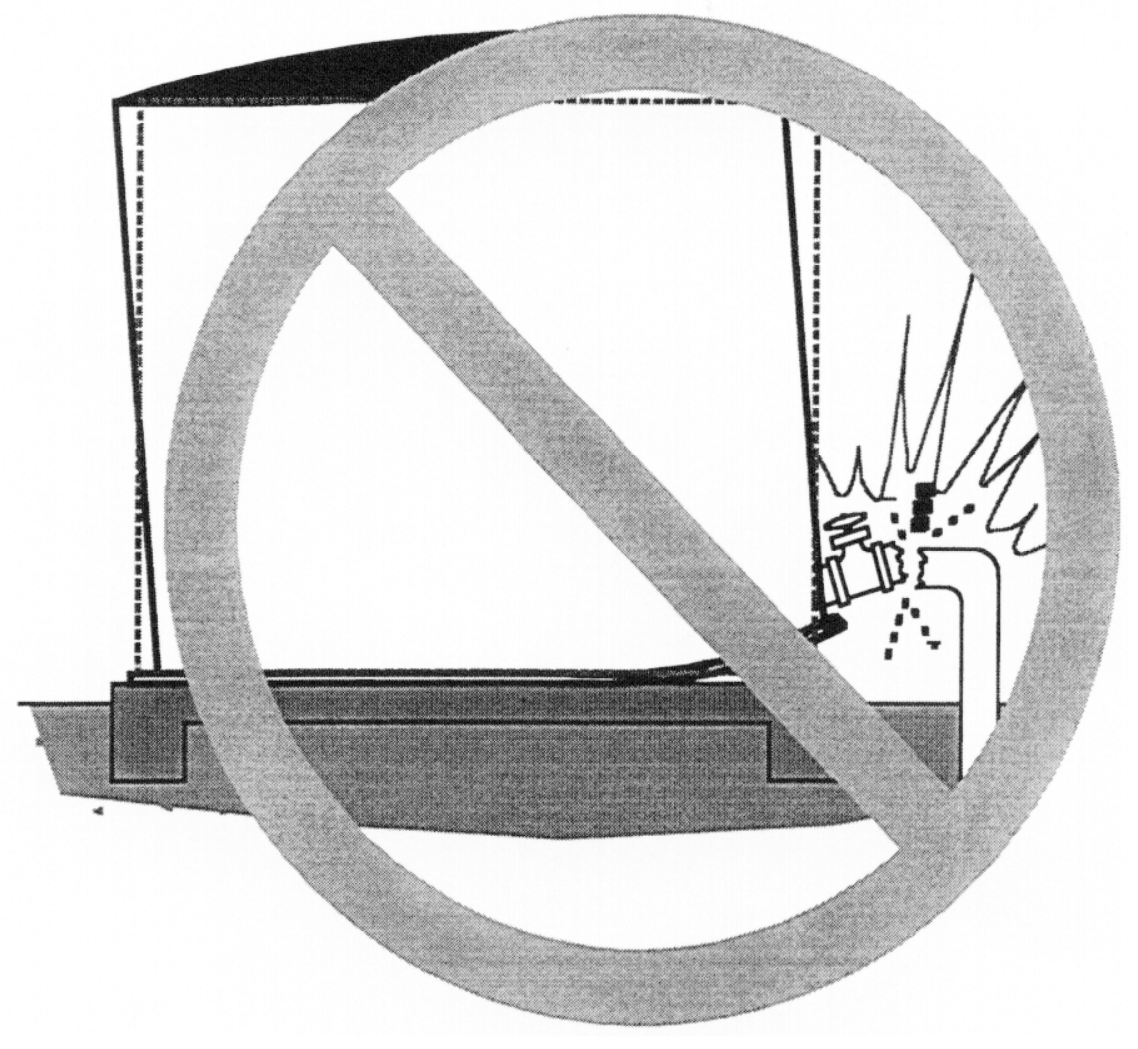

Figure 7.2-7 Pipe Break Potential for Unanchored Tanks 


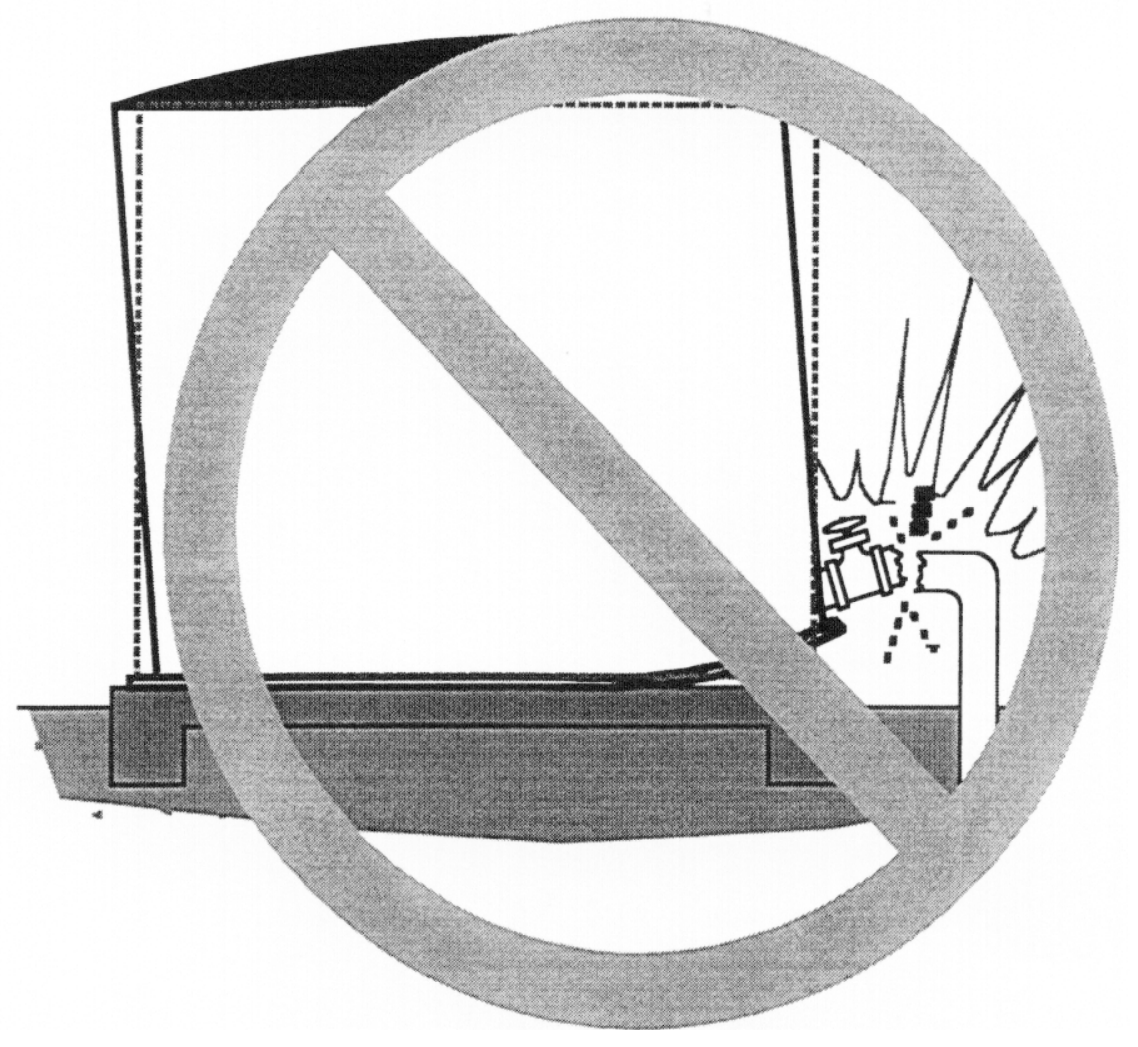

Figure 7.2-7 Pipe Break Potential for Unanchored Tanks 


\subsection{DOE GUIDANCE}

Guidance on the treatment of seismic interaction effects is included in DOE-STD-1021, "Natural Phenomena Hazards Performance Categorization Guidelines for Structures, Systems, and Components" (Ref. 7). This guidance focuses on "two over one" concerns and should be used to evaluate the seismic interaction effects discussed in Section 7.2. "Two over one" concerns, as discussed in DOE-STD-1021 and DOE-STD-3009 (Ref. 11), are those with a lower safety class structure, system, or component (SSC) located above, or able to interact with, a higher safety class SSC. Further detailed information on selecting performance and hazard categories is provided in References 7, 10, and 11 .

\subsubsection{System Interaction Effects ${ }^{11}$}

(a) An SSC that has been preliminary categorized in accordance with the basic performance categorization (PC) guidelines of Section 2.4 of Reference 7 (the source) shall have appropriate additional seismic mitigation requirements as provided in Paragraphs (b), (c) and (d) below, if its behavior by itself, or the multiple common-cause behavior of it with other SSCs may adversely affect the performance of other SSC (the target). These additional requirements will depend on the type of source behavior that causes adverse interaction with the target during or following an seismic event.

(b) If the source behavior that causes adverse interaction is within the acceptable behavior limits of the source (i.e., if the adverse interaction occurs before failure) adequate measures shall be taken to preclude such interaction and to ensure that the performance goal for the target is preserved. For example, assume that the postulated seismic deflection of a performance category (PC)-1 cabinet (source) is within its own acceptable behavior limits, but the cabinet can potentially impact and fail a PC-2 fire-suppression component (target). To prevent this adverse interaction, the cabinet support system or the cabinet itself can be stiffened/strengthened in such a way that the calculated deflection of the cabinet towards the target, when subjected to a seismic level corresponding to the performance category of the target, is less than the available clearance by a factor equal to the applicable design margin for the target. Alternatively, a barrier can be provided to preclude the adverse interaction and to protect the target. Such a barrier shall be designed to withstand seismic effects combined with the interaction effects from the source (in this case the impact from the PC-1 cabinet). To ensure that the target performance goal is preserved, the barrier shall be placed in the same performance category as the target (in this case PC-2).

(c) If the adverse interaction is possible only after the source fails or exceeds its acceptable behavior limits, either of the following two requirements shall be met to preclude adverse interaction:

(i) The source shall have additional seismic requirements corresponding to the performance category of the target, if the failure probability of the target, given the failure of the source, is greater than one percent. If the implementation of this criteria is judged not to be cost-effective, the additional seismic mitigation requirements for the source shall be in accordance with Table 7.3-1. In either case, these additional requirements can be restricted to the source failure mode related to the adverse interaction effects.

(ii) Adequate measures shall be taken to preclude adverse interaction and to ensure that the performance goal for the target is preserved. Examples of acceptable measures

11 Based on Section 2.5 of DOE-STD-1021 (Ref. 7) 
Table 7.3-1 System Interaction Effects on Performance Categorization (Reference 7)

\begin{tabular}{|c|c|c|c|}
\hline $\begin{array}{c}\text { Performance } \\
\text { Category of Target } \\
\text { SSC(1) }\end{array}$ & $\begin{array}{c}\text { Preliminary Performance } \\
\text { Category of Source } \\
\operatorname{SSC}^{(2)}\end{array}$ & $\begin{array}{c}\text { Range or Limit of } \\
\text { Target Failure Probability } \\
\text { Due to Interaction (3) } \\
\text { (p) }\end{array}$ & $\begin{array}{c}\text { Revised NPH } \\
\text { Requirements of } \\
\text { Source SSC }(4)\end{array}$ \\
\hline PC-4 & $\begin{array}{l}\text { PC-3 } \\
\text { PC-2 } \\
\text { PC-1 }\end{array}$ & $\begin{array}{c}p>10 \% \\
p \leq 10 \% \\
p>10 \% \\
1 \%<p \leq 10 \% \\
p \leq 1 \% \\
p>10 \% \\
1 \%<p \leq 10 \% \\
p \leq 1 \%\end{array}$ & $\begin{array}{c}P C-4 \\
P C-3^{(5)} \\
P C-4 \\
P C-3 \\
P C-2^{(5)} \\
P C-4 \\
P C-3 \\
P C-1^{(5)}\end{array}$ \\
\hline PC-3 & $\begin{array}{l}\text { PC-2 } \\
\text { PC-1 }\end{array}$ & $\begin{array}{l}p>10 \% \\
p \leq 10 \% \\
p>10 \% \\
p \leq 10 \%\end{array}$ & $\begin{array}{c}\text { PC-3 } \\
\text { PC-2 }{ }^{(5)} \\
\text { PC-3 } \\
\text { PC-1 }\end{array}$ \\
\hline PC-2 & PC-1 & $\begin{array}{l}p>10 \% \\
p \leq 10 \%\end{array}$ & $\begin{array}{c}\mathrm{PC}-2 \\
\mathrm{PC}-1^{(5)}\end{array}$ \\
\hline
\end{tabular}

SSC - Structure, System, or Component

NPH - Natural Phenomena Hazard

PC - Performance Category

Notes: (1) If the target consists of more than one SSC, the highest performance category of the group shall be considered here.

(2) This is the preliminary performance category of the source SSC before considering system interaction effects. Note that $P C-0$ is not considered here because a PC- 0 SSC cannot have any adverse effect on the performance of PC-1 through PC-4 SSCS

(3) This is the approximate probability of exceedance of acceptable behavior limit for the target SSC given that the source SSC will fail and interact with target SSC due to NPH effects.

Thus, if the target is a PC-4 SSC that may be adversely affected by the failure of a PC-2 SSC (source), and if the target failure probability due to this interaction is greater than $10 \%$, then one of the methods of precluding the interaction will be to subject the source to additional NPH requirements corresponding to PC-4 (see also note 4 below).

(4) The source SSC shall be designed/evaluated to those requirements of the revised performance category that are essential for precluding adverse interaction with the target (in other words, it is not necessary to satisfy the functional requirements of the source SSC when subjected to these additional NPH requirements unless essential for precluding adverse interaction).

The basis for determining the revised NPH requirements for the source SSC is that the performance goal of the target SSC shall not be compromised because of system interaction effects, i.e. the product of the performance goal for the revised source performance category and the target failure probability must not be more than the performance goal of the target SSC. However, to account for uncertainties in determining target failure probabilities, the limiting values in the 3rd column of the table have been selected conservatively (i.e. lower than the values computed on the above basis).

(5) For these cases, consideration of interaction effects does not require additional NPH requirements for the source SSC. 
are: stiffening/strengthening of the source structure or support system, relocating the source and/or the target, installing barriers, installing new components, modifying existing components, or any combination of these measures.

(d) If the behavior or failure of a source can adversely affect the performance of more than one target, the source shall have additional seismic requirements corresponding to the highest performance category that is determined by applying the rules provided in Paragraphs (a), (b), and (c) above separately for each target.

\subsubsection{Determination of System-Interaction-Related Target Failure Probability ${ }^{12}$}

To account for adverse system interaction, the determination of failure probability of the target component given the failure of the source component is required. Depending on the physical and functional complexity of the target and the nature of its interaction with the source, the level of effort in determining this target failure probability can vary. Following the" graded approach" philosophy, the level of rigor with which such failure probabilities are to be determined should depend on the safety significance and the preliminary performance category of the target, the hazard category of the facility, and the relative cost of various methods of determining target failure probabilities.

In the following paragraphs two methods of determining or estimating target failure probabilities are presented in order of decreasing rigor.

\section{(a) Systematic Analysis Method}

Target failure probabilities can be determined using a systematic analysis approach by constructing a fault-tree of the scenario. If justifiable from cost-benefit considerations, this may be a desirable method when necessary data is available. Generally, it should be used when the failure of the target is dependent on a large and complex chain of events that may follow the failure of the source, or to qualify a large system in its entirety. Component-bycomponent application of this method is unlikely to be cost-effective.

\section{(b) Approximate Method}

In this method, the effects of source failure on target are modeled approximately, but rationally, considering possible scenarios identified by review of system design. Even though such models are approximate, their analyses provide good "order-of magnitude" type of data that are often adequate for the purpose. Examples of the use of this method are given in Section 7.3.4.

\subsubsection{Application of System Interaction Rules ${ }^{13}$}

The consideration of adverse effects of system interaction of one component or system (source) on the other (target) is very important in determining performance categories of SSCs. Adverse interaction effects can be different for different systems. Examples of common adverse interaction effects are:

(i) Structural Failure and Falling (see Section 7.2.2): Inadequately designed, inadequately anchored, and unanchored components may fail, slide, and/or topple and fall on or bump into other components that are not designed to withstand such interaction effects.

12 Based on Section 3.8 of DOE-STD-1021 (Ref. 7)

13 Based on Section 3.9 of DOE-STD-1021 (Ref. 7) 
(ii) Proximity and Impact (see Section 7.2.1): Adjacent components may impact each other causing damage if the clearance between them is inadequate for seismic - induced deflections. Such adverse interaction may occur even if the deflection of the source is within its design limits.

(iii) Differential Displacement (see Section 7.2.3): A target distribution system (e.g., vital cable trays, pipes, ventilation ducts) may span between different structural systems (source). Differential displacement may be within acceptable behavior limits for the individual structures, but may still affect the distribution systems adversely.

(iv) Mechanical or Electrical Failure (see Section 7.2.4): The failure of a source mechanical or electrical component may impair the safety function of another component or system (e.g., the failure of a valve in a non-safety water distribution system causing flooding that shortcircuits a safety class electrical motor).

Paragraph (b) of Section 7.3.1 provides the general requirements for precluding interaction that can occur before the source fails or reaches its acceptable behavior limits. Paragraph (c) of Section 7.3.1 provides three options to meet the requirements for precluding adverse interaction that can occur only when the source fails. The following paragraphs provide additional discussions on these three options:

(a) The first guideline in Paragraph (c)(i) of Section 7.3.1 is the most conservative of the three options, because it requires additional seismic requirements if the failure probability of the target exceeds only $1 \%$. But it can also be most costly, since it may require upgrading the SSC. Hence, this guideline should be used when:

(i) upgrading of the source does not involve a significant design change, or

(ii) the existing source design has an adequate margin to withstand the same seismic level as the target.

(b) The second option in Paragraph (c)(i) of Section 7.3.1 requires the determination of target failure probability values, and depending on these values, the source may or may not need to be subjected to additional seismic requirements (see Table 7.3-1).

This guideline should be used if the application of conservative "one-percent" rule cannot be justified from cost-benefit considerations. For example, if it is determined that the application of the "one percent" rule will require a PC-1 source to have seismic requirements equivalent to a $\mathrm{PC}-4 \mathrm{SSC}$ resulting in expensive design changes, the use of Table 7.3-1 should be considered to reduce unnecessary conservatism.

(c) The third option given in Paragraph (c)(ii) of Section 7.3.1 requires the use of a barrier to prevent the source from interacting with the potential target. Very often this can be the most practical and cost-effective option. The barrier must be placed in the same performance category as the target, and be designed to withstand the interaction effects from the source in addition to the seismic loads. 


\subsubsection{Examples of Categorization Using System Interaction Rules ${ }^{14}$}

This subsection provides few examples of the application of categorization rules considering system interaction effects as provided in Section 7.3.1.

(a) Example 1

Consider an emergency diesel generator in a Hazard Category 2 facility that is classified as a safety system using appropriate DOE orders and general design criteria. The diesel, generator, and all their support systems (e.g., fuel, lubrication, cooling water, and DC power systems) that perform a safety function should be evaluated as PC-3 in accordance with the provisions of Section 2 of Reference 7.

Consider the fluorescent light (source) hung directly above the diesel. For this case, assume that the light is not needed for required operator actions following a seismic event. Hence its preliminary performance category is PC-1. Diesels themselves are fairly rugged, and a falling lightweight object, like the light fixture is unlikely to damage them. However, there are some possible weak spots, particularly in the peripheral support systems (e.g., lubrication lines) that might be damaged and result in system failure. Assume that, in accordance with Section 3.8 of Reference 7, the failure probability of the diesel resulting from the falling light fixture is estimated to be approximately $25 \%$. (This probability assumes the lighting fixture will fall. No credit is given at this stage for its design.) Following Paragraph (c)(ii) of Section 7.3.1, the lightning fixture should then be placed in PC-3.

\section{(b) Example 2}

Consider a case in which batteries for an uninterruptible power supply (UPS) in a Hazard Category 3 facility are in the same room with a 2000-gallon water storage tank. The UPS is classified as a safety system but the water storage tank is not. The UPS batteries (and their rack, connections, and the surrounding room structure) should be evaluated as PC-2 in accordance with the provisions of Section 2 of Reference 7.

Initially, the water storage tank might be considered as PC-1 (i.e., preliminary performance category). However, a systems-interaction check discloses that UPS batteries will short out during water immersion if only 1000 gallons of water flood the room. Thus, in accordance with criterion given in Paragraph (a)(i) of Section 7.3.1, the 2000-gallon tank should have the same performance category as the UPS batteries, that is, PC-2.

But what if the water was stored instead in ten 200-gallon tanks? The individual failure of each tank would not fail the UPS. However, if "multiple common-cause failure" is considered, one could reason that all ten tanks would be affected in the same way by the seismic event and simultaneous failure of several tanks might occur, leading to flooding of the batteries. Thus, each 200-gallon tank should also be placed in PC-2 in accordance with the provisions of Section 2 of Reference 7.

\section{(c) Example 3}

Consider a 100-foot-tall smoke stack for a laundry building at a DOE site that is not part of any safety system. However, its failure (from winds or earthquakes) would be costly and could injure workers, so initially it would be classified as Preliminary PC-1. Consider that there is a single Hazard Category (HC) 3 safety system component (say a PC-2 outside pump) that is 90 feet from

14 Based on Section 3.10 of DOE-STD-1021 (Ref. 7) 
the base of the stack. A systems interaction analysis may assume that the stack would fall in essentially one piece and would fail the pump if it hits it. But the stack is equally probable to fall in any radial direction and the target size of the pump is small, fitting into a 2 degree angle. It is concluded that the probability of the stack hitting the component is less than $1 \%$. Thus in accordance with Paragraph c(ii) of Section 7.3.1, the stack can be retained in PC-1.

\section{(d) Example 4}

Consider a Hazard Category 1 facility that relies heavily on operator actions, rather than seismically-qualified instrumentation and automatic control systems, to maintain a safe-state following a design basis earthquake. According to Section 2 of Reference 7, safety system SSCs of this facility should be placed in PC-4. In addition, SSCs needed to permit required operator actions following a design basis earthquake must also be classified as PC-4.

As an example, assume that one earthquake procedure written for this facility requires that an operator would go inside the pump room to read a water level gauge (which is seismicallyqualified), and then relay this information to the control room via a system of walkie-talkies (assume that inside telephone lines are not seismically qualified). Items needed to permit this action, and thus which must meet PC -4 criteria, include all access doors (deformation of the door frames may be critical), emergency lightning and communication systems (the storage of flashlights and walkie-talkies could become a seismic design consideration), and any water or steam line whose seismic failure would be hazardous to the operator.

\section{$7.4 \quad$ EVALUATION OF INTERACTION EFFECTS 15}

The SCEs should identify and evaluate all credible and significant interactions in the immediate vicinity of the equipment listed on the SEL. This includes consideration of seismic interactions on the equipment itself and on any connected distribution lines (e.g., instrument air lines, electrical cable, and instrumentation cabling) which are in the vicinity of the item of equipment. Evaluation of interaction effects should consider detrimental effects on the capability of equipment and systems to function; taking into account equipment attributes such as mass, size, support configuration, and material hardness in conjunction with the physical relationships of interacting equipment, systems, and structures. In the evaluation of proximity effects and overhead or adjacent equipment failure and interactions, the effects of intervening structures and equipment which would preclude impact should be considered. The effects of fire, flooding or exposure to fluids from ruptured vessels and piping should also be examined.

As summarized in this chapter, the considerations for seismic interaction effects include the following:

1. Soft targets free from impact by nearby equipment or structures.

2. If equipment contains sensitive essential relays, equipment free from all impact by nearby equipment or structures.

3. Attached lines have adequate flexibility.

4. No collapse of overhead equipment, distribution systems, or masonry walls.

5. Equipment is free from credible and significant seismic-induced flood and spray concerns.

15 Based on Section D.5 of SQUG GIP (Ref. 1) 
6. No credible seismic-induced fire concerns.

7. No other "two over one" concerns as defined in DOE-STD-1021.

8. No other concerns.

Good housekeeping within a facility can prevent many possible sources of seismic interaction. Miscellaneous equipment or supplies such as carts, ladders, brooms, and dollies can be easily stored such that they do not become sources of seismic interaction. In addition, the general arrangement of the facility and its contents can be developed to accommodate clearances and "stayout" zones for the equipment being evaluated.

Damage from interaction in earthquakes is from unusual circumstances or from generic, simple details such as open hooks on suspended lights. The SCEs should spend most of their time evaluating: 1) unusual impact situations, and 2) lack of proper anchorage or bracing. The SCEs should not be concerned much with interaction issues due to piping and other system or structural component failures. 


\section{Part III}

\section{Seismic Evaluation Procedures Adopted Directly from the SQUG GIP}




\section{EQUIPMENT CLASS EVALUATIONS USING CAVEATS FOR THE REFERENCE SPECTRUM AND / OR GERS}

Chapter 8 contains a summary of equipment class descriptions based on earthquake experience data and generic seismic testing data. These descriptions and the rest of the information in Chapter 8 is from Appendix B of Revision 2 of the SQUG GIP (Ref. 1). Any modifications from the corresponding sections of Appendix $B$ are denoted in Chapter 8 with words in italics (such as this introduction to Chapter 8). An item of equipment must have the same general characteristics as the equipment in the earthquake experience equipment class or the generic seismic testing equipment class to apply the methodology in Chapter 8. The intent of this rule is to preclude items of equipment with unusual designs and characteristics that have not demonstrated seismic adequacy in earthquakes or tests.

"Caveats" are defined as the set of inclusion and exclusion rules that represent specific characteristics and features particularly important for seismic adequacy of a particular class of equipment. Chapter 8 contains a summary of the caveats for the earthquake experience equipment class and for the generic seismic testing equipment class. If the caveats are satisfied, then the capacity of the equipment class can be represented by the Reference Spectrum and/or the GERS. For these equipment classes, extensive use of earthquake experience and test data permits the rigorous definition of the equipment capacity and evaluation of the seismic adequacy of the equipment. The equipment capacity determined in Chapter 8 is compared to the seismic demand using the provisions of Chapter 5.

The "intent" of the caveats should be met when evaluating an item of equipment as they are not fixed, inflexible rules. Engineering judgment may be used to determine whether the specific seismic concern addressed by the caveat is met. Chapter 8 provides brief discussions of the intent of the caveats. When specific cases are identified where the intent of the caveats are considered to be met, but the specific wording of the caveat rule is not, the reason for this conclusion should be documented.

Note that the caveats in Chapter 8 are not necessarily a complete list of every seismically vulnerable detail that may exist since it is impossible to cover all such situations by meaningful caveats. Instead, the SCEs should exercise their judgment and experience to seek out suspicious details or uncommon situations (not specifically covered by the caveats) which may make equipment vulnerable to earthquake effects. For example, the SCEs should note any areas of concern within the "box" which could be seismically vulnerable such as added attachments, missing or obviously inadequate anchorage of components, heavy objects mounted on the equipment, and components that are known to be seismically sensitive.

The summaries of the equipment class descriptions and caveats in Chapter 8 are based on information contained in References 19,35, and 40. Additional information on seismic experience data is contained in Chapter 9d of Reference 32. The SCEs should use the summaries in Chapter 8 only after first thoroughly reviewing and understanding the background of the equipment classes and bases for the caveats as described in these references. These references provide more details (such as photographs of the data base equipment) and more discussion than summarized in Chapter 8. Note that in some cases, clarifying remarks have been included in Chapter 8 that are not contained in the reference documents. These clarifying remarks include such things as the reason for including a particular caveat, the intent of the caveat, and recommended allowable limits for stress analysis. The remarks are also based on experience gained during SQUG GIP reviews at operating nuclear power plants and DOE seismic evaluations at DOE facilities and they serve to help guide the SCEs in their judgment. 
Certain important caveats from the reference documents are not included in Chapter 8 because they are covered in other sections of the DOE Seismic Evaluation Procedure. These caveats include:

- Equipment should be adequately anchored and base isolation should be carefully evaluated (see Chapter 6).

- Seismic interaction concerns, such as flexibility of attached lines, should not adversely affect the equipment (see Chapter 7).

- Relays for which chatter is not acceptable should be specifically evaluated. Note that although the primary responsibility for conducting the relay evaluation is the Lead Relay Reviewer, the SCEs should be alert for any seismically induced systems effects that may lead to loss of function or malfunction of the equipment being evaluated (see Chapter 11).

In addition, caveats discussing a limiting fundamental frequency of $8 \mathrm{~Hz}$ are not included in Chapter 8 because this limiting frequency does not apply with the provisions of Chapter 5. Table 8-1 lists the numbers of the SQUG GIP caveats which have been removed for Chapter 8 of the DOE Seismic Evaluation Procedure.

Table 8.1 SQUG GIP (Ref. 1) Caveats which are Removed for Chapter 8

\begin{tabular}{|c|c|c|}
\hline $\begin{array}{c}\text { Section in } \overline{D O E} \\
\text { Seismic Evaluation } \\
\text { Procedure }\end{array}$ & $\begin{array}{c}\text { SQUG GIP (Ref. I) } \\
\text { Bounding Spectrum } \\
\text { Caveats Removed for } \\
\text { Chapter } 8\end{array}$ & $\begin{array}{l}\text { SQUG GIP (Ref. 1) } \\
\text { GERS Caveats Removed } \\
\text { for Chapter } 8\end{array}$ \\
\hline 8.1 .1 & $\# 9$ & \\
\hline 8.1 .2 & $\# 9, \# 10$, and \#11 & \\
\hline 8.1 .3 & \#10 and \#11 & $\# 6, \# 7$, and \#8 \\
\hline 8.1 .4 & $\# 10$ and \#11 & $\# 7, \# 8$, and \#9 \\
\hline 8.1 .5 & \#6 and \#7 & \\
\hline 8.1 .6 & \#11 and \#12 & \\
\hline 8.1 .7 & \#7 and \#8 & \\
\hline 8.1 .8 & $\# 8, \# 9$, and $\# 10$ & $N / A$ \\
\hline 8.1 .9 & $\# 5, \# 6, \# 7$, and \#8 & \\
\hline 8.1 .10 & $\# 4$ & $N / A$ \\
\hline 8.2 .1 & $\# 8$ & \\
\hline 8.2 .2 & $\# 7$ & \\
\hline 8.2 .3 & $\# 5$, \#6, \#7, and \#8 & $N / A$ \\
\hline 8.2 .4 & $\# 4, \# 5$, and \#6 & $N / A$ \\
\hline 8.2 .5 & $\# 3, \# 4$, and \#5 & N/A \\
\hline 8.2 .6 & $\# 2, \# 3, \# 4$, and $\# 5$ & $N / A$ \\
\hline 8.2 .7 & $\# 3, \# 4, \# 5$, and \#6 & $\overline{N / A}$ \\
\hline 8.2 .8 & $\# 3, \# 4, \# 5$, and \#6 & $\overline{N / A}$ \\
\hline 8.2 .9 & $\# 5$, \#6, \#7, and \#8 & N/A \\
\hline 8.2 .10 & $\# 5, \# 6$, and \#7 & $N / A$ \\
\hline
\end{tabular}

Chapter 8 is organized by equipment class as listed in Table 2.1-2. For each equipment class, the class description and the caveats applicable to the Reference Spectrum are given first. A plot of the Reference Spectrum is provided in Chapter 5. Next, the class description and the caveats applicable to the GERS are given, when available. Some equipment classes have more than one GERS while other classes have none. A plot of the GERS follows the caveats for each applicable equipment class. While the GERS typically define a higher capacity, the GERS caveats are more restrictive than the reference spectrum caveats. 


\subsection{ELECTRICAL EQUIPMENT}

\subsubsection{BATTERIES ON RACKS ${ }^{1}$}

The seismic capacity for the equipment class of Batteries on Racks (BAT) (see Figure 8.1.1-1) may be based on earthquake experience data, provided the intent of each of the caveats listed below is met. This equipment class includes both storage batteries and their supporting structures. Most battery systems consist of lead-acid storage batteries mounted in series on steel-frame racks or wooden racks.

A battery is a group of electro-chemical cells interconnected to supply a specified voltage of DC power. Individual battery weights typically range from about 50 to 450 pounds. Batteries are used to supply a steady source of DC power for circuits in control and instrumentation systems, to power DC starter motors for emergency engine-generators, and to provide DC power to inverters for uninterruptible power systems.

Lead-acid storage batteries are the most prevalent type of battery and are the subject of this equipment class. The basic components of a lead-acid battery cell are the electrode element, cell cover, cell jar, electrolyte, and flame arrestor. The electrode elements are the key components of the battery system.

There are four basic types of lead-acid storage batteries which are distinguished by the construction of their positive plates. These four types are: calcium flat plate, Planté or Manchex, antimony flat plate, and tubular. Since there are no examples of antimony flat plate and tubular batteries in experience data, they are excluded from the equipment class. The Planté or Manchex battery is one of the older designs of batteries. It is constructed of heavy lead plate with either a series of horizontal cross-ribs attached to the plate (Planté plate design), or a matrix of spiral buttons inserted into the plate (Manchex design).

Battery racks are normally frames of steel channels, angles, and struts that support the batteries above the floor. Racks can be multi-rowed, multi-tiered, or multi-stepped. Multi-rowed racks are adjacent rows of batteries all at the same level. Multi-tiered racks are vertical rows of batteries mounted directly above each other. Multi-stepped racks have each succeeding row of batteries located above and to the rear of the previous row.

The shelf that supports the batteries typically consists of steel channels running longitudinally that are, in turn, supported by transverse rectangular frames of steel angles. The racks are usually braced by diagonal struts along either the front or rear face for longitudinal support. The rack members are connected by a combination of welds and bolts.

Well-designed battery racks include a restraining rail running longitudinally along the front and the rear of the row of batteries and wrapping around the ends of the row. The rails are located at about mid-height of the battery, and can prevent accidental overturning of the batteries, or overturning from earthquake loadings.

The battery (including the cell jar and enclosed plates, the supporting rack, electrical connections between batteries (bus bar), and attached electrical cable) are included in the Batteries on Racks equipment class.

1 Section B.15 of SQUG GIP (Ref. 1) 


\subsubsection{Reference Spectrum Caveats - Batteries On Racks}

The Reference Spectrum (RS) represents the seismic capacity of Batteries on Racks (BAT) if the batteries and racks meet the intent of the following inclusion and exclusion rules. Note, however, that when the specific wording of a caveat rule is not met, then a reason for concluding that the intent has been met should be provided on the SEWS.

BAT/RS Caveat 1 - Earthquake Experience Equipment Class. The batteries and racks should be similar to and bounded by the BAT class of equipment described above. The equipment class descriptions are general and the SCEs should be aware that worst case combinations of certain parameters may not be represented in the generic equipment class. These worst case combinations may have reduced seismic capacity and should be carefully evaluated on a case-by-case basis.

BAT/RS Caveat 2 - Plates of the Battery Cells Are Lead-Calcium Flat-Plate or They Are of Planté or Manchex Design. The plates of the battery must be of the lead-calcium flat-plate or the Planté or Manchex design. These are the only battery cell types included in the earthquake experience equipment class.

BAT/RS Caveat 3 - Each Individual Battery Weighs Less Than 450 Pounds. Individual battery cells should weigh less than about 450 pounds. This is the upper bound weight of the battery cells included in the earthquake experience equipment class.

BAT/RS Caveat 4 - Close-Fitting. Crush-Resistant Spacers Between Cells. There should be closefitting, crush-resistant spacers between the cells, which fill about two-thirds of the vertical space between the cells. The concern is that the batteries without spacers can rock and collide during the earthquake causing malfunction and damage.

BAT/RS Caveat 5 - Batteries Restrained by Side and End Rails. The battery racks should have end and side rails incorporated in the design. The end and side rails should also be close fitting against the cells (with shims, if needed). The concern is that batteries on racks without end and side rails may tip or slide off the rack.

BAT/RS Caveat 6 - Battery Racks Have Longitudinal Cross Bracing. The racks should have longitudinal cross bracing unless engineering judgment or analysis shows that such bracing is not needed. The concern is that racks without cross bracing may not be able to transfer the lateral seismic loads to the base support. Simple bounding hand calculations are recommended to show that the structural components of the rack are capable of transferring these loads. The capacity of rack steel members may be calculated following AISC Part 2 (Ref. 81) allowable stresses.

BAT/RS Caveat 7 - Racks Constructed of Wood To Be Evaluated. Battery racks constructed of wood should be specially evaluated. The concern is that racks constructed of wood may be more vulnerable to seismic loads than steel racks. Evaluation of the rack should consider industry accepted structural design standards for wood construction, using extreme load allowable stresses as appropriate.

BAT/RS Caveat 8 - Batteries Greater Than 10 Years Old To Be Evaluated. Batteries that are more than 10 years old should be identified as outliers. The concern with the aging of batteries is that some models have been shown by shake table testing to be susceptible to structural and or metallurgical changes with time that result in either structural failure or reduced capacity after vibration.

BAT/RS Caveat 9-Any Other Concerns? SCEs should seek out suspicious details or uncommon situations not specifically covered by the caveats which could adversely affect the seismic capacity of the batteries on racks. 


\subsubsection{GERS Caveats - Batteries on Racks}

The seismic capacity for the equipment class of Batteries on Racks (BAT) may be based on generic testing data, provided the intent of each of the caveats listed below is met. This equipment class includes storage battery sets of the lead-calcium type supported on racks with rail restraints. Each battery set consists of multiple lead-acid cells (nominal 2 volts each) interconnected by rigid bus connectors. Rows or groups of cells are connected by flexible bus connectors. The racks have either a two-step or single-tier configuration with longitudinal cross-braces. The racks have rail restraints to keep the batteries in place. There are snug-fitting spacers between the cells and, if needed, shims between the cells and rails. This equipment class covers typical stationary lead-acid battery cells used in facilities.

The GERS (see Figure 8.1.1-2) represent the seismic capacity of Batteries on Racks (BAT) if the batteries and racks meet the intent of the following inclusion and exclusion rules. Note, however, that when the specific wording of a caveat rule is not met, then a reason for concluding that the intent has been met should be provided on the SEWS.

BAT/GERS Caveat 1 - Generic Seismic Testing Equipment Class. The batteries and racks should be similar to and bounded by the BAT class of equipment described above. The equipment class descriptions are general and the SCEs should be aware that worst case combinations of certain parameters may not be represented in the generic equipment class. These worst case combinations may have reduced seismic capacity and should be carefully evaluated on a case-by-case basis.

BAT/GERS Caveat 2 - Reference Spectrum Caveats Apply. The batteries on racks should meet all the caveats given for the Reference Spectrum. This caveat is included to cover the vulnerabilities identified for the earthquake experience equipment class. Those GERS caveats which are the same as the Reference Spectrum caveats are not repeated below.

BAT/GERS Caveat 3 - Lead-Calcium Plates. The plates of the battery cell should be lead-calcium. Lead-calcium battery cells are the only type included in the generic seismic testing equipment class.

BAT/GERS Caveat 4 - Supported on Two-Step or Single-Tiered Racks with Longitudinal CrossBraces. The batteries should be supported on two-step racks or single-tier racks which have longitudinal cross-braces as supplied by the battery manufacturer (review of manufacturer's submittals is sufficient). A row of batteries should be restrained by double rails in front, back and on the ends, symmetrically placed with respect to the cell center of gravity. The concerns addressed by this caveat are that racks may not be able to transfer the lateral seismic loads to the base support, and that the natural frequencies of the rack may be lower than those in the generic seismic testing equipment class.

If the battery rack is custom made and/or does not have longitudinal cross-braces supplied by the manufacturer, then the intent of this caveat can be satisfied by showing that the racks have adequate strength (i.e., within 1.6 times normal AISC allowable stress limits) and have natural frequencies above about $8 \mathrm{~Hz}$ horizontal and $20 \mathrm{~Hz}$ vertical. If the natural frequency of the rack is below these values, then a realistic amplification through the rack to the center of gravity of the batteries should be included when determining the amplified response of the batteries for comparison to the GERS (for this case the GERS represents the battery capacity).

If the racks only have a single rail, then this rail should be evaluated to determine whether it will hold the cells in place and prevent significant relative motion between cells. 


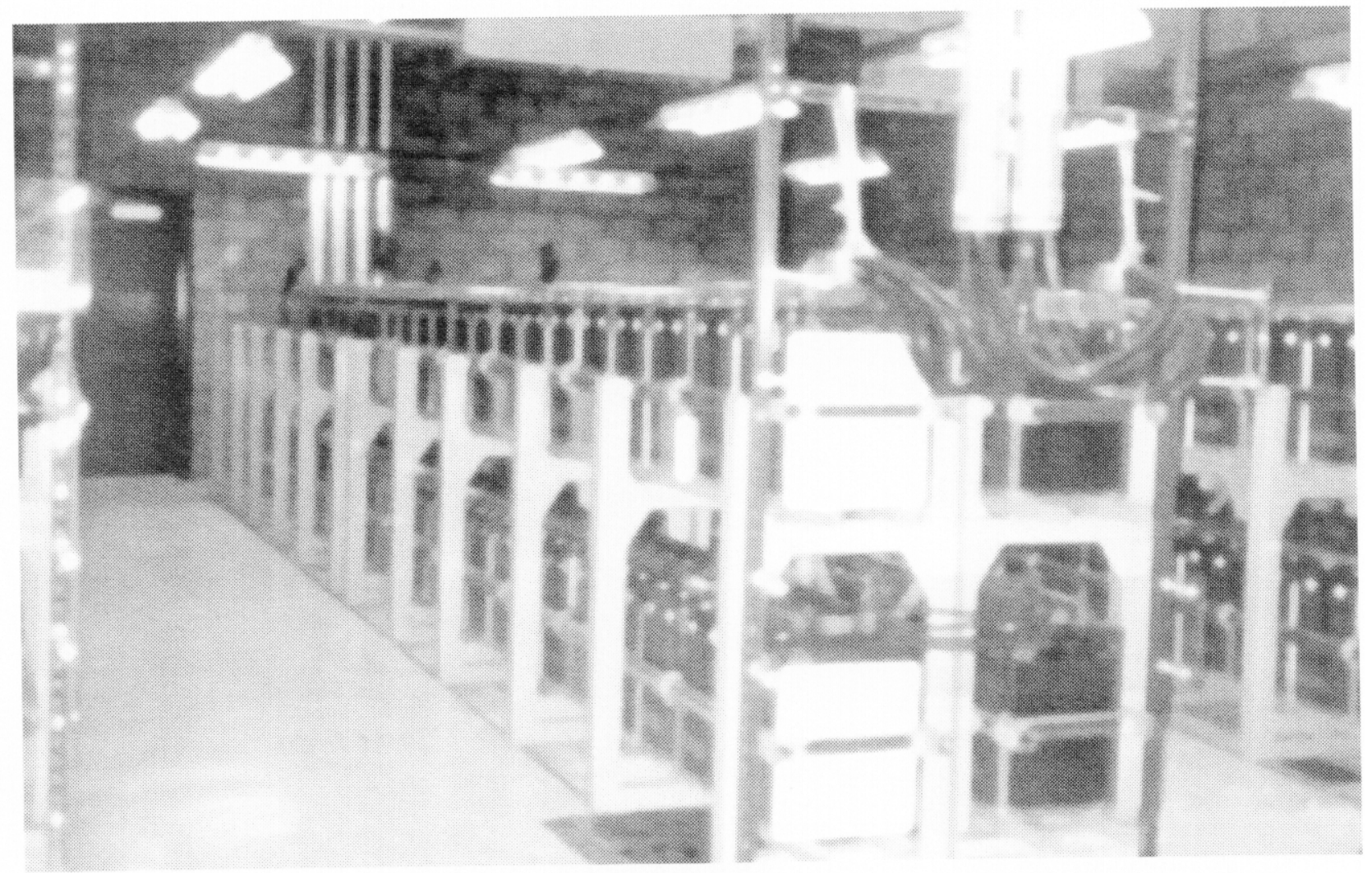

Figure 8.1.1-1 Batteries on Racks from the Earthquake Experience Database 


\section{Batteries on Racks \\ GERS-BAT.4 \\ $8 / 1 / 86$}

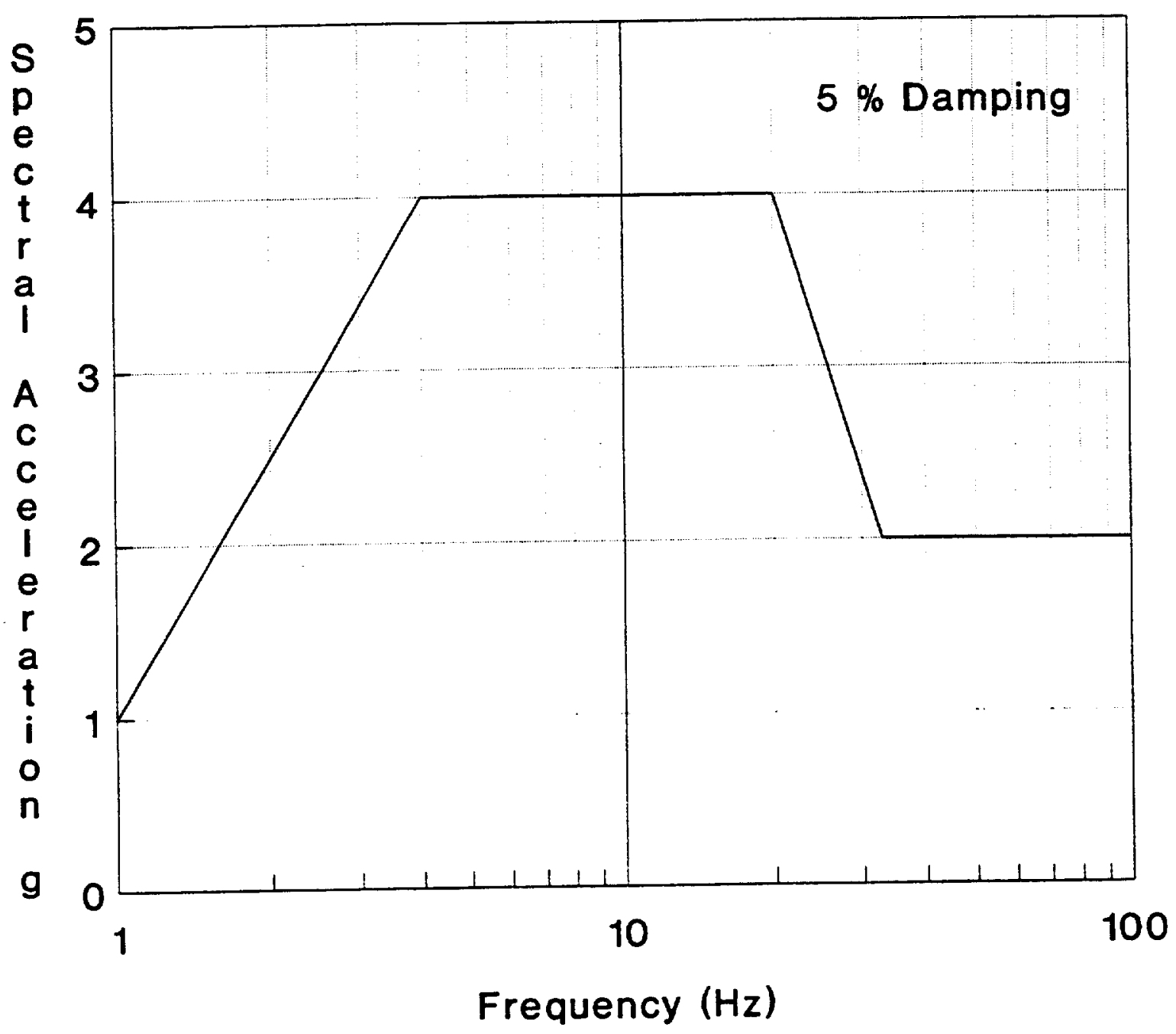

\begin{tabular}{l|cccc} 
Frequency $(\mathrm{Hz})$ & 1 & 4 & 20 & 33 \\
\hline Batteries on Racks $(\mathrm{g})$ & 1.0 & 4.0 & 4.0 & 2.0
\end{tabular}

Figure 8.1.1-2 Generic Equipment Ruggedness Spectra (GERS) for Batteries on Racks (Reference 40) (Figure B.15-1 of SQUG GIP, Reference 1) 


\subsubsection{MOTOR CONTROL CENTERS ${ }^{2}$}

The seismic capacity for the equipment class of motor control centers (MCCs) (see Figure 8.1.2-1) may be based on earthquake experience data, provided the intent of each of the caveats listed below is met. This equipment class includes control and electrical fault protection systems for motors powered at 600 volts or less (typically 480 volts). Motor controllers are mounted in sheet metal cubicles with controller cubicles typically assembled into stacks which are lined up side-by-side and bolted together to form a motor control center. This equipment class includes motor controllers mounted in individual cubicles on racks or walls as well as freestanding MCCs.

Individual motor controllers are normally mounted in a sheet metal box that can be removed from its cubicle in the motor control center. Motor controllers are arranged in vertical stacks or sections attached to each other within the MCC assembly. The individual components of the motor controller are attached to the sides and rear face of the box. Motor controller cubicles typically include the following types of components: molded case circuit breaker (or disconnect switch), magnetic contactors, a control transformer, fuses, push buttons, and pilot lights.

The motor controller cubicles are typically arranged in vertical stacks within an MCC assembly. Each stack is a separate sheet metal enclosure, usually reinforced at its corners by overlapped sheet metal or steel angle framework. Stacks are bolted together through adjacent sheet metal side walls or steel framework.

Motor control centers may be either single- or double-sided. Double-sided MCCs have controller cubicles on both the front and rear face of the cabinet, with vertical bus bars routed through a center compartment between the front and rear stacks of controller cubicles. Single-sided MCCs typically route electrical connections through vertical raceways along the sides of each stack section.

Motor control centers may be either freestanding units or form part of a more complex assembly. In many cases, MCCs are included in an assembly with switchgear, distribution panels, and/or transformers. Another alternative to the freestanding motor control center is the wall- or rackmounted motor control cubicle. Within these cubicles, motor control components are bolted to the inner faces of the wall in the same manner as in a small control or instrument cabinet. Access to the cubicle is usually through a swinging door that forms the front face of the cubicle.

MCC cabinet dimensions are generally standardized. Most MCC sections (stacks) are typically 20 to 24 inches wide, and 90 inches tall. The depth of each section typically varies from about 18 to 24 inches. Typical weight of each section is less than about 650 pounds.

MCC cabinets can weigh up to about 800 pounds per section for assemblies consisting of at least two adjacent cabinet sections which are bolted together. Narrower depth MCC cabinets should be top braced or attached to the wall.

The construction of motor control centers is typically governed by industry standards such as those developed by the National Electrical Manufacturers Association (NEMA) and Underwriters' Laboratories (UL) (e.g., NEMA ICS-6 (Ref. 82), UL-508 (Ref. 83)). These standards define minimum sheet metal thickness as a function of wall area between reinforcement.

Motor control center assemblies represented in the equipment class contain motor starters (contactors), disconnect switches, and, in some cases, over-current relays. They also contain distribution panels, automatic transfer switches, and relay/instrumentation compartments, and include attachments such as junction boxes, conduit and cables. Motor controllers are represented

2 Section B.1 of SQUG GIP (Ref. 1) 
in a variety of mounting configurations ranging from individual mounted controllers to $\mathrm{MCC}$ assemblies in outdoor enclosures.

\subsubsection{Reference Spectrum Caveats - Motor Control Centers}

The Reference Spectrum (RS) represents the seismic capacity of a Motor Control Center (MCC) if the MCC meets the intent of the following inclusion and exclusion rules. Note, however, that when the specific wording of a caveat rule is not met, then a reason for concluding that the intent has been met should be provided on the SEWS.

MCCIRS Caveat 1 - Earthquake Experience Equipment Class. The MCC should be similar to and bounded by the MCC class of equipment described above. The equipment class descriptions are general and the SCEs should be aware that worst case combinations of certain parameters may not be represented in the generic equipment class. These worst case combinations may have reduced seismic capacity and should be carefully evaluated on a case-by-case basis.

MCC/RS Caveat 2 - Rating of $600 \mathrm{~V}$ or Less. The MCC should have a $600 \mathrm{~V}$ rating or less. This is the upper limit voltage rating of MCCs in the earthquake experience equipment class.

MCCIRS Caveat 3 - Adjacent Cabinets Bolted Together. Adjacent cabinets which are close enough to impact each other and sections of a multi-bay cabinet assembly should be bolted together if any of these cabinets contains essential relays as defined in Chapter 11. The concern addressed in this caveat is that unbolted cabinets could respond out of phase to one another and impact each other during an earthquake. This would cause impact loadings and high frequency vibration loadings which could cause any essential, impact-sensitive relays to chatter.

MCC/RS Caveat 4 - Attached Weight of 100 Pounds or Less. Equipment and their enclosures (but not conduit) mounted externally to cabinets and supported by them should have a weight less than about 100 pounds for a cabinet assembly, i.e., a combination or a lineup of a number of individual adjacent cabinets, bays, or frames. The concern is that the center of gravity of the cabinet will be raised too high, the total weight of the cabinets will be too large, or large eccentric weights will introduce excessive torsion. This concern is directed primarily toward equipment which is attached to the cabinet but is not normally supplied with the MCC and thereby possibly not included in the earthquake experience equipment class. The load path for the attached component through the cabinet should be carefully examined. In addition, its attachment should be reviewed to ascertain whether the attached component may become a seismic interaction hazard source. Conduit was deleted from this caveat since conduit supported above an MCC is well represented by the earthquake experience data. Additional support of the cabinet and attached equipment will alleviate these concerns and satisfy the intent of this caveat.

For the purposes of anchorage checking, the effective weight of any attached conduit and equipment should be included in the cabinet weight.

MCCIRS Caveat 5 - Externally Attached Items Rigidly Anchored. Externally attached items should be rigidly attached to the cabinet. The concern addressed by this caveat is that these items could impact the cabinet and possibly lead to relay chatter, or impact other components of the MCC as a seismic interaction hazard. As an example, some electrical cabinets have small, externally attached panels mounted on hinges to the main cabinet frame. During seismic motion the externally attached panel may swing and cause significant impact loading to the electrical panel.

MCC/RS Caveat 6 - General Configuration Similar to NEMA Standards. The general configuration of the cabinets should be similar to those constructed to NEMA Standards. The MCC does not have to conform exactly to the NEMA standards but should be similar with regard to the gage of the steel, internal structure and support. This caveat is intended to preclude unusual 
designs not covered by the equipment class (thin gage material, flimsy internal structure, etc.). In general, cabinets manufactured by the major manufacturers of MCCs conform to this caveat if they have not been modified. Cabinets which are less than 18-inches tall and are not top braced are outliers.

MCCIRS Caveat 7 - Cutouts Not Large. Cutouts in the lower half of the cabinet sheathing should be less than 6 inches wide and 12 inches high. A second concern is that the shear load from the earthquake will not be able to be transferred through the shear walls to the anchorage. There are many standard MCCs that exceed this caveat; however, in many cases, the area around the cutout is reinforced with additional plate or steel members alleviating the concern of shear transfer. This caveat is of more concern for cutouts modifying the standard design that are not reinforced.

MCC/RS Caveat 8 - Doors/Buckets Secured. All doors and drawout buckets should be secured by a latch or fastener. The concern addressed by this caveat is that the doors and drawout buckets could open during an earthquake and repeatedly impact the housing, causing internal components such as relays and contactors to malfunction or chatter.

MCCIRS Caveat 9 - Any Other Concerns? The SCEs should seek out suspicious details or uncommon situations not specifically covered by the caveats which could adversely affect the seismic capacity of the MCC.

\subsubsection{GERS Caveats - Motor Control Centers}

The seismic capacity for the equipment class of MCCs may be based on generic testing data, provided the intent of each of the caveats listed below is met. This equipment class includes control and electrical fault protection systems for motors powered at $600 \mathrm{VAC}$ (480 VAC nominal), 250 VDC, or less. MCCs in the testing equipment class typically include several enclosure sections which are normally about 20 inches wide, about 20 inches deep, and about 90 inches high. These sections are fabricated of 14 gage $(0.0747$ inches thick) or heavier steel sheets and are supported at the floor on base channels which are either integral with the MCC frame or are external members connected by internal bolts to the MCC frame. Multiple MCC sections may be grouped together to make widths to 120 inches or greater. The weight per section of these MCCs ranges from 200 to 800 pounds.

The types of components typically housed within MCCs in the equipment class include contactors, overload relays, various types of other relays, circuit breakers, disconnect switches, control or distribution transformers, and panelboards. MCCs may also have indicator lamps and meters mounted on them.

The GERS (see Figure 8.1.2-2) represent the seismic capacity of a Motor Control Center (MCC) if the MCC meets the intent of the following inclusion and exclusion rules. Note, however, that when the specific wording of a caveat rule is not met, then a reason for concluding that the intent has been met should be provided on the SEWS.

MCC/GERS Caveat 1 - Generic Seismic Testing Equipment Class. The MCC should be similar to and bounded by the MCC class of equipment described above. The equipment class descriptions are general and the $S C E S$ should be aware that worst case combinations of certain parameters may not be represented in the generic equipment class. These worst case combinations may have reduced seismic capacity and should be carefully evaluated on a case-by-case basis.

MCC/GERS Caveat 2 - Reference Spectrum Caveats. The MCC should meet all the caveats given for the Reference Spectrum. This caveat is included to cover the vulnerabilities identified for the earthquake experience equipment class. Those GERS caveats which are the same as the Reference Spectrum caveats are not repeated below. 
MCC/GERS Caveat 3 - Floor-Mounted Cabinet. The MCC should be floor-mounted. This is the mounting configuration for all MCCs in the generic seismic testing equipment class.

MCC/GERS Caveat 4 - Weight Less Than 800 Pounds. The maximum weight per vertical section should be less than about 800 pounds. This is the upper bound weight of MCCs in the generic seismic testing equipment class.

MCC/GERS Caveat 5 - Anchored Through Base Channel. The MCC should be anchored through a base channel integral to the MCC frame or an external base channel which is connected to the MCC frame by internal bolts. The intent of this caveat is to avoid anchoring MCCs through flimsy or flexible sections in which significant bending of sheet metal could occur during an earthquake.

MCC/GERS Caveat 6 - Load Path Check. To use the "Function After" GERS, the load transfer path from the anchorage to base frame of the MCC should be checked for strength and stiffness. In particular, the following load path elements should be checked for adequacy. There should be stiff anchorage connections for each section to secure the unit to the floor, e.g., 4 anchors for a single MCC cabinet or 2 anchors for interior cabinets in a multi-cabinet assembly if these anchors are located near the shear wall of the cabinet and adjacent cabinets are bolted together. If the MCC frame is connected to external base structural members provided by the manufacturer with internal mounting bolts, then there should be at least four of these internal mounting bolts per section, and these bolts should be at least 3/8 inches in diameter. Any sheet metal cabinet components used for anchorage should have reinforcement. Excessive eccentricities in the internal load path which allow significant bending of sheet metal should be evaluated separately for strength and stiffness.

MCC/GERS Caveat 7 - "Function During" GERS. The "Function During" GERS can be used only if all the relays within the MCC have GERS greater than $4.5 \mathrm{~g}$ within the amplified spectral region. For this caveat, the term "relays" does not include contactors and other starter components. Auxiliary contacts of contactors require a separate relay evaluation as described in Chapter 11 if they are used for external control or lockout signals.

MCC/GERS Caveat 8 - "Function After" GERS. The "Function After" GERS can be used if it can be demonstrated that the starters can be reset. The Relay Functionality Review in Chapter 11 describes the guidelines for evaluating the acceptability of resetting relays and starters. Note that, in general, both system tolerance of the changed state and operator availability for manual reset should be shown.

MCC/GERS Caveat 9 - Adjacent Cabinets Bolted Together. Adjacent cabinets and sections of a multi-bay cabinet assembly should be bolted together, including those that do not contain essential relays. Adjacent cabinets and sections of multi-bay cabinet assemblies were bolted together when tested for this generic seismic testing equipment class. 


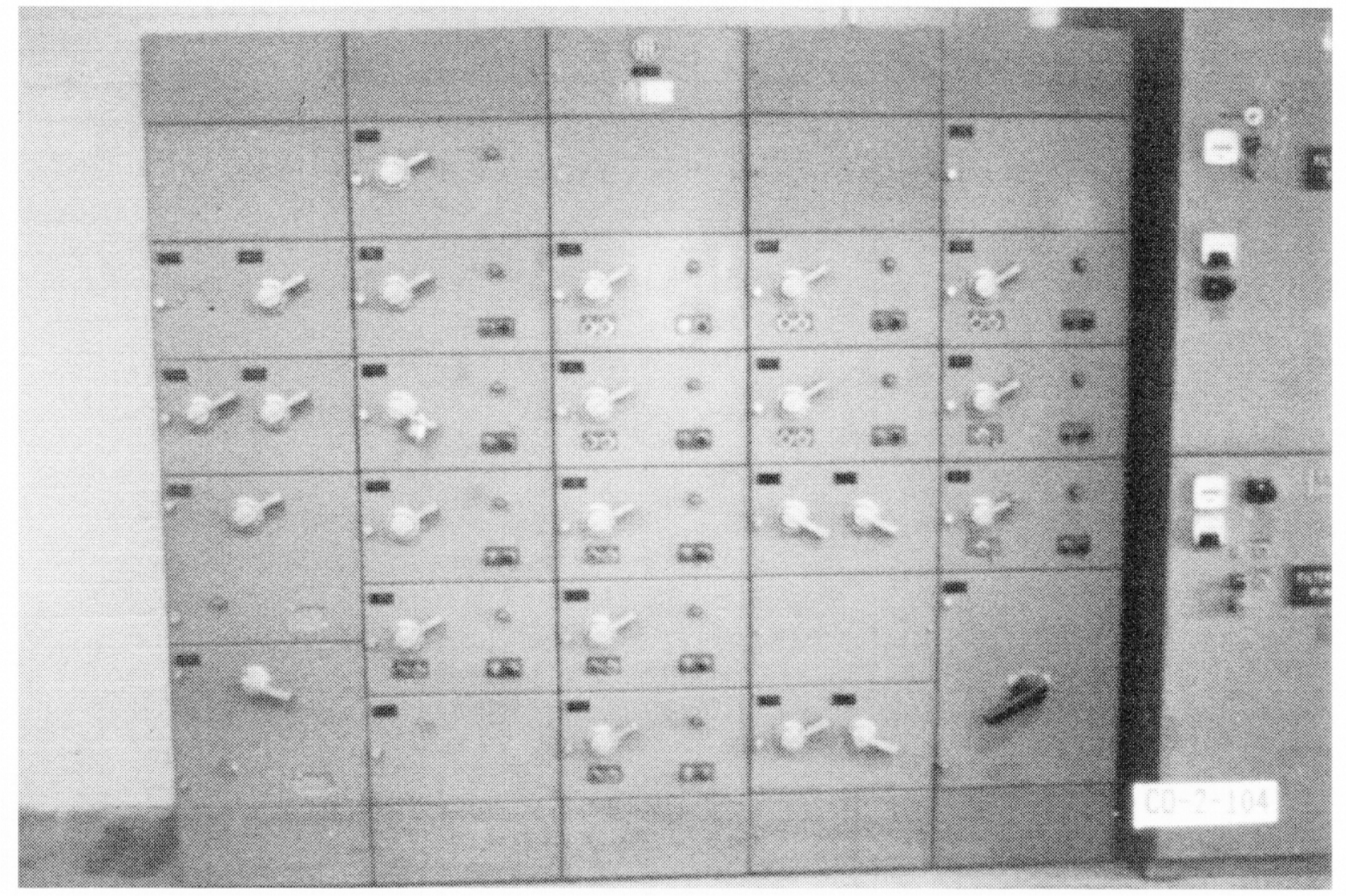

Figure 8.1.2-1 Motor Control Center from the Earthquake Experience Database 
Motor Control Centers

GERS-MCC.9

2/1/91

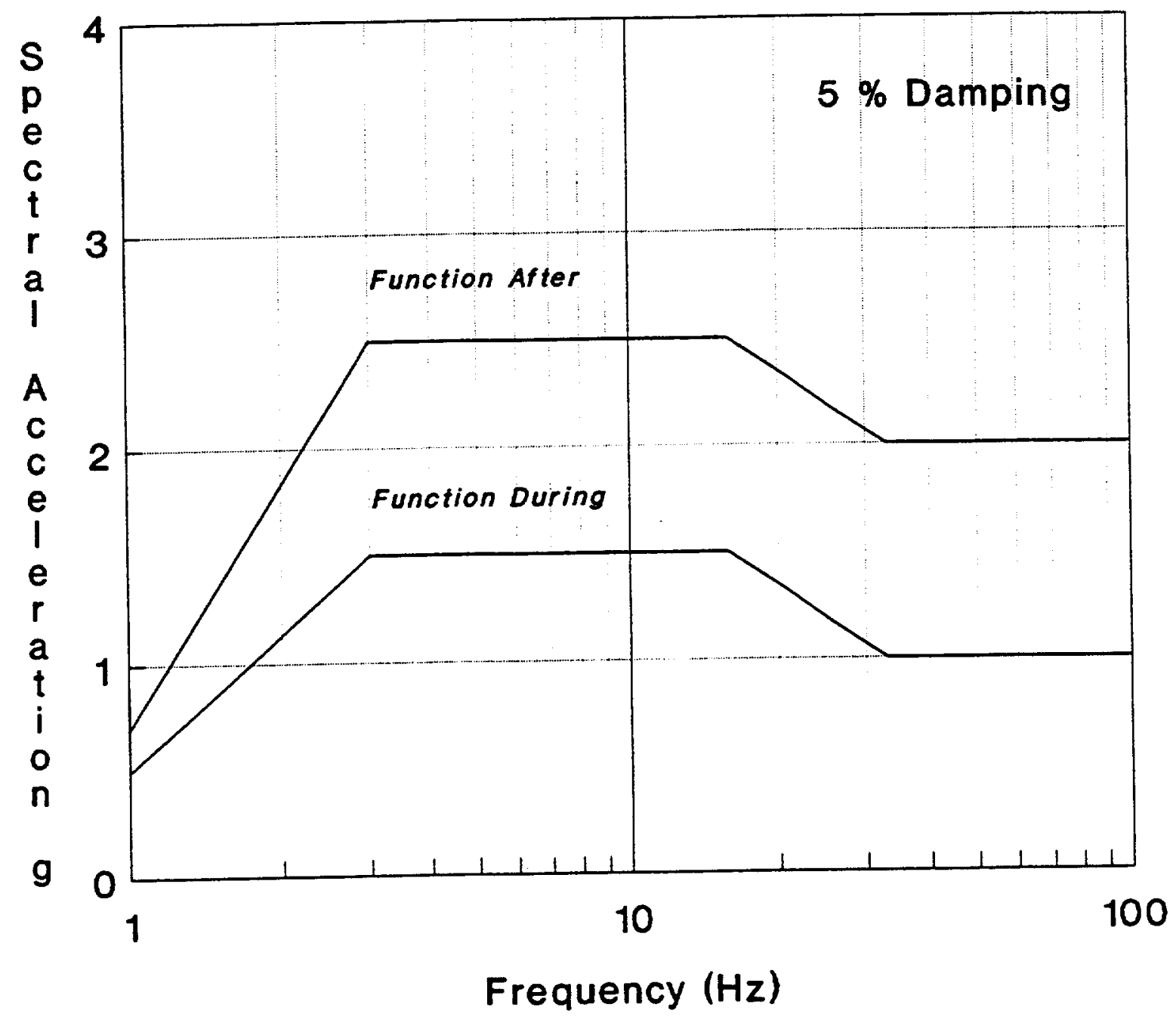

\begin{tabular}{l|llll} 
Frequency $(\mathrm{Hz})$ & 1 & 3 & 16 & 33 \\
\hline Function Alter $(g)$ & 0.7 & 2.5 & 2.5 & 2.0 \\
Function During $(g)$ & 0.5 & 1.5 & 1.5 & 1.0
\end{tabular}

Figure 8.1.2-2 Generic Equipment Ruggedness Spectra (GERS) for Motor Control Centers (Reference 40) (Figure B.1-1 of SQUG GIP, Reference 1) 


\subsubsection{LOW-VOLTAGE SWTCHGEAR ${ }^{3}$}

The seismic capacity for the equipment class of low voltage switchgear (LVS) assemblies (see Figure 8.1.3-1) may be based on earthquake experience data, provided the intent of each of the caveats listed below is met. This equipment class consists of one or more circuit breakers and associated control relays, instrumentation, disconnect switches, and distribution buses mounted in a sheet metal enclosure. The term "low voltage switchgear" is associated with circuits of 600 volts or less, typically 440 to 480 volts in modern industrial facilities.

Switchgear assemblies are composed of vertical sections which normally contain stacks of two to four circuit breaker cubicles. The vertical section is a sheet metal enclosure welded to a framework of steel angles or channels. Each section includes a circuit breaker or other control devices in a forward compartment and bus connections for the primary circuits in the rear compartment.

A section of a switchgear assembly is typically 90 inches in height and 60 inches in depth. The width of each section ranges from 20 to 36 inches, depending on the size of the circuit breaker it contains. A typical section weighs about 2000 pounds. Individual sections are bolted together through adjoining walls to form an assembly. LVS assemblies normally include at least one cubicle that serves as a metering compartment. The compartment typically contains ammeters, voltmeters, relays, and transformers.

Most low-voltage circuit breakers are the drawout type. They are mounted on a roller/rail support system that allows them to be disconnected from their primary contacts at the rear, and drawn forward out of their sheet metal enclosure for maintenance. While in operation, the circuit breaker clamps to bus bars in the rear of the switchgear assembly. Additional positive attachment of the breaker to its enclosure is made by a mechanical jack or racking mechanism which slides the breaker in or out of its operating position.

The circuit breaker can include the following types of components: spring-actuated electric contacts, a closing solenoid, various types of tripping devices (overcurrent, shunt, under voltage), fuses, and auxiliary switches.

Low-voltage breakers may be combined in assemblies with transformers, distribution panels, medium voltage breakers, and motor controllers. Circuit breakers, relays, instrumentation, the switchgear assembly enclosure, internal transformers, attachments such as junction boxes, and attached conduit and cables are included in the Low-Voltage Switchgear equipment class.

\subsubsection{Reference Spectrum Caveats - Low-Voltage Switchgear}

The Reference Spectrum (RS) represents the seismic capacity of a Low-Voltage Switchgear (LVS) if the switchgear meets the intent of the following inclusion and exclusion rules. Note, however, that when the specific wording of a caveat rule is not met, then a reason for concluding that the intent has been met should be provided on the SEWS.

LVS/RS Caveat 1 - Earthquake Experience Equipment Class. The low-voltage switchgear should be similar to and bounded by the LVS class of equipment described above. The equipment class descriptions are general and the SCEs should be aware that worst case combinations of certain parameters may not be represented in the generic equipment class. These worst case combinations may have reduced seismic capacity and should be carefully evaluated on a case-by-case basis.

LVS/RS Caveat 2 - Rating of $600 \mathrm{~V}$ or Less. The low voltage switchgear should have a $600 \mathrm{~V}$ rating or less. This is the upper bound voltage rating of LVS in the earthquake experience equipment class.

3 Section B.2 of SQUG GIP (Ref. 1) 
LVS/RS Caveat 3 - Side-to-Side Restraint of Breaker. The support structure for circuit breakers of the drawout type should have side-to-side restraint to limit relative motion with respect to the cabinet. The concern is to prevent damage or disconnection of secondary contacts. Restraint may be provided by the breaker support structure or by a special lateral restraint device.

LVS/RS Caveat 4 - Adjacent Cabinets Bolted Together. Adjacent cabinets which are close enough to impact each other and sections of multi-bay cabinet assemblies should be bolted together if any of these cabinets contain essential relays as defined in Chapter 11. The concern addressed in this caveat is that unbolted cabinets could respond out of phase to one another and impact each other during an earthquake. This would cause additional impact loadings and high frequency vibration loadings which could cause any essential relays to chatter.

LVS/RS Caveat 5 - Attached Weight of 100 Pounds or Less. Equipment and their enclosures (but not conduit) mounted externally to cabinets and supported by them should have a weight less than about 100 pounds for a cabinet assembly, i.e., a combination or a lineup of a number of individual adjacent cabinets, bays, or frames. The concern is that the center of gravity of the cabinet will be raised too high, the total weight of the cabinets will be too large, or large eccentric weights will introduce excessive torsion. The concern is directed primarily for equipment not normally supplied with the switchgear and thereby possibly not included in the earthquake experience equipment class. The load path of the attached component through the cabinet should be carefully examined. In addition, its attachment should be reviewed to ascertain whether the attached component may become a seismic interaction hazard source. Conduit was deleted from the caveat since conduit supported above switchgear is well represented by the earthquake experience data. Additional support of the cabinet and attached equipment will alleviate these concerns and satisfy the intent of this caveat.

For the purposes of anchorage checking, the effective weight of any attached conduit and equipment should be included in the cabinet weight.

LVS/RS Caveat 6 - Externally Attached Items Rigidly Anchored. Externally attached items should be rigidly attached to the cabinet. The concern addressed by this caveat is that these items could impact the cabinet and possibly lead to relay chatter, or impact other components of the switchgear as a seismic interaction hazard. As an example, some electrical cabinets have small, externally attached panels mounted on hinges to the main cabinet frame. During seismic motion the externally attached panel may swing and cause significant impact loading to the electrical panel.

LVS/RS Caveat 7 - General Configuration Similar to ANSI C37.20 Standards. The general configuration of the cabinets should be similar to those constructed to ANSI C37.20 Standards (Ref. 84). The switchgear does not have to conform exactly to ANSI standards but should be similar with regard to the gage of the steel, internal structure and support. This caveat is intended to preclude unusual designs not covered by the equipment class (thin gage material, flimsy internal structure, etc.) In general, cabinets manufactured by the major manufacturers of switchgear conform to this caveat if they have not been modified.

LVS/RS Caveat 8 - Cutouts Not Large. Cutouts in the lower half of cabinet sheathing should be less than $30 \%$ of the width of the side panel, and the height of the cutout should be less then $60 \%$ of the width of the side panel. This caveat also applies to side panels between multi-bay cabinets. Cutout restrictions do not apply to the bus transfer compartment if the remaining part of the enclosure conforms with the cutout limitation. The concern of this caveat is that the shear load from the earthquake will not be able to be transferred through the shear walls to the anchorage. Reinforcement around the cutout with additional plate or steel members may alleviate the concern of shear transfer. 
LVS/RS Caveat 9 - Doors Secured. All doors should be secured by a latch or fastener. The concern addressed by this caveat is that loose doors could repeatedly impact the housing and be damaged or cause internal components such as relays to malfunction or chatter.

LVS/RS Caveat 10 - Any Other Concerns? SCEs should seek out suspicious details or uncommon situations not specifically covered by the caveats which could adversely affect the seismic capacity of the switchgear.

\subsubsection{GERS Caveats - Low-Voltage Switchgear}

The seismic capacity for the equipment class of LVS may be based on generic testing data, provided the intent of each of the caveats listed below is met. This equipment class includes steel enclosures containing several draw-out type circuit breakers, bus bars, protective/auxiliary relays, and meters. Units have a maximum rating of $600 \mathrm{VAC}$ or $250 \mathrm{VDC}$. The metal enclosure sections are typically 20 to 30 inches wide, 60 inches deep, and 80 to 90 inches high. They are fabricated of 14 gage ( 0.0747 inches thick) or heavier steel sheet metal and framed with angles or other formed members, with anchorage provisions included in the base frame. The weight per section of the switchgear assembly ranges from 1000 to 1600 pounds. The units should be mounted within ANSI-type metal enclosures with either welded or bolted anchorage. To exclude specialty-type switchgear, the equipment class is limited to the following three manufacturers: ITE/Brown Boveri, Westinghouse, or General Electric.

The GERS (see Figure 8.1.3-2) represent the seismic capacity of a Low-Voltage Switchgear (LVS) if the switchgear meets the intent of the following inclusion and exclusion rules. Note, however, that when the specific wording of a caveat rule is not met, then a reason for concluding that the intent has been met should be provided on the SEWS.

LVS/GERS Caveat 1 - Generic Seismic Testing Equipment Class. The low voltage switchgear should be similar to and bounded by the LVS class of equipment described above. The equipment class descriptions are general and the SCES should be aware that worst case combinations of certain parameters may not be represented in the generic equipment class. These worst case combinations may have reduced seismic capacity and should be carefully evaluated on a case-bycase basis.

LVS/GERS Caveat 2 -Reference Spectrum Caveats Apply. The switchgear should meet all the caveats given for the Reference Spectrum. This caveat is included to cover the vulnerabilities identified for the earthquake experience equipment class. Those GERS caveats which are the same as the Reference Spectrum caveats are not repeated below.

LVS/GERS Caveat 3 - Floor-Mounted Switchgear. The low voltage switchgear must be housed within a floor-mounted ANSI-type enclosure. This ensures consistency with enclosures included in the generic seismic testing equipment class.

LVS/GERS Caveat 4 - No Specially-Designed Switchgear. The GERS are not applicable to specially-designed or custom made switchgear, such as those which have been used in some reactor trip systems. To preclude their use, the switchgear should be manufactured by either ITE/Brown Boveri, Westinghouse, or General Electric. These are the manufacturers which produced the switchgear included in the generic seismic testing equipment class.

LVS/GERS Caveat 5 - Weight Per Section Less than 1600 Pounds. The maximum weight per section should be less than about 1600 pounds. This is the upper bound weight limit of LVS in the generic seismic testing equipment class. 
LVS/GERS Caveat 6 - Vertical Restraint in the Form of Stops or Brackets. To utilize the $2.5 \mathrm{~g}$ GERS level, vertical restraint in the form of stops or brackets should be provided to prevent uplift of the circuit breaker so that the wheels do not come disengaged from rails.

LVS/GERS Caveat 7 - Reinforcement of Outside Corners of End Units. To utilize the $2.5 \mathrm{~g}$ GERS level, the outside base frame corners of the outer switchgear cabinets in a lineup should have certain enhancements to improve their seismic ruggedness. For Westinghouse type switchgear, the outside base frame corners of the outer switchgear cabinets in a lineup should be reinforced. For the other types of switchgear, the manufacturers (GE, ITE) should be consulted to determine what enhancements, if any, should be included in their switchgear cabinets to give them this seismic ruggedness level and then check whether these enhancements have been included on these units.

LVS/GERS Caveat 8 - Adjacent Cabinets Bolted Together. Adjacent cabinets and sections of a multi-bay cabinet assembly should be bolted together. Adjacent cabinets and sections of multi-bay cabinet assemblies were bolted together when tested for this generic seismic testing equipment class. 


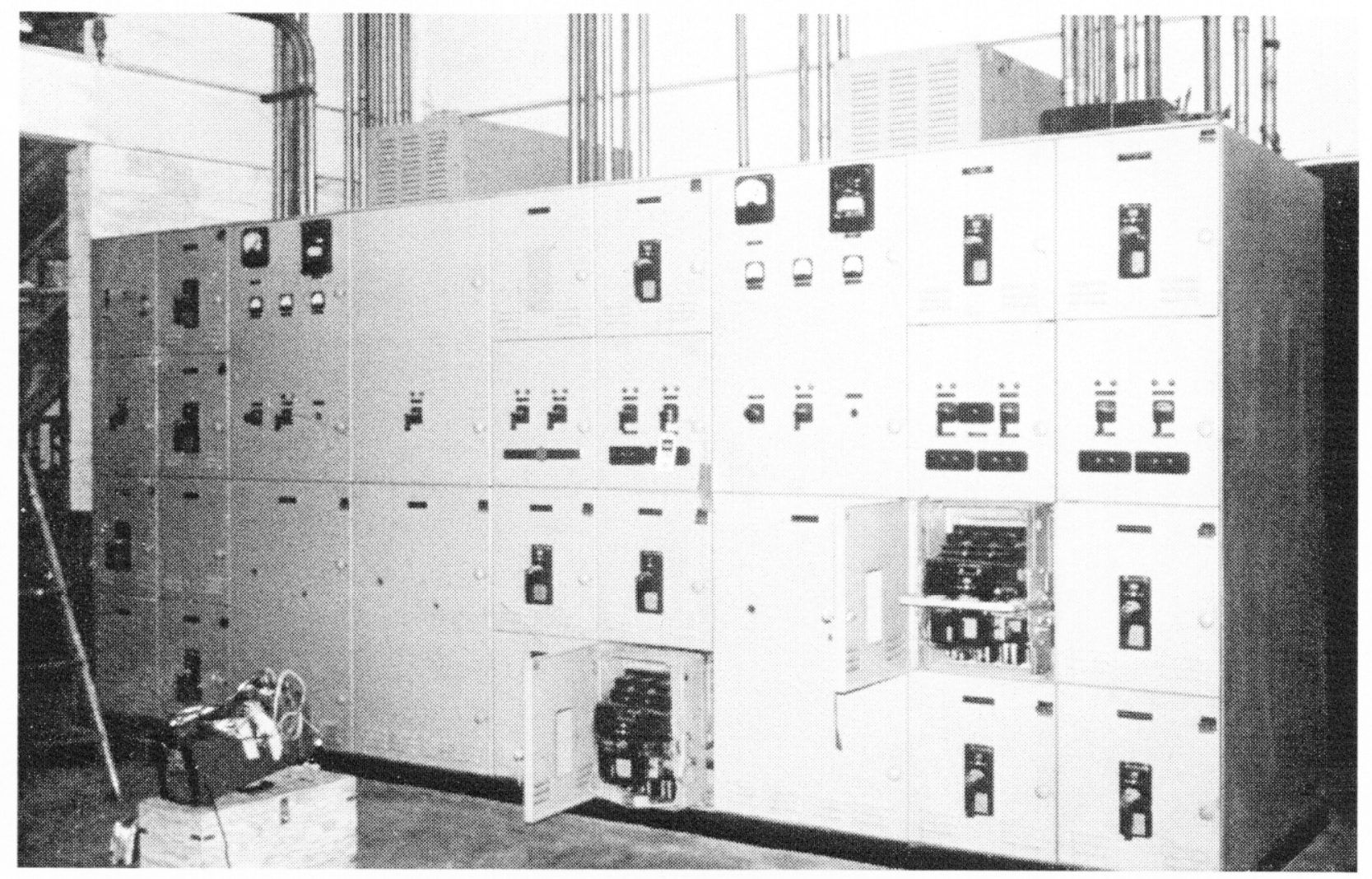

Figure 8.1.3-1 Low-Voltage Switchgear from the Earthquake Experience Database 
Low Voltage Switchgear

GERS-MVS/LVS.7 (Low Voltage)

2/1/91

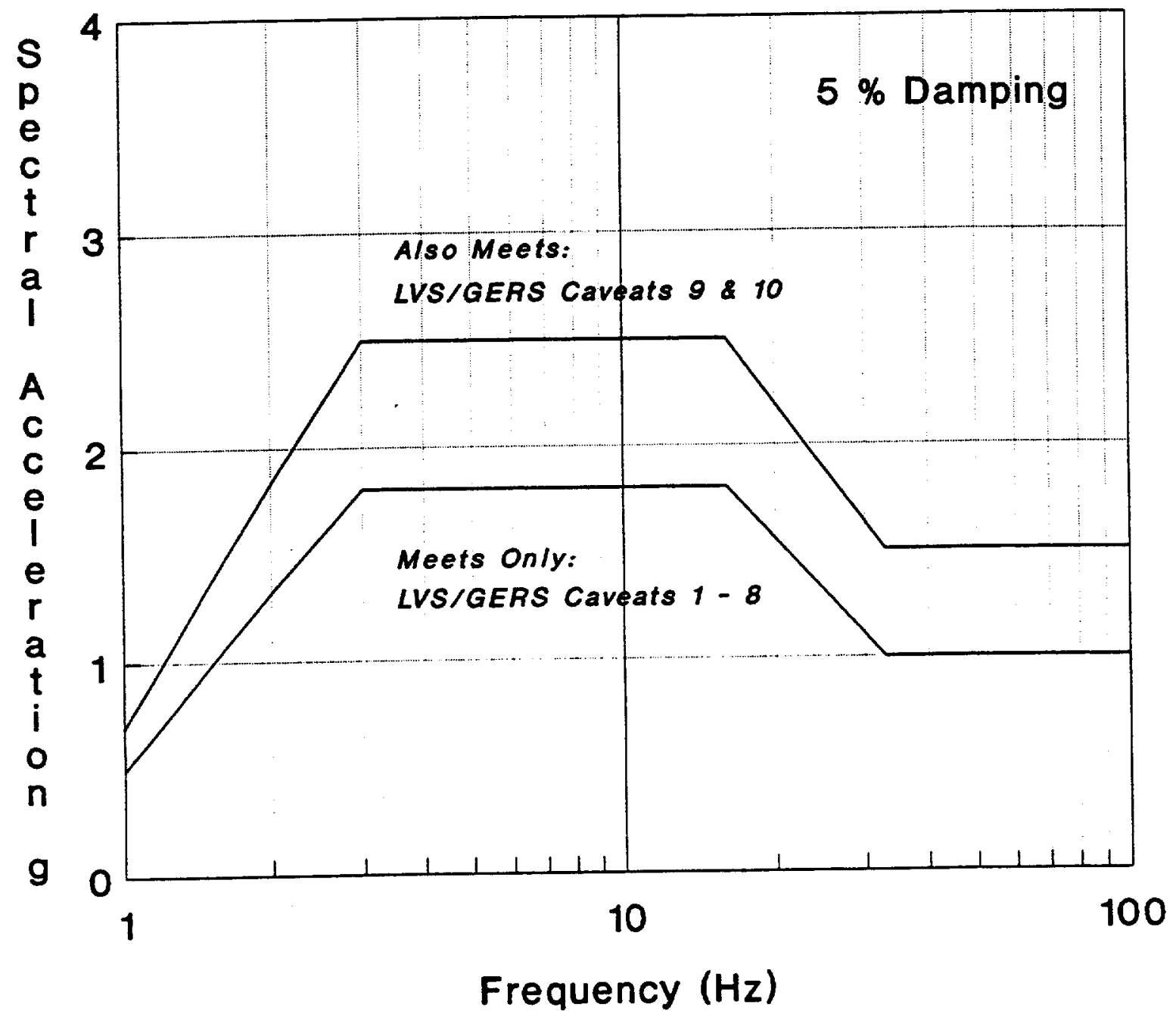

\begin{tabular}{l|cccc} 
Frequency $(H z)$ & 1 & 3 & 16 & 33 \\
\hline Meets LVS/GERS Caveats $1-10(0)$ & 0.7 & 2.5 & 2.5 & 1.5 \\
Meets LVS/GERS Caveats $1-8(g)$ & 0.5 & 1.8 & 1.8 & 1.0
\end{tabular}

Figure 8.1.3-2 Generic Equipment Ruggedness Spectra (GERS) for Low-Voltage Switchgear (Reference 40) (Figure B.2-1 of SQUG GIP, Reference 1) 


\subsubsection{MEDIUM-VOLTAGE SWITCHGEAR ${ }^{4}$}

The seismic capacity for the equipment class of medium voltage switchgear (MVS) assemblies (see Figure 8.1.4-1) may be based on earthquake experience data, provided the intent of each of the caveats listed below is met. This equipment class consists of one or more circuit breakers and associated control relays and instrumentation mounted in a sheet metal enclosure. The equipment class includes electrical switching and fault protection circuit breakers for systems powered between 2400 and 4160 volts. Medium-voltage circuit breakers are mounted in sheet metal cabinets which are bolted together, side-by-side, to form a switchgear assembly.

Medium-voltage circuit breakers or load interrupter switches are often integrated into unit substations that may include a transformer (typically $4160 / 480$ volt), a set of low-voltage switchgear, or a distribution switchboard. The switchgear assembly also may include internal transformers, junction boxes, and attached conduit and cables. The basic component of a medium voltage switchgear assembly is a metal-clad enclosure, typically containing a circuit breaker compartment in a lower section and a metering compartment in an upper section. The rear of the enclosure is a separate compartment for primary electrical connections. The enclosure consists of sheet metal panels welded to a supporting frame of steel angles or channels. Individual enclosures are typically 90 inches in height and approximately 90 inches in depth. The width of an enclosure typically varies from 24 to 36 inches, depending on the size of the circuit breaker within. The weight of a metal-clad enclosure ranges from 2000 to 3000 pounds, with the circuit breaker itself weighing from 600 to 1200 pounds.

Electro-mechanical relays are mounted either to the swinging doors at the front of the enclosure, or to the interior of the metering compartment. Relays are typically inserted through cutouts in the door and secured by screws through a mounting flange into the sheet metal. The metering compartment may also contain components such as ammeters, voltmeters, hand switches, and small transformers.

The medium-voltage circuit breakers commonly used in facility applications include the drawouttype air-magnetic circuit breakers, and stationary load interrupter switches. Each type is discussed in this section.

Drawout, air-magnetic circuit breakers are mounted on rollers to allow them to be wheeled in and out of their individual sheet metal enclosures. There are two general types of drawout circuit breakers: the horizontally-racked model and the vertically-racked model.

The horizontally-racked model has clamping bus connections at its rear. It is racked into operating position by a mechanical jack that rolls the circuit breaker into contact with the bus connections at the rear of its enclosure and secures it in place. The weight of the circuit breaker rests on the floor.

Vertically-racked circuit breakers roll into position within their enclosure and are then engaged by a jack built into the walls of the enclosure. The jack lifts the circuit breaker several inches above the floor, until the clamping connections atop the circuit breaker contact the bus connections at the top of the enclosure. The weight of the circuit breaker is then supported on the framework of the sheet metal enclosure. Lateral restraint of the circuit breaker should be provided by the cabinet framing and not solely by the jack lifts.

Air-magnetic circuit breakers typically include the following types of components: spring-actuated contacts, tripping devices, auxiliary switches, and fuses. Typical capacities for medium voltage circuit breakers range from 1200 to 3000 amperes.

4 Section B.3 of SQUG GIP (Ref. 1) 
Load interrupter switches perform the load connecting and interrupting function of circuit breakers, but do not include the same capabilities of electrical fault protection. Interrupter switches are bolted into sheet metal enclosures and are therefore designated as stationary devices. Like air-magnetic circuit breakers, interrupter switches usually operate with spring-actuated contacts to ensure quick opening of the primary circuit.

\subsubsection{Reference Spectrum Caveats - Medium-Voltage Switchgear}

The Reference Spectrum $(R S)$ represents the seismic capacity of a Medium-Voltage Switchgear (MVS) if the switchgear meets the intent of the following inclusion and exclusion rules. Note, however, that when the specific wording of a caveat rule is not met, then a reason for concluding that the intent has been met should be provided on the SEWS.

MVS/RS Caveat 1 - Earthquake Experience Equipment Class. The switchgear should be similar to and bounded by the MVS class of equipment described above. The equipment class descriptions are general and the $S C E s$ should be aware that worst case combinations of certain parameters may not be represented in the generic equipment class. These worst case combinations may have reduced seismic capacity and should be carefully evaluated on a case-by-case basis.

MVS/RS Caveat 2 - Rating between $2.4 \mathrm{KV}$ and $4.16 \mathrm{KV}$. The switchgear should have a rating between $2.4 \mathrm{KV}$ and $4.16 \mathrm{KV}$. This is the typical voltage range of MVS of this earthquake experience equipment class.

MVS/RS Caveat 3 - Transformers Restrained from Relative Motion. Potential transformers and/or control power transformers mounted on the switchgear should have restraints that limit relative motion of the transformers to prevent damage or disconnection of contacts. In particular, trunnionmounted transformers should have positive vertical restraint to keep the trunnion pin in its cradle. Positive vertical restraint of the trunnion pin is not required if the seismic demand at the base of the switchgear cabinet is less than or equal to about 1/2 of the Reference Spectrum.

MVS/RS Caveat 4 - Adjacent Cabinets Bolted Together. Adjacent cabinets which are close enough to impact each other and sections of multi-bay cabinet assemblies should be bolted together if any of these cabinets contain essential relays as defined in Chapter 11. The concern addressed in this caveat is that unbolted cabinets could respond out of phase to one another and impact each other during an earthquake. This would cause additional impact loadings and high frequency vibration loadings which could cause the essential relays to chatter.

MVS/RS Caveat 5 - Attached Weight of 100 Pounds or Less. Equipment and their enclosures (but not conduit) mounted externally to cabinets and supported by them should have a weight less than about 100 pounds for a cabinet assembly, i.e., a combination or a lineup of a number of individual adjacent cabinets, bays, or frames. The concern is that the center of gravity of the cabinet will be raised too high, the total weight of the cabinets will be too large, or large eccentric weights will introduce excessive torsion. The concern is directed primarily for equipment not normally supplied with the switchgear and thereby possibly not included in the earthquake experience equipment class. The load path for the attached component through the cabinet should be carefully examined. In addition, its attachment should be reviewed to ascertain whether the attached component may become a seismic interaction hazard source. Conduit was deleted from the caveat since conduit supported above switchgear is well represented in the seismic experience data base. Additional support of the cabinet and attached equipment will alleviate these concerns and satisfy the intent of this caveat.

For the purposes of anchorage checking, the effective weight of any attached conduit and equipment should be included in the cabinet weight. 
MVS/RS Caveat 6 - Externally Attached Items Rigidly Anchored. Externally attached items should be rigidly attached to the cabinet. The concern addressed by this caveat is that these items could impact the cabinet and possibly lead to relay chatter or impact other components of the switchgear as a seismic interaction hazard. As an example, some electrical cabinets have small, externally attached panels mounted on hinges to the main cabinet frame. During seismic motion the externally attached panel may swing and cause significant impact loading to the electrical panel.

MVS/RS Caveat 7 - General Configuration Similar to ANSI C37.20 Standards. The general configuration of the cabinets should be similar to those constructed to ANSI C37.20 Standards (Ref. 84). The switchgear does not have to conform exactly to ANSI standards but should be similar with regard to the gage of the steel, internal structure and support. This caveat is intended to preclude unusual designs not covered by the equipment class (thin gage material, flimsy internal structure, etc.). In general, cabinets manufactured by the major manufacturers of switchgear conform to this caveat if they have not been modified.

MVS/RS Caveat 8 - Cutouts Not Large. Cutouts in the lower half of cabinet sheathing should be less than $30 \%$ of the width of the side panel, and the height of the cutout should be less than $60 \%$ of the width of the side panel. This caveat also applies to side panels between multi-bay cabinets. Cutout restrictions do not apply to the bus transfer compartment if the remaining part of the enclosure conforms with the cutout limitations. The concern of this caveat is that the shear load from the earthquake will not be able to be transferred through the shear walls to the anchorage. Reinforcement around the cutout with additional plate or steel members may alleviate the concern of shear transfer.

MVS/RS Caveat 9 - Doors Secured. All doors should be secured by a latch or fastener. The concern addressed by this caveat is that the doors could open during an earthquake, and the loose door could repeatedly impact the housing and be damaged or cause internal components such as relays to malfunction or chatter.

MVS/RS Caveat 10 - Any Other Concerns? SCEs should seek out suspicious details or uncommon situations not specifically covered by the caveats which could adversely affect the seismic capacity of the switchgear.

\subsubsection{GERS Caveats - Medium-Voltage Switchgear}

The seismic capacity for the equipment class of metal clad medium-voltage switchgear may be based on generic testing data, provided the intent of each of the caveats listed below is met. This equipment class includes steel panel enclosures containing several wheel-mounted draw-out type circuit breakers, bus bars, auxiliary/ protective relays, transformers, switches, and meters. Units are medium voltage rated at 5000 VAC. Circuit breakers which must be jacked up to engage (vertical lift) into the connected position are not included in this class. The equipment in the GERS equipment class include ANSI C37.20 enclosures whose nominal section sizes are 30 inches wide, 60 inches deep, and 90 inches high. They are fabricated of 12 gage (0.1046 inches thick) or heavier steel sheet metal and framed with angles or other formed members, with anchorage provisions included in the base frame. Widths of MVS can range between 24 inches and 42 inches. Some cubicles can be essentially empty, while other cubicles can house very heavy circuit breaker units. In general, a single cubicle which houses a circuit breaker can typically weigh between 3000 and 5000 pounds. The MVS GERS equipment class covers most medium voltage switchgear used in facilities for overcurrent protection in primary voltage (normally $4160 \mathrm{VAC}$ ) distribution systems.

The GERS (see Figure 8.1.4-2) represent the seismic capacity of a Medium-Voltage Switchgear (MVS) if the switchgear meets the intent of the following inclusion and exclusion rules. Note, however, that when the specific wording of a caveat rule is not met, then a reason for concluding that the intent has been met should be provided on the SEWS. 
MVS/GERS Caveat 1 - Generic Seismic Testing Equipment Class. The switchgear should be similar to, and bounded by, the MVS class of equipment described above. The equipment class descriptions are general and the SCEs should be aware that worst case combinations of certain parameters may not be represented in the generic equipment class. These worst case combinations may have reduced seismic capacity and should be carefully evaluated on a case-by-case basis.

MVS/GERS Caveat 2 - Reference Spectrum Caveats Apply. The switchgear should meet all the caveats given for the Reference Spectrum. This caveat is included to cover the vulnerabilities identified for the earthquake experience equipment class. Those GERS caveats which are the same as the Reference Spectrum caveats are not repeated below.

MVS/GERS Caveat 3 - Floor-Mounted Switchgear. The medium-voltage switchgear should be housed within a floor-mounted ANSI-type enclosure. This ensures consistency with the enclosures included in the generic seismic testing equipment class.

MVS/GERS Caveat 4 - No Specially-Designed Switchgear. The GERS are not applicable to specially-designed or custom made switchgear, such as those which have been used in some reactor trip systems. Specially-designed switchgear are not included in the generic seismic testing equipment class.

MVS/GERS Caveat 5 - No Jack-Up or Vertical-Lift Type Breakers. The breakers should be the wheel-mounted type and not a jack-up or vertical-lift type. This is the only breaker configuration represented in the generic seismic testing equipment class.

MVS/GERS Caveat 6 - Weight Per Section Less than 5000 Pounds. The maximum weight per vertical breaker section should be less than about 5000 pounds (review of manufacturer's submittals is sufficient). This is the upper bound weight limit of sections included in the generic seismic testing equipment class.

MVS/GERS Caveat 7 - Vertical Restraint of Breaker. To utilize the $2.5 \mathrm{~g}$ GERS level, vertical restraint in the form of stops or brackets should be provided to prevent uplift of the circuit breaker so that the wheels do not become disengaged from the rails.

MVS/GERS Caveat 8 - Horizontal Restraint of Arc Chutes. To utilize the $2.5 \mathrm{~g}$ GERS level, horizontal restraint of the circuit breaker arc chutes should be provided. This restraint may take the form of blocks between adjacent arc chutes and between arc chutes and the wall or frame of the cabinet.

MVS/GERS Caveat 9 - Relay Model Excluded. The $2.5 \mathrm{~g}$ level GERS can not be used for Westinghouse medium-voltage switchgear if the " $\mathrm{Y}$ " anti-pump relay is a Beaver Type $\mathrm{Z}$.

MVS/GERS Caveat 10 - Separate Evaluation of Racking Mechanism. Breaker positioning or racking mechanisms should be evaluated. There should be side-to-side restraint of the breaker to prevent secondary/auxiliary breaker contacts from opening. The evaluation may consist of a visual inspection by the $S C E s$. This caveat is intended to address potential damage or operational problems due to excessive relative motion between the drawout breaker and the switchgear cabinet frame as observed in an example from the generic seismic test data.

MVS/GERS Caveat 11 -Adjacent Cabinets Bolted Together. Adjacent cabinets and sections of a multi-bay cabinet assembly should be bolted together. Adjacent cabinets and sections of multi-bay cabinet assemblies were bolted together when tested for this generic seismic testing equipment class. 


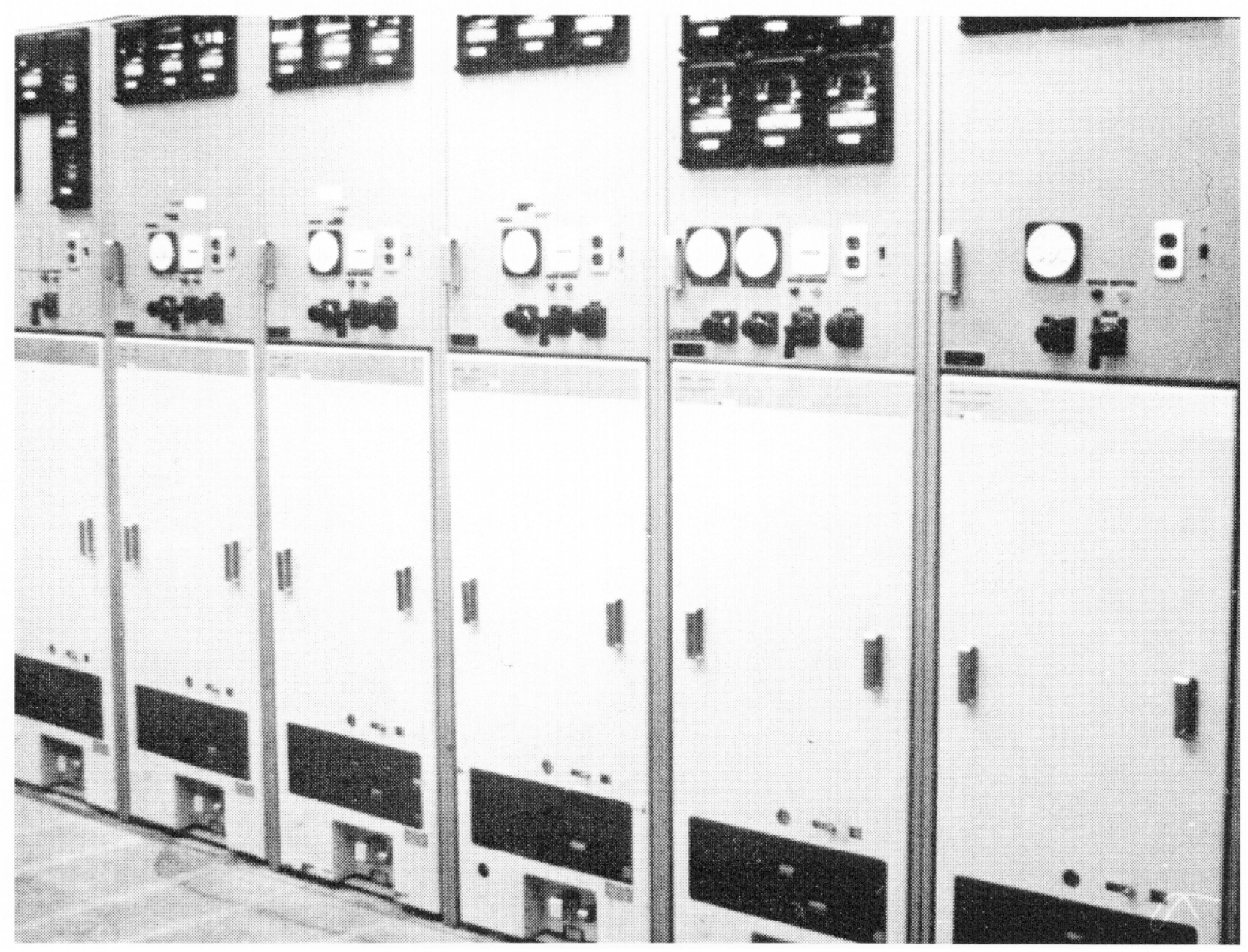

Figure 8.1.4-1 Medium-Voltage Switchgear from the Earthquake Experience Database 
Medium Voltage Switchgear

GERS-MVS/LVS.7 (Medium Voltage)

$2 / 1 / 91$

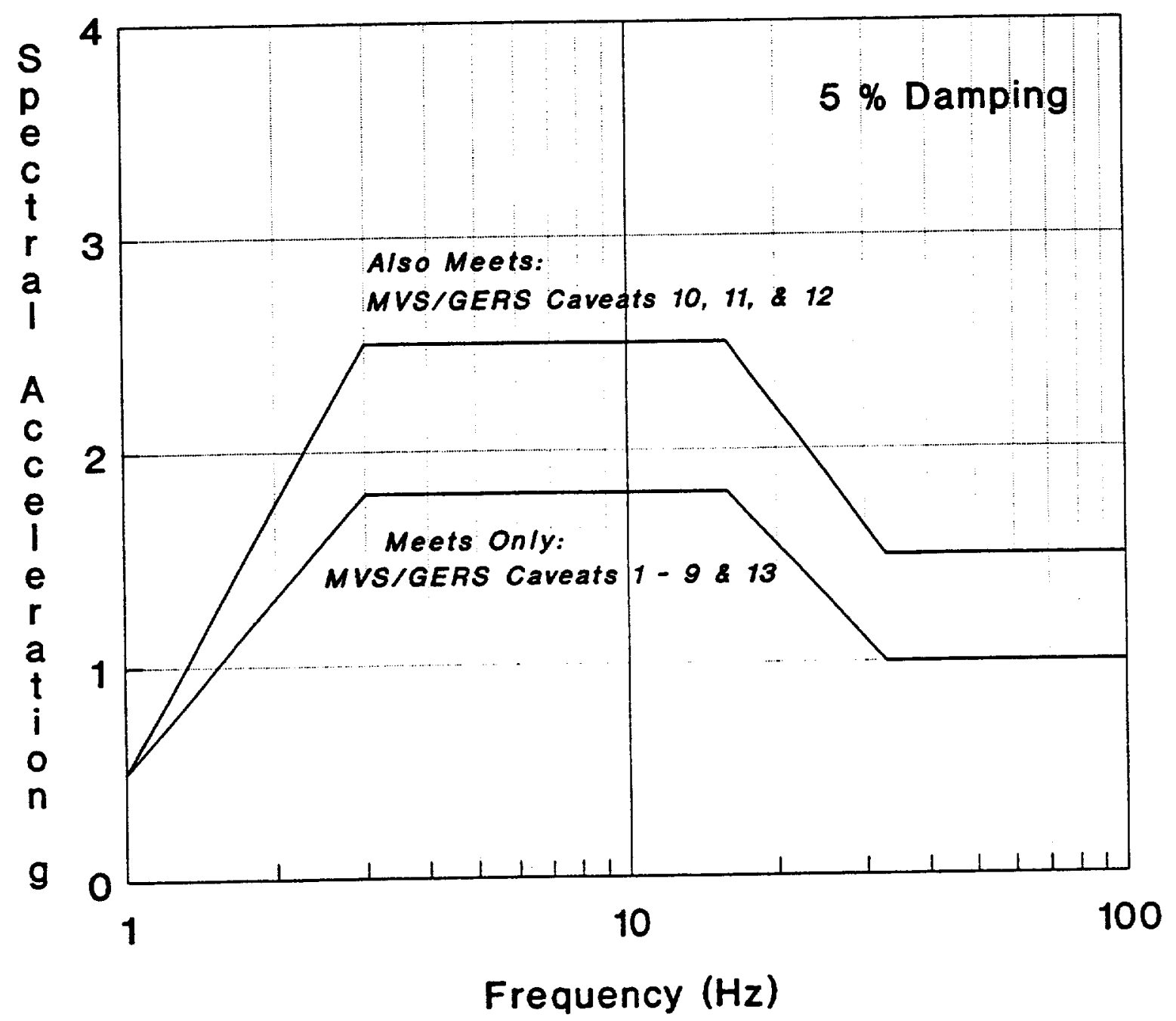

\begin{tabular}{l|cccc} 
& \multicolumn{3}{c}{} & \\
Frequency $(\mathrm{Hz})$ & 1 & 3 & 16 & 33 \\
\hline Meets MVS/GERS Caveats $1-13(\mathrm{~g})$ & 0.5 & 2.5 & 2.5 & 1.5 \\
Meets MVS/GERS Caveats $9 \& 13(\mathrm{~g})$ & 0.5 & 1.8 & 1.8 & 1.0
\end{tabular}

Figure 8.1.4-2 Generic Equipment Ruggedness Spectra (GERS) for MediumVoltage Switchgear (Reference 40) (Figure B.3-1 of SQUG GIP, Reference 1) 


\subsubsection{DISTRIBUTION PANELS 5}

The seismic capacity for the equipment class of Distribution Panels (DP) (see Figure 8.1.5-1) may be based on earthquake experience data, provided the intent of each of the caveats listed below is met. This equipment class consists of circuit breakers or fusible disconnect switches mounted in vertical stacks within sheet metal cabinets. The function of distribution panels is to distribute low voltage AC or DC power from a main circuit to branch circuits, and to provide overcurrent protection. Distribution panels typically serve AC power systems ranging up to 600 volts and DC power systems ranging up to 250 volts.

Two types of distribution panels are found in facility electrical systems: switchboards and panelboards. Although switchboards and panelboards perform the same function, they differ in construction and application. Switchboards are typically floor-mounted assemblies, while panelboards are usually wall-mounted. Switchboards usually distribute larger quantities of power than panelboards.

Distribution switchboards are freestanding cabinets containing stacks of circuit breakers or fusible switches. They have assemblies of circuit breakers or switches mounted into shelf-like cubicles. Electrical connections are normally routed through enclosed cable compartments in the rear of the cabinet. A switchboard will sometimes include a main circuit breaker and a power metering section mounted in separate compartments within the cabinet. Switchboards are often incorporated into substation assemblies that include motor control centers, transformers, and switchgear. In typical applications, the completely enclosed (safety) switchboard is almost exclusively used. These switchboards are completely enclosed in a sheet metal casing. Switchboard dimensions are standardized with individual sections ranging from 20 to 40 inches in depth and width. The height is generally 90 inches. Switchboard sections can weigh up to 500 pounds.

Distribution panelboards are defined by the National Electric Code (NEC) (Ref. 85) as panels which include buses, switches, and automatic protective devices designed for the control or distribution of power circuits. Panelboards are placed in a cabinet or cutout box which is mounted in or against a wall and accessible only from the front. The assembly of circuit breakers contained in a panelboard is normally bolted to a steel frame, which is in turn mounted to the rear or sides of the panelboard enclosure. Individual circuit breakers are either bolted or plugged into the steel chassis. A cable gutter typically runs along the side of the circuit breaker chassis. Panelboards have a wide range of cabinet sizes. Typical dimensions for wall-mounted units are 20 to 40 inches in height and width, and 6 to 12 inches in depth. Weights for wall-mounted panelboards typically range from 30 to 200 pounds.

Industry standards developed by the National Electrical Manufacturers Association and the Underwriters Laboratories (e.g., NEMA ICS-6 (Ref. 82), UL-508 (Ref. 83)), are maintained for the construction of distribution panel enclosures. These standards determine the minimum structural framing and sheet metal thickness for distribution panel enclosures as a function of sheet metal area between supports or reinforcing.

The Distribution Panel equipment class includes the circuit breakers, fusible switches, metering compartments, switchboard/panelboard enclosure and internals, and attached conduit.

\subsubsection{Reference Spectrum Caveats - Distribution Panels}

The Reference Spectrum (RS) represents the seismic capacity of a Distribution Panel (DP) if the panel meets the intent of the following inclusion and exclusion rules. Note, however, that when the specific wording of a caveat rule is not met, then a reason for concluding that the intent has been met should be provided on the SEWS.

5 Section B.14 of SQUG GIP (Ref. 1) 
DP/RS Caveat 1 - Earthquake Experience Equipment Class. The distribution panel should be similar to and bounded by the DP class of equipment described above. The equipment class descriptions are general and the $S C E s$ should be aware that worst case combinations of certain parameters may not be represented in the generic equipment class. These worst case combinations may have reduced seismic capacity and should be carefully evaluated on a case-by-case basis.

DP/RS Caveat 2 - Contains only Circuit Breakers and Switches. The distribution panel should only contain circuit breakers and switches. The concern is that other seismically vulnerable components not normally associated with a distribution panel may have been added. Other components contained within the panel should be evaluated on a case-by-case basis. This case-bycase evaluation may include use of earthquake experience, test data or component specific qualification data as discussed in Chapter 12, Outlier Evaluation.

DP/RS Caveat 3 - Doors Secured. All doors, latches or screwdriver-operated door fasteners should be secured. The concern addressed by this caveat is that the doors could open during an earthquake and the loose door could repeatedly impact the housing and be damaged or cause internal components to malfunction or chatter.

DP/RS Caveat 4 - Adjacent Cabinets Bolted Together. Adjacent cabinets which are close enough to impact each other and sections of multi-bay cabinet assemblies should be bolted together if any of these cabinets contain essential relays as defined in Chapter 11. The concern addressed in this caveat is that unbolted cabinets could respond out of phase to one another and impact each other during an earthquake. This would cause additional impact loadings and high frequency vibration loadings which may result in malfunction or chatter of internal components.

DP/RS Caveat 5 - General Configuration Similar to NEMA Standards. The general configuration of the distribution panel should be similar to those constructed to NEMA Standards. The unit does not have to conform exactly to NEMA Standards, but should be similar with regard to the gage of steel, internal structure and support. This caveat is intended to preclude unusual designs not covered by the equipment class (thin gage material, flimsy internal structure, etc.). In general, units manufactured by the major manufacturers of distribution panels conform to this caveat if they have not been modified.

DP/RS Caveat 6 - Any Other Concerns? SCEs should seek out suspicious details or uncommon situations not specifically covered by the caveats which could adversely affect the seismic capacity of the panel.

\subsubsection{GERS Caveats - Distribution Panels}

The seismic capacity for the equipment class of Distribution Panels (or load centers) may be based on generic testing data, provided the intent of each of the caveats listed below is met. This equipment class consists of individual molded-case circuit breakers and fused disconnect switches housed in NEMA-type floor and wall enclosures. Units are low voltage rated at 600 VAC (480 VAC nominal) or 250 VDC. A distribution panel receives its electrical power from the facility distribution system and distributes this power to each of the circuit breakers and fused disconnect switches by an internal arrangement of vertical and horizontal bus bars. This equipment class covers distribution panels which contain circuit breakers and switches. For panels which contain an occasional relay or motor starter, the GERS only applies to the remainder of the panel and components mounted on the panel, not to the relay or motor starter. The evaluation of relays and motor starters is covered in Chapter 11.

Floor-mounted (freestanding) distribution panels are denoted as Switchboards (NEMA Standard Publication No. PB2 (Ref. 86)). The typical floor enclosure is 90 inches high, 36 inches wide, and 20 inches deep. 
Wall-mounted (either flush or surface mount) distribution panels are denoted as Panelboards (National Electrical Code NFPA/ANSI No. 70 (Ref. 85)). Wall-mounted enclosures vary in size, with nominal dimensions ranging up to 48 inches high, 24 inches wide, and 12 inches deep.

The GERS (see Figure 8.1.5-2) represent the seismic capacity of a Distribution Panel (DP) (Switchboard or Panelboard) if the panel meets the intent of the following inclusion and exclusion rules. Note, however, that when the specific wording of a caveat rule is not met, then a reason for concluding that the intent has been met should be provided on the SEWS.

DP/GERS Caveat 1 - Generic Seismic Testing Equipment Class. The distribution panel should be similar to and bounded by the DP class of equipment described above. The equipment class descriptions are general and the SCEs should be aware that worst case combinations of certain parameters may not be represented in the generic equipment class. These worst case combinations may have reduced seismic capacity and should be carefully evaluated on a case-by-case basis.

DP/GERS Caveat 2 - Reference Spectrum Caveats Apply. The panel should meet all the caveats given for the Reference Spectrum. This caveat is included to cover the vulnerabilities identified for the earthquake experience equipment class. Those GERS caveats which are the same as the Reference Spectrum caveats are not repeated below.

DP/GERS Caveat 3 - Freestanding, Designated Switchboard. The Switchboard GERS can be used only if the unit is freestanding and designated as a switchboard by the manufacturer; otherwise the Panelboard GERS should be used. A review of manufacturer's submittals and parts list is sufficient. These two subclasses (Switchboard and Panelboard) have different seismic capacity based on the generic seismic test data.

DP/GERS Caveat 4 - Circuit Breaker Model Excluded. The GERS cannot be used for distribution panels that contain the Westinghouse "Quicklag" Type E circuit breakers. This circuit breaker model has been shown to trip at levels below the $2.5 \mathrm{~g}$ GERS. A review of manufacturer's submittals and parts listed is sufficient to determine whether this type of circuit breaker is used.

DP/GERS Caveat 5 - Adjacent Cabinets Bolted Together. Adjacent cabinets and sections of a multi-bay cabinet assembly should be bolted together. Adjacent cabinets and sections of multi-bay cabinet assemblies were bolted together when tested for this generic seismic testing equipment class. 


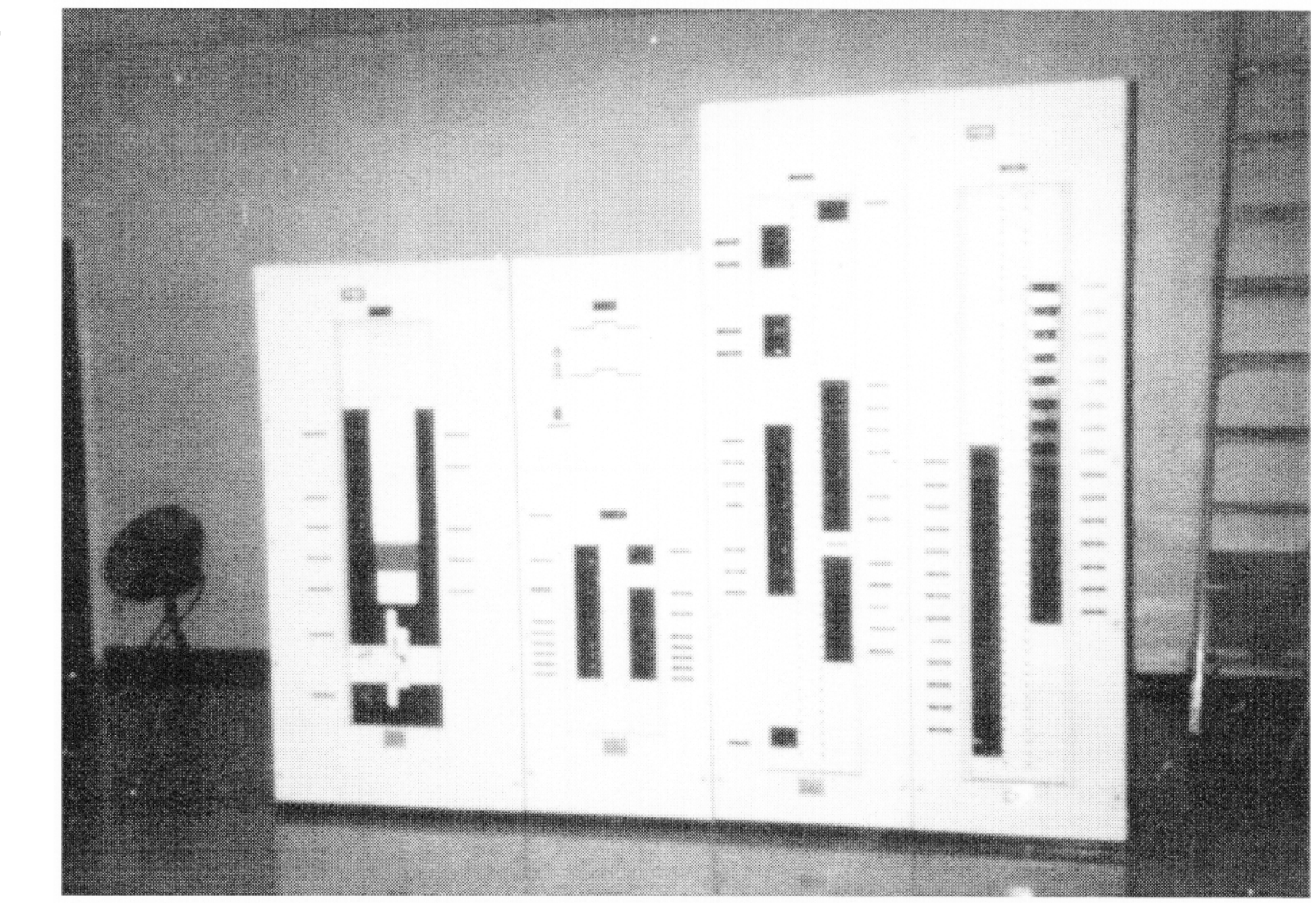

Figure 8.1.5-1 Distribution Panels from the Earthquake Experience Database 
Distribution Panels

GERS-DSP.7

$12 / 1 / 90$

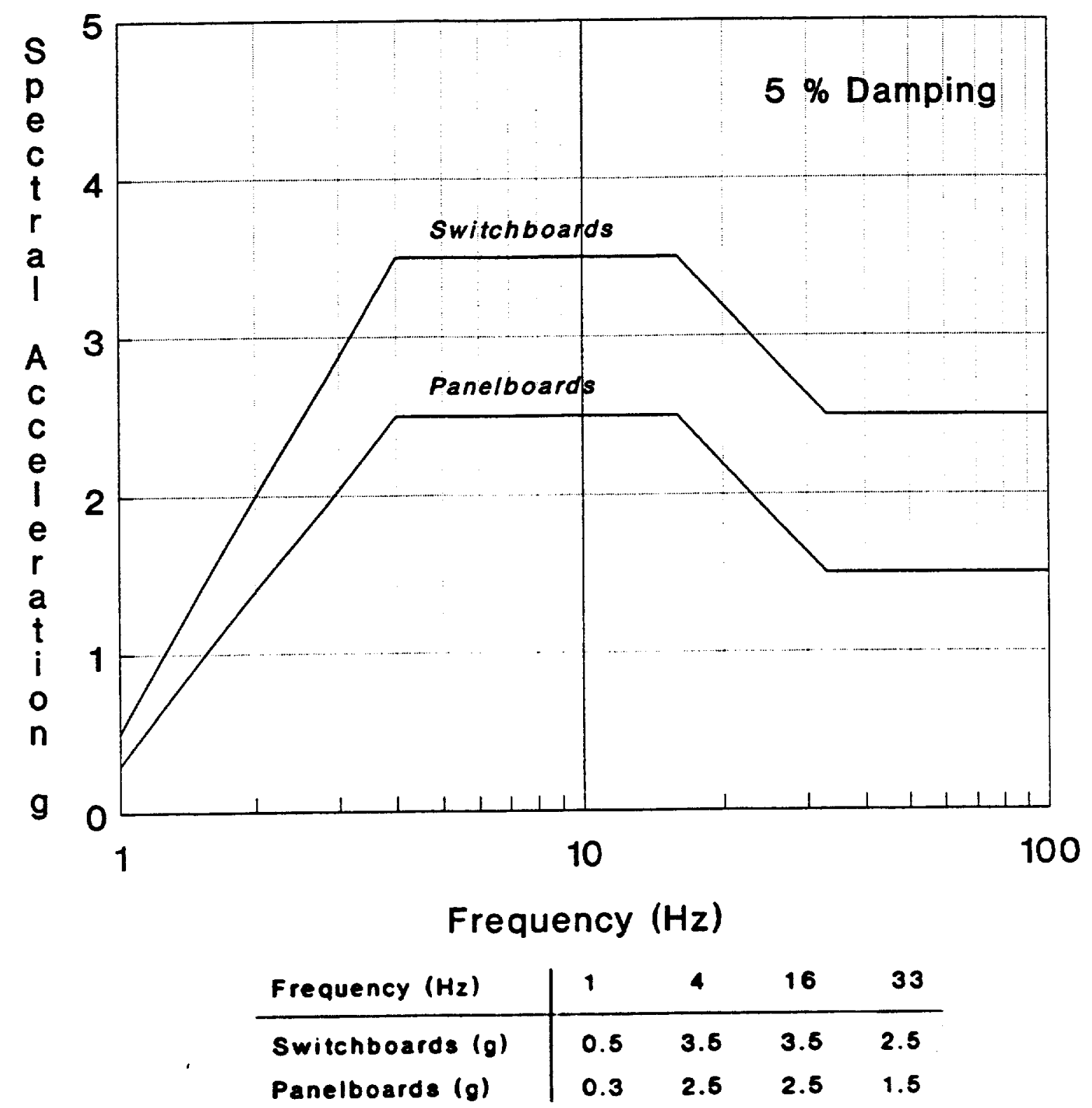

Figure 8.1.5-2 Generic Equipment Ruggedness Spectra (GERS) for Distribution Switchboards and Panelboards (Reference 40) (Figure B.14-1 of SQUG GIP, Reference 1) 


\subsubsection{TRANSFORMERS 6}

The seismic capacity for the equipment class of transformers (TRN) (see Figure 8.1.6-1) may be based on earthquake experience data, provided the intent of each of the caveats listed below is met. This equipment class includes the unit substation type, typically $4160 / 480$ volts, and the distribution type, typically $480 / 120$ volts. Main power transformers with primary voltages greater than about 13,800 volts are not included in this equipment class. Small transformers that are components of electrical equipment, such as motor control centers or control panels, are also not included in this equipment class but are addressed as components of other classes of electrical equipment.

Unit substation transformers step power down from the medium voltage levels (typically 4160 volts for use in large mechanical equipment) to lower voltage levels (typically 480 volts) for use in smaller equipment. Distribution transformers usually step power from the 480 volt level to the 120 to 240 volt level to operate small mechanical equipment, battery chargers, or lighting systems.

Unit substation transformers included in the equipment class can be freestanding or attached to motor control centers or switchgear assemblies. They typically have primary voltages of 2400 to 4160 volts, and secondary voltages of 480 volts. This transformer type may be either liquid- or air-cooled. Liquid-cooled units typically consist of a rectangular steel tank filled with oil or a similar insulating fluid. The transformer coils are submerged in a liquid bath which provides cooling and insulation within the steel tank casing. Most liquid-filled transformers have one or more radiator coils attached to the side of the transformer.

Air-cooled or dry-type unit substation transformers are similar in size and construction to liquidcooled units, except the transformer coils are mounted in a ventilated steel enclosure, rather than a liquid bath. Larger air-cooled unit substation transformers may have small fans mounted to their enclosures for forced air cooling.

The casings of both liquid-cooled and air-cooled unit substation transformers have typical overall dimensions of 60 to 100 inches in height, and 40 to 100 inches in width and depth. The weights of these units range from 2000 to 15,000 pounds.

Distribution transformers typically have primary voltages of 480 volts stepping down to secondary voltages of 120 to 240 volts. This type of transformer is almost always air-cooled. The construction of distribution transformers is essentially the same as that of unit substation transformers, except for a difference in size. The sizes of typical distribution transformers range from small wall-mounted or cabinet-mounted units that have overall dimensions of about 10 inches in height, width, and depth, and weights of 50 to 100 pounds; to larger units that are typically floor-mounted with dimensions ranging up to the size of unit substation transformers and weights ranging up to 5000 pounds.

The transformer equipment class includes the enclosure along with the internals and attached cable and conduit.

\subsubsection{Reference Spectrum Caveats - Transformers}

The Reference Spectrum (RS) represents the seismic capacity of a Transformer (TRN) if the transformer meets the intent of the following inclusion and exclusion rules. Note, however, that when the specific wording of a caveat rule is not met, then a reason for concluding that the intent has been met should be provided on the SEWS.

6 Section B.4 of SQUG GIP (Ref. 1) 
TRN/RS Caveat 1 - Earthquake Experience Equipment Class. The transformer should be similar to and bounded by the TRN class of equipment described above. The equipment class descriptions are general and the SCEs should be aware that worst case combinations of certain parameters may not be represented in the generic equipment class. These worst case combinations may have reduced seismic capacity and should be carefully evaluated on a case-by-case basis.

TRN/RS Caveat 2 - Rating of $4.16 \mathrm{KV}$ or Less. The transformer should have a $4.16 \mathrm{KV}$ rating or less. This is the upper bound voltage rating of transformers included in the earthquake experience equipment class.

TRN/RS Caveat 3 - Transformer Coils Positively Restrained Within Cabinet. For floor-mounted dry and oil-type units, the transformer coils should be positively restrained within their cabinet so that relative sliding and rocking motions between the transformer coil and their cabinet is kept to an acceptable level. The concern is that excessive relative motions may damage the wiring yoke, or that the coils may come in contact with their cabinet which may result in a short circuit or damage to the electrical insulation. This caveat especially applies to transformers whose installation procedure recommends that bolts used to anchor the coils during shipping be removed. If the unit is factory-sealed or constructed so that removing shipping anchors is precluded, no internal inspection is necessary.

TRN/RS Caveat 4 - Coils Top Braced or Analyzed for Large Transformers. Large transformers of $750 \mathrm{kVA}$ or larger should also have the top of the coils braced by a structural frame or should be analyzed for adequate restraint. If the unit is factory-sealed or constructed so that removing shipping anchors is precluded, no internal inspection is necessary.

TRN/RS Caveat 5 - Clearance Between Energized Component and Cabinet. For $750 \mathrm{kVA}$ transformers and larger, there should be at least a 2-inch gap between the energized component and the upper portion of the transformer cabinet. If the gap is less than 2 inches, it should be evaluated by analysis that there is sufficient gap and/or there should be provisions for relative lateral displacement to preclude contact between the energized component and the cabinet. The concern is that without adequate clearance, transformers could be shorted out during the earthquake and thereby rendered inoperable.

TRN/RS Caveat 6 - Adequate Slack in High Voltage Leads. For 750 kVA transformers and larger, the connection between the high voltage leads and the first anchor point should accommodate at least a 3-inch relative displacement, or should be analyzed for adequate slack for relative displacement.

TRN/RS Caveat 7 - Wall-Mounted Units Anchored Close to Enclosure Support. The transformer coil contained in wall-mounted units should have engineered anchorage and be anchored to its enclosure near the enclosure support surface. The concern is that a well-engineered load path should exist for earthquake loadings from the transformer coil (which is relatively massive), through the enclosure, and to the enclosure support. If the transformer coil is not anchored to the enclosure near the enclosure support surface, a calculation can be performed to show that the earthquake loadings can be transferred to the anchorage.

TRN/RS Caveat 8 - Weak-Way Bending. The base assembly of floor-mounted units should be properly braced or stiffened such that lateral forces in any direction do not rely on weak-way bending of sheet metal or thin webs of structural steel shapes. If unbraced or unstiffened steel webs are used, they should be specially evaluated so that adequate strength and stiffness is ensured. 
TRN/RS Caveat 9 - Adjacent Cabinets Bolted Together. Adjacent cabinets which are close enough to impact each other, and sections of multi-bay cabinet assemblies should be bolted together if any of these cabinets contains essential relays as defined in Chapter 11. The concern addressed in this caveat is that unbolted cabinets could respond out of phase to one another and cause impact loadings and high frequency vibration loadings which could cause any impact sensitive essential relays to chatter.

TRN/RS Caveat 10 - Doors Secured. All doors should be secured by a latch or fastener. The concern addressed by this caveat is that the doors could open during an earthquake, and the loose door could repeatedly impact the housing and be damaged or cause internal components such as relays to malfunction or chatter.

TRN/RS Caveat 11 - Any Other Concerns? SCEs should seek out suspicious details or uncommon situations not specifically covered by the caveats which could adversely affect the seismic capacity of the transformer.

\subsubsection{GERS Caveats - Transformers}

The seismic capacity for the equipment class of Transformers may be based on generic testing data, provided the intent of each of the caveats listed below is met. This equipment class includes only dry-type transformers. The equipment in the GERS equipment class is limited to units which range from 7.5 to $225 \mathrm{KVA}$ capacity with either single- or three-phase voltage ratings of 120-480 volts AC. These transformers are housed in NEMA-type (Ref. 82 ) metal enclosures which can be either wall-mounted or floor-mounted.

The GERS (see Figure 8.1.6-2) represent the seismic capacity of a Transformer (TRN) if the transformer meets the intent of the following inclusion and exclusion rules. Note, however, that when the specific wording of a caveat rule is not met, then a reason for concluding that the intent has been met should be provided on the SEWS.

TRN/GERS Caveat 1 - Generic Seismic Testing Equipment Class. The transformer should be similar to and bounded by the TRN class described above. The equipment class descriptions are general and the SCEs should be aware that worst case combinations of certain parameters may not be represented in the generic equipment class. These worst case combinations may have reduced seismic capacity and should be carefully evaluated on a case-by-case basis.

TRN/GERS Caveat 2 - Reference Spectrum Caveats Apply. The transformer should meet all the caveats given for the Reference Spectrum. This caveat is included to cover the vulnerabilities identified for the earthquake experience equipment class. Those GERS caveats which are the same as the Reference Spectrum caveats are not repeated below.

TRN/GERS Caveat 3 - Only Dry-Type Transformer. The transformer should be a dry-type unit. Oil-filled units are excluded as they are not included in the generic seismic testing equipment class.

TRN/GERS Caveat 4 - NEMA-Type Enclosure. The transformer should be housed within a wallor floor-mounted NEMA-type enclosure (review of manufacturer's submittals is sufficient). This is the enclosure type represented by the generic seismic testing equipment class.

TRN/GERS Caveat 5 - Voltage Rating of 120-480 VAC. The transformer should have a single- or three-phase voltage rating of $120-480$ volts AC (review of manufacturer's submittals or transformer name-plate is sufficient).

TRN/GERS Caveat 6 - Capacity of 7.5 to $225 \mathrm{KVA}$. The transformer should have a capacity of 7.5 to $225 \mathrm{KVA}$ (review of manufacturer's submittals or transformer name-plate is sufficient). 
TRN/GERS Caveat 7 - Weight of $180-2000$ Pounds. The transformer should weigh between 180 and 2000 pounds (review of the manufacturer's submittals or transformer name-plate is sufficient).

TRN/GERS Caveat 8 - Transformer Internal Supports. The internal supports should provide positive attachment of the transformer components (a force transfer path for seismic loads is necessary).

TRN/GERS Caveat 9 - Sufficient Clearance Between Bare Conductors and Enclosure. The clearance between any bare conductor and the transformer enclosure should be at least 3/8 inch. The concern is that without adequate clearance, transformers could be shorted out during the earthquake and thereby rendered inoperable.

TRN/GERS Caveat 10 - Adjacent Cabinets Bolted Together. Adjacent cabinets and sections of a multi-bay cabinet assembly should be bolted together. Adjacent cabinets and sections of multi-bay cabinet assemblies were bolted together when tested for this generic seismic testing equipment class. 


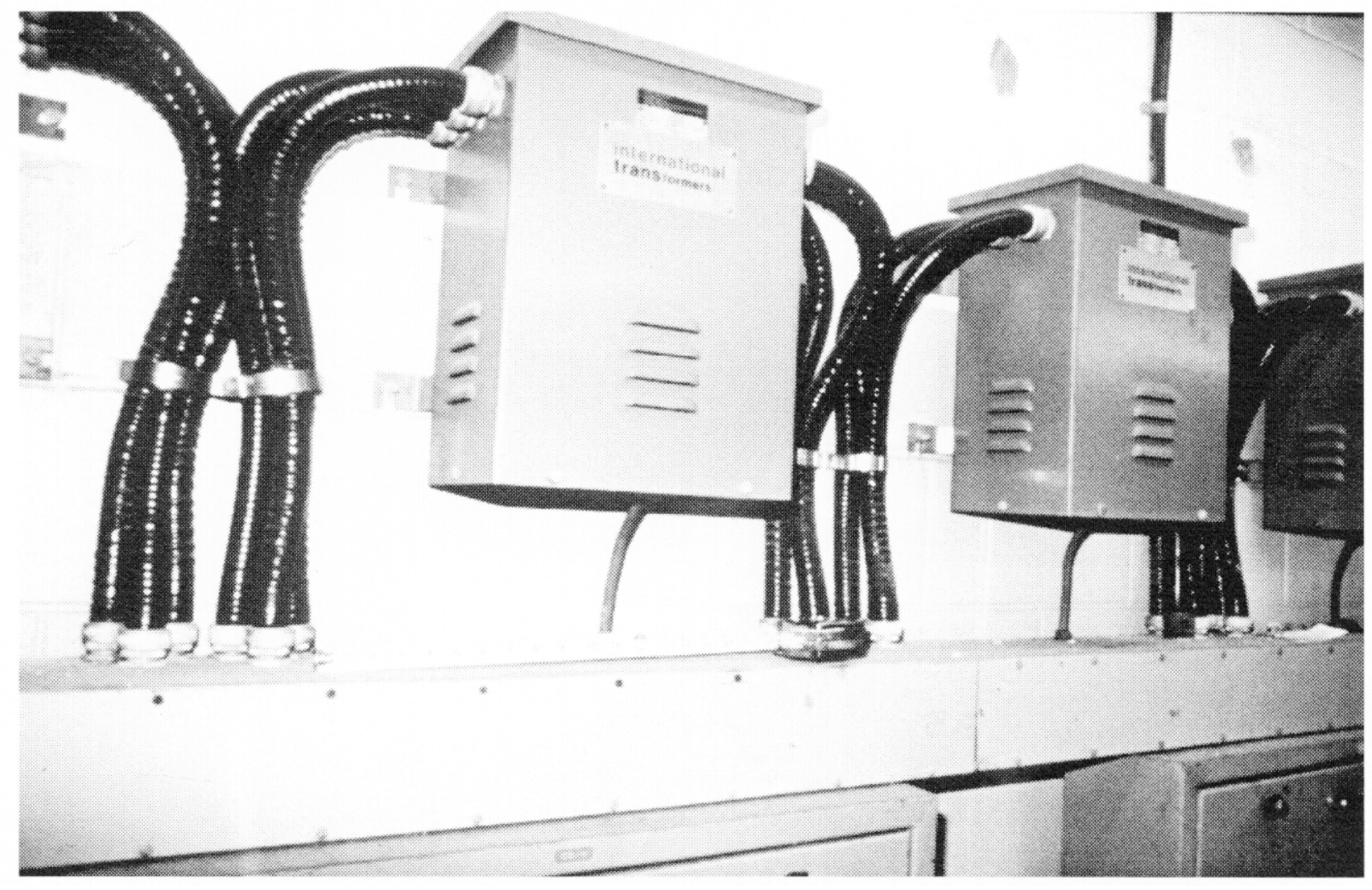

Figure 8.1.6-1 Transformers from the Earthquake Experience Database 
Transformers

GERS-TR.4

$12 / 1 / 90$

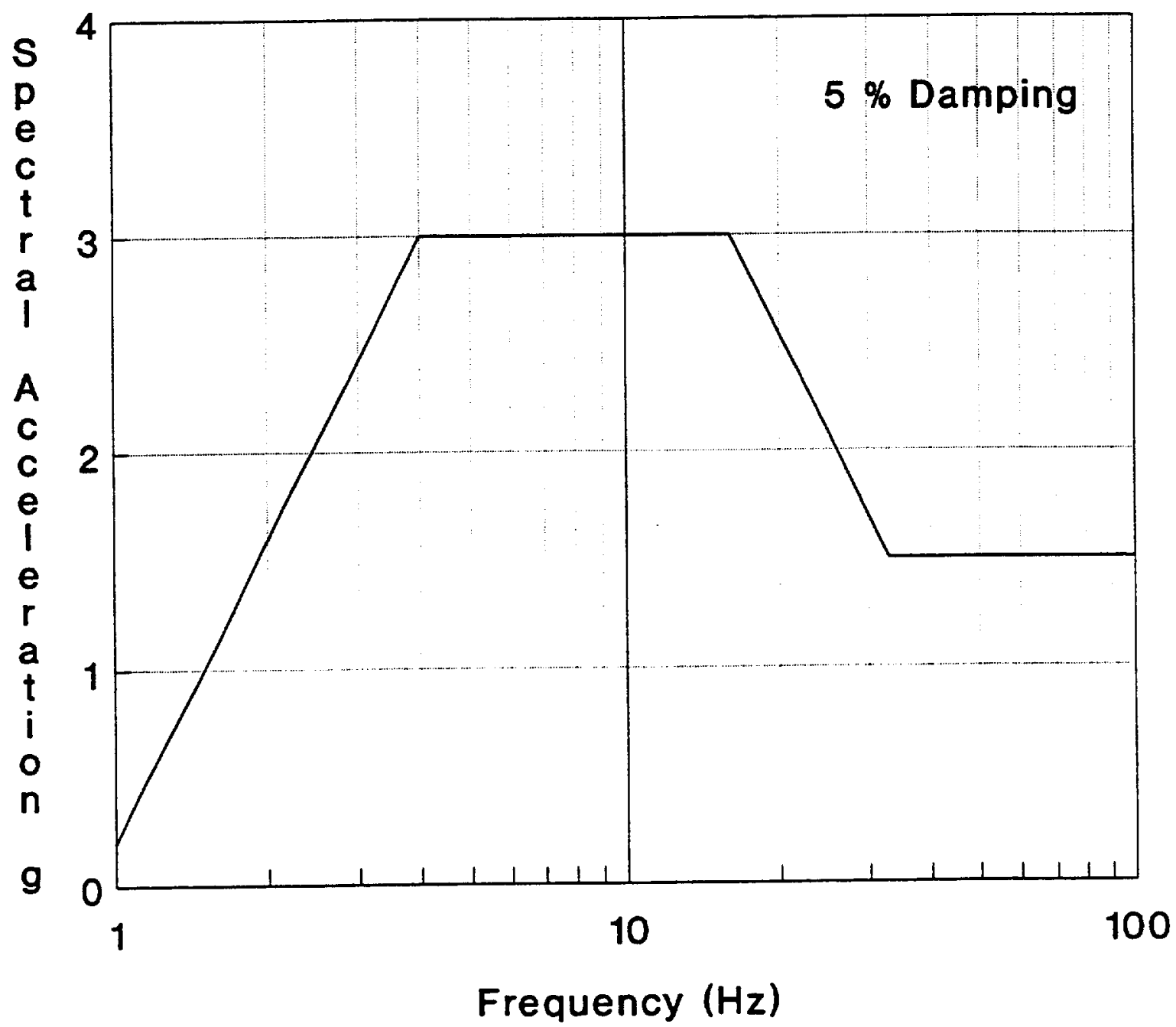

\begin{tabular}{l|cccc} 
Frequency $(\mathrm{Hz})$ & 1 & 4 & 16 & 33 \\
\hline Dry-Type Transformers $(g)$ & 0.2 & 3.0 & 3.0 & 1.5
\end{tabular}

Figure 8.1.6-2 Generic Equipment Ruggedness Spectra (GERS) for Dry-Type Transformers (Reference 40) (Figure B.4-1 of SQUG GIP, Reference 1) 


\subsubsection{BATTERY CHARGERS AND INVERTERS ${ }^{7}$}

The seismic capacity for the equipment class of Battery Chargers and Inverters (BCI) (see Figure 8.1.7-1) may be based on earthquake experience data, provided the intent of each of the caveats listed below is met. Chargers and Inverters are grouped into a single equipment class since they perform similar (although electrically inverse) functions, contain similar components, and are packaged in similar cabinets. Solid-state battery chargers are assemblies of electronic components whose function is to convert AC input into DC output. Inverters are assemblies whose function is to convert DC input into AC output. Battery chargers and inverters are normally housed in flooror wall-mounted cabinets.

The most common applications for both battery chargers and inverters are as components of an uninterruptible power supply (UPS). A typical UPS consists of a solid-state inverter, a battery charger, a set of lead-acid storage batteries, and an automatic transfer switch. Chargers serve the station batteries which provide a DC power source to controls, instrumentation and switchgear. A portion of the DC power from the batteries is routed through inverters which provide a source of $\mathrm{AC}$ power to critical equipment.

The primary electrical function of a battery charger is accomplished using a rectifier. Most battery chargers are based on solid-state rectifiers consisting of semiconductors. This equipment class is limited to solid-state battery chargers and inverters.

The primary components of battery chargers include solid-state diodes, transformer coils, capacitors, electronic filters, and resistors. In addition, the primary components are usually protected from electrical faults by molded case circuit breakers and fuses. The internal components are normally bolted either to the rear panel or walls of a cabinet, or to interior panels or steel frames mounted within a cabinet. The front panel of the cabinet typically contains instrumentation and controls, including ammeters, voltmeters, switches, alarms, and control relays. Inverters contain primary components similar to those found in battery chargers. Virtually all inverters use solid state components.

Battery chargers and inverters are typically mounted in separate cabinets, but they are sometimes supplied as an assembly of two adjoining cabinets. The smallest units are wall-mounted or rackmounted with typical dimensions of 10 to 20 inches in height, width, and depth, and typical weights of 50 to 200 pounds. Typical cabinet dimensions for larger floor-mounted units are 20 to 40 inches in width and depth, and 60 to 80 inches in height. The weights of the floor-mounted chargers and inverters range from several hundred to several thousand pounds. Typical AC voltages to battery chargers and from inverters range from 120 to 480 volts. Voltages in DC power typically range from 24 to 240 volts.

Industry standards are maintained for the construction of cabinets by the National Electrical Manufacturers Association (Ref. 82) and Underwriters Laboratories (Ref. 83). These standards determine the minimum structural framing and sheet metal thickness for charger and inverter cabinetry as a function of size.

Solid-state inverters and battery chargers are included in the equipment class in freestanding, rackmounted, and wall-mounted configurations. The Battery Charger and Inverter equipment class includes the sheet metal enclosure, all internal components, junction boxes, and attached cable or conduit.

7 Section B.16 of SQUG GIP (Ref. 1) 


\subsubsection{Reference Spectrum Caveats - Battery Chargers and Inverters}

The Reference Spectrum (RS) represents the seismic capacity of a Battery Charger or Inverter (BCI) if the equipment meets the intent of the following inclusion and exclusion rules. Note, however, that when the specific wording of a caveat rule is not met, then a reason for concluding that the intent has been met should be provided on the SEWS.

BCI/RS Caveat 1 - Earthquake Experience Equipment Class. The battery charger or inverter should be similar to and bounded by the BCI class of equipment described above. The equipment class descriptions are general and the SCEs should be aware that worst case combinations of certain parameters may not be represented in the generic equipment class. These worst case combinations may have reduced seismic capacity and should be carefully evaluated on a case-bycase basis.

BCI/RS Caveat 2 - Solid State Type. The battery charger or static inverter should be a solid-state type. The solid-state electrical construction is the primary type included in the earthquake experience equipment class. The concern is that electronics which are not of the solid state variety (glass tubes, etc.) are vulnerable to earthquake damage.

BCI/RS Caveat 3 - Transformer Mounted Near Base of Floor-Mounted Units. For floor-mounted units, the transformer, which is the heaviest component of this equipment, should be positively anchored and mounted near the base of the cabinet. If not mounted near the base, then the load path should be specially evaluated. The concern is that the lateral earthquake loads on the transformer will not be properly transferred to the equipment base. The load path evaluation may use judgment or simple calculations to ensure that the structure can transfer these loads.

BCI/RS Caveat 4 - No Reliance on Weak-Way Bending of Steel Plate or Structural Steel Shapes. The base assembly of floor-mounted units should be properly braced or stiffened such that lateral forces in any direction do not rely on weak-way bending of sheet metal or thin webs of structural steel shapes. If such unbraced or unstiffened steel webs exist, they should be investigated and evaluated for adequacy by the $S C E s$ to check the strength and stiffness.

BCI/RS Caveat 5 - Load Path Check for Wall-Mounted Units. If the battery charger or inverter is a wall-mounted unit, the transformer supports and bracing should be visually reviewed for a proper load path to the rear cabinet wall. Lateral earthquake loads on the heavy transformer need to be properly transferred to the anchorage.

BCI/RS Caveat 6 - Doors Secured. All doors should be secured by a latch or fastener. The concern addressed by this caveat is that the doors could open during an earthquake and the loose door could impact the housing and be damaged or cause internal components to malfunction.

BCI/RS Caveat 7 - Adjacent Cabinets Bolted Together. Adjacent cabinets which are close enough to impact each other, and sections of multi-bay cabinet assemblies should be bolted together if any of these cabinets contains essential relays as defined in Chapter 11. The concern addressed in this caveat is that unbolted cabinets could respond out of phase to one another and cause impact loadings and high frequency vibration loadings which could cause any impact sensitive essential relays to chatter.

BCIRS Caveat 8 - Any Other Concerns? SCEs should seek out suspicious details or uncommon situations not specifically covered by the caveats which could adversely affect the seismic capacity of the battery charger or inverter. 


\subsubsection{GERS Caveats - Battery Chargers and Inverters}

The seismic capacity for the equipment class of both Battery Chargers and Inverters may be based on generic testing data, provided the intent of each of the caveats listed below is met. Battery charger units range from 25 to 600 amp capacity with either single- or three-phase voltage ratings of 24 to 250 volts DC and 120 to 480 Volts AC. The units utilize solid-state technology (siliconcontrolled rectifier, SCR) in both the main circuits and the power controls. Major components include protective circuit breakers, transformers, power supply, SCR, filter, and various alarm relays, and control circuits. The units are housed in NEMA-type floor- or wall-mounted enclosures. This equipment class includes typical battery chargers used in facilities for float charging of lead-acid storage battery sets.

DC to AC inverter units included in the GERS data base range from 0.5 to $15 \mathrm{KVA}$ capacity with either single- or three-phase voltage ratings of 120 volts DC and 120 to 480 volts AC. The units utilize solid-state technology (silicon-controlled rectifier, SCR), and have protective circuit breakers, transformers, frequency control circuitry, various alarm relays and SCR power control circuits as major components. The units are housed in NEMA-type floor-mounted enclosures. This equipment class covers typical 120 VDC inverters used in facilities for critical power supply.

The GERS (see Figures 8.1.7-2 and 8.1.7-3) represents the seismic capacity of a Battery Charger or Inverter (BCI) if the equipment meets the intent of the following inclusion and exclusion rules. Note, however, that when the specific wording of a caveat rule is not met, then a reason for concluding that the intent has been met should be provided on the SEWS.

BCI/GERS Caveat 1 - Generic Seismic Testing Equipment Class. The battery charger or inverter should be similar to and bounded by the BCI class of equipment described above. The equipment class descriptions are general and the SCES should be aware that worst case combinations of certain parameters may not be represented in the generic equipment class. These worst case combinations may have reduced seismic capacity and should be carefully evaluated on a case-bycase basis.

BCI/GERS Caveat 2 - Reference Spectrum Caveats Apply. The battery charger or inverter should meet all the caveats given for the Reference Spectrum. This caveat is included to cover the vulnerabilities identified for the earthquake experience equipment class. Those GERS caveats which are the same as the Reference Spectrum caveats are not repeated below.

BCI/GERS Caveat 3 - SCR Power Controls Within NEMA-Type Enclosure. The battery charger or inverter should be a solid-state unit with SCR power controls (C\&D, PCP, or Exide for battery chargers) (Elgar, Solid State Controls, Staticon for inverters). Battery charger units should be wall- or floor-mounted within a NEMA-type enclosure (review of manufacturer's submittals is sufficient). Only floor mounted inverter units are permitted. The enclosure does not have to conform exactly to NEMA standards but should be similar with regard to the gage of the steel, internal structure and support. The purpose of this caveat is to ensure similarity with the power controls and enclosure type of the generic seismic testing equipment class.

BCI/GERS Caveat 4 - Battery Charger Size and Capacity Range. Battery Charger size and capacity should be within the following range: 24 to $250 \mathrm{VDC}, 120$ to $480 \mathrm{VAC}, 25$ to 600 amps; and weight in the range of 150 to 2,850 pounds with wall-mounted units limited to 600 pounds (review of manufacturer's submittals or Battery Charger nameplate is sufficient). This represents the size and capacity limits of the generic seismic testing equipment class. 
BCV/GERS Caveat 5 - Inverter Size and Capacity Range. Inverter size and capacity should be within the following range: $120 \mathrm{VDC}, 120$ to $480 \mathrm{VAC}, 0.5$ to $15 \mathrm{KVA}$; and weight in the range of 300 to 2,000 pounds. (Review of manufacturer's submittals or inverter nameplate is sufficient.) This represents the size and capacity range of the generic seismic testing equipment class.

BCI/GERS Caveat 6 - Cutouts Require Separate Evaluation. Heavy components should, in general, be located in the lower half of the enclosure height and either supported from the base or rear panel. If cutouts are adjacent to support points for heavy internal components, a separate evaluation is required. The concern is that the seismic load will not be able to be transferred through the shear panels to the anchorage.

BCL/GERS Caveat 7 - Adjacent Cabinets Bolted Together. Adjacent cabinets and sections of a multi-bay cabinet assembly should be bolted together. Adjacent cabinets and sections of multi-bay cabinet assemblies were bolted together when tested for this generic seismic testing equipment class. 


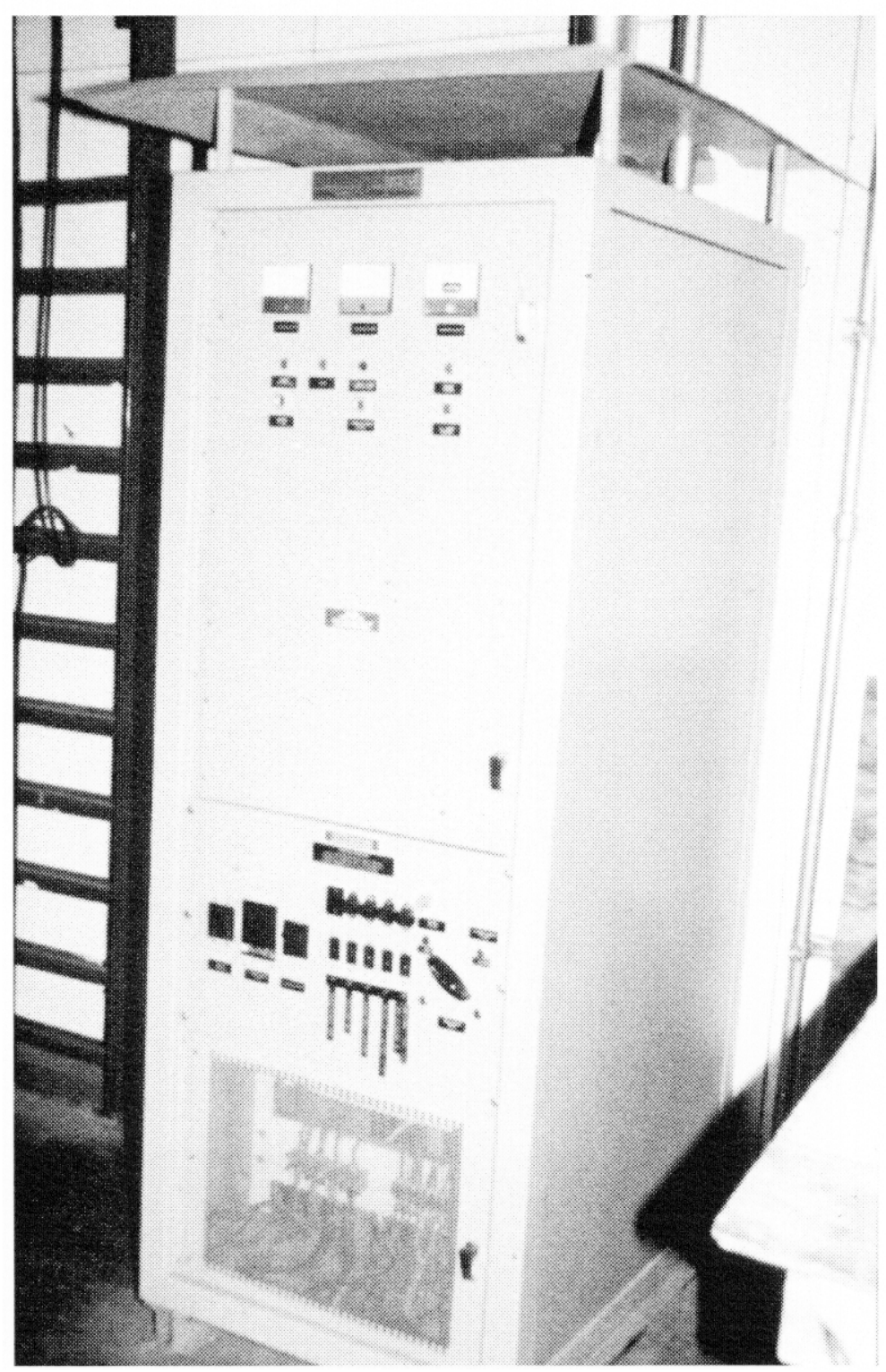

Figure 8.1.7-1 Inverter from the Earthquake Experience Database 


\section{Battery Chargers and Inverters \\ GERS-BC.3 \\ $6 / 1 / 88$}

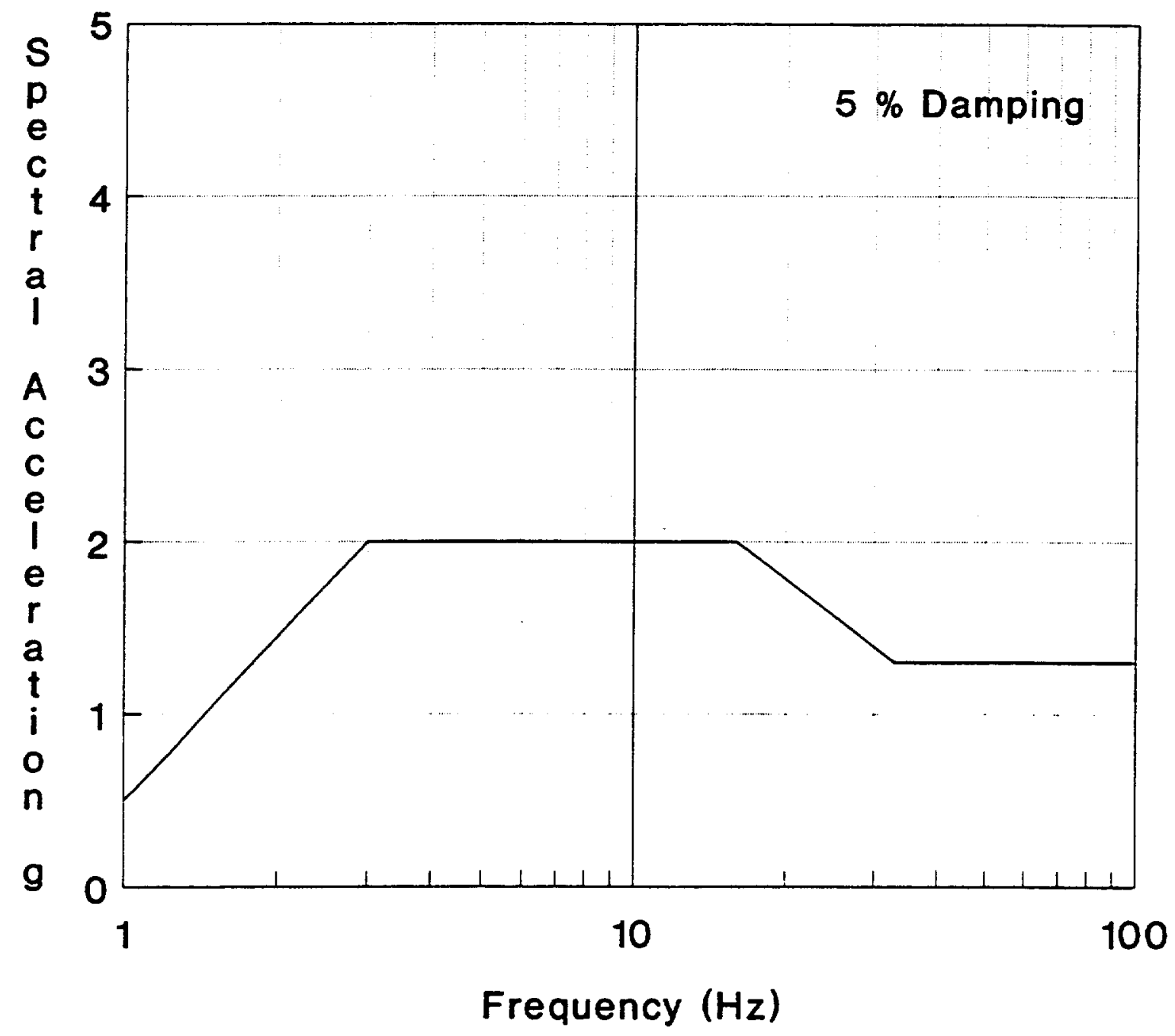

\begin{tabular}{l|llll} 
Frequency $(\mathrm{Hz})$ & 1 & 3 & 16 & 33 \\
\hline Battery Chargers $(\mathrm{g})$ & 0.5 & 2.0 & 2.0 & 1.3
\end{tabular}

Figure 8.1.7-2 Generic Equipment Ruggedness Spectra (GERS) for Battery Chargers (Reference 40) (Figure B.16-1 of SQUG GIP, Reference 1) 
Battery Chargers and Inverters

GERS-INV. 4

$6 / 1 / 88$

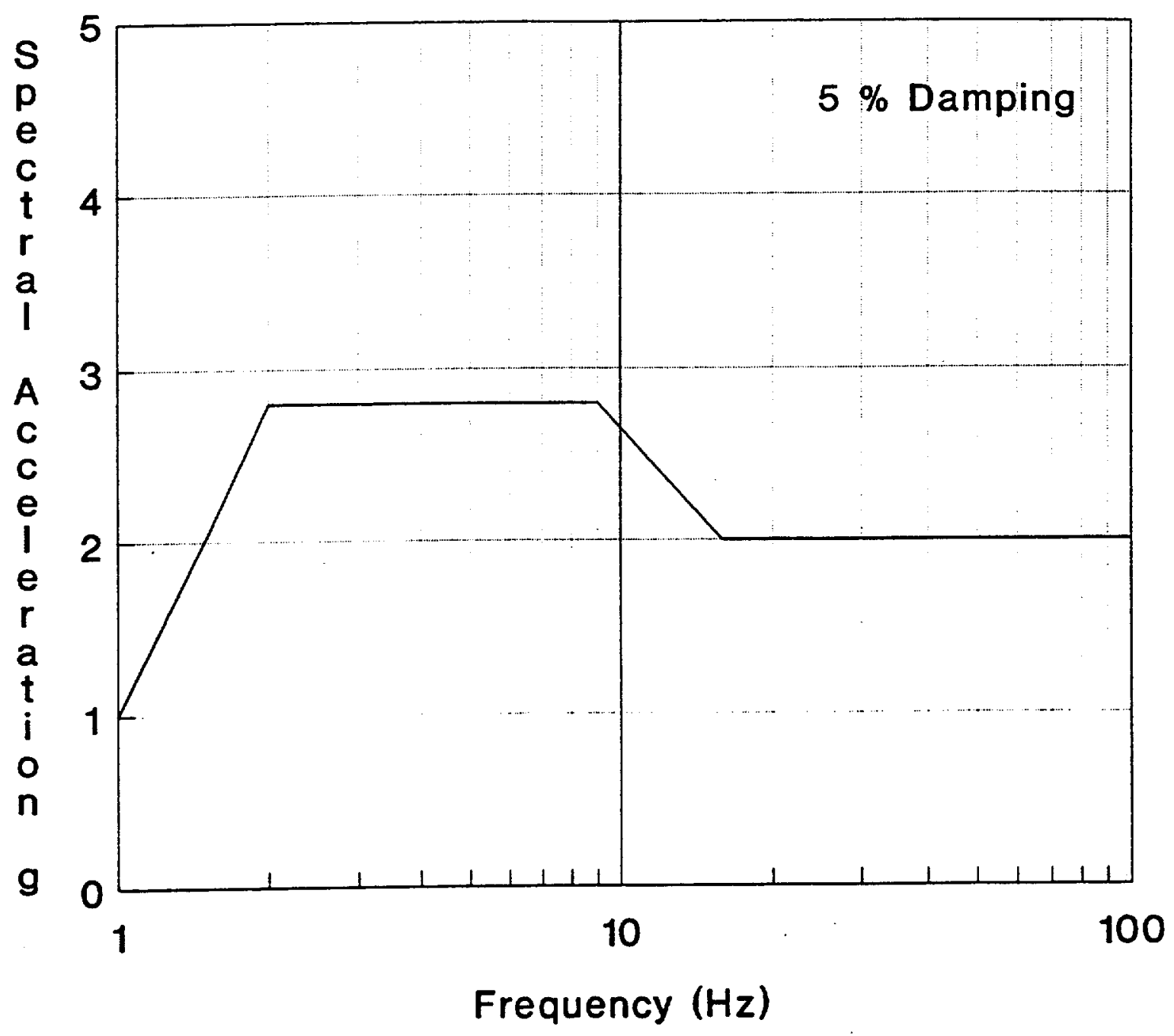

\begin{tabular}{l|lllll} 
Frequency $(\mathrm{Hz})$ & 1 & 2 & 9 & 16 & 33 \\
\hline Inverters $(g)$ & 1.0 & 2.8 & 2.8 & 2.0 & 2.0
\end{tabular}

Figure 8.1.7-3 Generic Equipment Ruggedness Spectra (GERS) for Inverters
(Reference 40) (Figure B.16-2 of SQUG GIP, Reference 1) 


\subsubsection{INSTRUMENTATION AND CONTROL PANELS ${ }^{8}$}

The seismic capacity for the equipment class of Instrumentation and Control Panels (I\&C) (see Figure 8.1.8-1) may be based on earthquake experience data, provided the intent of each of the caveats listed below is met. This equipment class includes all types of electrical panels that support instrumentation and controls. This equipment class includes both the sheet metal enclosure and typical control and instrumentation components mounted on or inside the enclosure.

Instrumentation and control panels and cabinets create a centralized location for the control and monitoring of electrical and mechanical systems. In addition to main control panels, local instrumentation and control panels are sometimes distributed throughout the facilities, close to the systems they serve.

Instrumentation and control panels and cabinets have a wide diversity of sizes, types, functions, and components. Panel and cabinet structures generally consist of a steel frame supporting sheet metal panels to which instrumentation and control components are bolted or clamped. Cabinet structures range from a single panel, braced against or built into a wall, to a freestanding cabinet enclosure. These enclosures are generally categorized as either switchboards or benchboards as described below.

A vertical switchboard is a single reinforced sheet metal instrument panel, which is either braced against an adjacent wall or built into it. An enclosed switchboard is a freestanding enclosed sheet metal cabinet with components mounted on the front face, and possibly on the interior walls. The front or rear panel is usually hinged as a single or double swinging door to allow access to the interior. A dual switchboard consists of two vertical panels braced against each other to form a freestanding structure, with components mounted to both front and rear panels. The sides are usually open, and the two panels are joined by cross members spanning between their tops. A duplex switchboard is similar to a dual switchboard, except that it consists of a panel fully enclosed by sheet metal on all sides, with access through doors in the two side panels.

A benchboard consists of a control desk with an attached vertical panel. A control desk has components mounted on the desk top, and interior access through swinging doors in the rear. The single panel is similar to a vertical switchboard and is normally braced against or built into a wall. A dual benchboard is similar to a dual switchboard, but the lower half of the front panel is a desk console. A duplex benchboard is similar to a duplex switchboard, a totally enclosed panel, but with a desk console in the lower half of the front panel.

Panel and cabinet enclosures normally consist of steel angles, channels, or square tubes welded together, with sheet metal siding attached by spot welds. Large panels are typically made of individual sections bolted together through adjoining framing. The cabinet may or may not include a sheet metal floor or ceiling.

Electronic or pneumatic instrumentation or control devices attached to sheet metal panels or within sheet metal cabinets are included in the equipment class. The Instrumentation and Control Panels equipment class includes the sheet metal enclosure, switches, push buttons, panel lights, indicators, annunciators, gauges, meters, recorders, relays (provided they meet relay requirements), controllers, solid-state circuit boards, power supplies, tubing, wiring, and terminal blocks.

\section{There are no GERS for Instrumentation and Control Panels}

8 Section B.20 of SQUG GIP (Ref. 1) 


\subsubsection{Reference Spectrum Caveats - Instrumentation and Control Panels}

The Reference Spectrum $(R S)$ represents the seismic capacity of Instrumentation and Control Panels (I\&C) if the panel or cabinet meets the intent of the following inclusion and exclusion rules. Note, however, that when the specific wording of a caveat rule is not met, then a reason for concluding that the intent has been met should be provided on the SEWS.

I\&C/RS Caveat 1 - Earthquake Experience Equipment Class. The panel or cabinet should be similar to and bounded by the I\&C class of equipment described above. The equipment class descriptions are general and the SCEs should be aware that worst case combinations of certain parameters may not be represented in the generic equipment class. These worst case combinations may have reduced seismic capacity and should be carefully evaluated on a case-by-case basis.

I\&C/RS Caveat 2 - Evaluate Computers and Programmable Controllers Separately. Computers and programmable controllers should be evaluated separately. The concern is that the subclass of computers and programmable controllers is so diverse that they may not be adequately represented by the earthquake experience data. Computers and programmable controllers should therefore be evaluated on a case-by-case basis.

I\&C/RS Caveat 3 - Evaluate Strip Chart Recorders Separately. Strip chart recorders should be evaluated separately. The concern is that long, narrow recorders which are cantilevered off the panel may not have adequate structural support. Strip chart recorders are commonly supported on compression-type mounting brackets supplied by the manufacturer. These types of support brackets are inherently rugged and generally adequate for transfer of seismic loads. If there are no support brackets, or the support system appears to be a custom design, or the SCEs have any concerns regarding the adequacy of the bracket, then the support system should be subject to further evaluation.

I\&C/RS Caveat 4 - Structural Adequacy. The steel frame and sheet metal should be evaluated for adequacy. Engineering judgment may be used to determine that an adequate load path exists to transfer the lateral earthquake loads to the foundation.

I\&C/RS Caveat 5 - Adjacent Cabinets or Panels Bolted Together. Adjacent cabinets or panels which are close enough to impact each other and sections of multi-bay assemblies should be bolted together if any of these assemblies contain essential relays as defined in Chapter 11. The concern addressed in this caveat is that unbolted cabinets or panels could respond out of phase to one another and impact each other during an earthquake. This would cause additional impact loadings and high frequency vibration loadings which could cause any essential relays to chatter.

I\&C/RS Caveat 6 - Drawers or Equipment on Slides Restrained. Drawers or equipment on slides should be restrained to prevent them from falling out during seismic motion. The concern is that the components in the drawer could slide and become damaged, or slide out and fall onto some other fragile essential component in the vicinity. A latch or fastener should secure these sliding components.

I\&C/RS Caveat 7 - Doors Secured. All doors should be secured by a latch or fastener. The concern addressed by this caveat is that loose doors could repeatedly impact the housing and be damaged or cause internal components such as relays to malfunction or chatter.

I\&C/RS Caveat 8 - Any Other Concerns? SCEs should seek out suspicious details or uncommon situations not specifically covered by the caveats which could adversely affect the seismic capacity of the cabinet or panel. 


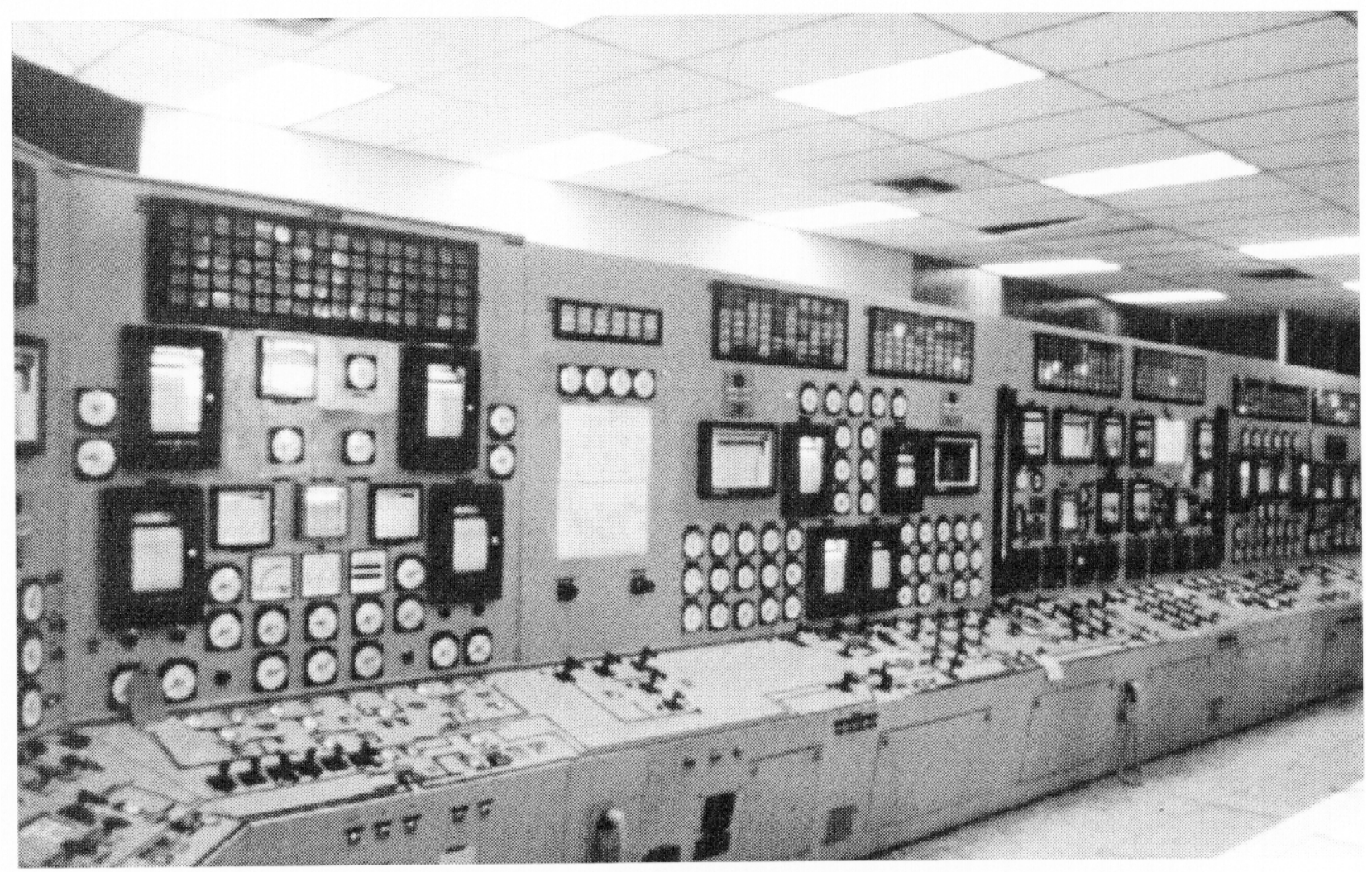

Figure 8.1.8-1 Instrumentation and Control Panels from the Earthquake Experience Database 


\subsubsection{INSTRUMENTS ON RACKS 9}

The seismic capacity for the equipment class of Instruments on Racks (IR) (see Figure 8.1.9-1) may be based on earthquake experience data, provided the intent of each of the caveats listed below is met. This equipment class consists of steel frames that provide mounting for local controls and instrumentation, such as signal transmitters to remote control panels. Instrument racks typically consolidate transducer or control signals from several equipment items in their immediate vicinity.

Instrument racks usually consist of steel members (typically steel angle, pipe, channel, or Unistrut) bolted or welded together into a frame. Components are attached either directly to the rack members or to metal panels that are welded or bolted to the rack. Floor-mounted instrument racks typically range from 4 to 8 feet in height, with widths varying from 3 to 10 feet, depending on the number of components supported on the rack. A simpler configuration of an instrument rack is a single floor-mounted post supporting one or two components. Wall-mounted and structural column-mounted racks are often used for supporting only a few components.

Control system components mounted on instrument racks may include electronic systems used for functions such as temperature monitoring, starting, stopping, and throttling electric motors, and monitoring electric power. Pneumatic system components mounted on instrument racks may be used for monitoring fluid pressure, liquid level, fluid flow, and for adjusting pneumaticallyactuated control valves. Electronic control and instrumentation system components mounted on instrument racks include transmitters that convert a pneumatic signal from the transducer to an electric signal for transmission to the main control panel.

Typical components supported on instrument racks include: pressure switches, transmitters, gauges, recorders, hand switches, manifold valves, and solenoid valves. Attachments to instrument racks include steel or plastic tubing, conduit, and junction boxes.

Freestanding, wall-mounted, and structural column-mounted instrument racks of bolted and welded steel construction are included in the equipment class along with the components mounted on them. Both pneumatic and electronic components, as well as associated tubing, wiring, and junction boxes, are included in the Instruments on Racks equipment class.

\subsubsection{Reference Spectrum Caveats - Instruments on Racks}

The Reference Spectrum (RS) represents the seismic capacity of Instruments on Racks (IR) if the instruments and racks meet the intent of the following inclusion and exclusion rules. Note, however, that when the specific wording of a caveat rule is not met, then a reason for concluding that the intent has been met should be provided on the SEWS.

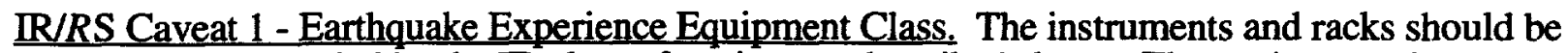
similar to and bounded by the IR class of equipment described above. The equipment class descriptions are general and the SCEs should be aware that worst case combinations of certain parameters may not be represented in the generic equipment class. These worst case combinations may have reduced seismic capacity and should be carefully evaluated on a case-by-case basis.

IR/RS Caveat 2 - Evaluate Computers and Programmable Controllers Separately. Computers and programmable controllers should be evaluated separately. The concern is that the subclass of computers and programmable controllers is so diverse that they may not be adequately represented by the earthquake experience equipment class. Computers and programmable controllers should therefore be evaluated on a case-by-case basis. Component specific test data for computers and programmable controllers may be used to resolve this concern.

9 Section B.18 of SQUG GIP (Ref. 1) 
IR/RS Caveat 3 - Structure Adequate. The steel frame and sheet metal structure should be evaluated in the walkdown for adequacy. Engineering judgment may be used to determine that an adequate load path exists to transfer the lateral earthquake loads to the foundation.

IR/RS Caveat 4 - Adjacent Racks Bolted Together. Adjacent racks which are close enough to impact each other and sections of multi-bay assemblies should be bolted together if any of these assemblies contain essential relays as defined in Chapter 11 . The concern addressed in this caveat is that adjacent, unbolted racks could respond out of phase to one another and impact each other during an earthquake. This would cause additional impact loadings and high frequency vibration loadings which could cause essential relays to chatter.

IR/RS Caveat 5 - Any Other Concerns? SCEs should seek out suspicious details or uncommon situations not specifically covered by the caveats which could adversely affect the seismic capacity of the instrument rack.

\subsubsection{GERS Caveats - Instruments on Racks}

The seismic capacity for the equipment class of Instruments on Racks may be based on generic testing data, provided the intent of each of the caveats listed below is met. This equipment class includes four kinds of transmitters: pressure, temperature, level, and flow. The racks for these instruments are not covered in the generic seismic testing equipment class. Transmitters are used to transmit signals received from transducers which monitor operating conditions. The transmitters send electric signals to control panels for use by safety systems, facility control systems, alarm systems and operator displays. Some transmitters are designed for remote rack or control panel mounting while others are mounted adjacent to the transducer. The term "transmitter" is also used for the transducer/signal conditioner combination when the transducer and signal conditioner are integral. This is the usual case for flow, pressure, and level transmitters. Temperature transmitters are usually remote from the transducer. In general, transmitters range in size from a few pounds to about 40 pounds; however, the majority of the transmitters weigh only a few pounds. The largest physical dimension of a transmitter is usually less than about 12 inches.

The GERS (see Figure 8.1.9-2) represent the seismic capacity of a pressure, temperature, level, or flow transmitter if the transmitter meets the intent of the following inclusion and exclusion rules. Note, however, that when the specific wording of a caveat rule is not met, then a reason for concluding that the intent has been met should be provided on the SEWS.

IR/GERS Caveat 1 - Generic Seismic Testing Equipment Class. The transmitter should be similar to and bounded by the IR class of equipment described above. The equipment class descriptions are general and the SCEs should be aware that worst case combinations of certain parameters may not be represented in the generic equipment class. These worst case combinations may have reduced seismic capacity and should be carefully evaluated on a case-by-case basis.

IR/GERS Caveat 2 - Reference Spectrum Caveats Apply. The transmitter and its supporting rack, when present, should meet all the caveats given for the Reference Spectrum. This caveat is included to cover the vulnerabilities identified for the earthquake experience equipment class. Those GERS caveats which are the same as the Reference Spectrum caveats are not repeated below.

IR/GERS Caveat 3 - Component is a Pressure, Temperature, Level, or Flow Transmitter. The component should be a pressure, temperature, level, or flow transmitter. These are the components included in the generic seismic testing equipment class. 
IR/GERS Caveat 4 - Specific Transmitter Models Included. There is a wide diversity of transmitter types and mechanical properties. Specific manufacturer/models were tested for function during an earthquake. The tested transmitters in the generic seismic testing equipment class include: Foxboro E96, E13, E916; Devar 18-119; Rosemount 1151, 1152, 442; Robertshaw 161; Love 48, 54, 8100, 1106; Kepco PCX; Travis P8, P24.

This caveat may be satisfied for other models of transmitters by performing a case-by-case evaluation of similarity to one of the above models.

IR/GERS Caveat 5 - Seismic Induced System Changes Should be Evaluated. Transmitters are sometimes sensitive to system perturbations. The concern is that the earthquake may induce system changes (i.e., pressure, flow, and level variation) which may have the same effect on the system being controlled as if the transmitter malfunctioned. For example, a level switch used to measure the oil level in the crank case of an emergency diesel-generator (EDG) may be tripped during an earthquake when the oil is sloshing. This reading may inadvertently cause the EDG to trip off line. This caveat is also addressed in the Relay Functionality Review in Chapter 11.

IR/GERS Caveat 6 - No Vacuum Tubes. Vacuum tubes should not be used as internal electrical components. The concern is that glass tubes are especially vulnerable to earthquake damage.

IR/GERS Caveat 7 - All Mounting Bolts in Place. All external mounting bolts (transmitter to bracket and bracket to support) should be in place. This is the condition under which the transmitters were tested during the generic seismic tests.

IR/GERS Caveat 8 - Evaluation of Amplified Response. The transmitters which were tested were attached directly to the shake table. Therefore realistic amplification through the rack (or other supporting structure) to the transmitter should be included when determining the amplified response of the transmitter-to-rack interface for comparison to the GERS. The basis for this amplification factor should be documented.

IR/GERS Caveat 9 - Rack Requires Separate Evaluation. The transmitters were tested separately from the rack, therefore in order use the GERS capacity curves which are higher than the Reference Spectrum, an evaluation of the rack should be made. The evaluation should show that the structural components of the rack are capable of transferring the earthquake loads to the anchorage. This evaluation may depend upon the engineering judgment of the SCES and may not require a formal calculation.

IR/GERS Caveat 10-Adjacent Racks Bolted Together. Adjacent racks and sections of multi-bay rack assemblies should be bolted together. 


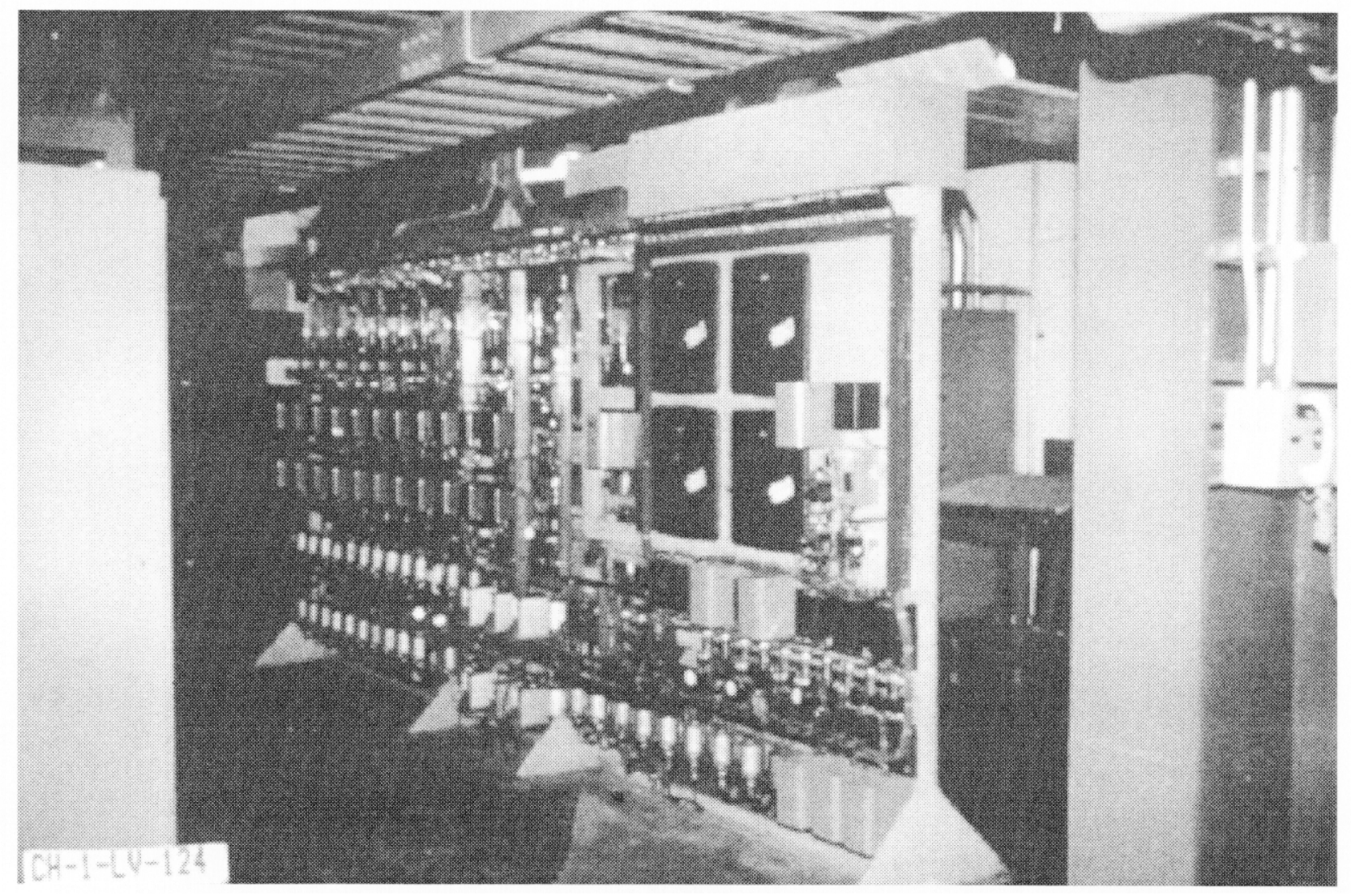

Figure 8.1.9-1 Instruments on Racks from the Earthquake Experience Database 
Instruments on Racks

GERS-PT. 4

$6 / 1 / 88$

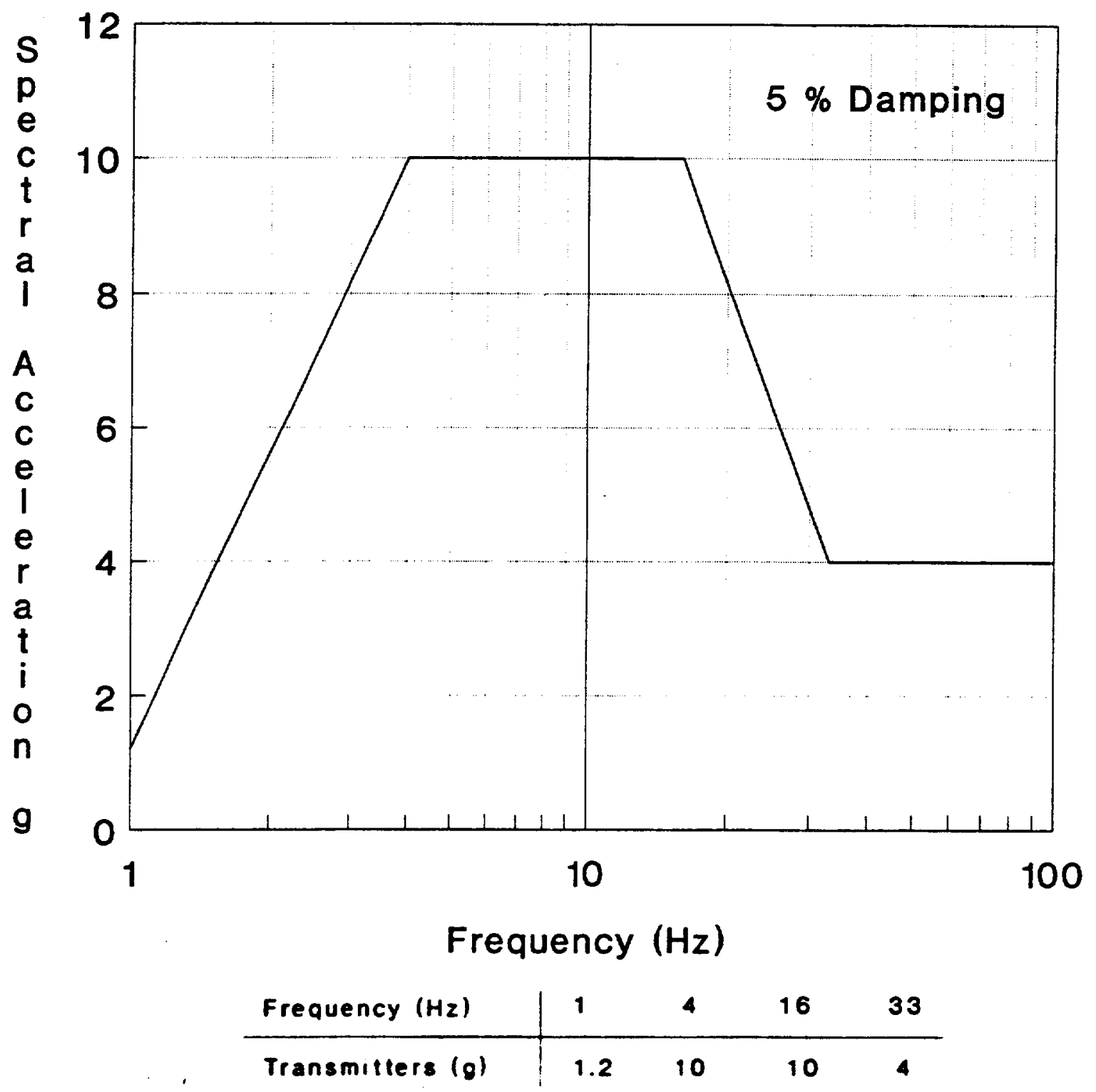

Figure 8.1.9-2 Generic Equipment Ruggedness Spectra (GERS) for Transmitters (Reference 40) (Figure B.18-1 of SQUG GIP, Reference 1) 


\subsubsection{TEMPERATURE SENSORS ${ }^{10}$}

The seismic capacity for the equipment class of Temperature Sensors (TS) (see Figure 8.1.10-1) may be based on earthquake experience data, provided the intent of each of the caveats listed below is met. This equipment class includes thermocouples and resistance temperature detectors (RTDs) that measure fluid temperature and typically are mounted within or on piping or tanks. Thermocouples are probes consisting of two dissimilar metal wires routed through a protective sleeve that produce a voltage output proportional to the difference in temperature between the hot junction and the lead wires (cold junction). RTDs are similar in construction to thermocouples, but their operation is based on variation in electrical resistance with temperature. RTDs and thermocouples are connected to pressure vessel boundaries (piping, tanks, heat exchangers, etc.) using threaded joints. The sensor's sheath will often be inserted into a thermowell or outer protective tube that is permanently mounted in the pipe or tank. A thermowell allows the thermocouple or RTD to be removed without breaking the pressure boundary of the pipe or tank.

Sensors are typically linked to transmitters mounted on nearby instrument racks, which amplify the electronic signal generated in the sensors, and transmit the signal to a remote instrument readout.

The Temperature Sensors equipment class includes the connection head, threaded fitting, sheath or protective tube, thermowell, and attached wires.

There are no GERS for Temperature Sensors.

\subsubsection{Reference Spectrum Caveats - Temperature Sensors}

The Reference Spectrum (RS) represents the seismic capacity of a Temperature Sensor (TS) if the sensor meets the intent of the following inclusion and exclusion rules. Note, however, that when the specific wording of a caveat rule is not met, then a reason for concluding that the intent has been met should be provided on the SEWS.

TS/RS Caveat 1 - Earthquake Experience Equipment Class. The temperature sensor should be similar to and bounded by the TS class of equipment described above. The equipment class descriptions are general and the SCEs should be aware that worst case combinations of certain parameters may not be represented in the generic equipment class. These worst case combinations may have reduced seismic capacity and should be carefully evaluated on a case-by-case basis.

TS/RS Caveat 2 - No Possibility of Detrimental Differential Displacement. Detrimental differential displacement between the mounting of the connection head and the mounting of the temperature sensor should not occur. The concern is that the differential displacement may cause the wiring to be pulled out of the sensor.

TS/RS Caveat 3 - Solid State Electronics. The electronics associated with the temperature sensor should be solid state (i.e., no vacuum tubes). The earthquake experience equipment class only includes solid-state electronics for temperature sensors. The concern is that electronics that are not of the solid-state variety (glass tubes, etc.) are vulnerable to earthquake damage.

TS/RS Caveat 4 - Any Other Concerns? SCEs should seek out suspicious details or uncommon situations not specifically covered by the caveats which could adversely affect the seismic capacity of the temperature sensor.

10 Section B.19 of SQUG GIP (Ref. 1) 


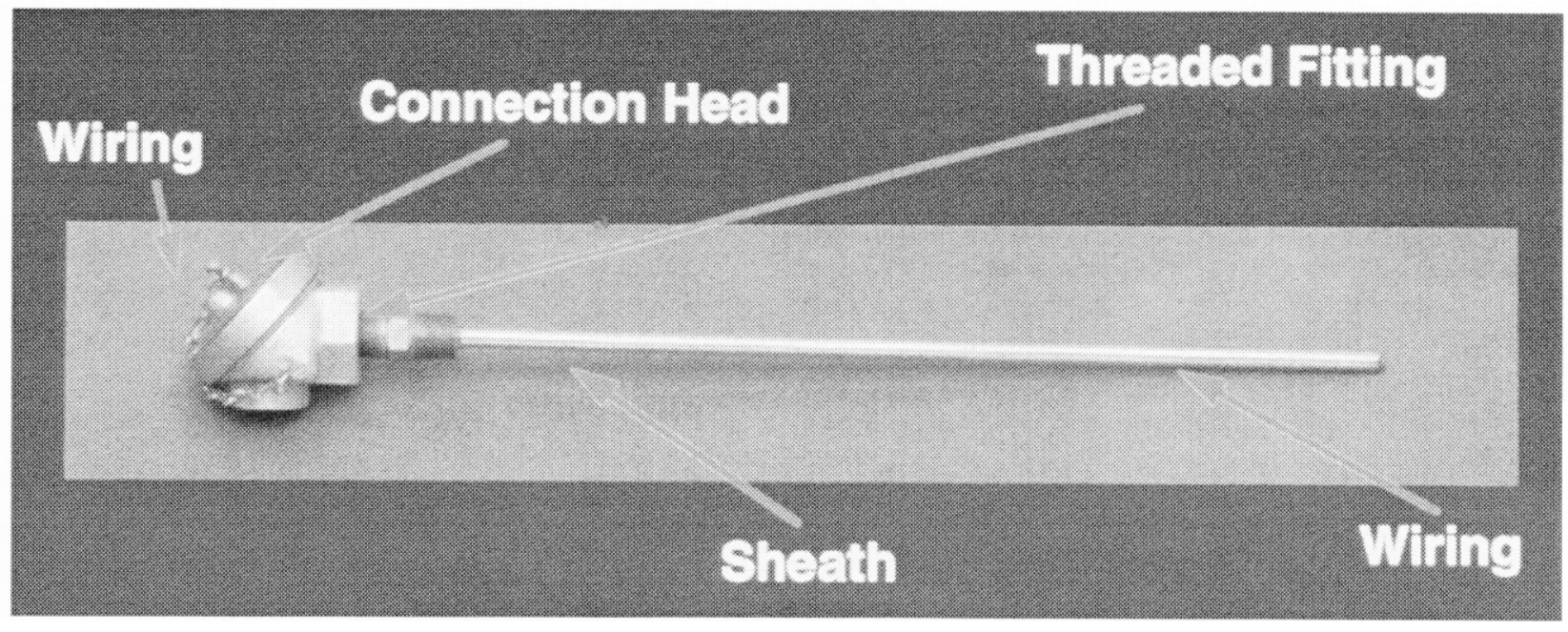

\section{Figure 8.1.10-1 Temperature Sensor}




\subsection{MECHANICAL EQUIPMENT}

\subsubsection{FLUID-OPERATED / AIR-OPERATED VALVES ${ }^{1}$}

The seismic capacity for the equipment class of Fluid-Operated Valves (FOV) and Air-Operated Valves (AOV) (see Figure 8.2.1-1) may be based on earthquake experience data, provided the intent of each of the caveats listed below is met. This equipment class includes a wide diversity of valve sizes, types, and applications, which are actuated by air, water, or oil. Liquid-operated (i.e., hydraulic) piston valves are not included in the FOV class of equipment because they have not been reviewed in sufficient detail to be included.

The main types of fluid-operated valves are diaphragm-operated, piston-operated, and pressure relief valves. The most common type of fluid-operated valve found in facility applications is a spring-opposed, diaphragm-operated pneumatic valve. The bell housing contains a diaphragm (usually a thin, steel membrane) which forms a pressure barrier between the top and bottom sections of the housing. The position of the actuated rod (or valve stem) is controlled by a return spring and the differential pressure across the diaphragm. The actuated rod position, in turn, controls the position of the valve. A yoke supports the bell housing and connects it to the valve body. A solenoid valve or, on larger valves, a pneumatic relay controls the air pressure difference across the diaphragm. This solenoid valve or pneumatic relay is often mounted directly to the operator yoke.

Piston-operated valves are similar to diaphragm-operated valves, with a piston replacing the diaphragm as the valve actuator. The piston typically acts in opposition to a spring to control the position of the valve.

Pressure relief valves are also included in this equipment class. Pressure relief valves balance confined fluid pressure against the force of a spring. The actuating force in a pressure relief valve is supplied by the fluid that is confined by the valve. Fluid-operators are typically cantilevered either above or to the side of the valves they serve. The valve and actuator can form a continuous body, or the actuator can be attached to the valve through a flanged, threaded, or ring clamp connection.

The valve, the operator, the inlet and outlet lines up to their first support on the building or nearby structure, and peripheral attachments (air lines, pneumatic relays, control solenoids, and conduit) are included in the Fluid-Operated Valve equipment class. The valve may be of any type, size, or orientation.

\subsubsection{Reference Spectrum Caveats - Fluid-Operated Valves}

The Reference Spectrum (RS) represents the seismic capacity of a Fluid-Operated Valve (FOV) if the valve meets the intent of the following inclusion and exclusion rules. Note, however, that when the specific wording of a caveat rule is not met, then a reason for concluding that the intent has been met should be provided on the SEWS.

FOV/RS Caveat 1 - Earthquake Experience Equipment Class. The valve should be similar to and bounded by the FOV class of equipment described above. The equipment class descriptions are general and the SCES should be aware that worst case combinations of certain parameters may not be represented in the generic equipment class. These worst case combinations may have reduced seismic capacity and should be carefully evaluated on a case-by-case basis.

1 Section B.7 of SQUG GIP (Ref. 1) 
FOV/RS Caveat 2 - Valve Body Not of Cast Iron. The valve body should not be made of cast iron. The intent of this caveat is to avoid the brittle failure mode of cast iron as evidenced by the poor performance of some cast iron components in past earthquakes. It is not necessary to determine the material of the valve body unless it appears to the SCES that the body is made of cast iron. It is suggested that the material of a flanged valve be checked. In such cases, if the valve is indeed cast iron, the intent of this caveat is satisfied if seismic stresses in the valve body due to piping loads are low (for example, less than $20 \%$ of specified minimum ultimate tensile strength).

FOV/RS Caveat 3 - Valve Yoke Not of Cast Iron for Piston-Operated Valves and Spring-Operated Pressure Relief Valves. The yoke of piston-operated valves and spring-operated pressure relief valves should not be made of cast iron. The intent of this caveat is to avoid the brittle failure mode of cast iron as evidenced by the poor performance of some cast iron components in past

earthquakes. It is not necessary to determine the material of the valve yoke unless it appears to the $S C E s$ that the yoke is made of cast iron. In such cases, if the yoke is indeed cast iron, this caveat may be satisfied by performing a stress analysis of the valve for a $3 \mathrm{~g}$ load applied at the center of gravity of the operator in the yoke's weakest direction. If the yoke stress is low (for example, less than $20 \%$ of specified minimum ultimate strength), then the intent of the caveat is satisfied.

FOVIRS Caveat 4 - Mounted on 1-Inch Diameter Pipe Line or Greater. The valve should be mounted on a pipe line of at least 1-inch diameter. This is the lower bound pipe size supporting FOVs in the earthquake experience equipment class. The concern is that valves with heavy operators on small lines may cause an overstressed condition in the adjacent piping. To satisfy the intent of this caveat a stress analysis (that accounts for the valve operator eccentricity) may be used to show that the pipe stress adjacent to the valve is low. There is no concern if the valve, the operator, and the line (if smaller than 1 inch) are well-supported and anchored to the same support structure.

FOV/RS Caveat 5 - Valve Operator Cantilever Length for Air-Operated Diaphragm Valves, SpringOperated Pressure Relief Valves, and Light Weight Piston-Operated Valves. The distance from the centerline of the pipe to the top of the operator or cylinder should not exceed the distance given in Figure 8.2.1-2 corresponding to the diameter of the pipe. This figure bounds the pipe diameter and operator length combinations included in the earthquake experience equipment class. The concern is that longer operator lengths may lead to excessive valve yoke stress.

As a second screen to evaluate the operator weight and length, Figure 8.2.1-3 may be used instead of the limits given in Figure 8.2.1-2 provided: (1) the yoke is not of cast iron (Caveat 3 applies), and (2) the operator length does not exceed about $30 \%$ beyond the limits of Figure 8.2.1-2.

As a third option, this caveat may also be satisfied by performing a stress analysis consisting of applying a $3 \mathrm{~g}$ load at the center of gravity of the operator in the yoke's weakest direction. If the yoke stresses are low and the relative deflections are small (to ensure that shaft binding will not occur) then the caveat is satisfied.

Alternately, an in-situ static test may be conducted to demonstrate seismic adequacy. In these tests, a static force equal to three times the operator weight should be applied approximately at the center of gravity of the operator, in each of the three orthogonal principal axes of the yoke (nonconcurrently). Such tests should include demonstration of operability, i.e., the valve can open and close, following the application of the static loads. Note that all of the other limitations still apply.

A mockup test stand may be used provided that the details are similar to those in the facility. If there are numerous valves, a rational test program may be developed to envelop the valve configurations in the facility. 
FOVIRS Caveat 6 - Valve Operator Cantilever Length for Substantial Piston-Operated Valves. For piston-operated valves which are of substantial weight, the distance from the centerline of the pipe to the top of the operator or cylinder and the weight of the operator should not exceed the values given in Figure 8.2.1-3 corresponding to the diameter of the pipe. This figure represents the pipe diameter and operator weight/length combinations included in the earthquake experience equipment class. The concern is that longer operator lengths or heavier operator weights may lead to excessive valve yoke stress.

To meet the intent of this caveat the operator length or weight may be extrapolated by as much as $30 \%$ beyond that given in Figure $8.2 .1-3$ provided the product of the weight times the lever arm does not exceed the limits of Figure 8.2.1-3.

If the ground motion spectra for the site is below the Reference Spectrum, over the entire frequency range possible for the piping and valve network, the operator weight or distance to the top of the operator can be increased by the ratio of the spectra. The cantilever length or the operator weight should not be increased by more than about $30 \%$ beyond the limits of Figure 8.2.1-3.

Another option for satisfying this caveat is to perform a stress analysis that consists of applying a $3 \mathrm{~g}$ load at the center of gravity of the operator in the yoke's weakest direction. If the yoke stresses are low and the relative deflections are small (to ensure that shaft binding will not occur) then the caveat is satisfied. Alternately, as discussed in FOV/RS Caveat 5 above, a static test may be performed.

FOV/RS Caveat 7 - Actuator and Yoke Not Independently Braced. The valve actuator and yoke should not be independently braced to the structure or supported by the structure unless the pipe is also braced to the same structure immediately adjacent to the valve. The concern is that if the operator is independently supported from the valve and attached piping, then the operator may act as a pipe support during seismic motion and attract considerable load through the yoke and possibly fail the yoke or bind the shaft. In addition, if both the operator and the valve/pipe are restrained, and if they are both not tied back to the same structure, then differential motion of support points may lead to high seismic loads and possible binding of the shaft. If either of these concerns are noted, then a special evaluation should be conducted to demonstrate low stress and small deflections.

FOVIRS Caveat 8 - Any Other Concerns? SCEs should seek out suspicious details or uncommon situations not specifically covered by the caveats which could adversely affect the seismic capacity of the valve.

\subsubsection{GERS Caveats - Air-Operated Valves}

The seismic capacity for the equipment class of air-operated valves may be based on generic testing data, provided the intent of each of the caveats listed below is met. This equipment class consists of spring-opposed, diaphragm-type pneumatic actuators which are designed to operate both gate and globe valves. They range in size from 12 to 40 inches in height (pipe centerline is reference position) with weights up to 500 pounds. The valves within this class are for 3 -inch and smaller pipe sizes with design pressures less than 2,500 psi. A pneumatic actuator generally consists of a reinforced rubber diaphragm enclosed in a steel housing. The valve stem and diaphragm are attached so that any diaphragm movement results in valve movement. A solenoid valve controls the admission of high pressure air (100 to $150 \mathrm{psi}$ ) to the diaphragm housing. A return spring supplies sufficient counter force to close or open the valve when air pressure is not pushing on the diaphragm. The yoke of this class of pneumatic actuator is an integral part of the unit which is directly bolted to the valve bonnet. The valve body, bonnet, and yoke material should be carbon steel. The active components of the actuator are the solenoid valve, limit switches, and a pressure 
regulator, all of which are yoke-mounted appurtenances. This equipment class covers virtually all air-operated diaphragm valves used in small bore piping systems.

The GERS (see Figure 8.2.1-4) represent the seismic capacity of an Air-Operated Valve (AOV) if the valve meets the intent of the following inclusion and exclusion rules. Note, however, that when the specific wording of a caveat rule is not met, then a reason for concluding that the intent has been met should be provided on the SEWS.

AQV/GERS Caveat 1 - Generic Seismic Testing Equipment Class. The valve should be similar to and bounded by the AOV class of equipment described above. The equipment class descriptions are general and the $S C E s$ should be aware that worst case combinations of certain parameters may not be represented in the generic equipment class. These worst case combinations may have reduced seismic capacity and should be carefully evaluated on a case-by-case basis.

AQV/GERS Caveat 2 - Reference Spectrum Caveats Apply. The valve should meet all the caveats given for the Reference Spectrum. This caveat is included to cover the vulnerabilities identified for the earthquake experience equipment class. Those GERS caveats which are the same as the Reference Spectrum caveats are not repeated below.

AOV/GERS Caveat 3 - Only Diaphragm-Type Air Operated Valves. The air-operated gate or globe valve should have a spring-opposed, diaphragm-type pneumatic actuator. This equipment class does not include piston-operated, pressure relief valves, or other diaphragm-type valves powered by fluids other than air. These valve types are the only types included in the generic seismic testing equipment class.

AOV/GERS Caveat 4 - Evaluation of Amplified Response. The valves and operators were tested with the valve fixed to the shake table. Therefore realistic amplification through the piping system should be included when determining the amplified response of the valve-to-pipe interface for comparison to the GERS.

AOV/GERS Caveat 5 - No Impact Allowed. A separate evaluation should be done to assure that the valve and operator will not impact surrounding structures and components as a result of pipe flexibility. The concern is that impact may damage the valve, operator, yoke, stem, or attached components. This type of damage has occurred in past earthquakes and is also identified as a seismic interaction concern.

AOV/GERS Caveat 6 - Nominal Pipe Size 1 to 3 Inches. The nominal pipe size of the valve should be within the range of 1 to 3 inches. This is the pipe size range included in the generic seismic testing equipment class.

AOV/GERS Caveat 7 - Carbon Steel Valve Body, Bonnet and Yoke. The valve body, bonnet, and yoke should all be carbon steel. Cast iron components are not covered by the GERS. It is not necessary to determine the material used for the valve body, bonnet, or yoke unless it appears to the SCEs that cast iron may have been used. 


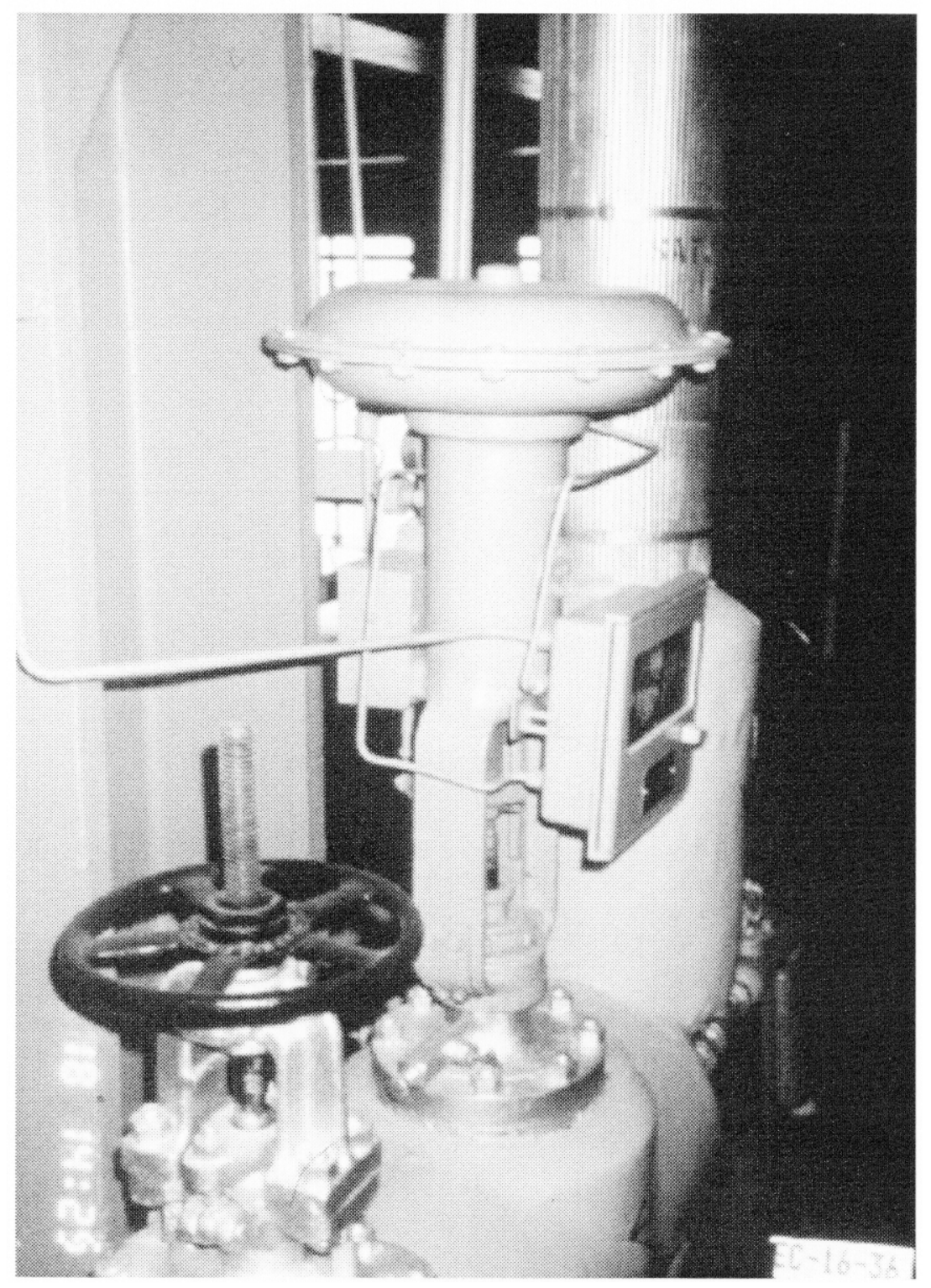

Figure 8.2.1-1 Air-Operated Valve from the Earthquake Experience Database 
Fluid-Operated Valves

Light Valve Operator

Cantilever Limits

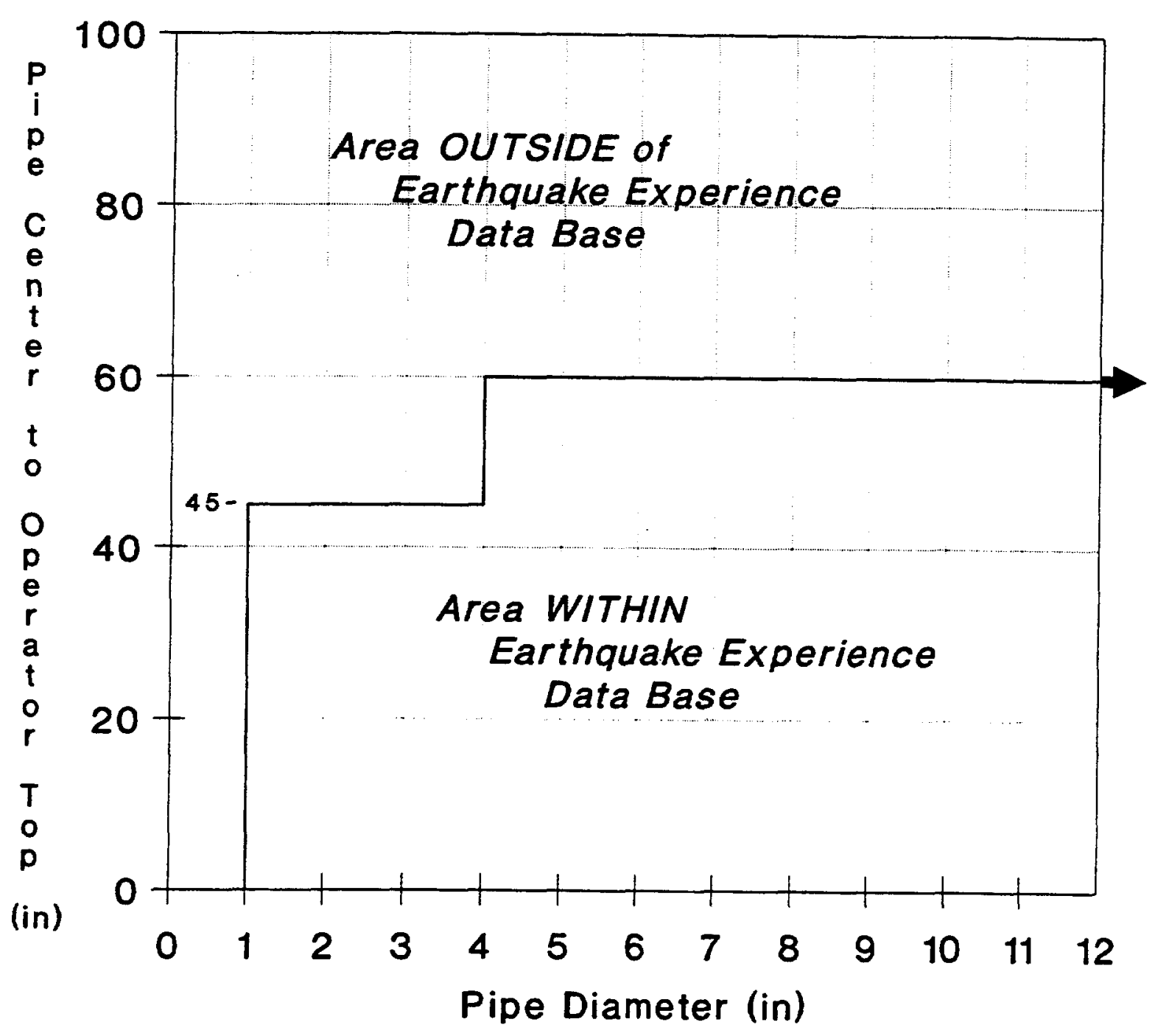

Figure 8.2.1-2 Valve Operator Cantilever Length Limits for Air-Operated Diaphragm Valves, Spring-Operated Pressure Relief Valves, and Piston-Operated Valves of Light-Weight Construction (Reference 19) (Figure B.7-1 of SQUG GIP, Reference 1) 
Fluid-Operated Valves

Heavy Valve Operator

Cantilever Limits

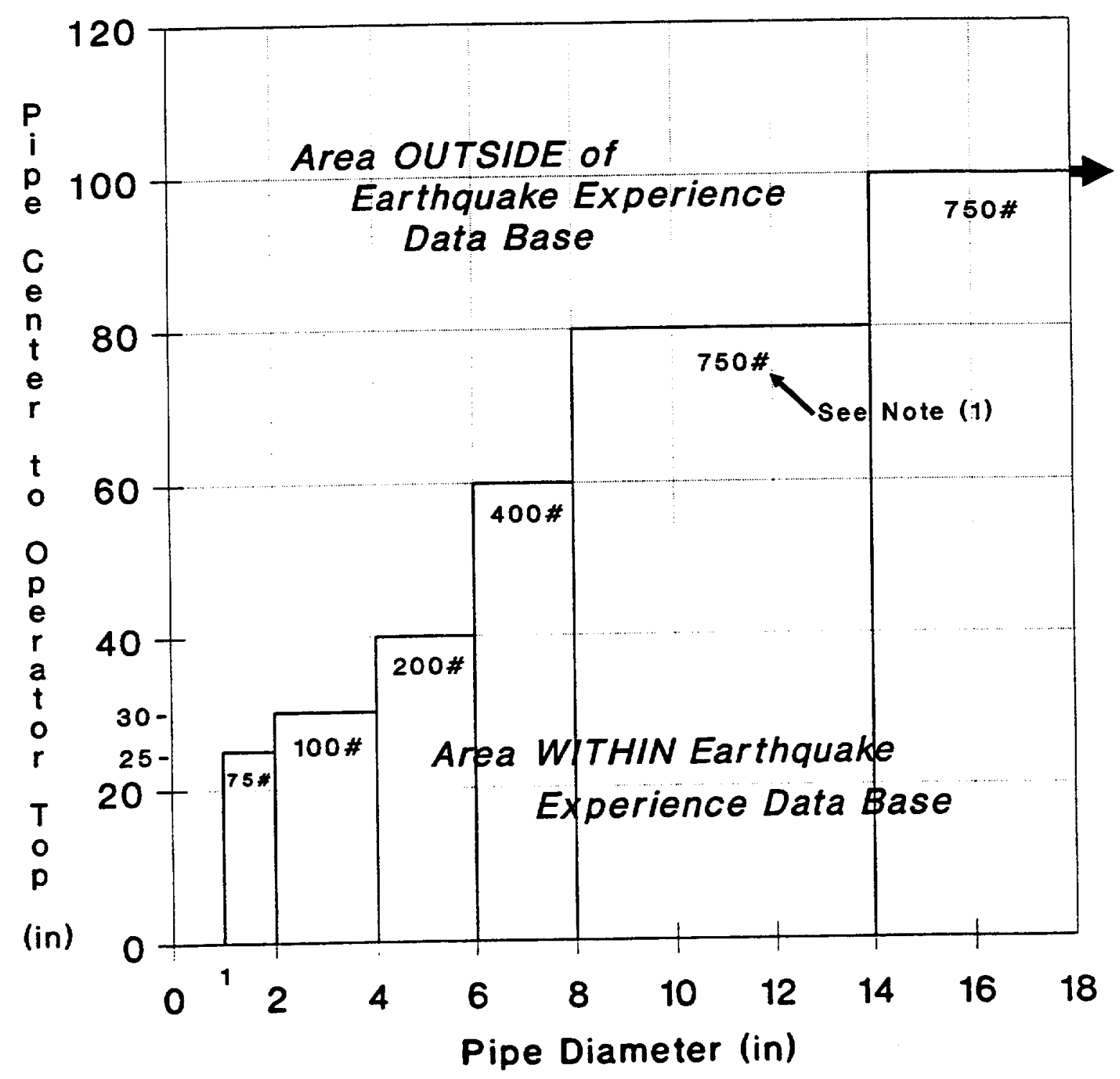

(1) Approximate Maximum Operator Weights Given for Various Ranges of Pipe Diameter

Figure 8.2.1-3 Valve Operator Cantilever Length Limits for Piston-Operated Valves of Substantial Weight and Construction (Reference 19) (Figure B.7-2 of SQUG GIP, Reference 1) 
Fluid-Operated Valves

GERS-AOV.4

$12 / 1 / 90$

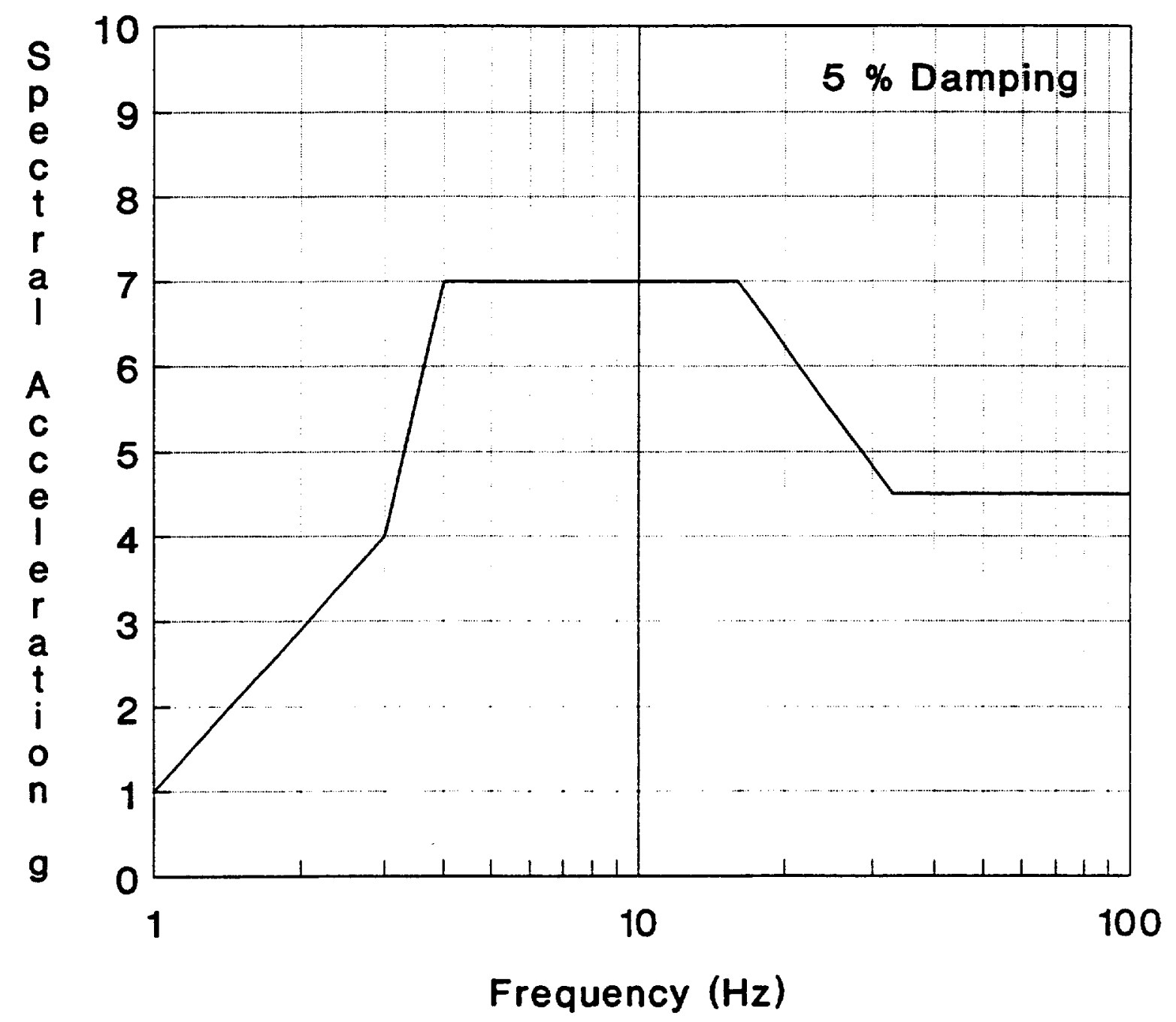

\begin{tabular}{l|lllll} 
Frequency $(\mathrm{Hz})$ & 1 & 3 & 4 & 16 & 33 \\
\hline Air-Operaled Valves $(\mathrm{O})$ & 1.0 & 4.0 & 7.0 & 7.0 & 4.5
\end{tabular}

Figure 8.2.1-4 Generic Equipment Ruggedness Spectra (GERS) for Air-Operated Valves (Reference 40) (Figure B.7-3 of SQUG GIP, Reference 1) 


\subsubsection{MOTOR-OPERATED / SOLENOID-OPERATED VALVES ${ }^{2}$}

The seismic capacity for the equipment class of Motor-Operated Valves (MOV) (see Figure 8.2.21) may be based on earthquake experience data, provided the intent of each of the caveats listed below is met. This equipment class includes a wide diversity of sizes, types, and applications.

Components of a motor-operated valve include a motor operator with a control box, gear box, and drive motor. The gear box includes the gears which link the valve actuation to the drive motor shaft. Local controls typically include a relay for actuating the primary circuit to the motor, and torque and limit switches for coordinating the drive motor and the valve position. Valve operators may have a local motor controller built into the operator housing. The valve actuator shaft typically passes through the steel support frame or yoke. The valve which is actuated by a motor operator may be of any type, size, or orientation.

Motor operators may be mounted in any position (e.g., cantilevered vertically above, below, or to the side of the valve). The yoke, which connects the operator to the valve body, may take the form of a steel pipe enclosing the actuator shaft or a frame of welded beams. The attachments of the motor-gearbox to the yoke and the yoke to the valve are typically bolted flange connections, threaded connections, or ring clamps. In some applications, motor operators are mounted at a remote location above the valve.

The equipment class of motor-operated valves includes all valves actuated by an electric motor. The valve, the operator, and the inlet and outlet lines and attached conduit up to their first support on the building or nearby structure are included in the Motor-Operated Valve equipment class.

The seismic capacity for the equipment class of Solenoid-Operated Valves (SOV) (see Figure 8.2.2-2) may be based on earthquake experience data, provided the intent of each of the caveats listed below is met. This equipment class includes a wide diversity of sizes, types, and applications.

Solenoid operators are smaller and lighter than motor operators. Solenoid-operated valves are actuated by passing an electrical current through a coil, thereby creating a magnetic field which opens or closes the valve. Solenoid operators are generally more compact than motor operators with less of a cantilevered mass supported from the valve body. In addition, solenoid-operated valves are typically mounted on smaller diameter lines than MOVs.

The equipment class of solenoid-operated valves includes all valves actuated by a solenoid. The valve, the operator, and the inlet and outlet lines and attached conduit up to their first support on the building or nearby structure are included in the Solenoid-Operated Valve equipment class.

\subsubsection{Reference Spectrum Caveats - Motor-Operated Valves}

The Reference Spectrum ( $R S$ ) represents the seismic capacity of a Motor-Operated Valve (MOV) if the valve meets the intent of the following inclusion and exclusion rules. Note, however, that when the specific wording of a caveat rule is not met, then a reason for concluding that the intent has been met should be provided on the SEWS.

MOVIRS Caveat 1 - Earthquake Experience Equipment Class. The valve should be similar to and bounded by the MOV class of equipment described above. The equipment class descriptions are general and the SCES should be aware that worst case combinations of certain parameters may not be represented in the generic equipment class. These worst case combinations may have reduced seismic capacity and should be carefully evaluated on a case-by-case basis.

2 Section B.8 of SQUG GIP (Ref. 1) 
MOV/RS Caveat 2 - Valve Body Not of Cast Iron. The valve body should not be made of cast iron. The intent of this caveat is to avoid the brittle failure mode of cast iron as evidenced by the poor performance of some cast iron components in past earthquakes. It is not necessary to determine the material of the valve body unless it appears to the SCEs to be made of cast iron. It is suggested that the material of flanged valves be checked. In such cases, if the valve is indeed cast iron, the intent of this caveat is satisfied if seismic stresses in the valve body due to piping loads are low (for example, less than $20 \%$ of specified minimum ultimate tensile strength).

MOVIRS Caveat 3 - Valve Yoke Not of Cast Iron. The yoke of the motor-operated valve should not be made of cast iron. The intent of this caveat is to avoid the brittle failure mode of cast iron as evidenced by the poor performance of some cast iron components in past earthquakes. It is not necessary to determine the material of the valve yoke unless it appears to be cast iron to the SCEs. In such cases, if the yoke is indeed cast iron, this caveat may be satisfied by performing a stress analysis of the valve for a $3 \mathrm{~g}$ load applied at the center of gravity of the operator in the yoke's weakest direction. If the yoke stress is low (for example, less than $20 \%$ of specified minimum ultimate strength), then the intent of the caveat is satisfied.

MOV/RS Caveat 4 - Mounted on 1-Inch Diameter Pipe Line or Greater. The valve should be mounted on a pipe line of at least 1-inch diameter. This is the lower bound pipe size supporting MOVs in the earthquake experience equipment class. The concern is that valves with heavy operators on small lines may cause an overstressed condition in the adjacent piping. To satisfy the intent of this caveat a stress analysis (that accounts for the valve operator eccentricity) may be used to show that the pipe stress adjacent to the valve is low. There is no concern if the valve, the operator, and the line (if smaller than 1 inch) are well supported and anchored to the same support structure. This caveat does not apply to SOVs, which typically are installed on air lines smaller than 1 inch.

MOVIRS Caveat 5 - Valve Operator Cantilever Length for Motor-Operated Valves. The distance from the centerline of the pipe to the top of the operator or cylinder and the weight of the operator should not exceed the values given in Figure 8.2.2-3 corresponding to the diameter of the pipe. This bounds the earthquake experience equipment class. The concern is that longer operator lengths may lead to excessive valve yoke stress.

To meet the intent of this caveat the operator length or weight may be extrapolated by as much as $30 \%$ beyond that given in Figure 8.2.2-3 provided the product of the weight times the lever arm does not exceed the limits of Figure 8.2.2-3. If the ground motion spectra for the site is below the Reference Spectrum, over the entire frequency range possible for the piping and valve network, the operator weight or distance to the top of the operator can be increased by the ratio of the spectra. The cantilever length or the operator weight should not be increased by more than about $30 \%$ beyond the limits of Figure 8.2.2-3.

As an option, this caveat may also be satisfied by performing a stress analysis consisting of applying a $3 \mathrm{~g}$ load at the center of gravity of the operator in the yoke's weakest direction. If the yoke stresses are low and the relative deflections are small (to ensure that shaft binding will not occur) then the caveat may be shown to be satisfied.

Alternatively, an in-situ static test may be conducted to demonstrate seismic adequacy. In these tests, a static force equal to three times the operator weight should be applied approximately at the center of gravity of the operator, non-concurrently in each of the three orthogonal principal axes of the yoke. Such tests should include demonstration of operability, i.e., the valve can open and close, following the application of the static loads. Note that all of the other limitations still apply. 
A mockup test stand may be used provided that the details are similar to those in the facility. If there are numerous valves, a rational test program may be developed to envelop the valve configurations in the facility.

MOV/RS Caveat 6 - Actuator and Yoke Not Independently Braced. The valve actuator and yoke should not be independently braced to the structure or supported by the structure unless the pipe is also braced to the same structure immediately adjacent to the valve. The concern is that if the operator is independently supported from the valve and attached piping, then the operator may act as a pipe support during seismic motion and attract considerable load through the yoke and possibly fail the yoke or bind the shaft. In addition, if both the operator and the valve/pipe are restrained, and if they are both not tied back to the same structure, then differential motion of support points may lead to high seismic loads and possible binding of the shaft. If either of these concerns are noted, then a special evaluation should be conducted to demonstrate low stress and small deflections.

MOV/RS Caveat 7 - Any Other Concerns? SCEs should seek out suspicious details or uncommon situations not specifically covered by the caveats which could adversely affect the seismic capacity of the valve.

\subsubsection{Reference Spectrum Caveats-Solenoid-Operated Valves}

The Reference Spectrum (RS) represents the seismic capacity of a Solenoid-Operated Valve (SOV) if the valve meets the intent of the following inclusion and exclusion rules. Note, however, that when the specific wording of a caveat rule is not met, then a reason for concluding that the intent has been met should be provided on the SEWS.

SOV/RS Caveat 1 - Earthquake Experience Equipment Class. The valve should be similar to and bounded by the SOV class of equipment described above. The equipment class descriptions are general and the SCEs should be aware that worst case combinations of certain parameters may not be represented in the generic equipment class. These worst case combinations may have reduced seismic capacity and should be carefully evaluated on a case-by-case basis.

SOVIRS Caveat 2 - Valve Body Not of Cast Iron. The valve body should not be made of cast iron. The intent of this caveat is to avoid the brittle failure mode of cast iron as evidenced by the poor performance of some cast iron components in past earthquakes. It is not necessary to determine the material of the valve body unless it appears to the SCEs to be made of cast iron. It is suggested that the material of flanged valves be checked. In such cases, if the valve is indeed cast iron, the intent of this caveat is satisfied if seismic stresses in the valve body due to piping loads are low (for example, less than $20 \%$ of specified minimum ultimate tensile strength).

SOV/RS Caveat 3 - Valve Yoke Not of Cast Iron. The yoke of the motor-operated valve should not be made of cast iron. The intent of this caveat is to avoid the brittle failure mode of cast iron as evidenced by the poor performance of some cast iron components in past earthquakes. It is not necessary to determine the material of the valve yoke unless it appears to be cast iron to the SCES. In such cases, if the yoke is indeed cast iron, this caveat may be satisfied by performing a stress analysis of the valve for a $3 \mathrm{~g}$ load applied at the center of gravity of the operator in the yoke's weakest direction. If the yoke stress is low (for example, less than $20 \%$ of specified minimum ultimate strength), then the intent of the caveat is satisfied.

SOV/RS Caveat 4-Valve Operator Cantilever Length. The distance from the centerline of the pipe to the top of the operator or cylinder and the weight of the operator should not exceed the values given in Figure 8.2.2-3 corresponding to the diameter of the pipe. This bounds the earthquake experience equipment class. The concern is that longer operator lengths may lead to excessive valve yoke stress. 
To meet the intent of this caveat the operator length or weight may be extrapolated by as much as $30 \%$ beyond that given in Figure 8.2.2-3 provided the product of the weight times the lever arm does not exceed the limits of Figure 8.2.2-3.

If the ground motion spectra for the site is below the Reference Spectrum, over the entire frequency range possible for the piping and valve network, the operator weight or distance to the top of the operator can be increased by the ratio of the spectra. The cantilever length or the operator weight should not be increased by more than about $30 \%$ beyond the limits of Figure 8.2.2-3.

As an option, this caveat may also be satisfied by performing a stress analysis consisting of applying a $3 \mathrm{~g}$ load at the center of gravity of the operator in the yoke's weakest direction. If the yoke stresses are low and the relative deflections are small (to ensure that shaft binding will not occur) then the caveat may be shown to be satisfied.

Alternatively, an in-situ static test may be conducted to demonstrate seismic adequacy. In these tests, a static force equal to three times the operator weight should be applied approximately at the center of gravity of the operator, non-concurrently in each of the three orthogonal principal axes of the yoke. Such tests should include demonstration of operability, i.e., the valve can open and close, following the application of the static loads. Note that all of the other limitations still apply.

A mockup test stand may be used provided that the details are similar to those in the facility. If there are numerous valves, a rational test program may be developed to envelop the valve configurations in the facility.

SOV/RS Caveat 5 -Actuator and Yoke Not Independently Braced. The valve actuator and yoke should not be independently braced to the structure or supported by the structure unless the pipe is also braced to the same structure immediately adjacent to the valve. The concern is that if the operator is independently supported from the valve and attached piping, then the operator may act as a pipe support during seismic motion and attract considerable load through the yoke and possibly fail the yoke or bind the shaft. In addition, if both the operator and the valve/pipe are restrained, and if they are both not tied back to the same structure, then differential motion of support points may lead to high seismic loads and possible binding of the shaft. If either of these concerns are noted, then a special evaluation should be conducted to demonstrate low stress and small deflections.

SOVIRS Caveat 6 - Any Other Concerns? SCEs should seek out suspicious details or uncommon situations not specifically covered by the caveats which could adversely affect the seismic capacity of the valve.

\subsubsection{GERS Caveats - Motor-Operated Valves}

The seismic capacity for the equipment class of electric motor operators for valves (MOV) may be based on generic testing data, provided the intent of each of the caveats listed below is met. This equipment class includes operators designed to control the five major types of valves (gate, globe, plug, ball, and butterfly). They range in weight from 150 pounds up to 3,500 pounds. A valve operator consists of a metal housing which connects to the valve body by a flange or yoke and contains limit switches, a torque switch, an electric motor, a clutch, gears, and bearings. For this class of equipment, the motor controls (reversing starter, overload relays, and push-button station) should be located in a remote location (usually a motor control center). For some valve configurations, the valve actuators are mounted on secondary reducers resulting in the actuator being eccentric and cantilevered from the valve body. For these configurations, a special seismic bracket supplied by the manufacturer is required. The mounting position of the valve operator is with the motor horizontal and the limit switch compartment horizontal or vertical as specified by the 
manufacturer. These positions will insure the proper distribution of lubricants through the internal working component of the units. This equipment class covers virtually all motor-driven valve operators used in facilities.

The MOV GERS (see Figure 8.2.2-4) represent the seismic capacity of an electric Motor Operator for a Valve (MOV) if the operator meets the intent of the following inclusion and exclusion rules. Note, however, that when the specific wording of a caveat rule is not met, then a reason for concluding that the intent has been met should be provided on the SEWS.

MOV/GERS Caveat 1 - Generic Seismic Testing Equipment Class. The electric motor-driven valve operator should be similar to and bounded by the MOV class of equipment described above. The equipment class descriptions are general and the SCEs should be aware that worst case combinations of certain parameters may not be represented in the generic equipment class. These worst case combinations may have reduced seismic capacity and should be carefully evaluated on a case-by-case basis.

MOV/GERS Caveat 2 - Reference Spectrum Caveats Apply. The operator should meet all the caveats given for the Reference Spectrum for the MOV class of equipment. This caveat is included to cover the vulnerabilities identified for the earthquake experience equipment class. Those GERS caveats which are the same as the Reference Spectrum caveats are not repeated below.

MOV/GERS Caveat 3 - Evaluation of Amplified Response. The GERS were based on tests in which the operators were mounted directly to the shake table and not on a valve yoke structure or a valve. Therefore realistic amplification through the piping system and valve should be included when determining the seismic demand at the operator-to-valve interface for comparison to the GERS. Note also that the MOV GERS apply only to the operator; the seismic adequacy of the valve and its yoke should be evaluated separately.

MOV/GERS Caveat 4 - Motor Axis Horizontal. The motor axis should be horizontal and the limit switch compartment should be horizontal or vertical (definition of orientation directions provided in manufacturer's submittals). These were the positions of the motor axis and limit switch compartment in the generic seismic testing equipment class shake table tests.

MOV/GERS Caveat 5 - No Impact Allowed. A separate evaluation should be done to assure that the operator will not impact surrounding structures and components as a result of pipe flexibility. The concern is that impact may damage the operator, yoke, stem, or attached components. This type of damage has occurred in past earthquakes and is also identified as a seismic interaction concern.

MOV/GERS Caveat 6 - Motor Controls Remotely Located. The motor controls (reversing starter, overload relays, and push-button station) should be remotely located and separately evaluated. The motor controls were not located on the valve operators during the GERS testing and are therefore not included in the generic seismic testing equipment class.

MOV/GERS Caveat 7 - Seismic Brackets for Side-Mounted Actuators. Side-mounted valve actuators attached to secondary reducers should have seismic brackets as supplied by the manufacturer (review of manufacturer's submittals is sufficient). The actuators in the GERS tests that were tested in this orientation had seismic brackets.

MOV/GERS Caveat 8 - Manufactured by Limitorque or Rotork. The operator should be manufactured by either Limitorque or Rotork. These are the MOV manufacturers included in the generic seismic testing equipment class. 
MOV/GERS Caveat 9 - Tighten Loose Valve-to-Operator Bolts. Any missing or loose valve-tooperator bolts which are noticed during the walkdown should be replaced or retightened; a tightness check is not required.

\subsubsection{GERS Caveats - Solenoid-Operated Valves}

The seismic capacity for the equipment class of solenoid-operated valves (SOV) may be based on generic testing data, provided the intent of each of the caveats listed below is met. This equipment class consists of a combination of two basic functional units: 1) a solenoid actuator (electromagnet) with its plunger (or core), and 2) a valve body containing an orifice in which a disc or plug is positioned to stop or allow flow. The valve is opened or closed by movement of the magnetic plunger which is drawn into the solenoid when the coil is energized. Solenoid valves can be either two-way, three-way or four-way valves. In the direct acting two-way solenoid valve, the solenoid acts directly on the valve stem to open or close the valve. Three-way solenoid valves are principally used as pilot valves to alternately apply pressure to and exhaust pressure from a diaphragm valve actuator. Four-way solenoid valves are often used for controlling double-acting pneumatic or hydraulic cylinders. The valves range in weight from a few pounds to 45 pounds and are made of either forged brass or steel. The valves within this class are for pipe sizes which are 1 inch or less in diameter and for design pressures less than 600 psi. This equipment class covers virtually all solenoid-operated valves used in small bore piping or process air systems.

The SOV GERS (see Figure 8.2.2-5) represent the seismic capacity of a Solenoid-Operated Valve if the valve meets the intent of the following inclusion and exclusion rules. Note, however, that when the specific wording of a caveat rule is not met, then a reason for concluding that the intent has been met should be provided on the SEWS.

SOV/GERS Caveat 1 - Generic Seismic Testing Equipment Class. The valve should be similar to and bounded by the SOV class of equipment described above. The equipment class descriptions are general and the SCEs should be aware that worst case combinations of certain parameters may not be represented in the generic equipment class. These worst case combinations may have reduced seismic capacity and should be carefully evaluated on a case-by-case basis.

SOV/GERS Caveat 2 - Reference Spectrum Caveats Apply. The valve should meet all the caveats given for the Reference Spectrum for the class of equipment. This caveat is included to cover the vulnerabilities identified for the earthquake experience equipment class. Those GERS caveats which are the same as the Reference Spectrum caveats are not repeated below.

SOV/GERS Caveat 3 - Evaluation of Amplified Response. The valves and operators were tested with the valve fixed to the shake table. Therefore realistic amplification through the piping system should be included when determining the amplified response of the valve-to-pipe interface for comparison to the GERS.

SOV/GERS Caveat 4 - No Impact Allowed. A separate evaluation should be done to assure that the valve and operator will not impact surrounding structures and components as a result of pipe flexibility. The concern is that impact may damage the valve, operator, yoke, stem, or attached components. This type of damage has occurred in past earthquakes and is also identified as a seismic interaction action concern.

SOV/GERS Caveat 5 - Nominal Pipe Size 1 Inch or Less. The nominal pipe size of the valve should be 1 inch or less. This is the upper bound pipe size included in the generic seismic testing equipment class. 
SOV/GERS Caveat 6 - Forged Brass or Steel Valve Body. The valve body should be made of either forged brass or steel. Other materials are not covered by the generic seismic testing equipment class.

SOV/GERS Caveat 7 - Orientation of Solenoid Housing. The solenoid housing should be oriented in accordance with the manufacturer's recommendations for the specific model (review of manufacturer's submittals is sufficient). GERS testing was performed with the solenoid housing in the recommended orientation.

SOV/GERS Caveat 8 - Overall Height Not to Exceed 12 Inches. The overall height of the valve (pipe centerline to top of solenoid housing) should not exceed 12 inches. This is the upper bound height limit included in the generic seismic testing equipment class.

SOV/GERS Caveat 9 - Separate Evaluation of Main Valve Controlled By SOV. When the Solenoid-Operated Valve is a pilot valve in a valve assembly, the main valve should be evaluated separately. Note that the amplified response spectra at the attachment point of the SOV should be used in the SOV evaluation as discussed in SOV/GERS Caveat 3.

SOV/GERS Caveat 10 - Lower ZPA for ASCO Type 206-381. For ASCO Type 206-381 solenoid valves, the GERS with a $3.5 \mathrm{~g}$ ZPA should be used. 


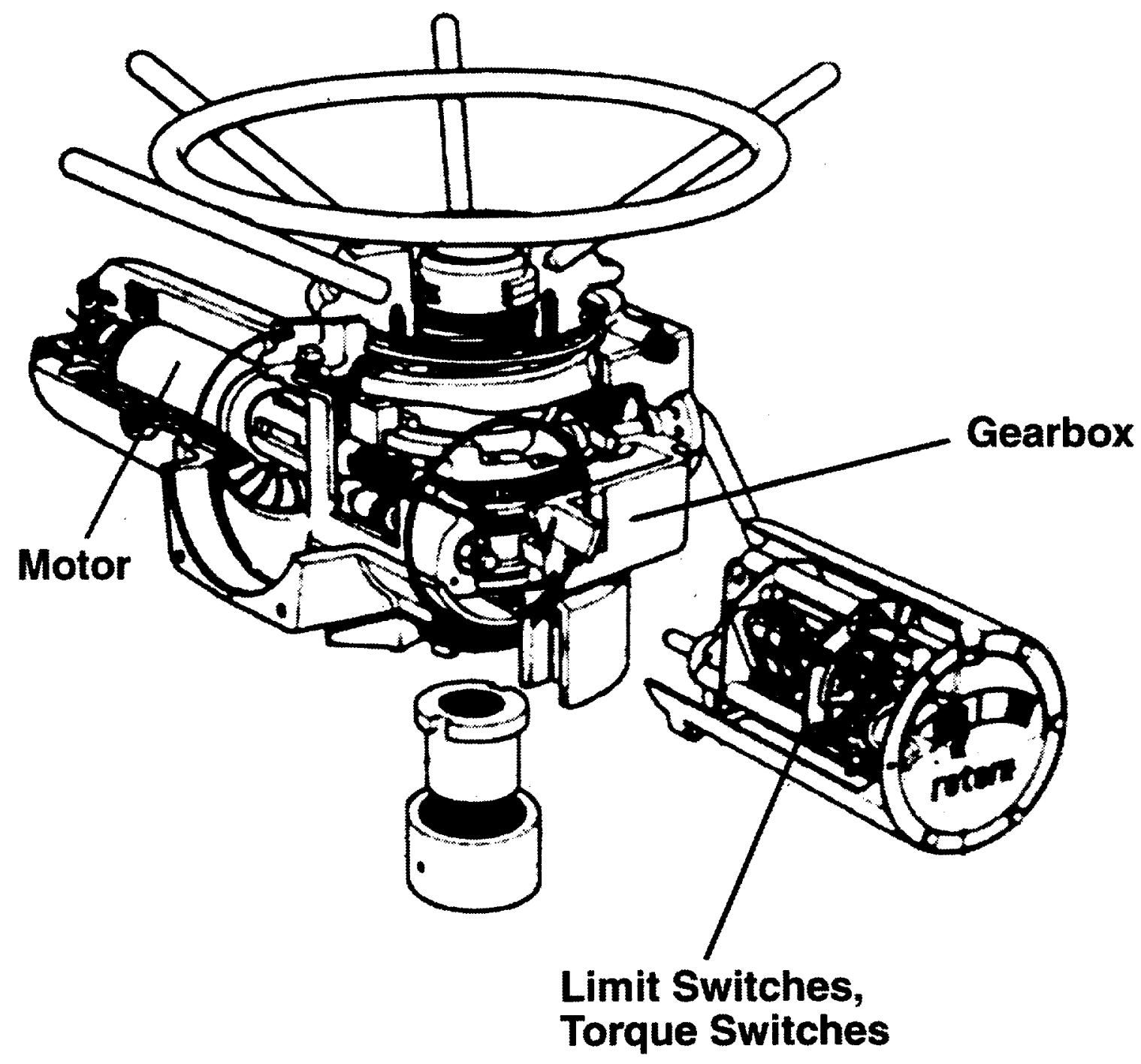

Figure 8.2.2-1 Motor-Operated Valve 


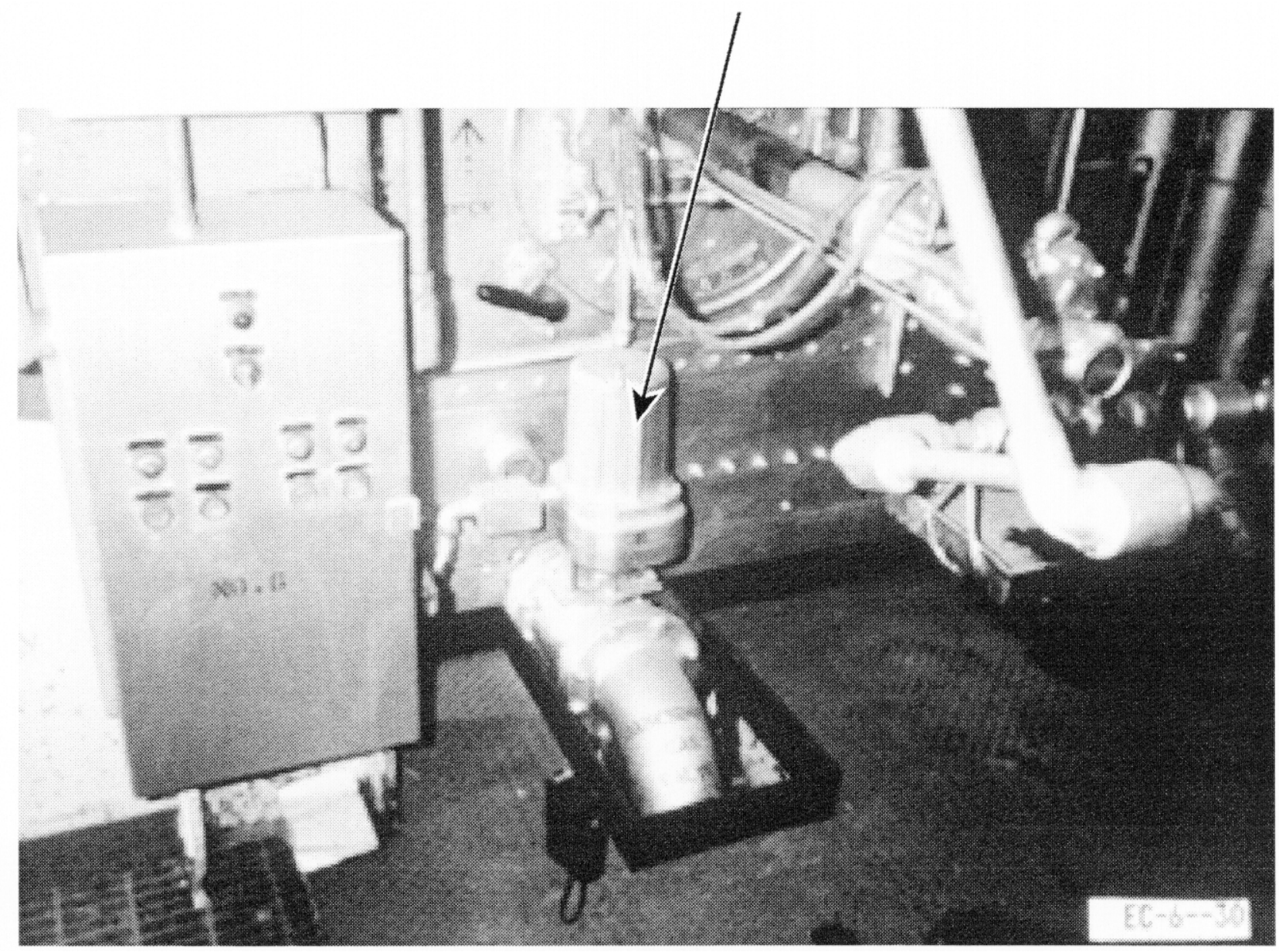

Figure 8.2.2-2 Solenoid-Operated Valve from the Earthquake Experience Database 
Motor-Operated and Solenoid-

Operated Valves

Heavy Valve Operator

Cantilever Limits

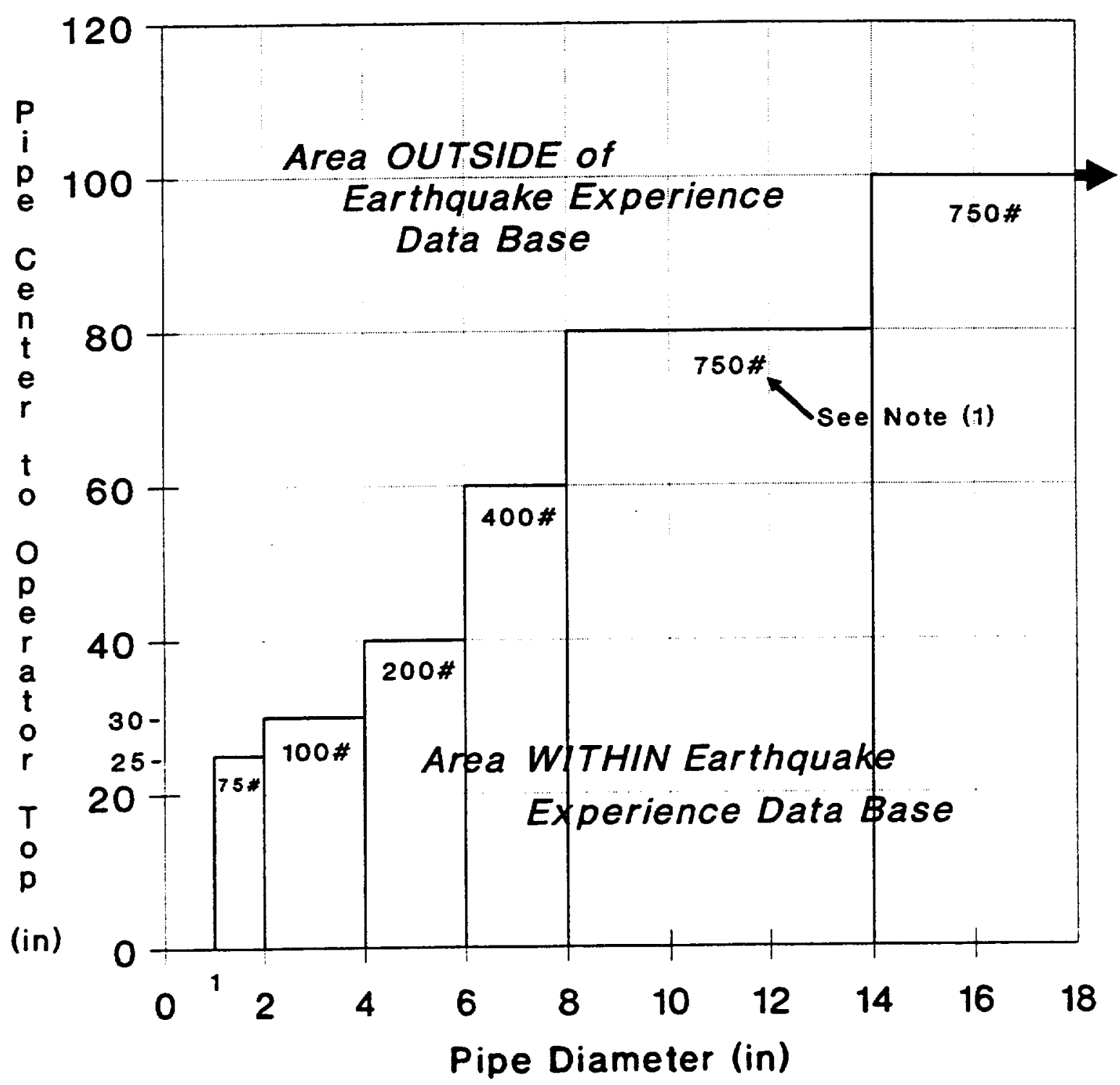

(1) Approximate Maximum Operator Weights Given for Various Ranges of Pide Diameter

Figure 8.2.2-3 Valve Operator Cantilever Length Limits for Motor-Operated Valves (Figure B.8-1 of SQUG GIP, Reference 1) 
Motor-Operated and Solenoid-

Operated Valves

GERS-MOV.4

$12 / 1 / 90$

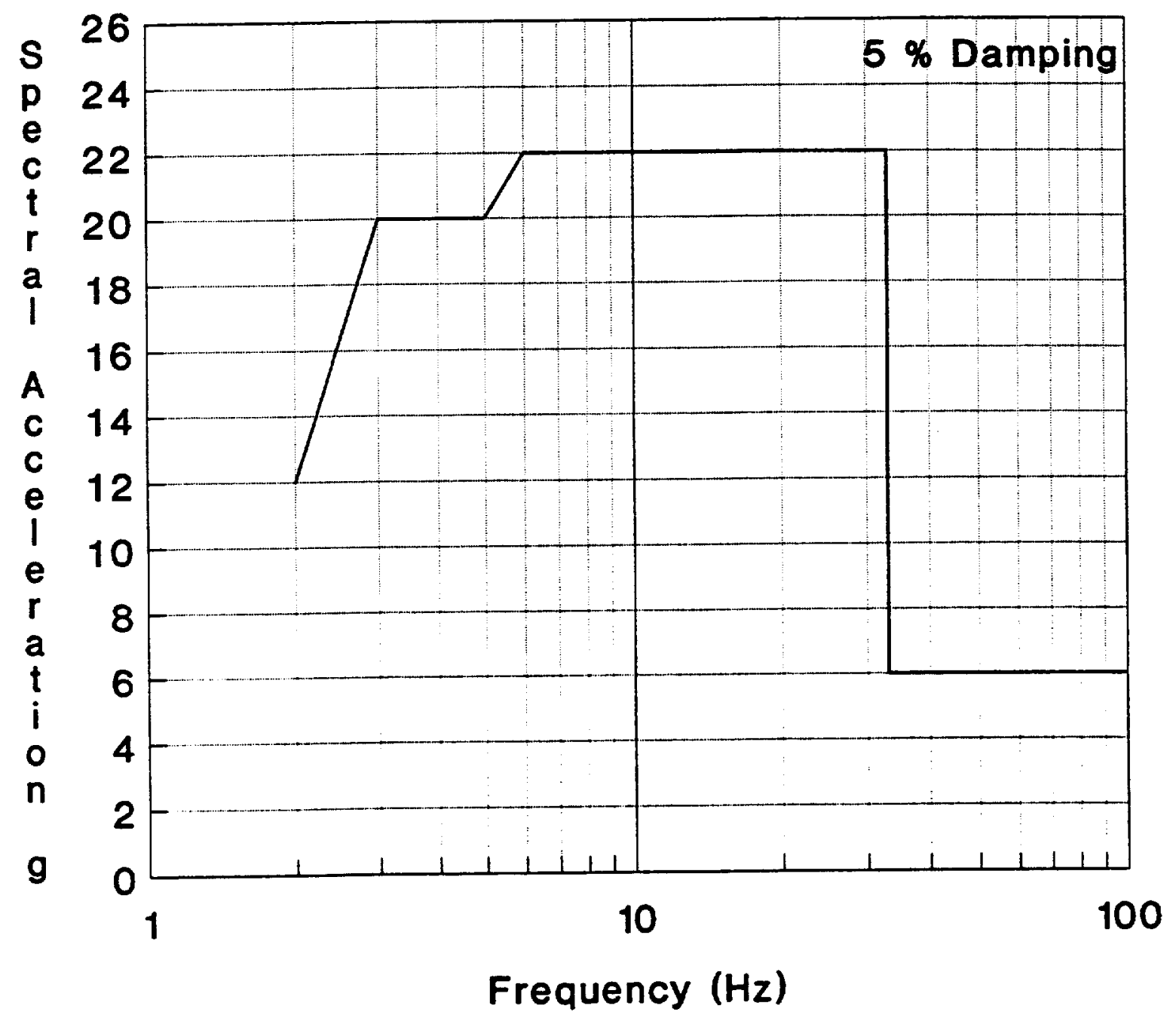

\begin{tabular}{l|llllll} 
Frequency $(H z)$ & 2 & 3 & 6 & 6 & 33 & 33 \\
\hline Motor Operators for Valves $(g)$ & 12 & 20 & 20 & 22 & 22 & 6
\end{tabular}

Figure 8.2.2-4 Generic Equipment Ruggedness Spectra (GERS) for Motor Operators On Valves (Reference 40) (Figure B.8-2 of SQUG GIP, Reference 1) 
Motor-Operated and Solenoid-

Operated Valves

GERS-SV.3

$12 / 1 / 90$

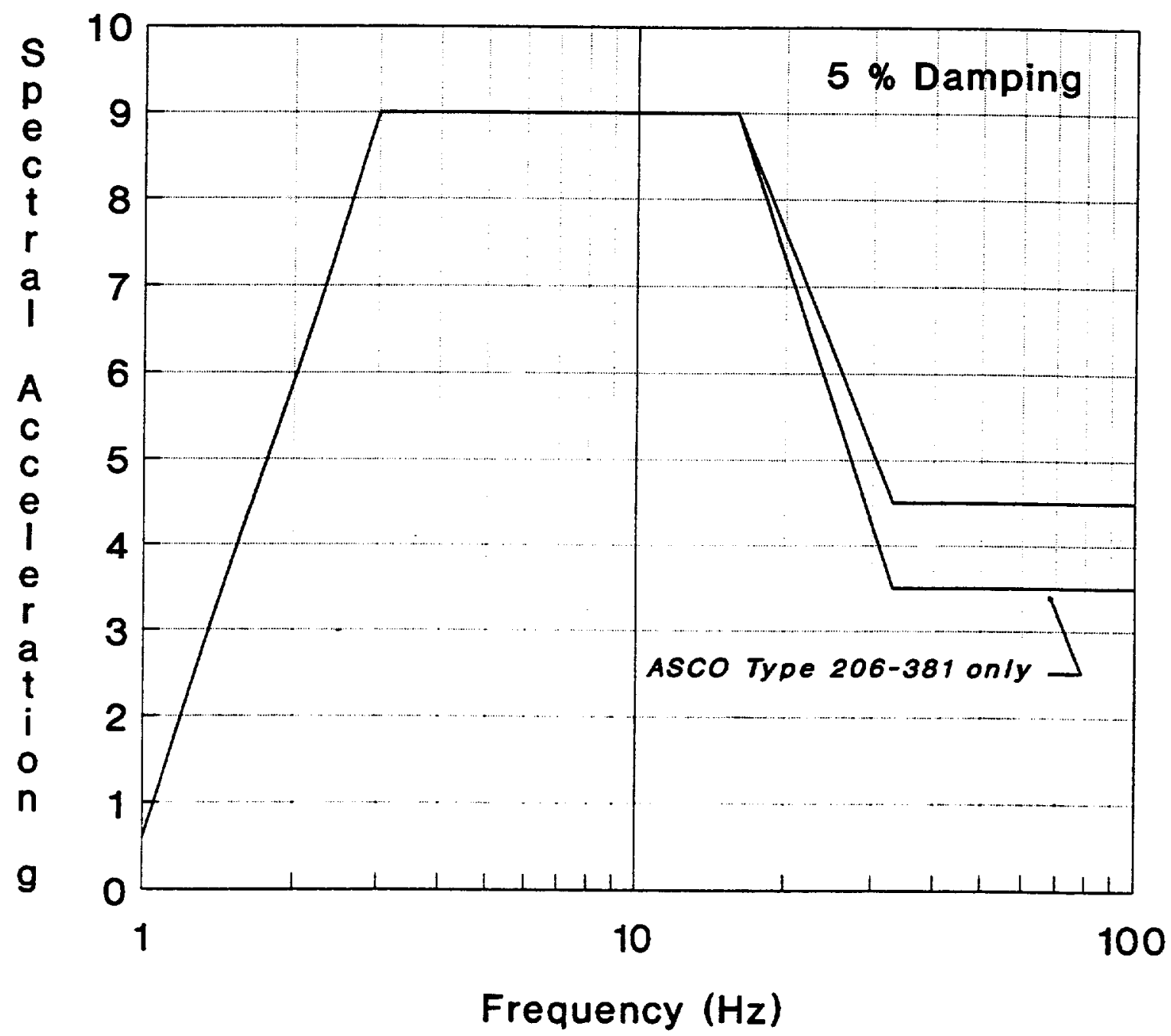

\begin{tabular}{l|cccc} 
Frequency $(\mathrm{Hz})$ & 1 & 3 & 16 & 33 \\
\hline Solenoid Valves - General $(g)$ & 0.6 & 0.0 & 9.0 & 4.5 \\
ASCO Type 206-381 only $(g)$ & 0.6 & 9.0 & 9.0 & 3.5
\end{tabular}

Figure 8.2.2-5 Generic Equipment Ruggedness Spectra (GERS) for SolenoidOperated Valves (Reference 40) (Figure B.8-3 of SQUG GIP, Reference 1) 


\subsubsection{HORIZONTAL PUMPS 3}

The seismic capacity for the equipment class of Horizontal Pumps (HP) (see Figure 8.2.3-1) may $b e$ based on earthquake experience data, provided the intent of each of the caveats listed below is met. This equipment class includes all pumps commonly found in applications which have their axes aligned horizontally. The class includes pumps driven by electric motors, reciprocating piston engines, and steam turbines. The common peripheral components such as conduit, instrumentation, and suction and discharge lines up to their first support on the building or nearby structure are included in this equipment class.

Pumps can generally be categorized as either kinetic (rotary impeller) or positive displacement types. Kinetic pumps move fluid using the kinetic energy of a rotating impeller. Positive displacement pumps move fluid by volumetric displacement.

Single-stage kinetic pumps typically include a single impeller that moves fluid primarily by centrifugal force. The suction port is normally mounted along or near the impeller axis, and the discharge port is mounted near the periphery. Pumps may range in size from fractional horsepower units, with capacities of a few gallons per minute (gpm), to units requiring several thousand horsepower, with capacities of tens of thousands of gpm.

Multi-stage kinetic pumps include two or more impellers working in series on a single shaft. Depending on the impeller design, multi-stage pumps move fluid using either centrifugal force toward the periphery of the impeller, or propeller force along the axis of the impeller. The impeller is surrounded by a stationary casing or volute that directs the flow from the discharge of one impeller to the intake of the next.

Kinetic pumps are usually powered by electric motors with the pump and motor sharing the same shaft through a close-coupled connection. Larger multi-stage pumps sometimes couple the motor and pump through a gearbox, which allows the pump and motor to turn at different speeds.

Single-stage pumps are occasionally belt-driven, with the motor mounted to the side, or even atop the pump casing. Smaller, single-stage pumps sometimes mount the motor and impeller within the same casing. Larger pumps, both single- and multi-stage, normally have the motor and pump in separate casings, with both casings anchored to the same steel skid. Kinetic pumps may also be powered by engines or steam turbines.

Reciprocating-piston positive displacement pumps are similar in design to reciprocating-piston air compressors. They include an electric motor that powers a set of piston impellers through a shaft or belt connection. The piston impellers are usually mounted within a cast block that also contains the piston crank shaft and valve mechanism.

Rotary-screw positive displacement pumps are somewhat similar to multi-stage kinetic pumps, except that the screw impeller moves fluid axially through volume displacement rather than through a transfer of kinetic energy from the impeller to the fluid. The screw impeller is normally powered by an electric motor through a close-coupled shaft.

Kinetic and positive displacement horizontal pumps driven by electric motors, engines, and turbines are represented in the range from 5 to $2300 \mathrm{hp}$ and 45 to $36,000 \mathrm{gpm}$. Submersible pumps are not included in this equipment class.

There are no GERS for Horizontal Pumps.

3 Section B.5 of SQUG GIP (Ref. 1) 


\subsubsection{Reference Spectrum Caveats - Horizontal Pumps}

The Reference Spectrum (RS) represents the seismic capacity of a Horizontal Pump (HP) if the pump meets the intent of the following inclusion and exclusion rules. Note, however, that when the specific wording of a caveat rule is not met, then a reason for concluding that the intent has been met should be provided on the SEWS.

HP/RS Caveat 1 - Earthquake Experience Equipment Class. The horizontal pump should be similar to and bounded by the HP class of equipment described above. The equipment class descriptions are general and the SCES should be aware that worst case combinations of certain parameters may not be represented in the generic equipment class. These worst case combinations may have reduced seismic capacity and should be carefully evaluated on a case-by-case basis.

HP/RS Caveat 2 - Driver and Pump on Rigid Skid. The driver and pump should be connected by a rigid base or common skid. The concern is that differential displacement between the pump and driver may cause shaft misalignment. If they are not mounted on a rigid skid, the potential for differential displacement between the driver and pump should be specially evaluated.

HP/RS Caveat 3 - Thrust Bearings in Both Axial Directions. Thrust restraint of the shaft in both axial directions should exist. The concern arose from shake table testing on pumps without thrust bearings that performed poorly. In general, pumps from U.S. manufacturers have such axial thrust restraint so that explicit determination is not necessary; however, any indication to the contrary should be investigated.

HP/RS Caveat 4 - Check of Long Unsupported Piping. Brief consideration should be given to identify situations where the horizontal pump may be affected by gross pipe motion, differential displacement, and excessive nozzle loads. The concern is that excessive force on pump nozzles could potentially break the pump nozzle or cause sufficient pump case distortion to cause binding, or fail the anchorage. These excessive forces are uncommon and need only be considered if there is a long section of unsupported pipe or a heavy valve attached to the pipe near the pump.

HP/RS Caveat 5 - Any Other Concerns? SCES should seek out suspicious details or uncommon situations not specifically covered by the caveats which could adversely affect the seismic capacity of the pump. 


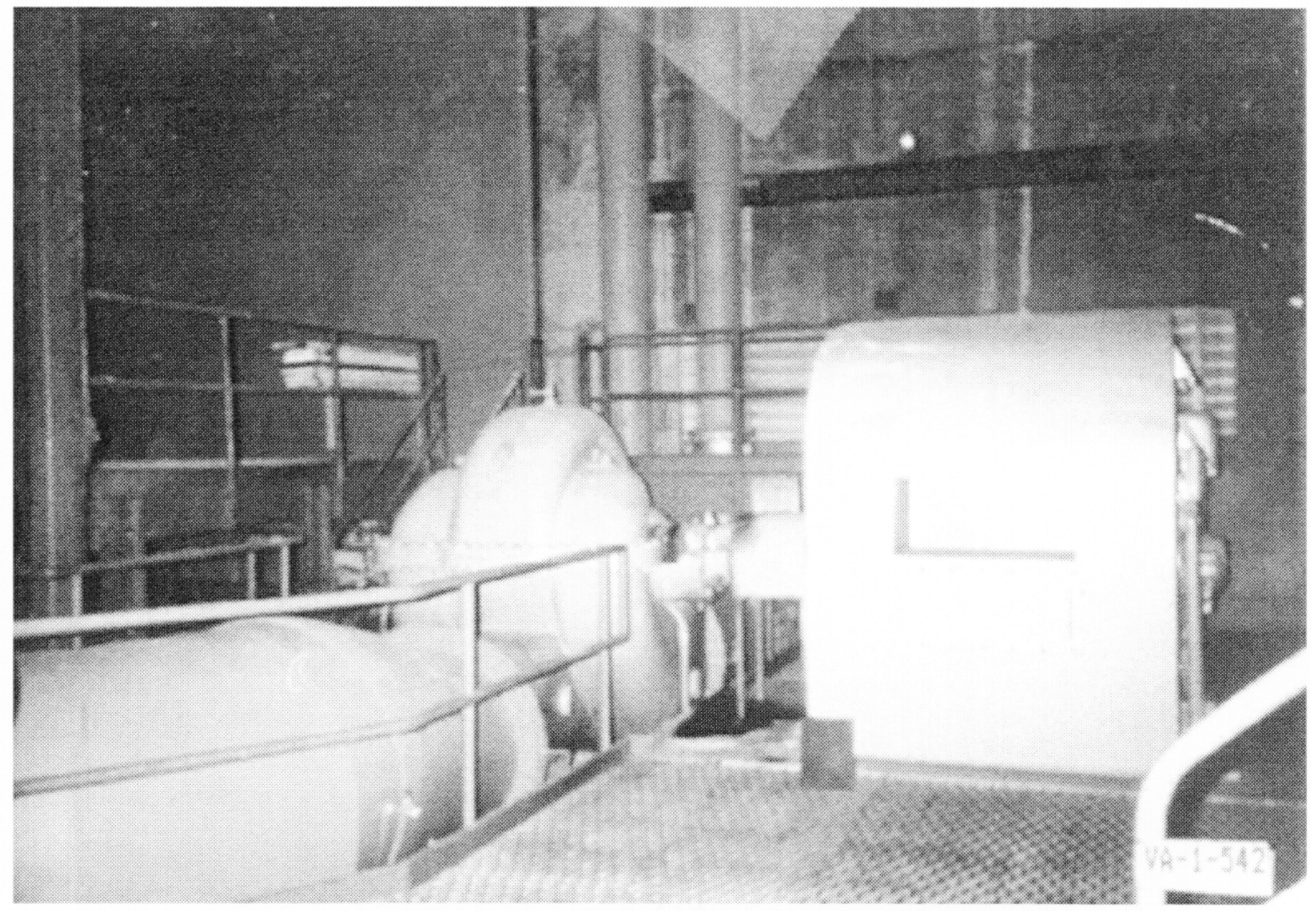

Figure 8.2.3-1 Horizontal Pump from the Earthquake Experience Database 


\subsubsection{VERTICAL PUMPS ${ }^{4}$}

The seismic capacity for the equipment class of Vertical Pumps (VP) (see Figure 8.2.4-1) may be based on earthquake experience data, provided the intent of each of the caveats listed below is met. This equipment class includes pumps with the impeller drive shaft mounted in a vertical (as opposed to horizontal) direction. Vertical pumps are typically powered by an electric drive motor, vertically aligned, and mounted atop a steel or cast-iron support frame that is anchored to a concrete base pad.

The two general types of vertical pumps represented in the earthquake experience equipment class are deep-well pumps and centrifugal pumps. Motor sizes range from 5 to $7000 \mathrm{hp}$ and flow rates range from 95 to $16,000 \mathrm{gpm}$.

Deep-well turbine type pumps have the pump impeller attached to the bottom of a long vertical drive shaft extending beneath the pump base plate. The pump drive shaft is enclosed in a steel or cast iron casing which extends below the pump base plate. The pump impeller is mounted in a contoured housing or bowl at the base of the casing. The casing or suction pipe is immersed in a well and opened at the bottom for fluid inlet.

A variation of the deep-well turbine pump is the can-type pump. The casing that encloses the impeller drive shaft is, in turn, enclosed by an outer casing or can. Fluid feed to the pump flows through an inlet line, usually mounted in the support frame above the pump base plate. The can forms an annular reservoir of fluid that is drawn into the impeller at the base of the inner casing.

Deep-well pumps range in size from fractional horsepower units to pumps of several thousand horsepower. The casings, cantilevered below the base plate, have typical lengths of 10 to 20 feet. The most massive component of the pump is normally the drive motor, which may weigh several tons.

Single-stage centrifugal pumps are configured with the impeller mounted above the base plate, directly beneath the drive motor. The impeller is housed in a casing that is usually part of the support frame for the drive motor. Instead of drawing fluid from a well or can beneath the pump base plate, the fluid inlet is a piping attachment aligned with a centerline of the impeller drive shaft. The discharge line is tangential to the periphery of the centrifugal impeller casing. Smaller centrifugal pumps are sometimes mounted directly on the piping system they serve.

The pump, drive motor, associated instrumentation and controls attached to the pump, and attached piping and conduit up to their first support on the building or nearby structure are included in the vertical pump equipment class. The equipment class does not include submersible pumps.

There are no GERS for Vertical Pumps.

\subsubsection{Reference Spectrum Caveats - Vertical Pumps}

The Reference Spectrum (RS) represents the seismic capacity of a Vertical Pump (VP) if the pump meets the intent of the following inclusion and exclusion rules. Note, however, that when the specific wording of a caveat rule is not met, then a reason for concluding that the intent has been met should be provided on the SEWS.

VPIRS Caveat 1 - Earthquake Experience Equipment Class. The vertical pump should be similar to and bounded by the VP class of equipment described above. The equipment class descriptions are general and the SCEs should be aware that worst case combinations of certain parameters may

4 Section B.6 of SQUG GIP (Ref. 1) 
not be represented in the generic equipment class. These worst case combinations may have reduced seismic capacity and should be carefully evaluated on a case-by-case basis.

VPIRS Caveat 2 - Cantilever Impeller Shaft Less Than 20 Feet Long. The impeller shaft and casing should not be cantilevered more than 20 feet below the pump mounting flange. This type of cantilever vertical pump should have a radial bearing at the bottom of the casing to support the impeller shaft. Twenty (20) feet represents the upper bound length of cantilever shafts of vertical pumps in the earthquake experience equipment class. The concern is that pumps with longer lengths may be subject to misalignment and bearing damage due to excessive lateral loads, damage to the impeller due to excessive displacement, and damage due to interfloor displacement on multifloor supported pumps. Either individual analysis or use of another method as a means of evaluating vertical pumps should be used when the shaft cantilever length exceeds 20 feet. The evaluation should address the concerns of excessive shaft and casing stresses and deflection of the impeller drive shaft.

VPIRS Caveat 3 - Check of Long Unsupported Piping. Brief consideration should be given to identify situations where the vertical pump may be affected by gross pipe motion, differential displacement, and excessive nozzle loads. The concern is that excessive force on pump nozzles could potentially break the pump nozzle or cause sufficient pump case distortion to cause binding, or fail the anchorage. These excessive forces are uncommon and need only be considered if there is a long section of unsupported pipe or a heavy valve attached to the pipe near the pump.

VPIRS Caveat 4 - Any Other Concerns? SCEs should seek out suspicious details or uncommon situations not specifically covered by the caveats which could adversely affect the seismic capacity of the pump. 


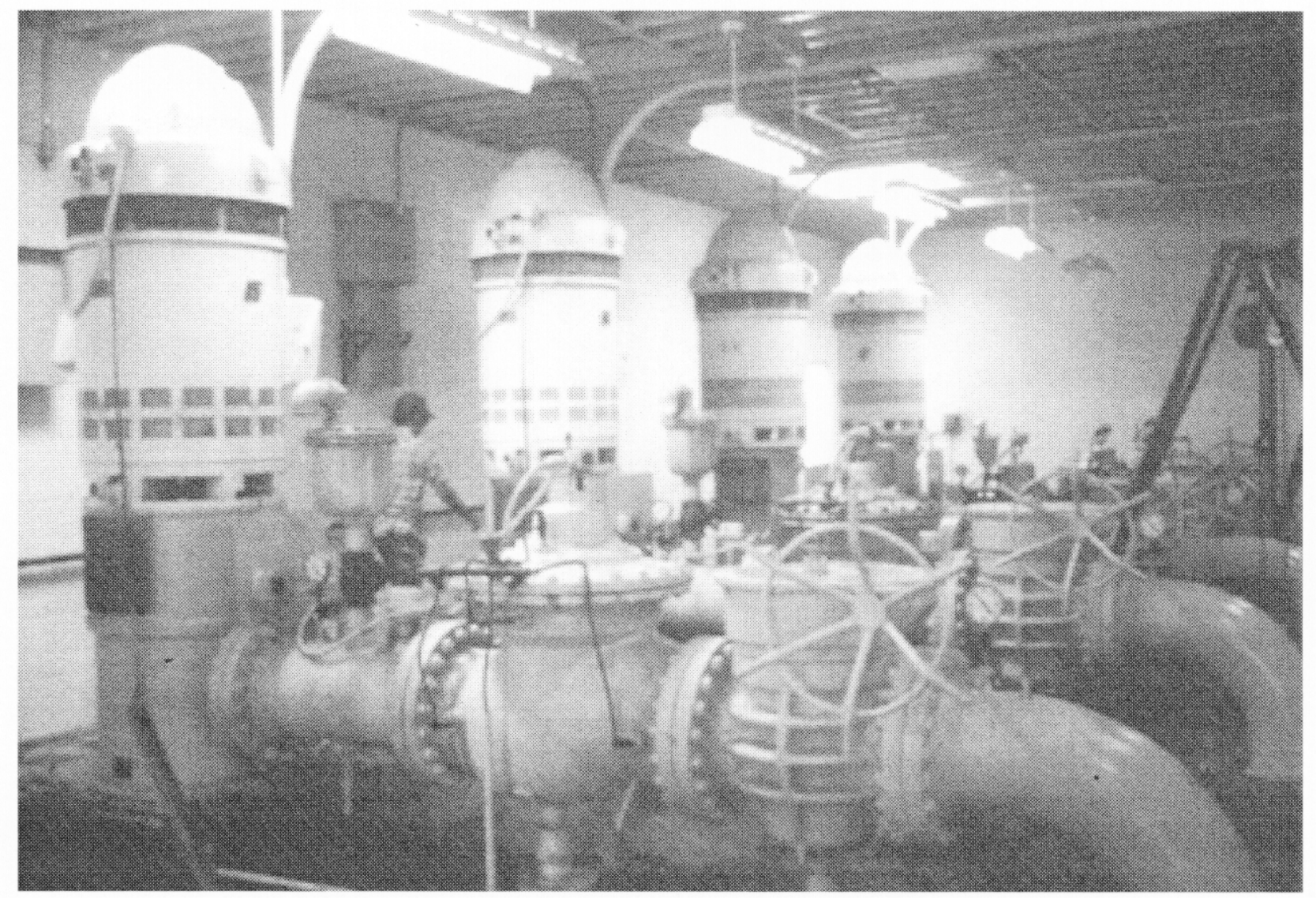

Figure 8.2.4-1 Vertical Pumps from the Earthquake Experience Database 


\subsubsection{CHILLERS ${ }^{5}$}

The seismic capacity for the equipment class of Chillers (CHL) (see Figure 8.2.5-1) may be based on earthquake experience data, provided the intent of each of the caveats listed below is met. This equipment class includes skid-mounted units comprised of components such as a compressor, a condenser, an evaporator, and a control and instrumentation panel. Chillers condense refrigerant or chill water for indoor climate-control systems which supply conditioned air for equipment operating environments and for personnel comfort.

Compressors draw vaporized refrigerant from the evaporator and force it into the condenser. The compressor of a chiller unit may be either the centrifugal or the reciprocating piston type.

Condensers are heat exchangers which reduce the refrigerant from a vapor to a liquid state. Chiller condensers are usually shell- and tube-type heat exchangers, with refrigerant on the shell side. Evaporators are tube bundles over which refrigerant is sprayed and evaporated, the inverse function of the condenser. Evaporator tubes can have either finned or plain surfaces. Control panels provide local chiller system monitoring and control functions. Typical components include: oil level switches/gauges, temperature switches/gauges, pressure switches/gauges, undervoltage and phase protection relays, and compressor motor circuit breakers.

Chiller components may be arranged in a variety of configurations. Typically the evaporator and condenser are mounted in a stacked configuration, one above the other, with the compressor and the control panel mounted on the side. Variations of this arrangement include the side-by-side configuration, with the compressor usually mounted above the condenser and evaporator, or a configuration with all components mounted side by side on the skid. Components are usually bolted to a supporting steel skid, which is, in turn, bolted to a concrete pad. Attachments to chillers include piping for routing cooling water or refrigerant to the unit, electrical conduit, and instrumentation and control lines. Chiller weights range up to about 40,000 lbs.

The compressor, condenser, evaporator, local control panel, support framing, and attached piping, instrument lines, and conduit which are attached to the same skid are included in the Chiller equipment class.

\section{There are no GERS for Chiller Units.}

\subsubsection{Reference Spectrum Caveats - Chillers}

The Reference Spectrum (RS) represents the seismic capacity of a Chiller (CHL) if the chiller meets the intent of the following inclusion and exclusion rules. Note, however, that when the specific wording of a caveat rule is not met, then a reason for concluding that the intent has been met should be provided on the SEWS.

CHL/RS Caveat 1 - Earthquake Experience Equipment Class. The chiller should be similar to and bounded by the CHL class of equipment described above. The equipment class descriptions are general and the SCEs should be aware that worst case combinations of certain parameters may not be represented in the generic equipment class. These worst case combinations may have reduced seismic capacity and should be carefully evaluated on a case-by-case basis.

CHL/RS Caveat 2 - No Reliance on Weak-Way Bending of Steel Plate or Structural Steel Shapes. The evaporator and condenser tanks should be reasonably braced between themselves for lateral forces parallel to the axis of the tanks without relying on weak-way bending of steel plate or webs of structural steel shapes. The concern is that in weak-way bending the structure will not be capable of transferring the lateral earthquake loads. If weak-way steel plate bending must be relied

5 Section B.11 of SQUG GIP (Ref. 1) 
on to brace the upper tank, then the adequacy of the steel components should be specially evaluated for adequate strength and stiffness.

CHIRS Caveat 3 - Any Other Concerns? SCEs should seek out suspicious details or uncommon situations not specifically covered by the caveats which could adversely affect the seismic capacity of the chiller. 


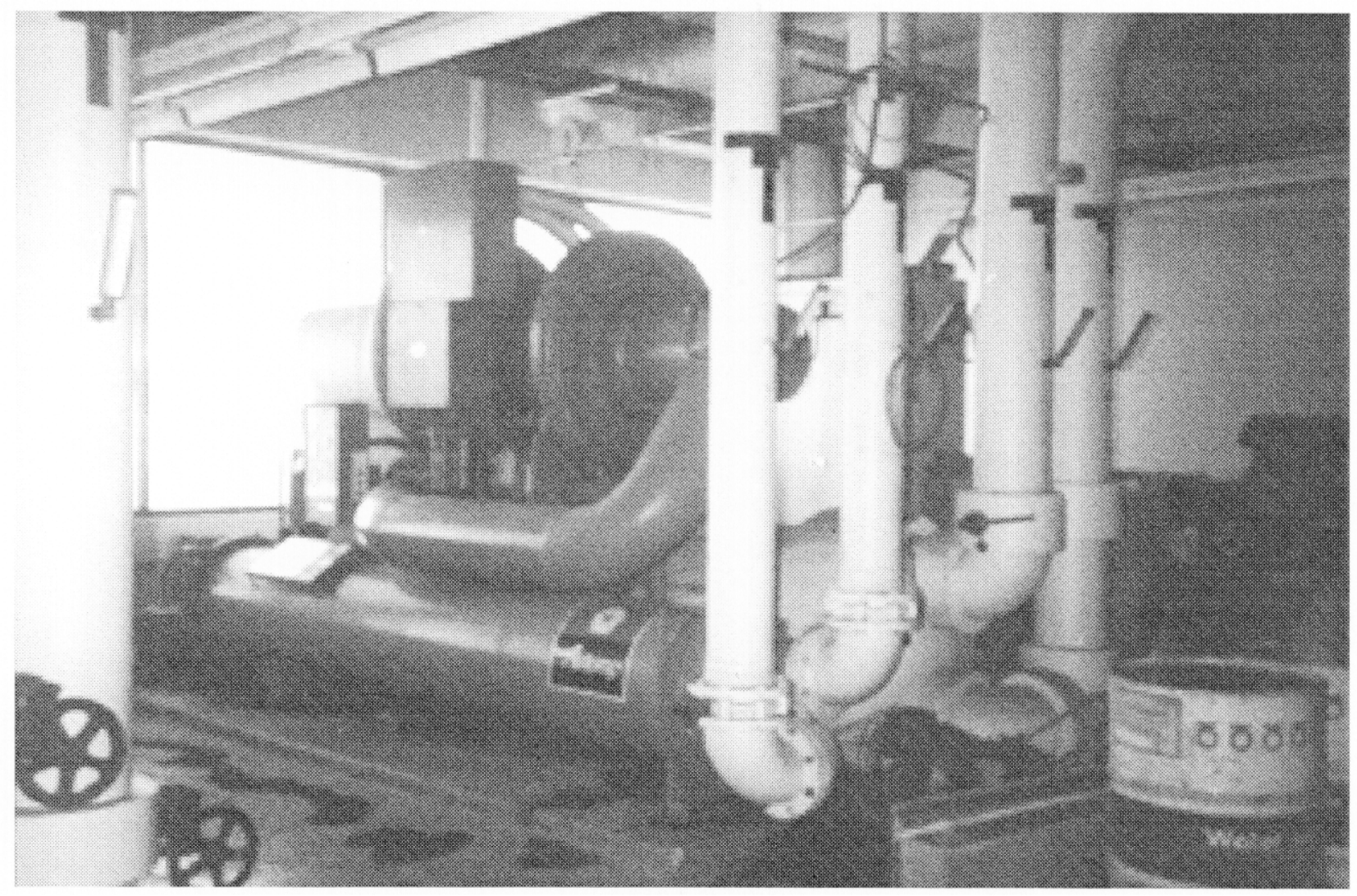

Figure 8.2.5-1 Chillers from the Earthquake Experience Database 


\subsubsection{AIR COMPRESSORS 6}

The seismic capacity for the equipment class of Air Compressor (AC) (see Figure 8.2.6-1) may be based on earthquake experience data, provided the intent of each of the caveats listed below is met. This equipment class includes freestanding air compressors together with attached components such as air intakes, air receiver tanks, local control panels, conduit, and discharge lines. Air compressors can be generally categorized as reciprocating piston or rotary screw. The equipment class of air compressors encompasses a wide range of sizes, configurations, and applications. Air compressors typically include as components: electric drive motor, piston- or impeller-driven compressor, air receiver tank, air intake filter, air aftercooler, moisture separator, lubrication system, and the control and instrument panel. Large compressors typically include water jackets to cool the compressor casing and the air aftercoolers, while smaller units are typically cooled by natural or fan-assisted convection to the surrounding air.

Air compressors supply operating pressure to pneumatic instrumentation and control systems, in particular to diaphragm-operated valves. Air compressors also charge pressurized air receiver tanks that serve the pneumatic starting systems for emergency engine-generators.

Compressor configurations in the equipment class include air receiver tank-mounted reciprocating piston or rotary screw compressors, skid-mounted reciprocating piston or rotary screw compressors, and freestanding reciprocating piston compressors.

Reciprocating piston compressors are constructed much like an automobile engine, with pistons encased in cast steel cylinders compressing the gas, and a system of timed valves controlling the inlet and discharge. Drive motor sizes typically range from fractional horsepower to over 100 horsepower. Piston air compressors generally have one or two cylinders but may include more. Cylinders are normally supported on a cast iron crankcase, which encloses the rotating crankshaft, linked either directly to the electric motor through a drive shaft, or indirectly through a belt linkage. Smaller reciprocating piston compressors are commonly mounted atop an air receiver tank.

Rotary screw compressors replace the reciprocating piston with a set of helical screws, typically encased in a cast iron block. The components and attachments of the air compressor are similar to reciprocating piston units except that the system of timed intake and discharge valves are not required. The most common configuration has the air compressor mounted on top of its air receiver tank. The units are usually not large, ranging in capacity from about 1 to $100 \mathrm{cfm}$ (cubic feet per minute of discharge air), with drive motors typically ranging from fractional horsepower up to $30 \mathrm{hp}$. Tank-mounted rotary screw compressors typically range in weight from about 200 to 2500 pounds.

Reciprocating piston and rotary screw compressors may also be mounted on a steel skid. The skid may be either open or enclosed in a sheet metal housing. The skid is normally constructed of a welded steel frame with the compressor, drive motor, receiver tank, control panel, and other components bolted to the frame in some convenient configuration. Skid-mounted compressors typically range in capacity up to about $2000 \mathrm{cfm}$, with drive motors of up to about $300 \mathrm{hp}$. Skidmounted compressors typically range in weight from about 2000 to 8000 pounds.

Freestanding compressors are usually the reciprocating piston type with one or two cylinders normally cantilevered from a crankcase. The crankcase may form the primary support for all components, or it may be mounted on a steel or cast iron pedestal. Freestanding compressors include the largest units typically found in facility applications, ranging in capacity up to about $4000 \mathrm{cfm}$, with drive motors up to about $1000 \mathrm{hp}$. Freestanding compressors range in weight from small units on the order of about 500 pounds to units as large as 10 tons.

6 Section B.12 of SQUG GIP (Ref. 1) 
The Air Compressor equipment class includes the piston- or impeller-driven compressor, drive motor, air receiver tank, and attached cooling coils and air intakes, attached air discharge lines, instrument lines, and attached conduit (up to the first support away from the unit).

\section{There are no GERS for Air Compressors}

\subsubsection{Reference Spectrum Caveats - Air Compressors}

The Reference Spectrum (RS) represents the seismic capacity of an Air Compressor (AC) if the compressor meets the intent of the following inclusion and exclusion rules. Note, however, that when the specific wording of a caveat rule is not met, then a reason for concluding that the intent has been met should be provided on the SEWS.

ACIRS Caveat 1 - Earthquake Experience Equipment Class. The air compressor should be similar to and bounded by the $A C$ class of equipment described above. The equipment class descriptions are general and the SCEs should be aware that worst case combinations of certain parameters may not be represented in the generic equipment class. These worst case combinations may have reduced seismic capacity and should be carefully evaluated on a case-by-case basis.

AC/RS Caveat 2 - Any Other Concerns? SCEs should seek out suspicious details or uncommon situations not specifically covered by the caveats which could adversely affect the seismic capacity of the compressor. 


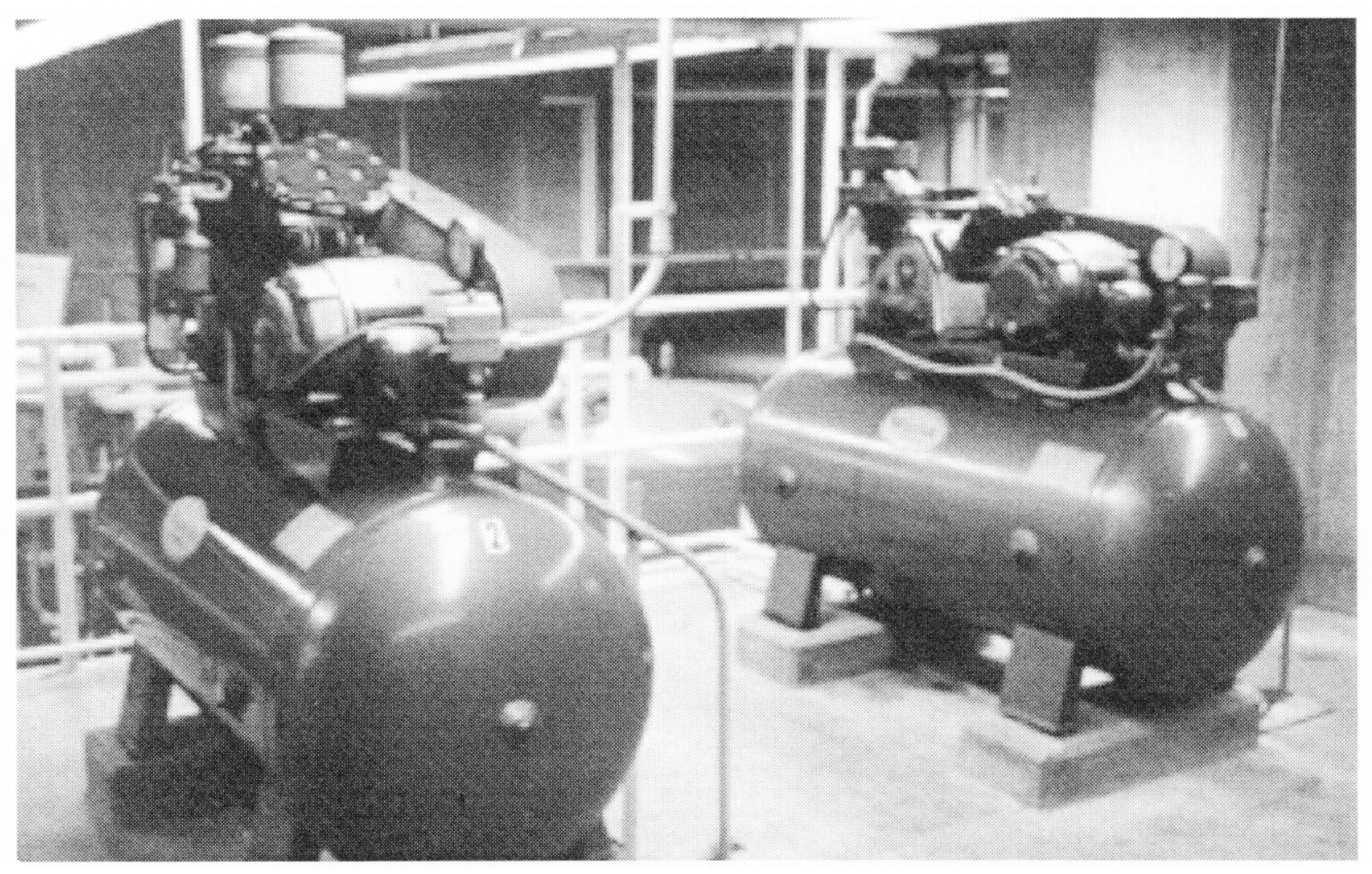

Figure 8.2.6-1 Air Compressors from the Earthquake Experience Database 


\subsubsection{MOTOR-GENERATORS?}

The seismic capacity for the equipment class of Motor-Generators (MG) (see Figure 8.2.7-1) may be based on earthquake experience data, provided the intent of each of the caveats listed below is met. This equipment class includes motors and generators that are coupled into a motor-generator set (M-G set). Motor-generator sets are structurally similar to horizontal pumps, which consist of an electric motor connected to a pump through a shaft. Motor-generators are basically two motors connected through a common shaft. M-G sets normally include either an AC or DC motor attached through a direct drive shaft to an AC or DC generator. A large flywheel is often mounted at one end of the shaft for storage of rotational inertia, to prevent transient fluctuations in generator output. Usually, both the motor and generator in an M-G set are mounted to a common drive shaft and bolted to a steel skid. Smaller sets sometimes house the motor and generator within the same casing. Motor-generator sets typically range in weight from about 50 to 5000 pounds.

The motor, generator, flywheel, and attached conduit are included in the Motor-Generator equipment class.

\section{There are no GERS for Motor-Generator sets.}

\subsubsection{Reference Spectrum Caveats-Motor-Generators}

The Reference Spectrum (RS) represents the seismic capacity of a Motor-Generator (MG) if the motor-generator meets the intent of the following inclusion and exclusion rules. Note, however, that when the specific wording of a caveat rule is not met, then a reason for concluding that the intent has been met should be provided on the SEWS.

MG/RS Caveat 1 - Earthquake Experience Equipment Class. The motor-generator should be similar to and bounded by the MG class of equipment described above. The equipment class descriptions are general and the SCES should be aware that worst case combinations of certain parameters may not be represented in the generic equipment class. These worst case combinations may have reduced seismic capacity and should be carefully evaluated on a case-by-case basis.

MG/RS Caveat 2 - Driver and Driven Component on Rigid Skid. The main driver and the driven component should be connected by a rigid base or common skid. The concern is that differential displacement between the driver and the driven component may bind the shaft or lead to excessive bearing wear. If they are not mounted on a rigid skid, the potential for differential displacement between the main driver and the driven component should be specially evaluated.

MG/RS Caveat 3 - Any Other Concerns? SCEs should seek out suspicious details or uncommon situations not specifically covered by the caveats which could adversely affect the seismic capacity of the motor-generator.

\footnotetext{
7 Section B.13 of SQUG GIP (Ref. 1)
} 


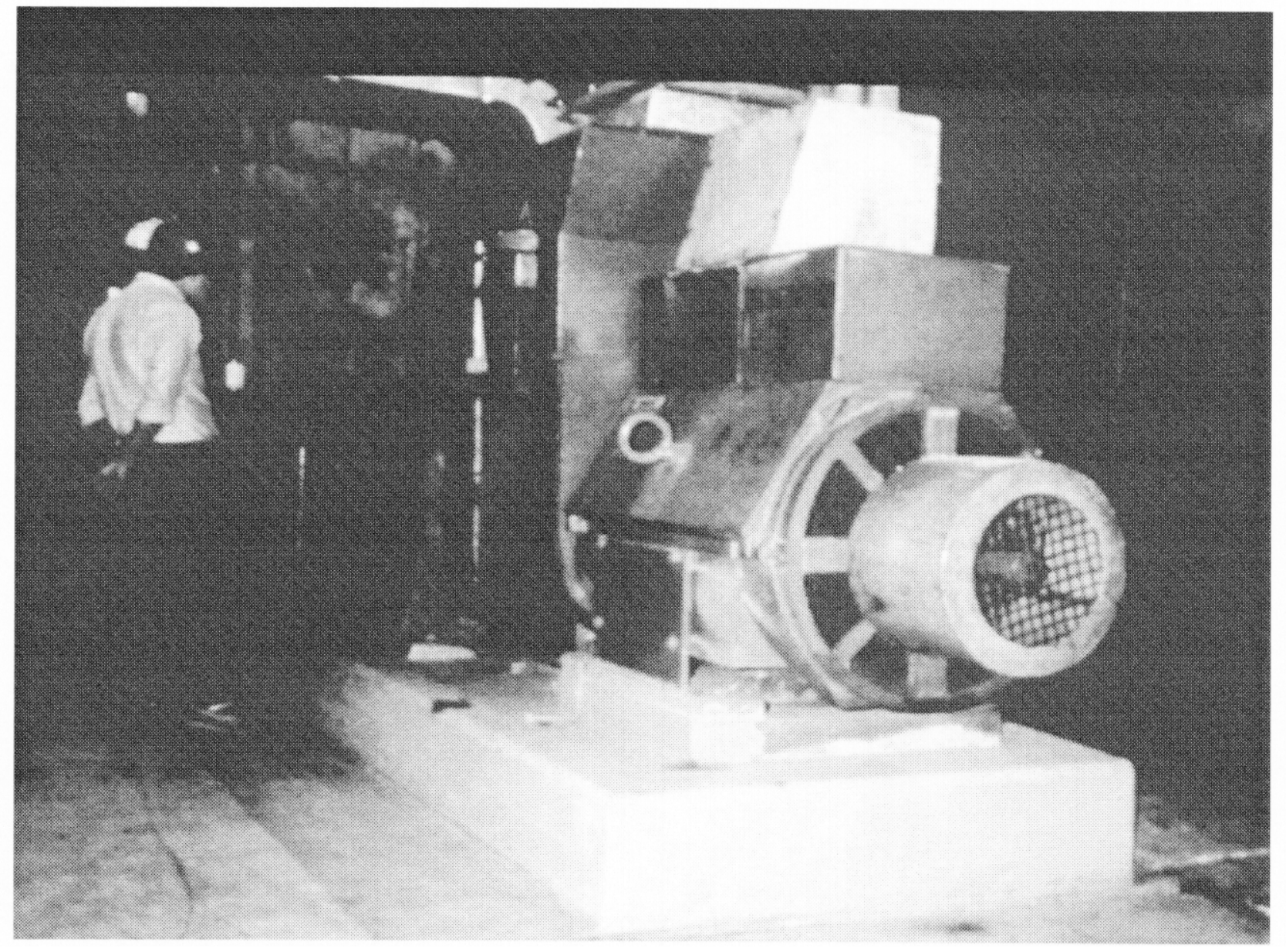

Figure 8.2.7-1 Motor-Generator from the Earthquake Experience Database 


\subsubsection{ENGINE-GENERATORS 8}

The seismic capacity for the equipment class of Engine-Generators (EG) (see Figure 8.2.8-1) may be based on earthquake experience data, provided the intent of each of the caveats listed below is met. This equipment class includes a wide range of sizes and types of generators driven by piston engines. Turbine driven generators are not included in this equipment class. Engine-Generators are emergency power sources that provide bulk $\mathrm{AC}$ power in the event of loss of off-site power.

In typical applications, generators range from $200 \mathrm{KVA}$ to $5000 \mathrm{KVA}$; electrical output is normally at 480,2400 , or 4160 volts. Generators are typically the brushless rotating-field type with either a rotating rectifier exciter or a solid-state exciter and voltage regulator. Reciprocatingpiston engines are normally diesel-fueled, although engines may operate on natural gas or oil. In typical applications, piston engines range from tractor-size to locomotive-size, with corresponding horsepower ratings ranging from about 400 to 4000 horsepower.

Engine-generators normally include the piston engine and generator in a direct shaft connection, bolted to a common steel skid. The skid or the engine block also supports peripheral attachments such as conduit, piping, and a local control and instrumentation panel.

The engine-generator system also includes peripheral components for cooling, heating, starting, and monitoring operation, as well as supplying fuel, lubrication, and air. The peripheral components may or may not be mounted on or attached directly to the engine-generator skid. If they are not mounted on the skid, they should be evaluated separately.

There are no GERS for Engine-Generators.

\subsubsection{Reference Spectrum Caveats - Engine-Generators}

The Reference Spectrum (RS) represents the seismic capacity of an Engine-Generator (EG) if the generator meets the intent of the following inclusion and exclusion rules. Note, however, that when the specific wording of a caveat rule is not met, then a reason for concluding that the intent has been met should be provided on the SEWS.

EG/RS Caveat 1 - Earthquake Experience Equipment Class. The engine-generator should be similar to and bounded by the EG class of equipment described above. The equipment class descriptions are general and the SCEs should be aware that worst case combinations of certain parameters may not be represented in the generic equipment class. These worst case combinations may have reduced seismic capacity and should be carefully evaluated on a case-by-case basis.

EG/RS Caveat 2 - Driver and Driven Component on Rigid Skid. The driver and the driven component should be connected by a rigid support or common skid. The concern is that differential displacement between the driver and the driven component may bind the shaft or lead to excessive bearing wear. If they are not mounted on a rigid skid, the potential for differential displacement between the driver motor and driven component should be evaluated.

EG/RS Caveat 3 - Any Other Concerns? SCEs should seek out suspicious details or uncommon situations not specifically covered by the caveats which could adversely affect the seismic capacity of the generator.

8 Section B.17 of SQUG GIP (Ref. 1) 


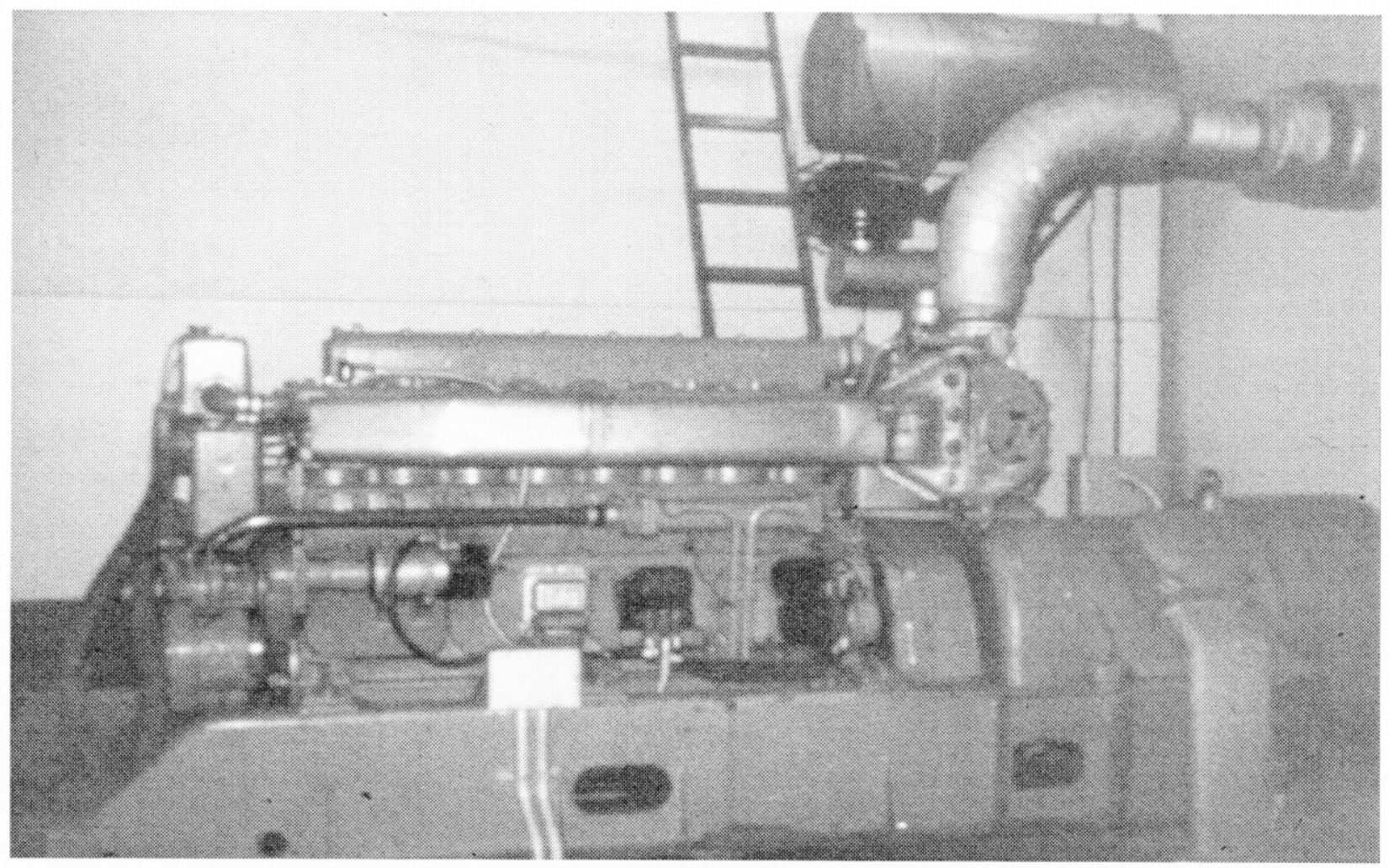

Figure 8.2.8-1 Engine-Generator from the Earthquake Experience Database 


\subsubsection{AIR HANDLERS 9}

The seismic capacity for the equipment class of Air Handlers (AH) (see Figure 8.2.9-1) may be based on earthquake experience data, provided the intent of each of the caveats listed below is met. This equipment class includes sheet metal enclosures containing (as a minimum) a fan and a heat exchanger. Air handlers are used for heating, dehumidifying or chilling, and distributing air.

The basic components of an air handler include a fan and a coil section. Small capacity, simple air handlers are often referred to as fan-coil units. Additional components such as filters, air-mixing boxes, and dampers are included in more elaborate air handlers. Fans (normally centrifugal) produce air flow across the coil for heat transfer. Coils act as heat exchangers in an air handler. Cooling coils are typically rectangular arrays of tubing with fins attached. Filters are typically mounted in steel frames which are bolted together as part of a modular system. Mixing boxes are used as a plenum for combining two airstreams before channeling the resulting blend into the air handler unit. Dampers are rotating flaps provided in the inlet or outlet sides of the air handler to control the flow of air into or out of the fan.

Air handlers are typically classified as being either a draw-through or a blow-through type. Drawthrough air handlers have the heat exchanger (coil) upstream of the fan, whereas the blow-through design locates the coil downstream. Air handler enclosures normally consist of sheet metal welded to a framework of steel angles or channels. Typical enclosures range in size from two feet to over ten feet on a side, with weights ranging from a few hundred pounds to several thousand pounds. Large components, such as fans and coils, are typically bolted to internal frames which are welded to the enclosure framing. Fans may be located in a variety of orientations with respect to the coil unit.

Air handlers typically include a system of attached ducts which provide for the intake and discharge of air. Additional attachments to air handlers include piping and cooling water or refrigerant, electrical conduit, and instrumentation lines. Self-contained air conditioning units are a variation of air handlers, in which the sheet metal enclosure includes a small refrigeration unit. Note that large centralized chillers are addressed as a separate equipment class.

Air handler configurations range from large floor-mounted units to smaller units suspended on rod hangers from ceilings. The sheet metal enclosure, fans and motors, heat exchanger coils, air filters, mixing boxes, dampers, attached ducts, instrument lines, and conduit are included in the Air Handler equipment class.

\section{There are no GERS for Air Handlers.}

\subsubsection{Reference Spectrum Caveats - Air Handlers}

The Reference Spectrum (RS) represents the seismic capacity of an Air Handler (AH) if the air handler meets the intent of the following inclusion and exclusion rules. Note, however, that when the specific wording of a caveat rule is not met, then a reason for concluding that the intent has been met should be provided on the SEWS.

AH/RS Caveat 1 - Earthquake Experience Equipment Class. The air handler should be similar to and bounded by the AH class of equipment described above. The equipment class descriptions are general and the SCEs should be aware that worst case combinations of certain parameters may not be represented in the generic equipment class. These worst case combinations may have reduced seismic capacity and should be carefully evaluated on a case-by-case basis.

9 Section B.10 of SQUG GIP (Ref. 1) 
AH/RS Caveat 2 - Anchorage of Internal Component. In addition to reviewing the adequacy of the unit's base anchorage, the attachment of heavy internal equipment of the air handler must be assessed. SCEs may exercise considerable engineering judgment when performing this review. Internal vibration isolators should meet the requirements for base isolators in Chapter 6.

AH/RS Caveat 3 -Doors Secured. All doors should be secured by a latch or fastener. The concern addressed by this caveat is that the doors could open during an earthquake, and the loose door could repeatedly impact the housing and be damaged or cause internal components such as relays to malfunction or chatter. In addition, the door may act as an integral structural member and may need to be latched to provide both stiffness and strength to the unit.

AHVRS Caveat 4 - No Possibility of Excessive Duct Distortion Causing Binding or Misalignment of Internal Fan. If the air handling unit contains a fan, then the possibility of excessive duct distortion during an earthquake should be considered for its effect on binding or misalignment of the fan. This need only be considered in cases of long unsupported ducts near the air handling unit or relatively stiff ducts subjected to significant relative motion. A special evaluation should be conducted to evaluate for this failure mode if these conditions are considered to be significant by the SCES.

AH/RS Caveat 5 - Any Other Concerns? SCEs should seek out suspicious details or uncommon situations not specifically covered by the caveats which could adversely affect the seismic capacity of the air handler. 


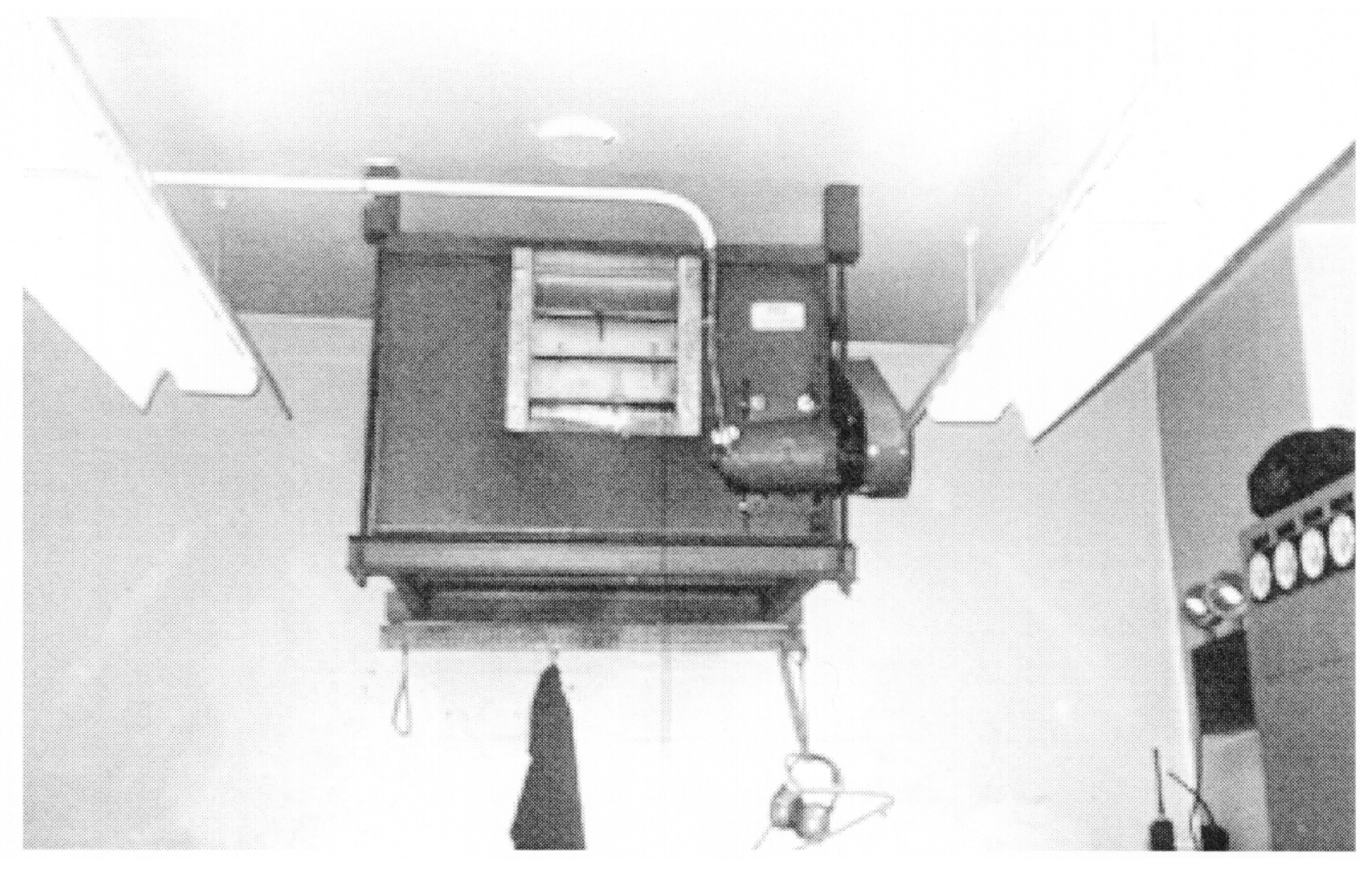

Figure 8.2.9-1 Air Handler from the Earthquake Experience Database 


\subsubsection{FANS 10}

The seismic capacity for the equipment class of Fans (FAN) (see Figure 8.2.10-1) may be based on earthquake experience data, provided the intent of each of the caveats listed below is met. This equipment class includes both freestanding and duct-mounted fans. Fans that are components of other classes of equipment such as air handlers are handled by other respective equipment classes and need not be specifically evaluated here. Blowers and exhausters are included in this equipment class.

Typical differential pressures for fans range from $1 / 2$ inch to 5 inches of water. Some centrifugal fans can have differential pressures ranging up to 12 inches of water. Air flow rates typically range from less than 1000 cubic feet per minute $(\mathrm{cfm})$ to flows on the order of $50,000 \mathrm{cfm}$. Corresponding fan drive motors typically range from $1 \mathrm{hp}$ to $200 \mathrm{hp}$. Typical weights of fan units range from 100 to 1000 pounds, depending on capacity and design details. The two basic types of fans in this equipment class include axial fans and centrifugal fans.

Axial fans are used in relatively low pressure applications such as building HVAC systems or cooling towers. Propeller fans and vane-axial fans are the two major types of axial fans. Propeller axial fans consist of two or more blades assembled on a central shaft and revolving within a narrow mounting-ring. Propeller fans are often mounted to a wall or ceiling. Vane-axial fans have an impeller wheel, typically with four to eight blades, mounted to a central shaft within a cylindrical casing. Vane-axial fans are generally used in higher pressure, higher flow applications than propeller fans. Vane-axial fans include a set of guide vanes mounted either before or after the impeller that streamline the air flow for greater efficiency. A variation of vane-axial design is the tube-axial fan, which includes the higher pressure impeller wheel mounted within a cylindrical casing, but without the provision of vanes.

Certain axial fan designs include multiple impellers for increased pressure boost. Axial-flow fans are normally mounted inside cylindrical ducting, supported by radial struts running from the duct wall to the duct centerline. Electric drive motors are usually mounted along the duct centerline immediately upstream of the impeller. The impeller and drive shaft are normally cantilevered from the motor. Alternate designs mount the motor on the outside of the duct with a belt connection between the motor and the impeller drive shaft.

Centrifugal fans are divided into three major categories depending upon the position of their blades. The three blade positions are: forward-curved, radial, and backward-inclined. Forwardcurved centrifugals have blades inclined toward the direction of rotation at the tip. These fans produce high flow volumes at low static pressures. Radial-blade centrifugals have their blades positioned on the radii extending from their axis of rotation. Backward-inclined fans are a type of centrifugal fan and have their blades inclined opposite to the direction of rotation at the tip.

Centrifugal fans typically have a cylindrical intake duct centered on the fan shaft and a square discharge duct directed tangentially from the periphery of the fan. A variation of the centrifugal fan is the tubular centrifugal fan which redirects the discharged air in the axial direction. As with axialflow fans, centrifugal fans can have the electrical drive motor mounted either directly on the fan shaft, or outside of the fan casing with a belt drive to the fan. The impeller and drive shaft may have either a single-point support, where they are cantilevered from the motor, or a two-point support, where the shaft is supported both at the motor and at an end bearing.

The fan impeller and its enclosure, drive motor, attached ducting, mounted louvers, and attached conduit and instrumentation lines are included in the Fan equipment class.

10 Section B.9 of SQUG GIP (Ref. 1) 
There are no GERS for Fans.

\subsubsection{Reference Spectrum Caveats - Fans}

The Reference Spectrum (RS) represents the seismic capacity of a Fan (FAN) if the fan meets the intent of the following inclusion and exclusion rules. Note, however, that when the specific wording of a caveat rule is not met, then a reason for concluding that the intent has been met should be provided on the SEWS.

FAN/RS Caveat 1 - Earthquake Experience Equipment Class. The fan should be similar to and bounded by the FAN class of equipment described above. The equipment class descriptions are general and the SCEs should be aware that worst case combinations of certain parameters may not be represented in the generic equipment class. These worst case combinations may have reduced seismic capacity and should be carefully evaluated on a case-by-case basis.

FAN/RS Caveat 2-Drive Motor and Fan Mounted on Common Base. The driver and fan should be connected by a common base or attached in a way to limit differential displacement. The concern is that differential displacement between the driver motor and fan may cause shaft misalignment. If the driver motor and fan are not mounted on a common base, then the potential for differential displacement should be specially evaluated.

FAN/RS Caveat 3 - Long Shafts Should be Supported at Fan and at Motor. Axial fans with long shafts between the motor and fan should have the shaft supported at the fan and at the motor. The concern is shaft misalignment. If the shaft is not supported in both locations, then a special evaluation should be conducted. The potential earthquake displacement of the shaft should be determined and compared to the operability displacement limits of the fan.

FAN/RS Caveat 4 - No Possibility of Excessive Duct Distortion Causing Binding or Misalignment of Fan. The possibility of excessive duct distortion during an earthquake should be considered for its effect on binding or misalignment of the fan. This need only be considered in cases of long unsupported ducts near the fan or relatively stiff ducts subjected to significant relative support motion. A special evaluation should be conducted to evaluate for this failure mode if these conditions are considered to be significant by the SCEs.

FAN/RS Caveat 5 - Any Other Concerns? SCEs should seek out suspicious details or uncommon situations not specifically covered by the caveats which could adversely affect the seismic capacity of the fan. 


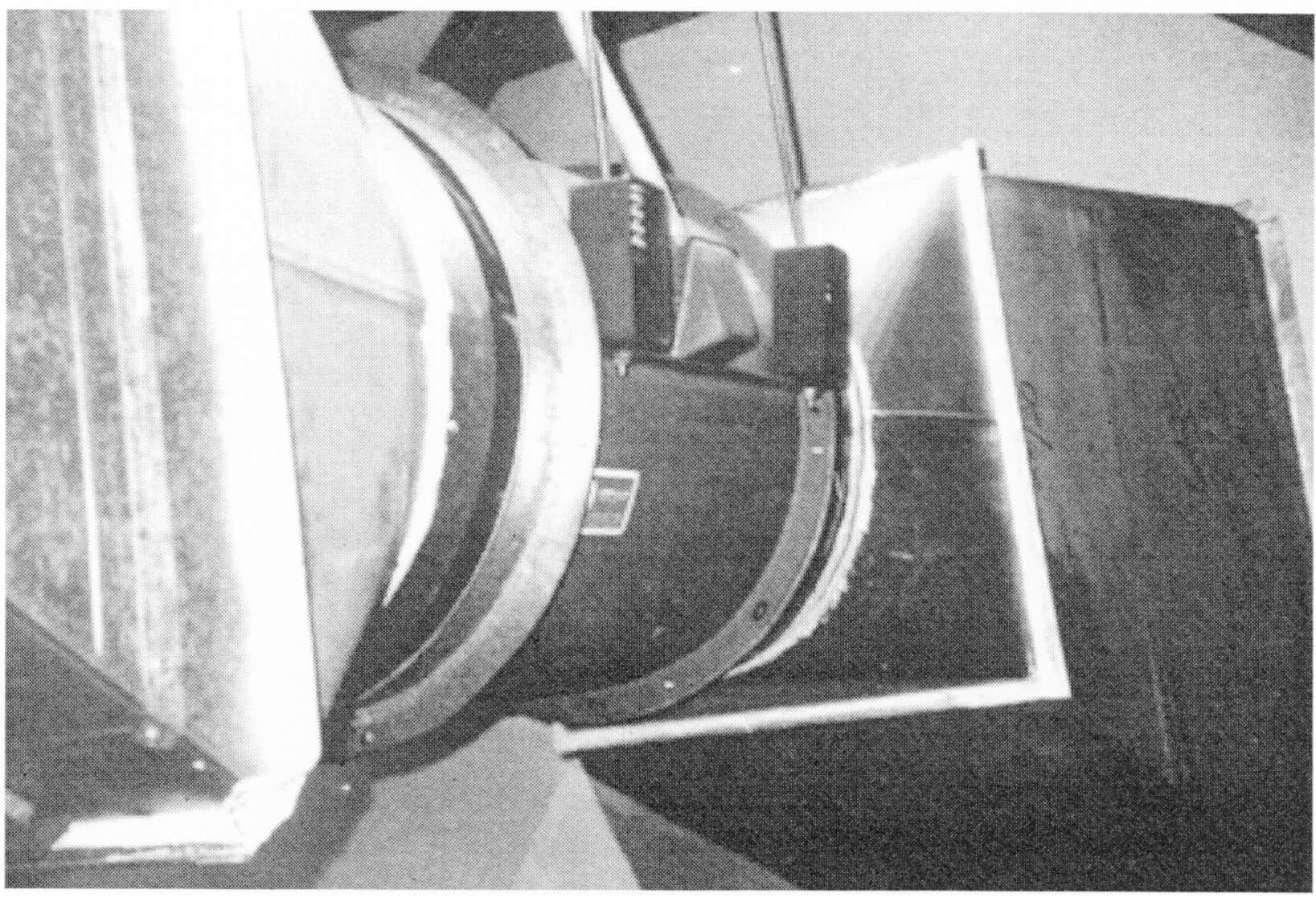

Figure 8.2.10-1 Fan from the Earthquake Experience Database 


\section{EQUIPMENT CLASS EVALUATIONS USING SCREENING PROCEDURES}

Chapter 9 contains a summary of equipment class descriptions and parameters based on earthquake experience data, test data, and analytical derivations. The screening procedures in Chapter 9 are from Chapters 7 and 8 of Revision 2 of the SQUG GIP (Ref. 1). Any modifications from the corresponding sections of Chapters 7 and 8 are denoted in Chapter 9 with words in italics (such as this introduction to Chapter 9). An item of equipment must have the same general characteristics as the equipment in the evaluation procedures. The intent of this rule is to preclude items of equipment with unusual designs and characteristics that have not demonstrated seismic adequacy in earthquakes or tests.

The screening procedures for evaluating the seismic adequacy of the different equipment classes in Chapter 9 cover those features which experience has shown can be vulnerable to seismic loadings. These procedures are a step-by-step process through which the important equipment parameters and dimensions are determined, seismic performance concerns are evaluated, the equipment capacity is determined, and the equipment capacity is compared to the seismic demand.

The screening procedures in Chapter 9 are based on information contained in References 42, 46, 47, and 50. The SCEs should use the information in Chapter 9 only after first thoroughly reviewing and understanding the background of the equipment classes and bases for the screening procedures as described in these references. These references provide more details and more discussion than summarized in Chapter 9. In some cases, clarifying remarks not contained in the reference documents have been included in Chapter 9. These clarifying remarks are based on experience gained during $S Q U G$ GIP reviews at operating nuclear power plants and DOE seismic evaluations at DOE facilities and they serve to help guide the SCEs apply their judgment.

The screening procedures in Chapter 9 are from Revision 2 of the SQUG GIP and Table 2.1-3 lists the equipment classes in Chapter 9. 


\subsection{ABOVEGROUND TANKS}

\subsubsection{VERTICAL TANKS}

\subsubsection{Introduction and Scope of Vertical Tanks ${ }^{1}$}

This section describes the guidelines which should be used for evaluating the seismic adequacy of those vertical tanks which are listed in the SEL as identified in Chapter 4. These guidelines are intended only for use on existing vertical tanks and are not to be used for new installations. The guidelines contained in this section are based on Reference 42 . Note, however, that to provide consistency with the remainder of the DOE Seismic Evaluation Procedure some of the nomenclature and symbols used in this section are slightly different than those used in Reference 42.

The screening evaluations described in this section for evaluating the seismic adequacy of vertical tanks cover those features of vertical tanks which experience has shown can be vulnerable to seismic loadings. These evaluations include the following features:

- Check that the shell of large, flat-bottom, vertical tanks will not buckle. Loadings on these types of tanks should include the effects of hydrodynamic loadings and tank wall flexibility.

- Check that the anchor bolts and their embedments have adequate strength against breakage and pullout.

- Check that the anchorage connection between the anchor bolts and the tank shell (e.g., saddles, legs, chairs, etc.) have adequate strength.

- Check that the attached piping has adequate flexibility to accommodate the motion of large, flat-bottom, vertical tanks.

Two SCEs (as defined in Chapter 3) should review these evaluations to determine that they meet the intent of these guidelines. This review should include a field inspection of the tank, the anchorage connections, and the anchor bolt installation against the guidelines described in this section and Chapter 6.

The derivation and technical justification for the guidelines in this section were developed specifically for large, flat-bottom, cylindrical, vertical storage tanks. The types of loadings and analysis methods described in this section are considered to be appropriate for these types of vertical tanks; however, a generic procedure cannot cover all the possible design variations. Therefore, it is the responsibility of the SCEs to assess the seismic adequacy of other design features not specifically covered in this section.

Other types of vertical tanks (e.g., vertical tanks supported on skirts and structural legs) which are not specifically covered by the guidelines in this section, should be evaluated by the SCEs using an approach similar to that described in this section. Reference 42 provides guidelines for evaluating vertical tanks on legs or skirts. Likewise, facilities may use existing analyses which evaluate the seismic adequacy of its vertical tanks in lieu of the DOE Seismic Evaluation Procedure, provided the SCEs determine that these other analyses address the same type of loading as the DOE Seismic Evaluation Procedure (e.g., hydrodynamic loading on the flexible wall of vertical, flat-bottom tanks, etc.) and the same failure modes (e.g., shell buckling of vertical, flat-bottom tanks, etc.).

1 Sections 7.2, 7.3, and 7.3.1 of SQUG GIP (Ref. 1) 
The screening guidelines described in this section were developed to simplify the complex dynamic fluid-structure interaction analyses for large vertical tanks. To accomplish this, it was necessary to make certain simplifying assumptions and to limit the range of applicability of the guidelines. Many vertical tanks in DOE facilities fall within the restrictions and range of values for which the screening guidelines were developed. Those vertical tanks which are not covered by these screening guidelines or do not pass these simple, but conservative, screening guidelines must be considered outliers. Outliers are evaluated in accordance with Section 9.1.1.8. For flat bottom vertical cylindrical tanks, a preferred method of treating outliers is through the use of Reference 29 ("Seismic Design and Evaluation Guidelines for the Department of Energy High-Level Waste Tanks and Appurtenances", BNL 52361). Even though this document was prepared for underground, high-level waste tanks, it covers the evaluation of seismic demand and capacity of aboveground tanks in a general and rigorous manner. Alternately, one can use the guidance of Appendix $H$ of Reference 18.

The screening guidelines described in this section are based on using $4 \%$ damped ground or floor response spectra (see Section 5.2) for overturning moment and shear loadings on the tanks. The slosh height of the fluid surface for vertical tanks is based on using $1 / 2 \%$ damped ground or floor response spectra. If $4 \%$ and $1 / 2 \%$ damped response spectra are not directly available, then they may be estimated by scaling from spectra at other damping values using the standard technique described in Appendix A of Reference 19 or Section 6.4.2.

This section covers the following topics for vertical tanks:

- Scope of vertical tanks

- Seismic demand applied to vertical tanks (Section 9.1.1.2)

- Overturning moment capacity calculation (Section 9.1.1.3)

- Shear load capacity vs. demand (Section 9.1.1.4)

- Freeboard clearance vs. slosh height (Section 9.1.1.5)

- $\quad$ Attached piping flexibility (Section 9.1.1.6)

- Tank foundation (Section 9.1.1.7)

The type of vertical tanks covered by the screening guidelines are large, cylindrical tanks whose axis of symmetry is vertical and are supported, on their flat bottoms, directly on a concrete pad or a floor. A section through a typical large vertical tank is shown in Figure 9.1.1-1. (Note: All figures and tables applicable to vertical tanks are grouped together at the end of Section 9.1.1). The range of parameters and assumptions which are applicable when using the guidelines to evaluate large vertical tanks are listed in Table 9.1.1-1. The nomenclature and symbols used for vertical tanks are listed in Table 9.1.1-2.

The guidelines assume that the tank shell material is carbon steel (ASTM A36 or A283 Grade C) or stainless steel (ASTM A240 Type 304) or aluminum. The number of bolts used to anchor down the tank is assumed to be 8 or more cast-in-place anchor bolts or J-bolts made of regular-strength or high-strength carbon steel (ASTM A36 or A307 or better material A325). These bolts are assumed to be spaced evenly around the circumference of the tank. These assumptions and the range of parameters given in Table 9.1.1-1 have been selected to cover the majority of vertical storage tanks in DOE facilities. 


\subsubsection{Seismic Demand Applied to Vertical Tanks ${ }^{2}$}

The seismic demand applied to vertical tanks in the screening guidelines is based on using the maximum horizontal component of the ground or floor response spectra (see Section 5.2). The tank should be evaluated for the condition where it is filled with fluid to the maximum level to which the tank is filled during operation; this is the most severe loading condition for typical tanks at $D O E$ facilities. Other types of loads, such as nozzle loads, are not considered in this screening method since they are typically very small compared to the tank inertial loads.

The horizontal response of fluid-filled vertical tanks has been found to be reasonably represented by two modes of response. One is a low frequency mode called the sloshing mode, in which the contained fluid sloshes within the tank. The other is a high frequency mode wherein the structure and fluid move together, called the impulsive mode. Previously, tank walls were assumed to be rigid in determining the response from these two modes. More recent work has shown that while the assumption is appropriate for the sloshing mode, it is not appropriate for the impulsive mode. For large, thin-walled tanks, the tank may deform under the impulsive mode pressures and vibrate at frequencies in the amplified response range of earthquake motion $(2$ to $20 \mathrm{~Hz}$ ). These screening guidelines account for fluid-structure interaction in the impulsive mode.

These hydrodynamic loads on the tank are characterized in the screening guidelines in terms of the tank overturning moment $(M)$ and the base shear load $(Q)$. By using certain simplifying assumptions and limiting the range of applicability, these loads can be determined using the stepby-step procedure given below.

Step 1 - Determine the following input data (where practical, as-built drawings should be used or a walkdown should be performed to gather data on the tank):

Tank Material:

R (Nominal radius of tank) [in.]

$\mathrm{H}^{\prime} \quad$ (Height of tank shell) [in.]

$t_{\min }$ (Minimum shell thickness along the height of the tank shell $\left(\mathrm{H}^{\prime}\right)$, usually at the top of the tank) [in.]

$t_{s}$ (Minimum thickness of the tank shell in the lowest $10 \%$ of the shell height $\mathrm{H}^{\prime}$ ) [in.]

$\sigma_{\mathrm{y}} \quad$ (Yield strength of tank shell material) [psi]

$\mathrm{h}_{\mathrm{c}} \quad$ (Height of shell compression zone at base of tank - usually height of chair) [in.]

$\mathrm{E}_{\mathrm{s}} \quad$ (Elastic modulus of tank shell material) [psi]

$\mathrm{V}_{\mathrm{s}}$ (Average shear wave velocity of soil for tanks located at grade) [ft/sec]

2 Section 7.3.2 of SQUG GIP (Ref. 1) 
Fluid:

$\gamma_{\mathrm{f}} \quad$ (Weight density of fluid in tank) [lbf/in $\left.{ }^{3}\right]$

$\mathrm{H}$ (Height of fluid at the maximum level to which the tank will be filled) [in.]

$h_{f} \quad$ (Height of freeboard above fluid surface at the maximum level to which the tank will be filled) [in.]

Bolts:

N (Number of anchor bolts)

d (Diameter of anchor bolt) [in.]

$h_{b}$ (Effective length of anchor bolt being stretched - usually from the top of the chair to embedded anchor plate) [in.]

$\mathrm{E}_{\mathrm{b}} \quad$ (Elastic modulus of anchor bolt material) [psi]

Loading:

Ground or floor response spectrum (see Section 5.2) acceleration at $4 \%$ damping for overturning moment and shear loadings on tanks and at $1 / 2 \%$ damping for fluid slosh height.

Step 2 - Calculate the following ratios and values:

H / R

$t_{s} / \mathbf{R}$

$\sum^{n} \mathrm{t}_{\mathbf{i}} \mathrm{h}_{\mathrm{i}}$

$t_{\mathrm{av}}=\frac{\mathrm{i}=1}{\mathrm{H}^{\prime}}$ (Thickness of the tank shell averaged over the linear height of the tank shell $\left(\mathrm{H}^{\prime}\right)$ ) [in.]

Where:

$\mathrm{n}=$ total number of sections of the tank shell with different thicknesses

$\mathrm{i}=$ counter digit

$\mathrm{t}_{\mathrm{i}}=$ thickness of the $\mathrm{i}^{\mathrm{th}}$ section of the tank shell [in.]

$h_{i}=$ height of the $i^{\text {th }}$ section of the tank shell [in.]

$\mathrm{H}^{\prime}=$ total height of tank shell [in.]

Note that $\sum_{i=1}^{n} h_{i}=H^{\prime}$ 


$$
\begin{aligned}
& t_{e f}=\frac{t_{a v}+t_{m i n}}{2} \quad \text { (Effective thickness of tank shell) [in.] } \\
& \begin{aligned}
t_{e f} / R \\
A_{b}=\frac{\pi d^{2}}{4} \quad \text { (Cross-sectional area of embedded anchor bolt) [in }{ }^{2} \text { ] }
\end{aligned} \\
& t^{\prime}=\left(\frac{N A_{b}}{2 \pi R}\right)\left(\frac{E_{b}}{E_{s}}\right) \quad \begin{array}{l}
\text { (Equivalent shell thickness having the same cross sectional } \\
\text { area as the anchor bolts) [in.] }
\end{array} \\
& c^{\prime}=\left(\frac{t^{\prime}}{t_{s}}\right)\left(\frac{h_{c}}{h_{b}}\right) \quad \text { (Coefficient of tank wall thicknesses and lengths under stress) } \\
& W=\pi R^{2} H \gamma_{f} \quad \text { (Weight of fluid in tank) [lbf] }
\end{aligned}
$$

Confirm that the parameters, values, and ratios determined in these first two steps are within the ranges given in Table 9.1.1-1. If they are, then the procedure given in this section is applicable to the subject vertical tank; proceed to Step 3. If the tank does not meet this guideline, classify the tank as an outlier and proceed to Section 9.1.1.8.

Step 3 - Determine the fluid-structure modal frequency for vertical carbon steel tanks containing water.

$\mathrm{F}_{\mathrm{f}}[\mathrm{Hz}]$ (from Table 9.1.1-3)

by entering Table 9.1.1-3 with:

$$
\begin{array}{ll}
\mathrm{R} \text { [in] } & \text { (from Step 1) } \\
\mathrm{t}_{\text {ef }} / \mathrm{R} & \text { (from Step 2) } \\
\mathrm{H} / \mathrm{R} & \text { (from Step 2) }
\end{array}
$$

Alternatively, enter Figure 9.1.1-2 with $t_{e f} / R$ and $H / R$ to obtain $F_{f}$. Then compute $F_{f}$ :

$$
F_{f}=F_{f}^{\prime}\left[\frac{1200}{R}\right]
$$

This frequency is for carbon steel tanks containing water. For other tank material (stainless steel or aluminum) with modulus of elasticity $E_{s}$ (psi) and fluid other than water with weight density $\gamma_{f}$ $\left[\mathrm{lbf} / \mathrm{in}^{3}\right]$, the frequency $F_{f}(s, f)$ may be computed from $F_{f}$, determined above, as follows:

$$
F_{f}(s, f)=F_{f} \sqrt{\frac{0.0361}{\gamma_{f}}} \sqrt{\frac{E s}{30 \times 10^{6}}}
$$


Step 4 - Determine the spectral acceleration $\left(\mathrm{Sa}_{\mathrm{f}}\right)$ for the fluid-structure modal frequency. (See Sections 5.2 and 6.4.2 for a discussion of input spectral acceleration.) Enter the 4\% damped horizontal ground or floor response spectrum (the maximum horizontal component) for the surface on which the tank is mounted, with the fluid-structure modal frequency:

$$
F_{f}[\mathrm{~Hz}] \text { (from Step 3) }
$$

and determine the maximum spectral acceleration:

$\mathrm{Sa}_{\mathrm{f}}[\mathrm{g}]$ (from horizontal 4\% damped response spectrum)

over the following frequency $(F)$ range:

$$
0.8 \mathrm{~F}_{\mathrm{f}}<\mathrm{F}<1.2 \mathrm{~F}_{\mathrm{f}}
$$

For tanks with concrete pads founded on ground, soil-structure interaction (SSI) effects on frequency $F_{f}$, and thus on $\mathrm{Sa}_{\mathrm{f}}$, must be accounted for if $\mathrm{V}_{\mathrm{s}}$ is less than $3,500 \mathrm{ft} / \mathrm{sec}$. The SSI effects on frequency may be computed explicitly by appropriate methods as discussed in Reference 42 , or by the following simplified procedure:

(a) If frequency $F_{f}$ is smaller than the frequency at the peak of the applicable ground response spectrum, SSI effects may be ignored.

(b) If frequency $F_{f}$ is larger than the peak frequency of the spectrum, then use the peak spectrum value for $\mathrm{Sa}_{\mathbf{f}}$.

Step 5 - Determine the base shear load (Q). Enter Figure 9.1.1-3 with:

$$
\begin{array}{ll}
\text { H / R } & \text { (from Step 2) } \\
\mathrm{t}_{\mathrm{ef}} / \mathrm{R} & \text { (from Step 2) }
\end{array}
$$

and determine the base shear load coefficient:

$$
\mathrm{Q}^{\prime} \quad \text { (from Figure 9.1.1-3) }
$$

Compute the shear load at the base of the tank:

$$
\mathrm{Q}=\mathrm{Q}^{\prime} \mathrm{W} \mathrm{Sa}_{\mathrm{f}}[\mathrm{lbf}]
$$

Step 6 - Determine the base overturning moment (M). Enter Figure 9.1.1-4 with:

$$
\begin{array}{ll}
\text { H / R } & \text { (from Step 2) } \\
t_{\text {ef }} / \mathrm{R} & \text { (from Step 2) }
\end{array}
$$

and determine the base overturning moment coefficient:

$$
\mathrm{M}^{\prime} \quad \text { (from Figure 9.1.1-4) }
$$

Compute the overturning moment at the base of the tank:

$$
\mathrm{M}=\mathbf{M}^{\prime} \mathrm{WH} \mathrm{Sa} \text { [in-lbf] }
$$

This completes the determination of the seismic demand applied to a vertical tank. 


\subsubsection{Overturning Moment Capacity Calculation ${ }^{3}$}

The seismic capacity of the tank shell and its anchorage to resist the overturning moment (M) calculated above is determined as explained below. The overturning moment is resisted by compression in the tank wall and tension in the anchor bolts. The overturning moment capacity is thus controlled by shell buckling on one side and anchor bolt capacity on the other side. The analysis procedure described below calculates the capacity of the shell to withstand buckling, assuming the anchor bolts stretch inelastically. The assumption of allowing the anchor bolts to stretch inelastically is used in these screening guidelines to distribute the overturning moment more evenly among several anchor bolts.

The overturning moment capacity calculation is broken down into four parts. First, the anchor bolt capacity is determined by the procedure given in Section 6.3 for cast-in-place bolts or J-bolts and is taken as the bolt yield capacity. Note, however, that the anchor bolt load using this allowable is subject to evaluation that there is adequate strength in the bolt chair and its connection to the shell to carry the anchor bolt yield capacity.

Therefore, the second part of the overturning moment capacity calculation is to determine the anchorage connection capacity. If it is determined that the anchorage connection assembly has lower capacity than that determined for the anchor bolt itself, then this lower capacity should be used. The failure mode governing the connection capacity should also be determined, i.e., is it ductile or brittle. For a brittle failure mode, the moment capacity is determined without allowing inelastic stretching (yielding) of the bolt.

The third part is to calculate the compressive axial buckling stress capacity of the tank shell. The fourth and final part is to determine the controlling overturning moment capacity using the calculated bolt tension capacity and tank shell buckling capacity and compare this to the overturning moment seismic demand determined in Step 6.

\subsection{Bolt Tensile Capacity ${ }^{4}$}

Step 7 - Determine bolt tensile load capacity, $P_{\mathbf{u}}(\mathrm{lbf})$, per guidelines for cast-in-place bolts in Section 6.3. This value should reflect any effects of less than minimum embedment, spacing, and edge distance as well as concrete cracking as detailed in Section 6.3. The bolt capacities from Section 6.3 are based on the weak link being the anchor bolt rather than the concrete such that the postulated failure mode is ductile. Compute the allowable bolt stress, $F_{b}$ (psi):

$$
\mathrm{F}_{\mathrm{b}}=\frac{\mathrm{P}_{\mathrm{u}}}{\mathrm{A}_{\mathrm{b}}}[\text { Psi] }
$$

where:

$$
\begin{aligned}
& \mathrm{P}_{\mathrm{u}}=\text { bolt tensile load capacity [lbf] (from Section 6.3) } \\
& \left.\mathrm{A}_{\mathrm{b}}=\text { cross-sectional area of embedded anchor bolt [in }{ }^{2}\right] \text { (from Step 2) }
\end{aligned}
$$

If the Section 6.3 criteria are not met for the anchorage, then the concrete is considered the weak link in the load path and the postulated failure mode is brittle. Determine an appropriate reduced allowable anchor bolt stress $\left(F_{r}\right)$ per applicable code requirements or, alternately, classify the tank as an outlier and proceed to Section 9.1.1.8 after completing all the evaluations in this section.

3 Section 7.3.3 of SQUG GIP (Ref. 1) 


\subsection{Anchorage Connection Capacity ${ }^{5}$}

In the previous step for determining bolt tensile capacity, it is assumed that the anchorage connection details are adequate for the bolt to develop its yield capacity in tension, and subsequently deform in a ductile manner. For this type of ductile behavior to occur, it should be possible to transfer loads at least equal to the anchor bolt allowable capacity to the tank wall local to the anchor bolts, the connection between the tank wall and the anchor bolt chair, and the anchor bolt chair itself.

The purpose of this check is to determine if the capacity of the load path is greater than the tensile capacity, $\mathrm{P}_{\mathrm{u}}$, of the anchor bolt. The evaluation guidelines given in this section are taken from Reference 42 which primarily uses the design guidelines developed by the American Iron and Steel Institute (Reference 87). Figure 9.1.1-5 shows a typical detail of a vertical tank anchor bolt chair. The chair includes two vertical stiffener plates welded to the tank wall. A top plate, through which the bolt passes, transfers loads from the bolt to the stiffeners which, in turn, transfer the loads into the tank wall. Figure 9.1.1-6 depicts two other less commonly-used anchor chair details. The detail shown in Figure 9.1.1-6(b) is an example of a poor anchorage connection design and is unlikely to satisfy the strength criteria for the connection. The procedure for checking the capacities of the various components of the anchorage connection is given below. This procedure applies to the typical chair assembly shown in Figure 9.1.1-5. A similar approach can be used for other types of anchor bolt chairs, however appropriate equations should be used. In particular the tank shell stress equation given below in Step 9 is only applicable for the type of chair assembly shown in Figure 9.1.1-5.

If each of the anchorage connection components meets the acceptance criteria defined below, then the bolt tensile capacity determined in the previous Step 7 is limiting. If, however, any of the components does not meet these guidelines, the reduced anchor bolt tension capacity represented by the equivalent value of anchor bolt allowable stress $\left(F_{r}\right)$, as calculated here, should be used. Note that, if the failure mode of the weak link is nonductile, the procedure for computing $\mathbf{M}_{\text {cap }}$ (in Section 9.1.1.3.4) is slightly different. Typically, plate or weld shear failure is considered nonductile, while tension yielding of the bolt or plastic bending failure is considered ductile. For the purposes of these guidelines, nonductile failure modes are classified as "brittle".

The procedure given below, Steps 8 through 11, is for carbon steel material (for tanks, connection elements and bolts), and is based on allowable stresses (adjusted for earthquake loading) per AISC specifications. Adjustments should be made for other material such as stainless steel and aluminum for the allowable stress per applicable codes. The symbols used in the equations given in these steps are defined in Figure 9.1.1-5.

Step 8 - Top Plate. The top plate transfers the anchor bolt load to the vertical stiffeners and the tank wall. The critical stress in the top plate occurs between the bolt hole and the free edge of the plate (the area identified by dimension $\mathrm{f}$ in Figure 9.1.1-5). This bending stress is estimated using the following equation. Note that if the top plate projects radially beyond the vertical plates, no more than $1 / 2$ inch of this projecting plate can be included in the dimension $f$ used in the following equation. The maximum bending stress in the top plate is:

$$
\sigma=\frac{(0.375 g-0.22 d) P_{u}}{f c^{2}} \quad[p s i]
$$

\begin{tabular}{ll}
\hline 4 & Section 7.3.3.1 of SQUG GIP (Ref. 1) \\
5 & Section 7.3.3.2 of SQUG GIP (Ref. 1)
\end{tabular} 
The top plate is adequate if the following guideline is satisfied:

$$
\sigma<\mathrm{f}_{\mathrm{y}}
$$

If the top plate does not meet this guideline, it is considered to fail in a ductile manner; therefore a load reduction factor:

$$
\frac{f_{y}}{\sigma}
$$

should be computed and multiplied by the anchor bolt allowable tensile stress $\left(\mathrm{F}_{\mathrm{b}}\right)$ :

$$
F_{r}=F_{b}\left(\frac{f_{y}}{\sigma}\right)[p s i]
$$

This reduced allowable anchor bolt stress should then be used to compute the overturning moment capacity in Section 9.1.1.3.4.

Step 9 - Tank Shell Stress. The anchor bolt loads are transferred into the tank shell as a combination of direct vertical load and out-of-plane bending moment (due to the eccentricity between the bolt centerline and the tank wall). A check of shell stresses is considered necessary only for large, flat-bottom, vertical storage tanks because of past experience with such tanks in earthquakes. Note that the stress equation given below is only applicable for the type of chair assembly shown in Figure 9.1.1-5.

The maximum bending stress in the tank shell is:

$$
\sigma=\frac{P_{u}}{t_{s}{ }^{2}}\left[\frac{1.32 \mathrm{Z}}{\frac{1.43 \mathrm{a} \mathrm{h}^{2}}{R \mathrm{t}_{\mathrm{s}}}+\left(4 \mathrm{a} \mathrm{h}^{2}\right)^{0.333}}+\frac{0.031}{\sqrt{\mathrm{Rt}_{\mathrm{s}}}}\right] \quad \text { [psi] }
$$

where:

$$
Z=\frac{1.0}{\frac{0.177 \mathrm{a} \mathrm{t}}{\sqrt{R t_{s}}}\left[\frac{t_{b}}{t_{s}}\right]^{2}+1.0}
$$

Note: The terms $a, t_{b}, t_{s}$, and $R$ in the above equation should all be in units of inches to be consistent with the proportionality factor of 0.177 which, as used in this equation, has units of $\left[\right.$ in. $\left.^{-1}\right]$.

The tank shell is adequate if the following guideline is satisfied:

$$
\sigma<\mathrm{f}_{\mathrm{y}}
$$


If the tank shell does not meet this guideline, it is considered to fail in a ductile manner; therefore a load reduction factor:

$$
\frac{f_{y}}{\sigma}
$$

should be computed and multiplied by the anchor bolt allowable tensile stress $\left(\mathrm{F}_{\mathrm{b}}\right)$.

$$
F_{r}=F_{b}\left(\frac{f_{y}}{\sigma}\right)[p s i]
$$

This reduced allowable anchor bolt stress should then be used to compute the overturning moment capacity in Section 9.1.1.3.4.

Step 10 - Vertical Stiffener Plates. The vertical stiffener plates are considered adequate for shear stress, buckling, and compressive stress if the following three guidelines are satisfied:

- $\quad \frac{k}{j}<\frac{95}{\sqrt{\frac{f_{y}}{1000}}}$

- $\mathrm{j}>0.4(\mathrm{~h}-\mathrm{c})$ and $\mathrm{j}>0.5 \mathrm{in}$.

- $\quad \frac{\mathrm{P}_{\mathrm{u}}}{2 \mathrm{kj}}<21,000 \mathrm{psi}$

If the vertical stiffener plates do not meet these guidelines, then the anchorage connection will fail in a nonductile manner before the anchor bolts will yield. For the purposes of these guidelines, nonductile failure modes are classified as "brittle". Determine an appropriate reduced allowable anchor bolt stress $\left(\mathrm{F}_{\mathrm{r}}\right)$ per applicable code requirements, and compute the overturning moment capacity in Section 9.1.1.3.4. Alternately, classify the tank as an outlier and proceed to Section 9.1.1.8 after completing the remainder of the evaluations in this section.

Step 11 - Chair-to-Tank Wall Weld. The load per linear inch of weld between the anchor bolt chair (i.e., the top plate plus the vertical stiffener plates) and the tank wall is determined from the following equation for an inverted U-weld pattern of uniform thickness:

$$
W_{w}=P_{u} \sqrt{\left[\frac{1}{a+2 h}\right]^{2}+\left[\frac{e}{a h+0.667 h^{2}}\right]}
$$


The weld is adequate if the following guideline is satisfied:

$$
\mathrm{w}_{\mathrm{w}} \leq \frac{30,600 \mathrm{t}_{\mathrm{w}}}{\sqrt{2}}
$$

where $30,600 \mathrm{psi}$ in the above equation is the allowable weld strength.

If the chair-to-tank wall weld does not meet this guideline, then the anchorage will fail in a nonductile manner before the anchor bolts will yield. For the purposes of these guidelines, nonductile failure modes are classified as "brittle." Determine an appropriate reduced allowable anchor bolt stress $\left(\mathrm{F}_{\mathrm{r}}\right)$ per applicable code requirements, and compute the overturning moment capacity in Section 9.1.1.3.4. Alternately, classify the tank as an outlier and proceed to 9.1.1.8 after completing the remainder of the evaluations in this section.

This completes the evaluation of the anchorage connection capacity for vertical tanks.

\subsection{Tank Shell Buckling Capacity ${ }^{6}$}

The compressive axial buckling stress capacity of the tank shell is most likely limited by the "elephant-foot" buckling mode near the base of the tank wall. Another possible buckling mode for vertical tanks is the "diamond-shape" buckling mode. Both of these buckling modes are dependent upon the hydrodynamic and hydrostatic pressure acting at the base of the tank which is determined below:

$\underline{\text { Step } 12}$ - Determine the fluid pressure for elephant-foot buckling $\left(P_{e}\right)$ by entering Figure 9.1.1-7 with:

$\mathrm{Sa}_{\mathrm{f}}$ [g] (from Step 4)

$H / R \quad$ (from Step 2)

and determine the pressure coefficient for elephant-foot buckling of the tank:

$\dot{P}_{e}^{\prime} \quad$ (from Figure 9.1.1-7)

Compute the fluid pressure at the base of the vertical tank for elephant-foot buckling:

$$
P_{e}=P_{e}^{\prime} \gamma_{f} R \text { [psi] }
$$

$\underline{\text { Step } 13}$ - Determine the elephant-foot buckling stress capacity factor

$$
\sigma_{\mathrm{pe}}[\mathrm{ksi}] \quad \text { (from Figure 9.1.1-8) }
$$

by entering Figure 9.1.1-8 with:

$$
\begin{array}{ll}
P_{e} \quad[p s i] & \text { (from Step 12) } \\
t_{s} / R & \text { (from Step 2) }
\end{array}
$$

6 Section 7.3.3.3 of SQUG GIP (Ref. 1) 
Convert $\sigma_{\mathrm{pe}}$ into units of psi by multiplying by 1000 . This value of $\sigma_{\mathrm{pe}}$ is for carbon steel. For other material, use the following formula:

$$
\sigma_{\mathrm{pe}}=\frac{0.6 \mathrm{E}_{\mathrm{s}}}{\left(\mathrm{R} / \mathrm{t}_{\mathrm{s}}\right)}\left[1-\left(\frac{\mathrm{P}_{\mathrm{e}} \mathrm{R}}{\sigma_{\mathrm{y}} \mathrm{t}_{\mathrm{s}}}\right)^{2}\right]\left[1-\frac{1}{1.12+\mathrm{S}_{1}^{1.5}}\right]\left[\frac{\mathrm{S}_{1}+\left(\sigma_{\mathrm{y}} / E\right)\left(30 x 10^{6}{ }_{p s i} / 36,000 \mathrm{psi}\right)}{\mathrm{S}_{1}+1}\right][\mathrm{psi}]
$$

where:

$$
\begin{aligned}
& S_{1}=\frac{R}{400 t_{s}} \\
& \sigma_{y}=\text { yield strength of tank shell material [psi] (from Step 1) } \\
& E_{S}=\text { elasticity modulus of tank shell material [psi] (from Step 1) } \\
& t_{s}=\begin{array}{l}
\text { minimum thickness of tank shell in the lowest 10\% of the shell } \\
\text { height ( } H^{\prime} \text { ) [in.] (from Step 1) }
\end{array} \\
& R=\text { nominal radius of tank [in.] (from Step 1) } \\
& P_{e}=\begin{array}{l}
\text { fluid pressure at the base of tank for elephant-foot buckling of tank } \\
\text { shell [psi] (from Step 12) }
\end{array}
\end{aligned}
$$

Step 14 - Determine the fluid pressure for diamond-shape buckling $\left(P_{d}\right)$ by entering Figure 9.1.1-9 with:

$$
\begin{array}{ll}
\mathrm{Sa}_{\mathrm{f}}[\mathrm{g}] & \text { (from Step 4) } \\
\mathrm{H} / \mathrm{R} & \text { (from Step 2) }
\end{array}
$$

and determine the pressure coefficient for diamond-shape buckling of the tank:

$$
P_{d}^{\prime} \quad \text { (from Figure 9.1.1-9) }
$$

Compute the fluid pressure at the base of the vertical tank for diamond-shape buckling:

$$
P_{d}=P_{d}^{\prime} \quad \gamma_{f} R \quad[p s i]
$$

Step 15 - Determine the diamond-shape buckling stress capacity factor:

$$
\sigma_{\mathrm{pd}}[\mathrm{ksi}] \quad \text { (from Figure 9.1.1-10) }
$$

by entering Figure 9.1.1-10 with:

$$
\begin{array}{ll}
\mathrm{P}_{\mathrm{d}} \quad[\mathrm{psi}] & \text { (from Step 14) } \\
\mathrm{t}_{\mathrm{s}} / \mathrm{R} & \text { (from Step 2) }
\end{array}
$$


Convert $\sigma_{\mathrm{pd}}$ into units of psi by multiplying by 1000 .

This value of $\sigma_{\mathrm{pd}}$ is for carbon steel. For other material use the following formula:

where:

$$
\sigma_{\mathrm{pd}}=(0.6 \gamma+\Delta \gamma) \frac{E_{\mathrm{s}}}{R / t_{\mathrm{s}}}
$$

$$
\begin{aligned}
& \gamma=1-0.73\left(1-\mathrm{e}^{-\phi}\right) \\
& \phi=\frac{1}{16} \sqrt{\frac{\mathrm{R}}{\mathrm{t}_{\mathrm{s}}}} \\
& \mathrm{E}_{\mathrm{s}}=\text { elastic modulus of tank shell material [psi] (from Step 1) } \\
& \mathrm{R}=\text { nominal radius of tank [in.] (from Step 1) } \\
& \mathrm{t}_{\mathrm{s}}=\text { minimum thickness of tank shell in the lowest 10\% of the shell height (H') [in.] } \\
& \Delta \gamma=\text { (from Step 1) }
\end{aligned}
$$

$\underline{\text { Step } 16}$ - Select the allowable buckling stress, $\sigma_{\mathrm{c}}$, as $72 \%$ of the lower value of $\sigma_{\mathrm{pe}}$ or $\sigma_{\mathrm{pd}}$ :

$$
\sigma_{\mathrm{c}}=0.72\left[\min \cdot\left(\sigma_{\mathrm{pe}}, \sigma_{\mathrm{pd}}\right)\right] \quad[\mathrm{psi}]
$$

\subsection{Overturning Moment Capacity ${ }^{7}$}

Step 17 - The overturning moment capacity of the tank, $M_{\text {cap }}$, is dependent upon whether the postulated weak link failure mode is ductile or brittle.

A ductile failure mode is defined as one in which the weak link is one of the following:

- $\quad$ Anchor bolt stretching (Step 7)

- $\quad$ Chair top plate bending (Step 8)

- $\quad$ Tank shell bending (Step 9)

A brittle mode of failure is defined as one in which the weak link is one of the following:

- $\quad$ Concrete cone failure (Step 7)

- Chair stiffener plate shear or buckling failure (Step 10)

- Chair-to-tank wall weld shear failure (Step 11)

7 Section 7.3.3.4 of SQUG GIP (Ref. 1) 
(a) Determine the base overturning moment coefficient for ductile failure:

$$
\mathbf{M}_{\text {cap }}^{\prime} \text { [dimensionless] (from Figure 9.1.1-12) }
$$

by entering Figure 9.1.1-12 with:

$$
\begin{array}{lll}
c^{\prime} & \text { [dimensionless] (from Step 2) } \\
\sigma_{c} & \text { [psi] } \quad \text { (from Step 16) } \\
F_{b}= & \text { smaller of } F_{b} \text { (from Step 7) or } F_{r} \text { (from Steps } 8 \text { or 9) } & \text { [psi] } \\
h_{c} & \text { [in] } \quad(\text { from Step 1) } \\
h_{b} & \text { [in] } \quad \text { (from Step 1) }
\end{array}
$$

If the postulated weak link failure mode is ductile go to Step (c) below. If the postulated weak link failure mode is brittle, continue on to Step (b) below.

(b) If the postulated weak link failure mode is brittle, then enter Table 9.1.1-4 with:

$$
c^{\prime} \text { [dimensionless] (from Step 2) }
$$

and determine the base overturning moment coefficient for the elastic limit:

$$
\mathbf{M}_{\text {cap }}^{\prime} \text { [dimensionless] (from Table 9.1.1-4) }
$$

Compare the $M_{\text {cap }}^{\prime}$ value determined above with the $M_{\text {cap }}^{\prime}$ value determined in Step (a) above and select the lower of the two values for use in Step (c) below.

(c) Compute $\mathrm{M}_{\text {cap }}$ :

$$
M_{\text {cap }}=\left(M_{\text {cap }}^{\prime}\right)\left(2 F_{b}\right)\left(R^{2} t_{s}\right)\left(h_{b} / h_{c}\right)
$$

using:

$$
\begin{array}{lll}
M_{\text {cap }}^{\prime} & \text { [dimensionless] } & \begin{array}{l}
\text { (from Step 17(a) for ductile failure mode or 17(b) } \\
\text { for brittle failure mode) }
\end{array} \\
F_{b} & = & \text { smaller of } F_{b} \text { or } F_{r} \text { (from Steps 7, 8, 9, 10, or 11) [psi] } \\
R & \text { [in.] } & \text { (from Step 1) } \\
t_{s} & \text { [in.] } & \text { (from Step 1) } \\
h_{b} & \text { [in.] } & \text { (from Step 1) } \\
h_{c} & \text { [in.] } & \text { (from Step 1) }
\end{array}
$$


Step 18 - Compare the overturning moment capacity of the tank ( $M_{\text {cap }}$, from Step 17) with the overturning moment (M, from Step 6). If

$$
\mathrm{M}_{\text {cap }} \geq \mathbf{M}
$$

then the tank is adequate for this loading; proceed to Step 19. If the tank does not meet this guideline, classify the tank as an outlier and proceed to Section 9.1.1.8 after completing the remainder of the evaluations in this section.

\subsubsection{Shear Load Capacity vs. Demand ${ }^{8}$}

The seismic capacity of the tank to resist the shear load $(Q)$ is determined below. The shear load is assumed to be resisted by sliding friction between the tank base plate and the supporting foundation material. The base shear load capacity is therefore a function of the friction coefficient and the pressure on the base plate. A friction coefficient of 0.55 is used in the screening guidelines. The pressure on the base plate is made up of hydrostatic pressure from the weight of the contained fluid less the hydrodynamic pressure from the vertical component of the earthquake. The hydrodynamic pressure from the horizontal component (from overturning moment) of the earthquake is ignored since its net or average pressure distribution over the entire base plate is zero. The weight of the tank shell is conservatively neglected.

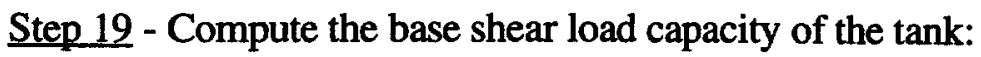

$$
\mathrm{Q}_{\text {cap }}=0.55\left(1-0.21 \mathrm{Sa}_{\mathrm{f}}\right) \mathrm{W}
$$

using:

$$
\begin{array}{lll}
\mathrm{Sa}_{\mathrm{f}} & {[\mathrm{g}]} & \text { (from Step 4) } \\
\mathrm{W} & {[\mathrm{lbf}]} & \text { (from Step 2) }
\end{array}
$$

$\underline{\text { Step } 20}$ - Compare the base shear load capacity of the tank ( $Q_{\text {cap }}$, from Step 19) with the shear load $(\mathrm{Q}$, from Step 5). If

$$
\mathrm{Q}_{\text {cap }} \geq \mathrm{Q}
$$

then the tank is adequate for this loading; proceed to Step 21. If the tank does not meet this guideline, classify the tank as an outlier and proceed to Section 9.1.1.8 after completing the remainder of the evaluations in this section.

This procedure assumes that no shear load is carried by the anchor bolts. Note that this assumption is theoretically valid only if there is a slight gap between the hole in the tank base and the anchor bolt; this is usually the case.

8 Section 7.3.4 of SQUG GIP (Ref. 1) 


\subsubsection{Freeboard Clearance vs. Slosh Height 9}

The screening guidelines described above are based on the assumption that there is enough freeboard clearance available between the liquid surface and the tank roof such that the tank roof is not subjected to significant forces from sloshing liquid. The procedure given below simply compares the freeboard clearance to the slosh height; this is considered to be $a$ reasonable approach to prevent roof damage even though some contact may occur.

Step 21 - The slosh height is given by the following equation:

$$
h_{s}=0.837 \mathrm{RSa}_{\mathrm{s}}
$$

where:

$$
\begin{aligned}
& \mathrm{R}=\text { nominal radius of tank [in.] (from Step 1) } \\
& \mathrm{Sa}_{\mathrm{s}}=\text { spectral acceleration (1/2\% damping) of the ground or floor (see Section } \\
& \text { 5.2) on which the tank is mounted at the frequency of the sloshing mode } \\
& \left(\mathrm{F}_{\mathrm{s}}\right. \text {, determined below). }
\end{aligned}
$$

In calculating the slosh height from this equation, the $\mathrm{Sa}_{\mathrm{s}}$ value must be obtained from the input demand spectrum at the sloshing mode frequency, $F_{s}$, and damping value of $1 / 2 \%$. Care should be exercised in assuring that the spectrum values are accurately defined in the sloshing mode frequency range, typically for $0.5 \mathrm{~Hz}$ to $0.2 \mathrm{~Hz}$. The sloshing mode frequency can be calculated from the following equation:

$$
\mathrm{F}_{\mathrm{s}}=\frac{1}{2 \pi} \sqrt{\frac{1.84 \mathrm{G}}{\mathrm{R}} \tanh \left(\frac{1.84 \mathrm{H}}{\mathrm{R}}\right)}
$$

where:

$$
\begin{array}{lll}
\mathrm{G} & = & \text { acceleration of gravity } \\
& = & 386.4 \quad\left[\mathrm{in} / \mathrm{sec}^{2}\right] \\
\mathrm{R} & \text { [in.] } & \text { (from Step 1) } \\
\mathrm{H} & \text { [in.] } & \text { (from Step 1) }
\end{array}
$$

Alternately, determine the slosh height by entering Table 9.1.1-5 with:

$$
\begin{array}{llll}
\mathrm{H} / \mathrm{R} & & \text { (from Step 2) } \\
\mathrm{R} & \text { [in.] } & \text { (from Step 1) }
\end{array}
$$

and determine the slosh height of the fluid in the tank for a ZPA of $1 \mathrm{~g}$ at the base of the tank:

$$
\mathrm{h}_{\mathrm{s}}^{\prime} \quad \text { [in.] (from Table 9.1.1-5) }
$$

9 Section 7.3.5 of SQUG GIP (Ref. 1) 
In calculating the slosh height given in Table 9.1.1-5, it has been assumed that for an input spectrum normalized to a $\mathrm{ZPA}$ of $1 \mathrm{~g}$, the $\mathrm{Sa}_{\mathrm{s}}(1 / 2 \%$ damping) values vary linearly from $0.75 \mathrm{~g}$ at $0.5 \mathrm{~Hz}$ to $0.4 \mathrm{~g}$ at $0.2 \mathrm{~Hz}$.

Compute the slosh height of the fluid in the tank for the ZPA of the ground or floor on which the tank is mounted:

$$
h_{s}=h_{s}^{\prime} \mathbf{Z P A}
$$

using:

$$
h_{s}^{\prime} \quad \text { [in.] (from above) }
$$

ZPA [g] (from horizontal response spectrum (see Section 5.2))

Step 22 - Determine the available freeboard above the fluid surface at the maximum level to which the tank will be filled $\left(h_{\mathrm{f}}\right.$, in.).

For conical tank roofs, measure the freeboard from the fluid surface to the intersection of the wall and the roof (a distance $\mathbf{R}$ from the tank centerline).

For tanks with a domed roof, measure the freeboard from the fluid surface to the point where the roof surface is at a distance of $0.9 \mathrm{R}$ from the tank centerline.

Compare the available freeboard $\left(h_{f}\right)$ to the slosh height of the fluid $\left(h_{s}\right.$, from Step 21). If

$$
h_{\mathrm{f}} \geq \mathbf{h}_{\mathrm{s}}
$$

then the tank is adequate for this condition; proceed to Step 23. If the tank does not meet this guideline, classify the tank as an outlier and proceed to Section 9.1.1.8 after completing the remainder of the evaluations in this section.

\subsubsection{Attached Piping Flexibility ${ }^{10}$}

For evaluation of large, flat-bottom, cylindrical, vertical tanks, the loads imposed on the tank due to the inertial response of attached piping can be neglected. It is considered that these piping loads have very little effect on the loads applied to the anchorage of large, flat-bottom tanks compared to the large hydrodynamic inertial loads from the tank and its contents. However, the relative motion between the tank and the piping presents a potential failure mode for the attached piping which could result in rapid loss of the tank's contents. This has occurred under certain circumstances in past earthquakes. Therefore this concern is addressed by requiring adequate flexibility in the piping system to accommodate tank motion as described below and in Section 7.2.3. In addition, the inertial loads should be considered for nozzles.

Step 23 - Flexibility of Attached Piping. The SRT should be aware that the analytical evaluation method for vertical tanks allows for a limited amount of base anchorage inelastic behavior. This, in turn, means that there may be uplift of the tank during seismic motion. When performing facility evaluations of tank anchorage, the SRT should assess attached piping near the base of the tank to ensure that the piping has adequate flexibility to accommodate any anticipated tank motion. Near the top of the tank, there will be considerably more motion and any attached piping should have substantial flexibility.

10 Section 7.3.6 of SQUG GIP (Ref. 1) 


\subsubsection{Tank Foundation ${ }^{11}$}

The screening guidelines contained herein are for use with all types of tank foundations typically found in $D O E$ facilities except ring-type foundations. Ring foundations should be identified as outliers and evaluated separately.

An acceptable outlier evaluation method for ring-type foundations is to check the tank overturning resistance and the adequacy of the rebar in the foundation. The overturning resistance may be checked by using the energy method to compute how much the tank and attached ring foundation lift up and whether there is adequate flexibility in the tank floor, shell, and associated welds, as well as any attached piping.

This completes the seismic evaluation for vertical tanks.

\subsubsection{Outliers $^{12}$}

An outlier is defined as a vertical tank which does not meet the screening guidelines for:

- Buckling of the shell of large, flat-bottom, vertical tanks,

- Adequacy of anchor bolts and their embedments,

- Adequacy of anchorage connections between the anchor bolts and the tank shell, or

- Flexibility of piping attached to large, flat-bottom, vertical tanks.

When an outlier is identified, proceed to Chapter 12, Outlier Identification and Resolution, and document the cause(s) for not meeting the screening guidelines on an Outlier Seismic Evaluation Sheet (OSES).

Note that all of the screening guidelines should be evaluated (i.e., go through all the steps in this procedure) so that possible causes for a vertical tank being classified as an outlier are identified before proceeding to Chapter 12 to resolve it.

The screening guidelines given in this section are intended for use as a generic screen to evaluate the seismic adequacy of vertical tanks. Therefore, if a vertical tank fails this generic screen, it may not necessarily be deficient for seismic loading; however, additional outlier evaluations are needed to show that it is adequate. When a vertical tank which is covered by this section fails to pass the screening guidelines, refined analyses could be performed which include use of more realistic or accurate methods instead of the simplified, generic analysis methods used in the section and Reference 42. Other generic methods for resolving outliers are provided in Chapter 12.

The preferred approach for evaluating flat-bottom, cylindrical, vertical tanks which are outliers is to follow the procedures given in Reference 29. This reference was primarily developed for underground waste storage tanks, but the approaches needed for the inner tank of a dual wall system are identical to those needed for aboveground tanks. Chapter 4 of Reference 29 describes the methodology for evaluating tank seismic demand including liquid-structure interaction effects and Chapter 5 of Reference 29 describes criteria for assessing structural capacity. The guidelines presented in Reference 29 are general enough to handle any geometrical configuration, material properties, and anchorage conditions (including unanchored tanks) as long as the vertical tank has a flat bottom and is cylindrical. Alternatively, one can use the guidance of Appendix $\mathrm{H}$ of Reference 18.

11 Section 7.3.7 of SQUG GIP (Ref. 1)

12 Section 7.5 of SQUG GIP (Ref. 1) 


\subsubsection{Documentation ${ }^{13}$}

The results of the engineering evaluations and field inspections performed using the guidelines in this section should be retained in the facility's files.

The results of the evaluations and inspections should also be documented by completing a Screening and Evaluation Data Sheet (SEDS) as described in Section 13.4 and a Screening Evaluation Work Sheet (SEWS) as described in Section 13.2.

If any of the screening guidelines contained in this section cannot be met, the vertical tank should be classified as an outlier. The Outlier Seismic Evaluation Sheet (OSES), discussed in Chapters 12 and 13 should be completed to document the cause(s) for not meeting the screening guidelines.

13 Section 7.6 of SQUG GIP (Ref. 1) 
Table 9.1.1-1 Applicable Range of Parameters and Assumptions for Vertical Tanks (Table 7-1 of SQUG GIP, Ref. 1)

\begin{tabular}{|c|c|c|}
\hline Tank Material $^{1}$ & \multicolumn{2}{|c|}{ Carbon or Stainless Steel, Aluminum } \\
\hline Tank Fluid Content & \multicolumn{2}{|c|}{ Water or similar } \\
\hline Nominal Radius of Tank & $\mathbf{R}$ & $\begin{array}{ll}= & 5 \text { to } 35 \mathrm{ft} \\
& (60 \text { to } 420 \mathrm{in})\end{array}$ \\
\hline Height of Tank Shell & $\mathrm{H}^{\prime}$ & $\begin{array}{l}=10 \text { to } 80 \mathrm{ft} \\
(120 \text { to } 960 \mathrm{in} .)\end{array}$ \\
\hline $\begin{array}{l}\text { Height of Fluid at the Maximum } \\
\text { Level to Which the Tank Will be Filled }\end{array}$ & $\mathrm{H}$ & $\begin{array}{l}=\quad 10 \text { to } 80 \mathrm{ft} \\
(120 \text { to } 960 \text { in. })\end{array}$ \\
\hline $\begin{array}{l}\text { Minimum Thickness of the Tank Shell } \\
\text { in the Lowest } 10 \% \text { of the Shell Height }\left(H^{\prime}\right)\end{array}$ & $t_{s}$ & $=3 / 16$ to $1 \mathrm{in}$. \\
\hline $\begin{array}{l}\text { Effective Thickness of Tank Shell Based } \\
\text { on the Mean of the Average Thickness }\left(t_{\text {av }}\right) \\
\text { and the Minimum Thickness }\left(t_{\text {min }}\right)\end{array}$ & $t_{e f}$ & $=3 / 16$ to $1 \mathrm{in}$. \\
\hline Diameter of Anchor Bolt ${ }^{2}$ & d & $=1 / 2$ to 2 in. \\
\hline Number of Anchor Bolts ${ }^{3}$ & $\mathbf{N}$ & $=8$ or more \\
\hline $\begin{array}{l}\text { Tank Wall Thickness (at Base)-to-Tank } \\
\text { Radius Ratio }\end{array}$ & $t_{s} / R$ & $=\quad 0.001$ to 0.01 \\
\hline $\begin{array}{l}\text { Effective Tank Wall Thickness-to-Tank } \\
\text { Radius Ratio }\end{array}$ & $\mathrm{t}_{\mathrm{ef}} / \mathrm{R}$ & $=0.001$ to 0.01 \\
\hline Fluid Height-to-Tank Radius Ratio & $H / R$ & $=\quad 1.0$ to 5.0 \\
\hline
\end{tabular}

\section{Assumptions:}

1 The tank material is assumed to be carbon steel (ASTM A36 or A283 Grade C), stainless steel (ASTM A240 Type 304), aluminum, or better material.

2 Anchor bolts are assumed to be cast-in-place or J-bolts and made of regular-strength or highstrength carbon steel (ASTM A36 or A307 or better material A325).

3 Anchor bolts are assumed to be evenly spaced around the circumference of the tank. 


\section{Table 9.1.1-2 Nomenclature Used for Vertical Tanks \\ (Table 7-2 of SQUG GIP, Ref. 1)}

Symbol Description [Units]

$A_{b} \quad$ Cross-sectional area of embedded anchor bolt [in. $\left.{ }^{2}\right]$

a - Width of chair top plate parallel to shell (see Figure 9.1.1-5) [in.]

b - Depth of chair top plate perpendicular to shell (see Figure 9.1.1-5) [in.]

c - Thickness of chair top plate (see Figure 9.1.1-5) [in.]

$c^{\prime} \quad$ - Coefficient of tank wall thicknesses and lengths under stress [dimensionless]

d - Diameter of anchor bolt [in.]

$\mathrm{E}_{\mathrm{s}} \quad-\quad$ Elastic modulus of tank shell material [psi]

$E_{b} \quad-\quad$ Elastic modulus of anchor bolt material [psi]

e - Eccentricity of anchor bolt with respect to shell outside surface (see Figure 9.1.1-5) [in.]

F - Frequency $[\mathrm{Hz}]$

$F_{b} \quad-\quad$ Allowable tensile stress of bolt [psi]

$F_{f} \quad-\quad$ Frequency of fluid-structure interaction mode $[\mathrm{Hz}]$

$\mathrm{F}_{\mathrm{r}} \quad$ - Reduced allowable tensile stress of bolt [psi]

$\mathrm{F}_{\mathrm{s}} \quad-\quad$ Sloshing mode frequency $[\mathrm{Hz}]$

f - Distance from outside edge of chair top plate to edge of hole (see Figure 9.1.1-5) [in.]

$f_{y} \quad$ Minimum specified yield strength of shell, chair, saddle, or base plate material [psi]

G - Acceleration of gravity $\left[386.4 \mathrm{in} / \mathrm{sec}^{2}\right]$

g - Distance between vertical plates of chair (see Figure 9.1.1-5) [in.]

H - Height of fluid at the maximum level to which the tank will be filled (see Figure 9.1.1-1) [in.] 


\section{Table 9.1.1-2 (Continued)}

Symbol Description [Units]

$H^{\prime} \quad$ - Height of tank shell (see Figure 9.1.1-1) [in.]

h - Height of chair (see Figure 9.1.1-5) [in.]

$h_{b} \quad$ - Effective length of anchor bolt being stretched (usually from top of chair to embedded anchor plate) (see Figure 9.1.1-1) [in.]

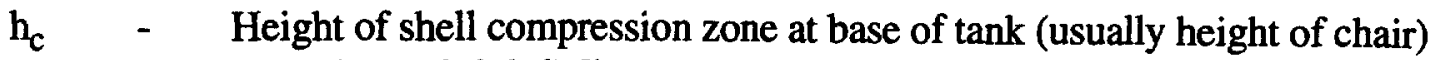
(see Figure 9.1.1-1) [in.]

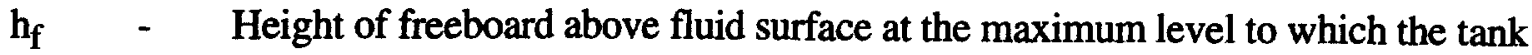
will be filled (see Figure 9.1.1-1) [in.]

$h_{s} \quad-\quad$ Slosh height of fluid in tank [in.]

$h_{s}{ }^{\prime} \quad-\quad$ Slosh height of fluid for a ZPA of $1 \mathrm{~g}$ applied at tank base [in.]

j - Thickness of chair vertical plate (see Figure 9.1.1-5) [in.]

k - Width of chair vertical plate (see Figure 9.1.1-5). Use average width for tapered plates [in.].

M - Overturning moment at base of tank [in-lbf]

$\mathbf{M}^{\prime} \quad$ - Base overturning moment coefficient [dimensionless]

$M_{\text {cap }} \quad$ Overturning moment capacity of tank [in-lbf]

$\mathbf{M}_{\text {cap }}^{\prime}$ - Base overturning moment capacity coefficient [dimensionless]

$\mathrm{N} \quad-\quad$ Number of anchor bolts [dimensionless]

$\mathrm{P}_{\mathrm{e}} \quad$ - Fluid pressure at base of tank for elephant-foot buckling of tank shell [psi]

$\mathrm{P}_{\mathrm{e}}{ }^{\prime} \quad$ - Pressure coefficient for elephant-foot buckling [dimensionless]

$P_{d} \quad-\quad$ Fluid pressure at base of tank for diamond-shape buckling of tank shell [psi]

$P_{d}{ }^{\prime} \quad$ - Pressure coefficient for diamond-shape buckling [dimensionless] 


\section{Table 9.1.1-2 (Continued)}

Symbol Description [Units]

$P_{u} \quad$ - Allowable tensile load of anchor bolt [lbf]

Q - Shear load at base of tank [lbf]

$Q^{\prime} \quad$ - Base shear load coefficient [dimensionless]

$\mathrm{Q}_{\text {cap }}$ - Base shear load capacity of tank [lbf]

$\mathrm{R} \quad$ - Nominal radius of tank [in.] (see Figure 9.1.1-1)

r - Least radius of gyration of vertical stiffener plate cross-sectional area about a centroidal axis [in.]

$S_{1} \quad$ Coefficient of tank radius to shell thickness $\left(\frac{R}{400 t_{s}}\right)$ [dimensionless]

Sa - Spectral acceleration of ground or floor $[\mathrm{g}]$

$\mathrm{Sa}_{\mathrm{f}}$ - Spectral acceleration (4\% damping) of the ground or floor on which the tank is mounted at the frequency of the fluid-structure interaction mode $\left(\mathrm{F}_{\mathrm{f}}\right)[\mathrm{g}]$

$\mathrm{Sa}_{\mathrm{s}}-\quad$ Spectral acceleration (1/2\% damping) of the ground or floor on which the tank is mounted at the frequency of the sloshing mode $\left(\mathrm{F}_{\mathrm{s}}\right)[\mathrm{g}]$

$t_{a v} \quad-\quad$ Thickness of the tank shell averaged over the linear height of the tank shell $\left(H^{\prime}\right)$ [in.]

$t_{b} \quad-\quad$ Thickness of bottom or base plate of tank (see Figure 9.1.1-5) [in.]

$t_{e f} \quad-\quad$ Effective thickness of tank shell based on the mean of the average thickness $\left(t_{a v}\right)$ and the minimum thickness $\left(t_{\min }\right)$ [in.]

$t_{\min }-\quad$ Minimum shell thickness anywhere along the height of the tank shell $\left(\mathrm{H}^{\prime}\right)$, usually at the top of the tank [in.]

$t_{s} \quad-\quad$ Minimum thickness of the tank shell in the lowest $10 \%$ of the shell height $\left(\mathrm{H}^{\prime}\right)$ [in.] 


\section{Table 9.1.1-2 (Continued)}

\section{Symbol_Description [Units]}

$t_{w} \quad-\quad$ Thickness of leg of weld [in.]

$t^{\prime} \quad$ - Equivalent shell thickness having the same cross-sectional area as the anchor bolts [in.]

$\mathrm{V}_{\mathrm{s}} \quad$ - Average shear wave velocity of soil for tanks founded at grade [ft/sec]

W - Weight of fluid contained in tank [lbf]

$W_{t} \quad-\quad$ Weight of tank without fluid [lbf]

$\mathrm{W}_{\mathrm{w}} \quad$ - Average shear load on weld connecting anchor bolt chair to tank shell per unit length of weld (i.e., total shear load on chair divided by total length of chair/shell weld) [lbf/in. of weld]

Z - Tank shell stress reduction factor [dimensionless]

ZPA - Zero period acceleration $[\mathrm{g}]$

B - Percentage damping [\%]

$\gamma \quad$ - Buckling coefficient $\left[1-0.73\left(1-\mathrm{e}^{-\phi}\right)\right]$ [dimensionless]

$\gamma_{f} \quad-\quad$ Weight density of fluid in tank $\left[\mathrm{lbf} / \mathrm{in}^{3}\right]$

$\Delta \gamma \quad-\quad$ Increase factor for internal pressure; given in Figure 9.1.1-11

$\sigma \quad-\quad$ Stress at a point [psi]

$\sigma_{c} \quad-\quad$ Stress at which shell buckles [psi]

$\sigma_{\mathrm{pe}} \quad-\quad$ Stress at which shell buckles in elephant-foot pattern [psi]

$\sigma_{\mathrm{pd}} \quad$ - Stress at which shell buckles in diamond-shape pattern [psi]

$\sigma_{\mathrm{y}} \quad$ - Yield strength of tank shell material [psi]

$\phi \quad-\quad$ Buckling coefficient $\left[(1 / 16)\left(\mathrm{R} / \mathrm{t}_{\mathrm{s}}\right)^{1 / 2}\right]$ [dimensionless] 
Table 9.1.1-3 Fluid-Structure Impulsive Mode Frequencies $\left(F_{p}, \mathrm{~Hz}\right)$ for Vertical Carbon Steel Tanks Containing Water (Reference 42)

(Table 7-3 of SQUG GIP, Ref. 1)

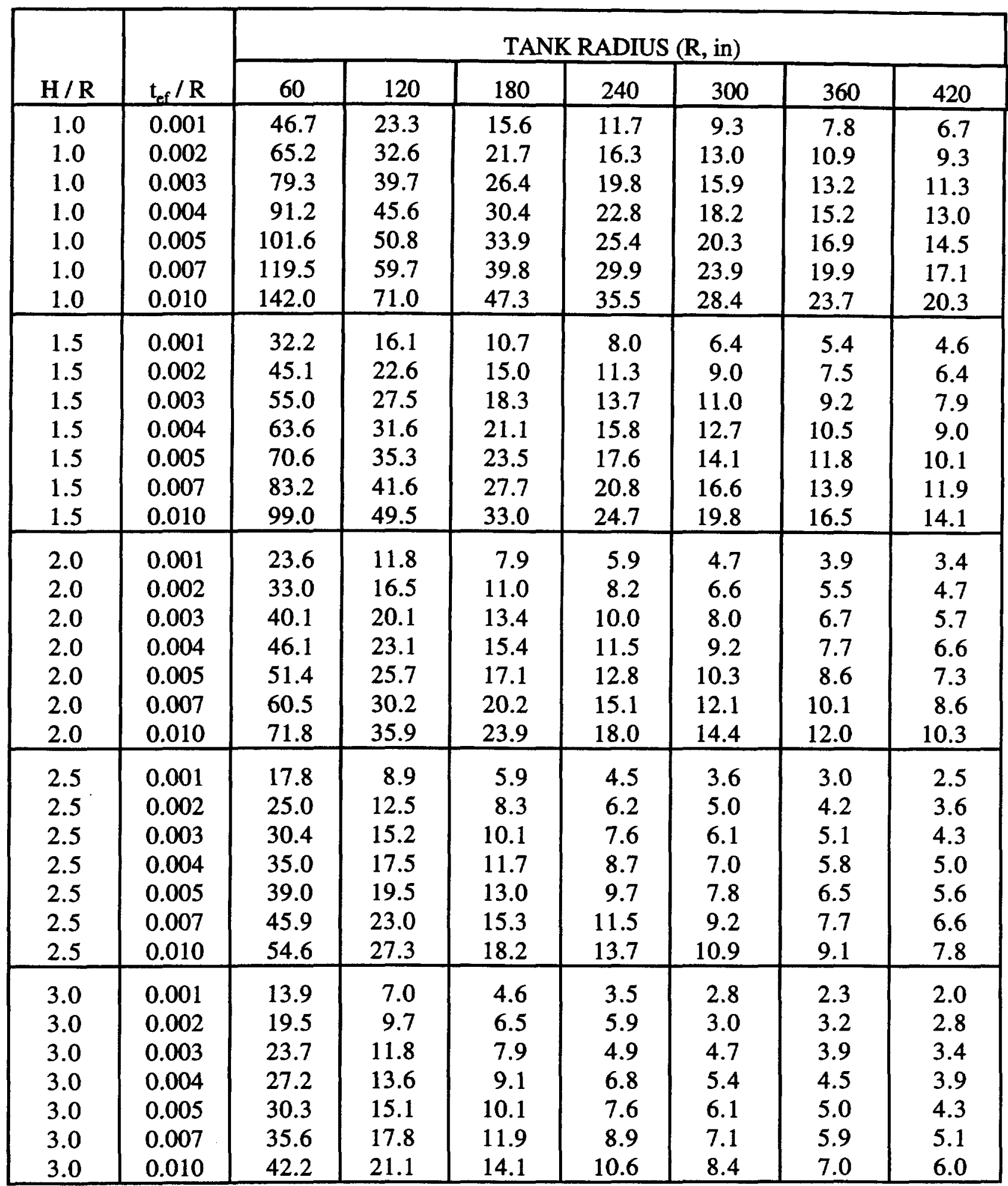


Table 9.1.1-3 (Continued)

\begin{tabular}{|c|c|c|c|c|c|c|c|c|}
\hline & & \multicolumn{7}{|c|}{ TANK RADIUS (R, in) } \\
\cline { 3 - 9 } $\mathrm{H} / \mathrm{R}$ & $\mathrm{t}_{\text {ef }} / \mathrm{R}$ & 60 & 120 & 180 & 240 & 300 & 360 & 420 \\
\hline 3.5 & 0.001 & 11.2 & 5.6 & 3.7 & 2.8 & 2.2 & 1.9 & 1.6 \\
3.5 & 0.002 & 15.5 & 7.8 & 5.2 & 3.9 & 3.1 & 2.6 & 2.2 \\
3.5 & 0.003 & 18.8 & 9.4 & 6.3 & 4.7 & 3.8 & 3.1 & 2.7 \\
3.5 & 0.004 & 2.16 & 10.8 & 7.2 & 5.4 & 4.3 & 3.6 & 3.1 \\
3.5 & 0.005 & 24.0 & 12.0 & 8.0 & 6.0 & 4.8 & 4.0 & 3.4 \\
3.5 & 0.007 & 28.2 & 14.1 & 9.4 & 7.0 & 5.6 & 4.7 & 4.0 \\
3.5 & 0.010 & 33.4 & 16.7 & 11.1 & 8.3 & 6.7 & 5.6 & 4.8 \\
\hline 4.0 & 0.001 & 9.1 & 4.6 & 3.0 & 2.3 & 1.8 & 1.5 & 1.3 \\
4.0 & 0.002 & 12.6 & 6.3 & 4.2 & 3.2 & 2.5 & 2.1 & 1.8 \\
4.0 & 0.003 & 15.2 & 7.6 & 5.1 & 3.8 & 3.0 & 2.5 & 2.2 \\
4.0 & 0.004 & 17.4 & 8.7 & 5.8 & 4.4 & 3.5 & 2.9 & 2.5 \\
4.0 & 0.005 & 19.3 & 9.7 & 6.4 & 4.8 & 3.9 & 3.2 & 2.8 \\
4.0 & 0.007 & 22.6 & 11.3 & 7.5 & 5.7 & 4.5 & 3.8 & 3.2 \\
4.0 & 0.010 & 26.7 & 13.4 & 8.9 & 6.7 & 5.3 & 4.5 & 3.8 \\
\hline 4.5 & 0.001 & 7.5 & 3.8 & 2.5 & 1.9 & 1.5 & 1.3 & 1.1 \\
4.5 & 0.002 & 10.3 & 5.2 & 3.4 & 2.6 & 2.1 & 1.7 & 1.5 \\
4.5 & 0.003 & 12.4 & 6.2 & 4.1 & 3.1 & 2.5 & 2.1 & 1.8 \\
4.5 & 0.004 & 14.2 & 7.1 & 4.7 & 3.5 & 2.8 & 2.4 & 2.0 \\
4.5 & 0.005 & 15.7 & 7.9 & 5.2 & 3.9 & 3.1 & 2.6 & 2.2 \\
4.5 & 0.007 & 18.3 & 9.2 & 6.1 & 4.6 & 3.7 & 3.1 & 2.6 \\
4.5 & 0.010 & 21.6 & 10.8 & 7.2 & 5.4 & 4.3 & 3.6 & 3.1 \\
\hline 5.0 & 0.001 & 6.2 & 3.1 & 2.1 & 1.6 & 1.2 & 1.0 & 0.9 \\
5.0 & 0.002 & 8.5 & 4.2 & 2.8 & 2.1 & 1.7 & 1.4 & 1.2 \\
5.0 & 0.003 & 10.2 & 5.1 & 3.4 & 2.5 & 2.0 & 1.7 & 1.5 \\
5.0 & 0.004 & 11.6 & 5.8 & 3.9 & 2.9 & 2.3 & 1.9 & 1.7 \\
5.0 & 0.005 & 12.8 & 6.4 & 4.3 & 3.2 & 2.6 & 2.1 & 1.8 \\
5.0 & 0.007 & 14.9 & 7.4 & 5.0 & 3.7 & 3.0 & 2.5 & 2.1 \\
5.0 & 0.010 & 17.5 & 8.7 & 5.8 & 4.4 & 3.5 & 2.9 & 2.5 \\
\hline
\end{tabular}


Table 9.1.1-4 Base Overturning Moment Capacity Elastic Limit Values (Reference 42) (Table 7-4 of SQUG GIP, Ref. 1)

\begin{tabular}{|c|c|c|}
\hline$c^{\prime}$ & $\left(\frac{\sigma_{c}}{F_{b}}\right)\left(\frac{h_{c}}{h_{b}}\right)$ & $M_{\text {cap }}^{\prime}$ \\
\hline 0.01 & 0.052 & 0.0231 \\
\hline 0.02 & 0.081 & 0.0454 \\
\hline 0.05 & 0.147 & 0.1092 \\
\hline 0.10 & 0.230 & 0.2087 \\
\hline 0.15 & 0.300 & 0.3045 \\
\hline 0.20 & 0.358 & 0.3932 \\
\hline 0.40 & 0.560 & 0.7271 \\
\hline
\end{tabular}

Table 9.1.1-5 Slosh Height of Water $\left(h_{s}^{\prime}\right.$, in)in Vertical Tanks for 1G Lateral Acceleration Reference 42) (Table 7.4 of SQUG GIP, Ref. 1)

\begin{tabular}{|c|c|c|c|c|c|c|c|}
\hline & \multicolumn{7}{|c|}{ TANK RADIUS (R, in.) } \\
\cline { 2 - 8 } H/R & 60 & 120 & 180 & 240 & 300 & 360 & 420 \\
\hline 1.0 & 39.0 & 60.2 & 78.7 & 95.5 & 111.5 & 126.7 & 141.4 \\
\hline 1.5 & 39.6 & 61.2 & 79.8 & 96.8 & 112.9 & 128.3 & 143.2 \\
\hline 2.0 & 39.7 & 61.3 & 79.9 & 97.1 & 113.2 & 128.5 & 143.4 \\
\hline 2.5 & 39.7 & 61.3 & 80.0 & 97.1 & 113.2 & 128.6 & 143.4 \\
\hline 3.0 & 39.7 & 61.3 & 80.0 & 97.1 & 113.2 & 128.6 & 143.4 \\
\hline 3.5 & 39.7 & 61.3 & 80.0 & 97.1 & 113.2 & 128.6 & 143.4 \\
\hline 4.0 & 39.7 & 61.3 & 80.0 & 97.1 & 113.2 & 128.6 & 143.4 \\
\hline 4.5 & 39.7 & 61.3 & 80.0 & 97.1 & 113.2 & 128.6 & 143.4 \\
\hline 5.0 & 39.7 & 61.3 & 80.0 & 97.1 & 113.2 & 128.6 & 143.4 \\
\hline
\end{tabular}




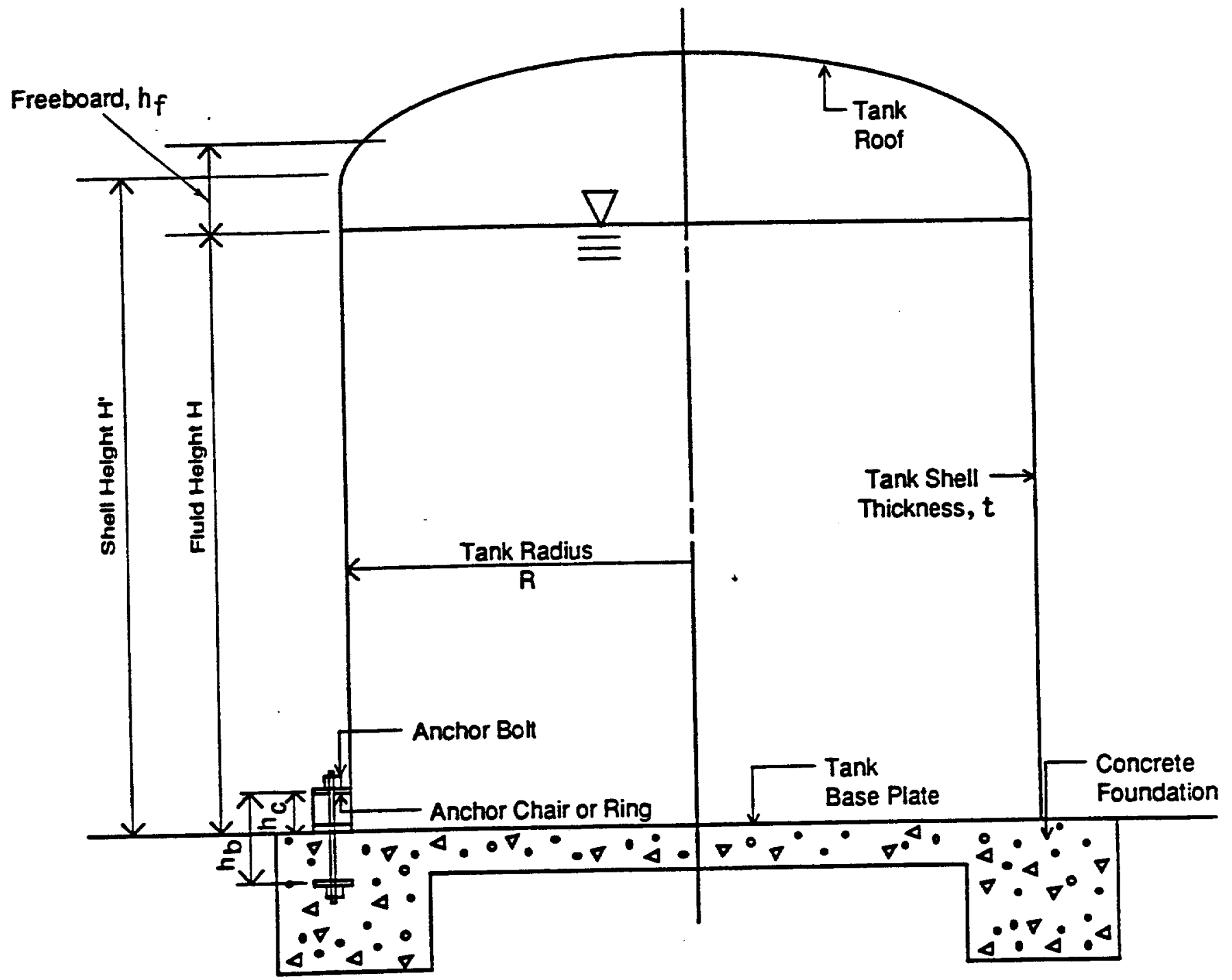

Figure 9.1.1-1 Large Vertical Tank (Reference 42) (Figure 7-1 of SQUG GIP, Reference 1) 


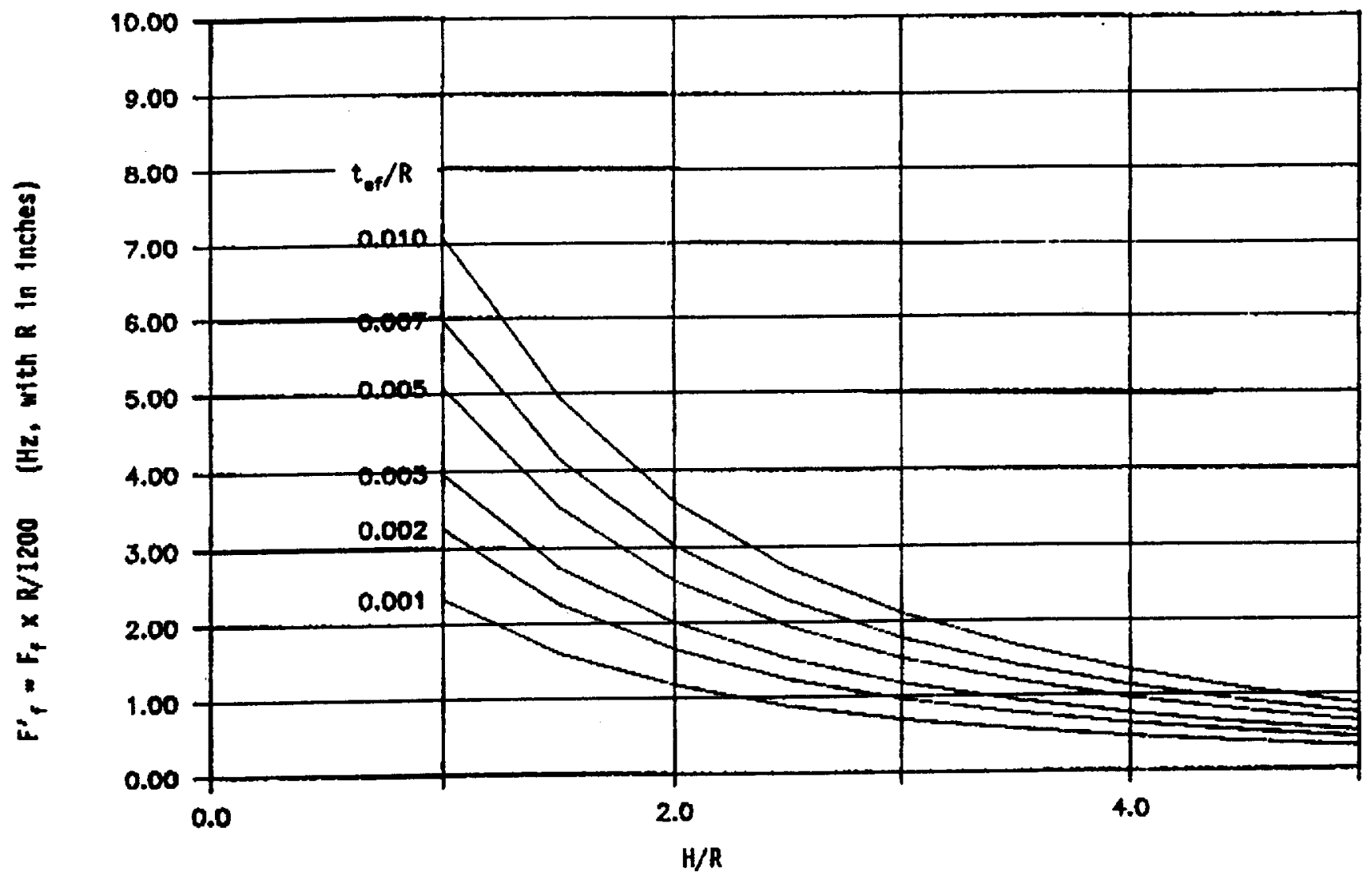

Figure 9.1.1-2 Fluid-Structure Impulsive Mode Frequency Coefficient for Vertical Carbon Steel Tanks Containing Water (Reference 42) 


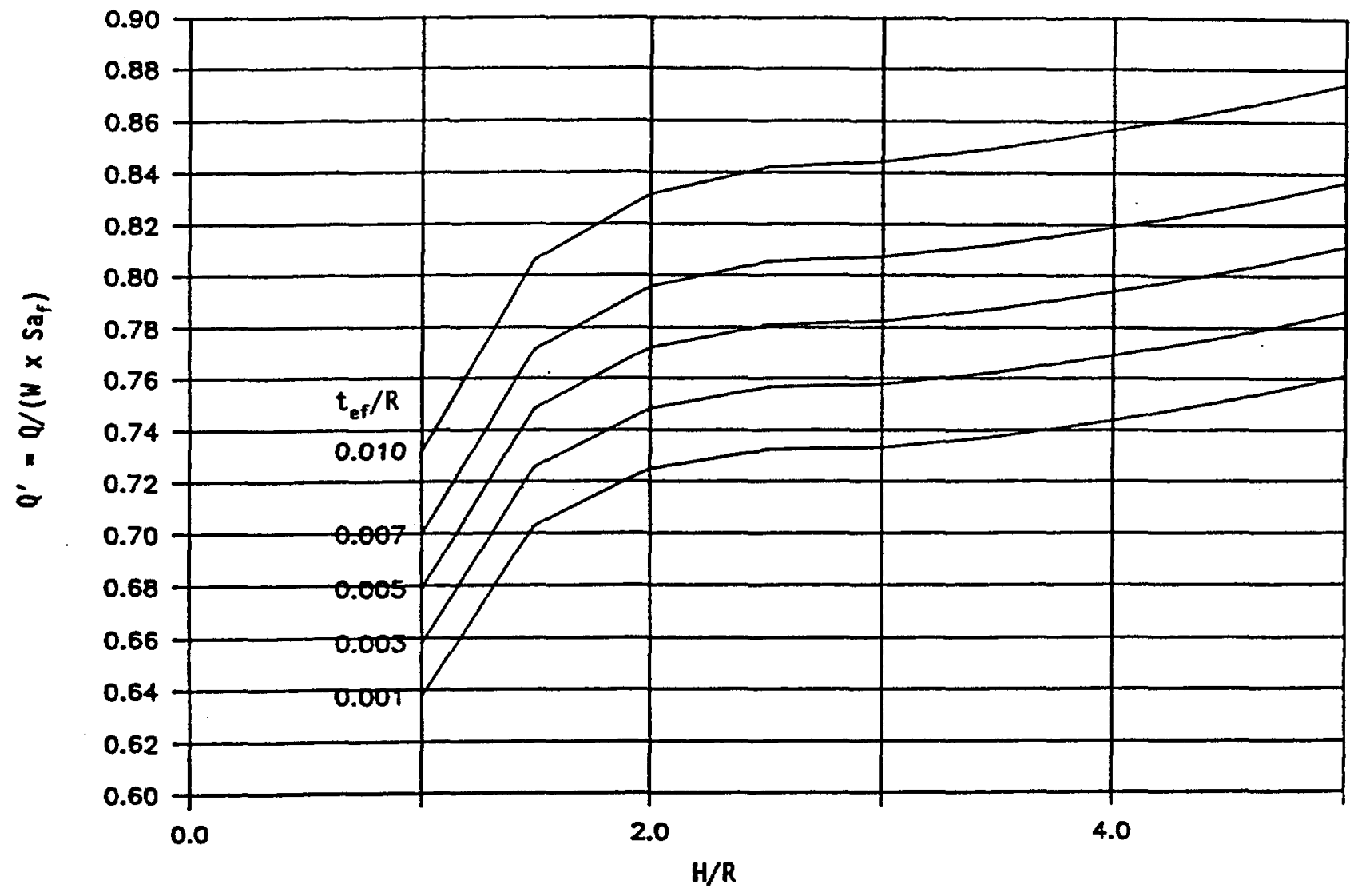

Figure 9.1.1-3 Base Shear Load Coefficient for Vertical Tanks (Reference 42) (Figure 7-3 of SQUG GIP, Reference 1) 


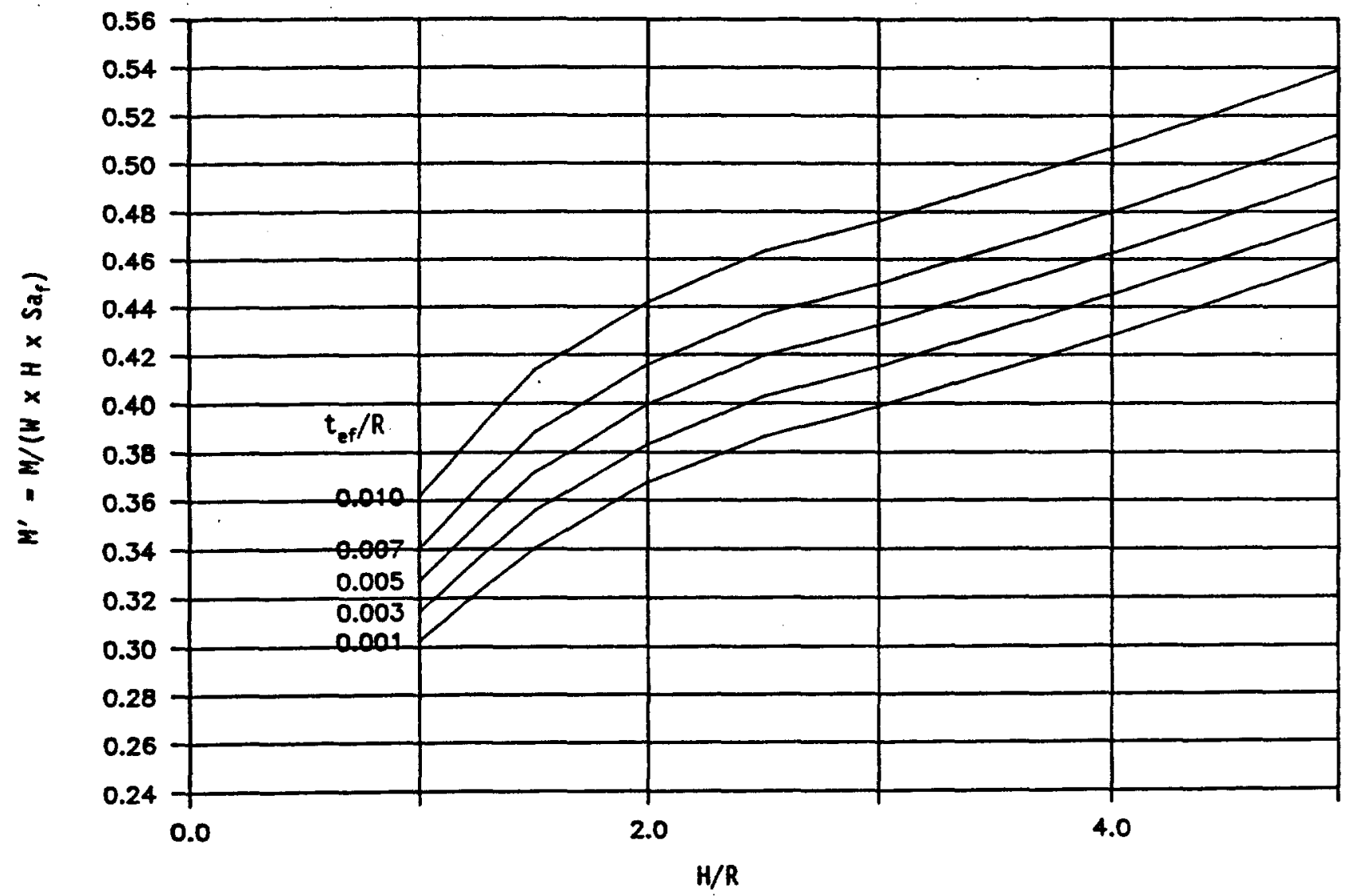

Figure 9.1.1-4 Base Overturning Moment Coefficient for Vertical Tanks (Reference 42) (Figure 7-4 of SQUG GIP, Reference 1) 


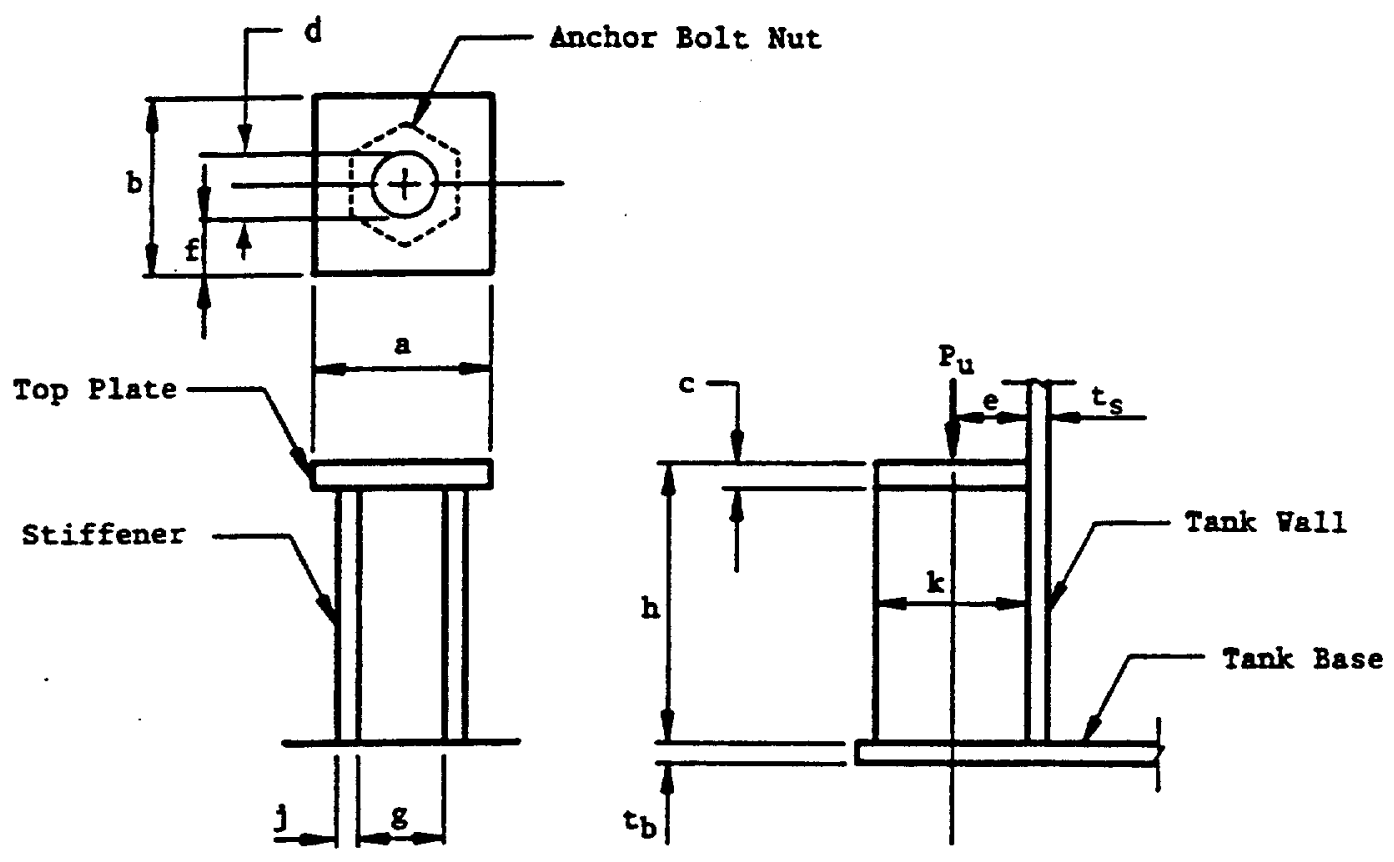
(a) Typical Plan and
(b) Side View

Figure 9.1.1-5 Typical Anchor Bolt Chair (Reference 42) (Figure 7-5 of SQUG GIP, Reference 1)

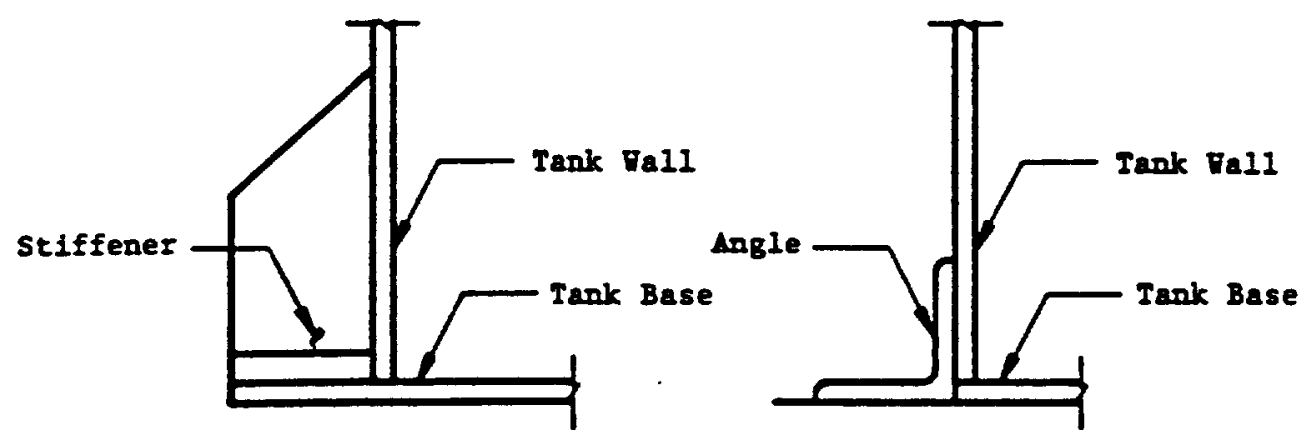

(a) Side View

(b) Angle Chair

Side View

Figure 9.1.1-6 Alternate Anchor Bolt Chair (Reference 42) (Figure 7-6 of SQUG GIP, Reference 1) 


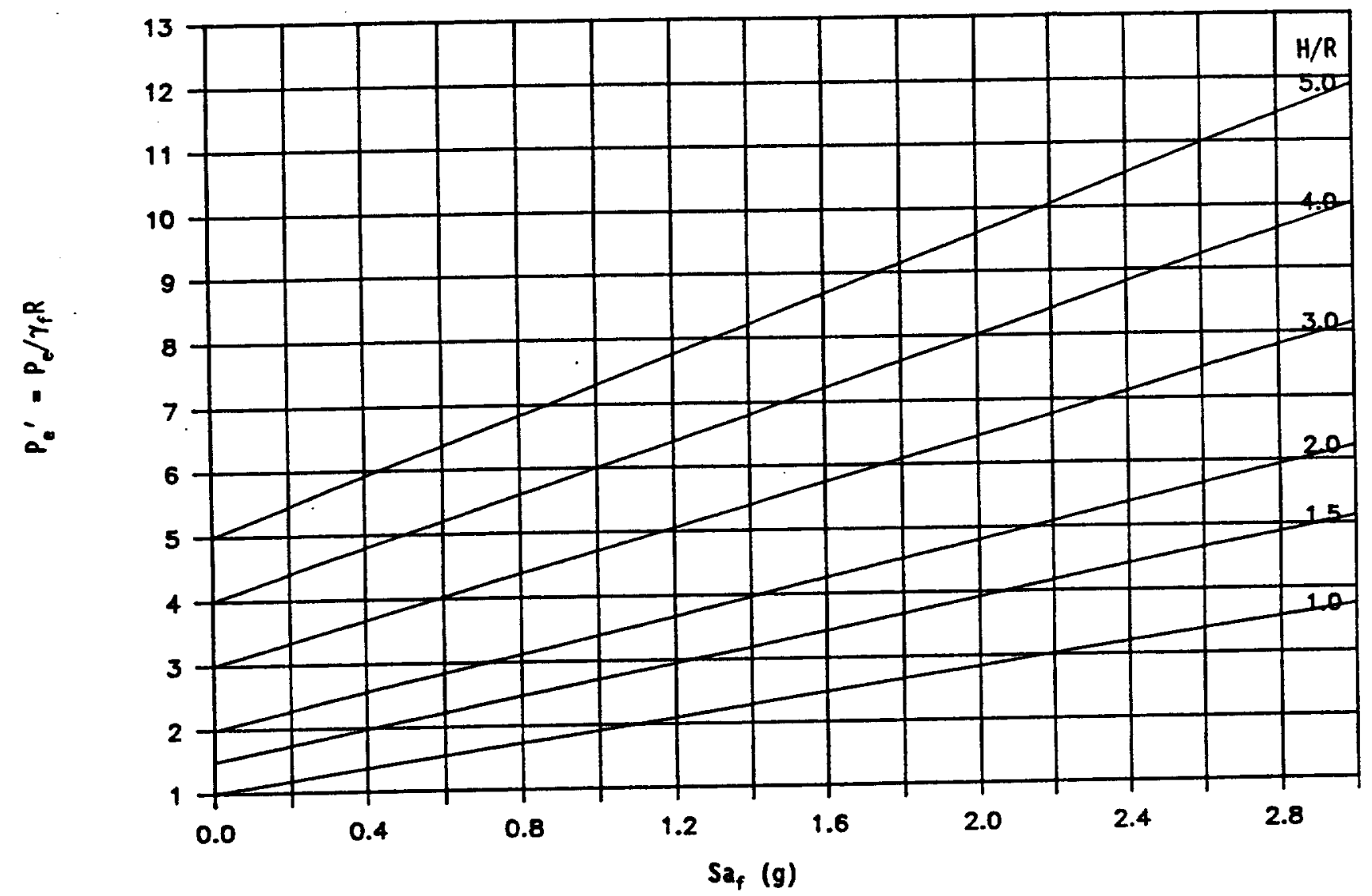

Figure 9.1.1-7 Pressure Coefficient for Elephant-Foot Buckling of Vertical Tanks (Reference 42) (Figure 7-7 of SQUG GIP, Reference 1) 


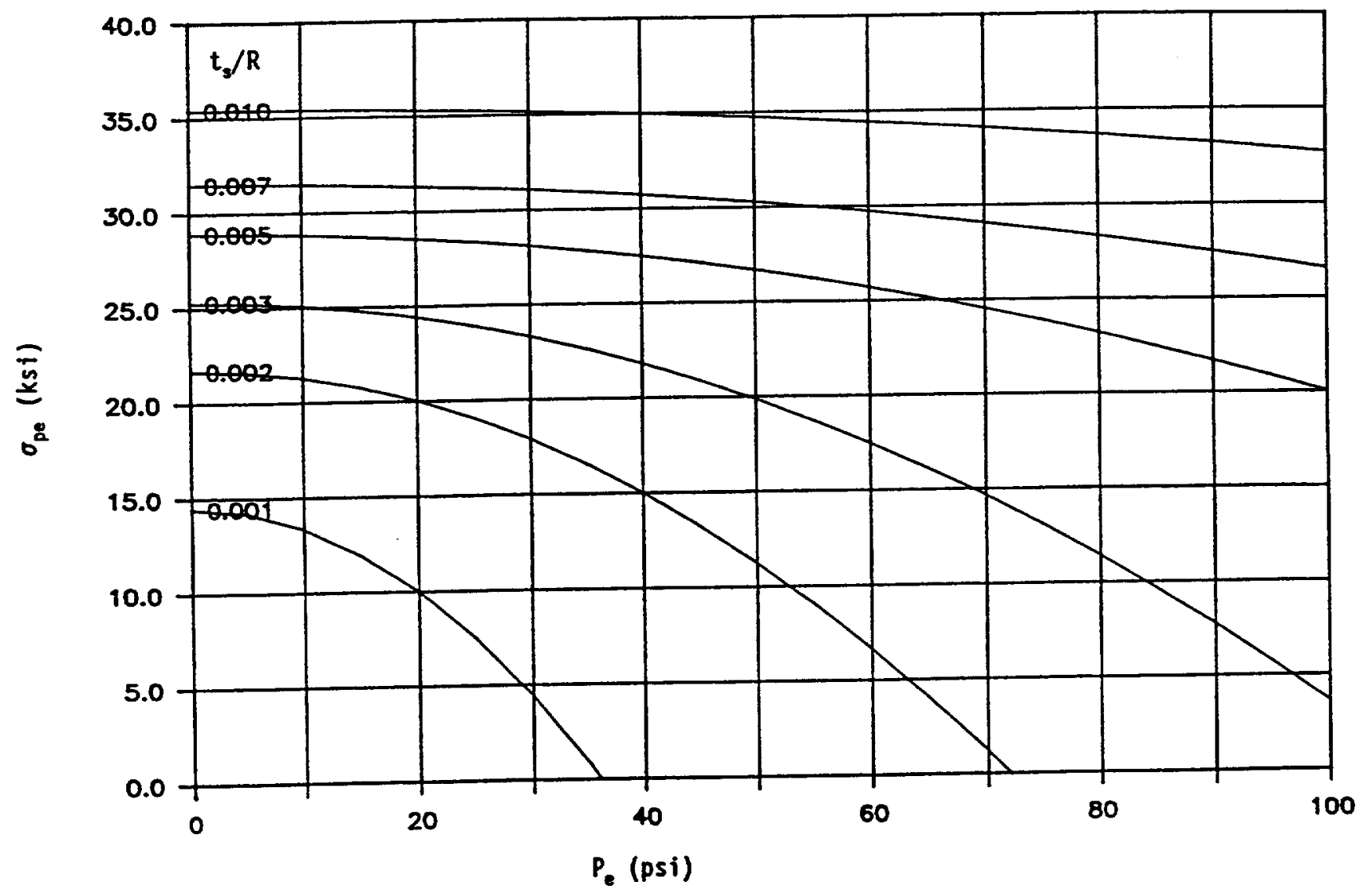

Figure 9.1.1-8 Compressive Axial Stress Capacity for Vertical Tanks, Elephant-Foot Bucking (Steel, E $=30,000$ psi, $\sigma_{y}=36,000$ psi) (Reference 42) (Figure 7-8 of SQUG GIP, Reference 1) 


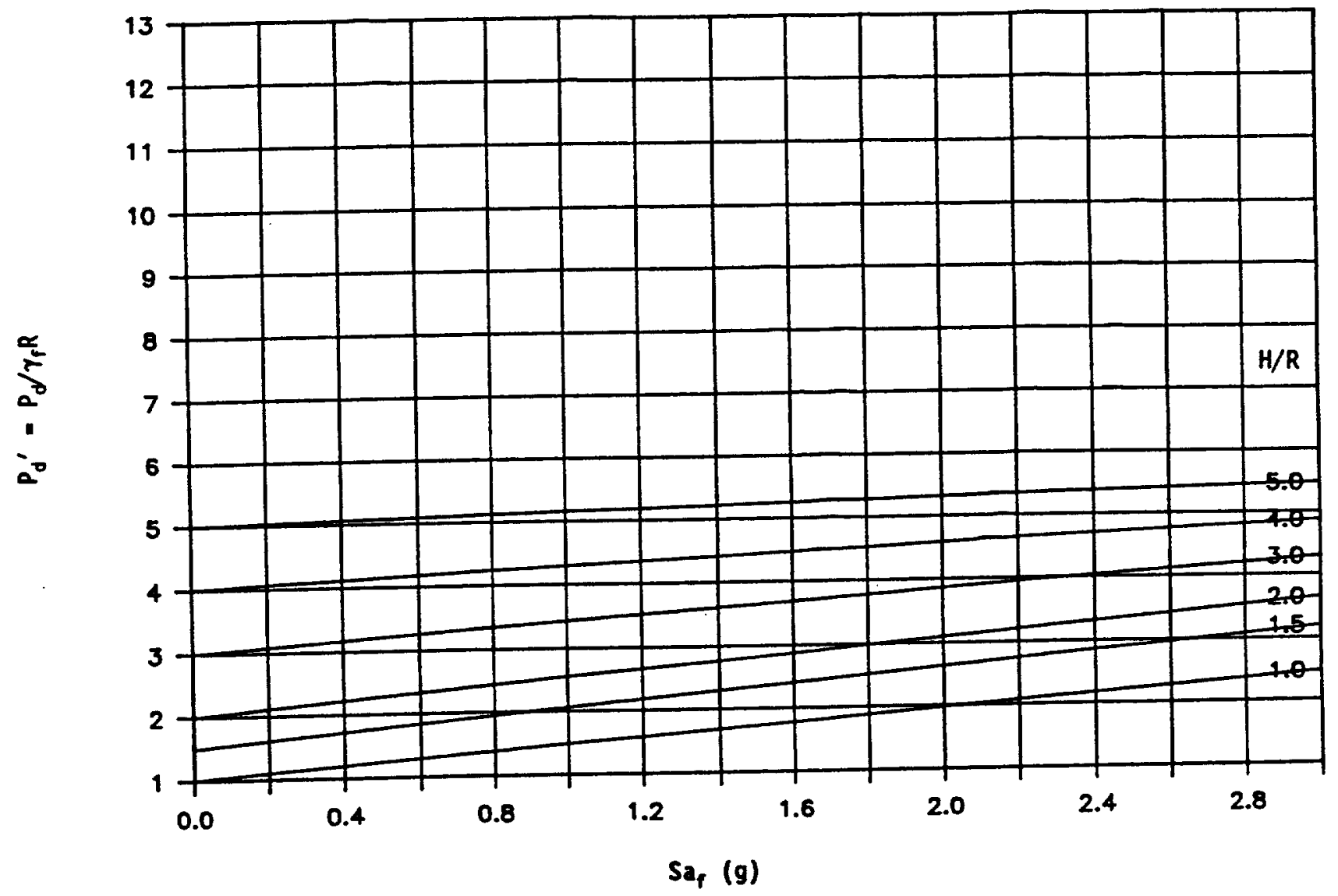

Figure 9.1.1-9 Pressure Coefficient for Diamond-Shape Buckling of Vertical Tanks (Reference 42) (Figure 7-9 of SQUG GIP, Reference 1) 


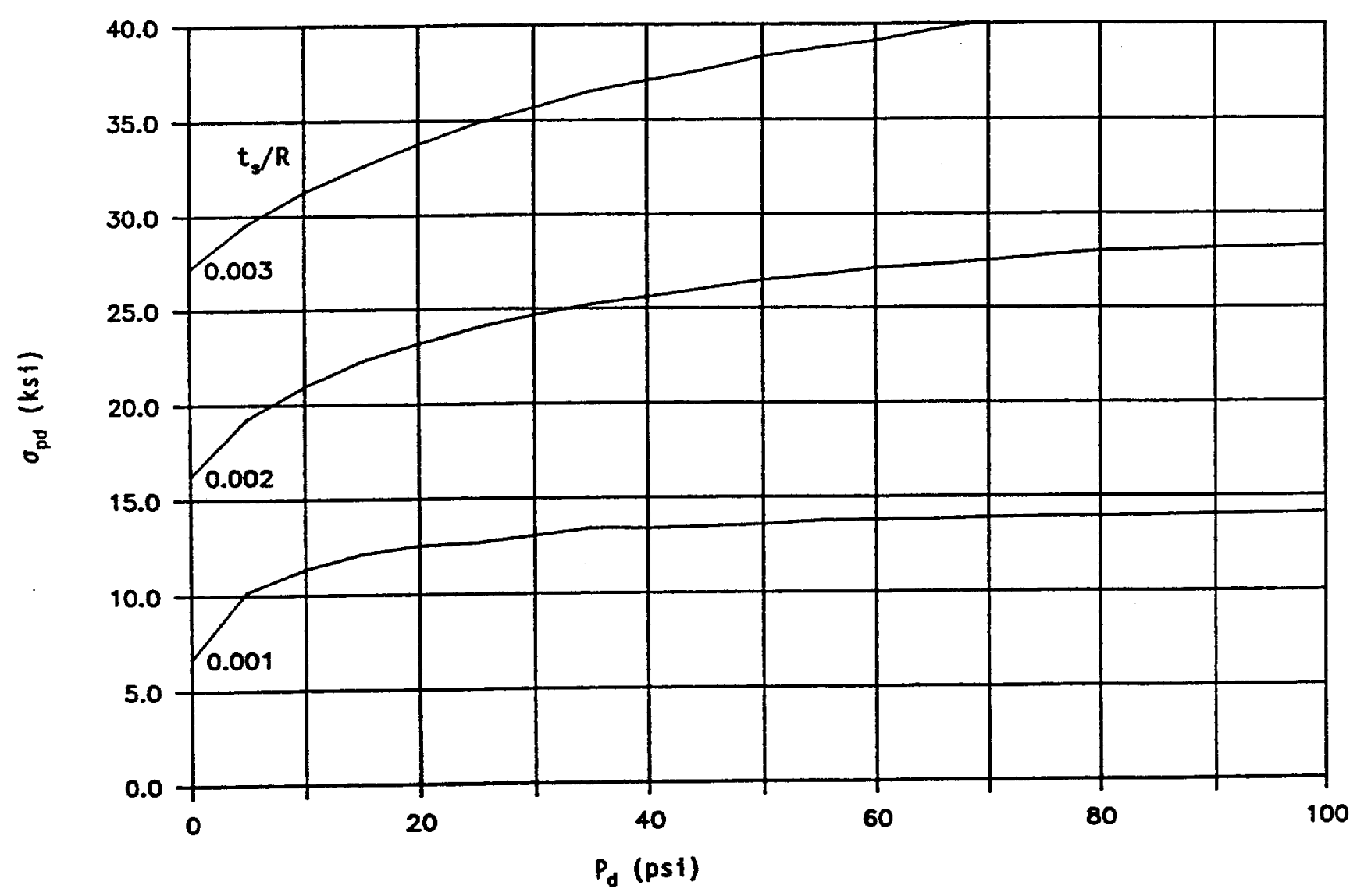

Figure 9.1.1-10 Compressive Axial Stress Capacity for Vertical Tanks, DiamondShape Buckling (Steel, E = 30,000 psi) (Reference 42) (Figure 7-10 of SQUG GIP, Reference 1) 


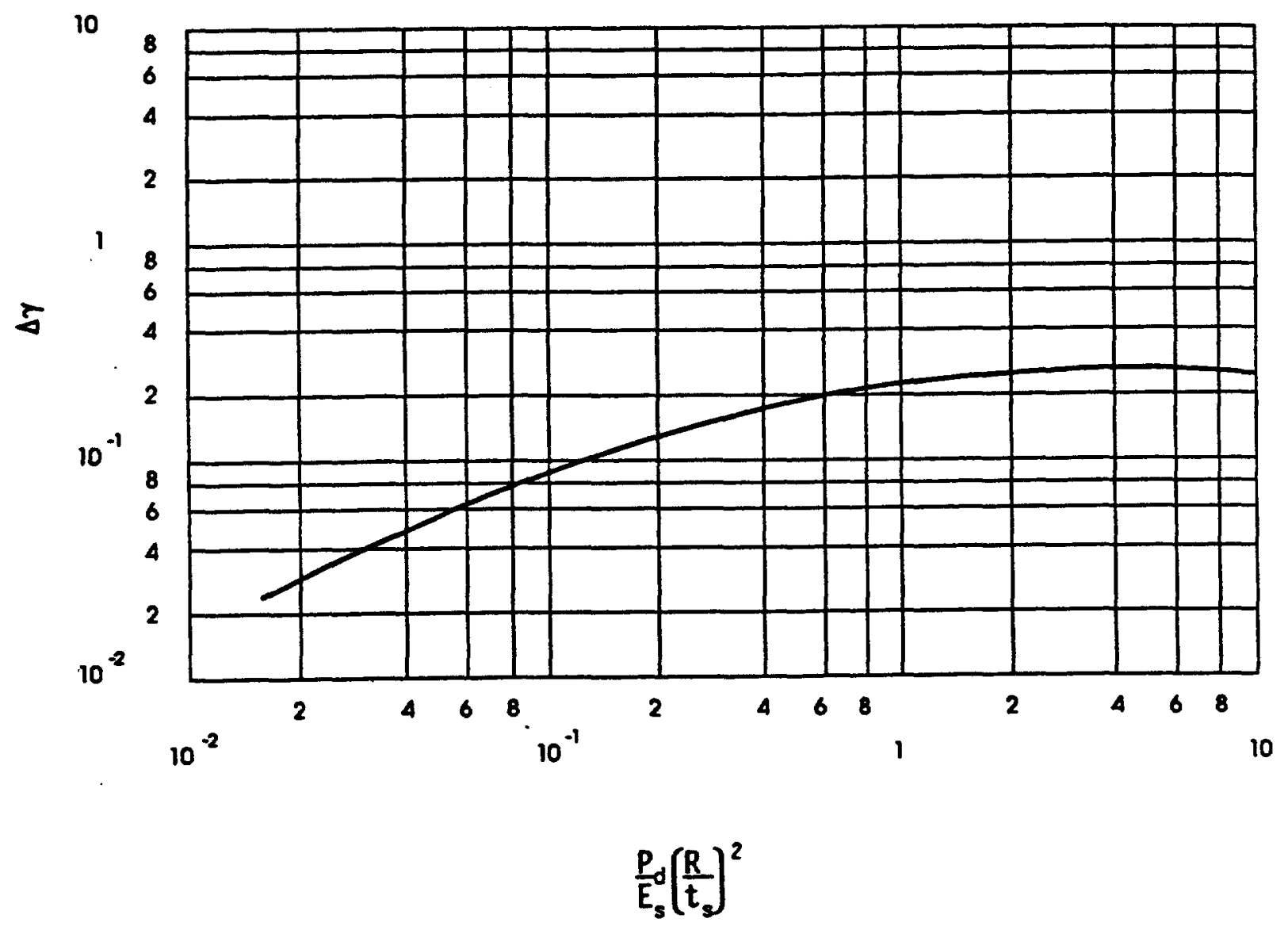

Figure 9.1.1-11 Increase Factor $\Delta \gamma$ for Diamond-Shape Buckling (Reference 42) (Figure 7-11 of SQUG GIP, Reference 1) 


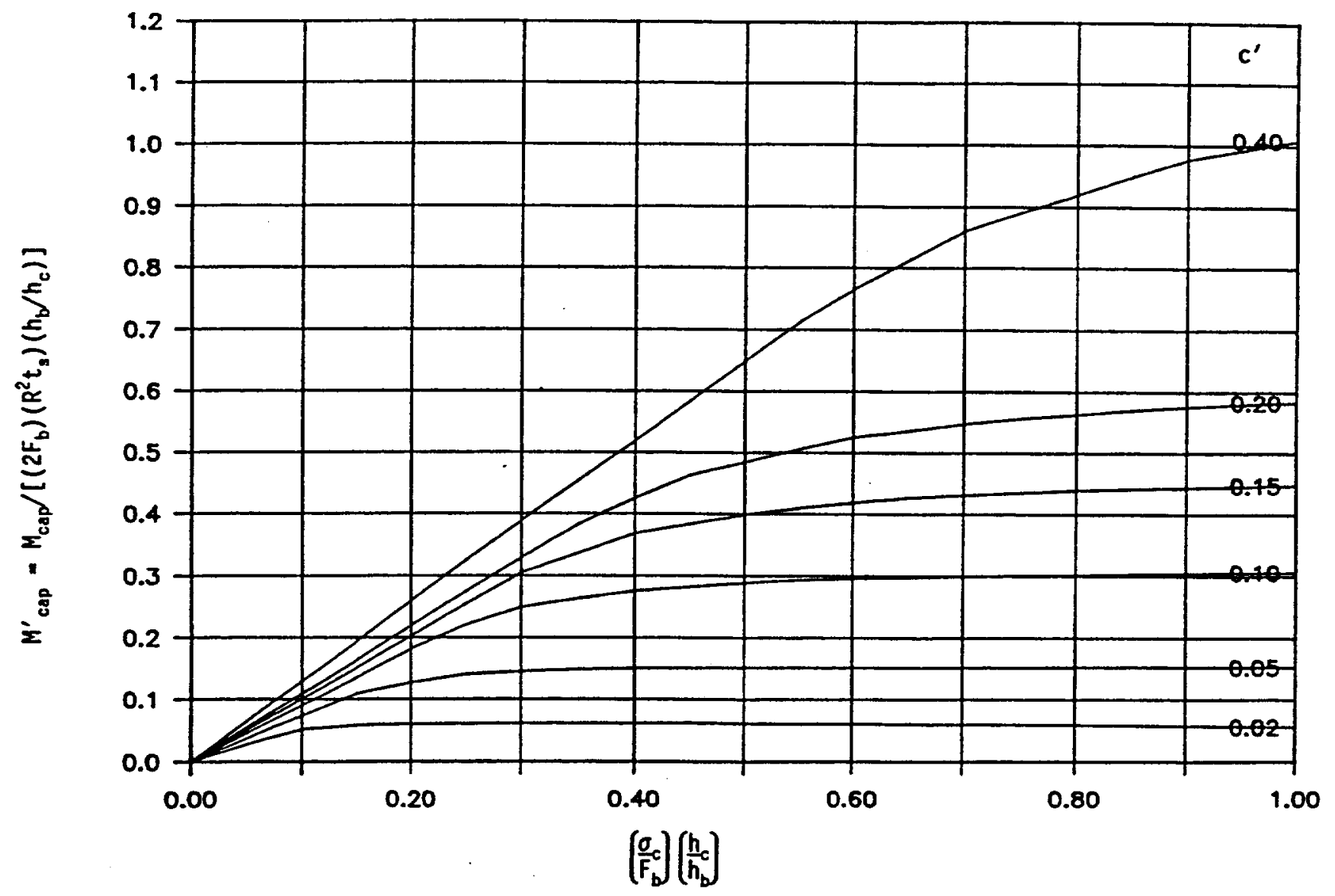

Figure 9.1.1-12 Base Overturning Moment Capacity Coefficient for Vertical Tanks (Reference 42) (Figure 7-12 of SQUG GIP, Reference 1) 


\subsubsection{HORIZONTAL TANKS AND HEAT EXCHANGERS}

\subsubsection{Introduction and Scope of Horizontal Tanks and Heat Exchangers ${ }^{14}$}

This section describes the guidelines which should be used for evaluating the seismic adequacy of those horizontal tanks and heat exchangers which are listed in the SEL as identified in Chapter 4. These guidelines are intended only for use on existing horizontal tanks and heat exchangers and are not to be used for new installations. The guidelines contained in this section are based on Reference 42. Note, however, that to provide consistency with the remainder of the DOE Seismic Evaluation Procedure some of the nomenclature and symbols used in this section are slightly different than those used in Reference 42.

The screening evaluations described in this section for evaluating the seismic adequacy of horizontal tanks and heat exchangers cover those features of horizontal tanks and heat exchangers which experience has shown can be vulnerable to seismic loadings. These evaluations include the following features:

- Check that the anchor bolts and their embedments have adequate strength against breakage and pullout.

- Check that the anchorage connection between the anchor bolts and the tank shell (e.g., saddles, legs, chairs, etc.) have adequate strength.

Two SCEs (as defined in Chapter 3) should review these evaluations to determine that they meet the intent of these guidelines. This review should include a field inspection of the tank, the anchorage connections, and the anchor bolt installation against the guidelines described in this section and Chapter 6.

The derivation and technical justification for the guidelines in this section were developed specifically for horizontal cylindrical tanks and heat exchangers with support saddles made of plates. The types of loadings and analysis methods described in this section are considered to be appropriate for these types of horizontal tanks and heat exchangers; however, a generic procedure cannot cover all the possible design variations. Therefore, it is the responsibility of the SCEs to assess the seismic adequacy of other design features not specifically covered in this section. For example, the guidelines in this section do not specifically include a check of the stress in the weld connecting the steel support saddles to the shell of a horizontal tank or heat exchanger since this weld is typically very strong compared to other parts of the saddle and its anchorage. However, if the $S R T$ finds there to be very little weld attaching these parts, then this weld should be evaluated for its seismic adequacy.

Other types of horizontal tanks and heat exchangers which are not specifically covered by the guidelines in this section, should be evaluated by the SCEs using an approach similar to that described in this section. Likewise, facilities may use existing analyses which determine the seismic adequacy of its horizontal tanks and heat exchangers in lieu of the DOE Seismic Evaluation Procedure, provided the SCEs determine that these other analyses address the same type of loading as the DOE Seismic Evaluation Procedure and the same failure modes.

The screening guidelines described in this section were developed to further simplify the equivalent static analysis procedure for smaller horizontal tanks. To accomplish this, it was necessary to make certain simplifying assumptions and to limit the range of applicability of the guidelines. Many horizontal tanks and heat exchangers in DOE facilities fall within the restrictions and range of values for which the screening guidelines were developed. However, for those horizontal tanks

14 Sections 7.2, 7.4, and 7.4.1 of SQUG GIP (Ref. 1) 
and heat exchangers which are not covered by, or do not pass the screening guidelines, it may be possible to perform tank-specific evaluations, using the approach described in Reference 42 , to evaluate the seismic adequacy of the horizontal tank or heat exchanger.

The screening guidelines described in this section are based on using $4 \%$ damped ground or floor response spectra (see Section 5.2) for overturning moment and shear loadings on the tanks. If $4 \%$ damped response spectra are not directly available, then they may be estimated by scaling from spectra at other damping values using the standard technique described in Appendix $A$ of Reference 19 or Section 6.4.2.

This section describes the scope of horizontal tanks and heat exchangers and range of parameters which are covered by the screening guidelines and the analysis procedure for determining the seismic demand on, and the seismic capacity of horizontal tanks and heat exchangers including their supports and anchorage.

The types of tanks covered by the screening guidelines in this section are cylindrical steel tanks and heat exchangers whose axes of symmetry are horizontal and are supported on their curved bottom by steel saddle plates. These types of tanks will be called "horizontal tanks" throughout this section. A typical horizontal tank on saddles is shown in Figure 9.1.2-1. (Note: All the figures and tables applicable to horizontal tanks are grouped together at the end of Section 9.1.2). The range of parameters and assumptions which are applicable when using the guidelines to evaluate horizontal tanks are listed in Table 9.1.2-1. The nomenclature and symbols used for horizontal tanks are listed in Table 9.1.2-2.

The screening guidelines are based on the assumption that the horizontal tanks are anchored to a stiff foundation which has adequate strength to resist the seismic loads applied to the tank. All the base plates under the saddles are assumed to have slotted anchor bolt holes in the longitudinal direction to permit thermal growth of the tank, except for the saddle at one end of the tank which is fixed. The saddles are assumed to be uniformly spaced a distance $S$ apart, with the two ends of the tank overhanging the end saddles a maximum distance of $S / 2$. These assumptions and the range of parameters given in Table 9.1.2-1 have been selected to cover the majority of horizontal tanks and heat exchangers in DOE facilities.

\subsubsection{Seismic Demand/Capacity of Horizontal Tanks ${ }^{15}$}

A simple, equivalent static method is used to determine the seismic demand on and capacity of the anchorage and the supports for horizontal tanks. This approach is similar to the seismic demand/capacity evaluations described in Chapter 6 for other types of equipment requiring anchorage evaluation (switchgear, transformers, pumps, battery chargers, etc.). Note that it is not necessary to evaluate the seismic adequacy of the shell of horizontal tanks or the shell-to-support welds since these items are normally rugged enough to withstand the loads which can be transmitted to them from the anchor bolts and support saddles.

The screening guidelines contained in this section specifically address only the seismic loads due to the inertial response of horizontal tanks. If, during the Screening Evaluation and Walkdown of a tank, the SCEs determine that the imposed nozzle loads due to the seismic response of attached piping may be significant, then these loads should be included in the seismic demand applied to the anchorage and supports of the tank. There is some discussion provided on this subject for piping loads applied to horizontal pumps in Section 8.2.3 HP/RS Caveat 4; this discussion is also applicable to horizontal tank evaluations.

15 Section 7.4.2 of SQUG GIP (Ref. 1) 
The guidelines in this section are in the form of tables, charts, and a few simple calculations to determine the seismic capacity of horizontal tanks in terms of the peak acceleration the tanks can withstand. This peak acceleration capacity is assumed to be composed of a uniform acceleration capacity, $\lambda$, in the two horizontal directions, and $2 / 3 \lambda$ in the vertical direction. The screening guidelines include the effect of combining the three directions of acceleration by the square-root-ofthe-sum-of-squares (SRSS) method. The seismic acceleration capacity, $\lambda$, is then compared with either the ZPA or the peak of the 4\% damped, horizontal floor response spectrum (see Section 5.2), depending on whether: (1) the horizontal tank is rigid in the vertical or traverse direction (i.e., whether the tank shell acts as a rigid or flexible beam between the saddles); or (2) the horizontal tank and its support system is rigid in the longitudinal direction.

The seismic adequacy of the following critical parts of horizontal tanks are evaluated in these screening guidelines:

- Anchor bolts and their concrete embedment

- Base plate bending

- Base plate-to-saddle weld

- Saddle bending and compression

Step-By-Step Procedure for Horizontal Tanks

Step 1 - Determine the following input data. See Figure 9.1.2-1 for location of some of these dimensions.

Tank: $\quad$ D $\quad$ (Diameter of tank) [ft.]

L (Length of tank) [ft.]

$\mathrm{t} \quad$ (Thickness of tank shell) [in.]

$\mathrm{W}_{\mathrm{tf}} \quad$ (Weight of tank plus fluid) [lbf]

$\gamma_{\mathrm{t}}$ or $\gamma_{\mathrm{h}} \quad$ (Weight density of horizontal tank or heat exchanger including fluid) [lbf/ $\mathrm{ft}^{3}$ ]

$\mathrm{H}_{\mathrm{cg}} \quad$ (Height of center-of-gravity of tank and fluid above the floor where the tank is anchored) [ft.]

Saddles: $\quad \mathrm{S} \quad$ (Spacing between support saddles) [ft.]

h (Height of saddle plate from the bottom of the tank to the base plate) [in.]

G (Shear modulus of saddle plate and stiffener material) [psi]

$\mathrm{E}$ (Elastic modulus of saddle plate and stiffener material) [psi]

NS (Number of saddles) 
Base Plate: $t_{b}$

$\mathbf{f}_{\mathbf{y}}$

$\mathbf{t}_{\mathbf{w}}$

$e_{s}$

Bolts: NL

$D^{\prime}$

NB (Number of anchor bolts at each bolt location)

d (Diameter of anchor bolt) [in.]

(Thickness of base plate under saddle) [in.]

(Minimum specified yield strength of saddle base plate) [psi]

(Thickness of leg of weld between saddle and base plate) [in.]

(Eccentricity from the anchor bolt centerline to the vertical saddle plate) [in.]

NL (Number of bolt locations on each saddle)

(Distance between extreme anchor bolts in base plate of saddle) [ft.]

Loading: $\quad$ Floor response spectrum at 4\% damping (see Section 5.2)

Confirm that the parameters and values determined in this step are within the range of applicable parameters given in Table 9.1.2-1. If they are, then the procedure given in this section is applicable to the subject horizontal tank; proceed to Step 2. If the horizontal tank does not meet this guideline, classify the tank as an outlier and proceed to Section 9.1.2.3.

Step 2 - Determine the anchor bolt tension and shear load allowables from Section 6.3, accounting for the effects of embedment, spacing, edge distance, and cracking in concrete, as discussed in Section 6.3.

$$
\begin{array}{lll}
\mathrm{P}_{\mathrm{u}}{ }^{\prime} & \text { [lbf }] & \text { (from Section 6.3) } \\
\mathrm{V}_{\mathrm{u}}{ }^{\prime} & \text { [lbf }] & \text { (from Section 6.3) }
\end{array}
$$

Step 3 - Determine the base plate bending strength reduction factor (RB). The width of the base plate that is stressed in bending is conservatively assumed to be equal to twice the distance between the centerline of the bolt and the vertical saddle plate; i.e., $2 \mathrm{e}_{\mathrm{s}}$. The strength reduction factor is determined by taking the ratio of the base plate yield strength $\left(f_{y}\right)$ over the maximum bending stress $(\sigma)$ :

$$
R B=\frac{f_{y}}{\sigma}=\frac{f_{y} t_{b}^{2}}{3 P_{u}^{\prime}}
$$

Step 4 - Determine the base plate weld strength reduction factor (RW). The length of weld assumed to carry the anchor bolt load is taken to be equal to twice the distance from the bolt centerline to the vertical saddle plate; i.e., $2 \mathrm{e}_{\mathrm{s}}$. The strength reduction factor is the ratio of the weld allowable strength $(30,600 \mathrm{psi})$ over the weld stress $(\sigma)$ :

$$
R W=\frac{30,600 \mathrm{psi}}{\sigma}=\frac{2 \sqrt{2} \mathrm{t}_{\mathrm{w}} \mathrm{e}_{\mathrm{s}}(30,600 \mathrm{psi})}{\mathrm{P}_{\mathrm{u}}^{\prime}}
$$


Step 5 - Determine the anchorage tension allowable using the strength reduction factors. The tension allowable anchorage load is based on the smaller of the strength reduction factors for base plate bending or base plate weld:

$$
P_{u}=P_{u}^{\prime} \times(\text { Smaller of: } R B \text { or } R W)[l b f]
$$

The shear allowable anchorage load is:

$$
\mathrm{V}_{\mathrm{u}}=\mathrm{V}_{\mathrm{u}}^{\prime}[\mathrm{lbf}]
$$

Step 6 - Calculate the following ratios and values:

$$
\begin{aligned}
& \alpha=\mathrm{P}_{\mathrm{u}} / \mathrm{V}_{\mathrm{u}} \\
& \mathrm{W}_{\mathrm{b}}=\frac{\mathrm{W}_{\mathrm{tf}}}{\mathrm{NS} \cdot \mathrm{NL} \cdot \mathrm{NB}} \\
& \mathrm{V}_{\mathrm{u}} / \mathrm{W}_{\mathrm{b}} \\
& \mathrm{H}_{\mathrm{cg}} / \mathrm{D}^{\prime} \\
& \mathrm{H}_{\mathrm{cg}} / \mathrm{S} \\
& \mathrm{F}_{1}=\sqrt{(\mathrm{NS})^{2}+1} \\
& \mathrm{~F}_{2}=\sqrt{\mathrm{NL}^{2}\left(\frac{\mathrm{H}_{\mathrm{cg}}}{\mathrm{D}^{\prime}}\right)^{2}+\left(\frac{2}{3}\right)^{2}+\left(\frac{\mathrm{H}_{\mathrm{cg}}}{\mathrm{S}}\right)^{2}\left(\frac{(\mathrm{NS})^{2}}{(\mathrm{NS}-1)^{2}}\right)}
\end{aligned}
$$

Step 7 - Determine the acceleration capacity of the tank anchorage. The acceleration capacity $(\lambda)$ of the tank anchorage is defined as the smaller of the two anchorage acceleration capacities $\lambda_{1}$ or $\lambda_{\mathrm{u}}$ :

$$
\begin{aligned}
& \lambda_{1}=\left(\frac{\mathrm{V}_{\mathrm{u}}}{\mathrm{W}_{\mathrm{b}}}\right)\left(\frac{1}{\mathrm{~F}_{1}}\right) \quad[\mathrm{g}] \\
& \lambda_{\mathrm{u}}=\frac{\frac{\mathrm{V}_{\mathrm{u}}}{\mathrm{W}_{\mathrm{b}}}+\frac{0.7}{\alpha}}{\left(\frac{0.7}{\alpha}\right) \mathrm{F}_{2}+\mathrm{F}_{1}} \quad[\mathrm{~g}] \\
& \lambda=\left(\text { Smaller of } \lambda_{1} \text { or } \lambda_{\mathrm{u}}\right) \text { [g] }
\end{aligned}
$$


Step 8 - Determine whether the tank is rigid or flexible in the transverse and vertical directions. Enter Figure 9.1.2-2 (for horizontal tanks with weight density $\gamma_{\mathrm{t}} \leq 75 \mathrm{lbf} / \mathrm{ft}^{3}$ ) or Figure 9.1.2-3 (for horizontal heat exchangers with weight density $\gamma_{\mathrm{h}} \leq 180 \mathrm{lbf} / \mathrm{ft}^{3}$ ) with:

$$
\begin{array}{ll}
\text { D } & \text { (Diameter of tank) [ft.] } \\
t & \text { (Thickness of tank shell) [in.] }
\end{array}
$$

and determine the maximum saddle spacing for rigid transverse and vertical frequency response (i.e., $\mathrm{F}_{\text {trans. }} \geq 33 \mathrm{~Hz}$ ):

$$
\mathrm{S}_{\mathrm{c}} \quad \text { [ft.] (from Figure 9.1.2-2 or 9.1.2-3) }
$$

If the maximum saddle spacing $\left(\mathbf{S}_{c}\right)$ is more than or equal to the actual spacing (S):

$$
\mathrm{S}_{\mathrm{c}} \geq \mathrm{S}
$$

then the tank is rigid in the transverse and vertical directions, otherwise it is flexible.

Step 9 - Determine whether the tank is rigid or flexible in the longitudinal direction. The rigidity of the one saddle not having slotted holes in its base plate controls the frequency response of the tank in the longitudinal direction. The longitudinal stiffness $\left(\mathrm{k}_{\mathrm{s}}\right)$ of the tank is determined by assuming the saddle plate and its stiffeners bend with a fixed (built-in) connection at the tank and a pinned connection at the base plate. The moment of inertia $\left(\mathrm{I}_{\mathrm{yy}}\right)$ of the cross-sectional area of the saddle plate and its stiffeners should be determined at a cross-section just below the bottom of the cylindrical tank. Compute the resonant frequency of the tank in the longitudinal direction using the following equation:

$$
\mathrm{F}_{\text {long. }}=\frac{1}{2 \pi} \sqrt{\frac{\mathrm{k}_{\mathrm{s}} \mathrm{g}}{\mathrm{W}_{\mathrm{tf}}}}
$$

Where the saddle stiffness $\left(\mathbf{k}_{\mathbf{s}}\right)$ is:

$$
k_{s}=\frac{1}{\frac{h^{3}}{3 E I_{y y}}+\frac{h}{A_{s} G}} \quad[l b f / i n]
$$

If the longitudinal resonant frequency ( $\mathrm{F}_{\text {long }}$ ) is greater than or equal to about $33 \mathrm{~Hz}$ :

$$
F_{\text {long. }} \geq 33 \mathrm{~Hz}
$$

then the tank is rigid in the longitudinal direction, otherwise it is flexible. 
Step 10 - Determine the seismic demand acceleration and compare it to the capacity acceleration. If the tank is rigid in all three directions; i.e.,

$$
\begin{aligned}
& S_{c} \geq S \text { and } \\
& F_{\text {long. }} \geq 33 \mathrm{~Hz}
\end{aligned}
$$

then determine the ZPA from the $4 \%$ damped floor response spectrum (maximum horizontal component). See Sections 5.2 and 6.4.2 for a discussion of input spectral acceleration.

\section{ZPA [g] (from 4\% damped floor response spectrum at $33 \mathrm{~Hz}$ )}

and compare it to the acceleration capacity of the tank anchorage:

$$
\lambda \text { [g] (from Step 7) }
$$

If

$$
\lambda \geq \mathrm{ZPA}
$$

then the tank anchorage is adequate; proceed to Step 11. If the tank anchorage does not meet this guideline, classify the tank as an outlier and proceed to Section 9.1.2.3 after completing the remainder evaluations in this section.

If the tank is flexible in any of the three directions, i.e.,

$$
\begin{aligned}
& S_{c}<S \text { or } \\
& F_{\text {long. }}<33 \mathrm{~Hz}
\end{aligned}
$$

then determine the spectral peak acceleration (SPA) ${ }^{16}$ from the $4 \%$ damped floor response spectrum (maximum horizontal component):

\section{SPA [g] (from peak of $4 \%$ damped response spectrum)}

and compare it to the acceleration capacity of the tank anchorage:

$$
\lambda \quad[g] \quad(\text { from Step 7) }
$$

$$
\text { If } \quad \lambda \geq \text { SPA }
$$

then the tank anchorage is adequate; proceed to Step 11. If the tank anchorage does not meet this guideline, classify the tank as an outlier and proceed to Section 9.1.2.3 after completing the remainder of the evaluations in this section.

16 This horizontal tank evaluation procedure uses the assumption that the tank is full of water. This assumption always results in a conservative evaluation when the peak of the response spectrum is used to estimate the seismic demand acceleration. If, however, the SCEs elect to determine the fundamental natural frequency of the tank more accurately, and use a spectral acceleration corresponding to a frequency less than the frequency at the peak of the demand spectrum, then they should also consider the case where the tank may not be full. For seismic demand spectra with sharp increases over small frequency changes, the seismic demand load for evaluation of the tank anchorage (weight $x$ spectral acceleration) may be greater for the partially filled tank than for the full tank. 
Step 11 - Check the saddle stresses. Longitudinal shear is the main load that the saddle and its stiffeners must carry if the other saddles have slotted anchor bolt holes in the base plate. Except for small tanks, the saddle which carries the longitudinal earthquake shear loading should have stiffeners to resist this weak axis bending. In addition to the longitudinal shear load, there are several other loads in the other directions which should be considered; these other loads are carried equally by all the saddles. The loads to include in determining the stresses in the saddle and its stiffeners are listed below.

- Longitudinal seismic loads

- Vertical compression load from dead weight

- Vertical seismic loads

- $\quad$ Overturning moment from transverse seismic load

The stresses in the saddle and its stiffeners should be determined in accordance with the combined compression and bending provisions of Part 1 of the AISC Manual of Steel Construction (Ref. 81). If the stresses are less than or equal to $1.7 \times$ AISC allowables (for earthquake loading), then the saddle is adequate and hence the tank is satisfactory for seismic loadings. If the saddle stresses exceed the AISC allowable, then classify the tank as an outlier and proceed to Section 9.1.2.3.

This completes the seismic evaluation for horizontal tanks.

\subsubsection{Outliers $^{17}$}

An outlier is defined as a horizontal tank or heat exchanger which does not meet the screening guidelines for:

- Adequacy of anchor bolts and their embedments, or

- Adequacy of anchorage connections between the anchor bolts and the tank shell.

When an outlier is identified, proceed to Chapter 12, Outlier Identification and Resolution, and document the cause(s) for not meeting the screening guidelines on an Outlier Seismic Evaluation Sheet (OSES).

Note that all of the screening guidelines should be evaluated (i.e., go through all the steps in this procedure) so that all possible causes for a horizontal tank or heat exchanger being classified as an outlier are identified before proceeding to Chapter 12 to resolve it.

The screening guidelines given in this section are intended for use as a generic screen to evaluate the seismic adequacy of horizontal tanks and heat exchangers. Therefore, if a horizontal tank or heat exchanger fails this generic screen, it may not necessarily be deficient for seismic loading; however, additional outlier evaluations are needed to show that it is adequate. Such analyses could include use of the principles and guidelines contained in this section and in Reference 42 for those types of horizontal tanks and heat exchangers not covered herein. When a horizontal tank or heat exchanger which is covered by this section fails to pass the screening guidelines, refined analyses could be performed which include use of more realistic or accurate methods instead of the simplified, generic analysis methods used in the section and Reference 42. Other generic methods for resolving outliers are provided in Chapter 12.

17 Section 7.5 of SQUG GIP (Ref. 1) 


\subsubsection{Documentation ${ }^{18}$}

The results of the engineering evaluations and field inspections performed using the guidelines in this section should be retained in the facility's files.

The results of the evaluations and inspections should also be documented by completing a Screening Evaluation Data Sheet (SEDS) as described in Section 13.4 and a Screening Evaluation Work Sheet (SEWS) as described in Section 13.2.

If any of the screening guidelines contained in this section cannot be met, the horizontal tank or heat exchanger should be classified as an outlier. The Outlier Seismic Evaluation Sheet (OSES), discussed in Chapters 12 and 13, should be completed to document the cause(s) for not meeting the screening guidelines.

${ }^{18}$ Section 7.6 of SQUG GIP (Ref. 1) 
Table 9.1.2-1 Applicable Range of Parameters and Assumptions
for Horizontal Tanks (Table 7-6 of SQUG GIP, Ref. 1)

\begin{tabular}{|c|c|c|}
\hline Diameter of Tank ${ }^{1}$ & $\mathrm{D}$ & $=1$ to $14 \mathrm{ft}$. \\
\hline Length of Tank & $\mathbf{L}$ & $=4$ to $60 \mathrm{ft}$. \\
\hline $\begin{array}{l}\text { Height of Center-of-Gravity of Tank and } \\
\text { Fluid Above the Floor Where the Tank is Anchored }\end{array}$ & $\mathrm{H}_{\mathrm{cg}}$ & $=1$ to $12 \mathrm{ft}$. \\
\hline Number of Saddles ${ }^{2}$ & NS & $=2$ to 6 \\
\hline Spacing Between Support Saddles ${ }^{3}$ & & $=3$ to $20 \mathrm{ft}$. \\
\hline Number of Bolting Locations ${ }^{4}$ per Saddle ${ }^{5}$ & NL & $=2$ or 3 \\
\hline Number of Anchor Bolts per Bolting Location & $\mathrm{NB}$ & $=1$ to 2 \\
\hline $\begin{array}{l}\text { Distance Between Extreme Anchor Bolts } \\
\text { in Base Plate of Saddle }\end{array}$ & $D^{\prime}$ & $=1$ to $12 \mathrm{ft}$. \\
\hline Ratio of Tank C.G. Height-to-Saddle Spacing & $\mathrm{H}_{\mathrm{cg}} / \mathrm{S}$ & $=0.5$ to 2.0 \\
\hline $\begin{array}{l}\text { Ratio of Tank C.G. Height-to-Distance } \\
\text { Between Extreme Anchor Bolts }\end{array}$ & $\mathrm{H}_{\mathrm{cg}} / \mathrm{D}^{\prime}$ & $=0.5$ to 2.0 \\
\hline Weight Density of Horizontal: & & \\
\hline - Tanks (including fluid) & $\gamma_{\mathrm{t}}$ & $=60$ to $75 \mathrm{lbf} / \mathrm{ft}^{3}$ \\
\hline - Heat Exchangers (including fluid) & & $=130$ to $180 \mathrm{lbf} / \mathrm{ft}^{3}$ \\
\hline
\end{tabular}

\section{Assumptions:}

1 Tanks are assumed to be cylindrical, horizontally oriented, and made of carbon steel.

2 Tanks are assumed to be supported on carbon steel plate saddles.

3 Saddles are assumed to be uniformly spaced a distance $S$ apart with the tank overhanging the end saddles a distance $S / 2$.

4 One or two anchor bolts are assumed at each bolting location.

5 All the base plates under the saddles are assumed to have slotted anchor bolt holes in the longitudinal direction to permit thermal growth of the tank, except for the saddle at one end of the tank which is fixed. 


\section{Table 9.1.2-2 Nomenclature Used for Horizontal Tanks \\ (Table 7-7 of SQUG GIP, Ref. 1)}

\section{Symbol Description [Units]}

$\mathrm{A}_{\mathrm{s}} \quad-\quad$ Cross-sectional area of saddle plate and its stiffeners (see Figure 9.1.2-1) [in. ${ }^{2}$ ]

D - Diameter of tank (see Figure 9.1.2-1) [ft.]

D' - Distance between extreme anchor bolts in base plate of a saddle (see Figure 9.1.2-1) [ft.]

d - Diameter of anchor bolt [in.]

E - Elastic modulus of saddle plate and stiffener material [psi]

e - Eccentricity (distance) from the anchor bolt centerline to the vertical saddle plate (see Figure 9.1.2-1) [in.]

$\mathrm{F}_{\text {long. }}$ - Resonant frequency of tank in longitudinal direction $[\mathrm{Hz}]$

$F_{\text {trans. }}$ - Resonant frequency of tank in transverse/vertical direction [Hz]

$F_{1} \quad-\quad$ Coefficient [dimensionless]

$\mathrm{F}_{2} \quad-\quad$ Coefficient [dimensionless]

$f_{y} \quad-\quad$ Minimum specified yield strength of shell, chair, saddle, or base plate material [psi]

G - Shear modulus of saddle plate and stiffener material [psi]

g - Acceleration of gravity $\left[386 \mathrm{in} / \mathrm{sec}^{2}\right]$

$\mathrm{H}_{\mathrm{cg}} \quad$ - Height of center-of-gravity of tank and fluid above the floor where the tank is anchored [ft.]

h - Height of saddle plate from the bottom of the tank to the base plate (see Figure 9.1.2-1) [in.]

$I_{y y} \quad-\quad$ Moment of inertia of cross-sectional area of saddle plate and its stiffeners about axis Y-Y (see Plan of Support S1 in Figure 9.1.2-1) [in. ${ }^{4}$ ]

$\mathrm{k}_{\mathrm{s}} \quad$ - Stiffness of the saddle plate and its stiffeners in the direction of the longitudinal axis of the tank [lbf/in]

L - - Length of tank (see Figure 9.1.2-1) [ft.] 


\section{Table 9.1.2-2 (Continued)}

NB - Number of anchor bolts at each bolt location [dimensionless]

NL - Number of bolt locations on each saddle [dimensionless]

NS - Number of saddles [dimensionless]

$P_{u} \quad-\quad$ Allowable tensile load of tank anchorage [lbf]

$P_{u}{ }^{\prime} \quad$ - Allowable tensile load of anchor bolt [lbf]

RB - Strength reduction factor for base plate bending [dimensionless]

RE - Strength reduction factor for an anchor bolt near an edge [dimensionless]

RS - Strength reduction factor for closely spaced anchor bolts [dimensionless]

RW - Strength reduction factor for base plate weld [dimensionless]

$\mathrm{S} \quad$ - $\quad$ Spacing between support saddles (see Figure 9.1.2-1) [ft.]

$\mathrm{S}_{\mathrm{c}} \quad-\quad$ Maximum saddle spacing for rigid tank $\left(\mathrm{F}_{\text {trans. }} \geq 30 \mathrm{~Hz}\right)[\mathrm{ft}$.

SPA - Spectral peak acceleration [g]

$t \quad$ - Thickness of tank shell [in.]

$t_{b} \quad-\quad$ Thickness of base plate under saddle [in.]

$t_{w} \quad$ - Thickness of leg of weld [in.]

$V_{u} \quad-\quad$ Allowable shear load of tank anchorage [lbf]

$V_{u}^{\prime} \quad$ - Allowable shear load of anchor bolt [lbf]

$W_{b} \quad-\quad$ Weight of tank per anchor bolt,

$$
\mathrm{W}_{\mathrm{b}}=\frac{\mathrm{W}_{\mathrm{tf}}}{\mathrm{NS} \cdot \mathrm{NL} \cdot \mathrm{NB}} \quad[\mathrm{lbf}]
$$




\section{Table 9.1.2-2 (Continued)}

Symbol

$\mathrm{W}_{\mathrm{tf}} \quad$ - Weight of tank plus fluid [lbf]

ZPA - Zero period acceleration [g]

$\alpha$ - Ratio of tensile to shear allowable anchorage load,

$\alpha=\frac{\mathrm{P}_{\mathrm{u}}}{\mathrm{V}_{\mathrm{u}}}$ [dimensionless]

$\gamma_{h} \quad-\quad$ Weight density of horizontal heat exchanger including fluid $\left[\mathrm{lbf} / \mathrm{ft}^{3}\right]$

$\gamma_{\mathrm{t}} \quad$ - Weight density of horizontal tank including fluid $\left[\mathrm{lbf} / \mathrm{ft}^{3}\right]$

$\lambda \quad-\quad$ Acceleration capacity of tank anchorage $[\mathrm{g}]$

$\lambda_{1} \quad$ L Lower acceleration capacity of tank anchorage [g]

$\lambda_{u} \quad-\quad$ Upper acceleration capacity of tank anchorage [g]

$\sigma \quad-\quad$ Stress [psi] 

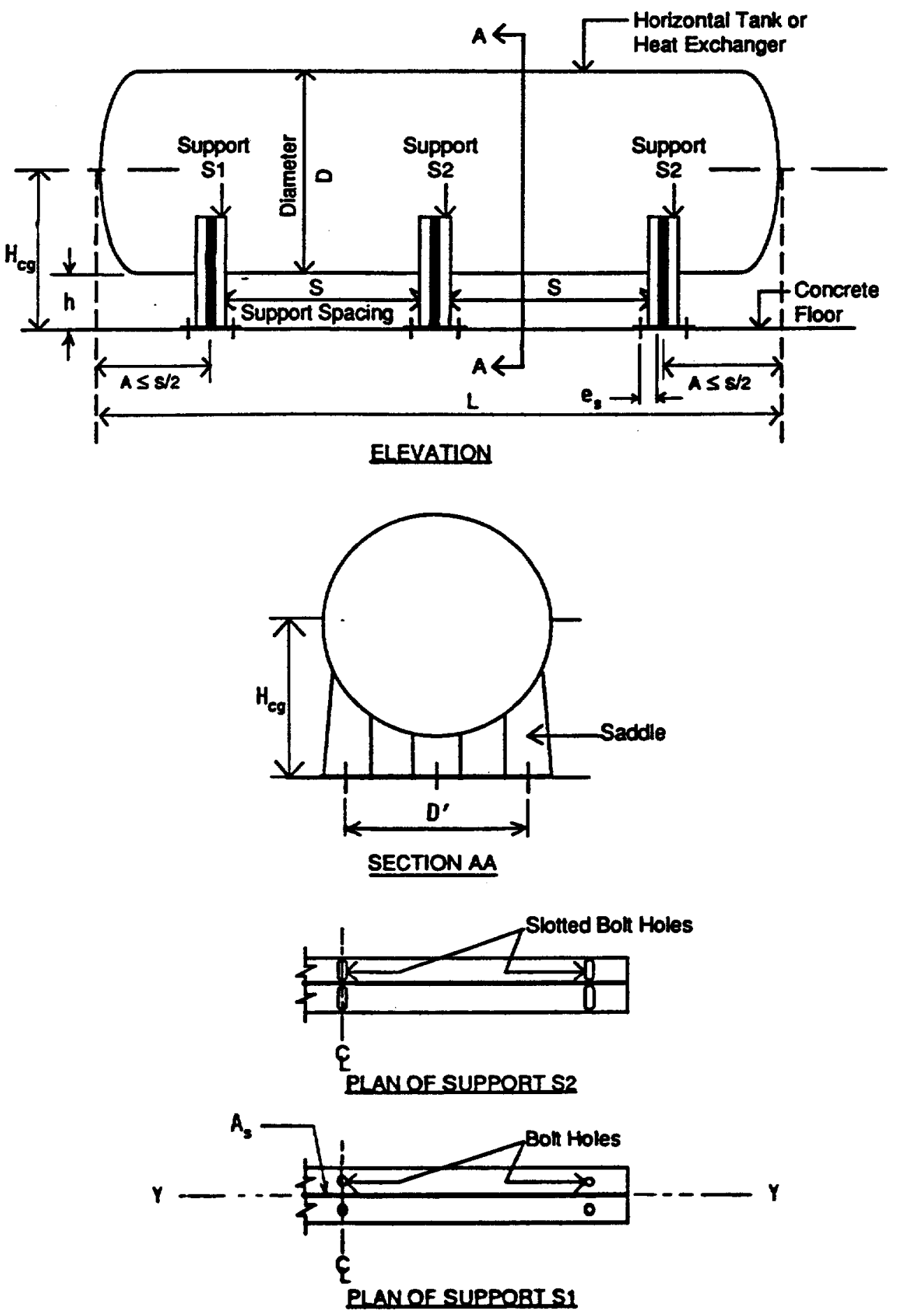

Figure 9.1.2-1 Horizontal Tank or Heat Exchanger (Reference 42) (Figure 7-13 of SQUG GIP, Reference 1) 


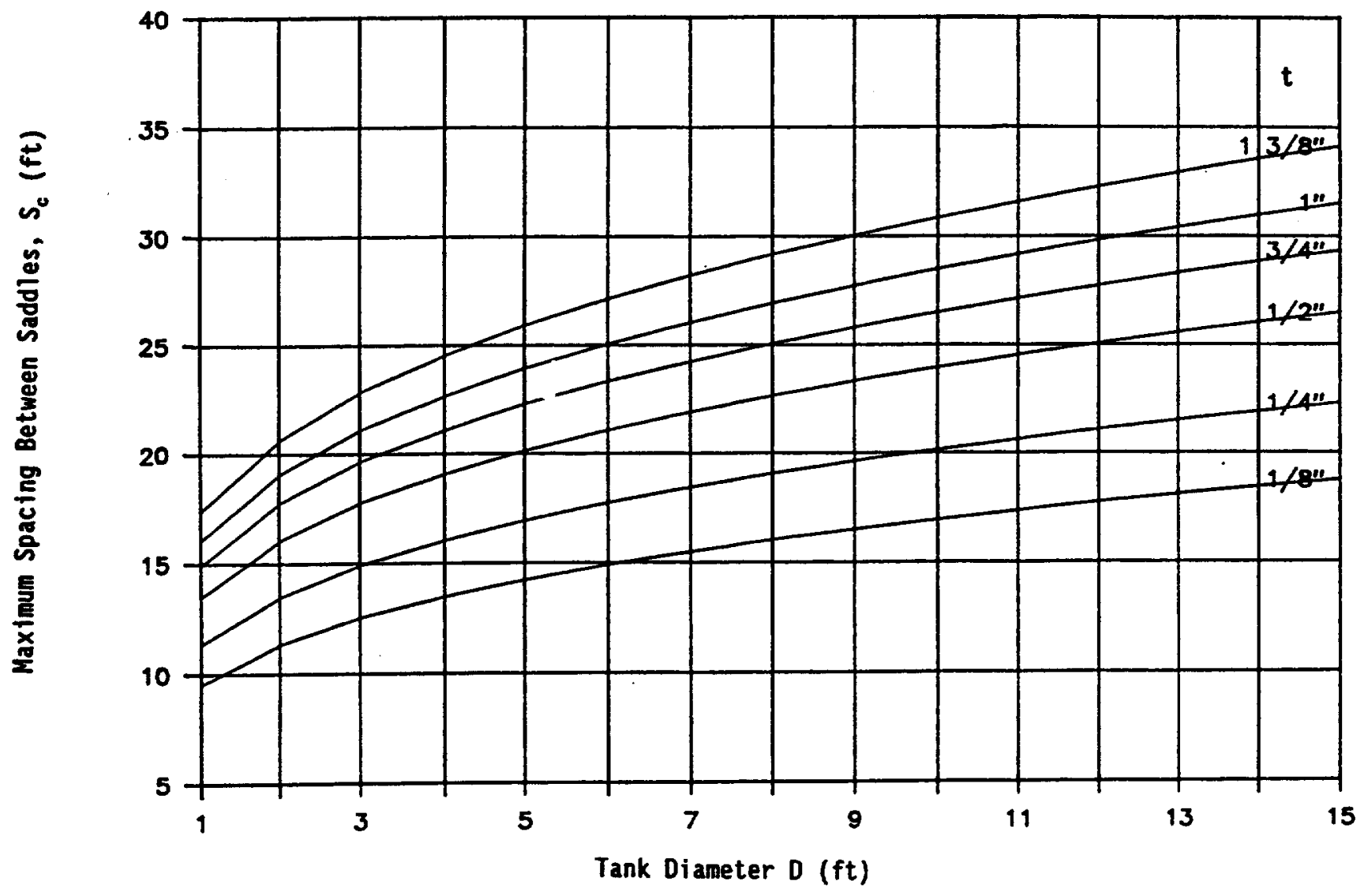

Figure 9.1.2-2 Maximum Saddle Spacing for Rigid $\left(F_{\text {trans. }} \geq 30 \mathrm{~Hz}\right)$ Horizontal Tanks $\left(\gamma_{t} \leq 75 \mathrm{lbf} / \mathrm{ft}^{3}\right)$ (Reference 42) (Figure 7-14 of SQUG GIP, Reference 1) 


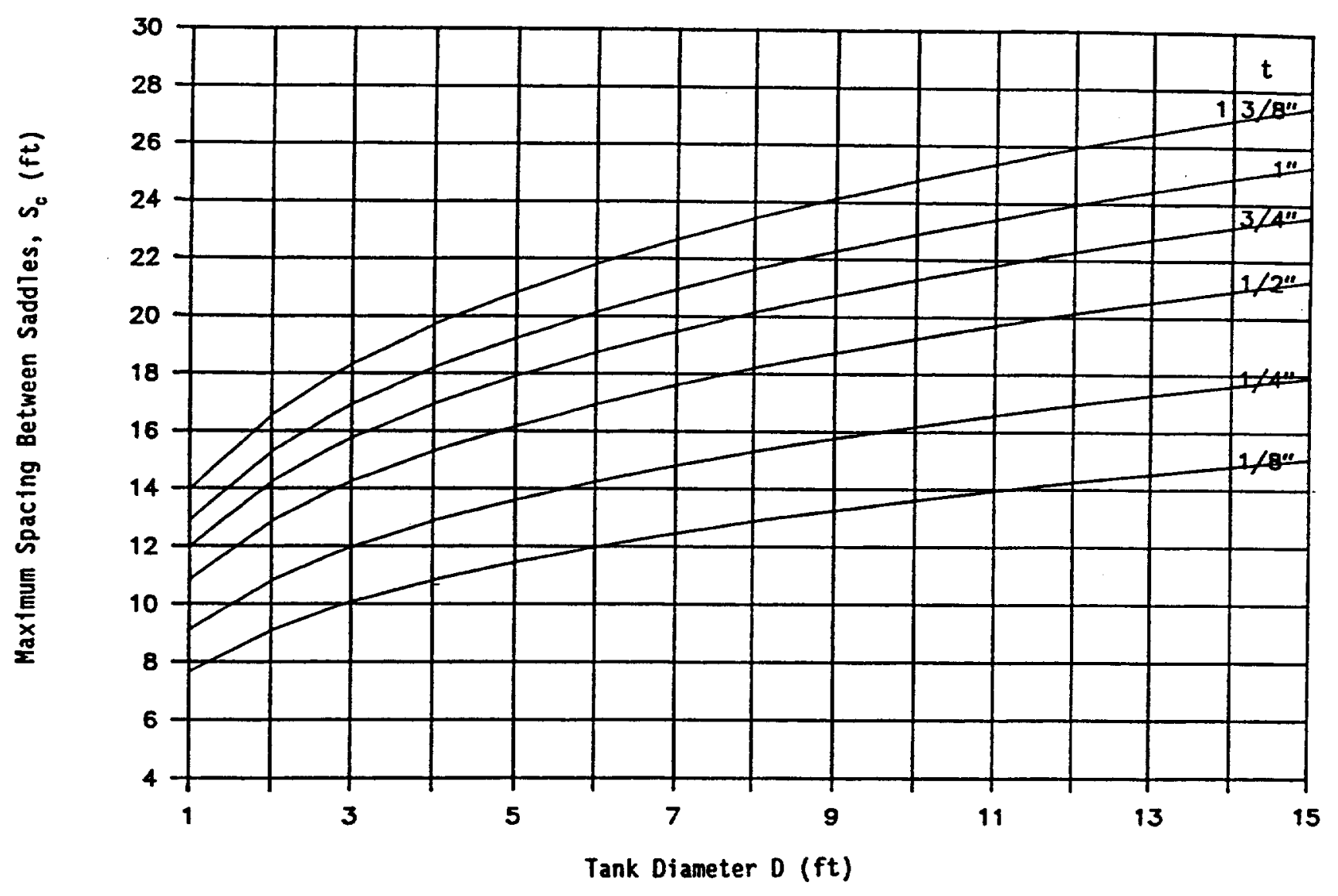

Figure 9.1.2-3 Maximum Saddle Spacing for Rigid $\left(F_{\text {trans. }} \geq 30 \mathrm{~Hz}\right)$ Horizontal Heat Exchangers $\left(\gamma_{\mathrm{h}} \leq 180 \mathrm{lbf} / \mathrm{ft}^{3}\right)$ (Reference 42) (Figure 7-15 of SQUG GIP, Reference 1) 



\subsection{RACEWAY SYSTEMS}

\subsubsection{CABLE AND CONDUIT RACEWAY SYSTEMS ${ }^{1}$}

\subsubsection{Introduction}

The purpose of this section is to describe the Cable and Conduit Raceway Review which should be used to screen out from further consideration the cable and conduit raceways which can be shown to be seismically adequate.

The Cable and Conduit Raceway Review consists of: (1) a facility walkdown in which the raceways are evaluated against a set of Walkdown Guidelines, and (2) an analytical check of selected worst-case supports using a set of Limited Analytical Review Guidelines. Those portions of the raceway systems which do not pass these screening guidelines are classified as outliers and should be evaluated separately using alternative methods. Some acceptable alternative methods for evaluating certain types of outliers are given. The remainder of this Introduction summarizes the elements of the Cable and Conduit Raceway Review.

\section{Basis for Screening Procedure}

The screening procedure contained in this section is based primarily on the use of earthquake experience and shake table test data. With few exceptions, raceway systems have exhibited superior performance in past earthquakes and in shake table tests. This successful performance has occurred despite the fact that most of the raceway systems in the data base had not been designed for earthquakes. This section of the DOE Seismic Evaluation Procedure provides guidance for understanding those aspects of raceway construction that provide acceptable performance and those features that might lead to poor performance.

Other more refined or sophisticated seismic qualification techniques may be used to evaluate the seismic adequacy of cable and conduit raceway systems; however, these other methods are generally not described in detail in this document. Some acceptable methods, based on standard engineering principles with consistent factors of conservatism, are included herein for evaluating certain types of outliers to the screening procedure.

\section{Seismic Review Guidelines}

The seismic review guidelines contained in this section are applicable to steel and aluminum cable tray and conduit support systems at any elevation in a DOE facility, provided the Reference Spectrum (shown in Section 5.3.1) envelopes the largest horizontal component of the 5\% damped, in-structure response spectrum (see Section 5.2) for that elevation.

Cable and conduit raceway systems are considered seismically adequate if, during and following a $D B E$, the electrical cables being supported by the raceway systems can continue to function and the raceway systems continue to maintain overhead support as defined in this section. Minor damage, such as member buckling or connection yielding, is considered acceptable behavior. The following guidelines are provided in this section:

- Walkdown Guidelines - The purpose of the walkdown guidelines is to evaluate that the raceway systems are bounded by the earthquake experience and shake table test data bases. This is done by checking the raceway systems against a set of "Inclusion Rules." Guidelines are also provided to assess "Other Seismic Performance Concerns" which could result in unacceptable damage. Guidance is also provided for selecting worst-case samples of the

1 Section 8.0 of SQUG GIP (Ref. 1) 
raceway support systems in the facility for which "Limited Analytical Reviews" should be performed. Finally, the walkdown should be used to evaluate that there are no seismic spatial interactions which could adversely affect the performance of the raceway system. Section 9.2.1.2 covers these Walkdown Guidelines.

- Limited Analytical Review Guidelines - The purpose of the Limited Analytical Review is to check that selected worst-case, representative samples of the raceway support systems in the facility are at least as rugged under seismic loadings as those in the earthquake experience and shake table test data bases that performed well. If these samples do not pass this Limited Analytical Review, further evaluations should be conducted and the sample expanded as appropriate. Section 9.2.1.3 covers these Limited Analytical Review Guidelines.

The background for these guidelines is described in Reference 42. A summary of available experience data from earthquakes and shake table tests can be found in Reference 46. Additional background on the philosophy behind several aspects of the guidelines are included in Reference 50. These references should be studied in conjunction with the guidelines in this section before conducting the seismic adequacy review of raceway systems.

\section{Outlier Resolution}

An outlier is defined as a raceway hardware feature which does not meet the Inclusion Rules, has significant Other Seismic Performance Concerns, or does not satisfy the Limited Analytical Review Guidelines contained in this section. An outlier may be adequate for seismic loadings, however, additional evaluations should be performed or alternative methods used beyond the scope of the screening evaluation procedure contained in this section. Section 9.2.1.4 describes some of the acceptable methods for evaluating raceway outliers. These additional evaluations and alternative methods should be thoroughly documented to permit independent review.

\section{Seismic Capability Engineers}

The screening guidelines for performing facility walkdowns and limited analytical reviews should be applied by a Seismic Review Team (SRT) consisting of at least two Seismic Capability Engineers (SCEs) who meet the qualification and training guidelines given in Chapter 3 . These engineers are expected to exercise engineering judgment based upon the guidelines given in this section and the background and philosophy used to develop these guidelines as described in References 46,47 , and 50 . They should understand those aspects of raceway construction that provide acceptable performance and those features that may lead to poor performance.

When resolving outliers, it is especially important that the SCEs exercise professional judgment when applying the guidelines contained in this section since these guidelines are generic in nature to cover a wide range of applications. The $S R T$ should be satisfied that the specific raceway system under review is adequately supported, based upon an understanding of the background and philosophy used to develop the guidelines in this section.

\section{Scope of Review}

The scope of review includes all the cable and conduit raceway systems in the facility which support electrical wire for equipment on the Seismic Equipment List (SEL), as developed in Chapter 4.

In some older facilities it may be difficult to identify which raceways support the power, control, and instrumentation wiring for individual items of equipment. If this detailed information is not available, then all the cable and conduit raceway systems in the facility which could carry wiring for equipment on the $S E L$ should be reviewed using the guidelines contained in this section. 


\section{Organization of Section}

The remainder of this section is organized as follows:

- Section 9.2.1.2 contains the Walkdown Guidelines for conducting seismic adequacy reviews of as-installed conduit, cable trays, and their support systems.

- Section 9.2.1.3 contains the Limited Analytical Review Guidelines for checking the seismic adequacy of a bounding sample of the facility raceway support systems.

- Section 9.2.1.4 contains a summary of additional evaluations and alternative methods for assessing the seismic adequacy of raceway outliers.

- Section 9.2.1.5 contains guidelines on how to document the results of the Cable and Conduit Raceway Review.

\subsubsection{Walkdown Guidelines ${ }^{2}$}

Guidelines for conducting a seismic adequacy review of as-installed conduit, cable trays, and their support systems are presented in this section. The review has two purposes. The first is to check the raceway systems against certain Inclusion Rules to show the facility raceway systems are within the envelope of the earthquake experience and shake table test data bases. Guidelines are also provided to assess Other Seismic Performance Concerns which could result in unacceptable damage.

The second purpose of the review is to select representative, worst-case samples of the raceway supports in the facility on which Limited Analytical Reviews will be performed. The samples selected should encompass the diversity of the facility's support systems. The guidelines for performing the Limited Analytical Review are covered in Section 9.2.1.3.

\subsection{General Walkdown Procedure ${ }^{3}$}

The general walkdown procedure given in this subsection describes a method for performing detailed screening and assessment of conduit and cable tray systems for seismic adequacy. This evaluation relies in part upon engineering judgment which should be exercised during the facility walkdown. This engineering judgment should be based on a good understanding of the performance of raceway systems in past earthquakes and in shake table tests.

The individuals on the raceway evaluation walkdown team should meet the requirements for SCEs as defined in Chapter 3. The walkdown should be conducted by one or more SRT, each consisting of at least two SCEs. The SRT should have a clear understanding and working knowledge of the screening guidelines presented below and have studied References 46,47 , and 50 thoroughly. They should also become familiar with the raceway design and construction practices of the facility, as well as with the general facility layout, raceway routing, and the design of raceway systems which cross building separations.

It is expected that the SRT will spend from one to two weeks in the facility. The duration may vary depending on the number of SRTs, the size of the facility, the complexity and accessibility of the facility raceway systems, and so forth.

2 Section 8.2 of SQUG GIP (Ref. 1)

3 Section 8.2.1 of SQUG GIP (Ref. 1) 
It is recommended that the SRT take general notes, including rough sketches or photographs, as appropriate, of typical system attributes. More detailed notes should be taken to document decisions and evaluations made in the field. Walkdowns may be conducted on an area-by-area, system-by-system, or run-by-run basis. Time should be set aside on a daily basis for the SRT to review notes and sketches; to collect facility drawings or information, if needed; and to check selected supports by preliminary calculations, if warranted. Recommended documentation for the review is discussed in Section 9.2.1.5.

During the facility walkdown, the SRT should (1) evaluate that the cable and conduit raceway systems meet the Inclusion Rules given in Section 9.2.1.2.2; (2) note and evaluate any of the Other Seismic Performance Concerns given in Section 9.2.1.2.3; (3) select a sample of representative worst-case raceway supports as described in Section 9.2.1.2.4; and (4) judge whether there are any seismic spatial interactions which could adversely affect the performance of the raceway system as outlined in Section 9.2.1.2.5. The distinction between the first two walkdown objectives is explained below.

The Inclusion Rules identify the important limits of the earthquake experience and shake table test data bases and certain undesirable details which, if violated, could significantly compromise the seismic adequacy of a raceway system.

The SRT should visually inspect the raceway systems within the scope of review to determine whether the general construction practice in the facility is in agreement with the Inclusion Rules. The SRT should examine in detail several supports or spans of each different configuration type at a variety of locations in the facility. In addition, the SRT should actively seek out problems and be alert for and evaluate any instances of non-compliance with the Inclusion Rules noticed as part of the walkdown.

If it appears that any of the Inclusion Rules are not met, then the SRT should investigate that portion of the raceway system in sufficient detail so that the team is convinced they understand the extent of the identified condition. That portion of the raceway system should then be classified as an outlier and evaluated using the guidelines given in Section 9.2.1.4.

The Other Seismic Performance Concerns given in Section 9.2.1.2.3 represent less significant or less well-defined conditions which should be evaluated during the facility walkdown. They are included in the guidelines of this section as representative of the type of concerns which the SRT should look for and evaluate to determine whether they could significantly compromise the seismic adequacy of the raceway system.

It is not necessary for all of the raceway systems in the facility to be inspected in detail for the Other Seismic Performance Concerns. Instead, the SRT should note and evaluate any of these concerns, if and when they are noticed as a part of the walkdown.

If it appears that any of the other Seismic Performance Concerns are not met, then the SRT should exercise their engineering judgment in assessing whether the condition significantly compromises the seismic adequacy of the raceway system. If it appears that the area of concern is not significant, then the SRT should note the condition on the walkdown documentation and provide a written explanation for their conclusion. However, if, in their judgment, the area of concern is significant, then that portion of the raceway system should be classified as an outlier and evaluated in a manner similar to an Inclusion Rule outlier.

In many cases, the facility walkdown may be conducted from the floor level. In some cases however, it may be necessary to examine the raceway system more closely if vision from the floor is obstructed. As different support configurations are observed during the facility walkdown, the SRT should examine them to familiarize themselves with the construction and details of the 
raceway system. When any suspect condition is observed which may violate one of the Inclusion Rules or may represent a significant Other Seismic Performance Concern, then a closer examination should be carried out.

In general, the level of effort of the review should be enough to give the SRT confidence in the seismic adequacy of the facility raceway systems. Ultimately the SRT is responsible for the seismic evaluations. Their sound engineering judgment is the key to successful execution of these guidelines so that the review is both safety-effective and cost-effective. In this spirit, these guidelines are only guidelines, not requirements; the sound engineering judgment of the SRT is the most important factor, particularly when evaluating the seismic adequacy of outliers.

\subsection{Inclusion Rules ${ }^{4}$}

The Inclusion Rules in this section identify the important limits of the earthquake experience and shake table test data bases and certain undesirable details which, if violated, could significantly compromise the seismic adequacy of a raceway system. These Inclusion Rules should be evaluated using the general walkdown procedure given in Section 9.2.1.2.1.

Rule 1 - Cable Tray Span. The length of unsupported cable tray between adjacent supports should not exceed about 10 feet in the direction of the run. When the cable tray extends beyond the last support in a run, it should not cantilever out (overhang) beyond this support more than $1 / 2$ the maximum unsupported span length, i.e., about 5 feet. This span and cantilever overhang were selected because they are supported by earthquake experience data.

Rule 2 - Conduit Span. The length of unsupported conduit in the direction of the run between adjacent supports, or the length of unsupported conduit cantilevered out from the last support in a run should not exceed the spans and overhangs given in the following table. These spans and overhangs were selected because they are supported by earthquake experience data and are consistent with the National Electrical Code (Reference 88).

\begin{tabular}{|c|c|c|}
\hline $\begin{array}{c}\text { Conduit } \\
\text { Size } \\
\text { (inches) }\end{array}$ & $\begin{array}{c}\text { Approximate Maximum } \\
\text { Spans Between } \\
\text { Adjacent Supports } \\
\text { (feet) }\end{array}$ & $\begin{array}{c}\text { Approximate Maximum } \\
\text { Cantilever Overhang } \\
\text { (feet) }\end{array}$ \\
\hline $1 / 2$ and 3/4 & 10 & 5 \\
\hline 1 & 12 & 6 \\
\hline $1-1 / 4$ and 1-1/2 & 14 & 7 \\
\hline 2 and 2-1/2 & 16 & 8 \\
\hline 3 and larger & 20 & 10 \\
\hline
\end{tabular}

Rule 3 - Raceway Member Tie-downs. For cantilever bracket-supported systems, cable trays and conduit should be secured to their supports so the trays or conduit cannot slide and fall off the supports. Normal industrial friction type hardware, such as the "z-clip" commonly used for cable trays, is a sufficient means of attachment.

4 Section 8.2.2 of SQUG GIP (Ref. 1) 
Systems do not have to be secured to every support, unless the supports are at the maximum spacing described above. For example, consider a 60 -foot length of cable tray. If there is a support at each end and the interior supports are at the maximum span of 10 feet described in Rule 1, then the raceway system should be tied down at all seven supports in the 60 -foot run. If there are more than seven supports, the trays need to be secured to only about seven of these supports in any 60-foot run, regardless of how many additional supports there actually are in the run.

Rule 4 - Channel Nuts. Channel nuts used with light metal framing systems should have teeth or ridges stamped into the nuts where they bear on the lip of a channel as shown in Figure 9.2.1-1.

Rule 5 - Rigid Boot Connection. Strut systems supported by "boots" or similar rigid devices, especially facility-specific designs, should be evaluated on a case-by-case basis. Shake table tests have shown that a rigid boot overhead connection detail, as shown in Figure 9.2.1-2(a), has a significantly-reduced vertical load-carrying capacity in seismic motion. Any gap between the vertical support member and the boot prevents the development of high clamping forces in the connection and thus causes a significantly reduced load-carrying capacity. Cable tray test specimens with this detail have collapsed in shake table tests.

A rigid boot connection with gaps can be upgraded to an acceptable connection by using a through bolt as shown in Figure 9.2.1-2b). This connection has been shown to be acceptable by shake table tests.

Rule 6 - Beam Clamps. Beam clamps should not be oriented in such a way that gravity loads are resisted only by the clamping or frictional forces developed by the clamps. The earthquake experience data base includes many examples of beam clamps attached to the lower flange of structural steel beams such that the gravity loads are resisted by bearing of the inside top of the clamp on the top of the lower flange of the beam. On the other hand, beam clamps oriented so gravity load is resisted only by the clamping frictional force, as shown in Figure 9.2.1-3, might loosen and slip off in an earthquake and possibly cause a collapse.

Rule 7 - Cast-Iron Anchor Embedment. Threaded rod-hanger anchor embedments constructed of cast iron should be specially evaluated since there is a potential for a brittle failure mode. Facility documentation should be used to determine whether anchor embedments are cast iron. The earthquake experience data base includes examples where heavily-loaded rod hangers threaded into cast-iron inserts failed. The cast-iron anchor detail is shown in Figure 9.2.1-4. Failure modes included anchor pullout and anchor fracture where rods were only partially threaded into the anchor.

\subsection{Other Seismic Performance Concerns ${ }^{5}$}

The Other Seismic Performance Concerns in this section represent less significant or less welldefined conditions which should be evaluated during the facility walkdown. They are included in the guidelines of this raceway evaluation section as representative of the type of concerns which the SRT should be looking for during the facility walkdown. When one of these Other Seismic Performance Concerns is found, the SRT should determine whether the area of concern could significantly compromise the seismic adequacy of the raceway system. These seismic concerns should be evaluated using the general walkdown procedure given in Section 9.2.1.2.1.

5 Section 8.2.3 of SQUG GIP (Ref. 1) 
Concern 1 - Anchorage. The SRT should pay close attention to the review of anchorage for the raceway supports. The team should pay particular attention to system anchorage for heavilyloaded supports. When the type of anchorage detail cannot be determined by visual inspection, other methods of determining the anchorage detail may be used, provided the SRT is convinced they understand the actual details. For example, the facility design drawings, construction records, or procurement specifications may provide the unknown details. If overhead welds are not visible (for example, they are covered by fire retardant), other similar supports without the coating can be inspected, or as-installed facility documentation reviewed to gain understanding of the weld adequacy. Similarly, if the anchorage for large junction boxes is not visible (for example, if the box is flush mounted to a wall), then other boxes that can be readily opened or reviewed may be inspected instead, or facility installation specifications may be reviewed to provide the unknown details. Small, lightweight junction boxes need not be specifically anchored if they are not required to act as conduit supports (i.e., they may be included within conduit spans as defined in Rule 2 of Section 9.2.1.2.2).

Adequacy of other types of anchorage such as plastic inserts or lead shield plugs for cable tray systems are not covered by these guidelines. However, the adequacy of anchorage such as plastic inserts or lead shield plugs on lightly-loaded conduit supports rigidly attached to a wall may be evaluated on a case-specific basis by using manufacturers' information, performing facility-specific tests, or performing proof tests. In addition, anchorage adequacy for lightly-loaded conduit supports which are rigidly attached to a wall with less than about 15 pounds dead load may be evaluated by giving the conduit a tug by hand.

Concern 2 - Cracks in Concrete. Visible large cracks, significantly spalled concrete, serious honeycomb or other gross defects in the concrete to which the cable tray or conduit supports are attached should be evaluated for their potential effects on anchorage integrity during an earthquake. The walkdown team should include supports of raceways anchored into concrete with gross defects in the sample selected for the Limited Analytical Review (Section 9.2.1.3).

Concern 3 - Corrosion. Excessive corrosion of cable trays, conduit, supports, or anchorage should be evaluated for its potential effect on structural integrity. Evaluations should consider the alternative of estimating the strength reduction due to corrosion, if appropriate.

Concern 4 - Sag of Conduit and Cable Trays. There should not be a noticeable sag of the conduit or cable tray. As a general guideline, noticeable sags are defined as about 1 inch of deflection in a span with a length of 10 feet. If a noticeable sag is found, its cause should be determined before concluding corrective action is required. For example, the sag may have occurred during construction, have no relation to structural integrity, and thus not require any corrective measures. The walkdown team should include supports of raceways sagging due to heavy loads in the sample selected for the Limited Analytical Review (Section 9.2.1.3).

Concern 5 - Broken or Missing Components. Broken or missing cable tray and conduit components should be repaired or replaced. Locations where cable is routed near rough, sharp edges such as sheet metal cutouts should be evaluated for their potential to cause insulation damage in an earthquake.

Concern 6 - Restraint of Cables. Any cables above the top of the side rail should be restrained to keep them in the tray during an earthquake. Isolated cables in the center of the tray do not have to be restrained. If cables are not restrained, they should be evaluated to determine if they are a credible earthquake hazard to themselves (through flopping or falling out of the trays and becoming pinched or cut) or whether they are a hazard to nearby facility features (for example, by impacting a fragile component). 
When cable trays have vertical drops of more than about 20 feet and flapping of the cables during an earthquake might cause pinching or cutting of the cables or impact with nearby fragile equipment, the cables should be restrained to keep them in the tray.

Concern 7 - Aging of Plastic Cable Ties. There is concern that old cable ties which are made of plastic-type materials may not have sufficient strength as a result of aging. Cable ties are frequently used to restrain cables within cable trays. If restraining straps are required on vertical drops or when trays are filled above the top of their side rails and those restraining straps are of a plastic-type material, then the walkdown engineers should make a brief qualitative evaluation by physically pulling or tugging on a few of the straps or enclosed cables to ensure that the straps have not become brittle. If the straps break or easily fail under this simple test, then their effectiveness in an earthquake is obviously questionable and they should be replaced in those areas where they are needed.

Concern 8 - Hard Spots. Occasional stiff supports in long flexible runs of cable trays or conduit should be evaluated to determine if the seismic movement of the run could cause the stiff support to fail. This concern is mainly associated with longitudinal motion. Cable tray or conduit systems with a long run of supports that are relatively flexible in the longitudinal direction may also contain a support that is relatively stiff as shown in Figure 9.2.1-5. The stiff support may thus be subjected to considerable load and fail due to loads from earthquake-induced, longitudinal movement of the cable tray or conduit run. Where the stiff support is located around the bend from the long run, the flexibility and ductility of the bend in the tray or conduit will typically prevent failure of the stiff support from being a credible event. The SRT should review Reference 49 which provides examples of undamaged, long raceway runs from the earthquake experience data base.

The Limited Analytical Review Guidelines in Section 9.2.1.3 include an evaluation for fatigue effects of fixed-end rod hanger trapeze supports. The walkdown team should note instances of occasional short, fixed-end rod hangers (stiff supports) in raceway runs with predominantly longer, more flexible supports. These should be specially evaluated for possible failure due to fatigue using the Rod Hanger Fatigue Evaluation methodology given in Section 9.2.1.3.5. Rod hanger trapeze support systems which are eccentrically-braced should also be similarly evaluated.

\subsection{Selection of Sample for Limited Analytical Review ${ }^{6}$}

The purpose of this subsection is to provide guidelines for selecting representative, worst-case samples of raceway supports on which Limited Analytical Reviews will be performed. The samples should include representative samples of the major different types of raceway support configurations in the facility. The sample size will vary with the diversity and complexity of the design and construction of each specific facility's raceway support system. As a general guideline, 10 to 20 different sample supports should be selected.

Before the samples are selected, the SCEs should become familiar with the Limited Analytical Review Guidelines in Section 9.2.1.3 and should review the sample evaluations contained in Reference 47.

During the facility walkdown, notes should be taken which describe the basis for selection of each sample. The location of the selected sample should be noted, and detailed sketches of the as-installed support should be made. As-built sketches should include the support configuration, dimensions, connection details and anchorage attributes, member sizes, and loading. Any additional information that may be considered relevant to the seismic adequacy of the sample support should be noted in detail.

6 Section 8.2.4 of SQUG GIP (Ref. 1) 
The SCEs should seek out the most heavily-loaded raceway support for each configuration. Deep cable fill, long spans, sagging raceways, multiple tier systems, top supports at vertical runs, and fire protective coatings are indicators of heavy load. Of particular importance are raceway support systems that appear to have possibly more load than originally designed for. These can be identified by the presence of other facility components attached to the raceway support, such as pipe supports, HVAC duct supports, and tack welded-on conduit supports.

Conduit and cable tray supports with anchorage that appear marginal for the supported weight are good candidates for sample evaluation. Anchorage of undersized welds, incomplete welds, or welds of poor quality should also be included as samples. When overhead miscellaneous support steel, such as steel angle, is used specifically as an anchor point to support the raceways, its anchorage to the building structure should also be reviewed, and included as part of the sample, especially if its anchorage appears to be the weak link in the load path back to the structure. In addition, the sample should also include worst-case large junction boxes that are also used to support conduit, if the anchorage for the box appears to be marginal for the supported weight. As an example, cable trays and junction boxes in electrical penetration areas may be good candidates as these can become heavily loaded.

It may facilitate decision-making processes in the facility if some sample or bounding calculations are performed prior to walkdowns. As an example, simple screening tables can be developed which list anchor capacities and raceway system weights. These tables would enable rapid assessment of certain anchors appearing marginal for the supported load.

\subsection{Seismic Interaction ${ }^{7}$}

The SCEs should use the seismic interaction assessment guidelines given in Chapter 7 to look for and evaluate potential seismic interaction hazards. The interaction concerns to be addressed include potential proximity effects, structural failure and falling, and flexibility of attached cables. As an example, raceway systems attached to or in the vicinity of unanchored components, or unrestrained block walls, should be noted and evaluated.

It may also be necessary to evaluate the seismic interaction effect of a single isolated raceway support which could fail and fall onto a nearby fragile item of equipment listed on the SEL.

\subsubsection{Limited Analytical Review Guidelines ${ }^{8}$}

This subsection describes the Limited Analytical Review which should be performed on cable tray and conduit supports. Analytical review calculations should be conducted to evaluate the structural integrity of the raceway supports chosen as representative, worst-case samples of the facility raceway support systems. The Limited Analytical Review Guidelines given in this section address structural integrity by correlation with raceway support systems that performed well in past earthquakes. The purpose of the calculations is not to estimate actual seismic response and system performance during an earthquake. Rather, the purpose of the calculations is to show that cable tray and conduit supports are at least as rugged as those that performed well as evidenced by past experience. It is important to understand the difference between these two purposes.

The Limited Analytical Review Guidelines are primarily based on the back-calculated capacities of raceway supports in the seismic experience data base. The checks of these guidelines are formulated to ensure that cable tray and conduit supports are seismically rugged, consistent with the seismic experience success data. The checks include the use of static load coefficients, plastic

7 Section 8.2.5 of SQUG GIP (Ref. 1)

8 Section 8.3 of SQUG GIP (Ref. 1) 
behavior structural theory, and engineering judgment. Reference 50 should be read by the SCEs since it provides considerable discussion and background information on the philosophy for the analytical review process.

The analytical checks and evaluations discussed in this section are as follows:

- Dead Load Check (Section 9.2.1.3.1)

- Vertical Capacity Check (Section 9.2.1.3.2)

- Ductility Check (Section 9.2.1.3.3)

- Lateral Load Check (Section 9.2.1.3.4)

- Rod Hanger Fatigue Evaluations (Section 9.2.1.3.5)

- Floor-to-Ceiling Support Evaluations (Section 9.2.1.3.6)

- Base-Mounted Support Evaluations (Section 9.2.1.3.7)

Allowable capacities and raceway system weights are also discussed in this section.

The relationship between the above analytical checks for suspended raceway support systems is shown in a logic diagram in Figure 9.2.1-6. It is suggested that this figure be used while reading the following descriptions of these analytical checks.

The raceway supports should pass a normal engineering dead load design review to working stress level allowable loads. This Dead Load Check is described in Section 9.2.1.3.1. This is the only check needed for rigid, wall-mounted supports. Rigid-mounted conduit and cable trays are inherently very stable and subject to minimal seismic amplification. A detailed dead load design review of these systems provides ample margin for seismic effects. The working stress level allowable loads which should be used are described in Section 9.2.1.3.8. Supports not meeting the dead load check should be considered as outliers. If a support does not meet the Dead Load Check, but is not required in order to meet the span Inclusion Rules \#1 and \#2 of Section 9.2.1.2.2, then the adjacent supports should be checked, with the support in question assumed to be not present.

All raceway supports except rigid-mounted conduit and cable trays, and base-mounted raceway supports should also pass a Vertical Capacity Check of 3 times dead load. This is described in Section 9.2.1.3.2. The Vertical Capacity Check ensures that the vertical capacity to dead load demand ratio is at least as high as those of support systems in the earthquake experience data base that performed well.

The Ductility Check is described in Section 9.2.1.3.3. As shown in Figure 9.2.1-6, supports characterized as ductile do not require an explicit lateral load check. Instead, seismic ruggedness for ductile supports is assured by the Vertical Capacity Check (Section 9.2.1.3.2). The high vertical capacity of the ductile data base raceway supports is the main attribute credited for their good seismic performance.

Supports that may not respond to seismic loads in a ductile manner should be checked for lateral load capacity. The Lateral Load Check, described in Section 9.2.1.3.4, is in the form of an equivalent static lateral load coefficient. Because this static coefficient is derived from the earthquake experience data base, it is considered applicable to ground motion consistent with the 
Reference Spectrum shown in Section 5.3.1. A method for scaling down the load coefficient for sites with lower ground motion response spectra is provided in Section 9.2.1.3.4.

The simple equivalent static lateral load method becomes overly conservative for suspended supports with long drop vertical support members from overhead. This is because calculated moments at the ceiling connection become very large. Unless the vertical support member is very rigid, lateral load effects may be limited by seismic response peak displacements. Section 9.2.1.3.4 provides a method for determining more realistic, deflection-controlled lateral loads for evaluation of these cases.

Although rod hanger trapeze supports may be characterized as ductile for seismic loading, the fatigue life of the threaded rod hangers may limit seismic capacity when fixed-end connections are subject to large bending strains. Rod Hanger Fatigue Evaluations should be done using the guidelines in Section 9.2.1.3.5 for rod hanger trapeze supports with fixed-end rods.

The checks described above and illustrated in the Figure 9.2.1-6 logic diagram directly apply only to seismic evaluations of suspended (and wall-mounted) raceway supports. Similarly, simple evaluation methods may also be applied to floor-to-ceiling supports and base-mounted supports, as long as consideration is given to lack of pendulum restoring force effects and instabilities that may arise from plastic hinge formation.

Floor-to-Ceiling Support Evaluations are discussed in Section 9.2.1.3.6. Ductility arguments may only be used if the support's base mount can be neglected (i.e., treating the support as if it is suspended). When the base mount is required to help resist vertical load, Lateral Load Checks of the top and bottom connections, as well as buckling capacity checks of the vertical support member, are warranted.

Base-Mounted Support Evaluations are discussed in Section 9.2.1.3.7. These supports cannot be characterized as inherently ductile, and strength checks are required for both equivalent lateral and longitudinal loads. In addition, the base connection hardware details should be reviewed for rigidity. Slight connection slips that may lead to acceptable behavior for suspended systems can result in an additional overturning moment due to P-delta effects (i.e., eccentric loadings) for base-mounted supports and should be reviewed.

If a support fails to meet the Limited Analytical Review Guidelines, then it should be considered to be an outlier. Further analyses or tests may be performed on this outlier to demonstrate its seismic ruggedness as described in Section 9.2.1.4.

If supports of the worst-case sample selection do not meet the Limited Analytical Review checks (i.e., are outliers), then the review team should develop an understanding of what supports in the facility are impacted by this analysis result.

The Vertical Capacity and Lateral Load Checks should be done using realistic capacity allowables as discussed in Section 9.2.1.3.8.

The raceway system weights that should be used for these Limited Analytical Reviews are described in Section 9.2.1.3.9.

\subsection{Dead Load Check ${ }^{9}$}

Back-analysis of raceway supports in the data base indicates that most systems have adequate dead load design. A detailed dead load design review of the worst-case sample conduit and cable tray

9 Section 8.3.1 of SQUG GIP (Ref. 1) 
supports should be conducted using normal design working stress allowable loads. The check should consider the as-installed configuration, connection detailing, and loading condition of the raceway support. All components such as bracket members, support members, conduit clamps, internal framing connections, and support anchorage should be checked. All system eccentricities, including load to anchor point eccentricity, should be considered, excluding evaluation of clip angle bending stresses. (Note, however, that clip angle bending stress should be considered during evaluation of base connections of floor-mounted supports as discussed in Section 9.2.1.3.7). Loads from other attached systems, such as piping or ducting, should be considered.

This is the only check recommended for cable tray and conduit supports directly mounted to or rigidly cantilevered from an adjacent structural wall. These support types have been shown to be inherently rugged by past experience. The mounting configuration is generally rigid for lateral response, so dynamic amplification of seismic motion is minimal. Performing a detailed dead load design review for these support types ensures adequate margin for seismic loads.

Consideration should also be given to the seismic adequacy of the wall to which cable tray and conduit raceway supports are attached. Reinforced concrete structural walls are not a concern. With the exception of very light conduit, anchorage into transite walls (asbestos fiber board) and gypsum board partitions should be considered outliers. Masonry walls should be checked to evaluate that they have been reviewed for seismic adequacy as described in Section 10.5.1. The anchor capacities in Section 6.3 cannot be used for expansion anchors in masonry block walls (especially if the anchorage are installed in hollow block cores or mortar joints) or in nonstructural material; reduced values should be used. The anchorage of partition walls and shielding walls should be checked.

\subsection{Vertical Capacity Check $^{10}$}

This check concentrates on the support anchorage, focusing on the weak link in the support anchorage load path. Back-analysis of conduit and cable tray support systems in the data base indicates that most supports have relatively high, vertical anchorage capacity. The high capacities are inherent in standard available connection hardware used for raceway support systems. The high vertical capacity is one of the primary design attributes that is given credit for good seismic performance. The Vertical Capacity Check evaluates whether the vertical capacity to dead load demand ratio is in the range of support systems in the data base that performed well. The high vertical capacity provides considerable margin for horizontal earthquake loading.

This Vertical Capacity Check is only applicable to raceway supports suspended from overhead. The Vertical Capacity Check is an equivalent static load check, in which the support is subjected to 3.0 times Dead Load in the downward direction, using the capacities discussed in Section 9.2.1.3.8. This check is limited to the primary raceway support connections and the anchorage of suspended support systems. It is not necessary to evaluate clip angle bending stress or secondary support members. Base-mounted supports are not subject to this check (see Section 9.2.1.3.7); however, the lower support member of floor-to-ceiling configurations should be checked for buckling if the upper connection cannot resist 3.0 times Dead Load by itself as discussed in Section 9.2.1.3.6.

Eccentricities resulting in anchor prying and eccentricities between vertical support members and anchor points should, in general, be ignored. This concept is the result of back-analyses of data base cable tray supports and is consistent with limit state conditions observed in test laboratories.

10 Section 8.3.2 of SQUG GIP (Ref. 1) 
For cantilever bracket support types, the eccentricity of the cantilevered dead load should be ignored. Even if overhead moment capacity is completely lost, the vertical support integrity is maintained, as the support balances itself with the center of mass below the anchor point. It is important to realize that this calculational method is only used to demonstrate seismic adequacy by comparison with experience data. It is not expected, and it has not been shown by the experience data, that a support will end up in this deformed position after an earthquake on the order of the Reference Spectrum shown in Figure 5.3-2 divided by 1.5 .

For trapeze frame and rod-hung supports, load distribution between the two vertical framing members should be considered if the center of the load is significantly distant from the centerline of the support frame. The bending strength and stiffness of frame members should be checked for transfer of the load between anchor bolts when overhead support is provided by light metal framing with anchor bolts spaced at relatively large intervals and when multiple anchor bolts are needed to resist the vertical load.

For most conduit and cable tray support systems, the anchorage is the weak link in the load path. For these support systems the Vertical Capacity Check is simply a comparison of anchor capacity to 3.0 times the supported load.

The 3.0 times dead load static coefficient should not be reduced if the in-structure response spectrum (see Section 5.2) for that facility is less than the Reference Spectrum shown in Figure 5.3-2. This is because there are only a few supports in the earthquake experience data base which have back-calculated vertical capacities less than 3.0 times Dead Load. If the 3.0 times Dead Load guideline is not met, then the support should be classified as an outlier. Resolution of the outlier can be accomplished by the methods described in Section 9.2.1.4.

\subsection{Ductility Check ${ }^{11}$}

An evaluation should be conducted of the supports selected for review to characterize their response to lateral seismic motion as either ductile or potentially non-ductile. Supports suspended only from overhead may be characterized as ductile if they can respond to lateral seismic motion by swinging freely without degradation of primary vertical support connections and anchorage. Ductile, inelastic performance such as clip angle yielding or vertical support member yielding is acceptable so long as deformation does not lead to brittle or premature failure of overhead vertical support.

Review of typical conduit and cable tray support systems in the earthquake experience and shake table test data bases indicates that many overhead mounted support types are inherently ductile for lateral seismic motion. Back-analysis of many data base conduit and cable tray supports predicts yielding of members and connections. These data base systems performed well, with no visible signs of distress. Ductile yielding of suspended supports results in a stable, damped swaying response mode. This is considered to be acceptable seismic response.

The ductility review of anchorage connection details is most important for rigid-type suspended raceway supports. Supports with rigid, non-ductile anchorage that do not have the capacity to develop the plastic strength of the vertical support members can possibly behave in a non-ductile fashion. Examples include large tube steel supports welded to overhead steel with relatively light welds, or rigid supports welded to large base plates and outfitted with relatively light anchorage. These types of support systems are not well represented in the data base.

11 Section 8.3.3 of SQUG GIP (Ref. 1) 
The seismic design of certain raceway support members may have been controlled by high frequency requirements rather than design loads, yet anchors may have been sized by the design loads. These types of supports may have low seismic margin due to loads placed on the support which were not considered by the original design. Supports with rigid, non-ductile anchorage are subject to further horizontal load strength review (see Section 9.2.1.3.4).

Examples of ductile and non-ductile raceway support connection details and configurations are shown in Figures 9.2.1-7 and 9.2.1-8, respectively, and are described below.

Standard Catalog Light Metal. Strut Framing Members. Clip Angles, and Bolts With Channel Nuts. The seismic experience data include many examples of unbraced supports suspended from overhead, constructed of standard catalog light metal, strut framing channels, clip angles, and bolts with channel nuts as shown in Figures 9.2.1-7A, B, C, and D. The good performance of these support types indicates that they may be characterized as ductile. This is even true of supports constructed of standard catalog light metal strut framing, gusseted, clip angle connections. Review of shake table tests of raceway support systems shows that slight slipping of channel nuts due to prying action of gusseted clip angles leads to acceptable behavior for suspended supports. The tests show that once the overhead moment connection is relaxed by this slippage, the support system is free to swing without additional degradation of the overhead connection.

Welded Steel Members. The philosophy of acceptable seismic response involving clip angle connection yielding for supports constructed of light metal, strut framing is extended to supports constructed of welded steel members as shown in Figure 9.2.1-7F. If an anchor point connection weld is stronger than the vertical member, then a plastic hinge will be able to form in the vertical member, allowing ductile response without weld failure. A support is seismically rugged so long as overhead support is maintained. In this case, plastic hinge action in the vertical member prevents transmission of loads capable of failing the welded anchorage point. For open channel structural sections, an all-around fillet weld whose combined throat thicknesses exceed the thickness of the part fastened, may be considered capable of developing the plastic hinge capacity of the open channel section vertical member. If the plastic hinge capacity of the framing support member exceeds the capacity of the weld, as shown in Figures 9.2.1-8A and B, then a brittle failure is possible, which is not acceptable seismic performance. For light metal, strut framing members, welded connections are likely to be non-ductile and thus not capable of developing plastic moment capacity of the framing member.

Ceiling Connection Plate Secured with Expansion Anchors. Raceway supports with overhead anchorage provided by a plate attached to concrete with expansion anchors may also be shown to be ductile. The anchorage may be characterized as ductile if it is stronger than the plastic flexural strength of the vertical support member. A simple anchor moment capacity estimate may be used, by multiplying the bolt pullout capacity times the distance between the bolts or center of bolt groups. In some cases, it may be possible to demonstrate ductility if the ceiling connection plate is the weak link in the anchorage load path. This is similar to the case of clip angle bending. The key to characterizing a support as ductile or non-ductile is reviewing the anchorage load path, and determining if the weak link responds in a ductile or brittle manner.

Braced Cantilever Bracket and Trapeze Frame Supports. The presence of a diagonal brace in a support, as shown in Figures 9.2.1-8E and F, has the potential of significantly increasing the pullout loads on anchorage when the support is subjected to horizontal motion. This is a function of the support geometric configuration, the realistic capacity of the brace, and the realistic capacity of the anchorage. Non-ductile behavior is possible when the brace reaction to horizontal load plus dead load has the capability of exceeding the primary the support anchor capacity. If a brace buckles or has a connection failure before primary support anchor capacity is reached, then the support may be considered as ductile. Braced supports are subject to further horizontal load capability review in Section 9.2.1.3.4 with a focus on primary support anchorage. 
Unbraced Rigid Trapeze Frames. Trapeze frames constructed as moment-resisting frames, such as those with a number of stiff cross-beam members welded to the two vertical supports as shown in Figure 9.2.1-8D, have the potential of significantly increasing the pullout loads on anchor bolts when the frame is subject to horizontal motion. Non-ductile behavior is possible when the rigid frame anchor point reactions to horizontal load exceed the anchor capacity. Unbraced rigid trapeze frames are subject to further horizontal load strength review in Section 9.2.1.3.4 with focus on anchorage.

Floor-Mounted Supports. Plastic behavior of floor-mounted supports may lead to structural instability. Ductility, as defined by these guidelines, only applies to suspended systems. Floor-mounted supports are characterized as non-ductile, and are subject to further horizontal strength review in Sections 9.2.1.3.6 and 9.2.1.3.7 with focus on stability.

Rod Hanger Trapeze Supports. Supports constructed of threaded steel rods with fixed-end connection details at the ends of the rods behave in a ductile manner under horizontal motion; however, relatively short rods may undergo very large strains due to bending imposed by horizontal seismic motion, at the fixed ends of the rods. Low cycle fatigue may govern response. Rod hanger trapeze supports with short, fixed-end rods should be evaluated for low cycle fatigue effects in Section 9.2.1.3.5.

No further review of horizontal response capability is required of supports characterized as ductile. Only the support vertical capacity need be evaluated, as discussed in Sections 9.2.1.3.1 and 9.2.1.3.2. If a support is characterized as non-ductile or has questionable ductility, then its lateral load capacity should be evaluated, as discussed in Section 9.2.1.3.4, as shown in the logic diagram for making these decisions in Figure 9.2.1-6.

\subsection{Lateral Load Check ${ }^{12}$}

A Lateral Load Check should be performed for the bounding case raceway supports that are characterized as potentially non-ductile. The Lateral Load Check is in the form of an equivalent static lateral load coefficient. The Lateral Load Check compares the ratio of horizontal load capacity divided by dead load demand (for potentially non-ductile supports) to the same ratios for support systems in the seismic experience data base that performed well. Because many of these data base raceway systems were subjected to earthquake ground motions that may have been greater than the Design Basis Earthquake for many facilities, provisions for scaling down the equivalent static horizontal loads are given below.

If a support is ductile, then no further review of horizontal response capability is required, and the support may be shown to be seismically rugged by the Vertical Capacity Check Section 9.2.1.3.2). If a support is non-ductile or has questionable ductility, then it should be analyzed for one of the following transverse load conditions:

- Dead load plus a $2.0 \mathrm{~g}$ horizontal acceleration in the transverse direction. The horizontal acceleration may be scaled down linearly by multiplying $2.0 \mathrm{~g}$ by the maximum ratio of the in-structure response spectrum (see Section 5.2) spectral acceleration for the facility divided by the corresponding spectral acceleration of the Reference Spectrum shown in Figure 5.3-2.

- Dead load plus a transverse acceleration of 2.5 times the Zero Period Acceleration (ZPA) of the floor response spectrum (see Section 5.2) for the anchor point in the facility where the raceway system is attached.

12 Section 8.3.4 of SQUG GIP (Ref. 1) 
For these loading conditions, only the tributary mass corresponding to dead load on the support should be considered. If large junction boxes are included in the worst-case sample, then the lateral load coefficients described above may be used as the seismic demand and the anchorage evaluated following the guidelines of Chapter 6.

The loading condition selected should be used consistently for all the facility raceway support systems selected as samples in any particular building. Different methods may be used for different structures. For example, the floor ZPA scaling method may be preferable for rock-founded structures or soil-founded structures for which realistic floor response spectra may be available. The scaled $2.0 \mathrm{~g}$ method may be preferable for soil-founded structures, such as diesel generator buildings, for which realistic floor response spectra may not be available.

The simple equivalent static load coefficient method may be too conservative for supports with long drops from the ceiling anchorage to the raceways. The static coefficient method predicts very high connection bending moments in these cases. In this case, the bending moment imposed on the ceiling connection may be limited by peak seismic deflection and not seismic accelerations. This is consistent with observations of back-calculated static coefficient capacities from the experience data. The lowest back-calculated capacities were often from supports with long drops and were not considered representative (i.e., they were not used to attempt to justify a static coefficient less than $2.0 \mathrm{~g}$ ).

If the support has long vertical members and has low natural frequency, then an alternative loading condition of dead load plus reaction forces due to a realistic estimate for seismic deflection imposed in the transverse direction may be used. A conservative estimate for seismic deflection may be obtained by using floor spectral displacement at a lower bound frequency estimate considering only single degree of freedom pendulum response of the support.

For diagonally-braced supports with ductile overhead anchorage, the load reaction imposed on the support anchorage during the Lateral Load Check does not need to exceed the buckling capacity of the brace or its connections. For example, if it is shown that a brace buckles at $0.80 \mathrm{~g}$ lateral load, then this load should be used for the Lateral Load Check and not $2.0 \mathrm{~g}$. For diagonally-braced supports where the anchorage is not ductile, the portion of the lateral load that is not resisted by the brace should be redistributed as bending stress to the overhead connection. The loads in the diagonal brace will cause additional vertical and horizontal loads on the anchorage, which should be accounted for.

An upper and lower bound estimate should be used for buckling capacity of the brace, whichever is worse, for the overhead anchorage. There is considerable variation in test data capacity for light metal strut framing connections. An upper bound estimate of 2.0 times the realistic capacities discussed in Section 9.2.1.3.8 can be used for these connection types.

\subsection{Rod Hanger Fatigue Evaluations ${ }^{13}$}

Shake table tests have shown that the seismic capacity of fixed-end rod hanger trapeze supports is limited by the fatigue life of the hanger rods. Rod hanger trapeze supports should be evaluated for possible fatigue effects if they are constructed with fixed-end connection details. This fatigue evaluation should be done in addition to the checks described in the previous sections.

13 Section 8.3.5 of SQUG GIP (Ref. 1) 
Fixed-end connection details include double-nutted rod ends at connections to flanges of steel members, rods threaded into shell-type concrete expansion anchors, and rods connected by rod coupler nuts to nonshell concrete expansion anchors. Fixed-end connection details also include rods with lock nuts at cast-in-place light metal strut channels and rod coupler nuts welded to overhead steel.

This section describes a screening method for evaluating rod hangers for fatigue based on the use of rod fatigue bounding (capacity) spectra (shown in Figure 9.2.1-9) and generic rod fatigue evaluation screening charts (shown in Figures 9.2.1-10 to 9.2.1-14). This screening method is based upon generic, bounding case fatigue evaluations in Reference 48.

The screening charts are directly applicable to hangers constructed of manufactured all-thread rods in raceway system runs with uniform length hangers. The charts may also be used for evaluation of supports constructed of field-threaded rods, and for short, isolated fixed-end rod hangers in more flexible systems with relatively much longer rod hangers; guidance is given later in this section on how to adjust the parameters when evaluating these special cases.

\section{Manufactured All-Thread Rods}

The fatigue evaluation for short, fixed-end rod hangers (manufactured all-thread) in trapeze supported raceway runs with all of the rods of uniform length, should proceed as follows:

- Obtain the 5\% damped floor response spectrum (see Section 5.2) for the location of the support.

- $\quad$ Enter Figure 9.2.1-9 which contains Rod Fatigue Bounding (Capacity) Spectrum anchored to $0.33 \mathrm{~g}, 0.50 \mathrm{~g}$, and $0.75 \mathrm{~g}$. Select a spectrum which envelopes the floor response spectrum. If the selected spectrum does not entirely envelop the floor response spectrum, then select a spectrum that envelops the floor response spectrum at the resonant frequency of the support.

Support resonant frequency may be estimated as follows:

$$
\mathrm{f}_{\text {support }}=\frac{1}{2 \pi} \sqrt{\frac{\mathrm{K}_{\mathrm{s}}}{\mathrm{M}_{\mathrm{s}}}}
$$

Where:

$$
\begin{aligned}
& \mathrm{M}_{\mathrm{S}}=\mathrm{W} / \mathrm{g} \\
& \mathrm{K}_{\mathrm{S}}=2\left(12 \mathrm{E} \mathrm{I} / \mathrm{L}^{3}\right)+\mathrm{W} / \mathrm{L} \\
& \mathrm{W}=\text { total dead weight on the pair of rod supports } \\
& \mathrm{g}=\text { gravitational constant } \\
& \mathrm{E}=\text { elastic modulus of steel } \\
& \text { I = moment of inertia of rod root section } \\
& \mathrm{L}=\text { length of rod above top tier }
\end{aligned}
$$


- $\quad$ Enter one of the Fatigue Evaluation Screening Charts shown in Figures 9.2.1-10 to 9.2.1-14 corresponding to the diameter of the threaded rod. Focus on the curve associated with the acceleration $(0.33 \mathrm{~g}, 0.50 \mathrm{~g}$, or $0.75 \mathrm{~g}$ ) of the Rod Fatigue Bounding Spectrum selected in the previous step. These charts do not directly apply to field-threaded rods (see discussion below).

- Compare the rod hanger length ( $\mathrm{L}$, length of rod above top tier) and rod hanger weight $(\mathrm{W}$, total dead weight on the pair of rod supports) with acceptable combinations of length and weight on the screening charts. Acceptable regions of the Fatigue Evaluation Screening Charts are below and to the right of the Screening Chart curve selected in the previous step.

If the support parameters are within acceptable regions on the Fatigue Evaluation Screening Chart, then the rod hanger support is seismically adequate.

The screening charts also include the 3 times Dead Load limit associated with the Vertical Capacity Check (Section 9.2.1.3.2) which can be used to facilitate evaluation of expansion anchors (based on reduction factor of 0.75 for anchor capacity determination in Section 6.3) for rod hanger trapeze supports.

\section{Field-Threaded Rods}

Rod fatigue tests have shown that field-threaded rods have less fatigue life than all-thread, manufactured rods. The evaluation method for field-threaded rods proceeds the same way as for manufactured threaded rods, except that adjusted weights and lengths should be used for comparison with the Fatigue Evaluation Screening Charts. For field-threaded rods, enter the Screening Charts with double the actual weight and $2 / 3$ the actual length of the rods. If these modified parameters are in acceptable regions of the Screening Charts, then the rod hanger is seismically adequate.

\section{Isolated. Short. Fixed-End Rod Hangers}

If an isolated, short, fixed-end rod hanger is used in a system with predominantly longer, more flexible hangers, a special evaluation should be conducted that decouples the response effects of the short isolated rod. The special evaluation method is as follows:

- $\quad$ Estimate the frequency of the support system, neglecting the isolated, short rod. The frequency estimation formula given above may be used. The length of the longer rods should be used in the formula.

- $\quad$ Enter the applicable Fatigue Evaluation Screening Chart (Figures 9.2.1-10 to 9.2.1-14) which corresponds to the Rod Fatigue Bounding Spectrum (Figure 9.2.1-9) that envelops the facility floor response spectrum (5\% damping) (see Section 5.2) at the frequency of interest which was calculated in the previous step.

- Back-calculate an equivalent weight for evaluation of the isolated short rod hanger, using the frequency of the longer rod hanger supports, with the following formula:

$$
W_{\text {equiv. }}=\frac{24 E I g}{(2 \pi f)^{2} L^{3}-g L^{2}}
$$

- $\quad$ Enter the appropriate Fatigue Evaluation Screening Chart (Figures 9.2.1-10 to 9.2.1-14) by using the above calculated equivalent weight and the length of the isolated short rod hanger. 
If these parameters are in an acceptable region on the Fatigue Evaluation Screening Chart, then the isolated, short, fixed-end rod hanger is seismically adequate.

Reference 48 may be reviewed to obtain an understanding of the analytical methods used to develop the Fatigue Evaluation Screening Charts. When using the charts, the simple equations given in this section for calculating response frequency should be used for consistency since these are the same equations used to generate the screening charts (i.e., the screening charts are based on the simplified results obtained from detailed fatigue analysis, considering capacities determined by component test results).

\subsection{Floor-to-Ceiling Support Evaluations ${ }^{14}$}

Floor-to-ceiling supports may be evaluated as suspended raceway supports if they can meet the previous Limited Analytical Review Checks by conservatively neglecting the floor connection and anchorage.

Seismic ruggedness for floor-to-ceiling supports that depend on the floor connection may be evaluated as follows. The checks described here ensure seismic adequacy by showing that the supports maintain high vertical capacity, demonstrate ductility, and maintain connection shear resistance.

The lower vertical support column member should be checked for buckling. The imposed buckling load should be the portion of 3.0 times Dead Load that cannot be resisted by the overhead anchorage. In addition, the support should be subject to a Lateral Load Check. The imposed lateral load static coefficient should be obtained as described in Section 9.2.1.3.4. The top and bottom connections and anchors should be checked for dead load plus the equivalent static lateral load reactions. Clip angle bending stresses may be ignored. The support columns themselves do not have to be checked for lateral loading; however, the entire support should be checked for design dead load as described in Section 9.2.1.3.1.

\subsection{Base-Mounted Support Evaluations $^{15}$}

Base-mounted supports present a different case than suspended supports in that, with excessive deflections and inelastic response effects, the base-mounted supports tend to become unstable whereas suspended supports have increased pendulum restoring force. The checks which should be performed include a detailed Dead Load Check and Lateral Load Check non-concurrently in both orthogonal directions, including P-delta effects if base hardware slip may be anticipated. Pdelta effects include the second order increases in base overturning moment due to additional eccentricity of the supported dead load during seismic deflections of the support. These P-delta effects may become significant if the connection hardware at the base of the support does not remain rigid. Base hardware slips that should be considered are discussed below. Reference 50 provides considerable discussion on the philosophy of the base-mounted support evaluations.

A detailed, Dead Load Check should be performed, similar to the check described in Section 9.2.1.3.1. The only exception is that clip angle bending stresses should be evaluated at the base connections. Base flexibility associated with clip angle inelastic behavior may lead to increased deflection and subsequent P-delta effects and possibly instability.

14 Section 8.3.6 of SQUG GIP (Ref. 1)

15 Section 8.3.7 of SQUG GIP (Ref. 1) 
A Vertical Capacity Check should not be conducted since the philosophy behind the Vertical Capacity Check only applies to ductile, suspended raceway supports. A Dead Load plus equivalent static Lateral Load Check should be performed instead, for loading non-concurrently in both orthogonal directions.

The equivalent static lateral load should be determined as outlined in Section 9.2.1.3.4. The Lateral Load Check should evaluate all members, connections, and anchors associated with the primary support frame and its bracing (if present). Realistic capacities should be used for the evaluation. If brace members (lower bound capacity estimate) cannot resist all of the lateral load, the portion of load exceeding the brace capacity may be transmitted to the base and resisted by the base moment capacity.

If light metal strut framing clip angle construction is used, bolt (with channel nut) slip of $1 / 16$ inch should be considered for P-delta evaluation. If the nominal capacities given in Section 6.3 are used for nonshell expansion anchors, anchor bolt slip of 1/8 inch should be considered for P-delta evaluation. For P-delta evaluation, all these bolt slips should be used to obtain an estimate for maximum possible base connection rotation.

Using this base rotation, and considering the displacement due to the flexibility of the vertical support post, a deflection of the raceways should be calculated. This additional deflection times dead load provides the effective P-delta base moment. If this moment is more than about $5 \%$ of the total moment from the Dead Load plus Lateral Load Check, it should be included in the Dead Load plus Lateral Load Check.

Torsional moments at the base of the support post that may result from lateral or longitudinal load checks may be ignored. Stresses in the support brackets due to longitudinal loading may also be ignored. These forces resulting from longitudinal loading are not considered realistic due to raceway member framing action and inelasticity of other components in the load resistance chain such as restraining clips. The goal of the lateral and longitudinal checks is to demonstrate seismic ruggedness.

\subsection{Allowable Capacities ${ }^{16}$}

The allowable capacities which can be used in the Limited Analytical Review are discussed in this section. For the Dead Load Check (Section 9.2.1.3.1), normal engineering design working stress allowable capacities should be used. For example, the capacities defined in Part 1 of the AISC Specification for Steel Design (Ref. 81) can be used.

More realistic allowable capacities can be used for the remainder of the checks in the Limited Analytical Review (Sections 9.2.1.3.2 to 9.2.1.3.7).

The remainder of this subsection defines these capacities for expansion anchors, cast-in-place anchors, embedded plates and channels, welds, steel bolts, structural steel, and other support members.

Capacity values for expansion anchors are provided in Section 6.3. The guidelines for using these anchorage capacities should be followed, including edge distance, bolt spacing, and inspection procedures. Note that tightness checks need not be conducted for anchor bolts of supports which resist tensile force under dead load. Tightness checks are waived because suspended and some wall-mounted raceway systems cause these types of anchorage to be subjected to constant tension under dead load and therefore the anchorage are, in effect, continuously proof-tested. The tightness checks should be carried out, however, for floor-mounted support anchors.

16 Section 8.3.8 of SQUG GIP (Ref. 1) 
Capacity values for embedded steel which uses headed studs are given in Section 6.3. These capacities should be used along with the generic guidelines contained in Chapter 6 . For cast-inplace embedments, other than those which use headed studs, the capacity may be determined using the approach discussed in Section 6.2.6, Embedment Steel and Pads.

The facility design or as-built drawings for cast-in-place anchors and steel plates should be reviewed to obtain details on these anchorage types. Anchor capacities for cast-in-place light metal strut framing channels should be taken as the manufacturer's catalog values with published factors of safety, or may be determined by available test information with appropriate factors of safety.

Capacities for welds, structural steel, and steel bolts should be taken as defined in Part 2 of the AISC Specification for Steel Design (Ref. 81). Capacity values for light metal strut framing hardware are taken as the manufacturer's recommended design values, including the published factor of safety. This factor of safety is considered sufficient to encompass the lower bounds of strength values, such as may result from minor product variation or low bolt torque.

When upper-bound strength estimates are required, such as in ductility reviews or limit state evaluations, the manufacturer's catalog capacities should be increased. A recommended upper bound estimate for bolts with channel nuts is double the manufacturer's published design values.

Tests may be used to establish realistic, ultimate capacities of raceway components. Appropriate factors of safety should be used with these test results. Dynamic tests should be performed to establish ultimate capacities of friction-type connections in most cases.

\subsection{Raceway System Weights ${ }^{17}$}

Cable tray weights may be estimated as 25 pounds per square foot for a standard tray with 4 inches of cable fill. It is suggested that the cable trays be considered to be completely full during the initial attempt at using the screening guidelines described above. Linear adjustment may be made for trays with more and less cable fill. Sprayed-on fireproof insulation may be conservatively assumed to have the same unit weight by itself as the cable in the tray it covers.

Estimated weights for steel and aluminum conduit may be taken as follows:

\begin{tabular}{|c|c|c|}
\hline \multirow{2}{*}{$\begin{array}{c}\text { Conduit } \\
\text { Diameter } \\
\text { (inches) }\end{array}$} & \multicolumn{2}{|c|}{$\begin{array}{c}\text { Conduit Weight } \\
\text { Including Cable } \\
\text { (pounds per foot) }\end{array}$} \\
\hline $1 / 2$ & Steel & Aluminum \\
\hline $3 / 4$ & 1.0 & 0.5 \\
\hline 1 & 1.4 & 0.7 \\
\hline $1-1 / 2$ & 2.2 & 1.1 \\
\hline 2 & 3.6 & 1.8 \\
\hline $2-1 / 2$ & 5.1 & 2.8 \\
\hline 3 & 8.9 & 5.2 \\
\hline 4 & 12.8 & 7.9 \\
\hline 5 & 16.5 & 9.5 \\
\hline
\end{tabular}

Conservative estimates should be made for the weights of other miscellaneous items attached to the raceway support, such as HVAC ducting, piping, and lighting.

17 Section 8.3.9 of SQUG GIP (Ref. 1) 


\subsubsection{Outliers $^{18}$}

An outlier is defined as a raceway hardware feature which does not meet one or more of the screening guidelines contained in this section. Namely, an outlier:

- Does not meet the Inclusion Rules given in Section 9.2.1.2.2,

- Has significant Other Seismic Performance Concerns as given in Section 9.2.1.2.3,

- Has potential adverse seismic interaction hazard as given in Section 9.2.1.2.5, or

- Does not satisfy the Limited Analytical Review Guidelines given in Section 9.2.1.3.

When an outlier is identified, proceed to Chapter 12, and document the cause(s) for not meeting the screening guidelines on an Outlier Seismic Evaluation Sheet (OSES).

The screening criteria given earlier in this section are intended for use as a generic basis to evaluate the seismic adequacy of cable and conduit raceway systems. If a raceway hardware feature fails this generic screen, it may not necessarily be deficient for seismic loading; however, additional evaluations are needed to show that it is adequate. Some of the additional evaluations and alternate methods for demonstrating seismic adequacy are summarized below. Additional details are also found in the previous subsections where these generic screening guidelines are described. Other generic methods for resolving outliers are found in Chapter 12.

In some cases it may be necessary to exercise engineering judgment when resolving outliers, since strict adherence to the screening guidelines in the previous subsections is not absolutely required for raceway support systems to be seismically adequate. These judgments, however, should be based on a thorough understanding of the background and philosophy used to develop these screening guidelines as described in References 46, 47, and 50. The justification and reasoning for considering an outlier to be acceptable should be based on mechanistic principles and sound engineering judgment.

The screening guidelines contained in the previous subsections have been thoroughly reviewed by industry experts to ensure that they are appropriate for generic use; however, the alternative evaluation methods and engineering judgments used to resolve outliers are not subject to the same level of peer review. Therefore, the evaluations and judgments used to resolve outliers should be thoroughly documented so that independent reviews can be performed if necessary.

\subsection{Cable Tray Span ${ }^{19}$}

As discussed in Inclusion Rules 1 and 2, the span lengths given there are not necessarily rigid requirements. For example, an isolated cable tray span of about 13 feet may be acceptable if the tray is lightly loaded and of rugged construction (for example, the tray meets the NEMA standards in Reference 89 and the cable loading is no more than one-half that in Table 3-1 of Reference 89).

\subsection{Conduit $\operatorname{Span}^{20}$}

An isolated conduit overspan may be acceptable if its vertical deflection is limited by other facility features in proximity. In addition, 3.0 times dead load vertical static load tests can be used to show that an isolated overspan is acceptable.

18 Section 8.4 of SQUG GIP (Ref. 1)

19 Section 8.4.1 of SQUG GIP (Ref. 1)

20 Section 8.4.2 of SQUG GIP (Ref. 1) 


\subsection{Raceway Member Tie-downs ${ }^{21}$}

Tie-downs should be installed until Inclusion Rule 3 is satisfied. As an alternative, analyses or a static lateral pull test of the lateral load-carrying capacity of the as-built trays or conduit can be performed to show that the trays or conduit are not capable of falling off the support. The amount of static lateral force used in this evaluation should be consistent with one of the options in the Lateral Load Check given in Section 9.2.1.3.4. It is preferable, and usually not a difficult maintenance activity, to add missing raceway member tie-downs.

\subsection{Channel Nuts ${ }^{22}$}

Channel nuts without teeth should be replaced with nuts with teeth or an extensive facility-specific dynamic testing program can be performed to show that the channel nuts without teeth are capable of carrying the anticipated seismic load.

\subsection{Rigid Boot Connection ${ }^{23}$}

Rigid boots are considered to be outliers even when there is only a small gap between the boot and the member it supports. If the boot was field assembled in such a way that no gaps exist and the boot fits the member tightly, then this connection can be considered acceptable. The basis for the finding that there are no gaps should be thoroughly documented. One simple fix to a rigid boot with gaps is to replace the individual bolts with one through bolt.

\subsection{Beam Clamps $^{24}$}

The clamp should be replaced with a positive connection or the clamp oriented so that gravity loads are not resisted by the clamping friction; however, if supported loads are less than about 15 pounds, the adequacy of an isolated clamp oriented in the wrong direction can simply be evaluated by tugging and shaking it by hand.

If an entire run of small conduit with light support dead loads (less than about 15 pounds per support) is anchored with beam clamps which resist dead load only by clamping friction, then a sufficient number of supports representative of the entire conduit run should be tugged to evaluate adequacy.

\subsection{Cast-Iron Anchor Embedments ${ }^{25}$}

Cast-iron anchor embedments should be replaced with an acceptable anchorage or the support braced horizontally and the stress in the anchor kept very low.

\subsection{Analytical Outliers ${ }^{26}$}

Outliers that do not satisfy the Limited Analytical Review guidelines, as illustrated in Figure 9.2.16 , can be evaluated further using more detailed analytical models of the raceway system or testing to demonstrate that the raceways are as rugged as required. Remember, however, that the analytical guidelines only have to be satisfied in an approximate manner. For example, if a support has a capacity of only 2.7 times Dead Load rather than the desired 3.0 times Dead Load, the SRT

\footnotetext{
Section 8.4.3 of SQUG GIP (Ref. 1)

Section 8.4.4 of SQUG GIP (Ref. 1)

Section 8.4.5 of SQUG GIP (Ref. 1)

Section 8.4.6 of SQUG GIP (Ref. 1)

Section 8.4.7 of SQUG GIP (Ref. 1)

Section 8.4.8 of SQUG GIP (Ref. 1)
} 
performing the screening evaluation may still find the support acceptable based on their professional judgment. Examples of acceptable outlier evaluation methods include Limit State Evaluations, Lateral Load Evaluations, Redundancy and Consequence Evaluations, and Support Upgrades. These methods are described below.

Limit State Evaluation. A limit state evaluation may be used to resolve ductile supports that do not meet the Vertical Capacity Check (3.0 times Dead Load) in Section 9.2.1.3.2. The Vertical Capacity Check provides a quick, generic means for assuring seismic ruggedness, consistent with the experience data. However, for certain configurations of raceway support systems, especially unbraced rod hanger trapeze systems, the Vertical Capacity Check may be too conservative.

The principle behind the Limit State Check is that the support anchorage capacity need only be greater than the maximum possible reactions from plastic hinge formation in the support, while the support is also subjected to dead load. This principle only applies to supports that are suspended from above and that are characterized as ductile, following the guidelines of Section 9.2.1.3.3.

The Limit State Evaluation provides a check of anchorage and anchorage connection capacity. The seismic demand applied to the anchor point using the limit state evaluation method is based on dead load plus anchor reaction due to formation of plastic hinges at credible support joint locations. Realistic upper bound estimates should be used for the support joint plastic hinge moment capacities, based on test results if possible.

The basic philosophy for the Limit State Check is that for ductile supports suspended from the overhead, anchor connection capacity need only exceed the maximum possible reactions resulting from the plastic hinges developed in the support, plus dead loads.

For rod hanger trapeze supports with fixed-end connection details, the Limit State Check is straightforward. The anchor capacity should be greater than dead load reaction plus the reaction from plastic hinges formed in the hanger rods at fixed-end connections. For multiple tier hangers, as a first approximation, plastic hinge formation may be assumed at all joints at all tiers. If the lateral deflection corresponding to onset of all these plastic hinges is excessive, such as if it is greater than the peak floor spectral displacement, then a more refined evaluation may be conducted. This may be accomplished by considering a realistic deflected shape for those locations where credible plastic hinges can be formed.

For threaded rods, the plastic hinge moment capacity should be consistent with those observed in the rod hanger fatigue tests (see Reference 48). The plastic moment capacity may be calculated using the rod hanger's cross-sectional moment of inertia based on the root diameter of the threaded section, a 1.7 shape factor, and a $90 \mathrm{ksi}$ apparent yield stress. For example, the plastic moment capacity of a 1/2-inch diameter threaded rod may be taken as 1,010 inch-pounds.

The anchorage shear load for the Limit State Evaluation may be calculated by estimating a point of inflection in the limit state deflection shape. For example, for a rod hanger trapeze support, the point of inflection may be taken as the mid-point between the top tier cross beam and the overhead anchorage.

Limit State Evaluations of light metal strut framing trapeze supports constructed with clip angles may assume that plastic hinges develop in all clip angles, with the strut framing members remaining rigid. The anchorage capacity should be greater than dead load reaction, plus frame reaction at the anchor point due to the formation of plastic hinges at all clip angles, plus reaction due to local prying action at the anchor due to a plastic moment in its clip angle. 
The local prying anchor load may be taken as the connection ultimate moment capacity divided by the distance between anchors for double clip angle connections. For single clip connections, the moment may be divided by the distance from the anchor bolt to the far edge of the light metal strut framing vertical member. The moment capacities for clip angle connections can be very difficult to estimate by calculation so it is better to base these moment capacities on test data if possible.

Lateral Load Evaluation. The Lateral Load Check of Section 9.2.1.3.4 may be used to evaluate outliers that do not meet the Vertical Capacity Check (3.0 times dead load) in Section 9.2.1.3.2. This is most applicable to supports characterized as non-ductile in Section 9.2.1.3.3, but may also be used for ductile supports.

Redundancy and Consequence Evaluation. Isolated cases of an outlier support which does not meet the Limited Analytical Review Guidelines described in Section 9.2.1.3 may be resolved if the adjacent raceway support system has high redundancy, and if a postulated failure of the support in question has no adverse consequence to facility safety, e.g., it will not fall on safety-related equipment and damage it. High redundancy can be demonstrated by showing that the adjacent supports are suspended and meet the Vertical Capacity Check ( 3 times Dead Load) of Section 9.2.1.3.2, and either the Ductility Check of Section 9.2.1.3.3 or the Lateral Load Check of Section 9.2.1.3.4.

"Isolated" means that it is not acceptable for as many as every other support to fail to meet the guidelines. In other words, there should be at least two supports, each of which meets the guidelines of Section 9.2.1.3.2 and either Section 9.2.1.3.3 or Section 9.2.1.3.4, between each "isolated" support.

The "consequence" of a failed isolated support should also be evaluated to determine whether there is any undesirable effect on nearby equipment. Engineering judgment should be used by the $S C E S$ to make this evaluation. If it is not credible for the support to swing away or fall, then there is no safety consequence. If it is credible for the support to swing away or fall, then it should be treated as a source of seismic interaction. In this case, there is no safety consequence if there are no fragile, safety-related targets in the vicinity or below.

Acceptance of worst-case, bounding supports by the Redundancy and Consequency Evaluation described above does not provide, by itself, sufficient insight into the seismic ruggedness of the facility's raceway support systems. Rather, this option should be used during the walkdown to screen out isolated instances of supports which appear marginal, so as to exclude them from the bounding case sample.

Support Upgrade. For certain supports which do not meet the Limited Analytical Review Checks, it may be preferable to strengthen these supports rather than expend resources on more refined analyses and evaluations.

When upgrading raceway supports, the facility may wish to use the Limited Analytical Review guidelines in this section as the starting point in the design process. It is recommended that new designs or retrofit designs use additional factors of safety, especially for anchorage, since the incremental added cost for larger anchor bolts is not significant but it leads to significantly larger seismic margin. 


\subsubsection{Documentation ${ }^{27}$}

A summary package should be assembled to document and track the SCE's evaluation activities. Suggested documentation should include records of the facility areas evaluated, the dates of the walkdowns, the names of the engineers conducting the evaluations, and a summary of results. Recommended Seismic Evaluation Work Sheets (SEWS) for the summary package are given in Chapter 13. Outlier Seismic Evaluation Sheets (OSES) are also given in Chapter 13. Included in the SEWS are:

Separate summary sheets should be completed for each designated room number or facility location where evaluations are conducted. The sheets include reminders, as a checklist, for primary aspects of the evaluation guidelines; however, the walkdown engineers should be familiar with all aspects of the seismic evaluation guidelines during screening reviews and not rely solely on the checklist. The SCEs who sign these sheets are ultimately responsible for the seismic evaluations conducted.

Analytical Review Data Sheet for recording information on the supports selected as the worst-case, representative samples.

Chapter 13 describes the Outlier Seismic Evalution Sheet. When collecting these data, the SCEs should record ample information so that repeated trips to the facility are not required for final outlier resolution.

Photographs may be used to supplement documentation, as required. When used as formal documentation for the summary packages, photographs should be clearly labeled for identification.

27 Section 8.5 of SQUG GIP (Ref. 1) 


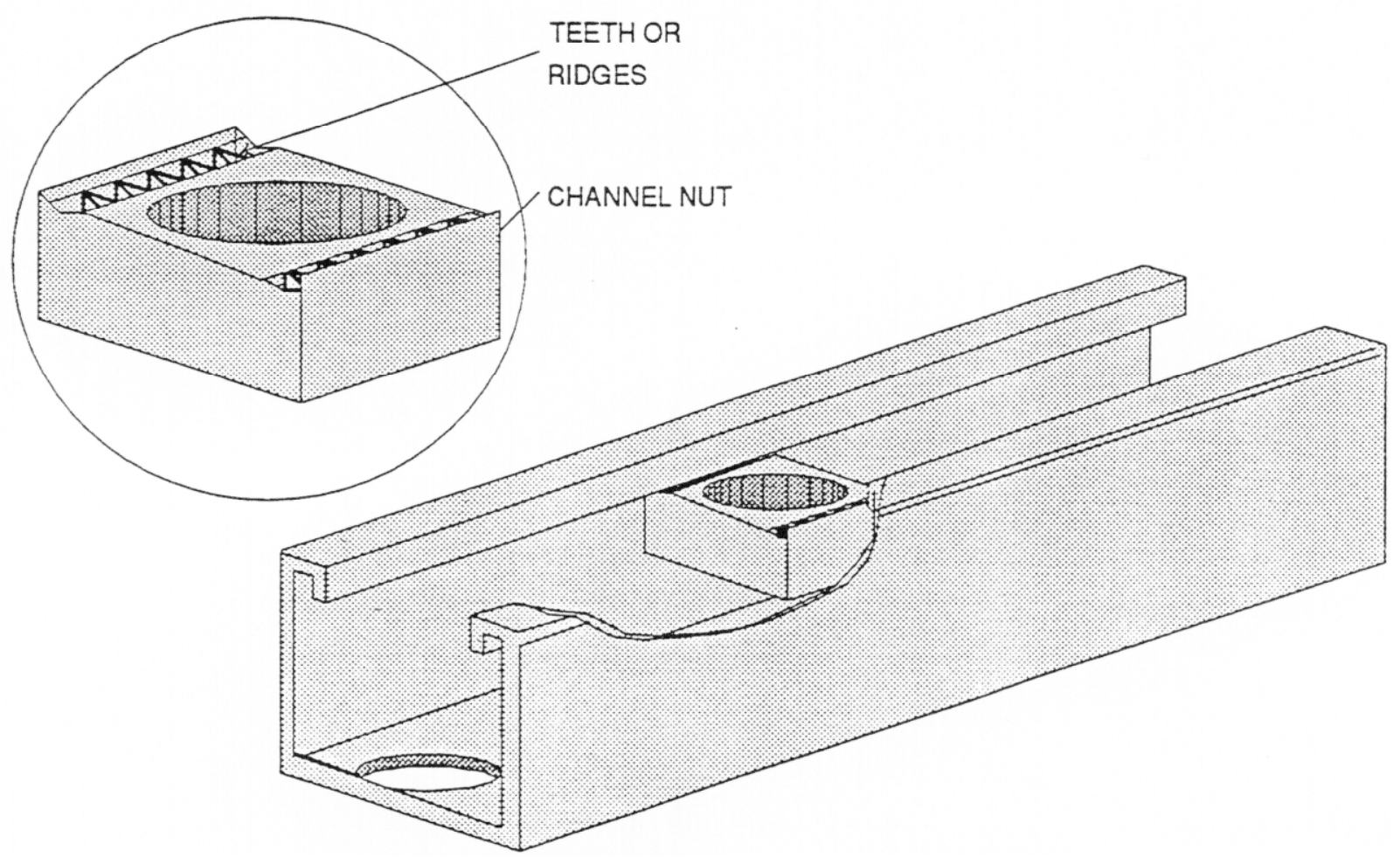

Figure 9.2.1-1 Channel Nut with Teeth or Ridges in Light Metal Framing Strut (Reference 47) (Figure 8-1 of SQUG GIP, Reference 1) 
(a)

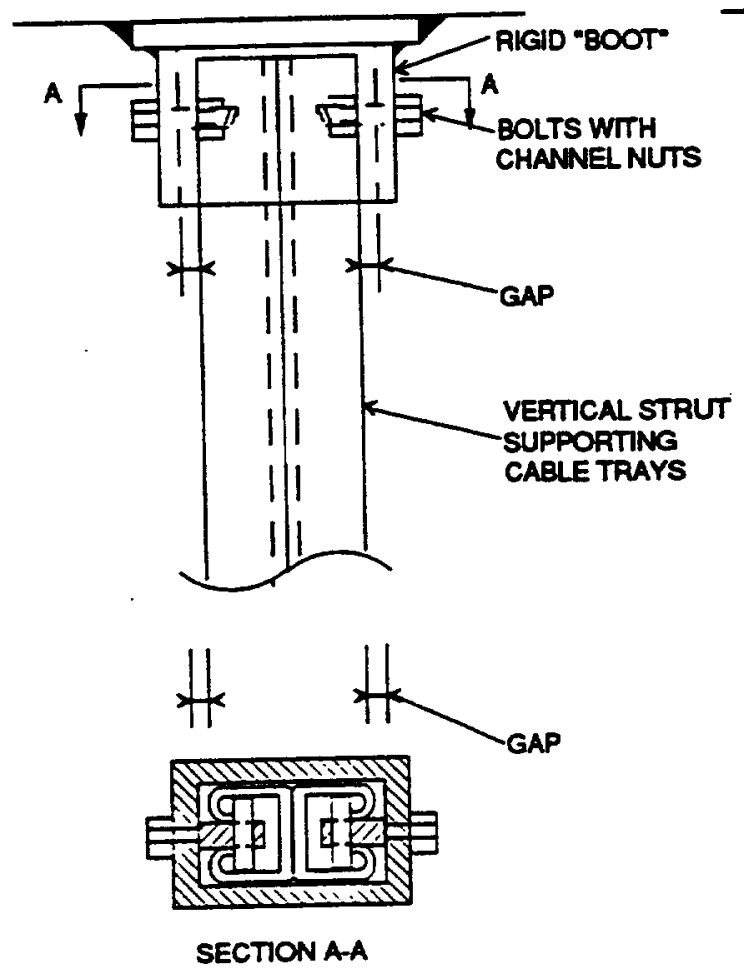

(b)

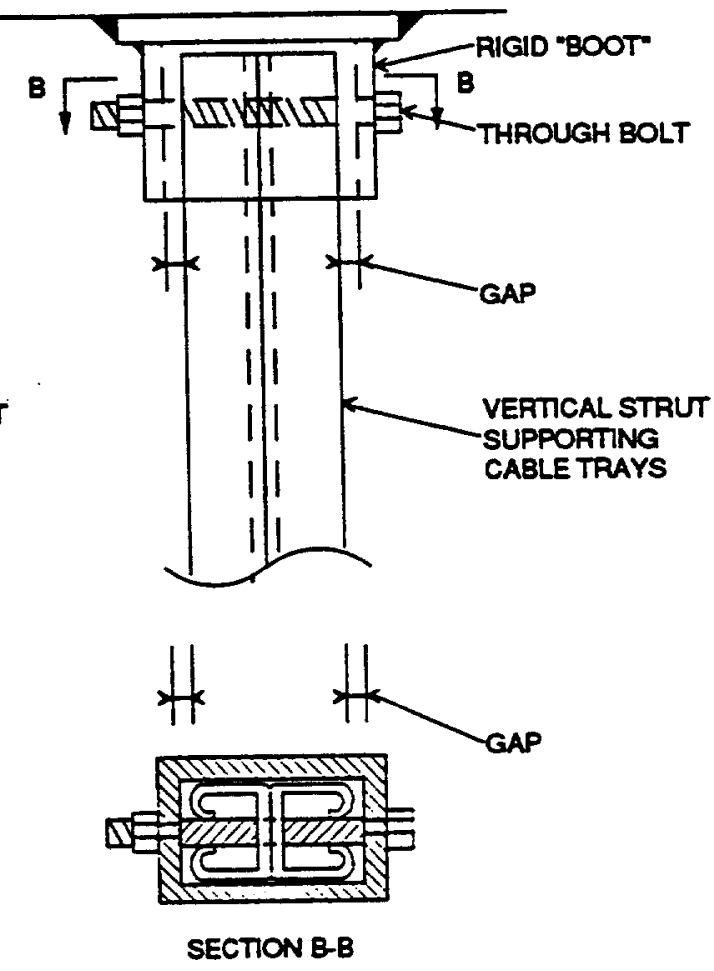

(a) Rigid "Boot" Connection Detail That Failed in Shake Table Test (b) Addition of a Through Bolt Corrected the Design Flaw.

Note: The size of the gap is exaggerated for emphasis. Any size gap, no matter how small, is a possible concern.

Figure 9.2.1-2 Rigid Boot Connection Details (Reference 47) (Figure 8-2 of SQUG GIP, Reference 1) 


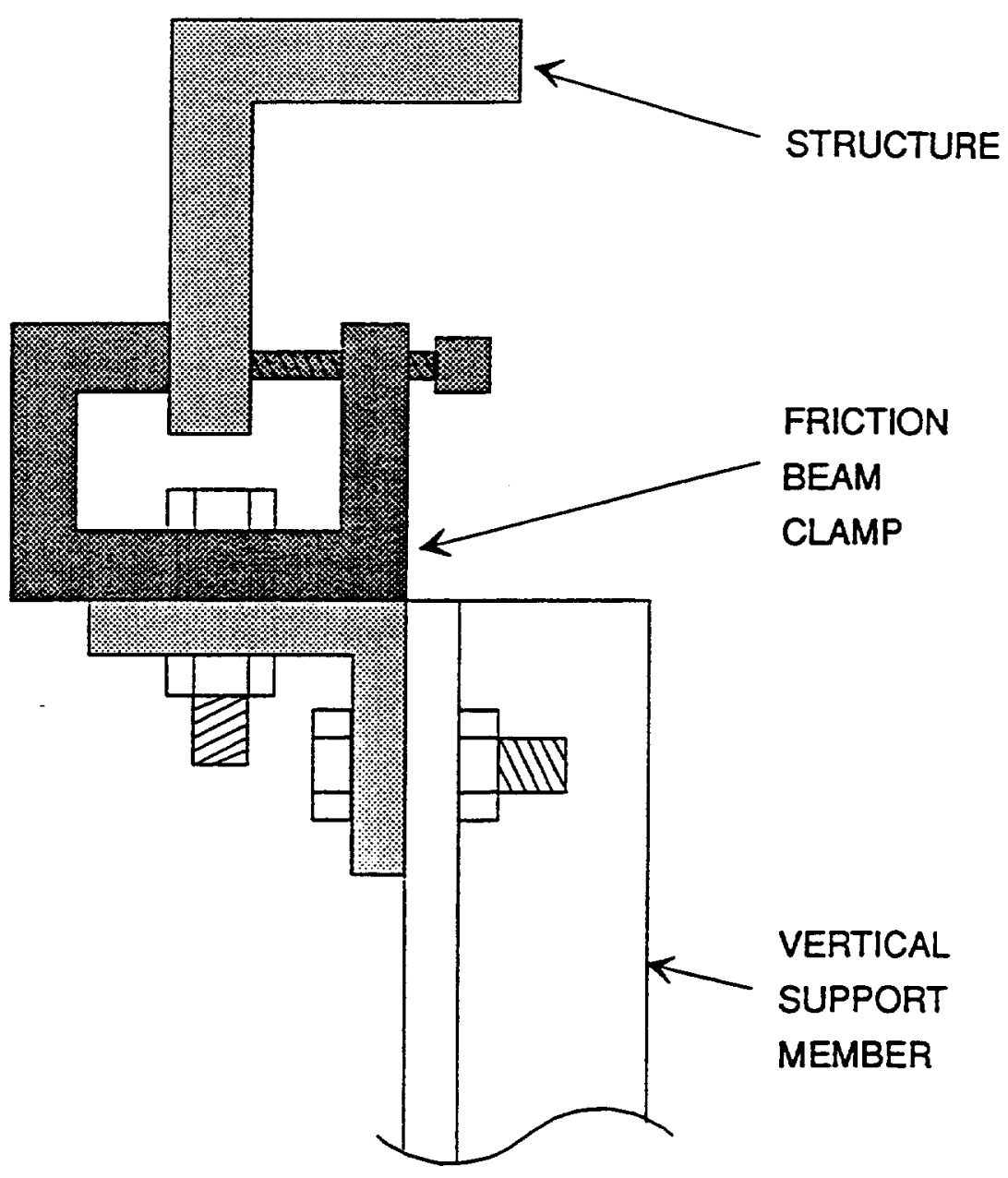

Note: This arrangement may loosen and slip, resulting in support collapse.

Figure 9.2.1-3 Beam Clamps Oriented with Dead Load Resisted Only by Clamping Friction (Reference 47) (Figure 8-3 of SQUG GIP, Reference 1) 


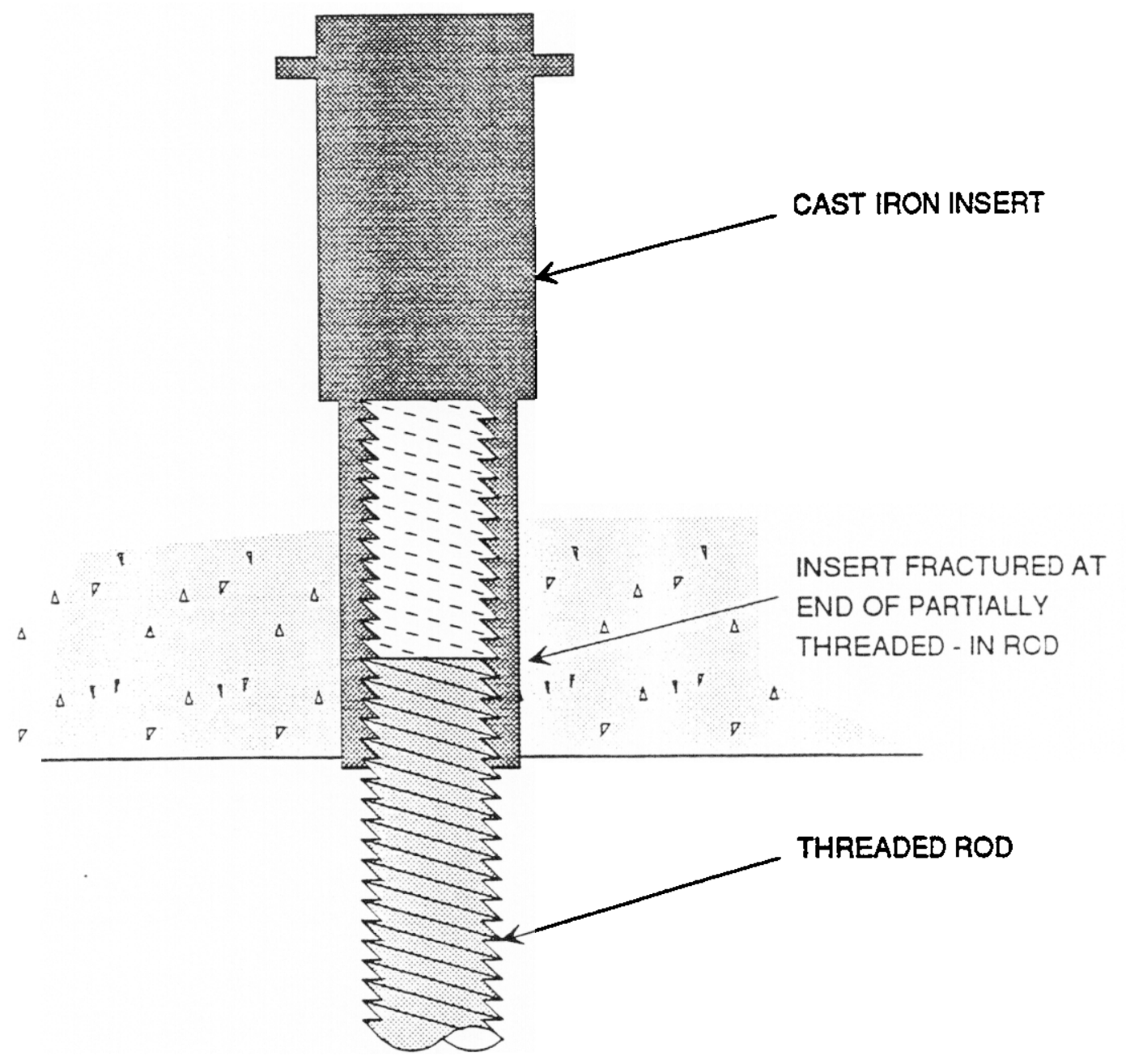

Figure 9.2.1-4 Cast-Iron Anchorage Detail that Failed at the Pacific Bell Alhambra Station, 1987 Whittier Earthquake (Reference 47) (Figure 8-4 of SQUG GIP, Reference 1) 

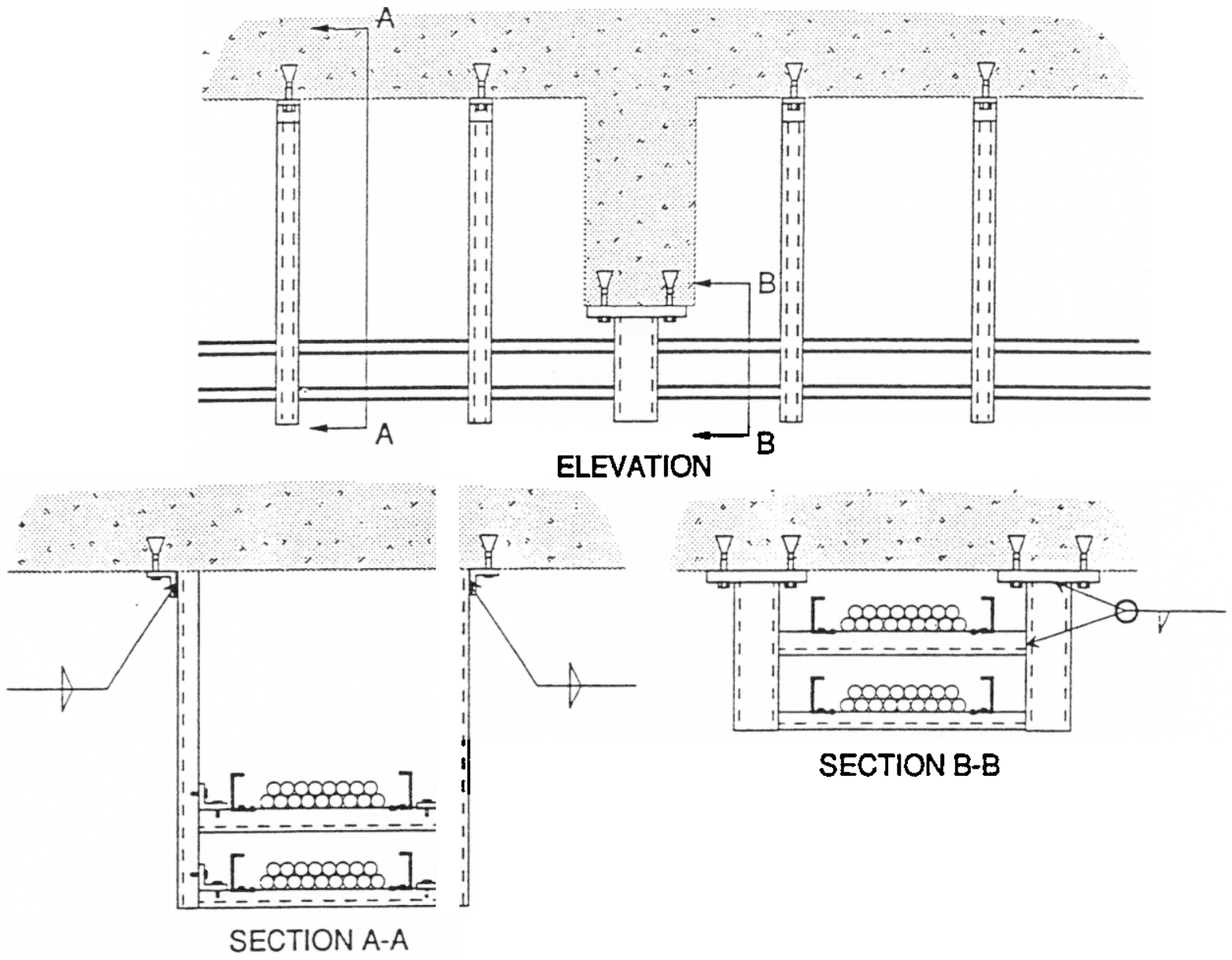

Note: The short, stiff support may attract considerable load from longitudinal motion during an earthquake.

Figure 9.2.1-5 Short, Stiff Support in a System of Longer, More Flexible Supports (Reference 47) (Figure 8-5 of SQUG GIP, Reference 1) 


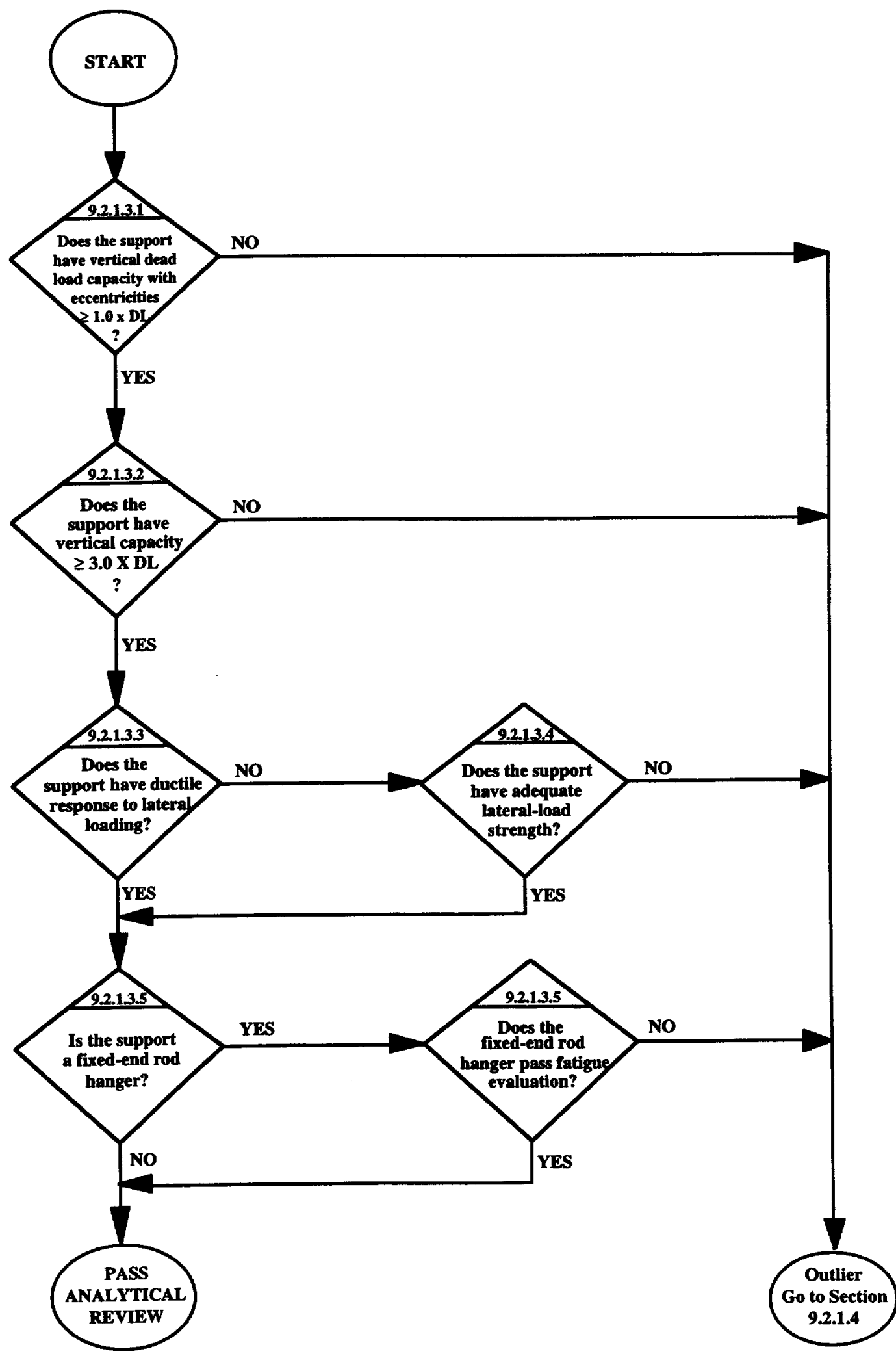

Figure 9.2.1-6 Logic Diagram for Limited Analytical Review of Suspended Raceway Supports (Figure 8-6 of SQUG GIP, Reference 1) 

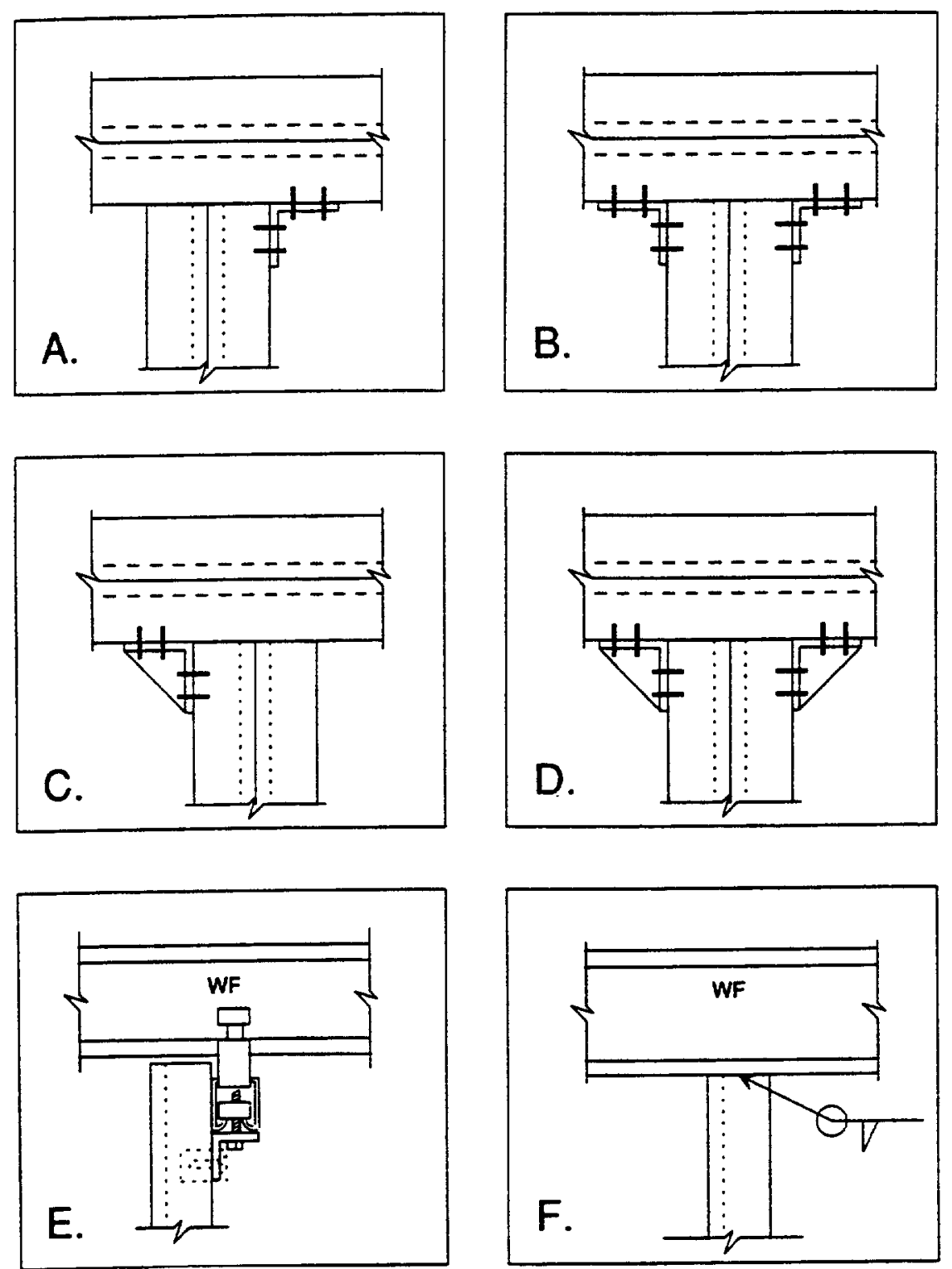

Notes: - Connections A, B, C, and D are ductile connections of standard catalog, light metal, strut framing systems.

- Connection $\mathrm{E}$ is a properly oriented beam clamp, configures as a pin-ended connection. Pin-ended connections are considered ductile.

- Connection $F$ is an all-around fillet weld on a structural steel angle section. If combined weld throat thickness is larger than the steel angle flange thickness, this may be considered a ductile connection.

- Connections $C$ and $D$ are ductile if the vertical bolts are into steel members as shown. If the vertical bolts are into concrete, the connections may not be ductile and should be checked.

Figure 9.2.1-7 Examples of Inherently Ductile Raceway Support Connection Details and Configurations (Reference 47) (Figure 8-7 of SQUG GIP, Reference 1) 

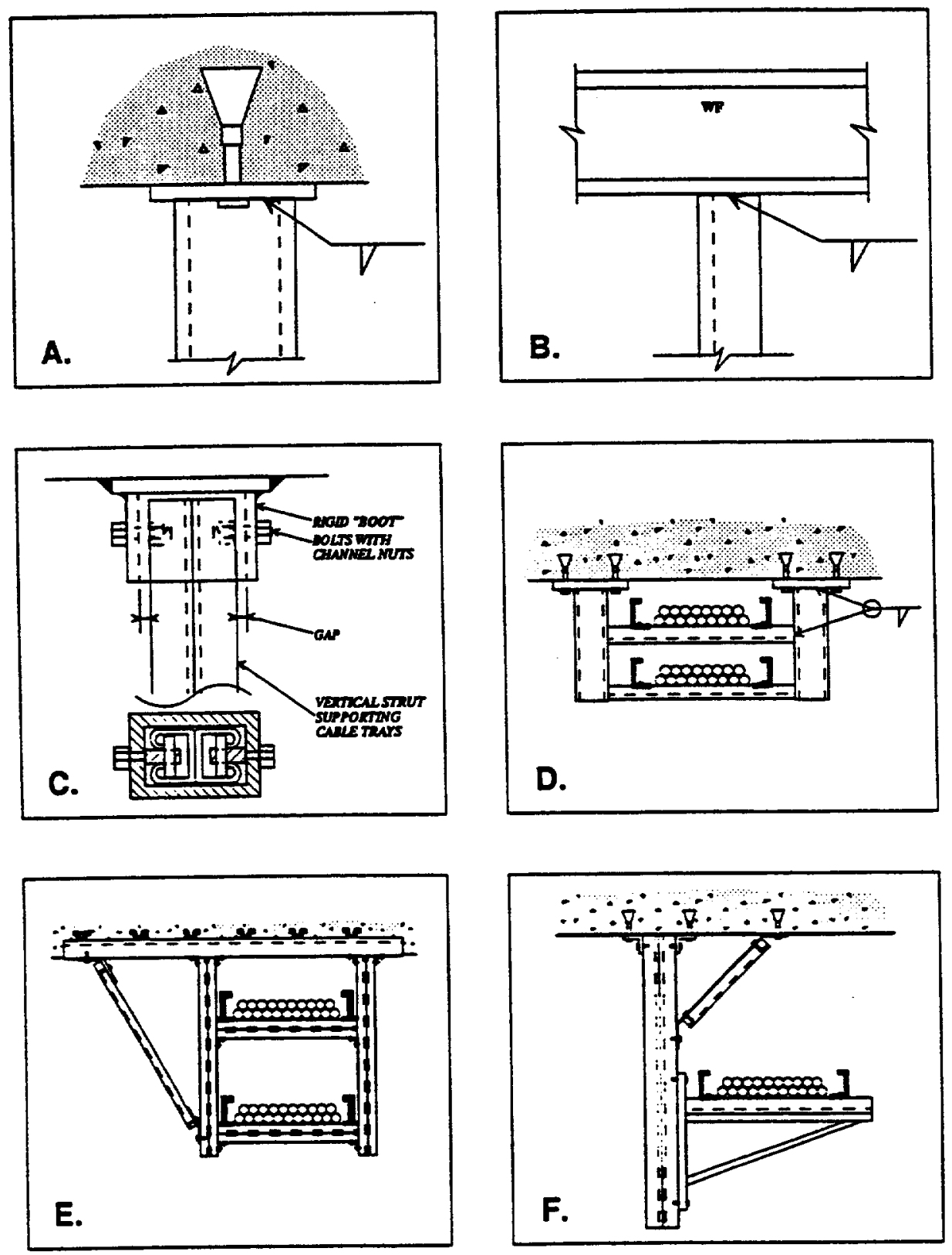

Notes: - Connections A and B are partially welded connection details. Partial welds cannot develop the plastic moment capacity of the vertical member, and are considered non-ductile.

- Connection $C$ is the non-ductile rigid boot connection.

- Connection D is a rigid moment-resisting frame and should be checked for horizontal load.

- Connections $\mathrm{E}$ and $\mathrm{F}$ are diagonally braced, and should be checked for horizontal load.

Figure 9.2.1-8 Examples of Potentially Non-Ductile Connection Details and Configurations (Reference 47) (Figure 8-8 of SQUG GIP, Reference 1) 


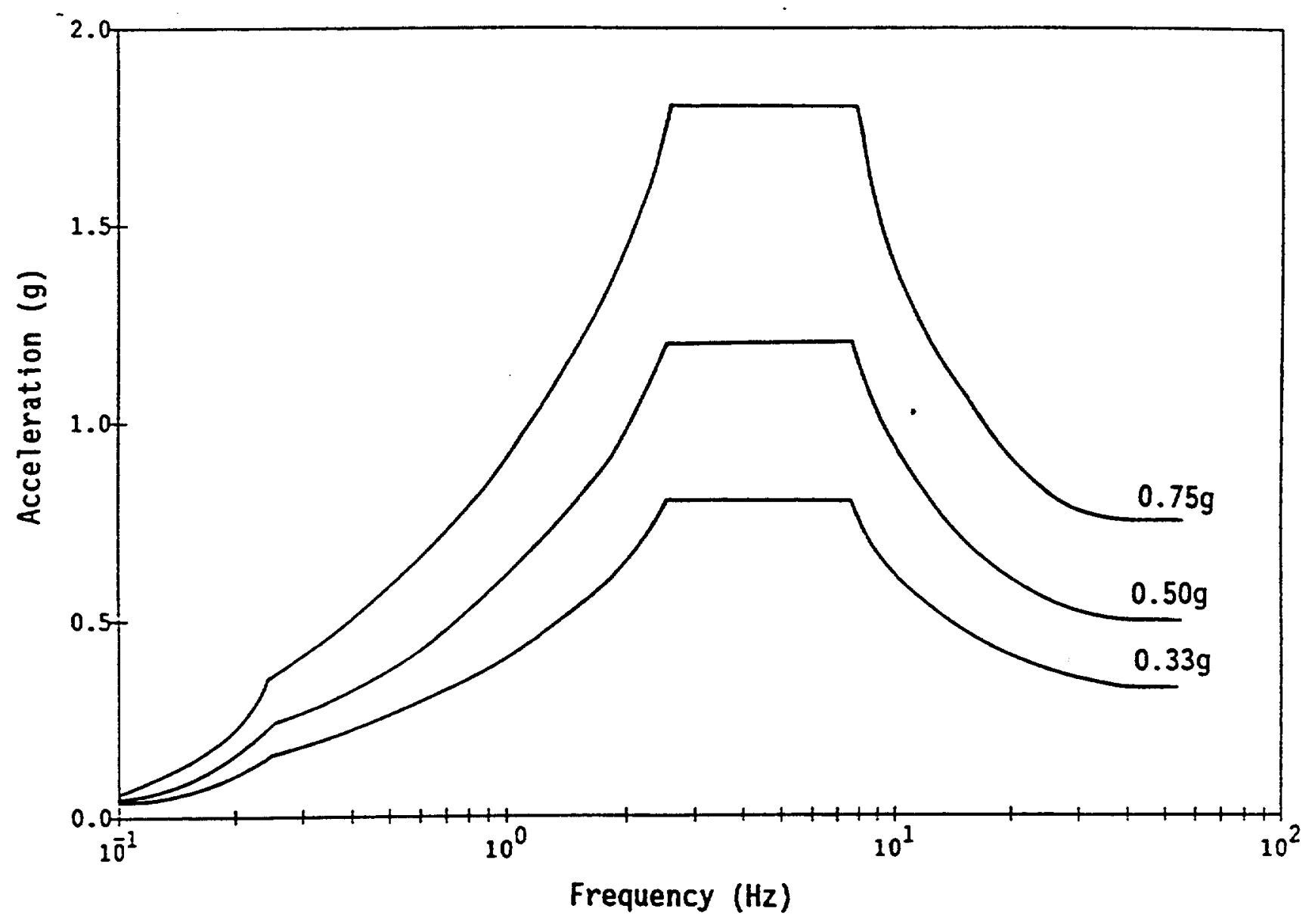

Figure 9.2.1-9 Rod Fatigue Bounding (Capacity) Spectrum Anchored to 0.33g, $0.50 \mathrm{~g}$, and $0.75 \mathrm{~g}$ (Reference 47) (Figure 8-9 of SQUG GIP, Reference 1) 


\section{1/4" THREADED RODS}

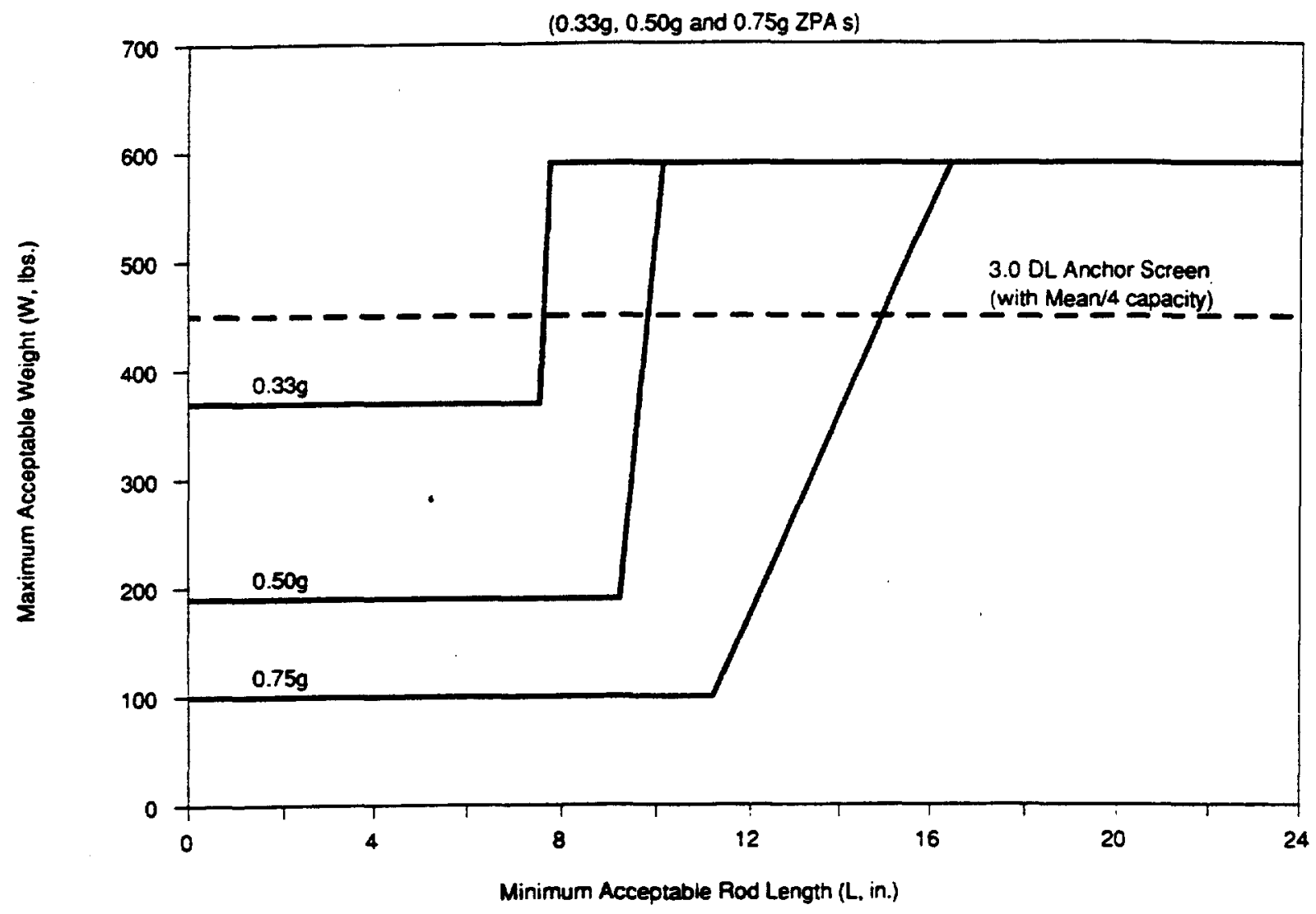

Notes: - "W" corresponds to the total dead weight of the support (i.e., carried by both rods).

- " $\mathrm{L}$ " corresponds to the clear length above the top tier.

Figure 9.2.1-10 Fatigue Evaluation Screening Chart for 1/4-Inch Diameter Manufactured All-Thread Rods (Reference 47) (Figure 8-10 of SQUG GIP, Reference 1) 


\section{3/8" THREADED RODS}

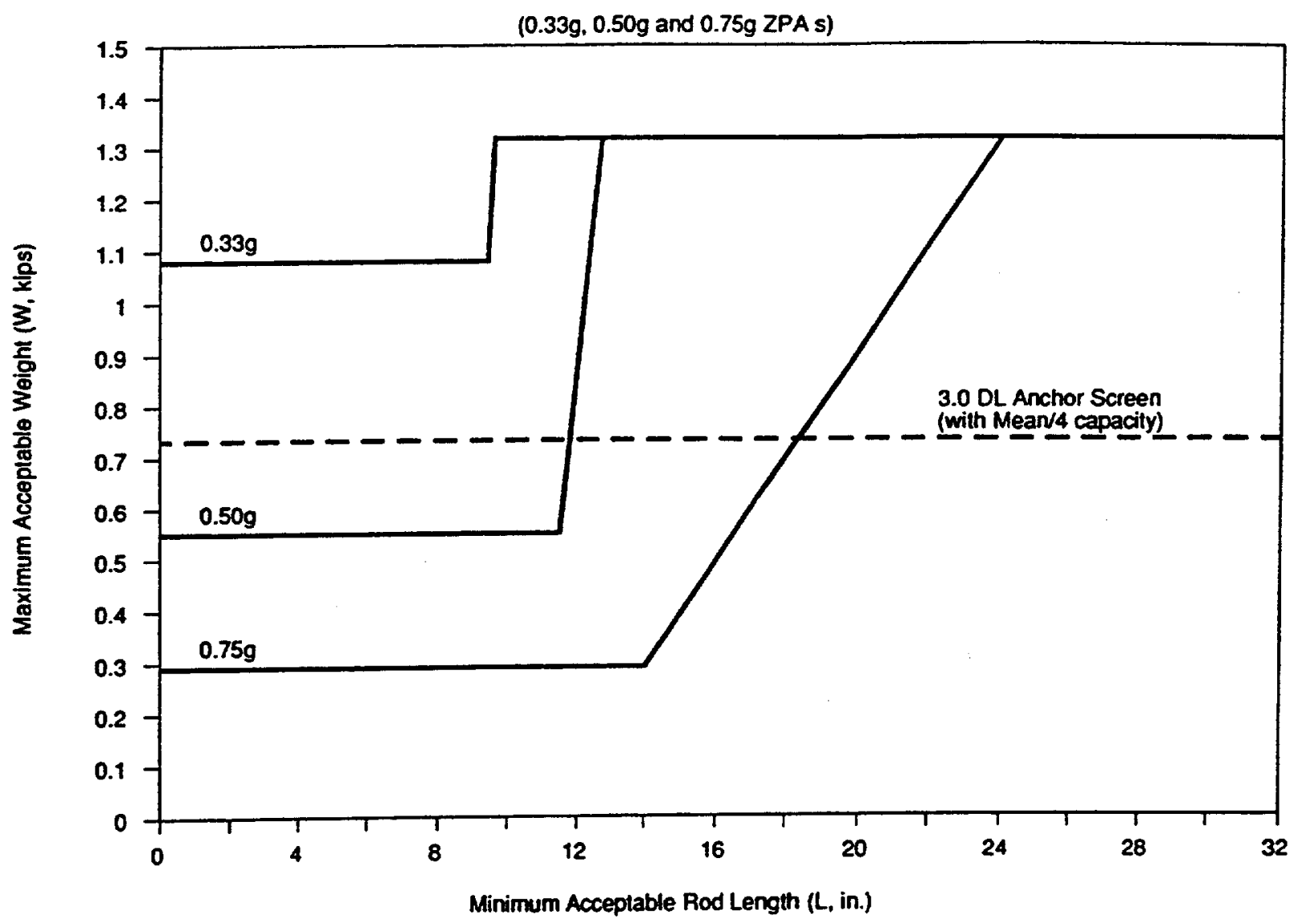

Notes: - "W" corresponds to the total dead weight of the support (i.e., carried by both rods).

- $\quad \mathrm{L}$ " corresponds to the clear length above the top tier.

Figure 9.2.1-11 Fatigue Evaluation Screening Chart for 3/8-Inch Diameter Manufactured All-Thread Rods (Reference 47) (Figure 8-11 of SQUG GIP, Reference 1) 


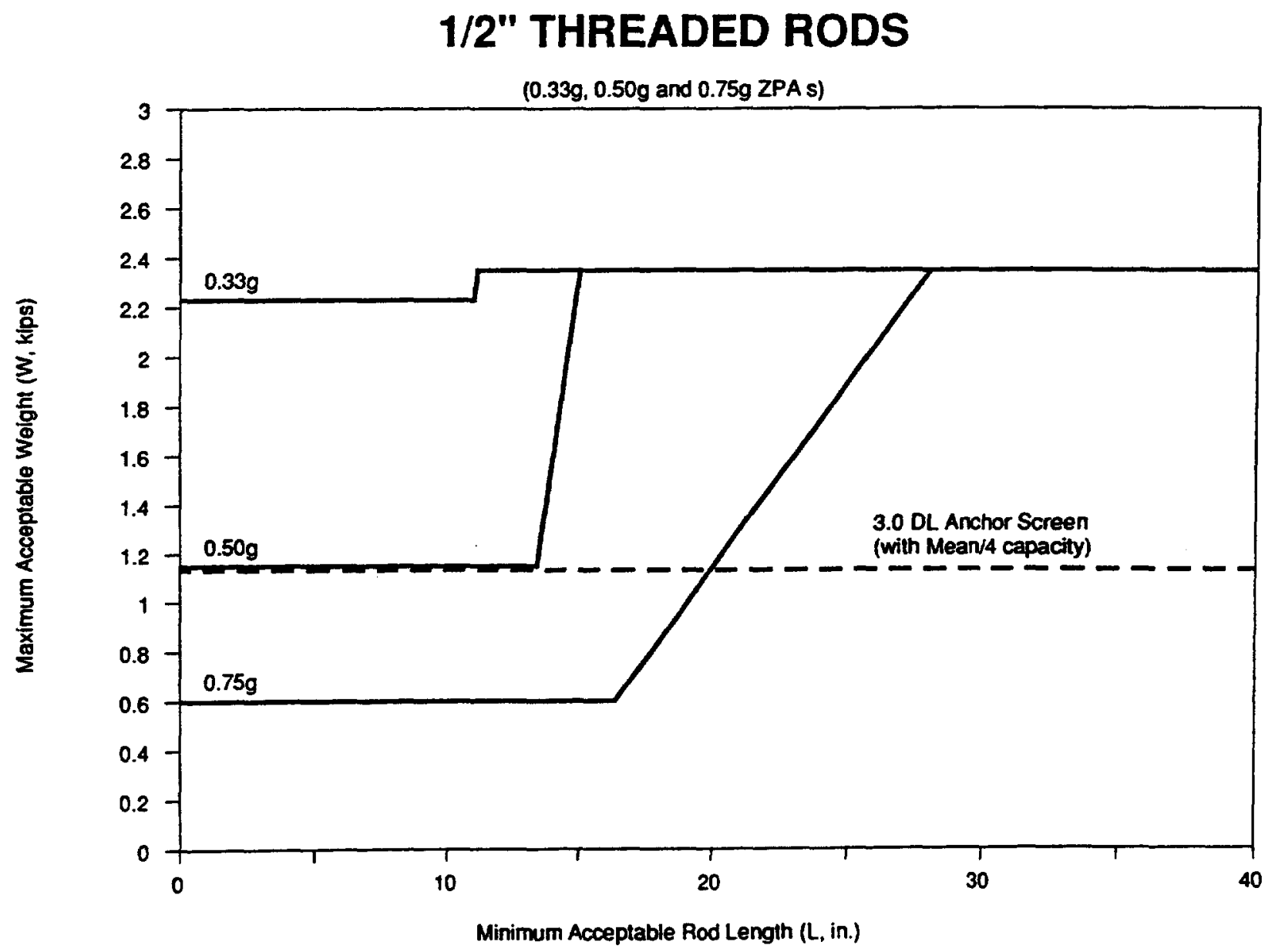

Notes: - "W" corresponds to the total dead weight of the support (i.e., carried by both rods).

- " $\mathrm{L}$ " corresponds to the clear length above the top tier.

Figure 9.2.1-12 Fatigue Evaluation Screening Chart for 1/2-Inch Diameter Manufactured All-Thread Rods (Reference 47) (Figure 8-12 of SQUG GIP, Reference 1) 


\section{5/8" THREADED RODS}

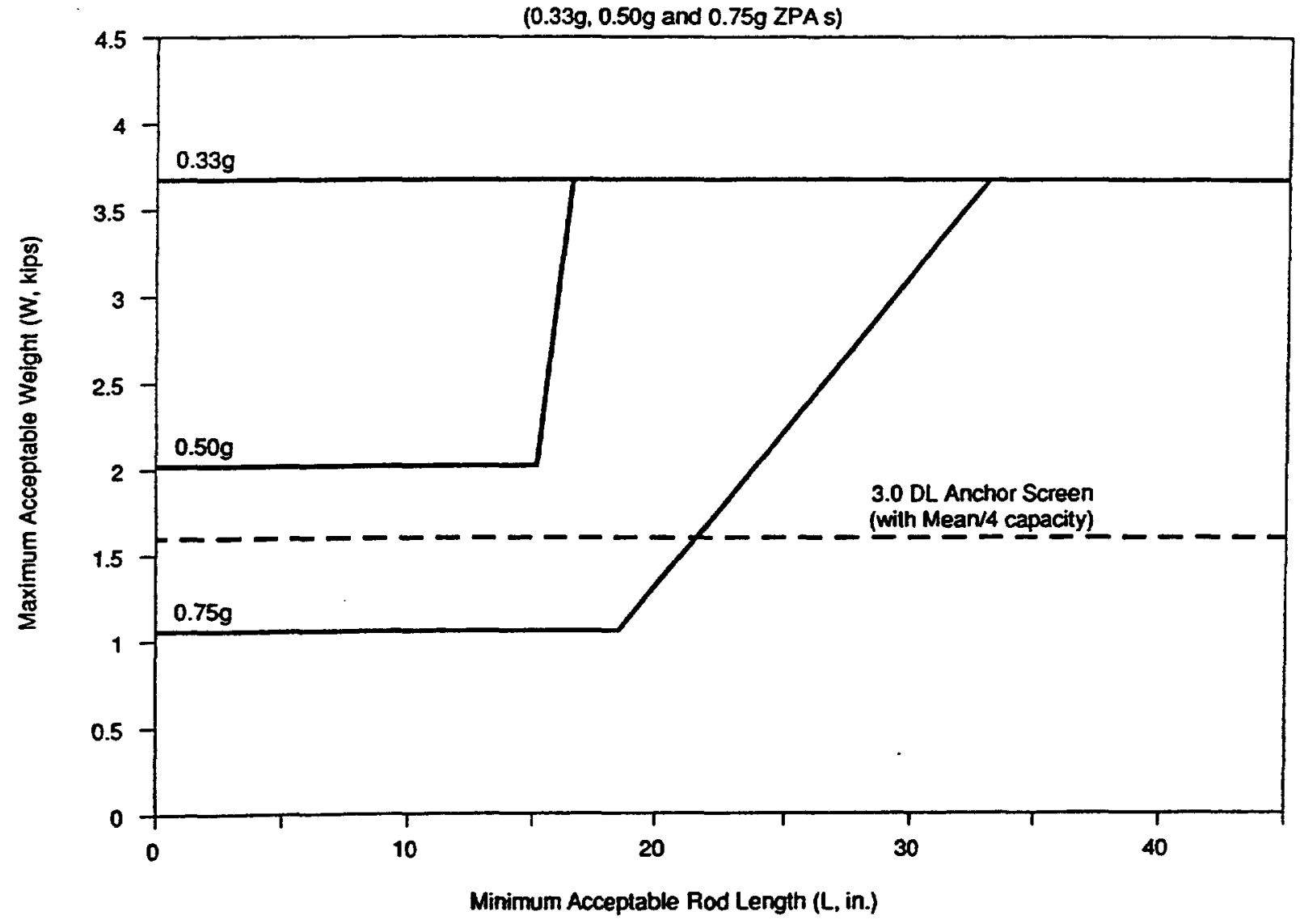

Notes: - "W" corresponds to the total dead weight of the support (i.e., carried by both rods).
- "L" corresponds to the clear length above the top tier.

Figure 9.2.1-13 Fatigue Evaluation Screening Chart for 5/8-Inch Diameter Manufactured All-Thread Rods (Reference 47) (Figure 8-13 of SQUG GIP, Reference 1) 


\section{3/4" THREADED RODS}

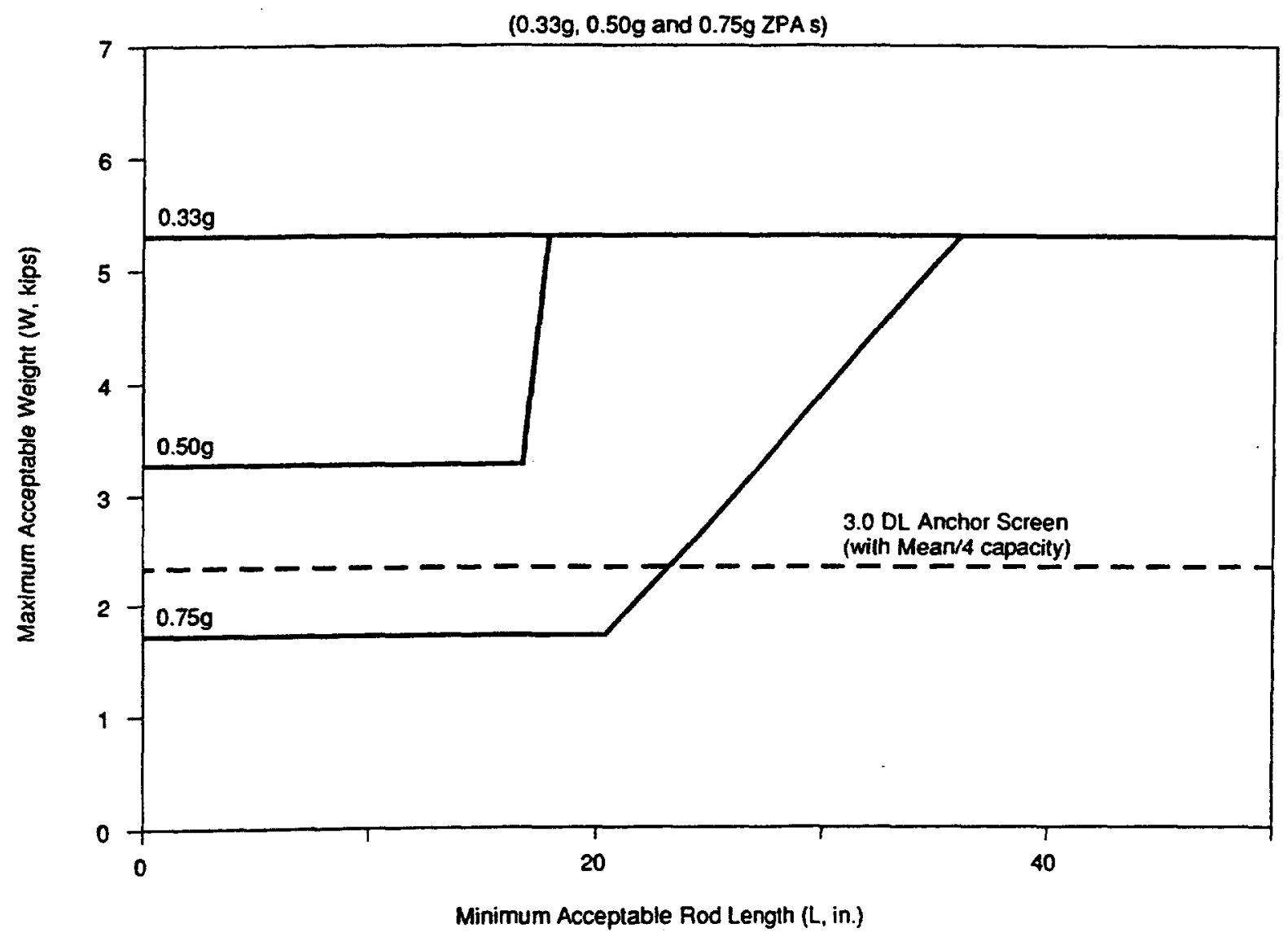

Notes: - "W" corresponds to the total dead weight of the support (i.e., carried by both rods).
- "L" corresponds to the clear length above the top tier.

Figure 9.2.1-14 Fatigue Evaluation Screening Chart for 3/4-Inch Diameter Manufactured All-Thread Rods (Reference 47) (Figure 8-14 of SQUG GIP, Reference 1) 


\section{Part IV}

\section{Seismic Evaluation Procedures Developed Uniquely for the DOE}





\section{EQUIPMENT CLASS EVALUATIONS USING SCREENING PROCEDURES OR GENERAL GUIDELINES}

Chapter 10 contains a summary of equipment class descriptions and parameters based on earthquake experience data, test data, and analytical derivations. The classes of equipment contained in Chapter 10 are not from the SQUG GIP (Ref. 1). Much of the information in Chapter 10 is from DOE references. Table 2.1-4 lists the principal references and authors for the sections in Chapter 10. An item of equipment must have the same general characteristics as the equipment in the screening procedures and general guidelines. The intent of this rule is to preclude items of equipment with unusual designs and characteristics that have not demonstrated seismic adequacy in earthquakes or tests.

The screening procedures in Sections 10.1.1, 10.4.1, and 10.5.1, for evaluating the seismic adequacy of piping, HVAC ducts, and unreinforced masonry (URM) walls respectively, cover those features which experience has shown can be vulnerable to seismic loading. These procedures are a step-by-step process through which the important equipment parameters and dimensions are determined, seismic performance concerns are evaluated, the equipment capacity is determined, and the equipment capacity is compared to the seismic demand. Sections 10.1.1 and 10.4.1 have been technically reviewed and used extensively at several DOE sites including Savannah River Site and Rocky Flats Environmental Technology Center.

The general guidelines for evaluating the seismic adequacy of the equipment classes in the other sections of Chapter 10 cover those features which experience has shown can be vulnerable to seismic loading. The sections contain practical guidelines and reference to documents that can be used to implement an equipment strengthening and upgrading program. The relatively simple seismic upgrades are designed to provide cost-effective methods of enhancing the seismic safety of the equipment classes in Chapter 10. Sections 10.3.1 and 10.1.2 summarize information from portions of a DOE document that has undergone extensive technical review. Sections 10.2.1, 10.2.2, 10.2.3, 10.3.2, 10.5.2, and 10.5.3, on the other hand, are based on walkdown and seismic strengthening efforts at several DOE sites including Los Alamos National Laboratory and Lawrence Livermore National Laboratory. 


\subsection{PIPING SYSTEMS}

\subsubsection{PIPING}

This section is the "Procedure for the Seismic Evaluation of Piping Systems Using Screening Criteria", WSRC-TR-94-0343 (Ref. 59) which was developed by the Westinghouse Savannah River Company. Some of the background material for this section is contained in References 52 through 55 and the technical review of this section is summarized in Reference 27.

\subsubsection{Objective}

This procedure may be used to evaluate the seismic adequacy of piping systems within the Scope, Section 10.1.1.2, and subject to the Cautions, Section 10.1.1.3.

The procedure may be used alone or with the rest of the DOE Seismic Evaluation Procedure, depending on the piping system's required function, listed in Table 10.1.1-1.

Table 10.1.1-1 Procedures Applicable to Required Piping System Functions

\begin{tabular}{|c|c|c|c|c|c|}
\hline FUNCTIONS & $\begin{array}{c}\text { Delivers } \\
\text { Flow? }\end{array}$ & $\begin{array}{l}\text { Equipment } \\
\text { Operating? }\end{array}$ & $\begin{array}{c}\text { Leak } \\
\text { Tight? }\end{array}$ & $\begin{array}{l}\text { Not } \\
\text { Fall? }\end{array}$ & PROCEDURE \\
\hline Operability & Yes & Yes & Yes & Yes & $\begin{array}{c}\text { Piping Screens and DOE Seismic } \\
\text { Evaluation Procedure } \\
\text { for Equipment }\end{array}$ \\
\hline $\begin{array}{l}\text { Maintain } \\
\text { Integrity of } \\
\text { Pressure } \\
\text { Boundary }\end{array}$ & No & No & Yes & No & $\begin{array}{c}\text { Piping Screens and DOE Seismic } \\
\text { Evaluation Procedure } \\
\text { for Equipment Anchorage }\end{array}$ \\
\hline $\begin{array}{c}\text { Position } \\
\text { Retention }\end{array}$ & No & No & No & Yes & Subset of Piping Screens \\
\hline
\end{tabular}

Features of a piping system that do not meet the screening criteria are called outliers. Outliers must be resolved through further evaluations (see Chapter 12), or be considered a potential source of seismically induced failure. Outlier evaluations, which do not necessarily require the qualification of a complete piping system by stress analysis, may be based on one or more of the following: simple calculations of pipe spans, search of the test or experience data, vendor data, industry practice, or other appropriate methodology. 


\subsubsection{Scope}

This procedure applies to existing (installed), safety or non-safety related, above ground metallic piping or tubing systems constructed of materials listed in ASME B31.1 (Ref. 90), ASME B31.3 (Ref. 91), NFPA (Ref. 92), or AWWA (Ref. 93), with the following restrictions:

1. Pipe materials must be ductile at service temperatures. Cast iron materials are excluded. Non ferrous alloys with a specified ultimate tensile strength (UTS) of less than $30 \mathrm{ksi}$ are excluded. Welded aluminum materials are excluded. Soldered joints are outliers.

2. Diameter-to-thickness ratio (D/t) of pipe must be 50 or less. In terms of pipe thickness $(t)$, the thickness must be greater than the diameter (D) divided by 50 .

3. Operating temperature must be below $250^{\circ} \mathrm{F}$, but above $-20^{\circ} \mathrm{F}$.

4. The facility's Seismic Demand Spectrum (SDS) must meet the requirements of Chapter 5.

\section{Commentary}

1. While the focus of seismic experience has been mostly on welded steel piping, there is no evidence that welded piping constructed of metals other than gray cast iron has performed poorly in past earthquakes. Test and earthquake experience of piping systems is contained in References 94 through 99.

Except for aluminum, non ferrous pipe materials allowed by the ASME B31.3 (Ref. 91) code have UTS of $30 \mathrm{ksi}$ or better. Welded aluminum is excluded since many grades of aluminum alloy have low specified ultimate and yield strengths, and tend to have low fatigue strength and limited ductility in the heat affected zone.

The screens may be used for copper piping. The UTS of weldable grades of copper and bronze piping exceeds $30 \mathrm{ksi}$. Copper tubing and piping can also be brazed, and a properly brazed joint is stronger than the pipe.

Soldered joints operating at ambient or higher temperatures exhibit, with time, a reduced strength. At cryogenic temperatures they tend to become brittle. Soldered joints, unlike brazed joints, must be considered outliers.

Pipe materials must be ductile at service temperatures, having total elongation at rupture greater than $10 \%$. Table 10.1.1-2 shows such properties for common piping materials at room temperature. When judging material ductility, the review team must consider the effect of material degradation on these properties, particularly the potential for reduced elongation caused by lowered ductility.

Cast iron or brittle elements in a ductile piping system are outliers, but they may be accepted (by other appropriate procedures) if proven to be located in low seismic stress areas, and not susceptible to impact.

Seismic induced deflection or loads at groove type mechanical joints shall be limited to vendor listed allowables or test based limits.

Dynamic seismic testing of threaded joint pipe sections indicates that they are prone to leakage under large rotations. For threaded joints, the span between lateral supports, in Section 10.1.1.10, have been reduced accordingly. 
2. The seismic testing and earthquake experience data is mostly from standard or thick wall pipe. The screening criteria apply directly to piping systems with a $D / t$ ratio of 50 or less.

3. Below $250^{\circ} \mathrm{F}$, thermal expansion loads are small for the purpose of seismic evaluation. The review team should identify unusually stiff piping configurations where the $250^{\circ} \mathrm{F}$ rule is questionable. Materials lose ductility at low temperatures. Therefore, piping operating below $-20^{\circ} \mathrm{F}$ are considered outliers.

4. Limiting the screening criteria to the specified free field horizontal spectral acceleration is a precaution introduced to remain within the scope of earthquake experience data for equipment.

Table 10.1.1-2 Typical Properties of Common B31.3 Piping, Tubing, Fitting, and Support Members Materials at Room Temperature

\begin{tabular}{|c|c|c|c|c|c|}
\hline DESCRIPTION & MATERIAL & $\begin{array}{c}\text { BASIC } \\
\text { ALLOWABLE } \\
\text { (ksi) }\end{array}$ & $\begin{array}{c}\text { YIELD } \\
\text { STRENGTH } \\
\text { (ksi) }\end{array}$ & $\begin{array}{l}\text { ULTIMATE } \\
\text { STRENGTH } \\
\text { (ksi) }\end{array}$ & $\begin{array}{l}\text { ELONGATION } \\
\text { IN 2" DIA. } \\
\text { ROUND SPECI. } \\
\quad(\text { min. } \%)\end{array}$ \\
\hline Structural Steel & A36 & 17.8 & 36.0 & $58.0-80.0$ & $20-23$ \\
\hline Carbon Steel Pipe & $\begin{array}{c}\text { A53, } \\
\text { GR. B }\end{array}$ & 20.0 & 35.0 & 60.0 & $22-23$ \\
\hline $\begin{array}{l}\text { Carbon Steel } \\
\text { (Forged Fitt.) }\end{array}$ & $\begin{array}{c}\text { 1A105, FR. } \\
\text { CL-70 }\end{array}$ & 23.3 & 36.0 & $\overline{70.0}$ & $18-30$ \\
\hline $\begin{array}{c}\text { Carbon Steel } \\
\text { (Seamless Pipe) }\end{array}$ & $\begin{array}{l}\text { A106, } \\
\text { GR. B }\end{array}$ & 20.0 & 35.0 & 60.0 & $16-30$ \\
\hline Pipe Fitting & $\begin{array}{c}\text { A234 } \\
\text { GR. WPB }\end{array}$ & 20.0 & 35.0 & 60.0 & $14-30$ \\
\hline Carbon Steel Bolt & $\begin{array}{l}\text { A307, } \\
\text { GR. B }\end{array}$ & 13.7 & 36.0 & $60.0-100.0$ & 18 \\
\hline $\begin{array}{c}\text { Stainless Steel } \\
\text { Pipe }\end{array}$ & $\begin{array}{l}\text { A312, GR. } \\
\text { TP-304L }\end{array}$ & 16.7 & 25.0 & 70.0 & $25-35$ \\
\hline Copper Tube & $\begin{array}{l}\begin{array}{l}\text { Various } \\
\text { types }\end{array} \\
\end{array}$ & $6.0-15.0$ & $9.0-40.0$ & $30.0-50.0$ & 25 \\
\hline Red Brass Pipe & $\begin{array}{c}\text { B43 } \\
\text { Temp. } 061\end{array}$ & 8.0 & 12.0 & 40.0 & 35 \\
\hline
\end{tabular}

\subsubsection{Cautions}

1. The screening criteria are not meant to be a design tool. The applicable code should be used at the design and layout stage. The screening criteria are not equivalent to compliance with the seismic design requirements of ASME B31.1 (Ref. 90), ASME B31.3 (Ref. 91), ASME 
Boiler and Pressure Vessel Code Section III (Ref. 100), NFPA-13 (Ref. 92), AWWA (Ref. 93), AISC (Ref. 81), or AISI (Ref. 101). An existing piping system may comply with the screening criteria but not with the design codes' seismic requirements, and vice-versa.

If a piping system has been designed and constructed to comply with the seismic design provisions of a reference code, it is not necessary to evaluate its seismic adequacy using this procedure. However, the review team may chose to address the provisions of screens 10.1.1.7 "Internal Degradation", 10.1.1.8 "External Corrosion" and 10.1.1.18 "Interaction with other structures" of this procedure, since these considerations are not typically addressed in design codes.

If seismic loads were not included in the original code design of the piping system, the review team may evaluate the seismic adequacy of the non-seismically installed piping system using this procedure, with approval from the owner and/or jurisdiction as appropriate. As an alternative, the review team may evaluate the seismic adequacy of the installed system using the seismic design provisions of the reference code.

2. Application of the screening criteria must reflect the consensus of a seismic review team of two or more degreed engineers, each engineer having the following qualifications (see Section 3.2.2):
a. a minimum of five years experience in seismic design and qualification of piping systems and support structures
b. capability to apply sound engineering judgment, based on the knowledge of the behavior of piping systems in actual earthquakes and seismic tests.

3. Qualified users of the screening criteria must complete a training course (see Section 3.2.2) and successfully pass an examination (as appropriate) in the following topics:
a. content and intent of the screening criteria (Ref. 101)
c. piping and pipe hanger standards
d. piping materials and degradation mechanisms
e. support anchorage rules of the DOE Seismic Evaluation Procedure
f. earthquake and seismic test experience data for piping systems
b. piping and pipe support design requirements of ASME B31.1 (Ref. 90), ASME B31.3 (Ref. 91), NFPA-13 (Ref. 92), AWWA (Ref. 93), AISC (Ref. 81), and AISI

4. The screening criteria rely on the considerable body of piping test, earthquake data and analytical design practice to screen and identify the following key attributes which may lead to seismically induced failures of piping systems:

a. Material condition: Poor construction details and material degradation are at the source of many seismic failures observed in piping systems. Construction quality and material condition are thoroughly covered in the screens. 
b. Anchor motion: Excessive anchor motion propagated through equipment and headers has resulted in seismic failures of piping systems. The screens provide for protection against excessive anchor motion.

c. Brittle features: Brittle materials and certain fittings and joints are screened out to avoid non-ductile piping systems.

d. Interactions: Experience data shows several failures traceable to seismic interactions on the piping systems the potential for interactions. Screens are provided to assess the potential for credible and significant interactions.

\subsubsection{Documentation}

The review team shall complete a Piping Seismic Evaluation Work Sheet (SEWS 10.1.1 in Chapter 13) for each piping system. Similar piping systems may be documented in a single SEWS 10.1.1.

The technical basis for judging each screening criterion shall be described on attached sheets and cross referenced in the corresponding notes column of the SEWS 10.1.1.

Written calculations shall be sufficiently detailed to clarify the purpose of the calculation and the conclusion. All assumptions shall be noted.

The method and calculations to resolve outliers shall be documented.

The purpose of each screening criterion is included in this procedure and explained in the required training course.

For each piping system, a complete documentation package will be assembled consisting of the PSEWS with attached notes and calculations, sketches, and photographs.

Documentation should be sufficient for independent review by an experienced piping engineer trained in the application of this procedure.

\subsubsection{Required Input}

1. Piping System ID

Record the appropriate piping identification numbers, such as line numbers, chronological numbers, calculation numbers, equipment list item numbers, etc.

2. System Description and Fluid Boundaries

Piping system descriptions such as system, subsystem, or line number must clearly communicate the scope of the seismic review (boundary points) on a flow diagram sketch. All branch lines shall be identified, and seismic/non-seismic fluid boundaries shall be noted.

3. Piping System Function and Contents

The contents and function of the piping system during and after the earthquake must be described and categorized as operability, integrity of pressure boundary or position retention (refer to Table 10.1.1-1). For operability, identify active equipment. 
4. Piping Layout and Structural Boundaries

Isometric sketches, based on visual inspection, must be sufficient for piping engineers to visualize system response and calculate approximate span equivalent lengths.

Structural boundaries, along with support types and locations shall be noted. If adjacent walls or structures are relied on for seismic restraint, these features shall also be noted. Inline equipment and concentrated masses shall be noted where they contribute to significant weight.

5. Piping System Location and Reference Drawings

Record the piping system location, such as building, floor or room number.

If the piping system spans different buildings or floors, note all locations.

A list of reference drawing numbers and revisions used in the evaluation, such as flow diagrams, piping arrangement diagrams, isometrics, equipment drawings, etc. is required. A separate sheet may be used if needed.

6. Piping Materials and Sizes

List all pipe materials, sizes (nominal pipe size and schedule or thickness) and the references used to determine this information (such as specifications or drawings).

7. Weights

Linear weight $(\mathrm{lb} / \mathrm{ft})$ of piping and contents must be recorded for each size of pipe. Noted contents (liquid, gas, air, steam, etc.) must be the same as expected during a postulated earthquake.

Note the linear weight $(\mathrm{lb} / \mathrm{ft})$ of insulation and the references used to determine this information (such as specifications or drawings). Record weight of in-line components and eccentricities, as necessary.

8. Concurrent Pressure and Temperature

Specify the pressure and temperature conditions expected concurrent with the postulated earthquake. The pressure values will be used in the component rating screen (refer to Construction Quality). The temperature must be below $300^{\circ} \mathrm{F}$ for the screens to apply (refer to Applicability Section).

9. Input Response Spectra (see Section 5.2)

The input response spectra are used in several screens and may be necessary for the resolution of outliers.

The review team shall document the appropriate ground and/or floor response spectra, applicable references, and status (final or preliminary). Final response spectra are required to finalize the evaluation.

The ground response spectra (at 5\% damping) shall be used for piping supported from grade. (see Section 5.2) 
The floor (in-structure) response spectra (at 5\% damping) shall be used for piping supported above grade. (see Section 5.2)

If the piping terminal ends are at large flexible equipment, seismic anchor motion of the equipment nozzles shall be considered.

If the piping spans between buildings, the relative anchor motions shall be considered. Relative building movements shall be obtained from the building structural analysis.

10. Applicability

Limits and conditions as given in the Applicability section must be met, to ensure that the material, size $(\mathrm{D} / \mathrm{t})$, temperature $\left(250^{\circ} \mathrm{F}\right.$ and $\left.-20^{\circ} \mathrm{F}\right)$ and input acceleration of evaluated piping is appropriate for this screening procedure.

\subsubsection{Construction Quality (Screen 1)}

Screen 1 - Piping, components and supports shall be undamaged and of good construction.

\section{Commentary}

An assessment shall be conducted of the design, welding, and fabrication quality, as well as all visible damage to the piping and the supports, prior to applying the screening criteria.

The piping system must have been fabricated and examined in accordance with ASME B31.1 (Ref. 90), ASME B31.3 (Ref. 91), AWWA (Ref. 93), or NFPA (Ref. 92).

Pressure ratings for branch connections and fittings shall be checked for adequacy. Systems with pressures in excess of that allowed for ANSI B16.5 (Ref. 102) class 2500 are considered outliers.

Standard pipe fittings manufactured to specifications must have the same pressure rating as their corresponding size and schedule of straight pipe. Unreinforced branch connections, or pipe fittings or couplings unlisted in the applicable standards, or which lack stated pressure ratings, could have significantly lower pressure ratings and seismic capability than their complementary straight pipes, in which case they are outliers.

The piping and supports shall be visually inspected for adequate quality of design, fabrication, installation and maintenance. Instances of poor quality shall be noted. Where piping is not accessible for direct visual examination (covered with insulation, located in inaccessible areas, etc.), construction quality may be based on as-built construction and maintenance records confirmed to be up-to-date.

Signs of poor construction quality or subsequent damage include:

1. excessive distortion of piping or supports

2. brazed joints, apparently of good quality, but without a thin layer of brazing or solder visible where the tube extends beyond the fitting socket

3. uneven, undersized or damaged welds

4. unusual or temporary repairs 
5. evidence of interference having caused significant bearing, scratch marks or distortion to the pipe metal or to components

6. a pipe dislodged from its support so that the weight of the pipe is distributed unevenly on the hangers or saddles

7. the deformation of a thin vessel wall in the vicinity of a pipe attachment

8. pipe supports forced out of position by expansion or contraction of the piping

9. the shifting of a base plate, breaking of a foundation, or shearing of foundation bolts of mechanical equipment to which piping is attached

10. missing nuts or bolts

11. signs of leakage (discoloration, dripping, wet surface)

12. cracks in connecting flanges or the cases of pumps or turbines to which piping is attached

13. deterioration of protective coatings, fireproofing or other periodic maintenance conditions

14. general physical damage

15. movement or deterioration of concrete footings

16. failure or loosening of foundation bolts

17. insecure attachment of brackets and beams to the support

18. restricted operation of pipe rollers or slide plates

19. insecure attachment or improper adjustment of pipe hangers

20. broken or defective pipe supports

21. oversized bolt holes

\subsubsection{Internal Degradation (Screen 2)}

Screen 2 - Piping and components shall be free of significant internal degradation.

\section{Commentary}

Significant degradation refers to that which may affect the pressure integrity of the piping system. The potential for internal degradation must be investigated and documented from two aspects.

1. the piping system operating performance records, and

2. a metallurgical assessment

It is unnecessary to perform new nondestructive surface or volumetric examinations of the piping system for this screen. The review of performance records and metallurgical assessments are to be based on existing data. If either source of information is unavailable or suggests potential internal degradation, the system must be classified as an outlier. 
If the condition of the piping system is judged adequate, but some degradation is expected to occur in the future, the system must be subjected to periodic in service inspection or evaluated for the effects of the expected degradation.

\subsection{Operating Performance Record}

The system cognizant engineer must identify and assess past maintenance, repairs and replacements performed on the piping system, or on similar systems, to judge if they indicate potential metallurgical or mechanical degradation mechanisms.

The system cognizant engineer must identify any history of abnormal events or loadings, such as flow induced vibration, water hammer, misalignment, binding, and excessive temperature cycling, to judge if they may have caused system degradation due to fatigue or localized yielding.

Evidence of pipe leakage, pipe repair, support failures, or abnormal vibration may indicate significant cyclic loading, which shall be resolved.

\subsection{Metallurgical Assessment}

The metallurgical assessment of the piping systems must be performed with the help of materials engineering. When considering materials, fluids and operating conditions, the materials engineer must judge the potential for reduced performance capability resulting from material degradation, erosion or corrosion.

\subsection{Guidance: Susceptible Areas}

The following areas are most susceptible to corrosion, erosion, and other forms of material degradation.

1. points at which condensation or boiling of acids or water is likely to occur

2. points at which acid carryover from process operations is likely to occur

3. points at which naphthenic or other organic acids may be present in the process stream

4. points at which high-sulfur streams at moderate-to-high temperatures exist

5. points at which high- and low-temperature hydrogen attack may occur

6. dead ends subject to turbulence, or where liquid-to-vapor interface or condensation occur

7. valve bodies and trim, fittings, ring grooves and rings, and flange facings

8. welded areas subject to preferential attack

9. catalyst, flue-gas, and slurry piping

10. steam systems where condensation occurs

11. ferrous and nonferrous piping subject to stress corrosion cracking

12. alkali lines subject to caustic embrittlement and resultant cracking 
13. areas near flanges or welded attachments that act as cooling fins, causing local corrosion because of temperature differences

14. locations where impingement or changes in fluid velocity can cause local accelerated corrosion or erosion

15. points of accidental contact or insulation breakdown that causes contact of dissimilar metals

16. an area where steam or electric tracing contacts piping handling material such as caustic soda, where concentrated heat can cause corrosion or embrittlement

17. an area immediately downstream of a chemical injection point, where localized corrosion might occur in the reaction zone

18. heat-affected zones (around and in welds) in non-post weld heat-treated carbon steel piping in amine service

19. dissimilar metal welds

20. piping subject to mechanical or flow induced vibration.

The potential for general corrosion or erosion that could result in pipe wall thinning shall be assessed. If wall thinning potential exists in the material or environment, sample measurements shall be taken. If the predicted thinning exceeds $20 \%$ of the pipe wall for the planned life of the piping system, the system is an outlier.

If stress corrosion cracking is likely, examinations shall be performed.

The hazard of embrittlement (due to hydrogen, hydrogen cracking, irradiation, thermal aging, etc.) for the planned life of the piping system shall be assessed. If it is possible for pipe ductility (total elongation at rupture) to be reduced by $10 \%$ or more, the system is an outlier.

\subsection{Guidance: Material Compatibility}

The following possible material conditions must be evaluated, along with other service specific conditions:

1. Carbon Steel, and Low and Intermediate Alloy Steels

a. possible embrittlement when handling alkaline or strong caustic fluids

b. possible hydrogen damage to piping material when exposed (under certain temperaturepressure conditions) to hydrogen or aqueous acid solution

c. possible stress corrosion cracking when exposed to wet hydrogen sulfide, and the further possibility of deterioration (sulfidation) in the presence of hydrogen sulfide at elevated temperatures

d. the need to limit maximum hardness of metals in applications subject to stress corrosion 
2. High Alloy (Stainless) Steels

a. possible stress corrosion cracking of austenitic stainless steels exposed to media such as chlorides and other halides either internally or externally as a result of improper selection or application of thermal insulation

3. Nickel and Nickel Base Alloys

a. possible stress corrosion cracking of nickel-copper alloy (70 Ni-20Cu) in hydrofluoric acid vapor if the alloy is highly stressed or contains residual stress from forming or welding

4. Copper and Copper Alloys

a. possible dezincification of brass alloys

b. susceptibility to stress-corrosion cracking of copper-based alloys exposed to fluids such as ammonia or ammonium compounds

c. possible unstable acetylene formation when exposed to acetylene

\subsubsection{External Corrosion (Screen 3)}

Screen 3 - Piping, components and supports shall be free of significant external corrosion.

\section{Commentary}

In reviewing the piping system for signs of corrosion, the seismic evaluation team must consult the materials engineer for questionable conditions.

Significant corrosion refers to metal thickness loss of more than $20 \%$. A surface discoloration or thin layer of rust does not harm structural integrity. Rust forms a surface coating which protects the inner metal from further corrosion.

A loss in thickness can be measured by comparing the pipe diameter at the corroded area with the original pipe diameter. The depth of pits can be determined with a depth gauge.

Stainless steel, copper, nickel, and their alloys are typically used in B31.3 (Ref. 91), and resist atmospheric corrosion. They may be accepted without further review. Iron and carbon (low alloy) steels, however, may be subject to attack, particularly in areas where moisture can accumulate. If piping is insulated and made of iron or carbon/low alloy steel, insulation should be removed at 3 accessible and susceptible points and the pipes inspected for corrosion.

Significant corrosion (uniform loss of more than $20 \%$ of metal thickness) can impair the ability of the supports or piping to carry loads. For supports, areas to consider include threaded sections and pipe-clamp or pipe-saddle interfaces. Local metal loss exceeding $20 \%$ of the wall thickness may be acceptable, but each occurrence must be evaluated.

\subsection{Atmospheric Corrosion}

When metals such as iron or steel are exposed to the atmosphere, they will corrode due to the presence of water or oxygen. Below $60 \%$ humidity, corrosion of iron and steel is negligible. To prevent atmospheric corrosion, it is necessary to protect the surface of the metal from water by means of a protective barrier or coating. 
The normal rate of atmospheric corrosion of unpainted steel in rural atmospheres is low, ranging from 0.001 to 0.007 inches per year. However in some atmospheres, a steel corrosion rate of 0.05 inches per year is possible. The rate of corrosion accelerates at any break in a protective coating because the exposed metal at the break becomes anodic to the remaining metal surface. At such breaks, deep pits will form.

Equipment which is located next to boiler or furnace stacks and exposed to corrosive gases such as sulfur dioxide and sulfur trioxide is subject to accelerated corrosion. These gases, dissolved in water condensate from flue gas, rain, or mist, form dilute acids which act as electrolytes. In addition, chlorides, hydrogen sulfide, cinders, fly ash, and chemical dusts present in industrial atmospheres may act in a similar manner.

\subsection{8.2 Corrosion Under Insulation and Fireproofing Materials}

Inadequate weatherproofing on piping allows moisture to penetrate to the underlying steel, where

hidden corrosion takes place. Such hidden corrosion is often severe in refrigeration systems. The skirts of all vessels, regardless of operating temperatures, are subject to severe corrosion under insulation or fireproofing. Cracks in fireproofing concrete, particularly at the top where the concrete ends, also allow moisture to penetrate and hidden corrosion to occur. Protective organic coatings may be useful, especially in seacoast areas where chlorides can come from the air rather than from the insulation. Inhibited insulation, or insulation free of water-soluble chlorides, should be used with austenitic ( 300 series) stainless steels to prevent stress corrosion cracking.

Defects in protective coatings and the waterproof coating of insulation will permit moisture to contact the piping. When defects are found in the waterproof coating of insulation, enough insulation should be removed to allow the extent and severity of corrosion to be determined. Sections of insulation should be removed from small connections, such as bleed lines and gauge connections, since these locations are particularly vulnerable to atmospheric attack due to the difficulty of sealing the insulation.

\subsection{Corrosion of Piping at Contact Points}

Piping installed directly on the ground suffers severe corrosion on the underside from dampness. If grass or weeds are allowed to grow beneath and around piping, the underside of the pipe will remain damp for long periods and will corrode. Lines laid directly on supports, or hung by clamps, often show crevice corrosion at the contact points.

Lines that sweat are susceptible to corrosion at support contact points, such as under clamps on suspended lines. Piping mounted on rollers or welded support shoes is subject to moisture accumulation and corrosion. Loss of vapor-sealing mastic from the piping insulation can result in local corrosion. Pipe walls inside open-ended trunnion supports are subject to corrosion. These points should be investigated.

\subsection{Corrosion of Structures}

Structures that provide crevices where water may enter and remain for long periods are subject to severe corrosion. Examples are structural members placed back to back, and platforms installed close to the tops of towers or drums. Structures located near furnace stacks and cooling towers are particularly susceptible to this type of attack. 


\subsection{Leakage}

The walkdown team must check for the possibility of leaking fluids, suggested by local discoloration or wet surfaces on the pipe or floor.

Bolted joints such as valve packings or flanges may leak. This is especially true for water lines following prolonged periods of sub-freezing weather. Performance records of frozen water pipes show incidents of leakage due to frozen water expanding through and distorting flange gaskets.

Leaks from bolted joints allow fluid to either collect on the pipe or drip onto other systems. In areas where leaks are encountered, the walkdown team should ensure either that the bolts and fluid are compatible or that the bolting has not been subjected to process fluid attack from gasket leakage.

\subsubsection{Span Between Vertical Supports (Screen 4)}

Screen 4 - Piping shall be well supported vertically.

\section{Commentary}

A piping system may be considered well supported for deadweight if the equivalent span length between vertical supports, for liquid or gas service, is as shown in Table 10.1.1-3, which lists acceptable vertical support spacing for this screen. The spans in this table correspond to $150 \%$ of the ASME B31.1 suggested pipe support spacing provided in Table 121.5. The ASME B31.1 values are based on a bending stress of $2300 \mathrm{psi}$ and a maximum sag of 0.1 inch. Since these values are low, it has been judged reasonable to use $150 \%$ of the ASME B31.1 span lengths for installed systems.

Table 10.1.1-3 Equivalent Span Between Vertical Supports

\begin{tabular}{|c|c|c|}
\hline $\begin{array}{c}\text { Nominal Pipe Size } \\
\text { (in) }\end{array}$ & $\begin{array}{c}\text { Liquid Service } \\
\text { (ft) }\end{array}$ & $\begin{array}{c}\text { Gas Service } \\
\text { (ft) }\end{array}$ \\
\hline 1 & 10 & 13 \\
\hline 2 & 15 & 19 \\
\hline 3 & 18 & 22 \\
\hline 4 & 21 & 25 \\
\hline 6 & 25 & 31 \\
\hline 8 & 28 & 36 \\
\hline 12 & 34 & 45 \\
\hline 16 & 40 & 52 \\
\hline 24 & 48 & 63 \\
\hline
\end{tabular}


The equivalent span length $\mathrm{Lei}_{\text {ei }}$ in a given direction $\mathrm{i}$ is defined as: $\mathrm{L}_{\mathrm{ei}}=\left(\mathrm{W}_{\mathrm{pi}}+\mathrm{W}_{\mathrm{ci}}\right) / \mathrm{W},[\mathrm{ft}]$

$\mathrm{W}_{\mathrm{pi}}=$ Weight of pipe length in span between consecutive supports in direction $\mathrm{i}$, including insulation and contents [lb]

$\mathrm{W}_{\mathrm{ci}}=$ weight of in line components in span [1b]

$\mathrm{W}=$ weight per unit length of pipe size, insulation and contents in span [lb/ft] The equivalent span length for gas service may be used for evaluating empty, normally dry, pipe spans.

Vertical loading can be resisted by engineered deadweight supports, or structures that are not considered deadweight supports, such as penetrations through walls, certain types of box beam horizontal restraints, and floor slabs.

The following vertical support configurations shall be considered outliers in seismic screening evaluations.

1. friction clamp connections

2. shallow pipe saddle support or pipe rolls

3. bottom support if not positively attached to the pipe and floor, and if the lateral movement of the pipe could possibly tip the support

4. pipe resting on a support, free to slide laterally so as to fall off the support

5. A clamp on a vertical riser without positive attachment to the pipe, such as lugs above the clamp.

\subsubsection{Span Between Lateral Supports (Screen 5)}

Screen 5 - Piping shall be sufficiently restrained in the lateral direction.

\section{Commentary}

A piping system may be considered sufficiently restrained in the lateral direction if the equivalent lateral span length for liquid or gas service does not exceed three times the spans in Table 10.1.1-3, which corresponds to 4.5 times the ASME B31.1 (Ref. 90) suggested vertical pipe support spacing. This span is to be divided by 2.3 (stress intensification factor for threaded joints) for pipe sections which contain threaded joints.

The 4.5 times the B31.1 deadweight spans for spacing of lateral restraints is consistent with the current draft ASME B31 Mechanical Design Committee Appendix on Seismic Design (Ref. 103). Seismic experience data has indicated that relatively long spans have experienced lower spectral accelerations and are more susceptible to displacement-induced damage. Therefore, actual spans between lateral supports will often be limited to less than 4.5 times the B31.1 deadweight spans by Screen 6 (anchor motion of headers), Screen 9 (equipment nozzle loads), or Screen 12 (pipe support). 
Lateral restraint may be provided either by an engineered lateral support, or by other means, such as:

\section{Interferences}

Lateral interferences will limit motion in piping routed along a wall or structural member. Although this restraint occurs in one direction only, it significantly restricts the response of the system to a reversing load.

\section{Box Beam}

A box beam, while not designed to provide horizontal restraint, will do so once the pipe moves through the gap and contacts the beam. When evaluating the effectiveness of a box beam's horizontal restraint potential, the gap on both sides of the pipe must be considered. Note that, should the pipe impact the vertical members of the beam, significant energy is dissipated and the frequency response of the system is modified.

\section{U-Bolts}

U-bolts provide significant horizontal restraint, even when the side load design capacity of the Ubolt is exceeded. Should the U-bolt yield under seismic stress, it will bend, resisting horizontal motion by tension. U-bolts should not be considered to provide longitudinal restraint along the pipe axis.

\section{Saddles}

There are generally two types of pipe saddle supports; a simple saddle on which the pipe merely rests, and that which includes a yoke (strap or U-bolt) to restrain the pipe in the saddle. A shallow simple saddle provides practically no horizontal restraint, and could permit the pipe to escape from its support during a seismic event. A deep saddle support will restrain the pipe in the lateral direction.

\section{Floor and Wall Penetrations}

Piping often passes through openings in floors, grating or walls. Since these openings are not designed as supports, gaps between the pipe and the structure exist. When made in floors or walls, the openings are usually secured by a sleeve; in gratings, a sleeve or a ring is used. These penetrations provide significant lateral restraint during dynamic seismic events and, like the box beam, prevent displacement, dissipate energy and modify system frequency.

\section{Rod Hangers}

The lateral support capacity of rod hangers is measurable as a function of the swing angle of the rod when subjected to a given lateral load. While this lateral support capacity is not provided by design, it can be important in practice. The length of the rod is significant because for shorter rods, the swing angle and resistance to horizontal displacement is greater. An effective lateral spring rate formula for short rod hangers is $W / 1$, where $W$ is the tributary weight on the rod and 1 is the length of the rod. 


\subsubsection{Anchor Motion (Screen 6)}

Screen 6 - Piping must have sufficient flexibility to accommodate the seismic motions of structures, equipment and headers to which it is attached.

\section{Commentary}

One of the most common causes of piping failure in strong motion earthquakes is seismic anchor motion (SAM) resulting from:

1. large displacement of unanchored tanks or equipment

2. failure of the tank or equipment anchorage

3. large differential motions of structures to which the piping is attached

4. large motions of header piping induced into smaller branch piping

5. differential movements due to soil settlements

SAM caused by these sources imposes large strains in rigid sections of the piping system. Most of the common piping failures are in pipes with non-welded connections to tanks, pumps, and larger header pipes which are insufficiently restrained.

In order to screen out SAM as a potential failure mode for piping, the following conditions must be evaluated; otherwise the effect of anchor motion must be calculated.

1. Tanks and equipment to which the piping attaches must be properly anchored to prevent sliding, rocking or overturning. Equipment anchorage shall be evaluated using Chapter 6 and Section 9.1 of the DOE Seismic Evaluation Procedure.

2. Tanks and equipment to which the piping attaches, and the supports for the tanks and equipment should be relatively stiff to minimize SAM.

Note: When vibration isolators are present, vibration isolators on equipment are a source of SAM, and must be evaluated as provided in Chapter 6 of the DOE Seismic Evaluation Procedure. If there are no seismic stops built into the isolators, the equipment will likely require the addition of seismic restraints to limit motion. If seismic stops are installed with the vibration isolators, the attached piping must be assessed for the maximum motion that can be realized before impacting the stops.

3. Piping rigidly attached to two different buildings, or substructures within a building, must be sufficiently flexible to accommodate the differential motion of the attachment points. Usually, structural displacements are relatively small, and the motion can be easily accommodated by pipe bending. Particular attention should be focused on piping that has its axial motion restrained at support points in two different structures

4. Header motion imposed on small branch lines must be assessed, or the header must be restrained near the branch.

The elastically calculated unintensified stress amplitude due to SAM (M/Z) may be limited to twice the material yield stress for screening purposes. When considering lateral movement of header pipes and restraint of branch pipes, it is necessary to define a lateral restraint, as discussed in Section 10.1.1.10, Lateral Span. 


\subsubsection{Mechanical Joints (Screen 7)}

Screen 7 - Piping shall not contain mechanical joints which rely solely on friction.

\section{Commentary}

The seismic experience data contains a number of instances where mechanical joints which rely on friction have leaked. While it is not clear whether this leakage was due to seismic anchor motion effects (already covered by an earlier screen), these joints must be classified as outliers pending further studies. Joint vendors may be contacted or tests may be conducted to obtain allowable loads, and simple span formulas may be used to estimate applied loads to be compared to the allowables.

\subsubsection{Flanged Joints (Screen 8)}

Screen 8 - Flanged joints shall withstand the expected seismic moments without leakage.

\section{Commentary}

Flanged joints have leaked under severe seismic loads, and sometimes may leak under normal service loads. If the flanged joint is a B16.5 (Ref. 102) flange adequate preload, and a rated pressure above the operating pressure, the flange is acceptable. Other flanged joints with lesser capacities should not be located in high stress areas. One method of assessing moment capacity at flanges is to determine excess pressure capability (rating minus operating pressure) and convert that into an equivalent moment. The rated pressure of flanged joints shall be established.

If there are indications of leakage at the joint in past service, the flanged joint is an outlier.

Slip-on flanges are only acceptable if located in areas of the piping system with estimated unintensified seismic stress less than approximately 10,000 psi.

\subsubsection{Equipment Nozzle Loads (Screen 9)}

Screen 9 - Equipment shall not be subjected to large seismic loads from the piping systems.

\section{Commentary}

To be considered operable, active equipment and components (such as pumps and valves) have to meet the requirements of the DOE Seismic Evaluation Procedure (refer to Table 10.1.1-1), in addition to the following requirements:

Equipment and component nozzles, except for valves that are stronger than the pipe, should be protected, by appropriate restraints, from excessive seismic loads, particularly where the equipment nozzle or joint is of smaller size than the pipe. The piping layout shall be reviewed to evaluate that large seismic loads are not reacted at the equipment nozzle. One potential problem is a long axial run of pipe not restrained from axial movement except at the equipment nozzle. If there is a possibility of large seismic loads, the unintensified bending stress at the nozzle shall be elastically evaluated and compared to twice the material yield stress.

Piping reaction loads at the nozzles of rotating equipment may affect their function. The seismic reaction loads imparted by the piping on the nozzle of the active (rotating) equipment shall be estimated. These loads shall be small (unintensified bending stress less than $6000 \mathrm{psi}$ ), or within the estimated capability of the equipment. 


\subsubsection{Eccentric Weights (Screen 10)}

Screen 10 - Eccentric weights in piping systems shall be evaluated.

\section{Commentary}

The adequacy of valves with eccentric operators shall be evaluated using the rules in Chapter 8 of the DOE Seismic Evaluation Procedure. Eccentric pipe segments, such as unsupported vents or drains, shall be evaluated using the peak spectral acceleration at 5\% damping (or a better estimate of the spectral acceleration at the pipe frequency) (see Section 5.2) and an allowable unintensified elastically calculated stress of twice the material yield stress.

\subsubsection{Flexible Joints (Screen 11)}

Screen 11 - Flexible joints shall be properly restrained to keep relative end movements within vendor limits.

\section{Commentary}

For unsupported flexible joints such as expansion joints, bellows, or flexible joints, the relative displacements need to be limited to prevent tearing or buckling the joint. Where manufacturer's limits can be exceeded, the Review Team should ensure the joint has sufficient mobility to absorb the seismic deflections. When such joints are adequately supported on either side this is not usually an issue.

If the configuration is such that excessive seismic movements at the expansion joint could tear or buckle the joint, the expansion joint is an outlier. Calculation of seismic displacements and comparison to established allowable displacements are required to resolve the outlier.

The seismic evaluation team may refer to the rules of the Expansion Joints Manufacturers Association (EJMA).

\subsubsection{Evaluation of Pipe Supports (Screen 12)}

Screen 12 - Pipe supports shall be capable of withstanding seismic loads without failure.

\section{Commentary}

Support failure refers to non-ductile rupture or complete loss of restraining function of the pipe support.

The review team shall evaluate the seismic load and capacity of supports judged to be prone to failure. The basis for the support selection shall be documented.

Examples of supports to be evaluated are:

- $\quad$ supports with largest spans or close to heavy components

- $\quad$ supports reacting the load from long axial runs

- short rods adjacent to longer rods

- $\quad$ stiff support in the midst of significantly more flexible supports (hard-spot) 
- $\quad$ supports with fewest or smallest anchor bolts

- $\quad$ gang supports reacting loads from several pipes

- $\quad$ supports not attached to structural steel or concrete (such a supports attached to other piping, cable tray or transite walls)

\subsection{Seismic Demand}

The calculation of horizontal and vertical seismic loading on pipe supports is based on the tributary weight of adjacent piping spans multiplied by one of the following factors:

1. For piping supported from grade, multiply by the peak of the $5 \%$ damped ground response spectrum. (see Section 5.2)

2. For piping supported above grade, multiply by the peak of the $5 \%$ damped floor (instructure) response spectrum. (see Section 5.2)

\subsection{Seismic Capacity}

Where failure is credible, the review team shall evaluate the seismic capacity of support members along the seismic load path. The capacity of support members, welds and joints may be estimated using AISC (Ref. 81) rules, multiplying the AISC allowables by 1.7 . Where manufacturer design limits are provided for standard pipe support elements (excluding anchor bolts in concrete), the seismic capacity may be taken as twice the design limit for members loaded in tension, bending or shear. For compression members, if the design limit is based on buckling, the seismic capacity shall be the same as the manufacturer design limit.

For cold-formed steel members, the stress allowables for seismic screening may be 1.7 times the AISI Specification for those members.

Anchorage shall be inspected, and capacity calculated and documented, using the rules of Chapter 6 of the DOE Seismic Evaluation Procedure.

The review team must take care to limit their calculations to credible failure modes which can hinder the function of the piping system. Limited yielding is, in most cases, not a credible failure mode.

An explicit calculation of weld capacities is not required if the welds are estimated to be the same size, and develop the same strength, as connecting members.

The fatigue capacity of threaded rod hangers with fixed-end connections to the wall or structural steel, may be evaluated using the fatigue evaluation screening charts for raceway supports in Section 9.2.1 of the DOE Seismic Evaluation Procedure.

\subsubsection{Interaction with Other Structures (Screen 13)}

Screen 13 - The piping being reviewed shall not be a source or target of interactions.

\section{Commentary}

A piping system subjected to seismic loads will displace or swing laterally, and may impact adjacent components. 


\subsection{Estimate of Displacement}

Without detailed analysis, lateral displacements or swing deflections of piping spans can be estimated.

An approximate formula to estimate pipe displacements (Sd [in]) at spectral acceleration $\left(\mathrm{S}_{\mathrm{a}}\right.$ $\left[\mathrm{in} / \mathrm{sec}^{2}\right]$ ) for a pipe frequency $\mathrm{f}[1 / \mathrm{sec}]$, is:

$$
S_{d}=1.3 S_{a} /(2 \pi f)^{2}
$$

where 1.3 is the mode participation factor for a simply supported beam. An approximate upper bound for a 0.3g Regulatory Guide 1.60 "Design Response Spectra for Seismic Design of Nuclear Power Plants" (Ref. 104) spectrum at low frequency (less than $0.25 \mathrm{~Hz}$ ) is about 28 " for $5 \%$ damping. Actual displacements of piping systems which meet the screens are rarely larger than 12".

\subsection{Estimate of Impact Consequences}

In all cases, the review team will have to carefully estimate the extent of pipe deflection and the component's capacity to absorb impact.

Generally, impact must be avoided if it affects the following components:

- $\quad$ active equipment (motors, fans, pumps, etc.)

- instrumentation

- tubing

- unstable or light weight structures

- electrical cabinets and panels

- $\quad$ sprinkler heads

Generally, impact may be of little consequence if it affects the following components:

- walls

- large frames or structures

- passive components (tank, check valve, etc.)

- pipes of approximately the same or larger diameter

In all cases, the review team must use judgment in estimating the extent of movement of the pipe under review and the capacity of the impacted equipment.

The review team shall visually inspect all structures and commodities located above the pipe and identify those hazards which are judged to be credible (may fall on the pipe) and significant (fall impact may cause pipe failure as defined in Table 10.1.1-1). The guidance in Chapter 7 of the DOE Seismic Evaluation Procedure for equipment interactions may be used for this evaluation. 


\subsubsection{UNDERGROUND PIPING}

\subsubsection{Scope}

This section addresses the seismic evaluation of underground, single wall, pressure piping made of steel, ductile iron, or copper material. Pipe materials must be ductile at service temperatures. Ductile pipe behavior requires joints which are stronger than the pipe. Arc-welded or properly brazed joints are examples of ductile pipe design. Oxy-acetylene welded joints in steel pipes must be considered an outlier and evaluated in accordance with Section 10.1.2.6.

Single or double containment piping (comprised of a core pipe contained inside a buried jacket pipe, as is commonly the case for radioactive waste transfer lines) are covered in Chapter 7 of Reference 29 ("Seismic Design and Evaluation Guidelines for the Department of Energy HighLevel Waste Tanks and Appurtenances," BNL 52361). This reference provides a rigorous methodology for evaluating underground piping. Additional guidance for evaluating underground piping is available in the "ASCE Guidelines for the Seismic Design of Oil and Gas Pipeline Systems" (Ref. 105) and ASCE 4 (Ref. 74).

Underground piping made of gray cast iron, non-ferrous alloys, welded aluminum, thermoplastics, fiberglass, reinforced concrete, and asbestos-cement may exhibit non-ductile behavior and must be considered an outlier. In addition, threaded joints, groove type mechanical joints, and flanged joints must be considered outliers as seismic induced displacements must be explicitly evaluated and compared to joint allowables. Mechanical joints which rely solely on friction are also considered outliers as they may have very low displacement capacity. Methods for dealing with outliers are described in Section 10.1.2.6.

\subsubsection{Pipe Condition Assessment}

The seismic evaluation of underground piping must include an assessment of the existing pipe condition with verification that there has not been significant degradation in the strength, ductility, wall thickness, and joint integrity. This assessment includes:

1. Confirmation of the compatibility of the pipe material, exterior coating, interior lining (where provided), with the conveyed fluid and the surrounding soil or backfill.

2. Examination of historical performance data and maintenance records for evidence of leakage or repairs.

3. A visual and volumetric examination of selected sections of the piping (which will have to be excavated at examination points) to confirm the soundness of materials and joints.

Should this assessment identify a problem with the existing pipe integrity, the piping should be considered an outlier. Piping designated as an outlier should be investigated over a larger extent of the pipe length than the selected sections to identify the entire extent of piping with the problem. Mitigation of piping integrity necessitates repair or replacement of the affected pipe length.

\subsubsection{Applied Loads}

Seismic loads acting on underground piping include wave passage directly inducing strains in the pipe, transient seismic anchor motion from differential movement of building or other structures to which the pipe is attached, and permanent seismic anchor motion from soil movements resulting from seismic induced liquefaction, lateral spreading, settlement, or landslides. Seismic loads are also induced by differential movement resulting from fault rupture intersecting underground pipe. 
Concurrent non-seismic loads might include internal pressure, soil overburden and surface loads, thermal expansion, and natural soil settlements.

\subsubsection{Evaluation of Piping for Wave Passage Induced Strains}

Typically, underground piping constructed of ductile materials and ductile joints can safely withstand strains induced by wave passage effects during an earthquake. In addition, underground piping constructed of ductile materials and ductile joints can safely withstand transient differential movements of underground portions of buildings or other underground attachment points during seismic wave passage. In general, no explicit analysis is required in these cases. Analyses or detailed evaluation is required for the following cases:

- impedance mismatch between soils, such as soft soil to stiff rock

- bends in the piping at which there can be stress concentration effects

- piping which passes through the interface of a building to its supporting soil

\section{- locations of excessive pipe corrosion}

It should be noted that there is one reported case of seismic wave propagation induced pipe failure to a corrosion free modern continuous welded steel pipeline. This case study is described in Reference 106 in which it is believed that the case study is the only documented case of wave passage damage to modern welded steel underground piping. This case has very extreme parameters, as discussed in the following paragraph, which should be considered when evaluating underground piping for wave passage effects and designated underground piping as an outlier. It is unlikely that a similar combination of circumstances exist at a DOE facility.

The pipe, which is discussed in Reference 106, was damaged in the 1985 Michoacan Earthquake. The pipe was a 42 inch diameter, $5 / 16$ inch wall thickness water pipe constructed in the early 1970 's of API $120 \mathrm{X}-42$ grade steel (yield stress $=42 \mathrm{ksi}$ ). The pipe centerline was about 6.4 feet below the ground surface. The soil profile consists of 130 feet very soft clay underlain by two stiffer strata of 260 feet and 1300 feet thickness atop rock. The pipe failure was wrinkling and tearing of the pipe wall. Three factors contributed to the failure of this pipe (1) the ground motion was dominated by Rayleigh waves as the earthquake source was very distant from the pipe location; (2) the peak ground velocity was very high for the acceleration level as the observed PGV/PGA was about $170 \mathrm{in} / \mathrm{sec} / \mathrm{g}$ instead of $48 \mathrm{in} / \mathrm{sec} / \mathrm{g}$ given by Newmark for alluvium; and (3) the soil was extremely soft with a shear wave propagation velocity of only about 130 feet per second.

Other examples of ductile underground piping subjected only to seismic wave propagation have demonstrated very good pipe performance. It is judged that the one case of observed damage resulted from a very unusual combination of circumstances. If conditions approach those described for this case, the ductile pipe must be designated an outlier and appropriate analyses can be used to evaluate this piping.

\subsubsection{Evaluation of Piping for Permanent Soil Movements}

Underground piping at sites subjected to permanent soil movements due to settlement, lateral spreading, liquefaction, landslides, or fault displacement must be considered an outlier. In these conditions, the pipe must be evaluated in the manner described in Section 10.1.2.6. 


\subsubsection{Qutlier Evaluation}

Underground piping designated as an outlier must be explicitly evaluated for the ability of the pipe and joints to withstand seismically-induced soil movements, either transient wave passage effects or permanent ground movements. The preferred approach is to evaluate pipe deformations imposed during earthquake motion and associated effects and to compare to strain criteria developed from full scale pipe tests. In some cases, pipe stresses are evaluated and compared to empirically determined stress limits. Analytical techniques must account for non-linear pipe behavior as acceptable strains may be beyond the elastic limit. Analytical techniques must also account for the non-linear stiffness of the soil surrounding the underground piping.

A method for estimating pipe strains induced during earthquake wave passage is completely described in Chapter 7 of Reference 29. The approach involves estimating axial strain and curvature of the ground during seismic wave passage. These strains may be transferred to long straight runs of buried piping by friction or bearing. Strains (or stresses) at elbows, bends, and tees are then determined by pseudo-static beam on elastic foundation analysis subjected to the axial strain and curvature of the surrounding soil. In such an analysis, the piping system, including both straight and curved sections, are modeled by relatively simple beams supported by linear Winkler springs representing the confinement of the surrounding soil. Similar analysis may be used to determine pipe response due to transient differential movements of buildings or other structures to which the pipe is attached/anchored. By this approach, strains and stresses may be determined for straight pipe, elbow, bend, and tee configurations, and at joints. The resulting strains or stresses should be compared to allowable levels depending on the ductility and strength of the pipe material and of the deformation capacity of joints.

For underground pipe at sites subject to permanent differential soil movement, considerable effort must be expended to establish the amount of movement, the rate of movement, the direction of movement, and the area impacted by the movement. In such cases, the preferred solution is to mitigate the soil such that movements do not occur or to reroute the pipe to avoid the affected area. If this is not possible, underground pipe evaluation is typically performed by conducting analysis of non-linear representations of the pipe and surrounding soil subjected to conservative estimates of the permanent ground deformation caused by settlement, spreading, liquefaction, or landslide. The resulting pipe response is compared to empirically based pipe strain criteria. In some cases, it may be possible to evaluate the pipe using the pseudo linear beam on elastic foundation analysis described in Chapter 7 of Reference 29 and discussed above for wave passage effects. Guidance on the evaluation of underground piping subjected to fault displacement is provided in Reference 105. The allowable strain criteria in Chapter 7 of Reference 29 is more conservative than that in Reference 105. 


\subsection{MECHANICAL EQUIPMENT}

\subsubsection{HEPA FILTERS}

This section describes general guidelines that can be used for evaluating and upgrading the seismic adequacy of HEPA Filters which are included in the Seismic Equipment List (SEL). The guidelines contained in this section are based on experience at Los Alamos National Laboratory as well as other DOE sites. Guidelines in this section cover those features of HEPA filters which experience has shown can be vulnerable to seismic loadings.

HEPA filters are generally used to prevent airborne radioactive material from being released to the environment. The environment may be a laboratory room, a facility, or external to a facility.

Filters attached to a glove box (see Figure 10.2.1-1) are used to limit the spread of radioactive material through out the ventilation system of a facility. By the "rule of the box" (see Section 2.1.3.4.1), these types of filters can be evaluated as part of the glove box. The evaluation of the equipment class of glove boxes is discussed in Section 10.2.2.

Filters which are used to scrub recirculating air in a facility or which scrub air that is released through the facility exhaust are generally found in filter plenums (see Figures 10.2.1-2 through 10.2.1-4). Filter plenums are generally similar to the equipment class of Air Handlers, which is discussed in Section 8.2.9, with the exceptions that there may not be a coil section and the fan may be external to the plenum structure. Therefore, the caveats given for Air Handlers in Section 8.2.9 can be used in the evaluation of HEPA filters. In addition, external fan units associated with filter plenums can be evaluated using the caveats given for the equipment class of Fans, as discussed in Section 8.2.10.

HEPA filters themselves are generally lightweight and firmly held in position to a frame by some type of restraining mechanism. Both the frame and the restraining mechanism need to be evaluated. The frame should be evaluated for overall stability and to determine if permanent deformations can take place that adversely affect the function of the filter bank. The restraining mechanisms should be reviewed to determine if the filters can come loose during an earthquake. Seismic evaluations should include not only the equipment the filters are installed in, but also the framing and restraining mechanisms within those pieces of equipment.

HEPA filters should also be reviewed for potential seismic interactions. One such interaction would be the effect of fire suppression water on the filter functionality. Should fire sprinklers activate during or following a seismic event and spray water on the HEPA filters, the HEPA will weaken and may fail to function as intended. In addition, should a seismic induced fire occur during or following an earthquake and the fire suppression fails to activate, heat from the fire could adversely affect the functionality of HEPA filters. 


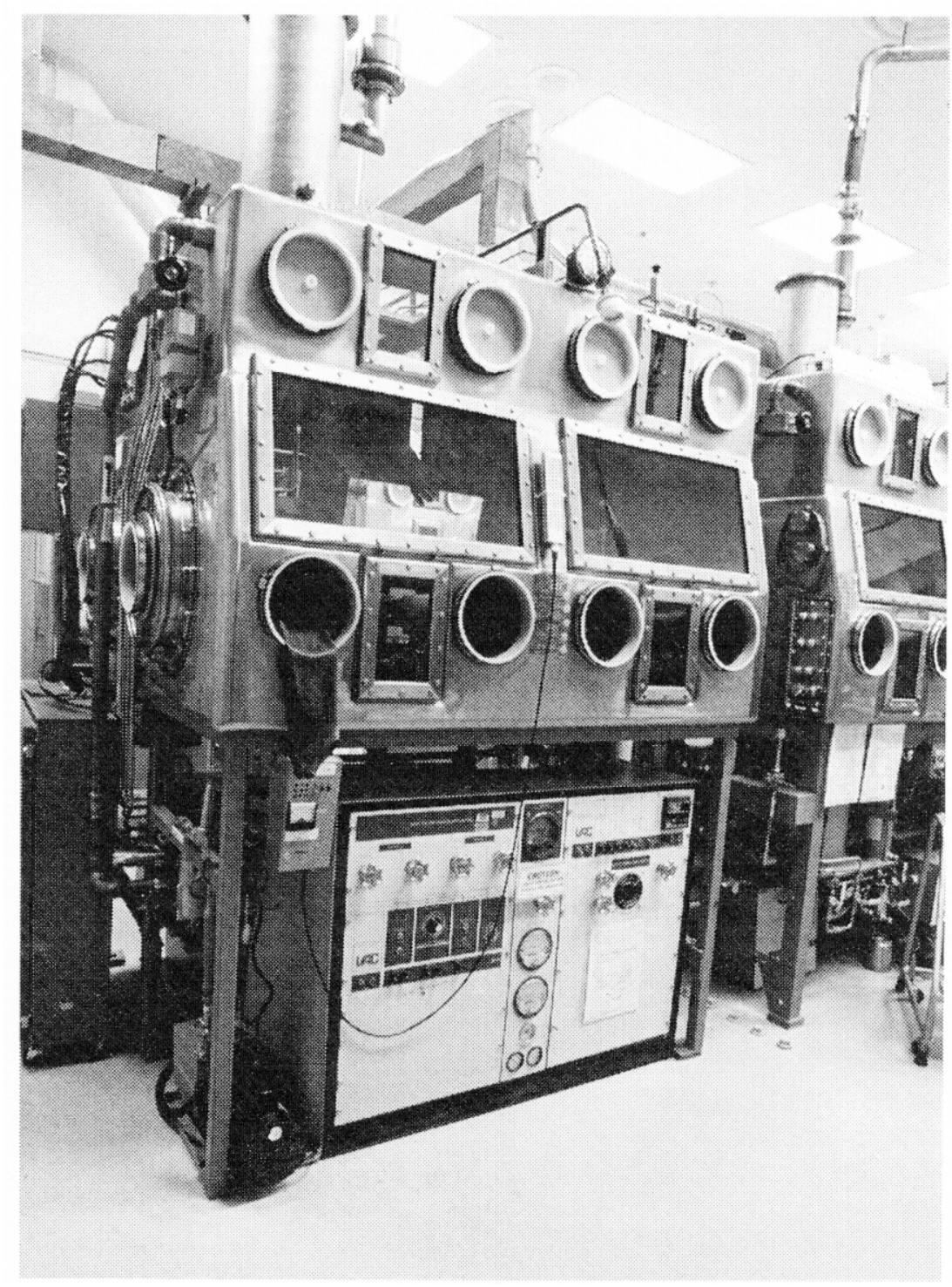

Figure 10.2.1-1 HEPA filters are contained in stainless steel canisters bolted to the tops of these glove boxes. 


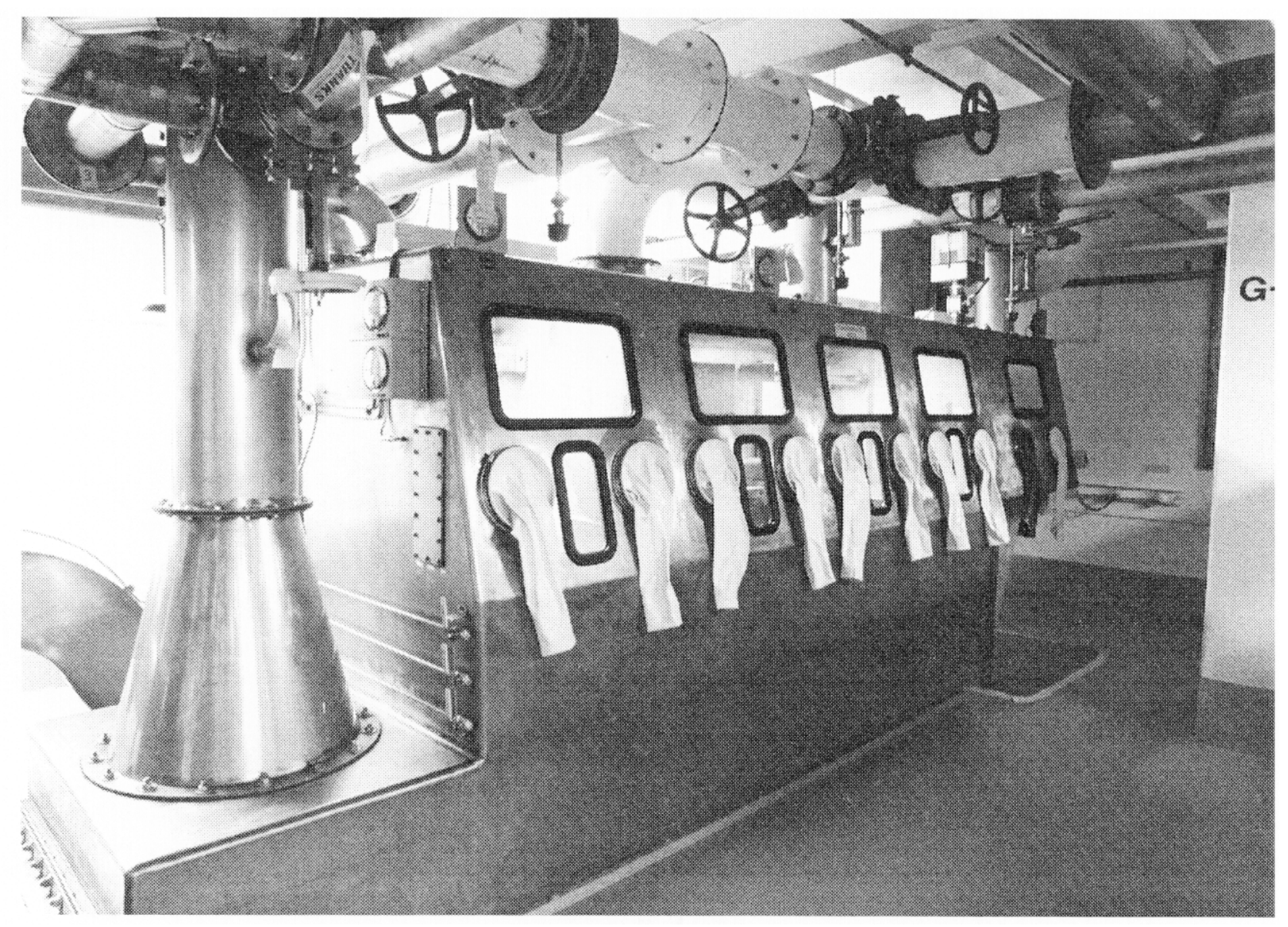

Figure 10.2.1-2 This filter plenum containing a series of HEPA filters is similar to a glove box. 


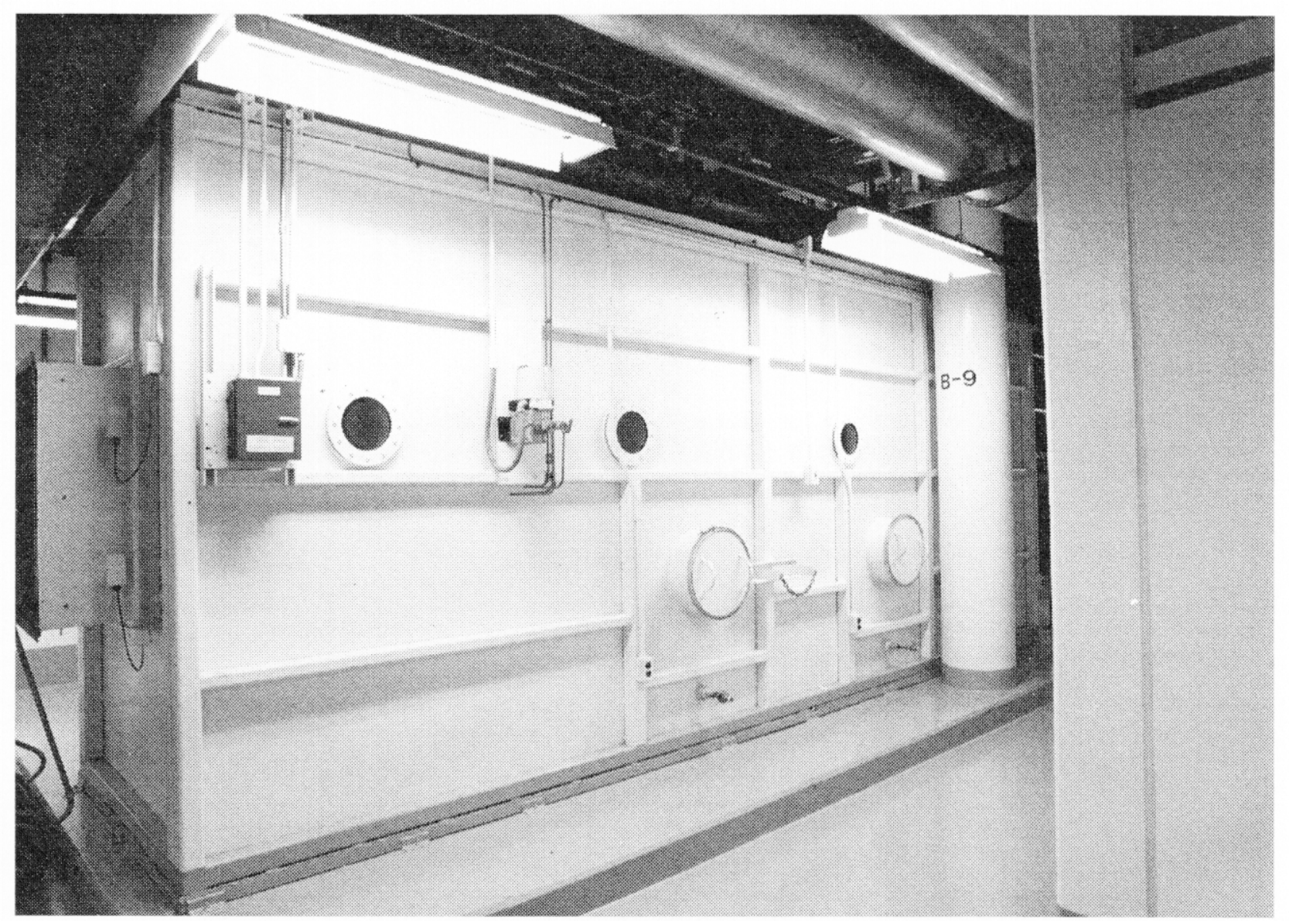

Figure 10.2.1-3 This filter plenum contains a series of HEPA filters and is constructed of structural steel tube frames with continuously welded steel plates for the walls, floor, and roof. 


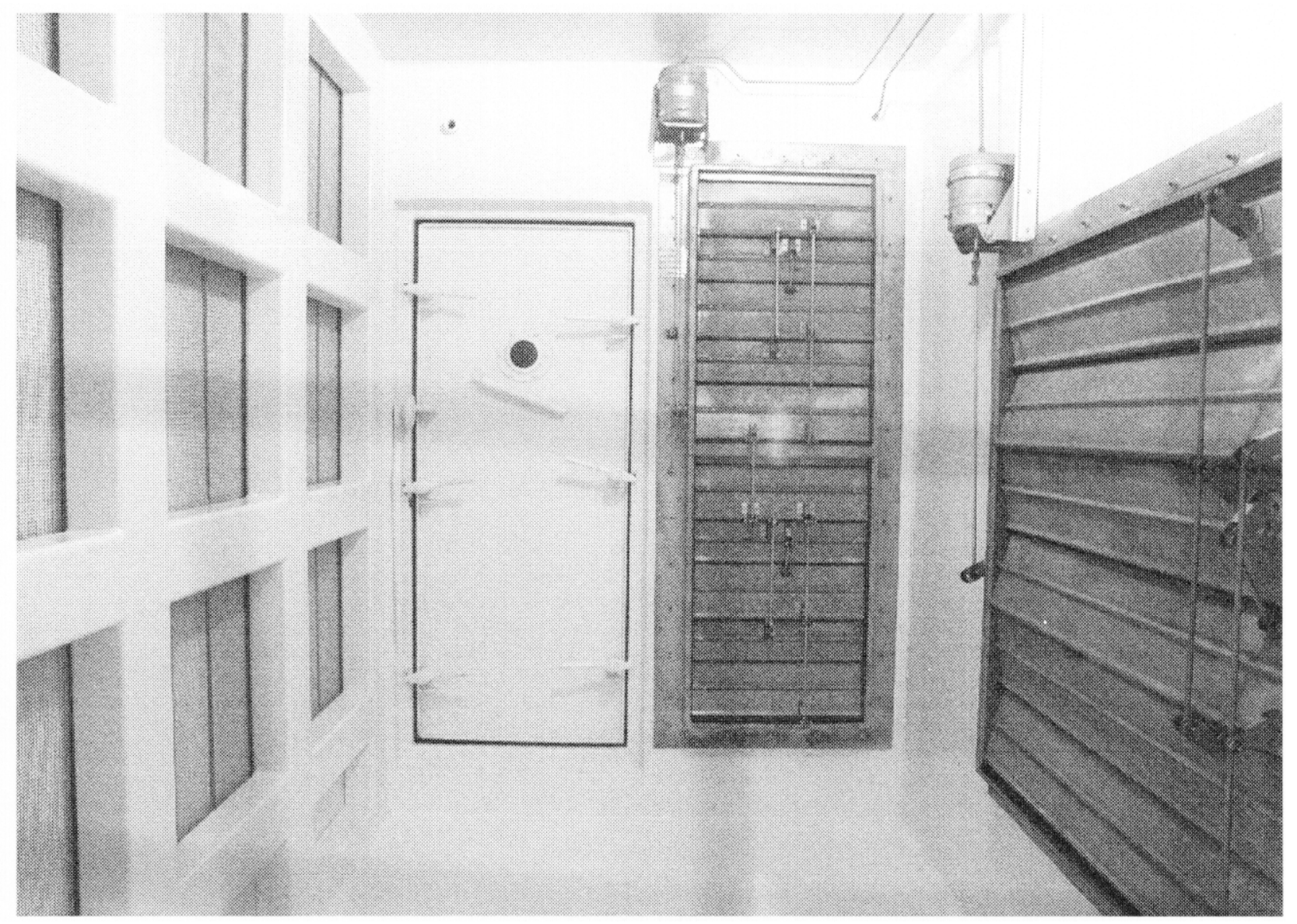

Figure 10.2.1-4 HEPA filters (on the left side of the photograph) are securely held to the structural steel tube frame by bolted clamps (not shown). Also shown are dampers which are typically associated with filter plenums. 


\subsubsection{GLOVE BOXES}

This section describes general guidelines that can be used for evaluating and upgrading the seismic adequacy of glove boxes which are included in the Seismic Equipment List (SEL). The guidelines contained in this section are based on analytical and walkdown experience at Los Alamos National Laboratory as well as other DOE sites. Guidelines in this section cover those features of glove boxes which experience has shown can be vulnerable to seismic loadings.

Glove boxes (see Figure 10.2.2-1) serve as primary confinement for radioactive or hazardous materials. As such, the pressure inside a glove box is less than the room pressure external to the glove box. Therefore, maintaining the pressure boundary is important when evaluating the seismic adequacy of glove boxes.

In evaluating glove boxes, the following five areas should be evaluated:

- $\quad$ seismic interaction effects, including flexibility of attached tubing and conduit and interaction with components or equipment located inside the glove box (heat sources, furnace, vacuum chamber, or flammable materials)

- load path

- supporting frame work

- leak tightness

- anchorage

As with other equipment, glove boxes are vulnerable to interaction effects. Windows, gloves and instrumentation tubing are all examples of fragile components associated with glove boxes that are prone to interaction effects. Interactions which should be considered include those that are both internal and external to the box. Externally, components such as power supplies and furnaces, which directly support glove box activities, should be restrained to prevent impact with windows (see Figure 10.2.2-2) and support frames. Internally, objects such as conveying systems and machining tools should be anchored to the box so that they cannot slide and tear gloves and break windows. Attached tubing and conduit need to have enough flexibility to accommodate the seismic motion of the glove box. Glove boxes which depend upon moment-resisting frame action for resistance of lateral seismic loads are more flexible than those using bracing and are therefore more susceptible to tubing and conduit failures. Additional guidance on evaluating the effects of seismic interaction is provided in Chapter 7.

The load path associated with the glove boxes needs to be evaluated. Load path refers to the manner in which inertial loads acting on the glove boxes and associated equipment are transferred through the glove box structure to the supporting framework, to the anchorage, and into the supporting structure. During seismic evaluations, the load path, including connections, should be carefully reviewed for adequate strength, stiffness, and ductility. Attachments, such as filtration devices and furnace wells, should be adequately anchored to the box. In addition, the box should be adequately attached to the supporting framework.

The supporting framework of glove boxes is one aspect of the evaluation in which structural calculations may be necessary to determine seismic adequacy. The framework should be reviewed for missing or altered (cutouts, notches or holes) members. Frames which rely on moment connections to provide lateral support and are constructed of unistrut or single angle legs have been found to be especially vulnerable. Braced frames are generally less vulnerable. 
As previously noted, glove boxes serve as primary confinement for radioactive or hazardous materials. As such, leak tightness is an important feature of the glove box system. Interaction effects, load path, and supporting framework, in particular the relative displacements with connections boxes and attachments, could jeopardize the integrity of the pressure boundary associated with a glove box.

As with most equipment, anchorage should be evaluated using the procedure in Chapter 6 . An area of concern which should be reviewed carefully is the gap between the bottom of the base plate and the floor. In many cases an individual glove box is part of a system or train of glove boxes in which one box is connected to another box. To maintain proper vertical alignment of the boxes, shims are typically used beneath the base plate (see Figure 10.2.2-3). These shims can introduce bending to the anchor bolts which can significantly reduce the capacity of the bolts. The reduction of bolt capacity due to bolt bending is briefly discussed in Chapter 6. 


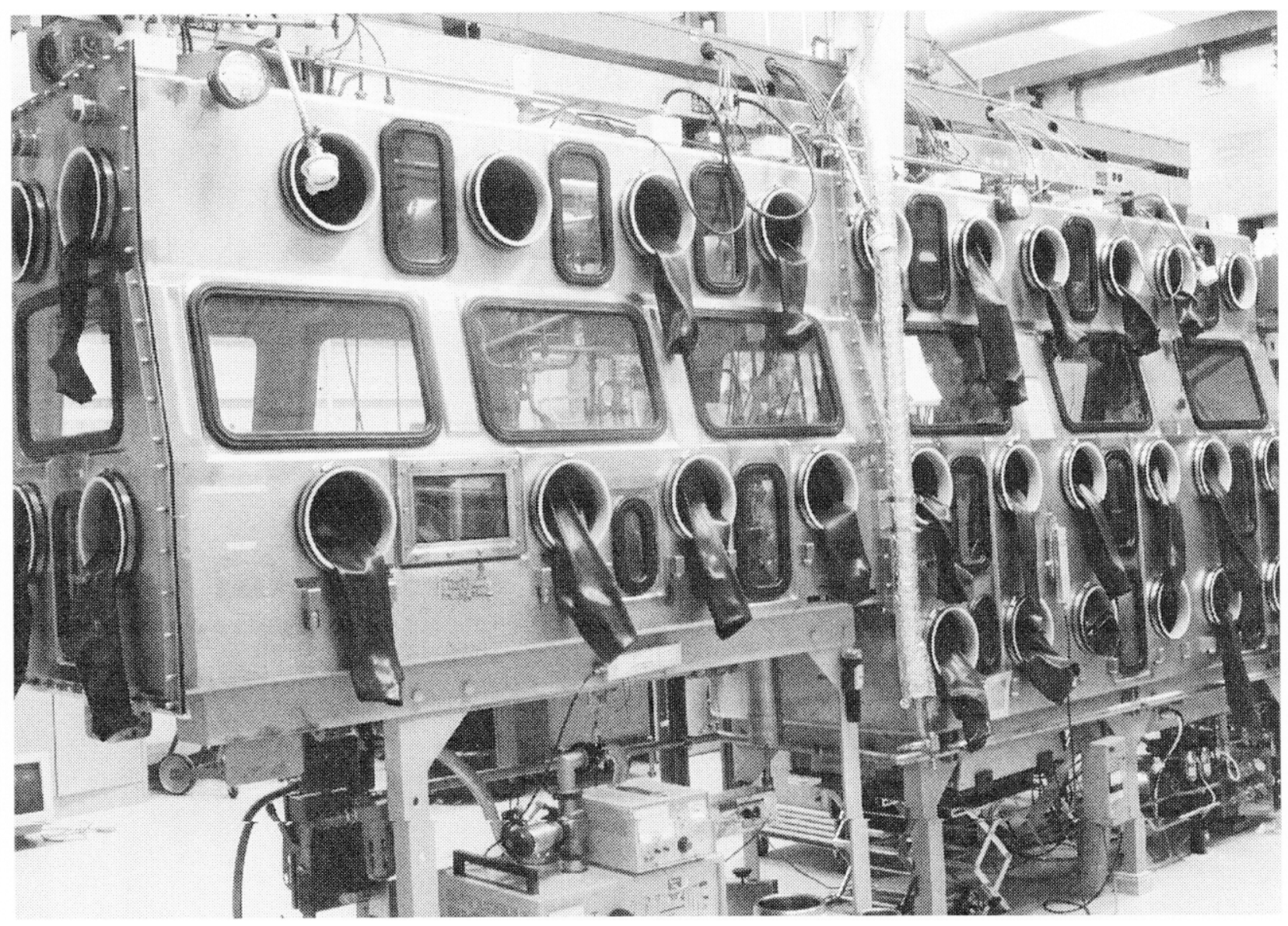

Figure 10.2.2-1 Shown is a typical glove box. This particular glove box is supported by a moment resisting frame composed of single angle legs. Frames of this type have been found to be vulnerable to seismic loads. 


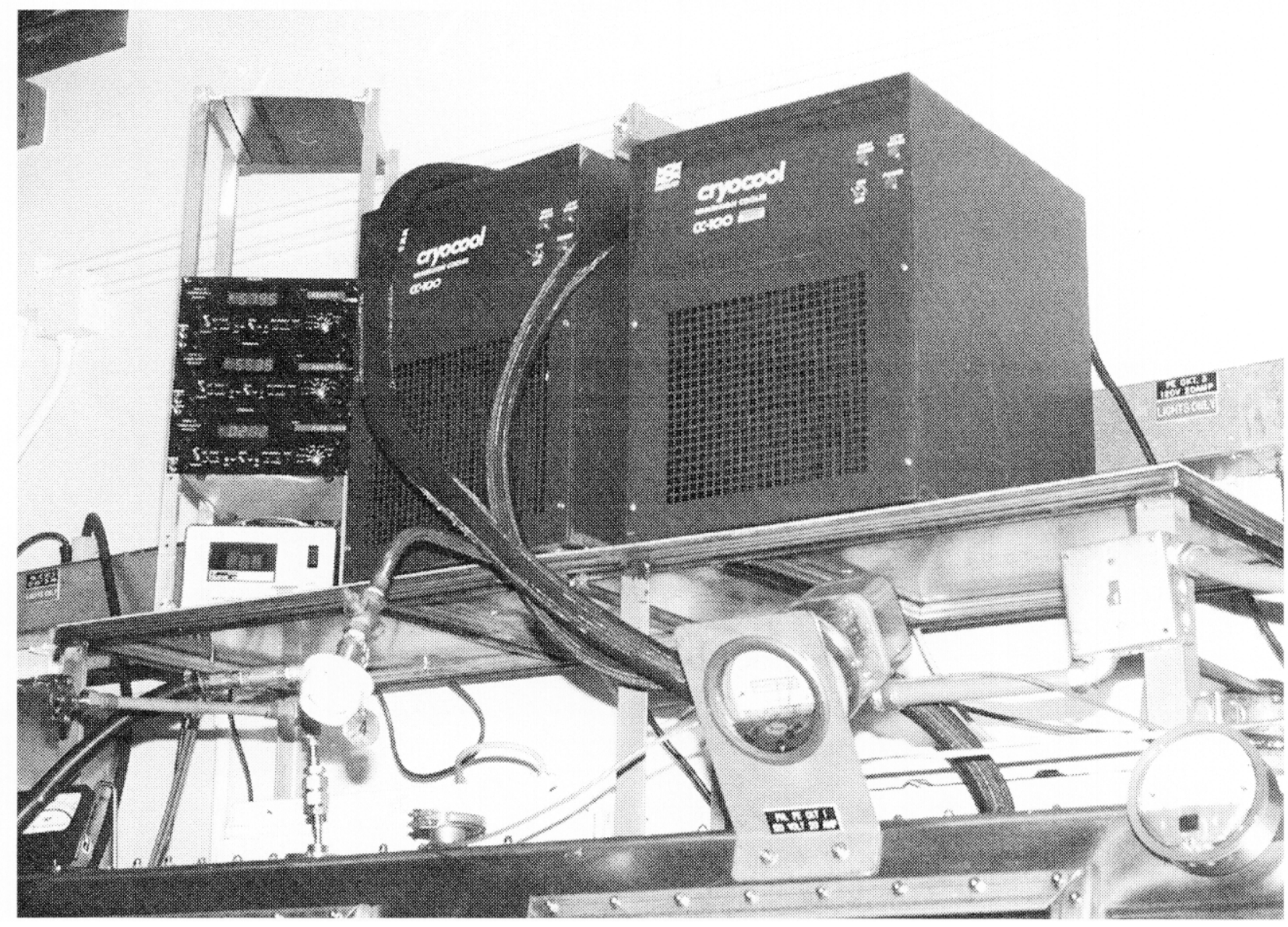

Figure 10.2.2-2 These refrigeration units support glove box activities. While the support stand is well supported on the top of the glove box, the units themselves are not anchored. During an earthquake, these units could slide off the support stand and impact a glove box window. 


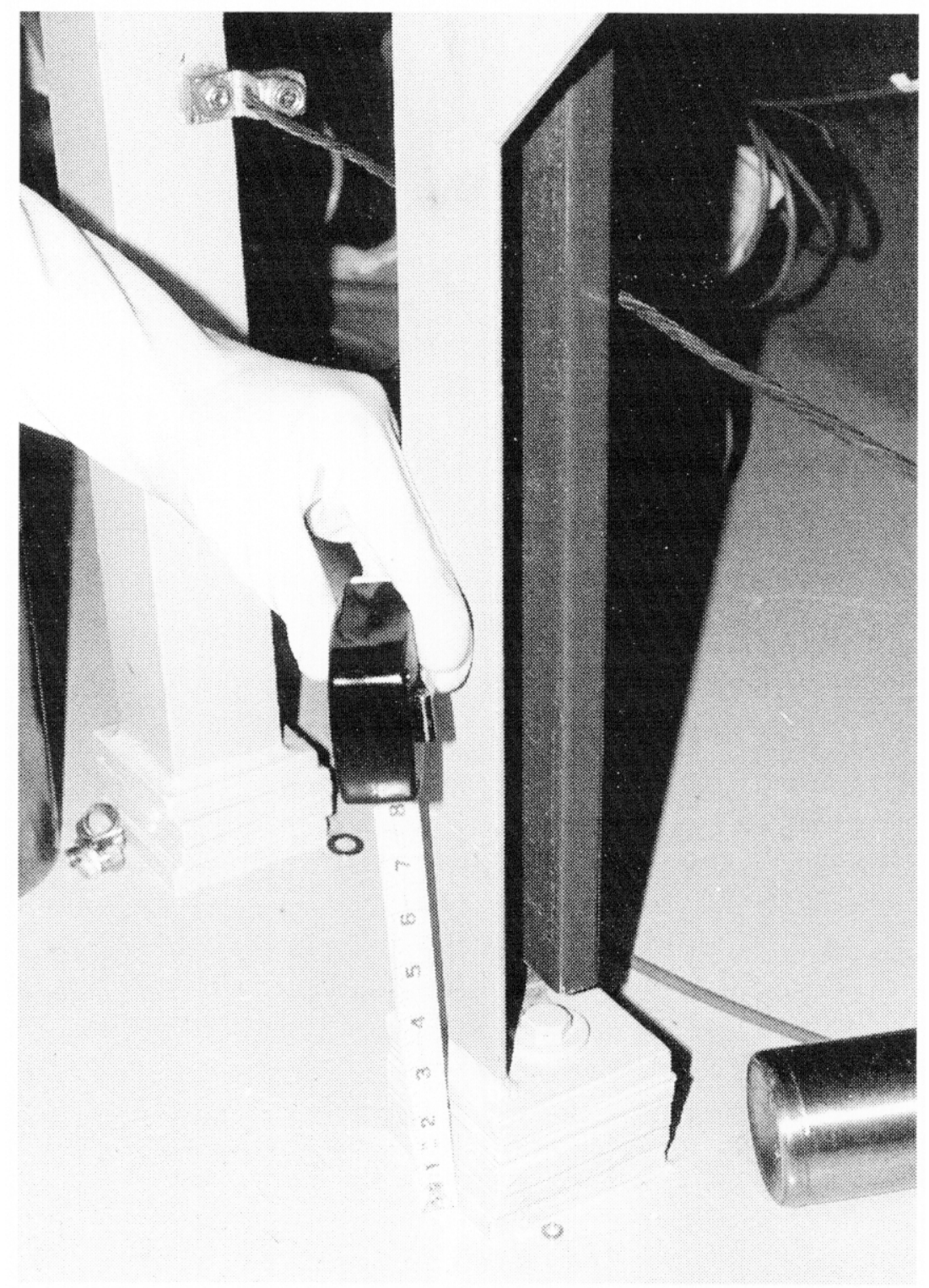

Figure 10.2.2-3 These legs have been shimmed to maintain proper vertical alignment of adjacent glove boxes. Excessive shim heights introduce bending to the anchor bolts which significantly decreases the bolt capacity. 


\subsubsection{MISCELLANEOUS MACHINERY}

This section describes general guidelines that can be used for evaluating and upgrading the seismic adequacy of miscellaneous machinery which is included in the Seismic Equipment List (SEL). The guidelines contained in this section are based on Section 4.9 of "Practical Equipment Seismic Upgrade and Strengthening Guidelines" (Ref. 60). Guidelines in this section cover those features of miscellaneous machinery which experience has shown can be vulnerable to seismic loadings.

Miscellaneous machinery is typically contained in a machine shop or maintenance facility. The machinery types in the facility include: lathes (see Figure 10.2.3-1), band saws (see Figure 10.2.3-2), drill presses (see Figure 10.2.3-3), and work bench mounted machinery.

Industrial grade machinery, such as that shown in Figures 10.2.3-1 to 10.2.3-3, is typically very rugged and does not experience significant damage during an earthquake as long as it is well anchored. The rugged machinery typically has an adequate load path for earthquake-induced lateral loads. Unanchored or inadequately anchored components can be susceptible to sliding, overturning, or component misalignment as shown in Figure 10.2.3-4.

Three general methods of evaluating and providing anchorage for shop and mechanical machinery are outlined below. The screening evaluation for anchor bolts is provided in Chapter 6 with the miscellaneous machinery typically treated as rigid. For miscellaneous machinery, the seismic evaluation should emphasize its anchorage.

- $\quad$ Anchor bolts should be provided through existing holes in machinery base. Bolt sizes should be the same as the size of the furnished holes and excessive amounts of shims should not be used.

- For tall, narrow, and/or top-heavy machinery which may overturn in a strong earthquake, anchors should be provided at all four corners, as shown in Figure 10.2.3-5.

- For short, wide, and/or bottom-heavy machinery which may slide but not overturn, bumpers should be provided at all four corners. As shown in Figure 10.2.3-5, bumpers should contact the edges of the machinery if possible. A resilient pad, such as neoprene, may be glued to the face of the angle to reduce impact loads.

Many miscellaneous machinery components are box-like units that simply rest on a concrete floor. A minimum of four anchor bolts should be provided for each item and the spacing between the anchor bolts should not exceed 4 feet. For machinery provided with base plates or structural members with holes intended for anchors, expansion anchors should be provided in these holes. Otherwise, new clips or angle can be either welded or bolted to the machinery and expansion anchors provided for the floor. For tall machinery, anchorage to a wall with adequate capacity in addition to that provided at the base can greatly increase the seismic capacity of the anchorage system.

There are many installation conditions for machinery in a machine shop or maintenance facility. General categories of the conditions include machinery on skids or wheels. Approaches which may be used to evaluate and upgrade the machinery in the two categories are presented below.

\section{Machinery on Skids}

Skids supporting machinery should be structural steel (or equivalent structural material) and the skids should be anchored to the floor slab with the machinery anchored to the skid. Stiffener plates should be supplied for steel skids which support heavy machinery to provide adequate 
stiffness to resist seismically induced lateral loads. Some recommended anchorage approaches are presented in Figure 10.2.3-6.

\section{Machinery on Wheels}

A number of different types of machinery, including maintenance machinery and computer consoles, are supported on casters or wheels. Without proper lateral restraint, machinery on wheels can roll around and damage other property and/or injure personnel. Wheel locks and an appropriate temporary restraining system, such as chains, should be provided for machinery that must remain mobile for operational purposes. Tall machinery should be anchored to the wall or roof at the top to prevent overturning. For more permanent items, floor or wall anchors should be installed, as shown in Figure 10.2.3-7. When anchoring to an existing wall, the capacity of the wall and the details of the structural connection of the wall and roof should be evaluated. If the wall is an unreinforced masonry (URM) wall, the provisions of Section 10.5.1 should be used. 


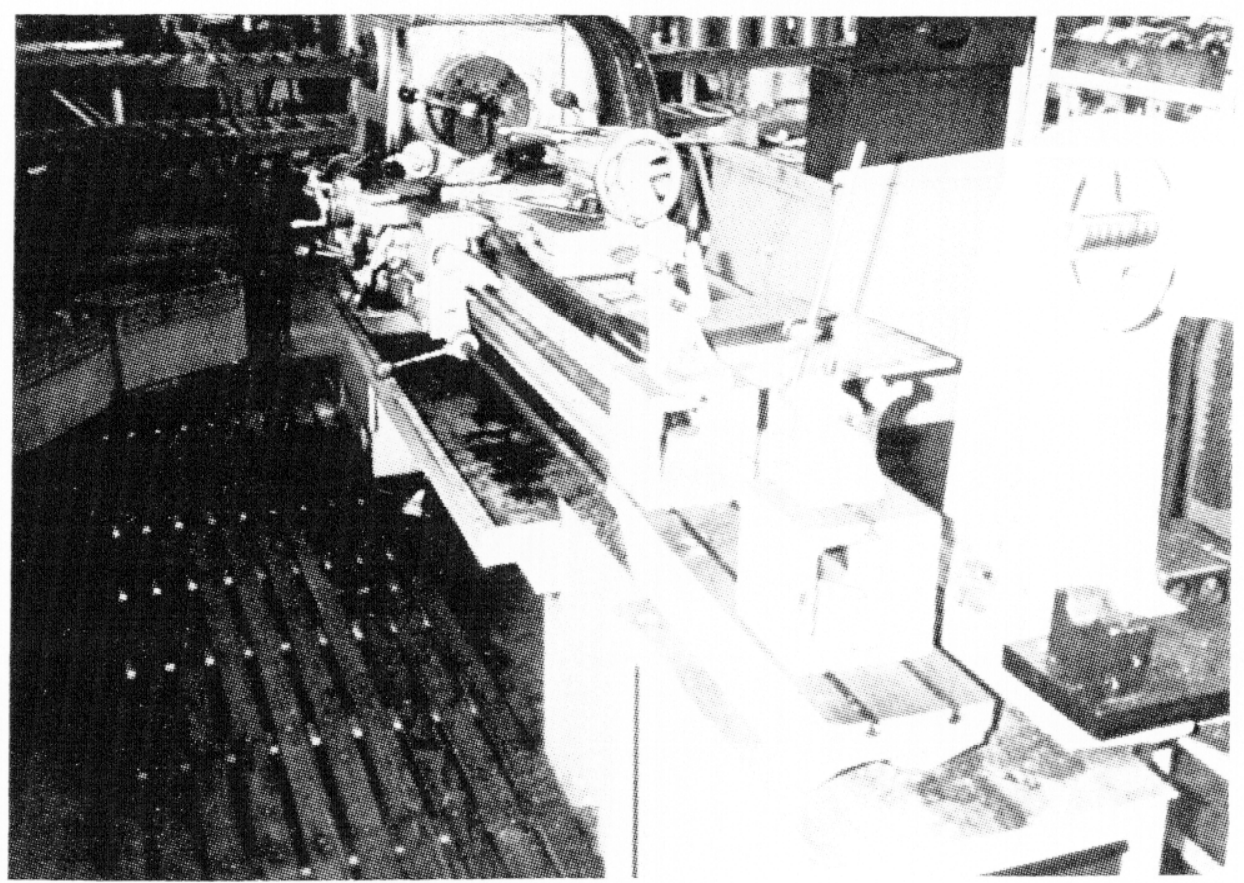

Figure 10.2.3-1 Unanchored Metal Lathe Susceptible to Sliding (Figure 4-69 of Reference 60) 

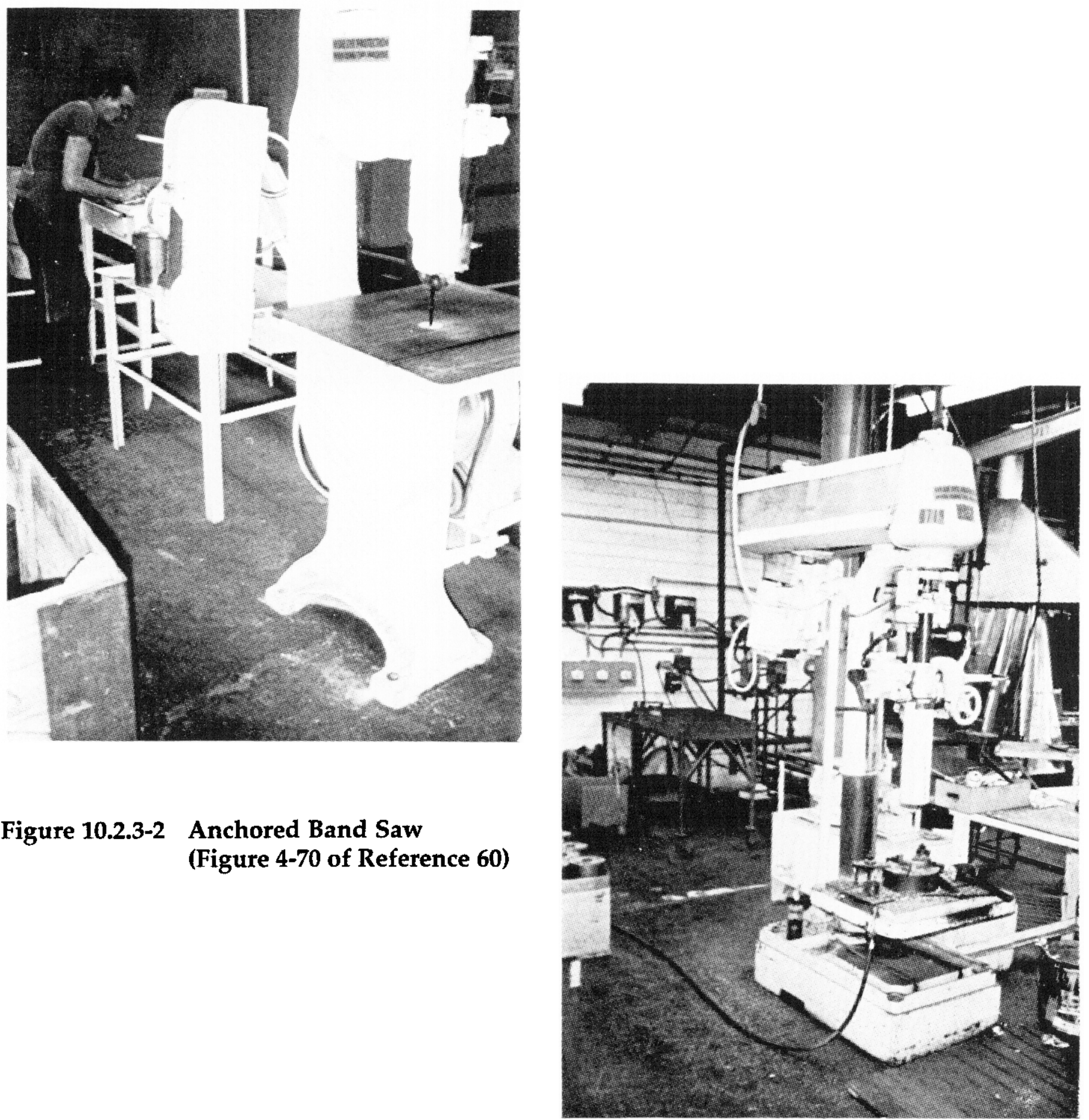

Figure 10.2.3-2 Anchored Band Saw (Figure 4-70 of Reference 60)

Figure 10.2.3-3 Unanchored Drill Press Susceptible to Overturning Damage (Figure 4-71 of Reference 60) 


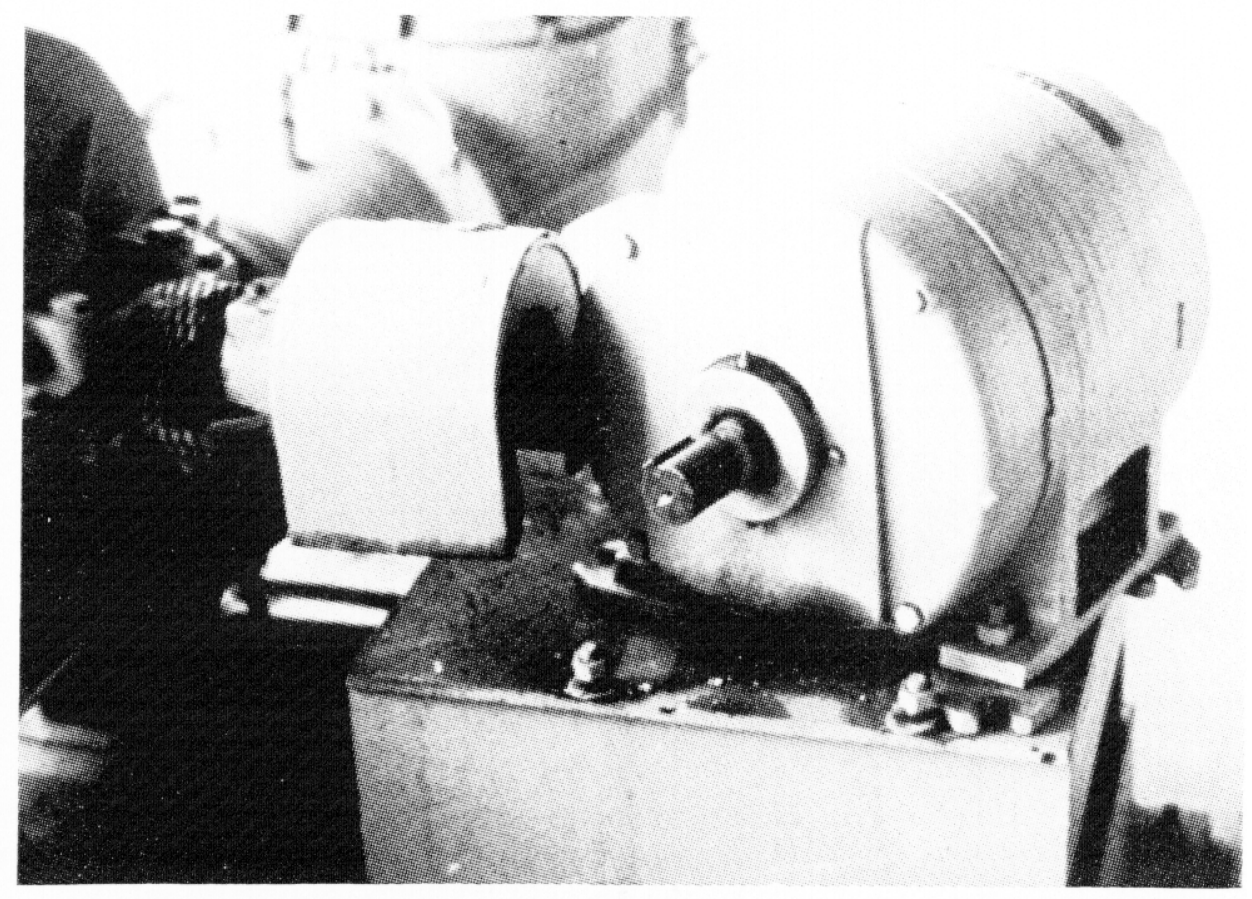

Figure 10.2.3-4 Misaligned Electrical Motor Resulting from Improper Anchorage (Figure 4-73 of Reference 60) 


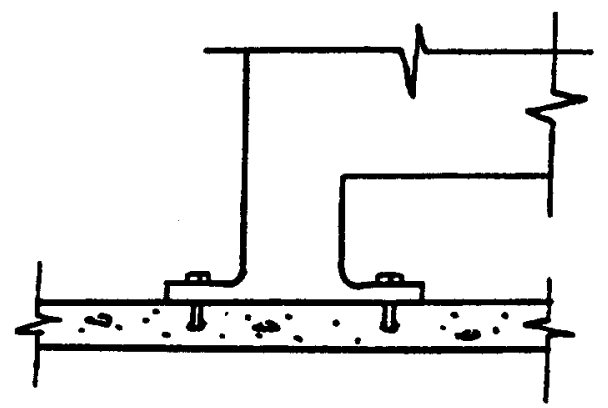

EQUIPMENT WITH FEET

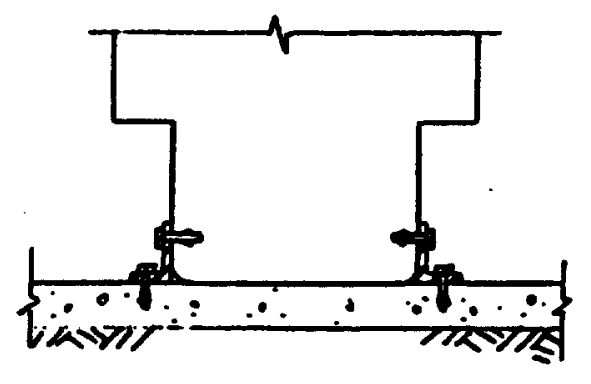

SOLID BASE EQUIPMENT

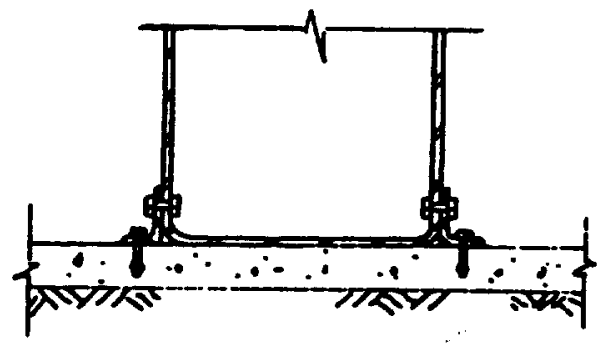

HOLLOW BASE EQUIPMENT

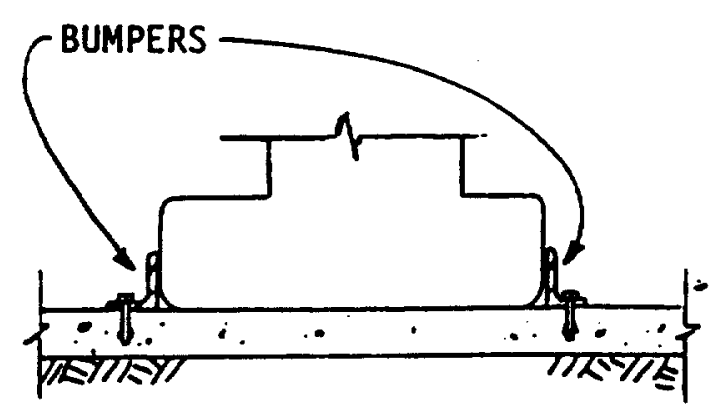

TYPICAL MATERIALS LIST:

L3 $5 \times 1 / 2^{n}$

3/4" ANCHOR BOLT

1/2" MACHINE BOLT

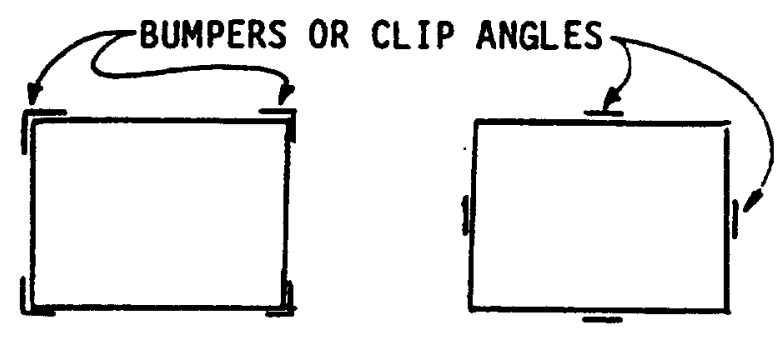

PLAN
PLAN

Figure 10.2.3-5 Approaches for Anchoring Machine Shop Equipment (Figure 4-74 of Reference 60) 

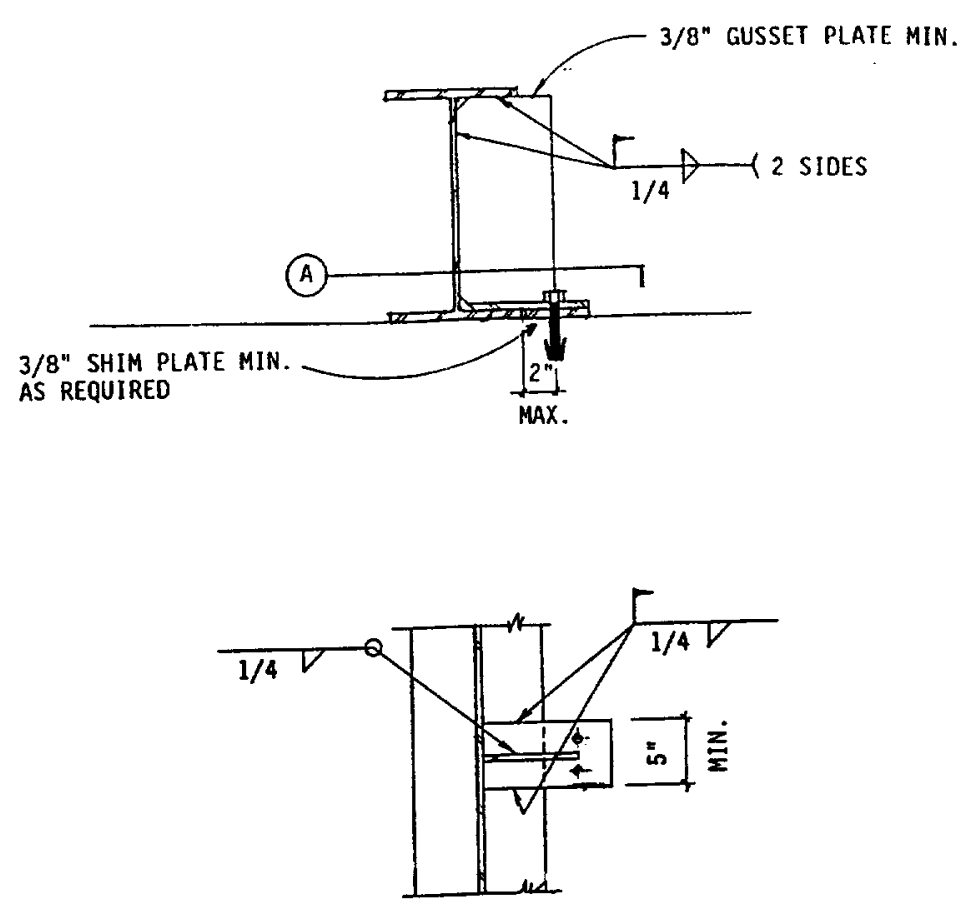

PLAN SECTION

(A)

RETROFIT SKID ANCHORAGE WHEN UPLIFT CAN OCCUR.

(TYPICALLY HEIGHT/DEPTH > 2)

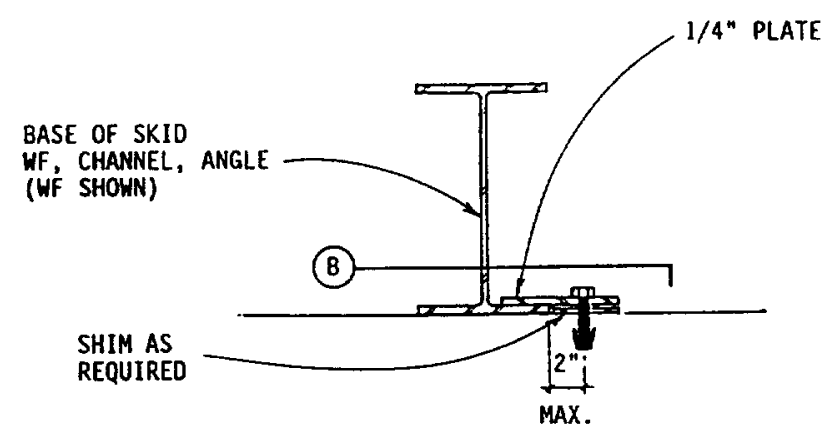

MIN. (4) 1/2" EXPANSION BOLT

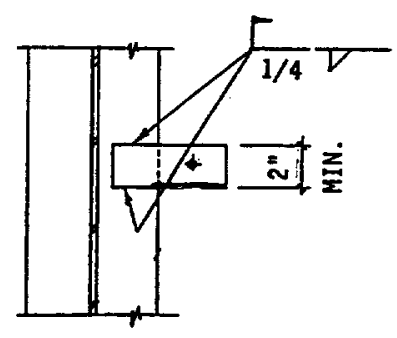

PLAN SECTION

(B)

RETROFIT SKID ANCHORAGE WHEN UPLIFT DOES NOT OCCUR (LOH PROFILE EQUIPMENT)

\section{Figure 10.2.3-6 Approaches for Anchoring Equipment Skids (Figure 4-76 of}

Reference 60) 

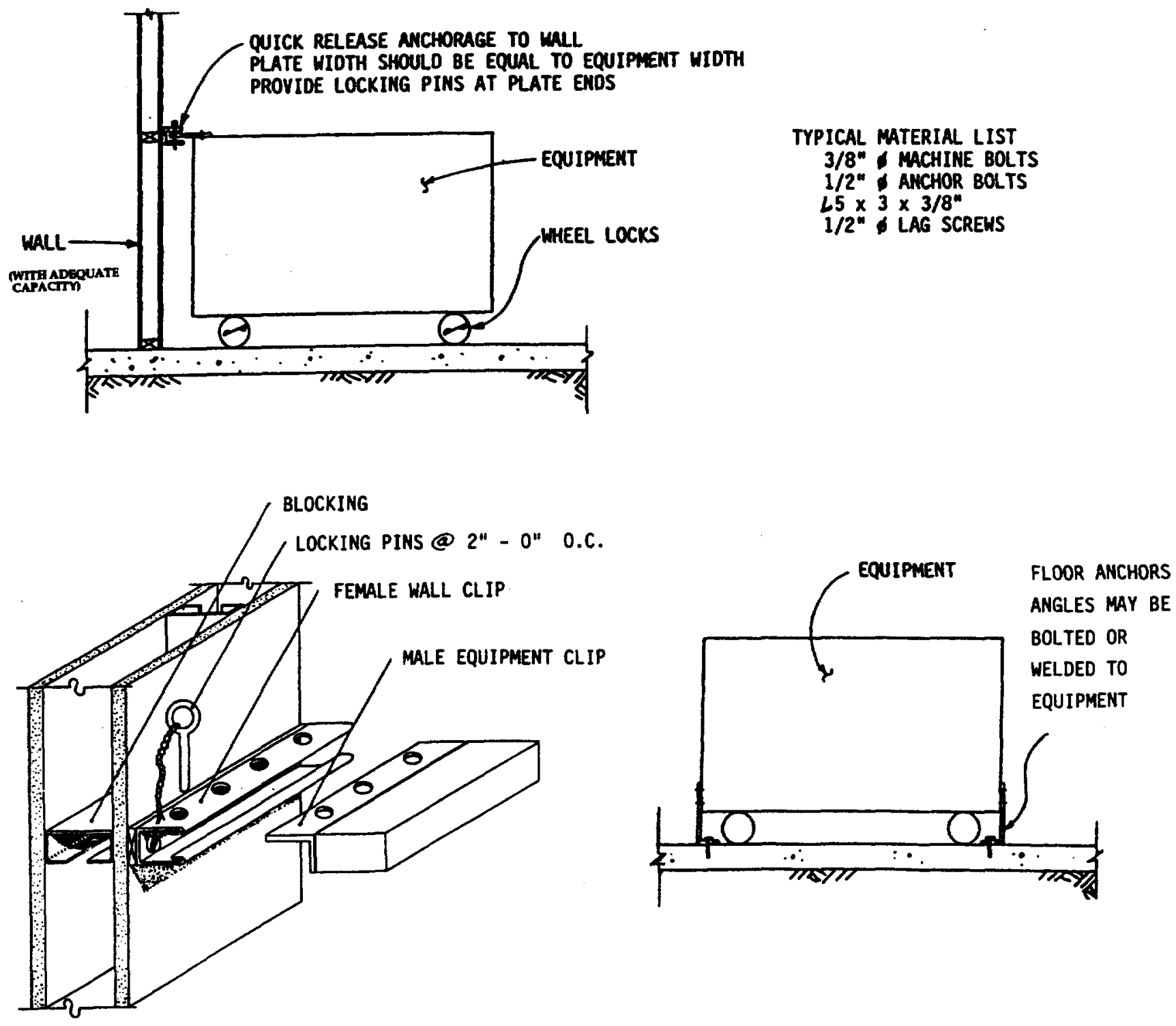

Figure 10.2.3-7 Approaches for Anchoring Equipment on Wheels (Figure 4-77 of Reference 60) 


\subsection{OTHER TANKS}

\subsubsection{UNDERGROUND TANKS}

Guidelines for considering earthquake loading for the design and evaluation of underground storage tanks can be found in Reference 29 ("Seismic Design and Evaluation Guidelines for the Department of Energy High-Level Waste Tanks and Appurtenances", BNL 52361). This document was prepared for high-level waste tanks and specifically covers the primary tank, secondary liner, concrete vault, transfer piping, and the other components required to maintain the confinement function of a tank farm. The guidelines are developed primarily for double-shell tanks since it is expected that all new tanks will be double-shell structures. However, these guidelines are also generally applicable to single-shell tanks.

The design and evaluation guidelines in Reference 29 include a definition of the design basis earthquake ground motion, simplified methods for determination of soil-structure and liquidstructure interaction effects, analytical techniques for evaluating seismic demand, and criteria for assessing structural capacity. Table 10.3.1-1 provides a road map to the various subjects addressed in Reference 29. The abstract states that these guidelines reflect the knowledge acquired in the last two decades in the areas of defining the ground motion and calculating hydrodynamic loads and dynamic soil pressures, and other loads for underground tank structures, piping, and equipment. Interpretation and implementation of the guidelines are illustrated through examples.

\section{Table 10.3.1-1 Use of "Seismic Design \& Evaluation Guidelines for the Department of Energy High-Level Waste Tanks \& Appurtenances" (Ref. 29)}

\begin{tabular}{|l|c|}
\hline \multicolumn{1}{|c|}{ Subject Matter } & $\begin{array}{c}\text { Chapter } \\
\text { and/or Appendix } \\
\text { from Reference 29 }\end{array}$ \\
\hline Seismic Design and Evaluation Criteria & Chapter 3 \\
\hline Evaluation of Tank Response & Chapter 4 \\
\hline Hydrodynamic Effects & Appendix B \\
\hline Liquid Viscosity Effects & Appendix C \\
\hline Soil-Structure Interaction & Appendix G \\
\hline Effect of Top Constraint & Chapter 5 \\
\hline Seismic Response Example & Appendix A \\
\hline Evaluation of Tank Capacity & Appendix F \\
\hline Seismic Capacity & Appendix D \\
\hline Inelastic Energy Absorption & Appendix E \\
\hline Buckling of Tanks & \\
\hline Effects of Sloshing Striking the Roof & \\
\hline Dimension Tolerance and Fabrication Details & Chapter 8 \\
\hline Associated Structures and Equipment & Chapter 7 and Appendix I \\
\hline Underground Piping (Section 10.1.2) & Equipment Qualification \\
\hline \multicolumn{2}{|l|}{}
\end{tabular}

As described in Chapter 3 of Reference 29 (see Table 10.3.1-1), the seismic guidelines for underground storage tanks are based on the same target performance goals upon which general seismic design and evaluation criteria for Department of Energy structures, systems, and components as given in DOE-STD-1020 (Ref. 6) are based. Deterministic, pseudo-linear seismic evaluation procedures are provided that are based on the DOE target performance goals. The 
document recognizes that there may be situations where explicit non-linear dynamic analysis of structures or soil columns may be necessary. It also recognizes cases, such as liquefaction analysis, where there may not be existing capacity standards consistent with the deterministic procedures. For these situations, a more general approach for complying with the target performance goals is discussed in which alternative design or evaluation techniques may be employed.

In addition to general seismic design and evaluation criteria, many subjects specifically addressing issues pertinent to underground storage tanks are covered by Reference 29 as illustrated by Table 10.3.1-1. In general, these subjects include evaluation of hydrodynamic effects in tanks, seismic capacity of tanks, evaluation of soil-vault interaction, and underground piping and conduits. Each of these areas is briefly described in the following paragraphs.

A critical element in the analysis of the seismic response of the tank-liquid system is the evaluation of the hydrodynamic pressures exerted against the tank wall and base. Once these pressures have been established, the corresponding forces and stresses in the tank may be determined with relative ease. Methods of evaluating hydrodynamic pressures for horizontal, rocking, and vertical components of earthquake ground motion are presented. In addition, sloshing motion of the free liquid surface is considered. These items are addressed in Chapter 4 and Appendix $\mathrm{G}$ of Reference 29 (see Table 10.3.1-1). Of special interest for waste storage tanks are the effects of inhomogeneous liquids within the tank or the influence of liquid viscosity on hydrodynamic effects which is addressed in Appendix B of Reference 29.

Assessment of the seismic capacity of tanks in Reference 29 considers observed failure modes for tanks in past earthquakes. Flat bottom vertical liquid storage tanks have sometimes failed with loss of contents during strong earthquake shaking. For tanks with radius to wall thickness ratios greater than about 600 or tanks with minimal or no anchorage, failure has often been associated with rupture of the tank wall near its connection to the base, due either to excessive tank wall buckling or bolt stretching and excessive base plate uplift. Both failure modes are primarily due to the dynamic overturning moment at the tank base from fluid pressure on the tank wall. Other common failure mode have been breaking of piping connected to a tank as a result of relative movement and severe distortion due to a soil failure (soil liquefaction, slope instability, or excessive differential settlement). Other failure modes, which are of much lesser importance either because of their general lack of occurrence or less severe consequences, but which deserve some attention, are: tank sliding, excessive hoop tensile stresses due to hydrodynamic pressures on the tank wall, damage to the roof due to insufficient freeboard for fluid sloshing, and damage to internal attachments from lateral and torsional fluid movements. Tank capacity evaluation is addressed in Chapter 5 and Appendices A, F, D, and E of Reference 29 as shown in Table 10.3.1-1.

Important considerations for soil-vault interaction are evaluation of the seismic input motion to the support points of the tank and the seismically induced pressures on the walls of the vault.

Evaluation of soil-vault (soil-structure) interaction must consider the vertical spatial variation of the free field ground motion and that the motion of the vault may differ from the free field motion. Guidelines for necessary soil properties and evaluation of soil structure interaction effects applied to underground tanks are presented in Chapter 6 and Appendix H of Reference 29.

Most underground waste process piping systems are encased (or double containment) piping systems. The inner pipe serves to transport the wastes and maintain the pressure boundary and the outer pipe provides secondary containment and is in direct contact with the surrounding soil. The design of underground piping systems and conduits must demonstrate the ability of the piping system to withstand strains and stresses caused by potential seismic movement of the surrounding soil in conjunction with stresses induced by other concurrent loads. Guidelines are provided to consider different aspects of seismically induced ground movements including: (1) abrupt relative 
displacements of the ground at faults; (2) ground failure of relatively large areas caused by liquefaction, landslides, gross surface movements, or collapse of voids at depth; (3) transient deformation of the ground during the earthquake due to wave passage effects; (4) inertial response of the inner piping system in response to induced movements of the outer piping; and (5) transient movements of anchor points or buildings connected to buried facilities. As shown in Table 10.3.1-1, underground piping is addressed in Chapter 7 and Appendix I of Reference 29. 


\subsubsection{CANISTERS AND GAS CYLINDERS}

This section describes general guidelines that can be used for evaluating and upgrading the seismic adequacy of canisters and gas cylinders which are included in the Seismic Equipment List (SEL). Guidelines in this section cover those features of canisters and gas cylinders which experience has shown can be vulnerable to seismic loadings.

Unanchored compressed gas cylinders will tip over at very low levels of ground shaking. If the reducing valve should snap off, the canister may become a high speed missile. In addition, escaping gas may represent a potential fire, explosion, or toxic gas hazard to nearby personnel.

Compressed gas cylinders often have a single safety chain located about mid-height (Figure 10.3.2-1). A single chain is not sufficient to prevent tipping during an earthquake. Examples of properly anchored cylinders are presented in Figures 10.3.2-2 and 10.3.2-3. In these figures, the gas cylinders have upper and lower safety chains, or restraints.

In the event of an earthquake, poorly restrained canisters and gas cylinders may fall and roll, spilling their contents, causing damage to other equipment, and/or injuring personnel. Methods of restraining them, including providing positive anchorage to a wall, storing them in well braced and anchored racks, or storing them horizontally on the floor, are shown in Figure 10.3.2-4. The supports for the canisters should be attached to walls that have adequate capacity to resist the seismic demand from the canisters. Adequate capacity typically results from two levels of support or a structural storage system that restrains moments. 


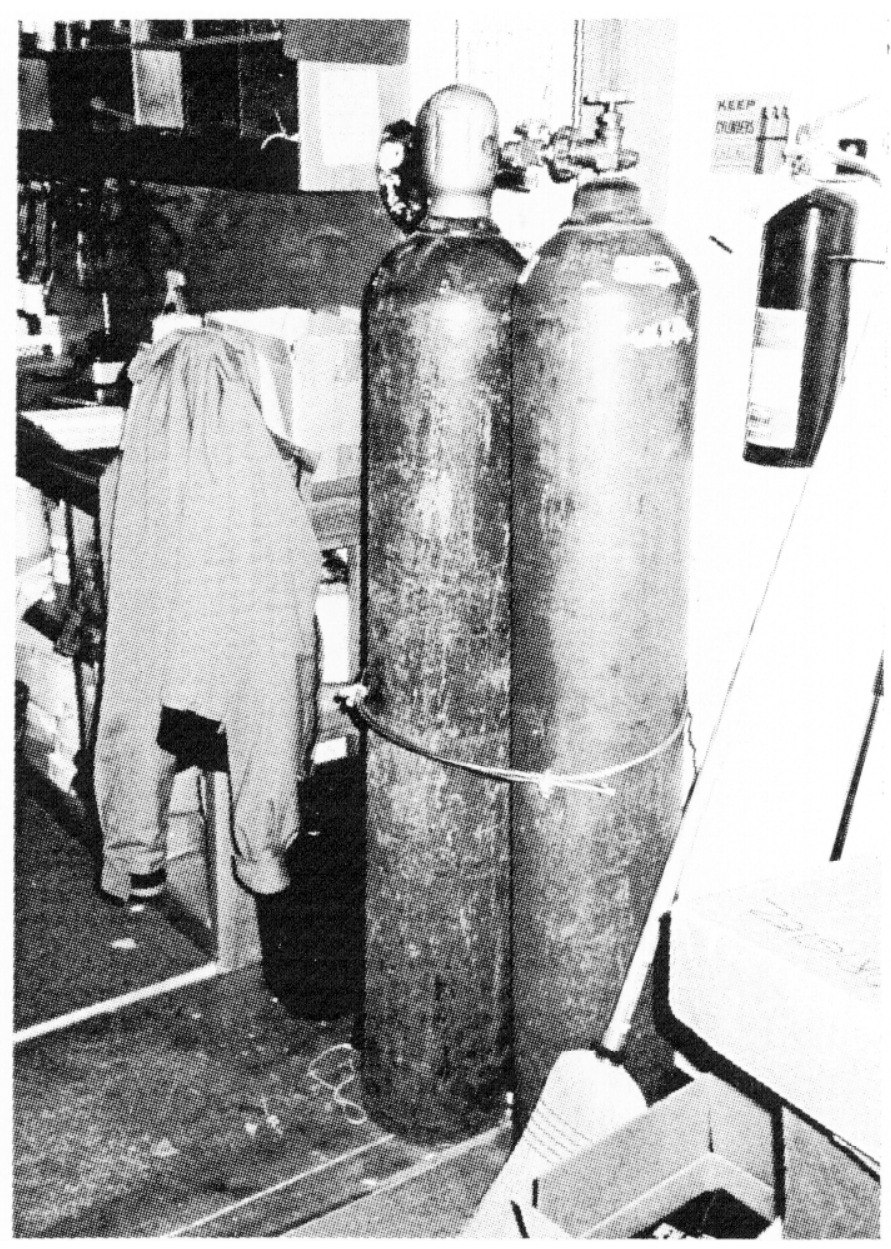

Figure 10.3.2-1 Compressed Gas Cylinder that is Inadequately Anchored with a Safety Chain Located at Midheight (Figure 4-55 of Reference 60) 


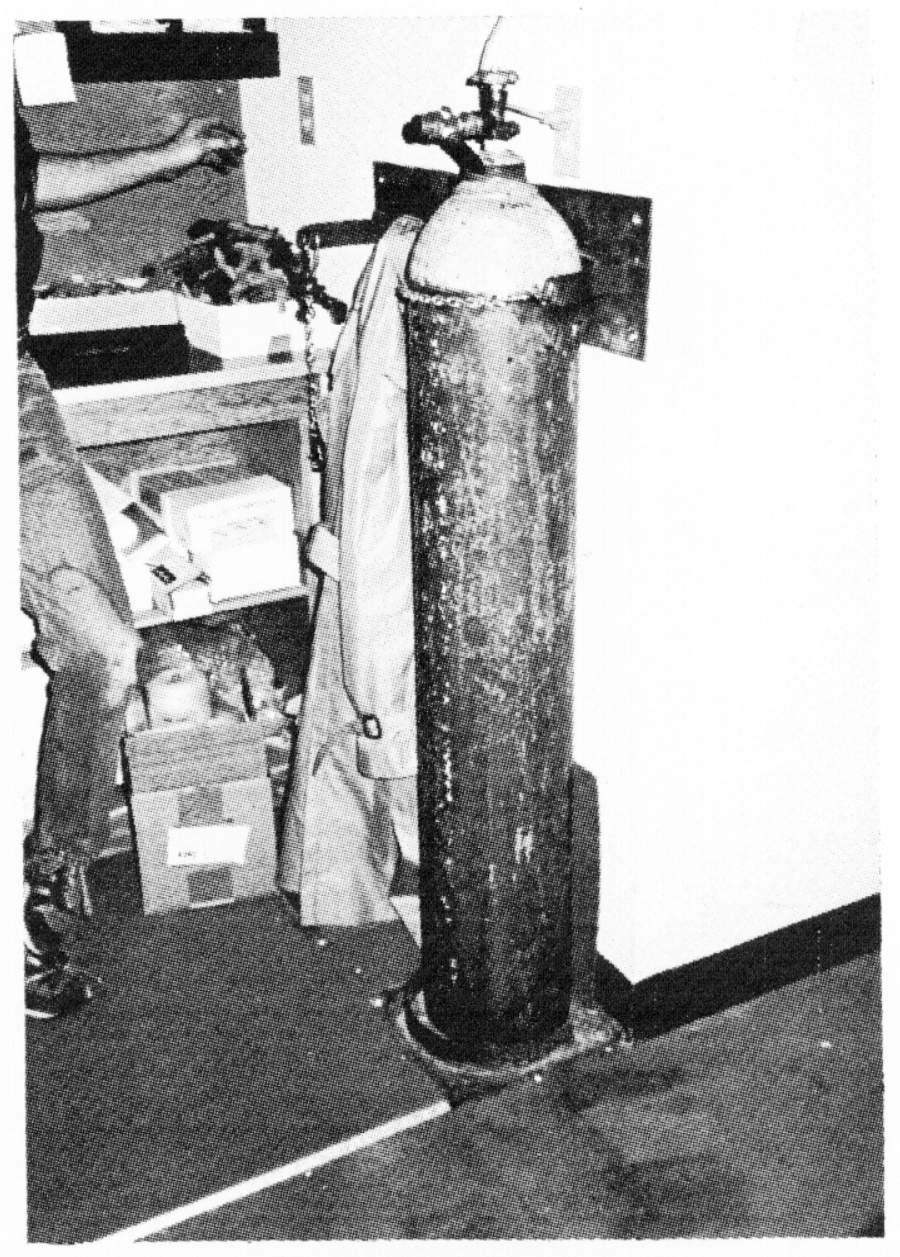

Figure 10.3.2-2 Adequately Anchored Compressed Gas Cylinder (Figure 4-57 of Reference 60) 


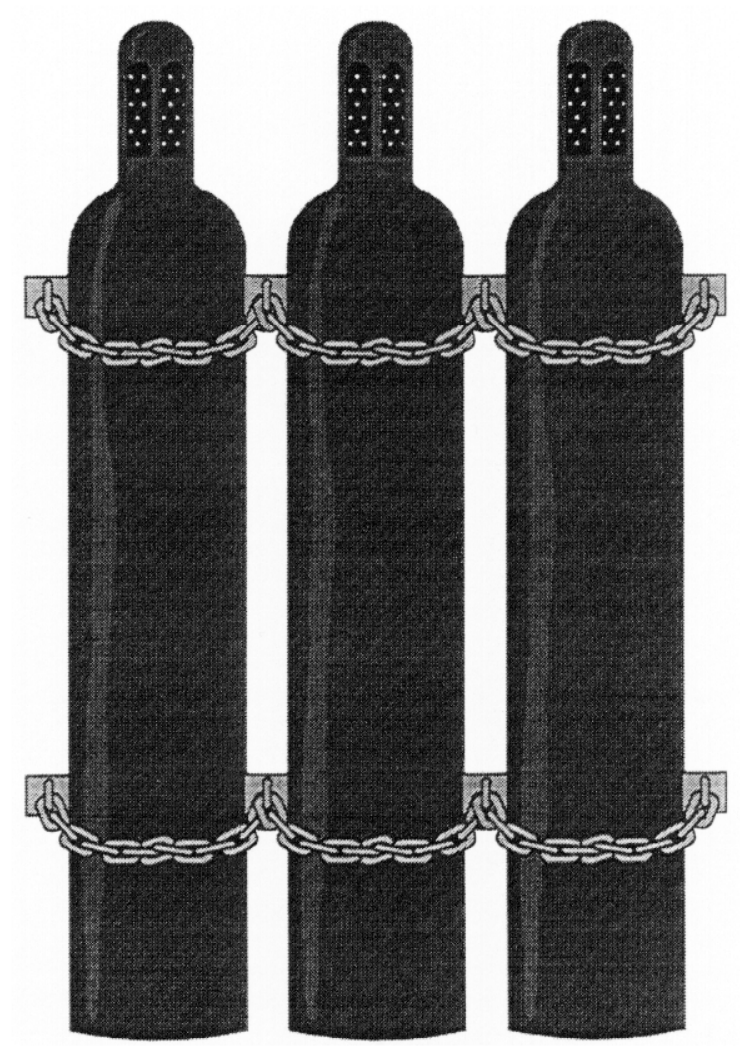

Figure 10.3.2-3 Upper and lower restraints are required for gas bottles. 

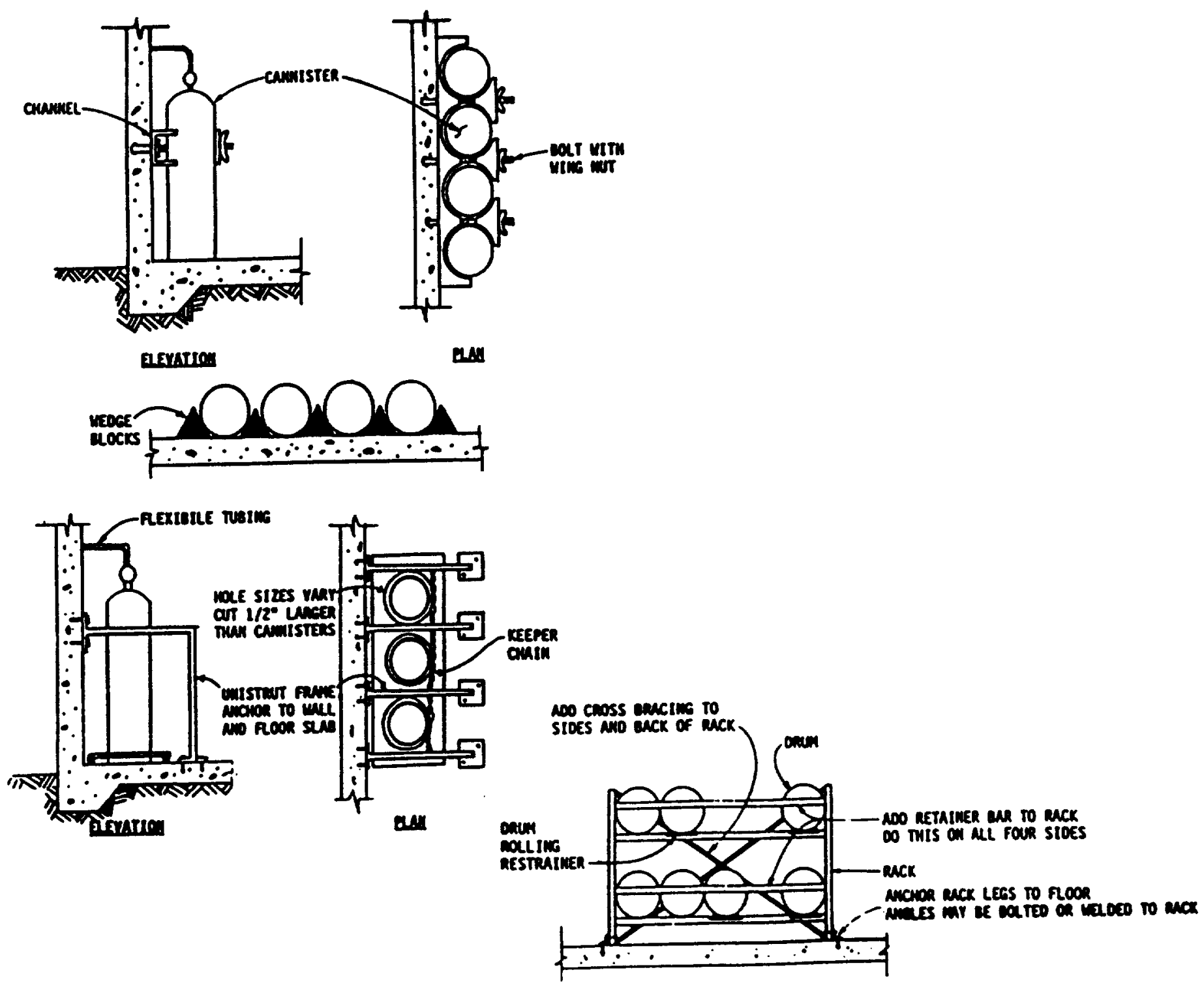

Figure 10.3.2-4 Approaches for Anchoring Canisters (Figure 4-56 of Reference 60) 


\subsection{DUCT SYSTEMS}

\subsubsection{HVAC DUCTS}

This section is the "Procedure for the Seismic Evaluation of Steel HVAC Duct" (Ref. 28) which was developed by the Westinghouse Savannah River Company and is based on information in Reference 107. It is limited to applications involving existing duct systems. For new design, the engineer is referred to other methods documented in References 108, 109, and 110. Additional information is contained in References 111 and 112.

\subsubsection{Scope}

This procedure provides seismic evaluation rules for existing rectangular or round steel HVAC duct. The objective of this evaluation procedure is to ensure a high confidence of acceptable seismic performance for the following:

- duct structural integrity

- material condition

- joint, seam, and stiffener design

- vertical and horizontal support bracing

- heavy components and appurtenances

- stiff branches

- duct pressure boundary integrity (if applicable)

- joint, seam, and stiffener design

- duct panel stress

- duct support bearing (point contact)

- flexible bellows

- duct support integrity

- material condition

- seismic capacity vs. demand

- support anchorage

- support details (load path)

- seismic interactions

The duct system seismic evaluation includes facility walkdown reviews and limited analytical reviews of bounding sample configurations. The relationship and typical sequence of these reviews is shown in a logic diagram in Figure 10.4.1-1.

Fans (including louvers) and air handlers (including dampers) are covered in Sections 8.2.10 and 8.2.9, respectively.

Duct mounted dampers that are not part of the fan or air handler assemblies and floor mounted filter housings and plenums must be evaluated separately and are not covered by this procedure.

\subsection{Industry Standards}

"HVAC Duct Construction Standard, Metal and Flexible", SMACNA (Ref. 113)

"Rectangular Industrial Duct Construction Standards", SMACNA (Ref. 114)

"Round Industrial Duct Construction Standards", SMACNA (Ref. 115) 


\subsection{Duct Loads}

(a) Duct weight

(b) Coating and insulation weight

(c) Positive (outward) or negative (inward) uniform pressure. Typically expressed in inches of water gage (wg), as a differential pressure relative to atmosphere ( 1 atmosphere $=0 \mathrm{wg}$ and $1 \mathrm{wg}=0.0361 \mathrm{psig}$ ).

(d) Weight of particulate accumulation in the duct.

(e) Weight of workmen or implements resting from time to time on the duct.

(f) Forces due to wind, for outdoor duct.

(g) Forces due to seismic events.

(h) Vibration from system operations.

Loads (a) through (f) are addressed in the SMACNA design standards.

Seismic loads ( $\mathrm{g}$ ) are evaluated by this procedure which is based on design standards, testing and seismic experience as documented in Reference 107.

Vibration loads (h) are typically evaluated and resolved after system start-up.

\subsection{Seismic Review Tearn}

The seismic review team shall consist of a minimum of 2 engineers certified in the use of the DOE Seismic Evaluation Procedure (see Section 3.2) and knowledgeable in the design requirements of the SMACNA standards. They shall document their review on Screening Evaluation and Work Sheets (SEWS) as described in this procedure. Each evaluation attribute shown on the SEWS form is described in Section 10.4.1.2 through 10.4.1.6 of this procedure.

\subsection{Duct System Boundary}

The duct system boundary establishes the scope of the configuration to be evaluated. These boundaries are determined based on consideration of system requirements and operational needs during or following a seismic event. For example, the HVAC system performance requirements following an earthquake may be to support environmental confinement of hazardous materials. In this case, pressure boundary integrity is important. The HVAC evaluation boundaries may terminate at system isolation points such as dampers. Furthermore, the evaluation scope might be limited to portions of the system that support filtration (e.g. HEPA which is also discussed in Section 10.2.1) and effluent exhaust.

In some cases, the performance objective of the HVAC system may be to convey air for the comfort and safety of building personnel. In this situation, duct structural integrity is the primary objective (instead of a high degree of pressure retention).

A Screening Evaluation Work Sheet (SEWS 10.4.1) may encompass a single run of duct, a duct system (several runs of duct with the same operating parameters) or a group of duct systems. The SEWS should describe, by sketch or system identification, the scope of ducts covered. 


\subsection{Evaluation Objectives (Pressure Boundary/Structural Integrity)}

Where only structural integrity is required, some leakage in or out of the duct is allowed, provided the duct retains its spatial configuration and does not fall. This procedure addresses the seismic structural integrity of the duct and its support system together with a review for potential seismic interactions.

Where pressure boundary integrity is required, the duct wall can not be breached and the duct joints and seams must remain pressure tight. An example is that of a HVAC duct that is used for conveyance of hazardous effluent gas to a HEPA filter. In general, confinement HVAC systems are configured so that the operating pressure for the hazardous gas is maintained at a negative pressure relative to the environment of the duct exterior. The safety requirements for such a configuration have very limited tolerance for duct leakage so as to preserve the duct system effectiveness and efficiency. Consequently, this duct would probably be classified as a safety related item (PC3 or PC4). This procedure augments the duct structural integrity evaluation requirements with additional criteria to provide a high degree of confidence that pressure boundary integrity will be maintained during a seismic event.

\subsection{Functionality Requirement}

HVAC duct systems may be required to function during a seismic event. In this case, spurious changes of equipment condition (such as accidental closing or opening of dampers, or loss of controls) are not permitted to occur.

HVAC duct systems may be allowed to malfunction during the period of seismic vibration, provided it can be reset (remotely or by local manual controls) to function after the seismic event.

\subsection{Bounding Sample Evaluation}

A group of duct systems may be evaluated based on a worst-case bounding sample review. For each attribute, the Seismic Review Team must select the worst-case configurations. For example, the review for stiffener spacing may be based on panels having the largest width, thinnest gage, greatest distance between stiffeners with the smallest section properties. The basis for the selected bounding sample(s) should be documented on the SEWS form.

\subsubsection{Evaluation for Structural Integrity}

\subsection{Duct Free of Damage. Defects, Degradation}

The HVAC duct system network should be visually inspected for damage, defects, and degradation. The inspection should also identify suspect repairs, missing parts, broken joints, poor workmanship and significant corrosion, particularly at duct joints.

\subsection{Duct Material and Stiffeners Comply with SMACNA}

A visual inspection of the ducts should confirm that the duct material, stiffeners and joints are in accordance with SMACNA (Ref. 113, 114, and 115).

In particular, the following attributes must be verified:

a. Materials should be rolled steel (below $650^{\circ} \mathrm{F}$ operating temperature), galvanized steel (below $400^{\circ} \mathrm{F}$ ), or stainless steel. 
b. Stiffeners should comply with SMACNA: steel shapes (Ref. 113 Page 1-23 and Ref. 114 Page 7-56 to 58), angle or bar reinforcement for round ducts (Ref. 115 Page 4f-2).

Fastening of the stiffener to the duct should be by tack weld, spot weld, bolt, screw or rivet, 12" max. spacing (Ref. 113 Page 1-48).

\subsection{Duct Joints and Seams Comply with SMACNA}

Joints and seams should conform to SMACNA standard configurations, and be positively attached, excluding friction or riveted joints. Acceptable transverse joint configurations are: Ref. 113 Page 1-35; Ref. 114 Page 8-7; Ref. 115 Pages 5-4 and 5-11 excluding sleeved (Figure 3), riveted (Figure 4) and draw band (Figure 5) joints. Acceptable longitudinal seam configurations are groove weld and fillet weld (Ref. 114 Pages 8-1 through 8-6; Ref. 113 Page 3-5), and lock type (Ref. 113 Page 1-40) excluding riveted seams.

\subsection{Duct Meets Support Span Criteria}

\subsection{SMACNA Rules}

SMACNA provides rules for the spacing of duct supports (Ref. 113 Page 4-3, Ref. 114 Page 9-7, and Ref. 115 Page 7-3), based on a maximum allowable bending stress in the duct wall of $8 \mathrm{ksi}$ for rectangular duct and $10 \mathrm{ksi}$ for circular duct.

For seismic loads, the same spacing criteria must be met, however an increase of the allowable bending stress by $33 \%$ is allowed provided the duct joints are type T-17 to T-24 (Ref. 113 Page 1-35).

\subsection{2.4.2 Computing the allowable support span length for rectangular duct:}

The SMACNA approximation for rectangular duct section properties is based on four 2" corners (Ref. 114 Page 9-7) and a bending stress $\left(\sigma=w \mathrm{~L}^{2} / 10\right.$ which is based on the average of simply supported and built-in moment). For duct with uniformly distributed load, the allowable span between consecutive vertical supports can be expressed as:

$L=\left[80 \mathrm{~F}_{\mathrm{b}} /(\mathrm{H}+\mathrm{W}) \rho \mathrm{K}_{\mathrm{R}}\right]^{1 / 2}$

where:

$\mathrm{Fb}=$ allowable bending stress (psi) [typically $8000 \mathrm{psi}$ for rectangular duct]

$\mathrm{H}, \mathrm{W}=$ height, width of duct (in) (see Figure 10.4.1-2)

$\rho=$ equivalent density of duct material (lb/in ${ }^{3}$ ). (Note - Include insulation and reinforcement mass contribution).

$\mathrm{K}_{\mathrm{R}}=$ parameter for rectangular duct in Section 10.4.1.6.1 (1/in $\left.{ }^{2}\right)$ 


\subsection{Computing the allowable support span length for circular duct:}

The SMACNA approximation for circular duct is based on a bending stress $\sigma=w \mathrm{~L}^{2} / 10$. For circular duct with uniformly distributed load, the corresponding allowable span between consecutive vertical supports can be expressed as:

$$
\mathrm{L}=\left(5 \mathrm{Fb}_{\mathrm{b}} \mathrm{D} / 2 \rho \mathrm{K}_{\mathrm{C}}\right)^{1 / 2}
$$

where:

$$
\begin{aligned}
& \mathrm{F}_{\mathrm{b}}=\text { allowable bending stress (psi) [typically } 10,000 \text { psi for circular duct] } \\
& \mathrm{D}=\text { duct diameter (in) } \\
& \rho=\text { equivalent density of duct material (lb/in }{ }^{3} \text { ). } \\
& \mathrm{K}_{\mathrm{C}}=\text { (Note -Include insulation and reinforcement mass contribution). }
\end{aligned}
$$

\subsection{Effect of concentrated weights}

Heavy in-line components, such as unsupported in-line dampers subject to seismic accelerations, exert an additional bending moment on the duct. The allowable support span must be reduced accordingly, to limit the bending stress to within the allowable $\mathrm{Fb}$.

Beam equations may be used to superimpose the distributed weight and the concentrated weight stress (see Section 10.4.1.6.3 for additional guidance).

\subsection{Duct Guided Against Sliding Off Supports}

Seismic experience indicates that HVAC duct can fail if it slides off its supports. The duct must be secured, by tie-downs or stops, if it can slide and fall off its supports.

\subsection{Heavy In-Line Components Properly Restrained}

Components mounted in-line on the duct work include fans, coolers, dryers, dampers, motor operators to dampers, and blowers.

In-line equipment must be positively attached to ductwork. Duct connections to heavy in-line components must be evaluated for structural capacity.

Support spans are to be reduced for heavy in-line components as discussed in Section 10.4.1.2.4.4.

In-line floor mounted equipment on vibration-isolation pads requires a separate evaluation based on failures recorded in the experience database. Guidance in performing this review is given in Chapter 6.

\subsection{Appurtenances Properly Attached}

Appurtenances to ducts include dampers, louvers, diffusers, and screens. Appurtenances must be positively attached to duct work (such as screwed or riveted) as opposed to slipped into place.

Duct connections to heavy cantilevered appurtenances must be evaluated for structural capacity. 


\subsection{No Stiff Branch With Flexible Headers}

Branch ducts must have sufficient flexibility to accommodate potential sway movement of a flexibly hung duct header.

In particular, the review should identify lateral duct branches rigidly supported off long runs of duct with no axial restraints. The axial movement of the header could damage the branch duct. Similarly, a duct on sway type supports (such as rod hung trapeze or rod hangers) could swing and rupture a rigidly supported branch duct.

\subsubsection{Evaluation for Pressure Boundary}

Duct which has to maintain a pressure boundary must meet all of the screens for structural integrity (Section 10.4.1.2) and the following supplementary requirements.

\subsection{Duct Joints and Seams Are of Rugged Type.}

In addition to the criteria for structural integrity (Section 10.4.1.2), transverse joint configurations T-1 to T-16 (Ref. 113 Page 1-35) are outliers for pressure boundary review. Similarly, all longitudinal seams that are not groove or fillet welded (Ref. 114 Pages 8-1 through 8-6; Ref. 113 Page 3-5) are considered to be outliers for pressure boundary review.

\subsection{Stiffeners and Joints Welded or Bolted to Duct}

Duct stiffeners and joint reinforcements shall be attached to the duct by intermittent welds or by bolts with a maximum spacing of 12 ". For rectangular duct, the maximum distance of a weld or bolt from the duct edge is 2", (Ref. 113 Page 1-48).

Intermittent welds are typically staggered on alternate sides of the stiffeners and shall be 1" to 3 " long (Ref. 114 Page 7-55).

\subsection{Duct Gage. Stiffeners Sized to Resist Seismic Load}

The Seismic Review Team shall verify the adequacy of the duct wall thickness (gage), stiffener size, and stiffener spacing in accordance with SMACNA (Ref. 113, 114, and 115), with the following provisions:

(a) The seismic accelerations generate uniform pressures acting on the duct in both + (internal pressure) and - (external pressure) directions. Due to the small deflections in duct wall, the scaled $2 \%$ damped accelerations must be used to evaluate stresses in duct walls.

(b) The stiffener deflection limits in SMACNA may be exceeded under seismic loads, provided the stiffener and the duct wall remain elastic. The SMACNA equations (Ref. 114 and 115) or the theory of plates and shells (Ref. 116) may be used for the stress analysis.

\subsection{No Potential for Puncture of Duct Wall}

Duct should not be supported on sharp edges or have point contacts with support members. Duct should be sufficiently restrained in the vertical and lateral directions, in accordance with the support span criteria for structural integrity, to avoid sliding or uplift impact. 


\subsection{Flexible Bellows Can Accommodate Motions}

Where flexible bellows are provided, potential seismic displacements must be compared to bellows capacity. Alternatively, the bellows must be guided to preclude significant seismic differential movements.

\subsubsection{Support Review}

\subsection{No Broken. Defective, or Degraded Hardware}

Duct supports shall be visually inspected for adequate fabrication and maintenance. Signs of poor construction quality or subsequent degradation include: distortion, dislodged or shifted support members, missing brackets, nuts or bolts, unusual or temporary repairs, cracks in concrete, etc.

\subsection{Support Member Capacity Exceeds Demand}

The Seismic Review Team shall evaluate the sample support configuration(s) likely to have the largest demand/capacity ratio.

\subsection{Seismic Demand}

Ductile Supports: HVAC duct supports suspended from overhead or sidewalls (i.e. not supported from the floor) and which can be classified as ductile, as defined in the DOE Seismic Evaluation Procedure, must be evaluated for vertical capacity. The demand shall be based on 5 times the dead load in the downward direction (Ref. 107 page 39 ). A high vertical capacity provides considerable margin for horizontal earthquake loading.

Non-Ductile Supports: HVAC duct supports not classified as ductile, must be evaluated for vertical and horizontal (lateral or axial) loads. The scaled $7 \%$ damped peak spectral acceleration should be used to calculate applied loads, unless the spectral acceleration (see Section 5.2) at the duct span resonant frequency is determined.

$$
\mathrm{F}_{\mathrm{a}}=\mathrm{WA}_{\mathrm{s}}
$$

where:

$$
\begin{aligned}
& \mathrm{W}=\text { tributary weight }(\mathrm{lbs}) \\
& \mathrm{A}_{\mathrm{S}}=\text { spectral acceleration }(\mathrm{g})
\end{aligned}
$$

Base-mounted supports represent a special type of non-ductile support. They are different than suspended supports in that base-mounted supports can become unstable when subjected to excessive lateral deflections or inelastic behavior since they don't have the pendulum restoring force attributes of suspended supports. Consequently, base-mounted support evaluations should include P-delta effects if there is the potential for base hardware slip. P-delta effects represent the second order increase in base overturning moment due to additional eccentricity of supported dead load during seismic deflections of the support. It is illustrated in Figure 10.4.1-3. Base plate flexibility (rotation) shall be postulated as applicable according to the following:

- shell expansion anchor slip of 1/8"

- channel nut slip of $1 / 16^{\prime \prime}$

- clip angle bending

Additional discussion of base mounted support evaluations for P-delta effects is found in References 47 and 50. 


\subsection{Seismic Capacity}

The support capacity shall be based on AISC (Ref. 81) including provisions to increase seismic allowable stresses by 1/3 (Ref. 81 Part 5 Section 1.5.6) and evaluation of potential for buckling.

HVAC duct supports consisting of rod hangers with fixed end connections shall be evaluated for fatigue (Ref. 47).

\subsection{Anchorage Adequacy}

For the bounding sample support configuration(s), the Seismic Review Team shall evaluate the support anchorage in accordance with Chapter 6 of the DOE Seismic Evaluation Procedure.

Anchor bolt installation (tightness) checks shall be performed for floor mounted supports as per Chapter 6 as well.

\subsection{Support Details}

Supports shall not include design details which have been a source of failure in past earthquakes such as beam clamps with no restraining strap, smooth channel nuts (without teeth or ridges) and cast-iron inserts.

\subsubsection{Sejsmic Interaction Review}

An evaluation shall be performed of potential seismic interaction hazards due to spatial proximity and differential motion between structures. Other seismic interaction evaluation considerations are identified in Chapter 7.

Free from Input by Nearby Equipment - Duct systems adjacent to other equipment should be evaluated for the consequences of interaction with moving items.

No Collapse of Overhead Equipment, Distribution Systems. or Masonry Walls - Duct Systems attached to or in the vicinity of unanchored components or unreinforced block walls should be evaluated for potential interaction.

Able to Accommodate Differential Displacements - Duct systems that span between different structures shall be evaluated to ensure adequate flexibility to accommodate relative movement of the structures. 


\subsubsection{Span Factors and Concentrated Weights}

\subsection{Span Factor for Rectangular Duct}

Horizontal run of duct:

$$
\begin{gathered}
\left.K_{R}=\left\{S_{h}^{2} R^{4} W^{2} /\left[\left(W^{2} / 2\right)-W+1\right)\right]^{2}+S_{v}^{2} H^{2} /\left[\left(H^{2} / 2\right)-H+1\right]^{2}\right\}^{1 / 2} \\
+H /\left[\left(H^{2} / 2\right)-H+1\right]
\end{gathered}
$$

where:

$$
\begin{aligned}
& S_{\mathrm{h}}=\text { horizontal spectral acceleration (see Section 5.2), lateral to duct (g) } \\
& \mathrm{S}_{\mathrm{v}}=\text { vertical spectral acceleration (g) (see Section 5.2) } \\
& \mathrm{R}_{\mathrm{W}}=\text { ratio of horizontal to vertical support spacing } \\
& \mathrm{W}=\text { width of duct (in) } \\
& \mathrm{H}=\text { height of duct (in) } \\
& \mathrm{K}_{\mathrm{R}}=\text { span factor }\left(1 / \mathrm{in}^{2}\right)
\end{aligned}
$$

Vertical run of duct:

$$
\left.K_{R}=\left\{S_{h W}^{2} R^{4} W^{2} /\left[\left(W^{2} / 2\right)-W+1\right)\right]^{2}+S_{h H}^{2} H^{2} /\left[\left(H^{2} / 2\right)-H+1\right]^{2}\right\}^{1 / 2}
$$

where:

$$
\begin{array}{ll}
\mathrm{S}_{\mathrm{hw}} & =\text { horizontal spectral acceleration (see Section 5.2), parallel to side } \mathrm{W}(\mathrm{g}) \\
\mathrm{S}_{\mathrm{hH}} & =\text { horizontal spectral acceleration (see Section 5.2), parallel to side } \mathrm{H}(\mathrm{g}) \\
\mathrm{R} & =\text { ratio of lateral support spacing in } \mathrm{S}_{\mathrm{hW}} \text { direction } 2 \text { to lateral } \\
& \text { support spacing in } \mathrm{S}_{\mathrm{hH}} \text { direction } 1 \\
\mathrm{~W} & =\text { width of duct (in) } \\
\mathrm{H} & =\text { height of duct (in) } \\
\mathrm{L} & =\text { maximum allowable support span in } \mathrm{S}_{\mathrm{hH}} \text { direction } 1 \text { (in) } \\
\mathrm{K}_{\mathrm{R}} & =\text { span factor }\left(1 / \mathrm{in}^{2}\right)
\end{array}
$$

\subsection{Span Factor for Circular Duct}

Horizontal run of duct:

$$
K_{c}=1+\left(S_{v}^{2}+R^{4} S_{h}^{2}\right)^{1 / 2}
$$

where:

$$
\begin{aligned}
& \mathrm{R}=\text { same as for horizontal rectangular duct } \\
& \mathrm{S}_{\mathrm{v}}=\text { same as for horizontal rectangular duct } \\
& \mathrm{S}_{\mathrm{h}}=\text { same as for horizontal rectangular duct } \\
& \mathrm{K}_{\mathrm{c}}=\text { span factor (dimensionless) }
\end{aligned}
$$


Vertical run of duct:

$$
K_{c}=\left(S_{h 1}^{2}+R^{4} S_{h 2}^{2}\right)^{1 / 2}
$$

where:

$$
\begin{array}{ll}
\mathrm{S}_{\mathrm{h} 1} & =\text { horizontal spectral acceleration (see Section 5.2) in direction } 1 \\
\mathbf{S}_{\mathrm{h} 2} & =\text { horizontal spectral acceleration (see Section 5.2) in direction } 2 \\
\mathbf{R} & =\text { ratio of lateral support spacing in direction } 2 \text { to lateral } \\
& \text { support spacing in direction } 1 \\
\mathrm{~L} & =\text { maximum allowable support span in direction } 1 \text { (in) } \\
\mathrm{K}_{\mathrm{C}} & =\text { span factor (dimensionless) }
\end{array}
$$

\subsection{Stress Equation}

Seismic and weight bending stress in a duct due to its distributed weight and the weight of a heavy in-line (duct-mounted) component located mid-span is given below. For a horizontal rectangular duct, the stress is computed to be:

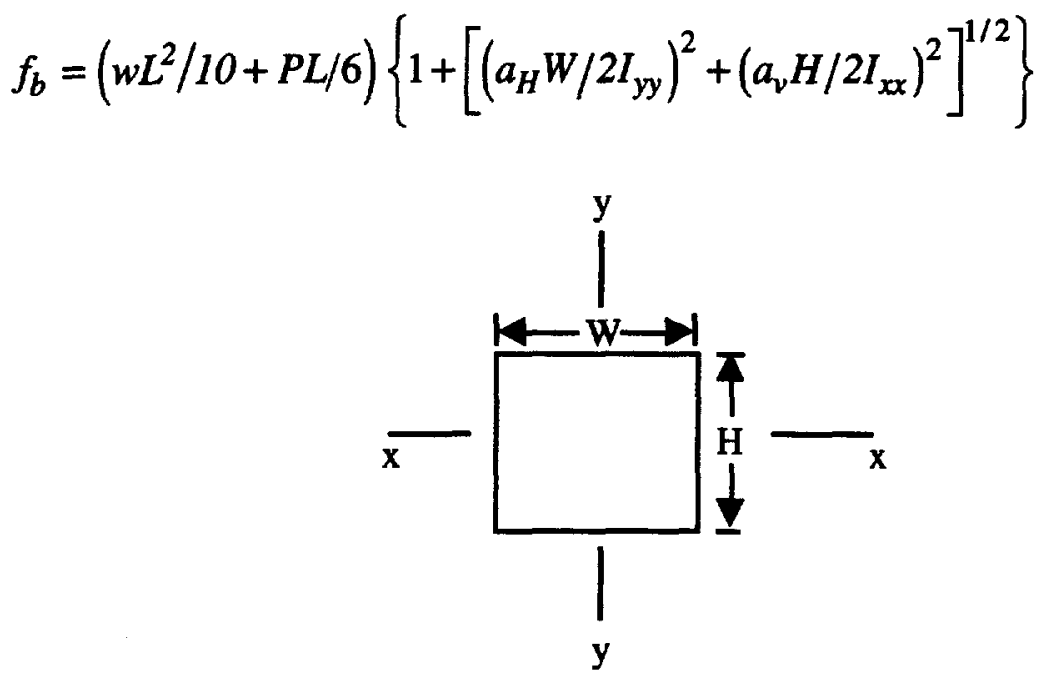

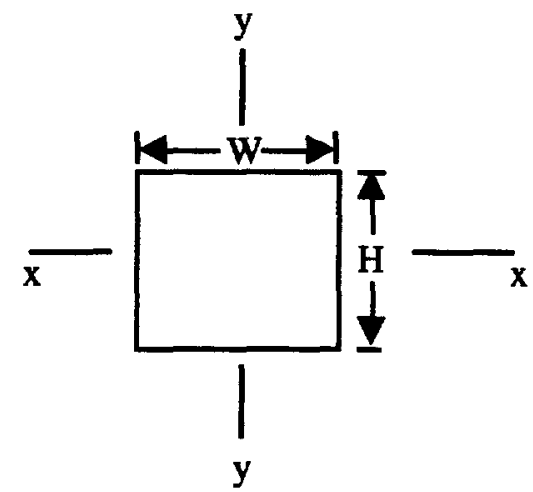

where:

$$
\begin{aligned}
& \mathrm{fb}=\text { total bending stress (psi) } \\
& \mathrm{w} \quad=\text { distributed wt of duct (lbs/in) } \\
& \mathrm{L} \quad=\text { length of duct span containing concentrated weight (in) } \\
& \mathrm{P}=\text { concentrated weight (lb) } \\
& \mathrm{a}_{\mathrm{H}}, \mathrm{a}_{\mathrm{y}} \quad=\text { horizontal and vertical accelerations }(\mathrm{g}) \\
& \mathrm{W}, \mathrm{H}=\text { width and height of duct (in) } \\
& \left.I_{x x}, I_{y y}=\text { moment of inertia of duct cross section (in } 4\right) \text {. } x x \text { axis } \\
& \text { is parallel to width } W \text {; yy axis is parallel to height } H \\
& \text { (see figure above) } \\
& \mathrm{R}=\text { ratio of horizontal to vertical support spacing }=1
\end{aligned}
$$

For a horizontal circular duct, the stress is computed using the above equation with $\mathrm{W}=\mathrm{H}=\mathrm{D}$ where $\mathrm{D}=$ outer diameter of duct (in). 


\subsection{Moments of Inertia for Rectangular Duct}

Based on the SMACNA rectangular duct approximation of 4 corner angle sections (Ref. 114 Page 9-7), the moment of inertia is:

$$
\begin{aligned}
& \mathrm{I}_{\mathrm{xx}}=4 \mathrm{t}\left(\mathrm{H}^{2}-2 \mathrm{H}+2\right) \\
& \mathrm{I}_{\mathrm{yy}}=4 \mathrm{t}\left(\mathrm{W}^{2}-2 \mathrm{~W}+2\right)
\end{aligned}
$$

where:

$$
\begin{array}{ll}
\mathrm{t} & =\text { duct thickness (in) } \\
\mathrm{H} & =\text { width of duct (in) } \\
\mathrm{W} & =\text { height of duct (in) }
\end{array}
$$

Note that the above equations include the $2 " \times 2$ " corners; hence, the $\mathrm{H}$ and $\mathrm{W}$ units must always be inches. If either $\mathrm{W}$ or $\mathrm{H}$ exceeds 72 in., the corresponding value used for calculating $\mathrm{I}_{\mathbf{X X}}$ and Iyy shall be 72 in. Moment of inertia and section modulus calculations shall be based on dimensions $\leq 72$ in. (Ref. 114 Page 9-7).

\subsection{Moments of Inertia for Round Duct}

$$
I_{x x}=I_{y y}=0.0491\left(D^{4}-d^{4}\right) \quad\left(i n^{4}\right)
$$

where:

$$
\begin{aligned}
& \mathbf{D}=\text { outer diameter of duct (in) } \\
& \mathbf{d}=\text { inner diameter of duct (in) }
\end{aligned}
$$




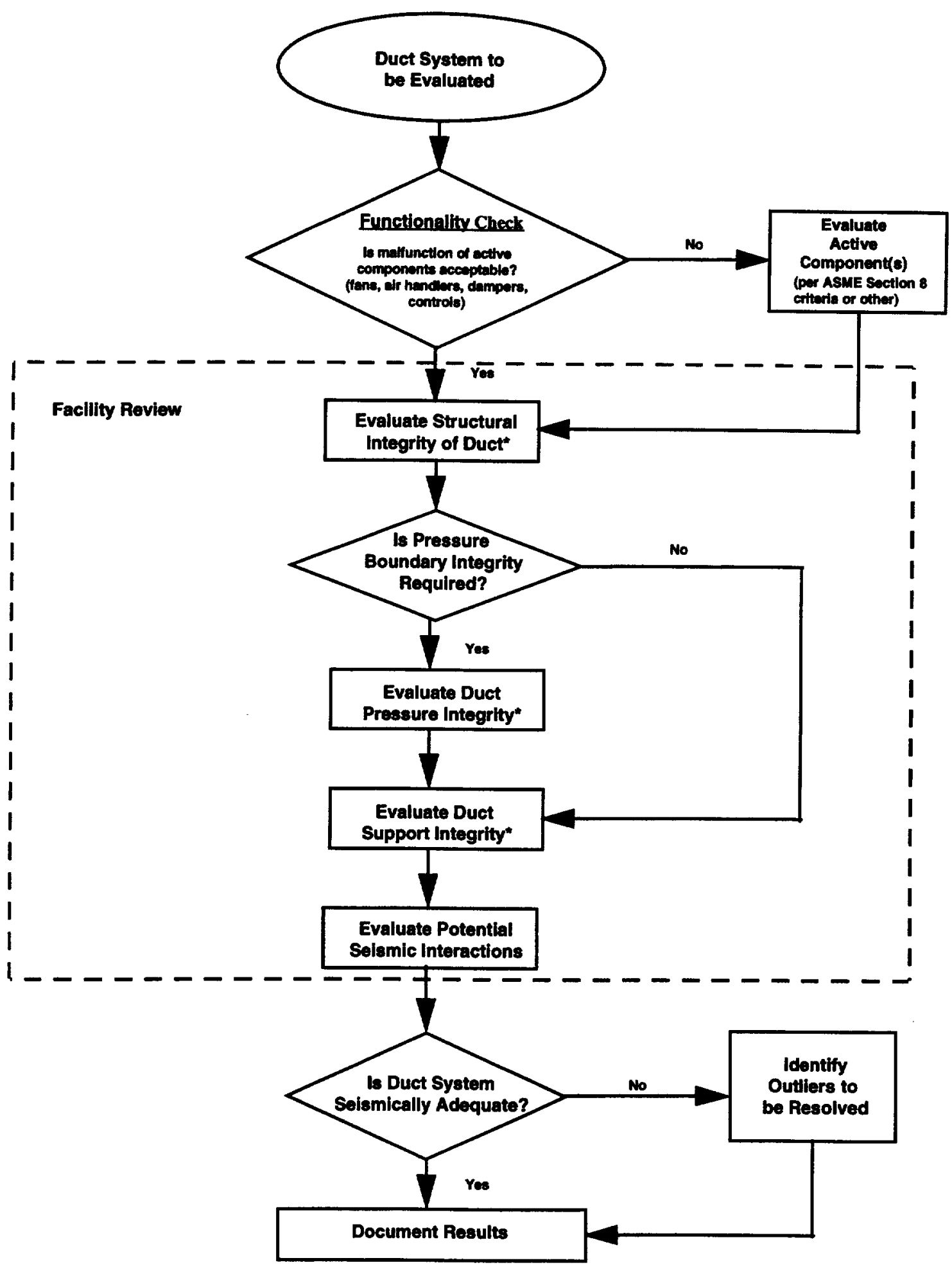

* Requires limited analytical review in addition to field walkdown.

Figure 10.4.1-1 Logic Diagram for Duct System Seismic Evaluation 


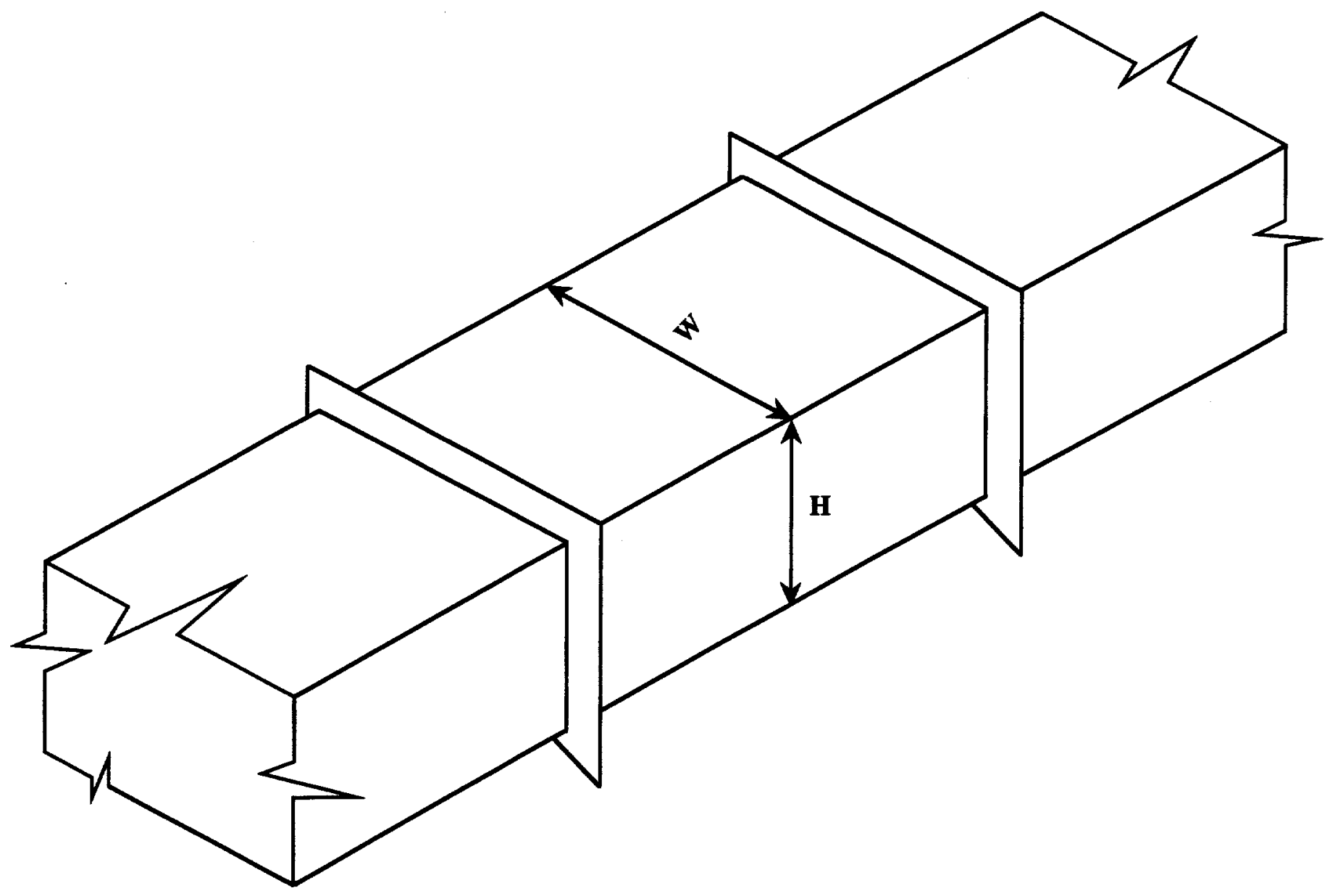

Figure 10.4.1-2 Typical Rectangular HVAC Duct Section 

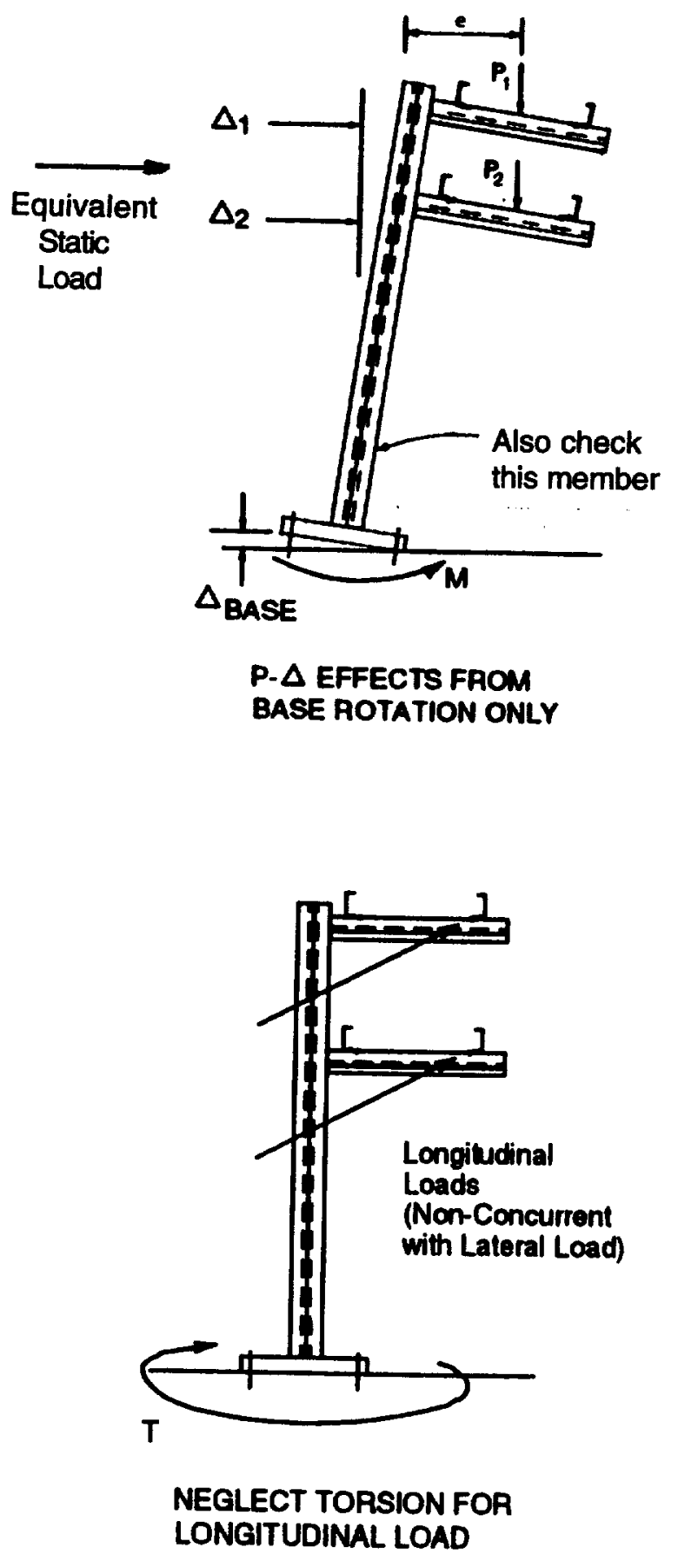

Figure 10.4.1-3 Floor Mounted Supports 


\subsection{ARCHITECTURAL FEATURES AND COMPONENTS}

\subsubsection{UNREINFORCED MASONRY (URM) WALLS}

This section provides guidance in evaluating unreinforced non-bearing masonry (URM) walls for seismic adequacy. It should be noted that the approaches presented herein address only the outof-plane behavior of non-bearing unreinforced masonry walls with respect to seismic loads. It is important to have a list of masonry walls selected before the Seismic Review Team (SRT) begins its seismic evaluation. The Seismic Capability Engineers (SCEs) that make up the SRT are not necessarily the ones expected to assemble the list of selected masonry walls for evaluation. That is a separate task to be performed by others (see Chapter 4).

The selected masonry wall is first examined by non-destructive evaluation (NDE) methods to determine if it is hollow or grouted solid. If the wall is found to be hollow in every cell (or only hollow in the cells that contain rebar), then it is considered to be unreinforced. If the wall is grouted solid in a specified minimum number of vertical cells, then it is further investigated by NDE methods to determine if it is either reinforced or unreinforced. If the wall is found to contain enough rebar to be categorized as reinforced, it is considered to be "out-of-scope" of the evaluation guidance provided in this module. If a URM wall is determined to be a load-bearing wall, it is also considered "out-of-scope" for this module. The URM walls included in the guidance herein are assumed to be either: (1) walls that in-fill a concrete or steel frame, or (2) partitions inside a concrete or steel-framed building.

One screening approach and three methods of URM wall evaluation for out-of-plane bending are presented in this module and are the following: (1) Screening based on height/thickness ratio, (2) The Elastic Method (also called the ACI working stress approach), (3) The Reserve Energy Method, and (4) The Arching Action Method. The Elastic Method is generally the most conservative and yields a relatively low capacity for the wall in question. The Arching Action Method provides the highest capacity for the wall. Both the Reserve Energy Method and the Arching Action Method are considered to be post-elastic approaches and account for additional wall strength after wall cracking. The methods are shown in Figure 10.5.1-1.

\subsubsection{List of Selected Masonry Walls}

This task should be performed by others before the Seismic Capability Engineers (SCEs) begin their URM wall evaluation. A list of selected masonry walls must be generated so that the SCEs can begin their evaluation of walls. The Seismic Equipment List (SEL) is discussed in Chapter 4. If masonry walls are included on the SEL, use that list.

Questions that should be addressed during the selection of masonry walls might include:

- Is seismic interaction credible?

- Is critical equipment in the vicinity of or attached to the masonry wall?

- Is the masonry wall in question used for:

- confinement of hazardous material?

- shielding?

- fire protection?

- security concerns?

A more detailed list of questions to be addressed can be found in Reference 117, Pages 18-21. 


\subsubsection{Type of Unreinforced Masonry Wall}

The three main types of masonry walls considered are:

- Concrete Masonry Unit (CMU)

- Hollow-Clay Tile (HCT)

- Brick

It will also make a difference whether each cell of the wall is grouted solid or left hollow. The hollow cell of masonry block will attract a smaller seismic loading, since it has less mass than the cell of masonry block which is fully grouted. If construction documents or installation records are not available, one must perform a non-destructive evaluation to determine the condition of the selected masonry wall. For determination of hollow cell vs. grouted cell, drilling a small hole through the face of the cell is one simple method. To ascertain whether only a few cells are grouted, check several consecutive blocks along a course of the selected wall. In some parts of the United States, insulation is placed in ungrouted cells of masonry walls. The weight of this insulation should be included when conducting the evaluations presented in this section.

It is also important to find out if the masonry wall is reinforced. The scope of the guidance in this section only includes unreinforced masonry walls. For detection of rebar, a hand-held ferromagnetic detector with a display meter or an audio signal can be easily used in many cases. An alternate method involves using imaging impulse radar. With either method, it is important to locate the positions of the following:

- vertical reinforcing steel and its approximate spacing

- horizontal reinforcing steel and its approximate spacing

An unreinforced masonry wall is a masonry wall in which the area of reinforcing steel is less than 25 percent of the minimum steel ratios required by the 1994 Uniform Building Code (UBC) for reinforced masonry (Ref. 69). Lightly or poorly reinforced walls are considered to be URM walls and can be evaluated by the methods presented in this Section.

\subsubsection{Determine Physical Condition of Wall}

As part of the seismic evaluation of the selected URM wall, it is important to examine the condition of mortar joints, openings, and existing cracks. If the mortar joints are not sound or if there are substantial cracks in the mortar or faces of the masonry units, the Elastic Method (ACI Working Stress Approach) in Section 10.5.1.5 may not be applicable.

The top connection is often not fully grouted and thus may be a free joint. Simple supports at the top and/or side should result from structural-steel angle "keepers" or dovetail slots in columns or overhead beams. There needs to be some positive means of carrying the out-of-plane load from the wall panel and into the support if it is to be considered a simple support boundary condition. If not, the wall may have to be evaluated as a cantilever.

\subsubsection{Screening Based on Height-to-Thickness Ratio}

A conservative screening approach based on the Elastic Method may be used to screen out walls from further evaluation. The top of the wall must be laterally supported to use this approach, there should be a tight fit between the supporting member, or suitable restraining members should be provided to prevent lateral motion of the top of the wall. 
The wall may be screened out if:

$$
\left(\frac{\mathrm{H}}{\mathrm{t}}\right)_{\text {actual }} \leq\left(\frac{\mathrm{H}}{\mathrm{t}}\right)_{\max }
$$

where:

$$
\left(\frac{H}{t}\right)_{\max }=\left(\frac{H}{t}\right)_{N} \frac{\alpha_{D}}{\sqrt{\frac{S_{A_{\max }}}{g}}}
$$

$\left(\frac{H}{t}\right)_{N}$ can be found in Table 10.5.1-1 as a function of actual wall thickness $t$

$$
\begin{aligned}
& \mathrm{H}=\text { wall height } \\
& \mathrm{t} \quad=\text { actual wall thickness } \\
& \alpha_{D}=\sqrt{150 / \rho} \text { or from Table 10.5.1-6 } \\
& \rho \quad=\text { weight density of masonry in \#/f } \mathrm{t}^{3} \\
& S_{A_{\max }}=\text { maximum spectral acceleration from 5\% damped input spectra for } \\
& \text { appropriate Performance Category and location above grade in facility } \\
& \mathrm{g}=\text { acceleration of gravity }
\end{aligned}
$$

Development of this screening approach is discussed in Section 10.5.1.8.

For walls that are not screened out by this process, continue with the analysis methods presented in Sections 10.5.1.5, 10.5.1.6, and 10.5.1.7.

\subsubsection{Elastic Method}

\section{Estimate Maximum Flexural Tensile Stress in URM Wall}

For the elastic method, this module makes extensive use of Reference 117. The following topics are considered in arriving at an estimate of the maximum flexural tensile stress in the URM wall:

- natural frequency prediction for a single-wythe, uncracked masonry wall,

- determine horizontal seismic acceleration,

- estimate maximum out-of-plane bending stress for a single-wythe, uncracked, masonry wall of height $\mathrm{H}$ and width $\mathrm{L}$

Multiple-wythe masonry walls with sufficient header courses to insure composite action can also be evaluated by this procedure. Header courses are used to tie single-wythe masonry walls together. 


\section{Determine boundary conditions of the selected URM wall}

To properly use the seismic guidance in this document, it is important to determine boundary conditions of the selected URM walls. Table 10.5.1-3 lists many combinations of boundary conditions, some of which include: 1 ) simply supported on all four edges; 2) simply supported on top and bottom, free on sides; and 3) simply supported on bottom and sides, free on top.

Cross walls will provide support to the wall sides. Using doorways as free edges may be appropriate. However, using a window as a free edge may be overly-conservative if the window is less than half of the height of the URM wall in question.

\section{Estimate the fundamental natural frequency of the wall}

Once the boundary conditions are verified, the fundamental natural frequency can be estimated as follows:

$$
f=\left(B_{f}\right)(F)\left(\alpha_{E}\right)\left(\alpha_{D}\right)\left(\alpha_{T}\right)
$$

- $\mathrm{f}$ has units of cycles per second $(\mathrm{Hz})$

- boundary condition factor, $B_{f}$ for fundamental frequency calculation from Table 10.5.1-3

- frequency factor, $\mathrm{F}$ from Table 10.5.1-4

- elastic modulus factor, $\alpha_{E}$ from Table 10.5.1-5

- weight density factor, $\alpha_{D}$ from Table 10.5.1-6

- orthotropic behavior adjustment factor, $\alpha_{\mathrm{T}}$ from Table 10.5.1-7

- special considerations (for cases of partial grouting, partially filled joints, and multi-wythe walls), see Table 10.5.1-8.

\section{Estimate the spectral acceleration of the wall}

If the wall is at the ground level, the site-specific 5\% damped ground response spectrum can be entered with the URM wall frequency to determine the spectral acceleration for the selected wall (see Section 5.2). If the wall is at a higher elevation in the building or if it has a basement, the appropriate floor spectrum should be used when determining the spectral acceleration of the selected wall.

Estimate the maximum flexural stress in the URM wall.

With the maximum flexural tensile stress tables, the estimated maximum flexural tensile stress for the selected wall can be scaled according to the wall spectral acceleration.

$$
\begin{aligned}
\sigma_{b} & =\left(B_{s}\right)(S)\left(A_{H}\right)\left(1 / \alpha_{D}\right)^{2} \\
& -\sigma_{b} \text { has units of pounds per square inch } \\
& -\quad \text { boundary condition factor, } B_{s} \text { from Table 10.5.1-9 }
\end{aligned}
$$


- $\quad$ stress factor, $S$ from Table 10.5.1-10

- horizontal seismic acceleration, $A_{H}$ (in g's)

- weight density factor, $\alpha_{D}$ from Table 10.5.1-6.

\section{Capacity by Elastic Method}

Compare the allowable stress, due to out-of-plane seismic loads, at mortar/masonry unit interface with the estimated maximum flexural tensile stress above.

When evaluating URM walls using the Elastic Method, the following should be considered:

1. ACI 530 Table 6.3.1.1 (Ref. 118) has conservative values of allowable flexural tensile stress. Only URM walls that are located in geographic regions with low values of seismic acceleration will meet these ACl 530 code values of allowable stress.

2. The location of maximum stress depends on the specific masonry wall boundary conditions. For example, the maximum moment and stresses in many cases will occur at the fixed boundary in the form of a negative moment. In-filled walls with simple supports at the edges will most likely have the maximum out-of-plane bending stress located near the center of the wall (approximately mid-height and mid-span).

3. Values that may be used for allowable flexural stress for good quality masonry, as stated in Ref. 117, are the following:

- 33 psi for hollow masonry

- 52 psi for solid or fully grouted masonry

4. If site-specific test data exist, a safety factor of 2 to 3 against measured flexural tensile stress at fracture should be applied to the test results and the safety factor chosen should be consistent with the scatter of the site-specific data (Ref. 117).

Example problems illustrating application of this method are shown in Section 10.5.1.10.

\subsubsection{Reserve Energy Method}

The formulas for screening non bearing unreinforced masonry walls are developed from the arching action method with the initial confining force at the top of the wall taken as zero, (Reference 119 and 120).

For the two rigid block rocking (see Figure 10.5.1-2), the spectral acceleration capacity, $\mathrm{S}_{\mathrm{AP}}$, is

$$
\frac{S_{\mathrm{AP}}}{\mathrm{g}}=6 \phi \frac{\mathrm{b}}{\mathrm{H}}\left(1-\frac{\delta_{\mathrm{H}}}{2 \mathrm{~b}}\right)
$$


For the cantilever wall (see Figure 10.5.1-3), the spectral acceleration capacity is

$$
\frac{S_{A P}}{g}=2 \phi \frac{b}{H}\left(1-\frac{\delta_{H}}{2 b}\right)
$$

where:

$$
\begin{aligned}
& \mathrm{g}=\text { acceleration of gravity } \\
& \phi=\text { capacity reduction factor (may be taken as } 0.67 \text { ) } \\
& \mathrm{t}=\text { actual wall thickness } \\
& \mathrm{b}=\text { effective wall thickness }=0.9 \mathrm{t} \\
& \mathrm{H}=\text { wall height } \\
& \delta_{\mathrm{H}}=\text { any specified out-of-plane displacement } \\
& \text { ( } \delta_{\mathrm{H}} \text { should be limited to no more than } \mathrm{b} \text { for wall stability) }
\end{aligned}
$$

The Spectral Acceleration Demand, $\mathrm{S}_{\mathrm{AD}}$, can be determined by the average of the $5 \%$ damped, peakbroadened floor spectra for the floors above and below the wall at the effective frequency, $\mathrm{f}_{\mathrm{e}}$ (see Section 5.2).

$$
\mathrm{f}_{\mathrm{e}}=\frac{1}{2 \pi} \sqrt{\frac{1.5\left(\frac{\mathrm{S}_{\mathrm{AP}}}{\mathrm{g}}\right) \mathrm{g}}{\delta_{\mathrm{H}}}}
$$

If $\quad \frac{S_{A P}}{g} \geq \frac{S_{A D}}{g}$, then the wall is acceptable.

If the capacity is less than the demand for all values of $\delta_{H}$ from 0 to b, the wall becomes an outlier. Wall displacement is the lowest $\delta_{\mathrm{H}}$ at which $\mathrm{S}_{\mathrm{AP}}=\mathrm{S}_{\mathrm{AD}}$.

The capacity trend using the Reserve Energy Method is shown in Figure 10.5.1-4. It can be seen that the ultimate capacity $S_{A P}$ occurs at low lateral displacement. However, the demand $S_{A D}$ is also likely to reduce at even a faster rate with increasing $\delta_{H}$ (see example problems) so that the largest ratio of $\left(\mathrm{S}_{\mathrm{AP}} / \mathrm{S}_{\mathrm{AD}}\right)$ is most likely to occur when $\delta_{\mathrm{H}}$ equals the stability limit $\mathrm{b}=0.9 \mathrm{t}$.

When evaluating URM walls using the Reserve Energy Method, the following should be considered:

1. Neglect cracking strength of the unreinforced masonry wall.

2. Assume an idealized rigid-body motion of the wall.

3. Assume that the URM wall is a non-load bearing wall. Load bearing walls can also be assessed by a more complex version of the Reserve Energy Method.

4. Failure of a URM wall is identified when the response exceeds the effective wall thickness $b$.

Example problems illustrating application of this method are shown in Section 10.5.1.10. 


\subsubsection{Arching Action Method}

Check for applicability of Arching Action. When this method can be justified, it provides the highest out-of-plane seismic capacity.

It is critical that the boundary conditions of the URM walls do not include any sionificant gaps $(>1 / 16$ inch) between the top of the selected URM wall and the beam or floor above for the Arching Action Method to apply. If gaps occur, then there may be limited, or reduced, ability for the wall to develop arching action. To take credit for arching action, it is also important to check the maximum allowable compressive stress in the masonry unit and compare it to the maximum stresses developed at the edges of critical masonry units (Ref. 119).

When the rotational restraints at the boundaries are considered, a higher capacity can be achieved for the URM wall. The rotational restraint due to the wall's horizontal displacement induces an arching mechanism (Ref. 119). This arching mechanism is illustrated in Figure 10.5.1-2.

Assuming rigid body rocking develops after the masonry wall has cracked at a location $\alpha \mathrm{H}$ above the base, as shown in Figure 10.5.1-2, the Reserve Energy method can be used to calculate the ultimate out-of-plane spectral acceleration capacity of a nonload bearing wall including arching action as:

$$
\frac{S_{A P}}{g}=\phi\left(\frac{b}{H}\right)\left[2 f_{p}\left(\frac{P_{R \delta}}{w H}\right)\left(1-\frac{\delta_{H}}{b}\right)+6\left(1-\frac{\delta_{H}}{2 b}\right)\right]
$$

where:

$$
\begin{aligned}
& \mathrm{g}=\text { acceleration of gravity } \\
& \phi \quad=\quad \text { capacity reduction factor (may be taken as } 0.67 \text { ) } \\
& \mathrm{t}=\text { actual wall thickness } \\
& \text { b }=\text { effective wall thickness }=0.9 \mathrm{t} \\
& \mathbf{H}=\text { wall height } \\
& f_{p}=1.03+3.0\left(\frac{e}{b}+0.5\right)^{0.65} \\
& \text { e } \quad=\quad \text { eccentricity of } P_{R} \text { (see Figure 10.5.1-2) } \\
& \mathrm{w}=\text { weight/unit area of masonry wall } \\
& \delta_{\mathrm{H}}=\text { any specified out-of-plane displacement. To take credit for arching action, } \\
& \delta_{H} \text { should not exceed } \delta_{p}
\end{aligned}
$$




$$
\begin{aligned}
& \delta_{\mathrm{p}}=\quad \text { out-of-plane displacement at which ultimate capacity is reached }=\frac{0.00045 \mathrm{H}^{2}}{f_{\mathrm{D}} t} \\
& \text { except } \frac{\delta_{p}}{b} \leq \frac{2 F_{e}}{\left(3-F_{e}\right)} \\
& f_{D}=\quad 1.0 \text { for concrete block and single wythe hollow clay tile walls } \\
& 1.5 \text { for double wythe hollow clay tile walls } \\
& \mathrm{F}_{\mathrm{e}}=\frac{\mathrm{e}}{\mathrm{b}}+0.5 \\
& \mathrm{P}_{\mathrm{R} \delta}=\quad \text { confining force at displacement } \delta_{\mathrm{H}} \\
& \text { (increases with displacement until the displacement } \delta_{p} \text { is reached at which the } \\
& \text { ultimate capacity occurs) } \\
& P_{R \delta}=P_{c} f_{R} \\
& P_{c}=\text { crushing capacity of block }=0.125 t f_{m}^{\prime} \\
& \dot{f_{m}}=\quad \text { ultimate compressive strength of masonry } \\
& \text { [analogus to ultimate compressive strength of concrete, } f_{c}^{\prime} \text {, } \\
& \text { typically } 1000-1500 \text { psi for concrete block (1350 psi typical), } \\
& \text { possibly as low as } 275 \text { psi for hollow clay tile] } \\
& f_{R}=\text { relative boundary element flexibility factor (See Section 10.5.1.9 for } \\
& \text { approach used to compute } f_{R} \text { ) } \\
& f_{R} \text { should not exceed }\left(1-\frac{w H}{P_{c}}\right) \text {. }
\end{aligned}
$$

The first term of the arching action capacity equation, shown above, defines the arching effect and generally dominates. For walls with large $H / t$ and small boundary stiffness (low $f_{R}$ ) the second term can become very significant.

Instability will occur when $\delta_{H}$ reaches 0.9 t. If $\delta_{H}$ substantially exceeds $\delta_{p}$, the wall should be assumed to have lost its in-plane capacity.

The increase in capacity over the Reserve Energy Method is shown in Figure 10.5.1-5.

The effective frequency $\mathrm{f}_{e}$ is:

$$
\mathrm{f}_{\mathrm{e}}=\frac{1}{2 \pi} \sqrt{\frac{1.5\left(\frac{\mathrm{S}_{\mathrm{AP}}}{\mathrm{g}}\right) \mathrm{g}}{\delta_{\mathrm{H}}}}
$$


The spectral acceleration demand, $\mathrm{S}_{\mathrm{AD}}$ can be determined from the average of the $5 \%$ damped, peak-broadened floor spectra (see Section 5.2) for the floors above and below the wall at the effective frequency $f_{e}$.

In order to determine $\delta_{H}$ for a given input response spectrum, start with a low $\delta_{H}$ and compute $S_{A P}, f_{e}$, and $S_{A D}$. Keep increasing $\delta_{H}$ until the spectral acceleration demand $S_{A D}$ at $f_{e}$ drops below the spectral acceleration capacity $S_{A P}$ corresponding to $\delta_{H}$. The lowest $\delta_{H}$ at which $S_{A P} \leq S_{A D}$ represents the appropriate $\delta_{H}$ for the given input response spectrum.

When $\delta_{\mathrm{H}}$ reaches $\delta_{\mathrm{p}}$, the masonry is assumed to crush sufficiently that arching benefit is lost. For larger $\delta_{\mathrm{H}}$ up to $0.9 \mathrm{t}$, the capacity may be conservatively estimated by the Reserve Energy Approach discussed in the previous subsection.

The ground motion level at which the wall is acceptable can be generally established by the larger of:

1. Elastic Method Capacity

2. Reserve Energy Method Capacity with $\delta_{H}=b=0.9 t$

3. Arching Method Capacity with $\delta_{\mathrm{H}}=\delta_{\mathrm{p}}$

It is always conservative to use the larger of these three capacities. In some cases, a greater $\left(S_{\mathrm{AP}} / \mathrm{S}_{\mathrm{AD}}\right)$ ratio might occur at lesser $\delta_{\mathrm{H}}$ values than the values defined above. However, in most cases, this increase is not sufficiently significant to warrant considering these intermediate $\delta_{H}$ values unless it is desired to have an estimate of the wall displacement for a given input spectrum.

Example problems illustration application of this method are in Section 10.5.1.10.

\subsubsection{Development of Screening Approach Based on Elastic Method}

A conservative screening approach has been developed to rapidly screen out walls from further analysis if they meet the screening criteria. This approach is based on the Elastic Method for walls simply supported top and bottom and free on both sides. The equations and terms used are those defined in subsection 10.5.1.5.

$$
\begin{aligned}
& \sigma_{b}=B_{s} S A_{H}\left(\frac{1}{\alpha_{D}}\right)^{2} \\
& A_{H}=S_{A_{\max }}=\text { Peak of the } 5 \% \text { damped response } \\
& \text { spectra for the site and Performance Category, (in } g \text { 's). } \\
& \text { Use the peak of the in-structure spectra if wall is not } \\
& \text { located at grade. } \\
& B_{s}=0.125 \text { for walls simply supported top and bottom } \\
& \text { and free on the side. }
\end{aligned}
$$




$$
\begin{aligned}
& \alpha_{D}=\sqrt{150 / \rho} \text { from Table } 10.5 .1-6 \\
& \sigma_{b}=33 \text { psi for hollow masonry and } 52 \text { psi for solid masonry }
\end{aligned}
$$

Therefore,

For hollow masonry:

$$
\mathrm{S}=\frac{\sigma_{\mathrm{b}} \alpha_{\mathrm{D}}^{2}}{\mathrm{~B}_{\mathrm{s}} \mathrm{A}_{\mathrm{H}}}=\frac{33 \alpha_{\mathrm{D}}^{2}}{0.125 \mathrm{~S}_{\mathrm{A}_{\max }}}=\frac{264 \alpha_{\mathrm{D}}^{2}}{\mathrm{~S}_{\mathrm{A}_{\max }}}
$$

or for solid masonry:

$$
\mathrm{S}=\frac{52 \alpha_{\mathrm{D}}^{2}}{0.125 \mathrm{~S}_{\mathrm{A}_{\max }}}=\frac{416 \alpha_{\mathrm{D}}^{2}}{\mathrm{~S}_{\mathrm{A}_{\max }}}
$$

and for solid masonry:

$$
\begin{aligned}
& S=H^{2} w \frac{c}{I^{\prime}} \text { from Table 10.5.1-10 } \\
& w=\rho t \\
& c=\frac{t}{2} \\
& I^{\prime}=\frac{t^{3}}{12} \\
& S=\frac{H^{2} \rho t\left(\frac{t}{2}\right)}{\frac{t^{3}}{12}}
\end{aligned}
$$

Therefore $S=6 \rho t\left(\frac{H}{t}\right)^{2}$

For hollow masonry, actual values for w and I' must be used.

Set

$$
\frac{264 \alpha_{D}^{2}}{S_{A_{\max }}}=H^{2} w_{\text {hollow }} \frac{c}{I_{\text {hollow }}^{\prime}}
$$

where $w_{\text {hollow }}$ and $\mathrm{I}_{\text {hollow }}$ are the actual values for hollow masonry used to develop stress factors, $\mathrm{S}$, in Table 10.5.1-10 
or for solid masonry

$$
\frac{416 \alpha_{D}^{2}}{S_{A_{\max }}}=6 \rho t\left(\frac{H}{t}\right)^{2}
$$

and determine $\left(\frac{H}{t}\right)$ from the smaller value. This becomes the developed values of $\left(\frac{H}{t}\right)_{N}$ presented in Table 10.5.1-1.

\subsubsection{Method of Calculating Boundary Member Flexibility Factor $f_{R}$}

The average value of $P_{R}$ along the length of the top beam can be approximated as shown in Figure 10.5.1-7. The load on the beam reaches the local block crushing capacity $P_{c}$ over length $a$ at each end of the beam, and is zero over the central region of the beam.

The length $a$ is from the end of the beam to point 1 of Figure 10.5.1-7 at which the upward displacement $\delta_{1}$ reaches

$$
\delta_{1}=\delta_{\mathrm{u}}-\delta_{\mathrm{g}}
$$

where $\delta_{\mathrm{g}}=$ height of any pre-existing gap between the beam and the top of the wall.

(Recall Arching Action may provide limited additional capacity if $\delta_{\mathrm{g}}>\frac{1}{16}$ in.)

Vertical displacement of a simply supported beam restrained against twisting due to arching of wall is:

$$
\delta_{1}=\underbrace{\frac{P_{c} L^{4}}{32 \mathrm{EI}_{\mathrm{B}}} \mathrm{f}_{\mathrm{R}}^{3}\left[1-\left(\frac{7}{12}\right) \mathrm{f}_{\mathrm{R}}\right]}_{\text {Flexural Term }}+\underbrace{\frac{\mathrm{P}_{c} \mathrm{e}_{\mathrm{b}}^{2} \mathrm{~L}^{2} \mathrm{f}_{\mathrm{R}}^{2}}{8 \mathrm{G} \mathrm{J}_{\mathrm{B}}}}_{\text {Torsion Term }}
$$

where:

$$
\begin{aligned}
& P_{c}=\text { crushing capacity of block } \\
& L=\text { length of beam and wall } \\
& I_{B}=\text { moment of inertia of beam } \\
& J_{B}=\text { polar moment of inertia of beam } \\
& E=\text { elastic modulus of beam } \\
& G=\text { shear modulus of beam } \\
& f_{R}=\text { beam flexibility factor } \\
& e_{b}=\text { eccentricity to load from beam centerline }
\end{aligned}
$$


Vertical displacement of wall due to horizontal displacement is calculated next.

As the wall blocks rock, the point at which $P_{R}$ is applied lifts and presses against the boundary beam. This wall uplift at the location of $P_{R}$ is given by:

$$
\delta_{u}=\delta_{H}\left(\frac{b}{H}\right) f_{p}
$$

Uplift Factor

$$
f_{p}=1.03+3.0\left(\frac{e}{b}+0.5\right)^{0.65}
$$

where $e$ is the load eccentricity measured from the center line of the wall (see Figure 10.5.1-8), and $b=0.9 t$ to account for block crushing.

Set vertical displacement of wall equal to vertical displacement of beam.

$$
\begin{aligned}
& \delta_{1}+\delta_{g}=\delta_{\mathrm{u}}=\delta_{\mathrm{H}}\left(\frac{\mathrm{b}}{\mathrm{H}}\right) \mathrm{f}_{\mathrm{p}} \\
& \text { or } \\
& \delta_{\mathrm{H}}=\left(\frac{\delta_{\mathrm{g}}+\delta_{1}}{\mathrm{f}_{\mathrm{p}}}\right) \frac{\mathrm{H}}{\mathrm{b}}
\end{aligned}
$$

Horizontal displacement of wall at ultimate capacity

$$
\begin{array}{ll}
\delta_{p}=\frac{.00045 \mathrm{~L}^{2}}{t} & \frac{\delta_{\mathrm{p}}}{\mathrm{b}} \leq \frac{2 \mathrm{~F}_{\mathrm{e}}}{3-\mathrm{F}_{\mathrm{e}}} \\
\delta_{\mathrm{H}} \leq \delta_{\mathrm{p}} &
\end{array}
$$

The value of $f_{R}$ can then be found by trial and error until the maximum permissible value of $f_{R}$ is reached.

The following procedure can be used:

Pick $\mathrm{f}_{\mathrm{R}}$, start low $\mathrm{f}_{\mathrm{R}}=0.1$, calculate $\delta_{1}$ from $(*)$ on the previous page, calculate $\delta_{\mathrm{H}}$ from $(* *)$ above and repeat until $\delta_{H}=\delta_{p}$

A tabular form is convenient

\begin{tabular}{l|l|l}
$\mathrm{f}_{\mathrm{R}}$ & $\delta_{1}$ & $\delta_{\mathrm{H}}$ \\
\hline & & \\
& & stop when $\delta_{\mathrm{H}}=\delta_{\mathrm{p}}$
\end{tabular}


The following data will assist the calculation

$C=f_{R}^{3}\left[1-\left(\frac{7}{12}\right) f_{R}\right]$

then $\quad \delta_{1}=\frac{\mathrm{P}_{\mathrm{c}} \mathrm{L}^{4}}{32 \mathrm{EI}_{\mathrm{B}}} \mathrm{C}+\frac{\mathrm{P}_{\mathrm{c}} \mathrm{e}_{\mathrm{b}}^{2} \mathrm{~L}^{2} \mathrm{f}_{\mathrm{R}}^{2}}{8 \mathrm{GJ}_{\mathrm{B}}}$

\begin{tabular}{c|l}
$\mathrm{f}_{\mathrm{R}}$ & \multicolumn{1}{|c}{$\mathrm{C}$} \\
\hline 0. & 0. \\
.1 & 0.000942 \\
.2 & 0.00707 \\
.3 & 0.0223 \\
.4 & 0.0491 \\
.5 & 0.0885 \\
.6 & 0.140 \\
.7 & 0.203 \\
.8 & 0.273 \\
.9 & 0.346 \\
1.0 & 0.417
\end{tabular}

The boundary member capacity must also be checked. Moment capacity $\mathbf{M}_{c}$ can place an upper limit on $f_{R}$. Torsion capacity $T_{c}$ can place an upper limit on $e_{b}$.

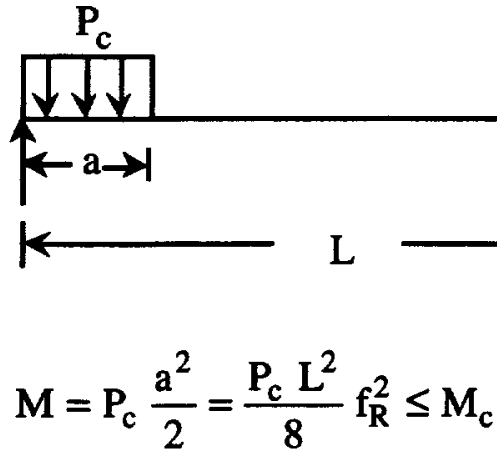

$$
\begin{aligned}
& f_{R} \leq\left(\frac{8 M_{c}}{P_{c} L^{2}}\right)^{1 / 2} \\
& T=P_{c} e_{b} a=\frac{P_{c} f_{R} L}{2} e_{b} \leq T_{c} \\
& e_{b} f_{R} \leq\left(\frac{2 T_{c}}{P_{c} L}\right) \\
& a=\frac{f_{R} L}{2}
\end{aligned}
$$




\subsubsection{Example Problems}

The following example problems are presented to demonstrate application of the methods in this section to a typical URM wall.

A 6 inch hollow concrete block wall at the Portsmouth Gaseous Diffusion Plant is evaluated by the Elastic, Reserve Energy, and Arching Action Methods using ground motion described by a Portsmouth Site Specific Spectra and a Newmark and Hall Generic Spectra (Ref. 72) for a soil site.

6" Concrete Block Wall

$$
\begin{aligned}
& f_{m}^{\prime}=1000 \mathrm{psi} \\
& H=12^{\prime}=144^{\prime \prime} \\
& L=18^{\prime}=216^{\prime \prime} \\
& \rho=135 \mathrm{lbs} / \mathrm{ft}^{3}
\end{aligned}
$$

Simply supported top and bottom, free on sides

Portsmouth Site with $0.15 \mathrm{~g}$ spectrum (see Figure 10.5.1-6A)

Screening Approach (Section 10.5.1.4)

$$
\begin{aligned}
& \left(\frac{\mathrm{H}}{\mathrm{t}}\right)_{\mathrm{actual}}=\frac{144}{5.625}=25.6 \\
& \mathrm{SA}_{\max }=0.4 \mathrm{~g} \text { (Portsmouth) } \\
& \mathrm{SA}_{\max }=2.12 \times .15=0.32 \mathrm{~g}(\text { Newmark \& Hall) } \\
& \alpha_{D}=\sqrt{\frac{150}{135}}=1.054 \\
& \left(\frac{H}{t}\right)_{\max }=\left(\frac{H}{t}\right)_{N} \frac{\alpha_{D}}{\sqrt{S_{A_{\max }}}} \\
& \left(\frac{H}{t}\right)_{N}=11.5 \text { for a } 6 " \text { wall from Table 10.5.1-1 } \\
& \left(\frac{\mathrm{H}}{\mathrm{t}}\right)_{\max }=\frac{(11.5)(1.054)}{\sqrt{0.4}}=19.17 \quad \text { (Portsmouth ground motion) } \\
& \left(\frac{\mathrm{H}}{\mathrm{t}}\right)_{\max }=\frac{(11.5)(1.054)}{\sqrt{0.32}}=21.43 \quad \text { (Newmark and Hall ground motion) }
\end{aligned}
$$




$$
\left(\frac{H}{t}\right)_{\text {actual }}>\left(\frac{H}{t}\right)_{\max }
$$

Wall is not screened out.

\section{Elastic Method (Section 10.5.1.5)}

Estimate seismic capacity from:

$$
\begin{aligned}
\sigma_{\mathrm{b}}=\mathrm{B}_{\mathrm{s}} S \mathrm{~A}_{\mathrm{H}}\left(\frac{1}{\alpha_{\mathrm{D}}}\right)^{2} \\
\sigma_{\mathrm{b}}=\sigma_{\mathrm{b}} \text { allowable }=33 \mathrm{psi} \\
\frac{\mathrm{H}}{\mathrm{L}}=\frac{12^{\prime}}{18^{\prime}}=0.67, \mathrm{~B}_{\mathrm{S}}=0.125 \text { from Table } 10.5 .1-9 \\
\mathrm{~S}=1245 \text { psi from Table } 10.5 .1-10 \\
\alpha_{\mathrm{D}}=\sqrt{\frac{150}{135}}=1.054 \\
\mathrm{~A}_{\mathrm{H}}=\mathrm{S}_{\mathrm{AP}}=\frac{\sigma_{\mathrm{b}} \alpha_{\mathrm{D}}^{2}}{\mathrm{~B}_{\mathrm{S}} \mathrm{S}}=\frac{(33)(1.054)^{2}}{(0.125)(1245)}=0.24 \mathrm{~g}
\end{aligned}
$$

Estimate frequency from:

$$
\begin{aligned}
& f=B_{f} F \alpha_{E} \alpha_{D} \alpha_{T} \\
& \frac{H}{L}=0.67, B_{f}=1.571 \text { from Table } 10.5 .1-3 \\
& 6 " \text { hollow concrete block, } H=12^{\prime}, F=6.70 \text { from Table } 10.5 .1-4 \\
& \alpha_{E}=1 \text { from Table } 10.5 .1-5 \\
& \alpha_{D}=1.054 \\
& \quad \alpha_{T}=0.97 \text { from Table } 10.5 .1-7 \text { for } 6 " \text { wall } \\
& f=(1.571)(6.70)(1)(1.054)(0.97)=10.8 \mathrm{~Hz} \\
& T=\frac{1}{f}=0.093 \mathrm{sec} \\
& S_{A D}=0.4 \mathrm{~g} \text { from } 0.15 \mathrm{~g} \text { Portsmouth } 5 \% \text { damped spectra at } 0.093 \mathrm{sec}
\end{aligned}
$$


Capacity to Demand Ratio $=\frac{S_{\mathrm{AP}}}{S_{\mathrm{AD}}}=\frac{0.24}{0.40}=0.6<1.0$

Wall Fails Elastically

The maximum elastic peak ground acceleration that will not fail the wall elastically is

$$
\mathrm{a}_{\mathrm{g}}=(0.60)(0.15 \mathrm{~g})=0.09 \mathrm{~g}
$$

Reserve Energy Method (Section 10.5.1.6)

$$
\text { b }=0.9 \mathrm{t}=0.9\left(6^{\prime \prime}\right)=5.4^{\prime \prime}
$$

(Note: 6" is the nominal wall thickness, the actual wall thickness should be used in the calculation).

$$
\begin{aligned}
& \frac{\mathrm{S}_{\mathrm{AP}}}{\mathrm{g}}=6 \phi \frac{\mathrm{b}}{\mathrm{H}}\left(1-\frac{\delta_{\mathrm{H}}}{2 \mathrm{~b}}\right) \\
& \frac{\mathrm{S}_{\mathrm{AP}}}{\mathrm{g}}=6(0.67)\left(\frac{5.4}{144}\right)\left[1-\frac{\delta_{\mathrm{H}}}{2(5.4)}\right] \\
& \frac{\mathrm{S}_{\mathrm{AP}}}{\mathrm{g}}=0.151\left[1-\frac{\delta_{\mathrm{H}}}{10.8}\right] \\
& \mathrm{f}_{\mathrm{e}}=\frac{1}{2 \pi} \sqrt{\frac{1.5 \mathrm{~S}_{\mathrm{AP}} \mathrm{g}}{\delta_{\mathrm{H}}}}=\frac{1}{2 \pi} \sqrt{\frac{(1.5) \mathrm{S}_{\mathrm{AP}}(386.4)}{\delta_{\mathrm{H}}}}=3.83\left(\frac{\mathrm{S}_{\mathrm{AP}}}{\delta_{\mathrm{H}}}\right)^{0.5} \mathrm{~Hz}
\end{aligned}
$$

Find $S_{A P}, f_{e}$, and $S_{A D}$ at various $\delta_{H}$ up to stability limit of 5.4".

Reserve Energy Results in tabular form:

\begin{tabular}{|c|c|c|c|c|c|c|}
\hline $\begin{array}{c}\delta_{\mathrm{H}} \\
\text { (inch) }\end{array}$ & $\begin{array}{c}\text { Capacity } \\
\mathrm{S}_{\mathrm{AP}} \\
(\mathrm{g})\end{array}$ & $\begin{array}{c}\text { Frequency } \\
\mathrm{f}_{\mathrm{e}} \\
(\mathrm{Hz})\end{array}$ & $\begin{array}{c}\text { Period } \\
\mathrm{T} \\
(\mathrm{sec})\end{array}$ & $\begin{array}{c}\text { Demand } \\
\mathrm{S}_{\mathrm{AD}} \\
(\mathrm{g})\end{array}$ & $\begin{array}{c}\text { Capacity/ } \\
\text { Demand } \\
\frac{\mathrm{S}_{\mathrm{AP}}}{\mathrm{S}_{\mathrm{AD}}}\end{array}$ & $\begin{array}{c}\mathrm{a}_{\mathrm{g}} \\
(\mathrm{g})\end{array}$ \\
\hline 0.2 & .148 & 3.29 & 0.30 & .215 & 0.69 & 0.10 \\
0.4 & .145 & 2.31 & 0.43 & .145 & 1.00 & $0.15^{(1)}$ \\
1.0 & .137 & 1.42 & 0.70 & .066 & 2.08 & 0.31 \\
2.0 & .123 & .95 & 1.05 & .036 & 3.42 & 0.51 \\
5.4 & .076 & .45 & 2.22 & .012 & 6.33 & $0.95^{(2)}$ \\
\hline
\end{tabular}

1. Wall displaces only 0.4 " for $0.15 \mathrm{~g}$ Spectrum

2. Wall reaches stability limit at $0.95 \mathrm{~g}$ Spectrum

Much greater capacity than for Elastic Method because spectrum drops quickly at lower frequencies. 


\section{Arching Action Method (Section 10.5.1.7)- Case 1}

Case 1: Simply supported steel W8 $\times 28$ beam centered on top of wall with no gap between beam and top of wall

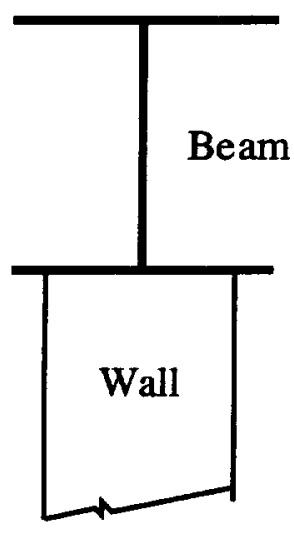

Negligible torsional resistance

web of beam lines up with

centerline of wall, $e_{0}=0$ (see Figure 10.5.1-8)

$$
\text { Use } \begin{aligned}
e & =0 \\
e_{b} & =0 \\
E & =29 \times 10^{6} \mathrm{psi} \\
I_{B} & =98 \mathrm{in} .^{4} \\
L & =216 \mathrm{in} .
\end{aligned}
$$

Masonry:

$$
\begin{aligned}
& \mathrm{f}_{\mathrm{m}}^{\prime}=1000 \mathrm{psi} \\
& \mathrm{P}_{\mathrm{c}}=.125 \mathrm{t} \mathrm{f}_{\mathrm{m}}^{\prime}=.125\left(6^{\prime \prime}\right)(1000 \mathrm{psi})=750 \# / \mathrm{in} . \\
& \mathrm{w}=\rho \mathrm{t}=(135)(0.5) \# / \mathrm{ft} 2=0.469 \mathrm{psi} \\
& \text { gap }=\delta_{\mathrm{g}}=0
\end{aligned}
$$

Vertical displacement of beam:

$$
\begin{aligned}
& \delta_{1}=\frac{P_{c} L^{4}}{32 E I_{B}} f_{R}^{3}\left\{1-\left(\frac{7}{12}\right) f_{R}\right\}=\frac{750 \# / \text { in. }(216 \text { in. })^{4}}{32\left(29 \times 10^{6} \mathrm{psi}\right)\left(98 \mathrm{in}^{4}\right)} f_{R}^{3}\left(1-.583 f_{R}\right) \\
& \delta_{1}=17.95^{\prime \prime} f_{R}^{3}\left(1-.583 f_{R}\right)=17.95 \mathrm{C}
\end{aligned}
$$


Displacement at ultimate capacity:

$$
\delta_{p}=\frac{.00045\left(144^{\prime \prime}\right)^{2}}{\left(6^{\prime \prime}\right)}=1.56^{\prime \prime}
$$

Set $\delta_{H} \leq \delta_{p}=1.56^{\prime \prime}$

Uplift factor:

$$
\begin{aligned}
& f_{p}=1.03+3.0\left(\frac{e}{b}+0.5\right)^{0.65}=1.03+3.0(.5)^{.65}=2.94 \\
& \delta_{u}=\delta_{H}\left(\frac{b}{H}\right) f_{p}=2.94\left(\frac{5.4}{144}\right) \delta_{H}=0.110 \delta_{H} \\
& \delta_{u}-\delta_{g}=\delta_{1} \\
& \delta_{u}=\delta_{1}=0.110 \delta_{H} \\
& \delta_{H}=\frac{\delta_{1}}{0.110}
\end{aligned}
$$

Maximum permissible $f_{R}$ :

$$
\mathrm{f}_{\mathrm{R}} \leq\left(1-\frac{w H}{\mathrm{P}_{\mathrm{c}}}\right) \leq 0.91
$$

Check steel W $8 \times 28$ beam A36 steel:

$$
\begin{aligned}
M_{C A P} & =\phi F_{y} Z_{x}=(0.9)(36 \mathrm{ksi})\left(27.2 \text { in. }^{3}\right)=881 \mathrm{k} \text {-in } \quad \text { (LRFD Method) } \\
\mathrm{f}_{\mathrm{R}} & \leq\left[\frac{8(881)}{.750(216)^{2}}\right]^{\frac{1}{2}}=0.45
\end{aligned}
$$

thus $\mathrm{f}_{\mathrm{R}} \leq 0.45$

$$
\begin{aligned}
& \mathrm{T}_{\mathrm{CAP}} \approx 0 \text { for wide flange held only on web at ends } \\
& \mathrm{e}_{\mathrm{b}}=0 \\
& \mathrm{e}=\mathrm{e}_{\mathrm{b}}-\mathrm{e}_{\mathrm{o}}=0-0=0
\end{aligned}
$$


Start by picking a $f_{R}=0.10$, calculate $\delta_{1}$ and $\delta_{H}$ until $\delta_{H}=\delta_{p}=1.56$ :

\begin{tabular}{|c|c|c|}
\hline$f_{\mathrm{R}}$ & $\begin{array}{c}\delta_{1} \\
\text { (in) }\end{array}$ & $\begin{array}{c}\delta_{\mathrm{H}} \\
\text { (in) }\end{array}$ \\
\hline 0.10 & .0169 & .154 \\
\hline 0.14 & .0452 & .411 \\
\hline 0.20 & .127 & 1.15 \\
\hline 0.22 & .167 & 1.52 \\
\hline 0.225 & .178 & 1.61 \\
\hline
\end{tabular}

$\max \delta_{\mathrm{H}}$ for arching (block begins to crush) $f_{R} \approx 0.222$

$$
\begin{aligned}
& P_{R \delta}=P_{c} f_{R} \\
& \frac{S_{A P}}{g}=\phi\left(\frac{b}{H}\right)\left[2 f_{p} \frac{P_{R \delta}\left(1-\frac{\delta_{H}}{b}\right)}{w H}+6\left(1-\frac{\delta_{H}}{2 b}\right)\right] \\
& \frac{S_{A P}}{g}=0.67\left(\frac{5.4}{144}\right)\left[2(2.94) \frac{750 \frac{\pi}{n} f_{R}\left(1-\frac{\delta_{H}}{5.4^{\prime \prime}}\right)}{.469 p s i(144 ")}+6\left(1-\frac{\delta_{H}}{10.8}\right)\right] \\
& \frac{S_{A P}}{g}=\underbrace{1.64 f_{R}\left(1-\frac{\delta_{H}}{5.4}\right)}_{\text {Arching }}+\underbrace{0.151\left(1-\frac{\delta_{H}}{10.8}\right)}_{\text {Reserve }} \\
& f_{e}=\quad 3.83\left(\frac{S_{A P}}{\delta_{H}}\right)^{0.5}
\end{aligned}
$$


Arching Action results:

\begin{tabular}{|c|c|c|c|c|c|c|c|}
\hline $\begin{array}{c}\delta_{\mathbf{H}} \\
\text { (inch) }\end{array}$ & $\begin{array}{c}\text { Capacity } \\
\mathrm{S}_{\mathrm{AP}} \\
(\mathrm{g})\end{array}$ & $\begin{array}{c}\text { Frequency } \\
\mathrm{f}_{\mathrm{e}} \\
(\mathrm{Hz}) \\
\end{array}$ & $\begin{array}{c}\text { Period } \\
T \\
(\sec )\end{array}$ & $\begin{array}{c}\text { Demand } \\
S_{\mathrm{AD}} \\
(\mathrm{g})\end{array}$ & $\frac{S_{\mathrm{AP}}}{S_{\mathrm{AD}}}$ & $\begin{array}{l}\mathrm{a}_{\mathrm{g}} \\
(\mathrm{g})\end{array}$ & \multirow{6}{*}{$\begin{array}{l}\text { Arching } \\
\text { Action }\end{array}$} \\
\hline 0.154 & 0.300 & 5.35 & .187 & .342 & 0.88 & 0.13 & \\
\hline 0.200 & & & & & 1.00 & 0.15 & \\
\hline 0.411 & 0.357 & 3.57 & .280 & .228 & 1.57 & 0.24 & \\
\hline 1.15 & 0.393 & 2.24 & .446 & .129 & 3.05 & 0.46 & \\
\hline 1.56 & 0.388 & 1.91 & .524 & .101 & 3.84 & 0.58 & \\
\hline 2.0 & 0.123 & .95 & 1.05 & .036 & 3.42 & 0.51 & Reserve \\
\hline 5.4 & 0.076 & .45 & 2.22 & .012 & 6.33 & 0.95 & \\
\hline
\end{tabular}

Wall displaces only 0.2 " for $0.15 \mathrm{~g}$ Spectrum (by interpolation) (Only about $50 \%$ of Reserve Energy deflection)

Stability limit is still $0.95 \mathrm{~g}$ Spectrum (Same as for Reserve Energy)

Not much benefit from arching because of flexibility of support beam and quick drop-off with lowering frequency for input spectrum.

\section{Arching Action Method (Section 10.5.1.7) - Case 2}

Case 2: Same wall, but supported by a large simply supported, torsionally restrained reinforced concrete beam with the following properties:

$$
\begin{array}{ll}
\mathrm{I}_{\mathrm{B}}=6000 \mathrm{in}^{4} & \mathrm{E}=3 \times 10^{6} \mathrm{psi} \\
\mathrm{J}_{\mathrm{B}}=7000 \mathrm{in}^{4} & \mathrm{G}=1.2 \times 10^{6} \mathrm{psi}
\end{array}
$$

see Figure 10.5.1-8

$$
\begin{aligned}
& e_{o}=0 \quad \mathrm{e} / \mathrm{b}=0.5 \\
& f_{p}=1.03+3.0(1.0)^{.65}=4.03 \\
& \delta_{u}-\delta_{g}=\delta_{1}=\delta_{H}\left(\frac{5.4^{\prime \prime}}{144^{\prime \prime}}\right)(4.03)=0.151 \delta_{H} \\
& \delta_{H}=\frac{\delta_{1}}{0.151}
\end{aligned}
$$$$
e_{b}=\frac{b}{2}-e_{o}=0.45 t-0=2.7^{\prime \prime}
$$ 


$$
\begin{aligned}
& \delta_{1}=\frac{P_{c} L^{4}}{32 E_{B}} f_{R}^{3}\left\{1-\frac{7}{12} f_{R}\right\}+\frac{P_{c} e_{b}^{2} L^{2} f_{R}^{2}}{8 G_{B}}
\end{aligned}
$$

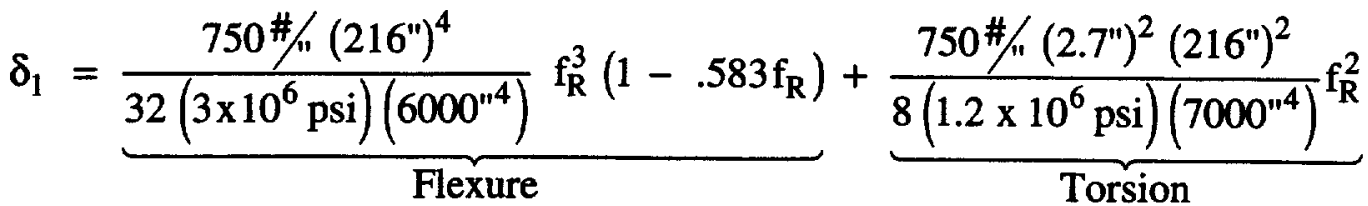

$$
\begin{aligned}
& \delta_{1}=2.83^{\prime \prime} \mathrm{f}_{\mathrm{R}}^{3}\left(1-.583 \mathrm{f}_{\mathrm{R}}\right)+(\approx 0)
\end{aligned}
$$

Maximum permissible $f_{R}$ :

$$
f_{R} \leq\left(1-\frac{w H}{P_{c}}\right)=1-\frac{.469 p s i\left(144^{\prime \prime}\right)}{750 \# / "}=0.91
$$

Check concrete beam $\left(12^{\prime \prime}\right.$ x 24" Deep, $A_{s} \geq 2$ in $\left.^{2}\right)$ with some torsional steel:

$$
\begin{gathered}
\mathrm{M}_{\mathrm{CAP}}=2000 \mathrm{k} \text {-in. } \\
\mathrm{f}_{\mathrm{R}} \leq\left[\frac{8(2000)}{.750(216)^{2}}\right]^{\frac{1}{2}}=0.68 \\
\text { thus } \mathrm{f}_{\mathrm{R}} \leq 0.68 \\
\mathrm{~T}_{\mathrm{CAP}}=120 \mathrm{k} \text {-in. } \\
\mathrm{e}_{\mathrm{b}} \mathrm{f}_{\mathrm{R}} \leq\left[\frac{2(120)}{.750(216)}\right]=1.48
\end{gathered}
$$

$e_{b}$ must be reduced below 2.7 " if $\mathrm{f}_{\mathrm{R}}$ exceeds $\frac{1.48}{2.7}=0.55$

\begin{tabular}{|c|c|c|}
\hline$f_{\mathrm{R}}$ & $\begin{array}{c}\delta_{1} \\
\text { (in.) }\end{array}$ & $\begin{array}{c}\delta_{\mathrm{H}}=\frac{\delta_{1}}{0.151} \\
\text { (in.) }\end{array}$ \\
\hline 0.20 & .0200 & 0.132 \\
\hline 0.25 & .0378 & 0.250 \\
\hline 0.30 & .0630 & 0.417 \\
\hline 0.40 & .139 & 0.920 \\
\hline 0.486 & & 1.56 \\
\hline 0.50 & .251 & 1.66 \\
\hline
\end{tabular}




$$
\begin{aligned}
& \frac{\mathrm{S}_{\mathrm{AP}}}{\mathrm{g}}=2.25 \mathrm{f}_{\mathrm{R}}\left(1-\frac{\delta_{\mathrm{H}}}{5.4}\right)+0.151\left(1-\frac{\delta_{\mathrm{H}}}{10.8}\right) \\
& \mathrm{f}_{\mathrm{e}}=3.83 \sqrt{\frac{\mathrm{S}_{\mathrm{AP}}}{\delta_{\mathrm{H}}}}
\end{aligned}
$$

\begin{tabular}{|c|c|c|c|c|c|c|c|}
\hline $\begin{array}{c}\delta_{\mathrm{H}} \\
\text { (inch) }\end{array}$ & $\begin{array}{l}\mathrm{S}_{\mathrm{AP}} \\
(\mathrm{g})\end{array}$ & $\begin{array}{c}\mathrm{f}_{\mathrm{e}} \\
(\mathrm{Hz})\end{array}$ & $\begin{array}{c}\mathrm{T} \\
(\mathrm{sec})\end{array}$ & $\begin{array}{l}\mathrm{S}_{\mathrm{AD}} \\
(\mathrm{g})\end{array}$ & $\frac{\mathbf{S}_{\mathrm{AP}}}{\mathrm{S}_{\mathrm{AD}}}$ & $\begin{array}{l}\mathrm{a}_{\mathrm{g}} \\
\text { (g) }\end{array}$ & \\
\hline 0.132 & .588 & 8.08 & 0.124 & 0.40 & 1.47 & 0.22 & \\
\hline 0.250 & .684 & 6.33 & 0.158 & 0.40 & 1.71 & 0.26 & \\
\hline 0.417 & .768 & 5.20 & 0.192 & 0.338 & 2.27 & 0.34 & Arching \\
\hline 0.920 & .885 & 3.76 & 0.266 & 0.245 & 3.61 & 0.54 & Action \\
\hline 1.56 & .907 & 2.92 & 0.342 & 0.191 & 4.75 & 0.71 & \\
\hline 2.0 & .123 & 0.95 & 1.05 & .036 & 3.42 & 0.51 & Reserve \\
\hline 5.4 & .076 & 0.45 & 2.22 & .012 & 13 & 0. & Energy \\
\hline
\end{tabular}

Wall displays only 0.13 inches for a $0.22 \mathrm{~g}$ input

However, stability limit is still $0.95 \mathrm{~g}$

Arching Action did not increase stability limit because of shape of input spectrum.

Comparison of results for Portsmouth input spectrum shape:

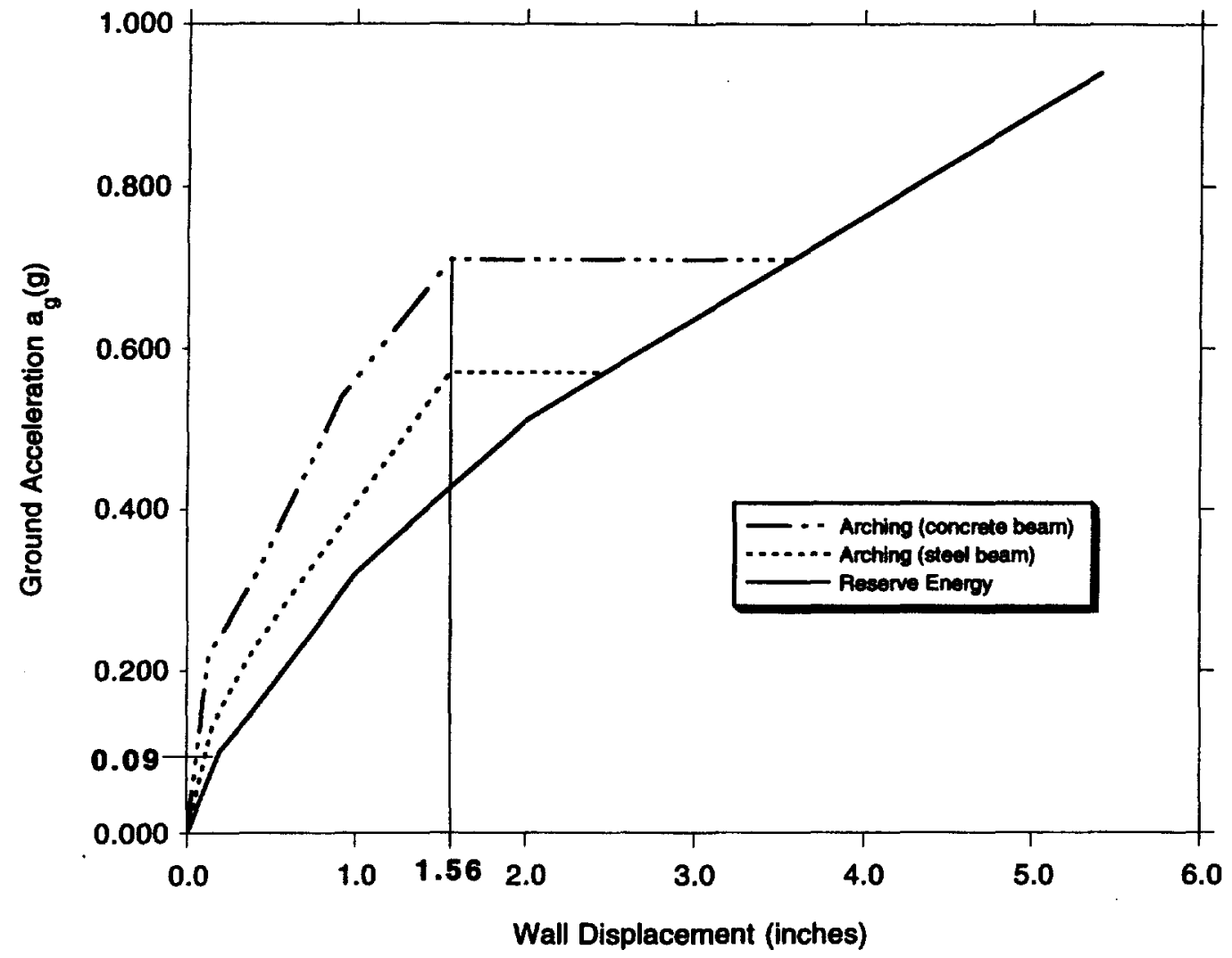


Rework same example with NUREG/CR-0098 (Ref. 72) input median spectrum for a soil site to illustrate the importance of the input spectrum shape on relative results.

Spectrum properties for 5\% damping are given below and shown in Figure 10.5.1-6B:

$$
\begin{array}{ll}
8 \mathrm{~Hz} \leq \mathrm{f} \leq 33 \mathrm{~Hz}: & \mathrm{S}_{\mathrm{AD}}=\mathrm{a}_{\mathrm{g}}(\mathrm{f} / 33 \mathrm{~Hz})^{-0.53} \\
1.64 \mathrm{~Hz} \leq \mathrm{f} \leq 8 \mathrm{~Hz} & \mathrm{~S}_{\mathrm{AD}}=2.12 \mathrm{a}_{\mathrm{g}} \\
0.25 \mathrm{~Hz} \leq \mathrm{f} \leq 1.64 \mathrm{~Hz} & \mathrm{~S}_{\mathrm{AD}}=1.29 \operatorname{sec~} \mathrm{f} \mathrm{a}_{\mathrm{g}} \\
\mathrm{f} \leq 0.25 \mathrm{~Hz} & \mathrm{~S}_{\mathrm{AD}}=5.08 \operatorname{sec~} \mathrm{f}^{2} \mathrm{a}_{\mathrm{g}}
\end{array}
$$

Elastic Method (Section 10.5.1.5)

\begin{tabular}{|c|c|c|c|c|}
\hline $\begin{array}{c}\delta_{H} \\
\text { (in.) }\end{array}$ & $\begin{array}{c}S_{\mathrm{AP}} \\
(\mathrm{g})\end{array}$ & $\begin{array}{c}f_{e} \\
(\mathrm{~Hz})\end{array}$ & $S_{A D} / a_{g}$ & $a_{g}=\underbrace{\left(\frac{S_{A P}}{S_{A D}}\right.}_{(g)} a_{g})$ \\
\hline 0.20 & .148 & 3.29 & 2.12 & 0.07 less than elastic \\
\hline 0.40 & .145 & 2.31 & 2.12 & 0.07 \\
\hline 1.0 & .137 & 1.42 & 1.83 & 0.07 \\
\hline 2.0 & .123 & .95 & 1.23 & 0.10 \\
\hline 5.4 & .076 & .45 & 0.58 & 0.13 \\
\hline
\end{tabular}

$$
\begin{aligned}
& \mathrm{S}_{\mathrm{AP}}=0.24 \mathrm{~g} \\
& \mathrm{f}=10.8 \mathrm{~Hz} \longrightarrow \mathrm{S}_{\mathrm{AD}}=1.81 \mathrm{a}_{\mathrm{g}}=0.27 \mathrm{~g} \\
& \frac{\mathrm{S}_{\mathrm{AP}}}{\mathrm{S}_{\mathrm{AD}}}=\frac{0.24}{0.27}=0.89<1.0 \\
& \mathrm{a}_{\mathrm{g}}=(0.89)(0.15 \mathrm{~g})=0.13 \mathrm{~g}
\end{aligned}
$$

Reserve Energy Method (Section 10.5.1.6)

Using previous results:

No value over Elastic Method

For NUREG/CR-0098 soil spectrum, wall becomes unstable when it exceeds $0.13 \mathrm{~g}$ elastic capacity, no advantage to Reserve Energy Method. (Spectrum has lots of low frequency) 
Arching Action Method (Section 10.5.1.7)-Case 1

Case 1 - Steel beam

Using previous results:

\begin{tabular}{|c|c|c|c|c|}
\hline $\begin{array}{c}\delta_{\mathrm{H}} \\
\text { (in.) }\end{array}$ & $\begin{array}{c}\mathrm{S}_{\mathrm{AP}} \\
(\mathrm{g})\end{array}$ & $\begin{array}{c}\mathrm{f}_{\mathrm{e}} \\
(\mathrm{Hz})\end{array}$ & $\mathrm{S}_{\mathrm{AD}} / \mathrm{a}_{\mathrm{g}}$ & $\mathrm{a}_{\mathrm{g}}=\left(\begin{array}{c}\frac{\mathrm{S}_{\mathrm{AP}}}{\mathrm{S}_{\mathrm{AD}}} \\
\mathrm{a}_{\mathrm{g}}\end{array}\right)$ \\
\hline .154 & .300 & 5.35 & 2.12 & 0.14 \\
.411 & .357 & 3.57 & 2.12 & 0.17 \\
1.15 & .393 & 2.24 & 2.12 & 0.18 \\
1.56 & .388 & 1.91 & 2.12 & 0.18 \\
\hline
\end{tabular}

Maximum $\mathrm{a}_{\mathrm{g}} \approx 1.4$ * Elastic capacity for NUREG/CR-0098 soil spectrum

Arching Action Method (Section 10.5.1.7) - Case 2

Case 2 - Concrete beam

Using previous results:

\begin{tabular}{|c|c|c|c|c|}
\hline $\begin{array}{c}\delta_{\mathrm{H}} \\
\text { (in.) }\end{array}$ & $\begin{array}{c}\mathrm{S}_{\mathrm{AP}} \\
(\mathrm{g})\end{array}$ & $\begin{array}{c}\mathrm{f}_{\mathrm{e}} \\
(\mathrm{Hz})\end{array}$ & $\mathrm{S}_{\mathrm{AD}} / \mathrm{a}_{\mathrm{g}}$ & $\begin{array}{c}\mathrm{a}_{\mathrm{g}}=\frac{\frac{\mathrm{S}_{\mathrm{AP}}}{\mathrm{S}_{\mathrm{AD}}}}{\mathrm{g}} \\
(\mathrm{g})\end{array}$ \\
\hline .132 & .588 & 8.08 & 2.11 & 0.28 \\
.250 & .684 & 6.33 & 2.12 & 0.32 \\
.417 & .768 & 5.20 & 2.12 & 0.36 \\
.920 & .885 & 3.76 & 2.12 & 0.42 \\
1.56 & .907 & 2.92 & 2.12 & 0.43 \\
\hline
\end{tabular}

Maximum $\mathrm{a}_{\mathrm{g}} \approx 3.3 *$ Elastic capacity for NUREG/CR-0098 soil spectrum 
Summary of Section 10.5.1.10

\begin{tabular}{|l|c|c|}
\hline \multirow{2}{*}{} & \multicolumn{2}{|c|}{ Factor Over Elastic a . Capacity } \\
\cline { 2 - 3 } & $\begin{array}{c}\text { Portsmouth } \\
\text { Spectrum }\end{array}$ & $\begin{array}{c}\text { NUREG/CR-0098 } \\
\text { Soil Spectrum }\end{array}$ \\
\hline Reserve Energy & 10.6 & 1.0 \\
Arching Case 1 (Steel Beam) & 10.6 & 1.4 \\
Arching Case 2 (Concrete Beam) & 10.6 & 3.3 \\
\hline
\end{tabular}

Whether Reserve Energy results in increased capacity over Elastic Method is highly sensitive to shape of input demand spectrum.

Increase in capacity from Arching Action is significantly influenced by stiffness of boundary element and shape of input demand spectrum. 
Table 10.5.1-1 $\left(\frac{H}{t}\right)_{N}$ versus Wall Thickness for use in URM Wall Screening (based on Sections 10.5.1.4 and 10.5.1.8)

\begin{tabular}{|c|c|c|c|}
\hline $\begin{array}{c}\text { Nominal URM } \\
\text { Wall Thickness }\end{array}$ & $\begin{array}{c}\text { Actual Concrete } \\
\text { Block Wall } \\
\text { Thickness }\end{array}$ & $\begin{array}{c}\text { Minimum Concrete } \\
\text { Block Flange } \\
\text { Thickness }\end{array}$ & $\left(\frac{\mathrm{H}}{\mathrm{t}}\right)_{\mathrm{N}}$ \\
\hline $4 "$ & $3.625^{\prime \prime}$ & $.75^{\prime \prime}$ & 13.5 \\
\hline $6^{\prime \prime}$ & $5.625^{\prime \prime}$ & $1.0^{\prime \prime}$ & 11.5 \\
\hline $8^{\prime \prime}$ & $7.625^{\prime \prime}$ & $1.25^{\prime \prime}$ & 10.0 \\
\hline $10^{\prime \prime}$ & $9.625^{\prime \prime}$ & $1.375^{\prime \prime}$ & 9.0 \\
\hline $12^{\prime \prime}$ & $11.625^{\prime \prime}$ & $1.5^{\prime \prime}$ & 8.0 \\
\hline
\end{tabular}


Table 10.5.1-2 DBE Ground Motion $S_{A_{\max }}$ from UBC Seismic Zone (May be used for PC 1 Structures, Systems, and Components, Ref. 6)

\begin{tabular}{|c|c|c|}
\hline DOE Site & Seismic Zone & $S_{A_{\max }}$ \\
\hline Kansas City & $2 \mathrm{~A}$ & 0.41 \\
\hline LANL & 2B & 0.55 \\
\hline Mound & 1 & 0.21 \\
\hline Pantex Plant & 1 & 0.21 \\
\hline Rocky Flats & 1 & 0.21 \\
\hline Sandia, Albuquerque & $2 \mathrm{~B}$ & 0.55 \\
\hline Sandia, Livermore & 4 & 1.10 \\
\hline Pinellas Plant & 0 & 0.10 \\
\hline Argonne-East & 0 & 0.10 \\
\hline Argonne-West & 2B & 0.55 \\
\hline Brookhaven & $2 \mathrm{~A}$ & 0.41 \\
\hline Princeton & $2 A^{\prime}$ & 0.41 \\
\hline INEL & 2B & 0.55 \\
\hline Feed Materials Production Center & 1 & 0.21 \\
\hline Oak Ridge & $2 \mathrm{~A}$ & 0.41 \\
\hline Paducah & $2 \mathrm{~A}$ & 0.41 \\
\hline Portsmouth & 1 & 0.21 \\
\hline Nevada Test Site & 3 & 0.83 \\
\hline Hanford & 2B & 0.55 \\
\hline LBL & 4 & 1.10 \\
\hline LLNL & 4 & 1.10 \\
\hline ETEC & 4 & 1.10 \\
\hline SLAC & 4 & 1.10 \\
\hline Savannah River & $2 \mathrm{~A}$ & 0.41 \\
\hline
\end{tabular}


Table 10.5.1-3 Boundary Condition Factors, $B_{F}$, for Fundamental Frequency Calculation

(Table 1 of Reference 117)

Top

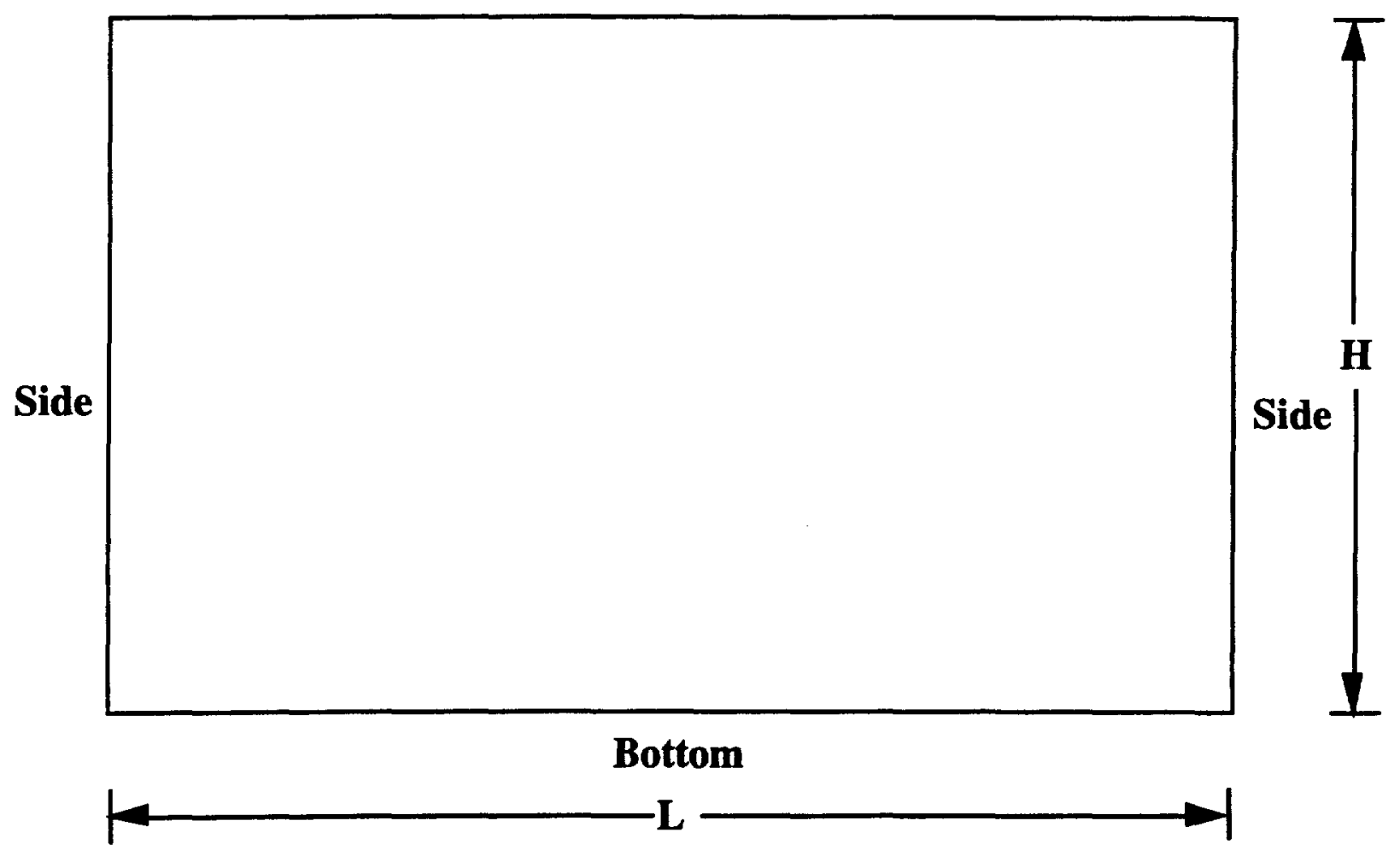

Case 1: Simple Support Top/Simple Support Bottom with Specified Combination of Side Supports

\begin{tabular}{|c|c|c|c|c|c|c|}
\hline $\mathrm{H} / \mathrm{L}$ & Free-Free & SS-Free & Fixed-Free & SS-SS & SS-Fixed & Fixed-Fixed \\
\hline$\leq 0.20$ & 1.571 & 1.571 & 1.571 & 1.571 & 1.571 & 1.571 \\
\hline 0.4 & 1.571 & 1.612 & 1.622 & 1.822 & 1.870 & 1.931 \\
\hline 0.667 & 1.571 & 1.698 & 1.748 & 2.270 & 2.480 & 2.765 \\
\hline 1.0 & 1.571 & 1.859 & 2.020 & 3.142 & 3.764 & 4.608 \\
\hline 1.5 & 1.571 & 2.182 & 2.677 & 5.106 & 6.769 & 8.968 \\
\hline 2.5 & 1.571 & 2.992 & 4.875 & 11.39 & 16.54 & 23.16 \\
\hline
\end{tabular}


Table 10.5.1-3 (Continued)

Case 2: Fixed Top/Fixed Bottom with Specified Combination of Side Supports

\begin{tabular}{|c|c|c|c|c|c|c|}
\hline $\mathrm{H} / \mathrm{L}$ & Free-Free & SS-Free & Fixed-Free & SS-SS & SS-Fixed & Fixed-Fixed \\
\hline$\leq 0.20$ & 3.561 & 3.561 & 3.561 & 3.561 & 3.561 & 3.561 \\
\hline 0.4 & 3.561 & 3.587 & 3.594 & 3.706 & 3.731 & 3.764 \\
\hline 0.667 & 3.561 & 3.638 & 3.664 & 3.986 & 4.116 & 4.299 \\
\hline 1.0 & 3.561 & 3.734 & 3.823 & 4.608 & 5.066 & 5.730 \\
\hline 1.5 & 3.561 & 3.944 & 4.254 & 6.221 & 7.666 & 9.672 \\
\hline 2.5 & 3.561 & 4.545 & 5.994 & 12.07 & 17.05 & 23.52 \\
\hline
\end{tabular}

Case 3: Simple Support Top/Fixed Bottom (or Vice-Versa) with Specified Combination of Side Supports

\begin{tabular}{|c|c|c|c|c|c|c|}
\hline $\mathrm{H} / \mathrm{L}$ & Free-Free & SS-Free & Fixed-Free & SS-SS & SS-Fixed & Fixed-Fixed \\
\hline$\leq 0.20$ & 2.454 & 2.454 & 2.454 & 2.454 & 2.454 & 2.454 \\
\hline 0.4 & 2.454 & 2.491 & 2.499 & 2.646 & 2.682 & 2.727 \\
\hline 0.667 & 2.454 & 2.558 & 2.593 & 3.008 & 3.175 & 3.407 \\
\hline 1.0 & 2.454 & 2.685 & 2.804 & 3.764 & 4.307 & 5.066 \\
\hline 1.5 & 2.454 & 2.951 & 3.349 & 5.579 & 7.144 & 9.260 \\
\hline 2.5 & 2.454 & 3.672 & 5.344 & 11.69 & 16.76 & 23.32 \\
\hline
\end{tabular}


Table 10.5.1-3 (Continued)

Case 4: Free Top/Fixed Bottom with Specified Combination of Side Supports

\begin{tabular}{|c|c|c|c|c|c|c|}
\hline $\mathrm{H} / \mathrm{L}$ & Free-Free & SS-Free & Fixed-Free & SS-SS & SS-Fixed & Fixed-Fixed \\
\hline$\leq 0.20$ & 0.560 & 0.560 & 0.560 & 0.560 & 0.560 & 0.560 \\
\hline 0.4 & 0.560 & 0.613 & 0.634 & 0.780 & 0.855 & 0.959 \\
\hline 0.667 & 0.560 & 0.704 & 0.793 & 1.190 & 1.488 & 1.891 \\
\hline 1.0 & 0.560 & 0.897 & 1.105 & 2.020 & 2.804 & 3.823 \\
\hline 1.5 & 0.560 & 1.103 & 1.786 & 3.932 & 5.833 & 8.243 \\
\hline 2.5 & 0.560 & 1.607 & 3.965 & 10.14 & 15.62 & 22.46 \\
\hline
\end{tabular}

Case 5: Free Top/Simple Support Bottom with Specified Combination of Side Supports

\begin{tabular}{|c|c|c|c|c|c|c|}
\hline $\mathrm{H} / \mathrm{L}$ & Free-Free* & SS-Free & Fixed-Free & SS-SS & SS-Fixed & Fixed-Fixed \\
\hline$\leq 0.2$ & 0 & 0.107 & 0.159 & 0.224 & 0.258 & 0.285 \\
\hline 0.4 & 0 & 0.210 & 0.257 & 0.479 & 0.587 & 0.727 \\
\hline 0.667 & 0 & 0.356 & 0.491 & 0.971 & 1.313 & 1.755 \\
\hline 1.0 & 0 & 0.536 & 0.854 & 1.859 & 2.685 & 3.734 \\
\hline 1.5 & 0 & 0.800 & 1.585 & 3.821 & 5.755 & 8.186 \\
\hline 2.5 & 0 & 1.313 & 3.834 & 10.08 & 15.57 & 22.42 \\
\hline
\end{tabular}

* Rigid Body Mode 
Table 10.5.1.4 Frequency Factors, F

(Table 2 of Reference 117)

\begin{tabular}{|c|c|c|c|c|c|c|c|c|c|c|}
\hline \multirow{2}{*}{$\begin{array}{l}\text { WALL } \\
\text { HEIGHT H }\end{array}$} & \multicolumn{9}{|c|}{ HOLLOW MASONRY THICKNESS } & \multicolumn{3}{|c|}{ SOLID MASONRY THICKNESS } \\
\hline & $4^{\prime \prime}$ & $6^{\prime \prime}$ & $8^{\prime \prime}$ & $10^{\prime \prime}$ & $12^{\prime \prime}$ & $4^{\prime \prime}$ & $66^{\prime \prime}$ & $8^{\prime \prime}$ & $10^{\prime \prime}$ & $12^{\prime \prime}$ \\
\hline $6^{\prime}$ & 17.4 & 26.8 & 36.5 & 45.8 & 55.1 & 13.5 & 20.9 & 28.3 & 35.8 & 43.2 \\
\hline $8^{\prime}$ & 9.81 & 15.1 & 20.5 & 25.7 & 31.0 & 7.57 & 11.8 & 15.9 & 20.1 & 24.3 \\
\hline $10^{\prime}$ & 6.28 & 9.65 & 13.1 & 16.5 & 19.8 & 4.85 & 7.52 & 10.2 & 12.9 & 15.5 \\
\hline $12^{\prime}$ & 4.36 & 6.70 & 9.13 & 11.4 & 13.8 & 3.37 & 5.22 & 7.08 & 8.94 & 10.8 \\
\hline $14^{\prime}$ & 3.20 & 4.92 & 6.71 & 8.41 & 10.1 & 2.47 & 3.84 & 5.20 & 6.57 & 7.94 \\
\hline $16^{\prime}$ & 2.45 & 3.77 & 5.14 & 6.44 & 7.75 & 1.89 & 2.94 & 3.98 & 5.03 & 6.07 \\
\hline $18^{\prime}$ & 1.94 & 2.98 & 4.06 & 5.09 & 6.13 & 1.50 & 2.32 & 3.15 & 3.97 & 4.79 \\
\hline $20^{\prime}$ & 1.57 & 2.41 & 3.29 & 4.12 & 4.96 & 1.21 & 1.88 & 2.55 & 3.22 & 3.88 \\
\hline $24^{\prime}$ & 1.09 & 1.68 & 2.28 & 2.86 & 3.45 & .841 & 1.31 & 1.77 & 2.23 & 2.70 \\
\hline $30^{\prime}$ & .698 & 1.07 & 1.46 & 1.83 & 2.21 & .538 & .836 & 1.13 & 1.43 & 1.73 \\
\hline
\end{tabular}

$$
\mathrm{F}=\left(1 / \mathrm{H}^{2}\right) *\left(\mathrm{EI} \mathrm{g}^{\prime} / \mathrm{w}\right)^{1 / 2}
$$

where

$\mathrm{H}=$ Wall Height (in)

$\mathrm{E}=$ Elastic Modulus $=1 \times 10^{6} \mathrm{\# / \textrm {in } ^ { 2 }}$

$I^{\prime}=$ Effective Plate Moment of Inertia (in ${ }^{4} /$ in)

$\mathrm{g}=$ Acceleration of Gravity $=386.4 \mathrm{in} / \mathrm{sec}^{2}$

$\mathbf{w}=$ Distributed Load per Unit Surface Area (\#/in $\left.{ }^{2}\right)$ based on masonry weight density $=150 \# / \mathrm{ft}^{3}$ 
The Frequency Factor, $F$, is based on $E=1 \times 10^{6}$ psi. To adjust $f$ for other values of $E$, $\alpha_{E}=\sqrt{E /\left(1 \times 10^{6}\right)}$. For masonry, $E$ is typically taken as $1000 f_{m}^{\prime}$, where $f_{m}^{\prime}$ is the compressive strength of the masonry unit/mortar combination. The typical range of $E$ is $0.7 \times 10^{6} \mathrm{psi}$ to $2.5 \times 10^{6} \mathrm{psi}$. Site-specific testing can be utilized to determine $\mathrm{E}$.

The following table shows $\alpha_{E} v s . E$ for the range of interest:

\begin{tabular}{|c|c|}
\hline$E(p s i)$ & $\alpha_{E}$ \\
\hline $0.5 \times 10^{6}$ & 0.71 \\
\hline $0.7 \times 10^{6}$ & 0.84 \\
\hline $0.9 \times 10^{6}$ & 0.95 \\
\hline $1.0 \times 10^{6}$ & 1.0 \\
\hline $1.25 \times 10^{6}$ & 1.12 \\
\hline $1.50 \times 10^{6}$ & 1.22 \\
\hline $1.75 \times 10^{6}$ & 1.32 \\
\hline $2.00 \times 10^{6}$ & 1.41 \\
\hline $2.25 \times 10^{6}$ & 1.50 \\
\hline $2.50 \times 10^{6}$ & 1.58 \\
\hline $2.75 \times 10^{6}$ & 1.66 \\
\hline $3.00 \times 10^{6}$ & 1.73 \\
\hline & \\
\hline & \\
\hline
\end{tabular}




\section{Table 10.5.1-6 Weight Density Factor $(\alpha D)$ \\ (Table 4 of Reference 117)}

The Frequency Factor, F, is based on a weight density, $\rho$, of $150 \# / \mathrm{ft}^{3}$ for the masonry material. Based on the density, the masonry block construction (solid vs. hollow), and the nominal block thickness (4", 6", 8", 10", 12"), the surface loading, w, is defined in \#/in ${ }^{2}$.

The density of masonry may vary over a wide range, depending on the application. By varying aggregate density and constituent ratios, $\rho$ can range from $75 \# / \mathrm{ft}^{3}$ to $200 \# / \mathrm{ft}^{3}$. For most DOE facilities, the reference value of $\rho=150 \# / \mathrm{ft}^{3}$ should be a suitable, slightly conservative value.

To account for cases where there is significant difference, based on site-specific design specifications or sample testing, the following table provides values of $\alpha_{D}$ vs. $p$ for the expected range of variation:

\begin{tabular}{|c|c|}
\hline$\rho\left(\# / \mathrm{ft}^{3}{ }^{3}\right)$ & $\alpha_{\mathrm{D}}$ \\
\hline 200 & 0.87 \\
\hline 175 & 0.93 \\
\hline 150 & 1.0 \\
\hline 125 & 1.10 \\
\hline 100 & 1.22 \\
\hline 75 & 1.41 \\
\hline
\end{tabular}

To adjust $f$ for other values of $\rho, \alpha_{D}=\sqrt{150 / \rho}$

\section{Additional Weight of Attachments}

To account for the additional weight of attachments to the wall, an effective weight density can be estimated as follows:

1. Estimate total weight of attachments, $\mathrm{WT}_{\mathrm{A}}$

2. Divide $\mathrm{WT}_{\mathrm{A}}$ by gross wall volume (HxLxt) to get effective increase in density

$$
\rho_{\mathrm{A}}=\mathrm{WT}_{\mathrm{A}} /(\mathrm{HLt})\left[\# / \mathrm{ft}^{3}\right]
$$

3. For solid masonry, effective total density is

$$
\rho=\rho_{\text {masonry }}+\rho_{A}
$$

4. For hollow masonry, effective total density is

$$
\rho=\rho_{\text {masonry }}+2\left(\rho_{A}\right)
$$

The factor of 2 on $\rho_{\mathrm{A}}$ for hollow masonry accounts for the fact that the net volume is approximately $50 \%$ of the gross volume.

5. Select factor $\alpha_{D}$ based on the effective total density. 
Table 10.5.1-7 Orthotropic Behavior Adjustment Factor $\left(\alpha_{T}\right)$

(Table 5 of Reference 117)

\section{A. Solid Masonry}

For solid masonry (including hollow masonry with completely grouted cells), isotropic out-of-plane bending behavior is expected. Consequently,

$$
\alpha_{\mathrm{T}}=1.0
$$

\section{B. Hollow Masonry}

Based on the geometry of the hollow masonry, the section properties resisting out-of-plane bending are different for bending about axes perpendicular to and parallel to the cell axis direction. Assuming completely mortared web joints between masonry units, the webs contribute to the bending resistance about an axis perpendicular to the cell axis direction. For bending about an axis parallel to the cell axis direction, the webs are considered to be ineffective; this results in a modest reduction of bending resistance, which is a function of the masonry unit thickness. The significance of this reduction on the out-of-plane natural frequency depends on the plate aspect ratio and the cell axis direction. The worst case reduction factors are provided in the table below for the range of masonry unit thicknesses:

\begin{tabular}{|c|c|}
\hline $\begin{array}{c}\text { Hollow Masonry } \\
\text { Unit Thickness (in.) }\end{array}$ & $\begin{array}{c}\alpha_{\mathrm{T}} \\
\text { (minimum value) }\end{array}$ \\
\hline $4^{\prime \prime}$ & 0.98 \\
\hline $6^{\prime \prime}$ & 0.97 \\
\hline $8^{\prime \prime}$ & 0.96 \\
\hline $10^{\prime \prime}$ & 0.94 \\
\hline $12^{\prime \prime}$ & 0.91 \\
\hline
\end{tabular}

A more accurate value for $\alpha_{T}$ can be determined by the following procedure:

1) Calculate the wall aspect ratio (AR), defined as the lineal dimension parallel to the cell axis divided by the lineal dimension perpendicular to the cell axis:

2) $\quad$ For $A R \leq 0.2$, use $\alpha_{T}=1.0$.

3) For $A R \geq 5.0$, use $\alpha_{T}(\mathrm{~min})=0.91$.

4) For $\mathrm{AR}=1.0$, use $\alpha_{\mathrm{T}}=0.5\left[1.0+\alpha_{\mathrm{T}}(\min )\right]$.

5) For $0.2<A R<1.0$, use linear interpolation between 1.0 and $0.5\left[1.0+\alpha_{T}(\min )\right]$.

6) For $1.0<A R<5.0$, use linear interpolation between $0.5\left[1.0+\alpha_{T}(\mathrm{~min})\right]$ and $\alpha_{T}$ $(\min )$. 


\section{Table 10.5.1-8 Special Considerations for Elastic Method (Table 6 of Reference 117)}

\section{A) Partial Grouting of Cells in Hollow Masonry}

If selected cells are grouted from top to bottom of the wall, in a regular pattern, then both wall mass and stiffness are increased. This would tend to decrease the applicable frequency factor, $F$. Therefore, the solid masonry values in Table 10.5.1-4 can be used as a conservative lower bound for F. Alternately, interpolation between the solid and hollow masonry values can be used, based on the percentage of cells filled.

\section{B) Partially Filled Mortar Joints}

\section{1) Solid Masonry}

This is an undesirable condition, which raises questions about the original construction workmanship. A technical basis for such construction should be investigated. In addition, a significant amount of in-situ sampling is probably required to characterize the mortar joints

2) Hollow Masonry

The original construction may not have specified mortaring of the webs in the bed joints. If this condition has been verified by in-situ sampling then the Orthotropic Behavior Adjustment Factor, $\alpha_{\mathrm{T}}$, is set to the appropriate minimum value from Table 10.5.1-5 in the calculation of the wall frequency. This effectively eliminates any contribution to bending stiffness from the webs.

Any other deviation from fully mortared joints is an undesirable condition. Refer to discussion above for solid masonry.

\section{C) Multi-Wythe and Composite Construction}

The possible combinations are too numerous to quantify. However, certain guidance can be provided for the assessment of such walls.

1) If adequate connectivity between wythes cannot be demonstrated, then each wythe must be treated as a separate wall. In this case, the formulas and data provided here should be applicable to each wythe.

2) Adequate connectivity should be verified by definitive design and fabrication documentation, supported by in-situ sampling.

3) The Boundary Condition Factor, $\mathbf{B}_{\mathbf{f}}$ from Table 10.5.1-3 is applicable to multiwythe and composite construction. A case-specific Frequency Factor, F, would have to be developed for composite bending behavior. 
Table 10.5.1-9 Boundary Condition Factors, $\mathbf{B}_{\mathbf{s}}$, for Maximum Bending Stress Calculation

(Table 7 of Reference 117)

Case 1: SS Top/SS Bottom

\begin{tabular}{|c|c|c|c|}
\hline $\mathrm{H} / \mathrm{L}$ & Free-Free Sides & SS-SS Sides & Fixed-Fixed Sides \\
\hline$\leq 0.20$ & 0.125 & 0.125 & 0.125 \\
\hline 0.4 & 0.125 & 0.110 & 0.122 \\
\hline 0.667 & 0.125 & 0.081 & 0.105 \\
\hline 1.0 & 0.125 & 0.048 & 0.070 \\
\hline 1.5 & 0.125 & 0.036 & 0.037 \\
\hline 2.5 & 0.125 & 0.018 & 0.013 \\
\hline
\end{tabular}

Case 2: Fixed Top/Fixed Bottom

\begin{tabular}{|c|c|c|c|}
\hline $\mathrm{H} / \mathrm{L}$ & Free-Free Sides & SS-SS Sides & Fixed-Fixed Sides \\
\hline$\leq 0.20$ & 0.083 & 0.083 & 0.083 \\
\hline 0.4 & 0.083 & 0.083 & 0.083 \\
\hline 0.667 & 0.083 & 0.082 & 0.076 \\
\hline 1.0 & 0.083 & 0.070 & 0.051 \\
\hline 1.5 & 0.083 & 0.047 & 0.034 \\
\hline 2.5 & 0.083 & 0.020 & 0.013 \\
\hline
\end{tabular}


Table 10.5.1-9 (Continued)

Case 3: SS Top/Fixed Bottom (or Vice-Versa)

\begin{tabular}{|c|c|c|c|}
\hline $\mathrm{H} / \mathrm{L}$ & Free-Free Sides & SS-SS Sides & Fixed-Fixed Sides \\
\hline$\leq 0.20$ & 0.125 & 0.125 & 0.125 \\
\hline 0.4 & 0.125 & 0.125 & 0.119 \\
\hline 0.667 & 0.125 & 0.110 & 0.095 \\
\hline 1.0 & 0.125 & 0.084 & 0.060 \\
\hline 1.5 & 0.125 & 0.050 & 0.034 \\
\hline 2.5 & 0.125 & 0.020 & 0.013 \\
\hline
\end{tabular}

Case 4: Free Top/Fixed Bottom

\begin{tabular}{|c|c|c|c|}
\hline H/L & Free-Free Sides & SS-SS Sides & Fixed-Fixed Sides \\
\hline$\leq 0.20$ & 0.50 & 0.50 & 0.50 \\
\hline 0.4 & 0.50 & 0.375 & 0.275 \\
\hline 0.667 & 0.50 & 0.227 & 0.173 \\
\hline 1.0 & 0.50 & 0.119 & 0.085 \\
\hline 1.5 & 0.50 & 0.055 & 0.037 \\
\hline 2.5 & 0.50 & 0.021 & 0.013 \\
\hline
\end{tabular}




\section{Table 10.5.1-9 (Continued)}

Case 5: Free Top/Simple Support Bottom

\begin{tabular}{|c|c|c|c|}
\hline $\mathrm{H} / \mathrm{L}$ & Free-Free Sides & SS-SS Sides & Fixed-Fixed Sides \\
\hline 0.2 & $*$ & 0.78 & 0.78 \\
\hline 0.4 & $*$ & 0.34 & 0.34 \\
\hline 0.667 & $*$ & 0.187 & 0.187 \\
\hline 1.0 & $*$ & 0.112 & 0.085 \\
\hline 1.5 & $*$ & 0.057 & 0.037 \\
\hline 2.5 & $*$ & 0.021 & 0.013 \\
\hline
\end{tabular}

* Unstable Condition 
Table 10.5.1-10 Stress Factors, $S$ (psi) (Table 8 of Reference 117)

\begin{tabular}{|c|c|c|c|c|c|c|c|c|c|c|}
\hline $\begin{array}{c}\text { WALL } \\
\text { HEIGHT } \\
\text { H }\end{array}$ & \multicolumn{9}{|c|}{ HOLLOW MASONRY THICKNESS } & \multicolumn{3}{l|}{ SOLID MASONRY THICKNESS } \\
\hline & $4^{\prime \prime}$ & $6^{\prime \prime}$ & $8^{\prime \prime}$ & $10^{\prime \prime}$ & $12^{\prime \prime}$ & $4^{\prime \prime}$ & $6^{\prime \prime}$ & $8^{\prime \prime}$ & $10^{\prime \prime}$ & $12^{\prime \prime}$ \\
\hline $6^{\prime}$ & 460 & 310 & 230 & 195 & 170 & 745 & 480 & 355 & 280 & 230 \\
\hline $8^{\prime}$ & 815 & 555 & 410 & 345 & 305 & 1,325 & 850 & 630 & 500 & 415 \\
\hline $10^{\prime}$ & 1,275 & 865 & 640 & 545 & 475 & 2,075 & 1,330 & 985 & 780 & 645 \\
\hline $12^{\prime}$ & 1,835 & 1,245 & 925 & 780 & 680 & 2,985 & 1,915 & 1,415 & 1,120 & 930 \\
\hline $14^{\prime}$ & 2,500 & 1,695 & 1,255 & 1,065 & 930 & 4,065 & 2,610 & 1,930 & 1,525 & 1,265 \\
\hline $16^{\prime}$ & 3,260 & 2,215 & 1,640 & 1,390 & 1,215 & 5,310 & 3,405 & 2,520 & 1,995 & 1,650 \\
\hline $18^{\prime}$ & 4,130 & 2,805 & 2,075 & 1,760 & 1,535 & 6,720 & 4,310 & 3,185 & 2,525 & 2,090 \\
\hline $20^{\prime}$ & 5,100 & 3,460 & 2,565 & 2,170 & 1,895 & 8,295 & 5,320 & 3,935 & 3,115 & 2,580 \\
\hline $24^{\prime}$ & 7,340 & 4,985 & 3,690 & 3,125 & 2,730 & 11,945 & 7,665 & 5,665 & 4,485 & 3,715 \\
\hline $30^{\prime}$ & 11,470 & 7,790 & 5,765 & 4,885 & 4,265 & 18,660 & 11,975 & 8,850 & 7,010 & 5,805 \\
\hline
\end{tabular}

$\mathrm{S}=\mathrm{H}^{2} *\left(\mathrm{wc} / \mathrm{I}^{\prime}\right)$

where $\mathrm{H}=$ Wall Height (in)

$I^{\prime}=$ Effective Plate Moment of Inertia (in $4 /$ in)

$c=$ Distance from Neutral Axis to Extreme Fiber (in)

$w=$ Distributed Load per Unit Surface Area (\#/in $\left.{ }^{2}\right)$ based on masonry weight density $=150 \# / \mathrm{ft}^{3}$ 


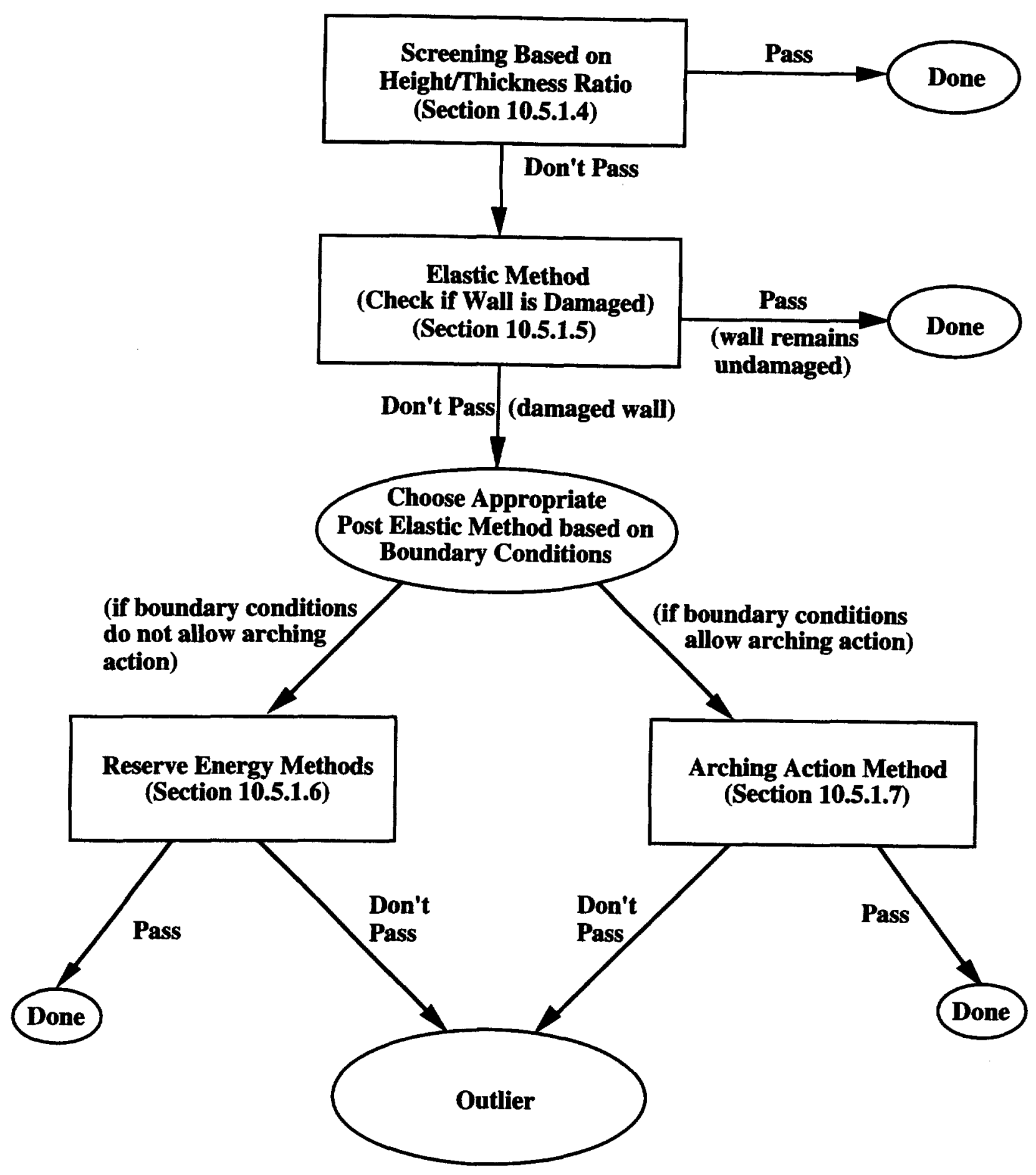

Figure 10.5.1-1 Methods for Evaluation of Out-of-Plane Bending of Non-Bearing Infill or Partition Unreinforced Masonry Walls in Section 10.5.1 


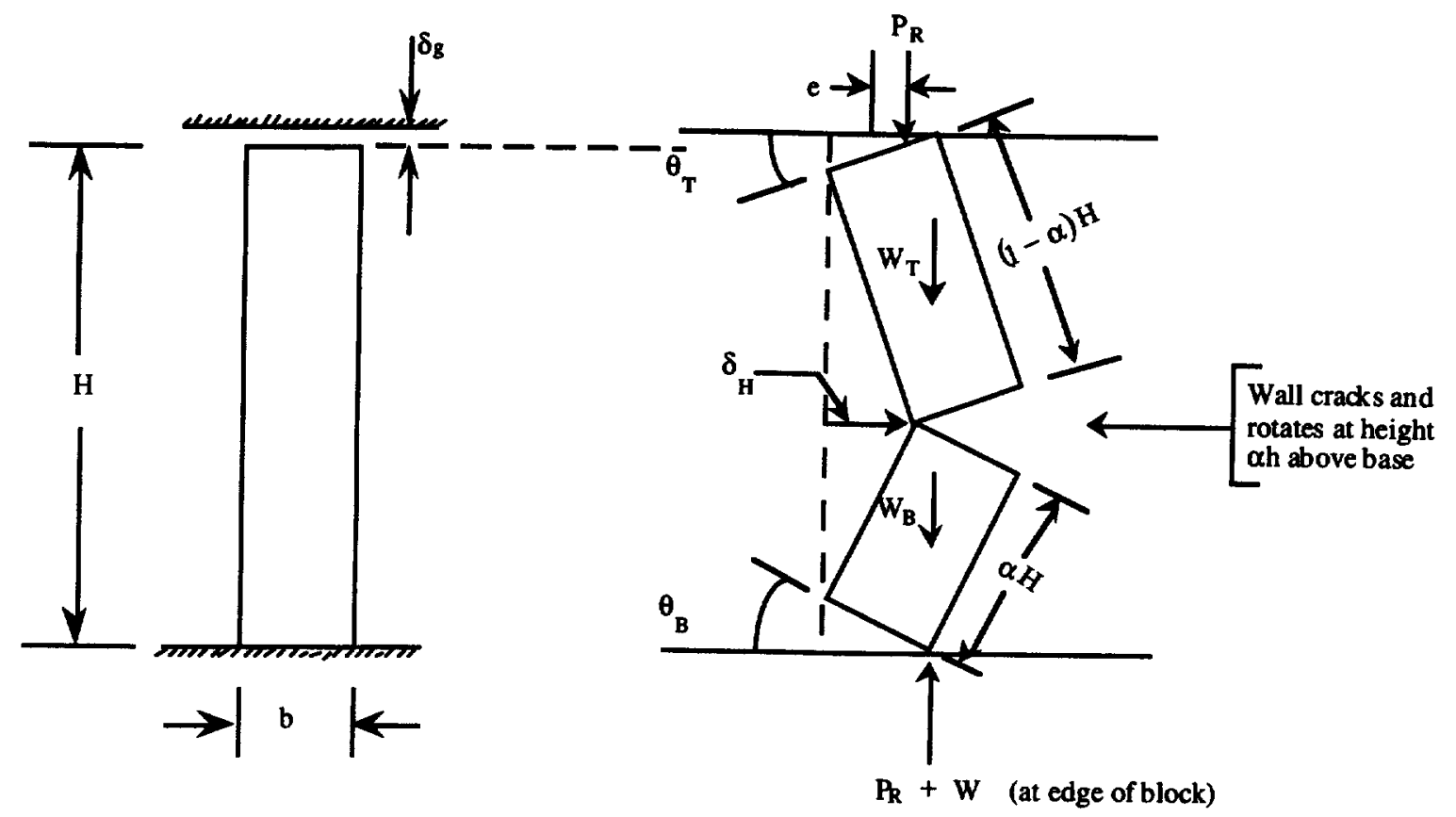

$$
\begin{aligned}
& P_{R}=\text { in-plane compressive force } \\
& \text { zero for Reserve Energy Method (non load bearing wall) } \\
& \text { increases with displacement for Arching Action Method } \\
& \mathrm{W}_{\mathrm{B}}=\mathrm{W} \alpha \\
& \mathrm{W}_{\mathrm{T}}=\mathrm{W}(1-\alpha) \\
& \mathrm{W}=\text { block wall weight } \\
& \alpha=\text { parameter which locates crack location } \\
& \text { e }=\text { load eccentricity from centerline of wall } \\
& \mathrm{H}=\text { wall height } \\
& \text { b }=\text { effective wall thickness }(=0.9 \text { actual wall thickness }) \\
& \delta_{\mathrm{H}}=\text { lateral displacement } \\
& \delta_{\mathrm{g}}=\text { gap between wall and upper support } \\
& \theta_{\mathrm{B}}=\text { angle of rotation of bottom block } \\
& \theta_{\mathrm{T}} \quad=\quad \text { angle of rotation of top block }
\end{aligned}
$$

Figure 10.5.1-2 Wall Properties for Reserve Energy and Arching Action 

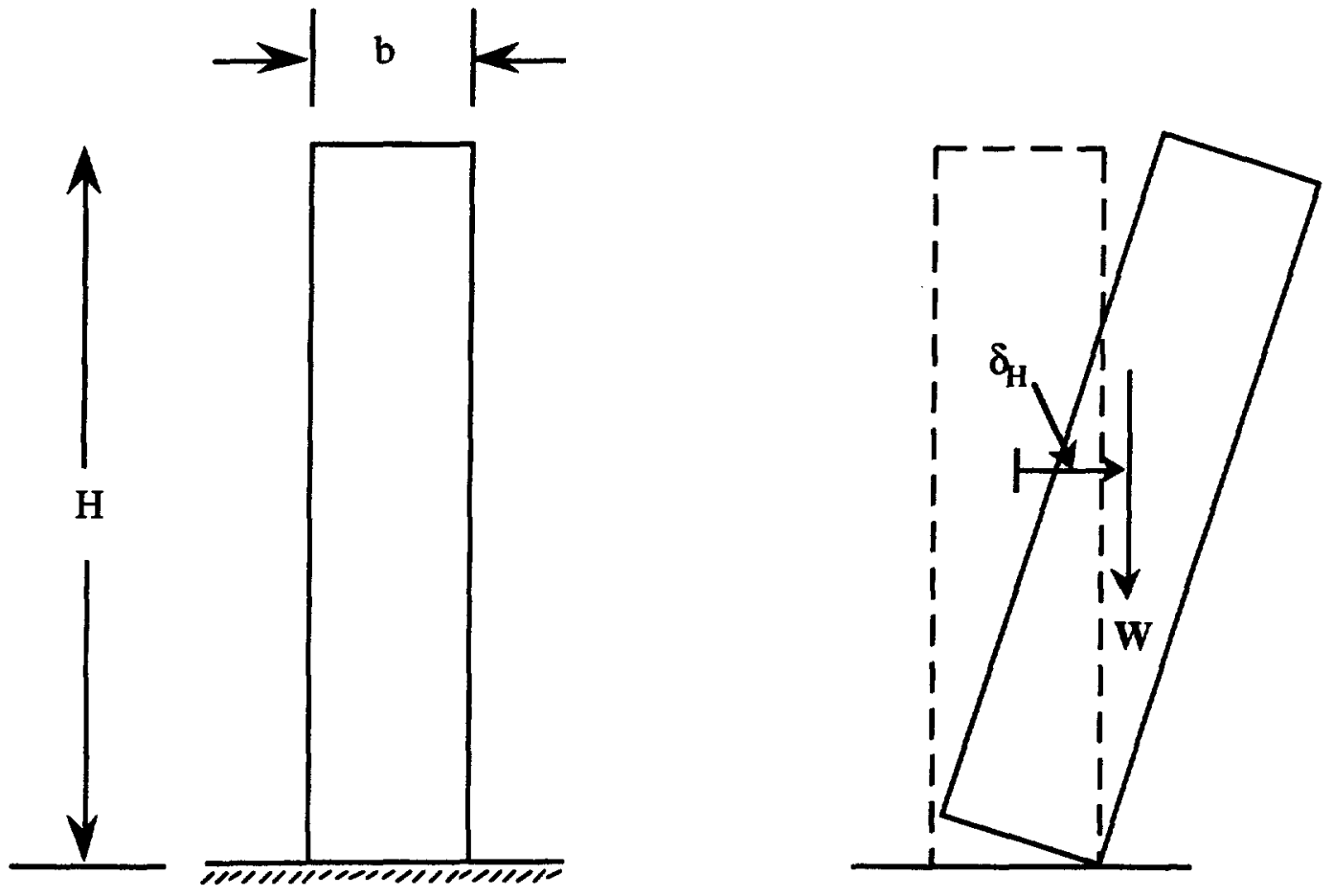

$$
\begin{aligned}
& \mathrm{W}=\text { Block wall weight } \\
& \mathrm{H}=\text { wall height } \\
& \mathrm{b}=\text { effective wall thickness }(=0.9 \text { actual wall thickness }) \\
& \delta_{\mathrm{H}}=\text { Lateral displacement }
\end{aligned}
$$

Figure 10.5.1-3 Properties for a Cantilever Wall for Reserve Energy Method (Large gap at top of wall, non load bearing, and no lateral restraint at top of wall) 


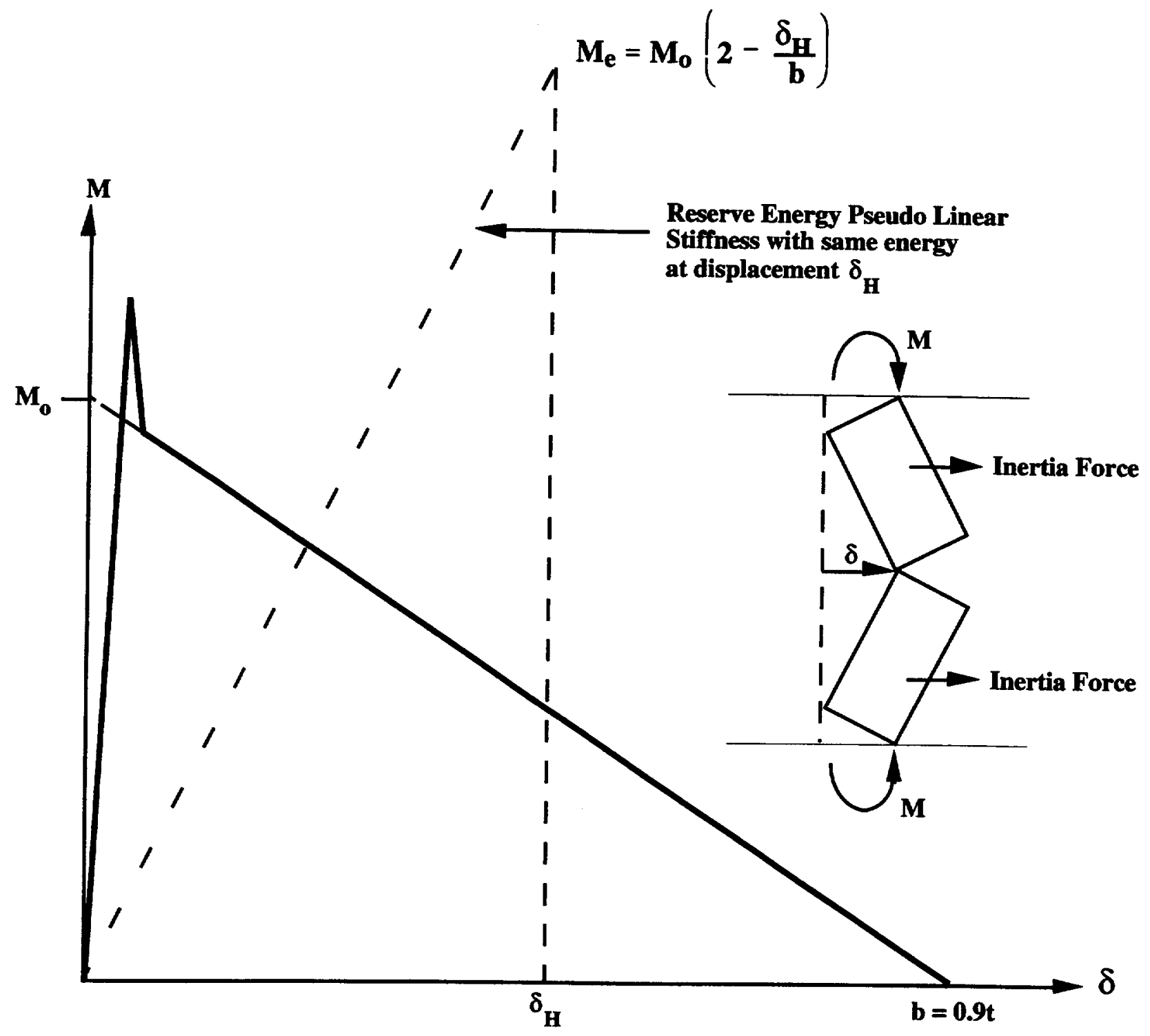

$$
\begin{array}{ll}
\mathrm{M} & =\text { restoring moment } \\
\mathbf{M}_{\mathrm{o}} & =\text { actual moment at zero displacement } \\
\mathrm{M}_{\mathrm{e}} & =\text { effective moment } \\
\mathrm{b} & =\text { effective wall thickness } \\
\mathrm{t} & =\text { actual wall thickness } \\
\delta_{1} \delta_{\mathrm{H}} & =\text { out-of-plane displacements }
\end{array}
$$

Figure 10.5.1-4 Restoring Force for Reserve Energy Method 


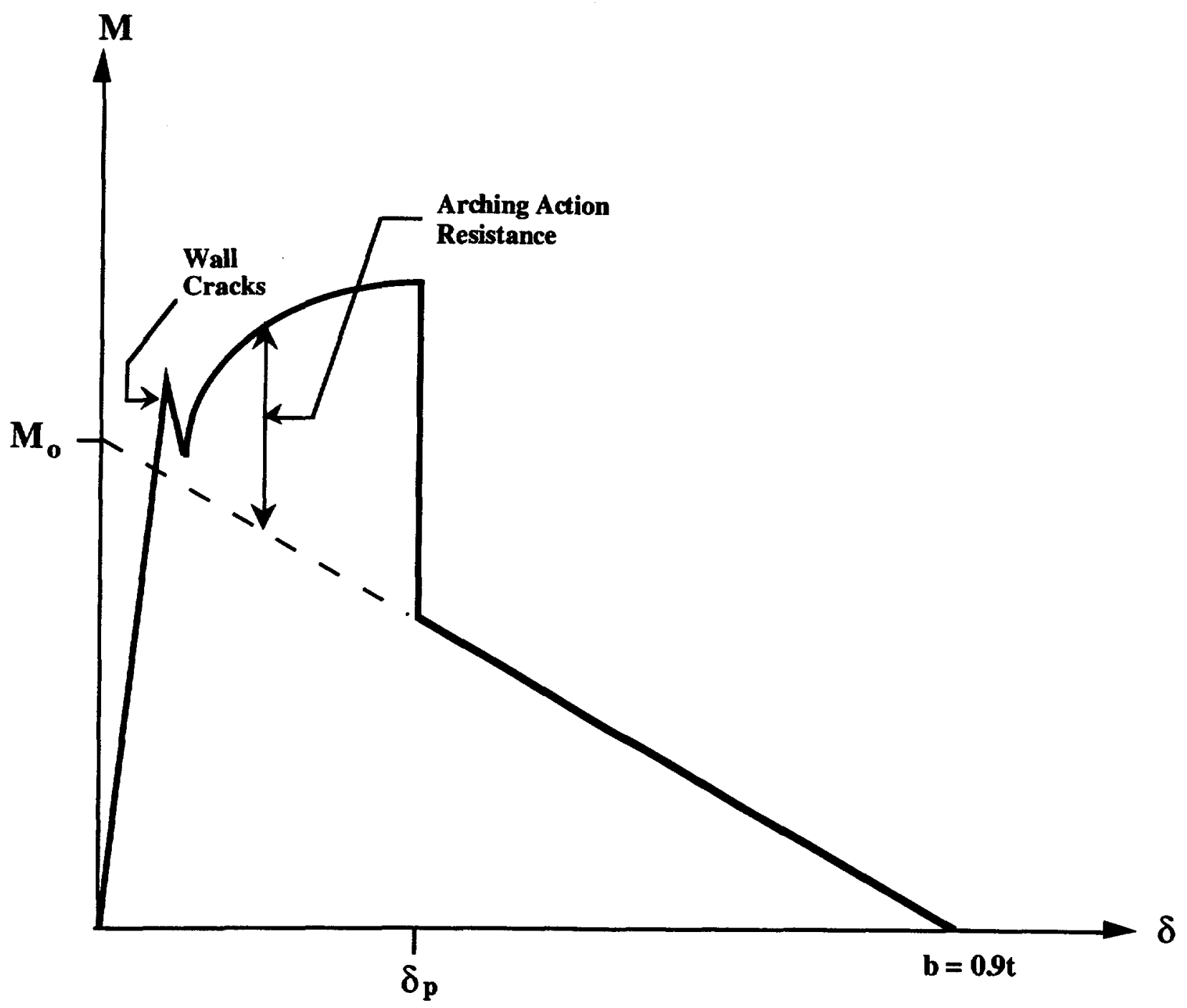

$$
\begin{aligned}
& \mathbf{M}=\text { restoring moment } \\
& \mathbf{M}_{\mathbf{o}}=\text { actual moment at zero displacement } \\
& \mathrm{b}=\text { effective wall thickness } \\
& \mathrm{t}=\text { actual wall thickness } \\
& \delta_{\mathrm{p}}=\text { out-of-plane displacement at which ultimate capacity is reached } \\
& \delta \quad=\text { out-of-plane displacement }
\end{aligned}
$$

Figure 10.5.1-5 Restoring Force for Arching Action Method 


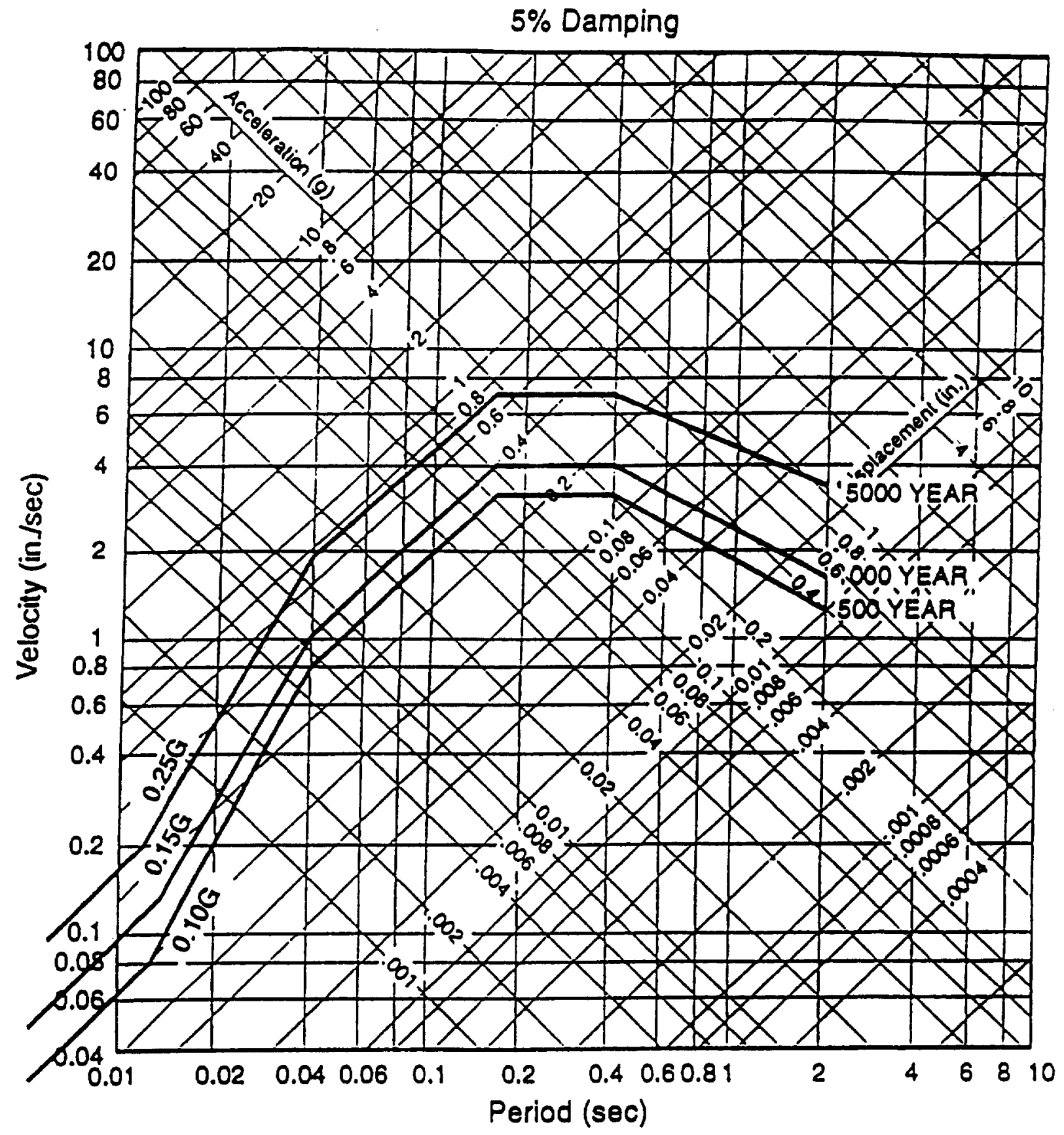

Figure 10.5.1-6A Portsmouth-Uniform Hazard Response Spectra for Horizontal Ground Motion 


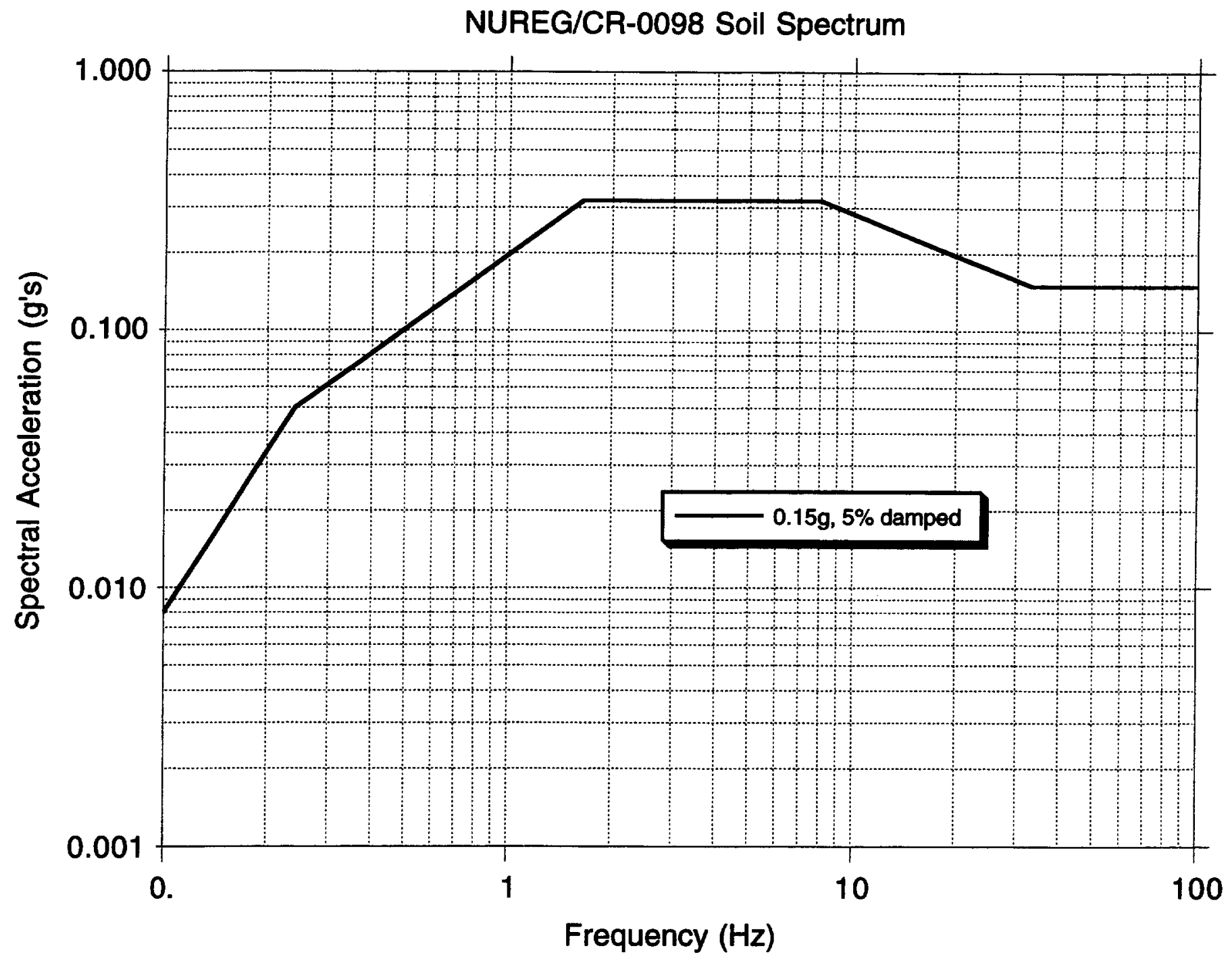

Figure 10.5.1-6B NUREG/CR-0098 Median Soil Spectrum (Ref. 72) 


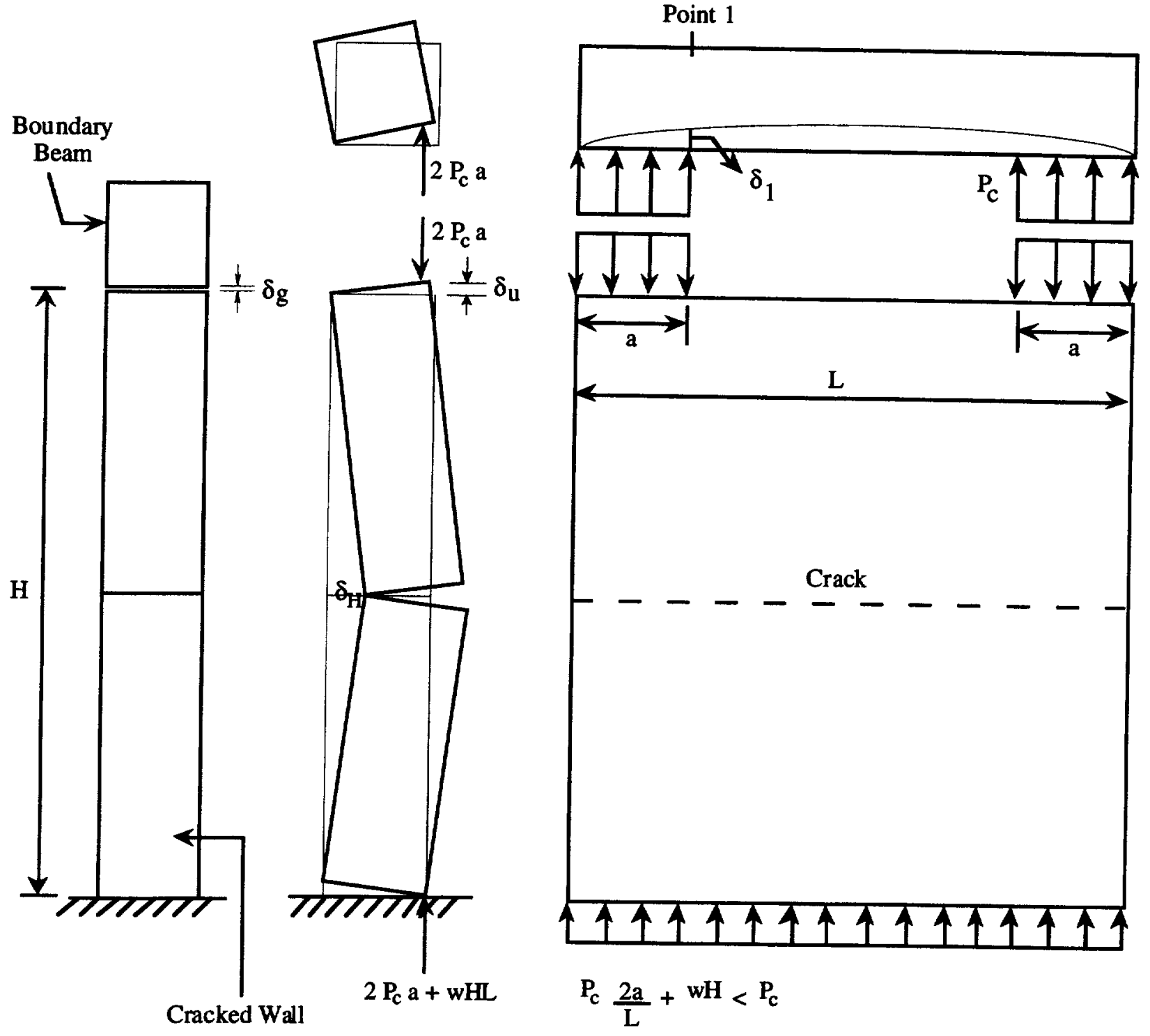

Figure 10.5.1-7 Arching Kinematics and Assumed Load Distribution along Length of Top Beam 


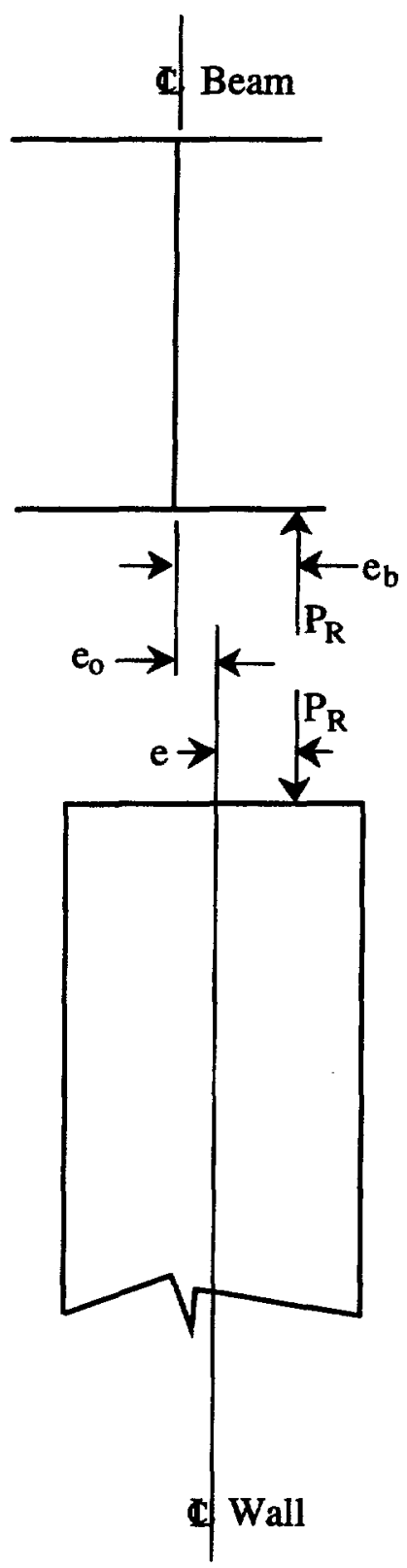
$e_{0}=$ offset between beam centerline and wall centerline

e $=$ eccentricity to load $P_{R}$ from wall centerline

$e_{b}=$ eccentricity to load $P_{R}$ from beam centerline

If beam twists more freely than top of wall rotates (typical for steel beam)

$$
\text { take } \quad \begin{aligned}
\mathrm{e}_{\mathrm{b}} & =0 \\
\mathrm{e} & =-\mathrm{e}_{\mathrm{o}}
\end{aligned}
$$

If twisting stiffness of beam is sufficiently large, then the beam twists less than the top of the wall rotates (typical for concrete beam)

$$
\begin{array}{ll}
\text { take } & \mathrm{e} \approx 0.45 \mathrm{~b}_{\mathrm{f}}-\mathrm{e}_{\mathrm{o}} \leq 0.45 \mathrm{t} \\
& \mathrm{e}_{\mathrm{b}}=\mathrm{e}+\mathrm{e}_{\mathrm{o}} \\
& \mathrm{b}_{\mathrm{f}}=\text { flange width of beam }
\end{array}
$$

Figure 10.5.1-8 Geometry of Beam, Wall, and Confining Force 


\subsubsection{RAISED FLOORS}

This section describes general guidelines that can be used for evaluating and upgrading the seismic adequacy of raised floors which are included in the Seismic Equipment List (SEL). The guidelines contained in this section are based on Section 4.4 of "Practical Equipment Seismic Upgrade and Strengthening Guidelines" (Ref. 60), Chapter 6 of "Data Processing Facilities: Guidelines for Earthquake Hazard Mitigation" (Ref. 121), and Chapter 9c of the "Seismic Safety Manual" (Ref. 32). In Chapter 6 of Reference 121, further detailed information on the seismic performance of raised floors and techniques for upgrading their seismic capacity is contained in the following sections: Descriptions of some of the more common floor systems and their strengths and weaknesses under earthquake loading; Specific guidelines for the seismic design, analysis, testing, and inspection of new raised floor systems; and Guidelines for analysis, retrofit design, and testing of existing raised access floors. Guidelines in this section of the DOE Seismic Evaluation Procedure cover those features of raised floors which experience has shown can be vulnerable to seismic loadings.

Because of extensive cabling requirements, components in computer facilities, data processing facilities, and control rooms are often supported on a raised floor with removable panels that may or may not be supported by stringers. A typical raise floor system is shown in Figure 10.5.2-1. A raised floor system forms the basic foundation or support for computer and data processing equipment, creates a space for a HVAC air plenum, and provides a protective shield for subfloor utilities vital to the operation of the equipment. The equipment supported on raised floors often costs hundreds of times more than the cost of the floor. Because of the cost of the equipment on a raised floor, earthquake-induced damage to the floor has a very high property loss potential. Furthermore, reconstruction of the collapsed floor and reinstallation of subfloor power, cooling, and signal cables could take a considerable amount of time. Potential damage evidenced in raised floor systems include buckling of support pedestals, buckling of floor panels, misalignment of floor penetrations, shifting of the entire floor system, and tipping of equipment supported by the floor.

For raised floor systems, the following seismic parameters should be evaluated:

- Seismic Demand Spectrum (SDS) at location of floor anchorage (see Section 5.2)

- dynamic stability or ability to withstand tipping and buckling capacity of pedestals

- type of anchorage system (leveling pads, skids, adhesives, clips, bolts, none)

- load path to load-bearing floor or foundation

- geometry and size (aspect ratio, height, width, length)

- relative strength and stiffness (stiff, flexible, strong, medium, weak)

- spacing of pedestals

- penetrations in the raised floor system

- operational considerations (weight being supported by floor, distribution of weight)

Large computer or control room raised floors may be susceptible to earthquake-induced damage due to tipping of the support pedestals. Figures 10.5.2-2 and 10.5.2-3 show examples of support pedestals that are typically slender, relatively long, and unanchored to the load-bearing floor or 
foundation. In addition, many raised floor systems lack lateral bracing between the pedestals (see Figure 10.5.2-4) which would provide horizontal stiffness.

To resist potential earthquake-induced damage, raised floor systems should be properly anchored by drilling holes in the base plates of supporting pedestals and installing anchor bolts. The anchor bolts can be evaluated using the procedures in Chapter 6 . Many raised floor systems use an adhesive to attach the pedestals to the load-bearing floor or foundation. Test results have indicated that this adhesive is not adequate for withstanding significant lateral motion.

Earthquake and test experience has indicated that the unbraced pedestals and the weld to the pedestal base plate are often too weak to transfer the required lateral loads. Bracing schemes as shown in Figures 10.5.2-5 should be provided to create moment-frame action of the raised floor systems, to increase the lateral stiffness of the raised floor system, and to avoid concerns about the weld to the pedestal base plate. Potential flexibility of the threaded screw connections and weak welds, such as tack welds, to the pedestal should be evaluated.

In addition to strengthening the raised floor support system, the penetrations in the floor systems should be carefully evaluated. In many cases, the equipment on the raised floor is not anchored so there needs to be adequate accommodations for movement of the equipment during an earthquake. If there are extensive floor penetrations, the equipment on the raised floor may roll into, tip on, or catch on the penetrations. This action may cause a large concentrated lateral overload on the floor system as well as cause local floor breakup due to panel buckling. The floor penetrations should be modified to prevent equipment entry or covered with special air vents that permit the equipment to traverse the floor without penetration. Special precautions may be required to anchor the equipment through the raised floor or tether it to prevent it from catching in the penetrations. For light equipment on a braced floor, connecting to the bracing at the stringers may be adequate restraint. The use of tethers is discussed below.

Strengthening of the raised floor will not necessarily provide a system capable of resisting the lateral loads associated with heavy computer or control equipment. Separate anchorage for these items of equipment should be provided. The most desirable strategy for upgrading the seismic capacity of computer equipment typically involves either floor anchorage, vertical bracing schemes, or the use of tethers. The anchorage of the equipment on the raised floor may be used for the following conditions:

- the equipment is relatively heavy

- analysis of the equipment indicates that it will tip

- the equipment is closely spaced and will impact

- the internal components have low vulnerability to vibratory motion

- the cabinet frame has sufficient strength and stiffness to support the equipment without supplemental bracing.

Because unbraced raised floors cannot carry significant lateral loads, independent anchorage and support for equipment meeting one or more of the conditions listed above should be to a loadbearing floor or foundation. With the independent support, the raised floor should not be part of the load path for the anchorage of large computer and control equipment. The base of the equipment should be evaluated to determine if it has adequate capacity to support the anchorage loads. 
An approach for independently securing equipment on top of a raised floor is to use under-floor cable tethers which allow for limited movement of the equipment. The cable tethers secure the equipment by providing a support path between a floor or load-bearing wall and the base of the equipment. As discussed in Reference 32, the following factors should be considered when using a tethering system:

- openings in the raised floor should have raised edges or curbs to prevent the base of the equipment from sliding into the opening

- the equipment should be stable against overturning when an appropriate coefficient of friction (judgment is required) is assumed between the raised floor and the base of the equipment

- there should be sufficient space between equipment to prevent seismic interactions

- elastomeric pads or bumpers may be used between closely spaced equipment

- the location of tether anchors and cable attachments to the equipment should consider the distribution of mass and stiffness within the equipment

- the design of the tether anchorage should consider the interaction with the raised floor if the cable becomes taut

- attached lines to the equipment should have sufficient slack to accommodate the constrained movement of the equipment

A second approach for independently anchoring computer equipment to a load-bearing floor or foundation is to use a separate support system, such as a diagonally - braced frame, for the equipment. This support system must be adequately anchored, have adequate lateral bracing, and have an appropriate load path from the equipment to the support system. If the equipment anchorage to the separate support system passes through an unbraced raised floor, interactions between the floor and the equipment anchorage should be considered. 


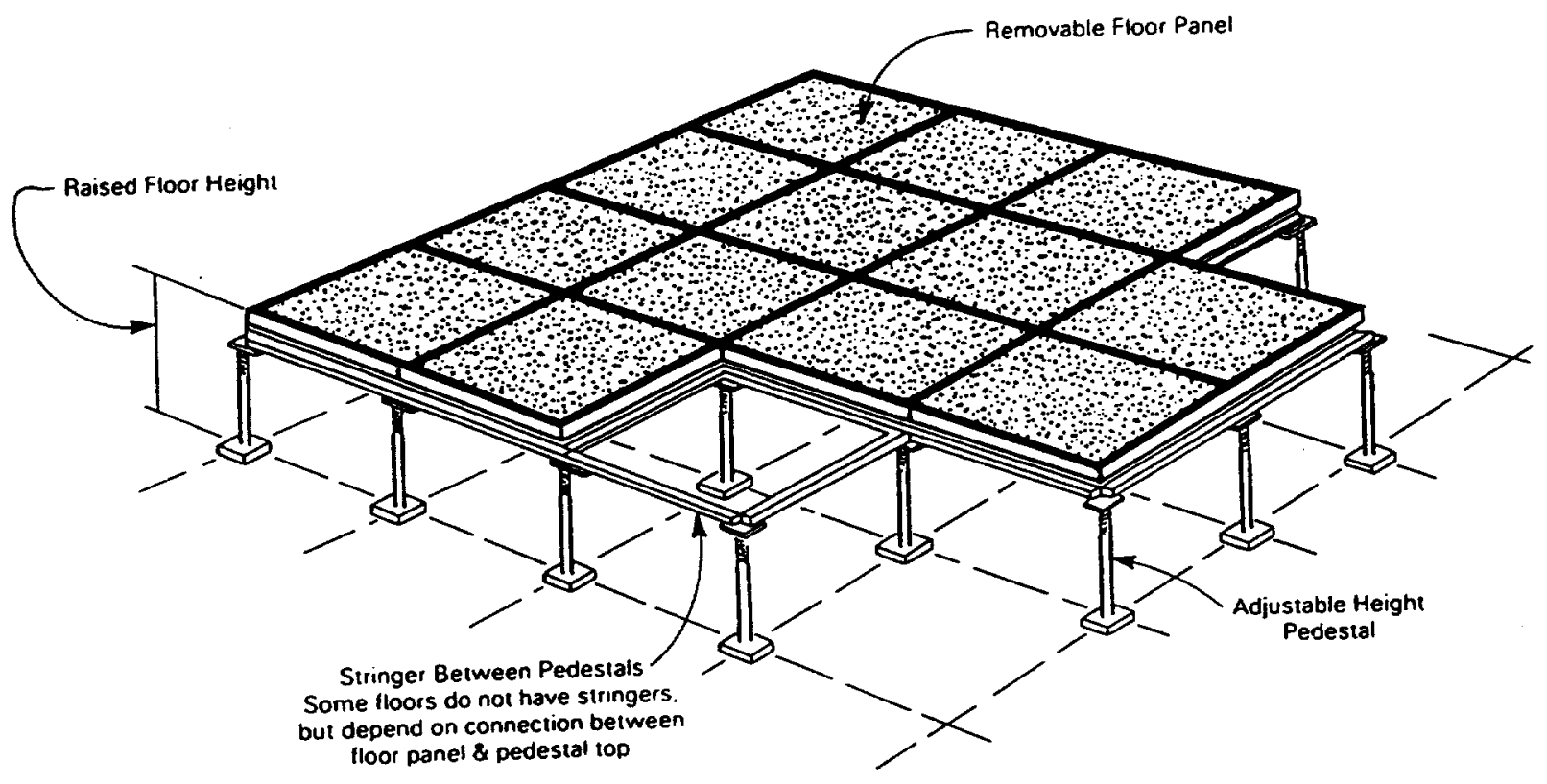

Figure 10.5.2-1 Raised Floor System (Figure 6.1 of Reference 121) 


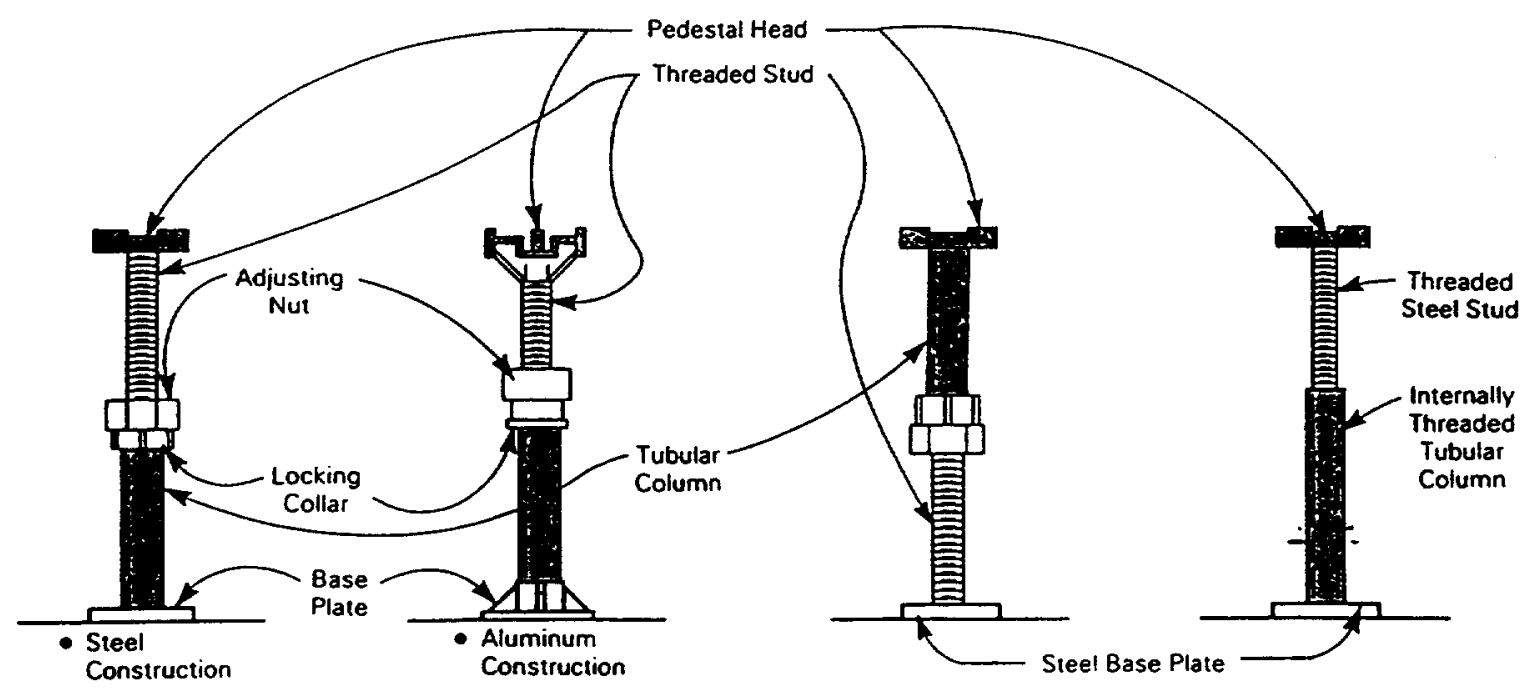

Figure 10.5.2-2 Pedestal Types (Figure 6.2 of Reference 121) 


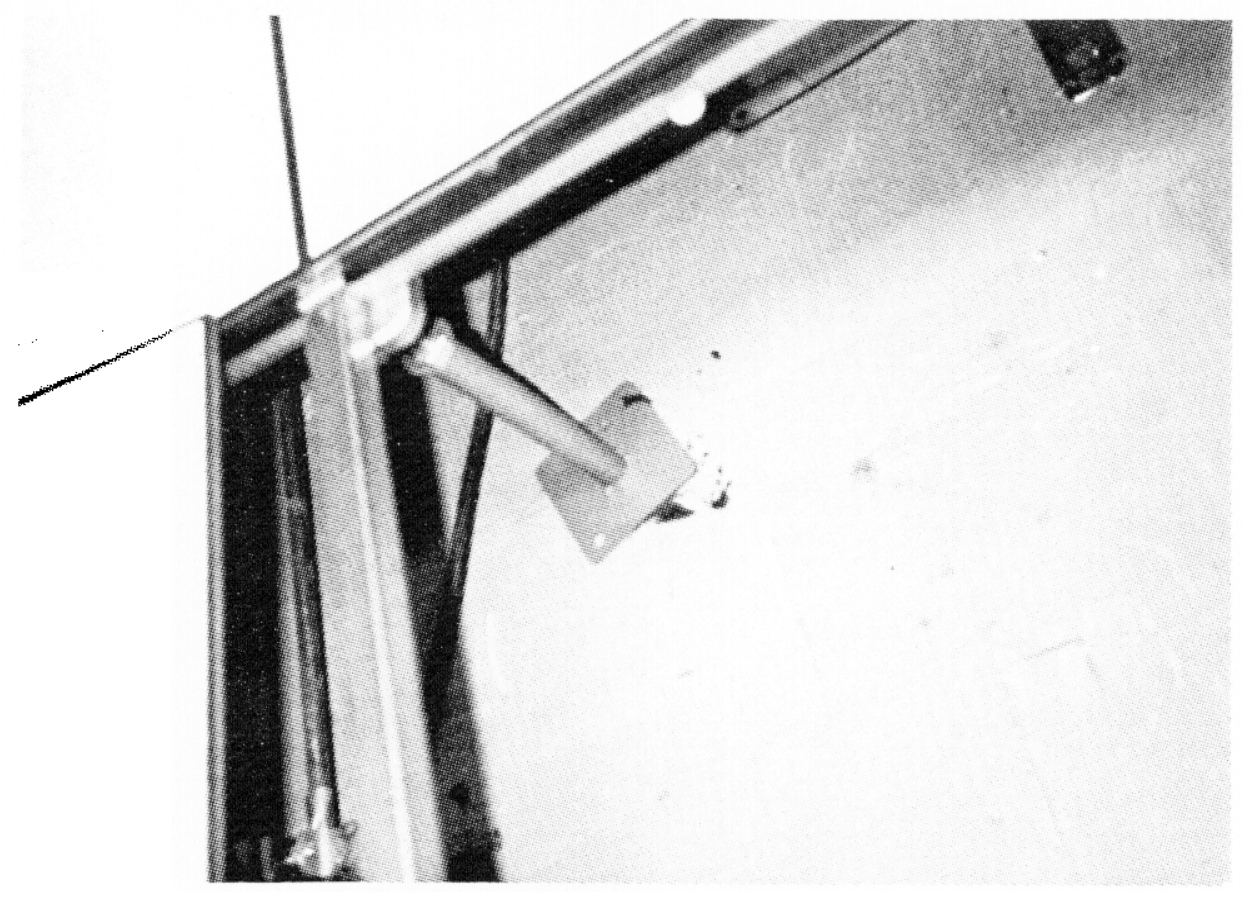

Figure 10.5.2-3 Raised Computer Floor Supported by Pedestal and Leveling Screw (Figure 4-30 of Reference 60)

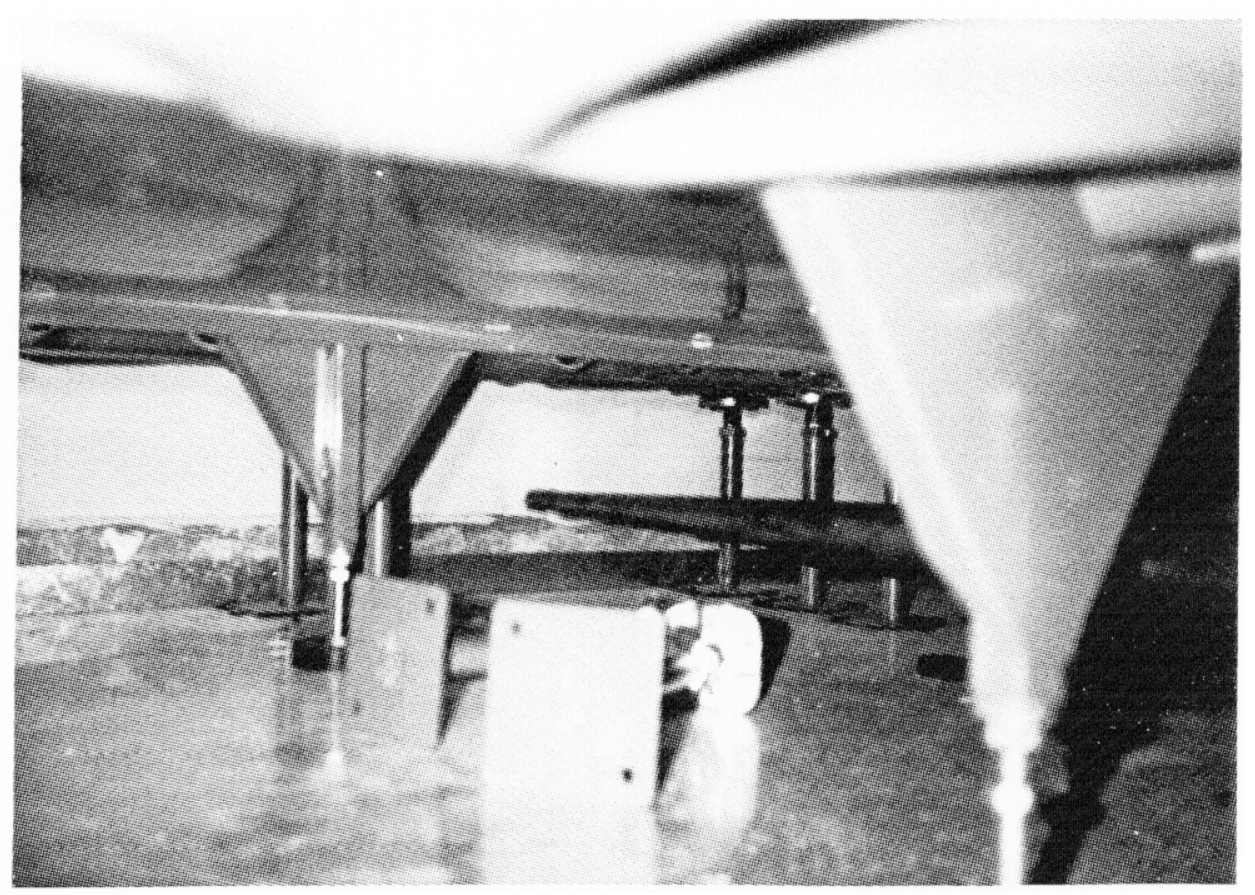

Figure 10.5.2-4 Raised Computer Floor Showing Lack of Lateral Bracing (Figure 4-31 of Reference 60) 


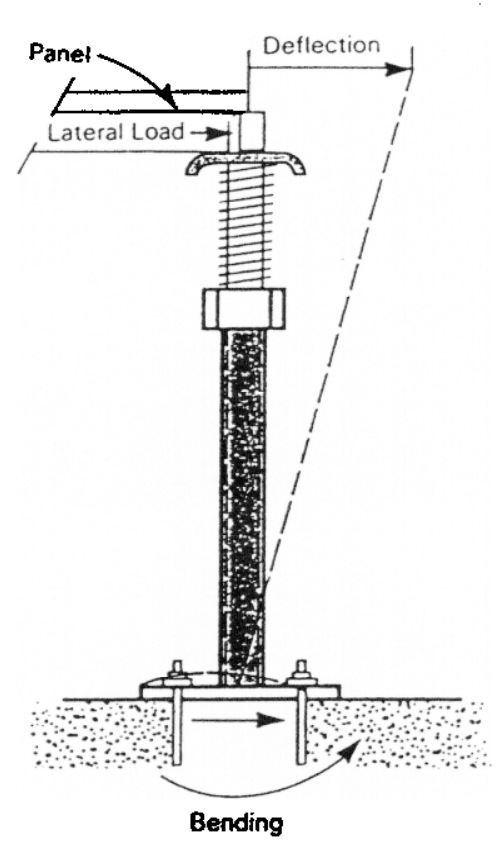

A. CANTILEVER PEDESTAL

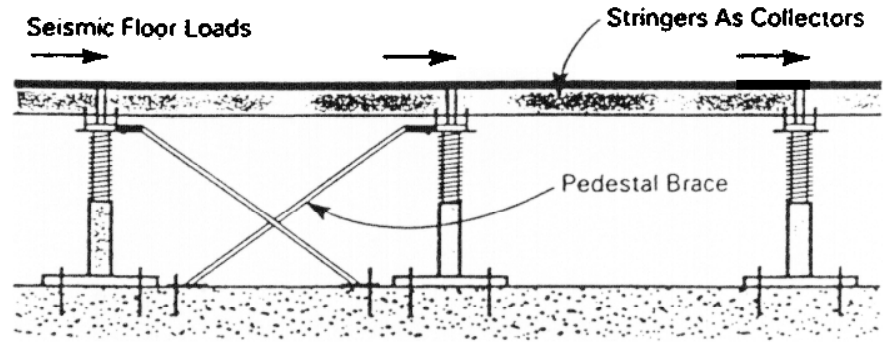

B. BRACED PEDESTAL SYSTEM
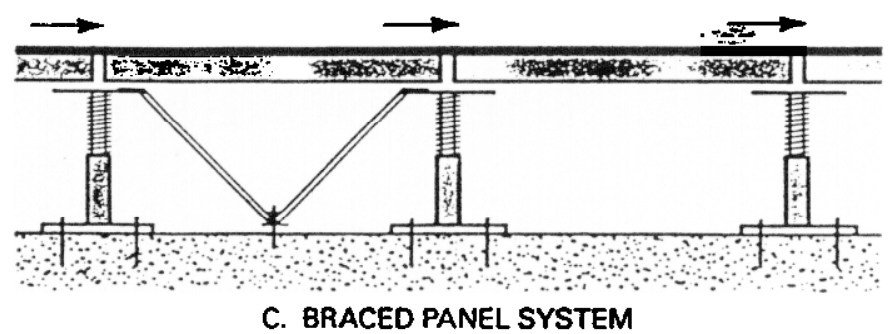

BRACED PANEL SYSTEM

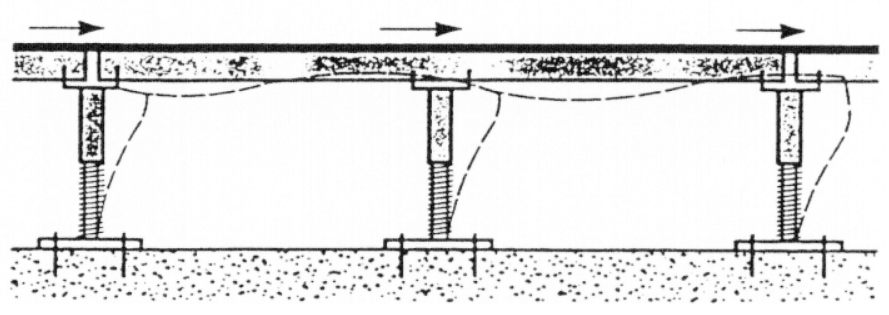

D. PEDESTAL - STRINGER FRAME

(NOT SHOWING BRACING)

Figure 10.5.2-5 Lateral Force Resisting Systems (Figure 6.5 of Reference 121) 


\subsubsection{STORAGE RACKS}

This section describes general guidelines that can be used for evaluating and upgrading the seismic adequacy of storage racks which are included in the Seismic Equipment List (SEL). The guidelines contained in this section are based on Sections 4.6.5 and 4.8 of "Practical Equipment Seismic Upgrade and Strengthening Guidelines" (Ref. 60). Guidelines in this section cover those features of storage racks which experience has shown can be vulnerable to seismic loadings.

Raw materials and finished products are typically stored on racks, in bins, or in stacks. Storage racks range from light metal shelving (see Figure 10.5.3-1) to heavy industrial grade shelving (see Figure 10.5.3-2). Inventory is extremely susceptible to earthquake-induced damage if racks or bins have no identifiable lateral load carrying system (see Figure 10.5.3-3). During an earthquake, items may slide off shelves or shelving may collapse which causes the contents to spill to the floor. If hazardous chemicals are involved, the resulting toxic chemical spill can be extremely dangerous and expensive to clean up.

The seismic evaluation of storage racks should emphasize the following considerations:

- anchorage

- structural capacity

- lateral bracing

- load path

- connection details

- restraints for contents

The structural capacity of a storage rack should be evaluated, especially its capacity for lateral loads. It may be difficult to determine the capacity of the rack without performing some calculations to determine member strengths and the modal, or stiffness, characteristics of the frame. Judgment may be required for determining the appropriate model for the connection details in a rack system. The connections in rack systems range from welded connections to slip joints. According to the provisions of Section 5.4, the capacity of the rack should be compared to the Seismic Demand Spectrum (SRS) at the anchorage location of the rack.

Storage racks should be evaluated to determine if they have adequate anchorage and if lateral bracing is present and of sufficient size to accommodate seismic loads. Tall racks should be anchored to walls with adequate capacity, the floor, and/or each other to prevent overturning. Most rack units have holes provided in their base plates and legs to accommodate anchor bolts. The screening evaluation for anchor bolts is provided in Chapter 6 . The capacity of the floor to resist the anchorage loads should be evaluated. Many rack systems are leveled with shims and the excessive use of shims may reduce the capacity of the anchorage for those systems. If the rack is anchored to an unreinforced masonry (URM) wall, the capacity of the wall should be evaluated according to the provisions of Section 10.5.1 including the lateral loads of the racks.

Since racks are relatively flexible, extensive use of lateral bracing is useful in increasing the seismic capacity of the rack and in limiting earthquake-induced damage. Bracing should be provided at the ends and along the back side as shown in Figure 10.5.3-4. In addition to bracing, the load path in the structure should be evaluated. The bracing should attach to the structural members of the rack and these members should have sufficient capacity to withstand the earthquake-induced lateral demand. Many racks are designed only for vertical loads, so the effects of lateral loads should be 
evaluated. Additional information on the seismic design of storage racks is available from the Rack Manufacturer's Institute. Finally, possible reductions in the structural capacity of a storage rack may result from improper assembly of the rack or damage from operational use, such as forklift damage. Manufacturer's data should be used to determine if the rack was properly assembled and is being used as designed.

Horizontal shelves that are structurally attached to the supporting frame members are preferred as part of the connection details in a storage rack. If the rack has removable shelves, these shelves cannot be considered part of the lateral force resisting system. Loose pieces of wood spanning between frames may fall during an earthquake and should be restrained. Heavier stock should be moved to lower shelves to prevent injury to personnel and to minimize damage. Whenever possible, restraint should be provided for equipment or stock that can slide off during earthquake motions. Methods of achieving restrain include installation of a steel angle (lip) at the front edge of each shelf or an elastic band or tensioned wire across the opening. If feasible, removable restraints can also be provided across the front of the rack to preclude materials from sliding off shelves as shown in Figure 10.5.3-4.

During an earthquake, the support structure for drums supported on a rack may collapse if it does not have adequate lateral bracing and seismic anchorage. Poorly restrained canisters and drums may fall and/or roll causing them to possibly spill their contents, to damage other equipment, and injure personnel. Methods of restraining them include providing positive anchorage to the floor or a wall with adequate capacity, storing them in well-braced and anchored racks, or storing them horizontally on the floor.

Storage bins are temporary storage containers stacked on top of each other. Bins are often stacked very high with no lateral supports. In a strong earthquake, the upper bins can fall causing damage to contents and pose a possible life safety hazard. Materials stored in bins or stacks should be assessed to determine their stability under earthquake loads. Often, the seismic requirements of these components is in direct conflict with operational requirements. However, if materials are extremely hazardous or are expensive to replace, mitigation measures should be considered to provide positive restraint. These measures might include the installation of permanent racks, minimizing stack heights to 2 or 3 layers in height, or restraining existing stacks through tiedowns. 


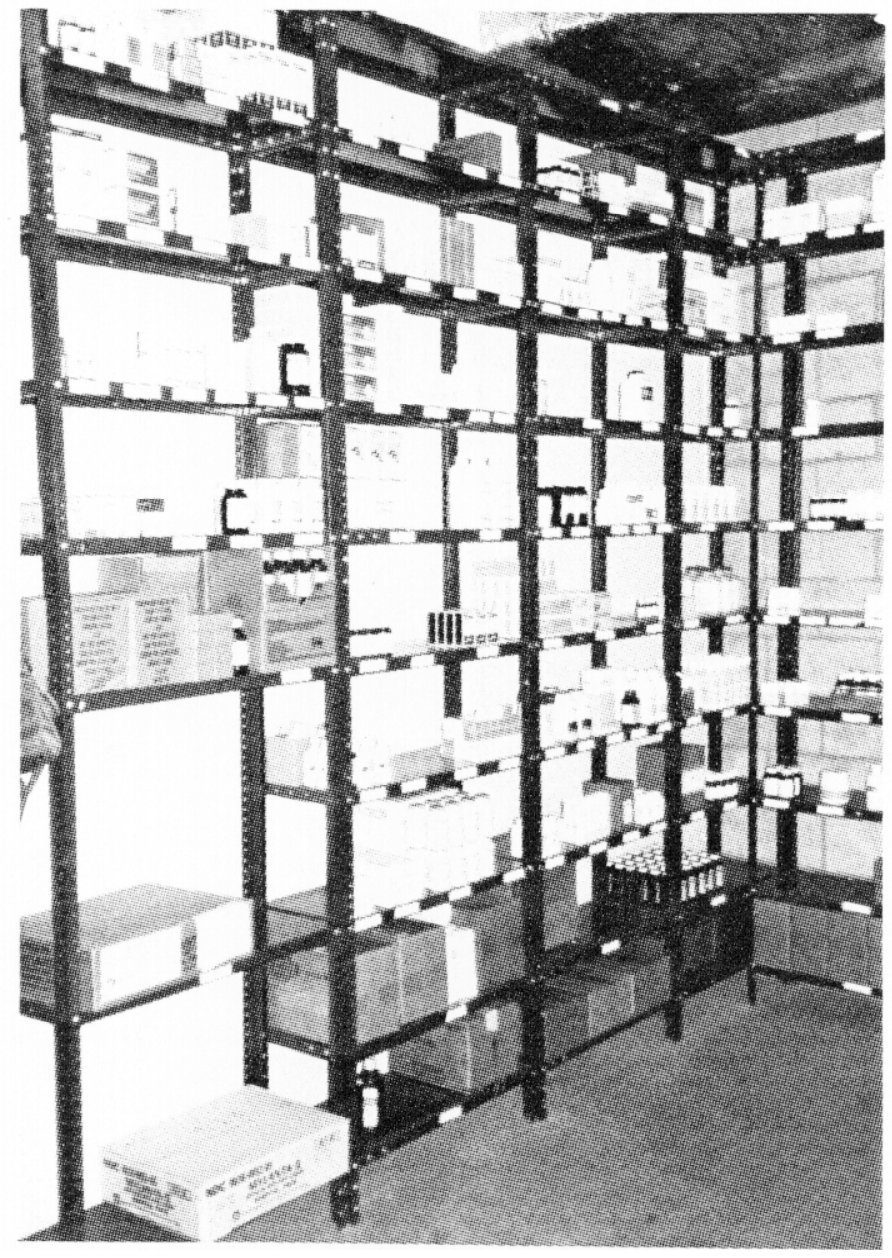




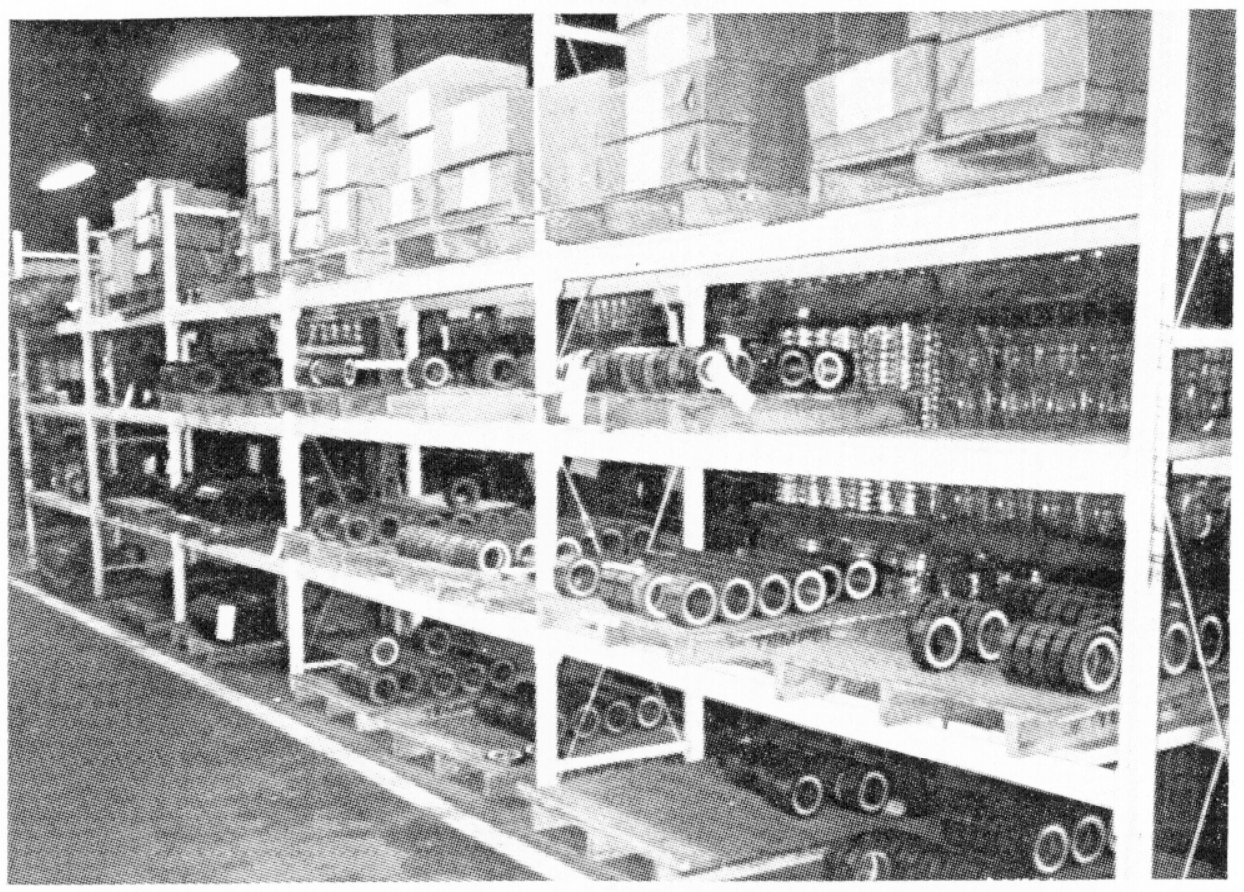

Figure 10.5.3-2 Unanchored Industrial Grade Shelving (Figure 4-63 of Reference 60)

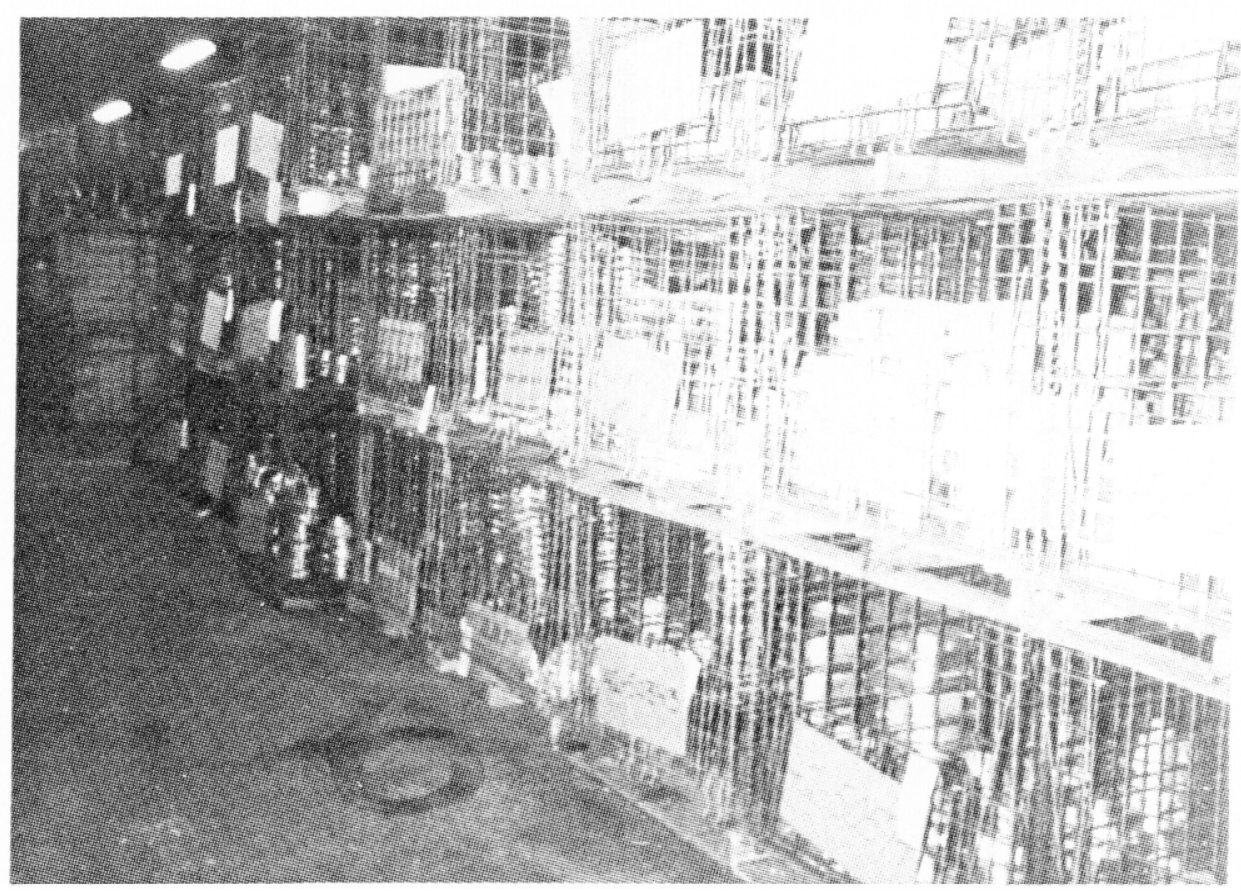

Figure 10.5.3-3 Unanchored Storage Bins (Figure 4-64 of Reference 60) 

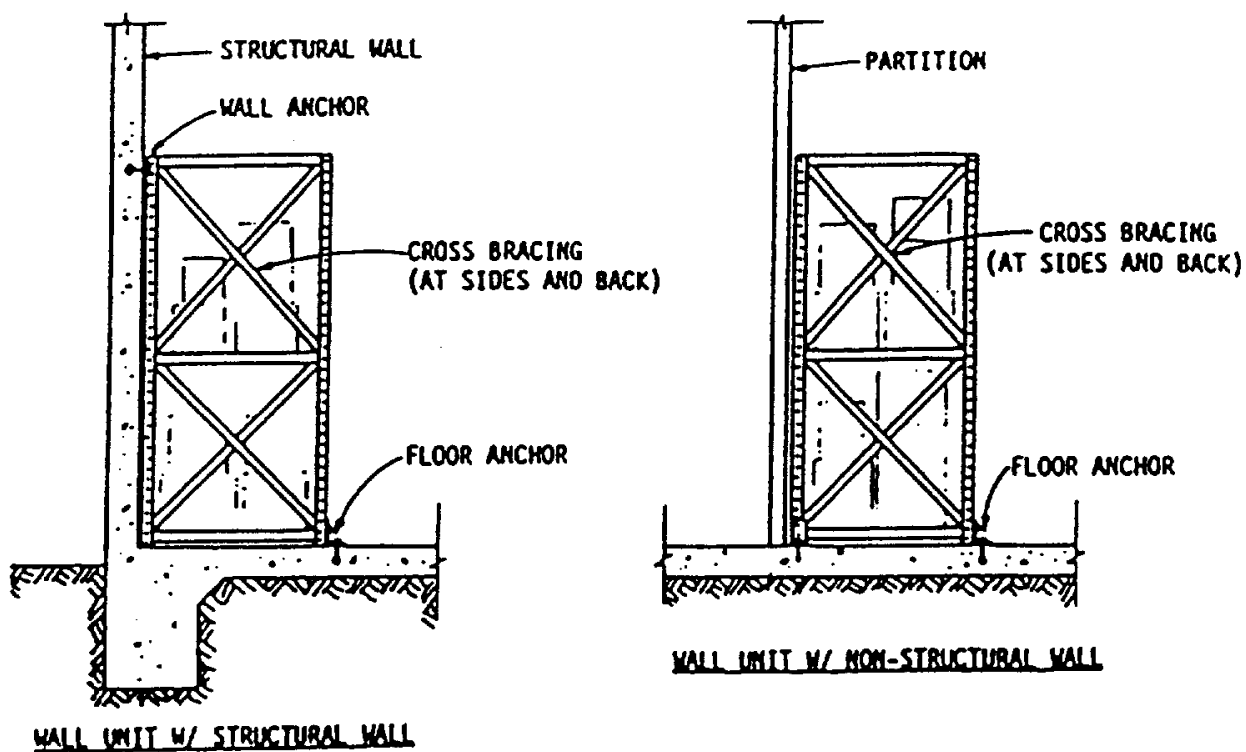

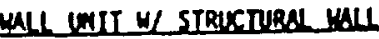
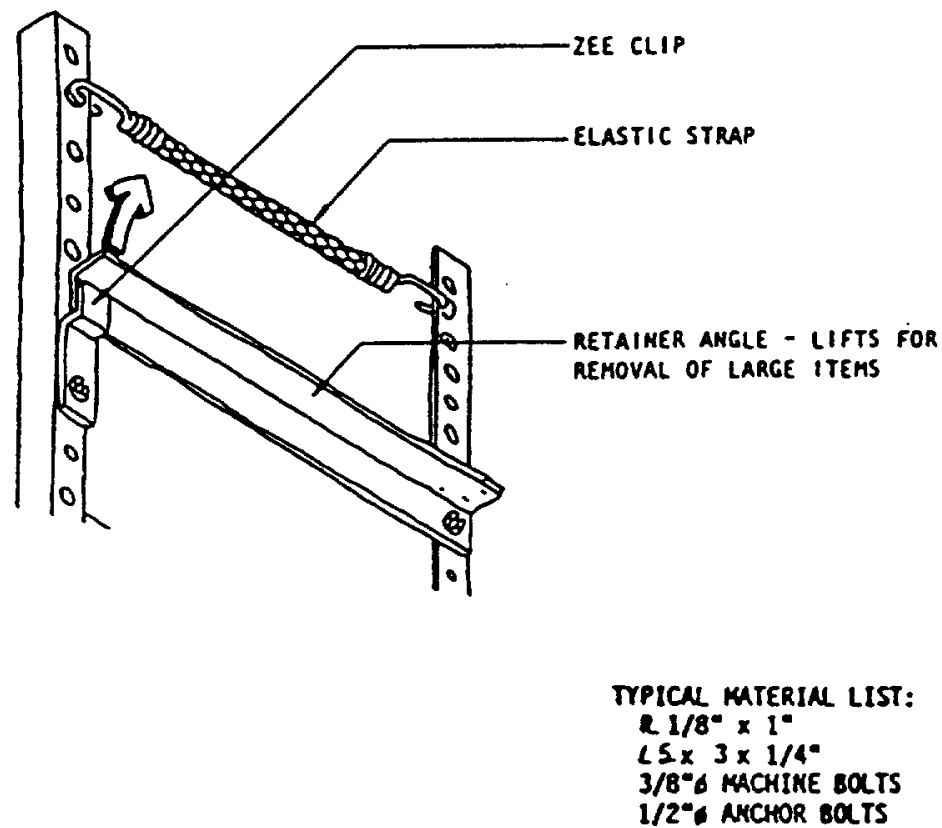

NKEES MAY BE BORTED OR VELDED TO SHEL VES

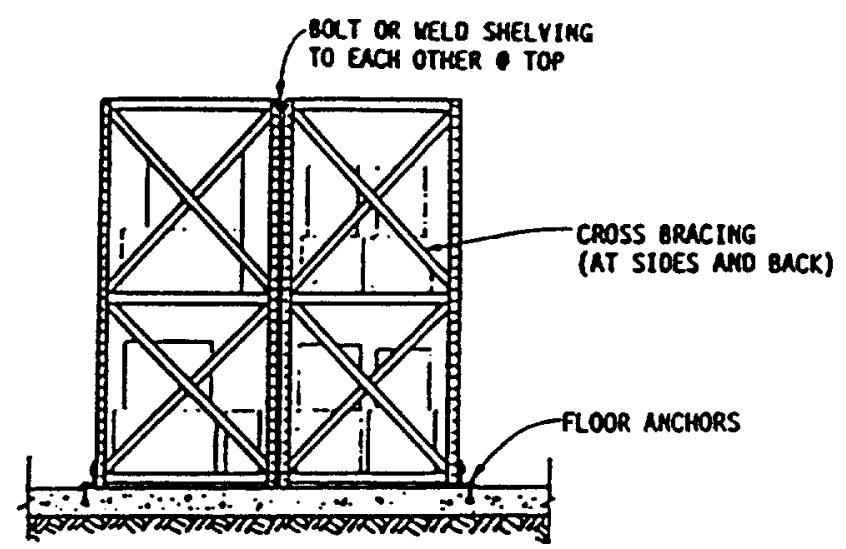

Figure 10.5.3-4 Approaches for Anchoring Storage Racks (Figure 4-67 of Reference 60) 


\section{Part V}

\section{Additional Seismic Evaluation Procedures Modified from the SQUG GIP}




\section{RELAY FUNCTIONALITY REVIEW}

\subsection{INTRODUCTION}

As part of the seismic evaluation of DOE facilities, it may be necessary to perform a relay seismic functionality review. The purpose of this review is to determine if the equipment listed on the Seismic Equipment List (SEL), as described in Chapter 4, could be adversely affected by relay malfunction in the event of a Design Basis Earthquake (DBE) and to evaluate the seismic adequacy of those relays for which malfunction is unacceptable. The term "relay malfunction" is used to designate relay chatter or inadvertent change-of-state of the electrical contacts in a relay, motor starter, or switch. The purpose of this section of the DOE Seismic Evaluation Procedure is to provide an overview of the relay evaluation procedure and describe the interfaces between other activities described in the DOE Seismic Evaluation Procedure and the relay evaluation.

Information on a detailed procedure for evaluating relays is contained in Section 6 of the SQUG GIP (Ref. 1) and in its supporting documents. The SCEs and relay evaluation personnel should not use the material in this chapter unless they have thoroughly reviewed and understood the information in Section 6 of the SQUG GIP and its supporting documents. The DOE Seismic Evaluation Procedure contains a condensed version of the detailed procedure in the SQUG GIP. In Sections 11.2 through 11.5 , the relay functionality review is intended to identify most of the essential relays that should be evaluated, to provide the procedure for evaluating those relays, and to be a cost effective approach for identifying "bad actors". Section 11.2 discusses three methods

for establishing the seismic capacity of relays and includes a list of low ruggedness relays. Section 11.3 provides two methods for determining the seismic demand on relays mounted in cabinets or other structures. The seismic capacity is compared to the seismic demand using the guidelines of Section 5.4. Section 11.4 provides information for conducting a walkdown as part of the relay evaluation. This walkdown can be incorporated as part of the Screening Evaluation and Walkdown described in Section 2.1.3. Finally, Section 11.5 discusses techniques for resolving relay outliers.

\subsection{SEISMIC CAPACITY OF RELAYS}

\subsubsection{Generic Seismic Test Data}

Seismic test data is available on a variety of types of relays. These data have been reduced to Generic Equipment Ruggedness Spectra (GERS) in Reference 44 which define seismic acceleration levels below which relays can be expected to function without chatter or other damage. The GERS are seismic response spectra within which a class or subclass of relays has functioned properly during shake-table tests. In some cases the GERS are based on "success" data (that is, seismic test spectra for which no relay malfunction occurred). In this case, the test spectra for one or more relays in a given class represent a lower bound of the seismic ruggedness of the class. In other cases, the GERS may be based on "fragility" data as the seismic response spectra in which failures or malfunctions occurred. In this case, the GERS represent an upper bound of the seismic ruggedness of the relay class. Where both success and fragility data are available for a given relay class, the GERS fall between the two spectra. Engineering judgment was used in developing the GERS level to smooth out sharp peaks and valleys in the test response spectra.

An example GERS for several auxiliary relay types is shown in Figure 11.2-1. A normalized GERS shape is illustrated at the top of this figure and GERS levels (i.e., the peak acceleration) for example relays are tabulated at the bottom of this figure. Complete sets of all available GERS for relays are given in Reference 44.

1 Based on Section 6.4.1 of SQUG GIP (Ref. 1) 


\subsubsection{Earthquake Experience Data ${ }^{2}$}

Data have been obtained on relay performance, specific failures, relay vulnerabilities, and other information from actual earthquake experience in industrial power plants and other facilities which have undergone significant earthquakes. This information has been used to identify unacceptable relay types such as those which are known to be susceptible to damage or chatter due to moderate shaking. Unacceptable relays and related contact devices that must be avoided are listed and considered in the screening procedure given in Reference 45. Based on earthquake experience data and on test data, solid state relays and mechanically-actuated switches are considered seismically rugged and need not be evaluated for relay chatter. Details and restrictions regarding the screening of both the low-ruggedness and high-ruggedness classes of control circuit devices are described in Reference 45.

Table 11.2-1 from Appendix E of Reference 45 provides a list of low ruggedness relays, or "bad actors". The relay evaluation procedure seismic demand determination and GERS cannot be applied to these relays because of their low seismic ruggedness or demonstrated sensitivity to high frequency vibration. Relays listed in Table 11.2-1 should be classified as outliers and case specific techniques or current qualification techniques must be utilized to demonstrate the adequacy of these relays.

\subsubsection{Relay-Specific Test Data ${ }^{3}$}

The GERS and earthquake experience data discussed above are expected to apply to many of installed relay types in essential circuits. Facility-specific and relay-specific seismic test data, where available, can also be used. This seismic test data is generally maintained by specific facilities and/or relay suppliers and has not been included in the relay GERS. It may be used on a relay-specific or facility-specific basis.

2 Based on Section 6.4.1 of SQUG GIP (Ref. 1)

3 Based on Section 6.4.1 of SQUG GIP (Ref. 1) 
Table 11.2-1 Low Ruggedness Relays (Appendix E of Reference 45)

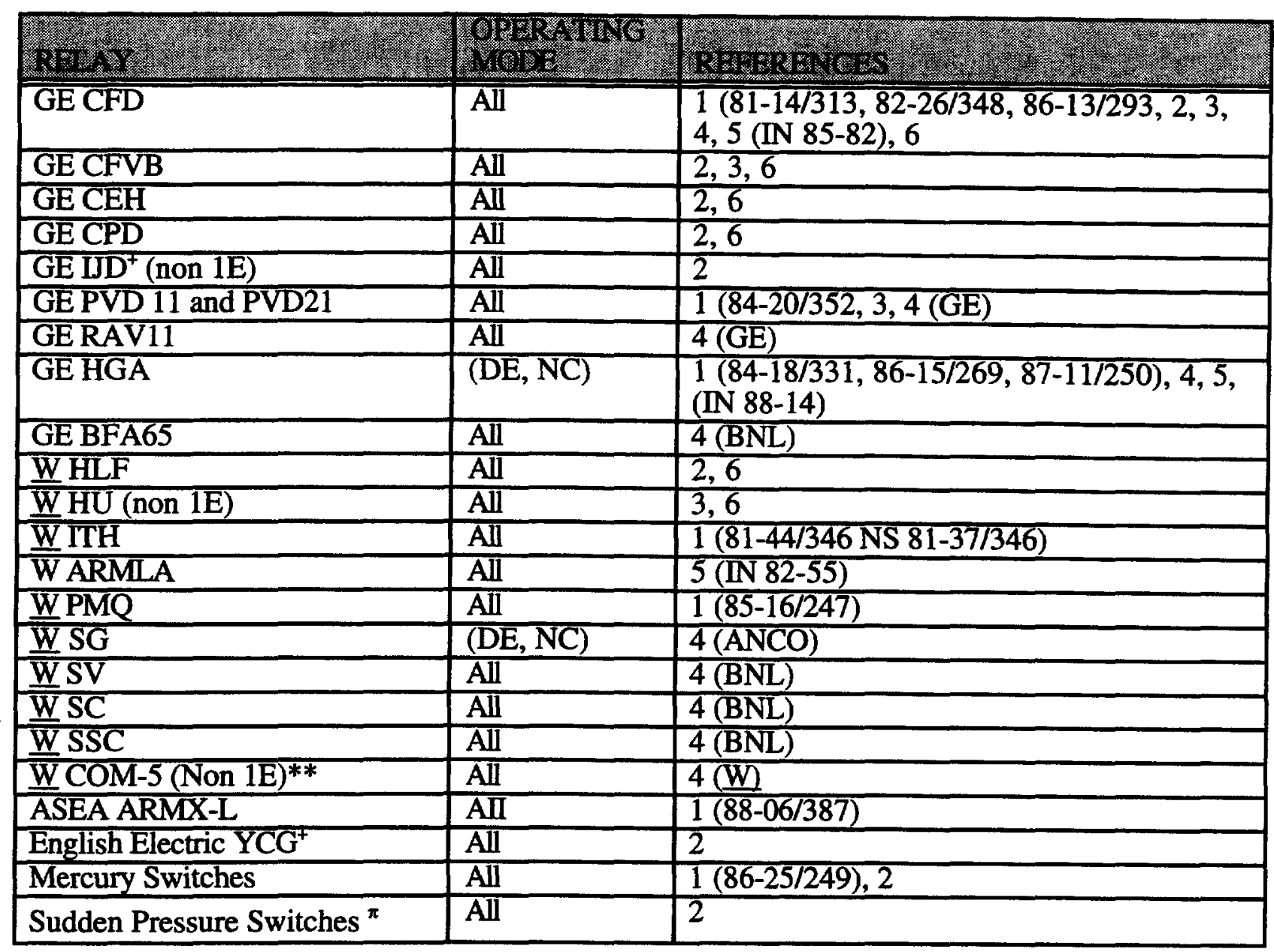

References:

1. LERS

2. Earthquake Experience Data

3. SAFEGUARDS Data

4. IEEE 501 Test Data

5. Notices, Bulletins, etc.

6. Induction cup or induction cylinder design

* $\mathrm{DE}=$ De-energized

$\mathrm{E}=$ Energized

$\mathrm{NC}=$ Normally Closed Contact

$\mathrm{NO}=$ Normally Open

All $=$ All Modes

+ Damage has occurred to this relay in an earthquake and it must be assumed that it will be inoperable following a DBE level earthquake.

$\pi \quad$ Transformer pressure surge sensing devices

** With SSC-T IITH unit 
Normalized Relay GERS

Auxiliary, Industrial Type 2 (300V)

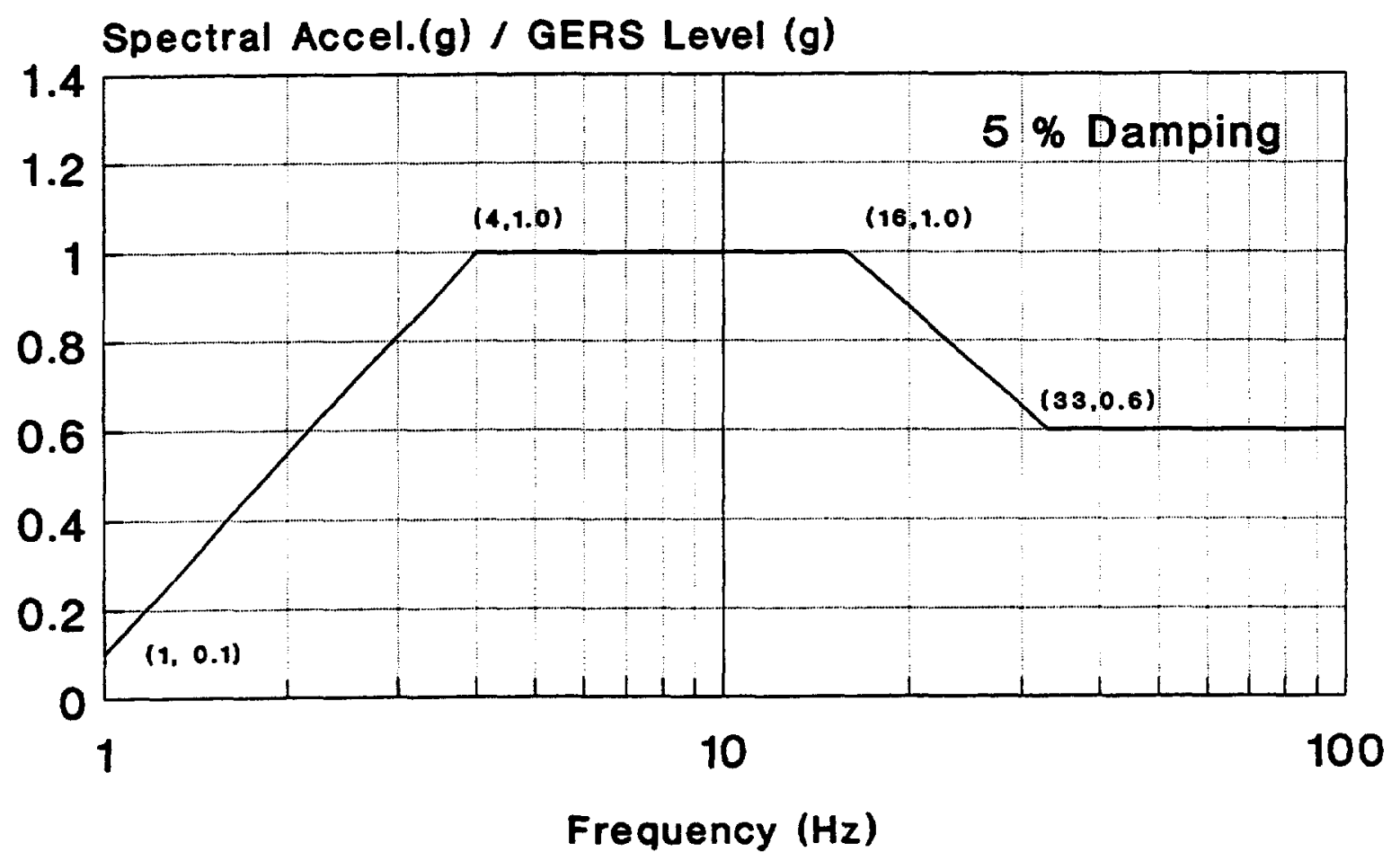

\begin{tabular}{|c|c|c|c|}
\hline \multirow[b]{2}{*}{$\begin{array}{l}\text { Type and Submodel } \\
\text { Identification }\end{array}$} & \multicolumn{3}{|c|}{ GERS Leve ${ }^{1}$} \\
\hline & $\frac{\mathrm{Nc}}{\mathrm{NO}^{2}}$ & $\frac{\mathrm{rate}^{2}}{N C^{2}}$ & $\frac{\text { Operate }}{\mathrm{NO} / \mathrm{NC}^{2}}$ \\
\hline Make \#1, Model A & 10 & 9 & 10 \\
\hline Make \#2, Model A & 10 & 9 & 10 \\
\hline Make \#2, Mode1 B & 10 & -3 & 10 \\
\hline Make 3 , Model A & 10 & 9 & 10 \\
\hline Make 14 , Model A & 10 & 5 & 10 \\
\hline Make $\$ 5$, Model A & 10 & 10 & 10 \\
\hline
\end{tabular}

1 "GERS Level" is the spectral acceleration (g) from 4 to $16 \mathrm{~Hz}$ for $5 \%$ damping.

2 "NO" = Normally Open; "NC" = Normally Closed; "NO/NC" = Change State.

$3 \quad$ " $"$ = Data not available.

Figure 11.2-1 Generic Equpment Ruggedness Spectra (GERS) for Auxiliary Relays (Reference 44) (Figure 6-2 of SQUG GIP, Reference 1) 


\subsection{SEISMIC DEMAND ON RELAYS}

This section has two methods for determining the seismic demand on relays. Seismic adequacy of essential relays can be confirmed by successful application of either one of these methods. Details on the methods for determining seismic demand on relays is contained in Section 6 of the SQUG GIP (Ref. 1) and in its supporting documents. After computing the seismic demand on the relays, the demand is then compared to the seismic capacity (discussed in Section 11.2) using the guidelines of Section 5.4.

\subsubsection{Use of In-Cabinet Amplification Factors ${ }^{4}$}

The first method for determining relay seismic demand is based on: (1) using a Seismic Demand Spectrum (SDS) at the base of the cabinet containing the relay and (2) multiplying this spectrum by an in-cabinet Amplification Factor (AF). To use this method, the essential relay should not be one of the low-ruggedness types listed in Table 11.2-1. The seismic demand on relays can be represented by an In-cabinet Demand Spectrum (IDS) which is computed using the following equation:

$$
\mathrm{IDS}=\mathrm{SDS} \times \mathrm{AF}
$$

Where:

$$
\begin{aligned}
& \text { SDS - } \quad \text { Seismic Demand Spectrum (SDS) as described in Section 5.2.3. The SDS } \\
& \text { AF - in-cabinet Amplification Factor, as given in Table 11.3-1, for various types }
\end{aligned}
$$

A relay is considered seismically adequate if the IDS is bounded by the relay capacity spectrum in the frequency ranges from $4-16 \mathrm{~Hz}$ and from $33 \mathrm{~Hz}$ and above, i.e., the zero period acceleration (ZPA). If the guidelines for this method cannot be applied, or the seismic demand is not bounded by the seismic capacity of the relay, then the following method can be used instead.

4 Based on Section 6.4.2 of SQUG GIP (Ref. 1) 
Table 11.3-1 In-Cabinet Amplification Factors for Use with Section 11.3.1 (Table 6-2 of SQUG GIP, Ref. 1)

\begin{tabular}{|c|c|}
\hline \multicolumn{1}{|c|}{ Type of Cabinet } & $\begin{array}{c}\text { In-Cabinet } \\
\text { Amplification } \\
\text { Factor (AF) }\end{array}$ \\
\hline $\begin{array}{c}\text { MCC-type cabinet } \\
\text { (defined in Appendix I of Reference 45) }\end{array}$ & 3 \\
\hline $\begin{array}{c}\text { Conventional control panel or benchboard } \\
\text { (defined in Appendix I of Reference 45) }\end{array}$ & $4.5^{2}$ \\
\hline $\begin{array}{c}\text { Switchgear-type cabinet or similar large unsupported panel } \\
\text { (defined in Appendix I of Reference 45) }\end{array}$ & 7 \\
\hline $\begin{array}{l}\text { Other type of cabinet, panel, or enclosure for which } \\
\text { cabinet-specific amplification data exists }\end{array}$ & 3 \\
\hline
\end{tabular}

1 The SCEs and relay evaluation personnel should not apply these amplification factors unless they have thoroughly reviewed and understood the information in Section 6 of the SQUG GIP (Ref. 1) and its supporting documents such as References 43 and 45.

2 To use an amplification factor of 4.5, the control panel or benchboard must meet the restrictions (or caveats) given in Reference 45, Appendix I, except that a $13 \mathrm{~Hz}$ lower bound fundamental frequency shall apply instead of the $11 \mathrm{~Hz}$ fundamental frequency specified by the relevant caveat in Reference 45, Appendix I, when assessing: devices located on internal independent racks, cantilevered appendages, such as cantilevered wing walls attached to a front face or side wall, and access doors which are part of a control panel or benchboard. Note that one intent of the control panel and benchboard caveats is to restrict use of this amplification factor to only those cabinets and panels which have all significant natural modes at $13 \mathrm{~Hz}$ and higher. The amplification factor is a function of the panel frequency with the most flexible panel mode typically being the diaphragm, or out-of-plane, mode.

3 For the "Other" type of cabinets, an effective broad-based amplification factor can be developed from appropriate test data. Reference 43 can be used for this purpose as a guide in which an effective in-cabinet amplification factor can be obtained by multiplying the measured peak amplification factor, for the location in the cabinet where the relay is mounted, times an appropriate reduction factor. Appropriate reduction factors are discussed in Reference 43; for typical, narrow peak amplification spectra, the reduction factor is 0.6 . 


\subsubsection{Use of In-Cabinet Response Spectra ${ }^{5}$}

In this method, the technique of computing relay seismic demand is the same as in Section 11.3.1 (i.e., the demand spectrum is bounded by the capacity spectrum in the frequency ranges from 4 $16 \mathrm{~Hz}$ and from $33 \mathrm{~Hz}$ and above) except that instead of using an in-cabinet amplification factor to determine the seismic demand on the relay, an in-cabinet response spectrum is used. To use this method, the essential relay should not be one of the low-ruggedness types listed in Table 11.2-1. For comparison to relay capacity spectrum, the in-cabinet response spectrum can be treated similar to the IDS of Section 11.3.1. There are two methods for developing in-cabinet response spectra, depending upon the type of equipment:

Control Room Benchboards and Panels. An amplified, in-cabinet response spectrum can be determined using the methodology and software described in Reference 43 for control room benchboards and panels. In this option, the cabinet or panel evaluated must meet the restrictions (or caveats) given in Reference 43. A $13 \mathrm{~Hz}$ lower bound frequency shall apply instead of the 11 $\mathrm{Hz}$ fundamental frequency specified by the relevant caveat in Reference 43 when assessing devices located on internal independent racks, cantilevered appendages such as cantilevered wing walls attached to a front face or side wall, and access doors which are part of a control panel or benchboard. Note that one intent of the control panel and benchboard caveats is to restrict use of this amplification factor to only those cabinets and panels that have all significant natural modes at $13 \mathrm{~Hz}$ or higher. The use of Reference 43 software should not be extended to other classes of equipment without the review and approval of the DOE.

Other Types of Equipment. For other types of cabinets and panels that are not covered by Reference 43, in-cabinet response spectrum can be determined using analytical and/or test methods which are suitable for the specific case. These other methods should be justified in the documentation of the Relay Functionality Review. This is equivalent to the case-specific analysis and/or test approach. Caution should be exercised when using this method to determine in-cabinet response spectra by considering the effects of local flexibility and mounting details such as local plastic deformation, slotted holes, fitted connections, etc.

\subsection{RELAY WALKDOWN 6}

Information on a detailed procedure for conducting relay walkdowns is contained in Section 6 of the SQUG GIP (Ref. 1) and in its supporting documents. The SCEs and relay evaluation personnel should refer to the details in these documents when conducting relay walkdowns. A walkdown should be performed as a part of the relay evaluation. The purposes of the relay walkdown are to:

- Obtain information needed to determine cabinet types which house essential relays and to determine the in-cabinet amplification, where needed, for the seismic capacity methods described above.

- Evaluate the seismic adequacy of the cabinets or enclosures which support the essential relays.

- Spot check mountings of essential relays.

- Spot check the essential relays to evaluate their types and locations, including checks for vulnerable relays (as listed in Table 11.2-1).

5 Based on Section 6.4.2 of SQUG GIP (Ref. 1)

6 Based on Section 6.5 of SQUG GIP (Ref. 1) 
These purposes can be accomplished during one walkdown or separately during different walkdowns. To accomplish the first purpose of the relay walkdown, the cabinets or panels which house essential relays should be identified and the information needed to determine in-cabinet amplification should be reviewed. A SCE and a Relay Reviewer (as discussed in Section 3.3.3) should accomplish this purpose. The serial and model number of the relays should be compared with the applicable relay numbers in References 43 and 45.

The second purpose, evaluation of the seismic adequacy of the cabinet or enclosure supporting the relay, should be done as a part of the Screening Evaluation and Walkdown as described in Section 2.1.3. Note that the cabinets or enclosures supporting essential relays should be identified prior to this walkdown.

The third purpose of the relay walkdown is to spot check relay mountings to confirm that relays are mounted in accordance with manufacturer's recommendations. The objective of the spot checks is to identify any abnormal or a typical relay mounting techniques. The specific number of relays to be checked is not quantified because the bulk of the relays addressed in the relay evaluation procedure are typically located in a few specific facility areas and can be easily checked. Most of the relays encountered in the relay evaluation can be checked by opening relay cabinets in the following areas:

- Control room

- Relay room or auxiliary control room

- Switchgear rooms

- Diesel generator control panel area

Spot checking relay mountings can be performed during a separate relay walkdown by personnel familiar with relay installation. Alternatively, relay mountings may be spot checked during the seismic walkdown when in-cabinet amplification information is gathered. Special preparation or training is not required for spot checking relay mountings. Indications such as proper relay label orientation, mounting bolts in place and tight, and whether the relay is snug in its mounting bracket are sufficient to judge the adequacy of the mounting; analytical checks are not intended except as a means to evaluate atypical mountings.

The fourth purpose of the relay walkdown is to confirm relay types and locations. This can be performed at the same time that the relay mountings are checked and by the same individuals. The approach for confirming relay types by the relay walkdown team includes noting relay types. observed in the cabinets and then comparing this with the relays identified on electrical drawings. It is important to note that relay mountings are considered to be standard and the circuit drawings are assumed to be correct and up-to-date. Spot checks of the relay mountings and relay types are a mechanism to confirm these assumptions. Any significant spot check discrepancies will necessitate more thorough relay inspections. 


\subsection{OUTLIERS 7}

An outlier is defined as an essential relay which does not meet the guidelines for:

- Relay seismic capacity and seismic demand as given in Sections 11.2 and 11.3

- $\quad$ Relay mounting as given in Section 11.4

Chapter 12, Outlier Identification and Resolution, is used when an outlier is identified and the cause(s) for not meeting the guidelines should be documented with the Outlier Seismic Evaluation Sheet (OSES) provided in Chapter 13. Methods are given in this section for use as a generic basis to evaluate the seismic adequacy of essential relays. Therefore, if an essential relay fails these generic methods, it may not necessarily be deficient for seismic loading; however, additional evaluations are needed to show that it is adequate. Some of the additional evaluations and alternative methods for demonstrating seismic adequacy are summarized below.

- Refine the seismic requirements and/or analyses.

- Test the relay and/or the cabinet in question.

- Re-design and modify the circuit to make the relay function nonessential.

- Relocate the relay to reduce the seismic demand imposed upon it.

- Replace the relay with a seismically qualified one.

- Stiffen the relay mounting.

- Use other justifiable approaches.

Generic methods for resolving outliers are also discussed in Chapter 12.

7 Based on Section 6.6 of SQUG GIP (Ref. 1) 


\section{OUTLIER IDENTIFICATION AND RESOLUTION}

\subsection{INTRODUCTION ${ }^{1}$}

The purpose of this chapter is to define the term outliers, how they should be identified and documented, and how they may be resolved. An outlier is an item of equipment that does not comply with all of the screening guidelines provided in the DOE Seismic Evaluation Procedure. The screening guidelines are intended to be used as a generic basis for evaluating the seismic adequacy of equipment at DOE facilities. If an item of equipment fails to pass these generic screens, it may still be shown to be adequate for seismic loading by additional evaluations.

This chapter describes how outliers should be identified and documented for equipment that does not pass the screening guidelines for:

- $\quad$ Electrical Equipment (Sections 8.1)

- Mechanical Equipment (Sections 8.2 and 10.2)

- $\quad$ Tanks (Sections 9.1 and 10.3)

- $\quad$ Piping, Raceway, and Duct Systems (Sections 9.2,10.1, and 10.4)

- $\quad$ Architectural Features and Components (Section 10.5)

- $\quad$ Relays (Chapter 11)

Several generic methods for resolving outliers are summarized in this chapter. Specific methods for addressing the different types of equipment are also discussed in the sections where the screening guidelines are described.

The chapter is organized as follows:

- A summary of generic methods for resolving outliers is contained in Section 12.2.

- Suggested methods for grouping and pooling of outliers from several different facilities for efficient reconciliation are provided in Section 12.3.

- The reasons for classifying an item of equipment as an outlier are described in Section 13.3 along with a description of how outliers should be documented.

\subsection{OUTLIER RESOLUTION ${ }^{2}$}

Several generic methods for resolving outliers are summarized below. Additional specific methods for addressing outliers for the different types of equipment are also discussed in the sections where the screening guidelines are described. The details for resolving outliers, however, are beyond the scope of this procedure. It is the responsibility of the facility to resolve outliers using their existing engineering procedures as they would resolve any other seismic concern.

It is permissible to resolve outliers by performing additional evaluations and applying engineering judgment to address those areas which do not meet the screening guidelines contained in this

1 Based on Section 5.0 of SQUG GIP (Ref. 1)

2 Based on Section 5.3 of SQUG GIP (Ref. 1) 
procedure. Strict adherence to the screening guidelines in the DOE Seismic Evaluation Procedure is not absolutely required; however, these additional outlier evaluations and the application of engineering judgment should be based on a thorough understanding of the screening guidelines contained in the DOE Seismic Evaluation Procedure and the background and philosophy used to develop these guidelines as given in the applicable references. The justification and reasoning for considering an outlier to be acceptable should be based on mechanistic principles and sound engineering judgment.

The screening guidelines have been thoroughly reviewed by experts to ensure that they are acceptable for generic use in DOE facilities; however, the resolution of outliers for individual facilities will not likely receive the same level of review as the generic screening guidelines. Therefore, it is recommended that the evaluations and judgments used to resolve outliers be thoroughly documented so that independent reviews can be performed if necessary as discussed in Section 2.2.

Some of the methods summarized below for resolving outliers build upon the earthquake experience and generic testing data discussed in the DOE Seismic Evaluation Procedure. Facility personnel may use the Screening Evaluation and Walkdown procedure described in Section 2.1.3 in applying earthquake experience or generic testing data which was not available during the initial walkdown for resolution of outliers or they may develop an alternative approach which best fits the circumstances of the specific outlier issue. Outlier issues may also be resolved using current procedures and criteria. As an alternative, facility personnel may choose to not perform corrective modifications or replacement of outliers. Instead, facility personnel must then explain to the DOE the safety implications of not modifying or replacing the outliers.

Methods which can be used to resolve outliers include the following:

1. The subject equipment and/or its anchorage may be fixed or modified to bring it within the scope of the DOE Seismic Evaluation Procedure or in compliance with some other seismic qualification method. For example, appropriate anchorage should be installed for equipment lacking adequate anchorage.

2. The subject equipment and/or its anchorage may be evaluated more rigorously to determine appropriate techniques for strengthening it in order to bring it within the scope of the DOE Seismic Evaluation Procedure or in compliance with some other seismic qualification method. For example, the equipment or its supports may be stiffened so that its resonant frequency is increased to a frequency where the seismic demand is less. Providing an upper lateral support to a floor-mounted item of equipment would typically increase the fundamental frequency to above $8 \mathrm{~Hz}$.

3. The subject equipment may be replaced with equipment which is covered by screening guidelines in the DOE Seismic Evaluation Procedure or has been seismically qualified by some other means.

4. Detailed engineering analyses may be performed to more carefully and/or accurately evaluate the seismic capacity of the equipment and/or the seismic demand to which it is exposed. For example, when using more accurate analytical procedures, consideration should be given to using "as-built" rather than specified minimum material properties for the equipment.

5. The earthquake experience equipment class may be expanded to include the equipment or specific equipment features of interest. The scope of the earthquake experience data which is documented in References 19 and 35 represents only a portion of the total data available. 
An expansion of the earthquake experience equipment classes beyond the scope included in Chapters $8,9,10$, and 11 could include a more detailed breakdown by type, model or manufacturer of a particular class of equipment, less restrictive requirements for inclusion within a class, or development of a sub-category with higher capacity.

Extension of the generic experience equipment classes beyond the descriptions in the DOE Seismic Evaluation Procedure is subject to DOE review and to an extensive peer review beyond what is discussed in Section 2.2. The external peer review for expanding the earthquake experience database should be of similar caliber as that required during the original development of the database. An extension of the earthquake experience database must satisify the requirements discussed in Section 1.4.4.

6. In-situ tests may be performed on the equipment of interest to determine more accurately the equipment dynamic properties.

7. Shake table tests may be performed on the same or similar equipment to check its seismic capacity or evaluate more carefully its dynamic properties.

8. Information not available during the Screening Evaluation and Walkdown may be obtained and used to meet the DOE Seismic Evaluation Procedure screening guidelines.

The most appropriate type of outlier evaluation will depend upon a number of factors, including the reason that the equipment failed the screening guidelines, whether the outlier lends itself to additional review of the earthquake experience or generic testing data or an additional analytical evaluation, the cost of design or hardware modifications, and how extensive the problem is in the facility and in other facilities. Any type of outlier evaluation will require peer review as discussed in Section 2.2. The DOE should be provided with a proposed schedule for complete resolution or future modifications and replacement of outliers. Documentation of the methods used by the facility for resolution of outlier issues and tracking of their implementation can be provided in the OSES as discussed in Section 13.3.

\subsection{GROUPING AND POOLING OF OUTLIERS 3}

Once an outlier has been identified and an OSES is prepared for that item of equipment, the OSES could then be placed in an appropriate outlier category or "basket". There could be one basket for each class of equipment for which there are outliers. Within each basket the outliers could be further divided into the various reasons that the equipment failed the screening evaluation (e.g., capacity vs. demand, caveats, anchorage, or interactions). The organization of the outliers in this manner can facilitate reconciliation of recurring outlier issues.

One method to efficiently reconcile recurring outliers in DOE facilities is for them to pool the outlier information obtained during walkdowns. One means of pooling this information is to tabulate the outliers, including the information contained on the SEDS and, if available, the method ultimately used to evaluate the seismic adequacy of the outlier. These tables may be generated and organized, using a data base management program. This summary may be distributed to DOE facilities so that common outliers may be evaluated using the experience obtained from other facilities. For example, one facility may have one or several unreconciled outliers that an SRT at another facility was able to evaluate. The facility with the unreconciled outliers may be able to employ a similar methodology if the detailed information used in the outlier resolution is shared. Outliers from several DOE facilities may also be resolved more cost-effectively using shared funding.

3 Based on Section 5.4 of SQUG GIP (Ref. 1) 



\section{DOCUMENTATION}

\subsection{INTRODUCTION 1}

This section describes the various types of documents that should be generated with the Screening Walkdown and Evaluation Procedure and how they relate to each other. This section also describes the types of information which could be submitted to the DOE. The following five major types of documents are used with the DOE Seismic Evaluation Procedure:

- Seismic Equipment List (SEL)

- Screening Evaluation Work Sheets (SEWS)

- Outlier Seismic Evaluation Sheets (OSES)

- Screening Evaluation and Data Sheets (SEDS)

- Equipment Seismic Evaluation Report (ESER)

The Seismic Equipment List (SEL) and supporting documents should describe the overall approach used in identifying the equipment listed in the SEL and the basis for selecting the listed equipment. In addition, the SEL and its supporting documentation should describe the method used for verifying the compatibility of the SEL with the facility operating procedures. Further guidance for developing the SEL is provided in Chapter 4 , which discusses the contents of and methods for generating the SEL.

The Screening Evaluation Work Sheets (SEWS), Outlier Seismic Evaluation Sheets (OSES), Screening Evaluation and Data Sheets (SEDS), and Equipment Seismic Evaluation Report (ESER) are discussed in Sections 13.2, 13.3, 13.4, and 13.5, respectively. Copies of the SEWS, OSES, and SEDS forms follow Section 13.5. The forms contained in the DOE Seismic Evaluation Procedure are suggested formats for documenting the information from the seismic evaluations. Other forms, which contain equivalent information to those discussed in this chapter, may be used to document the results of the seismic evaluations using this Procedure.

The extent of suggested documentation for the seismic evaluations is limited. The underlying reason is that the evaluations are to be done by highly-qualified individuals who have been trained in the use and application of the DOE Seismic Evaluation Procedure. For example, SCEs should have the background, experience, and training to make engineering judgments during the facility walkdown and thus avoid having to develop large quantities of backup documentation to record every decision made in applying the procedure. These SCEs are then held accountable for the scope, accuracy, and completeness of the Screening Evaluation and Walkdown process by signing that the results of the seismic evaluations are correct and accurate. One of these signatories should also be a licensed Professional Engineer, as discussed in Section 3.2.

\subsection{SCREENING,EVALUATION WORK SHEETS ${ }^{2}$}

The purpose of the Screening Evaluation Work Sheets (SEWS) is to provide a convenient summary and checklist of the seismic evaluation criteria described in the DOE Seismic Evaluation Procedure. During the seismic walkdown, the SEWS can serve as a tool for collecting and organizing the important information from the seismic evaluation. The SEWS, or a similar checklist, should be used during the facility walkdown to document the results of the evaluation.

1 Based on Section 9.0 of SQUG GIP (Ref. 1)

2 Based on Appendix G of SQUG GIP (Ref. 1) 
Equipment class caveats and guidelines are summarized on the SEWS. Other informal documentation may be used by the SCEs as aids during the Screening Evaluation and Walkdown. These may include calculations, sketches, photographs, and charts. The SEWS should not be used unless the user has a thorough understanding of the DOE Seismic Evaluation Procedure and the reference documents.

There are 26 SEWS for most of the classes of equipment discussed in the DOE Seismic Evaluation Procedure. The 26 SEWS correspond to the following classes of equipment and sections from Chapters 8 through 10:

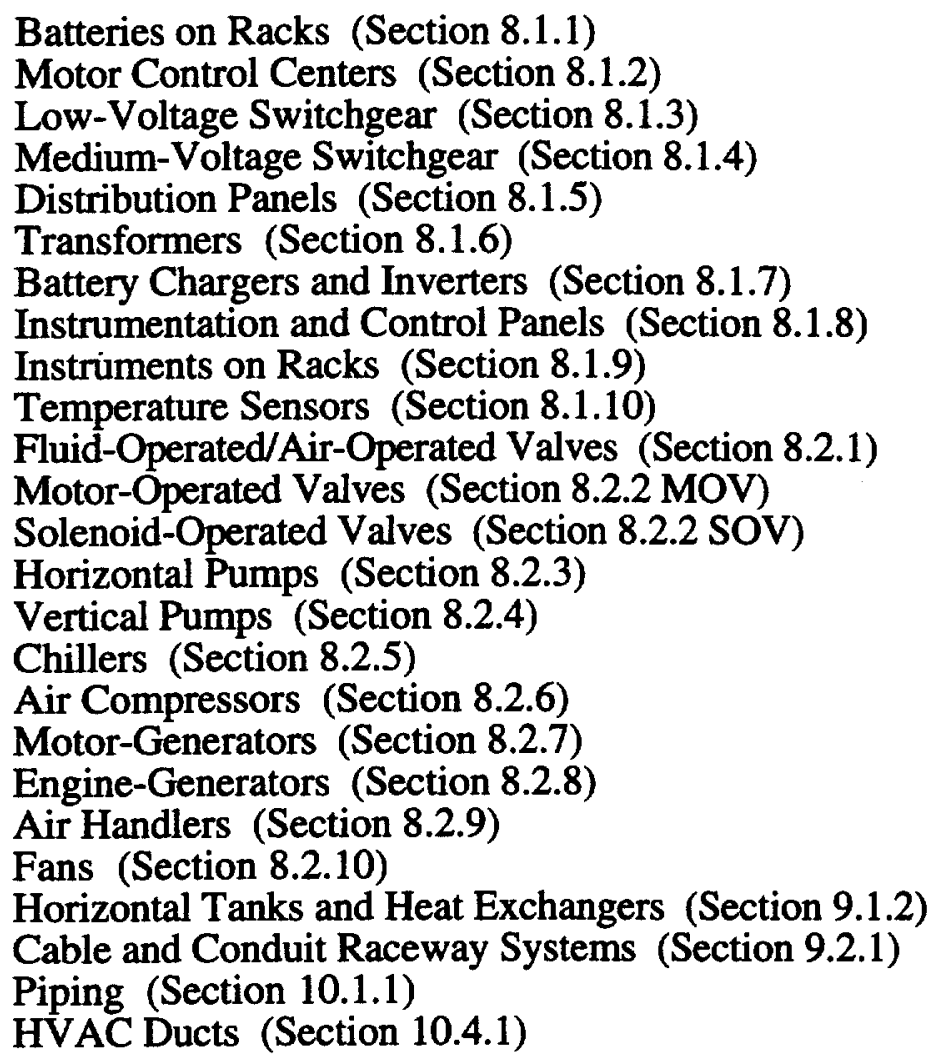

SEWS are not provided for several classes of equipment. For these classes of equipment, the SEWS for Section 10.X.X can be used as a template and the checklists provided in the sections for those classes of equipment can be used during the facility walkdown. SEWS are not provided for the following classes of equipment and sections:

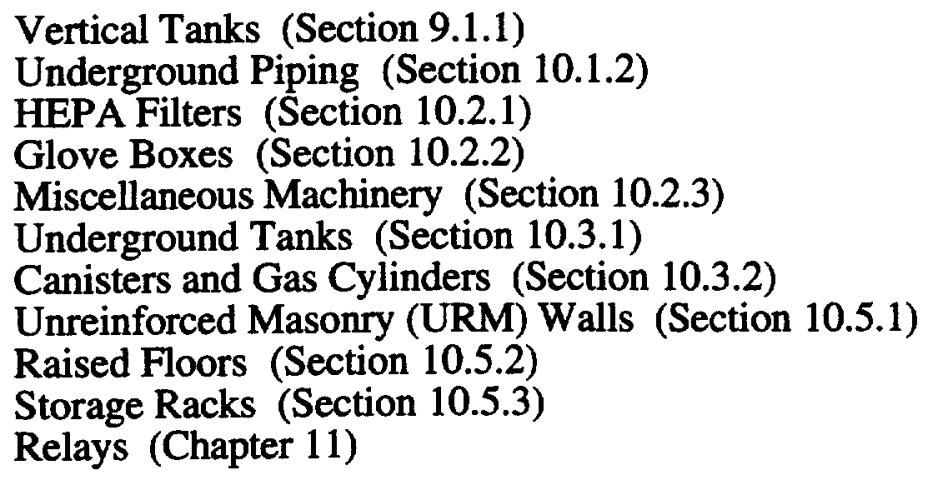


Most of the information at the top of each SEWS (equipment ID number, equipment description, equipment location, etc.) can be entered on the SEWS prior to the facility walkdown. If a data base program is used to develop the SEL, then the information at the top of each page of the SEWS can be printed directly from the data base file containing the SEL information.

The SEWS can be used as a checklist by circling the appropriate symbol in response to each statement. The meaning of the symbols is given below:

$\mathrm{Y}$ - Yes. This criterion is met. ("Y" is always the favorable response, i.e., all the "Y" symbols should be circled if an item of equipment is seismically adequate.)

$\mathrm{N}$ - No. This criterion is not met.

U - Unknown. It cannot be determined whether this criterion is met at this time. (This response can be used while the screening evaluation is in progress to identify criteria which must be evaluated later.)

N/A - Not Applicable. Some of the criteria may not apply for a particular item of equipment.

Some of the statements on the SEWS ask which of several alternatives is being used in the Screening Evaluation and Walkdown and the meaning of these symbols is self-explanatory. Likewise, when all the questions have a final response, the last question in each section of the SEWS can then be answered.

The SEWS also provide space to record information about the item of equipment, to document any comments the SCEs may wish to make, to document the reason why the intent of any caveats and guidelines are met without meeting the specific wording of the caveat rule, to sketch the equipment, and to sign off. In addition, the SEWS has a "Recommend Resolution" section to summarize the equipment evaluation. For equipment identified as an outlier, this section provides space to identify potential outlier resolution approaches. The resolution choices are:

- Maintenance action

- Further evaluation

- Retrofit design

- Other

- No further action required. Equipment is seismically adequate.

At the bottom of the SEWS are signature lines for all those performing the Seismic Evaluation and Walkdown. As discussed in Chapter 3, there should be at least two SCE signatories and one of the SCEs should be a licensed Professional Engineer. A signature on the SEWS indicates the SCE is in agreement with all the entries and conclusions entered on the sheet and all signatories should agree with all the entries and conclusions.

The SEWS are designed to be compatible with the Screening Evaluation Data Sheets (SEDS) discussed in Section 13.4 so that the summary information from the SEWS can be transferred directly to the SEDS. The responses to the final question in each section of the SEWS and the overall conclusion can be entered directly into the appropriate column in the SEDS discussed in Section 13.4 . 
The Outlier Seismic Evaluation Sheets (OSES) are used to document the reason(s) for an item of equipment identified as an outlier during a screening evaluation to fail the screening guidelines. A separate OSES should be completed for each item of equipment classified as an outlier as discussed in Chapter 12.

An item of equipment listed in the SEL, as described in Chapter 4, should be identified as an outlier if it does not meet the screening guidelines covered in the other sections of this procedure. If an item of equipment is identified as an outlier during a screening evaluation in one of the sections of the DOE Seismic Evaluation Procedure, then the reason(s) for failing to satisfy the screening guidelines can be documented on an Outlier Seismic Evaluation Sheet (OSES). Other documentation, such as the Screening Evaluation Work Sheets (SEWS) discussed in Section 13.3, also have provisions for outliers. A separate OSES should be completed for each item of equipment classified as an outlier. The information to be included in each of the four sections of the OSES is described below.

Section 1 of the OSES describes the item of equipment identified as an outlier. This is the same information as found in the first seven columns of the SEDS which is discussed in Section 13.4. On the OSES, however, more space is provided to describe the equipment so that more details can be included to facilitate later resolution of this outlier issue without requiring repeated trips into the facility.

Section 2 of the OSES defines those conditions which cause that item of equipment to be classified as an outlier. This section should identify which of the conditions is the cause for the item of equipment becoming an outlier. More than one condition may be the cause for the outlier. In addition, the reason(s) for the equipment being an outlier should be described in more detail. For example, the SCEs could indicate at what frequencies the demand exceeded the capacity.

Section 3 of the OSES can be used to provide a proposed method for resolving the outlier issue, based on the experience and detailed evaluation of that item of equipment by the SCEs or the Lead Relay Reviewer. This is an optional part of the outlier identification process. This section also provides space for supplying any additional information which may be used to implement the proposed method of resolution. This may include information such as an estimate of the fundamental natural frequency of the equipment.

\subsection{SCREENING EVALUATION AND DATA SHEETS ${ }^{4}$}

The results of the Screening Evaluation and Walkdown, as described in Section 2.1.3, should be documented on walkdown checklists. These checklists include the Screening Evaluation Work Sheets (SEWS) discussed in Section 13.2 and the Screening Evaluation Data Sheets (SEDS). Preparation of the SEDS includes a review of generic and facility-specific seismic documentation and a facility walkdown of the equipment listed on the SEL. The completed SEDS constitute a tabulated summary for the formal documentation of the Screening Evaluation and Walkdown and reflect the final judgment of the SCEs. The SEDS offer a convenient way for tabulating the significant information from the SEWS for all the equipment listed on the SEL.

The SEDS is arranged in rows and columns and each row contains one item of equipment listed in the SEL. The columns contain information about the equipment and the results of the Screening Evaluation and Walkdown. Guidelines for completing each of the columns are provided below.

3 Based on Section 5.2 of SQUG GIP (Ref. 1)

4 Based on Section 4.6 of SQUG GIP (Ref. 1) 
At the bottom of the SEDS are two sets of suggested signature blocks to be signed by those performing the Seismic Evaluation and Walkdown. The first block should be signed by all the SCEs who performed the Screening Evaluation and Walkdown. There should be at least two signatories and one of which should be a licensed Professional Engineer. A signature indicates the SCE is in agreement with all the entries and conclusions entered on the SEDS. All signatories should agree with all the entries and conclusions.

The second block for signatures at the bottom of the SEDS is for use by a safety professional, systems engineer, or operations engineer who may provide critical information to the SCEs during their seismic evaluation of the equipment. Examples of such information include how a piece of equipment operates or whether a feature on the equipment is needed to accomplish its safety function. Information of this type is particularly important if an item of equipment is found during the walkdown which should be added to the SEL. It is left to the SCEs to determine whether this second block of signatures is needed. Only the signature of the safety professional, systems engineer, or operations engineer should be documented on the SEDS and details of the information supplied to the SCEs need not be included.

Note that the completed SEDS reflect the final judgment of the SCEs. Prior to arriving at this final judgment, there may have been several walkdowns, calculations, and other seismic evaluations which form the basis for determining whether the equipment meets the screening guidelines contained in the DOE Seismic Evaluation Procedure.

Compilation of the information on the SEDS can be done using a data base management system. This makes it possible to manipulate the order in which the equipment is listed on the sheets. It may be convenient to use SEDS by location in the facility. This may optimize the routing of the SCEs during the walkdowns so that backtracking is minimized and separate teams of SCEs can cover different parts of the facility. After the walkdown is complete, the data base management system can be used to sort the equipment on the SEDS into lists of outliers or other categories of equipment.

The contents of each of the 16 columns of the SEDS are described below.

Columns 1 through 6 contain information for identifying and locating the equipment on the SEL.

Column 1 contains the equipment class number.

Column 2 contains the facility identification or tag number for the equipment. This is normally an alpha-numeric designation by which an item of equipment is uniquely identified in the facility. This identifier will permit direct access and a cross-reference to the existing facility files or data system for the item of equipment.

Column 3 contains both a designation of the facility system to which the equipment belongs and a description of the equipment. If the system designation is placed at the beginning of this field, then the equipment list can be sorted by system with a data base management system.

Column 4 identifies the building in which the equipment is located.

Column 5 contains the floor elevation from which the item of equipment can be viewed by the SCEs. 
Column 6 contains a designation of the location of the equipment within the building. An example of this is by building column line intersection, such as F-12. This indicates the intersection of column lines $\mathrm{F}$ and 12 . Alternatively, the room designation can be given; e.g., diesel generator room (DG room).

Columns 7 through 10 are used to document the source of the seismic capacity and the source of the seismic demand.

Column 7 contains the elevation at which the equipment is mounted; i.e., the elevation at which the equipment receives its seismic input (demand). This elevation should be determined by the SCEs during the walkdown. Note that this elevation may not be the same as the floor elevation given in Column 5.

Column 8 identifies the source of the seismic capacity. The following codes may be used:

DOC Component-Specific Seismic Qualification Documentation.

RS Reference Spectrum (for comparison to in-structure response spectra).

GERS Generic Equipment Ruggedness Spectra, GERS.

If the GERS are used, a number designation (XXX) should also be given to indicate which unique GERS is used. If seismic qualification documentation is used, reference to the documentation should be noted in Column 16.

Column 9 indicates the experience data factor, $\mathrm{F}_{\mathrm{ED}}$, for the equipment.

Column 10 indicates the method used to define the seismic demand. The following codes may be used:

$\begin{array}{ll}\text { SDS } & \text { Seismic Demand Spectrum } \\ \text { IDS } & \text { In-Cabinet Demand Spectrum }\end{array}$

If an in-structure response spectrum is used, a number designation should also be given to indicate which unique spectrum is used.

Columns 11 through 14 are used to document the results of the evaluation of the equipment against the four seismic screening guidelines: comparison of seismic capacity to seismic demand, caveat and guidelines compliance, anchorage adequacy, and seismic interaction.

Column 11 indicates whether capacity of the equipment exceeds the demand imposed on it. The following codes may be used:

Y Yes, capacity exceeds demand.

N No, capacity does not exceed demand.

U Unknown whether capacity exceeds demand. 
Column 12 indicates whether the equipment is within the scope of the earthquake/testing equipment class and meets the intent of all the caveats and guidelines for the equipment class. The following codes may be used:

Y Yes, the equipment is in the equipment class, and the intent of all applicable caveats and guidelines is satisfied.

$\mathrm{N}$ No, the equipment is not in the equipment class, or the intent of all applicable caveats and guidelines is not satisfied.

U Unknown whether the equipment is in the equipment class or whether the intent of all applicable caveats is satisfied.

N/A The earthquake/test equipment class and the caveats and guidelines are not applicable to this item of equipment.

Column 13 indicates whether the equipment anchorage meets the anchorage screening guidelines. The following codes may be used:

Y Yes, anchorage capacities equal or exceed seismic demand, and anchorage is free of gross installation defects and has adequate stiffness.

N No, anchorage capacities do not equal or exceed the seismic demand, or anchorage is not free of gross installation defects, or anchorage does not have adequate stiffness.

U Unknown whether anchorage capacities equal or exceed seismic demand, or whether anchorage is free of gross installation defects or has adequate stiffness.

N/A Anchorage guidelines are not applicable to this equipment; e.g., valves are not evaluated for anchorage.

Column 14 indicates whether the equipment is free of adverse seismic interaction effects. The following codes may be used:

$Y$ Yes, the equipment is free of interaction effects, or the interaction effects are acceptable and do not compromise the function of the equipment.

N No, the equipment is not free of adverse interaction effects.

U Unknown whether interaction effects will compromise the function of the equipment.

Columns 15 and 16 are used to document the overall result of the equipment evaluation and to record a note number for explaining anything unusual for an item of equipment. 
Column 15 indicates whether, in the final judgment of the SCEs, the seismic adequacy of the equipment is verified. Note that this judgment may be based on one or more walkdowns, calculations, and other supporting data. The following codes are used:

$\mathrm{Y}$ Yes, seismic adequacy has been verified, i.e., code "Y", for all the applicable screening guidelines:

(1) seismic capacity is greater than demand,

(2) the equipment is in the earthquake/test equipment class and the intent of all the caveats and guidelines is met,

(3) equipment anchorage is adequate, and

(4) seismic interaction effects will not compromise the function of the item of equipment.

$\mathrm{N}$ No, seismic adequacy does not meet one or more of the seismic evaluation criteria. Equipment is identified as an outlier requiring further effort in accordance with Chapter 12.

Note that there is no "Unknown" category in Column 15 since this column represents the final judgment by the SCEs. At this point, the item of equipment should be either verified to be seismically adequate $(\mathrm{Y})$ or found to be lacking in one or more areas $(\mathrm{N})$ and should be evaluated as an outlier in accordance with Chapter 12.

Column 16 can be used for explanatory notes. A number can be entered in this field which corresponds to a list of notes which provide additional information on the seismic evaluation of equipment. For example, a note could indicate that a solenoid-operated valve is mounted on the yoke of an air-operated valve (AOV) and is evaluated as a component mounted within the "box" of this AOV. This column should also be used to identify when the intent of any caveat and guidelines is met, but the specific wording of the rule is not needed.

\subsection{EQUIPMENT SEISMIC EVALUATION REPORT ${ }^{5}$}

The Equipment Seismic Evaluation Report (ESER) should summarize the equipment seismic evaluations which result from applying the procedures in the DOE Seismic Evaluation Procedure. The following information should be documented in the ESER:

- $\quad$ Resumes of the SCEs in the SRT.

- Description of the seismic design basis of the facility, description of the seismic demand of the facility including the design basis earthquake (DBE), identification of the performance category and function of the facility, description of the site characteristics, and basis for establishing the degree of uncertainty in the natural frequency of the building structure if unbroadened response spectra are used with frequency shifting of response peaks.

- List of systems and components in the SEL.

- $\quad$ Screening Evaluation and Walkdown documentation including the SEWS, OSES and SEDS.

- Notes, photographs, drawings, calculations, assumptions, and judgments, as appropriate, used to justify the Screening Evaluation and Walkdown.

5 Based on Section 9.4 of SQUG GIP (Ref. 1) 
- $\quad$ Results of the Screening Evaluation and Walkdown for equipment on the SEDS forms, including descriptions of any cases which specific caveats and guidelines are met by intent without meeting the specific wording of the caveat rule.

- Description of outliers on OSES and SEWS forms and explanations of the safety and operation implications of not resolving these outliers.

- $\quad$ Results of engineering evaluations, tests, calculations, equipment modifications, and equipment replacements as well as a proposed schedule to resolve outliers.

- Description of significant or programmatic deviations from the DOE Seismic Evaluation Procedure. 



\section{SCREENING EVALUATION WORK SHEET (SEWS)}

Equipment ID No.:

Equipment Class: Batteries on Racks

Equipment description:

System:

Equipment Location: Bldg.

Floor El. Room, Row/Col.

Manufacturer, model, etc.:

Battery type:

Individual Battery Weight:

Drawing No.:

Performance Category:

\section{Functionality Requirement}

Contact Lead Relay Reviewer to determine if item contains Essential Relays

For components whose function or structural integrity is required, complete all sections of this form.

For all other components, only anchorage evaluation is required.

\section{Seismic Capacity vs, Demand (Chapter 5)}

1. Seismic Capacity based on:

$\begin{array}{ll}\square & \text { Reference Spectrum } \\ \square & \text { GERS } \\ \square & \text { Existing documentation }\end{array}$

2. Elevation where equipment receives seismic input

Seismic Demand Spectrum (SDS) based on:

In-structure response spectrum (IRS) per DOE-STD-1020

$\square \quad$ Other in-structure response spectrum (determine appropriate experience data scale factor)

$\square \quad$ Design basis earthquake (DBE) per DOE-STD-1020

Other

Scale Factor (SF)

Does capacity exceed demand?

Experience Data Factor $\left(F_{E D}\right)$

Reference:

\section{Caveats $(\operatorname{section} 0.1 .1)$}

Reference Spectrum (Identify with an asterisk $\left(^{*}\right)$ those caveats which are met by intent without meeting the specific wording of the caveat rule and explain the reason for this conclusion in the COMMENTS section below)

1. Equipment is included in earthquake experience equipment class $\quad$ Y $\quad N \quad U \quad N / A$

2. Plates of the cells are lead-calcium flat-plate, Planté or of

Manchex design

3. Each individual battery weighs less than $\mathbf{4 5 0} \mathrm{lbs}$

4. Close-fitting, crush-resistant spacers fill two-thirds of

vertical space between cells

5. Cells restrained by end and side rails

$\begin{array}{llll}Y & N & U & N / A\end{array}$

$Y \quad N \quad U \quad N / A$

$\begin{array}{llll} & N & U & N / A\end{array}$

$Y \quad N \quad U \quad N / A$ 


\section{SCREENING EVALUATION WORK SHEET (SEWS) (Cont.)}

Equipment ID No.:

Equipment Class: Batteries on Racks

Equipment description:

Caveats (Cont)

6. Racks have longitudinal cross bracing

7. Wood racks evaluated to industry accepted standards

8. Batteries greater than 10 years old specifically evaluated for aging effects

9. Have you looked for and found no other adverse concerns?

Is the intent of all the caveats met for Reference Spectrum?

$\begin{array}{llll}Y & N & U & \text { N/A } \\ Y & N & U & \text { N/A } \\ Y & N & U & \text { N/A } \\ Y & N & U & \text { N/A } \\ Y & N & U & \text { N/A }\end{array}$

GERS (Identify with an asterisk (*) those caveats which are met by intent without meeting the specific wording of the caveat rule and explain the reason for this conclusion in the COMMENTS section below)

\begin{tabular}{|c|c|c|c|c|c|}
\hline 1. & Equipment is included in generic seismic testing equipment class & $\bar{Y}$ & $N$ & $\mathrm{U}$ & N/A \\
\hline 2. & Meets all Reference Spectrum caveats & $Y$ & N & $U$ & N/A \\
\hline 3. & $\begin{array}{l}\text { Plates of the cells are lead-calcium flat-plate design } \\
\text { (i.e., not Manchex design) }\end{array}$ & $Y$ & $\mathbf{N}$ & $U$ & N/A \\
\hline 4. & $\begin{array}{l}\text { Batteries supported on two-step racks or single-tier racks; } \\
\text { restrained by double side and end rails which are symmetrically } \\
\text { located with respect to the cell center-of-gravity }\end{array}$ & Y & $\mathbf{N}$ & $\mathrm{U}$ & N/A \\
\hline & tent of all the caveats met for GERS? & Y & $\mathbf{N}$ & $\mathrm{U}$ & N/A \\
\hline
\end{tabular}

\section{Anchorage (Chapter 6)}

1. Type of anchorage:

$\begin{array}{ll}\square & \text { expansion anchor } \\ \square & \text { cast-in-place bolt or headed stud anchor } \\ \square & \text { cast-in-place J-bolt } \\ \square & \text { grouted-in-place bolt } \\ \square & \text { welds to embedded steel on exposed steel } \\ \square & \text { lead cinch anchors } \\ \square & \text { Other } \\ \square & \text { N/A (no further anchorage considerations) }\end{array}$

2. Appropriate characteristics for anchorage type checked

(size, location, equipment characteristics)

3. Gap at threaded anchor less than $1 / 4$ inch

4. Base stiffness and no significant prying action requirements met

5. Equipment base strength and structural load path adequate

6. Embedment steel and pads requirements met

7. Embedment length requirements met

8. Anchor spacing requirements met

9. Edge distance requirements met

10. Concrete strength requirements met

$\begin{array}{llll}Y & N & U & \\ Y & N & U & \text { N/A } \\ Y & N & U & \\ Y & N & U & \\ Y & N & U & \text { N/A } \\ Y & N & U & \\ Y & N & U & \\ Y & N & U & \\ Y & N & U & \end{array}$




\section{SCREENING EVALUATION WORK SHEET (SEWS) (Cont.)}

Equipment ID No.:

Equipment Class: Batteries on Racks

Equipment description:

\section{Anchorage (Cont.)}

11. Concrete crack requirements met

12. Equipment with essential relays requirements met

13. Installation adequacy requirements met

14. No other concerns

Does anchorage capacity exceed demand?

$\begin{array}{llll}Y & N & U & \\ Y & N & U & N / A \\ Y & N & U & N / A \\ Y & N & U & \end{array}$

Reference:

\section{Interaction Effects (Chapter 7 )}

1. Soft targets free from impact by nearby equipment or structures

2. If equipment contains sensitive essential relays, equipment free from all impact by nearby equipment or structures

3. Attached lines have adequate flexibility

4. No collapse of overhead equipment, distribution systems, or masonry walls

5. Equipment is free from credible and significant seismic-induced flood and spray concerns

6. No credible seismic-induced fire concerns

7. No other "two over one" concerns as defined in DOE-STD-1021

8. No other concerns

$\begin{array}{lll}\mathrm{N} & \mathrm{U}\end{array}$

Is equipment free of interaction effects?

$\begin{array}{llll}Y & N & U & \text { N/A } \\ Y & N & U & \text { N/A } \\ Y & N & U & \text { N/A } \\ Y & N & & \text { N/A } \\ Y & N & & \text { N/A } \\ Y & N & & \text { N/A } \\ Y & N & & \text { N/A } \\ Y & N & U & \text { N/A } \\ Y & N & U & \end{array}$

\section{Comments}




\section{DOE Seismic Evaluation Procedure}

SEWS $8.1 .1(4$ of 4$)$

Sheet 4 of

\section{SCREENING EVALUATION WORK SHEET (SEWS) (Cont.)}

\begin{tabular}{l|l} 
Equipment ID No.: & Equipment Class: Batteries on Racks
\end{tabular}

Equipment description:

Comments (Cont)

Screening Walkdown(s):

Date Time Team Members

Recommend Resolution

- Maintenance action:

Further evaluation:

Retrofit design:

$\square \quad$ Other:

- No further action required. Equipment is seismically adequate.

All aspects of the equipment's seismic adequacy have been addressed.

Evaluation by:

Date:

(All team members) 


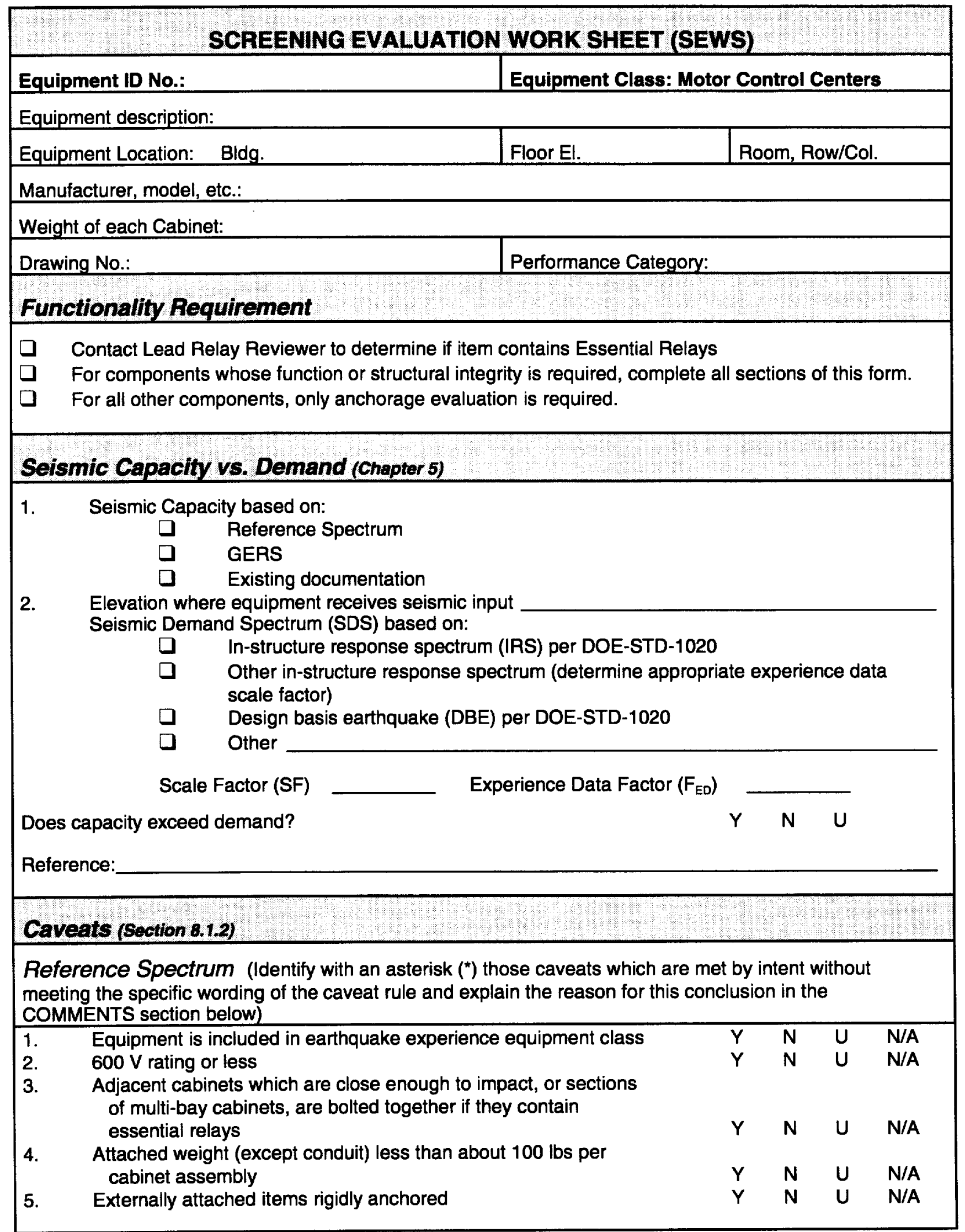




\section{SCREENING EVALUATION WORK SHEET (SEWS) (Cont.)}

\begin{tabular}{l|l} 
Equipment ID No.: & Equipment Class: Motor Control Centers
\end{tabular}

Equipment description:

\section{Caveats (Cont.)}

6. General configuration similar to NEMA standards

7. Cutouts in lower half less than 6 in. wide and 12 in. high

8. All doors secured by latch or fastener

9. Have you looked for and found no other adverse concerns?

Is the intent of all the caveats met for Reference Spectrum?

$\begin{array}{llll}Y & N & U & N / A \\ Y & N & U & N / A \\ Y & N & U & N / A \\ Y & N & U & N / A \\ Y & N & U & N / A\end{array}$

GERS (Identify with an asterisk (*) those caveats which are met by intent without meeting the specific wording of the caveat rule and explain the reason for this conclusion in the COMMENTS section below)

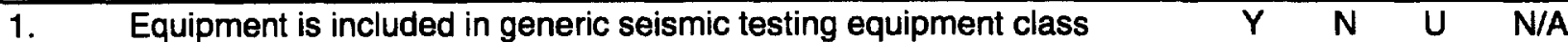

2. Meets all Reference Spectrum caveats

3. Floor-mounted cabinet

4. Average weight per section less than 800 pounds

5. Base anchorage utilizing MCC base channels

6. Adequate strength and stiffness in load transfer path from anchorage to base frame (only for "function after" GERS)

7. Essential relays have GERS $>4.5 \mathrm{~g}$ (only for "function during" GERS)

8. Able to reset starters (only for "function after" GERS)

9. Adjacent cabinets which are close enough to impact, or sections of

multi-bay cabinets, are bolted together.

Is the intent of all the caveats met for GERS?

$\begin{array}{llll}Y & N & U & N / A\end{array}$

$\begin{array}{llll}Y & N & U & N / A\end{array}$

$Y \quad N \quad U \quad N / A$

$\begin{array}{llll}Y & N & U & N / A\end{array}$

$\begin{array}{llll}Y & \mathrm{~N} & \mathrm{~N} / \mathrm{A}\end{array}$

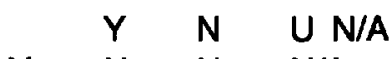

$Y \quad N \quad U \quad N / A$

\section{Anchorage (Chapter 6)}

1. Type of anchorage:

$\begin{array}{ll}\square & \text { expansion anchor } \\ \square & \text { cast-in-place bolt or headed stud anchor } \\ \square & \text { cast-in-place J-bolt } \\ \square & \text { grouted-in-place bolt } \\ \square & \text { welds to embedded steel on exposed steel } \\ \square & \text { lead cinch anchors } \\ \square & \text { Other } \\ \square & \text { N/A (no further anchorage considerations) }\end{array}$

2. Appropriate characteristics for anchorage type checked

(size, location, equipment characteristics)

3. Gap at threaded anchor less than 1/4 inch

4. Base stiffness and no significant prying action requirements met

5. Equipment base strength and structural load path adequate

6. Embedment steel and pads requirements met

7. Embedment length requirements met

8. Anchor spacing requirements met

9. Edge distance requirements met

10. Concrete strength requirements met

$\begin{array}{llll}Y & N & U & \\ Y & N & U & N / A \\ Y & N & U & \\ Y & N & U & \\ Y & N & U & N / A \\ Y & N & U & \\ Y & N & U & \\ Y & N & U & \\ Y & N & U & \end{array}$




\section{DOE Seismic Evaluation Procedure}

SEWS 8.1.2 ( 3 of 4$)$

Sheet 3 of

\section{SCREENING EVALUATION WORK SHEET (SEWS) (Cont.)}

Equipment ID No.:

Equipment Class: Motor Control Centers

Equipment description:

\section{Anchorage (Cont.)}

11. Concrete crack requirements met

12. Equipment with essential relays requirements met

13. Installation adequacy requirements met

14. No other concerns

$\begin{array}{lll}Y & N & U\end{array}$

$Y \quad N \quad U \quad N / A$

$\begin{array}{llll}Y & N & U & N / A\end{array}$

Does anchorage capacity exceed demand?

$Y \quad N \quad U$

N/A

Reference:

\section{Interaction Effects (Chapter 7)}

1. Soft targets free from impact by nearby equipment or structures

Y N U

2. If equipment contains sensitive essential relays, equipment free from all impact by nearby equipment or structures

3. Attached lines have adequate flexibility

4. No collapse of overhead equipment, distribution systems, or masonry walls

5. Equipment is free from credible and significant seismic-induced flood and spray concerns

6. No credible seismic-induced fire concerns

7. No other "two over one" concerns as defined in DOE-STD-1021

8. No other concerns

$\begin{array}{llll}\mathrm{N} & \mathrm{U} & \mathrm{N} / \mathrm{A}\end{array}$

Is equipment free of interaction effects?

$\begin{array}{llll}Y & N & U & N / A\end{array}$

$\begin{array}{llll}Y & N & U & N / A\end{array}$

$\begin{array}{lll}\mathrm{Y} N & \mathrm{~N}\end{array}$

$\begin{array}{lll}Y & N & N\end{array}$

$\begin{array}{lll}Y & N & N\end{array}$

$\begin{array}{lll}Y & N & N\end{array}$

$\begin{array}{llll}Y & N & U & N / A\end{array}$

$Y \quad N \quad U$

\section{Comments}




\section{DOE Seismic Evaluation Procedure}

SEWS $8.1 .2(4$ of 4$)$

Sheet 4 of

\section{SCREENING EVALUATION WORK SHEET (SEWS) (Cont.)}

Equipment ID No.:

Equipment Class: Motor Control Centers

Equipment description:

Comments (Cont)

Screening Walkdown(s):

Date Time Team Members

\section{Recommend Resolution}

- Maintenance action:

$\square \quad$ Further evaluation:

$\square \quad$ Retrofit design:

$\square \quad$ Other:

No further action required. Equipment is seismically adequate.

All aspects of the equipment's seismic adequacy have been addressed.

Evaluation by:

Date:

(All team members) 


\section{SCREENING EVALUATION WORK SHEET (SEWS)}

Equipment ID No.:

Equipment Class: Low-Voltage Switchgear

Equipment description:

Equipment Location: Bldg.

Floor El.

Room, Row/Col.

Manufacturer, model, etc.:

Weight of each Cabinet:

Drawing No.:

Performance Category:

\section{Functionality Requirement}

- Contact Lead Relay Reviewer to determine if item contains Essential Relays

- For components whose function or structural integrity is required, complete all sections of this form.

$\square \quad$ For all other components, only anchorage evaluation is required.

\section{Seismic Capacity vs. Demand (Chapter 5)}

1. Seismic Capacity based on:

$\begin{array}{ll}\square & \text { Reference Spectrum } \\ \square & \text { GERS } \\ \square & \text { Existing documentation }\end{array}$

2. Elevation where equipment receives seismic input

Seismic Demand Spectrum (SDS) based on:

In-structure response spectrum (IRS) per DOE-STD-1020

$\square \quad$ Other in-structure response spectrum (determine appropriate experience data scale factor)

$\square \quad$ Design basis earthquake (DBE) per DOE-STD-1020

$\square \quad$ Other

Scale Factor (SF)

Experience Data Factor $\left(F_{E D}\right)$

Does capacity exceed demand?

Y N U

Reference:

\section{Caveats (Section 8.1.3)}

Reference Spectrum (Identify with an asterisk (*) those caveats which are met by intent without meeting the specific wording of the caveat rule and explain the reason for this conclusion in the COMMENTS section below)

1. Equipment is included in earthquake experience equipment class

2. $\quad 600 \mathrm{~V}$ rating or less

3. Side-to-side restraint of draw-out circuit breakers is provided

$\begin{array}{llll}Y & N & U & N / A\end{array}$

4. Adjacent cabinets which are close enough to impact, or sections of multi-bay cabinets, are bolted together if they contain essential relays $\begin{array}{lllll}Y & N & U & N / A\end{array}$

5. Attached weight (except conduit) less than about $100 \mathrm{lbs}$ per cabinet assembly

6. Externally attached items rigidly anchored

7. General configuration similar to ANSI C37.20 standards 


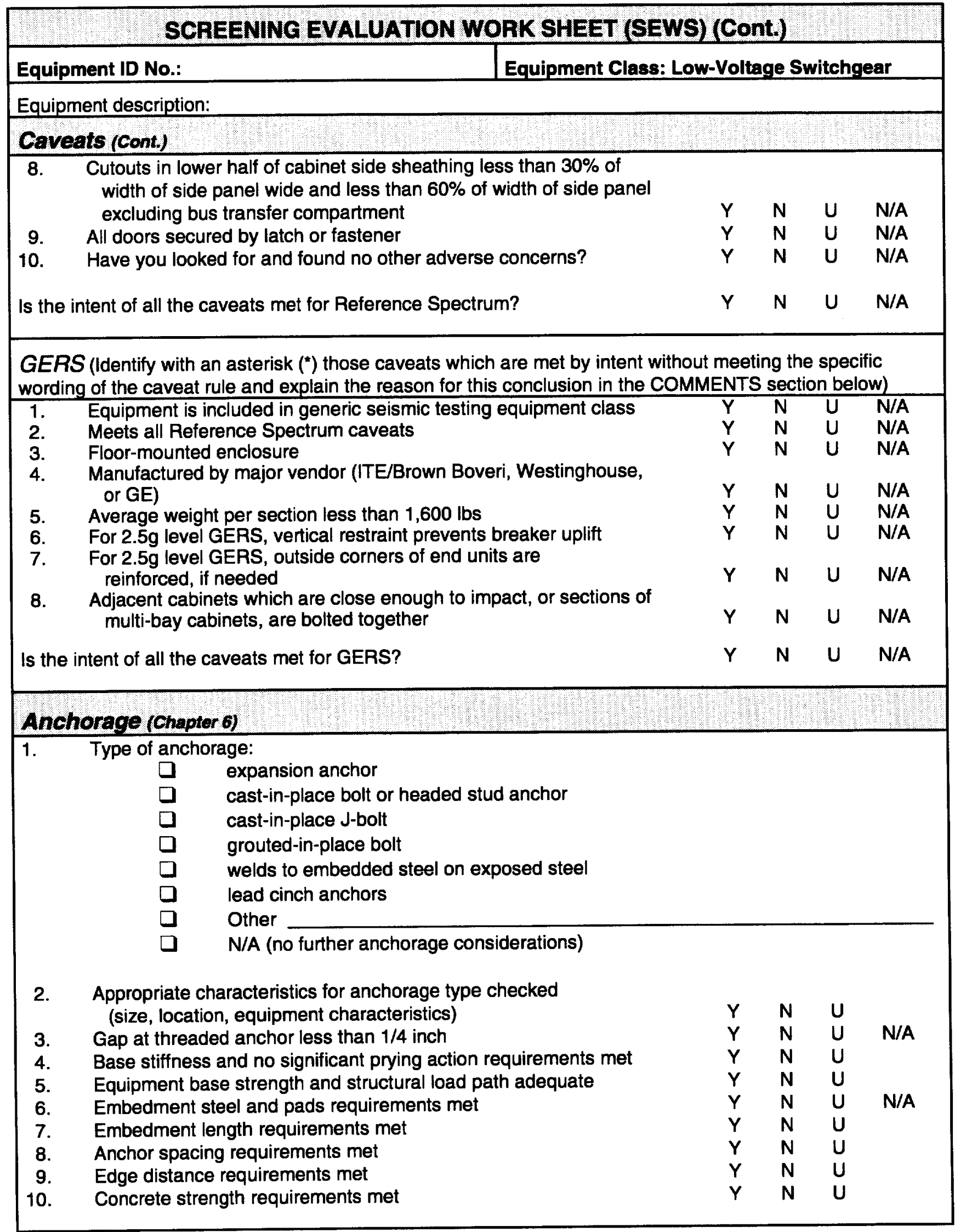




\section{SCREENING EVALUATION WORK SHEET (SEWS) (Cont.)}

Equipment ID No.: Equipment Class: Low-Voltage Switchgear

Equipment description:

\section{Anchorage (Cont)}

11. Concrete crack requirements met

12. Equipment with essential relays requirements met

13. Installation adequacy requirements met

14. No other concerns

Does anchorage capacity exceed demand?

$\begin{array}{llll}Y & N & U & \\ Y & N & U & N / A \\ Y & N & U & N / A \\ Y & N & U & \end{array}$

Reference:

\section{Interaction Effects (Chapter 7)}

1. Soft targets free from impact by nearby equipment or structures

2. If equipment contains sensitive essential relays, equipment free from all impact by nearby equipment or structures

3. Attached lines have adequate flexibility

4. No collapse of overhead equipment, distribution systems, or masonry walls

5. Equipment is free from credible and significant seismic-induced flood and spray concerns

6. No credible seismic-induced fire concerns

7. No other "two over one" concerns as defined in DOE-STD-1021

8. No other concerns

$\begin{array}{lll}\mathrm{N} & \mathrm{U}\end{array}$

Is equipment free of interaction effects?

$\begin{array}{llll}\mathrm{N} & \mathrm{U} & \mathrm{N} / \mathrm{A}\end{array}$

$\begin{array}{llll}Y & N & U & N / A\end{array}$

$\begin{array}{llll}Y & N & U & N / A\end{array}$

Y N N/A

$\begin{array}{lll}\mathrm{Y} N & \mathrm{~N} & \mathrm{~N} / \mathrm{A}\end{array}$

$Y \quad N \quad N / A$

$\begin{array}{lll}Y & N & N\end{array}$

$\begin{array}{llll}Y & N & U & N / A\end{array}$

$\begin{array}{lll}\mathrm{N} & \mathrm{U}\end{array}$

\section{Comments}


DOE Seismic Evaluation Procedure

SEWS 8.1 .3 (4 of 4)

Sheet 4 of

\section{SCREENING EVALUATION WORK SHEET (SEWS) (Cont.)}

Equipment ID No.:

Equipment Class: Low-Voltage Switchgear

Equipment description:

\section{Comments (Cont.)}

Screening Walkdown(s):

Date Time Team Members

\section{Recommend Resolution}

- Maintenance action:

Further evaluation:

- Retrofit design:

$\square \quad$ Other:

$\square \quad$ No further action required. Equipment is seismically adequate.

All aspects of the equipment's seismic adequacy have been addressed.

Evaluation by:

Date:

(All team members) 


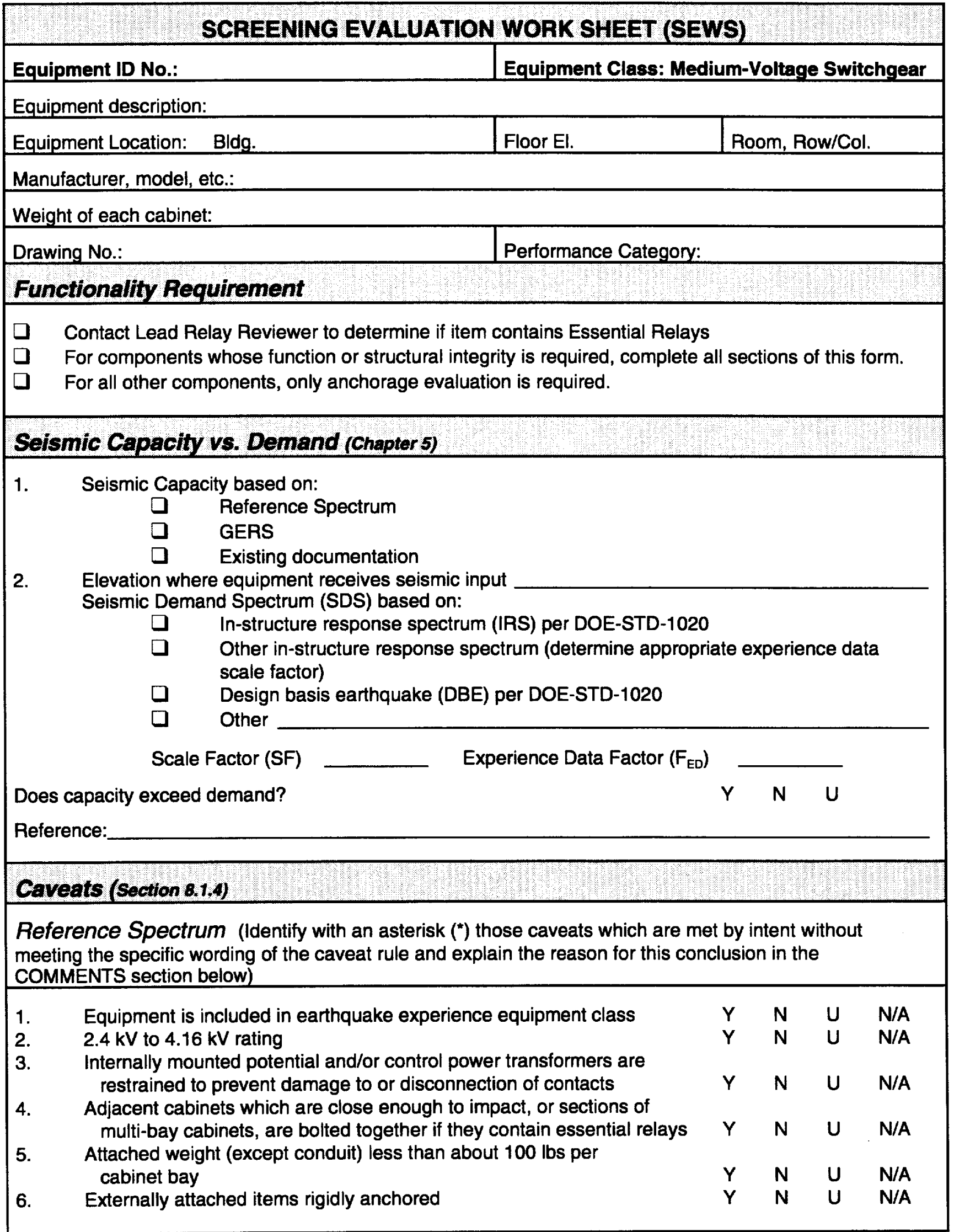




\section{SCREENING EVALUATION WORK SHEET (SEWS) (Cont.)}

Equipment ID No.:

Equipment Class: Medium-Voltage Switchgear

Equipment description:

\section{Caveats (Cont.)}

7. General configuration similar to ANSI C37.20 standards

8. Cutouts in lower half of cabinet side sheathing less than $30 \%$ of

width of side panel wide and less than $60 \%$ of width of side panel

excluding bus transfer compartment

9. All doors secured by latch or fastener

10. Have you looked for and found no other adverse concerns?

Is the intent of all the caveats met for Reference Spectrum?

$\begin{array}{llll}Y & N & U & N / A \\ Y & N & U & N / A \\ Y & N & U & N / A \\ Y & N & U & N / A \\ Y & N & U & N / A\end{array}$

GERS (Identify with an asterisk (*) those caveats which are met by intent without meeting the specific wording of the caveat rule and explain the reason for this conclusion in the COMMENTS section below)

1. Equipment is included in generic seismic testing equipment class

2. Meets all Reference Spectrum caveats

3. Floor-mounted enclosure

4. The switchgear is not a specially-designed type

5. Circuit breakers are truck-mounted type, not jack-up or vertical lift

6. Average weight per vertical section less than $5,000 \mathrm{lbs}$

7. For $2.5 \mathrm{~g}$ level GERS, vertical restraint prevents circuit breaker uplift

8. For $2.5 \mathrm{~g}$ level GERS, circuit break arc chutes are restrained horizontally

9. For $2.5 \mathrm{~g}$ level GERS, a Beaver Type $Z$ relay is not used in Westinghouse MV switchgear for the " $Y$ " anti-pump relay

10. Separate evaluation of breaker racking mechanism completed; seismic positioner or sufficient side-to-side restraints used

11. Adjacent cabinets which are close enough to impact, or sections of multi-bay cabinets, are bolted together

$\begin{array}{llll}Y & N & U & \text { N/A } \\ Y & N & U & \text { N/A } \\ Y & N & U & \text { N/A } \\ Y & N & U & \text { N/A } \\ Y & N & U & \text { N/A } \\ Y & N & U & \text { N/A } \\ Y & N & U & \text { N/A } \\ Y & N & U & \text { N/A } \\ Y & N & U & \text { N/A } \\ Y & N & U & \text { N/A } \\ Y & N & U & \text { N/A }\end{array}$

Is the intent of all the caveats met for GERS?

Y N U N/A

\section{Anchorage (Chapter 6)}

1. Type of anchorage:

$\begin{array}{ll}\square & \text { expansion anchor } \\ \square & \text { cast-in-place bolt or headed stud anchor } \\ \text { cast-in-place J-bolt } \\ \square & \text { grouted-in-place bolt } \\ \square & \text { welds to embedded steel on exposed steel } \\ \square & \text { lead cinch anchors } \\ \square & \text { Other } \\ \square & \text { N/A (no further anchorage considerations) }\end{array}$

2. Appropriate characteristics for anchorage type checked

(size, location, equipment characteristics)

$\begin{array}{lll}\mathrm{Y} & \mathrm{N}\end{array}$ 


\section{SCREENING EVALUATION WORK SHEET (SEWS) (Cont.)}

Equipment ID No.:

Equipment description:

\section{Anchorage (Cont.)}

3. Gap at threaded anchor less than 1/4 inch

4. Base stiffness and no significant prying action requirements met

5. Equipment base strength and structural load path adequate

6. Embedment steel and pads requirements met

7. Embedment length requirements met

8. Anchor spacing requirements met

9. Edge distance requirements met

10. Concrete strength requirements met

11. Concrete crack requirements met

12. Equipment with essential relays requirements met

13. Installation adequacy requirements met

14. No other concerns

$\begin{array}{llll}Y & N & U & \text { N/A } \\ Y & N & U & \\ Y & N & U & \\ Y & N & U & \text { N/A } \\ Y & N & U & \\ Y & N & U & \\ Y & N & U & \\ Y & N & U & \\ Y & N & U & \\ Y & N & U & \text { N/A } \\ Y & N & U & \text { N/A } \\ Y & N & U & \end{array}$

Does anchorage capacity exceed demand?

Y N U

Reference:

\section{Interaction Effects (Chapter 7)}

1. Soft targets free from impact by nearby equipment or structures

2. If equipment contains sensitive essential relays, equipment free from all impact by nearby equipment or structures

3. Attached lines have adequate flexibility

4. No collapse of overhead equipment, distribution systems, or masonry walls

5. Equipment is free from credible and significant seismic-induced flood and spray concerns

6. No credible seismic-induced fire concerns

7. No other "two over one" concerns as defined in DOE-STD-1021

8. No other concerns

$\begin{array}{llll}\mathrm{N} & \mathrm{U} & \mathrm{N} / \mathrm{A}\end{array}$

$\begin{array}{llll}Y & N & U & N / A\end{array}$

$\begin{array}{llll}Y & \mathrm{~N} & \mathrm{~N} / \mathrm{A}\end{array}$

Is equipment free of interaction effects?
$\begin{array}{llll}\mathrm{N} & \mathrm{U} & \mathrm{N} / \mathrm{A}\end{array}$

$\begin{array}{llll}Y & N & U & N / A\end{array}$

$Y \quad N \quad U \quad N / A$

$Y \quad N \quad N / A$

$\begin{array}{llll}Y & N & U & N / A\end{array}$

$\begin{array}{lll}\mathrm{N} & \mathrm{U}\end{array}$

\section{Comments}




\section{SCREENING EVALUATION WORK SHEET (SEWS) (Cont))}

Equipment ID No.:

Equipment Class: Medium-Voltage Switchgear

Equipment description:

\section{Comments (Cont.)}

Screening Walkdown(s):

Date Time Team Members

\section{Recommend Resolution}

D Maintenance action:

Further evaluation:

- Retrofit design:

$\square \quad$ Other:

$\square \quad$ No further action required. Equipment is seismically adequate.

All aspects of the equipment's seismic adequacy have been addressed.

Evaluation by:

Date:

(All team members) 


\section{SCREENING EVALUATION WORK SHEET (SEWS)}

Equipment ID No.:

Equipment Class: Distribution Panels

Equipment description:

System:

Equipment Location: Bldg.

Floor El.

Room, Row/Col.

Manufacturer, model, etc.:

Weight of each Panel:

Wall mounted:

Drawing No.:

Floor mounted:

Performance Category:

\section{Functionality Requirement}

- Contact Lead Relay Reviewer to determine if item contains Essential Relays

For components whose function or structural integrity is required, complete all sections of this form.

- For all other components, only anchorage evaluation is required.

\section{Seismic Capacity vs. Demand (Chapter 5)}

1. Seismic Capacity based on:

$\begin{array}{ll}\square & \text { Reference Spectrum } \\ \square & \text { GERS } \\ \square & \text { Existing documentation }\end{array}$

2. Elevation where equipment receives seismic input

Seismic Demand Spectrum (SDS) based on:

In-structure response spectrum (IRS) per DOE-STD-1020

$\square \quad$ Other in-structure response spectrum (determine appropriate experience data scale factor)

Design basis earthquake (DBE) per DOE-STD-1020

$\square \quad$ Other

Scale Factor (SF) _ Experience Data Factor $\left(F_{E D}\right)$

Does capacity exceed demand?

Reference:

\section{Caveats (Section 8.1.5)}

Reference Spectrum (Identify with an asterisk $\left(^{*}\right)$ those caveats which are met by intent without meeting the specific wording of the caveat rule and explain the reason for this conclusion in the COMMENTS section below)

\begin{tabular}{|c|c|c|c|c|c|}
\hline 1. & Equipment is included in earthquake experience equipment class & $\bar{Y}$ & $\mathbf{N}$ & $\bar{U}$ & N/A \\
\hline 2. & Contains only circuit breakers and switches & Y & N & U & N/A \\
\hline 3. & All latches and fasteners in door secured & $\mathrm{Y}$ & $\mathbf{N}$ & U & N/A \\
\hline 4. & $\begin{array}{l}\text { Adjacent cabinets which are close enough to impact, } \\
\text { or sections of multi-bay cabinets, are bolted together } \\
\text { if they contain essential relays }\end{array}$ & $Y$ & $N$ & $U$ & N/A \\
\hline 5. & Wall- or floor-mounted NEMA type-enclosure & Y & $\mathbf{N}$ & $U$ & N/A \\
\hline
\end{tabular}




\section{SCREENING EVALUATION WORK SHEET (SEWS) (Cont.)}

\section{Equipment ID No.:}

Equipment description:

\section{Caveats (Cont.)}

6. Have you looked for and found no other adverse concerns?

$\begin{array}{llll}Y & N & U & N / A \\ Y & N & U & N / A\end{array}$

Is the intent of all the caveats met for Reference Spectrum?

Y

GERS (Identify with an asterisk (") those caveats which are met by intent without meeting the specific wording of the caveat rule and explain the reason for this conclusion in the COMMENTS section below)

1. Equipment is included in generic seismic testing equipment class

2. Meets all Reference Spectrum caveats

$Y \quad N \quad U \quad N / A$

3. If Switchboard GERS used, item is freestanding and designated as

a switchboard by the manufacturer

4. No Westinghouse Quicklag Type E Breakers

5. Adjacent cabinets which are close enough to impact are bolted together

Is the intent of all the caveats met for GERS?

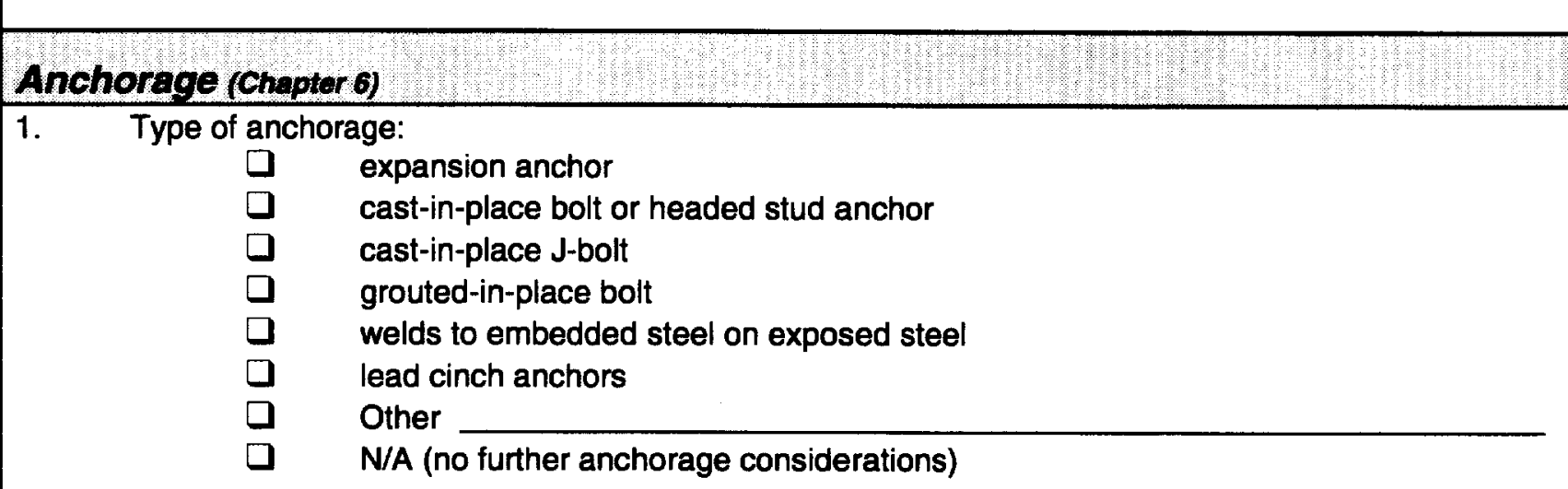

2. Appropriate characteristics for anchorage type checked

(size, location, equipment characteristics)

3. Gap at threaded anchor less than $1 / 4$ inch

4. Base stiffness and no significant prying action requirements met

5. Equipment base strength and structural load path adequate

6. Embedment steel and pads requirements met

7. Embedment length requirements met

8. Anchor spacing requirements met

9. Edge distance requirements met

10. Concrete strength requirements met

$\begin{array}{lll}Y & N & U \\ Y & N & U \\ Y & N & U \\ Y & N & U \\ Y & N & U \\ Y & N & U \\ Y & N & U \\ Y & N & U \\ Y & N & U\end{array}$




\section{SCREENING EVALUATION WORK SHEET (SEWS) (Cont.)}

Equipment ID No.:

Equipment Class: Distribution Panels

Equipment description:

\section{Anchorage (Cont.)}

11. Concrete crack requirements met

12. Equipment with essential relays requirements met

13. Installation adequacy requirements met

14. No other concerns

Does anchorage capacity exceed demand?

$\begin{array}{llll}Y & N & U & \\ Y & N & U & N / A \\ Y & N & U & N / A \\ Y & N & U & \end{array}$

Reference:

\section{Interaction Effects (Chapter 7)}

1. Soft targets free from impact by nearby equipment

or structures

2. If equipment contains sensitive essential relays, equipment free from all impact by nearby equipment or structures

3. Distribution lines have adequate flexibility

4. No collapse of overhead equipment, distribution systems, or masonry walls

5. Equipment is free from credible and significant seismic-induced flood and spray concerns

6. No credible seismic-induced fire concerns

7. No other "two over one" concerns as defined in DOE-STD-1021

8. No other concerns

$\begin{array}{lll}Y & \mathrm{~N}\end{array}$

Is equipment free of interaction effects?

$\begin{array}{llll}Y & N & U & \text { N/A } \\ Y & N & U & \text { N/A } \\ Y & N & U & \text { N/A } \\ Y & N & & \text { N/A } \\ Y & N & & \text { N/A } \\ Y & N & & \text { N/A } \\ Y & N & & \text { N/A } \\ Y & N & U & \text { N/A } \\ Y & N & U & \end{array}$

\section{Comments}


DOE Seismic Evaluation Procedure

SEWS 8.1 .5 (4 of 4$)$

Sheet 4 of

\section{SCREENING EVALUATION WORK SHEET (SEWS) (Cont.)}

Equipment ID No.:

Equipment Class: Distribution Panels

Equipment description:

\section{Comments (Cont.)}

Screening Walkdown(s):

Date Time Team Members

\section{Recommend Resolution}

- Maintenance action:

$\square \quad$ Further evaluation:

Retrofit design:

O Other:

No further action required. Equipment is seismically adequate.

All aspects of the equipment's seismic adequacy have been addressed.

Evaluation by:

Date:

(All team members) 


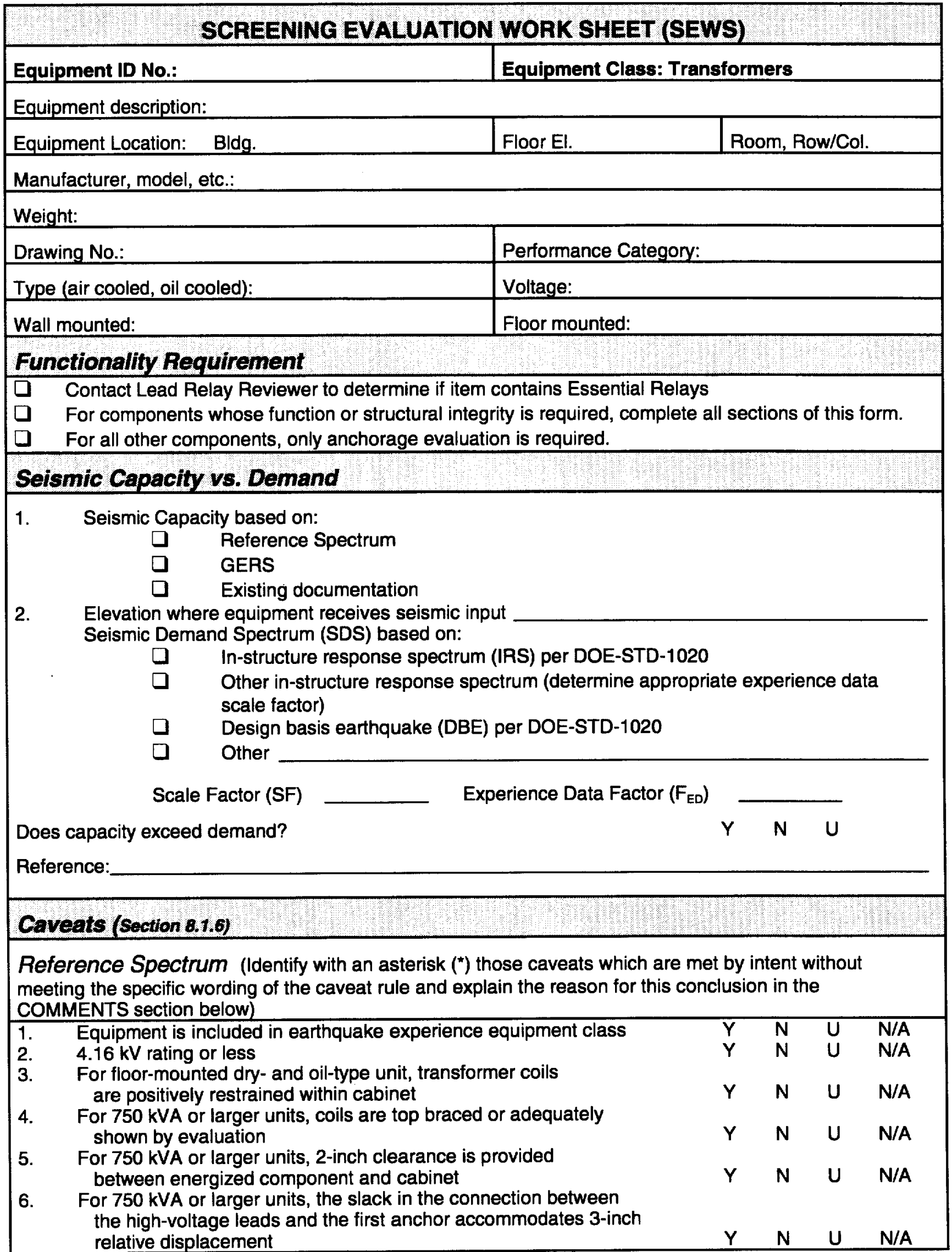




\section{SCREENING EVALUATION WORK SHEET (SEWS) (Cont.)}

Equipment ID No.:

Equipment Class: Transformers

Equipment description:

\section{Caveats (Cont.)}

7. For wall-mounted units, transformer coils anchored to enclosure near enclosure support surface

8. For floor-mounted units, anchorage does not rely on weak-way bending of cabinet structures under lateral forces

9. Adjacent cabinets which are close enough to impact are bolted together if they contain essential relays

10. All doors secured by latch or fastener

11. Have you looked for and found no other adverse concerns?

Is the intent of all the caveats met for Reference Spectrum?

$\begin{array}{llll}Y & N & U & \text { N/A } \\ Y & N & U & \text { N/A } \\ Y & N & U & \text { N/A } \\ Y & N & U & N / A \\ Y & N & U & \text { N/A } \\ Y & N & U & \text { N/A }\end{array}$

GERS (Identify with an asterisk (*) those caveats which are met by intent without meeting the specific wording of the caveat rule and explain the reason for this conclusion in the COMMENTS section below)

\begin{tabular}{llllll}
\hline 1. & Equipment is included in generic seismic testing equipment class & $Y$ & $N$ & $U$ & $N / A$
\end{tabular}

2. Meets all Reference Spectrum caveats

3. Dry-type unit (not oil-filled)

4. Wall- or floor-mounted NEMA-type enclosure

$5 . \quad 120$ to $480 \mathrm{VAC}$ rating

6. $\quad 7.5$ to $225 \mathrm{kVA}$ rating

7. 180 to 2,000 pounds weight

8. Internal supports provide positive attachment of transformer components

9. There is a sufficient clearance of $3 / 8$ inches between bare conductors and enclosure

10. Adjacent cabinets which are close enough to impact are bolted together Is the intent of all the caveats met for GERS?

$\begin{array}{llll}Y & N & U & \text { N/A } \\ Y & N & U & \text { N/A } \\ Y & N & U & \text { N/A } \\ Y & N & U & \text { N/A } \\ Y & N & U & \text { N/A } \\ Y & N & U & \text { N/A } \\ Y & N & U & \text { N/A } \\ Y & N & U & \text { N/A } \\ Y & N & U & \text { N/A } \\ Y & N & U & \text { N/A }\end{array}$

\section{Anchorage (chapter 6)}

1. Type of anchorage:

$\begin{array}{ll}\square & \text { expansion anchor } \\ \square & \text { cast-in-place bolt or headed stud anchor } \\ \square & \text { cast-in-place J-bolt } \\ \square & \text { grouted-in-place bolt } \\ \square & \text { welds to embedded steel on exposed steel } \\ \square & \text { lead cinch anchors } \\ \square & \text { Other } \\ \square & \text { N/A (no further anchorage considerations) }\end{array}$

2. Appropriate characteristics for anchorage type checked

(size, location, equipment characteristics)

3. Gap at threaded anchor less than $1 / 4$ inch

4. Base stiffness and no significant prying action requirements met

5. Equipment base strength and structural load path adequate

6. Embedment steel and pads requirements met

7. Embedment length requirements met

8. Anchor spacing requirements met

9. Edge distance requirements met

10. Concrete strength requirements met

$\begin{array}{llll}Y & N & U & \\ Y & N & U & N / A \\ Y & N & U & \\ Y & N & U & \\ Y & N & U & N / A \\ Y & N & U & \\ Y & N & U & \\ Y & N & U & \\ Y & N & U & \end{array}$




\section{DOE Seismic Evaluation Procedure}

SEWS 8.1.6 ( 3 of 4$)$

Sheet 3 of

\section{SCREENING EVALUATION WORK SHEET (SEWS) (Cont.)}

Equipment ID No.:

Equipment Class: Transformers

Equipment description:

Anchorage (Cont.)

11. Concrete crack requirements met

12. Equipment with essential relays requirements met

13. Installation adequacy requirements met

14. No other concerns

Does anchorage capacity exceed demand?

$\begin{array}{llll}Y & N & U & \\ Y & N & U & \text { N/A } \\ Y & N & U & \text { N/A } \\ Y & N & U & \end{array}$

Reference:

Interaction Effects (Chapter 7)

1. Soft targets free from impact by nearby equipment or structures

2. If equipment contains sensitive essential relays, equipment free from all impact by nearby equipment or structures

3. Attached lines have adequate flexibility

4. No collapse of overhead equipment, distribution systems, or masonry walls

5. Equipment is free from credible and significant seismic-induced flood and spray concerns

6. No credible seismic-induced fire concerns

7. No other "two over one" concerns as defined in DOE-STD-1021

8. No other concerns

$\begin{array}{lll}Y & N & U\end{array}$

Is equipment free of interaction effects?

$\begin{array}{llll}\mathrm{N} & \mathrm{N} & \mathrm{N} / \mathrm{A}\end{array}$

$\begin{array}{llll}Y & N & U & N / A\end{array}$

$Y \quad N \quad U \quad N / A$

$\begin{array}{llll}Y & N & U & \text { N/A }\end{array}$

$\begin{array}{llll}Y & N & U & N / A\end{array}$

$\begin{array}{lll}Y & N & N\end{array}$

$\begin{array}{lll}Y & N & N\end{array}$

$\begin{array}{llll}Y & N & U & N / A\end{array}$

$\begin{array}{lll}\mathrm{N} & \mathrm{U}\end{array}$

\section{Comments}




\section{DOE Seismic Evaluation Procedure}

SEWS 8.1.6 (4 of 4$)$

Sheet 4 of

\section{SCREENING EVALUATION WORK SHEET (SEWS) (Cont.)}

Equipment ID No.:

Equipment Class: Transformers

Equipment description:

Comments (cont)

Screening Walkdown(s):

Date Time Team Members

\section{Recommend Resolution}

- Maintenance action:

$\square \quad$ Further evaluation:

Retrofit design:

$\square$ Other:

a No further action required. Equipment is seismically adequate.

All aspects of the equipment's seismic adequacy have been addressed.

Evaluation by:

Date:

(All team members) 
Sheet 1 of

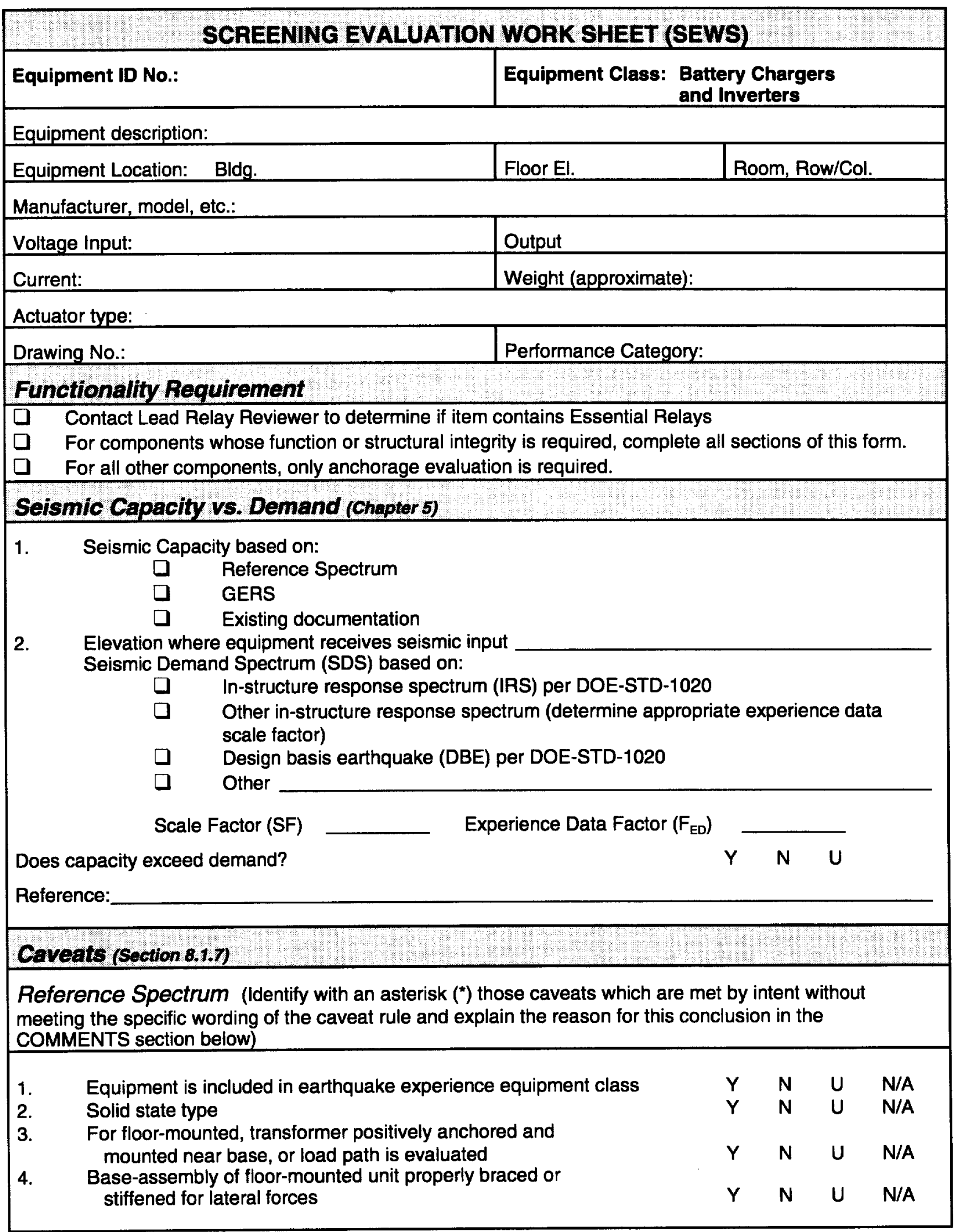




\section{SCREENING EVALUATION WORK SHEET (SEWS) (Cont.)}

Equipment ID No.:

Equipment Class: Battery Chargers

and Inverters

Equipment description:

Caveats (Cont)

5. For wall-mounted units, transformer supports and bracing

provide adequate load path to the rear cabinet wall

6. All latches and fasteners in doors secured

7. Adjacent cabinets which are close enough to impact are bolted

together if they contain essential relays

8. Have you looked for and found no other adverse concerns?

Is the intent of all the caveats met for Reference Spectrum?

$\begin{array}{llll}Y & N & U & N / A\end{array}$

$Y \quad N \quad U \quad N / A$

$\begin{array}{llll}Y & N & U & N / A\end{array}$

$\begin{array}{llll}Y & N & U & N / A\end{array}$

$\begin{array}{llll}\mathrm{N} & \mathrm{U} & \mathrm{N} / \mathrm{A}\end{array}$

GERS (Identify with an asterisk (*) those caveats which are met by intent without meeting the specific wording of the caveat rule and explain the reason for this conclusion in the COMMENTS section below)

1. Equipment is included in generic seismic testing equipment class

2. Meets all Reference Spectrum caveats

3. Silicon-controlled Rectifier (SCR) power controls; wall- or

floor-mounted NEMA-type enclosure

4. Within range of battery charger ratings:

$$
\begin{aligned}
24-250 & \text { VDC } \\
120-480 & \text { VAC } \\
25-600 & \text { amps } \\
150-2,850 & \text { pounds (floor mounted) } \\
150-600 & \text { pounds (wall mounted) }
\end{aligned}
$$

5. Within range of inverter ratings:

$$
\begin{aligned}
120 & \text { VDC only } \\
120-480 & \text { VAC } \\
0.5-15 & \text { kVA } \\
300-2,000 & \text { pounds }
\end{aligned}
$$

6. Heavy components are located in lower half of cabinet and

are supported from base or rear panel with no panel cutouts adjacent to attachment

7. Adjacent cabinets which are close enough to impact are bolted together

Is the intent of all the caveats met for GERS?

$\begin{array}{llll}Y & N & U & N / A\end{array}$

$Y \quad N \quad U \quad N / A$

$\begin{array}{llll}\mathrm{N} & \mathrm{U} & \mathrm{N} / \mathrm{A}\end{array}$

$\begin{array}{llll}Y & N & U & N / A\end{array}$

$\begin{array}{llll}Y & N & U & N\end{array}$

$\begin{array}{llll}Y & N & U & N / A\end{array}$

$\begin{array}{llll}Y & N & U & \text { N/A }\end{array}$

$Y \quad N \quad U \quad N / A$

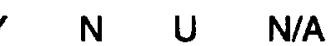

$\begin{array}{llll}Y & N & U & N / A\end{array}$

$\begin{array}{llll}Y & N & U & N / A\end{array}$

$Y \quad N \quad U \quad N / A$

Anchorage (Chapter 6)

1. Type of anchorage:

$\begin{array}{ll}\square & \text { expansion anchor } \\ \square & \text { cast-in-place bolt or headed stud anchor } \\ \square & \text { cast-in-place J-bolt } \\ \square & \text { grouted-in-place bolt } \\ \square & \text { welds to embedded steel on exposed steel } \\ \square & \text { lead cinch anchors } \\ \square & \text { Other } \\ \square & \text { N/A (no further anchorage considerations) }\end{array}$




\section{SCREENING EVALUATION WORK SHEET (SEWS) (Cont.)}

Equipment ID No.:

Equipment description:

\section{Anchorage (Cont)}

2. Appropriate characteristics for anchorage type checked

(size, location, equipment characteristics)

3. Gap at threaded anchor less than $1 / 4$ inch

4. Base stiffness and no significant prying action requirements met

5. Equipment base strength and structural load path adequate

6. Embedment steel and pads requirements met

7. Embedment length requirements met

8. Anchor spacing requirements met

9. Edge distance requirements met

10. Concrete strength requirements met

11. Concrete crack requirements met

12. Equipment with essential relays requirements met

13. Installation adequacy requirements met

14. No other concerns

Does anchorage capacity exceed demand?

$\begin{array}{llll}Y & N & U & \\ Y & N & U & \text { N/A } \\ Y & N & U & \\ Y & N & U & \\ Y & N & U & \text { N/A } \\ Y & N & U & \\ Y & N & U & \\ Y & N & U & \\ Y & N & U & \\ Y & N & U & \\ Y & N & U & \text { N/A } \\ Y & N & U & \text { N/A } \\ Y & N & U & \\ Y & N & U & \end{array}$

Reference:

\section{Interaction Effects (Chapter 7)}

1. Soft targets free from impact by nearby equipment

or structures

2. If equipment contains sensitive essential relays, equipment free from all impact by nearby equipment or structures

3. Attached lines have adequate flexibility

4. No collapse of overhead equipment, distribution systems, or masonry walls

5. Equipment is free from credible and significant seismic-induced flood and spray concerns

6. No credible seismic-induced fire concerns

7. No other "two over one" concerns as defined in DOE-STD-1021

8. No other concerns

is equipment free of interaction effects? 


\section{DOE Seismic Evaluation Procedure}

SEWS 8.1.7 (4 of 4)

Sheet 4 of

\section{SCREENING EVALUATION WORK SHEET (SEWS) (Cont.)}

Equipment ID No.:

Equipment Class: Battery Chargers

and Inverters

Equipment description:

Comments (Cont)

Screening Walkdown(s):

Date Time Team Members

\section{Recommend Resolution}

ㅁ Maintenance action:

Further evaluation:

$\square \quad$ Retrofit design:

Other:

No further action required. Equipment is seismically adequate.

All aspects of the equipment's seismic adequacy have been addressed.

Evaluation by:

Date:

(All team members) 


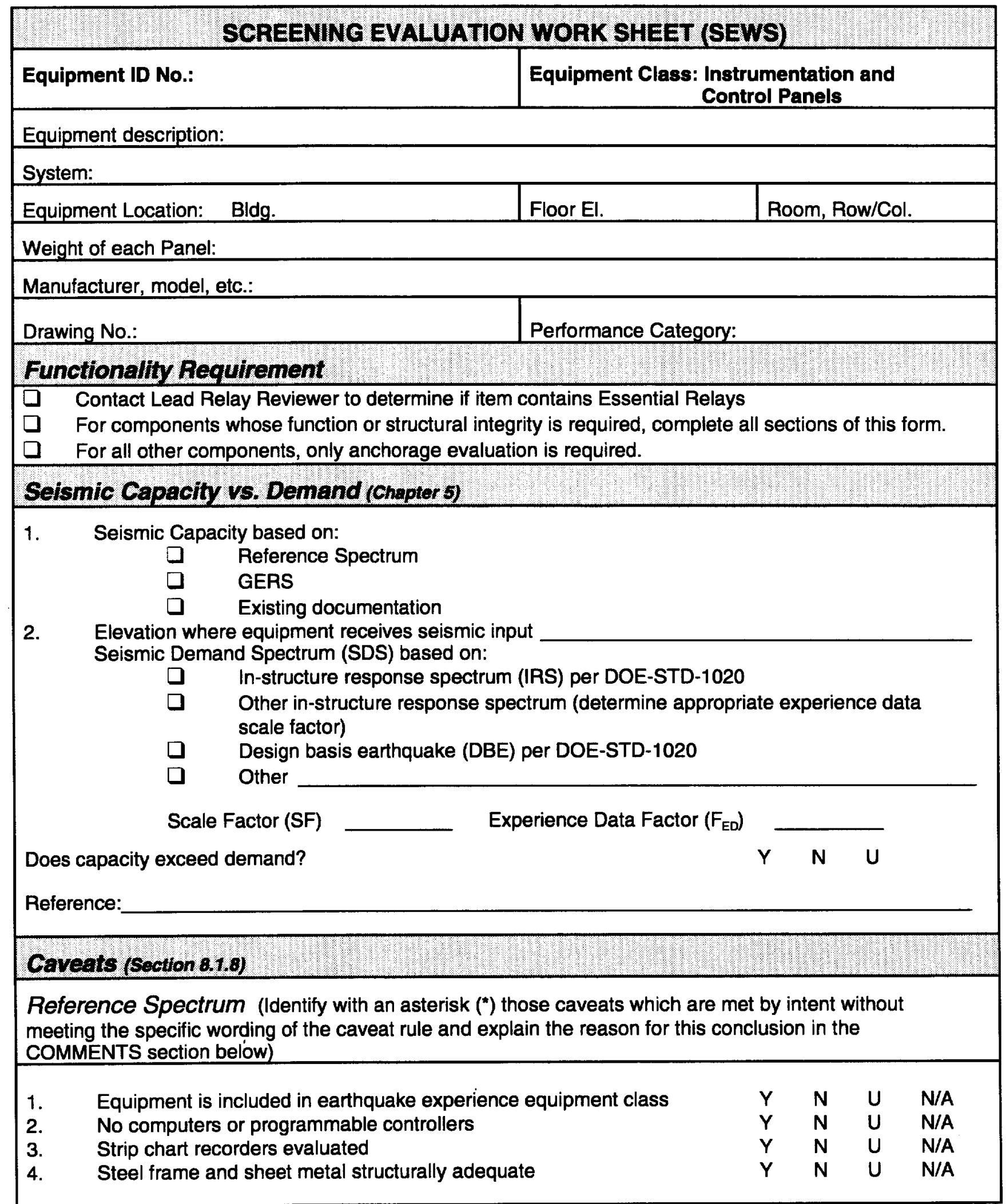




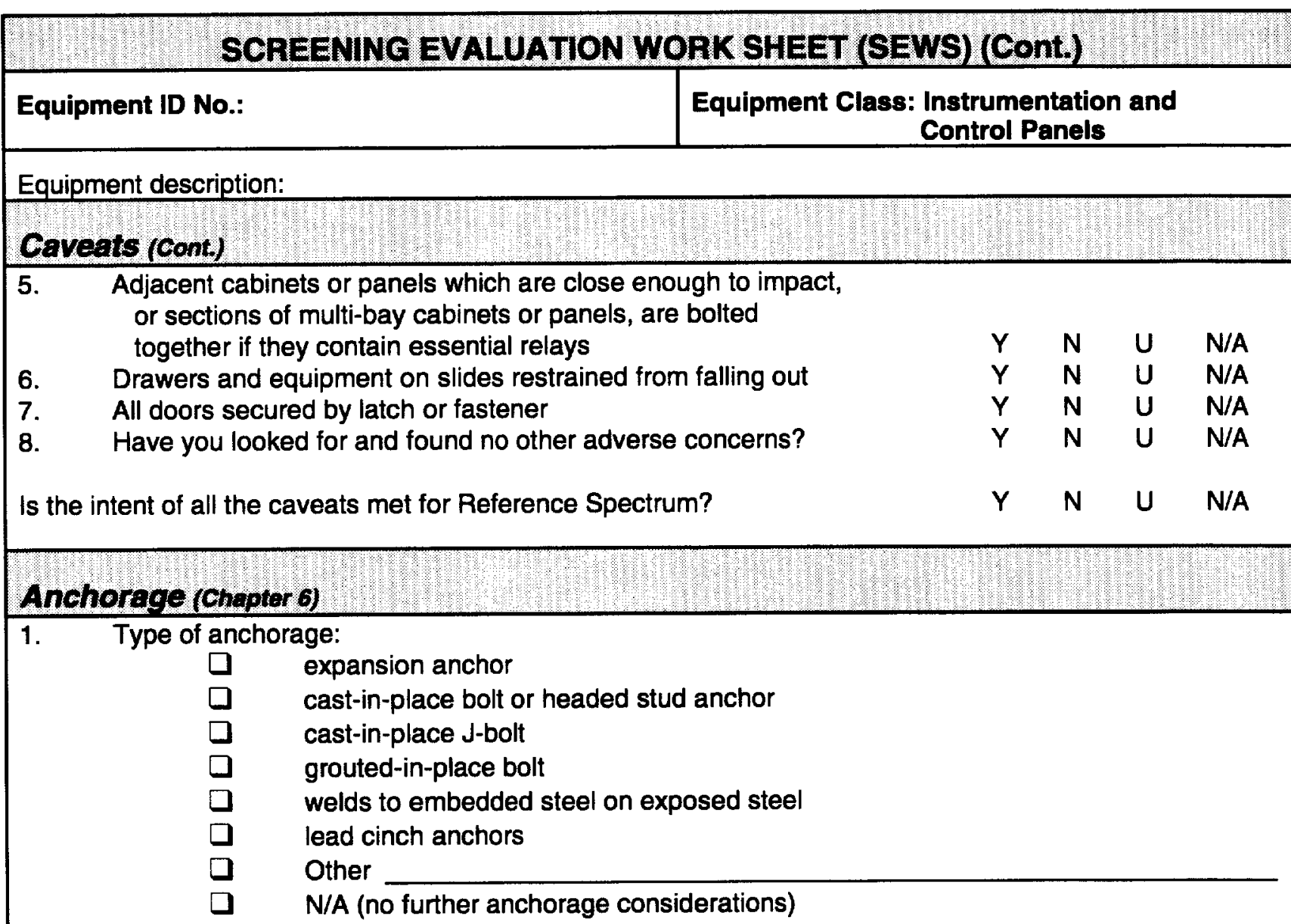

2. Appropriate characteristics for anchorage type checked

(size, location, equipment characteristics)

3. Gap at threaded anchor less than 1/4 inch

4. Base stiffness and no significant prying action requirements met

5. Equipment base strength and structural load path adequate

6. Embedment steel and pads requirements met

7. Embedment length requirements met

8. Anchor spacing requirements met

9. Edge distance requirements met

10. Concrete strength requirements met

11. Concrete crack requirements met

12. Equipment with essential relays requirements met

13. Installation adequacy requirements met

14. No other concerns

$\begin{array}{llll}Y & N & U & \\ Y & N & U & \text { N/A } \\ Y & N & U & \\ Y & N & U & \\ Y & N & U & N / A \\ Y & N & U & \\ Y & N & U & \\ Y & N & U & \\ Y & N & U & \\ Y & N & U & \\ Y & N & U & N / A \\ Y & N & U & N / A \\ Y & N & U & \\ Y & N & U & \\ Y & N & \end{array}$

Does anchorage capacity exceed demand?

Reference: 


\section{SCREENING EVALUATION WORK SHEET (SEWS) (Cont.)}

Equipment ID No.:

Equipment Class: Instrumentation and Control Panels

Equipment description:

Interaction Effects (Chaptern)

1. Soft targets free from impact by nearby equipment or structures

2. If equipment contains sensitive essential relays, equipment free from all impact by nearby equipment or structures

3. Attached lines have adequate flexibility

4. No collapse of overhead equipment, distribution systems, or masonry walls

5. Equipment is free from credible and significant seismic-induced flood and spray concerns

6. No credible seismic-induced fire concerns

7. No other "two over one" concerns as defined in DOE-STD-1021

8. No other concerns

Is equipment free of interaction effects?

$\begin{array}{llll}\mathrm{N} & \mathrm{U} & \mathrm{N} / \mathrm{A}\end{array}$

$\begin{array}{llll}Y & N & U & N / A\end{array}$

$Y \quad N \quad U \quad N / A$

Y N U N/A

$\begin{array}{llll}Y & N & U & N / A\end{array}$

$Y \quad N \quad N / A$

$Y \quad N \quad N / A$

$\begin{array}{llll}Y & N & \mathrm{~N} / \mathrm{A}\end{array}$

$\begin{array}{lll}Y & N & U\end{array}$

\section{Comments}




\section{DOE Seismic Evaluation Procedure}

SEWS 8.1 .8 (4 of 4$)$

Sheet 4 of

\section{SCREENING EVALUATION WORK SHEET (SEWS) (Cont.)}

Equipment ID No.:

Equipment Class: Instrumentation and Control Panels

Equipment description:

Comments (Cont)

Screening Walkdown(s):

Date Time $\quad \underline{\text { Team Members }}$

\section{Fecommend Resolution}

ㅁ Maintenance action:

Further evaluation:

$\square \quad$ Retrofit design:

$\square$ Other:

No further action required. Equipment is seismically adequate.

All aspects of the equipment's seismic adequacy have been addressed.

Evaluation by:

Date:

(All team members) 


\section{SCREENING EVALUATION WORK SHEET (SEWS)}

Equipment ID No.:

Equipment Class: Instruments on Racks

Equipment description:

Equipment Location: Bldg Floor El. Room, Row/Col.

Manufacturer, model, etc.:

Weight:

Drawing No.:

Performance Category:

\section{Functionality Requirement}

C Contact Lead Relay Reviewer to determine if item contains Essential Relays

- For components whose function or structural integrity is required, complete all sections of this form.

F For all other components, only anchorage evaluation is required.

\section{Seismic Capacity vs. Demand (Chapter 5)}

1. Seismic Capacity based on:
D Reference Spectrum
G GERS
Existing documentation

2. Elevation where equipment receives seismic input

Seismic Demand Spectrum (SDS) based on:
In-structure response spectrum (IRS) per DOE-STD-1020
- Other in-structure response spectrum (determine appropriate experience data scale factor)
Design basis earthquake (DBE) per DOE-STD-1020
a Other

Scale Factor (SF)

Experience Data Factor $\left(F_{\mathrm{ED}}\right)$

Does capacity exceed demand?

Y N U

Reference:

\section{Caveats (Section 8.1.9)}

Reference Spectrum (Identify with an asterisk ( ${ }^{*}$ ) those caveats which are met by intent without meeting the specific wording of the caveat rule and explain the reason for this conclusion in the COMMENTS section below)

1. Equipment is included in earthquake experience equipment class

2. No computers or programmable controllers

3. Steel frame and sheet metal structurally adequate

4. Adjacent racks which are close enough to impact, or sections

of multi-bay racks, are bolted together if they contain essential relays

5. Have you looked for and found no other adverse concerns?

$\begin{array}{llll}\mathrm{Y} & \mathrm{N} & \mathrm{U} & \mathrm{N} / \mathrm{A} \\ \mathrm{Y} & \mathrm{N} & \mathrm{U} & \mathrm{N} / \mathrm{A} \\ \mathrm{Y} & \mathrm{N} & \mathrm{U} & \mathrm{N} / \mathrm{A}\end{array}$

Is the intent of all the caveats met for Reference Spectrum?

$\begin{array}{llll}Y & N & U & N / A\end{array}$

$\begin{array}{llll}Y & N & U & N / A\end{array}$

is the intent of all the caveats met for Reference Spectum?

$\begin{array}{llll}Y & \mathrm{~N} & \mathrm{~N} / \mathrm{A}\end{array}$ 
Sheet 2 of

\section{SCREENING EVALUATION WORK SHEET (SEWS) (Cont.)}

Equipment ID No.:

Equipment Class: Instruments on Racks

Equipment description:

\section{Caveats (Cont)}

GERS (Identify with an asterisk (*) those caveats which are met by intent without meeting the specific wording of the caveat rule and explain the reason for this conclusion in the COMMENTS section below)

1. Equipment is included in generic seismic testing equipment class

2. Meets all Reference Spectrum caveats

3. Component is a pressure, temperature, level or flow transmitter

4. Component is one of the specific makes and models tested

5. Necessary function of component not sensitive to seismically induced system perturbations (e.g., sloshing)

6. No vacuum tubes

7. All external mounting bolts in place

8. Demand based on amplified portion of $3 \%$ damped floor response spectrum if estimated natural frequency of rack less than 33 Hertz

9. Rack capable of structurally transferring GERS level seismic loads to anchorage

10. Adjacent racks which are close enough to impact, or sections of multi-bay racks, are bolted together

$\begin{array}{llll}Y & N & U & \text { N/A } \\ Y & N & U & \text { N/A } \\ Y & N & U & \text { N/A } \\ Y & N & U & \text { N/A } \\ Y & N & U & \text { N/A } \\ Y & N & U & \text { N/A } \\ Y & N & U & \text { N/A } \\ Y & N & U & \text { N/A } \\ Y & N & U & \text { N/A } \\ Y & N & U & \text { N/A } \\ Y & N & U & \text { N/A }\end{array}$

Is the intent of all the caveats met for GERS?

\section{Anchorage (Chepter 6$)$}

1. Type of anchorage:

$\begin{array}{ll}\square & \text { expansion anchor } \\ \square & \text { cast-in-place bolt or headed stud anchor } \\ \square & \text { cast-in-place J-bolt } \\ \square & \text { grouted-in-place bolt } \\ \square & \text { welds to embedded steel on exposed steel } \\ \square & \text { lead cinch anchors } \\ \square & \text { Other } \\ \square & \text { N/A (no further anchorage considerations) }\end{array}$

2. Appropriate characteristics for anchorage type checked

(size, location, equipment characteristics)

3. Gap at threaded anchor less than $1 / 4$ inch

4. Base stiffness and no significant prying action requirements met

5. Equipment base strength and structural load path adequate

6. Embedment steel and pads requirements met

7. Embedment length requirements met

8. Anchor spacing requirements met

9. Edge distance requirements met

10. Concrete strength requirements met

$\begin{array}{llll}Y & N & U & \\ Y & N & U & N / A \\ Y & N & U & \\ Y & N & U & \\ Y & N & U & N / A \\ Y & N & U & \\ Y & N & U & \\ Y & N & U & \\ Y & N & U & \end{array}$




\section{SCREENING EVALUATION WORK SHEET (SEWS) (Cont.)}

Equipment ID No.:

Equipment Class: Instruments on Racks

Equipment description:

\section{Anchorage (Cont)}

11. Concrete crack requirements met

12. Equipment with essential relays requirements met

13. Installation adequacy requirements met

14. No other concerns

Does anchorage capacity exceed demand?

$\begin{array}{llll}Y & N & U & \\ Y & N & U & N / A \\ Y & N & U & N / A \\ Y & N & U & \end{array}$

Reference:

Interaction Effects (Chapter 7 )

1. Soft targets free from impact by nearby equipment or structures

2. If equipment contains sensitive essential relays, equipment free from all impact by nearby equipment or structures

3. Attached lines have adequate flexibility

4. No collapse of overhead equipment, distribution systems, or masonry walls

5. Equipment is free from credible and significant seismic-induced flood and spray concerns

6. No credible seismic-induced fire concerns

7. No other "two over one" concerns as defined in DOE-STD-1021

8. No other concerns

$\begin{array}{lll}\mathrm{N} & \mathrm{N}\end{array}$

Is equipment free of interaction effects?

$\begin{array}{llll}\mathrm{N} & \mathrm{U} & \mathrm{N} / \mathrm{A}\end{array}$

Y N U N/A

$\begin{array}{llll}Y & N & U & N / A\end{array}$

$\begin{array}{lll}\mathrm{N} & \mathrm{N} / \mathrm{A}\end{array}$

$\begin{array}{lll}Y & N & N\end{array}$

$\begin{array}{lll}Y & N & N\end{array}$

$\begin{array}{lll}Y & N & N / A\end{array}$

$\begin{array}{llll}Y & N & U & \text { N/A }\end{array}$

$Y \quad \mathrm{~N}$
$\mathbf{Y}$

\section{Comments}




\section{DOE Seismic Evaluation Procedure}

SEWS 8.1.9 (4 of 4)

Sheet 4 of

\section{SCREENING EVALUATION WORK SHEET (SEWS) (Cont)}

Equipment ID No.:

Equipment Class: Instruments on Racks

Equipment description:

Comments (Cont)

Screening Walkdown(s):

Date Time Team Members

\section{Recommend Resolution}

a Maintenance action:

- Further evaluation:

ㅁ Retrofit design:

Other:

[ No further action required. Equipment is seismically adequate.

All aspects of the equipment's seismic adequacy have been addressed.

Evaluation by:

Date:

(All team members) 


\section{SCREENING EVALUATION WORK SHEET (SEWS)}

Equipment ID No.:

Equipment Class: Temperature Sensors

Equipment description:

System:

Equipment Location: BIdg.

Floor El. Room, Row/Col.

Manufacturer, model, etc.:

Approximate Weight:

Drawing No.:

Performance Category:

\section{Functionality Requirement}

Contact Lead Relay Reviewer to determine if item contains Essential Relays

For components whose function or structural integrity is required, complete all sections of this form.

For all other components, only anchorage evaluation is required.

\section{Seismic Capacity vs. Demand (Chapter 5)}

1. Seismic Capacity based on:

$\begin{array}{ll}\square & \text { Reference Spectrum } \\ \square & \text { GERS } \\ \square & \text { Existing documentation }\end{array}$

2. Elevation where equipment receives seismic input

Seismic Demand Spectrum (SDS) based on:

In-structure response spectrum (IRS) per DOE-STD-1020

$\square \quad$ Other in-structure response spectrum (determine appropriate experience data scale factor)

Design basis earthquake (DBE) per DOE-STD-1020

$\square \quad$ Other

Scale Factor (SF)

Experience Data Factor $\left(F_{E D}\right)$

Does capacity exceed demand?

Y N U

Reference:

\section{Caveats (Section 8.1.10)}

Reference Spectrum (Identify with an asterisk (*) those caveats which are met by intent without meeting the specific wording of the caveat rule and explain the reason for this conclusion in the COMMENTS section below)

$\begin{array}{lllllll}\text { 1. } & \text { Equipment is included in earthquake experience equipment class } & Y & N & U & \text { N/A }\end{array}$

2. No possibility of detrimental differential displacement between mounting of connection head and mounting of temperature sensor

3. Associated electronics are all solid state (no vacuum tubes)

4. Have you looked for and found no other adverse concerns?

$\begin{array}{llll}\mathrm{N} & \mathrm{N} & \mathrm{N} / \mathrm{A}\end{array}$

$Y \quad N \quad U \quad N / A$

Is the intent of all the caveats met for Reference Spectrum?

$Y \quad N \quad U \quad N / A$

$\begin{array}{llll}\mathrm{N} & \mathrm{U} & \mathrm{N} / \mathrm{A}\end{array}$




\section{SCREENING EVALUATION WORK SHEET (SEWS) (Cont.)}

Equipment ID No.: Equipment Class: Temperature Sensors

Equipment description:

\section{Anchorage (Chapter 6)}

1. Type of anchorage:

$\begin{array}{ll}\square & \text { expansion anchor } \\ \square & \text { cast-in-place bolt or headed stud anchor } \\ \square & \text { cast-in-place J-bolt } \\ \square & \text { grouted-in-place bolt } \\ \square & \text { welds to embedded steel on exposed steel } \\ \square & \text { lead cinch anchors } \\ \square & \text { Other } \\ \square & \text { N/A (no further anchorage considerations) }\end{array}$

2. Appropriate characteristics for anchorage type checked

(size, location, equipment characteristics)

3. Gap at threaded anchor less than $1 / 4$ inch

4. Base stiffness and no significant prying action requirements met

5. Equipment base strength and structural load path adequate

6. Embedment steel and pads requirements met

7. Embedment length requirements met

8. Anchor spacing requirements met

9. Edge distance requirements met

10. Concrete strength requirements met

11. Concrete crack requirements met

12. Equipment with essential relays requirements met

13. Installation adequacy requirements met

14. No other concerns

$\begin{array}{llll}Y & N & U & \\ Y & N & U & \text { N/A } \\ Y & N & U & \\ Y & N & U & \\ Y & N & U & \text { N/A } \\ Y & N & U & \\ Y & N & U & \\ Y & N & U & \\ Y & N & U & \\ Y & N & U & \\ Y & N & U & \text { N/A } \\ Y & N & U & \text { N/A } \\ Y & N & U & \\ Y & N & U & \\ & & & \end{array}$

Does anchorage capacity exceed demand?

Reference:

\section{Interaction Effects (Chapter 7$)$}

1. Soft targets free from impact by nearby equipment or structures

2. If equipment contains sensitive essential relays, equipment free from all impact by nearby equipment or structures

3. Attached lines have adequate flexibility

4. No collapse of overhead equipment, distribution systems, or masonry walls

5. Equipment is free from credible and significant seismic-induced flood and spray concerns

6. No credible seismic-induced fire concerns

7. No other "two over one" concerns as defined in DOE-STD-1021

8. No other concerns

Is equipment free of interaction effects?

$\begin{array}{llll}Y & N & \mathrm{~N} & \end{array}$

$\begin{array}{llll}Y & N & U & N / A\end{array}$

$Y \quad N \quad U \quad N / A$

$\mathrm{Y} N \quad \mathrm{~N} / \mathrm{A}$

$\begin{array}{lll}Y & N & N\end{array}$

$\begin{array}{lll}Y & N & N\end{array}$

$\begin{array}{lll}Y & N & N\end{array}$

$\begin{array}{llll}Y & \mathrm{~N} & \text { N/A }\end{array}$

Y N U 


\section{SCREENING EVALUATION WORK SHEET (SEWS) (Cont.)}

Equipment ID No.:

Equipment description:

\section{Comments}

Screening Walkdown(s):

Date Time Team Members

\section{Recommend Resolution}

口 Maintenance action:

Further evaluation:

$\square \quad$ Retrofit design:

$\square$ Other:

No further action required. Equipment is seismically adequate.

All aspects of the equipment's seismic adequacy have been addressed.

Evaluation by:

Date:

(All team members) 


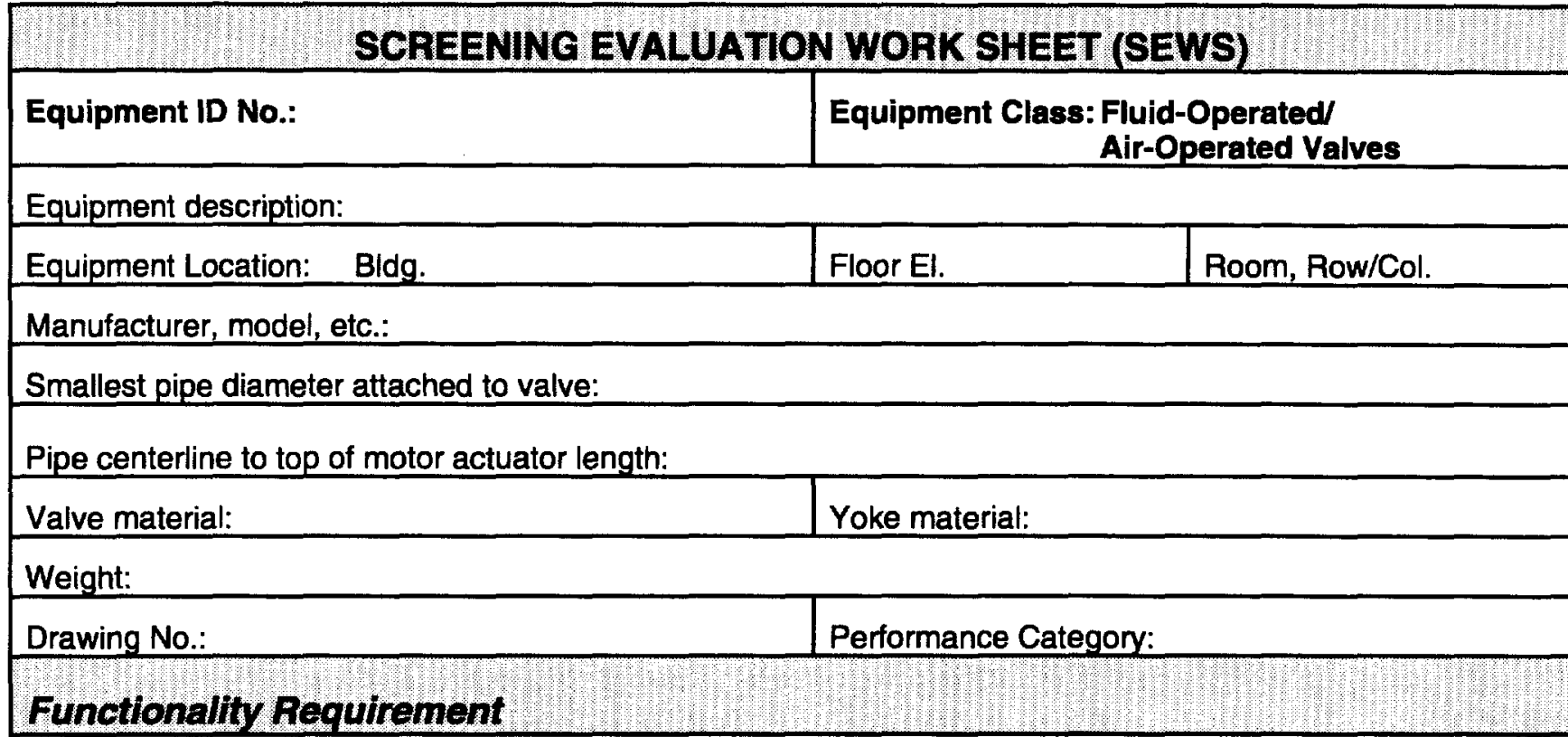

- Contact Lead Relay Reviewer to determine if item contains Essential Relays

- For components whose function or structural integrity is required, complete all sections of this form.

- For all other components, only anchorage evaluation is required.

\section{Seismic Capacity vs. Demand (Chapter 5)}

1. Seismic Capacity based on:
R Reference Spectrum
GERS
D Existing documentation

2. Elevation where equipment receives seismic input

Seismic Demand Spectrum (SDS) based on:
In-structure response spectrum (IRS) per DOE-STD-1020
Other in-structure response spectrum (determine appropriate experience data scale factor)
Design basis earthquake (DBE) per DOE-STD-1020
$\square$ Other

Scale Factor (SF)

Experience Data Factor $\left(F_{E D}\right)$

Does capacity exceed demand?

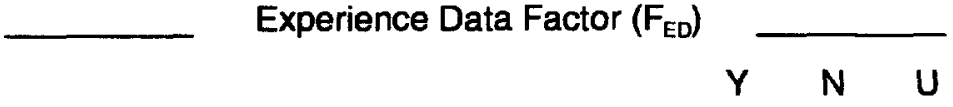

Reference:

\section{Caveats (Soction 8.2.1)}

Reference Spectrum Fluid-Operated Valves (Identify with an asterisk (*) those caveats which are met by intent without meeting the specific wording of the caveat rule and explain the reason for this conclusion in the COMMENTS section below)

\begin{tabular}{llllll}
\hline 1. & Equipment is included in earthquake experience equipment class & $\mathrm{Y}$ & $\mathrm{N}$ & $\mathrm{U}$ & N/A \\
2. & $\begin{array}{l}\text { No cast-iron body } \\
\text { 3. }\end{array}$ & $\mathrm{Y}$ cast-iron yoke (for spring-operated pressure relief or & $\mathrm{N}$ & $\mathrm{U}$ & N/A \\
& piston-operated valves) & $\mathrm{Y}$ & $\mathrm{N}$ & $\mathrm{U}$ & N/A
\end{tabular}




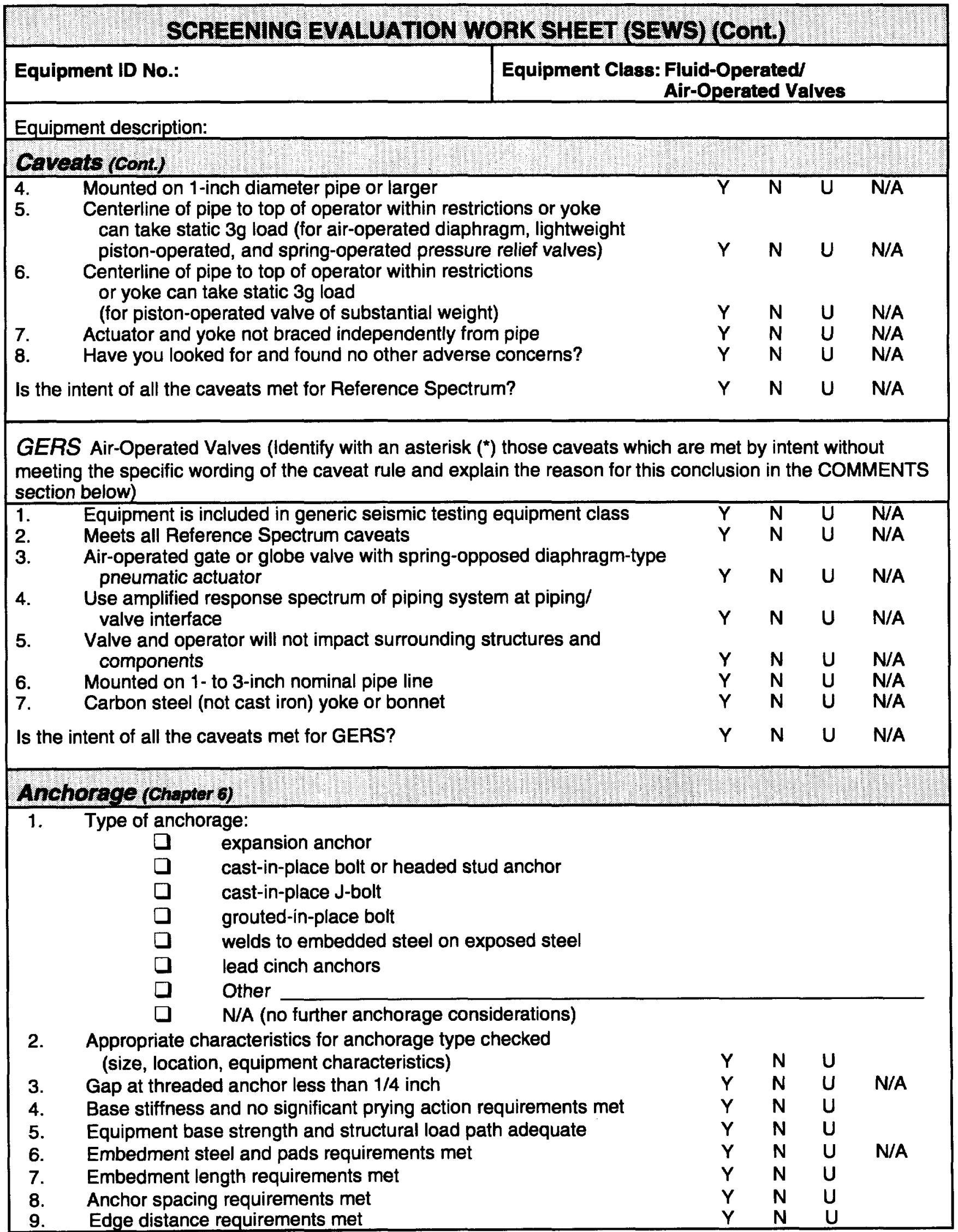


Sheet 3 of

\section{SCREENING EVALUATION WORK SHEET (SEWS) (Cont.)}

Equipment ID No.:

Equipment Class: Fluid-Operated/

Air-Operated Valves

Equipment description:

\section{Anchorage (Cont)}

10. Concrete strength requirements met

11. Concrete crack requirements met

12. Equipment with essential relays requirements met

13. Installation adequacy requirements met

14. No other concerns

Does anchorage capacity exceed demand?

$\begin{array}{llll}Y & N & U & \\ Y & N & U & \\ Y & N & U & \text { N/A } \\ Y & N & U & \text { N/A } \\ Y & N & U & \end{array}$

Reference:

\section{Interaction Effects (Chapter 7 )}

1. Soft targets free from impact by nearby equipment

or structures

2. If equipment contains sensitive essential relays, equipment free

from all impact by nearby equipment or structures

3. Attached lines have adequate flexibility

4. No collapse of overhead equipment, distribution systems,

or masonry walls

5. Equipment is free from credible and significant seismic-induced flood and spray concerns

6. No credible seismic-induced fire concerns

7. No other "two over one" concerns as defined in DOE-STD-1021

8. No other concerns

Y N U

Is equipment free of interaction effects?

$\begin{array}{llll}Y & N & U & \text { N/A } \\ Y & N & U & \text { N/A } \\ Y & N & U & \text { N/A } \\ Y & N & & \text { N/A } \\ Y & N & U & \text { N/A } \\ Y & N & & \text { N/A } \\ Y & N & & \text { N/A } \\ Y & N & U & \text { N/A } \\ Y & N & U & \end{array}$

\section{Comments}


SEWS 8.2.1 (4 of 4)

Sheet 4 of

\section{SCREENING EVALUATION WORK SHEET (SEWS) (Cont.)}

Equipment ID No.:

Equipment Class: Fluid-Operated/

Air-Operated Valves

Equipment description:

Comments (Cont)

Screening Walkdown(s):

Date $\quad \underline{T i m e} \quad$ Team Members

\section{Recommend Resolution}

口 Maintenance action:

$\square \quad$ Further evaluation:

$\square$ Retrofit design:

Other:

- No further action required. Equipment is seismically adequate.

All aspects of the equipment's seismic adequacy have been addressed.

Evaluation by:

Date:

(All team members) 
Sheet 1 of

\section{SCREENING EVALUATION WORK SHEET (SEWS)}

Equipment ID No.:

Equipment Class: Motor-Operated Valves

Equipment description:

Equipment Location: Bldg.

Floor El.

Room, Row/Col.

Manufacturer, model, etc.:

Smallest pipe diameter attached to valve:

Pipe centerline to top of motor actuator length:

Valve material:

Yoke material:

Weight:

Drawing No.:

Performance Category:

\section{Functionality Requirement}

- Contact Lead Relay Reviewer to determine if item contains Essential Relays

- For components whose function or structural integrity is required, complete all sections of this form.

- For all other components, only anchorage evaluation is required.

\section{Seismic Capacity vs. Demand (Chapter 5)}

1. Seismic Capacity based on:
$\square$ Reference Spectrum
G GERS
D Existing documentation

2. Elevation where equipment receives seismic input

Seismic Demand Spectrum (SDS) based on:

$\square \quad$ In-structure response spectrum (IRS) per DOE-STD-1020

$\square \quad$ Other in-structure response spectrum (determine appropriate experience data

scale factor)

Design basis earthquake (DBE) per DOE-STD-1020

O Other

Scale Factor (SF)

Experience Data Factor $\left(F_{E D}\right)$

Does capacity exceed demand?

Y N U

Reference:

Caveats (Section 8.22)

Reference Spectrum (Identify with an asterisk (*) those caveats which are met by intent without meeting the specific wording of the caveat rule and explain the reason for this conclusion in the COMMENTS section below)

1. Equipment is included in earthquake experience equipment class

2. No cast-iron body

3. No cast-iron yoke

$\begin{array}{llll}Y & N & U & \text { N/A } \\ Y & N & U & \text { N/A } \\ Y & N & U & \text { N/A }\end{array}$




\section{SCREENING EVALUATION WORK SHEET (SEWS) (Cont.)}

Equipment ID No:

Equipment Class: Motor-Operated Valves

Equipment description:

\section{Caveats (Cont)}

4. Mounted on 1-inch diameter pipe or larger

5. Centerline of pipe to operator within restrictions or yoke can take static $3 g$ load

6. Actuator and yoke not braced independently from pipe

7. Have you looked for and found no other adverse concerns?

Is the intent of all the caveats met for Reference Spectrum?

$\begin{array}{llll}\mathrm{N} & \mathrm{U} & \mathrm{N} / \mathrm{A}\end{array}$

$\begin{array}{llll}Y & N & \mathrm{~N} & \mathrm{~N} / \mathrm{A}\end{array}$

$\begin{array}{llll}Y & N & U & N / A\end{array}$

$\begin{array}{llll}Y & N & \mathrm{~N} / \mathrm{A}\end{array}$

Y N $\quad$ U N N

GERS (Identify with an asterisk (*) those caveats which are met by intent without meeting the specific wording of the caveat rule and explain the reason for this conclusion in the COMMENTS section below) (Note that GERS for this class apply to only motor operator and its connection to valve; valve itself and valve/pipe interface are not covered.)

1. Equipment is included in generic seismic testing equipment class

2. Meets all Reference Spectrum caveats

3. Use amplified spectrum of piping system and valve at valve/operator interface

4. Motor axis is horizontal

5. Valve and operator will not impact surrounding structures and components

6. Motor controls remotely located

7. If valve has side mounted actuator attached to secondary reducer,

$\begin{array}{llll}Y & N & U & N / A\end{array}$

$\begin{array}{llll}Y & N & U & N / A\end{array}$

$\begin{array}{llll}Y & N & U & N / A\end{array}$

$\begin{array}{llll}Y & \mathrm{~N} & \mathrm{U} & \mathrm{N} / \mathrm{A}\end{array}$

$\begin{array}{llll}Y & N & \mathrm{~N} / \mathrm{A}\end{array}$

$\begin{array}{llll}\mathrm{Y} & \mathrm{N} & \mathrm{U} & \mathrm{N} / \mathrm{A}\end{array}$

8. Manufactured by Limitorque or Rotork

9. Any loose or missing valve-to-operator bolts are tightened or replaced (tightness check not required)

Is the intent of all the caveats met for GERS?

$\begin{array}{llll}Y & N & U & N / A\end{array}$

\section{Anchorage (Chapter 6)}

1. Type of anchorage:

$\begin{array}{ll}\square & \text { expansion anchor } \\ \square & \text { cast-in-place bolt or headed stud anchor } \\ \square & \text { cast-in-place J-bolt } \\ \square & \text { grouted-in-place bolt } \\ \square & \text { welds to embedded steel on exposed steel } \\ \square & \text { lead cinch anchors } \\ \square & \text { Other } \\ \square & \text { N/A (no further anchorage considerations) }\end{array}$

2. Appropriate characteristics for anchorage type checked

(size, location, equipment characteristics)

3. Gap at threaded anchor less than $1 / 4$ inch

4. Base stiffness and no significant prying action requirements met

$\begin{array}{lll}Y & N & U \\ Y & N & U \\ Y & N & U\end{array}$




\section{SCREENING EVALUATION WORK SHEET (SEWS) (Cont)}

Equipment ID No.: Equipment Class: Motor-Operated Valves

Equipment description:

Anchorage (cont)

5. Equipment base strength and structural load path adequate

6. Embedment steel and pads requirements met

7. Embedment length requirements met

8. Anchor spacing requirements met

9. Edge distance requirements met

10. Concrete strength requirements met

11. Concrete crack requirements met

12. Equipment with essential relays requirements met

13. Installation adequacy requirements met

14. No other concerns

$\begin{array}{llll}Y & N & U & \\ Y & N & U & N / A \\ Y & N & U & \\ Y & N & U & \\ Y & N & U & \\ Y & N & U & \\ Y & N & U & \\ Y & N & U & \text { N/A } \\ Y & N & U & \text { N/A } \\ Y & N & U & \end{array}$

Does anchorage capacity exceed demand?

Y N U

Reference:

\section{Interaction Effects (Chapter ?)}

1. Soft targets free from impact by nearby equipment or structures

2. If equipment contains sensitive essential relays, equipment free from all impact by nearby equipment or structures

3. Attached lines have adequate flexibility

4. No collapse of overhead equipment, distribution systems, or masonry walls

5. Equipment is free from credible and significant seismic-induced flood and spray concerns

6. No credible seismic-induced fire concerns

7. No other "two over one" concerns as defined in DOE-STD-1021

8. No other concerns

Is equipment free of interaction effects?

$\begin{array}{llll}Y & N & U & N / A\end{array}$

$Y \quad N \quad U \quad N / A$

Y N N/A

$\begin{array}{llll}Y & N & U & N / A\end{array}$

$\begin{array}{lll}Y & N & N\end{array}$

$\begin{array}{lll}Y & N & N / A\end{array}$

$\begin{array}{llll}Y & N & U & \text { N/A }\end{array}$

Y N U

\section{Comments}




\section{DOE Seismic Evaluation Procedure}

\section{SCREENING EVALUATION WORK SHEET (SEWS) (Cont.)}

Equipment ID No.:

Equipment Class: Motor-Operated Valves

Equipment description:

Comments (Cont)

Screening Walkdown(s):

Date Time $\underline{\text { Team Members }}$

Recommend Resolution

- Maintenance action:

- Further evaluation:

$\square \quad$ Retrofit design:

$\square \quad$ Other:

- No further action required. Equipment is seismically adequate.

All aspects of the equipment's seismic adequacy have been addressed.

Evaluation by:

Date:

(All team members) 


\section{SCREENING EVALUATION WORK SHEET (SEWS)}

Equipment ID No.:

Equipment Class: Solenoid-Operated Valves

Equipment description:

\begin{tabular}{l|l|l}
\hline Equipment Location: Bldg. & Floor El. & Room, Row/Col.
\end{tabular}

Manufacturer, model, etc.:

Smallest pipe diameter attached to valve:

Pipe centerline to top of motor actuator length:

Valve material:

Yoke material:

Weight:

Drawing No.:

Performance Category:

\section{Functionality Requirement}

$\square \quad$ Contact Lead Relay Reviewer to determine if item contains Essential Relays

For components whose function or structural integrity is required, complete all sections of this form.

F For all other components, only anchorage evaluation is required.

\section{Seismic Capacity vs. Demand (Chapter 5)}

1. Seismic Capacity based on:

$\begin{array}{ll}\square & \text { Reference Spectrum } \\ \square & \text { GERS } \\ \square & \text { Existing documentation }\end{array}$

2. Elevation where equipment receives seismic input

Seismic Demand Spectrum (SDS) based on:

口 In-structure response spectrum (IRS) per DOE-STD-1020

$\square \quad$ Other in-structure response spectrum (determine appropriate experience data scale factor)

$\square \quad$ Design basis earthquake (DBE) per DOE-STD-1020

$\square$ Other

Scale Factor (SF) _ Experience Data Factor $\left(F_{E D}\right)$

Does capacity exceed demand?

Y N U

Reference:

\section{Caveats (section 8.2.2)}

Reference Spectrum (Identify with an asterisk (*) those caveats which are met by intent without meeting the specific wording of the caveat rule and explain the reason for this conclusion in the COMMENTS section below)

1. Equipment is included in earthquake experience equipment class

2. No cast-iron body

3. No cast-iron yoke

$\begin{array}{llll}Y & N & U & N / A \\ Y & N & U & N / A \\ Y & N & U & \text { N/A }\end{array}$




\section{SCREENING EVALUATION WORK SHEET (SEWS) (Cont)}

Equipment ID No.:

Equipment Class: Solenoid-Operated Valves

Equipment description:

\section{Caveats (Cont)}

4. Centerline of pipe to operator within restrictions or yoke can take

static $3 \mathrm{~g}$ load

5. Actuator and yoke not braced independently from pipe

6. Have you looked for and found no other adverse concerns?

Is the intent of all the caveats met for Reference Spectrum?

$\begin{array}{llll}Y & N & U & \text { N/A } \\ Y & N & U & \text { N/A } \\ Y & N & U & \text { N/A } \\ Y & N & U & \text { N/A }\end{array}$

GERS (Identify with an asterisk (*) those caveats which are met by intent without meeting the specific wording of the caveat rule and explain the reason for this conclusion in the COMMENTS section below) (Note that GERS for this class apply to only motor operator and its connection to valve; valve itself and valve/pipe interface are not covered.)

1. Equipment is included in generic seismic testing equipment class

2. Meets all Reference Spectrum caveats

3. Use amplified spectrum for piping system at piping/valve interface

4. Valve and operator will not impact surrounding structures and components

5. Nominal pipe size is 1 inch or less

6. Valve body is forged brass or steel

7. Housing oriented in accordance with manufacturer's recommendations

8. Height of valve (pipe centerline to top of housing) does not exceed 12 in.

9. If SOV is a pilot on a larger valve, use amplified response spectrum at attachment point of SOV to larger valve

10. Use 3.5g ZPA GERS for ASCO Type 206-381

Is the intent of all the caveats met for GERS?

$\begin{array}{llll}Y & N & U & N / A \\ Y & N & U & N / A \\ Y & N & U & N / A \\ Y & N & U & N / A \\ Y & N & U & N / A \\ Y & N & U & N / A \\ Y & N & U & N / A \\ Y & N & U & N / A \\ Y & N & U & N / A \\ Y & N & U & N / A \\ Y & N & U & \text { N/A }\end{array}$

\section{Anchorage (Chapter 6)}

1. Type of anchorage:

$\begin{array}{ll}\square & \text { expansion anchor } \\ \square & \text { cast-in-place bolt or headed stud anchor } \\ \text { cast-in-place J-bolt } \\ \square & \text { grouted-in-place bolt } \\ \square & \text { welds to embedded steel on exposed steel } \\ \square & \text { lead cinch anchors } \\ \square & \text { Other } \\ \square & \text { N/A (no further anchorage considerations) }\end{array}$

2. Appropriate characteristics for anchorage type checked

(size, location, equipment characteristics)

3. Gap at threaded anchor less than $1 / 4$ inch

4. Base stiffness and no significant prying action requirements met

5. Equipment base strength and structural load path adequate

6. Embedment steel and pads requirements met

$\begin{array}{llll}Y & N & U & \\ Y & N & U & N / A \\ Y & N & U & \\ Y & N & U & \\ Y & N & U & \text { N/A }\end{array}$




\section{SCREENING EVALUATION WORK SHEET (SEWS) (Cont.)}

Equipment ID No.:

Equipment Class: Solenoid-Operated Valves

Equipment description:

\section{Anchorage (Cont)}

7. Embedment length requirements met

8. Anchor spacing requirements met

9. Edge distance requirements met

10. Concrete strength requirements met

11. Concrete crack requirements met

12. Equipment with essential relays requirements met

13. Installation adequacy requirements met

14. No other concerns

Does anchorage capacity exceed demand?

Reference:

\section{Interaction Effects (Chapter 7)}

1. Soft targets free from impact by nearby equipment

$$
\text { or structures }
$$

2. If equipment contains sensitive essential relays, equipment free from all impact by nearby equipment or structures

3. Attached lines have adequate flexibility

4. No collapse of overhead equipment, distribution systems, or masonry walls

5. Equipment is free from credible and significant seismic-induced flood and spray concerns

6. No credible seismic-induced fire concerns

7. No other "two over one" concerns as defined in DOE-STD-1021

8. No other concerns

$\begin{array}{llll}Y & N & U & \\ Y & N & U & \\ Y & N & U & \\ Y & N & U & \\ Y & N & U & \\ Y & N & U & \text { N/A } \\ Y & N & U & \text { N/A } \\ Y & N & U & \\ Y & N & U & \end{array}$

Is equipment free of interaction effects?

$\begin{array}{llll}Y & N & U & \text { N/A } \\ Y & N & U & \text { N/A } \\ Y & N & U & \text { N/A } \\ Y & N & & \text { N/A } \\ Y & N & U & \text { N/A } \\ Y & N & & \text { N/A } \\ Y & N & & \text { N/A } \\ Y & N & U & \text { N/A } \\ Y & N & U & \end{array}$

\section{Comments}




\section{DOE Seismic Evaluation Procedure}

SEWS 8.2.2 SOV (4 of 4)

Sheet 4 of

\section{SCREENING EVALUATION WORK SHEET (SEWS) (Cont.)}

Equipment ID No.:

Equipment Class: Solenoid-Operated Valves

Equipment description:

Comments (cont)

\section{Recommend Resolution}

- Maintenance action:

ㅁ Further evaluation:

$\square \quad$ Retrofit design:

Other:

$\square \quad$ No further action required. Equipment is seismically adequate.

All aspects of the equipment's seismic adequacy have been addressed.

Evaluation by:

Date:

(All team members) 


\section{SCREENING EVALUATION WORK SHEET (SEWS)}

Equipment ID No.:

Equipment Class: Horizontal Pumps

Equipment description:

\begin{tabular}{l|l|l}
\hline Equipment Location: Bldg. & Floor El. & Room, Row/Col.
\end{tabular}

Manufacturer, model, etc.:

Drawing No.: Performance Category:

Weight:

Horsepower/Motor rating: RPM Head Flow rate

\section{Functionality Requirement}

- Contact Lead Relay Reviewer to determine if item contains Essential Relays

For components whose function or structural integrity is required, complete all sections of this form.

F For all other components, only anchorage evaluation is required.

\section{Seismic Capacity vs. Demand (Chapter 5)}

1. Seismic Capacity based on:

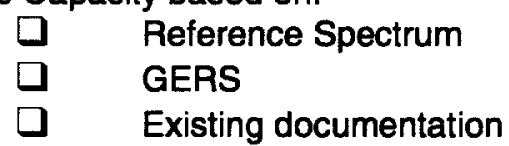

2. Elevation where equipment receives seismic input

Seismic Demand Spectrum (SDS) based on:
In-structure response spectrum (IRS) per DOE-STD-1020
$\square$ Other in-structure response spectrum (determine appropriate experience data scale factor)
Design basis earthquake (DBE) per DOE-STD-1020
$\square \quad$ Other

Scale Factor (SF)

Experience Data Factor $\left(F_{E D}\right)$

Does capacity exceed demand?

Y N U

Reference:

\section{Caveats (Section 8.2.3)}

Reference Spectrum (Identify with an asterisk (*) those caveats which are met by intent without meeting the specific wording of the caveat rule and explain the reason for this conclusion in the COMMENTS section below)

\begin{tabular}{llllll}
\hline 1. & Equipment is included in earthquake experience equipment class & $\mathrm{Y}$ & $\mathrm{N}$ & $\mathrm{U}$ & $\mathrm{N} / \mathrm{A}$ \\
2. & Driver and pump connected by rigid base or skid & $\mathrm{Y}$ & $\mathrm{N}$ & $\mathrm{U}$ & $\mathrm{N} / \mathrm{A}$ \\
3. & Shaft has thrust restraint in both axial directions & $\mathrm{Y}$ & $\mathrm{N}$ & $\mathrm{U}$ & $\mathrm{N} / \mathrm{A}$ \\
4. $\quad$ No risk of excessive nozzle loads such as gross pipe motion & & & & \\
& or differential displacement & $\mathrm{Y}$ & $\mathrm{N}$ & $\mathrm{U}$ & $\mathrm{N} / \mathrm{A}$ \\
5. $\quad$ Have you looked for and found no other adverse concerns? & $\mathrm{Y}$ & $\mathrm{N}$ & $\mathrm{U} / \mathrm{A}$ \\
& & $\mathrm{Y}$ & $\mathrm{N}$ & $\mathrm{U}$ & $\mathrm{N} / \mathrm{A}$ \\
Is the intent of all the caveats met for Reference Spectrum? &
\end{tabular}




\section{SCREENING EVALUATION WORK SHEET (SEWS) (Cont.)}

Equipment ID No.:

Equipment Class: Horizontal Pumps

Equipment description:

\section{Anchorage (Chapter o)}

1. Type of anchorage:

$\begin{array}{ll}\square & \text { expansion anchor } \\ \square & \text { cast-in-place bolt or headed stud anchor } \\ \square & \text { cast-in-place J-bolt } \\ \square & \text { grouted-in-place bolt } \\ \square & \text { welds to embedded steel on exposed steel } \\ \square & \text { lead cinch anchors } \\ \square & \text { Other } \\ \square & \text { N/A (no further anchorage considerations) }\end{array}$

2. Appropriate characteristics for anchorage type checked

(size, location, equipment characteristics)

3. Gap at threaded anchor less than 1/4 inch

4. Base stiffness and no significant prying action requirements met

5. Equipment base strength and structural load path adequate

6. Embedment steel and pads requirements met

7. Embedment length requirements met

8. Anchor spacing requirements met

9. Edge distance requirements met

10. Concrete strength requirements met

11. Concrete crack requirements met

12. Equipment with essential relays requirements met

13. Installation adequacy requirements met

14. No other concerns

Does anchorage capacity exceed demand?

$\begin{array}{llll}Y & N & U & \\ Y & N & U & N / A \\ Y & N & U & \\ Y & N & U & \\ Y & N & U & N / A \\ Y & N & U & \\ Y & N & U & \\ Y & N & U & \\ Y & N & U & \\ Y & N & U & \\ Y & N & U & N / A \\ Y & N & U & N / A \\ Y & N & U & \\ Y & N & U & \end{array}$

Reference:

\section{Interaction Efiects (Chaptor 7 )}

1. Soft targets free from impact by nearby equipment or structures

2. If equipment contains sensitive essential relays, equipment free from all impact by nearby equipment or structures

3. Attached lines have adequate flexibility

4. No collapse of overhead equipment, distribution systems, or masonry walls

5. Equipment is free from credible and significant seismic-induced flood and spray concerns

6. No credible seismic-induced fire concerns

7. No other "two over one" concerns as defined in DOE-STD-1021

8. No other concerns

$\begin{array}{llll}\mathrm{N} & \mathrm{U} & \mathrm{N} / \mathrm{A}\end{array}$

$\begin{array}{llll}Y & N & U & N\end{array}$

$Y \quad N \quad U \quad N / A$

Y N N/A

$Y \quad N \quad U \quad N / A$

$Y \quad N \quad N / A$

$\begin{array}{lll}Y & N & N\end{array}$

$Y \quad N \quad U \quad N / A$

Is equipment free of interaction effects? 


\section{DOE Seismic Evaluation Procedure}

SEWS 8.2.3 (3 of 3 )

Sheet 3 of

\section{SCREENING EVALUATION WORK SHEET (SEWS) (Cont.)}

Equipment ID No.:

Equipment Class: Horizontal Pumps

Equipment description:

\section{Comments}

Screening Walkdown(s):

Date Time $\quad \underline{\text { Team Members }}$

\section{Recommend Resolution}

- Maintenance action:

口 Further evaluation:

- Retrofit design:

Other:

- No further action required. Equipment is seismically adequate.

All aspects of the equipment's seismic adequacy have been addressed.

Evaluation by:

Date:

(All team members) 


\section{SCREENING EVALUATION WORK SHEET (SEWS)}

Equipment ID No.:

Equipment Class: Vertical Pumps

Equipment description:

Equipment Location: Bldg.

Floor El. Room, Row/Col.

Manufacturer, model, etc.:

Drawing No.: Performance Category:

Weight:

Horsepower/Motor rating: RPM Head Flow rate

\section{Functionality Requirement}

Contact Lead Relay Reviewer to determine if item contains Essential Relays

For components whose function or structural integrity is required, complete all sections of this form.

For all other components, only anchorage evaluation is required.

\section{Seismic Capacity vs. Demand (Chapter 5)}

1. Seismic Capacity based on:

$\begin{array}{ll}\square & \text { Reference Spectrum } \\ \square & \text { GERS } \\ \square & \text { Existing documentation }\end{array}$

2. Elevation where equipment receives seismic input

Seismic Demand Spectrum (SDS) based on:

In-structure response spectrum (IRS) per DOE-STD-1020

O Other in-structure response spectrum (determine appropriate experience data scale factor)

Design basis earthquake (DBE) per DOE-STD-1020

a Other

Scale Factor (SF)

Experience Data Factor $\left(F_{E D}\right)$

Does capacity exceed demand?

Y N $\quad$ U

Reference:

\section{Caveats (Section 8.2.4)}

Reference Spectrum (Identify with an asterisk (*) those caveats which are met by intent without meeting the specific wording of the caveat rule and explain the reason for this conclusion in the COMMENTS section below)

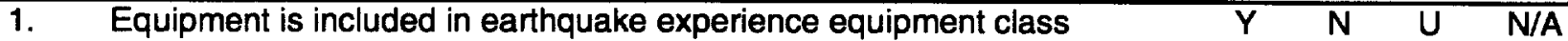

2. Casing and impeller shaft not cantilevered more than 20 feet, with radial bearing at bottom to support shaft

3. No risk of excessive nozzle loads such as gross pipe motion or differential displacement

4. Have you looked for and found no other adverse concerns?

$\begin{array}{llll}\mathrm{N} & \mathrm{N} & \mathrm{N} / \mathrm{A}\end{array}$

Is the intent of all the caveats met for Reference Spectrum?

$\begin{array}{llll}Y & N & U & N / A\end{array}$

$\begin{array}{llll}Y & N & U & N / A\end{array}$

Y N U N/A




\section{SCREENING EVALUATION WORK SHEET (SEWS) (Cont.)}

Equipment ID No.:

Equipment Class: Vertical Pumps

Equipment description:

\section{Anchorage (Chapter 6)}

1. Type of anchorage:

\begin{tabular}{ll}
$\square$ & expansion anchor \\
cast-in-place bolt or headed stud anchor \\
$\square$ & cast-in-place J-bolt \\
$\square$ & grouted-in-place bolt \\
$\square$ & welds to embedded steel on exposed steel \\
$\square$ & lead cinch anchors \\
$\square$ & Other \\
\hline & N/A (no further anchorage considerations)
\end{tabular}

2. Appropriate characteristics for anchorage type checked

(size, location, equipment characteristics)

3. Gap at threaded anchor less than $1 / 4$ inch

4. Base stiffness and no significant prying action requirements met

5. Equipment base strength and structural load path adequate

6. Embedment steel and pads requirements met

7. Embedment length requirements met

8. Anchor spacing requirements met

9. Edge distance requirements met

10. Concrete strength requirements met

11. Concrete crack requirements met

12. Equipment with essential relays requirements met

13. Installation adequacy requirements met

14. No other concerns

$\begin{array}{llll}Y & N & U & \\ Y & N & U & \text { N/A } \\ Y & N & U & \\ Y & N & U & \\ Y & N & U & \text { N/A } \\ Y & N & U & \\ Y & N & U & \\ Y & N & U & \\ Y & N & U & \\ Y & N & U & \\ Y & N & U & \text { N/A } \\ Y & N & U & \text { N/A } \\ Y & N & U & \\ Y & N & U & \\ & & & \\ & & & \end{array}$

Does anchorage capacity exceed demand?

Reference:

\section{Interaction Effects (chapter 7)}

\section{Soft targets free from impact by nearby equipment} or structures

2. If equipment contains sensitive essential relays, equipment free from all impact by nearby equipment or structures

3. Attached lines have adequate flexibility

4. No collapse of overhead equipment, distribution systems, or masonry walls

5. Equipment is free from credible and significant seismic-induced flood and spray concerns

6. No credible seismic-induced fire concerns

7. No other "two over one" concerns as defined in DOE-STD-1021

8. No other concerns

Is equipment free of interaction effects?

$\begin{array}{cccc}Y & N & U & \text { N/A } \\ Y & N & U & \text { N/A } \\ Y & N & U & \text { N/A } \\ Y & N & & \text { N/A } \\ Y & N & U & \text { N/A } \\ Y & N & & \text { N/A } \\ Y & N & & \text { N/A } \\ Y & N & U & \text { N/A } \\ Y & N & U & \end{array}$


DOE Seismic Evaluation Procedure

SEWS 8.2.4 ( 3 of 3 )

Sheet 3 of

\section{SCREENING EVALUATION WORK SHEET (SEWS) (Cont.)}

Equipment ID No.:

Equipment Class: Vertical Pumps

Equipment description:

\section{Comments}

Screening Walkdown(s):

Date Time Team Members

\section{Recommend Resolution}

Maintenance action:

Further evaluation:

Retrofit design:

Other:

- No further action required. Equipment is seismically adequate.

All aspects of the equipment's seismic adequacy have been addressed.

Evaluation by:

Date:

(All team members) 



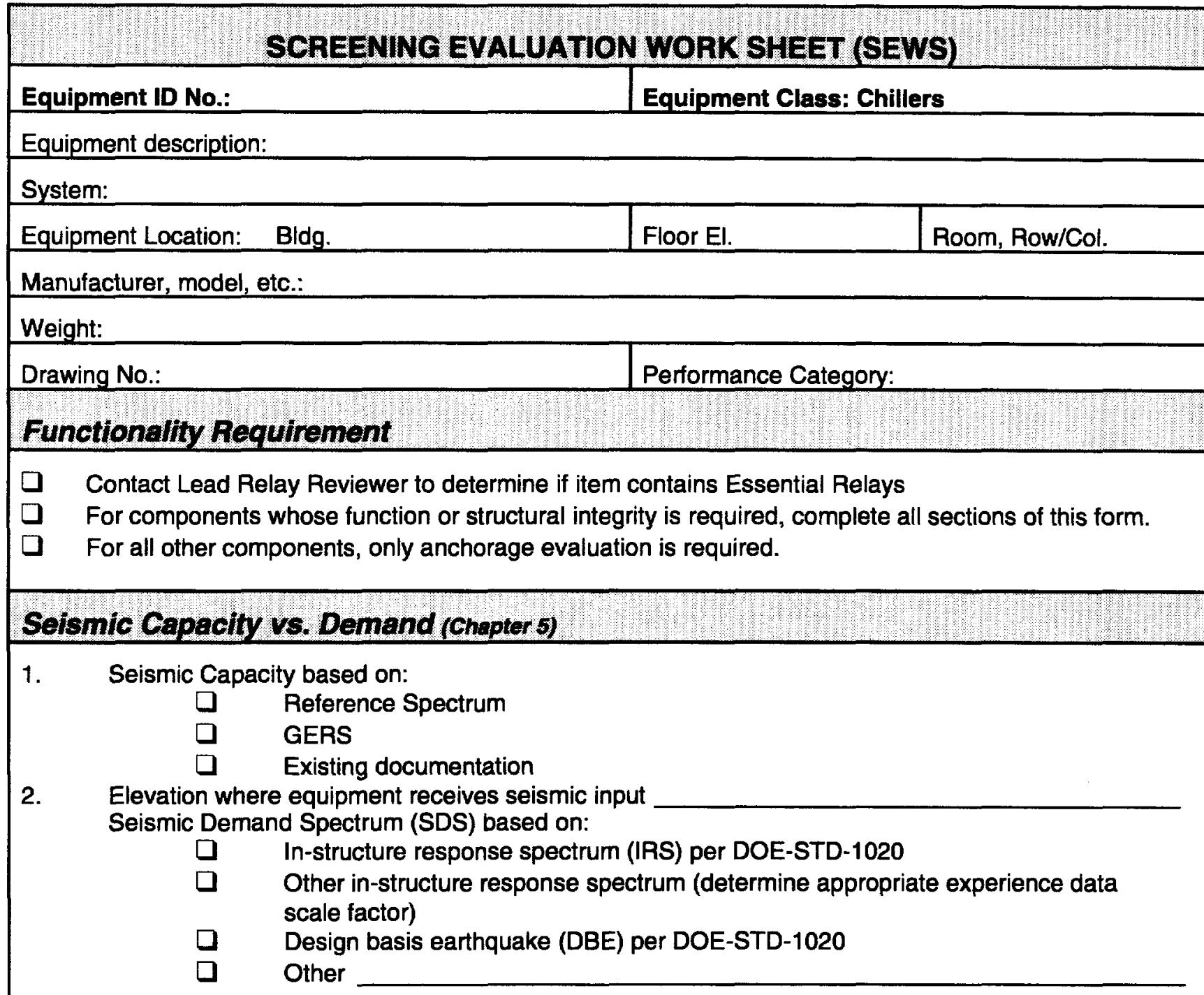

Scale Factor (SF)

Does capacity exceed demand?

Reference:

\section{Caveats (Section 8.2 .5 )}

Reference Spectrum (Identify with an asterisk $\left(^{*}\right)$ those caveats which are met by intent without meeting the specific wording of the caveat rule and explain the reason for this conclusion in the COMMENTS section below)

1. Equipment is included in earthquake experience equipment class

2. Evaporator and condenser tanks reasonably braced between themselves for lateral forces without relying on weak-way bending of steel plates or structural steel shapes

3. Have you looked for and found no other adverse concerns?

Is the intent of all the caveats met for Reference Spectrum?

$\begin{array}{llll}\mathrm{Y} & \mathrm{N} & \mathrm{U} & \mathrm{N} / \mathrm{A} \\ \mathrm{Y} & \mathrm{N} & \mathrm{U} & \mathrm{N} / \mathrm{A} \\ \mathrm{Y} & \mathrm{N} & \mathrm{U} & \mathrm{N} / \mathrm{A} \\ \mathrm{Y} & \mathrm{N} & \mathrm{U} & \mathrm{N} / \mathrm{A}\end{array}$




\section{SCREENING EVALUATION WORK SHEET (SEWS) (Cont.)}

Equipment ID No.:

Equipment Class: Chillers

Equipment description:

\section{Anchorage (Chapter 6 )}

$1 . \quad$ Type of anchorage:

$\begin{array}{ll}\square & \text { expansion anchor } \\ \square & \text { cast-in-place bolt or headed stud anchor } \\ \square & \text { cast-in-place J-bolt } \\ \square & \text { grouted-in-place bolt } \\ \square & \text { welds to embedded steel on exposed steel } \\ \square & \text { lead cinch anchors } \\ \square & \text { Other } \\ \square & \text { N/A (no further anchorage considerations) }\end{array}$

2. Appropriate characteristics for anchorage type checked

(size, location, equipment characteristics)

3. Gap at threaded anchor less than $1 / 4$ inch

4. Base stiffness and no significant prying action requirements met

5. Equipment base strength and structural load path adequate

6. Embedment steel and pads requirements met

7. Embedment length requirements met

8. Anchor spacing requirements met

9. Edge distance requirements met

10. Concrete strength requirements met

11. Concrete crack requirements met

12. Equipment with essential relays requirements met

13. Installation adequacy requirements met

14. No other concerns

$\begin{array}{lll}Y & N & U\end{array}$

$\begin{array}{lll}Y & N & U\end{array}$

$\begin{array}{lll}Y & N & U\end{array}$

$\begin{array}{lll}Y & N & U\end{array}$

$\begin{array}{lll}Y & N & U\end{array}$

$\begin{array}{lll}Y & N & U\end{array}$

N/A

$\begin{array}{lll}Y & N & U\end{array}$

$\begin{array}{lll}Y & N & U\end{array}$

$\begin{array}{lll}Y & N & U\end{array}$

$\begin{array}{lll}Y & N & U\end{array}$

$\begin{array}{lll}Y & N & U\end{array}$

$\begin{array}{lll}Y & N & U\end{array}$

Does anchorage capacity exceed demand?

Y N U

Reference:

\section{Interaction Effects (Chapter 7)}

1. Soft targets free from impact by nearby equipment or structures

2. If equipment contains sensitive essential relays, equipment free from all impact by nearby equipment or structures

3. Attached lines have adequate flexibility

4. No collapse of overhead equipment, distribution systems, or masonry walls

5. Equipment is free from credible and significant seismic-induced flood and spray concerns

6. No credible seismic-induced fire concerns

7. No other "two over one" concerns as defined in DOE-STD-1021

8. No other concerns 


\section{SCREENING EVALUATION WORK SHEET (SEWS) (CONt.)}

Equipment ID No.:

Equipment Class: Chillers

Equipment description:

\section{Comments}

Screening Walkdown(s):

Date Time Team Members

\section{Recommend Resolution}

Maintenance action:

$\square \quad$ Further evaluation:

D Retrofit design:

$\square$ Other:

No further action required. Equipment is seismically adequate.

All aspects of the equipment's seismic adequacy have been addressed.

Evaluation by:

Date:

(All team members) 
. 


\section{SCREENING EVALUATION WORK SHEET (SEWS)}

Equipment ID No.:

Equipment Class: Air Compressors

Equipment description:

System:

Equipment Location: Bldg.

Floor El. Room, Row/Col.

Manufacturer, model, etc.:

Weight:

Drawing No.:

Performance Category:

\section{Functionality Requirement}

- Contact Lead Relay Reviewer to determine if item contains Essential Relays

- For components whose function or structural integrity is required, complete all sections of this form.

F For all other components, only anchorage evaluation is required.

\section{Seismic Capacity vs. Demand (Chapter 5)}

1. Seismic Capacity based on:

$\begin{array}{ll}\square & \text { Reference Spectrum } \\ \square & \text { GERS } \\ \square & \text { Existing documentation }\end{array}$

2. Elevation where equipment receives seismic input

Seismic Demand Spectrum (SDS) based on:

I In-structure response spectrum (IRS) per DOE-STD-1020

$\square \quad$ Other in-structure response spectrum (determine appropriate experience data scale factor)

Design basis earthquake (DBE) per DOE-STD-1020

$\square \quad$ Other

Scale Factor (SF)

Experience Data Factor $\left(F_{\mathrm{ED}}\right)$

Does capacity exceed demand?

Y N U

Reference:

\section{Caveats (Section 8.2.6)}

Reference Spectrum (Identify with an asterisk $\left(^{*}\right)$ those caveats which are met by intent without meeting the specific wording of the caveat rule and explain the reason for this conclusion in the COMMENTS section below)

1. Equipment is included in earthquake experience equipment class

2. Have you looked for and found no other adverse concerns?

$\begin{array}{llll}Y & N & \mathrm{U} & \mathrm{N} / \mathrm{A}\end{array}$

$\begin{array}{llll}Y & N & U & N / A\end{array}$

Is the intent of all the caveats met for Reference Spectrum?

$\begin{array}{llll}\mathrm{N} & \mathrm{U} & \mathrm{N} / \mathrm{A}\end{array}$




\section{SCREENING EVALUATION WORK SHEET (SEWS) (Cont.)}

Equipment ID No.:

Equipment Class: Air Compressors

Equipment description:

\section{Anchorage (Chapter 6 )}

$1 . \quad$ Type of anchorage:

$\begin{array}{ll}\square & \text { expansion anchor } \\ \square & \text { cast-in-place bolt or headed stud anchor } \\ \square & \text { cast-in-place J-bolt } \\ \square & \text { grouted-in-place bolt } \\ \square & \text { weids to embedded steel on exposed steel } \\ \square & \text { lead cinch anchors } \\ \square & \text { Other } \\ \square & \text { N/A (no further anchorage considerations) }\end{array}$

2. Appropriate characteristics for anchorage type checked

(size, location, equipment characteristics)

3. Gap at threaded anchor less than $1 / 4$ inch

4. Base stiffness and no significant prying action requirements met

5. Equipment base strength and structural load path adequate

6. Embedment steel and pads requirements met

7. Embedment length requirements met

8. Anchor spacing requirements met

9. Edge distance requirements met

10. Concrete strength requirements met

11. Concrete crack requirements met

12. Equipment with essential relays requirements met

13. Installation adequacy requirements met

14. No other concerns

$\begin{array}{llll}Y & N & U & \\ Y & N & U & \text { N/A } \\ Y & N & U & \\ Y & N & U & \\ Y & N & U & \text { N/A } \\ Y & N & U & \\ Y & N & U & \\ Y & N & U & \\ Y & N & U & \\ Y & N & U & \\ Y & N & U & \text { N/A } \\ Y & N & U & \text { N/A } \\ Y & N & U & \\ Y & N & U & \\ & & & \\ \end{array}$

\section{Interaction Effects (Chapter 7)}

1. Soft targets free from impact by nearby equipment or structures

2. If equipment contains sensitive essential relays, equipment free from all impact by nearby equipment or structures

3. Attached lines have adequate flexibility

4. No collapse of overhead equipment, distribution systems, or masonry walls

5. Equipment is free from credible and significant seismic-induced flood and spray concerns

6. No credible seismic-induced fire concerns

7. No other "two over one" concerns as defined in DOE-STD-1021

8. No other concerns

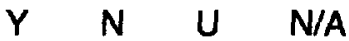

$\begin{array}{llll}Y & N & U & N / A\end{array}$

$\begin{array}{llll}Y & N & U & N / A\end{array}$

Y N N/A

$\begin{array}{llll}Y & N & U & N / A\end{array}$

$\begin{array}{llll}Y & N & U & N / A\end{array}$

$\begin{array}{llll}Y & N & U & N / A\end{array}$

$Y \quad N \quad U \quad N / A$

Is equipment free of interaction effects?

Y N U 


\section{DOE Seismic Evaluation Procedure}

SEWS 8.2.6 ( 3 of 3 )

Sheet 3 of

\section{SCREENING EVALUATION WORK SHEET (SEWS) (Cont.)}

Equipment ID No.:

Equipment Class: Air Compressors

Equipment description:

\section{Comments}

Screening Walkdown(s):

Date Time Team Members

\section{Recommend Resolution}

ㅁ Maintenance action:

$\square \quad$ Further evaluation:

$\square \quad$ Retrofit design:

$\square \quad$ Other:

$\square \quad$ No further action required. Equipment is seismically adequate.

All aspects of the equipment's seismic adequacy have been addressed.

Evaluation by:

Date:

(All team members) 



\section{SCREENING EVALUATION WORK SHEET (SEWS)}

Equipment ID No.:

Equipment Class: Motor-Generators

Equipment description:

System:

Equipment Location: Bldg.

Floor El. Room, Row/Col.

Manufacturer, model, etc.:

Weight:

Drawing No.:

Performance Category:

\section{Functionality Requirement}

$\square \quad$ Contact Lead Relay Reviewer to determine if item contains Essential Relays

For components whose function or structural integrity is required, complete all sections of this form.

For all other components, only anchorage evaluation is required.

\section{Seismic Capacity vs. Demand (Chapter 5)}

1. Seismic Capacity based on:
a Reference Spectrum
G GERS
[ Existing documentation

2. Elevation where equipment receives seismic input

Seismic Demand Spectrum (SDS) based on:
I In-structure response spectrum (IRS) per DOE-STD-1020
口 Other in-structure response spectrum (determine appropriate experience data scale factor)
Design basis earthquake (DBE) per DOE-STD-1020
O Other

Scale Factor (SF)

Experience Data Factor $\left(F_{E D}\right)$

Does capacity exceed demand?

Y N U

Reference:

\section{Caveats (Section 8.2.2)}

Reference Spectrum (Identify with an asterisk $\left(^{*}\right)$ those caveats which are met by intent without meeting the specific wording of the caveat rule and explain the reason for this conclusion in the COMMENTS section below)

\begin{tabular}{|c|c|c|c|c|c|}
\hline 1. & $\begin{array}{l}\text { Equipment is included in earthquake experience equipment class } \\
\text { Main driver and driven equipment connected by a riqid support }\end{array}$ & $\mathbf{Y}$ & $\mathbf{N}$ & U & N/A \\
\hline & or skid & $Y$ & $\mathrm{~N}$ & U & N/A \\
\hline & Have you looked for and found no other adverse concerns? & $\mathbf{Y}$ & $\mathbf{N}$ & $\mathbf{U}$ & N/A \\
\hline & ent of all the cave & $\mathbf{Y}$ & $\mathrm{N}$ & $\mathrm{U}$ & \\
\hline
\end{tabular}




\section{SCREENING EVALUATION WORK SHEET (SEWS) (Cont.)}

Equipment ID No.:

Equipment Class: Motor-Generators

Equipment description:

\section{Anchorage (Chopter 6)}

1. Type of anchorage:

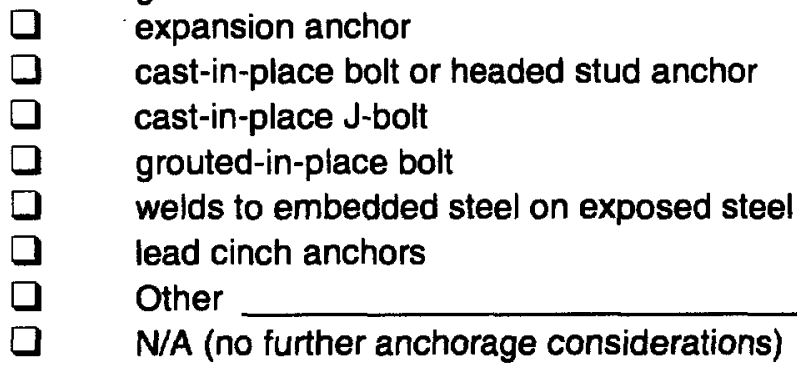

2. Appropriate characteristics for anchorage type checked

(size, location, equipment characteristics)

3. Gap at threaded anchor less than $1 / 4$ inch

4. Base stiffness and no significant prying action requirements met

5. Equipment base strength and structural load path adequate

6. Embedment steel and pads requirements met

7. Embedment length requirements met

8. Anchor spacing requirements met

9. Edge distance requirements met

10. Concrete strength requirements met

11. Concrete crack requirements met

12. Equipment with essential relays requirements met

13. Installation adequacy requirements met

14. No other concerns

$\begin{array}{lll}Y & N & U\end{array}$

$\begin{array}{lll}Y & N & U\end{array}$

$\begin{array}{lll}Y & N & U\end{array}$

N/A

$\begin{array}{lll}Y & N & U\end{array}$

$\begin{array}{lll}Y & N & U\end{array}$

N/A

$\begin{array}{lll}Y & N & U\end{array}$

$\begin{array}{lll}Y & N & U\end{array}$

$\begin{array}{lll}Y & N & U\end{array}$

$\begin{array}{lll}Y & N & U\end{array}$

$\begin{array}{lll}Y & N & U\end{array}$

$\begin{array}{lll}Y & N & U\end{array}$

$\begin{array}{lll}Y & N & U\end{array}$

N/A

N/A

Does anchorage capacity exceed demand?

Reference:

\section{Interaction Effects (Chepter 7}

1. Soft targets free from impact by nearby equipment or structures

2. If equipment contains sensitive essential relays, equipment free from all impact by nearby equipment or structures

3. Attached lines have adequate flexibility

4. No collapse of overhead equipment, distribution systems, or masonry walls

5. Equipment is free from credible and significant seismic-induced flood and spray concerns

6. No credible seismic-induced fire concerns

7. No other "two over one" concerns as defined in DOE-STD-1021

8. No other concerns

$\begin{array}{lll}\text { Is equipment free of interaction effects? } & Y & N \\ U\end{array}$ 
DOE Seismic Evaluation Procedure

SEWS 8.2.7 ( 3 of 3 )

Sheet 3 of

\section{SCREENING EVALUATION WORK SHEET (SEWS) (Cont.)}

Equipment ID No.:

Equipment Class: Motor-Generators

Equipment description:

\section{Comments}

Screening Walkdown(s):

Date $\quad \underline{T i m e} \quad$ Team Members

\section{Recommend Resolution}

a Maintenance action:

Further evaluation:

- Retrofit design:

$\square \quad$ Other:

No further action required. Equipment is seismically adequate.

All aspects of the equipment's seismic adequacy have been addressed.

Evaluation by:

Date:

(All team members) 



\section{SCREENING EVALUATION WORK SHEET (SEWS)}

Equipment ID No.:

Equipment Class: Engine-Generators

Equipment description:

System:

\begin{tabular}{l|l|l}
\hline Equipment Location: Bldg. & Floor El. & Room, Row/Col.
\end{tabular}

Manufacturer, model, etc.:

Weight:

Drawing No.:

Performance Category:

\section{Functionality Requirement}

$\square \quad$ Contact Lead Relay Reviewer to determine if item contains Essential Relays

- For components whose function or structural integrity is required, complete all sections of this form.

For all other components, only anchorage evaluation is required.

\section{Seismic Capacity vs. Demand (Chapter 5)}

1. Seismic Capacity based on:
$\square \quad$ Reference Spectrum
G GERS
$\square \quad$ Existing documentation

2. Elevation where equipment receives seismic input Seismic Demand Spectrum (SDS) based on:

In-structure response spectrum (IRS) per DOE-STD-1020

$\square$ Other in-structure response spectrum (determine appropriate experience data scale factor)

Design basis earthquake (DBE) per DOE-STD-1020

O Other

Scale Factor (SF)

Experience Data Factor $\left(F_{E D}\right)$

Does capacity exceed demand?

$\begin{array}{lll}\mathrm{N} & \mathrm{U}\end{array}$

Reference:

\section{Caveats (Section 8.2.8)}

Reference Spectrum (Identify with an asterisk (") those caveats which are met by intent without meeting the specific wording of the caveat rule and explain the reason for this conclusion in the COMMENTS section below)

1. Equipment is included in earthquake experience equipment class

2. Driver and driven equipment connected by a rigid support or common skid

3. Have you looked for and found no other adverse concerns?

Is the intent of all the caveats met for Reference Spectrum?

$\begin{array}{llll}Y & N & U & \text { N/A } \\ Y & N & U & \text { N/A } \\ Y & N & U & \text { N/A } \\ Y & N & U & \text { N/A }\end{array}$




\section{SCREENING EVALUATION WORK SHEET (SEWS) (Cont)}

Equipment ID No.:

Equipment Class: Engine-Generators

Equipment description:

\section{Anchorage (Chapter 6$)$}

1. Type of anchorage:

$\begin{array}{ll}\square & \text { expansion anchor } \\ \square & \text { cast-in-place bolt or headed stud anchor } \\ \square & \text { cast-in-place J-bolt } \\ \square & \text { grouted-in-place bolt } \\ \square & \text { welds to embedded steel on exposed steel } \\ \square & \text { lead cinch anchors } \\ \square & \text { Other } \\ \square & \text { N/A (no further anchorage considerations) }\end{array}$

2. Appropriate characteristics for anchorage type checked

(size, location, equipment characteristics)

3. Gap at threaded anchor less than $1 / 4$ inch

4. Base stiffness and no significant prying action requirements met

5. Equipment base strength and structural load path adequate

6. Embedment steel and pads requirements met

7. Embedment length requirements met

8. Anchor spacing requirements met

9. Edge distance requirements met

10. Concrete strength requirements met

11. Concrete crack requirements met

12. Equipment with essential relays requirements met

13. Installation adequacy requirements met

14. No other concerns

\begin{tabular}{llll}
$Y$ & $N$ & $U$ & \\
$Y$ & $N$ & $U$ & N/A \\
$Y$ & $N$ & $U$ & \\
$Y$ & $N$ & $U$ & \\
$Y$ & $N$ & $U$ & N/A \\
$Y$ & $N$ & $U$ & \\
$Y$ & $N$ & $U$ & \\
$Y$ & $N$ & $U$ & \\
$Y$ & $N$ & $U$ & \\
$Y$ & $N$ & $U$ & \\
$Y$ & $N$ & $U$ & $N / A$ \\
$Y$ & $N$ & $U$ & $N / A$ \\
$Y$ & $N$ & $U$ & \\
$Y$ & $N$ & $U$ & \\
& & & \\
\hline
\end{tabular}

Does anchorage capacity exceed demand?

Reference:

\section{Interaction Effects (Chapter 7)}

1. Soft targets free from impact by nearby equipment or structures

2. If equipment contains sensitive essential relays, equipment free from all impact by nearby equipment or structures

3. Attached lines have adequate flexibility

4. No collapse of overhead equipment, distribution systems, or masonry walls

5. Equipment is free from credible and significant seismic-induced flood and spray concerns

6. No credible seismic-induced fire concerns

7. No other "two over one" concerns as defined in DOE-STD-1021

8. No other concerns

Is equipment free of interaction effects?

$\begin{array}{llll}Y & N & U & \text { N/A } \\ Y & N & U & \text { N/A } \\ Y & N & U & \text { N/A } \\ Y & N & & \text { N/A } \\ Y & N & U & \text { N/A } \\ Y & N & & \text { N/A } \\ Y & N & & \text { N/A } \\ Y & N & U & \text { N/A } \\ Y & N & U & \end{array}$




\section{DOE Seismic Evaluation Procedure}

\section{SCREENING EVALUATION WORK SHEET (SEWS) (Cont.)}

\section{Equipment ID No.:}

Equipment description:

\section{Comments}

Screening Walkdown(s):

Date $\quad \underline{\text { Time }}$ Team Members

\section{Recommend Resolution}

- Maintenance action:

$\square \quad$ Further evaluation:

$\square \quad$ Retrofit design:

$\square \quad$ Other:

No further action required. Equipment is seismically adequate.

All aspects of the equipment's seismic adequacy have been addressed.

Evaluation by:

Date:

(All team members) 



\section{SCREENING EVALUATION WORK SHEET (SEWS)}

Equipment ID No.:

Equipment Class: Air Handlers

Equipment description:

System:

Equipment Location: Bldg. Floor El. Room, Row/Col.

Manufacturer, model, etc.:

Weight:

Drawing No.:

Performance Category:

\section{Functionality Requirement}

- Contact Lead Relay Reviewer to determine if item contains Essential Relays

- For components whose function or structural integrity is required, complete all sections of this form.

For all other components, only anchorage evaluation is required.

\section{Seismic Capacity vs. Demand (Chapter 5)}

1. Seismic Capacity based on:
- Reference Spectrum
G GERS
$\square \quad$ Existing documentation

2. Elevation where equipment receives seismic input

Seismic Demand Spectrum (SDS) based on:
In-structure response spectrum (IRS) per DOE-STD-1020
口 Other in-structure response spectrum (determine appropriate experience data scale factor)
$\square \quad$ Design basis earthquake (DBE) per DOE-STD-1020
Other

Scale Factor (SF)

Experience Data Factor $\left(F_{\mathrm{ED}}\right)$

Does capacity exceed demand?

Y N U

Reference:

\section{Caveats (Section 8.2.9)}

Reference Spectrum (Identify with an asterisk (") those caveats which are met by intent without meeting the specific wording of the caveat rule and explain the reason for this conclusion in the COMMENTS section below)

\begin{tabular}{|c|c|c|c|c|c|}
\hline $\begin{array}{l}1 . \\
2 .\end{array}$ & $\begin{array}{l}\text { Equipment is included in earthquake experience equipment class } \\
\text { Anchorage of heavy internal components is adequate; internal } \\
\text { vibration isolators have seismic stops to limit uplift and } \\
\text { lateral movement }\end{array}$ & $\bar{Y}$ & $\bar{N}$ & $\bar{U}$ & $\bar{N} / \mathrm{A}$ \\
\hline 3. & All doors secured by latch or fastener & $\mathbf{Y}$ & $\mathbf{N}$ & $\mathrm{U}$ & N/A \\
\hline 4. & $\begin{array}{l}\text { No possibility of excessive duct distortion causing binding or } \\
\text { misalignment of any internal fan }\end{array}$ & $\mathbf{Y}$ & $\mathbf{N}$ & $\mathrm{u}$ & \\
\hline 5. & Have you looked for and found no other adverse concerns? & $Y$ & N & $u$ & \\
\hline Is $t$ & tent of all the caveats met for Reference Spectrum? & $\mathbf{Y}$ & $\mathbf{N}$ & $\mathrm{U}$ & \\
\hline
\end{tabular}




\section{SCREENING EVALUATION WORK SHEET (SEWS) (Cont.)}

Equipment ID No.:

Equipment Class: Air Handlers

Equipment description:

\section{Anchorage (Chapter G)}

1. Type of anchorage:

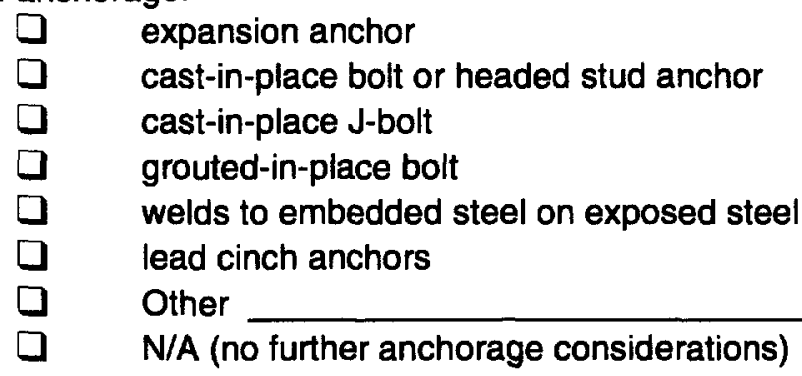

2. Appropriate characteristics for anchorage type checked

(size, location, equipment characteristics)

3. Gap at threaded anchor less than $1 / 4$ inch

4. Base stiffness and no significant prying action requirements met

5. Equipment base strength and structural load path adequate

6. Embedment steel and pads requirements met

7. Embedment length requirements met

8. Anchor spacing requirements met

9. Edge distance requirements met

10. Concrete strength requirements met

11. Concrete crack requirements met

12. Equipment with essential relays requirements met

13. Installation adequacy requirements met

14. No other concerns

$\begin{array}{llll}Y & N & U & \\ Y & N & U & \text { N/A } \\ Y & N & U & \\ Y & N & U & \\ Y & N & U & \text { N/A } \\ Y & N & U & \\ Y & N & U & \\ Y & N & U & \\ Y & N & U & \\ Y & N & U & \\ Y & N & U & \text { N/A } \\ Y & N & U & \text { N/A } \\ Y & N & U & \\ Y & N & U & \\ & & & \end{array}$

Does anchorage capacity exceed demand?

Reference:

\section{Interaction Effects (Chapter 7$)$}

\section{Soft targets free from impact by nearby equipment or structures}

2. If equipment contains sensitive essential relays, equipment free from all impact by nearby equipment or structures

3. Attached lines have adequate flexibility

4. No collapse of overhead equipment, distribution systems, or masonry walls

5. Equipment is free from credible and significant seismic-induced flood and spray concerns

6. No credible seismic-induced fire concerns

7. No other "two over one" concerns as defined in DOE-STD-1021

8. No other concerns

Is equipment free of interaction effects?
$\begin{array}{llll}\mathrm{N} & \mathrm{U} & \mathrm{N} / \mathrm{A}\end{array}$

$\begin{array}{llll}Y & N & U & N / A\end{array}$

Y N U N/A

Y N N/A

$\begin{array}{llll}Y & N & U & N / A\end{array}$

$\begin{array}{lll}Y & N & N\end{array}$

$\begin{array}{lll}Y & N & N\end{array}$

$Y \quad N \quad U \quad N / A$

Y N U 


\section{DOE Seismic Evaluation Procedure}

SEWS 8.2.9 (3 of 3$)$

Sheet 3 of

\section{SCREEWINC EVALUATION WORK SHEET (SEWS) (Cont.)}

\begin{tabular}{|l|l}
\hline Equipment ID No.: & Equipment Class: Air Handlers \\
\hline
\end{tabular}

Equipment description:

\section{Comments}

Screening Walkdown(s):

Date Time $\quad$ Team Members

\section{Recommend Resolvtion}

口 Maintenance action:

Further evaluation:

$\square \quad$ Retrofit design:

Other:

o further action required. Equipment is seismically adequate.

All aspects of the equipment's seismic adequacy have been addressed.

Evaluation by:

Date:

(All team members) 


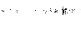




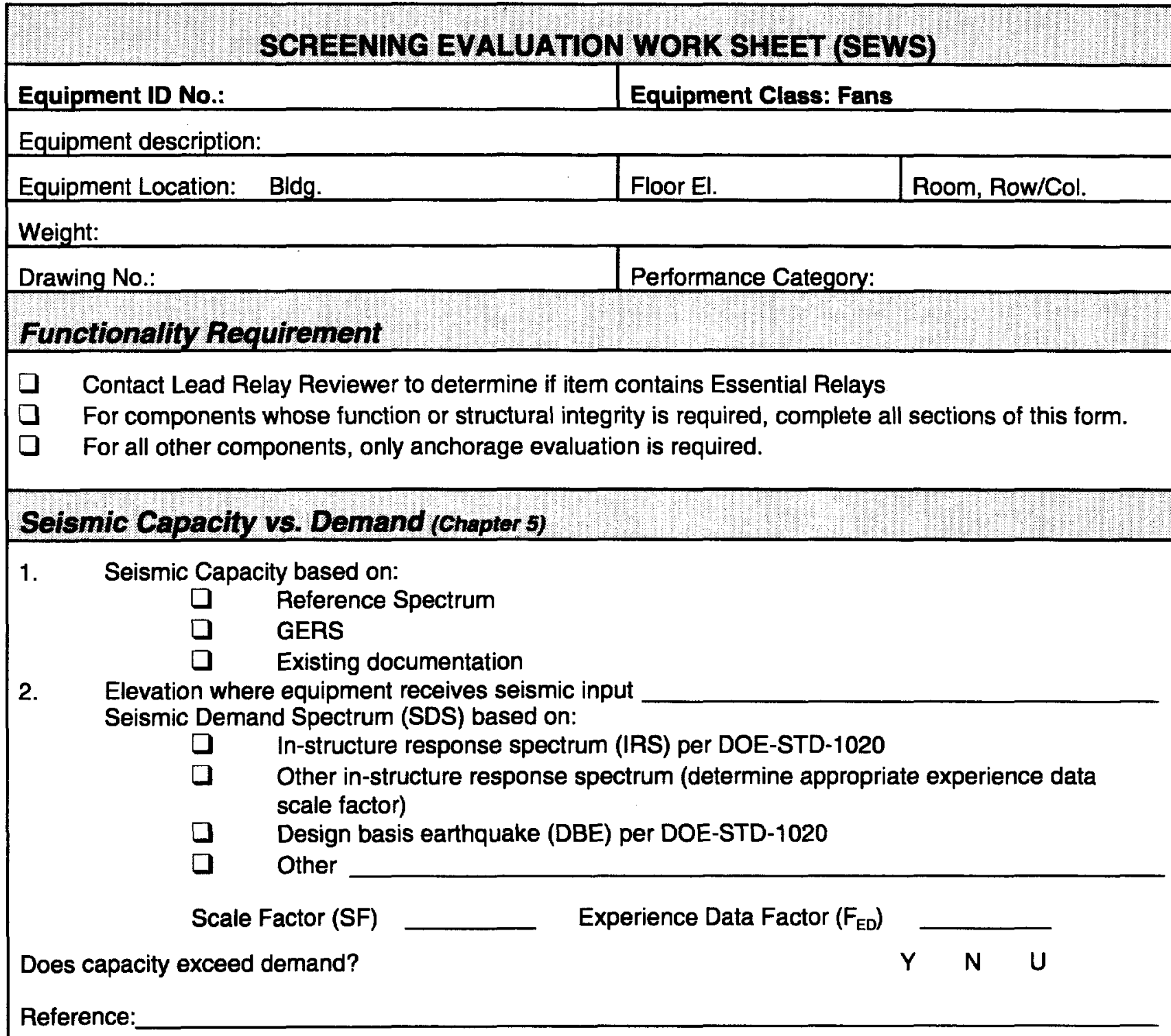

\section{Caveats (Section 8.2.10)}

Reference Spectrum (Identify with an asterisk (") those caveats which are met by intent without meeting the specific wording of the caveat rule and explain the reason for this conclusion in the COMMENTS section below)

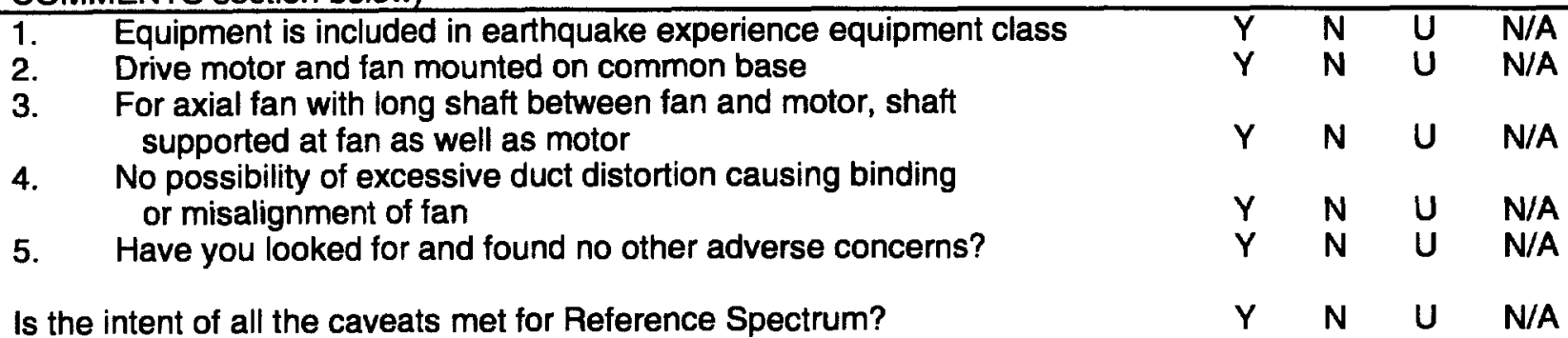




\section{SCREENING EVALUATION WORK SHEET (SEWS) (CONt)}

Equipment ID No.:

Equipment Class: Fans

Equipment description:

\section{Anchorage (Chapter 6)}

$1 . \quad$ Type of anchorage:

$\begin{array}{ll}\square & \text { expansion anchor } \\ \square & \text { cast-in-place bolt or headed stud anchor } \\ \square & \text { cast-in-place J-bolt } \\ \square & \text { grouted-in-place bolt } \\ \square & \text { welds to embedded steel on exposed steel } \\ \square & \text { lead cinch anchors } \\ \square & \text { Other } \\ \square & \text { N/A (no further anchorage considerations) }\end{array}$

2. Appropriate characteristics for anchorage type checked

(size, location, equipment characteristics)

3. Gap at threaded anchor less than $1 / 4$ inch

4. Base stiffness and no significant prying action requirements met

5. Equipment base strength and structural load path adequate

6. Embedment steel and pads requirements met

7. Embedment length requirements met

8. Anchor spacing requirements met

9. Edge distance requirements met

10. Concrete strength requirements met

11. Concrete crack requirements met

12. Equipment with essential relays requirements met

13. Installation adequacy requirements met

14. No other concerns

$\begin{array}{llll}Y & N & U & \\ Y & N & U & \text { N/A } \\ Y & N & U & \\ Y & N & U & \\ Y & N & U & N / A \\ Y & N & U & \\ Y & N & U & \\ Y & N & U & \\ Y & N & U & \\ Y & N & U & \\ Y & N & U & N / A \\ Y & N & U & N / A \\ Y & N & U & \end{array}$

Does anchorage capacity exceed demand?

$\begin{array}{lll}\mathrm{N} & \mathrm{U}\end{array}$

Reference:

\section{Interaction Effects (Chapter 7}

1. Soft targets free from impact by nearby equipment

or structures

2. If equipment contains sensitive essential relays, equipment free from all impact by nearby equipment or structures

3. Attached lines have adequate flexibility

4. No collapse of overhead equipment, distribution systems, or masonry walls

5. Equipment is free from credible and significant seismic-induced flood and spray concerns

6. No credible seismic-induced fire concerns

7. No other "two over one" concerns as defined in DOE-STD-1021

8. No other concerns

Is equipment free of interaction effects?

$\begin{array}{llll}Y & N & U & \text { N/A } \\ Y & N & U & \text { N/A } \\ Y & N & U & \text { N/A } \\ Y & N & & \text { N/A } \\ Y & N & U & \text { N/A } \\ Y & N & & \text { N/A } \\ Y & N & & \text { N/A } \\ Y & N & U & \text { N/A } \\ Y & N & U & \end{array}$




\section{SCREENING EVALUATION WORK SHEET (SEWS) (Cont.)}

Equipment ID No.:

Equipment description:

Comments

Screening Walkdown(s):

Date $\quad \underline{\text { Time }} \quad \underline{\text { Team Members }}$

\section{Recommend Resolution}

- Maintenance action:

Further evaluation:

$\square \quad$ Retrofit design:

$\square \quad$ Other:

$\square \quad$ No further action required. Equipment is seismically adequate.

All aspects of the equipment's seismic adequacy have been addressed.

Evaluation by:

Date:

(All team members) 


\section{SCREENING EVALUATION WORK SHEET (SEWS)}

\section{Equipment ID No.:}

Equipment Class: Horizontal Tanks and Heat Exchangers

Equipment description:

\begin{tabular}{|l|l|l}
\hline Equipment Location: Bldg. & Floor El. & Room, Row/Col. \\
\hline
\end{tabular}

Manufacturer, model, etc.:

Approximate weight:

Drawing No.:

Performance Category:

\section{Functionality Requirement}

Contact Lead Relay Reviewer to determine if item contains Essential Relays

For components whose function or structural integrity is required, complete all sections of this form.

$\square$ For all other components, only anchorage evaluation is required.

\section{Caveats (Section 9.1.2)}

(Identify with an asterisk $\left(^{*}\right)$ those steps which are met by intent without meeting the specific wording of the step and explain the reason for this conclusion in the COMMENTS section below)

Step 1 Parameters and values within range of applicable parameters

Y N U N/A

Step 2 Anchor bolt tension and shear load allowables determined

Y N U N/A

Step 3 Base plate bending strength reduction factor (RB) determined

$Y \quad N \quad U \quad N / A$

Step 4 Base plate weld strength reduction factor (RW) determined

$\begin{array}{llll}\mathrm{N} & \mathrm{U} & \mathrm{N} / \mathrm{A}\end{array}$

Step 5 Anchorage tension allowable determined using strength reduction factors

Step 6 Ratios and values calculated

Step 7 Acceleration capacity of tank anchorage determined

Step 8 Flexibility of tank in transverse and vertical directions determined

Step 9 Flexibility of tank in longitudinal direction determined

Step 10 Capacity acceleration exceeds seismic demand acceleration

Step 11 Saddle stresses checked

$\begin{array}{cccc}Y & N & U & N / A \\ Y & N & U & N / A \\ Y & N & U & N / A \\ Y & N & U & N / A \\ Y & N & U & N / A \\ Y & N & U & N / A \\ Y & N & U & \text { N/A }\end{array}$




\section{SCREENING EVALUATION WORK SHEET (SEWS) (Cont.)}

Equipment ID No.:

Equipment description:

\section{Anchorage (Chapter 6)}

1. Type of anchorage:

$\begin{array}{ll}\square & \text { expansion anchor } \\ \square & \text { cast-in-place bolt or headed stud anchor } \\ \square & \text { cast-in-place J-bolt } \\ \square & \text { grouted-in-place bolt } \\ \square & \text { welds to embedded steel on exposed steel } \\ \square & \text { lead cinch anchors } \\ \square & \text { Other } \\ \square & \text { N/A (no further anchorage considerations) }\end{array}$

2. Appropriate characteristics for anchorage type checked

(size, location, equipment characteristics)

3. Gap at threaded anchor less than 1/4 inch

4. Base stiffness and no significant prying action requirements met

5. Equipment base strength and structural load path adequate

6. Embedment steel and pads requirements met

7. Embedment length requirements met

8. Anchor spacing requirements met

9. Edge distance requirements met

10. Concrete strength requirements met

11. Concrete crack requirements met

12. Equipment with essential relays requirements met

13. Installation adequacy requirements met

14. No other concerns

\begin{tabular}{llll}
$Y$ & $N$ & $U$ & \\
$Y$ & $N$ & $U$ & $N / A$ \\
$Y$ & $N$ & $U$ & \\
$Y$ & $N$ & $U$ & \\
$Y$ & $N$ & $U$ & $N / A$ \\
$Y$ & $N$ & $U$ & \\
$Y$ & $N$ & $U$ & \\
$Y$ & $N$ & $U$ & \\
$Y$ & $N$ & $U$ & \\
$Y$ & $N$ & $U$ & \\
$Y$ & $N$ & $U$ & $N / A$ \\
$Y$ & $N$ & $U$ & $N / A$ \\
$Y$ & $N$ & $U$ & \\
$Y$ & $N$ & $U$ & \\
& & & \\
\hline
\end{tabular}

Does anchorage capacity exceed demand?

Reference:

\section{Interaction Efiects (Chapter 7 )}

1. Soft targets free from impact by nearby equipment or structures

2. If equipment contains sensitive essential relays, equipment free from all impact by nearby equipment or structures

3. Attached lines have adequate flexibility

4. No collapse of overhead equipment, distribution systems, or masonry walls

5. Equipment is free from credible and significant seismic-induced flood and spray concerns

$\begin{array}{llll}Y & N & U & \text { N/A } \\ Y & N & U & \text { N/A } \\ Y & N & U & \text { N/A } \\ Y & N & & \text { N/A } \\ Y & N & & \text { N/A }\end{array}$




\section{DOE Seismic Evaluation Procedure}

SEWS 9.1.2 (3 of 4)

Sheet 3 of

\section{SCREENING EVALUATION WORK SHEET (SEWS) (Cont.)}

Equipment ID No.:

Equipment Class: Horizontal Tanks and Heat Exchangers

Equipment description:

\section{Interaction Effects (Cont.)}

6. No credible seismic-induced fire concerns

7. No other "two over one" concerns as defined in DOE-STD-1021

8. No other concerns

Y N

$\begin{array}{lll}Y & N & N\end{array}$

$Y \quad N \quad U \quad N / A$

Is equipment free of interaction effects?

$\begin{array}{lll}\mathrm{N} & \mathrm{U}\end{array}$

\section{Comments}




\section{SCREENING EVALUATION WORK SHEET (SEWS) (Cont.)}

Equipment ID No.:

Equipment Class: Horizontal Tanks and Heat Exchangers

Equipment description:

Comments (cont)

Screening Walkdown(s):

Date Time Team Members

$\square \quad$ Maintenance action:

ㅁ Further evaluation:

$\square \quad$ Retrofit design:

$\square \quad$ Other:

No further action required. Equipment is seismically adequate.

All aspects of the equipment's seismic adequacy have been addressed.

Evaluation by:

Date:

(All team members) 
DOE Seismic Evaluation Procedure

SEWS 9.2.1 ( 1 of 6 )

Sheet 1 of

\section{SCREENING EVALUATION WORK SHEET (SEWS)}

Equipment ID No.:

Equipment Class: Cable and Conduit Raceway Systems

Cable tray/Conduit identification:

Systems:

Building:

Floor El. (S):

Location:

Performance Category:

Tray System or Conduit Boundary

Cable tray/Conduit description:

Description or sketch (attach sheets as necessary):

Functionality Requirement

Maintain electrical cable function

Maintain position 


\section{SCREENING EVALUATION WORK SHEET (SEWS) (Cont.)}

Equipment ID No.:

Equipment Class: Cable and Conduit Raceway Systems

Cable tray/Conduit identification:

Systems:

Selsmic Capacity vs. Demand (Chapter 5)

1. Seismic Capacity based on:
$\square \quad$ Reference Spectrum
G GERS
$\square \quad$ Existing documentation

2. Elevation where equipment receives seismic input

Seismic Demand Spectrum (SDS) based on:

$\square \quad$ In-structure response spectrum (IRS) per DOE-STD-1020

$\square \quad$ Other in-structure response spectrum (determine appropriate experience data scale factor)

Design basis earthquake (DBE) per DOE-STD-1020

口 Other

Scale Factor (SF)

Experience Data Factor $\left(F_{E D}\right)$

Does capacity exceed demand?

$\begin{array}{lll}\mathrm{N} & \mathrm{U}\end{array}$

Reference:

\section{Inclusion Rules Review (Section 9.2.1)}

1. Cable tray spans

Y $\quad N \quad U \quad N / A$

2. Conduit spans

$\begin{array}{llll}Y & N & \mathrm{~N} / \mathrm{A}\end{array}$

3. Tie downs

$\begin{array}{llll}Y & N & \mathrm{~N} / \mathrm{A}\end{array}$

4. Channel nuts

$\begin{array}{llll}Y & \mathrm{~N} & \mathrm{~N} / \mathrm{A}\end{array}$

5. Rigid boots

6. Beam clamps

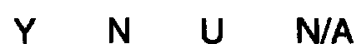

7. Cast-iron inserts

$Y \quad N \quad U \quad N / A$

Y N U N/A 


\section{SCREENING EVALUATION WORK SHEET (SEWS) (Cont.)}

Equipment ID No.:

Cable tray/Conduit identification:

Systems:

Ceneral Walkolown Review (Section 9.2.1)

1. Anchor bolts

$\begin{array}{llll}\mathrm{N} & \mathrm{U} & \mathrm{N} / \mathrm{A}\end{array}$

2. Concrete condition

$\begin{array}{llll}\mathrm{N} & \mathrm{U} & \mathrm{N} / \mathrm{A}\end{array}$

3. Corrosion

$\begin{array}{llll}Y & N & U & N / A\end{array}$

4. Sagging raceways

Y N U N/A

5. Broken or missing components

Y N U N/A

6. Restraint of cables

Y N U N/A

7. Aging of plastic ties

Y N U N/A

8. System hardspots

$\begin{array}{llll}Y & N & U & N / A\end{array}$

Welded connections

$\begin{array}{llll}\mathrm{N} & \mathrm{N} & \mathrm{N} / \mathrm{A}\end{array}$

Components and sharp edges

$\begin{array}{llll}\mathrm{N} & \mathrm{U} & \mathrm{N} / \mathrm{A}\end{array}$

Bare cables

Y N U N/A

Cable fill/ties

$\begin{array}{llll}\mathrm{N} & \mathrm{U} & \mathrm{N} / \mathrm{A}\end{array}$

Short rods

$\begin{array}{llll}Y & N & \text { U N }\end{array}$

\section{Interaction Effects (Chapter 7)}

1. Soft targets free from impact by nearby equipment

or structures

2. If equipment contains sensitive essential relays, equipment free from all impact by nearby equipment or structures

3. Attached lines have adequate flexibility

4. No collapse of overhead equipment, distribution systems, or masonry walls

5. Equipment is free from credible and significant seismic-induced flood and spray concerns

6. No credible seismic-induced fire concerns

7. No other "two over one" concerns as defined in DOE-STD-1021

8. No other concerns

$\begin{array}{llll}Y & N & U & N / A\end{array}$

$\begin{array}{llll}Y & N & U & N / A\end{array}$

$\begin{array}{llll}Y & N & U & N / A\end{array}$

Is equipment free of interaction effects?

Y N N/A

$\begin{array}{lll}Y & N & N\end{array}$

$\begin{array}{lll}Y & N & N\end{array}$

$Y \quad N \quad$ N/A

$Y \quad N \quad U \quad N / A$

$Y \quad N \quad U$ 


\section{DOE Seismic Evaluation Procedure}

SEWS 9.2.1 (4 of 6)

Sheet 4 of

\section{SCREENING EVALUATION WORK SHEET (SEWS) (Cont.)}

Equipment ID No.:

Equipment Class: Cable and Conduit Raceway Systems

Cable tray/Conduit identification:

Systems:

Analytical Review Support Selection 
DOE Seismic Evaluation Procedure

SEWS 9.2 .1 (5 of 6$)$

Sheet 5 of

SCREENING EVALUATION WORK SHEET (SEWS) (Cont.)

Equipment ID No.:

Equipment Class: Cable and Conduit Raceway

Systems

Cable tray/Conduit identification:

Systems:

Analytical Review Data Sheet

Room No.:

Selection No.:

Location:

Description and Sketch:

Additional Notes: 


\section{SCREENING EVALUATION WORK SHEET (SEWS) (Cont.)}

Equipment ID No.:

Cable tray/Conduit identification:

Systems:

\section{Comments}

Screening Walkdown(s):

Date Time Team Members

\section{Recommend Resolution}

口 Maintenance action:

- Further evaluation:

Detrofit design:

$\square$ Other:

- No further action required. Equipment is seismically adequate.

All aspects of the equipment's seismic adequacy have been addressed.

Evaluation by:

Date:

(All team members) 


\section{DOE Seismic Evaluation Procedure}

SEWS 10.1.1 (1 of 3)

Sheet 1 of

SCREENING EVALUATION WORK SHEET (SEWS)

Piping System ID No.: Equipment Class: Piping

Performance Category:

System Description and Fluid Boundaries:

\section{Piping System Function and Contents}

$\square$ Operability $\square$ Pressure Boudary $\square$ Position Retention

Piping Layout and Structural Boundaries

Piping System Location and Reference Drawings

Piping Materials and Sizes

Weights

Concurrent Pressure and Temperature

Input Response Spectra and SAM-Reterence

$\square$ Final $\square$ Preliminary

Applicability

$\square$ Ductile material $\square \mathrm{D} / \mathrm{t}<50 \quad \square-20^{\circ} \mathrm{F} \leq \mathrm{T} \leq 250^{\circ} \mathrm{F} \square$ Reference Spectra 


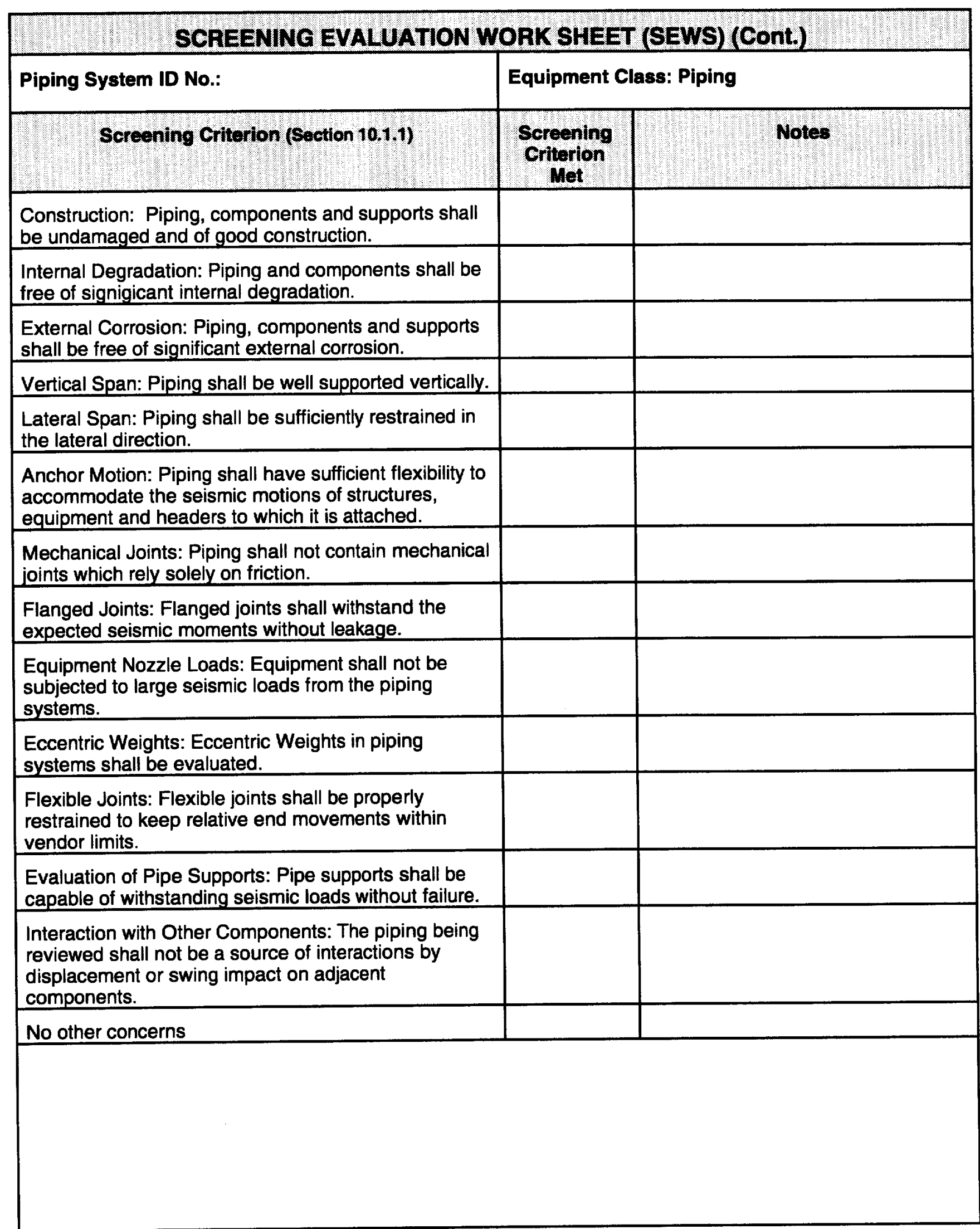




\section{DOE Seismic Evaluation Procedure}

SEWS 10.1.1 ( 3 of 3$)$

Sheet 3 of

\section{SCREENING EVALUATION WORK SHEET (SEWS) (Cont.)}

Piping System ID No.:

Equipment Class: Piping

\section{Comments}

Screening Walkdown(s):

Date Time Team Members

\section{Recommend Resolution}

Maintenance action:

Further evaluation:

口 Retrofit design:

$\square$ Other:

$\square \quad$ No further action required. Equipment is seismically adequate.

All aspects of the equipment's seismic adequacy have been addressed.

Evaluation by:

Date:

(All team members) 



\section{SCREENING EVALUATION WORK SHEET (SEWS)}

Equipment ID No.:

Equipment Class: HVAC Ducts

HVAC line identification:

System:

\begin{tabular}{|l|l|l|}
\hline Building: & Floor El. (s): & Location: \\
\hline Operating Pressure: & PSIG & Inches of water \\
\hline
\end{tabular}

Performance Category:

\section{Duct System Boundary}

Description or sketch (attach sheets as necessary):

\section{Functionality Requirement}

1. During seismic event

2. After seismic event

$\begin{array}{lll}Y & N & U \\ Y & N & U\end{array}$

\section{Structural Integrity Review (Section 10.4.1)}

1. Duct free of damage, defects, and degradation

2. Industry standard duct material and stiffeners are utilized

3. Industry standard duct joints are utilized

4. Support spans satisfy the criteria

5. Ducts are properly tied-down to the supports

6. Heavy in-line equipment is adequately restrained

7. Appurtenances are positively attached to duct

8. No stiff branch with flexible header

9. No other concerns

Are the above caveats met?

$\begin{array}{llll}Y & N & U & \text { N/A } \\ Y & N & U & \text { N/A } \\ Y & N & U & \text { N/A } \\ Y & N & U & \text { N/A } \\ Y & N & U & \text { N/A } \\ Y & N & U & \text { N/A } \\ Y & N & U & \text { N/A } \\ Y & N & U & \text { N/A } \\ Y & N & U & \text { N/A } \\ Y & N & U & \end{array}$




\section{SCREENING EVALUATION WORK SHEET (SEWS) (Cont.)}

Equipment ID No.:

Equipment Class: HVAC Ducts

HVAC line identification:

System:

\section{Pressure Boundary Integrity Review (Soction 10.4.1)}

Is any pressure boundary integrity required?

If the answer to the above question is NO, SKIP THIS SECTION

1. Duct joints are rugged

2. Stiffener spacings are within the guidelines

3. Bolted flanged joints satisfy SMACNA (Tables $\mathrm{G}$ and $\mathrm{H}$ ) requirements

4. No point supported round duct

5. Flexible bellows can accomodate motions

6. No additional concerns

Are the above caveats met?

Y N U

$\begin{array}{llll}Y & N & U & \text { N/A } \\ Y & N & U & \text { N/A } \\ Y & N & U & \text { N/A } \\ Y & N & U & \text { N/A } \\ Y & N & U & \text { N/A } \\ Y & N & U & \text { N/A } \\ Y & N & U & \end{array}$

\section{Support Review (Section 10.4.1)}

1. Beam Clamps are oriented to preclude slipping off the support, channel nuts have teeth or ridges, and no cast-iron inserts

2. Support memeber capacity exceeds demand

3. Does the anchorage appear adequate?

4. No broken or obviously defective hardware

5. No additional concerns

Are the above caveats met?

$\begin{array}{llll}Y & N & U & \text { N/A } \\ Y & N & U & \text { N/A } \\ Y & N & U & \text { N/A } \\ Y & N & U & \text { N/A } \\ Y & N & U & \text { N/A } \\ Y & N & U & \end{array}$

\section{Interaction Effects (Chapter 7 )}

1. Soft targets free from impact by nearby equipment or structures

2. If equipment contains sensitive essential relays, equipment free from all impact by nearby equipment or structures

3. Attached lines have adequate flexibility

4. No collapse of overhead equipment, distribution systems, or masonry walls

5. Equipment is free from credible and significant seismic-induced flood and spray concerns

6. No credible seismic-induced fire concerns

7. No other "two over one" concerns as defined in DOE-STD-1021

8. No other concerns

Is equipment free of interaction effects?

Y N U N/A

$\begin{array}{llll}Y & N & U & N\end{array}$

$\begin{array}{llll}Y & N & \mathrm{~N} / \mathrm{A}\end{array}$

Y N N/A

$\begin{array}{lll}Y & N & N\end{array}$

$Y \quad N \quad N / A$

$Y \quad N \quad N / A$

$\begin{array}{llll}Y & N & N & N / A\end{array}$

$\begin{array}{lll}\mathrm{N} & \mathrm{U}\end{array}$ 
DOE Seismic Evaluation Procedure

SEWS 10.4.1 (3 of 4$)$

Sheet 3 of

\section{SCREENING EVALUATION WORK SHEET (SEWS) (Cont.)}

Equipment ID No.:

Equipment Class: HVAC Ducts

HVAC line identification:

System:

Bounding Candidate Evaluation

Duct is not a candidate for bounding calculations

$\begin{array}{llll}Y & \mathrm{~N} & \mathrm{~N} / \mathrm{A}\end{array}$

Discussion: 


\section{DOE Seismic Evaluation Procedure}

SEWS 10.4 .1 (4 of 4$)$

Sheet 4 of

\section{SCREENING EVALUATION WORK SHEET (SEWS) (Cont)}

Equipment ID No.:

Equipment Class: HVAC Ducts

HVAC line identification:

System:

\section{Comments}

Screening Walkdown(s):

Date

Time

Team Members

\section{Recommend Resolution}

口 Maintenance action:

$\square \quad$ Further evaluation:

Retrofit design:

$\square$ Other:

a No further action required. Equipment is seismically adequate.

All aspects of the equipment's seismic adequacy have been addressed.

Evaluation by:

Date:

(All team members) 
DOE Seismic Evaluation Procedure

SEWS $10 . X . X(1$ of 2$)$

Sheet 1 of

\section{SOREENING EVALUATION WORK SHEET (SEWS)}

ID No.:

Equipment Class:

Building:

Performance Category:

Floor El. (s):

Location:

Description or Sketch

Functionality Requirement

1. During seismic event

2. After seismic event

$\begin{array}{lll}Y & N & U \\ Y & N & U\end{array}$ 


\section{SCREENING EVALUATION WORK SHEET (SEWS) (Cont.)}

ID No.:

Equipment Class:

Building:

Floor El. (s):

Location:

\section{Comments}

Screening Walkdown(s):

Date Time Team Members

\section{Recommend Resolution}

口 Maintenance action:

- Further evaluation:

Retrofit design:

Other:

- No further action required. Equipment is seismically adequate.

All aspects of the equipment's seismic adequacy have been addressed.

Evaluation by:

Date:

(All team members) 


\section{OUTLIER SEISMIC EVALUATION SHEET (OSES)}

1. OUTLIER IDENTIFICATION, DESCRIPTION, AND LOCATION

SEWS Form

Equipment ID Number

Equipment Class

Equipment Location: Building

Floor Elevation

Room or Row/Column

Base Elevation

Equipment Description

Performance Category

2. OUTLIER ISSUE DEFINITION

a. Identify all the screening guidelines which are not met. (Check more than one if several guidelines could not be satisfied.)

Mechanical and Electrical Equipment

Seismic Capacity vs. Demand

Reference Spectrum Caveats

GERS Caveats

Anchorage

Interaction Effects

Other

Essential Relays

Seismic Capacity vs. Demand

Interaction Effects

Mounting, Type, Location

Other
Tanks and Heat Exchangers

Caveats

Anchorage

Interaction Effects

Other

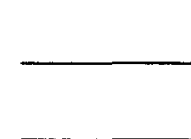

\section{Cable and Conduit Raceway Systems}

Seismic Capacity vs. Demand

Inclusion Rules Review

General Walkdown Review

Interaction Effects

Analytical Review

Other 


\section{OUTLIER SEISMIC EVALUATION SHEET (OSES) (CONt.)}

Piping

Screening Criterion

Other

\section{HVAC Ducts}

Structural Integrity Review

Pressure Boundary Integrity Review

Support Review

Interaction Effects

Analytical Review

Other

b. Describe all the reasons for the outlier (i.e., if all the listed outer issues were resolved, then the signatories would consider this item of equipment to be evaluated for seismic adequacy):

\section{PROPOSED METHOD OF OUTLIER RESOLUTION}

a. Define proposed method(s) of resolving outlier: 


\section{DOE Seismic Evaluation Procedure}

OSES (3 of 3$)$

Sheet 3 of

\section{OUTLIER SEISMIC EVALUATION SHEET (OSES) (Cont.)}

b. Provide information needed to implement proposed method(s) for resolving outlier:

c. Provide information on potential hardware upgrades: 
Sheet 1 of

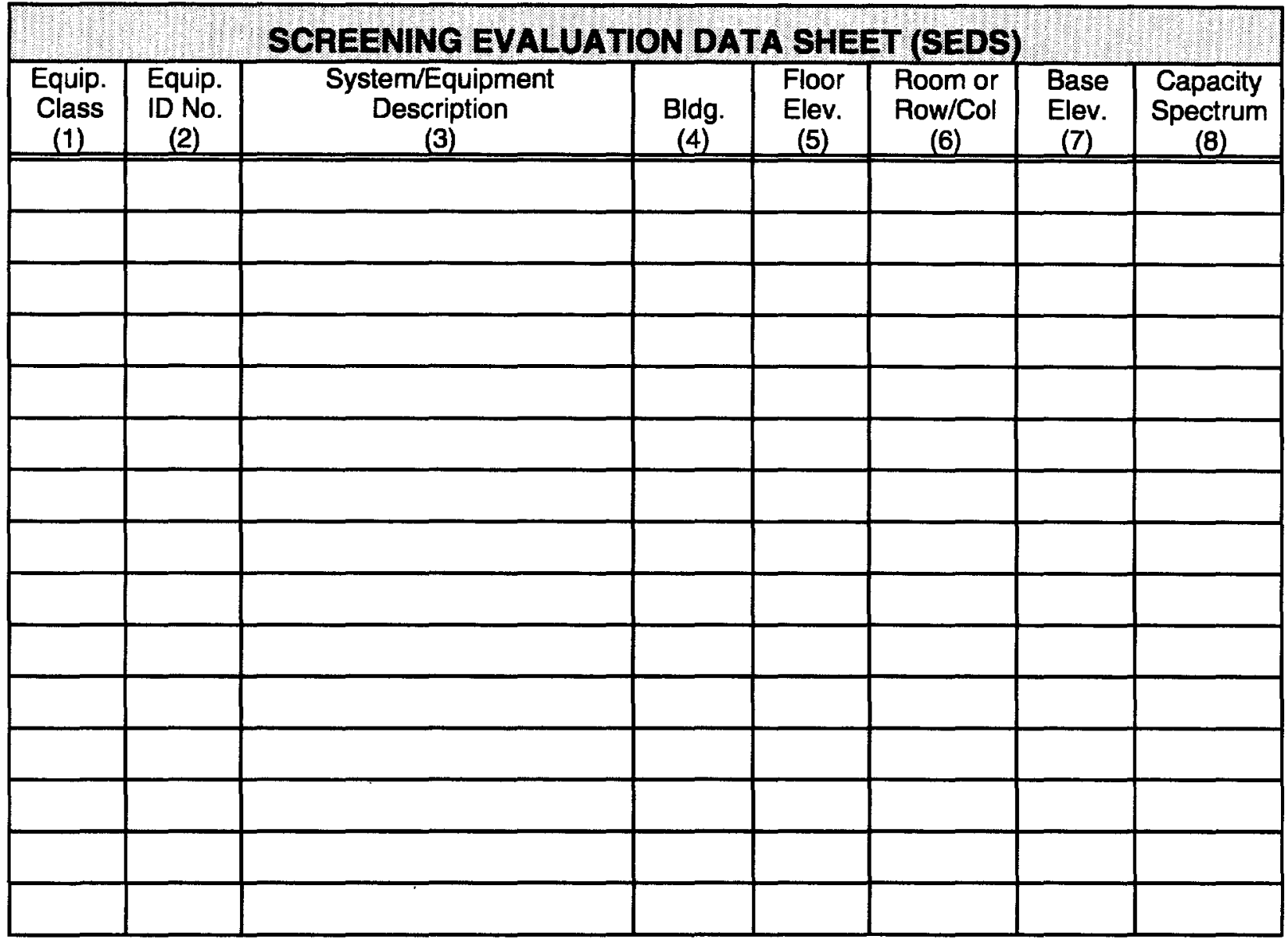

SIGNATURES:

All the information contained on this Screening Evaluation Data Sheet (SEDS) is, to the best of our knowledge and belief, correct and accurate. "All information" includes each entry and conclusion (whether evaluated to be seismically adequate or not).

Approved: All Seismic Capability Engineers on the Seismic Review Team should sign.

Print or Type Name

Print or Type Name

Print or Type Name
Signature

Signature

Date

Date
Signature

\section{Date}




\begin{tabular}{|c|c|c|c|c|c|c|c|}
\hline & SCF & BNING E & UATIO & ИАTA SHE & T ISED & (Cont,) & \\
\hline $\begin{array}{c}F_{E D} \\
(9)\end{array}$ & $\begin{array}{l}\text { Demand } \\
\text { Spectrum } \\
\text { (10) }\end{array}$ & $\begin{array}{c}\text { Cap > } \\
\text { Demand? } \\
(11)\end{array}$ & $\begin{array}{c}\text { Caveats } \\
\text { OK? } \\
\text { (12) }\end{array}$ & $\begin{array}{c}\text { Anchorage } \\
\text { OK? } \\
\text { (13) }\end{array}$ & $\begin{array}{l}\text { Interact. } \\
\text { OK? } \\
(14)\end{array}$ & $\begin{array}{l}\text { Equipment } \\
\text { OK? } \\
\text { (15) }\end{array}$ & $\begin{array}{c}\text { Notes } \\
\text { (16) }\end{array}$ \\
\hline & & & & & & & \\
\hline & & & & & & & \\
\hline & & & & & & & \\
\hline & & & & & & & \\
\hline & & & & & & & \\
\hline & & & & & & & \\
\hline & & & & & & & \\
\hline & & & & & & & \\
\hline & & & & & & & \\
\hline & & & & & & & \\
\hline & & & & & & & \\
\hline & & & & & & & \\
\hline & & & & & & & \\
\hline & & & & & & & \\
\hline & & & & & & & \\
\hline
\end{tabular}

\section{ADDITIONAL SIGNATURES:}

The information provided to the Seismic Capability Engineers regarding systems and operations of the equipment contained on this SEDS is, to the best of our knowledge and belief, correct and accurate.

Approved: $\quad$ Signature(s) of Systems or Operations Engineers are required if the Seismic Capability Engineers deem it necessary.

Print or Type Name

Print or Type Name

Print or Type Name
Signature

Signature

Signature
Date

Date 


\section{REFERENCES}

1. "Generic Implementation Procedure (GIP) for Seismic Verification of Nuclear Plant Equipment", Revision 2, Seismic Qualification Utility Group, prepared by Winston \& Strawn, EQE Incorporated, MPR Associates, Inc., Stevenson \& Associates, and URS Corporation / John A. Blume \& Associates, Engineers, February 14, 1992. (Volume 2 of DOE Binders)

2. "Supplement No. 1 to Generic Letter (GL) 87-02 that Transmits Supplemental Safety Evaluation Report No. 2 (SSER No. 2) on SQUG Generic Implementation Procedure, Revision 2, as Corrected on February 14, 1992 (GIP-2)", U.S. Nuclear Regulatory Commission, Washington, D.C., May 22, 1992. (Volume 2 of DOE Binders)

3. "Procedure for the Seismic Evaluation of SRS Systems Using Experience Data", SEP-6, Rev. 1, Westinghouse Savannah River Company, Aiken, South Carolina, February 14, 1992.

4. "Generic Implementation Procedure (GIP) for Seismic Verification of Nuclear Plant Equipment", Revision 3, Seismic Qualification Utility Group, being reviewed by the U.S. Nuclear Regulatory Commission, Rockville, Maryland.

5. DOE Order 420.1, "Facility Safety", U.S. Department of Energy, Washington, D.C., October 1996.

6. DOE-STD-1020-94 (CH-1), "Natural Phenomena Hazards Design and Evaluation Criteria for Department of Energy Facilities", U.S. Department of Energy, Washington, D.C., January 1996.

7. DOE-STD-1021-93 (CH-1), "Natural Phenomena Hazards Performance Categorization Guidelines for Structures, Systems, and Components", U.S. Department of Energy, Washington, D.C., January 1996.

8. DOE Order 5480.28, "Natural Phenomena Hazards Mitigation", U.S. Department of Energy, Washington, D.C., January 15, 1993.

9. DOE Order 5480.23, "Nuclear Safety Analysis Reports", U.S. Department of Energy, Washington, D.C., April 10, 1992.

10. DOE-STD-1027-92, "Hazard Categorization and Accident Analysis Techniques for Compliance with DOE Order 5480.23, Nuclear Safety Analysis Reports", U.S. Department of Energy, Washington, D.C., December 1992.

11. DOE-STD-3009-94, "Preparation Guide for U.S. Department of Energy Nonreactor Nuclear Facility Safety Analysis Reports", U.S. Department of Energy, Washington, D.C., July 1994.

12. IEEE-344-1975, "IEEE Recommended Practices for Seismic Qualification of Class 1E Equipment for Nuclear Power Generating Stations", Institute of Electrical and Electronics Engineers, 1975.

13. Unresolved Safety Issue (USI) A-46, "Seismic Qualification of Equipment in Operating Plants", U.S. Nuclear Regulatory Commission, Rockville, Maryland, December 1980. 
14. 10CFR100, Appendix A, Code of Federal Regulations - Energy, Office of the Federal Register - National Archives and Records Administration, Washington, D.C., 1990.

15. NRC Generic Letter 87-02, "Verification of Seismic Adequacy of Mechanical and Electrical Equipment in Operating Reactors, Unresolved Safety Issue (USI) A-46", U.S. Nuclear Regulatory Commission, Rockville, Maryland, February 19, 1987.

16. NUREG-1030, "Seismic Qualification of Equipment in Operating Nuclear Power Plants, Unresolved Safety Issue A-46", U.S. Nuclear Regulatory Commission, Washington, D.C., February 1987.

17. NUREG-1211, "Regulatory Analysis for Resolution of Unresolved Safety Issue A-46, Seismic Qualification of Equipment in Operating Plants", U.S. Nuclear Regulatory Commission, Washington, D.C., February 1987.

18. EPRI Report NP-6041, Revision 1, "A Methodology for Assessment of Nuclear Power Plant Seismic Margin", Electric Power Research Institute, Palo Alto, California, prepared by NTS Engineering and RPK Consulting, July 1991. (Volume 7 of DOE Binders)

19. SSRAP Report, "Use of Seismic Experience Data to Show Ruggedness of Equipment in Nuclear Power Plants", Revision 4, SAND92-0140 Part I, UC-523, Sandia National Laboratories, Albuquerque, New Mexico, prepared by Senior Seismic Review and Advisory Panel, February 28, 1991. (Volume 4 of DOE Binders)

20. "Program Plan for the Evaluation of Systems and Components in Existing DOE Facilities Subject to Natural Phenomena Hazards", Revision 0, U.S. Department of Energy, Washington, D.C., prepared by Lawrence Livermore National Laboratory and EQE Engineering Consultants, November 1992.

21. "NPH Steering Group Position on Use of Experience Data for DOE Seismic Evaluations and on Use of Walkthrough Field Guide and EPRI / SQUG Seismic Evaluation Material", Westinghouse Savannah River Company, Aiken, South Carolina, prepared by NPH Steering Group, May 25, 1993.

22. "Experience Based Seismic Evaluation By Rules in Lieu of Rigorous Analysis or Testing", Kennedy, R.P., Structural Mechanics Consulting, Yorba Linda, California, letter to R.C. Murray, September 2, 1996.

23. "Walkthrough Screening Evaluation Field Guide - Natural Phenomena Hazards at Department of Energy Facilities", UCRL-ID-115714 Rev. 2, Lawrence Livermore National Laboratory, Livermore, California, prepared by S.J. Eder, M.W. Eli, and M.W. Salmon, November 1993.

24. "Meeting Performance Goals by the Use of Experience Data", UCRL-CR-120813, S/CB235369, S/C-B298294, Lawrence Livermore National Laboratory, Livermore, California, prepared by M.W. Salmon and R.P. Kennedy, December 1, 1994.

25. "Generic Implementation Procedure for the Seismic Evaluation of Equipment in Existing U.S. Department of Energy Facilities - Draft", UCRL-ID-122109DR, Lawrence Livermore National Laboratory, Livermore, California, September 12, 1995.

26. "Seismic Evaluation Procedure for Equipment in U.S. Department of Energy Facilities Final Draft", UCRL-ID-122109DR Revision 1, Lawrence Livermore National Laboratory, Livermore, California, November 11, 1996. 
27. "Response to Comments Received by Letter of September, 1994 from Mr. E.A. Wais to Mr. G.A. Antaki", ECS-SSA-95-0130, Westinghouse Savannah River Company, Aiken, South Carolina, June 9, 1995.

28. "Procedure for the Seismic Evaluation of Steel HVAC Duct", ECS-SSA-95-0195, Westinghouse Savannah River Company, Aiken, South Carolina, November 7, 1995.

29. "Seismic Design and Evaluation Guidelines for the Department of Energy High-Level Waste Storage Tanks and Appurtenances", BNL 52361, K. Bandyopadhyay, et. al., Brookhaven National Laboratory, Upton, New York, October 1995.

30. "Review of DOE Seismic Evaluation Procedure", Budnitz, R.J., Kennedy, R.P., and Wyllie, L.A., Future Resources Associates, Inc., Berkeley, California, letter to R.C. Murray, March 14, 1997.

31. "Economies of Using Seismic Experience Data Qualification Methods at Department of Energy Facilities", F. Loceff, G. Antaki, and L. Goen, Fifth DOE Natural Phenomena Hazards Mitigation Conference, Denver, Colorado, November 1995.

32. "Seismic Safety Manual - A Practical Guide for Facility Managers and Earthquake Engineers", UCRL-MA-125085, Lawrence Livermore National Laboratory, Livermore, California, prepared by Eagling, D.G., September 1996.

33. "The January 17, 1994 Northridge Earthquake: Effects on Selected Industrial Facilities and Lifelines", UCRL-ID-120522 Rev. 0, Lawrence Livermore National Laboratory, prepared by M.W. Eli, S.C. Sommer, T.R. Roche, and K.L. Merz, February 1995.

34. "The Hyogo-Ken Nanbu Earthquake of January 17, 1995 - NRC/DOE Reconnaissance Report - Draft", Chokshi, N.C., et. al., U.S. Nuclear Regulatory Commission, Rockville, Maryland, 1996.

35. EPRI Report NP-7149, "Summary of the Seismic Adequacy of Twenty Classes of Equipment Required for Safe Shutdown of Nuclear Plants", Electric Power Research Institute, Palo Alto, prepared by EQE, Inc., March 1991. (Volume 4 of DOE Binders)

36. Earthquake Engineering Research Center (EERC), "Seismological and Engineering Aspects of the January 17, 1994 Northridge Earthquake: Abridge Slide Set", University of California at Berkeley, 1994.

37. blank

38. blank

39. EPRI Report NP-6628, "Procedure for Seismic Evaluation and Design of Small Bore Piping (NCIG-14)", Electric Power Research Institute, Palo Alto, California, prepared by Stevenson \& Associates and EQE Engineering, April 1990.

40. EPRI Report NP-5223, Revision 1, "Generic Seismic Ruggedness of Power Plant Equipment in Nuclear Power Plants", Electric Power Research Institute, Palo Alto, California, prepared by ANCO Engineers, Inc., February 1991. (Volume 4 of DOE Binders) 
41. EPRI Report NP-5228, Revision 1, "Seismic Verification of Nuclear Plant Equipment Anchorage", Volumes 1, 2, and 3, Electric Power Research Institute, Palo Alto, California, prepared by URS Corporation / John A. Blume \& Associates, Engineers, June 1991. (Volume 3 of DOE Binders)

42. EPRI Report NP-5228, Revision 1, "Seismic Verification of Nuclear Plant Equipment Anchorage", Volume 4, "Guidelines for Tanks and Heat Exchangers", Electric Power Research Institute, Palo Alto, California, prepared by URS Corporation / John A. Blume \& Associates, Engineers, June 1991. (Volume 3 of DOE Binders)

43. EPRI Report NP-7146, "Development of In-Cabinet Amplified Response Spectra for Electrical Benchboards and Panels", Electric Power Research Institute, Palo Alto, California, prepared by Stevenson \& Associates, December 1990. (Volume 5 of DOE Binders)

44. EPRI Report NP-7147, "Seismic Ruggedness of Relays", Electric Power Research Institute, Palo, Alto, California, prepared by ANCO Engineers, Inc., February 1991. (Volume 5 of DOE Binders)

45. EPRI Report NP-7148, "Procedure for Evaluating Nuclear Power Plant Relay Seismic Functionality", Electric Power Research Institute, Palo Alto, California, prepared by MPR Associates, Inc., December 1990. (Volume 5 of DOE Binders)

46. EPRI Report NP-7150, "The Performance of Raceway Systems in Strong Motion Earthquakes", Electric Power Research Institute, Palo Alto, California, prepared by EQE, Inc., March 1991. (Volume 6 of DOE Binders)

47. EPRI Report NP-7151, "Cable Tray and Conduit System Seismic Evaluation Guidelines", Electric Power Research Institute, Palo Alto, California, prepared by EQE, Inc., March 1991. (Volume 6 of DOE Binders)

48. EPRI Report NP-7152, "Seismic Evaluation of Rod Hanger Supports for Electrical Raceway Systems", Electric Power Research Institute, Palo Alto, California, prepared by EQE Engineering, Inc., March 1991. (Volume 6 of DOE Binders)

49. EPRI Report NP-7153, "Longitudinal Load Resistance in Seismic Experience Data Base Raceway Systems", Electric Power Research Institute, Palo Alto, California, prepared by EQE Engineering, Inc., March 1991. (Volume 6 of DOE Binders)

50. SSRAP Cable Tray Report, "Review Procedure to Assess Seismic Ruggedness of Cantilever Bracket Cable Tray Supports", Revision 3, SAND92-0140 Part II, UC-523, Sandia National Laboratories, Albuquerque, New Mexico, prepared by Senior Seismic Review and Advisory Panel, March 1, 1991. (Volume 6 of DOE Binders)

51. ATC-29, "Seismic Design and Performance of Equipment and Nonstructural Elements in Buildings and Industrial Structures - A Proposed Methodology for the Seismic Design of Rectangular Duct Systems", Bragagnolo, L.J., J.P. Conoscente, and S.J. Eder, EQE Engineering, San Francisco, California, 1991. (Volume 6 of DOE Binders)

52. "Guidelines for the Seismic Design of Fire Protection Systems", Benda, B. and Cushing, R., EQE Engineering, and Driesen, G.E., Westinghouse Savannah River Company, PVPVol. 214, DOE Facilities Programs and Systems Interaction with Linear and Non-Linear Techniques, American Society of Mechanical Engineers, 1991. (Volume 6 of DOE Binders) 
53. "Screening Criteria for the Verification of Seismic Adequacy of Piping Systems", Antaki, G.A., Westinghouse Savannah River Company, Hardy, G., EQE Engineering, and Rigamonti, G., United Engineers and Constructors, PVP-Vol. 214, DOE Facilities Programs and Systems Interaction with Linear and Non-Linear Techniques, American Society of Mechanical Engineers, 1991. (Volume 6 of DOE Binders)

54. "Advancement in Design Standards for Raceway Supports and its Applicability to Piping Systems", Dizon, J.O. and S.J. Eder, EQE Engineering, PVP-Vol. 210-1, Codes and Standards and Applications for Design and Analysis of Pressure Vessel and Piping Components, American Society of Mechanical Engineers, 1991. (Volume 6 of DOE Binders)

55. "Response Predictions for Piping Systems which have Experienced Strong Motion Earthquakes", Campbell, R.D., L.W. Tiong, and J.O. Dizon, EQE Engineering, Costa Mesa, California, 1991. (Volume 6 of DOE Binders)

56. "Procedure for Control and Use of SQUG/EPRI Material Licensed for Use on DOE Facilities", Lawrence Livermore National Laboratory, Livermore, California, August 9, 1995.

57. NUREG/CR-1489, UCRL-52746, "Best Estimate Method vs. Evaluation Method: A Comparison of Two Techniques in Evaluating Seismic Analysis and Design", Lawrence Livermore National Laboratory, Livermore, California, May 1980.

58. LLL-TB-026, "Seismic Safety Margins Research Program, Executive Summary Number 1 - Best Estimate vs. Evaluation Method", Lawrence Livermore National Laboratory, Livermore, California, (no date).

59. "Procedure for the Seismic Evaluation of Piping Systems Using Screening Criteria", Rev. 1, WSRC-TR-94-0343, Westinghouse Savannah River Company, Aiken South Carolina, June 1995.

60. "Practical Equipment Seismic Upgrade and Strengthening Guidelines", UCRL-15815, P/O 9227705, Lawrence Livermore National Laboratory, Livermore, California, prepared by EQE Incorporated, September 1986.

61. 10CFR830.120, Code of Federal Regulations - Energy, Office of the Federal Register National Archives and Records Administration, Washington, D.C., 1990.

62. DOE Order 5700.6C (CH-1), "Quality Assurance", U.S. Department of Energy, Washington, D.C., May 10, 1996.

63. "DOE Workshop on NPH Walkthrough Field Guide and SQUG/EPRI Seismic Evaluation Material", Lawrence Livermore National Laboratory, December 1992. (Volume 1 of DOE Binders)

64. 'DOE Training Course on SQUG/EPRI Walkdown Screening and Seismic Evaluation Material", Lawrence Livermore National Laboratory, Livermore, California, March 1994 and September 1995. (Volume 8 of DOE Binders)

65. "Workshop on the Seismic Evaluation of Piping Systems Using Screening Criteria", WSRC-TR-94-0343, Antaki, G.A., Westinghouse Savannah River Company, Aiken, South Carolina, November 1995. 
66. DOE Order 5480.30, "General Design Criteria for Nuclear Reactors", U.S. Department of Energy, Washington, D.C., January 1993.

67. DOE Order 5480.25, "Safety of Accelerator Facilities", U.S. Department of Energy, Washington, D.C. November 3, 1992.

68. DOE Order 5481.1B, "Safety Analysis and Review System", U.S. Department of Energy, Washington, D.C., September 1986.

69. International Conference of Building Officials, "Uniform Code for Building Conservation", Whittier, CA., 1994.

70. DOE-STD-1022-94, "Natural Phenomena Hazards Site Characterization Criteria", U.S. Department of Energy, Washington, D.C., 1994.

71. DOE-STD-1023-95, "Natural Phenomena Hazards Assessment Criteria", U.S. Department of Energy, Washington, D.C., 1995.

72. NUREG/CR-0098, "Development of Criteria for Seismic Review of Selected Nuclear Power Plants", by N. M. Newmark, et. al., May 1978.

73. "Basis for Seismic Provisions of UCRL-15910 - Draft", UCRL-CR-111478 DR, Sub. No. B192525, Lawrence Livermore National Laboratory, Livermore, California, prepared by R.P. Kennedy and S.A. Short, September 1992.

74. ASCE 4, "Seismic Analysis of Safety-Related Nuclear Structures and Commentary - Final Draft", American Society of Civil Engineers, New York, New York, January 1997.

75. FEMA 222A, "NEHRP Recommended Provisions for Seismic Regulations for New Buildings", Federal Emergency Management Agency, Building Seismic Safety Council, Washington, D.C., 1994.

76. "Design Safety Standards - Mechanical Engineering", M-012 Revision 7, Lawrence Livermore National Laboratory, Livermore, California, February 1993.

77. EPRI Report TR-102180, "Guidelines for Estimation or Verification of Equipment Natural Frequency", Electric Power Research Institute, Palo Alto, California, prepared by ANCO Engineers, Inc., March 1993.

78. EPRI Report TR-103960, "Recommended Approaches for Resolving Anchorage Outliers", Electric Power Research Institute, Palo Alto, California, prepared by URS / John A. Blume \& Associates, Engineers, June 1994.

79. ASTM A-307, "Standard Specification for Carbon Steel Bolts and Studs", American Society for Testing and Materials, 1994.

80. "Analysis of Laboratory Tests on Grouting of Anchor Bolts Into Hardened Concrete", M.A. Cones, Tennessee Valley Authority, November 1977.

81. "Manual of Steel Construction", 9th Edition, American Institute of Steel Construction, Inc., Chicago, Illinois, 1989. 
82. NEMA ICS-6, "Industrial Control and Systems Enclosures", National Electrical Manufacturers Association, 1993.

83. UL-508, "UL Standard for Safety Industrial Control Equipment", Underwriters Laboratory, 1993.

84. ANSI IEEE C37.20, "Standard for Metal-Enclosed Low-Voltage Power Circuit Breaker Switchgear", Institute of Electrical and Electronic Engineers, 1993.

85. NEC NFPA 70, "National Electrical Code", National Fire Protection Association, Quincy, Massachusetts, 1996.

86. NEMA PB2, "Deadfront Distribution Switchboards", National Electrical Manufacturers Association, 1989.

87. "Design of Plate Structures, Steel Plate Engineering Data - Volume 2", Part VII, Anchor Bolt Chairs, American Iron and Steel Institute, June 1985.

88. NFPA 70-1984, "National Electrical Code", National Fire Protection Association, Boston, Massachusetts, 1984.

89. NEMA Standard VE 1-1984, "Metallic Cable Tray Systems", National Electric Manufacturers Association, Washington, D.C., 1984,

90. ASME B31.1, "Power Piping”, American Society of Mechanical Engineers, New York, New York., 1995.

91. ASME B31.3, "Chemical Plant and Petroleum Refinery (Process) Piping", American Society of Mechanical Engineers, New York, New York, 1996.

92. NFPA-13, "Installation of Sprinkler Systems", National Fire Protection Association, Quincy, Massachusetts, 1996.

93. AWWA Manual M11, "Steel Pipe-A Guide for Design and Installation", American Water Works Association, Denver, Colorado, 1989.

94. NUREG-1061, "Report of the U.S. Nuclear Regulatory Commission Piping Review Committee", "Strong-Motion Earthquake Seismic Response and Damage to Aboveground Industrial Piping", "Summary and Evaluation of Historical Strong Motion Earthquake Seismic Response and Damage to Aboveground Industrial Piping", Volume 2 Addendum, U.S. Nuclear Regulatory Commission, Rockville, Maryland, April 1985.

95. EPRI NP-5617, "Recommended Piping Seismic Adequacy Criteria Based on Performance During and After Earthquakes", Volume 1 \& 2, Electric Power Research Institute, Palo Alto, California, February 1987, January 1988.

96. "Review of Seismic Response Data for Piping", Slagis, G.C., Grant 92-03, ASME Pressure Vessel Research Council, Welding Research Council, New York, New York, 1992.

97. “Application of Bounding Spectra to Seismic Design of Piping Based on the Performance of Above Ground Piping in Power Plant Subjected to Strong Motion Earthquakes", Stevenson, J.D., Stevenson \& Associates, Cleveland, Ohio, May 11, 1992. 
98. "Survey of Strong Motion Earthquake Effects on Thermal Power Plants in California with Emphasis on Piping Systems", Stevenson \& Associates, Cleveland, Ohio, March 31, 1993.

99. "Performance of Piping During the January 17, 1994, Northridge Earthquake", Freeland, D.J. and Roche, T.R., EQE Engineering Consultants, San Francisco, California, March 1994.

100. ASME Boiler and Pressure Vessel Code, American Society of Mechanical Engineers, New York, New York, July 1, 1992.

101. "Specification for the Design of Cold-Formed Steel Structural Members", American Iron and Steel Institute, Washington, D.C., 1979.

102. ANSI B16.5, "Pipe Flanges and Flanged Fittings", American National Standards Institute, 1988.

103. "ASME B31 Appendix on Seismic Design - Draft", American Society of Mechanical Engineers, New York, New York, 1996.

104. NRC Regulatory Guide 1.60, Revision 1, "Design Response Spectra for Seismic Design of Nuclear Power Plants", U.S. Nuclear Regulatory Commission, Rockville, Maryland, December 1973.

105. "ASCE Guidelines for the Seismic Design of Oil and Gas Pipeline Systems", American Society of Civil Engineers, New York, New York, 1984.

106. "Effects of the 1985 Michoacan Earthquake on Water Systems and other Buried Lifelines in Mexico", Technical Report NCEER-89-0009, Ayala, A.G. and M.J. O'Rourke, State University of New York at Buffalo, New York, March 8, 1989.

107. "Criteria for the Seismic Evaluation of Steel HVAC Ducts at SRS Facilities", WSRC Calculation No. T-CLC-G-00034, Westinghouse Savannah River Company, Aiken, South Carolina, prepared by EQE International, Inc., 1995.

108. ASME AG-1-1994, "Code on Nuclear Air and Gas Treatment", American Society of Mechanical Engineers, New York, New York, 1995.

109. "Advanced Light Water Reactor (ALWR) First-of-a-kind-Engineering (FOAKE) Project on The Performance of HVAC Ducts and Supports in Earthquakes and Tests", Advanced Reactor Corporation, prepared by EQE Engineering Consultants, April 1995.

110. "Advanced Light Water Reactor (ALWR) First-of-a-kind-Engineering (FOAKE) Project on Design Concepts for HVAC Ducting and Supports", Advanced Reactor Corporation, prepared by EQE Engineering Consultants, San Francisco, California, April 1995.

111. "Advanced Light Water Reactor (ALWR) First-of-a-kind-Engineering (FOAKE) Project on Design by Rule for Cable Trays and Conduit Systems", Advanced Reactor Corporation, prepared by EQE Engineering Consultants, January 1996.

112. "HVAC Ducts, Comparison of Section Properties by AISI and by SMACNA", EQE Engineering Consultants, San Francisco, California, March 21, 1995. 
113. "HVAC Duct Construction Standard, Metal and Flexible", SMACNA, Vienna, Virginia, 1985.

114. "Rectangular Industrial Duct Construction Standards", SMACNA, Vienna, Virginia, 1988.

115. "Round Industrial Duct Construction Standards", SMACNA, Vienna, Virginia, 1989.

116. "Theory of Plates and Shells", 2nd Edition, Timoshenko and Woinowsky-Krieger, McGraw Hill, San Francisco, California, 1959.

117. "Technical Guidance for the Seismic Review of Masonry Structures at DOE Nuclear Facilities", ERAD/RSM-1 (95), R.J. Morante, Brookhaven National Laboratory, Upton, New York, March 1995.

118. ACI 530-92/ASCE 5-92/TMS 402-92, "Building Code Requirements for Masonry Structures", American Concrete Institute, Detroit, Michigan, 1992.

119. "Comments on Out-of-Plane Capacity of Vertical Spanning In-Filled Hollow Clay Tile Block Walls", Kennedy, R. P. and Merz, K., Structural Mechanics Consulting, Yorba Linda, California, October 1995.

120. "Independent Review of Oak Ridge HCTW Test Program and Development of Seismic Evaluation Criteria", Revision 1, EQE and RPK Consulting, Irvine, California, October 1995 Draft.

121. "Data Processing Facilities: Guidelines for Earthquake Hazard Mitigation", FIMS, Inc., VSP Associates, Inc., June 1987. 
